\title{
Abstracts of the 51st Annual Conference of the Italian Society of Neurology
}

\section{CASE REPORTS \\ LISTERIA MONOCYTOGENES RHOMBENCEPHALITIS: A CHALLENGING DIAGNOSIS}

\author{
S. M. Angelocola, M. Acciarri, R. Angeloni, P. Cardinali, F. Forconesi, I. \\ Paolino, E. Pucci
}

\author{
Neurology Unit, "A. Murri" Hospital (Fermo)
}

Neurolisteriosis may manifest in humans as meningitis, meningoencephalitis, and rhombencephalitis. Compared to other pathogens, Listeria Monocytogenes ( $\mathrm{Lm})$ has a brainstem predilection. Contrary to the more common meningitis, Lm rhombencephalitis occurs rarely and mostly in healthy and immunocompetent people. We describe the case of a 71-year-old immunocompetent woman previously in good health. About a week before hospital admission, the patient experienced nausea, vomiting and diarrhea. Subsequently, dysarthria associated with mild binocular diplopia appeared. At the time of admission, neurological examination showed dysarthria and right sixth and seventh cranial nerve palsy. The meningeal irritation signs were negative and body temperature was $36^{\circ} \mathrm{C}$. She then performed a brain MRI with gadolinium, showing an extensive and clear-cut FLAIR signal alteration, affecting the pontine and mesencephalic tegmentus, without contrast enhancement; there was also a small focal diffusion signal alteration located in the midbrain-pons, on the right. The thiamine dosage, serum electrolytes and inflammatory markers were in range. During the hospitalization, she experienced worsening of vigilance and left hemiparesis. Because of the previous history of gastrointestinal infection, we made the suspiction of a post-infective Bickerstaff rhombencephalitis. The patient underwent a lumbar puncture that showed a mild mononuclear pleiocytosis with a little increase in proteins; virological tests were negative; the cultural exam didn't show bacterial growth. We then made a therapeutic attempt with steroids and a cycle of in vein immunoglobulins, without satisfactory results. There was a progressive further clinical worsening with fever appearence. We then started empirical antibiotic treatment with ceftriaxone and decided to repeat a brain RMN, which showed progression of the FLAIR hyperintense lesion with widespread involvement of the midbrain and basal ganglia. Finally, we repeated a CSF examination with evidence of negative culture test but positive film-array PCR for Listeria. Blood cultures showed the presence of Lm. Specific antibiotic treatment was then started but the clinical picture was very poor and the patient died after a few days. Our attempt is to hilight the importance of considering a possible infectious nature of brainstem lesions even in presence of non enhancing MRI alterations, negative CSF cultures and without systemic and laboratory signs of infection. If Listerial infection is not considered or ruled out with specific tests, late diagnosis and thereby late initiation of target antibiotic treatment will allow the disease to run its devastating natural course. The administration of corticosteroids or i.v. immunoglobulins would accelerate the progression of the disease.
References:

- L. Mandigers et al. A case report of iatrogenic deterioration of yet undiagnosed Rhombencephalitis; always be careful with corticoids. -BMC Clinical Pathology (2018);27;18:15

- Décard BF et al. Listeria rhombencephalitis mimicking a demyelinating event in an immunocompetent young patient. Multiple Sclerosis (2017) Jan;23(1):123-125

- Bartt R. et al. Listeria and atypical presentations of Listeria in the central nervous system. Semin Neurol. (2000);20(3):361-73

\section{PAINLESS WINGED SCAPULA AS THE ONSET OF A VERNET SYNDROME: A CASE REPORT}

S. M. Angelocola, M. Acciarri, R. Angeloni, P. Cardinali, F. Forconesi, I. Paolino, E. Pucci

Neurology Unit, "A. Murri" Hospital (Fermo)

The "Jugular Foramen Syndrome (JFS)" or "Vernet syndrome (VS)" refers to the paralysis of the IX, $\mathrm{X}$ and $\mathrm{XI}$ cranial nerves, crossing the jugular foramen at the skull base. The main etiologies are the following: vascular (jugular vein thrombosis, aneurysm of internal carotid artery), inflammatory (Wegener granulomatosis, Giant Cell arteritis), infectious (VZV, HSV), neoplastic or traumatic. We describe the case of a patient with subacute onset of painless atrophy of the left shoulder, in particular with a winged scapula increased by the abduction of the arm. The patient did not report prior painful symptoms, nor trauma on the shoulder and cervical spine. Moreover, he didn't have infectious-inflammatory episodes prior to the development of hyposthenia. His symptoms were initially attributed to a neuromuscular condition, therefore the patient initially performed the sampling of CPK levels and anti-AChR antibodies, with negative results. VES and PCR levels were in range. An electromyography was then scheduled. Subsequently, dysphagia and nasal voice also appeared, and the patient was then evaluated by an otolaryngologist, who found a paralysis of the left vocal cord and a hypomobility of the soft palate with reduction of the gag reflex on the left. The patient then performed a brain and skull base CT scan with evidence of a "widening" of the jugular foramen. He then underwent an electromyography, with the evidence of a lesion of the left accessory nerve with phenomena of total denervation of the trapezius muscle and partial denervation of the sternocleidomastoid muscle. The remaining muscles belonging to the brachial plexus were normal, as well as motor and sensory nerve conduction studies. A brain and cervical MRI with angio sequences showed an expansive process at the level of the left jugular foramen with a polylobulated appearance and irregular contrast enhancement. This lesion showed a mass effect with erosive phenomena at the skull base. The patient then underwent the mass removal; the definitive histological examination revealed a chondrosarcoma. Vernet Syndrome is a rare condition, attributable to various etiologies, which must always be kept in mind when we are faced with the involvement of multiple cranial nerves 
(especially bulbar). Some pathologies that enter into differential diagnosis are: brachial plexopathy, myasthania gravis, cranial inflammatory multineuropathy and other syndromes involving more than one cranial nerve (e.g. Collet-Sicard, Tapia, Avellis, Shmidt). If the onset is acute or subacute, Parsonnage Turner syndrome must be excluded.

References:

- P. Doneddu et al. Neuralgic amyotrophy mimicking Vernet syndrome. Journal of the Neurological Sciences (2016);362:230-231

- J. M Das et al. Jugular Foramen Syndrome (Vernet). NBCI Bookshelf (2020)

\section{EARLY COMBINED PERCUTANEOUS AORTIC VALVULOPLASTY AND CAROTID ENDARTERECTOMY IN A PATIENT WITH MINOR ISCHEMIC STROKE AND HIGH PERIOPERATIVE RISK}

\author{
A. Antonioni, N. Merli, R. Simonetti, A. Braccia, C. Azzini, A. De Vito \\ Neurology Department, Sant'Anna Hospital, University of Ferrara \\ (Ferrara)
}

Objectives: Here, we present the case of L.B., a 68-year-old Caucasian male patient who reported a sudden onset of right upper limb weakness and transient visual disturbance.

Materials and methods: Therefore, he was referred to ER of S. Anna University Hospital of Ferrara. His medical history included moderate aortic valve stenosis due to previous rheumatic heart disease at early age, dyslipidemia, hypertension and not significant epi-aortic trunks atherosclerosis. Arrived at the Hospital on May 24th 2020, neurological evaluation documented just a slight weakness of right upper limb (NIHSS 1); accordingly, he performed urgent brain CT, showing no acute lesions, and he was admitted to the Stroke Unit. A Carotid US was carried out, which showed an isohypoechogenic ulcerated plaque with hemodynamic stenosis of $70 \%$ at the origin of the left internal carotid artery; patient also underwent echocardiography, which documented an evolution of the previously known aortic valve stenosis in severe degree stenosis (left ventricular outflow tract diameter 2.3 $\mathrm{cm}$, Aortic valve area $0,87 \mathrm{~cm} 2$ ).

Results: Considered neurological and instrumental findings, an indication was given for very early carotid TEA, but this was contraindicated for the high operative risk linked to the cardiac condition. Hence, on May 26th 2020, the patient underwent a coronary angiography + balloon percutaneous aortic valvuloplasty (inflation of a $20 \mathrm{ml}$ balloon), as a bridge procedure in order to perform early carotid TEA intervention during the same day (after a few hours).

Discussion and Conclusion: These procedures were carried out without complications and the patient had full neurological recovery at discharge, one week later. Furthermore, coronary angiography showed a critical stenosis of the middle tract of the LAD with a subcritical stenosis of common trunk. Thus, the patient was listed for a CABG and definitive surgical aortic valve replacement.

References:

- Moraca RJ, Shah AA, Bailey SH et al "Combined carotid endarterectomy and transcatheter aortic valve replacement: Technique and outcomes”, J Card Surg (2018) May;33(5):265-269. doi: 10.1111/ jocs.13601

- Shunsuke S, Teruya N, Mitsuru M et al. Concomitant carotid endarterectomy and aortic valve replacement in a patient with high risk of perioperative stroke. Gen Thorac Cardiovasc Surg (2020) Feb;68(2):170-173.doi: 10.1007/s11748-018-1035-6

\section{A (VERY) EARLY ONSET PARKINSONISM}

S. Aramini ${ }^{1}$, S. Satolli ${ }^{1}$, R, De Micco ${ }^{1}$, A.L. Torella ${ }^{2}$, F. Del Vecchio

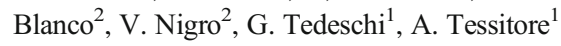

${ }^{1}$ Department of Advanced Medical and Surgical Science, University of Campania "Luigi Vanvitelli" (Napoli); ${ }^{2}$ UOSD Medical Genetics, Department of Precision Medicine, University of Campania "Luigi Vanvitelli" (Napoli)

A 51 years-old woman was admitted to our Neurology department, complaining of the poor control of her Parkinson's disease's (PD) symptoms. Her history started back when she was 11 years old, with an abrupt onset of gait disturbances and a dystonic posture in the left lower limb. She then progressively developed bilateral arm tremor and axial rigidity. She was diagnosed with $\mathrm{PD}$ at the age of 24, and then started dopaminergic medication, which was still ongoing when she was referred to our Clinic. Her personal history also revealed a psychiatric disorder treated with neuroleptics since the patient was about 20 years old. Her father presented cognitive impairment. At our clinical examination, she was presenting with unpredictable motor and non-motor levodopa-induced fluctuations, namely dystonic postures during the OFF-state and lower limbs dyskinesia during the $\mathrm{ON}$-state. The presence of a fixed dystonic posture of left lower limb and facial-faucial-finger myoclonus were also detected. We performed a brain MRI, showing diffuse cerebral atrophy; Dopamine Trasporter (DAT) SPECT revealed absent tracer activity in the putamen of both hemispheres. We also performed an extensive neuropsychological evaluation showing multiple cognitive deficit (MMSE: 21.31) and mood disturbances. Given the early symptoms onset, the presence of motor and non-motor features and the family history, we performed a next generation sequencing genetic testing, which revealed a double heterozygosis within the ATP13A2 gene (PARK9). These mutations have not been described yet and are potentially diagnostic for a Kufor-Rakeb syndrome. This case highlights the importance of considering genetic testing in the presence of family history for neurological disorders as well as early onset and occurrence of both motor and behavioral disturbances. The presence of facial-faucial-finger myoclonus is a clue clinical sign suggesting the diagnosis of Kufor-Rakeb syndrome.

\section{LATE-ONSET PURE CEREBELLAR ATAXIA MASKING MULTIPLE SYSTEM ATROPHY FOR 3 YEARS: A CASE REPORT}

G. Avino, P. Barbero, L. Mazzini, C. Varrasi, D. Vecchio, R. Cantello

Neurology Unit, Department of Translational Medicine, University of Piemonte Orientale (Novara)

Introduction: Multiple system atrophy (MSA) is an idiopathic neurodegenerative disease. Main clinical features are autonomous failure, parkinsonism, cerebellar ataxia and pyramidal symptoms. The subtype MSA-C presents predominant cerebellar features that are often preceded by autonomic dysfunctions. Imaging could support diagnosis: the so called "hot cross bun sign" of pons is one of the most distinctive findings. Our aim is to present a case of pure cerebellar ataxia and severe cerebellar atrophy that developed MSA-C 3 years later.

Materials and Methods: A 68 year-old woman presented with progressive cerebellar ataxia. A brain MRI that showed a severe cerebellar 
atrophy. At a first neurological examination there were no parkinsonian signs and no autonomic dysfunction. Genetic tests (Friedreich Ataxia, SCA1,2,3,6,7,8,12,17), EEG, EMG were negative. She arrived to our attention 3 years later. On physical examination she showed gaze paralysis in the vertical direction and saccadic breakdown of smooth pursuit. Bilateral dysmetria at finger to nose and heel to shin tests. Her gait was ataxic. Romberg test was negative. She also presented dysphagia and dysgraphia. Dizziness and urinary incontinence appeared in the previous last 10 months.

Results: Routine laboratory investigations were normal. We repeated Cerebral MRI confirming the cerebellar atrophy and showing "hot cross bun sign" that it was absent at the first MRI. Autonomic function test showed absence of detrusor contraction, orthostatic hypotension and no physiological nocturnal blood pressure dipping. The patient was diagnosed MSA-C.

Discussion and Conclusion: We presented a case of MSA with cerebellar symptoms for at least 3 years from the onset. The first neuroimaging was suggestive for a cerebellar atrophy that moved to perform genetic tests. The follow-up permitted to show autonomic dysfunction. A range of other disorders may present with MSA-C like features and therefore the differential diagnosis is not always straightforward. Symptoms during disease progression are still the clue to identify a specific neurodegenerative disease. This patient fulfils "probable MSA-C criteria" that is defined by the presence of cerebellar ataxia, dysarthria or oculomotor dysfunction, together with autonomic failure in the form of urinary incontinence or severe orthostatic hypotension. The "hot cross bun sign" clearly supports the diagnosis. In conclusion MSA-C should be considered in any patient with late-onset cerebellar ataxia with no other appreciable causes.

References:

- Gilman S, et al. Second Consensus Statement on the Diagnosis of Multiple System Atrophy. Neurology (2008);71(9):670-76

- Cicilet Soumya, et al. Hot Cross Bun and Bright Middle Cerebellar Peduncle Signs in Cerebellar Type Multiple System Atrophy. BMJ Case Reports (2017) Nov 1;2017:bcr2017220576

\section{FIBROCARTILAGINOUS EMBOLISM: AN UNCOMMON CAUSE OF SPINAL CORD INFARCTION}

P. Barbero, F. De Marchi, G. Avino, G. Strigaro, L. Mazzini, R. Cantello

Neurology, University of Piemonte Orientale (Novara)

Objectives: Fibrocartilaginous embolism (FCE) refers to a rare type of embolism occurring in the spinal cord. FCE originates by the migration of fibrocartilaginous nucleus pulposus material into the nearby vascular system. The material reaches the vasculature via three pathways: revascularization of the inter-vertebral disc by normal aging or degenerative disc disease, formation of Schmorl's nodes, or persistence of inter-vertebral disc neonatal vasculature. FCE can cause embolic infarction to the lung, brain, vertebrae and ribs. Signs and symptoms often develop after a minor or even unnoticed triggering event, such as lifting, straining or falling.

Materials and methods: We present the case of a 25-year-old woman admitted to our Neurological Department due to subacute onset of paraparesis, started the day before from the right leg; the patient reported back pain after heavy lifting. Her past medical history was irrelevant. The first neurological examination showed mild paraparesis, sensory level at T10, brisk lower limbs deep tendon reflexes with pathological spread and right Babinski sign. Muscle strength was normal in the neck and in upper limbs. Cranial nerve examination and higher cortical functions were normal.
Results: Spinal cord magnetic resonance imaging (MRI) showed multiple Schmorl's nodes, disc protrusion at T9-T10, and T2 hyper-intense lesion at T9, without contrast enhancement. Assuming an inflammatory etiology of the lesion, steroid therapy was started and the patient underwent a lumbar puncture. However, CSF analysis revealed no alterations, including normal protein and IgG index and absence of anti-MOG and anti-AQP4. Thus, the patient underwent a second spinal cord MRI, confirming the T2 hyper-intense lesion, with abnormal restricted diffusion, shifting the diagnostic hypothesis to an ischemic etiology. Consequently, antiaggregant treatment was started. The patient underwent several exams to identify cerebrovascular risk factors. Thrombophilic and autoimmune screening was negative, thorax and abdomen CT angiography, echocardiography, cervical arterial insonation and transcranial color doppler were unremarkable. Based on the clinical picture, the temporal relation with the trigger, the CSF findings and the presence of degenerative disc disease with Schmorl's node nearby the lesion, a diagnosis of suspected FCE was made.

Discussion and conclusions: FCE is considered a rare and underdiagnosed cause of spinal cord infarction. Diagnosis of FCE in based on clinical grounds with suggestive CSF analysis and spine MRI findings, and confirmed only with biopsy for histo-pathologic analysis. It is important considering the FCE as possible cause of spinal cord infarction for appropriate therapeutic management and prognosis.

References:

- Naiman JL, Donohue WL, Prichard JS. Fatal nucleus pulposus embolism of spinal cord after trauma. Neurology (1961);11:83-7

- AbdelRazek MA, Mowla A, Farooq S, Silvestri N, Sawyer R, Wolfe G. Fibrocartilaginous embolism: a comprehensive review of an under-studied cause of spinal cord infarction and proposed diagnostic criteria. J Spinal Cord Med. (2016); 39(2):146-54

- Mateen FJ Monrad PA, Hunderfund AN, Robertson CE, and Sorenson EJ. Clinically suspected fibrocartilaginous embolism: clinical characteristics, treatments, and outcomes. Eur J Neurol (2011);18(2):218-25

\section{IS PEDIATRIC RADIOLOGICALLY ISOLATED SYNDROME (RIS) A TREATABLE PATHOLOGICAL CONDITION? A CASE REPORT}

\author{
E. Barbuti, R. Nistri, A. Ianniello, S. Ruggieri, C. Pozzilli \\ MS Center, S'Andrea Hospital, Sapienza University (Roma)
}

Background/Aim: Radiologically isolated syndrome (RIS) describes asymptomatic individuals with incidental radiologic abnormalities suggestive of MS. Treatment of RIS is controversial, especially in pediatric age, but early treatment in selected patients might improve long-term outcome. We report a single RIS patient followed-up for 17 years in our MS Center.

Materials/Methods/Results: A 15-year-old female patient was referred to our Center in July 2002 because of atypical findings on brain MRI (altered signal intensity of semioval centre, trunk and genu of corpus callosum and retro-trigonal area bilaterally). The exam was requested because she presented with a several days-history of high fever, slight drowsiness and sleepiness. Neurological exam was normal and the decision was made to monitor the evolution of the disease with follow-up MRIs. In January 2003, brain MRI showed a new cortical parietal area of 
altered signal, without contrast enhancement. The cerebrospinal fluid analysis (March 2003) showed the presence of oligoclonal bands, with an IgG index of 3,0. Evoked potentials (March 2003) were all normal. The following MRIs in September 2003, September 2004 and May 2005 showed new small lesions (without enhancement), so intramuscular INFbeta1a was prescribed on compassionate use in May 2005. Because the drug was poorly tolerated, and MRIs were stable, the medication was stopped in February 2007. Due to MRI activity she restarted the treatment from November 2007 up to January 2010. Along this period annual MRIs remained stable. Then, she decided to interrupt the treatment but new lesions appeared in June 2010 and December 2010 brain MRIs. Treatment was started again in December 2010 with INF-beta1a and switched to the pegylated form in September 2015. All brain MRIs performed until October 2019 were stable.

Discussion: Our patient showed several features suggesting a high risk of conversion to MS (oligoclonal bands, pediatric onset, high cerebral lesion load and rapid accumulation of lesions in less than 3 years). The appearance of new brain MRI lesions was clearly related to the period of treatment interruption.

Conclusion: We do believe early treatment can be considered an appropriate choice in Pediatric RIS cases who satisfy dissemination in time and space according to the McDonald revised criteria (2017), especially considering that Pediatric MS has been shown to lead to significant disability over time.

\section{POST-MEASLES ENCEPHALOPATHY IN A YOUNG WOMAN WITH RECENT IMMUNOSUPPRESSIVE THERAPY: A CHALlENGING CASE}

F. Barone, G. Fisco, E. Cerulli Irelli, A. Morano, B. Orlando, M. Fanella, C. Di Bonaventura, A. Giallonardo

Department of Human Neurosciences, University "La Sapienza" (Roma)

Introduction: Neurological complications of measles infection represent insidious and often serious conditions, especially in vulnerable category, and can occur during the early phase but even after many years from the acute disease. The two late neurologic complications are the measles inclusion body encephalitis (MIBE) and the subacute sclerosing panencephalitis (SSPE). The first one strikes immunocompromised hosts after few months from acute infection, showing often a rapidly aggressive course. SSPE instead begins from 7 to 15 years after infection, presenting with insidious behavioural and cognitive dysfunction and a typical electroencephalographic periodic pattern synchronous to a massive myoclonus.

Materials and Method: We present the case of a 30 years old Italian woman who underwent a chemotherapy (R-CHOP) for B-cell Lymphoma in 2016. After few months from the clinical remission she contracted measles infection in an atypical form, perhaps due to her immunodeficiency. She wasn't vaccinated. Four months later she developed right facio-brachial motor seizure with progression to status epilepticus and coma. The neurological status at the resolution of SE consisted of right hemiplegia and hypoesthesia, and persistence of myoclonic movements at right leg. Repeated brain MRI showed several lesions in cortical and subcortical grey matter, with a relapsing-remitting pattern. Several EEG showed the presence of periodic epileptiform discharges in left hemisphere. A sub-acute measles encephalitis was suspected. CSF analysis and blood exam showed only the positivity of serum anti-measles IgM, three months after the acute phase.
Results: After one year from the acute phase the patient came to our attention for the onset of involuntary movements at the opposite hand (left). An EEG with back averaging showed the cortical source of myoclonus and a new MRI highlights a diffuse cortical atrophy without new lesions. CSF analysis was normal and blood exam showed the persistence of qualitative IgM measles antibodies.

Discussion: This case presents clinical findings of both MIBE and PESS, without a determinant discriminatory element. The atypical slight progression of the condition, with bilateral cortical involvement and diffuse atrophy, and the particular EEG pattern, can be seen as a progressive brain inflammatory condition, and other diagnosis have to be evaluated (Rasmussen encephalitis, Mitochondrial diseases or other Autoimmune Encephalopathies).

Conclusions: Late measles related encephalitis are rare and insidious conditions, especially in adults. Although this case shows several clinical characteristic suggestive for MIBE, its relatively slight progression and the absence of secure determinants such as brain biopsy leaves this case still unsolved.

References:

- C. Singer, A. E. Lang, Adult-Onset Subacute Sclerosing Panencephalitis: Case Reports and Review of the Literature. Mov Disor, (1997);12(3):342-53

- R. Buchanan, DJ. Bontious. Measles virus and associated central nervous system sequelae. Semin Pediatr. Neurol. (2012) Sep;19(3):107-14

- R.K. Garg. Subacute sclerosing panencephalitis. J Neurol (2008);255:1861-71

\section{OCRELIZUMAB FOR AGGRESSIVE MULTIPLE SCLEROSIS ONSET. A CASE REPORT}

\section{Bazzurri, E. Tsantes, E. Curti, A. Fiore, F. Granella}

Neurosciences Unit, Department of Medicine and Surgery, University of Parma (Parma)

Background: Multiple sclerosis (MS) has an extremely heterogeneous disease course. Relapsing course leading to rapid accrual of physical and cognitive disability, often despite treatment, is referred to as 'aggressive' disease. However, no consensus exists either in the definition of this type of MS, or in the best treatment strategy. Alemtuzumab, cladribine, cyclophosphamide and mitoxantrone are the most used agents. Rituximab has been tested in some cases, but its off-label use and the lack of phase 3 randomized clinical trials in MS may limit its employment. No clear reports of ocrelizumab in tumefactive, breakthrough disease are available in current literature.

Case report: A 34-year-old Caucasian woman, with no significant past medical history, was admitted to hospital with dizziness, nausea, vomiting, and mild gait ataxia. Brain MRI revealed multiple demyelinating lesions, many of which were infratentorial, showing tumefactive aspect and gadolinium enhancement. Cerebrospinal fluid analysis revealed presence of oligoclonal bands, while many rheumatologic, infectious and autoimmune diseases were excluded by biochemical tests, including antiAQP4 and anti-MOG antibodies. Despite treatment with intravenous methylprednisolone (IVMP) $1000 \mathrm{mg}$ for 5 days, her clinical conditions worsened. Extraocular movement impairment, vertical nystagmus, left facial palsy, right hemifacial numbness occurred and she was essentially bedridden due to intractable vomiting and severe ataxia (EDSS 8.0). After seven plasma exchanges, left facial and right limbs paresthesias appeared, while MRI revealed 8 new/enlarged 
lesions, the majority of which showing ring contrast-enhancement. She was treated with another IVMP cycle for seven days and finally underwent ocrelizumab treatment $(2 \times 300 \mathrm{mg}$ iv within two weeks). After this therapy, she started to recover and at discharge her neurological exam revealed facial paresthesias, right hypoacusia, horizontal nystagmus, slight vibration sense decrease in the four limbs, mild gait and left limbs ataxia (EDSS 3.0). During a 12-month follow-up she pursued ocrelizumab treatment; her clinical conditions continued to improve (EDSS 1.5) and two consecutive MRIs showed no new lesions and reduction in size of the pre-existing ones.

Conclusions: Our patient, with an aggressive MS onset, showed a very good response to ocrelizumab treatment. Ocrelizumab therapy should be considered in cases of rapidly evolving disease onset.

\section{W ALL-EYED B ILATERAL INTERNUCLEAR OPHTHALMOPLEGIA (WEBINO) AND AREA POSTREMA SYNDROME AS UNUSUAL PRESENTATION OF MULTIPLE SCLEROSIS: A CASE REPORT}

\author{
F. Bernocchi ${ }^{1}$, M. Di Donna ${ }^{1}$, M. Conti ${ }^{1}$, G. Cola ${ }^{1}$, G. Marfia ${ }^{2}$, A. \\ Martorana ${ }^{1}$, N. Mercuri ${ }^{1}$, A. Stefani ${ }^{1}$, F. Izzi $^{1}$
}

${ }^{1}$ Neurology Unit, Department of System Medicine, "Tor Vergata" University (Roma); ${ }^{2}$ Multiple Sclerosis Clinical and Research Unit, Department of System Medicine, "Tor Vergata" University (Roma)

Aim of the study: Our aim was to investigate a rare condition defined Wall-Eyed Bilateral Internuclear Ophthalmoplegia (WEBINO) and Area Postrema Syndrome (APS) in a 17-year-old patient.

Materials: We evaluated a 17-year-old boy, who was admitted to the Neurology Department of "Policlinico Tor Vergata", Rome, with a chief complaint of diplopia in primary position of gaze and lateralized gaze in both horizontal directions. He also presented nausea and vomiting with subsequent onset of vertigo and unsteadiness. At the admission, the neurological examination revealed left exotropia, impaired adduction in the lateral gaze, bilaterally, with dissociated nystagmus in the abducting eye, ad an impairment of convergence. We performed brain CT scan, brain MRI and spinal MRI, a lumbar puncture with the oligoclonal band array, evaluation of sensitive and visual evoked potentials (SEP and VEP) and the anti-AQP4 and anti-MOG assay. We also ruled out other autoimmune conditions and vitamin deficiency by blood samples.

Results: Brain CT scan was negative. On the contrary, brain MRI showed a small dorso-medial pontine hyperintensity with a mild contrast enhancement. Moreover, a lesion involving right posterior periventricular white matter and a lesion of left peritrigonal area, both characterized by a post contrastographic enhancement were found. Cervical MRI showed an additional oval hyperintensity in T2 sequences involving posterior columns. Lumbar puncture revealed a normal cell count and link index, but the oligoclonal band array evidenced the presence of at least 6 bands in $\mathrm{CSF}$ and no serum bands. Anti-AQP4 and anti-MOG assay was negative and the same for SEP and VEP. Other comorbidities were ruled out by laboratory testing.

Discussion: WEBINO is a rare syndrome, caused by lesions involving bilateral medial longitudinal fasciculus (MLF) and medial rectus subnucleus [1]. This rare condition is mainly associated to demyelinating diseases, first of all multiple sclerosis, and ischemic lesions [2]. We evaluated a patient with no other comorbidities and, based on the tests carried out during the hospitalization, we made a diagnosis of multiple sclerosis. The particular localization of the midbrain lesion involved bilateral MLF and caused nausea and vomiting, due to APS. We started a treatment with intravenous bolus of $1 \mathrm{~g}$ methylprednisolone for 5 days, with an almost complete regression of the symptoms. He was further evaluated after one month and started a chronic therapy with Interferon beta1A.

Conclusions: WEBINO and APS are uncommon disorders that may be the sole presentation of MS.

References:

1. T. Yoshinaga, K. Nakamura, K. Kaneko, A. Nakamura, A case report of WEBINO syndrome with convergence impair-ment. Journal of Neurology and Neurophysiology (2005);6:270

2. Wu YT, Cafiero-Chin M, Marques C. Wall-eyed bilateral internuclear ophthalmoplegia: review of pathogenesis, diagnosis, prognosis and management. Clin Exp Optom. (2015);98(1):25-30. doi: $10.1111 /$ cxo. 12200

\section{AN HETEROZIGOUS FUS MUTATION WITH A MONOMELIC PHENOTYPE AND MUSCLE INFLAMMATION}

\author{
F. Bianchi ${ }^{1}$, A. Fogli ${ }^{2}$, G. Ricci ${ }^{1}$, L. Fontanelli ${ }^{1}$, A. Galgani ${ }^{1}$, E. Bollani ${ }^{3}$, \\ G. Siciliano ${ }^{1}$
}

${ }^{1}$ Department of Clinical and Experimental Medicine, Neurological Clinic, University of Pisa (Pisa); ${ }^{2}$ Molecular Genetics Unit, Santa Chiara Hospital (Pisa); ${ }^{3}$ Neurology Unit, Portoferraio Hospital (Portoferraio-LI)

Background: Amyotrophic lateral sclerosis (ALS) is a fatal neurodegenerative disorder that affects lower and upper motor neurons. The study of ALS familial variants had pointed out that defects of RNA processing and protein clearance may contribute to disease development. Alterations of the Fused in Sarcoma FUS gene functionality have been put in relation with an array of neurodegenerative disorders, among which juvenile forms of familial ALS with an aggressive progression and an involvement of the lower motor neuron (LMN) as the prominent type at onset. Here, we describe the case of a 41-years-old man with a patent family track record for ALS that came to our attention for a history of progressive hyoposthenia and muscle hypotrophy of his right leg with signs of LMN impairment, with a heterozygous pathogenic mutation in the FUS gene. Remarkably, after one year of disease progression the patient still shows a monomelic involvement at clinical examination, while a muscle MRI at lower limbs showed a marked and quite spread inflammatory pattern involving muscles of the affected leg.

Aim: To describe a case of monomelic lower motor neuron disease in association with a FUS mutation with slow clinical progression after one year and with an inflammatory pattern on the affected leg

Materials and Methods: Exams included a high camp MRI, a muscular MRI of the lower limbs, an EMG-ENG, CSF searching for antibodies and for protein neurodegeneration and DNA tests for 5qSMA, non-5q SMA, and for familial ALS.

Results: A muscular MRI showed a pronounced edema mainly localized on the quadriceps and the tibialis anterior muscle of the right leg, while a diffuse chronic neurogenic pattern with acute denervation on the tibilias anterior was found at the electomyography. A heterozygous mutation in FUS gene, variants $\mathrm{R}>521 \mathrm{C}$ of exon 15 has been detected in the DNA analyses. A muscular biopsy has been scheduled to study the inflammatory pattern and to search any FUS aggregates in muscle.

Discussion and Conclusion: In the case here reported, the disease had only affected the right leg after one-year progression. This is peculiar in relation to the commonest form of FUS-related ALS, that typically causes an aggressive progression with precocious disability. Moreover, a strong inflammatory pattern has been revealed at the muscular MRI. FUS is 
known to be involved in the neuro-muscular junction and in muscular cells development. In the case here presented, the search of FUS aggregates in a muscular biopsy may shed light on the complex pathogenic mechanisms of FUS-related neurodegeneration.

References

- Panza F. et al. Development of disease-modifying drugs for frontotemporal dementia spectrum disorders. Nature Reviews Neurology (2020);1-16

- Picchiarelli G., Luc Dupuis. Role of RNA Binding Proteins with prion-like domains in muscle and neuromuscular diseases. Cell stress 4.4 (2020);4.4:76

- Naumann M. et al. Phenotypes and malignancy risk of different FUS mutations in genetic amyotrophic lateral sclerosis. Annals of Clinical and Translational Neurology (2019);6(12): 2384-94

\section{ATYPICAL INTRAPARENCHYMAL HEMORRHAGE DUE TO CEREBRAL MYCOTIC ANEURYSM RUPTURE: AN UNUSUAL PRESENTATION OF INFECTIVE ENDOCARDITIS}

\author{
E. Biassoni ${ }^{1,2}$, A. Murialdo ${ }^{1}$, D. Rizzi ${ }^{1}$, D. $\operatorname{Sassos}^{1}$, G. Ribizzi ${ }^{1}, \mathrm{~S}$. \\ Pretta $^{1}$, E. Pedemonte ${ }^{1}$, D. Farinini ${ }^{1}$, A. Schenone ${ }^{1,2}$
}

\begin{abstract}
${ }^{1}$ Division of Neurology, IRCCS Polyclinic San Martino Hospital (Genova); ${ }^{2}$ Department of Neuroscience, Rehabilitation, Ophthalmology, Genetics, Maternal and Child Health (DiNOGMI), University of Genoa, and Neurology Clinic Unit, IRCCS Polyclinic San Martino Hospital (Genova)
\end{abstract}

Background: Infective endocarditis (IE) is an endocardial heart layer infectious disease. The most frequent complications are neurological, including cerebral mycotic aneurysm (CMA) rupture, which can manifest with subarachnoid, intracerebral or intraventricular hemorrhage. Its formation seems due to septic embolization, which causes inflammation, weakness and enlargement of blood vessels.

Case report: We report a case of a 25 -year-old male, without significant medical history or neurological deficits, who arrived to emergency department for long lasted intermittent headache. Brain CT showed an atypical intraparenchymal hemorrhage in left parietal lobe. Angiography revealed a suspected mycotic pseudoaneurysm on left internal carotid artery distal parietal branch (M4-M5). Trans-thoracic echocardiogram showed a bicuspid aortic valve endocarditis determining severe regurgitation. Left ventricle dilatation without hypertrophy or systolic function impairment was also reported. Total body CT highlighted splenic hypodense area. Second brain CT showed a new abscess appearance finding in left parietal site. Orthopantomography revealed several suspected dental abscesses, subsequently extracted. Serial blood cultures were negative. The patient successfully underwent to neurosurgical clipping on left parietal M5 aneurysm. On control angiography not previously reported small suspected pseudoaneurysms on some branches of left superficial temporal artery were highlighted. Patient was subjected to aortic valve mechanical replacement. Cultural explanted valve examination resulted negative, but Bartonella quintana ribosomal RNA 16S was positive. Bartonella quintana serology was positive for both IgG and IgM. Targeted antibiotic and Warfarin anticoagulant therapies were set up.

Discussion: Since the patient's young age, we attributed his hemorrhage to a complication of underlying arteriovenous malformation or aneurysm. Possible IE signs of suspicion were: young age, absence of vascular risk factors, unexplained hemorrhagic lesion. Furthermore brain and spleen are the most frequent locations affected by septic embolization in left-sided IE and our patient presented both. Between genus Bartonella bacteria, Bartonella quintana is the main etiological agent of blood culture-negative IE and can cause prolonged and asymptomatic bacteremias. Frequently aortic valve is the most affected and clinical manifestations are subacute, which may explain why our patient had an already advanced clinical setting.

Conclusions: Although CMA rupture may be an uncommon IE presentation, it must be considered especially in young patients without vascular risk factors. Patients with suspected CMA finding should be screened with echocardiogram to exclude possible valvular vegetations and consequent septic embolizations. Epidemiological informations and clinical features should guide the etiological agent identification.

References:

- Sotero FD, Rosário M, Fonseca AC, Ferro JM. Neurological Complications of Infective Endocarditis. Current Neurology and Neuroscience Reports. (2019);19(5):23:1-8

- Habib G, Lancellotti P, Antunes MJ, Bongiorni MG, Casalta JP, Del Zotti F, et al. 2015 ESC Guidelines for the management of infective endocarditis. European Heart Journal. (2015);36: 3075-23

- Okaro U, Addisu A, Casanas B, Anderson B. Bartonella species, an emerging cause of blood-culture-negative endocarditis. Clinical Microbiology Reviews (2017);30:709-46

\section{HSV-2 ENCEPHALITIS OF THE ADULT: A CASE REPORT}

\author{
M. Bolchini ${ }^{1}$, B. Borroni ${ }^{1}$, C. Agosti $^{2}$, A. Padovani ${ }^{1}$
}

${ }^{1}$ Neurology Unit, Department of Clinical and Experimental Sciences, University of Brescia (Brescia); ${ }^{2}$ Neurology Unit, Department of Neurological and Vision Sciences, ASST Spedali Civili (Brescia)

Background: Herpes simplex virus 2 (HSV-2) is a rare causative agent of encephalitis in the adult, unlike HSV-1, and its often mild clinical presentation with subtle symptoms may lead to its diagnosis being missed. The aim of this case report is, therefore, to highlight the characteristics of this pathological condition to avoid an incorrect or missed diagnosis.

Case presentation: A 71-year-old male was admitted to our department for a seizure, which manifested as an episode of unresponsiveness and absence associated with clonic head movements. During the hospitalization the patient never showed fever, abnormal levels of inflammatory markers (WBC and CRP) or any neurological sign or symptom. The patient was studied with a head CT, which showed a very slight corticosubcortical hypodensity in the left temporo-polar and temporo-mesial area; he was further investigated with brain MRI, showing hyperintense signal in T2 and FLAIR sequences, hypointense in T1 sequences, in the same temporal area of the brain, suggestive for a low-grade glioma or, less likely, an encephalitic process. A lumbar puncture was performed; CSF was clear, proteins, glucose and cells were within normal range; CSF search for atypical or neoplastic cells was negative; search for autoantibodies suggestive for autoimmune encephalitis was negative as well, while CSF search for viral nucleic acids by PCR was positive for Herpes simplex virus 2 (HSV-2), therefore a diagnosis of HSV-2 encephalitis was made. Immediate treatment with acyclovir $(10 \mathrm{mg} / \mathrm{Kg}$ x3 die) was initiated, lasting for 21 days, according to current herpetic encephalitis treatment guidelines, and the patient was then discharged from hospital.

Discussion: HSV-2-related neurological infections are usually encephalitis in the newborn, as the virus is acquired through the birth canal, and benign aseptic recurrent meningitis (also called 
Mollaret's meningitis) in the adult. HSV-2 encephalitis of the adult is, therefore, an infrequent and insidious disease; as shown by this case report, it can present with mild or only slightly altered signs or symptoms, not suggestive for an infectious process, therefore diagnosis could be missed or delayed, with possible clinical worsening of patient conditions. MRI alterations in temporomesial area are quite common but these findings can also be present in other pathological conditions and could also be missing in some cases; CSF analysis is therefore mandatory, as PCR for search of HSV-2 DNA is the gold standard for diagnosis.

\section{GIANT CELL ARTERITIS AND ACUTE ISCHAEMIC STROKE AFTER COVID-19: REPORT OF A CASE}

\author{
I. Bonanni, I. Gandoglia, L. Strada, P. Mattioli, A. Assini, M. Del Sette
}

Neurology Unit, E.O. Ospedali Galliera (Genova)

Background: Horton's Arteritis or Giant Cell Arteritis (GCA), is a chronic vasculitis of large and medium sized vessels, involving mainly the extracranial branches of carotid arteries, in particular the temporal artery. Diagnostic criteria, established by the American College of Rheumatology, are: 1) Age 50 years or older 2)New-onset localized headache or localized head pain 3)Temporal artery tenderness to palpation or decreased pulsation 4) ESR of $50 \mathrm{~mm} / \mathrm{h}$ or higher 5)Positive arterial biopsy results. The presence of 3 or more criteria yields a diagnostic sensitivity of $93 \%$ and specificity of $91 \%$. Covid- 19 is an infectious disease caused by the newly discovered SARS-CoV2.

Case Report: A 55 years old latin american diabetic woman in late May 2020 developed Sars-Cov-2 infection, with fever and cough, without neurological complication, and with complete recovery in a month. Two months later she reported the occurrence of dull lateralized headache on the left side of the head. After few days she acutely presented dizziness and visual disturbances involving the right visual field. She did not seek medical attention because she had scheduled to fly form her country (Equador) to Italy, were she lives. After the arrival in Italy, she presented to the emergency room of our hospital complaining headache and visual loss of the right side of the visual field. CT scan showed an ischaemic lesion in the left occipital lobe, involving the vascular territory of left posterior cerebral artery. CT angiography demonstrated wall irregulaties of both posterior cerebral arteries, without vessels occlusions. She underwent a brain MRI, that confirmed the ischaemic nature of the occipital lesion and showed an additional hyperintense lesion in the left hippocampal cortex. Erythrocite sedimentation rate was over the threshold $(55 \mathrm{~mm} / \mathrm{h}$, normal value $0-30 \mathrm{~mm} / \mathrm{h})$. Color-Duplex examination temporal arteries showed increased diameter of right superficial temporal artery and hypoechoic wall thickening ("halo sign"). She fulfilled three criteria for the diagnosis of Giant Cell Arteritis and was treated with prednisone $(1 \mathrm{mg} / \mathrm{kg})$ with complete resolution of the headache in just one day. A follow up Duplex performed after seven days showed reduction of the halo sign. Follow-up is ongoing.

Conclusions: To our knowledge this is the first description of the occurrence of Giant Cell Arteritis shortly after the recovery from Covid-19. Although we cannot establish the coincidental or causal nature of the relationship between COVID-19 and GCA, this case supports the hypotesis that COVID19 could act as a trigger for autoimmune disease development.

References:

- Hunder GG, Bloch DA, Michel BA, Stevens MB, Arend WP, Calabrese LH, Edworthy SM, Fauci AS, Leavitt RY, Lie JT. The
American College of Rheumatology 1990 criteria for the classification of giant cell arteritis. Arthritis Rheum (1990)Aug;33(8):1122-8

- HR Niazkar, B Zibaee, A Nasimi, N Bahri The neurological manifestations of COVID-19: a review article. Neurol Sci (2020) Jun 1; $1-5$

- M Liu, Y Gao, Y Zhang, S Shi, Y Chen, J Tiana, The association between severe or dead COVID-19 and autoimmune diseases: A systematic review and meta-analysis. J Infect (2020) Jun. 2; 81(3):e93-e95

\section{A VERY RARE CAUSE OF ATAXIA}

F.P. Bonifacio ${ }^{1}$, M. Silvestro ${ }^{1}$, R. De Micco ${ }^{1}$, A. Tessa $^{2}$, F. M. Santorelli ${ }^{2}$, G. Tedeschi ${ }^{1}$, A. Tessitore ${ }^{1}$

${ }^{1}$ Department of Advanced Medical and Surgical Science, University of Campania "Luigi Vanvitelli" (Napoli); ${ }^{2}$ UOC of Molecular Medicine, Neurogenetics and Neuromuscular Diseases, IRCCS Stella Maris Foundation (Calambrone - PI)

We report the case of a 60 -year-old woman referred to our clinic for the slowly progressive onset, about 11 years ago, of right upper arm tremor, followed by global slowness, impaired gait, postural instability, urinary urge-incontinence and cognitive decline. She had no response to pramipexole (up to $0.52 \mathrm{mg}$ ) and inconstant benefit with levodopa/ carbidopa (total daily dose of 150). Her father suffered from a not better accounted gait disorder in late age; a sister suffered from not better accounted behavioral disturbance in late age. Her personal history revealed sensorineural hypoacusia since the age of 50 years, visual disturbances since the age of 28 years with a recent diagnosis of degenerative maculopathy, and chronic obstructive pulmonary disease. Neurological examination showed hypomimia, antero-flexion of the trunk, broadbased ataxic gate, postural instability, vertical gaze limitation, squarewave jerks, severe slow-frequency postural, action and rest tremor of the upper arms, dysmetria of the upper arms, bradykinesia of upper and lower arms, ecolalia. Blood examination were unremarkable. Brain MRI showed cerebral and cerebellar atrophy and basal ganglia mineralization. Nigro-striatal tracer uptake was normal. Genetic examination revealed c.1709C $>$ T (p.A570V) heterozygous mutation of DNMT1 gene. This mutation has been descripted as causative of Autosomal Dominant Cerebellar Ataxia, Deafness, and Narcolepsy (ADCADN). ADCADN is a very rare hereditary disease whose prevalence is unknown. Most cases have been described in Italian population. This case highlights the importance of considering genetic testing in the presence of familiar history. Even in the absence of narcolepsy the rare diagnosis of ADCADN should be considered when ataxia is associated to deafness.

\section{MOTOR FLUCTUATIONS AND LEVODOPA-INDUCED DYSKINESIAS IN A YOUNG ADULT CASE OF BPAN}

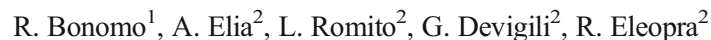

${ }^{1}$ Experimental Neurology Unit, School of Medicine and Surgery, University of Milano-Bicocca (Milano); ${ }^{2}$ Neurological Unit 1, Fondazione I.R.C.C.S. Istituto Neurologico Carlo Besta (Milano)

Background: Beta-propeller protein-associated neurodegeneration (BPAN) is a neurological disorder characterized by progressive iron accumulation in the substantia nigra and in the globus pallidus, resulting in a 
wide array of symptoms including cognitive decline, parkinsonism and dystonia.

Case: This 34-year-old right-handed lady was referred for a 2-year history of progressive slowing of movement associated with episodes of "spasms" affecting the left leg. Over the months, she gradually developed clumsiness of the left limbs, cognitive decline and social isolation. Concerning her past medical history, she suffered from early-childhood generalized seizures, which resolved with age. Developmental milestones were delayed and expressive language was limited. There was no family history of neurological disorders. On examination, she presented with generalized dystonia affecting the left limbs more than the right ones. Rapid alternating movements of the limbs were suggestive of bradykinesia. Gait was broad-based and severely affected by the dystonic posturing of the legs. Brain MRI disclosed bilateral hypointensity in the globus pallidus and in the substantia nigra on $\mathrm{T} 2 *$ sequences. SWI sequences confirmed iron accumulation. DaTSCAN brain images showed a bilateral reduced uptake in the striatum. Genetic screening revealed a mutation in the WDR45 gene consistent with a diagnosis of BPAN. She then started levodopa treatment for her parkinsonism with a beneficial effect on motor function. Nevertheless, after a few months, she developed disabling motor fluctuations (wearing-off phenomenon) and dyskinesias.

Discussion: Recent reports on BPAN patients have demonstrated widespread tau deposition, which might be suggestive of a tauopathy. The accumulation of tau aggregates typically represents a neuropathological feature of patients with atypical parkinsonism usually not responsive to levodopa. Conversely, this patient presented with parkinsonism with a significant motor response to levodopa and developed levodopa-induced motor fluctuation as observed in Parkinson's disease.

Conclusion: The observation of severe levodopa-induced motor complications is uncommon in taupathies and might suggest a new potential mechanism underlying BPAN.

\section{PARKINSONISM AND NIGROSTRIATAL DAMAGE SECONDARY TO CSF1R-RELATED ADULT ONSET LEUKOENCEPHALOPATHY WITH AXONAL SPHEROIDS AND PIGMENTED GLIA}

\author{
S. Bonvegna ${ }^{1}$, G. Straccia ${ }^{2}$, N. Golfrè Andreasi $^{1}$, G. Marucci $^{3}$, D. Di \\ Bella $^{4}$, A. Elia ${ }^{1}$, R. Cilia ${ }^{1}$, R. Eleopra ${ }^{1}$
}

${ }^{1}$ Department of Clinical Neuroscience, Foundation Neurological Institute Carlo Besta (Milano); ${ }^{2}$ Department of Medical Sciences and Advanced Surgery, University of Campania "Luigi Vanvitelli" (Napoli); ${ }^{3}$ Neuropathology Unit, Foundation Neurological Institute Carlo Besta (Milano); ${ }^{4}$ Unit of Medical Genetics and Neurogenetics, Foundation Neurological Institute Carlo Besta (Milano)

A 51-year-old woman presented with a one-year history of apathy, depression and behavioural disorder (hypersexuality) followed by progressive symmetrical parkinsonism. Parkinsonian features worsened with early development of axial signs, including mild dysphagia, and cognitive decline. When she was assessed by a neurologist experienced in movement disorders at the outpatient department, neurological examination revealed multi-domain dementia with frontal involvement (e.g., attention deficit, perseveration, ecopraxia, frontal-lobe release signs), vertical and horizontal gaze palsy and dysarthria with hypophonic and tachiphemic speech, stimulus-induced myoclonus, ideomotor apraxia of the right hand, retrocollis and left lateral flexion of the trunk, standing was almost impossible without help for severe spontaneous retropulsion and compromised postural reflexes, aided walking was instable, shuffling and impaired by freezing of gait. CT scan showed calcifications at corticosubcortical junction of frontal and parietal lobes. A severe bilateral reduction of striatal DAT density was present. Diagnostic hypothesis included a rapidly-progressive tauopathy, combining aspects of progressive supranuclear palsy, corticobasal syndrome, or frontotemporal dementia. However, some additional associated neurological signs, such as gazeevoked nystagmus, cerebellar dysmetria and diffuse hyperreflexia, prompted towards a different diagnosis. She was admitted to perform an extensive diagnostic protocol was performed. 3-Tesla brain MRI displayed a severe disruption of cerebral white matter with bilateral periventricular and subcortical fronto-temporo-parietal hyperintensity associated with atrophy of corpus callosum, consistent with an adult-onset leukodistrophy. A brain biopsy and genetic testing were performed: histopathologic examination of the specimen revealed the presence of neuro-axonal spheroids and multiple macrophages, confirming the suspicion of Adult-onset Leukoencephalopathy with axonal Spheroids and Pigmented glia (ALSP) and genetic testing completed the diagnosis by revealing a pathogenic mutation in the colony stimulating factor 1 receptor gene [c.2345G $>\mathrm{T}$ (p.R782L), novel mutation].[1,2] This case is of particular interest for its diagnostic challenge, due to its rarity and clinical complexity, initially misdiagnosed as 'mixed' tauopathy, underestimating additional subtle neurological signs. Coexistence of neurological signs highly suggestive of a degenerative parkinsonism (PSP/CBS-like) with atypical neurological findings (cerebellar and pyramidal), highlights the pivotal importance of an accurate neurological examination to define the most appropriate diagnostic assessment. Another interesting feature is the abnormal DAT uptake, which misled clinicians towards a primary parkinsonism. Here, the abnormal DAT imaging was caused by a microglia-related damage of the nigrostriatal pathway rather than a primary neurodegenerative process involving the SNc. The take-home message is that neurologists should always perform an exhaustive neurological examination in patients presenting with parkinsonism and critically assess instrumental findings.

References:

1. Konno T, Kasanuki K, Ikeuchi T, Dickson DW, Wszolek ZK. CSF1R-related leukoencephalopathy: A major player in primary microgliopathies. Neurology (2018);91:1092-1104

2. Sundal C, Fujioka S, Van Gerpen JA, et al. Parkinsonian features in hereditary diffuse leukoencephalopathy with spheroids (HDLS) and CSF1R mutations. Parkinsonism Relat Disord (2013);19:86977

\section{AN UNUSUAL CASE OF ISCHEMIC HAEMODYNAMIC STROKE DURING ACUTE RUPTURE OF SUBRENAL AORTIC ANEURYSM}

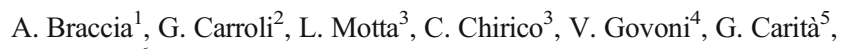
A. De Vito ${ }^{6}$

${ }^{1}$ Neurology Clinic, Department of Biomedical and Surgical Sciences, University of Ferrara (Ferrara); ${ }^{2}$ Neurology Department, University of Ferrara (Ferrara); ${ }^{3}$ Radiology Department, University of Ferrara (Ferrara); ${ }^{4}$ Neurological Clinic, St. Anna University-Hospital (Ferrara); ${ }^{5}$ Radiology Department, St. Anna University-Hospital (Ferrara); ${ }^{6}$ Stroke Unit, St. Anna UniversityHospital (Ferrara)

Objectives: We report the case of an ischemic haemodynamic stroke due to acute occlusion of the cervical right internal carotid artery (ICA) during 
an episode of acute hypotension caused by rupture of subrenal abdominal aortic aneurysm and secondary haemorrhagic shock.

Materials and methods: A 64-year-old man, with history of hypertension and smoking habit, was admitted to our ER at 11.45 am because of a syncopal episode preceded by back pain and followed by sudden left hemiparesis, with onset 1 hour before; first neurological examination revealed right gaze deviation, left hemianopsia, dysarthria, left hypoesthesia and severe left hemiparesis (NIH stroke scale, NIHSS 18). Non enhancedbrain CT (NECT) showed no significant ischemic lesions (ASPECTS 10), while CT-Angio revealed complete right cervical and intracranial carotid artery occlusion, while normal patency of the Willis branches was found. Due to the haemodynamic nature of the stroke and the heavy clot burden, we decided to bypass the systemic thrombolysis treatment and, at 12:30 $\mathrm{pm}$, the patient underwent cerebral angiography, confirming occlusion of middle and distal part of ICA; an endovascular thromboaspiration was performed, followed by angioplasty and stenting for residual preocclusive atherosclerotic stenosis of proximal ICA. During the procedure systemic hypoperfusion and severe hemodynamic instability became evident and required continue administration of vasoactive amines. For this reason, a thoraco-abdominal contrast-CT was performed, which revealed an acute rupture of subrenal abdominal aortic aneurysm. At 3:30 pm the patient underwent an emergent open surgical aortic repair requiring intraoperative intravenous administration of 5000 low-molecular-weight heparin units. At the end of the surgery $(7: 30 \mathrm{pm})$ a follow-up brain NECT and CT-Angio were repeated for monitoring the presence of procedure complications: these showed very early ischemic changes in the right middle cerebral artery (MCA) territory (ASPECTS 7) and acute in-stent occlusive thrombosis of the right ICA, so the patient promptly underwent a new, successful revascularization by endovascular thromboaspiration.

Results and discussion: After the procedure, the patient was transferred to the Intensive Care Unit and then to the Intensive Rehabilitation ward. At three months follow up he has a residual mild left hemiparesis $(\mathrm{mRS}=1)$.

Reference:

- Klijn CJ, Kappelle LJ. Haemodynamic stroke: clinical features, prognosis, and management. Lancet Neurol. (2010);9(10):10081017. doi:10.1016/S1474-4422(10)70185-X

\section{BROADENING THE PHENOTYPIC SPECTRUM OF CEREBRAL MANIFESTATIONS RELATED TO MUTATIONS OF THE LAMA2 GENE: A CLINICAL CASE WITH LATE-ONSET EPILEPTIC SEIZURES AND CEREBRAL LEUKOENCEPHALOPATHY}

\author{
F. Brigo, F. Rinaldi, A. Norami, V. Tavernelli, A. Bratti, R. Nardone, B. \\ Nucera
}

Department of Neurology, Hospital of Merano (SABES-ASDAA) (Merano-BZ)

Objectives: The LAMA2 gene, on chromosome 6q22-q23, encodes the heavy chain a2 (merosin) component of laminin, a membrane protein of skeletal muscle [1]. A mutation in homo/heterozygosity is responsible for congenital muscular dystrophy1 A (LAMA2-related muscular dystrophy). A classic form, with predominantly motor involvement, has been reported alongside an "atypical" form with mental retardation (7$12 \%)$, epileptic seizures $(8-20 \%)$, abnormalities of neuronal migration (4\%) [2]. Focal or generalized epileptics seizures occur with early-onset forms [3]. We describe a patient with late-onset epileptic seizures and cerebral leukoencephalopathy related to LAMA2 mutation.
Materials and methods. Case report. Results: 44-year-old man, without familiarity for neurological disorders, and with no history of febrile seizures. From the age of 10 months, he had a delay in reaching motor skills milestones, with weakness of the muscles of the pelvic girdle, which led to a diagnosis of "girdle muscular dystrophy". The clinical picture remained stable over the years, with a mild-moderate motor deficit and no cognitive delay. At the age of 37 years, the patients experienced focalonset seizures with impaired awareness, buccal automatisms, and postictal disorientation (without aphasia); the seizures lasted few minutes and occurred once or twice per month. The electroencephalographic recording showed epileptiform abnormalities in bilateral front-temporal regions, more pronounced on the right; the brain magnetic resonance imaging showed white matter lesions (leukoencephalopathy). The patient continued having seizures despite therapy with levetiracetam $3000 \mathrm{mg} /$ day, valproic acid $3000 \mathrm{mg} /$ day, used as monotherapy, or in combination. He eventually reached seizure freedom with carbamazepine $800 \mathrm{mg} /$ day and levetiracetam $2000 \mathrm{mg} /$ day. A LAMA2 mutation in heterozygosity (exons 5 and 55) was identified.

Discussion and Conclusions: This case, characterized by mild motor deficit and late-onset of epilepsy, expands the phenotypic spectrum of the cerebral manifestations related to LAMA2 mutations. In this patient it is reasonable to hypothesize a causal correlation between the leukoencephalopathy and the epileptic seizures.

References:

1. Geranmayeh F, Clement E, Feng LH, et al. Genotype-phenotype correlation in a large population of muscular dystrophy patients with LAMA2 mutations. Neuromuscul Disord. (2010);20(4):24150

2. Jones KJ, Morgan G, Johnston $\mathrm{H}$, et al. The expanding phenotype of laminin alpha2 chain (merosin) abnormalities: case series and review. J Med Genet. (2001);38(10):649-57

3. Camacho A, Núñez N, Dekomien G, Hernández-Laín A, de Aragón AM, Simón R. LAMA2-related congenital muscular dystrophy complicated by West syndrome. Eur J Paediatr Neurol. (2015);19(2):243-47

\section{EPILEPTIC SEIZURES FOLLOWING ILLICIT DRUG INTAKE: IS IT EPILEPSY?}

\author{
F. Brigo, F. Rinaldi, B. Nucera, A. Bratti, A. Norami, V. Tavernelli, R. \\ Nardone
}

Department of Neurology, Hospital of Merano (SABES-ASDAA) (Merano-BZ)

Objectives: The use of illicit drugs can reduce the epileptic threshold leading to epileptic seizures. EEG abnormalities in patients with seizures following illicit drug intake raise the question whether these seizures should be classified as epilepsy or as acute symptomatic seizures.

Materials and Methods: We describe two patients (Patient 1: female, 16 years-old. Patient 2: male, 19 years-old. Normal neurological findings, personal history unremarkable) with a generalized tonic-clonic seizure after taking illicit drugs (Patient 1: cocaine; Patient 2: ketamine and cannabis), without concurrent alcohol intake or sleep deprivation.

Results: In both cases the EEG showed generalized spike-andwave complexes on a normal background activity (Patient 2 had also photosensitivity). These findings were seen also in an EEG 
performed one week later. Both patients subsequently had a further generalized tonic-clonic seizure, unrelated to illicit drug or alcohol intake, or sleep deprivation. Magnetic resonance imaging of the brain was normal. In both cases, levetiracetam treatment was started and no further seizures occurred.

Discussion: Seizures after illicit drug intake should not be automatically regarded as acute symptomatic seizures, but require further diagnostic investigation. The use of illicit drugs can cause seizures in patients who were otherwise predisposed to idiopathic generalized epilepsy. These patients can develop further spontaneous seizures unrelated to illicit drug use, possibly due to a persistent lowering of the epileptic threshold. Patients with a first epileptic seizure following illicit drug use and generalized spikeand-wave complexes in the EEG should probably be diagnosed with idiopathic generalized epilepsy, and treated as such.

References:

- Koppel BS, Samkoff L, Daras M. Relation of cocaine use to seizures and epilepsy. Epilepsia. (1996);37:875-8

- Myers JA, Earnest MP. Generalized seizures and cocaine abuse. Neurology (1984);34:675-6

- Fisher RS, Acevedo C, Arzimanoglou A, Bogacz A, Cross JH, Elger CE, Engel J Jr, Forsgren L, French JA, Glynn M, Hesdorffer DC, Lee BI, Mathern GW, Moshé SL, Perucca E, Scheffer IE, Tomson T, Watanabe M, Wiebe S. ILAE official report: a practical clinical definition of epilepsy. Epilepsia (2014);55:475-82

\section{MACROACUSIA AND VISUAL DISTURBANCES IN ALICE IN WONDERLAND SYNDROME}

\section{F. Brigo, F. Rinaldi, B. Nucera, A. Norami, G. Zorzi, R. Nardone}

Department of Neurology, Hospital of Merano (SABES-ASDAA) (Merano-BZ)

Objectives: Alice in Wonderland syndrome (AIWS) is a disorder characterized by distortions of visual perception, the body schema, and the experience of time, which are attributed to functional and structural aberrations of the perceptual system.

Materials and Methods: We report a 9-year-old previously healthy boy with AIWS manifesting during a febrile illness.

Results: The patient experienced recurrent episodes of distorted vision (macropsia, microtelepsia, zoom vision, and palinopsia with illusory visual spread) and sudden increase in the volume of sounds (macroacusia). Neurological and opthalmological examination was normal; a comprehensive serological and laboratory testing showed only mild relative neutropenia, lymphocytosis and monocytosis, with normal inflammation parameters. A head magnetic resonance imaging and continuous electroencephalogram were normal. Symptoms gradually reduced in intensity and duration over the following two months and eventually disappeared completely.

Discussion: The symptom onset related to an acute febrile illness, the epidemiological context, the mild blood abnormalities typically encountered in viral infections, the benign course, and the exclusion of other causes, suggest an association with a viral infection (possibly due to Influenzavirus). Remarkably, this case manifested also with macroacusia, which has been exceptionally reported in AIWS. As our case shows, perceptual distortion in AIWS can involve somesthetic and other nonvisual modalities, including alterations in the sound perception

\section{INTRACRANIAL STENOSIS RESOLUTION IN A CHILD WITH SICKLE CELL ANAEMIA}

\author{
N. Brunelli ${ }^{1}$, B. Peer Mohamed ${ }^{2}$, M. Fuschi ${ }^{3}$, D. Simonato ${ }^{3}$, A. Qureshi ${ }^{4}$, \\ S. Mazzucco 5
}

${ }^{1}$ Neurology Unit, Campus Bio-Medico University of Rome (Roma); ${ }^{2}$ Department of Paediatrics, Milton Keynes University Hospital (Milton Keynes-UK); ${ }^{3}$ Neuroradiology Unit, Oxford University Hospitals NHS Foundation Trust (Oxford-UK); ${ }^{4}$ Paediatric Haematology and Oncology Service, Children's Hospital-Oxford University Hospital Foundation Trust (Oxford-UK); ${ }^{5}$ Wolfson Centre for Prevention of Stroke and Dementia, Nuffield Department of Clinical Neurosciences, University of Oxford (Oxford-UK)

Background: Sickle cell disease (SCD) is a leading cause of paediatric stroke worldwide, with long-term disability and cognitive impairment. [1] Clinical guidelines [2] recommend transcranial Doppler (TCD) surveillance between the age of 2 and 16 to detect children with time-averaged maximum mean velocity (TAMMV) $\geq 200 \mathrm{~cm} / \mathrm{s}$, who are at high risk of stroke, and should be started on transfusion treatment for primary prevention of stroke. [3]

Aim: We present a case of sickle cell anaemia with newly developed intracranial stenosis during TCD surveillance.

Case presentation: An 11-year-old boy with homozygous SCD was under annual TCD surveillance since the age of two at a UK district general hospital. His annual TCD TAMMV had been within the limits for "standard stroke risk" $(<170 \mathrm{~cm} / \mathrm{s})$ [2] until 2018, when it started to progressively decrease focally in the right Middle Cerebral Artery (MCA) to values $<70 \mathrm{~cm} / \mathrm{s}$. An MRA scan confirmed right MCA stenosis. According to guidelines, [3] he was then started on monthly blood transfusions. Seven months later, a followup MRA showed resolution of the previously demonstrated right MCA stenosis; TCD scan showed significant increase in right MCA TAMMV with normalised values, consistent with MRA results.

Discussion and Conclusion: To our knowledge, this is the first fully documented description of a case of pediatric SCD with MCA stenosis which developed under TCD surveillance in a child with previously normal velocities and regressed on transfusion therapy. This case supports that intracranial vasculopathy in children with SCD is a dynamic process that can be reversed if recognized at an early stage and treated in a timely manner with transfusion therapy.

References:

1. Ohene-Frempong, K. The Cooperative Study of Sickle Cell Disease. Cerebrovascular accidents in sickle cell disease: rates and risk factors. Blood (1998);91:288-94

2. Adams RJ, McKie VC, Hsu L, et al. Prevention of a first stroke by transfusions in children with sickle cell anemia and abnormal results on transcranial Doppler ultrasonography. N Engl J Med (1998); 339:5-11

3. DeBaun MR, Jordan LC, King AA, et al. American Society of Hematology 2020 guidelines for sickle cell disease: prevention, diagnosis, and treatment of cerebrovascular disease in children and adults. Blood Adv (2020); 4:1554-88

\section{BULBAR SYMPTOMS IN A YOUNG GIRL: A CHALLENGING DIAGNOSIS}

S. Cabras ${ }^{1}$, M. Grassano ${ }^{1}$, A. Bombaci ${ }^{1}$, A. Canosa $^{2}$, A. Calvo ${ }^{2}$, A. $\mathrm{Chiò}^{2}$, C. Moglia ${ }^{2}$ 
${ }^{1}$ Department of Neurosciences "Rita Levi Montalcini", University of Turin (Torino); ${ }^{2}$ Department of Neurosciences "Rita Levi Montalcini"; Neurology 1, Azienda Ospedaliero-Universitaria Città della Salute e della Scienza di Torino, University of Turin (Torino)

Objective: We report the case of a young girl, that represented a diagnostic, and, consequently, therapeutic challenge for neurologists.

Materials: Medical reports and discharge letters.

Methods: Review of the patient's medical history and personal interview with her mother.

Results: A 23-year-old girl presented with an important tongue oedema and consequent sialorrhea, speech and swallowing impairment after a recent tonsillectomy. After a first hypothesis of a local pharyngeal inflammation, treated with antibiotics and steroids, she developed neck weakness. Neurophysiologic studies were at first compatible with a neuromuscular junction disorder, although myasthenia specific antibodies resulted negative and she had no benefit from neither acetylcholinesterase inhibitors nor steroids. At a second examination, electromyography (EMG) showed tongue and limbs denervation. An inflammatory neuropathy was suspected, but she had no response to both immunoglobulin and plasmapheresis. Other investigations, including brain magnetic resonance, lumbar puncture, visual and brainstem auditory evoked potentials, total-body positron emission tomography (PET), tongue biopsy, infectious markers and autoantibodies, resulted normal. During hospitalization, moreover, she presented a dyspnoeic event followed by a generalized tonic-clonic seizure, with subsequent oral intubation and tracheostomy for mechanical ventilation. Meanwhile, she presented also an unexpected cardiovascular instability, then regressed. A genetic counselling was performed, suggesting the hypothesis of Brown-Vialetto-Van Laere syndrome and riboflavin supplementation was started ex juvantibus, without benefit. Genetic testing for juvenile Amyotrophic Lateral Sclerosis (ALS) was also requested. Four months later, neurologic examination showed lower and upper motor neuron signs and EMG confirmed a lower motor neuron disorder. Finally, genetic analysis demonstrated positivity for FUS (Fused in Sarcoma) mutation, confirming the suspect of juvenile ALS.

Discussion: Defining the diagnosis can be challenging, even with the help of instrumental investigations, that can result confounding in some cases. For example, low-frequency repetitive nerve stimulation and single-fiber EMG could be both positive in ALS cases, especially in rapid progressors when examining proximal muscles. Other factors complicating the diagnostic process in our case were the young age, not typical for ALS onset, the strange tongue oedema, instead of the classic atrophy, and the absence of upper motor neuron signs in the initial phase.

Conclusion: In cases with complex diagnostic definition, different therapeutic attempts could be considered, not only to try everything possible to contrast disabling symptoms, especially in young patients, but also to support the final diagnosis after excluding other possible causes. References:

- Calvo A, Moglia C, Canosa A, et al. De novo nonsense mutation of the FUS gene in an apparently familial amyotrophic lateral sclerosis case. Neurobiol Aging. (2014);35(6):1513.e7-1513.e1.513E11. doi:10.1016/ j.neurobiolaging.2013.12.028

- Li HF, Wu ZY. Genotype-phenotype correlations of amyotrophic lateral sclerosis. Transl Neurodegener. (2016);5:3. Published 2016 Feb 3. doi:10.1186/s40035-016-0050-8

\section{NEW-ONSET-REFRACTORY STATUS EPILEPTICUS IN ELDERLY PATIENT: WHAT IS UNDERNEATH?}

\author{
C. Calvello, E. Nardi Cesarini, N. Salvadori, M. Silla, L. Parnetti, C. \\ Costa
}

Neurology Clinic, Department of Medicine, University of Perugia (Perugia)

Introduction: The new-onset refractory status epilepticus (NORSE) is a clinical feature defined as the occurrence of refractory status epilepticus in a patient without active epilepsy, or a clear structural or metabolic cause. Most cases evolve to super RSE with unfavorable outcome. Autoimmune encephalitis is the most frequent identified cause but half of adult cases remain of unknown etiology. [1] It is worth highlighting that, in the unknown etiology of late onset epilepsy, AB42 represents the link between epilepsy and cognitive impairment. [2,3] We report a case of 76 years-old man with NORSE as first presentation of Alzheimer's disease.

Case report: A 76-year-old man presented with sudden speech arrest episodes was admitted to our department for further treatment. These episodes, lasting about 10-15 minutes, showed increased frequency in the last 48 hours. Patient was independent in the activity of daily life, suffering from hypertension, diabetes type II, chronic obstructive pulmonary disease, and recently coronary artery disease. He underwent brain CT scan, blood tests, ECG and echocardiogram with no relevant results. Video electroencephalography revealed epileptic seizures. During the registration, the patient presented speech arrest associated with staring, eyelid myoclonus, lip-smacking, and tardive impaired consciousness. The events lasted more than 10 minutes, such as to configure a non-convulsive status epilepticus. Continuous spike-wave in the front-temporal region characterized the ictal pattern. The seizures proved resistant to multiple antiseizure medications, at high doses including lorazepam, levetiracetam and valproic acid, for several days. At that stage, one bolus of lacosamide was administered with progressive improvement. $\mathrm{He}$ underwent on extensive workup: infectious and autoimmune disease was excluded. Given patient age and the unknown etiology of the status epilepticus, CSF dosing of amyloidosis, tauopathy and neurodegeneration biomarkers was performed. CSF profile displayed a clear AD-like profile. One month after discharge, he was seizure-free. The performance on neuropsychological tests revealed a multidomain cognitive impairment with corticalsubcortical pattern. The Neuropsychiatric Inventory revealed personality changes with presence of delusional beliefs, apathy and irritability. At functional evaluation, a clinical status of dementia was clearly detected.

Conclusion: In this case report we managed to diagnose late onset epilepsy, of unknown origin, clinically presented as NORSE. After excluding the most common causes of NORSE ${ }^{1}$, therefore, it is useful to dose CSF-ATN biomarkers and perform cognitive tests to highlight an underlying neurodegenerative disorder such as, in our case, Alzheimer's disease. [2,3]

References:

1. Hirsch LJ, Gaspard N, van Baalen A, et al. Proposed consensus definitions for new-onset refractory status epilepticus (NORSE), febrile infection-related epilepsy syndrome (FIRES), and related conditions. Epilepsia (2018);59:739-44

2. Jansen W.J, et al. Prevalence of cerebral amyloid pathology in persons without dementia: A meta-analysis. JAMA 2015 May 19;313(19):1924-38 
3. Costa, C., Parnetti, L., D’Amelio, M., Tozzi, A., Tantucci, M., Romigi, A., Siliquini, S., Cavallucci, V., Di Filippo, M., Mazzocchetti, P., Liguori, C., Nobili, A., Eusebi, P., Mercuri, N.B., Calabresi, P., Epilepsy, amyloid- $\beta$, and D1 dopamine receptors: a possible pathogenetic link? Neurobiol. Aging (2016);48:161-71

\section{A RARE CASE OF ATAXIC SYNDROME AND DEVELOPMENTAL DELAY}

\author{
I. Campini ${ }^{1}$, L. Magistrelli ${ }^{1}$, S. Mellone ${ }^{2}$, M. Giordano ${ }^{2}$, R. Cantello ${ }^{1}$ \\ ${ }^{1}$ Department of Translational Medicine, Section of Neurology, University \\ of Eastern Piedmont (Novara); ${ }^{2}$ Department of Health Sciences, Human \\ Genetics Laboratory, University of Eastern Piedmont (Novara)
}

Objective: To describe a rare cause of an ataxic and tremulous syndrome with cognitive delay.

Case description: Here we present a case of a 70 years-old female patient with diffuse tremors, ataxia and cognitive delay since infantry. Her tremors started at the age of 2 , along with motor and cognitive regression. Particularly, in the following years, she was unable to perform coordinate motor tasks and to learn without specific support. Furthermore, she presented dysmorphic features characterized by broad, flat nasal bridge and strabismus. No diagnosis was reached until she was referred to our attention because of a progressive worsening of the tremors, motor uncoordination and unbalance, which lead to several falls. Her past medical and familiar history were unremarkable. On neurological examination she had explosive and dysartric speech, reduced verbal fluency and incomplete time/space orientation. She also presented dysmetria at the 4 limbs. Furthermore, a subcontinuos rest and kinetic tremor involving head and the 4 limbs was present. Upstanding was possible only with constant bilateral assistance and she could make a few steps with an ataxic gait. On blood examinations serum calcium, TSH and ceruloplasmin were normal. At first a brain CT scan was performed, which was normal. A brain MRI showed hypoplasy of cerebellar vermis along with a mild diffuse gliosis. Considering the co-occurrence of the dimorphic feature and the cognitive impairment and the movement disorders we firstly performed an array-CGH that shows an interstitial deletion in the region 6q22.1-q22.31 (hg19:118,051,261-120,084,595) of approximately $2 \mathrm{Mb}$ encompassing 13 genes.

Discussion: The 6q22.1-q22.31 deletion is a rare copy number variation $(\mathrm{CNV})$ that has been associated with intellectual disability/ developmental delay, facial dysmorphism, and growth retardation. Neurological features mostly include epilepsy and rarely movement disorders such as myoclonus, tremor and ataxia. Structural abnormalities comprise above all thickening of corpus callosus and cerebellar vermis hypoplasia. Adult neurologists should be aware of the presence of these rare CNV syndromes since patients reach adulthood and the mixed clinical features may be difficult to recognize and fully characterize. Accordingly, these conditions should be included in the differential diagnosis of complex movement disorders syndromes.

\section{NATALIZUMAB AS RESCUE THERAPY IN A PATIENT WITH A SEVERE NEUROLOGICAL WORSENING AFTER FINGOLIMOD SUSPENSION}

F. Caputo, A. Introna, B. Orlando, L. Bollo, S. Landolfo, D. Paolicelli, P. Iaffaldano, M. Trojano
Department of Basic Medical Sciences, Neurosciences and Sense Organs, University of Bari Aldo Moro (Bari)

Background: The rebound syndrome (RS) in Multiple Sclerosis (MS) is described as a flare-up disease activity exceeding the pretreatment level after discontinuation of disease modifying therapies (DMTs). Although this phenomenon has been well described after Fingolimod (FTY) suspension, the choice of the best following DMT is still debated.

Case report: A 37-year-old man with a seventeen years history of relapsing-remitting MS (diagnosis in 2003) was initially treated with interferon-beta-1a $22 \mathrm{mcg}$ replaced by the highest dosage one year later due to two relapses (Expanded Disability Status Scale (EDSS) 2.5). In 2011, because of persistent clinical and radiological activity, it was proposed the switch to Natalizumab (NTZ), but the patient declined. In 2015, after a further relapse, FTY was started. During FTY treatment, MS remained clinically stable (EDSS 2.0) until to 2019 when three consecutive magnetic resonance imagings (MRIs) showed new demyelinating lesions. Therefore, in October 2019 FTY was discontinued, interferonbeta-1a $30 \mathrm{mcg}$ was administered during the washout period prior a higher-efficacy DMT. After FTY withdrawal, the patient rapidly developed left hemiparesis, ataxia and dizziness (EDSS 6.5). MRI showed new large enhancing demyelinating lesions in the brain and spinal cord. Two courses of $5 \mathrm{gr}$. intravenous methylprednisolone failed, so he received ten cycles of immunoadsorption (IA) with partial recovery (EDSS 6.0). However, two days after the last IA, the patient developed tetraparesis, dysarthria, dysphagia and urinary retention (EDSS 9.0) and subcontinous focal seizures treated with different antiepileptic drugs. The neurological status declined resulting in coma (Glasgow Coma Scale 3). The cerebrospinal fluid analysis was negative for JCV-PCR and other neuroinfections. After a further inefficacious $5 \mathrm{gr}$ of methylprednisolone, although the stratify JCV ab test positivity (index 1.68), considering the severe clinical conditions of the patient, NTZ was started. The clinical picture began to improve: the patient awoke and after four-monthly-infusions he was able to feed, keep the upright posture and make few steps with bilateral assistance (EDSS 6.5).

Discussion: Although the exact mechanism of RS after FTY withdrawal is unclear, it could determinate severe consequences, resulting in a high disability accrual and sometimes death. To date a standardized therapeutic management of this phenomenon has not been established yet, therefore which DMT and when to start it is a great challenge. Moreover, the following immunotherapy could become crucial especially in patients refractory to methylprednisolone and IA. This case report proves the efficacy of NTZ as rescue therapy in a patient with a dramatic neurological worsening after FTY discontinuation.

References:

- Hatcher SE, Waubant E, Nourbakhsh B, Crabtree-Hartman E, Graves JS. Rebound syndrome in patients with multiple sclerosis after cessation of fingolimod treatment. JAMA Neurol. (2016);73(7):790-4

- Członkowska A, Smoliński Ł, Litwin T. Severe disease exacerbations in patients with multiple sclerosis after discontinuing fingolimod. Neurol Neurochir Pol. (2017);51(2):156-62

- Barry B, Erwin AA, Stevens J, Tornatore C. Fingolimod Rebound: A Review of the Clinical Experience and Management Considerations. Neurol Ther [Internet]. (2019);8(2):241-50. Available from: https://doi.org/10.1007/s40120-019-00160-9 


\section{IATROGENIC CEREBRAL AMYLOID ANGIOPATHY 34 YEARS AFTER DURA MATER EMBOLIZATION OF A NASOPHARYNGEAL ANGIOFIBROMA}

P. Caroppo ${ }^{1}$, G. Marucci ${ }^{1}$, E. Maccagnano ${ }^{2}$, C. Gobbo ${ }^{3}$, I. Bizzozero ${ }^{1}$, P. Tiraboschi $^{1}$, V. Redaelli ${ }^{1}$, M. Catania ${ }^{1}$, G. Di Fede ${ }^{1}$, L. Caputi ${ }^{4}$, G. Giaccone $^{1}$

${ }^{1}$ Neurology V/Neuropathology, Fondazione IRCCS Istituto Neurologico Carlo Besta (Milano); ${ }^{2}$ Neuroradiology Unit, Fondazione IRCCS Istituto Neurologico Carlo Besta (Milano); ${ }^{3}$ Nuclear Medicine Unit, ASSTSettelaghi (Varese); ${ }^{4}$ Neurology IX, Cerebrovascular Diseases Unit, Fondazione IRCCS Istituto Neurologico Carlo Besta (Milano)

Objective: Cerebral Amyloid Angiopathy (CAA) is a neurodegenerative disorder characterized by the accumulation of $A \beta$ protein in small cerebral vessels that can cause spontaneous Intracerebral Hemorrhage (ICH) in the elderly. Early-onset ICH has been described in rare iatrogenic CAA cases, several years after a neurosurgical intervention or a traumatic brain injury. In some of these cases, the use of cadaveric dura mater grafts has been documented during the neurosurgical procedure $[1,2]$. In those cases dura mater would have acted as a source of $A \beta$ seeds causing the accumulation of $A \beta$ through a "prion-like" mechanism [3]. We report the clinical and neuroradiological features of a patient with probable iatrogenic CAA following exposure of dura mater extracts used to embolize a nasopharyngeal angiofibroma, 34 several years before the first hemorrhagic event.

Methods: The patient underwent a detailed clinical, CSF and neuroimaging (MRI, amyloid-PET) evaluation.

Results: The patient had an ICH at age 51 in occipito-parietal brain region. The brain MRI showed the ICH associated with multiple microbleeds suggesting a diagnosis of CAA. Microbleeds increased after 3 months from ICH. CSF biomarkers were consistent with cerebral amyloidosis showing a decrease of Abeta42 with normal tau e p-tau. The amyloid-PET with 18-F- flutemetamol confirmed the presence of widespread $A \beta$ cortical deposition. The neuropsychological evaluation did not show cognitive impairment. The clinical history revealed that at age of 17 , the patient underwent to a pre-surgical embolization of a nasopharyngeal angiofibroma by lyophilized dura foam, supporting the hypothesis of an iatrogenic cause of the disease.

Discussion and conclusion: We enlarge the series of iatrogenic CAA with documented dura mater use, that occurred several years before the first hemorrhagic event. In this case, the surgical procedure leading to dura exposure was the embolization of an extracranial lesion, supporting the transmission of the disease by intravascular route.

References:

1. Banerjee G, Adams ME, Jaunmuktane Z, et al. Early onset cerebral amyloid angiopathy following childhood exposure to cadaveric dura. Ann Neurol. (2019);85(2):284-90

2. Hervé D, Porché M, Cabrejo L, et al. Fatal A $\beta$ cerebral amyloid angiopathy 4 decades after a dural graft at the age of 2 years. Acta Neuropathol. (2018);135(5):801-03

3. Kovacs GG, Lutz MI, Ricken G, et al. Dura mater is a potential source of A $\beta$ seeds. Acta Neuropathol. (2016);131(6):911-23

\section{ATYPICAL CHRONIC INFLAMMATORY DEMYELINATING POLINEUROPATHY (CIDP) AND POEMS SYNDROME}

\author{
G. Carroli, D. Carlucci, A. Braccia, M. Pugliatti
}

Department of Biomedical and Surgical Sciences, University of Ferrara (Ferrara)

Background: POEMS syndrome (polyneuropathy, organomegaly, endocrinopathy, M-protein, skin changes) is a rare paraneoplastic syndrome in plasma cell proliferative disorders. The neurological involvement is characterized by a demyelinating often painful and rapidly disabling neuropathy with subacute onset. This finding is mandatory for diagnosis.

Case Presentation: A 45 years-old woman with a history of chronic inflammatory demyelinating polyneuropathy (CIDP) diagnosed in the previous year and poorly responsive to usual treatments (steroid and periodic plasmapheresis), was admitted to the Ferrara S. Anna University Hospital for a 5-day history of headache and blurred vision associated with worsening of previous tetraparesis. On admission, a bilateral papilledema in idiopathic intracranial hypertension was documented at the ophthalmologic evaluation and magnetic resonance imaging (MRI). Treatment with acetazolamide was started with no amelioration of the symptoms, and a ventriculo-peritoneal shunt was therefore performed with benefit on the visual defect. After extensive investigations which included a bone marrow biopsy, a diagnosis of POEMS was made based on the polyneuropathy, elevated lambda light chain level, splenomegaly, sclerotic bone lesions, papilledema/ascites and thrombocytosis. The patient was then referred to the Unit of Haematology and treated with chemotherapy (four cycles of Bortezepib, Cyclophosphamide and Dexamethasone). Autologous stem cell transplantation is now being considered.

Discussion: Our case report suggest that patients with a diagnosis of CIDP and no response to standard treatments should be investigated for a POEMS syndrome and start early on specific treatment.

References:

- Dispenzieri A. POEMS Syndrome: 2019 Update on diagnosis, riskstratification, and management. Am J Hematol. (2019) Jul;94(7):812-27

- Piccione EA et al. Nerve pathologic features differentiate POEMS syndrome from CIDP. Acta Neuropathol Commun. (2016) Oct 31;4(1):116

\section{BICKERSTAFF BRAINSTEM ENCEPHALITIS (BBE) AND ACUTE INFLAMMATORY DEMYELINATING POLIRADICULONEUROPATHY (AIDP): A CASE REPORT WITH DRAMATIC CLINICAL COURSE}

\author{
G. Carroli, M. Pugliatti
}

Department of Biomedical and Surgical Sciences, University of Ferrara (Ferrara)

Background: Bickerstaff brainstem encephalitis (BBE) is a rare autoimmune encephalitis clinically characterized by acute ophthalmoplegia, ataxia and altered state of consciousness. Together with acute inflammatory demyelinating poliradiculoneuropathy (AIDP) it belongs to a spectrum of post-infectious demyelinating diseases. Overlapping forms between BBE and AIDP (BBE/AIDP) are described in patients with lower limbs weakness and typical signs of BBE, suggesting a combined involvement of the central and the peripheral nervous systems.

Case Presentation: A 80-year-old man was admitted to the Ferrara S. Anna University Hospital Emergency Unit for acute onset of dysarthria and gait unsteadiness developed after a one day history of nausea and vomiting. 
The patient was drowsy, ataxic, with severe weakness of the lower limbs and areflexia. Brain CT and MRI scans were normal. Nerve conduction studies (NCS) showed slowed motor conductions with absent F-wave. Cerebrospinal fluid (CSF) analysis revealed an albumin-cytologic dissociation; serum and CSF analysis were negative for bacterial and viral infections. AIDP was diagnosed. By virtue of a rapid worsening of his clinical status over the following hours and which featured flaccid tetraplegia, external ophtalmoparesis, facial weakness and dysphagia, the overlap BBE/ AIDP was then considered. High-dose steroid therapy and a cycle of plasmapheresis were started but without benefit. On day 5 he dramatically worsened to coma and in need of mechanical ventilation, the patient was referred to the intensive care unit. A second brain MRI was performed which revealed symmetrical alterations of medulla oblungata with irregular contrast enhancement. Intravenous immunoglobulins (IVIG) were started without any clinical effect and the patient died after 10 days from admission.

Discussion: We report a case of encephalitis sharing the classical features of BBE with signs of acute peripheral involvement typically seen in AIDP. Only few cases of BBE/AIDP overlap are reported in literature, but the two disorders are deemed to be closely related. The coexistence of AIDP and BBE may give rise to different patterns of complications, response to therapy and clinical outcomes. New therapeutic strategies should be explored upon better understanding this clinical entity.

References:

- A. Michev et al. Bickerstaff Brainstem Encephalitis and overlapping Guillain-Barré syndrome in children: Report of two cases and review of the literature. European Journal of Paediatric Neurology 2019-0101, Fascicolo 23, Numero 1, Pagine 43-52

- Masaaki Odaka et al. Bickerstaffs brainstem encephalitis: clinical features of 62 cases and a subgroup associated with Guillain-Barré syndrome. Brain (2003) Oct; 126(Pt 10): 2279-90

\section{SERTRALINE-INDUCED TARDIVE DYSKINESIAS: A CASE REPORT}

S. M. Cartella, C. Terranova, S. Attardo, A. Laganà, G. Mento, A. Toscano, P. Girlanda

Department of Clinical and Experimental Medicine, University of Messina (Messina)

Background: Tardive dyskinesias are rhythmic, repetitive, stereotypic movements of the face, mouth and tongue occurring as a result of dopamine-receptor blocking agents. The mean prevalence is $25 \%$ in patients taking antipsychotics and risk factors are age, sex and ethnicity. Movement disorders have rarely been reported as a result of antidepressants use ( $2,8 \%$ of reports). Sertraline-induced tardive dyskinesias have infrequently been reported in pharmacovigilance databases and, as far as we know, this is the one of the few case descriptions.

Aim: To describe a case of a movement disorder induced by an antidepressant rarely reported before.

Patients and methods: A 84-years-old woman was admitted to our Neurology ward due to an ischaemic stroke. She was affected by anxious-depressive syndrome, treated with sertraline $50 \mathrm{mg} /$ day for 5 years. She underwent clinical examination, EEG, brain CT scan, MRI.

Results: Neurological examination showed right hemiparesis, oromandibular dyskinesias, present all day long and postural tremor in her upper limbs. Her history confirmed the tremor had been present for some years, whereas oral dyskinesias only for some weeks before. We ruled out epileptic status performing an EEG, basal ganglia alterations performing brain CT scan and MRI. After discontinuation of sertraline dyskinesias reduced. We decided not to use any drug to treat them because they did not interfere with the patient's functionality.

Discussion: Antidepressants use and movement disorders incidence was analysed in a pharmacovigilance study. The most significant association with all types of movement disorders and antidepressant was found with SSRIs. There are three possible underlying mechanisms. The first one is the most recognized one and is based on the chronic blockade of D2 receptor which leads to their up regulation. The second one is the neurodegenerative theory, based on drugs' neurotoxicity which leads to Reactive Oxygen Species production. Finally, the last hypothesis concerns synaptic plasticity. D2 receptor hypersensitivity leads to a change in the glutamatergic system, which leads to an imbalance between the two basal ganglia circuits. A genetic predisposition is involved, specifically cytochrome $\mathrm{P} 450$ or dopamine receptor polymorphisms.

Conclusion: Our data highlights the necessity for the clinician to be aware of an unusual but important side effect of antidepressants and therefore the necessity to carefully choose the right one, based on the patient's risk factors and predisposition to develop this kind of adverse effects.

References:

- Revet A, Montastruc F, Roussin A, Raynaud JP, Lapeyre-Mestre M, Nguyen TTH, Antidepressants and Movement Disorders: a postmarketing study in the world pharmacovigilance database, BMC Psychiatry (2020) Jun 16;20(1):308

- Frei K, Tardive dyskinesia: who gets it and why, Parkinsonism Relat Disord (2019) Feb;59:151-54

- Mörkl S, Seltenreich D, Letmaier M, Bengesser S, Wurm W, Grohmann R, et al. Extrapyramidal reactions following treatment with antidepressants: Results of the AMSP multinational drug surveillance programme. World J Biol Psychiatry (2020) Apr;21(4):308-16

\section{IPSILATERAL NON-STENOTIC CAROTID PLAQUE IS A POTENTIAL, UNRECOGNIZED CAUSE OF ESUS: TWO TEACHING CASES}

A. Cascio Rizzo ${ }^{1}$, G. Giussani ${ }^{1}$, A. Gatti ${ }^{1}$, R. Tortorella ${ }^{1}$, C. Ceresa ${ }^{1}$, A. Guccione $^{1}$, C. Motto ${ }^{1}$, L. Quilici ${ }^{2}$, A. Lista ${ }^{3}$, G. Pero ${ }^{2}$, E. Agostoni ${ }^{1}$

${ }^{1}$ Neurology \& Stroke Unit, ASST Grande Ospedale Metropolitano Niguarda (Milano); ${ }^{2}$ Neuroradiology, ASST Grande Ospedale Metropolitano Niguarda (Milano); ${ }^{3}$ Vascular Surgery, ASST Grande Ospedale Metropolitano Niguarda (Milano)

Carotid artery plaque is considered a possible cause of stroke according to TOAST classification, but only if stenosis is $>50 \%$. Non stenotic carotid plaques $(\leq 50 \%)$ are classified as stroke of undetermined source. Most cryptogenic strokes share the clinical and radiographic appearance of an embolic source of unknown source (ESUS). Most likely causes are covert AFib, atrial cardiopathy, unrecognized myocardial infarction, PFO, occult cancer and nonstenosing large-artery atherosclerosis. A substantial proportion of ESUS may be the result of large-artery atherosclerotic disease, unrecognized because it does not cause significant stenosis.

Case reports: We discuss two cases of nonstenotic carotid disease presenting with major ischemic stroke. A 68 years-old woman, with history of hypertension, diabetes, right parietal ischemic stroke and right carotid TIA, on clopidogrel therapy, developed acute left hemiparesis and sensory loss (NIHSS 13). Head-CTA showed a right MCA-M2 occlusion, primary mechanical thrombectomy was performed with benefit. 
No embolic sources were found. She was discharged on aspirin. Two months later she was again admitted because of acute left-sided weakness due to a right M2 branch occlusion. Mechanical thrombectomy was performed with complete recanalization of the vessel. Cerebral angiogram revealed a surface irregularity of the right carotid bulb without luminal narrowing, unrecognized at the previous angiography. At carotid ultrasound it appeared as mild isoechoic wall thickening with irregular surface. She underwent carotid endoarterectomy revealing a mild atherosclerotic plaque with irregular and ulcerated surface. A 48 years-old woman, with no vascular risk factors, acutely developed aphasia and right hemiplegia (NIHSS 22). Head-CTA showed occlusion of the left carotid apex with a doubtful floating thrombus at the carotid bulb. Mechanical thrombectomy was performed and angiography revealed an irregular surface of the carotid bulb wall, without thrombus, probably due to a mild atherosclerotic plaque. After complete evaluation no other sources of embolism were found. Aspirin and statin were started.

Discussion: Multiple studies have found a higher prevalence of nonstenosing atherosclerotic plaque ipsilateral to a cryptogenic stroke. $[1,2]$ High-risk plaque features such as intraplaque hemorrhage, plaque thickness, soft plaque, ulceration are related to strokes in general and ESUS in particular, regardless of the degree of stenosis. Moreover plaque surface irregularity on angiography is strongly associated with an increase in ipsilateral stroke risk irrespective of the stenosis degree. [3] These findings suggest that nonstenotic carotid plaque might play a role in stroke etiology, defining a subset of ESUS patients ("symptomatic nonstenotic carotid disease") who probably not benefit from anticoagulation but who need a targeted treatment and prevention strategy.

References:

1. Coutinho JM, Derkatch S, Potvin AR, Tomlinson G, Kiehl TR, Silver FL, et al. Nonstenotic carotid plaque on CT angiography in patients with cryptogenic stroke. Neurology (2016);87:665-72

2. Ospel J, Singh N, Marko M et al. Prevalence of Ipsilateral Nonstenotic Carotid Plaques on Computed Tomography Angiography in Embolic Stroke of Undetermined Source. Stroke (2020);51:1743-49

3. Rothwell PM, Gibson R, Warlow CP. Interrelation between plaque surface morphology and degree of stenosis on carotid angiograms and the risk of ischemic stroke in patients with symptomatic carotid stenosis. On behalf of the European Carotid Surgery Trialists Collaborative Group. Stroke (2000);31:615-21

\section{NERVE ULTRASOUND DIAGNOSTIC OF CHARCOT-MARIE- TOOTH DISEASE IN A CHILD WITH ORBITAL RHABDOMYOSARCOMA AND DRAMATIC VINCRISTINE- INDUCED AXONAL POLYNEUROPATHY}

\section{F. Castellani ${ }^{1}$, M. Cacciavillani ${ }^{2}$, M. Campagnolo ${ }^{1}$, A. Salvalaggio ${ }^{1}$, G. Bisogno $^{3}$, M. Corbetta ${ }^{1}$, C. Briani ${ }^{1}$}

${ }^{1}$ Departement of Neuroscience, University of Padua (Padova); ${ }^{2}$ Synlab, CEMES-EMG Lab (Padova); ${ }^{3}$ Department of Women's and Children's Health, University of Padua (Padova)

Vincristine is a chemotherapy agent belonging to the class of vinka alkaloids, whose common side effect is a length-dependent axonal sensory-motor polyneuropathy. Severe childhood worsening of an unknown hereditary neuropathy, with acute quadriplegia, vocal cord paralysis and dysphagia, triggered by vincristine have been described both in hematological and solid tumors. Charcot-Marie-Tooth $1 \mathrm{~A}$ (CMT1A) neuropathy is the most common autosomal dominant hereditary neuropathy, caused by duplication of the peripheral myelin protein 22 (PMP22) gene. It is characterized by painless, symmetric, slowly progressive motor and sensory neuropathy and by diffuse nerve enlargement at nerve ultrasound. Some CMT1A patients are asymptomatic or have only mild manifestations. We describe an 8-yr-old previously healthy child, who presented with acute weakness at four limbs, and occasional dysphagia after chemotherapy treatment, including vincristine, for an orbital rhabdomyosarcoma. After 4 weekly doses of vincristine she became wheelchair-bound, with distal motility abolished at four limbs, and reduced/absent tendon reflexes. She did not complain of sensory deficit or cranial nerve involvement, except for occasional dysphagia and partial ophthalmoplegia in the left eye secondary to the rhabdomyosarcoma. The child history was negative for fever or infection in the weeks preceding the symptoms onset. Needle electromyography and nerve conduction study showed a severe axonal sensory-motor polyneuropathy, with all the nerves inexcitable and denervation signs at four limbs. Nerve ultrasound showed enlargement at median, ulnar, peroneal, tibial, and sural nerves bilaterally, and at brachial plexus. Spine MRI was negative. The patient's mother reported to be affected by CMT1A. Chemotherapy continued but vincristine was discontinued. Genetic analysis in the child resulted positive for duplication of PMP22 gene. The presence of diffuse nerve enlargement in an axonal neuropathy is very rare. It is therefore likely that the child had an asymptomatic demyelinating CMT1A with diffuse nerve enlargement, that dramatically worsened to a severe axonal neuropathy after vincristine use. The possibility of a hereditary neuropathy should always be ruled out before starting vincristine therapy, through an accurate family history and neurological examination. If family history is negative, nerve ultrasound may be considered as non-invasive screening tool useful to prompt genetic testing.

References:

- Yiu EM, Brockley CR, Lee KJ, et al. Peripheral nerve ultrasound in pediatric Charcot-Marie-Tooth disease type 1A. Neurology (2015);84:569-74

- Padua L, Coraci D, Lucchetta M, et al. Different nerve ultrasound patterns in Charcot-Marie-Tooth types and hereditary neuropathy with liability to pressure palsies. Muscle Nerve (2018);57:E18-E23

\section{WITHDRAWAL OF FINGOLIMOD TREATMENT: RESULTS FROM A SINGLE-COHORT OBSERVATIONAL STUDY}

M. Cellerino ${ }^{1}$, E. Mancuso ${ }^{1}$, S. Lenti ${ }^{1}$, N. Bruschi ${ }^{1}$, G. Boffa ${ }^{1}$, C. Lapucci $^{2}$, L. Roccatagliata ${ }^{3}$, G. Novi ${ }^{4}$, A. Laroni ${ }^{1}$, E. Capello ${ }^{4}$, A. Uccelli $^{1}$, M. Inglese ${ }^{1}$

${ }^{1}$ Department of Neuroscience, Rehabilitation, Ophthalmology, Genetics, and Mother-Child Health (DINOGMI), University of Genoa (Genova); ${ }^{2}$ Experimental Neurosciences Laboratory, Ospedale Policlinico San Martino IRCCS (Genova) ${ }^{3}$ Department of Health Sciences (DISSAL), University of Genoa and Ospedale Policlinico San Martino IRCCS (Genova); ${ }^{4}$ Department of Neurology, Ospedale Policlinico San Martino IRCCS (Genova)

Background: Management of multiple sclerosis (MS) patients who discontinue fingolimod (FTY) is not established yet and breakthrough disease activity has been reported following fingolimod withdrawal. However, data regarding this phenomenon and its possible impact in the long-term are still sparse. 
Aims: To explore frequency of disease reactivation after FTY cessation in a single-center cohort and clinical/radiological characteristics of patients (pts) discontinuing FTY during (I) the wash-out period and (II) the first 12-months following a new treatment onset.

Methods: Data regarding relapses, Expanded Disability Status Scale (EDSS), MRI activity (new T2 and/or Gd-enhancing lesion) and lymphocyte count before and during FTY treatment, the wash-out period and the first 12-moths of a new treatment were retrospectively collected. Pts were grouped according to (I) discontinuation reason (inefficacy/adverse events/other reasons) and (II) disease activity during wash-out (no disease activity/at least one relapse or MRI activity/rebound). Differences in clinical/radiological characteristics or time to NEDA3-failure between groups were assessed with ANOVA, Chi-square and Kaplan-Meier estimator as appropriate.

Results: We included 71 pts [females:70\%; mean age and disease duration at FTY start:37.6 \pm 8.4 and $11 \pm 7.6$ years; median EDSS:3 (0-7); mean treatment duration:2.3 year]. $70 \%$ discontinued for inefficacy, $22 \%$ for adverse events, $8 \%$ for other reasons (pregnancy/pts's choice). During the wash-out $69 \%$ of pts remained stable, $21.2 \%$ had clinical/radiological activity, $9.8 \%$ had a rebound (mean wash-out period: $2.3,8.2,4.1$ months, respectively; $\mathrm{p}=0.03$ ). Age was lower in rebound vs stable pts $(28.5 \pm 4.9 \mathrm{vs} 39.4 \pm 8.3 ; \mathrm{p}=0.006)$. Discontinuation for inefficacy was observed in $70 \%$ of stable, $93 \%$ of clinically/radiologically active and $42 \%$ of pts with a rebound during wash-out $(\mathrm{p}<0.0001)$. No differences in time to NEDA3-failure during the first 12-months following a new treatment start were observed according to discontinuation reason or disease activity during wash-out (Log-Rank test: $\mathrm{p}=0.67$ and $\mathrm{p}=0.23$, respectively). Disease duration, EDSS, lymphocytes' count at FTY stop and lymphocytes' increase during wash-out did not differ according to disease activity during wash-out or response to following treatment.

Conclusions: Younger pts were more likely to have a rebound, while more frequent discontinuation for inefficacy and longer wash-out period were observed in pts with clinical/radiological activity during wash-out. Time to NEDA3-failure within the 12-months following a new treatment onset was not influenced by discontinuation reason or disease activity during wash-out. References:

- Hatcher, S. E., Waubant, E., Nourbakhsh, B., Crabtree-Hartman, E. \& Graves, J. S. Rebound syndrome in patients with multiple sclerosis after cessation of fingolimod treatment. JAMA Neurol. (2016);73:790-94

- Lapucci, C. et al. Different MRI patterns in MS worsening after stopping fingolimod. Neurol. Neuroimmunol. NeuroInflammation (2019) Apr 16;6(4):e566

- Frau J, Sormani MP, Signori A, et al. Clinical activity after fingolimod cessation: disease reactivation or rebound? Eur J Neurol. (2018);25(10):1270-75. doi:10.1111/ene.13694

\section{CEREBRAL VENOUS SINUS THROMBOSIS AS A COMPLICATION OF TAMOXIFEN USE IN A BREAST CANCER PATIENT: A CASE REPORT}

P. Cerrone, M. Vitale, S. Sciamanna, M. Di Giuseppe, A. Casalena, G. Manente, M. Assetta

Neurology and Stroke Unit, PO Mazzini ASL Teramo (Teramo)

Background and aim: Cerebral venous sinus thrombosis (CVST) is an unusual site of thrombosis and a rather uncommon form of stroke. Thrombosis of dural venous sinus and veins can lead to ischemic or haemorrhagic stroke, or both. CVST has an incidence particularly high in young adults, especially in women, and it is usually linked to hypercoagulable states, puerperium, oral contraceptives and malignancy. Tamoxifen is a selective estrogen receptor modulator (SERM), used in the treatment of breast cancer. SERMs could induce an acquired hypercoagulable state with an increased risk of thrombosis. We present a case of a patient treated with tamoxifen for breast cancer who developed CVST.

Case Report: A 47-year old women presented to the Emergency Department for a focal seizure with motor onset. She complained of moderate intensity headache since 10 days. Five months ago she underwent mastectomy for breast cancer and she started treatment with tamoxifen. Brain CT at admission showed two hyperdense areas with perilesional oedema in the right frontal cortex. During the first night after admission, the patient developed nausea and vomiting and headache intensity worsened. A brain CTA and MRI showed venous thrombosis in the superior sagittal sinus, left straight sinus and sigmoid sinus. D-dimer level was increased. There was no evidence of breast cancer recurrence. Oral anticoagulation with Warfarin was started and there was a rapid clinical improvement. The patient was discharged without symptoms and neurological complications. Tamoxifen was discontinued.

Discussion: Considering the history of the patient, the first diagnostic hypothesis was cerebral hematomas due to brain metastases. Symptoms and signs of endocranial hypertension, associated with seizure and patient's characteristics (sex, age, pharmacological history), lead to the hypothesis of CVST, confirmed with CTA and MRI. In the absence of other risk factors for venous thrombosis, in this patient CVST may be linked to the hypercoagulable state due to tamoxifen use.

Conclusions: Tamoxifen use has an increased risk of thromboembolic complications. Even if CVST is an infrequent cause of stroke, clinicians should consider it in the diagnostic approach of women treated with SERMs, presenting with signs of endocranial hypertension and/or focal neurological deficits.

References:

- M. Capecchi, M. Abbattista and I. Martinelli; Cerebral venous sinus thrombosis. J Thromb Haemost (2018); 16: 1918-31

- A.E.A. Dahm, N. Iversen, B. Birkenes, A. Hansen Ree and P.M. Sandset; Estrogens, selective estrogen receptor modulators, and a selective estrogen receptor down-regulator inhibit endothelial production of tissue factor pathway inhibitor 1; BMC Cardiovasc Disord. (2006);6:40

\section{LONGITUDINALLY EXTENSIVE MYELITIS: LOOKING THROUGH THE LUNG}

\author{
A. Cicia, M. Lucchini
}

Institute of Neurology, Catholic University of the Sacred Heart (Roma)

Neurosarcoidosis is an uncommon clinical entity, even in patients with systemic sarcoidosis, and it can arise with a wide variety of clinical pictures. Its presentation as a longitudinal extensive myelitis is rare and difficult to be identified, as it may mimic other inflammatory neurologic diseases such as multiple sclerosis (MS) and neuromyelitis optica spectrum disorder (NMOSD). We report the case of a 60-year-old patient with sensitive disturbances and pyramidal signs, with no past medical history nor any other systemic symptoms. The spinal MRI demonstrated a longitudinally extensive transverse myelitis with a characteristic dorsal subpial and central canal enhancement which marked out, in axial sequencies, the so-called "trident sign". A chest CT scan, performed to exclude paraneoplastic or systemic infiltrative diseases, showed 
bilaterally multiple enlarged lymph nodes with central colliquation in mediastinal and hilar stations. The histopathological examination of transbronchial needle biopsy showed non-caseating granulomatous lymphadenitis, consistent with the diagnosis of sarcoidosis. The patient was treated with methylprednisolone and mycophenolate mofetil, with a significant improvement of the clinical picture and the almost complete disappearance of the inflammatory lesion in the six following months; no relapses occurred in the two following years. The diagnostic process in neurosarcoidosis may be challenging. Laboratory analyses have low sensibility and specificity (e.g. hyperprotidorrachia in cerebrospinal fluid or serum ACE elevation) and sometimes are even confounding, (e.g. anti-AQP-4 IgG positivity, which could represent a false positive or reflect an overlapping NMOSD on neurosarcoidosis). The histological examination of involved sites is necessary to a definite diagnosis. Given the absence of univocal laboratory, clinical and imaging findings, our case suggests that the detection of the trident sign on spinal MRI in patients with subacute myelitis represents an important clue to widen the diagnostic process and raise suspicion of sarcoidosis.

References:

- Flanagan EP, Kaufmann TJ, Krecke KN, Aksamit AJ, Pittock SJ, Keegan BM, et al. Discriminating long myelitis of neuromyelitis optica from sarcoidosis. Annals of Neurology (2016) Mar;79(3):437-47

- Zalewski NL, Krecke KN, Weinshenker BG, Aksamit AJ, Conway $\mathrm{BL}, \mathrm{McKeon} \mathrm{A}$, et al. Central canal enhancement and the trident sign in spinal cord sarcoidosis. Neurology (2016) Aug 16;87(7):743-4

- Jolliffe EA, Keegan BM, Flanagan EP. Trident sign trumps Aquaporin-4-IgG ELISA in diagnostic value in a case of longitudinally extensive transverse myelitis. Multiple sclerosis and Related Disorders (2018) Jul;23:7-8

\section{JAK2-MUTATION THROMBOCYTHEMIA AS A RISK FACTOR FOR CEREBRAL AND CARDIOLOGICAL ISCHAEMIC EVENTS}

\section{P. Cimino, M. Melis, G. C. Primicerio, L. Polizzi, G. Defazio}

Department of Medical Science and Public Health, Neurology Unit, University of Cagliari (Cagliari)

Background and aims: Essential thrombocythemia (ET) is an often underdiagnosed myeloproliferative disorder associated with an increased risk of thrombotic and haemorrhagic events [1] and early diagnosis is fundamental for their prevention. A gain of function mutation of Janus kinase 2 (JAK2) gene, caused by the exchange of valine by phenylalanine at position $617(\mathrm{~V} 617 \mathrm{~F})$, has been identified in $55 \%$ of patients with ET $[1,2]$.

Materials and methods: We present a 51-year-old male patient admitted to the hospital because of acute onset of dysarthria and facial palsy. A similar transient event had been described the day before. The patient did not report risk factors for cerebrovascular disease except previous smoking habit and new detection of thrombocythemia, blood hypertension and hypercholesterolemia. Brain MRI highlighted several acute ischemic lesions in the vascular territory of the right middle cerebral artery (MCA). Carotid ultrasound did not reveal significant stenosis while transcranial doppler ultrasound (TCD) and CT angiography (CTA) of intracranial vessels demonstrated a segmental stenosis $>70 \%$ of right MCA in M1 tract. No laboratory evidence of autoimmune diseases or coagulopathies was found. The presence of IgG against Varicella-Zoster virus was detected, but IgM and viral DNA were both negative. Double antiplatelet therapy with aspirin and clopidogrel was started together with smoking cessation and pharmacological treatment of blood hypertension and hypercholesterolemia. Cardiological investigations showed a silent chronic ischemic heart disease, and angioplasty with coronary stent implant was therefore performed in election. Molecular biology analysis identified the JAK2 V617F mutation. A cytoreductive therapy with hydroxyurea was introduced because of a platelet count of $950,000 /$ microL. Three-monthtreatment with hydroxyurea and double antithrombotic therapy yielded partial recanalization of the right MCA at sonographic check.

Discussion and conclusion: This case report confirms that ET may increase the risk of both cerebrovascular and cardiological ischaemic events, regardless of other possible vascular risk factors. Early diagnosis and adequate therapy of ET are crucial to reduce relapse and limit morbidity and mortality in this population $[1,3]$.

References:

1. Tania Filipa Pereira Batista, Paula Ferreira Manuel, Antonio Carvalho Correia Essential thrombocythemia- a predisponent factor for stroke Rev Assoc Med Bras (2019);65(6):772-74

2. E. Ong, F. Barraco, N. Nighoghossian, A. Praire, V. Desestret, L. Derex, A. Vighetto, D. Biotti Cerebrovascular events as presenting manifestations of Myeloproliferative Neoplasm Revue Neurologique (2016) 172: 703-08

3. Yuji Kato, Takeshi Hayashi, Yoshihide Sehara, Ichiro Deguchi, Takuya Fukuoka, Hajime Maruyama, Yohsuke Horiuchi, Yuito Nagamine, Hiroyasu Sano and Norio Tanahashi Ischemic Stroke with Essential Thrombocythemia: A Case Series Journal of Stroke and Cerebrovascular Diseases (2015);24: 890-93

\section{RHEUMATOID ARTHRITIS PRESENTING AS ASEPTIC LEPTOMENINGITIS: A CASE REPORT}

\author{
E. Cioffi, G. Di Pietro, F. Pauri, F. Fattapposta
}

Department of Human Neurosciences, Sapienza University of Rome (Roma)

Introduction: Rheumatoid arthritis (RA) is one of the most prevalent chronic inflammatory diseases. It primarily involves joints, but it can include extraarticular manifestations, such as pulmonary involvement or vasculitis. Also, it can manifest with a range of neurologic abnormalities: cervical spine instability, carpal tunnel syndrome, peripheral vasculitis of vasa nervorum, and sensorimotor axonal neuropathy. Rare central nervous system manifestations are represented by central rheumatoid vasculitis and rheumatoid meningitis [1,2].

Methods: We report the case of a 79-year old female patient suffering from Leber Hereditary Optic Neuropathy presenting with a subacute decline of cognitive functions and right-sided tonic involuntary movements. After admission, examination revealed a right-sided facio-brachial weakness (MRC 4/5), brisk reflexes and bilateral Hoffmann sign, recurrent tonic involuntary movements of the right arm, motor impersistence and mnesic deficit (MMSE 17/30). A brain MRI showed a leptomeningeal enhancement in right frontal, bilateral cingulate and left parietal cortex consistent with leptomeningitis. The patient underwent: lumbar puncture comprehensive of cytochemical, infective and anatomopathological study; EEG; screening for paraneoplastic and autoimmune etiologies (TC total-body, thyroid echography, mammography, transvaginal echography, tumor markers, serologic autoimmune panel, dermatologic, oculistic and rheumatologic examination). 
Results: CSF revealed 8 nucleated cells; EEG, TC total-body, mammography, transvaginal echography, tumor markers, dermatologic and oculistic evaluations were normal. Thyroid echography showed a lesion proved to be a TIR 2 on fine-needle aspiration. Autoimmune screening disclosed only a high titer of anti-rheumatoid factor (anti-RF) and anticyclic citrullinated peptide (anti-CCP) associated with elevated Erythrocyte Sedimentation Rate and C-reactive Protein. ACE levels were normal.

Discussion: Given the evidence of an aseptic leptomeningitis, the exclusion of other possible neoplastic or autoinflammatory conditions and the presence of anti-RF and anti-CCP antibodies, a possible rheumatoid meningeal pathology was hypothesized. Intravenous prednisone was started followed by oral prednisone tapering dose. A meningeal biopsy was planned but not performed due to the progressive clinical improvement. Biopsy, in other literature cases, did not show specific pathological hallmarks [3]. Treatment led to stable remission of previously noted neurological deficit. A 3 months follow-up brain MRI showed a complete resolution.

Conclusion: In our case, in the setting of an aseptic leptomeningitis associated with anti-RF and anti-CCP antibodies, the clinical-radiological improvement after steroids administration and the exclusion of other possible hypotesis led us to the diagnosis of Rheumatoid leptomeningitis. Even if rare, Rheumatoid leptomeningitis should be considered in the diagnostic workup of aseptic leptomeningitis, as this could be the early and unique manifestation of this rheumatologic pathology.

References:

1. Bhattacharyya S, Helfgott SM. Neurologic complications of systemic lupus erythematosus, Sjögren syndrome, and rheumatoid arthritis. Semin Neurol (2014);34:425

2. Nadeau SE. Neurologic manifestations of connective tissue disease. Neurol Clin. (2002); 20:151

3. I. Padjen, M. Mayer, M. Habek, D. Kolenc, S. Dotlic. Redefining a diagnosis: from meningeal plasma cell granuloma to rheumatoid meningitis. Report of a patient follow-up. Neurol Sci. (2015);36:1047-48

\section{PARANEOPLASTIC IMMUNE-MEDIATED NECROTIZING MYOPATHY PRESENTING WITH THIGHS INVOLVEMENT: A CASE REPORT OF A B-CELL LYMPHOMA}

\section{E. Cipriano, D. Vecchio, C. Varrasi, R. Bruna, S. Pittau, R. Cantello}

Department of Traslational Medicine, University of Piemonte Orientale (Novara)

Background: Immune-mediated necrotizing myopathies (IMNMs) are recognized among the idiopathic inflammatory myopathies. The paraneoplastic form is reported in less than 100 cases worldwide. Diagnosis is supported by clinical symptoms, muscle biopsy, serum creatine kinase (CK) level, specific antibodies, electromyography (EMG) and imaging. Our case is paraneoplastic IMNM at diagnosis of B-cell lymphoma.

Case Description and Requested Exams: A fifty-four years old Chinese patient presented to emergency department for typical atrial flutter and described an history of two months persistent cough, mild serotine fever, night sweating, weight loss and symmetric weakness at the lower limbs with walking difficulties. Chest X-Ray showed lung thickening so the patient was treated with antibiotics without benefits. Multiple blood test for infections were negative. PET showed multiple areas of accumulation in muscles. Electromyography and electroneurography were performed showing an acute myogenic suffering. EGDS showed a sessile lesion of the duodenum that was biopsied, quadriceps muscle biopsy had been executed too.

Results: Gastric biopsy diagnosed a high grade gastric B lymphoma. Quadriceps biopsy showed moderate primitive muscular suffering with necrotizing and inflammatory aspects and no direct infiltration. These muscular aspects along with clinical features and laboratory findings were suggestive for the diagnosis of IMNM associated with gastric B lymphoma. The patient was transferred to the hematology department to start a chemotherapy. First cycle of treatment reduced CK and lactate but considering the idea of a paraneoplastic IMNM he is actually on prednisolone $25 \mathrm{mg}$ die.

Discussion: IMNM in the context of tissue disease may be severely underdiagnosed. Often the first symptoms of IMNM comes after the diagnosis of malignancies or during the chemotherapy. Instead in our case weakness and elevated CK where relevant from the beginning. Only with PET and duodenal biopsy it was impossible to understand if the muscular accumulation was sign of a secondary infiltration of the lymphoma or a muscular primitive pathology. In cases like this double biopsy is mandatory to allow using steroid after chemotherapy.

Conclusion: In our case walking difficulties were the first symptoms of paraneoplastic myositis in the context of B cell lymphoma. Considering in the diagnosis not only malignant infiltration of the muscle but also IMNM is challenging but must be done. An early recognition of the skeletal muscle deficit along with other typical organ involvement are crucial findings to a correct framing of the disease, and if B-symptoms are presents, searching for tumor is mandatory to start early the chemotherapy.

References:

- W Stenzel, H-H Goebel, E Aronica, Review: immune-mediated necrotizing myopathies-A heterogeneous group of diseases with specific myopathological features. Pathol Appl Neurobiol, (2012) Dec, 38(7):632-46

- Pinal-Fernandez, M. Casal-Dominguez, A. L. Mammen, ImmuneMediated Necrotizing Myopathy. Curr Rheumatol Rep. (2018) Mar; 20(4):21

\section{MAGNETIC RESONANCE SPECTROSCOPY AS A MARKER OF RESPONSE TO DOPAMINERGIC TREATMENT IN PARKINSON'S DISEASE}

R. Ciurleo, L. Bonanno, F. Corallo, S. Marino, G. Di Lorenzo, P. Bramanti

Neurobioimaging Laboratory, IRCCS Centro Neurolesi Bonino-Pulejo (Messina)

Objective: To evaluate the influence of dopaminergic therapy on metabolic profile of cortico-basal ganglia loops in de novo patients with Parkinson's Disease (PD) by using proton Magnetic Resonance Spectroscopy (1H-MRS).

Background: PD is the most common neurodegenerative movement disorder; its treatment is symptomatic. One of the major challenges in PD is the development of disease-modifying therapies, such as neuroprotective agents. Few dopaminergic drugs, among which monoamine oxidase $\mathrm{B}$ inhibitors and even levodopa, have been proposed to have a diseasemodifying effect. So far, the main outcome measure used in clinical trials to detect disease progression has been the motor part of the UPDRS-III, which does not allow to distinguish between symptomatic and diseasemodifying drug effects. MRS is a noninvasive imaging technique for 
exploration in vivo of intracellular metabolic status, and may provide a neuroimaging objective biomarker of pathological processes or response to therapeutic intervention in PD.

Materials/Methods: We enrolled PD patients who were divided into 6 groups of 20 patients each, according to the dopaminergic treatment prescribed at the baseline visit (levodopa, ropinirole, pramipexole, rotigotine, rasagiline and selegiline). At the baseline visit and 6,12 and 18 months after, all patients underwent clinical and neuropsychological evaluations as well as structural MRI and 1H-MRS. Healthy controls underwent MR evaluation at baseline and 18 months after.

Results: PD patients, compared to healthy controls, showed significantly lower concentrations of NAA, Cho and mIn and significantly higher concentrations of Glu in the globus pallidus. NAA concentration was significantly lower in patients than in healthy controls in the motor and prefrontal cortices, while Cho level showed a trend to decrease only in the motor cortex. After dopaminergic treatment, at all follow-ups we observed a significant increase in levels of NAA, Cho and mIn for patients treated with levodopa, rasagiline and selegiline and a significant decrease in Glu level for patients treated with levodopa and rasagiline in the globus pallidus. At all follow-ups, NAA level in the motor and prefrontal cortices was increased significantly for patients treated with levodopa, rasagiline and selegiline and Cho level showed in the motor cortex a trend to increase only for patients treated with rasagiline and selegiline. In these 3 patient groups, NAA concentration change correlated positively with UPDRS-III scores.

Discussion/Conclusions: Levodopa, rasagiline and selegiline induce a significant and long-term restoration of neurometabolites, suggesting a possible neuroprotective role that could be responsible in turn for the partial recovery of cortico-ganglia-basal functions.

References:

- Olanow CW, Rascol O, Hauser R, Feigin PD, Jankovic J, Lang A, Langston W, Melamed E, Poewe W, Stocchi F, Tolosa E; ADAGIO Study Investigators. A double-blind, delayed-start trial of rasagiline in Parkinson's disease. N Engl J Med. (2009) Sep 24;361(13):126878

- Fahn S. A new look at levodopa based on the ELLDOPA study. J Neural Transm Suppl. (2006);(70):419-26

- Pålhagen S, Heinonen E, Hägglund J, Kaugesaar T, Mäki-Ikola O, Palm R; Swedish Parkinson Study Group. Selegiline slows the progression of the symptoms of Parkinson disease. Neurology (2006) Apr 25;66(8):1200-6

\section{CREUTZFELDT-JAKOB DISEASE (CJD) PRESENTING AS CORTICAL BASAL SYNDROME (CBS): A CASE REPORT}

\author{
F. Colucci ${ }^{1}$, G. Rosa $^{2}$, A. De Vito ${ }^{3}$
}

${ }^{1}$ Department of Biomedical and Specialist Surgical Sciences, Section of Neurological, Psychiatric and Physicological Science, St. Anna Hospital, University of Ferrara (Ferrara); ${ }^{2}$ Biomedical and Specialty Surgical Sciences, University of Ferrara (Ferrara); ${ }^{3}$ Unit of Neurology, Ferrara University Hospital (Ferrara)

Background: The cortical basal syndrome is a combination of an asymmetric parkinsonian syndrome with cortical signs. These symptoms might underlie different etiologies as Cortical-basal Degeneration (CBD), Fronto-Temporal Dementia (FTD), Progressive Supranuclear Palsy (PSP), Alzheimer's disease (AD), vascular and paraneoplastic encephalopathies. We report a case of CBS later diagnosis as probably sporadicCJD. Therefore, CJD should be included within the differential diagnosis.
Case presentation: A seventy-six-year-old Italian female, affected by depression and subclinical hypothyroidism, had a 2-month history of progressive gait disturbance associated with global slowness, left upper limb jerky-tremor, and cognitive-behavioral changes, mainly memory loss and "telegraphic" speech. On depth examination, she had extrapyramidal features (akinesia and general plastic rigidity, left > right), left limbs stimulus-sensitive myoclonus, left alien foot phenomena, gaze and bilateral upper limbs apraxia (mainly on the left side), hyperreflexia, and startle reaction to tactile face stimuli. On admission, levodopa did not improve the symptoms; neurophysiology studies showed exaggerated left-limbs Long Loop Reflexes (LLR) to ulnar nerve stimulation, absence of both giant somesthetic evoked potentials and cortical potentials preceding myoclonus in back-averaging studies; MRI detected diffuse high signal intensity on T2-weighted images and restriction on DWI sequences in the cerebral cortex, mainly on the right hemisphere. CJD was suspected and the CSF analysis showed elevated Tau-protein levels and normal 143-3 protein levels, using the ELISA test.

Results: During admission, the patient had a rapid cognition and gait abilities decline. She was bedridden, dumb, and unresponsive. The SCJD suspect was high, and the CSF analysis through RT-QuIC detected a higher 14-3-3 protein level. The patient was discharged into hospice care and she died one month later. Brain biopsy is still ongoing.

Conclusions: We reported a case of probably SCJD, initially mimicking CBS. The two diseases share many of the same symptoms. Therefore our findings suggest that the presence of progressive dementia associated with limb apraxia, extrapyramidal features, and involuntary movements should prompt a screen for CJD. From literature, there are often long delays between the symptoms onset and the diagnosis of SCJD, probably due to the variability and unspecificity of early symptoms and signs. RTQuIC might help in the diagnosis.

References:

- Boeve B.F., Lang A.E., Litvan I. Corticobasal degeneration and its relationship to progressive supranuclear palsy and frontotemporal dementia. Ann. Neurol. (2003);54:S15-S19

- Paterson RW, Torres-Chae CC, Kuo AL, et al. Differential diagnosis of Jakob-Creutzfeldt disease. Arch Neurol. (2012);69(12):1578-82

- Green AJE. RT-QuIC: a new test for sporadic CJD. Pract Neurol. (2019);19(1):49-55

\section{COVID-19 IN A MULTIPLE SCLEROSIS PATIENT TREATED WITH OCRELIZUMAB}

\author{
M. Z. Conti, M. Radaelli, V. Barcella, M. Grimoldi, M. Rottoli, M. Sessa
}

Neurology, ASST Papa Giovanni XXIII (Bergamo)

Objective: The emergence of the COVID-19 pandemic represents a new challenge for neurologist treating Multiple Sclerosis (MS), due to the immunosuppressive effect of some disease-modifying therapies (DMT). We describe a case of COVID-19 pneumoniae in a patient with MS treated with ocrelizumab, a B-cell depleting therapy.

Materials and Methods: Case report

Results: R.M.is a 43-year old woman affected by relapsing remitting MS since 2006. No other comorbidity are reported. She was treated with glatiramer acetate till 2011 and then with Natalizumab till 2018 (this treatment was stopped for high PML risk). She started ocrelizumab on december 2018. She received three courses drug, the last infusion was performed on December 2019. On 23 February 2020 she developed fever, cough and diarrhea. She was initially treated at home with oral 
azithromycin without any improvement therefore she was admitted on pneumological ward on 31 March. The CT chest revealed an interstitial pneumoniae. The diagnosis was confirmed trough positive SARSCoV-2 RT-PCR in bronchoalveolar lavage fluid obtained by bronchoscopy due to repeated negative nasopharyngeal swabs and she was treated with i.v. ceftriaxone $2 \mathrm{gr} / \mathrm{die}$, i.v. methylprednisolone $80 \mathrm{mg}$, hydroxychlorokine, low-molecular-weight-heparin and supported by oxygen therapy at low flow (4LO2/min). She was discharged on 8 April and again hospidalized on 17 April because of recurrence of fever and dyspnea. The chest CT revealed an initial improvement of the pneumonia. She received an additional course of oral azithromycin $500 \mathrm{mg} /$ die for 8 days and oral steroid with complete remission without any sequaele within two weeks. Anti SARS-CoV2 IgG antibodies tested on 26 of June were negative. At last haematological evaluation performed on June 19 the CD20 were $0 \%$, immunoglobulin level was normal. No clinical and radiological reactivation of MS was observed.

Discussion and conclusions: The use of antiCD20s therapies during the pandemic phase of SARS-CoV-2 have raised major concerns. The good outcome of our patient showed that despite the use of ocrelizumab she was able to have an adequate immuneresponse to the infection. However the absence of antibodies against SARS-CoV2 can be the results of the selective B-cell depleting effect of the drug and might have possible implication on the risk of a reinfection and of the humoral response to vaccination.

\section{References:}

- V. Meca-Lallana, C. Aguirre, B. Rio, L. Cardenoso, T. Alarcon, J. Vivancos. COVID-19 in 7 multiple sclerosis patients in treatment with anti-CD20 therapies. Multiple Sclerosis and Related Disorders (2020) Jun 15; 44:102306

- R. Hughes, R. Pedotti, H. Koendgen. COVID-19 in persons with multiple sclerosis treated with ocrelizumab - A pharmacovigilance case series. Multiple Sclerosis and Related Disorders. (2020) Jul; 42: 102192

- G. Novi, M. Mikulska, F. Briano, F. Toscanini, F. Tazza, A. Uccelli, M. Inglese. COVID-19 in a MS patient treated with ocrelizumab: does immunosuppression have a protective role? Multiple Sclerosis and Related Disorders (2020) Jul;42:102120

\section{MULTI-ORGAN INVOLVEMENT AFTER A MILD COVID-19 ACUTE PHASE IN AN ELDERLY AND FRAIL PATIENT: A CASE REPORT}

M. Cortinovis ${ }^{1}$, F. Schiano di Cola ${ }^{1}$, A. Scalvini ${ }^{1}$, R. Rao ${ }^{1}$, L. Rozzini ${ }^{1}$, A. Castiello ${ }^{2}$, P. Garyfallidis ${ }^{2}$, G. Giovannini ${ }^{1}$, M. Mutti ${ }^{2}$, E. Baldelli ${ }^{1}$, M. Benini ${ }^{3}$, A. Benussi ${ }^{1}$, S. Cotti Piccinelli ${ }^{1}$, M. Gamba ${ }^{4}$, S. Gipponi ${ }^{1}$, I. Libri $^{1}$, S. Masciocchi ${ }^{1}$, A. Pezzini ${ }^{1}$, A. Pilotto ${ }^{1}$, L. Poli ${ }^{1}$, E. Premi ${ }^{4}$, I. Volonghi $^{1}$, R. Gasparotti ${ }^{5}$, M. Ferrara ${ }^{5}$, E. Gavazzi ${ }^{6}$, R. Maroldi ${ }^{6}$, A. Padovani $^{1}$

\footnotetext{
${ }^{1}$ Department of Clinical and Experimental Sciences, Neurology Clinic, University of Brescia (Brescia); ${ }^{2}$ Cardiology Consultancy Service, Spedali Civili Hospital, ASST Spedali Civili (Brescia); ${ }^{3}$ Department of Clinical and Experimental Sciences, Neurology Clinic, University of Bologna (Bologna); ${ }^{4}$ Vascular Neurology, Stroke Unit, Spedali Civili Hospital, ASST Spedali Civili, (Brescia); ${ }^{5}$ Neuroradiology Unit, Spedali Civili Hospital, ASST Spedali Civili (Brescia); ${ }^{6}$ 2nd Radiology Unit, Spedali Civili Hospital, ASST Spedali Civili (Brescia)
}

COVID-19 has become an important public health issue and has been linked to multi-organ involvement through systemic inflammation. Frail people are particularly at risk of death through the acute phase of the disease mainly due to respiratory distress syndrome (ARDS). Despite much had been written about the acute phase, little is known about proinflammatory state post COVID-19. Here we describe the case of a multimorbid frail 86-year old patient that suffered from a mild form of COVID-19 forty days before, presenting to the emergency department for acute ischaemic stroke with concomitant pulmonary embolism and myopericarditis. The three diseases were then assumed to be related to the previous SARS-CoV-2 infection. This case highlights that also when the viral infection is resolved, a generalized inflammatory status may still be ongoing, potentially harming CNS, heart and the vascular system, even after a mild acute course of disease. Patient who had suffered from COVID-19, especially the elderly with high comorbidities, should be closely monitored to prevent and treat thrombotic and inflammatory complications.

\section{HYPERACUTE EXTENSIVE SPINAL CORD INFARCTION AND NEGATIVE SPINE MAGNETIC RESONANCE IMAGING: A TIME-SENSITIVE MATTER}

G. Costamagna ${ }^{1}$, M. Meneri ${ }^{2}$, E. Abati ${ }^{1}$, R. Brusa ${ }^{2}$, D. Velardo ${ }^{2}$, D. Gagliardi ${ }^{1}$, E. Mauri ${ }^{2}$, C. Cinnante ${ }^{3}$, N. Bresolin ${ }^{1}$, G. Comi ${ }^{1}$, S. Corti ${ }^{1}$, I. Faravelli ${ }^{1}$

${ }^{1}$ Department of Pathophysiology and Transplantation, University of Milan (Milano); ${ }^{2}$ Neurology Unit, Foundation IRCCS Ca' Granda Ospedale Maggiore Policlinico (Milano); ${ }^{3}$ Neuroradiology Unit, Foundation IRCCS Ca' Granda Ospedale Maggiore Policlinico (Milano)

A 51-year-old woman presented to our Emergency Department with acute severe abdominal pain, nausea, vomiting, sudden-onset of bilateral leg weakness with diffuse sensory loss, and paresthesia on the trunk and legs. She denied recent vaccinations, infections, traumatic injuries, strenuous physical exertion, and Valsalva maneuver before symptom onset. ECG, blood tests, toxicology screen, brain CT scan, and aortic CT angiography were normal. On neurological examination, she showed severe paraparesis and a T6 sensory level. CSF analysis was unremarkable. A spine MRI with gadolinium performed at 9 hours after symptom onset was negative. The patient was admitted to our Neurology Unit and she developed paresthesia and weakness in her left hand and forearm and an ipsilateral Horner's syndrome. Due to the persistence of a clinical picture suggestive of acute myelopathy, an MRI of the spine was repeated after 72 hours showing a "pencil-like" hyperintensity mainly involving the grey matter from $\mathrm{T} 1$ to $\mathrm{T} 6$ on $\mathrm{T} 2$ sequence, mildly hypointense on $\mathrm{T} 1$ and with restricted diffusion. Vertebral marrows from $\mathrm{C} 7$ to T4 were hyperintense on $\mathrm{T} 2$, presented gadolinium enhancement on postcontrast $\mathrm{T} 1$ and restricted diffusion. Comprehensive serologic screening for viral infections, autoimmunity (including MOG and AQP4), inherited and acquired prothrombotic states were negative. Copper, vitamin B12, and angiotensin-converting enzyme levels were normal. Cancer, systemic vasculitis, and cardiac anomalies were ruled out by appropriate diagnostic workup. A diagnosis of spinal cord infarction (SCI) was suspected. The patient was given salicylic acid $(100 \mathrm{mg} /$ day), prophylactic lowmolecular-weight heparin, and began neuromotor rehabilitation. Two months later, a follow-up neurological examination revealed a severe spastic paraparesis, no evident sensory level and poor sphincteric control with a distended bladder. At 18 months, she was able to walk with assistance and recovered partial sphincter control.

Discussion: SCI accounts for only 1-2\% of all ischemic strokes and 5$8 \%$ of acute myelopathies [1]. Regardless of its relative infrequence, SCI should be suspected in every patient with acute myelopathic symptoms, 
even without associated vascular risk factors. MRI is important in ruling out other causes and in diagnosing SCI. However, sensitivity and specificity of spine MRI with T2-weighted imaging and DWI may be imperfect in confirming the diagnosis, even with extensive spinal cord lesions and particularly in the hyperacute setting [2]. Thus, the patient's history and careful examination suggesting a potential vascular syndrome involving the spinal cord override an initially negative MRI and should not delay timely and appropriate management.

References:

1. Vargas, M. I. et al. Advanced magnetic resonance imaging (MRI) techniques of the spine and spinal cord in children and adults. Insights Imaging (2018);9:549-57

2. Kramer, C. L. Vascular Disorders of the Spinal Cord. Continuum (Minneap Minn) (2018);24:407-26

\section{ISOLATE HYPOGLOSSAL NERVE PALSY AS RARE CAUSE OF CERVICAL OSTEOPHYTE}

\author{
M. S. Cotelli ${ }^{1}$, B. Borroni ${ }^{2}$, M. Stivala ${ }^{3}$, F. Manelli ${ }^{4}$, P. Donati ${ }^{4}$, M. \\ Michelini $^{4}$, F. Uva ${ }^{4}$, M. Bianchi ${ }^{1}$, P. Civelli ${ }^{1}$, V. Bertasi ${ }^{1}$, A. \\ Padovani ${ }^{2}$, M. Turla ${ }^{1}$
}

${ }^{1}$ Neurology Unit, Azienda Socio Sanitaria Territoriale Valcamonica Esine (Esine-BS); ${ }^{2}$ Department of Clinical and Experimental Sciences, Center for Neurodegenerative Disorders, Neurology Unit, University of Brescia (Brescia); ${ }^{3}$ Neurosurgery Unit, Spedali Civili di Brescia (Brescia); ${ }^{4}$ Emergency Unit, Azienda Socio Sanitaria Territoriale Valcamonica Esine (Esine-BS)

Introduction: The hypoglossal nerve is a pure motor nerve that innervates intrinsic as well as extrinsic muscles of the tongue. The nerve can be damaged anywhere during its course. Localizing the lesion could allow the clinician to narrow possible etiological factors. Isolate hypoglossal nerve palsy (HNP) is a rare finding, because of its proximity to other important anatomical structures throughout its pathway, and is described in several case reports and a very small case series. The literature reports multiple IHP etiologies. Skull base tumor (metastasis, or primitive malignant or benign tumor) is the most frequent. Trauma is the second, comprising cranial trauma (occipital condyle fracture) sustained in road accidents and surgical trauma (neck surgery, prolonged cervical hyperextension during anesthesia). Thirdly, vascular etiologies notably comprise medial carotid artery dissection. Joint and auto-immune pathologies (cervical rheumatoid arthritis, occipito-atloid cyst) and occipital hinge deformity (Arnold-Chiari malformation) have also been implicated. In children and adolescents, infectious mononucleosis should be investigated. Finally, in certain cases no etiology can be determined.

Materials and Methods: We report the case of a 64 years-old woman, evaluated due to gradually progressive slurred speech and swallowing deficits. Neurological examination showed tongue deviation to the left on protrusion, with left 12 th cranial nerve palsy. There was no involvement of 9th, 10th or 11th cranial nerves.

Results: Brain Magnetic Resonance Imaging was unremarkable. Cervical Magnetic Resonance Imaging and Computer Tomography scan of the neck showed cervical osteophyte (C2) facing the left hypoglossal canal. Elettroneurography confirmed isolate unexcitability of left 12th nerve. Neurosurgery approach was discussed, but then excluded for surgical risk.

Conclusion: In conclusion, HNP is infrequent and usually symptomatic of skull base pathology. To the best of our knowledge, only two few reports have been published concerning isolated hypoglossal compression by osteophyte, together with two cases of a small series.

Discussion: History-taking and meticulous examination guide rational selection of the radiological examinations (combination of magnetic resonance imaging and high resolution computer tomography scan of the skull base) is key to diagnose this rare cause of 12 th cranial nerve palsy. References:

- Patron V, Roudaut PY, Lerat J, Vivent M, Bessède JP, Aubry K. Isolated hypoglossal palsy due to cervical osteophyte. Eur Ann Otorhinolaryngol Head Neck Dis. (2012) Feb;129(1):44-6

- Boban M, Brinar VV, Habek M, Rados M. Isolated hypoglossal nerve palsy: a diagnostic challenge. Eur Neurol. (2007);58(3):17781

- Patro SN, Torres C, Riascos R. An unusual case of isolated hypoglossal nerve palsy secondary to osteophytic projection from the atlanto-occipital joint. Neuroradiol J. (2014) Jun;27(3):361-4

\section{CEREBELLAR AGENESIS IN A LIVING PATIENT: CASE REPORT}

M. S. Cotelli ${ }^{1}$, B. Borroni ${ }^{2}$, P. Lavezzi ${ }^{3}$, F. Manelli $^{4}$, N. Del Villano ${ }^{4}$, G. Tomasini $^{4}$, M. Michelini ${ }^{4}$, M. Bianchi ${ }^{1}$, P. Civelli ${ }^{1}$, V. Bertasi ${ }^{1}$, R. Furloni $^{5}$, A. Padovani ${ }^{2}$, M. Turla ${ }^{1}$

${ }^{1}$ Neurology Unit, Azienda Socio Sanitaria Territoriale Valcamonica (Esine-BS); ${ }^{2}$ Department of Clinical and Experimental Sciences, Center for Neurodegenerative Disorders, Neurology Unit, University of Brescia (Brescia); ${ }^{3}$ Radiology Unit, Azienda Socio Sanitaria Territoriale Valcamonica (Esine-BS); ${ }^{4}$ Emergency Unit, Azienda Socio Sanitaria Territoriale Valcamonica (Esine-BS); ${ }^{5}$ Medicine Unit, Azienda Socio Sanitaria Territoriale Valcamonica (Esine-BS)

Introduction: Cerebellar agenesis (CA) is an extremely rare entity. There is a debate in the literature as to whether a normal or near normal life is possible in case of complete CA. While supporters of one side of the debate say that such patients always have severe motor deficits, others believe the myth that they may not have any observable symptoms. Most cases with CA are diagnosed in the prenatal or early postnatal period and are associated with other central nervous system abnormalities. Only few children or adults with this disorder have so far been published. A malformative or disruptive etiology may be responsible from CA. In primary $\mathrm{CA}$, there is a malformation of the developmental process; where in acquired cases, there is disruption of the cerebellum in the prenatal or perinatal period due to hemorrhage, ischemia, or other factors. CA with disruptive etiology is usually accompanied by additional brain abnormalities More recently two genes, pancreatic transcription factor $1 \mathrm{~A}$ (PTF1A), a key regulator of cerebellar neurogenesis, and collagen IV A1 (COL4A1), have been particularly implicated in predisposing to disruptive cerebellar development. Another potential cause for the infant's cerebellar agenesis is thought to be due to in utero infection.

Materials and Methods: We report a case of subtotal cerebellar agenesis associated with encephalic trunk hypoplasia in a 53 years-old Caucasian man. His familiar history was negative, while his medical history was positive for mild developmental delay, alternating exotropia, bilateral neurosensorial deafness, arterial hypertension, recurrent warts. His neurological examination showed blurred speech, alternating exotropia, hypertelorism, palatine malformation. He doesn't suffer from dysphagia, dyspnea or drooling. He also presented bilateral dysmetria and dysdiadochokinesia, ataxic gait. He used trolley walker aid and bilateral plantars. 
Results: Brain computer tomography confirmed subtotal cerebellar agenesis associated with encephalic trunk hypoplasia. Genetic analysis was not performed (he lives in a protected structure due to the absence of relatives) and magnetic resonance wasn't performed due to claustrophobia.

Discussions: We briefly reported clinical and brain computer tomography characteristics of an adult patient diagnosed with a rare entity (few case reports of living patients have been reported in literature). Patients with CA present with a variety of developmental, clinical, and mental abnormalities. Although they may lead a simple life, they suffer various degrees of cognitive, intellectual, and fine motor skill problems.

Conclusions: These cases emphasize the role of the cerebellum in normal motor, language, and mental development.

References:

- Glickstein M Cerebellar agenesis. Brain (1994) Oct; 117 ( Pt 5):1209-12

- Hamilton RL, Grafe MR Complete absence of the cerebellum: a report of two cases. Acta Neuropathol. (1994); 88(3):258-61

- Gupta A, Malik GK, Gupta A, Saksena S, Gupta RK MR demonstration of complete cerebellar and corpus callosum agenesis. Pediatr Neurosurg. (2007); 43(1):29-31

\section{RENDU OSLER WEBER DISEASE: CASE REPORT FROM A NEUROLOGICAL POINT OF VIEW}

M. S. Cotelli ${ }^{1}$, M. Scarsi ${ }^{2}$, S. Elia ${ }^{3}$, M. Bianchi ${ }^{1}$, V. Bertasi ${ }^{1}$, P. Civelli ${ }^{1}$, F. Manelli ${ }^{4}$, E. Atzeni ${ }^{4}$, M. Briganti ${ }^{4}$, P. Donati ${ }^{4}$, R. Furloni ${ }^{2}$, B. Borroni $^{5}$, A. Padovani ${ }^{5}$, M. Turla ${ }^{1}$

${ }^{1}$ Neurology Unit, Azienda Socio Sanitaria Territoriale Valcamonica (Esine-BS); ${ }^{2}$ Medicine Unit, Azienda Socio Sanitaria Territoriale Valcamonica (Esine-BS); ${ }^{3}$ Radiology Unit, Azienda Socio Sanitaria Territoriale Valcamonica (Esine-BS); ${ }^{4}$ Emergency Unit, Azienda Socio Sanitaria Territoriale Valcamonica (Esine-BS); ${ }^{5}$ Department of Clinical and Experimental Sciences, Center for Neurodegenerative Disorders, Neurology Unit, University of Brescia (Brescia)

Introduction: Osler-Weber-Rendu disease or hereditary hemorrhagic telangiectasia (HHT) is an autosomal dominant condition. Nearly $20 \%$ of the cases do not have a family history. It is characterized by multiple mucocutaneous telangiectasias, which represent small arterio-venous malformations that frequently tend to bleeds (nose bleeds, gastrointestinal bleeds), with possible iron deficiency anemia. This disease puts patients at risk for life-threatening complications from having arteriovenous malformations in several organ systems. Diagnosis of HHT is made on the basis of the Curaçao Criteria that includes the following factors: epistaxis, telangiectasia, visceral lesions and family history and is confirmed by the presence of at least three of these manifestations.

Materials and Methods: We report the case of a 68 years-old Caucasian woman who received diagnosis of HHT. She was evaluated due to recurrent epistaxis, leading to generalized weakness and severe anemia. General exam showed bilateral hand and face telangiectasias. Neurological examination resulted normal. She presented paleness of the skin and mucous membrane. Her medical history was positive for epistaxis and migraine without aura. Her familial history was unremarkable. She performed gastroscopy, which showed multiple antral angiodysplasias with mucosal erosions. Brain magnetic resonance showed A1-A2 anterior cerebral artery ectasia, with $3 \mathrm{~mm}$ aneurysm. It also showed left cerebellar venous angioma, small ischemic lacunae in right nucleus caudatus, a temporal left cerebral softening and symmetrical high signal intensity in the globi pallidi on T1-weighted MR images (possible manganese accumulation). Cortico-subcortical atrophy was also reported. Abdomen ultrasound revealed hepatic hemangioma in segment VII. Abdomen tomography confirmed the presence of bilateral arteries ectasia. She performed neuropsychological evaluation was normal except for visuospatial and attentive functions, which were compromised. Blood exams before blood transfusion showed marked anemia.

Results: On the basis of Curaçao Criteria diagnosis of definite HHT was performed.

Conclusions and Discussions: We reported a sporadic case of OslerWeber-Rendu Disease. We suggested to our patient to evaluate to perform genetic evaluation even if all relatives have been reported as asymptomatic. HHT should be suspected when evaluating patients with persistent epistaxis, expecially when leading to acute anemia. Neurologists should evaluate familial history for cerebrovascular disorders or malformations, pathological history of atypical headache or cerebrovascular disorders, and should suggest the use of brain magnetic resonance for cerebrovascular malformations screening in adults with possible or definite HHT using a protocol with and without contrast administration and using sequences that detect blood products, to maximise sensitivity as recommended by international guidelines.

References:

- Macri A, Wilson AM, Shafaat O, Sharma S. Osler-Weber-Rendu Disease (Hereditary Hemorrhagic Telangiectasia, HHT). StatPearls [Internet]. Treasure Island (FL): StatPearls Publishing; (2020) Jan-. 2019 Dec 2

- Labeyrie PE, Courthéoux P, Babin E, Bergot E, Touzé E, Pelage JP. Neurological involvement in hereditary hemorrhagic telangiectasia. J Neuroradiol. (2016) Jul;43(4):236-45

- Brinjikji W, Iyer VN, Sorenson T, Lanzino G. Cerebrovascular Manifestations of Hereditary Hemorrhagic Telangiectasia. Stroke (2015) Nov;46(11):3329-37

\section{TREATMENT OF EPILEPTIC STATUS WITH LACOSAMIDE IN A PATIENT WITH POLYCYSTIC LIPOMEMBRANOUS OSTEODYSPLASIA WITH SCLEROSING LEUKOENCEPHALOPATHY (PLOSL)}

M. S. Cotelli ${ }^{1}$, R. Furloni ${ }^{2}$, M. Bianchi ${ }^{1}$, P. Civelli ${ }^{1}$, V. Bertasi ${ }^{1}, \mathrm{~F}$. Manelli $^{3}$, N. Del Villano ${ }^{3}$, M. Michelini ${ }^{3}$, F. $\mathrm{Uva}^{3}$, G. Tomasini ${ }^{3}$, B. Borroni $^{4}$, A. Padovani ${ }^{4}$, M. Turla ${ }^{1}$

${ }^{1}$ Neurology Unit, Azienda Socio Sanitaria Territoriale Valcamonica (Esine-BS); ${ }^{2}$ Medicine Unit, Azienda Socio Sanitaria Territoriale Valcamonica (Esine-BS); ${ }^{3}$ Emergency Unit, Azienda Socio Sanitaria Territoriale Valcamonica (Esine-BS); ${ }^{4}$ Department of Clinical and Experimental Sciences, Center for Neurodegenerative Disorders, Neurology Unit, University of Brescia (Brescia)

Introduction: Nasu-Hakola disease (NHD; polycystic lipomembranous osteodysplasia with sclerosing leukoencephalopathy or PLOSL) is a rare disease characterized by bone cysts and fractures, frontal lobe syndrome, and progressive presenile dementia. NHD may be the prototype of primary microglial disorders of the CNS or, as they have been coined, "microgliopathies". Diagnosis can be established on the basis of clinical and radiological findings. Molecular analysis of affected families revealed mutations in the DAP12 (TYROBP) or TREM2 genes. The clinical course of PLOSL can be divided into four stages: (1) The latent stage is characterized by normal early development. (2) The osseous stage is 
characterized by pain and tenderness, usually following strain or injury. Fractures are typically diagnosed several years later, most commonly in the bones of the extremities. (3) In the early neurologic stage, a change of personality begins to develop insidiously. Affected individuals show a frontal lobe syndrome leading to serious social problems. (4) The late neurologic stage is characterized by progressive dementia and loss of mobility. Death usually occurs before age 50 years.

Materials and Methods: We report the case of a 49 years-old Caucasian affected by PLOSL stage 4 (late neurological stage) whose clinical history was positive for epilepsy treated with valproic acid $100 \mathrm{mg}$ bid and levetiracetam $1000 \mathrm{mg}$ at 8 hour and 1500 at 20 hours. She had been recently in-hospitalized due to right lower limb dermohypodermitis, Clostridioides Difficile infection plus Pseudomonas Aeruginosa sepsis. She was in-hospitalized due to persistent involuntary movements at right hemisoma. Brain computer tomography without contrast showed cortical atrophy associated with basal ganglia calcifications. Electroencephalography was suitable partial convulsive epileptic status. Blood electrolytes resulted altered (hypokaliemia and hyponatremia). Neurological examination showed global aphasia, spastic tetraparesis with lower limbs deformities, cachexia, brisk deep tendon reflexes and bilateral extensor plantar, Frontal release signs were all strongly positive.

Results: First attempt to treat epileptic status was performed with benzodiazepines, but it was unsuccessful. She was treated with intravenous lacosamide $200 \mathrm{mg}$ followed by maintaining dose of $100 \mathrm{mg}$ bid, with resolution of epileptic status. Correction of electrolytes was also performed. Vital parameters resulted all normal.

Conclusions and Discussions: Epileptic status epilepticus, may be considered as a possible manifestation of NHD. Due to the rarity of disease there isn't a consensus about treatment. Due to successful outcome in our patient we suggest that intravenous lacosamide can be considered in treatment of epileptic status in PLOSL disease.

References:

- Bianchin MM, Capella HM, Chaves DL, Steindel M, Grisard EC, Ganev GG, da Silva Júnior JP, Neto Evaldo S, Poffo MA, Walz R, Carlotti Júnior CG, Sakamoto AC. Nasu-Hakola disease (polycystic lipomembranous osteodysplasia with sclerosing leukoencephalopathy-PLOSL): a dementia associated with bone cystic lesions. From clinical to genetic and molecular aspects. Cell Mol Neurobiol. (2004) Feb;24(1):1-24

- Montalbetti L, Soragna D, Ratti MT, Bini P, Buscone S, Moglia A. Nasu-Hakola disease: a rare entity in Italy. Critical review of the literature. Funct Neurol. (2004) Jul-Sep;19(3):171-9

- Madry H, Prudlo J, Grgic A, Freyschmidt J Nasu-Hakola disease (PLOSL): report of five cases and review of the literature. Clin Orthop Relat Res. (2007) Jan;454:262-9

\section{SLC52A2-LINKED BROWN-VIALETTO-VAN LAERE SYNDROME: A CLINICAL CASE}

\author{
S. Cotti Piccinelli ${ }^{1}$, F. Novara ${ }^{2}$, B. Risi ${ }^{1}$, E. Baldelli ${ }^{1}$, N. Necchini ${ }^{1}$, A.

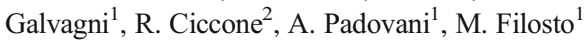

\footnotetext{
${ }^{1}$ ASST Spedali Civili of Brescia, University of Brescia (Brescia); ${ }^{2}$ Microgenomics Laboratory (Pavia)
}

Objectives: Brown-Vialetto-Van Laere Syndrome (BVS) is an extremely rare disorder characterized by sensorineural deafness, lower cranial nerve and lower motor neuron involvement and impaired respiratory function. Autonomic dysfunctions, epilepsy, optic atrophy and pigmentary retinitis may be also present in a variable combination. Pathogenic variants in the
SLC52A2 and SLC52A3 genes encoding for human riboflavin transporters have been associated to the disease. Several studies have reported improvement in clinical outcome with riboflavin supplementation especially in younger patients. We here report on clinical and genetic findings of a patients affected with BVS.

Patient and Methods: A 42 years-old man was suffering from severe hearing loss, chronic respiratory failure and optic atrophy since childhood. Neurological examination showed weakness and hypotrophy of the intrinsic muscles of the hands, ataxic gait, mild cerebellar signs in the limbs, absence of ankle jerk reflexes, severe hearing loss and visual acuity reduction with erratic eye movements. Neurophysiological studies, muscle biopsy and genetic analysis by clinical exome were conducted in order to achieve a diagnosis.

Results: Chronic neurogenic signs were detected on EMG and muscle biopsy. Genetic analysis revealed two novel heterozygote variants (c.353C > A; p.Ala118Asp and c.863C > T; p.Ala288Val) in the SLC52A2 gene. Oral riboflavin $10 \mathrm{mg} / \mathrm{kg} /$ day was started which was further increased to $20 \mathrm{mg} / \mathrm{kg}$ /day after a week and patient was followed-up.

Discussion: BVS is difficult to diagnose because its rarity, variable age of onset and heterogeneous clinical presentation. SLC52A2-linked BVS is much less common than the SLC52A3-related disease and is mainly characterized by sensorineural hearing loss, early-onset sensory ataxia and nystagmus, optic atrophy and weakness of neck extension and upper limbs. The course and prognosis of the disease are variable and may be influenced by the initiation of riboflavin treatment.

Conclusions: Since BVS is a potentially treatable condition, an early diagnosis should be actively sought because riboflavin may be an effective therapy and shift the clinical course. An early therapeutic attempt with riboflavin appears to be crucial as diaphragmatic paralysis may be irreversible.

\section{ISCHEMIC STROKE IN DOWN SYNDROME: INTRAVENOUS THROMBOLYSIS AND BLEEDING RISK}

\author{
C.T. Currò ${ }^{1,2}$, C. Dell'Aera ${ }^{1}$, M. Cotroneo ${ }^{1}$, C. Casella ${ }^{1}$, P. La Spina ${ }^{1}$, \\ M.C. Fazio ${ }^{1}$, F. Grillo ${ }^{1}$, A. Toscano ${ }^{2}$, R. F. Musolino ${ }^{1}$ \\ ${ }^{1}$ Stroke Unit, Department of Medical and Experimental Medicine, \\ University of Messina (Messina); \\ ${ }^{2}$ Department of Neurosciences, University of Messina (Messina)
}

Down syndrome (DS) is related with both ischemic stroke (IS) and cerebral hemorrhage $(\mathrm{CH})$. We reported a case of IS in DS underwent intravenous thrombolysis (IVT). A 30-year-old man with DS presented impairment of speech and right limbs strength at 3.00 a.m. He was conducted to a spoke center, he had left-side head and look deviation, right-side hemianopsia, hypoestesia, inattention and right limbs paresis, NIHSS 10. Brain CT, MRI and an Angio-CT showed an acute ischemic lesion due a left cerebral posterior artery occlusion. IVT was effectuated at 6.41 a.m. and he was transferred to our center. Another MRI showed a wide left temporo-occipital-thalamic-capsular lesion with minimal ischemic penumbra and thrombectomy did not perform for this reason and the thrombus distal position. Laboratory test revealed hypercholesterolemia. Supraaortic vessels color-doppler-ultrasound did not reveal any alterations. Transcranial doppler with bubble study showed a patent foramen ovale. Admission ECG individuated a sinusal arythmia but 24 hour-holter ECG was normal. Transthoracic echocardiogram did not reveal any alterations. $\mathrm{CT}$ on the second day showed only the ischemia, but CT on the fifth day evidenced some microbleedings in ischemic area. On seventh day, head 
and look deviation disappeared, strength impairment was improved (NIHSS 7) and he was transferred to a rehabilitation centre. Modified Rankin scale at 90 days was 3. In literature only a unique case of IVT in a DS patient was reported and it was complicated by hemorrhagic transformation (HT) [1] as our case. DS indeed has a three-fold higher IS risk but it is also associated with $\mathrm{CH}$ [2]. An important risk factor for hemorrhagic transformation could be cerebral amyloid angiopathy but an MRI study did not perform in literature case and it did not show sign related with this condition in our patients. High rate of hemorrhagic surgery complications and many cases of lung hemorrhage [3] were reported In literature that can occur also in early age. We could suppose the existence of a systemic vascular fragility condition which is independent or could precede amyloid angiopathy. This fragility could explain HT in our patient and importance of weighting IS severity and possible HT in DS. References:

1. Gallerini S, Chiti A, Gialdini G, et al Intravenous thrombolysis for ischaemic stroke in Down syndrome. Neurological Sciences (2012);33:1491-92

2. Sobey CG, Judkins CP, Sundararajan V, et al Risk of major cardiovascular events in people with down syndrome. PLoS ONE (2015);10:1-11

3. Boylan MR, Kapadia BH, Issa K, et al Down Syndrome Increases the Risk of Short-Term Complications After Total Hip Arthroplasty. Journal of Arthroplasty (2016);31:368-72

\section{POSTINFECTIOUS ADEM AND SIMULTANEOUS POLYRADICULONEUROPATHY TREATED WITH PLASMA EXCHANGE COMBINED TO HIGH DOSE STEROID: A CASE REPORT}

C. Cutelle', D. Cereda, S. Diamanti, V. Polonia, B. Storti, I. Appollonio, C. Ferrarese

Department of Neurology, S. Gerardo Hospital, University of MilanoBicocca (Monza)

Background: Acute disseminated encephalomyelitis (ADEM) is an immune-mediated inflammatory disorder of the central nervous system (CNS), which is usually preceded by a systemic infection. Co-occurrence of ADEM with peripheral nervous system (PNS) involvement is reported, but probably underestimated because it is often masked by the presence of severe CNS damage.

Case report: A 58 years-old man developed pharyngitis treated with antibiotic. After three weeks, he complained vertigo, postural instability, hypotension with several syncopes and, some days later, dysphagia and dysphonia. Neurological examination revealed second degree nystagmus, right facial paresis, dysarthric and hypernasal speech, hypoelicitable reflexes and ataxic gait with the need for support. Right IX cranial nerve plasy was demonstrated by fibroscopy. Brain MRI detected two hyperintense lesions on $\mathrm{T} 2 \mathrm{~W}$ images, one in the right dorsal bulb including the cerebellar peduncle and one in the right cingulate, with gadolinium ringshaped enhancement on T1W images. Spinal cord MRI was negative. The brain lesions explained most of the symptoms but not the bilateral ataxia and the autonomic dysfunction, suggesting further investigations. Electromyoneurography evidenced prolonged F-wave latencies and active denervation, compatible with assonal polyradiculoneuritis. Analysis of the cerebrospinal fluid showed albumin-cytological dissociation with mild blood-brain barrier damage. Infective, autoimmune and paraneoplastic screening was negative. Suspecting a postinfectious disimmune disease involving both the CNS and PNS, a combined protocol of Plasma exchange
(PE) and high dose intravenous methylprednisolone on alternate days was administrated with fully clinical and radiological recovery. During a two years follow-up no other neurological symptoms occurred.

Discussion: We described an adult patient affected by postinfectious monophasic ADEM associated to acute polyradiculoneuropathy. The clinical presentation imposed a wide spectrum of examinations and a follow-up to rule out differential diagnosis such as infections of the nervous system, vasculitis, paraneoplastic syndromes, other demyelinating diseases or systemic autoimmune disorders. In some of these conditions simultaneous involvement of the PNS is possible. In several case-series ADEM with additional polyneuropathy is related to poor outcome and low responsiveness to steroid mono-therapy. However, guidelines or comparison studies between therapeutic options still does not exist. In our patient a combined protocol of $\mathrm{PE}$ and high dose steroid on alternate days, never described in literature at the same regime in ADEM patients, determined the complete recovery. Prompt recognition of concomitant polyradiculoneuritis, even if partially or totally masked by CNS symptoms, could promote an earlier effective treatment of ADEM and, therefore, a better neurological outcome.

References:

- Tenembaum S, Chitnis T, Ness J et al, Acute disseminated encephalomyelitis. Neurology (2007);68(16 Suppl 2):S23-36

- Marchioni E, Ravaglia S, Piccolo G et al, Postinfectious inflammatory disorders: subgoups based on prospective follow-up. Neurology (2005);65(7):1057-65

- Cortese A, Franciotta D, Alfonsi E et al, Combined central and peripheral demyelination: clinical features, diagnostic findings, and treatment. Journal of the Neurological Sciences (2016);363:182-7

\section{A CASE OF HANDL SYNDROME}

S. Dagostino ${ }^{1}$, G. Cossu ${ }^{2}$, G. Fenu ${ }^{2}$, V. Oppo ${ }^{2}$, P. Cimino ${ }^{3}$, G. Cadeddu ${ }^{3}$, A. Musu ${ }^{3}$, M. Melis ${ }^{2}$

${ }^{1}$ Emergency Department, Neurology Department, AOU Cagliari, University of Cagliari (Cagliari); ${ }^{2}$ Department of Neurology and Stroke Unit, Hospital "G. Brotzu" (Cagliari); ${ }^{3}$ Department of Medical Sciences and Public Health, Institute of Neurology, University of Cagliari and AOU Cagliari (Cagliari)

Objectives: To describe a 50 years old man with HaNDL Syndrome (syndrome of transient headache and neurologic deficits with cerebrospinal fluid lymphocytosis) who presented with stroke like symptoms.

Case Report: A 50 years old man presented with sudden right-sided headache, throbbing in quality, followed by left sided paresthesias and aphasia, which lasted less than one hour. He reported mild fever with gastro-intestinal symptoms three days before admission. He underwent surgery in 2010 for a right epidural post-traumatic hematoma and was affected by Crohn's Disease. On admission the patient was alert, well oriented and cooperative, with no focal neurological signs. Vitals were normal. During hospital stay he experienced three episodes of headache exacerbation, accompanied by aphasia and confusional state. These attacks lasted around 4 hours, with complete regression of the symptoms. In one occasion such confusional state consisted in agitation and aggressivity, which required deep pharmacological sedation. Brain CT and CTA, Brain MRI and blood tests were unremarkable. Electroencephalogram showed slowing of background activity, with right predominance, and without epileptic activity. CSF analysis showed 75 cells/mm3 with protein concentration of $151 \mathrm{mg} / \mathrm{dL}$. Microbiological analysis for viruses and 
bacteria were negative. Search for onco-neural and anti neuronal surface antibodies was also negative. A treatment with high dosis IV Metilprednisolone was introduced and the patient achieved full clinical recovery. He was discharged with diagnosis of HaNDL Syndrome.

Discussion: HaNDL syndrome or pseudomigraine with Lymphocytosis is a medical condition that has been described since the ' 80 s [1] but it's still currently underdiagnosed. It is characterized by one or more episodes of severe headache, transient neurologic deficits, and lymphocytic pleocytosis in the CSF. It is a benign condition, with excellent prognosis, but it can present like serious medical conditions, such as stroke and meningoencephalitis [2]. Extensive differential diagnosis should be sought before this diagnosis of exclusion can be reached. Findings in various reported cases suggest a migrainous patophysiology, although the pleocytosis, monophasic course and frequent viral prodrome suggest an infectious/inflammatory trigger [3].

References:

1. Bartleson JD, Swanson JW, Whisnant JP. A migrainous syndrome with cerebrospinal fluid pleocytosis. Neurology (1981); 31:1257

2. Gómez-Aranda F, Cañadillas F, Martí-Massó JF, et al. Pseudomigraine with temporary neurological symptoms and lymphocytic pleocytosis. A report of 50 cases. Brain (1997); 120 (Pt 7):1105

3. Kappler J, Mohr S, Steinmetz H. Cerebral vasomotor changes in the transient syndrome of headache with neurologic deficits and CSF lymphocytosis (HaNDL). Headache (1997); 37:516

\section{INFLUENZA B VIRUS ENCEPHALITIS DURING TREATMENT WITH FINGOLIMOD FOR MULTIPLE SCLEROSIS: A CASE REPORT}

A. de Falco, S. Peluso, S. Ascione, R. Bruno, G. Cerullo, G. Grimaldi, M. Lieto, M. Mazzaferro, S. Montella, P. Ripa, V. Romano, R. Scala, A. Topa, V. Palma

Neurology Unit, Ospedale del Mare Hospital ASL Napoli 1 Centro (Napoli)

Introduction: Human influenza B virus (IBV) is a negative singlestranded RNA virus, responsible for seasonal epidemics of self-limited acute respiratory infections. Rare and severe complications can involve the central nervous system. We describe an IBV-related encephalitis in a patient treated with fingolimod for relapsing-remitting multiple sclerosis (RRMS)

Case report: A 34-years-old male patient went to our observation for a syncopal episode, followed by confusion and behavioural alterations. In the previous 4 days, fever and flu-like symptoms were reported from his family. The patient was on treatment with fingolimod for the last 7 years. On admission, neurological examination showed confusion and psychomotor agitation. Neither meningeal signs nor other neurological deficits were present. Laboratory examination showed lymphopenia and a mild $\mathrm{CPK}$ and transaminases increase. Blood and urine culture tests were negative. EEG showed diffuse slowing background activity without epileptiform discharge. A brain MRI revealed mild cortical diffuse hyperintensities on DWI and FLAIR sequences, more evident in right frontal cortex. Multiple foci of T2 hyperintensity, related to MS pathology, were detected in the periventricular and juxtacortical white matter. Cerebral 18F-FDG PET revealed a mild reduction of tracer uptake in the left frontal and mesial temporal cortex. Cerebrospinal fluid (CSF) analysis revealed only slight hyperproteinorrachia. CSF RT-PCR and CSF culture test did not detect common virus and bacteria. Neuronal surface and onconeuronal antibodies were not detected. Nasopharyngeal swab revealed positive PCR for IBV. The patient started a treatment with oseltamivir. During hospitalization, his cognitive functions and mental status progressively improved. On hospital discharge, the patient showed normal consciousness and orientation and neuropsychological evaluation evidenced only mild alterations in visuospatial long-term memory, abstract reasoning and frontal functions.

Discussion: Fingolimod is a sphingosine-1-phosphate receptor modulator and has been the first approved oral drug for RRMS treatment. Although it has long been considered at low risk of opportunistic infections due to a preservation of immuno-surveillance, serious infections have been reported. According to recent data, in patients with MS taking fingolimod the rate of infections is higher than in the general population and in MS patients under treatment with other immunomodulatory drugs. In this context, we are the first to describe a case of encephalitis associated with IBV during treatment with fingolimod.

Conclusion: Immunomodulation in MS represents a risk factor for opportunistic and not opportunistic infections. Among these infections, IBV encephalitis has to be considered strengthening the recent vaccination recommendations for MS patients including yearly influenza vaccinations.

References:

- Cervera C. Infections and fingolimod. Rev Neurol. (2012) Aug 16;55(4):227-37

- Luna G, Alping P, Burman J, Fink K, Fogdell-Hahn A, Gunnarsson $\mathrm{M}$ et al. Infection Risks Among Patients with Multiple Sclerosis Treated With Fingolimod, Natalizumab, Rituximab, and Injectable Therapies. JAMA Neurol. (2020);77(2):184-91

- Farez MF, Correale J, Armstrong AJ, Rae-Grant A, Gloss D, Donleyet $\mathrm{D}$ et al. Practice guideline update summary: Vaccine preventable infections and immunization in multiple sclerosis. Neurology (2019);93:1-11

\section{NON-CONVULSIVE STATUS EPILEPTICUS AS A PRESENTING SYMPTOM OF NONALCOHOLIC WERNICKE'S ENCEPHALOPATHY WITH CEREBRAL CORTICAL LESIONS}

\author{
A. de Falco ${ }^{1}$, M. Mazzaferro ${ }^{1}$, R. Scala ${ }^{1}$, M. Lieto ${ }^{1}$, S. Montella ${ }^{1}$, M. \\ Prudente $^{2}$, V. Palma ${ }^{1}$
}

${ }^{1}$ Neurology Unit, Ospedale del Mare Hospital ASL Napoli 1 Centro (Napoli); ${ }^{2}$ Neuroradiology Unit, Ospedale del Mare Hospital ASL Napoli 1 Centro (Napoli)

Introduction: Wernicke encephalopathy (WE) is a neurological disorder due to thiamine (vitamin B1) deficiency characterized by ocular signs, mental-status changes and ataxia. Thiamine deficiency leads to brain lesions in vulnerable regions with high thiamine content and turnover. WE is frequently associated with chronic alcohol use but can also be caused by non-alcoholic precipitating illnesses including cancer. Epileptic seizures are an exceedingly rare symptom of WE. Here we present a first report of nonalcoholic WE presenting with nonconvulsive status epilepticus (NCSE).

Case Report: The patient was a 58-year-old woman with advanced pancreatic cancer complicated by peritoneal carcinomatosis. She performed ileostomy for sub-occlusion and she had a two-months history of inadequate oral and parenteral feeding without thiamine replacement. Neurological evaluation was performed for mental status alteration with impaired alertness. Neurological examination showed drowsiness and 
lethargy without eyes movement abnormalities. Blood serum analysis showed normal values except hemoglobin $9.9 \mathrm{~g} / \mathrm{dl}$ and sodium 133 $\mathrm{mEq} / \mathrm{l}$. An EEG revealed subcontinuous high voltage sharp waves and spikes in right temporal areas diffusing to the right hemisphere. After diazepam $10 \mathrm{mg}$ iv administration, EEG showed disappearance of epileptic discharges and improved background activity. Diagnosis of focal NCSE was made and levetiracetam 2 gr daily was started. Patient showed mild improvement in neuropsychological status. She performed brain MRI showing diffuse DWI hyperintensities in brainstem, in periacqueductal grey, in cerebellum, in thalamic and sub-thalamic nuclei bilaterally, in mammillary bodies, and in cortical cortex involving frontal, rolandic and interhemispheric areas without meningeal enhancement as for WE with cortical involvement. Cerebrospinal fluid analysis showed normal appearance, high protein level $(217 \mathrm{mg} / \mathrm{dl}), 8$ cells and normal glucose. Cytologic analysis did not revealed malignant cells. Serum onconeural antigens antibodies were negative. Diagnosis of WE was made and thiamine $600 \mathrm{mg}$ daily was started. Patient did not presented seizures or epileptic activity on follow-up EEG but gradually worsened for the advanced oncologic disease and died 7 days later.

Discussion and conclusion: Compared to alcoholic WE, nonalcoholic WE leads to a high proportion of atypical brain lesions and a lower proportion of classic triad symptoms as in our case. Epileptic seizures are an exceedingly rare symptom in WE with an incidence of $0 \%-3.1 \%$ even in patients with WE involving the cortex but they could be the presenting symptom. Seizures are mainly tonic-clonic generalized seizures but can also be focal with or without consciousness involvement. NCSE can be an insidious presenting symptom which needs to be considered in patients with suspected WE.

References:

- Galvin R, Bråthen G, Ivashynka A, et al. EFNS guidelines for diagnosis, therapy and prevention of Wernicke encephalopathy. Eur J Neurol. (2010);17(12):1408-1418. doi:10.1111/j.14681331.2010.03153.x

- Shang W, Chen X, Li X, Chen H, Tang S, Hong H. Epileptic seizures in nonalcoholic Wernicke's encephalopathy: a case report and literature review. Metab Brain Dis. (2017);32(6):2085-2093. doi:10.1007/s11011-017-0106-1

- Fu K, Tian L, Xue W, Teng W. Epileptic seizures as the first symptom of Wernicke's encephalopathy with cerebral cortical lesions. J Clin Neurosci. (2017);40:50-52. doi:10.1016/j.jocn.2017.01.016

\section{MULTIPLE SCLEROSIS AND AMYOTROPHIC LATERAL SCLEROSIS ASSOCIATED TO A TBK1 MUTATION: A CASE REPORT}

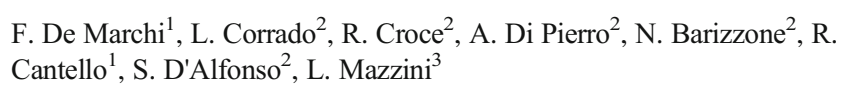

${ }^{1}$ Department of Neurology, University of Piemonte Orientale (Novara); ${ }^{2}$ Department of Health Sciences Interdisciplinary Research Center of Autoimmune Diseases, University of Piemonte Orientale (Novara); ${ }^{3}$ Department of Neurology and ALS Centre, University of Piemonte Orientale (Novara)

Background: Mutations in TANK-binding kinase 1 (TBK1) are typically associated with Amyo-trophic Lateral Sclerosis / Frontotemporal Dementia (ALS/FTD). TBK1 is a serine / threonine protein kinase involved in many signaling pathway acting as key player in autophagy and innate immunity. Insufficient regulation of TBK1 activity can lead to autoimmune, neurodegenerative diseases or tu-morigenesis. Many
ALS-linked TBK1 mutations lead to a premature stop codons and TBK1 haploin-sufficiency that is predicted to impair autophagy.

Material: A 52-year-old man presented with a two-year history of progressive dysarthria, dysphagia and right arm hypotrophy. He had a history of relapsing-remitting multiple sclerosis (MS) (age of diagnosis: 26 years), started with optic neuritis and balance disturbances, in treatment with natalizumab for two years. His family history was positive for neurological disorders (younger sister with optic neuritis, and father with myasthenia gravis). Neurological examination disclosed upper cingulate hypotrophy and weakness, bilaterally, with diffuse fasciculations, dysphagia and dysarthria. Neurocognitive evaluation shown frontotemporal mild cognitive impairment. EMG exam showed marked degeneration of lower motor neurons, as described in Motor Neuron Diseases. The diagnosis of Amyotrophic Lateral Sclerosis was made and he started a drug therapy with riluzole. The disease progression was rapidly progressive with anarthria and dyspnea at rest after 6 months, requiring Non-Invasive Ventilation for 12-14 hours/day.

Results: The patient was screened for mutations in a multi-gene panel including 25 genes associated with ALS and FTD. Genetic analysis revealed the presence of c.922 C>T, p.R308X (NM_013254) mutation in heterozygous status in exon 8 of TBK1 gene. The variant is classified as pathological (ACMG 2015) and to date never reported in public databases (1000Genomes, GNomAD). No further pathological variants were found in the remaining analyzed ALS genes.

Discussion: We identified a novel pathogenic TBK1 variant in a proband presenting ALS and MS phenotype. TBK1 is involved in a variety of ALS-relevant pathways such as autophagy and neuroinflammation leading to the accumulation of protein aggregates, autophagosomes and damaged mitochondria in motor neurons and a crossover in the pathogenic mechanisms between ALS and MS can be hypothesized. Although we cannot exclude that the two phenotypes are due to different causes and their concomitance is due to a coincidence, on the other hand it is tempting to speculate that the TBK1 mutation, causative of the typical ALS phenotype evident in this patient might have contributed to the susceptibility to MS and other autoimmune multifactorial diseases presented in the patient and his family.

References:

- Oakes JA, Davies MC, Collins MO. TBK1: a new player in ALS linking autophagy and neuroinflammation. Mol Brain (2017);10(1):5. Published 2017 Feb 2. doi:10.1186/s13041-0170287-x

- Ismail A, Cooper-Knock J, Highley JR, et al. Concurrence of multiple sclerosis and amyo-trophic lateral sclerosis in patients with hexanucleotide repeat expansions of C9ORF72. J Neurol Neurosurg Psychiatry (2013);84(1):79-87. doi:10.1136/jnnp-2012303326

\section{INTRACRANIAL HYPERTENSION AS THE FIRST MANIFESTATION OF MULTIPLE SCLEROSIS}

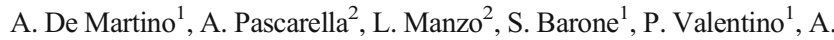 \\ Gambardella $^{1}$, F. Bono ${ }^{2}$
}

${ }^{1}$ Institute of Neurology, Magna Græcia University (Catanzaro); ${ }^{2}$ Center for Headache and Intracranial Pressure Disorders, A. O. U. "Mater Domini" (Catanzaro)

Objective: About $80 \%$ of multiple sclerosis (MS) patients suffer from headache. Recently it has been reported that headache may be the first clinical manifestation of MS [1]. Although the increased risk of headache 
in patients with MS is now recognized, the secondary etiology of MSassociated headache has been poorly studied. Indeed, the possibility that intracranial hypertension (IH) is the cause of headache in MS patients has been rarely considered [2]. Here we report four patients presenting IH syndrome as the first manifestation of MS.

Methods: In this series of cases we report clinical data from patients who had severe headache as the first clinical manifestation of MS. All patients underwent a general and neurological evaluation. Clinical features of headache, MIDAS disability scale, HIT-6, cutaneous allodynia scale, visual analogue scale, Beck inventory for depression, and Hamilton scale for anxiety were assessed. All patients underwent enhanced brain and spine MRi and cerebral MR venography (VMR). The cerebrospinal fluid was analyzed and the presence of oligoclonal bands was tested. In addition, patients had their CSF pressure measured by 1-hour lumbar CSF pressure monitoring via spinal puncture needle [2]. Diagnosis of IH and MS was made according to validated diagnostic criteria.

Results: Four obese patients had a severe headache, mainly nocturnal, that had recently emerged with associated visual disturbances and dizziness. Brain MRI showed in three patients empty sella, perioptic subarachnoid space distension and flattening of the posterior sclera. VMR showed transverse sinus stenosis. The cerebrospinal fluid analysis was normal with absence of the oligoclonal bands, whereas the opening CSF pressure was high and abnormal pressure pulsations (B waves) were present. Diagnosis of idiopathic IH associated with transverse stenosis was made. Medical treatment produced an improvement in symptomatology. However, neurological deficits were observed in the following months. At that time cerebral and spinal MRI and the presence of oligoclonal bands allowed us to make a diagnosis of relapsing remitting MS.

Conclusion: These preliminary data suggest that IH syndrome may be the first clinical manifestation of MS. Early recognition of headache due to CSF hypertension secondary to MS could avoid a misdiagnosis as idiopathic IH and could reduce latency at the final diagnosis, allowing adequate and prompt treatment. Cerebral venous outflow disturbances associated with IH could be the relationship between IH and MS [3]. Our data indicate that further studies are needed to better understand the relationship between these two diseases.

References:

1. Gebhardt M, Kropp P, Hoffmann F, Zettl UK. Headache at the Time of First Symptom Manifestation of Multiple Sclerosis: A Prospective, Longitudinal Study. Eur Neurol. (2018);80(3-4):115120. doi:10.1159/000494092

2. Bono F, Curcio M, Rapisarda L, et al. Cerebrospinal Fluid PressureRelated Features in Chronic Headache: A Prospective Study and Potential Diagnostic Implications. Front Neurol. (2018);9:1090. Published 2018 Dec 18. doi:10.3389/fneur.2018.01090

3. D'haeseleer M, Cambron M, Vanopdenbosch L, De Keyser J. Vascular aspects of multiple sclerosis. Lancet Neurol. (2011);10(7):657-666. doi:10.1016/S1474-4422(11)70105-3

\section{PARKINSONISM, HYPOPARATHYROIDISM AND BRAIN CALCIFICATIONS: AN UNUSUAL PHENOTYPE ASSOCIATED WITH M.3243A>G MUTATION}

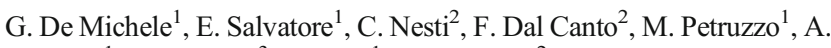 \\ De Rosa ${ }^{1}$, S. Cocozza ${ }^{3}$, A. Filla ${ }^{1}$, F. Santorelli ${ }^{2}$
}

${ }^{1}$ Department of Neuroscience, Reproductive and Odontostomatological Sciences, Federico II University (Napoli); ${ }^{2}$ Department of Molecular Medicine \& Neurogenetics, IRCCS Stella Maris Foundation (Pisa);
${ }^{3}$ Department of Advanced Biomedical Sciences, Federico II University (Napoli)

Introduction: The pathogenic variant $\mathrm{m} .3243 \mathrm{~A}>\mathrm{G}$ is the most frequent mitochondrial DNA mutation. It can be associated with different clinical syndromes, including Mitochondrial Encephalopathy Lactic Acidosis and Stroke-like episodes (MELAS) and Maternally Inherited Deafness and Diabetes (MIDD) [1]. However, many patients exhibit a phenotype that does not fall within the criteria for the classical mitochondrial syndrome, and there is also major overlap between the different classical phenotypes. Here, we describe a patient harbouring m.3243A $>\mathrm{G}$ that exhibits an unusual phenotype, characterized by parkinsonism, hypoparathyroidism with massive brain calcifications, associated to diabetes and deafness.

Case report: A 66-year-old man presented to our department for a 6year history of progressive fatigue, generalised slowness, dysarthria and dysphagia. His medical history was relevant for diabetes mellitus type 2 (since he was 35-year-old), sensorineural deafness, severe constipation with episodes of pseudo-obstruction, and smoking. He also had right hip replacement. His mother and 6/7 his siblings had diabetes, while 5/7 had also deafness. On examination, his gait was slow and unsteady, with little steps. There was limitation of vertical eye movement, marked dysarthria, mild dysphagia, hypomimia with mild oral dyskinesia. He also had bradykinesia, plastic hypertonia, diffuse muscle atrophy. Tendon reflexes were decreased. Sensation was normal. Routine laboratory test showed mild anemia and hyperglycemia. Brain CT revealed massive calcifications in basal ganglia, corona radiata and centrum semiovale, occipital cortex, mesencephalon and cerebellar hemispheres. There was also moderate cerebral and cerebellar atrophy. PTH was low $(12,4 \mathrm{pg} / \mathrm{ml}, \mathrm{nv} 14-$ 87), while blood calcium, phosphorus and $D$ vitamin were normal, but he was already in treatment with calcitriol. In view of the clinical findings, as well as of the family history, genetic test for the m.3243A $>\mathrm{G}$ was performed on blood leucocytes and urinary sample. PCR-RFLP revealed m.3243A $>$ G heteroplasmy ( $2 \%$ in blood leucocytes, $47 \%$ in urine). Two sisters of the patient, affected by diabetes and deafness, were also screened for $\mathrm{m} .3243 \mathrm{~A}>\mathrm{G}$ in leucocytes and urines, and were found to harbour heteroplasmic m.3243A>G (respectively 1 and 10\%; 0,3 and $6 \%)$.

Discussion: This case shows that $\mathrm{m} .3243 \mathrm{~A}>\mathrm{G}$ can be responsible of more complex phenotypes than MELAS and MIDD. Indeed, besides deafness and diabetes, our patient presented hypoparathyroidism with extensive brain calcifications and parkinsonism. His gastrointestinal symptoms (constipation with pseudo-obstruction) can also be attributed to mitochondrial dysfunction [2]. The phenotype variability within the families is only partially explained by the different levels of heteroplasmy [3].

References:

1. Nesbitt V, Pitceathly RD, Turnbull DM, Taylor RW, Sweeney MG, Mudanohwo EE, Rahman S, Hanna MG, McFarland R. The UK MRC Mitochondrial Disease Patient Cohort Study: clinical phenotypes associated with the m.3243A $>$ G mutation-implications for diagnosis and management. J Neurol Neurosurg Psychiatry (2013) Aug;84:936-8

2. Suzuki J, Iwata M, Moriyoshi H, Nishida S, Yasuda T, Ito Y. Familial Pernicious Chronic Intestinal Pseudo-obstruction with a Mitochondrial DNA A3243G Mutation. Intern Med. (2017);56:1089-93

3. Grady JP, Pickett SJ, Ng YS, et al. mtDNA heteroplasmy level and copy number indicate disease burden in m.3243A $>$ G mitochondrial disease. EMBO Mol Med. (2018);10:e8262 


\section{SUBACUTE COGNITIVE DECLINE IN A PATIENT WITH IDIOPATHIC DELAYED-ONSET EDEMA AFTER STN-DBS FOR PARKINSON'S DISEASE}

T. De Santis ${ }^{1}$, S. Lalli ${ }^{1}$, D. Calandrella ${ }^{1}$, A. Cocco $^{1}$, A. Ampollini ${ }^{2}$, F. Motta $^{2}$, E. Mailland ${ }^{2}$, M. Grimaldi ${ }^{3}$, L. Politi ${ }^{3}$, M. Egidi ${ }^{2}$, P. Amami ${ }^{1}$, A. Albanese $^{1}$

${ }^{1}$ Neurology Unit, Humanitas Clinical and Research Hospital (RozzanoMI); ${ }^{2}$ Neurosurgery Unit, San Carlo Hospital, ASST Santi Paolo e Carlo (Milano); ${ }^{3}$ Department of Neuroradiology, Humanitas Clinical and Research Hospital (Rozzano-MI)

Objective: Idiopathic delayed-onset edema (IDE) is a rare, selflimiting complication of DBS procedures. It is defined as a spontaneous edema diffusing along the DBS lead that occurs approximately $72 \mathrm{~h}$ after implant, and is not associated with trauma, vascular events or signs of infection [1]. Typical symptoms of IDE are apathy, confusion and seizures, although asymptomatic cases have also been reported. We describe symptoms, diagnostic tests and outcome of a patient with IDE after subthalamic nucleus (STN) DBS.

Methods and Materials: A patient with IDE was followed-up for 4 months after implant. Evaluation was performed 1 month (T1), 3 months (T2) and 4 months (T3) after surgery performing in each session motor assessment (MDS-UPDRS III), EEG and brain MRI. Furthermore, brain metabolism was evaluated with 18-FDG PET. Neuropsychological tests assessing executive domains (digit span backward, trial making test, Stroop test, modified Wisconsin card sorting test, phonemic fluency), visuospatial abilities (Rey complex figure test) and memory (Rey 15-item memory test) were administered.

Case History: A 55-year-old man with a 10-year history of Parkinson's disease was admitted one month after implant, as he developed severe apathy, memory impairment and apraxia. These symptoms started three days after surgery, when stimulation had not yet been activated. Cerebrospinal fluid examination revealed slightly increased protein concentration with normal cell count and no evidence for viral or bacterial infections. Brain MRI showed bilateral edema and micro-hemorrhages along the DBS electrode at the level of the left thalamus and supra-adjacent white matter with no associated significant mass effect. Left side was more involved. The patient was assessed without turning on stimulation and followed-up for 4 months after implant, when DBS was turned on. During this time, motor and cognitive performances gradually improved. At T3 brain edema and microhemorrhages disappeared. EEG at $\mathrm{T} 1$ showed diffused theta slowing with focal delta activity over left frontotemporal regions. At T2 and T3 delta activity had disappeared and EEG displayed only diffuse and symmetrical theta activity. Brain metabolism, assessed with 18FDG PET, was globally decreased, particularly in left temporal and parietal lobes. This picture did not change over time.

Conclusion: IDE is considered a temporary and self-limiting adverse event of STN-DBS. To our knowledge, this is the first case report showing persistence of EEG and 18FDG PET abnormalities notwithstanding recovery of clinical symptomatology.

References:

1. C.de Cuba et al. Idiopathic delayed-onset edema surrounding deep brain stimulation leads: Insights from a case series and systematic literature review, Parkinsonism Relat Disord. (2016);32:108-15

\section{RAPIDLY PROGRESSIVE COGNITIVE DETERIORATION AS PARANEOPLASTIC ANTINEURONAL ANTIBODIES AB TR/ DNER - MEDIATED MANIFESTATION OF RELAPSING HODGKIN'S LYMPHOMA}

\author{
M. G. Di Donna, F. Bernocchi, M. Bagnato, C. Bonomi, G. Di Mauro, M. \\ Conti, F. Marrama, C. Rocchi, M. Pierantozzi, N. Mercuri
}

Neurology Institute, Policlinico Tor Vergata Hospital (Roma)

Aim of the study: Our aim was to investigate rapidly progressive cognitive deterioration as paraneoplastic Antineuronal Antibodies $\mathrm{Ab} \mathrm{Tr} /$ DNER - mediated manifestation of relapsing Hodgkin's Lymphoma.

Material: We evaluated a 64-year-old man, who was admitted to the Neurology department of "Policlinico Tor Vergata", Rome, with a sudden onset of non-fluent aphasia, after a 2 weeks insidious presentation of behavioural symptoms described as emotional blunting and depressed mood. The patient had a history of nodular sclerosis Hodgkin Disease, treated 2 years before with surgery, CT and RT cycles. At the admission, the neurological examination revealed fluctuating lack of orientation and confusion (the patient couldn't recognize his own child at times), cognitive and motor slowing, memory loss with confabulation, aphasia nominum, semantic circumlocutions, ideomotor and constructional apraxia (MMSE on admission 11.8/30). Frontal release signs, gait apraxia, slight postural and kinetic tremor in both hands were found. We performed brain angioCT scan, brain MRI, EEG, blood samples and a lumbar puncture looking for infectious, autoimmune or degenerative conditions together with vitamin deficiency.

Results and Discussion: In the Emergency Room, the clinical presentation was concerned as stroke aphasia: AngioTc scan showed an occlusion in left MCA territory and flectadol was prescribed. On the contrary, brain MRI documented the absence of any recent ischemic lesion and so we excluded the hypothesis of stroke aphasia and considered rapidly progressive dementia. The EEG showed few non-significant theta waves on bilateral centre-temporal regions. Low serum level of B9 were found, but the implementation did not improved the clinical conditions. CSF analysis showed the presence of $52.00 / \mathrm{mmc} \mathrm{WBC}$, with some activated T-CD4 lymphocytes; CSF biomarkers for Alzheimer, Creutzfeldt-Jakob, Neurosyphilis and viral markers were negative. The research of antineuronal Antibodies in serum found $\mathrm{Ab}$ anti-Tr/DNER positivity and a tb-PET-CT was performed, revealing the presence of widely spread relapsed hematologic neoplasia. Second line chemiotherapy resulted in clinical improvement of neurologic syndrome (2 months after treatment MMSE 24/30), such as regression of neoplastic disease.

Conclusion: Hodgkin's Lymphoma (HL) is associated with several paraneoplastic neurological syndromes (PNs), which can often be the only presenting feature of underlying disease, posing a diagnostic challenge. Except for paraneoplastic cerebellar degeneration in HL, other PNs are uncommon and have only been reported as isolated case reports or short series. However, when diagnosed on time, it may afford an opportunity for the institution of individual treatment with favorable outcome. References:

Carr I. The Ophelia syndrome: memory loss in Hodgkin's disease. Lancet (1982);1(8276):844-45

- Briani C, Vitaliani R, Grisold W, et al. PNS Euronetwork. Spectrum of paraneoplastic disease associated with lymphoma. Neurology (2011);76(8):705-10

- Laffon M, Giordana C, Almairac F, Benchetrit M, Thomas P. Anti$\mathrm{Hu}$-associated paraneoplastic limbic encephalitis in Hodgkin lymphoma. Leuk Lymphoma. (2012);53(7):1433-34 


\section{PRENATAL EXPOSURE TO HEAVY METALS AND NEURODEVELOPMENTAL DISORDERS: EPIDEMIOLOGICAL AND TOXICOLOGICAL ASPECTS}

\section{F. Di Gaudio, M. Scaglione}

School of Medicine and Surgery, University Hospital Paolo Giaccone, University Palermo (Palermo)

Aim: High levels of xenobiotics have been associated with several pathological conditions. The hypothesis of their etiopathogenetic role in the large family of neurodevelopmental disorders is increasingly frequent. This prospective study, conducted in CQRC, involved 217 mother-infant couples, selected in the Sicilia, and aimed to verify any correlations between metallic pollution of the mother and the newborn development. It is essential to have, first of all, suitable matrices, accurate measurement systems and a significant and representative statistical population. The study aims to provide further information on the neuro-psycho-motor outcome, following the baby in life outside the cradle.

Materials: The study looked at a large panel of heavy metals, reports accurate analytical data carried out in Mass Spectrometry, use the most suitable biological matrices for the purpose. For each mother-infant couple, metal levels were measured in the mother's hair, just before delivery, and in the umbilical cord blood at the birth of the baby.

Methods: Blood was drawn was obtained by using a syringe without needle and collected on metal-free tubes (Vacuette ${ }^{\circledR}$ Greiner) after discarding the first $3 \mathrm{ml}$, to limit any possible contamination. The measurement of the metals was performed by using Inductively Coupled Plasma Mass Spectrometry (ICPMS) of the Thermo Fisher. All the laboratory materials used were made by polyethylene and or Teflon. Each sample was subjected to a preliminar mineralization process. An instrumental Performance Report Test was obtained in order to verify the operative conditions both in STANDARD and KED modes by using a TUNE A solution at $10 \mu \mathrm{g} / \mathrm{L}(\mathrm{Li}, \mathrm{Be}, \mathrm{Bi}, \mathrm{Ce}, \mathrm{Co}, \mathrm{In}$,) in $\mathrm{HNO} 3$ $1 \%$ for covering the entire range of masses to be analysed. Data analysis was performed by Regional Quality laboratory Center, of the Sicilian Government Health Department, using geometric mean and categorical variables.

Results: Levels of metal in umbilical cord blood sample are not closely related to mother levels. This suggests that each placenta shows a peculiar permeability to the passage of metals.

Discussions: By combining, in each pair, the metal levels in the two samples, high risk for neurodevelopmental disorders pairs mother-child were identified, i.e. pairs in which both samples were found to have values beyond the range for one or more metals.

Conclusions: Long-term follow-up is required in order to verify a correlation between the high-risk phenotype and the onset of neurodevelopmental disorders.

References:

- M. Torre, L. Genova Gaia, M. G. Calevo, M. Wong, F. Di Gaudio, M. Raso, S. Barco, G. Cangemi. Blood metal levels after minimally invasive repair of pectus excavatum: is it time to change? European Journal of Cardio-Thoracic Surgery- Submitted 21.06.2020

- S. Orecchio, D. Amorello, M. Raso, S. Barreca, C. L. F. Di Gaudio, Determination of trace elements in gluten-free food for celiac people by ICP-MS. Microchemical Journal (2014); 116

\section{CAVERNOUS SINUS SYNDROME: UNRAVELING THE GORDIAN KNOT}

E. Di Sabatino ${ }^{1}$, I. Corbelli ${ }^{1}$, C. Gallina ${ }^{2}$, P. Chiarini ${ }^{2}$, M. Hamam ${ }^{3}$, G. Fiorucci $^{4}$, A. Falorni ${ }^{5}$, P. Giovenali ${ }^{6}$, C. Castrioto ${ }^{4}$, G. Guercini ${ }^{3}$, L. Parnetti $^{1}$, M. Di Filippo ${ }^{1}$, L. Gaetani ${ }^{1}$

${ }^{1}$ Neurology Unit, Department of Medicine, University of Perugia, Santa Maria della Misericordia Hospital (Perugia); ${ }^{2}$ Neuroradiology Unit, Santa Maria della Misericordia Hospital (Perugia); ${ }^{3}$ Neuroangiography Unit, Santa Maria della Misericordia Hospital (Perugia); ${ }^{4}$ Neurosurgery Unit, Santa Maria della Misericordia Hospital (Perugia); ${ }^{5}$ Endocrinology Unit, Department of Medicine, University of Perugia, Santa Maria della Misericordia Hospital (Perugia); ${ }^{6}$ Pathology Unit, Santa Maria della Misericordia Hospital (Perugia)

Introduction: Cavernous sinus (CS), also known as "the anatomical jewel box" for the considerable nerves and vessels it contains, can be affected by several vascular, inflammatory, traumatic or neoplastic diseases that give rise to CS syndrome (i.e. ophthalmoplegia, chemosis, proptosis, and trigeminal sensory loss) [1]. Although CS syndrome can be easily recognized, the diagnosis of the underlying disease could be a neurological challenge.

Case report: A 68-years-old woman was admitted in our Clinic because of a complete III cranial nerve palsy, chemosis, exophthalmos and pain in the right eye. She was on edoxaban because of atrial fibrillation, and she referred a familiar history of venous thrombosis. In order to rule out vascular and inflammatory causes of CS syndrome, she underwent a contrast-enhanced brain MRI and MR angiography that revealed an enlarged pituitary gland suggestive of adenoma, without significant compression on the right CS, and a cerebral angiography that demonstrated blood flow absence in the right $\mathrm{CS}$, suggestive of thrombosis. A screening for thrombophilia showed mutation of the MTHFR gene, and a possible antiphospholipid syndrome. A total body CT scan and an initial screening for neoplastic causes of CS syndrome were negative [1]. Enoxaparin followed by warfarin were started without improvement. Two weeks later, the patient underwent a control brain MRI that showed an enlarged lateral-convex right CS due to intra-CS, brain-parenchyma isointense material, without contrast enhancement. The MRI also showed a concomitant pituitary stalk thickening and enhancement, suggestive of hypophysitis [2]. Glucocorticoids were started with initial improvement, followed by a progressive worsening after discharge with appearance of IV, VI and V1 cranial nerves involvement. On MRI, right CS was more enlargedand, therefore, the possibility of CS infections and malignancies was further considered. CSF examination was negative for bacterial and mycotic infections. Eventually, transsphenoidal biopsy of CS and pituitary gland was performed, showing CS diffuse large B cell lymphoma with normal pituitary gland. A total body FDG-PET showed systemic localizations of lymphoma. The patient was then referred to the Hematology unit, and a proper treatment was started.

Conclusions: This case is paradigmatic of the complexity in the management of parasellar diseases. Although the semeiology of CS syndrome can be easily recognized, the underlying etiology could be difficult to identify. In our case, only the execution of transsphenoidal biopsy allowed to "cut the Gordian knot" and made a definite diagnosis of CS lymphoma.

References:

1. Bakan AA et al, Cavernous Sinus: A Comprehensive Review of its Anatomy, Pathologic Conditions, and Imaging Features, Clin Neuroradiol. (2014);25(2):109-25 
2. Gubbi S. et al. Hypophysitis: An update on the novel forms, diagnosis and management of disorders of pituitary inflammation. Best Pract Res Clin Endocrinol Metab. (2019); 12:101371

\section{INCIDENTAL MRI LESIONS SUGGESTIVE OF MULTIPLE SCLEROSIS ASSOCIATED WITH SYMPTOMATIC SUPRATENTORIAL ARACHNOID CYST}

\section{G. Falcicchio, M. Trojano, I. Simone}

Department of Basic Medical Sciences, Neuroscience and Sense Organs, University of Bari "Aldo Moro" (Bari)

Introduction: Arachnoid cysts (ACs) are considered benign congenital anomalies consisting of a wall lined by arachnoid cells and intraarachnoid collections of cerebrospinal fluid and they account for $1 \%$ of all intracranial lesions. Although almost all ACs remain silent, in some cases they may become symptomatic. Association with multiple sclerosis (MS) is an incidental event.

Case report: A 31-year-old woman came to the MS Centre for an incidental imaging finding of multiple white matter encephalic lesions without any associated symptoms. Her medical history was unremarkable until one year before when she developed slurred speech and occasional left facial and brachial paresthesias lasting two months. For these symptoms a brain magnetic resonance imaging (MRI) was performed showing a voluminous $\mathrm{AC}$ in left frontal, temporal and parietal lobes with mass effect and $7 \mathrm{~mm}$ midline shift. Furthermore multiple bilateral peritrigonal, frontal and occipital white matter T2-lesions without contrast enhancement were present. The patient underwent ventriculo-cystocysternostomy with complete regression of the symptoms. The cyst gradually reduced and the midline shift almost disappeared. Serial brain MRIs showed an increased number of inactive FLAIR-lesions. The last one however revealed a new active plaque in the right paratrigonal temporal lobe. For this reason, the patient was referred to our MS centre for further evaluation. At neurological assessment nystagmus, left hyperreflexia and lower limbs hypopallesthesia were detected. All haematological analyses, including autoimmunity, were in range. Virological screening was not suggestive of active infection. Evoked potentials were normal. A new active lesion at D1 was detected at the MRI check. A dose of 3 grams of methylprednisolone was administered. Diagnosis of MS was performed and disease-modifying therapy (DMT) is ongoing.

Discussion: The neurological symptoms reported by our patient were related to the large AC, in fact they completely disappeared after surgery. Nevertheless, the attention to the incidental MRI lesions suggestive of demyelinating disease (Radiologically Isolated Syndrome) led us to conclude for a diagnosis of MS as demonstrated by spatial and temporal dissemination of MRI plaques without clinical evidence.

References:

- Osama Shukir Muhammed Amin and Sa'ad Seud Shwani Asymptomatic supratentorial arachnoid cyst and symptomatic demyelination plaques: which one needs treatment? BMJ Case Reports (2010); 1-3

- Logan C et al. Arachnoid Cysts - Common and Uncommon Clinical Presentations and Radiological Features. Journal of Neuroimaging in Psychiatry and Neurology (2016);1(2):79-84

- D.G. Grosset et al. Missed Diagnosis - Single central nervous system lesions can simulate multiple sclerosis. Postgraduate Medical Journal (1989);65:656- 59

\section{SYMPTOMATIC HYPOPHONIA IN PATIENT WITH EMBOLIC STROKE AND AORTIC ARCH ANEURYSM: A CASE REPORT}

\author{
P. Falco, F. Fattapposta
}

Department of Human Neuroscience, La Sapienza University (Roma)

In this report, we present the case of a 65 years old man who came to our attention with a wake-up paresis of the left leg (NIHSS 2); he reported a two-weeks history of dysphagia for fluids and hypophonia/ disphonia, with symptoms slowly progression. An urgent brain MRI was performed, that showed two small ischemic strokes in the right hemisphere, one in the lenticular-external capsule and the other one in the peri-trigonal region, without DWI/PWI mismatch. In our neurology ward, we performed a dysphagia test, which resulted negative; an ORL faringo-laryngoscopy evaluation was made, showing an hypomobile left vocal cord. In order to exclude a left recurrent laryngeal nerve paresis, a contrast chest-CT scan was performed, which showed a large aneurysm (three $\mathrm{cm}$ ) of the inferior wall of the aortic arch, with a parietal thrombotic apposition. Therefore, the patient underwent an elective surgical exclusion of the aneurysm with implantation of a personalized-engineered mechanical prothesis. One year later there was an improvement of clinical conditions and neurologic examination showed only a slight hypophonia. Moreover, the patient didn't have any other ischaemic event. We concluded for a wake-up embolic stroke caused by a symptomatic aortic arch aneurysm that first manifested with a subacute paresis of the left recurrent laryngeal nerve.

References:

- Zangirolami AC, Oliveira FV, Tepedino MS. Ortner's Syndrome: Secondary Laryngeal Paralysis Caused by a Great Thoracic Aorta Aneurysm. Int Arch Otorhinolaryngol. (2015);19(2):180-82

- Teixido MT, Leonetti JP. Recurrent laryngeal nerve paralysis associated with thoracic aortic aneurysm. Otolaryngol Head Neck Surg. (1990);102(2):140-44

\section{AQUAPORIN-4 ANTIBODY POSITIVE NEUROMYELITIS OPTICA SPECTRUM DISORDER IN AN ELDERLY PATIENT WITH HYPERCKEMIA SUCCESSFULLY TREATED WITH PLASMA EXCHANGE AND RITUXIMAB}

\author{
A. Farina ${ }^{1}$, S. Casagrande ${ }^{1}$, L. Massacesi ${ }^{1}$, A. Barilaro ${ }^{2}$
}

${ }^{1}$ Department of Neuroscience, Drug and Child Health, University of Florence (Firenze); ${ }^{2}$ Neurology 2, AOU Careggi (Firenze)

Introduction: The discovery of anti-aquaporin 4 (AQP4) antibodies as the pathogenic marker for neuromyelitis optica spectrum disorder (NMOSD) has widened the spectrum of the disease allowing the description of unexpected clinical manifestations and age groups. Clinical onset in the very elderly is rare (1\% above 80 years) [1]. AQP4 is expressed in the skeletal muscle and hyperCKemia has been reported as a possible manifestation of the disease [2]. A rare of AQP4 positive NMOSD in an octogenarian man presenting with myelitis and hyperCKemia is herewith reported.

Case Report: An 83-year-old man developed gait imbalance, ascending numbness in lower limbs extending to abdomen over few days, urinary retention and fecal incontinence. 2-week episode of nausea with persistent hiccups 5 months before the presenting event was reported in clinical history, which was also remarkable for atrial fibrillation, chronic renal failure and glucose intolerance. Neurological examination showed 
D6 sensory level and severe gait ataxia (EDSS 7.5). Blood tests revealed increased CPK levels (>13.000) and thrombocytopenia. Spinal cord MRI demonstrated longitudinally extensive myelitis from D2 to D9. Cerebrospinal fluid analysis showed normal cell count $(4$ cells/ $\mathrm{mcl})$ and mild protein increase $(0.58 \mathrm{~g} / \mathrm{l})$. Oligoclonal bands were absent. Brain MRI showed a small lesion in the area postrema. AQP4 antibodies were detected in serum and myositis antibody panel was negative. CPK levels returned to normal during hospitalization. Screening for occult malignancies was negative. Patient's symptoms progressed despite high dose IV steroid treatment, which led to poor blood glucose control requiring high dose insulin. Plasma exchange was performed without complications and with neurological improvement. At discharge, sphincter dysfunction was completely absent, with amelioration of gait ataxia (EDSS 6.5). Rituximab was started as maintenance treatment. At 6-months follow-up, the patient was relapse-free and neurologically stable. He did not report any signs or symptoms of infections despite complete B cell depletion and hypogammaglobulinemia, and insulin was suspended after glucose levels normalization.

Discussion: To the best of our knowledge, this is the oldest case of NMOSD presenting with hyperCKemia ever reported. Efficacy and safety data of NMOSD therapies in the elderly are limited. The use of steroids in this age group may be limited by the presence of comorbidity. The experience of a favorable outcome in our case supports the use of both plasma exchange and rituximab as effective and well-tolerated therapeutic options in elderly patients with autoimmune diseases such as NMOSD [3].

References:

1. Niino M, Uesugi H, Takahashi T, Fukazawa T, Minami N, Tashiro J, Fujiki N, Doi S, Kikuchi S. Recurrent brainstem lesions mimicking infarctions in an elderly patient with neuromyelitis optica spectrum disorder. Intern Med. (2012);51(7):809-12

2. He D, Zhang A, Li Y, Cai G, Li Y, Guo S. Autoimmune aquaporin4 induced damage beyond the central nervous system. Mult Scler Relat Disord. (2017) Nov;18:41-46

3. Basic-Jukic N, Brunetta B, Kes P. Plasma exchange in elderly patients. Ther Apher Dial. (2010) Apr;14(2):161-5

\section{FINGOLIMOD IN PEDIATRIC MULTIPLE SCLEROSIS: THREE CASE REPORTS}

\section{A. N. Ferilli, L. Papetti, M. Valeriani}

Department of Neuroscience, Bambino Gesù Children's Hospital, IRCCS (Roma)

Background and Objective: Treatment for pediatric-onset multiple sclerosis (POMS) currently reflects treatment for adult-onset MS, despite some differences in its clinical course. First-line treatment of POMS generally consists of interferon $\beta$-1a or glatiramer acetate, with therapies such as the oral immunomodulator fingolimod reserved for second-line treatment. In cases of severe disease, fingolimod can be proposed as first-line therapy. This paper presents three case histories of patients with POMS, and highlights the different uses of fingolimod within the POMS treatment algorithm.

Methods and Results: First and third cases are examples of escalation therapy, both in females aged 16 to 17 years, with fingolimod administered second-line following disease progression. The second case is an example of using fingolimod as a first-line therapy, given to a 12-year-old male with severe disease.
Conclusion: All three cases, over a period of approximately one year after initiation of fingolimod treatment, there was no further disease progression and no adverse events were recorded.

\section{STROKE-LIKE EPISODES AND CORTICOSPINAL TRACT HYPERINTENSITY: A RARE AND CHALLENGING CASE OF PARANEOPLASTIC SYNDROME WITH ANTI-YO ANTIBODIES}

\section{Filippi, N. Bruschi, F. Tazza, A. Laroni, M. Grandis}

Department of Neuroscience, Rehabilitation, Ophthalmology, Genetics, Maternal and Child Health, University of Genoa and IRCCS AOU San Martino-IST (Genova)

Anti-yo paraneoplastic encephalitis is a rare condition, typically characterized by subacute development of cerebellar ataxia, most frequently in women with pelvic or breast tumors. Brain magnetic resonance imaging (MRI) is often normal in early stages, with cerebellar atrophy seen later. We herein report a rare presentation of anti-yo encephalitis with atypical clinical and neuroimaging features. A 74-year-old woman was admitted to our clinic in March 2019 because of acute aphasia and weakness on the left side; the patient underwent intravenous thrombolysis with complete clinical recovery; the day after, a brain MRI scan showed one lesion with restricted diffusion in the white matter adjacent to the right lateral ventricle. In the next months, she presented further transient stereotyped episodes of language disturbance and weakness on the left side and a progressive cognitive decline and worsening ataxia, and she was once more admitted to our clinic. Brain contrast-enhanced MRI in September 2019 revealed the persistence of the previously described lesion, with partial restricted diffusion and expansion along the ipsilateral corticospinal tract, likely due to Wallerian degeneration. This lesion was clearly visible inT2/FLAIR, without contrast enhancement in T1. Routine blood work, nerve conduction velocities and needle electromyography were unremarkable, whereas electroencephalography revealed the presence of slow waves particularly in the right hemisphere, where brain positron emission tomography (PET) reported a severe hypometabolism. Cerebrospinal fluid (CSF) examination showed abnormal protein level $(0.8 \mathrm{~g} / \mathrm{L})$ and oligoclonal bands in serum and CSF ("mirror pattern"). Extensive workout with total body computed tomography (CT) and whole body PET scan unveiled ovarian cancer. Anti-yo antibodies were detected in CSF. The patients was treated with intravenous immunoglobulin, surgical intervention and adjuvant chemotherapy which is still ongoing. The last MRI scan showed persistence of the lesion with unchanged volume but a less intense signal. Few cases have been reported so far of Anti-Yo paraneoplastic syndrome with pseudobulbar affect, frontal executive functional impairment and motor neuron disease in addition to progressive cerebellar impairment [1-2]; multifocal inflammatory changes in cerebellum, brainstem, spinal cord and nerve rootlets have been described [3]. However, the association of stroke-like episodes with MRI evidence of progressive corticospinal tract hyperintensity has never been described, and expands the clinical spectrum of anti-Yo -associated syndromes.

References:

1. M. Le May, S. Dent Anti-Yo antibody-mediated paraneoplastic cerebellar degeneration associated with cognitive affective syndrome in a patient with breast cancer: a case report and literature review. Curr Oncol. (2018) Dec;25(6):e585-e591

2. B Jane Distad, Michael D Weiss Paraneoplastic Motor Neuron Disease Associated With Purkinje Cell Autoantibody Type 1. J Clin Neuromuscul Dis. (2010) Sep;12(1):36-41 
3. A McKeon, JA. Tracy Purkinje Cell Cytoplasmic Autoantibody Type 1Accompaniments the Cerebellum and Beyond Arch Neurol. (2011);68(10):1282-89. Published online June 13, 2011. doi:10.1001/archneurol.2011.128

\section{CLUSTER HEADACHE AS THE SOLE MANIFESTATION OF MULTIPLE SCLEROSIS. REPORT OF A CASE}

\author{
A. Fiore ${ }^{1}$, E. Tsantes ${ }^{1}$, E. Curti ${ }^{1}$, V. Bazzurri ${ }^{1}$, F. Granella ${ }^{2}$
}

${ }^{1}$ Neurosciences Unit, Department of Medicine and Surgery, University of Parma (Parma); ${ }^{2}$ Multiple Sclerosis Centre, Department of General Medicine, Parma University Hospital (Parma)

Background: Headache is more frequent in patients with multiple sclerosis (MS) than in the general population. Tension-type headache and migraine without aura are the most common primary headaches in MS, while cluster headache $(\mathrm{CH})$ is considered exceptional [1]. Here we report a unique case of $\mathrm{CH}$ in a MS patient showing a brainstem lesion contralateral to headache.

Case Report: A 54-year-old woman complained since 2 years of attacks of severe pain located in the right maxillary-zygomatic region. The attacks occurred 2-4 times a day, often starting at night, and lasted 30-60 minutes. There were no local autonomic signs, but the patient was restless and agitated during the attacks. In 2017 trigeminal neuralgia was diagnosed and a treatment with carbamazepine $600-800 \mathrm{mg}$ /day was started, leading to a reduction of the attacks frequency. A brain MRI showed two T2-hyperintense lesions, one in the left periventricular area and the other in the left periaqueductal gray matter region. After two years, the patient decided to reduce the dosage of carbamazepine to $200 \mathrm{mg}$ /day and the frequency of the attacks increased. As a consequence, she was evaluated at the emergency room by a neurologist, who suggested to increase the carbamazepine dose to $600 \mathrm{mg} /$ day and to repeat a bran MRI, that confirmed the infratentorial lesion and showed multiple focal demyelinating areas in the supratentorial white matter. In 2019 the patient was admitted to our Day Hospital. The neurological and clinical examinations were normal. After a re-assessment of the pain characteristics, we made a chronic cluster-like headache diagnosis and introduced prophylactic therapy with verapamil $360 \mathrm{mg} /$ day, obtaining a significant decrease of the attacks frequency. A spinal MRI revealed no demyelinating plaques and the biochemical and immunoserological analyses were within normal range. Cerebrospinal fluid (CSF) analysis showed the presence of oligoclonal bands. Based on MRI results and the presence of CSFoligoclonal bands, a diagnosis of multiple sclerosis was made.

Conclusions: This is the second case in the literature in which $\mathrm{CH}$ is the sole clinical manifestation of MS and the first case with a brainstem lesion contralateral to the pain side. Patient's brainstem lesion affected the dorsal longitudinal fasciculus, that contains fibers connecting the hypothalamus, brainstem parasympathetic nuclei and preganglionic autonomic neurons, apparently all involved in CH attacks' mechanisms [2]. Since neuroimaging showed a lesion contralateral to $\mathrm{CH}$ pain, we hypothesized the presence of projections between hypothalamus and the contralateral trigeminocervical complex, besides the known ipsilateral connection.

References:

1. La Mantia L, Prone V. Headache in multiple sclerosis and autoimmune disorders. Pain (2013); 154 (12), 2691-99

2. Dantas S A F, Alho E J L, da Silva J J, Mendes Neto N N, Talamoni Fonoff E, Hamani C. Deep brain stimulation modulates hypothalamic-brainstem fibers in cluster headache: case report. Journal of Neurosurgery (2020);132: 717-20

\section{ISCHEMIC STROKES AND MULTIPLE PERIPHERAL VENOUS THROMBOSIS: A COMPLEX CASE OF SICKLE CELL ANEMIA PATIENT}

G. Fiume, C. Dell'Aera, L. Ferraù, F. Grillo, M. Cotroneo, C. Casella, M. Fazio, P. La Spina, A. Toscano, R. Musolino

Department of Clinical and Experimental Medicine, University of Messina (Messina)

Aims: To describe the diagnostic and therapeutic pathways in a patient affected by Sick Cell Anemia (SCA) with recurrent ischemic strokes and multiple peripheral venous thrombosis.

Case Report: A 45 years-old woman with a history of SCA in transfusion therapy was admitted in a peripheral center in October 2019 for global aphasia, deviation gaze, and right homonymous lateral hemianopia, hemiparesis, neglet and hypoesthesia (NIHSS 19). She underwent brain CT and intravenous thrombolysis $(0,9 \mathrm{mg} / \mathrm{kg})$ and transferred to our Stroke Unit where mechanical thromboectomy for middle left cerebral artery occlusion (M2 tract) was performed. Sickle Hemoglobin $(\mathrm{HbS})$ at admission was $38.8 \%$, $\mathrm{HbA} 9 \mathrm{~g} \%$ and G.R. $2.920 .0000 \mathrm{~mm} 3$ and the patient underwent one session of eritroexchange (EEX). Four days after admission, the patient developed cephalic vein thrombosis of right limb confirmed by Color Doppler and fondaparinux at dose of $2,5 \mathrm{mg} /$ die was started. Transthoracic echocardiogram, cardiac MRI, coronary angiography, Holter-ECG, Color Doppler of the supraaortic vessels and molecular analysis for genetic thrombophilia carried out during the stay were normal. Other two sessions of EEX were made and the patient was discharged at a rehabilitation facility with non-fluent aphasia and right facio-brachial hemiparesis (NIHSS 5). Thirty days after first ischemic stroke the patient presented a worsening of aphasia and right hemiparesis. Brain MRI showed new left front-insular and temporo-parietal ischemic lesions and the patient underwent a session of EEX. Five days after second admission a popliteus-femoral-iliac left venous axis and pulmonary veins thrombosis showed at chest and abdomen CT angiography have occurred and fondaparinux was increased at $7,5 \mathrm{mg} / \mathrm{die}$. In the following days other three sessions of EEX were made and after fifteen days a caval filter was positioned. At six months of follow-up the patient didn't have other relapses.

Discussion and Conclusions: SCA is a hemolytic disorder associated with a hypercoagulable state. [1] More than 33\% of deaths in SCA patients are caused by vascular occlusions. [2] There are few data on secondary stroke prevention in adult patients, but initial treatment with EEX after a ischemic stroke appears to be the first choice in terms of effectiveness in preventing recurrence. [3] Moreover, peripheral venous thrombosis can accompany and complicate the SCA patient's picture and for this reason it's important to stratify the risk of thromboembolism and adopt the right therapy. This case demonstrates how regular EEX, an adequate anticoagulant therapy and, in addition, a caval filter positioning, allow to reduce the recurrence of cerebral and venous thrombotic events.

References:

1. Naik RP, Streiff MB, Haywood CJ, Nelson JA and Lanzkron S. Venous thromboembolism in adults with sickle cell disease: A serious and under-recognized complication. Am J Med. (2013); 126(5): 443-49

2. Stuart MJ and Nagel RL. Sickle-cell disease. Lancet (2004);364:1343-60

3. AIEOP - Linee guida per la gestione della malattia drepanocitica in età pediatrica in Italia. 2018. III edizione. 
ERENUMAB DURING PREGNANCY: A CASE REPORT IN A PATIENT WITH CHRONIC MIGRAINE

\author{
L. Fofi, G. Egeo, C. Aurilia, P. Barbanti
}

Headache and Pain Unit, IRCCS San Raffaele (Roma)

Background: Migraine affects women of childbearing age. Erenumab is a fully human monoclonal antibody targeting the calcitonin gene-related (CGRP) receptor with a very favorable efficacy/tolerability profile in migraine prevention. Very limited data are available on its effects in pregnancy.

Case presentation: Here we describe the case of a 37-years-old woman who became pregnant during erenumab treatment for chronic migraine. At the time of her first visit at our Unit, she reported 18 headache days/month - despite concomitant treatment with valproate $300 \mathrm{mg}$ b.i.d. - and 5 prior preventative treatments failures. We started erenumab $70 \mathrm{mg}$ therapy, tapering down valproate. Erenumab reduced migraine frequency to 7 days/month since the first administration. On the followup visit scheduled for the fifth erenumab administration, the patient informed us of her pregnancy, documented by appropriate serological test the day before. Erenumab was immediately stopped. The last erenumab $70 \mathrm{mg}$ dose had been taken during the first two weeks of pregnancy. Serological fetal DNA test was normal. Migraine episodes increased up to 10 days/month during the I pregnancy trimester, progressively disappeared over the II-III trimesters, and gradually reappeared from the 34th week of pregnancy throughout delivery and breastfeeding. No adverse event or complication occurred during pregnancy. The baby, delivered by vaginal birth, was in healthy conditions.

Conclusions: In this case report, we document no complications, abnormalities, or adverse events in both the mother and the child, and a physiological course of pregnancy, delivery and breastfeeding following erenumab exposure during pregnancy.

References:

- Goadsby PJ, Reuter U, Hallström Y, et al. A con- trolled trial of erenumab for episodic migraine. N Engl J Med. (2017);377:2123-32

- Tepper S, Ashina M, Reuter U, et al. Safety and efficacy of erenumab for preventive treatment of chronic migraine: A randomised, double-blind, placebo-controlled phase 2 trial. Lancet Neurol. (2017);16:425-34

- Bussiere JL, Davies R, Dean C, Xu C, Kim KH, Vargas HM, Chellman GJ, Balasubramanian G, Rubio-Beltran E, MaassenVanDenBrink A, Monticello TM. Nonclinical safety evaluation of erenumab, a CGRP receptor inhibitor for the prevention of migraine. Regul Toxicol Pharmacol. (2019) Aug;106:224-238. doi: 10.1016/j.yrtph.2019.05.013. Epub 2019 May 11

\section{NEUROMA OF INFERIOR ALVEOLAR NERVE: A TREATABLE MIMIC OF REFRACTORY TRIGEMINAL NEURALGIA}

\author{
F. Fortunato ${ }^{1}$, M. Trimboli ${ }^{1}$, L. Fortunato ${ }^{2}$, A. Gambardella ${ }^{1}$ \\ ${ }^{1}$ Institute of Neurology, Magna Graecia University (Catanzaro); ${ }^{2}$ Institute \\ of Odontostomatology, Magna Graecia University (Catanzaro)
}

Introduction: Trigeminal neuralgia $(\mathrm{TN})$ is characterized by recurrent unilateral brief electric shock-like pain that is abrupt in onset and termination. [1] The diagnosis of TN is mostly clinical, and especially $\mathrm{TN}$ of the third branch can pose diagnostic challenge with atypical odontalgia or other causes of facial pain. [2-3] Here, we report a case of neuroma involving the inferior alveolar nerve that mimicked idiopathic refractory $\mathrm{TN}$. Its surgical removal has led to complete and sustained pain freedom.

Case description: A 71-year-old man presented to our clinic because of facial pain strongly suggestive of refractory TN that had started 5 years earlier. The pain was located at the left part of his jaw, on the mandibular division of the trigeminal nerve and persisted over the following years, despite several medications including oxcarbazepine pregabalin, gabapentin, and baclofen. Neurological examination was normal, Brain 3T MR study showed no intracranial masses or any neurovascular conflict in the cerebellopontine cistern. Venous and arterial MR angiography were normal, as also the blink reflex. His current treatment was gabapentin $900 \mathrm{mg} /$ day and even the add-on of carbamazepine $800 \mathrm{mg} /$ day in combination with amitriptyline failed to give any benefit. There were no relevant dental interventions prior to the onset of pain. Intraoral examination was unremarkable; radiological investigation revealed an oval lesion adjacent to the left mental foramen that was surgically removed. Histological examination revealed a fibrous tissue with a foreign body giant cell reaction, organized around amorphous brownish material. There was also a multifocal proliferation of bundles of nerves in a haphazard arrangement, without new bone formation. The fibrous reaction peripherally involved fibers of striated muscle tissue. All these features were characteristic of traumatic neuroma combined with a foreign body reaction. Surgical removal of the neuroma has led to complete and sustained pain freedom, without recurrence of the lesion in the following 18 months.

Discussion and conclusion: This case emphasizes the diagnostic challenge that can pose facial pain involving the mandibular branch for even the most experienced neurologist. It also reinforces the necessity for a thorough evaluation of all possible causes of refractory facial pain, before recommending more invasive therapeutic procedures. In our patient, indeed, the routine intraoral radiological examination allowed us to reach the diagnosis of neuroma, whose removal resolved the disabling symptoms.

References:

1. Headache Classification Committee of the International Headache Society (IHS). The International Classification of Headache Disorders, 3rd edition. Cephalalgia (2018);38:1-211

2. Duvall JR, Robertson CE. Clinical Reasoning: A misdiagnosis of atypical trigeminal neuralgia. Neurology (2019);93:124-31

3. Ko AL, Lee A, Raslan AM, Ozpinar A, McCartney S, Burchiel KJ. Trigeminal neuralgia without neurovascular compression presents earlier than trigeminal neuralgia with neurovascular compression. J Neurosurg (2015);123:1519-27

\section{RAPIDLY PROGRESSIVE COGNITIVE IMPAIRMENT WITH ACQUIRED LEUKOENCEPHALOPATHY: REPORT OF TWO CASES WITH RARE CAUSES OF WHITE MATTER DISORDERS}

\author{
S. Franciotta ${ }^{1}$, S. Zywicki ${ }^{1}$, E. Monai ${ }^{1}$, M. G. Anglani ${ }^{2}$, A. Cagnin ${ }^{1,3}$
}

${ }^{1}$ Neurology Unit, University Hospital of Padua (Padova);

${ }^{2}$ Neuroradiology Unit, University Hospital of Padua (Padova);

${ }^{3}$ Department of Neurosciences, Medical School, University of Padua (Padova)

Background: Acquired causes of white matter (WM) disorders present with a variety of neurological symptoms. Subacute onset of rapidly 
progressive cognitive decline and MRI findings of WM changes represent a diagnostic challenge particularly when rare conditions are involved.

Objectives: To describe imaging features of rare neurological disorders presenting in adulthood with similar WM changes and discuss differential diagnosis.

Methods: We report on two patients, a 64-year-old male and a 72year-old female, presenting with rapidly progressive cognitive impairment and extensive T2-weighted WM abnormalities at clinical onset. Both patients underwent a thorough diagnostic work-up comprehensive of CSF analysis, extensive immunological and microbiological assessment, whole body imaging with full-body CT-scan and full-body and brain 18FDG PET-MRI. In both patients a brain biopsy was performed.

Results: In both cases WM changes increased in extension and new lesions appeared in the following 2-4 months from clinical onset. In the first case MRI imaging showed sparse DWI hyper-intensities of WM lesions suggestive for hypercellularity, without contrast enhancement, and vascular abnormalities on MRA and conventional angiography; in the second patient, multiple microhaemorrhages appeared in follow-up MRI studies within WM regions of T2 hyper-intensity. CSF analysis revealed lymphocytic pleocytosis without monoclonal expansion and increased protein concentration. Repeated cycles of IV high dose steroids were administered with transient clinical benefit. In both cases immunosuppressive treatment with cyclophosphamide was attempted, with no clear response. Brain biopsy was inconclusive in both patients. The first patient died of pneumonia after 3 months from disease onset; the second patient worsened until death in 10 months. On autopsy, Intravascular Large B Cell Lymphoma (IVLBCL) was diagnosed in the first patient and Acute Hemorrhagic Leukoencephalitis (AHLE) in the second one.

Conclusions: Similar rapidly changing WM abnormalities on brain MRI are neuroimaging findings which can underlie different rare neurological disorders, making the differential diagnosis difficult. In these cases, repeated brain biopsy is often necessary to achieve a diagnosis. Brain imaging features, although non-specific, along with clinical and biological data, could give hints for suspecting the rare conditions here described.

\section{ATYPICAL CLINICAL PRESENTATION OF CADASIL IN YOUNG AFRICAN MAN}

\section{G. Galli ${ }^{1}$, E. Virgilio ${ }^{2}$, C. Montabone ${ }^{1}$, D. Vecchio ${ }^{3}$, R. Cantello ${ }^{1}$}

\begin{abstract}
${ }^{1}$ Neurology Unit, Department of Translational Medicine, University of Piemonte Orientale, Azienda Ospedaliera-Universitaria Maggiore della Carità (Novara); ${ }^{2}$ Neurology Unit, Department of Translational Medicine, Phd program in Medical Sciences and Biotechnologies, Department of Translational Medicine, University of Piemonte Orientale, Azienda Ospedaliera-Universitaria Maggiore della Carità (Novara); ${ }^{3}$ Neurology Unit, Department of Translational Medicine, Phd program in Medical Sciences and Biotechnologies, Department of Translational Medicine, Interdisciplinary Research Center of Autoimmune Diseases (IRCAD), Department of Health Sciences, University of Piemonte Orientale, Azienda Ospedaliera-Universitaria Maggiore della Carità (Novara)
\end{abstract}

Introduction: CADASIL is a heritable cause of stroke and vascular dementia in adults, caused by mutations in NOTCH3 gene. It is characterized by subcortical ischaemic events, migraine with aura, cognitive impairment and psychiatric disorders. T2 white matter (WM) abnormalities at MRI can precede symptoms onset (except for migraine) by 10-15 years. We present a 33-year-old Nigerian man who developed progressive and severe spastic palsy of the right limbs, within a year, later diagnosed with CADASIL.

Case Report: At first neurological examination the patient presented with mild palsy of right arm and severe palsy of lower limbs with spasticity (Babinski sign and achilleus clonus). Deambulation gradually deteriorated with necessity of support. First symptoms started one year prior when the patient was still in Nigeria. His medical history was unremarkable and no family history of neurological disease was reported. MRI revealed severe leukoencephalophaty with multiple confluent T2 WMhyperintensities and lacunar lesions in periventricular regions, anterior temporal lobes as well as midbrain and pons with no contrast enhancement. An extensive infective, rheumatologic and metabolic screening was performed without pathological findings; no modifiable cerebrovascular risk factors were found and lumbar puncture was unremarkable. Metachromatic leukodystrophy, Fabry, Krabbe and Adrenoleukodistrophy diseases were excluded. Due to significant linguistic barrier, neuropsychological evaluation was not possible. Finally, genetic test for NOTCH3 was performed revealing the presence of R588C mutation - exon 11 -. Accordingly, CADASIL diagnosis was made.

Discussion and Conclusions: Progressive spastic hemiparesis normally is not suggestive for CADASIL, usually characterized by migraine with aura, followed by acute neurological deficits due to ischaemic events and MRI abnormalities. Motor disabilities and dementia usually appear later on. We cannot exclude mild cognitive impairment in our patient due to linguistic barrier. Moreover, the frequencies of NOTCH3 mutations are not completely known among African population and few cases are described in literature, in contrast with European and Asian population. Mutations in exon 11 are common in Italian population but to our knowledge R558C mutation is not described in African population. Mutations outside domains 1-6 might predispose to a milder phenotype, however our case was associated with early severe leukoencephalophaty. Our case highlights the importance to consider CADASIL diagnosis in the differential diagnosis of African patients, even with atypical clinical presentations or absent family history. If MRI is highly evocative a search for NOTCH3 mutation should be recommended.

References:

- R. O Akinyemi, M. O Owolabi, M. Ihara3 et al. Stroke, Cerebrovascular Diseases and Vascular Cognitive Impairment in Africa. Brain Res Bull. (2019) February; 145: 97-108. doi:10.1016/ j.brainresbull.2018.05.018

- J. W. Rutten, H. G. Dauwerse, G. Gravesteijn et al. Archetypal NOTCH3 mutations frequent in public exome: implications for CADASIL. Annals of Clinical and Translational Neurology (2016); 3(11): 844-53 doi: 10.1002/acn3.344

- O. Ross, A. Soto-Ortolaza, M. Heckman et al. NOTCH3 Variants and Risk of Ischemic Stroke. PLOS One (2013);8(9):e75035

\section{CEREBRAL MICROBLEEDS: A COMMON FINDING IN PATIENTS WITH CONGENITAL HEART DISEASE AFTER CARDIAC SURGERY}

\author{
S. Gallo Cassarino, G. Barbiera, M. Perini, L. Bet
}

IRCCS Policlinico San Donato, Neurology Unit, University of Milan (Milano)

Case report: A Caucasian woman of 22 years old presented to the Emergency Room due to acute onset, about four hours before, of visual disturbance, numbness and hyposthenia of left limbs, followed by her usual headache. She suffered of migraine with and without aura and 
had a history of Tetralogy of Fallot surgically treated at 5 months old. Because of her history of migraine, a month before she performed a brain MRI, including T2* sequences, that documented the presence of more than 10 cerebral microbleeds (CMBs) in both lobar and lacunar sites, and a chronic ischemic lesion in the left caudate nucleus (head). The neurological evaluation showed a mild hypoesthesia of the left arm, improved compared to the onset, a mild hyposthenia of left limbs and lateralised migraine (right side) associated with nausea. The visual disturbance, described as a light waves not clearly localized, resolved within 30 minutes from onset. The brain CT was negative for acute cerebrovascular events. Migraine with atypical aura was hypothesised, but migraine stroke could not be excluded due to persistence of motor symptoms. Nevertheless, considering the presence of a high burden of CMBs, thrombolysis was excluded. ASA $1000 \mathrm{mg}$ and metoclopramide $10 \mathrm{mg}$ IV were administered with headache improvement, but numbness and hyposthenia of left arm persisted. The patient was admitted in the Neurology Unit. Sensitive symptoms resolved within 24 hours whereas motor deficits within 72 hours. Brain MRI with intracranial vessel study was performed, showing no acute ischemic lesions and confirmed the presence and location of multiple CMBs in bilateral cortical (frontal, parietal, temporal and occipital) and subcortical (pulvinar, cerebellar cortex and cerebellar peduncles) sites. The MRI was compared with the previous one and no increase of CMBs was pointed out.

Discussion: The presence of CMBs has been described in pediatric and adult patients with congenital heart disease (CHD), included Tetralogia of Fallot, after cardiac surgery [1,2]. The hypothesis is that cardiac surgery may affect the brain through cerebral micro- o macroembolism and hypoperfusion. In particular, percutaneous valvereplacement manoeuvres, that implicate valve manipulation, balloon expansion, and vessels mechanical stress could generate small cerebral embolic infarcts. Therefore, these findings should be interpreted as chronic correlates of an acute injury rather than expression of haemorrhagic diathesis. Based on this remark, we think that CMBs in surgically treated CHD should not rule out thrombolyisis in otherwise eligible patients.

References:

1. Codari M, Papini GDE, Melazzini L, Pluchinotta FR, Secchi F, Carminati M, Frigiola A, Chessa M, Sardanelli F. Does Tetralogy of Fallot affect brain aging? A proof-of-concept study. PLoS One (2018) Aug 21;13(8):e0202496. doi: 10.1371/ journal.pone.0202496. eCollection 2018. PubMed PMID: 30130369; PubMed Central PMCID: PMC6103512

2. Kim PPC, Nasman BW, Kinne EL, Oyoyo UE, Kido DK, Jacobson JP. Cerebral Microhemorrhage: A Frequent Magnetic Resonance Imaging Finding in Pediatric Patients after Cardiopulmonary Bypass. J Clin Imaging Sci. (2017) Jul 6;7:27. doi: 10.4103/ jcis.JCIS_29_17. eCollection 2017. PubMed PMID: 28781924; PubMed Central PMCID: PMC5523507

\section{BILATERAL PTOSIS AND DYSPHAGIA IN A 73-YEAR-OLD WOMAN: A LATE-DIAGNOSED OCULOPHARYNGEAL MUSCULAR DYSTROPHY}

\section{S. Gallo, R. Cantello, D. Vecchio}

Neurological Department, University of Piemonte Orientale, "Maggiore della Carità" University Hospital (Novara)

Introduction: Oculopharyngeal muscular dystrophy (OPMD) is a rare disorder characterized by progressive eyelid ptosis, dysphagia and further development of proximal limb weakness. Disease presentation is usually during the 5-6th decade. OPMD is an autosomal dominant muscular disease caused by expansions of the GCG trinucleotide in the polyadenylate binding-protein nuclear 1 (PABPN1) gene. We are presenting a case of OPMD with a late onset of oculopharyngeal symptoms and delayed diagnosis.

Case report: A 73-year-old woman presented with progressive bilateral ptosis and swallowing difficulties started 2 years before. When questioned, she reported mild proximal limb weakness which had started 8 years before that affected her ability in climbing stairs (not her walking). Her family history revealed her mother and her maternal uncle had mild bilateral ptosis. The neurological examination showed: bilateral ptosis around $60 \%$ with no fatigability, limitation of the eye motility to the left and symmetric muscles weakness as follows (according to MRC scale): deltoid, biceps, extensor digitorum, EDB 3/5; iliopsoas 4/5. Neurophysiological studies excluded neuromuscular junction disorder and showed myopathic features at needle testing. The clinical picture and the familiar history suggested a muscle autosomal disorder. Results: Genetic testing revealed indeed a heterozygous (GCN)14/Ala14 [(GCG)10(GCA)3(GCG)] mutation that led to the diagnosis of OPMD.

Discussion and conclusion: This case report pictures a rare autosomal muscular dystrophy in the elderly that showed an unusual late onset of the oculopharyngeal symptoms compared to limb muscular weakness. OPMD is a rare disease that must be considered in patients that present late-onset features that could resemble myasthenia gravis with no fatigability. An important clue for the diagnosis is provided by family history of ptosis or dysphagia.

References:

- Mirabella M, Silvestri G, de Rosa G, Di Giovanni S, Di Muzio A, Uncini A, et al. GCG genetic expansions in Italian patients with oculopharyngeal muscular dystrophy. Neurology (2000);54:608-14

- Chen AW, Wu SL, Cheng WL, et al. Dysphagia with fatal choking in oculopharyngeal muscular dystrophy: Case report. Medicine (Baltimore) (2018);97(43):e12935.doi:10.1097/ MD.0000000000012935

\section{UNILATERAL ONSET OF BILATERAL FACIAL PALSY, A RARE CASE OF NEUROSARCOIDOSIS}

\author{
C. Gallo, D. Vecchio, C. Varrasi, P. Naldi, R. Cantello
}

Department of Neurology, University of Piemonte Orientale (Novara)

Introduction: Facial nerve palsy is the most common cranial nerve disorder. We present the case of a 52-year-old woman with acute onset of unilateral right facial nerve palsy who subsequently developed facial diplegia. Chest Xray findings suggested a systemic disease. Lymph node needle biopsy and ACE test confirmed the diagnosis of neurosarcoidosis.

Case Report: Our patient presented to Emergency department with acute right peripheral facial nerve palsy. The neurological examination also demonstrated right tongue deviation, impaired sensation to the right side of the face, dysgeusia and mild weakness at the upper right arm. Her past medical history included situational depression with anxiety and mild tension-type headache occurring since adolescence. A cerebrovascular accident was ruled out by emergent brain $\mathrm{CT}$ scan and subsequent brain MRI. Routine chest X-ray showed multiple bilateral lymphadenopathies confirmed by chest CT. We searched for occult neoplasia, performing a mammography and breast ultrasound (BI-RADS 1 bilaterally, meaning the mammogram was negative) and planning a total body PET scan. Meanwhile the patients was discharged on antiaggregant therapy. After a week she presented left peripheral facial nerve palsy. 
Following readmission, a lumbar puncture was performed with CSF analysis showing slight increase in cell count ( 17 cells $/ \mathrm{mm} 3$ - monocytes) and normal protein level. The PET scan showed multiple supraclavicular, paratracheal, epiaortic, paraesophageal and parahilar adenopathies with a high metabolic component. Serological tests including ANCA, ANA, ESR and serology for tuberculosis were negative. After consultation with the rheumatologist, suspecting a granulomatous disorder, corticosteroid therapy was started with partial clinical benefit over four days. In order to characterize the lymph node alterations, a needle biopsy was performed via bronchoscopy showing epithelioid macrophages in granulomatous aggregation. Histological finding were compatible with sarcoidosis. ACE value was increased (67.7 U / L - normal range 8.0-52.0). The patient was therefore diagnosed with neurosarcoidosis.

Discussion: Facial nerve involvement is frequent in the setting of neurosarcoidosis, and bilateral palsy occurs in $15 \%$ to $25 \%$ of cases. Facial diplegia therefore warrants investigations directed to unveil signs of systemic disorder, especially pulmonary involvement through chest $\mathrm{CT}$ and gallium scintigraphy in selected cases. As in our case, radiological findings, together with the clinical history, could suggest a diagnosis of sarcoidosis, which must then be confirmed by hystopathological examination. Acute onset unilateral facial palsy evolving into a bilateral deficit, radiologic findings and the response to steroid therapy strongly support the diagnosis of bilateral facial involvement as the first manifestation of systemic sarcoidosis.

\section{References:}

- J. C. Hoyle, C. Jablonski, H. B. Newton. Neurosarcoidosis: clinical review of a disorder with challenging inpatient presentations and diagnostic considerations. Neurohospitalist (2014) Apr;4(2):94-101

- Zimmermann J, Jesse S, Kassubek J, Pinkhardt E, Ludolph AC. Differential diagnosis of peripheral facial nerve palsy: a retrospective clinical, MRI and CSF-based study. J Neurol (2019) Oct;266(10):2488-94

- S. Bolat et al. Fluorodeoxyglucose positron emission tomography (FDG-PET) is useful in the diagnosis of neurosarcoidosis. J Neurol Sci (2009) Dec 15;287(1-2):257-9

\section{CONTRAST-INDUCED ENCEPHALOPATHY FOLLOWING CEREBRAL ANGIOGRAPHY IN A PATIENT WITH ACUTE ISCHEMIC STROKE}

\author{
F. Garri ${ }^{1}$, A. Pieroni ${ }^{2}$, M. Saiani ${ }^{1}$, M. Caravello ${ }^{3}$, M. Munari ${ }^{3}$, F. Viaro ${ }^{1}$ \\ ${ }^{1}$ Department of Neurology, Azienda Ospedaliera of Padua (Padova); \\ ${ }^{2}$ Department of Neurology, University of Padua (Padova); ${ }^{3}$ Neuro- \\ Intensive Unit, Azienda Ospedaliera of Padua (Padova)
}

Contrast-induced encephalopathy (CIE) is a very rare complication of iodinated contrast agents during angiography. Very few cases are reported in literature, most commonly related to coronary angiography. To the best of our knowledge no cases of CIE occurring after a cerebral angiography in patients with acute ischemic stroke (AIS) have ever been reported. A 50-year-old man with a previous history of ischemic cardiopathy, diabetes and hypertension was admitted to our emergency department due to the acute onset of severe aphasia and weakness in the right arm (NIHSS 7). Brain CT-scan showed a dense media sign on the left side. He was treated with i.v. rTPA (onset to treatment time of 2 hours 45 minutes). After rTPA, NIHS score was 5. Intracranial CT angiography disclosed an occlusion of left M2 segment so the patient underwent endovascular treatment (EVT) without recanalization (TICI 0). Right after EVT, his neurological status was gradually deteriorating with progression to right hemiplegia, acute onset of right hemianopsia and right sided neglect (NIHSS 22). The day after he suffered motor focal to tonic-clonic seizure with persistent altered consciousness and Levetiracetam was started. Shortly after the patient was admitted to the intensive care unit due to coma and respiratory insufficiency. He was treated conservatively with hydration and neurological conditions reverted to the baseline (NIHSS 6) after 9 days. A repeat CT scan showed edema in the entire left hemisphere. Accordingly, beside the known ischemic lesion, MRI confirmed an extensive left gyral swelling and hyperintensity on FLAIR and DWIsequences, without change on ADC maps. Diffuse gyral contrastenhancement was also detected. Lumbar puncture was performed and CSF chemical and microbiological analysis were unremarkable. Repeat Brain-MRI showed resolution of the left hemispheric edema. This presentation seemed most consistent with CIE. CIE can result in a broad clinical spectrum varying from confusion to acute onset of neurological deficits and even coma, making the clinical scenario of AIS highly complex. We emphasize the importance of awareness of this rare neurologic complication especially in cases of unexplained neurologic deterioration after contrast injection and a distinct imaging pattern of significant ipsilateral hemisphere edema, which cannot be exclusively explained by stroke.

\section{COVID-19 AND ANTI-N-METHYL-D-ASPARTATE RECEPTOR ENCEPHALITIS: A CASE REPORT}

G. Gelosa, D. Facchetti, M. Brioschi, L. Saraceno, A. Guccione, A. Innocenti, C. Ceresa, G. Giussani, E. Agostoni

Neurology, ASST Grande Ospedale Metropolitano Niguarda (Milano)

Severe Acute Respiratory Syndrome Coronavirus- 2 is responsible for the current pandemic outbreak of coronavirus disease 2019 (COVID-19), and neurological manifestations have been reported, including cases of meningoencephalitis [1]. Here we report the case of a 23 y.o. male who developed an autoimmune encephalitis with NMDA antibodies in the context of a COVID-19 pneumonia. He presented with prominent acute psychiatric signs, including agitation, aggressiveness, imperative hallucinations, grandiose delusions. Patient was apiretic on admission. Occasional use of cocaine, phencyclidine and marijuana "crispy" was reported. Laboratory analysis were unremarkable. Urine analysis resulted positive for cannabinoids. He was admitted to the psychiatry ward, intramuscular aripiprazole and quetiapine were started. Mild fever and desaturation were reported in the first days. On further investigation, fever and flu-like symptoms (nausea, muscle pain and tiredness) were reported one week before admission. Lung CT scan and nasopharyngeal swab confirmed COVID-19 interstitial right lobe pneumonia. Serum IL-6 was increased. Oxygen therapy, darunavir/cobicistat, hydroxychloroquine were started, but progressive neurological deterioration was reported, showing reduced levels of consciousness, axial and limb rigidity, involuntary mouth movements, abnormal upper limbs postures, with bilateral forearm antigravitational movements, and hands dystonic postures, with fingers extension and flexed wrists. Nasogastric tube was introduced for dysphagia. Brain MRI and CSF analysis were normal at baseline and after 4-week, CSF SARS-CoV-2 PCR was negative in both occasions. Serial EEG did not show epileptiform activity. Autonomic dysfunction included hyperpyrexia at $40{ }^{\circ} \mathrm{C}$, high blood pressure and hyperhidrosis. Neuroleptic therapy was withdrawn, iv dantrolene therapy was ineffective, and he remained in a catatonic, lethargic state. COVID-19 pneumonia was clinically in resolution, supported by serial negative Covid-19 swabs. Autoimmune encephalitis was postulated and methylprednisolone 1 gram daily was introduced for 5 days, followed by prednisone $75 \mathrm{mg}$. 
Fever resolved and a gradual improvement of vigilance was noted. A comprehensive autoimmune panel returned anti-NMDA receptor antibodies CSF positivity. Brain FDG PET showed severe diffuse bilateral hypometabolism. IVIg $0.4 \mathrm{~g} / \mathrm{kg} /$ day were administered for 5 days, showing significant improvement both in alertness and limb rigidity. Twomonth follow-up confirms patient oriented and cooperative. Spontaneous speech is reduced, apathy, partial memory and attentional deficits are reported. Limb tone is normal. He reaches the standing position autonomously, walking with support of a physiotherapist. We postulate that COVID-19 infection might have induced an autoimmune process producing NMDA autoantibodies, through molecular mimicry mechanisms, as already reported in literature for other viral infections as HSV-1, EBV and Japanese encephalitis [2-3].

References:

1. Moriguchi T., Harii N., Goto J., Harada D., Sugawara H., Takamino J., et al. A first Case of Meningitis/Encephalitis associated with SARS-Coronavirus-2. International Journal of Infectious Diseases (2020); 94:55-58

2. Dorcet G., Benaiteau M., Bost C. Mengelle C., Bonneville F., Martin-Blondel G., Pariente J. Two Cases of Late-Onset AntiNMDAr Auto-Immune Encephalitis After Herpes Simplex Virus 1 Encephalitis. Front Neurol (2020);11:38

3. Ma J., Han W., Jiang L. Japanese encephalitis-induced anti-Nmethyl-d-aspartate receptor encephalitis: A hospital-based prospective study. Brain Dev (2020);42(2):179-184

\section{CEREBRAL VENOUS SINUS THROMBOSIS AS ONSET OF SLE}

P. Ghiglione, J. Maistrelli, M. Rosso, C. Bianco, E. Bucciantini, M. Bongioanni

\section{Neurology, ASL CN1 (Savigliano-CN)}

Introduction: We describe a case of a 31 years old indian woman admited in our hospital in february for the sudden onset of headache, nausea and left hemiplegia. A CT brain scan and intracerebral angio TC that showed a right frontal ipodensity and a thrombosis of the superior sagittal sinus. The patient was immediately treated with Warfarin. No risk factors were found.

Method: During hospitalization an MRI with angio MRI that confirmed the superior sagittal sinus thrombosis and blood test showed the presence of antiphosholipid antibodies and lupus anticoagulant.

Result: Hemiplegia improved with after physiotherapy but subsequently she manifested arthralgia and the rheumatologist confirmed the diagnosis of Systemic lupus erythematosus (SLE) and introduced steroids and idrossiclorochine. In july the neurological exam was unremarkable.

Conclusion: The cerebral venous sinus Thrombosis (CVST) is a major cause of stroke in young patiens; it's strongly related with female gender, contraceptive use, protrombotic states, malignancy and infiammatory diseases. SLE is a cronic autoimmune inflammatory disease more frequent in females. Central nervous system involvement range from 3 to $20 \%$ presenting with headache, seizures, psychosis and cerebrovascular disorders. CVST is a rare complication of SLE (around 1,5\%), and more rarely the first presentation of SLE.

References:

- J. Ferro et al. Cerebral Venous Thrombosis. Stroke (2016);35:664670
- Duman T. et al. A multicenter study of 1144 patients with cerebrale venous thrombosis, the VENOST. Stroke (2017);26:834-41

\section{MOYAMOYA SYNDROME (MMS) WITH SEVERE IRON DEFICIENCY ANEMIA (SIDA) AND ANTIPHOSPHOLIPID SYNDROME (APS) IN YOUNG ADULT WITH RECURRENT ACUTE ISCHEMIC STROKE (AIS)}

\author{
F. Giammello ${ }^{1}$, F. Grillo ${ }^{1}$, L. Ferraù ${ }^{1}$, C. Dell'Aera ${ }^{1}$, C. Casella ${ }^{1}$, M. \\ Cotroneo $^{1}$, M. Fazio ${ }^{1}$, P. La Spina ${ }^{1}$, F. Atzeni' ${ }^{2}$, A. Toscano ${ }^{1}$, R. \\ Musolino ${ }^{1}$
}

${ }^{1}$ Department of Clinical and Experimental Medicine, University of Messina (Messina); ${ }^{2}$ Rheumatology Unit, University of Messina (Messina)

Aim: To describe a diagnostic pathway of unusual case of moyamoya syndrome (MMS) with severe iron deficiency anemia (SIDA) and antiphospholipid syndrome (APS) in young adult with recurrent acute ischemic stroke (AIS) in the context of rare causes of stroke.

Case report: We report the case of a 39-year-old woman with multiple bilateral ischemic lesions due to recurrent AIS. She had a history of smoking, throbbing headaches, primary arterial hypertension, dyslipidemia and pregnancies characterized by gestosis with a miscarriage and threatened miscarriages. Serial magnetic resonance imaging showed AIS in right precentral area, in right frontoparietal and left frontal lobes. Digital subtraction angiography showed reduced caliber of the left internal carotid artery with filiform aspect in the terminal segment and reduced caliper in right ICA and proximal M1 segment of ipsilateral middle cerebral artery. Laboratory tests revealed SIDA and a mild increase of anticardiolipin antibodies (aCL). A therapy with high-dose methylprednisolone, iron supplementation and vitamin $\mathrm{K}$ antagonist (VKA) plus low-dose aspirin (LDA) has been started and the patient did not have any relapse in 5-month follow up.

Discussion: The radiological findings were suggestive for an autoimmune or degenerative vasculopathy in early stage moyamoya-like. The diagnosis of MMS secondary to APS was defined by specific autoantibody positivity confirmed 12 weeks after first laboratory assessment. The formation of aCL might be associated with an unidentified systemic disease underlying MMS and promotes further thrombosis with recurrent ischaemic events [1]. Patients with APS with arterial thrombosis have a higher risk of recurrence in the same vascular bed. The EULAR recommendations for the management of APS in adults agreed to increase INR target to 3-4, or INR 2-3 with LDA in addition to VKA [2]. The association of MMS with SIDA has been reported occasionally in early childhood. Reduced deformability of microcytic red cells may obstruct vasa vasorum of the large vessels, leading to vessel wall ischemia and intimal hyperplasia, and subsequent vasculopathy. Anemia may also worsen regional hypoxia in areas of decreased cerebral perfusion, resulting in mismatch between oxygen demand and endarterial supply, leading to ischemia and infarction [3].

Conclusions: Etiopathogenetic mechanisms of MMS are largely unknown. If the definite APS is comorbid with MMS, then any antiplatelet, anticoagulant, or surgical treatments should be administered with caution. When aPL appears in MMS, other underlying comorbidities should be considered. To our knowledge, the association between SIDA and MMS has never been described in adults. 


\section{References:}

1. Wang, Z, Fu, Z, Wang, J, Cui, H, Zhang, Z, Zhang, B. Moyamoya syndrome with antiphospholipid antibodies: a case report and literature review. Lupus (2014);23:1204-06

2. Tektonidou MG, Andreoli L, Limper M, Amoura Z, Cervera R, Costedoat-Chalumeau N, Cuadrado MJ, Dörner T, Ferrer-Oliveras R, Hambly K, Khamashta MA, King J, Marchiori F, Meroni PL, Mosca M, Pengo V, Raio L, Ruiz-Irastorza G, Shoenfeld Y, Stojanovich L, Svenungsson E, Wahl D, Tincani A, Ward MM (EULAR recommendations for the management of antiphospholipid syndrome in adults. Ann Rheum Dis (2019);78(10):1296-1304

3. Meena et al. Moyamoya Syndrome Associated with Severe Iron Deficiency Anemia in a Young Child. Pediatric Hematology and Oncology (2012);29(4):368-71

\section{HETEROZYGOUS VARIANTS IN POLR3A AND HEREDITARY SPASTIC PARAPLEGIA: DESCRIPTION OF TWO CASES}

\author{
G. Gianferrari ${ }^{1}$, J. Mandrioli ${ }^{1}$, N. Fini ${ }^{1}$, I. Martinelli ${ }^{1}$, E. Zucchi ${ }^{1}$, C. \\ Simonini ${ }^{2}$
}

${ }^{1}$ Neurology, Baggiovara Hospital (Modena); ${ }^{2}$ Baggiovara Hospital (Modena)

Background: Beside lower limb spasticity and weakness, deep sensorial disorder and urinary dysfunction, a variety of neurological and systemic symptoms have been recognized in hereditary spastic paraplegia (HSP), and a growing number of new genetic subtypes were recently described [1]. Despite extensive efforts, several patients with HSP and cerebellar ataxias, remain genetically unexplained. POLR3-related disorders include several clinically overlapping diseases caused by recessive mutations including early-childhood-onset hypomyelinating leukodystrophy, 4H-leukodystrophy and other disease. Recently, a novel and milder phenotype consisting of adolescent-onset spastic ataxia, has been proposed to be specific of intronic mutation c.1909+ $22 \mathrm{G}>\mathrm{A}$ in POLR3A gene [2]. We discuss two cases of spastic ataxia related to a heterozygous mutation in intronic region of POLR3A gene (c.1909+ 22G>A) associated with another heterozygous novel mutation in the same gene.

Case report: Two unrelated patients presented with gait clumsiness or unsteadiness at 49 (female) and 30 (male) years old respectively, without a known family history of neurological diseases. A predominantly spastic type gait was observed in the female patient, without ocular or sensitive abnormalities. In the younger male patient ataxia predominated over spasticity, with reduced vibration sense at the lower limbs. Saccadic pursuits were also detected. Both patients had widespread brisk deep tendon reflexes, with bilateral Babinski sign. Bilateral pes cavus was present in the female, scoliosis and knee arthrosis in the male. Unlike previously described, our patients didn't have gonadal dysfunction [2-3], cognitive impairment [2-3], postural and kinetic limbs tremor [2-3], distal lowerlimb acrocyanosis [2-3], dental abnormalities or myopia [2]. Electrophysiological studies showed abnormal lower-limb somatosensory evoked potentials with increase central latency, and increased central conduction time in motor evoked potentials, whereas motor and sensory nerve conduction studies and electromyography were normal. 3D FLAIR MRI images showed bilateral hyperintensity along the superior cerebellar peduncles extending from the dentate nucleus up to the midbrain. NGS showed a heterozygous mutation in intronic region of POLR3A gene (c.1909+22G>A) associated with another heterozygous novel mutation in the same gene (c.2788-2A $>\mathrm{T}$ in the female; c.2394T>A (p.C798*) in the male). In silico prediction showed that single nucleotide variant c. $1909+22 \mathrm{G}>\mathrm{A}$ is likely pathogenic.

Discussion and conclusion: Our cases may suggest a role of compound heterozygous mutations of POLR3A in hereditary ataxia/HSP and underline the importance of deep geno- and phenotyping of patients with HSP to better understand underlying pathomechanisms, recalling the importance of the non-coding variants. Other studies will be needed to confirm these data.

References:

1. P. V. S. de Souza Hereditary spastic paraplegia: clinical and genetic hallmarks. The cerebellum (2017):525-51

2. M. Minnerop Hypomorphic mutations in POLR3A are a frequent cause of sporadic and recessive spastic ataxia. Brain (2017);156178

3. J. Infante POLR3A-related spastic ataxia: new mutations and a look into the phenotype. Journal of Neurology (2020):324-30

\section{INTERNAL CAROTID DISSECTION PRESENTING AS SYMPTOMATIC MULTIPLE CRANIAL NEUROPATHIES: A CASE REPORT}

C. Giliberto ${ }^{1}$, F. Govone ${ }^{1}$, E. Brahimi ${ }^{1}$, G. Turano ${ }^{1}$, E. Nako ${ }^{1}$, V. Marotta $^{1}$, L. Gozzoli ${ }^{2}$, A. Di Sapio ${ }^{1}$

${ }^{1}$ Department of Neurology, Regina Montis Regalis Hospital (Mondovì$\mathrm{CN}$ ); ${ }^{2}$ Department of Radiology, SS Annunziata Hospital (Savigliano$\mathrm{CN})$

Background: Craniocervical artery dissection is an important cause of cerebrovascular events in young and middle aged patients. It is caused by an intimal tear followed by dissection of media towards the cranial direction. It usually follows a trauma but can also be spontaneous or associated with underlyng connective tissue disease or fibromuscular dysplasia. In rare cases, when cervicocranial artery dissection extends from intima to adventitia, it can develop an intramural hematoma that can induce external compression of bordering lower cranial nerves. Here, we describe a case of cervical Intracranial Carotid Artery Dissection (ICAD) presenting with unilateral IX, X and XII cranial neuropathies.

Case report: A 43 years old man presented in emergency department complaining swallowing and speech difficulties, preceded the day before by transient numbness of the left half of the face and mild headache. He had no significant medical history and at the time none injury was reported. On physical examination, his tongue on protrusion was slightly deviated on the left with evidence of hypotrophy on its left side, deviation of the uvula on the right side with asymmetry of soft palate, dysartria and dysphagia. He underwent a laryngeal flexible pan-endoscopic examination, which showed hypomobility of left side of larynx with preserved respiratory space. Therefore, clinical findings were consistent with IX, X and XII cranial nerve neuropathies. Laboratory tests did not showed abnormalities and a routine head CTscan was normal. Color duplex US of the neck did not show any pathological flow disorder. Angio-TCscan of the neck showed a stenosis of $65 \%$ in left cervical ICA. An MRI of head and neck with magnetic resonance angiography (MRA) was arranged for further evaluation. These findings confirmed a narrowing of the true lumen at the distal extracranial segment of the left internal carotid artery and posteriorly, at the same level, semilunar area of hyperintensity on T1 and T2-weighted imaging suggestive of ICAD. According to the neuroradiological data the patient was treated with antiaggregant therapy and 
parenteral nutrition. After 15days the patient began gradually to swallow. At 2 months-follow-up the patient reported a significant improvement of his symptoms with almost full recovery of swallowing and speech.

Conclusion: Our patient's presenting symptoms were suggestive of multicranial neuropathies secondary to external ICAD. In rare cases, cervical ICAD can be responsible of vessel enlargement leading to compression of the lower cranial nerves located in the parapharingeal space. Therefore, ICAD should be considered in the differential diagnosis in multicranial nerve palsy.

References:

- English SW, Passe TJ, Lindel EP et al. Multiple cranial neuropathies as a presentation of spontaneous internal carotid artery dissection: a case report and literature review. J Clinical Neuroscience (2018);50:129-31

- Rodallec MH, Marteau V, Gerber S et al. Craniocervical arterial dissection: spectrum of imaging findings and differential diagnosis. Radiographics (2008);6:711-28

\section{AN UNUSUAL CASE OF MYASTHENIA-LIKE PRESENTATION IN ERDHEIM-CHESTER DISEASE}

F. Girolamo ${ }^{1}$, E. Lauricella ${ }^{2}$, A. D'amati ${ }^{3}$, G. Ingravallo ${ }^{3}$, M. Foresio ${ }^{4}$, D. Ribatti $^{1}$, M. de Tommaso ${ }^{1}$, M. Cives ${ }^{2}$

${ }^{1}$ Department of Basic Medical Sciences, Neurosciences and Sense Organs, University of Bari School of Medicine (Bari); ${ }^{2}$ Department of Biomedical Sciences and Human Oncology, University of Bari School of Medicine (Bari); ${ }^{3}$ Department of Emergency and Organ Transplantation (D.E.T.O.), University of Bari School of Medicine (Bari); ${ }^{4}$ Urology, SS Annunziata Hospital (Taranto)

Aim: To report an unusual neurological presentation of Erdheim-Chester disease $(E C D)$ in order to increase the awareness of this rare, protean disease.

Methods: A 42-year-old man presented with a 6-year history of mild evening fatigability in his four limbs followed by motor and cognitive symptoms associated with cerebellar atrophy, dentate nuclei, and dentatothalamic pathway degeneration.

Results: Magnetic resonance imaging revealed hyperintense signals in $\mathrm{T} 2$ and FLAIR sequences within the pons, cerebellar white matter, dentate nuclei and globi pallidi in the absence of any contrast enhancement. Whole-body bone scintigraphy with 99Tc- methylene diphosphonate and fluorodeoxyglucose-positron emission tomography both revealed symmetric uptake in the lower extremities a finding suggestive of a diagnosis of ECD. Histological examination revealed diffuse infiltration of CD 68+ histiocytes with foamy cytoplasms in the presence of BRAFV600E activating mutation in tumor cells.

Conclusion: In patients with myasthenia-like symptoms who test negatively for myasthenia gravis, neurodegenerative diseases, and disorders of the hypothalamus, a diagnosis of ECD should be taken into consideration.

References:

- Bhatia, A. Hatzoglou, V. Ulaner, G. Rampal, R. Hyman, D. M. Abdel-Wahab, O. Durham, B. H. Dogan, A. Ozkaya, N. Yabe, M. Petrova-Drus, K. Panageas, K. S. Reiner, A. Rosenblum, M. Diamond, E. L. Neurologic and oncologic features of ErdheimChester disease: a 30-patient series. Neuro Oncol. (2020);22:979-92

- Cives, M. Simone, V. Rizzo, F. M. Dicuonzo, F. Cristallo Lacalamita, M. Ingravallo, G. Silvestris, F. Dammacco, F.
Erdheim-Chester disease: a systematic review. Crit Rev Oncol Hematol. (2015);95:1-11

- Haroche, J. Cohen-Aubart, F. Amoura, Z. Erdheim-Chester disease. Blood (2020);135:1311-18

\section{PROTON PUMP INHIBITORS-INDUCED EXTRAPYRAMIDAL SYMPTOMS WORSENING IN PARKINSON'S DISEASE}

A. Giugno ${ }^{1}$, A. Quattrone ${ }^{1}$, A. Mechelli ${ }^{1}$, I. Sammarra ${ }^{1}$, M. Morelli ${ }^{1}$, A. Guido $^{2}$, R. Calabria ${ }^{2}$, A. Gambardella ${ }^{1}$, G. Arabia ${ }^{1}$

${ }^{1}$ Institute of Neurology, Magna Graecia University (Catanzaro); ${ }^{2}$ Service of Endoscopic Surgery, Unit of Emergency Surgery, Magna Graecia University (Catanzaro)

Objectives: To describe a case of a patient with Parkinson's Disease (PD) with proton pump inhibitors (PPIs)-induced extrapyramidal symptoms worsening.

Materials: A 72-years old woman, with a 17-year history of PD started with unilateral bradykinesia and rigidity, who developed motor fluctuations and dyskinesias during disease course. Neurologic evaluation showed camptocormia, bradykinesia and rigidity in the upper and in the lower limbs, postural and rest tremor in the upper limbs, troublesome truncal and limbs dyskinesias. She was candidate for levodopacarbidopa intestinal gel (LCIG) procedure performed with percutaneous endoscopic gastrostomy and jejunal tube (PEG-J).

Methods: Clinical assessment included Unified Parkinson Disease Rating Scale (UPDRS), with motor examination (UPDRS-ME) at baseline and after LCIG administration. After a clinical evaluation with nasointestinal LCIG infusion, the patient underwent LCIG infusion via PEG-J. A direct $\mathrm{x}$-ray of abdomen verified the correct placement of the PEG-J.

Results: At baseline, the total UPDRS and the UPDRS-ME were of 85 and of 49 respectively. The patient was treated with LCIG via PEG-J, with a daily dose of $1256 \mathrm{mg}$. After LCIG, the total UPDRS and the UPRS-ME were of 72 and 36 respectively. Because of erosive gastritis, she was prescribed with proton pump inhibitors (PPIs), but rigidity and bradykinesia worsened. Despite the administration of extra bolus doses, the total UPDRS and the UPDRS-ME returned to the baseline levels. After PPIs withdrawing, rigidity and bradykinesia improved. Thereafter, we treated patient with anti-acids.

Discussion: Levodopa (L-DOPA) is the most effective treatment of PD. Its absorption is mediated by a sodium-dependent L-neutral amino acid (LNAA) carrier. Several factors may influence L-DOPA absorption, such as $\mathrm{pH}$ changes, often due to concomitant treatment with anti-acids or PPIs [1]. As previously reported, amino acids transported by the LNAA system seems to stimulate omeprazole-sensitive $\mathrm{H}+\mathrm{K}+\mathrm{ATPase}$, enhancing the gastric acid production. $\mathrm{H}+\mathrm{K}+\mathrm{ATPase}$ and LNAA system are part of a mechanism which produces the amino acid uptake [2]. We could hypothesize that PPIs, inhibiting $\mathrm{H}+\mathrm{K}+\mathrm{ATPase}$ and reducing the gastric acid production, may inhibit the uptake of L-DOPA.

Conclusions: Our case demonstrated that PPIs, despite are not believed to interfere with L-DOPA[3], could alter L-DOPA absorption. Anti-acids, with a short-lasting mechanism, could replace PPIs.

References:

1. LeWitt PA. Levodopa therapy for Parkinson's disease: Pharmacokinetics and pharmacodynamics. Movement Disorders (2015);30(1):64-72

2. Kirchhoff P, Dave MH, Remy C, Kosiek O, Busque S.M, Dufner M, Geibel J.P, Verrey F., Wagner C.A. An amino acid transporter 
involved in gastric acid secretion. Pflugers Arch - Eur J Physiol, (2006);451(6):738-48

3. Patel D, Bertz R, Ren S, Boulton DW, Någård M. A Systematic Review of Gastric Acid-Reducing Agent-Mediated Drug-Drug Interactions with Orally Administered Medications. Clinical Pharmacokinetics (2020);59(4):447-62

\section{CLINICAL AND RADIOLOGICAL FEATURES OF POSTERIOR CORTICAL ATROPHY (PCA) IN A GRN MUTATION CARRIER: A CASE REPORT}

\author{
M. Giunta ${ }^{1}$, I. Libri ${ }^{1}$, E. Premi ${ }^{2}$, C. Brattini ${ }^{1}$, B. Paghera ${ }^{3}$, S. Archetti ${ }^{4}$, R. \\ Gasparotti $^{5}$, A. Padovani ${ }^{1}$, B. Borroni ${ }^{1}$, A. Benussi ${ }^{1}$
}

${ }^{1}$ Neurology Unit, Department of Clinical and Experimental Sciences, University of Brescia (Brescia); ${ }^{2}$ Stroke Unit, Department of Clinical and Experimental Sciences, Spedali Civili Hospital (Brescia); ${ }^{3}$ Nuclear Medicine Department, University of Brescia (Brescia); ${ }^{4}$ Biotechnology Laboratory, Department of Diagnostics, Spedali Civili Hospital (Brescia); ${ }^{5}$ Neuroradiology Unit, University of Brescia (Brescia)

Introduction and Objectives: Posterior Cortical Atrophy (PCA) is a rare neurodegenerative syndrome, defined by a distinctive clinicalradiological profile, with Alzheimer's disease pathology accounting for the majority of cases. Here we present the case of a patient with impairment of visual and constructional abilities as initial manifestations.

Method: Patient underwent a multidimensional assessment, including neuropsychological evaluation, structural and functional imaging and genetic screening.

Results: Neurological and neuropsychological assessment showed an impairment of constructive and visuo-spatial skills, associated to dyscalculia, simultanagnosia, optic ataxia and oculomotor apraxia. In according to latest consensus criteria, a diagnosis of Posterior Cortical Atrophy was made. Coherent with clinical findings, structural and functional imaging showed a peculiar pattern of atrophy with primary involvement of right parietooccipital cortices, whereas CSF biochemical analysis did not reveal a profile compatible with Alzheimer's pathology. Genetic screening pointed out a known pathogenic GRN mutation.

Discussion: For the first time we present a case of PCA in a GRN mutation carrier in whom a concomitant Alzheimer's pathological process was excluded. Consequently, although lacking histological data, our case suggests GRN-related pathology causative of PCA

Conclusion: Through this report we want to provide further proof for a new neurodegenerative pathway leading to PCA, extending the clinical spectrum of GRN associated syndromes.

References:

- Crutch SJ, Schott JM, Rabinovici GD, et al. Consensus classification of posterior cortical atrophy. Alzheimers Dement. (2017);13(8):87084. doi:10.1016/j.jalz.2017.01.014

- Beck J, Rohrer JD, Campbell T, et al. A distinct clinical, neuropsychological and radiological phenotype is associated with progranulin gene mutations in a large UK series. Brain. (2008);131(3):706-20

- Jacova C, Hsiung GY, Tawankanjanachot I, et al. Anterior brain glucose hypometabolism predates dementia in progranulin mutation carriers. Neurology (2013);81(15):1322-1331. doi:10.1212/ WNL.0b013e3182a8237e

\section{CNS INFECTION MIMICKING ANOREXIA NERVOSA? MALNUTRITION AS “THE CHICKEN OR THE EGG” DILEMMA}

\author{
R. Infante ${ }^{1}$, M. Piatti ${ }^{1}$, M. Frigo ${ }^{1}$, R. Xhani ${ }^{1}$, F. Morini ${ }^{2}$, S. Foresti ${ }^{2}$, C. \\ Ferrarese $^{3}$
}

${ }^{1}$ Neurology Unit, San Gerardo Hospital (Monza); ${ }^{2}$ Infectious Diseases Unit, San Gerardo Hospital (Monza); ${ }^{3}$ School of Neurology, University of Milano-Bicocca (Monza)

Objectives: To describe a case of CNS infection potentially mistaken for a Primary Eating Disorder.

Materials: 46-year-old, female, employee, lives alone. Medical history known for depression (never on medications) and anaemia (on iron therapy). She presented severe weight loss within a year (body weight of 28,8 $\mathrm{kg}$ for 1,58 $\mathrm{m}$ of height), Amenorrhea and Anorexia Nervosa typical skin findings, such as hair effluvium, lanugo, hypertrichosis, brittle nails, and white scars (but no Russel's sign). In a couple of weeks, she developed memory problems, along with sporadic headache and low-grade fever. First-line tests resulted in hyponatremia, a normal CT-scan and a further suggestion to start with Levosulpride. One week later, she experienced an abrupt worsening of her neurological status with mental confusion, anosognosia and speech disorder with features of aphasia, leading her to ER.

Methods and Results: She underwent blood tests (CRP 3,9 mg/dl) and CT scan (supra-tentorial hydrocephalus with interstitial oedema), followed by angio-CT (calibre reduction in left M1-M2 and bilateral A1 segments, with no alterations of venous sinuses) and NMR (possible cytotoxic oedema of middle corpus callosum body; contrast agent was not performed due to agitation). Few hours after the admission, she developed mild neck rigidity. Lumbar puncture revealed $390 \mathrm{mg} / \mathrm{dl}$ proteins, $18 \mathrm{mg} / \mathrm{dl}$ glucose and 13 cell elements (lymphocytes and PMNs). Among microbiological testing on CSF, she was found PCRpositive for M. Tuberculosis. She started intravenous Isoniazid, Rifampicin, Ethambutol, Pyrazinamide, and parenteral nutrition support. After an episode of acute abdomen, a total-body CT Scan concluded for disseminated tuberculosis (spread to kidneys, lungs, and CNS). After few weeks, control CT scan showed ventricular reduction and the patient had slow symptoms regression along with a significant increased appetite. To be noted that, analysing her personal history, a psychiatric evaluation excluded Primary Eating Disorder, even in an atypical form.

Discussion: This is one of a few existing cases of CNS infection masked by the clinical picture of an Eating disorder. CNS Tuberculosis is often a challenging diagnosis, especially when symptoms may be confused with metabolic conditions within severe malnutrition. In this case, without a third-party supervision on eating and/or purging behaviours, both personal and familial settings did not help us discriminating if malnutrition was an Egg (eating disorder as the cause) or a Chicken (consequence of the infection).

References:

- Leonard JM et al. Central Nervous System tuberculosis. Microbiol Spectr. (2017) Mar;5(2). doi: 10.1128/microbiolspec.TNMI7-00442017

- Caplan DB 1990 et al. Organic diseases mimicking atypical eating disorders. Clin Pediatr (Phila). (1990) Oct;29(10):614. doi: 10.1177/ 000992289002901015

- Backer H Dobmeier M Landthaler M Skin Changes in Anorexia Nervosa. Hautarzt (2007) Mar;58(3):265-72; quiz 273. doi: $10.1007 / \mathrm{s} 00105-007-1296-\mathrm{y}$ 


\section{ANTI-CONTACTINE-1 ANTIBODIES IN CHRONIC INFLAMMATORY DEMYELINATING POLYNEUROPATHY: A CHALLENGE TREATMENT STRATEGY}

\section{Lazzari, D. Vecchio, E. Virgilio, R. Cantello}

Clinical Neurology, Department of Translational Medicine, University of Piemonte Orientale (Novara)

Background and Aims: Chronic inflammatory demyelinating polyneuropathy (CIDP) is an acquired autoimmune disorder of peripheral nerves, characterized by electrodiagnostic features of demyelination and an often good respond to glucocorticoids [1]. A poor response to treatment could suggest a variant with specific underlying pathogenetic mechanism. Infact different autoantibodies may be related to peculiar clinical features and outcomes [2].

Case Presentation (Material and Methods, Results): We describe the case of a 44-year-old male complaining of progressive distal numbness started from lower limbs, then involving the hands over two weeks. His family history was negative and he was diagnosed of Hashimoto thyroiditis one year before. At his first neurological visit, his standing was imbalanced and his gait stomping. A postural tremor was present at the upper limbs. He showed a symmetric proximal and distal muscle weakness (scored according to MRC): 5/5 strenght in arm abduction, forearm flexion and wrist extension, $3 / 5$ finger extension, 4/5 leg flexion, 3/5 EDB. He had hypopallesthesia from the elbows and at lower limbs with a glove and stocking dysesthesia. His osteotendinous reflexes were absent. There were no evidence of fasciculations nor muscle atrophy. The electroneurographic study showed a sensorimotor demyelinating polyneuropathy and an albuminocytologic dissociation was found on cerebrospinal fluid. A diagnosis of CIDP was so established and the patient started a first course of intravenous high-dose corticosteroid, with initial response. Then the patient had a relapsing-remitting course, treated with other two steroid courses, intravenous immunoglobulin and rehabilitation for the subsequent eight months. No strategy was definitively effective, meanwhile azathioprine was started at $150 \mathrm{mg} /$ daily. At that time, according to the poor response to treatment, the worsening of the patient, become almost wheelchair-bound, and the presence of tremor, further investigations were performed or repeated: including $\mathrm{HbA} 1 \mathrm{C}$, liver and thyroid function, serum protein electrophoresis, immunofixation, hepatitis and HIV antibodies, Quantiferon test, chest X-ray, amyloidosis genetic testing, anti-ganglioside antibodies, anti-neurofascin140 and anti-Contactine-1 (CNTN1) antibodies, that were found.

Discussion and Conclusion: Despite our patient fulfilled CIDP criteria, several red flags led us to suspect a rarer subtype. The clinical phenotype of CNTN1-CIDP is not fully established, but it is often severe and predominantly motor with tremor [2]. This latter symptom and the lack of response to therapies were a clue to search for CNTN1 antibodies. Our patient has actually regained the walking ability. We are treating him with azathioprine and IVIG courses, still considering the option of rituximab in case of inefficacy.

References:

1. Dyck PJ et al. Chronic inflammatory polyradiculoneuropathy. Mayo Clin Proc. (1975);50(11):621

2. Mathey EK et al. Chronic inflammatory demyelinating polyradiculoneuropathy: from pathology to phenotype. J Neurol Neurosurg Psychiatry (2015) Sep;86(9):973-85
A CASE REPORT OF REFRACTORY ANTI-NMDAR ENCEPHALITIS TREATED WITH BORTEZOMIB WITH ASSOCIATED MOVEMENT DISORDERS CONTROLLED WITH TRAMADOL

S. M. Lazzarin ${ }^{1}$, M. Vabanesi ${ }^{1}$, G. Cecchetti ${ }^{1}$, G. Fanelli ${ }^{2}$, F. Minicucci ${ }^{2}$, M. Filippi ${ }^{3}$

${ }^{1}$ Neurology Unit and Neurophysiology Unit, IRCCS San Raffaele Scientific Institute (Milano); ${ }^{2}$ Neurophysiology Unit, IRCCS San Raffaele Scientific Institute (Milano); ${ }^{3}$ Neuroimaging Research Unit, Institute of Experimental Neurology, Division of Neuroscience; Neurology Unit and Neurophysiology Unit, IRCCS San Raffaele Scientific Institute; Vita-Salute San Raffaele University (Milano)

Objectives: Anti-N-methyl-D-aspartate receptor (NMDAR) encephalitis is a rare and potentially severe autoimmune disease. Available diseasemodifying therapies include corticosteroids, immunoglobulins, plasma exchange, rituximab and cyclophosphamide. Only few data are available about the management of non-responder patients and of encephalitisassociated movement disorders.

Case description: A 29-year-old, previously healthy, woman was admitted to another hospital due to a secondarily generalized seizure; she was discharged with normal brain CT and MRI scans. After one week, she experienced a new-onset, severe headache, nausea and vomit. Few days later, she was referred to our ED due to another seizure. Brain MRI findings were non-specific. During hospitalization, seizures persisted and she developed acute psychosis. Lumbar puncture was normal except for lymphocytic pleiocytosis $(136 / \mu \mathrm{L})$. Hightitre anti-NMDAR antibody positivity was detected on serum and CSF. Full-body CT-scan, pelvic ultrasound and MRI were negative for malignancy. She received first IVIG cycle and seven daily methylprednisolone boluses. Despite first-line immunotherapy, she developed catatonia, coma and orofacial dyskinesias. EEG showed "extreme delta brush" pattern. Second-line immunotherapy with rituximab and cyclophosphamide was attempted. Seizures and movement disorders were refractory to full-dose treatment with seven antiepileptic drugs. After ICU admission, she was treated with propofol and midazolam. Further attempts with plasma exchange, repeat IVIG and rituximab showed no benefit, despite CD19 depletion. At 3 months, brain MRI/PET study showed parieto-occipital hypometabolism. A rescue therapy with bortezomib was attempted, resulting in reduced antibody titre in CSF and serum. Motor manifestations were rhythmic, worsening with higher levels of arousal. Combined EEG-EMG polygraphy suggested subcortical origin. After unsuccessful trial with tetrabenazine, therapy with tramadol was started; after two days, marked reduction of dyskinesias was observed, allowing weaning of sedation. After four bortezomib cycles and intensive rehabilitation, at 8 months from onset, she was discharged, could walk without assistance, showed mild cognitive deficits and minimal dysarthria.

Conclusions: Hyperkinetic movement disorders, such as orofacial dyskinesias and myorhythmias, are frequently associated with antiNMDAR encephalitis, and have been linked to the impairment of glutamatergic transmission in inhibitory GABAergic interneurons. Evidence from multiple case reports [1] for efficacy of tramadol, an opioid and noncompetitive NMDAR inhibitor, in reducing these movement disorders, supports the hypothesis of autoantibody-mediated "gain-of-toxic-function" genesis of movement disorders. Standard immune-modulating therapies, targeting mainly replicating B cells, are often ineffective in severe 
cases, reflecting the persistence of long-lived plasma cells. Bortezomib, a proteasome inhibitor targeting plasma cells, currently used in multiple myeloma, may prove useful as rescue therapy in severe anti-NMDAR encephalitis [2].

References:

1. Seifi A, Kitchen DL Management of dyskinesia in anti-NMDAR encephalitis with tramadol. Clin Neurol Neurosurg (2016);147:105-107

2. Behrendt V, Krogias C, Reinacher-Schick A, Gold R, Kleiter I Bortezomib treatment for patients with Anti-N-Methyl-daspartate receptor encephalitis. JAMA Neurol (2016); 73(10):1251-53

\section{NEURO-PHYSIOLOGICAL AND NEURO-ANATOMICAL MARKERS OF VISUAL BEHAVIORS IN DISORDERS OF CONSCIOUSNESS}

M. Leonardi ${ }^{1}$, F. Magnani ${ }^{1}$, D. Rossi Sebastiano ${ }^{2}$, L. D'Incerti ${ }^{3}$, D. Guido $^{1}$, G. Marotta ${ }^{4}$, R. Benti ${ }^{4}$, S. Tirelli ${ }^{1}$, A. Bersano ${ }^{5}$, D. Duran ${ }^{2}$, S. Ferraro $^{3}$, L. Minati ${ }^{3}$, A. Nigri ${ }^{3}$, C. Rosazza ${ }^{3,6}$, S. Bianchi Marzoli ${ }^{7}$, D. Sattin ${ }^{1}$

${ }^{1}$ Neurology, Public Health, Disability Unit, Fondazione IRCCS Istituto Neurologico Carlo Besta (Milano); ${ }^{2}$ Neurophysiology and Diagnostic Epileptology Unit, Fondazione IRCCS Istituto Neurologico Carlo Besta (Milano); ${ }^{3}$ Neuroradiology Unit, Fondazione IRCCS Istituto Neurologico Carlo Besta (Milano); ${ }^{4}$ Department of Nuclear Medicine, Fondazione IRCCS Ca' Granda Ospedale Maggiore Policlinico (Milano); ${ }^{5}$ Neurology Unit, Fondazione IRCCS Istituto Neurologico Carlo Besta (Milano); ${ }^{6}$ Department of Humanistic Studies (DISTUM), University of Urbino Carlo Bo (Urbino); ${ }^{7}$ Neuro-Ophthalmology Unit, Istituto Auxologico Italiano (Milano)

Objective: Distinguishing Vegetative from Minimally Conscious State (VS; MCS) is a challenge due to the difficulty in relating observed behaviors with underlying brain activity. The visual responses included in the visual subscale of the Coma Recovery Scale-revised (CRS-r) are informative of the change from VS to MCS. Specifically, the visual pursuit represents a cognitive mediated behavior indicative of MCS diagnosis, whilst patients in VS only present reflexive visual blink [1]. Despite the importance of visual behaviors for Disorders of Consciousness (DoCs), few studies explored the functional and structural integrity of the visual system in these patients. With this aim, we compared patients manifesting cognitive mediated and reflexive visual behavior.

Materials: Forty-two DoC patients (mean age $\pm \mathrm{SD}=49.36 \pm 18.18$ ) showing only visual blink, and twelve showing visual pursuit (mean age $\pm \mathrm{SD}=54.24 \pm 26.28$ ) were enrolled in the study. All of the patients had a diagnosis of VS except for the visual subscale of the CRS-r. For each participant, we collected flash Visual Evoked Potentials (fVEPs), structural Magnetic Resonance Imaging (MRI) of the visual system, and Positron Emission Tomography with 2-deoxy-2-[fluorine-18] fluoro-Dglucose (FDG-PET) variables including Standardized Uptake Value maps of striate and extra-striate visual cortices.

Method: We explored differences between groups by performing a series of Mann Whitney U test and Fisher's exact Test considering fVEPs, MRI, and FDG-PET data as dependent variables of interest one at a time.

Results: Patients manifesting visual pursuit showed a better fVEPs response, greater integrity at the level of right primary visual area (V1) and optic radiation, and a difference for the cluster localized in the right calcarine cortex and lingual gyrus.

Discussion: Despite the role of V1 in conscious visual processing is still controversial, our results highlighted the importance of primary visual areas for conscious visual behaviors, a data further supported by evidence deriving from patients with blindsight and studies on visual tasks in healthy individuals $[2,3]$. The right lateralization of neuroimaging results is supported by the right-hemisphere dominance for top-down modulation in visuo-spatial processing. As for the fVEPs data, our results indicated how differences can be detected even by easily applicable exams, as our fVEPs data were not able to detect higher-order visual processing differently from other studies.

Conclusions: Multimodal measures would be adopted as they can help clinicians for diagnostic purposes when a doubt on visual behaviors emerged.

\section{References:}

1. Vanhaudenhuyse, A., Giacino, J., Schnakers, C., Kalmar, K., Smart, C., Bruno, M.A., Gosseries, O., Moonen, G., \& Laureys, $\mathrm{S}$. Blink to visual threat does not herald consciousness in the vegetative state. Neurology (2008);71:1374-75

2. Azzopardi P., Hock H.S. Illusory motion perception in blindsight. Proc. Natl. Acad. Sci. U. S. A. (2011);108:876-81

3. Hurme, M., Koivisto, M., Revonsuo, A., Railo, H. V1 activity during feedforward and early feedback processing is necessary for both conscious and unconscious motion perception. Neuroimage (2019);185:313-21

\section{AUTOIMMUNE GLIAL FIBRILLARY ACIDIC PROTEIN ASTROCITOPATHY MIMICKING INFECTIOUS ENCEPHALITIS}

I. Libri ${ }^{1}$, S. Masciocchi ${ }^{1}$, I. Volonghi ${ }^{1}$, L. Poli ${ }^{1}$, R. Iorio ${ }^{2}$, A. Benussi ${ }^{1}$, A. Padovani $^{1}$

${ }^{1}$ Neurology Unit, Department of Clinical and Experimental Sciences, University of Brescia (Brescia); ${ }^{2}$ Neurology Institute, Department of Neuroscience, University Cattolica del Sacro Cuore (Roma)

Background: Autoimmune glial fibrillary acidic protein (GFAP) astrocytopathy is a novel corticosteroid-responsive meningoencephalomyelitis, first described in 2016, with a wide spectrum of clinical presentations. It is commonly diagnosed in individuals over 40 years old without gender predominance. We here present the case of GFAP astrocytopathy mimicking infectious encephalitis in a young woman.

Case presentation: A 20-year-old Caucasian woman was admitted to our neurology ward due to altered state of consciousness, mild paraparesis, tremor to extremities, opsoclonus and rigour nucalis. The past medical history was unremarkable. The patient reported influenzalike symptoms, weight loss and amenorrhea for over three months. To rule out infectious meningoencephalitis we performed a lumbar puncture that revealed a cerebrospinal fluid (CSF) lymphocytic pleyocitosis (74 cells $/ \mu \mathrm{L}, \mathrm{n} . \mathrm{v}<5 / \mu \mathrm{L})$, hyperproteinorrachia $(1252 \mathrm{mg} / \mathrm{dL}$, n.v $<450 \mathrm{mg} /$ dL) with normal glycorrhachia (51 mg/dL, n.v 50-100 mg/dL). An empiric antiviral (aciclovir) and antibiotic (ceftriaxone, ampicillin) therapy was introduced without clinical improvement. The EEG showed slow wave abnormalities especially localized in the left cerebral hemisphere. Brain and spinal cord magnetic resonance (MRI) demonstrated diffuse T2 hyperintensity of white matter, radial perivascular gadolinium enhancement, medullary swelling with marked enhancement of the cauda. Fundus 
examination revealed bilateral papilledema. CSF, blood and urine infectious screening were negative. In suspicion of autoimmune etiology of encephalitis, a second lumbar puncture was performed. CSF diagnostics revealed overlapping physical-chemical analysis. CSF oligoclonal bands and an elevated IgG index were detected. Furthermore, testing for autoantibodies specific to neuronal cell surface proteins/synaptic receptors (NMDAR, AMPAR, LGI1, CASPR2, GABAAR and GABABR) and onconeural antigens (anti $\mathrm{Hu}, \mathrm{Yo}, \mathrm{Ri}$, Amphiphysin, CV2, MA2) was negative. In relation to MRI findings, patient's CSF and serum were tested for GFAP-IgG autoantibodies by indirect immunofluorescence assays on mouse brain sections and GFAP-expressing HEK293 cells yielding a positive result. Paraneoplastic aetiology was excluded with total body CT scan. The patient was treated with high-dose methylprednisolone for 5 consecutive days with a good response. In relation to the response to immunosuppressive therapy, a second cycle of steroid therapy and IV immunoglobulins was performed. The patient was discharged with pyramidal signs and mild tremor. At one-month follow-up, MRI showed a clear improvement of the neuroradiological features with resolution of the areas of enhancement.

Conclusions: Our case report supports the importance for a prompt detection of GFAP antibodies in patients with meningoencephalitis-like symptoms but with specific MRI findings like perivascular radial gadolinium enhancement which is responsive to immunosuppressive therapy. References:

- Boyan Fang et al. Autoimmune Glial Fibrillary Acidic Protein Astrocytopathy. JAMA Neurol. (2016);73(11):1297-1307

- Raffaele Iorio et al. Clinical and immunological characteristics of the spectrum of GFAP autoimmunity: a case series of 22 patients. J Neurol Neurosurg Psychiatry (2018) Feb;89(2):138-146

\section{FUNCTIONAL CONVERGENCE SPASM: AN UNEXPECTED FINDING DURING NEUROLOGICAL EXAMINATION}

C. Lobianco ${ }^{1}$, G. Mastroianni ${ }^{1}$, S. Gasparini ${ }^{1,2}$, M. Ascoli ${ }^{2}$, V. Cianci ${ }^{1}$, C. Paleolologo ${ }^{1}$, G. Tripodi ${ }^{1}$, S. Neri ${ }^{2}$, T. Garcea ${ }^{2}$, U. Aguglia ${ }^{1,2,3}$, E. Ferlazzo $1,2,3$

${ }^{1}$ Regional Epilepsy Centre, Great Metropolitan "Bianchi-MelacrinoMorelli Hospital" (Reggio Calabria); ${ }^{2}$ Department of Medical and Surgical Sciences, Magna Græcia University of Catanzaro (Catanzaro); ${ }^{3}$ Institute of Molecular Bioimaging and Physiology, National Research Council (Catanzaro)

Case report: A 52-year-old woman came to our attention for therapeutic assessment of focal epilepsy due to cortical dysplasia. Neurological examination was normal except for the appearance, during ocular motility assessment, of convergent strabismus in the right eye with horizontal diplopia, lasting a few seconds. This manifestation was not reported before by the patient and recurred only during ocular motility evaluation. Video-EEG recording during convergence spasm did not show ictal activity. MRI confirmed the presence of left occipital cortical dysplasia in the absence of other lesions. Neuropsychological tests revealed depression with somatoform disorder suggesting a psychogenic nature of convergence spasm. At 6-month follow-up the patient did not report spontaneous convergence spasms.

Discussion: Convergence spasm is characterized by intermittent episodes of convergence, miosis and accommodation. It may be a manifestation of brainstem pathology in the setting of different neurologic diseases (e.g.: multiple sclerosis, stroke, Wernicke-Korsakoff syndrome, etc..) but, more often, it represents a functional neurological disorder [1]
To our knowledge, the coexistence of convergence spasm and focal epilepsy has never been reported.

Reference:

1. Fekete R, Baizabal-Carvallo JF, Ha AD, Davidson A, Jankovic J. Convergence spasm in conversion disorders: prevalence in psychogenic and other movement disorders compared with controls. J Neurol Neurosurg Psychiatry (2012);83(2):202-04

\section{ARTERIAL TORTUOSITY SYNDROME AND TRANSIENT ISCHEMIC ATTACKS}

M. Locatelli ${ }^{1}$, S. Cotti Piccinelli ${ }^{1}$, E. Premi ${ }^{2}$, S. Bonacina ${ }^{1}$, N. Gilberti ${ }^{2}$, V. Vergani ${ }^{2}$, M. Gamba ${ }^{2}$, R. Spezi ${ }^{2}$, I. Delrio ${ }^{2}$, M. Bernier ${ }^{3}$, A. Costa ${ }^{2}$, M. Ravanelli ${ }^{4}$, M. Colombi $^{5}$, R. Gasparotti ${ }^{6}$, A. Padovani ${ }^{1}$, M. Magoni ${ }^{2}$

${ }^{1}$ Neurology Unit, Department of Clinical and Experimental Sciences, Brescia University (Brescia); ${ }^{2}$ Stroke Unit, Vascular Neurology Unit, Spedali Civili (Brescia); ${ }^{3}$ Martinos Center for Biomedical Imaging, Harvard Medical School, Massachusetts General Hospital (Charlestown-USA); ${ }^{4}$ Radiology Unit, Department of Medical-Surgical Specialties, Radiological Sciences and Public Health, Brescia University (Brescia); ${ }^{5}$ Division of Biology and Genetics, Department of Molecular and Translational Medicine, Brescia University (Brescia); ${ }^{6}$ Neuroradiology Unit, Department of Medical-Surgical Specialties, Radiological Sciences and Public Health, Brescia University (Brescia)

Arterial Tortuosity Syndrome (ATS) is a rare, autosomal recessive connective tissue disease characterized by elongated and tortuous large and medium-sized arteries. It usually presents in infancy with cardiovascular manifestations, like coarctation of the isthmus aortae, aortic dilatation or tortuosity, pulmonary hypertension and cardiac failure. Other manifestations include aneurysm formation and arterial dissection which may involve cerebrovascular circulation [1]. However, only few cases of cerebrovascular events related to this syndrome have been described so far. We report the case of a 33-years-old man diagnosed with ATS since childhood, carrying the homozygous c.1334delG (p.Gly445-Glufs*40) SLC2A10 pathogenic variant $[2,3]$. The first clinical manifestation consisted of cardiac arrest at delivery, successfully treated with cardiopulmonary resuscitation. During childhood and adolescence, he suffered of dyspnea, diaphoresis, fainting, recurrent pneumonia, vomiting and failure to thrive. At 19 years old he was diagnosed with aortic sinus dilation with moderate aortic valve incompetence and treated with surgical ascending aorta replacement. At 33 years old he experienced an episode of sudden expressive aphasia and right hemiparesis, spontaneously recovered in 15 minutes. During transport to hospital, another analogous episode occurred, lasting about 10 minutes. A further episode occurred at the emergency room. Neurological evaluation showed central deficit of the VII right cranial nerve, mild right hemiparesis, mild expressive aphasia and mild dysarthria (National Institute of Health Stroke Scale, NIHSS=6). Brain CT showed no hyperacute signs of ischemia. Intravenous infusion of recombinant tissue plasminogen activator (rtPA) was started, with complete symptoms regression. Brain Magnetic Resonance Imaging (MRI) showed absence of acute ischemic lesions, while intracranial and supra-aortic trunks Magnetic Resonance Angiography (MRA) showed marked vessels tortuosity, without signs of dissection, aneurysmal dilatation or significant stenoses. The patient was diagnosed with recurrent transient ischemic attacks (TIA) and discharged with Aspirin $100 \mathrm{mg}$ daily. Only a few cases of juvenile stroke in ATS patients are reported in literature $[1,3]$. Our report strengthens the 
relationship between ATS and juvenile cerebrovascular events, suggesting the importance of an extensive study of body vessels in these cases. Moreover, the greater predisposition to cerebrovascular events in ATS could suggest a benefit from more aggressive primary and secondary prevention therapy. Furthermore, to our knowledge, this is the first reported case of intravenous rTPA administration in an ATS patient. The treatment was effective and safe, supporting its use in ATS patients.

References:

1. Beyens A., Albuisson J., Boel A. et al. Arterial tortuosity syndrome: 40 new families and literature review. Genetics in Medicine (2018) Oct;20(10):1236-45

2. Coucke P. J., Willaert A., Wessels M. W., et al. Mutations in the facilitative glucose transporter GLUT10 alter angiogenesis and cause arterial tortuosity syndrome. Nature Genetics (2006);38(4):452-57

3. Gardella R., Zoppi N., Assanelli D., Muiesan M. L., Barlati S., Colombi M. Exclusion of candidate genes in a family with arterial tortuosity syndrome. American Journal of Medical Genetics (2004);126A(3):221-28

\section{MARBURG TYPE MULTIPLE SCLEROSIS WITH ATYPICAL MRI PRESENTATION}

L. Mancinelli ${ }^{1}$, Y. Bartolini ${ }^{1}$, M. Cataldi ${ }^{2}$, C. Bomprezzi ${ }^{1}$, A. Tomassini ${ }^{3}$, M. Antonelli ${ }^{4}$, F. Gianno ${ }^{4}$, D. Bartolini ${ }^{5}$, M. Ruggiero ${ }^{2}$, M. Longoni ${ }^{1}$

${ }^{1}$ Neurology Unit, Local Health Agency of Romagna (Cesena); ${ }^{2}$ Neuroradiology Unit, Local Health Agency of Romagna (Cesena); ${ }^{3}$ Neurosurgery Unit, Local Health Agency of Romagna (Cesena); ${ }^{4}$ Department of Radiological, Oncological and Pathological Sciences, Sapienza University (Roma); ${ }^{5}$ Anatomic Pathology Unit, Local Health Agency of Romagna (Cesena)

Introduction: Marburg type is a rare variant of Multiple Sclerosis (MS) characterized by a severe and progressive course leading to exitus in few months. On Magnetic Resonance Imaging (MRI) scans the typical presentation consists of pseudotumoral demyelinating lesions [1].

Materials and methods: We report the case of a 53-years-old woman who presented at our emergency department complaining of progressive confusion, memory impairment and right hemianopia. The gadolinumenhanced brain MRI scan revealed a large irregular and predominantly rim-enhanced lesion involving the left forceps major and extended to the adjacent peritrigonal white matter, lateral ventricular ependymal lining and splenium of the corpus callosum, with an inverted NAA-Cho and NAA-Cr ratios and presence of lipids and lactate at spectroscopy. Spinal cord MRI scan was normal and CSF analysis showed mild increase in protein and a pattern 3 at serum-CSF isoelettrofocusing. A complete laboratory workup as well as total body computed tomography scan and positron emission tomography did not suggest other conditions. Anti-myelin oligodendrocyte glycoprotein (MOG) antibodies were absent in both CSF and serum. Brain lymphoma was firstly hypothesized and the patient underwent brain biopsy revealing histiocytic-macrophagic infiltrate supportive of an inflammatory-demyelinating lesion. CSF and brain biopsy were negative for JCV DNA. The subsequent course was of an unrelenting progression with increasing confusion and right arm apraxia followed by progressive right hemiparesis and lastly by mutacism, tetraparesis, diffuse pain with hyperalgesia. No clinical effect was achieved despite treatment with high dose steroids, intravenous immunoglobulins and rituximab, as confirmed by the follow-up MRI scans, always showing symmetrical growth of the lesion with significant rim enhancement and peripheral patchy diffusion restriction, particularly at the leading edge. The patient died 5 months after disease onset and a brain autopsy study confirmed the diagnosis of a fulminant demyelinating disease like Marburg type MS.

Discussion and conclusions: The atypical feature of this fulminant MS case lies in its MRI appearance and evolution, showing a classic infiltrative growth pattern, characteristic of malignancies, and a peripheral Gd enhancement with patchy diffusion restriction particularly at the leading edge, evoking progressive multifocal leucoencephalopaty. Even though infiltrative is one of the four possible MRI pattern of pseudotumoral demyelinating lesions, in fulminant MS - Marburg variant - Capello has described that follow-up MRI usually reveals the shrinkage of the lesion after treatment with steroids or, on the contrary, the appearance of new areas of demyelination. Our patient did not present none of either $[1,2]$.

References:

1. Capello E., Mancardi G.L. Marburg type and Balò's concentric sclerosis: rare and acute variants of multiple sclerosis. Neurol Sci. (2004) Nov;25 Suppl 4:S361-3

2. Seewann A., Enzinger C., Filippi M., et al. MRI characteristics of atypical idiopathic inflammatory demyelinating lesions of the brain: A review of reported findings. J Neurol. (2008) Jan;255(1):1-10. Epub 2007 Nov 15

\section{ANTI-MOG-ASSOCIATED DEMYELINATING DISORDERS: TWO SIDES OF THE SAME COIN}

G. T. Maniscalco ${ }^{1}$, G. Alfieri $^{1}$, P. Candelaresi $^{1}$, W. Di Iorio ${ }^{1}$, M. Napolitano $^{1}$, A. Ranieri ${ }^{1}$, R. Renna ${ }^{1}$, M. Rippa ${ }^{1}$, S. Salvatore ${ }^{1}$, A. Sagnelli $^{1}$, G. Servillo ${ }^{1}$, V. Manzo ${ }^{1}$, S. Ferrari ${ }^{2}$, S. Mariotto ${ }^{2}$

${ }^{1}$ Neurological Clinic and Stroke Unit, A. Cardarelli Hospital (Napoli); ${ }^{2}$ Neurology Unit, Department of Neuroscience, Biomedicine, and Movement Sciences, University of Verona (Verona)

Background: Anti-myelin oligodendrocyte glycoprotein antibodiesassociated disorders (MOGAD) are new emerging diseases with heterogeneuos course, treatment response, and prognosis.

Case report: We herein present 2 cases with antibodies to MOG, one with a cerebellar/brainstem monophasic syndrome which partially improved after treatment, and the other with an optic neuritis onset then relapsed with cortical encephalitis and presented a subsequent complete recovery. We further discuss elements possibly associated with disease heterogeneity and influencing treatment choices.

Conclusions: MOGAD is an extremely variable disease which can relapse and accumulate disability over time. An early diagnosis and correct timely treatment is fundamental to improve clinical outcome.

References:

- Jarius, S. et al. MOG-IgG in NMO and related disorders: A multicenter study of 50 patients. Part 1: Frequency, syndrome specificity, influence of disease activity, long-term course, association with AQP4-IgG, and origin. J. Neuroinflammation (2016); 13:1-16

- Cobo-Calvo, A. et al. Clinical spectrum and prognostic value of CNS MOG autoimmunity in adults: The MOGADOR study. Neurology (2018); doi:10.1212/WNL.0000000000005560

- Mariotto, S. et al. Relevance of antibodies to myelin oligodendrocyte glycoprotein in CSF of seronegative cases. Neurology (2019); doi:10.1212/WNL.0000000000008479 


\section{INTRACRANIAL VENOUS HYPERTENSION AND SLEEP APNEA IN SCLEROSTEOSIS: DIAGNOSTIC AND THERAPEUTIC IMPLICATIONS}

\author{
L. Manzo ${ }^{1}$, A. Pascarella ${ }^{1}$, L. Rapisarda ${ }^{1}$, C. Bombardieri ${ }^{2}$, R. Procopio ${ }^{3}$, \\ A. Gambardella ${ }^{4}$, F. Bono ${ }^{1}$ \\ ${ }^{1}$ Headache Center, Neurology Unit, Magna Graecia University \\ (Catanzaro); ${ }^{2}$ Neuroradiology Unit, A.O.U. Mater Domini (Catanzaro); \\ ${ }^{3}$ Institute of Molecular Bioimaging and Physiology, N.R.C. (Catanzaro); \\ ${ }^{4}$ Insitute of Neurology, Magna Graecia University (Catanzaro)
}

Objective: The neurological features of patients and disease carriers of sclerosteosis have not been well characterized [1-2]. We aimed to identify the neurological features of the first Italian family with sclerosteosis.

Materials and methods: We investigated neurological features, CSF pressure and neuroimaging findings in patients with sclerosteosis and in disease carriers.

Results: In the first Italian family with sclerosteosis genetic tests detected the p.Gln24X (c.70C>T) homozygous mutation of SOST gene [3] in the proband, and an heterozygous mutation among 9/13 siblings. Moreover, nocturnal polysomnography showed a condition of Obstructive Sleep Apnea Syndrome (OSAS). Brain MRI and MR venography displayed partial empty sella, flattening of posterior aspect of the globes, distension of subarachnoid perioptic space, bilateral transverse sinus stenosis, presence of Chiari malformation. We also performed 1hour lumbar CSF pressure monitoring through lumbar spinal needle which gave evidence of an elevated intracranial pressure with an high opening pressure. Cranial CT showed a massive and sclerotic thickening of neurocranial and splanchnocranial bones, optic ducts stenosis, absence of mastoid pneumatisation bilaterally.

Discussion: The severe hyperostosis of the skull caused chronic headache secondary to intracranial hypertension due to venous hypertension and obstructive sleep apnea syndrome in adult homozygotes. Moreover, minor hyperostosis of the skull was associated with headache and snoring in heterozygotes. The finding of an occipital transosseous intracranialextracranial venous drainage indicated a compensatory mechanism of venous hypertension and intracranial hypertension.

Conclusions: Our findings suggest that accommodation of cerebral venous drainage, and alleviation of airway obstruction might represent additional therapeutic interventions to mitigate intracranial hypertension of patients with sclerosteosis.

References:

1. Hamersma H, Gardner J, Beighton P. The natural history of sclerosteosis. Clin Genet (2003);63:192-97

2. Truswell AS. Osteopetrosis with syndactyly; a morphological variant of Albers-Schonberg's disease. J Bone Joint Surg Br (1958);40-B:209-18

3. Balemans W, Patel N, Ebeling $M$ et al. Identification of a $52 \mathrm{~kb}$ deletion downstream of the SOST gene in patients with van Buchem disease. J Med Genet (2002):39:91-97

\section{AT THE END OF THE CARPAL TUNNEL A DIAGNOSIS OF BALT- LYMPHOMA PARANEOPLASTIC POLYNEUROPATHY}

\author{
J. Marotta ${ }^{1}$, L. Larocca ${ }^{2}$, V. Riso ${ }^{1}$, G. Presicce ${ }^{1}$, G. Granata $^{1}$ \\ ${ }^{1}$ Istitute of Neurology, Catholic University of Sacred Heart (Roma); \\ ${ }^{2}$ Istitute of Anatomic Pathology, Catholic University of Sacred Heart \\ (Roma)
}

Objectives: Bronchus associated lymphoid tissue lymphoma (BALT) is a type a Non-Hodgkin Lymphoma (NHLS), located under respiratory tract epithelia. It is diagnosed by biopsy, done with bronchoscopy or computer tomography-guided, showing the characteristic lesions of bronchus. The aim of this case-report is to describe a paraneoplastic polyneuropathy associated with BALT.

Materials and methods: We report the case of a 56 years old woman with paresthesia in both hands after an operation for carpal tunnel on the right hand without benefit. The clinical examination showed a bilateral deficit of both median and ulnar innervated hand muscles, graded F 4 according to MRC scale. A mild deficit of common extensor fingers was also evident (F 4.5). Moreover, we found absent deep tendon reflexes at four limbs. She underwent neurophysiological studies, neuroimaging (thorax CT and cervical MRI), blood and immunological essays and thyroid function tests.

Results: The neurophysiological examination showed an axonal sensory-motor polyneuropathy with myelinopathic component. Blood exams were normal excluding a positivity for anti GD1b and anti GM1 antibodies. A cervical MRI showed a small thoracic lesion confirmed by chest CT. That lesion did not improved even after antibiotic therapy so the patient was asked to make a bronchoscopy with biopsy. The histologic examination documented the presence of a thick lymphocytic infiltrate, relatively monomorphic, represented in large prevalence of B lymphocytes indicative of "lymphoma of marginal extranodal zone type BALT". The bone marrow biopsy that was normal and a total body PET with 18F-FDG excluded other localization of the lymphoma. The patient started the treatment for BALT with the association Rituximab plus Bendamustina with total remission of the pathology.

Discussion: Together with the remission of BALT the patient experienced an improvement of neurological symptoms with only a residual mild hypoesthesia localized to the fingertips of both hands. The follow-up neurophysiological examination documented an improvement of the polyneuropathy with a partial recovery of the axonal damage and the disappearance of the demyelinating signs.

Conclusion: Lymphomas's paraneoplastic neuropathies are fully described in literature and, although rare, an association between paraneoplastic polyneuropathies and antiganglioside antibodies (especially GD1b and anti GM1) is also reported. A clear relationship between the kind of polyneuropathy (from a neurophysiological point of view) and antiganglioside antibodies is still missing and in our case there was a mixed damage, axonal and myelinopathic. To the best of our knowledge, this is the first description of paraneoplastic polyneuropathy associated with BALT.

References:

- Borekci S, Ozbalak M, Ersen E, Akı H, Cem AR M, Umut S. Bronchus Associated Lymphoid Tissue Lymphoma Presenting with Immunodeficiency and Multiple Pulmonary Nodules. Case Rep Pulmonol. (2017):4804378

- Wanleenuwat P, Iwanowski P, Kozubski W. Antiganglioside antibodies in neurological diseases. J Neurol Sci. (2020) Jan 15;408:116576

- Shihashi G, Yagi T, Suzuki S et al. Immune-mediated Neuropathy With Anti-disialosyl IgM Antibodies in Diffuse Large B-cell Lymphoma: A Case Report and Literature Review Intern Med. (2015);54(13):1647-51 


\section{SIMULTANEOUS 18F-FDG-PET/3T-MRI STUDY SHOWS BRAINSTEM HYPERMETABOLISM IN A PATIENT WITH ESSENTIAL PALATAL TREMOR}

\author{
O. Marsico ${ }^{1}$, I. Sammarra ${ }^{1}$, P. Viola ${ }^{2}$, D. Pisani ${ }^{2}$, M. Trimboli ${ }^{1}$, G. \\ Chiarella $^{2}$, G. Cascini ${ }^{3}$, A. Labate ${ }^{1}$, A. Gambardella ${ }^{1}$
}

${ }^{1}$ Institute of Neurology, Magna Graecia University (Catanzaro); ${ }^{2}$ Unit of Audiology, Magna Graecia University (Catanzaro); ${ }^{3}$ Department of Nuclear Medicine, Magna Graecia University (Catanzaro)

Aims: Essential palatal tremor (EPT), previously referred as palatal myoclonus, is a rare involuntary movement disorder characterized by rhythmic and continuous jerks of soft palate [1]. While symptomatic palatal tremor (SPT) is typically caused by brainstem or cerebellar lesions [2], pathogenetic mechanism underlying essential PT remains debated [1]. Here we reported a simultaneous brain fluorine-18-fluorodeoxyglucosepositron emission tomography (18F-FDG-PET)/3T- magnetic resonance imaging (MRI) study in a woman with EPT.

Materials: A 54-year-old woman presented to our Neurological Department reporting an isolated manifestation of bilateral ear clicking started at the age of 20 years. This click was present during voluntary movements and at rest, absent during sleep and worsening during stressful situations. She referred to be able to suppress it temporarily. Over time, this continuous noise deteriorated her life quality and she developed major depression and anxiety disorder, treated with escitalopram and diazepam. The neurological examination revealed palatal movements lifting posterior soft palate, including the uvula, synchronous to an audible outside clicking noise, resembling a clockwork mechanism, and to bilateral twitching of nasal muscles. Rate was irregular, at about 20 per minute.

Methods: A full blood panel, urine tests, EEG, surface electromyography, multimodal evocated potentials, blink reflex study, audiometric evaluation, video-laryngoscopy, brain 3T-MRI and 18F-FDG-PET/3TMRI were performed.

Results: Oropharyngeal evaluation revealed rhythmic and brief contraction of posterior soft palate, associated with bilateral sensorial hearing loss on high frequency. Video-laryngoscopy recorded soft palatal lifting movements and myoclonic contraction of tensor veli palatini. Surface electromyography on nasal muscles showed bilateral contractions, synchronous with ear click. EEG, multimodal evocated potentials, blink reflex study and 3T brain MRI were all normal. Lack of inferior olive nuclei change signal or enlargement on MRI ruled out SPT [2]. A diagnosis of EPT was made. The simultaneous 18F-FDG-PET/3T-MRI study showed an area of increased glucose uptake in the lower brainstem, nearby the inferior olive nuclei bilaterally, more on the right side. DISCUSSION: This is the first application of simultaneous 18F-FDG-PET/3T-MRI in EPT. Our study demonstrated the hypermetabolism in the brainstem without any structural lesion on brain MRI. Of interest, a similar brainstem hypermetabolism, corresponding to the inferior olive, was already recognized in SPT. Overall, these findings strongly favour the so-called central generator theory of both EPT and SPT [1].

Conclusion: Our results illustrate that the lower brainstem encompassing the inferior olivary nuclei may be the generator of EPT.

References:

1. Zadikoff C, Lang AE, Klein C. The 'essentials' of essential palatal tremor: a reappraisal of the nosology. Brain (2006);129(Pt4):832-40

2.Gambardella A, Zappia M, Valentino P, Aguglia U, Fera F, Pardatscher K, Quattrone A. Action palatal tremor in a patient with primary intestinal lymphoma. Mov Disord. (1997);12(5):794-97

\section{PROPRIOCEPTIVE-INDUCED SEIZURES IN A SUBJECT WITH NON-KETOTIC HYPERGLYCEMIA}

G. Mastroianni, E. Ferlazzo, S. Gasparini, M. Ascoli, C. Cianci, S. Neri, T. Garcea, C. Lobianco, G. G Tripodi, U. Aguglia

Regional Epilepsy Centre, Great Metropolitan Hospital (Reggio Calabria)

Introduction: Proprioceptive-induced seizures are reflex seizures commonly triggered by active or passive movements. Seizures are typically focal, and the involvement of the sensorimotor area of the contralateral hemisphere to the clinical seizure onset is constant [1].

Case report: A 64-year-old man came to our attention for recurrent, short-lasting (about 20 seconds) episodes of right leg jerks triggered by raising from the chair and walking, started a few hours before at awakening. He was affected by type 2 diabetes mellitus but he had spontaneously stopped treatment with insulin 7 days before. He was fully alert, and presented polydipsia, polyuria and two episodes of vomiting. Inter-ictal neurological examination and neuropsychological tests were normal. Video-polygraphic recording allowed to record several focal seizures characterized by short-lasting ( 25 to 40 seconds) clonic jerks of the right lower limb triggered by raising from the chair, extending the leg on the thigh, as well as tapping the patellar tendon. EEG revealed rhythmic spikes over the central vertex derivations; EMG channels showed rhythmic, synchronous contractions of right lower limb muscles (Figure1). Blood glucose level was $752 \mathrm{mg} / \mathrm{dl}$, sodium level was $130 \mathrm{mEq} / \mathrm{l}$, osmolarity was $325 \mathrm{mOsm} / 1$. Urinary ketone bodies were absent. Brain MRI was normal. Hydration and normalization of glycaemia by intravenous and subcutaneous insulin lead to prompt seizure control. Seizures never recurred at 6-month follow-up.

Conclusion: Proprioceptive-induced seizures are rare and most commonly occur as a transient phenomenon with non-ketotic hyperglycemia [2]. Antiepileptic drugs are useless while insulin and hydration represent the main treatments. New-onset proprioceptive-induced seizures need fast medical and neurologic evaluation to identify subjects with non-ketotic hyperglycemia.

References:

1. Italiano D, Ferlazzo E, Gasparini S, et al. Generalized versus partial reflex seizures: a review. Seizure (2014);23:512-20. DOI: 10.1016/ j.seizure.2014.03.014

2. Brick JF, Gutrecht JA, Ringel RA. Reflex epilepsy and nonketotic hyperglycemia in the elderly: a specific neuroendocrine syndrome. Neurology (1989);39:394-99. DOI: 10.1212/wnl.39.3.394

\section{HEAD TREMOR AND CEREBELLAR INVOLVEMENT IN POLR3A RELATED SPASTIC ATAXIA}

F. Matrone ${ }^{1}$, A. Bisecco ${ }^{1}$, A. d'Ambrosio ${ }^{1}$, M. Cirillo ${ }^{1}$, R. Docimo ${ }^{1}$, M. Risi $^{1}$, F. Santorelli ${ }^{2}$, G. Tedeschi ${ }^{1}$, A. Gallo ${ }^{1}$

${ }^{1}$ AOU L. Vanvitelli, University of Campania L. Vanvitelli (Napoli); ${ }^{2}$ IRCCS Fondazione Stella Maris (Pisa)

Introduction: Hereditary cerebellar ataxias and hereditary spastic paraplegias are heterogeneous neurologic disorders, characterized by Purkinje cells and spinocerebellar tracts degeneration or pyramidal tract degeneration, respectively. The advent of next-generation sequencing has recently revolutionized the classification of these two entities, sharing not only overlapping phenotypes and underlying genes, but also common cellular 
pathways disfunction, leading to the concept of spastic ataxia spectrum. Mutations in POLR3A are a recently recognized cause of autosomal recessive spastic ataxias: RPC1, the gene product of POLR3A, contributes to the catalytic activity of PolIII, its dysfunction leading to an extreme vulnerability of myelin homeostasis. Previous studies have also linked this gene to hypomyelinating leukodystrophies. The recently discovered intronic variant (c. $1909+22 \mathrm{G}>\mathrm{A}$ ) has conversely been associated to a novel uniform phenotype, characterized by adolescent-onset progressive spastic ataxia with tremor, involvement of central sensory tracts and characteristics imaging features (FLAIR hyperintensities along the superior cerebellar peduncles, cervical cord atrophy and hypoplasia of the corpus callosum).

Case reports: A 47 years-old woman was admitted to our department for a slowly progressive gait and balance disorder associated with head tremor. Neurological examination revealed ataxo-spastic gait, brisk reflexes (with right Babinski sign), dysarthria and lower limbs deep sensory loss. Electrophysiological studies showed bilateral dysfunction of both sensory and motor pathways. The MRI revealed: i) cervical spinal cord atrophy, ii) thin corpus callosum, iii) T2 hyperintensity ob bilateral superior cerebellar peduncles. Her brother, a 41 years-old man, was admitted to our department for a 20 -years history of slowly progressive gait impairment and postural instability. His neurological examination showed an overt spastic paraparesis, brisk reflexes, dysarthria, dysdiadokokinesia and lower limbs deep sensory loss. Electrophysiological studies revealed bilateral lower limbs sensory dysfunction. The MRI showed spinal cord and cerebellar atrophy with thin corpus callosum and T2 hyperintensity in bilateral superior cerebellar peduncles. Secondary causes of ataxia and spasticity (like inflammatory, infectious, paraneoplastic conditions and vitamin deficiencies) were ruled out in both. Segregation genetic analysis with Whole Exome Sequencing technique performed in the two sibilings revealed double heterozygosity in POLR3A gene $(\mathrm{G} .1909+22 \mathrm{G}>\mathrm{A}, \mathrm{C} .3733 \mathrm{C}>\mathrm{T})$ which is pathogenic for the recently described phenotype of autosomal recessive spastic ataxia.

Discussion and conclusions: These case reports highlight the importance of considering mutations in POLR3A in patients with juvenileonset, slowly-progressive, spastic ataxias, particularly if associated with tremor and deep sensory loss, and with characteristics MRI features, like T2 hyperintensity of superior cerebellar peduncles, cervical cord atrophy and corpus callosum hypoplasia.

References:

- Synofzik M, Schüle R. Overcoming the divide between ataxias and spastic paraplegias: Shared phenotypes, genes, and pathways. Mov Disord. (2017);32(3):332-345. doi:10.1002/mds.26944

- Minnerop M, Kurzwelly D, Wagner H, et al. Hypomorphic mutations in POLR3A are a frequent cause of sporadic and recessive spastic ataxia [published correction appears in Brain. 2017 Dec 9;:]. Brain (2017);140(6):1561-1578. doi:10.1093/brain/ awx095

- Rydning SL, Koht J, Sheng Y, et al. Biallelic POLR3A variants confirmed as a frequent cause of hereditary ataxia and spastic paraparesis. Brain (2019);142(4):e12. doi:10.1093/brain/ awz041

\section{SPINAL CORD INVOLVEMENT IN CADASIL: A CASE REPORT}

F. Matrone, A. d'Ambrosio, A. Bisecco, R. Capuano, M. Risi, S. Satolli, M. Cirillo, G. Tedeschi

AOU L. Vanvitelli, University of Campania L. Vanvitelli (Napoli)
Introduction: Cerebral autosomal dominant arteriopathy with subcortical infarcts and leukoencephalopathy (CADASIL) is an inherited small vessel disease. It is caused by mutations of the NOTCH3 gene, located on the 19 p13 chromosome, which encodes a receptor expressed in smooth muscle cells of blood vessels and pericytes. The disease can manifest with migraine with aura, recurrent cerebral ischemic events, psychiatric disturbance and progressive cognitive impairment. The main MRI findings are leukoencephalopathy and subcortical infarcts with involvement of external capsules and anterior pole of temporal lobes. Spinal cord involvement, albeit rare, has been documented since 2011 and could be either the expression of the small vessel disease as well as an inflammatorydriven process. Genetic analysis is the gold standard for the diagnosis. To date, more than 230 different mutations located in 20 different exons have been reported.

Case report: A 54-year-old woman was admitted to our department for a slowly progressive gait and balance disorder, associated with impaired speech, urge incontinence. She also complained of slowly progressive cognitive impairment and abulia. Her medical history was remarkable for migraine without aura and bipolar disorder. Her family history revealed psychosis in matrilinear relatives. Abnormal findings in her neurological examination were moderate spasticity in lower limbs, hyperreflexia (with right Hoffmann and Babinski sign) and bilateral dysmetria/ dysdiadochokinesia. Neuropsychological evaluation revealed mild cognitive impairment (with involvement of working, short-term memory and executive functions). Routine blood tests (including thrombophilia and a comprehensive autoimmune screening) were unremarkable. The brain and spinal cord MRI showed bilateral and symmetrical leukoencephalopathy with involvement of basal ganglia and external capsule and some cerebral microbleeds; multiple lesions were also present in the brainstem, cerebellum and spinal cord, both in posterior and lateral columns. CSF (cytology, biochemistry, PCR for viruses and oligoclonal bands) was unremarkable. Analysis of NOTCH3 gene showed c.619C $>$ T missense mutation and CADASIL was diagnosed.

Discussion and conclusions: To our knowledge, this is the first case of diffuse spinal cord involvement reported in a patient with CADASIL related to c.619C $>\mathrm{T}$ missense mutation. This case highlights the importance of considering CADASIL in the diagnostic work-up of patients with symmetric leukoencephalopathy and atypical clinical presentation, including those with involvement of the spinal cord.

References:

- Bentley P, Wang T, Malik O, et al. CADASIL with cord involvement associated with a novel and atypical NOTCH3 mutation. J Neurol Neurosurg Psychiatry (2011);82(8):855-860. doi: 10.1136/ jnnp.2010.223297

- Xiromerisiou G., Marogianni C., Dadouli K, et al. Cerebral autosomal dominant arteriopathy with subcortical infarcts and leukoencephalopathy revisited Genotype-phenotype correlations of all published cases Neurol Genet Jun (2020);6(3):e434; DOI: 10.1212/NXG.0000000000000434

- Jing X., Jiang W., Gan L. et al. CADASIL with spinal cord involvement: a case report and literature review. J Neurol (2019);266:2330 2333 https://doi.org/10.1007/s00415-019-09436-4

\section{A PECULIAR CASE OF INTERNAL CAROTID DISSECTION: "DO NOT FORGET EAGLE'S SYNDROME!"}

\author{
P. Mattioli ${ }^{1}$, G. Baldino ${ }^{2}$, I. Gandoglia ${ }^{3}$, M. Del Sette $^{3}$
}


${ }^{1}$ Clinical Neurology, Dept. of Neuroscience (DINOGMI), University of Genoa, San Martino Polyclinic Hospital (Genova); ${ }^{2}$ Vascular and Endovascular Surgery Unit, Galliera Hospital (Genova); ${ }^{3}$ Neurology Unit, Galliera Hospital (Genova)

Background: Eagle's Syndrome (ES) is a rare condition, due to an elongation of styloid process of temporal bone or a calcification of stylohyoid ligament, that can cause pharyngeal and facial pain $[1,2]$. Two variants of the syndrome have been described: classical and vascular ones [2]. Vascular variant is characterized by symptoms due to the vascular conflict between carotid artery and styloid process [2]. Despite only $0.16 \%$ of people present ES, this syndrome has been associated with carotid artery dissection [1,2].

Materials and Methods: We report the case of a man affected by the vascular variant of Eagle syndrome, who come to our attention for a symptomatic left internal carotid artery (ICA) dissection.

Results: A fifty-six-year-old man, with an unremarkable medical history, except for uncontrolled hypertension and metabolic syndrome, came to the emergency room of Galliera Hospital because of an episode of loss of consciousness followed by transient confusion and dysarthria. A brain Computerized Tomography (CT) did not show any acute cerebrovascular lesion. Twenty-four hours later, a brain CT Angio (CTA) showed a dissection of left ICA, associated with a hypertrophy of the styloid processes (more prominent on the left side). The dissection was then confirmed by carotid Duplex imaging. We started anticoagulant therapy. After two weeks a brain Magnetic Resonance Imaging was performed, confirming the dissection of left ICA. No cause of secondary hypertension was found, and the patient fully recovered. Until today no further neurological events occurred and clinical and radiological follow up is keeping up.

Discussion: This case underlines the importance of considering ES as a potential cause of ICA dissection. In fact, although this syndrome remains uncommon, its association with ICA dissection has been described $[1,2]$. Moreover, CT and CT-angio were adequate and affordable imaging technique that allows to obtain either a diagnosis and further data for an eventual surgical plan [3].

Conclusion: Even though ES is a rare syndrome it has to be considered within the differential diagnosis of ICA dissection's causes $[1,2]$.

References:

1. Subedi R, Dean R, Baronos S, Dhamoon A. Carotid artery dissection: A rare complication of Eagle syndrome. BMJ Case Rep. (2017);2017:bcr2016218184

2. Baldino G, Di Girolamo C, De Blasis G, Gori A. Eagle Syndrome and Internal Carotid Artery Dissection: Description of Five Cases Treated in Two Italian Institutions and Review of the Literature. Ann Vasc Surg. (2020) https://doi.org/10.1016/j.avsg.2020.02.033

3. Scavone G, Caltabiano DC, Raciti MV, Calcagno MC, Pennisi M, Musumeci AG, et al. Eagle's syndrome: a case report and CT pictorial review. Radiol Case Reports. (2019);14:141-5

\section{EARLY BRAIN MRI FEATURES IN A PATIENT WITH MIXED CONNECTIVE TISSUE DISEASE}

G. Mazza ${ }^{1}$, S. Bartalini ${ }^{1}$, A. Rossi ${ }^{1}$, M. Stromillo ${ }^{1}$, A. Cerase ${ }^{2}$, S. Dallagiacoma $^{1}$, N. De Stefano ${ }^{1}$

${ }^{1}$ Unit of Neurology, Department of Medicine, Surgery and Neurosciences, A.U.O. Senese (Siena); ${ }^{2}$ Unit of Neuroimaging, Diagnostic and Functional Neuroradiology, Department of Neurological and Movement Sciences, A.U.O. Senese (Siena)
Background: Mixed connective tissue disease (MCTD) is characterized by clinical features of systemic lupus erythematosus, scleroderma and polymyositis occurring sequentially, in the presence of high titers of antibody U1 small nuclear RNP. The nervous system involvement is rare and occurs in MCTD in approximately 10 20\% of patients as cerebrovascular disease, peripheral neuropathy and mielopathy [1].

Clinical case: We report a 60-year-old man who was referred to our unit for headache, asthenia and mild proximal upper limbs myalgia. Brain CT-scan showed multiple white matter lesions with contrast enhancement in both hemispheres. Medical history was silent with the exception of Raynaud phenomenon and, occasionally, puffy fingers. Neurological examination was unremarkable. Brain MRI showed supratentorial and infratentorial white matter lesions with diffuse contrast enhancement.

Methods: Serum and liquoral autoimmunity, paraneoplastic and microbiological test, PET-CT, nailfold digital capillaroscopy (NDC), conventional angiography of intracranial vessels and brain biopsy were performed.

Results: Laboratory investigations were remarkable for positive antiribonuclear protein (RNP) antibody at high titer in the serum. Extensive infectious disease workup was reproducibly negative. CSF analysis showed high protein with normal glucose and cell count. PETCT evidenced an area of glucidic hypermetabolism in the musculotendinous structures of the right shoulder of phlogistic nature compatible with focal myositis. NDC findings was suggestive of active angiitis. Conventional angiography did not show significant signs of CNS vasculitis. A brain biopsy showed chronic transmural inflammation involving small vessels wall with reactive astrocytosis, lymphocytic cellular infiltrates, focal granulomatous inflammation compatible with CNS vasculitis.

Discussion: The presence of anti RNP, the Raynaud Phenomenon, the capillaroscopy's and PET-TC results as well as the CNS vasculitis led us to conclude for the diagnosis of MCTD. At present, there are four coexisting diagnostic criteria for MCTD and we used those provided by Alarcón-Segovia.

Conclusions: Brain MRI of CNS vasculitis is generally characterised by ischaemic lesions of different ages. The simultaneous contrast enhancement of most of the brain lesions was found in our patient and should be interpreted as an early, unusual feature of angiitis of the CNS [2-3].

References:

1. Nadeau S. E. Neurologic Manifestations of Connective Tissue Disease. Neurologic Clinics (2002);20(1):151-78

2. C. Salvarani, R.D. Brown Jr, G G Hunder. Adult primary central nervous system vasculitis Lancet (2012); 380: 767-77

3. A. Charil et al. MRI and the diagnosis of multiple sclerosis: expanding the concept of "no better explanation". Lancet Neurol (2006);5:841-52

\section{THE BROAD SPECTRUM OF VOLTAGE-GATED CHANNEL (VGKC) COMPLEX AUTOIMMUNITY: A RARE CASE OF MORVAN SYNDROME}

\author{
V. Mazzoleni ${ }^{1}$, S. Cotti Piccinelli ${ }^{1}$, E. Baldelli ${ }^{1}$, N. Zoppi ${ }^{1}$, I. Volonghi ${ }^{1}$, \\ C. Agosti ${ }^{1}$, P. Liberini ${ }^{1}$, U. Leggio ${ }^{2}$, B. Borroni ${ }^{1}$, A. Benussi ${ }^{1}$, A. \\ Padovani $^{1}$
}

${ }^{1}$ Neurology Unit, Department of Clinical and Experimental Sciences, University of Brescia (Brescia); ${ }^{2}$ Neurophysiology Unit, Department of Neurological and Vision Sciences, ASST Spedali Civili (Brescia) 
Background: Among neurologic autoimmune disorders, Morvan Syndrome (MoS) is a rare condition, characterised by the coexistence of central nervous system (CNS) manifestations, autonomic dysfunctions and peripheral nerve hyperactivity. This syndrome is often associated with serum and/or cerebrospinal fluid (CSF) VGKC complex antibodies positivity. The major target of these antibodies is contactin-associated protein 2 (CASPR2) and, sometimes, leucine-rich glioma-inactivated 1 (LGI1). Thymoma occurs in around 50\% of the patients. It is reported that immunomodulatory treatment allows clinical improvements of this potentially life-threating condition.

Case Report: An otherwise healthy 22-years old man presented at the Emergency Department, reporting painful back cramps, which subsequently disseminated at lower limbs and widespread muscle twitching. These symptoms arose 6 weeks before, and worsen over time. Additionally, he developed a progressive short-term memory disfunction, culminating in a complete amnesia on the events of the previous weeks. He also reported severe insomnia, episodes of low-grade fever, hyperhidrosis and weight loss. Neurological examination revealed prominent fasciculations of both legs, with normal muscle tone and strength of the limbs. Furthermore, we detected a persistent tachycardia, blood pressure fluctuations and a mild hyponatremia. Brain and spinal cord Magnetic Resonance Imaging (MRI), electroencephalogram and the cerebrospinal fluid (CSF) analysis were all unremarkable. Electrophysiological studies showed normal nerve conduction, whereas electromyography (EMG) revealed bilateral hyperexcitability of neuromuscular plaque and myokymic discharges at gastrocnemius. Serological studies, (via cellbased assay), showed the presence of serum CASPR2 and LIG1 antibodies, however, the CSF antibodies analysis was negative. Other autoantibodies against surfaces antigens and neuromuscular plaque tested negative. The clinical presentation and the antibody positivity pointed to the diagnosis of Morvan syndrome. We excluded thymoma and other malignancy, relying on negative CT scan of chest-abdomen-pelvis and scrotal US. The patient clinically improved after a 5-days cycle of high dose steroids, followed by further 5-days of plasma exchange and oral steroid therapy. Concerning the symptomatic therapy, lamotrigine $50 \mathrm{mg}$ daily was introduced as a membrane-stabilising drug.

Discussion: This case reports a typical presentation of an extremely uncommon syndrome. Despite its rarity, the clinical suspicion should arise when peripheral signs are coupled with autonomic dysfunction and CNS involvement. Within the diagnostic workup, a whole body imaging is crucial to detect the frequently associated neoplasms and adjust the treatment, accordingly. In line with encouraging results from literature, we chose plasma exchange as therapeutic approach, obtaining a dramatic clinical benefit, since the second administration.

\section{References:}

- Irani S.R., Pettingill P., Kleopa K.A., Schiza N., Waters P., Mazia C., Zuliani L., Watanabe O., Lang B., Buckley C., Vincent A., Morvan syndrome: Clinical and serological observations in 29 cases. Ann Neurol. (2012);72:241-55

- Boyko M, Au KLK, Casault C, de Robles P, Pfeffer G. Systematic review of the clinical spectrum of CASPR2 antibody syndrome. J Neurol. (2020);267(4):1137-46

- Abou-Zeid E, Boursoulian LJ, Metzer WS, Gundogdu B. Morvan syndrome: a case report and review of the literature. J Clin Neuromuscul Dis. (2012);13(4):214-27

\section{ATROPHY OF SUPERIOR CEREBELLAR PEDUNCLES IN SCA7: A CLINICO-RADIOLOGICAL CASE REPORT}

\author{
A. Mechelli ${ }^{1}$, A. Quattrone ${ }^{1}$, I. Martino ${ }^{2}$, A. Piro ${ }^{2}$, A. Giugno ${ }^{1}$, I.

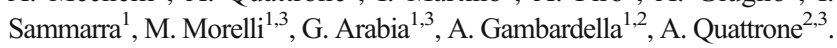

${ }^{1}$ Institute of Neurology, University Magna Graecia (Catanzaro); ${ }^{2}$ Neuroimaging Unit, Institute of Bioimaging and Molecular Physiology, National Research Council (CNR-IBFM) (Catanzaro) ${ }^{3}$ Neuroscience Research Centre, University Magna Graecia (Catanzaro)

Objectives: Spinocerebellar ataxia type 7 (SCA7) is an autosomal dominant neurodegenerative disorder, related to the group of CAG/ polyglutamine (polyQ) expansion disorders, characterized by progressive cerebellar ataxia, slow saccadic eye movements and progressive blindness [1]. A morphometric brain MR imaging study was performed on a SCA7 patient in order to highlight novel elements of atrophy in this disease.

Materials: We report the case of a 23-year-old male subject, who developed visual acuity deficit and cone dystrophy from age 16, ataxia and mild dysphagia at age 18 . His father developed mild gait impairment around the age of 60. Our patient underwent a neurological clinical evaluation that confirmed limb ataxia, dysmetria and gait impairment, with internationally approved clinical scales for ataxia assessment with the following scores: ICARS (International Cooperative Ataxia Rating Scale) 28, SARA (Scale for the assessment and rating of ataxia) 16, Brief ataxia rating scale (BARS) 9; a battery of tests for color vision (Ishihara test, Farnsworth D15 test, City University Colour Vision test) whose results where compatible with cone dystrophy; a full neuropsychological evaluation indicating long-term and short-term memory impairment and visuo-spatial deficits; video-oculographic measurements for quantitative ocular movements assessment that showed reduced mean saccadic amplitude and peak velocity compared to control subjects; a brain 3T MR imaging examination that highlighted mild cerebellar atrophy. Consistently with the above-mentioned data, a genetic research for SCA7 gene mutation showed $75 \pm 2$ CAG triplets in the ATXN7 gene (wild-type alleles: 4-35 CAG triplets [1]).

Methods: In order to highlight possible novel elements of atrophy in this disease, morphometric MRI measures of the brainstem structures were manually acquired on T1-weighted images according to Quattrone et al. [2] methods and compared with values of 38 control subjects.

Results: From morphometric analyses, we found that superior cerebellar peduncles (SCPs) width value $(2.26 \mathrm{~mm})$ was lower than healthy controls (mean $\pm \mathrm{SD}: 3.77 \mathrm{~mm} \pm 0.45$ ). Interestingly, our patient's value was similar to SCPs values of PSP-RS patients ( $2.67 \mathrm{~mm} \pm 0.42$ ), according to a recent study [2], thus suggesting a similar degree of atrophy of this structure.

Discussion: In our patient, along with the typical brain atrophy pattern found in SCA7 patients [3], morphometric MRI data highlight atrophy in SCPs.

Conclusions: We suggest that the analysis of the SCPs could precede and direct the genetic analysis in similar clinical phenotypes and that the involvement of this brainstem structure should be evaluated with the same methodology also in other SCA.

References:

1. Niewiadomska-Cimicka A, Trottier Y Molecular Targets and Therapeutic Strategies in Spinocerebellar Ataxia Type 7. Neurotherapeutics (2019);16:1074-96

2. Quattrone A, Caligiuri ME, Morelli M et al. Imaging counterpart of postural instability and vertical ocular dysfunction in patients with PSP: A multimodal MRI study. Parkinsonism Relat Disord (2019);63:124-30

3. Seidel K, Siswanto S, Brunt ER, den Dunnen W, Korf HW, Rub U Brain pathology of spinocerebellar ataxias. Acta Neuropathol. (2012);124:1-2 


\section{CREUTZFELDT-JAKOB DISEASE PRESENTING AS STROKE- LIKE ONSET OF CORTICOBASAL SYNDROME}

\author{
V. Melas ${ }^{1}$, G. Floris ${ }^{1}$, M. Melis ${ }^{1}$, M. Sechi ${ }^{1}$, G. Cadeddu ${ }^{1}$, E. Casaglia ${ }^{1}$, \\ G. Primicerio ${ }^{1}$, S. Vascellari ${ }^{2}$, A. Serra ${ }^{3}$, G. Defazio ${ }^{1}$
}

${ }^{1}$ Department of Medical Science and Public Health, Neurology Unit, University of Cagliari (Cagliari); ${ }^{2}$ Department of Biomedical Sciences, University of Cagliari (Cagliari); ${ }^{3}$ Nuclear Medicine, Department of Medical Science, University of Cagliari (Cagliari)

Background and aims: Creutzfeldt-Jakob disease (CJD) is the most common human prion disease. It is characterized by mean age of onset in the sixth decade, cognitive deterioration and an average survival of six months from symptoms onset $[1,2]$. However, there are some atypical presentations of this disease leading to possible misdiagnosis [2]. We describe a rare case of SCJD with acute stroke-like onset and clinical features of corticobasal syndrome (CBS).

Materials and methods: A 76 y.o. woman was admitted to the Neurology Department of AOU Cagliari because of an acute onset right upper limb weakness and ataxia associated to hypoesthesia and dysarthria. Family hystory was unremarkable, while personal history was positive for hypertension, hypercolesterolemia and severe aortic valve stenosis. The clinical scenario was suggestive for acute stroke, however over the hospitalization the patient developed dystonic postures of the right upper limb with "alien hand" syndrome, mood changes, cognitive deterioration, myoclonus and ideomotor apraxia. Brain MRi documented left insula and parietal lobe ribbon-like cortical-DWi-restriction while SPET showed left-hemisfericcortex extensive hypoperfusion. Moreover, CSF showed negative 14-3-3 protein, but increased values of total tau protein $(1700 \mathrm{pg} \backslash \mathrm{ml}$, reference range $<500 \mathrm{pg} \backslash \mathrm{ml}$ ). A typical triphasic-waves EEG pattern was present and CSF RT-QuIC was positive. According to international diagnostic criteria a diagnosis of probable CJD characterized by features of CBS was made [3].

Discussions and conclusions: Atypical features or acute-onset of focal symptoms in CJD may cause diagnostic delay. Stroke-like unilateral neurologic signs like paresis or speech disturbances have already been described, possibly associated to DWi hyperintensities. In these cases the aspect of cortical ribboning irrespective for vascular territories may suggest an alternative diagnosis [2], thus avoiding inappropriate treatment. The reported presentation of CJD as corticobasal syndrome is very rare and associated with worse prognosis [2]. This report supports the possibility of a non-cognitive onset of SCJD, suggesting that rapidly progressive neurological signs, even without initial cognitive impairment, should be considered as clinical presentation of CJD.

References:

1. Brian S Appleby et al., Creutzfeldt-Jakob disease. www.uptodate.com @2020 UpToDate

2. Baiardi S. et al., Unusual Clinical Presentations Challenging the Early Clinical Diagnosis of Creutzfeldt-Jakob Disease. J Alzheimers Dis. (2018);64(4):1051-1065. doi: 10.3233/JAD180123

3. Mackenzie G., Will R. Creutzfeldt-Jakob disease: recent developments. F1000Res (2017);6:2053

\section{UNUSUAL SMALL FIBER NEUROPATHY RELATED TO IDIOPATHIC HYPEREOSINOPHILIC SYNDROME: A CASE REPORT}

E. Merico ${ }^{1}$, A. Govoni ${ }^{1}$, L. Chico ${ }^{1}$, E. Schirinzi ${ }^{1}$, R. Calabrese ${ }^{1}$, C. Baldini ${ }^{2}$,

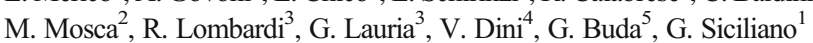

${ }^{1}$ Neurological Clinic, Department of Clinical and Experimental Medicine, University of Pisa (Pisa); ${ }^{2}$ Rheumatology Unit, Department of Clinical and Experimental Medicine, University of Pisa (Pisa); ${ }^{3} 3 \mathrm{rd}$ Neurology Unit and Skin Biopsy Peripheral Neuropathy and Neuropathic Pain Centre, Carlo Besta Neurological Institute, IRCCS Foundation (Milano); ${ }^{4}$ Dermatology Unit, Department of Clinical and Experimental Medicine, University of Pisa (Pisa); ${ }^{5}$ Hematology Unit, Department of Clinical and Experimental Medicine, University of Pisa (Pisa)

Objectives: We present a case of a 21-year-old woman with small-fiber neuropathy (SFN) associated to systemic idiopathic hypereosinoplilic syndrome.

Materials and methods: Patient was admitted to the rheumatology unit of the Santa Chiara Hospital in Pisa: medical history, physical examination, autoimmunity test, infectious disease screening, gastroscopy, cephalorachid fluid (CSF), electroneurographic and imaging data were collected and the skin biopsy was performed.

Results: The patient's first complaints were the appearance of itchy rush to the limbs and trunk with painless lymphadenopathy of the neck and groin, and persistent lack of appetite and vomiting with consequent weight loss of $13 \mathrm{~kg}$ in forty days. After a temporaneous disappearance of the rash, neurological symptoms begin with burning pressure allodynia and spontaneous painful dysesthesia in the feet. Blood test showed eosinophilic leukocytosis. Allergic causes, celiac, autoimmune and infectious diseases, solid tumors, hematological malignancies, and porphyria were excluded, while serum transamines increased. Gastroscopy revealed gastroectasia and modest thickening of the jejunal loops' walls, moderate atrophy of the duodenal mucosa with intraepithelial lymphocytes. Brain and spinal cord MRI were normal. Electrophysiological studies showed axonal sensory neuropathy on the sural nerve bilaterally, unavoidable $\mathrm{H}$ reflex recorded from the left soleus and sympathetic skin responses almost absent in the lower left limb; nerves ultrasound highlighted smallsized vague, while CSF analysis showed cytological-albumin dissociation. Skin biopsy was decisive in confirming a severe picture of small nerve fibers degeneration. After a combined therapeutic attempt with intravenous immunoglobulin (IVIG, $0.4 \mathrm{mg} / \mathrm{kg}$ day for 5 day once a month for 3 months) and steroids, she progressively improved, with resolution of vomiting episodes, resumption of regular nutrition and important reduction of painful dysesthesia.

Discussion: SFN is a disorder clinically dominated by neuropathic pain and autonomic complaints. It can be associated to various pathogenc mechanisms (genetic, autoimmune, toxic or infectious) but in more than $50 \%$ of cases no etiology can be identified. Some evidence suggests that dysimmunity can cause large and small fiber neuropathy. Among the most common manifestations of hypereosinophilic syndrome numerous neurologic lesions have been described, both affecting central and peripheral nervous systems, but this is the first case with systemic involvement of small unmyelinated nerve fiber.

Conclusions: In this case idiopathic hypereosinophilic syndrome was the likely cause of atopic dermatitis and small fiber pathology compatible with dysimmune neuropathy, as confirmed by the clinical response to IVIG and steroid administration.

\section{ENCEPHALOPATHY AS UNIQUE NEUROLOGICAL AND SYSTEMIC MANIFESTATION OF SJOGREN'S SYNDROME: A CASE REPORT}

\author{
A. Miscioscia, F. Rinaldi, P. Riguzzi, M. Puthenparampil, P. Gallo
}

Department of Neuroscience, Padua University (Padova) 
Background: Primary Sjögren's syndrome (pSS) is a frequent rheumatological disorder mostly affecting the exocrine glands. Peripheral nervous system is involved up to one-third of patients and central nervous system is much less common, especially manifestations like encephalopathy. We present our case of an isolated encephalopathic onset of a pSS with no other neurological and systemic manifestation, providing clinical and instrumental features that lead to a correct diagnosis.

Case Report: A 85-year-old man presented to our attention with a history of progressive drowsiness, time and space disorientation, postural instability evolving since the previous month. Neurological examination furthermore revealed left eyelid ptosis, right central facial palsy, limbs stiffness and Babinski sign on left foot. A first cerebral MRI showed multiple white matter T2 hyperintensities, with either juxtacortical, periventricular or brainstem location, as well as in thalamus and hypothalamus, but no abnormal contrast enhancement was detected. Blood tests revealed normal leucocyte count, increased ESR $(36 \mathrm{~mm} / \mathrm{h})$ with normal PCR $(5 \mathrm{mg} / \mathrm{L})$. Serological immunological screening showed the positivity of anti-SS-A (anti-Ro) antibodies. CSF and serum ACE dosage resulted in normal range. Detailed microbiological screening in blood and CSF resulted unremarkable. CSF examination showed high protein levels (146 mg\%, CSF/ serum albumin ratio 26.3), lymphocytic pleocytosis (101 mononucleated cells $/ \mu \mathrm{L}$ ). IsoElectroFocusing on serum and CSF proteins depicted the presence of a mirror IgG pattern with additional CSF-restricted IgG oligoclonal bands (Pattern III). Thoracic high-resolution CT was unremarkable as well as the whole body FDG PET-CT. On the basis of laboratory and radiological findings we suspected Sjogren's syndrome with CNS involvement. Although our patient didn't mention any ocular or oral dryness, Schirmer test was performed revealing a decreased tear production ( $5 \mathrm{~mm} / 5 \mathrm{~min}$ in the right eye). 2016 ACR-EULAR criteria were finally achieved and diagnosis of "Sjogren's syndrome with CNS involvement resulting in encephalopathy" was made. Therapy with high doses of intravenous methylprednisolone (1g daily) for three days was started, followed by steroid tapering. We observed an immediate improvement state of consciousness, cognitive skills and walking ability. After one month, brain MRI demonstrated reduction of alterations previously observed.

Conclusion: Clinicians should always consider pSS in patients presenting with encephalopathy, even when history of sicca syndrome or other neurological or systemic manifestation is absent. MRI features as "MS-like lesions" and laboratory features as "anti-SS-A autoantibodies" should lead to a concrete suspect and, finally, criteria for diagnosis of Sjogren's Syndrome should be verified.

References:

- Shiboski CH, Shiboski SC, Seror R, Criswell LA, Labetoulle M, Lietman TM, et al. 2016 American College of Rheumatology/ European League Against Rheumatism classification criteria for primary Sjögren's syndrome: a consensus and data-driven methodology involving three international patient cohorts. Arthritis Rheumatol. (2017);69:35-45

- Fauchais AL, Magy L, Vidal E. Central and peripheral neurological complications of primary Sjögren's syndrome. Presse Med (2012); 41: 485-93

- Delalande S, de Seze J, Fauchais AL, Hachulla E, Stojkovic T, Ferriby D, Dubucquoi S, Pruvo JP, Vermersch P, Hatron PY. Neurologic manifestations in primary Sjögren syndrome: a study of 82 patients. Medicine (Baltimore). (2004) Sep;83(5):280-91

\section{HEALTHCARE RESOURCE UTILIZATION AND COSTS FOR EXTENDED INTERVAL DOSING OF NATALIZUMAB IN RELAPSING REMITTING MULTIPLE SCLEROSIS}

\author{
M. Moccia ${ }^{1}$, I. Loperto ${ }^{2}$, L. Santoni ${ }^{3}$, S. Masera ${ }^{4}$, A. Carotenuto ${ }^{1}$, M.
} Triassi $^{2}$, V. Brescia Morra ${ }^{1}$, R. Palladino ${ }^{2}$

${ }^{1}$ Department of Neurosciences, Federico II University (Napoli); ${ }^{2}$ Department of Public Health, Federico II University (Napoli); ${ }^{3}$ Health Economics \& Pricing, Biogen Italy (Milano); ${ }^{4}$ Medical Affairs, Biogen Italy (Milano)

Objective: Natalizumab is approved to be administered every 4 weeks (standard interval dosing, SID) in relapsing remitting multiple sclerosis (RRMS). Recently, extended interval dosing (EID) has been used by some physicians to reduce progressive multifocal leukoencephalopathy (PML) risk1. However, the extent to which EID can impact on healthcare resources and costs remains unknown.

Methods: In this population-based study conducted in the Campania Region of Italy, from 2015 to 2017, we included RRMS patients exposed to Natalizumab for at least 18 months. Healthcare data from hospital discharges, drug prescriptions, outpatient consultations and costs were derived from Regional Administrative Databases. EID was defined as $\leq 15$ infusions in the last 18 months; SID was defined as $>15$ infusions in the last 18 months 2 . We used logistic regression, Poisson regression and linear regression models, as appropriate, to study the association between SID/EID and healthcare resources utilization and costs. In the absence of clinical data, the covariates were age, sex, year of treatment start $(<2015,2015$ or 2016), and Charlson comorbidity index, which weights chronic comorbidities to define an overall risk.

Results: Out of 426 patients treated with Natalizumab, we included 208 patients. 157 patients were on SID $(75.5 \%$; age $=36.5 \pm 10.8$ years; female $=68.1 \%$ ) with mean $\pm \mathrm{SD}$ of $31.3 \pm 2.4$ days infusion interval and $29.9 \pm 2.4$ months of treatment, whilst 51 were on EID $(24.5 \%$; age $=33.7 \pm 11.1$ years; female $=72.5 \%)$ with 43.1 \pm 4.8 days infusion interval and $33.5 \pm 3.2$ months of treatment. Compared with SID, Natalizumab EID was associated with lower MS outpatient visits $(\mathrm{p}=0.01)$ and related costs $(\mathrm{p}=0.03)$, and lower natalizumab costs $(p<0.01)$, without changes in other MS and non-MS-related healthcare resource utilization and costs.

Conclusions: Analyzing Regional Administrative Databases in Campania Region, Natalizumab EID seems to be associated with $23 \%$ reduced direct treatment costs, without additional healthcare burden for Italian National Healthcare Service. Limitations include the inability to adjust for clinical measures and potential selection bias.

References:

- Zhovtis Ryerson L, Frohman TC, Foley J, et al. Extended interval dosing of natalizumab in multiple sclerosis. J Neurol Neurosurg Psychiatry (2016);87:885-89

- Zhovtis Ryerson L, Foley J, Chang I, et al. Risk of natalizumabassociated PML in patients with MS is reduced with extended interval dosing. Neurology (2019);93:e1452-e1462

\section{A PATIENT WITH A MENINGIOMA OF THE FORAMEN MAGNUM: IS THIS PSEUDODYSTONIA?}

V. Moschella, E. Pennisi, C. Altavista

Unit of Neurology, San Filippo Neri Hospital (Roma) 
Background: Cervical dystonia is a form of focal dystonia characterized by head/neck movements/postures inducing head deviation, sometimes with head tremor and often with sensory trick [1]. Traditionally related to basal ganglia dysfunction, actually dystonia is considered a network disorder, involving also brainstem and cerebellum [2]. Pseudodystonia [3] is contemplated when clinical, imaging, laboratory or electrophysiological investigations are not compatible with dystonia; it could be caused by musculoskeletal, sensory or motor pathways disorders or can represent a compensatory posture; it is associated with abnormalities in regions different from those typically involved in dystonia network. Discrimination between dystonia and pseudodystonia becomes less clear when a lesion involves part of the dystonia network. Lesions of posterior fossa are rarely cause of dystonia and are especially described in paediatric population.

Aims: To describe an adult with cervical dystonia and meningioma of foramen magnum.

Results: When she was 50-years old, CG insidiously started complaining cervical pain with muscular contracture. She was evaluated in a movement disorders centre and performed brain MRI, reported as normal. Diagnosis of cervical dystonia was made and she started botulinum toxin, with mild benefit. One year later, she came to our botulinum treatment centre. Neurological examination showed mild laterocaput to the right, mild anterocollis and mild retrocaput. Tremor was absent but she showed small oscillations during left head rotation. Touching the nape ameliorated posture and head movements, resembling a sensory trick. Rotation of head to the left evocated right neuropathic pain, similar to occipital neuralgia. The right part of the tongue showed mild atrophy. There was no history of neuroleptic exposure or family history for dystonia or parkinsonism. Patient was treated with botulinum toxin (right sternocleidomastoideus and bilateral trapezius and splenius), with discrete benefit. Brain MRI, integrated with CT scan of skull base, revealed homogeneous enhancing mass lesion, with a diameter of $35 \mathrm{~mm}$, on the right part of foramen magnum, osteolysis of right occipital condyle with deformity of atlo-occipital joint and mild compression of medulla oblongata. Lesion was removed and was histologically diagnosed as a transitional meningioma WHO grade I. Patient showed a rapid improvement of neurological condition with reduction of neuralgic pain and movement limitation.

Discussion: Distinction from dystonia and pseudodystonia could be clinically difficult as indeed topographic relations between dystonia network and spinal accessory nerve. Since dystonia could be secondary and tractable, also in adults, scrupulous research of atypical clinical and radiological elements, with periodic reassessment, is essential in diagnostic process.

References:

1. Defazio G, Albanese A, Pellicciari R, Scaglione CL, Esposito M, Morgante F, Abbruzzese G, Bentivoglio AR, Bono F, Coletti Moja M, Fabbrini G, Girlanda P, Lopiano L, Pacchetti C, Romano M, Fadda L, Berardelli A. Expert recommendations for diagnosing cervical, oromandibular, and limb dystonia. Neurol Sci. (2019) Jan;40(1):89-95. doi: 10.1007/s10072-018-3586-9

2. Corp DT, Joutsa J, Darby RR, Delnooz CCS, van de Warrenburg BPC, Cooke D, Prudente CN, Ren J, Reich MM, Batla A, Bhatia KP, Jinnah HA, Liu H, Fox MD. Network localization of cervical dystonia based on causal brain lesions. Brain. (2019) Jun 1;142(6):1660-1674. doi: 10.1093/brain/awz1 12

3. Berlot R, Bhatia KP, Kojović M. Pseudodystonia: A new perspective on an old phenomenon. Parkinsonism Relat Disord. (2019) May;62:44-50. doi: 10.1016/j.parkreldis.2019.02.008

\section{INCLUSION BODY MYOSITIS: RHABDOMYOLYSIS AS AN ATYPICAL MISLEADING ONSET}

G. Nicocia, C. Bonanno, A. Pugliese, A. Migliorato, G. Vita, A. Toscano, C. Rodolico

Department of Clinical and Experimental Medicine, Unit of Neurology and Neuromuscular Diseases, University of Messina (Messina)

Introduction: Inclusion body myositis (IBM) is the most common idiopathic degenerative and inflammatory myopathy in people over 50 years old. The hallmark clinical presentation is slowly progressive, often asymmetric, weakness of quadriceps, finger and wrist flexor muscles.

Aim: We report the case of 75 years-old man, diagnosed with IBM who suffered, as presenting manifestation, from rhabdomyolysis.

Case Report: A 75 years-old man self-referred to the Emergency Department for generalized fatigue associated with heart palpitations and cough. The electrocardiogram showed atrial fibrillation. Treatment with intravenous amiodarone was promptly started with conversion to sinus rhythm. The patient was addressed to an out-patient cardiological consultation after a few weeks. Laboratory tests revealed raised serum level of creatine kinase (10000 U/L, n.v. 0 - 200 U/L), elevated erythrocyte sedimentation rate (ERS rate) and myoglobinuria. Because of hyperCKemia patient was referred to Our Department of Neurology. Recent medical history revealed a 15-days of dark urine ("dark orange") without muscle pain, fatigue and other muscular symptoms Admission neurological examination showed no abnormalities. After one week of hospitalization, neck flexors and proximal lower limbs weakness were detected. Electromyography (EMG) showed a myopathic pattern on both upper and lower limbs muscles. The pathognomonic IBM signs revealed by muscle biopsy from left vastus lateralis muscle guided us to the diagnosis.

Discussion and Conclusion: To our knowledge, there are no reported cases of IBM presenting as defined rhabdomyolysis. This case allows to expand the currently knowledge of possible different onset of IBM which has to be included in the differential diagnosis of rhabdomyolysis.

\section{SEVERE DISEASE REACTIVATION AFTER TRANSPLANTATION OF HUMAN NEURAL STEM CELLS IN SECONDARY PROGRESSIVE MULTIPLE SCLEROSIS PATIENT}

\author{
C. G. Nicoletti ${ }^{1}$, D. Landi ${ }^{1}$, G. Cecchi ${ }^{1}$, G. Cola ${ }^{1}$, G. Mataluni ${ }^{1}, \mathrm{~N}$. \\ Mercuri $^{2}$, G. Marfia ${ }^{3}$
}

${ }^{1}$ Multiple Sclerosis Clinical and Research Unit, Department of Systems Medicine, Tor Vergata University (Roma); ${ }^{2}$ Neurology Unit, Department of Systems Medicine, Tor Vergata University (Roma); ${ }^{3}$ Multiple Sclerosis Clinical and Research Unit, Department of Systems Medicine, Tor Vergata University, Unit of Neurology and Neurorehabilitation, IRCCS Neuromed (Roma, Pozzilli-IS)

Objective: A wide variety of preclinical studies, using experimental autoimmune encephalomyelitis model of multiple sclerosis (MS) have recently shown that grafted cells with different origins including mesenchymal stem cells, neural precursor and induced-pluripotent stem cells have the ability to repair CNS lesions and to recover functional neurological deficits. Cell transplantation offers an important therapeutic solution to prevent progression to irreversible disability in those patients with MS not responder to commercially available disease modifying treatments. 
Several trials are ongoing in human MS but efficacy and safety of transplant needs to be established. We describe a case of severe MS reactivation after fetal neural stem cells transplant.

Materials and methods, results: A 53-year-old female patient was diagnosed with Relapsing - Remitting MS in July 2005 with onset in 1996 characterized by retrobulbar optic neuritis. Two infusions of Mitoxantrone followed the 2005 hospitalization; then she started Interferon $\beta-1 b$ (IFN $\beta-1 b)$ in January 2006 until July 2011. Because of radiological and clinical progression, in September 2012, she started therapy with Fingolimod which was interrupted in October 2016 due to diagnosis of secondary profession and disability worsening. In the 2019, she was enrolled in the trial 'Phase I multicentric study of allogenic of intracerebroventricular human neural stem cells transplantation for the experimental treatment of secondary progressive multiple sclerosis patients.'. The patient received, through intraventricular implantation, human neural stem cells from aborted fetuses, in July 2019. She took Prednisone $60 \mathrm{mg} / \mathrm{die}$ in the following month and Tacrolimus 0,1 $\mathrm{mg} / \mathrm{kg} / \mathrm{die}$ for six months after. Monthly clinical, instrumental and laboratory follow-ups followed. Each of follow-up MRIs after transplant showed new unexpected gadolinium enhancing lesions, despite she received two cycles of I.v. methylprednisolone. Once completed the study, she came back to our attention complaining of neurological worsening in the previous months (EDSS 7.5).

Discussion: Neural stem cells are generally expected to exert beneficial anti-inflammatory environment in nervous system and neuroprotective effect leading to clinical and pathological improvement. The stem cells can do this by differentiating into glial or neural cells, or indirectly, stimulating microglia towards an anti-inflammatory type. Nevertheless, in our case, recurrent and severe reactivation of brain inflammation was found in a previously stable patient after implant of non-self-cells. We can speculate that immune reaction against implanted cells triggered abnormal disease rebound or bystander brain damage.

Conclusion: We need more knowledge about the mechanisms of action of neural cell transplantation. This case also highlights the challenge of the optimal next therapy for the patient.

References:

- Butti E, Bacigaluppi M, Chaabane L, Ruffini F, Brambilla E, Berera G, Montonati C, Quattrini A, Martino G. Neural Stem Cells of the Subventricular Zone Contribute to Neuroprotection of the Corpus Callosum after Cuprizone- Induced Demyelination. J Neurosci. (2019) Jul 10;39(28):5481-92

- Genc B, Bozan HR, Genc S, Genc K. Stem Cell Therapy for Multiple Sclerosis. Adv Exp Med Biol. (2019);1084:145-74

- J Quandt JA, Bratincsak A, Shahar T, Eusebi F, Sherman LS, Mattson MP, Martin R, Rao MS. Disease progression after bone marrow transplantation in a model of multiple sclerosis is associated with chronic microglial and glial progenitor response. J Neuropathol Exp Neurol. (2007) Jul;66(7):637-49

\section{AUTOIMMUNE MYOCARDITIS IN PATIENT WITH MULTIPLE SCLEROSIS}

\section{R. Nistri, E. Barbuti, C. Pozzilli, S. Ruggieri, A. Ianniello}

Neurology, Sant'Andrea Hospital, Sapienza University (Roma)

Introduction: Patients with MS have an increased risk of cardiovascular events like left ventricular dysfunctions and sudden unexpected death in MS compared to general population.
Clinical case: We describe a case of myocarditis in a 34-year-old male patient with relapsing remitting multiple sclerosis and unremarkable past medical history. After the onset of the disease with diplopia in 2011 he started Interferon beta-1b until December 2014. Due to the presence of MRI activity, patient was switched to Teriflunomide and stopped in May 2015 without washout as he desired to have a child. He was then treated with Dimethyl fumarate with radiological and clinical efficacy (EDSS 2). In April 2016, an ECG performed for other reasons showed diffuse inversions of $\mathrm{T}$ waves, so he underwent further cardiological tests. A first cardiac echocardiography showed an ejection fraction (EF) of $70 \%$, but a 24-hour ECG monitoring showed ectopic supraventricular and ventricular extrasystoles, and a run of non-sustained ventricular tachycardia. Subsequently, a cardiac MR (CMR) showed an EF of $56 \%$, and findings of a subacute myocarditis. In October, a second CMR showed worsening of the left ventricle function with EF of $43 \%$. After several stable controls, in March 2019 another CMR showed a worsened EF $(31 \%)$ and a myocarditis with a post-myocarditis cardiomyopathy. Therefore, the patient, although asymptomatic, underwent a myocardial biopsy, which revealed a post-inflammatory cardiomyopathy without fraying. All the virologic and autoimmune screenings were negative, with the exception of positivity for Parvovirus B19 on myocardial tissue, which was not associated with a positive serology. Patient was discharged with a diagnosis of autoimmune myocarditis. Thus, the patient was treated with Azathioprine $125 \mathrm{mg}$ daily and Prednisone (50 mg gradually reduced until suspension). In June 2019 an echocardiography showed a little improvement of EF (36\%), but after an Ergometric stress test revealed a symptomatic tachycardia the implant of an ICD became necessary. During the treatment with Azathioprine, patient's neurological examination and MRI were unchanged.

Discussions: This is the first case of "autoimmune myocarditis" described in a patient treated with Dimethyl fumarate, which instead was tested in experimental autoimmune myocarditis in rats. 3 cases of cardiological sudden deaths have been described with teriflunomide.

Conclusions: We described a very rare cardiac complication occurred without any clinical evidence, in a patient with a stable form of MS. The severity of the myocarditis and the ease of misdiagnosis suggest the utility of performing an ECG on a regular basis.

\section{A PERIPHERALLY INDUCED MOVEMENT DISORDER: THE BREATHING ARM SYNDROME}

\author{
D. Norata, T. Corradetti, F. Lupidi, P. Di Bella, M. Silvestrini
}

Neurological Clinic, AOU Ospedali Riuniti (Ancona)

Introduction: After a lesion in the brachial plexus or cervical nerve roots, the motor neurons originally destined to the diaphragm can regenerate aberrantly and re-innervate arm/shoulder muscles, which may contract involuntarily, synchronously with inspiration, thus the definition of breathing arm syndrome.

Case report: We performed an ENG-EMG study in a 47-year-old woman that suffered from involuntary circling movements of the right scapular region. The simultaneous recording of intercostal muscles showed that the movements were synchronized to inspiration and lead to diagnose a breathing arm syndrome.

Discussion: The patient movement is clinically classified as a dystonia. There are several clinical evidences of borderline conditions between dystonia and peripheral synkinesias. The involvement of basal ganglia in dystonia's pathogenesis itself has been recently reviewed within a more sophisticated scheme, where dystonia may result from an injury of the 
nervous system in general. This may complicate even more the distinction between dystonia and peripheral synkinesia.

Conclusions: Despite the trend to group together different types of movement disorder, it is still necessary to make a precise diagnosis, in order to avoid diagnostic errors and allow future therapeutic possibilities. References:

- Swift TR, Leshner RT, Gross JA. Arm-diaphragm synkinesis: electrodiagnostic studies of aberrant regeneration of phrenic motor neurons. Neurology (1980);30(4):339-344. doi:10.1212/ wnl.30.4.339

- Yldiz FG, Nurlu G, Temucin CM. A Missed EMG Finding of Brachial Plexopathy: Breathing Arm. J Neurol Sci. (2016);(33):227-32

- Chan Chun Kong D, Breiner A, Wolff G. Idiopathic respiratory synkinesis: A case series. Muscle Nerve (2020);61(1):E8-E9. doi:10.1002/mus.26733

\section{A 6-MONTH LASTING NEW ONSET REFRACTORY STATUS EPILEPTICUS (NORSE): CLINICAL EVOLUTION AND LONG- TERM OUTCOME}

N. Orlandi ${ }^{1}$, G. Giovannini ${ }^{1}$, M. Cioclu ${ }^{1}$, J. Rossi ${ }^{1}$, G. Turchi ${ }^{1}$, A. Marudi $^{2}$, E. Bertellini' ${ }^{2}$, G. d'Orsi ${ }^{3}$, S. Meletti ${ }^{1}$

${ }^{1}$ Neurology Unit, OCB Hospital, Azienda Ospedaliera-Universitaria of Modena (Modena); ${ }^{2}$ Intensive Care Unit, OCB Hospital, Azienda Ospedaliera-Universitaria of Modena (Modena); ${ }^{3}$ Epilepsy Centre, Clinic of Nervous System Diseases, Riuniti Hospital (Foggia)

Introduction: New Onset Refractory Status Epilepticus (NORSE), is a rare and challenging condition occurring in previously healthy patients, whose etiology often remains undiscovered and with unfavorable outcome [1]. We report the electroclinical and neuroradiological evolution of an extremely prolonged case of super-refractory myoclonic status epilepticus.

Material and Methods: Continuous EEG monitoring was performed during the hospitalization as well as serial MRI scans (details in figure 1), neurological evaluation, and extensive serological/CSF investigations.

Results and discussion: A 38 years-old healthy woman with a prodrome of fever, vomiting and diarrhea was admitted to our ICU for refractory and generalized seizures requiring deep sedation. CSF examination and serological tests for viral and autoimmune aetiologies were unremarkable, except for a low IgM positivity against Toscana virus. Prolonged politherapy with antiseizures drugs, anesthetics and first line immunotherapy was performed as well as trials with intravenous allopregnenolone, chetogenic diet and hypothermia (details in figure 2). Nearly 6 months after hospital admission SE finally resolved, the patient gradually recovered to a partially responsive state and was transferred to a rehabilitation facility with severe tetraplegia. In a twoyear follow-up she developed pharmaco-resistant epilepsy, subcortical myoclonus and severe cognitive impairment.

Conclusion: This represents the longest case of NORSE with final status resolution. Despite initial failure, prolonged and combined treatment with multiple synergistic mechanisms should not be withdrawn as may lead to recovery. However, severe neurological sequelae were developed, and future research should focus on new disease modifying treatment.
Reference:

1. Gaspard N, Hirsch LJ, Sculier C et al. New-onset refractory status epiepticus (NORSE) and febrile infection-related epilepsy syndrome (FIRES): State of the art and perspectives. Epilepsia (2018) Apr 59(4):745-52

\section{SPINDLE COMA IN METABOLIC ENCEPHALOPATHY. A CASE REPORT}

B. Orlando, G. Iliceto, F. Caputo, G. Procida, F. Lapenna, G. Libro, M. Trojano

Department of Basic Medical Sciences, Neuroscience and Sense Organs, University of Bari (Bari)

Background: Spindle coma (SC) describes an electro-clinical entity with sleep-like electroencephalogram (EEG) pattern that appears in comatose patients. The prognostic implications of SC are related to aetiology and EEG reactivity.

Case Report: A 59-year-old Greek woman with fulminant hepatitis related to Hepatitis B viral infection was admitted in a comatose state to a general intensive care unit (ICU) in her country; she was intubated with Propofol and Remifentanil sedation. Emergency liver transplantation (LT) was considered the most effective treatment option and at 15 days after symptoms onset she was transferred to ICU of Polyclinic Hospital of Bari. Neurological assessment was required in order to verify the possibility of performing the LT and to obtain potential early prognostic factors; clinical examination revealed a Glasgow Coma Score (GCS) of 3, bilateral myosis, reduced pupillary light reflex, flaccid quadriplegia. The brain computed tomography scan was normal and transcranial Doppler examination excluded intracranial hypertension. Serial EEGs were acquired over a period of 24-48 hours, decreasing the dose of each respective sedatives to exclude possible interfering of iatrogenic factors. The EEG performed at the end of the sedation period disclosed severe decreased of brain activity in amplitude, theta and delta background with superimposed of intermittent sleep-like spindle patterns of 9 to 14 Hertz and sporadic triphasic waves, without reactivity to noxious stimuli. The patient persisted GCS of 3. Despite the role of SC as a prognostic marker is controversial, it is generally associated with a good prognosis when coma is due to hepatic encephalopathy; thus, based on electrophysiological diagnosis of SC, the indication for a LT was made. After LT surgery a close neurological and EEG follow-up showed a progressive improvement either neurological than electrophysiological condition. EEG at 5 days after LT documented a generalized alpha-coma pattern without background reactivity with concomitant GCS of 3. EEG at 10 days revealed a marked improvement in absence of any pathological patterns. According to EEG activity, level of consciousness was normal although remaining neurologic examination showed flaccid tetraparesis due to critical illness polyneuropathy confirmed on electromyography. The patient was discharged to the hospital with a GCS of 15, addressed to rehabilitation center.

Conclusion: This case report highlights that the aetiology of SC is a key factor for outcome. SC due to hepatic encephalopathy has a good outcome despite critical neurological conditions and can provide diagnostic and prognostic information in patients with deep coma, as in our case. References:

- Cologana V, Schabusa M, Ledouxc D, Moonend G, Maqueta P, Laureysa S. Sleep in disorders of consciousness. Sleep Med Rev (2010);14:97-105 
- Husain A. Electroencephalographic assessment of coma. J Clin Neurophysiol (2006);23:208-20

\section{MONOCULAR DIPLOPIA IN IDIOPATHIC INTRACRANIAL HYPERTENSION: CASE REPORT AND LITERATURE REVIEW}

\author{
G. R. Palmieri, M. Sansone, R. De Simone, M. Moccia
}

Department of Neuroscience, University of Naples Federico II (Napoli)

Purposes: Here we describe a case of monocular diplopia in Idiopatic Intracranial Hypertension (IIH). Diplopia is the double-vision of a single object, and can be binocular or monocular [1]. The latter is caused by neurological disorders (i.e., oculomotor nerve palsies, ocular myopathies, and neuromuscular junction disorders), causing a misalignment of the visual axes, with images falling on the fovea of the fixating eye and on the extra-foveal retina of the non-fixating eye. On the contrary, monocular diplopia is generally explained by intraocular pathology (i.e., refractive errors, ocular media abnormalities, dry eyes), causing the image of a single object to fall, at the same time, on the fovea and on the extrafoveal retina of the same eye.

Materials and Methods: We reported on a 22-year old woman presenting with abrupt-onset monocular diplopia, along with recurring 15minute episodes of tensive headache. She did not report on previous history of headaches, concomitant treatments, or drug abuse. She was obese, with reported weight gain over the past months (body mass index was $40.8 \mathrm{Kg} / \mathrm{m} 2$ ), and was recently diagnosed with binge eating disorder.

Results: Diagnosis of Idiopathic Intracranial Hypertension (IIH) was based on the presence of papilledema and elevated cerebrospinal fluid (CSF) pressure. Monocular diplopia resolved after CSF subtraction [2].

Discussion: This case is specifically interesting because monocular diplopia responded to CSF subtraction, in absence of any other ophthalmological or psychiatric causes, suggesting it was directly due to $\mathrm{IIH}$. Mechanisms of monocular diplopia in IIH are unknown. We might hypothesize that the extravasation of the CSF from the enlarged optic nerve, through nerve fibers, into the macula, can be responsible for changes in dioptric media, and subsequent monocular diplopia, as already suggested for epiretinal membrane maculopathy. Epiretinal membranes are caused by the stretch of the dural sheath by the CSF, that allows the access of migratory glial cells to the pre-retinal surface around the optic nerve, and can occur in IIH, further suggesting a pathogenetic association $[1,3]$.

Conclusions: We described a case of monocular diplopia as a presenting symptom of IIH and discussed diagnostic issues of this possiblyunderestimated symptom in neurology clinical practice.

References:

1. Danchaivijitr C, Kennard C. Diplopia and eye movement disorders. Neurol Pract. (2004);75(4)

2. Friedman DI, Liu GT, Digre KB. Revised diagnostic criteria for the pseudotumor cerebri syndrome in adults and children. Neurology (2013);81(13):1159-65

3. Hiscott P, Wong D, Grierson I. Challenges in ophthalmic pathology: The vitreoretinal membrane biopsy. Eye (2000);14(4):549-59

\section{LV-PACNS: TRANSCRANIAL ULTRASOUND AS NON- INVASIVE FOLLOW-UP EXAM}

M. Parisi ${ }^{1}$, A. Introna ${ }^{1}$, M. Petruzzellis ${ }^{2}$, D. Mezzapesa ${ }^{2}$, F. Rizzo ${ }^{1}$, G. Scaglione $^{1}$, N. Loizzo ${ }^{1}$, M. Savarese ${ }^{2}$, I. Simone ${ }^{1}$, M. Trojano ${ }^{1}$
${ }^{1}$ Department of Basic Medical Sciences, Neurosciences and Sense Organs, University of Bari "Aldo Moro" (Bari); ${ }^{2}$ Stroke Unit, Neurology Unit, Department of Basic Medical Sciences- Neurosciences and Sense Organs, General Hospital of Bari (Bari)

Introduction: The primary angiitis of the central nervous system (PACNS) is an uncommon disorder with unknown causes which involves brain and spinal cord. The diagnosis remains challenging for unspecific imaging findings and absence of standardized diagnostic criteria.

Case Report: We present the case of a 43-year-old man with a past medical history of smoking, hypertension, Obstructive Sleep Apnea and untreated impaired fasting glucose. No substance abuse was reported. In February 2020 for repeated episodes of transient diplopia and right facial-brachial paraesthesia, he underwent brain computed tomography (CT) and Angiography (CTA), which was unremarkable for parenchymal lesions but showed an irregular aspect of intracranial arteries. A subsequent Transcranial Color Doppler (TCCD) examination did not reveal abnormal findings. On May 2020 he was admitted to our department because of drowsiness and mild dysarthria followed one week later by unusual psychomotor slowdown and apathy. Neurological examination was remarkable for mild left partial facial palsy, severe hypophonia and psychomotor slowdown. Brain $\mathrm{CT}$ and CTA showed right thalamic ischemic lesion and left anterior thalamic subacute lesion with an irregular profile of middle cerebral arteries (MCAs). At this time, TCCD examination showed severe increase of MCAs velocity suggesting vasculitis. Brain MRI with angiography (MRA) and digital subtraction angiography (DSA) revealed diffuse segmental narrowing, mainly affecting MCAs and left Anterior Cerebral Artery (ACA). Laboratory examination and autoimmune study were normal. Thus, large-vessels PACNS (LV-PANCS) was suspected and methylprednisolone ( $1 \mathrm{gr} /$ day for 5 days) was started followed by gradual tapering off with prednisone to $1 \mathrm{mg} / \mathrm{Kg}$. At the end of steroid bolus, a new MRA and ultrasound examination reported the persistence of narrowing of main intracranial arteries, despite a mild benefit on hypophonia. Therefore, we started treatment with cyclophosphamide $1 \mathrm{gr}$ IV monthly. After two infusions TCCD examination showed a mild improvement of MCAs velocities and normalization of left ACA findings, although neurological status was unchanged.

Discussion: Diagnosis of LV-PANCS is focused on DSA and MRA. In our case, the ultrasound (US) features were consistent with more invasive vascular imaging. In this setting, we underline the importance of TCCD as unique non-invasive vascular imaging technique, suitable for repeated follow-up, extremely useful for monitoring disease evolution and treatment efficacy. In conclusion, we suggest the importance of US examination in the follow-up of LV-PANCS.

References:

- C. Salvarani, R. Brown, R. D. Christianson et al. Adult primary central nervous system vasculitis treatment and course: Analysis of one hundred sixty-three patients. Arthritis and Rheumatology (2015);67(6):1637-45

- C. Salvarani, R. Brown, K.T. Calamia et al. Primary central nervous system vasculitis: Analysis of 101 patients. Annals of Neurology (2007);62)5):442-51

- Hajj-Ali R.A., Singhal A. B., Benseler S., Molloy E., Calabrese L. H. Primary angiitis of the CNS. The Lancet Neurology (2011);10(6):561-72 


\section{PARANEOPLASTIC LAMBERT EATON MYASTHENIC SYNDROME AND CEREBELLAR DEGENERATION ASSOCIATED WITH MERKEL CELL CARCINOMA OF UNKNOWN PRIMARY SITE: A CASE REPORT}

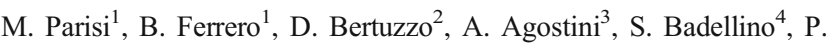 \\ Quaglino $^{3}$, L. Lopiano ${ }^{1}$
}

${ }^{1}$ Department of Neuroscience "Rita Levi Montalcini", University of Turin (Torino); ${ }^{2}$ Department of Neurology, Cardinal Massaia Hospital (Asti); ${ }^{3}$ Medical Sciences Department, Dermatologic Clinic, University of Turin (Torino); ${ }^{4}$ Department of Oncology, University of Turin (Torino)

Aims: Paraneoplastic cerebellar degeneration (PCD) and Lambert-Eaton myasthenic syndrome (LEMS) are both uncommon disorders that can be associated with any cancer. We describe a case of a Merkel cell Carcinoma with neurological symptoms as first manifestation.

Materials: A 59-years-old man, with no comorbidities, was admitted to our hospital because of the subacute onset of diplopia, speech disorder and gait abnormality, in the last 2 weeks. He presented horizontal nystagmus, marked dysarthria, trunk ataxia, dysmetria, preserved muscular strength in all limbs, difficulty standing and wide-based gait. Myotatic reflexes were diffusely hypoactive.

Method: Cerebrospinal fluid analysis showed the presence of raised protein level. A brain and spine MRI were performed: the exam showed contrast enhancement of cauda equina's roots on T1 weighted sequence. At the nerve conduction studies, we noticed global CMAP amplitude reduction in all four limbs. Tibial $\mathrm{F}$ waves were absent. Based on the suspect of an incomplete form of Miller Fisher Syndrome, a cycle of intravenous immunoglobulin (IGIV).

Results: No clinical improvement was seen and afterward we repeated the neurophysiologic study and adding a $3 \mathrm{~Hz}$ repetitive nerve stimulation test recording from nasalis muscle that showed a significant decrement of the CMAP amplitude. The $30 \mathrm{~Hz}$ RNS test recording from abductor digiti minimi showed an increment in CMAP amplitude more than $100 \%$ and the diagnosis of presynaptic disorder was made. Anti-VGCC antibodies were present in the serum and the total body PET-CT scan showed multiple pelvic lymph nodes enhancement. Excisional biopsy and immunohistochemical analysis revealed a poorly differentiated carcinoma positive for anti-synaptophysin, anticytokeratin AE1/AE3, cytokeratin 20 and chromogranin A.

Discussion: Since no evidence of a primary site was found at skin or subcutaneous tissue, a diagnosis of Merkel Cells Carcinoma metastasis from an unknown primary origin was made. Cerebellar symptoms were related to Cerebellar Paraneoplastic Degeneration. The patient was referred to a multidisciplinary evaluation and the patient underwent a radiation therapy. After 4 months a PET scan showed no progression and we saw mild clinical and neurophysiological improvement. The identification of two paraneoplastic syndromes allowed to make the diagnosis of an aggressive neuroendocrinal tumor with a poor prognosis, especially when metastatic.

Conclusion: This report aims to underline the complexity around paraneoplastic syndromes associated with MCC and the outstanding importance of clinical neurophysiology: deepened neurophysiological studies could unmask rare disorders and help in anticipating the diagnosis and the treatment of aggressive cancers, improving survival and quality of life.

References:

- Iyer JG, Parvathaneni K, Bhatia S, Tarabadkar ES, Blom A, Paraneoplastic syndromes (PNS) associated with Merkel cell carcinoma (MCC): A case series of 8 patients highlighting different clinical manifestations. Journal of American Dermatology (2016); 75(3):541-47
- Pavolucci L, Giannini G, Giannoccaro MP, Foschini MP, Lang B, Avoni P, Tinuper P, Vincent A, Liguori R. Paraneoplastic cerebellar degeneration and lambert-eaton myasthenia in a patient with merkel cell carcinoma and voltage-gated calcium channel antibodies. Muscle Nerve (2017) Nov;56(5):998-1000

- Kontis E, Vezakis A, Pantiora E, Stasinopoulou S. Merkel cell carcinoma of unknown primary site; case presentation and review of the literature. Annals of Medicine and Surgery, (2015);4(4):434-37

\section{VESSEL WALL IMAGING IN THUNDERCLAP HEADACHES: A RCVS CASE}

\section{U. Pensato ${ }^{1}$, L. Cirillo ${ }^{2}$, V. Favoni ${ }^{2}$, G. Pierangeli ${ }^{2}$, P. Cortelli ${ }^{2}$, S. Cevoli ${ }^{2}$}

${ }^{1}$ Department of Biomedical and NeuroMotor Sciences (DIBINEM), University of Bologna (Bologna); ${ }^{2}$ IRCCS Institute of Neurological Sciences of Bologna, University of Bologna (Bologna)

Background: Reversible Cerebral Vasoconstriction Syndrome (RCVS) is characterized by recurrent short-lasting thunderclap headaches, usually triggered by intense physical activity or exposure to abrupt temperature changes, variably associated with focal deficits and/or seizures [1]. Conventional catheter angiography and non-invasive vascular are limited by similar luminal abnormalities shared by other pathologies.

Case: A 26-year-old woman present with two-days history of recurrent episodes of thunderclap headache (two hours each). Her medical history revealed cannabis daily use and episodic migraine. Neurological and CSF examinations were unremarkable, while vascular neuroimaging revealed segmental vasoconstriction in multiple intracranial arteries in addition to moderate asymmetric and focal wall enhancement. She was treated with oral Nimodipine, advised to avoid potential triggers and discontinue cannabis daily use. Her headache and neuroradiological findings resolved completely within three months, confirming a diagnosis of RCVS.

Discussion: Conventional vascular imaging assess only the vascular lumen, often resulting in overlapping patterns between different pathologies involving intracranial arterial walls. In contrast, Vessel Wall Imaging is a non-invasive technique which may reveal supportive findings. Indeed, it usually shows multiple vessel walls thickening associated with a concentric and marked persistent enhancement in CNS vasculitis, whereas thickening with no or minimal focal enhancement is observed in RCVS [2]. References:

1. Ducros A. Reversible cerebral vasoconstriction syndrome. Handb Clin Neurol. (2014);121:1725-41

2. Mossa-Basha M, Shibata DK, Hallam DK, de Havenon A, Hippe DS, Becker KJ, et al. Added Value of Vessel Wall Magnetic Resonance Imaging for Differentiation of Nonocclusive Intracranial Vasculopathies. Stroke (2017);48(11):3026-33

\section{PHYSIOTHERAPEUTIC TREATMENT OF A PATIENT WITH DEMYELINATING DISEASE OF THE POPLITEAL SCIATIC NERVE IN MS DISEASE}

\author{
M. Perini ${ }^{1}$, S. Vernocchi ${ }^{2}$, R. Laezza ${ }^{3}$, P. Manfroi ${ }^{3}$, S. Ipsaro Passione ${ }^{3}$ \\ ${ }^{1}$ Humanitas Institute (Milano); ${ }^{2}$ Internal Medicine, Abbiategrasso \\ Hospitals (Abbiategrasso-MI); ${ }^{3}$ Associazione Interstudi Europeo, \\ Ostrava University (Chiasso-CH)
}


Neuropathies in the lower limbs can affect balance and physical strength, compromising the patient's quality of life. We treat a case of sciaticpopliteal neuropathy in a patient affected by SM. Among the most frequent causes of peripheral neuropathy of popliteal sciatica we have compressive pathology including the reduction of the fibular tunnel in which it starts or from the mass or cyst formation that includes it. In the differential diagnosis we considered the L5-L4 discopathies, the medullary canal stenosis, the irritating syndrome of L5 which have been described, with EMG and ENG. We describe the singular case that is presented in our outpatient observation of a 42-year-old young woman. She is a married patient, with a daughter, no systemic disease, smoker 7p/y, normal weight, regular physical activity. One year ago, after an episode of parestesias at left side, she performed a brain MRI that showed multiple periventricular demyelinating lesions compatible with Multiple Sclerosis diagnosis. No specific immunotherapy was stated up now. It comes with a selected neurological symptomatology with a deficit in the extension of the big toe of the foot, a diffuse paraesthesia in the back of the foot that has oriented towards a compromise of the peroneal nerve. Vertebral and pelvic MRI shows deformation and flattening of the femoral head and alteration of the signal affecting the muscle bundles of the rectus femoral muscle to refer to a contractual phenomenon and oedematous imbibition. The EMG shows marked axonal and myelin pain affecting the destroyed deep peroneal nerve associated with axonal neurogenic muscle pain affecting the anterior tibial muscles and short extensor of the external fingers in the presence of active denervation. This figure points to an important neuro-praxis damage to the SPE. Electromyography has shown a demyelinating pathology excluding the compressive form, the story has confirmed the demyelinating disease. A steroid therapy was started. We moreover set up a rehabilitation program that affected both the functional and specific dysfunctional areas. An attempt was made to recover joint widths, muscle strength, use the techniques of proprioceptive facilitation according to Kabat and cognitive exercise according to Perfetti, active and passive mobilization. In addition, efforts have been made to improve skills. The short-term result over 3 weeks is quite positive, a full recovery has been achieved over 9 months. A rare case of demyelinating pathology confined to an isolated nerve in a MS patient.

References:

- E. Viel. Il metodo kabat. Facilitazione neuromuscolare propriocettiva. Editore Marrapese. (1997)

- Puccini P. Perfetti C. Lo sviluppo del sistema funzionale della manipolazione (analisi strutturale) SIMFER 12, Riassunti, (1981)

\section{EXPLORING THE DARK SIDE OF CEREBROVASCULAR DISEASE: SUSAC'S SYNDROME}

\author{
D. Pezzini ${ }^{1}$, L. Poli ${ }^{1}$, I. Volonghi ${ }^{1}$, S. Cotti Piccinelli ${ }^{1}$, E. Gatti ${ }^{2}$, A. \\ Pezzini $^{1}$, A. Padovani ${ }^{1}$
}

\begin{abstract}
${ }^{1}$ Department of Clinical and Experimental Sciences, Neurology Clinic, University of Brescia (Brescia); ${ }^{2}$ Neuroradiology Unit, University of Brescia (Brescia)
\end{abstract}

Background: Susac's syndrome (SuS) is a rare disease characterized by an occlusive microangiopathy affecting the precapillary arterioles of the brain, retina and inner ear, presumably mediated by an underlying autoimmune mechanism. The full clinical spectrum comprises a triad of manifestations, including CNS dysfunction, multiple recurrent retinal arterial branch occlusions (BRAOs) and sensorineural hearing loss (SNHL). The average time from symptoms onset to triad completion is 21 weeks but it can take up to 10 years, with 3 distinct patterns of clinical course: monophasic, polyphasic and, rarely, chronic-continuous [1]. To date, the optimal treatment of SuS is unknown.

Case Presentation: A 40-year-old man with a 10-year history of fluctuating hypoacusia and tinnitus was hospitalized because of subacute onset of confusion. The patient appeared poorly aware of the events occurred in previous days, fatuous, distrustful and with a busy attitude, and a tangential, sometimes inconsistent uninformative speech. He reported worsening of hypoacusia, reappearance of tinnitus, and headache. Brain magnetic resonance imaging (MRI) showed multiple small lesions hyperintense on T2-weighted images and miliariform contrast enhancement in the white matter of both the supratentorial and infratentorial structures along with multifocal small hyperintense foci that involve mainly the central part of the corpus callosum (snowballs like). Cerebrospinal fluid (CSF) examination revealed an inflammatory pattern (proteins, $4395 \mathrm{mg} / \mathrm{L}$, normal range 150-450 mg/L; glucose, $58 \mathrm{mg} / \mathrm{dL}$, normal range $50-100 \mathrm{mg} / \mathrm{dL}$; cells, $13 / \mu \mathrm{L}$, normal range $<4 / \mu \mathrm{L}$ ). Screening for CNS infections was negative. Cognitive assessment revealed global impairment (MOCA 18/30). The diagnosis of SuS was made based on the coexistence of bilateral low frequency SNHL at tonal audiometric test and multiple BRAOs in peripheral sectors associated with poor peripheral ischemic phenomena at fundus fluorescein angiography (FFA). Clinical data together with neuroimaging findings were consistent with the diagnosis of definite SuS, according to Kleffner et al. [2] criteria. The patient was treated with acetyl salycilic acid 100 $\mathrm{mg}$ /day and methylprednisolone $1 \mathrm{gr} /$ day for 5 days, followed by prednisone $1 \mathrm{mg} / \mathrm{Kg} /$ day, with gradual clinical improvement.

Conclusion: Our case demonstrates that progressive SNHL over many years may precede the presentation of SuS triad. Although a few patients have spontaneous remission and no evidence-based therapeutic approach is currently available, immunosuppressive treatment is strongly recommended to prevent irreversible organ damage, leading to dementia, blindness and deafness. However, the best regimen and timing still need to be defined. Similarly, whether prophylactic treatment with anticoagulants or antiplatelet agents may be beneficial is currently unknown.

References:

1. Sauma J, Rivera D, Wu A, et al., Susac's syndrome: an update. Br J Ophtalmol (2020);10:1-6

2. Kleffner I, Dörr J, Ringelstein M, et al., Diagnostic criteria for Susac syndrome, J Neurol Neurosurg Psychiatry (2016);87:128795

\section{AUTOSOMAL DOMINANT CEREBELLAR ATAXIA WITH DEAFNESS AND NARCOLEPSY (ADCA-DN): CLINICAL AND POLYSOMNOGRAPHIC FOLLOW UP OF 4 ITALIAN KINDREDS}

\author{
E. Postiglione ${ }^{1}$, E. Antelmi ${ }^{2}$, F. Pizza ${ }^{1,3}$, E. Mignot ${ }^{4}$, G. Plazzi ${ }^{3,5}$
}

${ }^{1}$ Department of Biomedical and Neuromotor Sciences (DIBINEM), University of Bologna (Bologna); ${ }^{2}$ Neurology Unit, Movement Disorders Division, Department of Neurosciences, Biomedicine and Movement Sciences, University of Verona (Verona); ${ }^{3}$ IRCCS Istituto delle Scienze Neurologiche di Bologna (Bologna); ${ }^{4}$ Stanford University Center for Sleep Sciences, Department of Psychiatry and Behavioral Sciences, Stanford University School of Medicine (Palo Alto-USA); ${ }^{5}$ Department of Biomedical, Metabolic and Neural Sciences, University of Modena and Reggio-Emilia (Modena)

Objectives: DNMT1 gene mutations have been associated with two autosomal dominant degenerative diseases with multi systemic involvement 
including central and peripheral nervous system. Narcolepsy with cataplexy is a core feature of the ADCA-DN phenotype, however, narcolepsy has been also reported in Hereditary Sensory and Autonomic Neuropathy (HSAN 1E). We report the follow up of three Italian ADCA-DN kindreds already described adding a new Italian ADCA-DN family.

Materials and methods: Seven patients underwent repeated extensive evaluation encompassing polysomnographic investigations, auditory functions and peripheral nervous system including skin biopsy, ophthalmological studies neurophysiological examination, and cerebrospinal fluid (CSF) hypocretin-1 (hcrt-1) assay. Three patients were reviewed from clinical records.

Results: The first symptom (30\% hypoacusia; $20 \%$ excessive daytime sleepiness (EDS)/cataplexy) arose at a mean age of 31.33 years (y) (25 43). Cerebellar ataxia, neurosensorial hypoacusia, sensorial neuropathy, optical atrophy was documented in 7/10 (mean onset age 38.6 y), in 7/10 (mean onset age $33.6 \mathrm{y}$ ), in $4 / 8$, and in $3 / 7$ patients, respectively. Severe psychiatric comorbidity ( $4 / 7$ patients) and cognitive impairment (3/6 patients) appeared later (mean age of 38.8 and of $46.7 \mathrm{y}$, respectively). MSLT showed short sleep latency and multiple SOREMPs in 3 and 4 out of 7 patients, while nocturnal polysomnography (PSG) disclosed increased periodic limb movements during sleep (PLMS - 42.9\%), REM sleep behaviour disorder (RBD - 28.6\%), and reduced sleep efficiency (28.7\%) at first evaluation. CSF hort-1 levels were $233.84 \pm 7.93 \mathrm{pg} / \mathrm{mL}$, with $2 / 7$ patients around the cut-off of $110 \mathrm{pg} / \mathrm{mL}$. Follow up at $5.42 \pm 1.14$ years revealed increase in cataplexy, and reduction of sleep efficiency (clearer in younger patients), sleep figures deterioration, increased limb movements (LM), PLMS and RBD (more evident in older patients).

Discussion and conclusion: Narcoleptic symptoms are the key complaints to seek neurological consultation in ADCA-DN. Across disease course, a multifaceted deterioration of sleep included increased LM, PLMS, RBD up to a "status dissociatus". CSF hort-1 levels were variably reduced, confirming the involvement of the hypocretin system within a widespread neurodegeneration.

\section{THE SPECTRUM OF CNS INFLAMMATORY DEMYELINATING DISEASE IN CHILDREN: AN UNUSUAL CASE REPORT OF NEURO-BEHÇET'S DISEASE OCCURRING AT 2 YEARS?}

\author{
M. Pozzato ${ }^{1}$, F. Minoia ${ }^{2}$, C. Bulgaro ${ }^{3}$, A. Tozzo ${ }^{4}$, F. Andreetta ${ }^{5}$, C. \\ Bana $^{6}$, G. Filocamo ${ }^{2}$, N. Bresolin ${ }^{7}$, C. Agostoni ${ }^{2}$, S. Barbieri ${ }^{6}$, F. \\ Martinelli Boneschi ${ }^{7}$, R. Dilena ${ }^{6}$
}

\begin{abstract}
${ }^{1}$ Foundation IRCCS Ca'Granda Ospedale Maggiore Policlinico, University of Milan (Milano); ${ }^{2}$ Pediatric Unit, Fondazione IRCCS Ca' Granda Ospedale Maggiore Policlinico (Milano); ${ }^{3}$ Pediatric Unit, Fondazione IRCCS Ca' Granda Ospedale Maggiore Policlinico, University of Milan (Milano); ${ }^{4}$ Infantile Neuropsichiatry Unit, Pediatric Neuroscience Department, IRCCS Fondazione Istituto Neurologico (Milano); ${ }^{5}$ U.O. Neurology IV, Neuroimmunology Laboratory, IRCCS Fondazione Istituto Neurologico (Milano); ${ }^{6}$ Neuropathophysiology Unit, Fondazione IRCCS Ca' Granda Ospedale Maggiore Policlinico (Milano); ${ }^{7}$ Neurology Unit \& MS Centre, Dino Ferrari Centre, Neuroscience Section, Department of Pathophysiology and Transplantation, Fondazione IRCCS $\mathrm{Ca}^{\prime}$ Granda Ospedale Maggiore Policlinico, University of Milan (Milano)
\end{abstract}

Introduction: Inflammatory demyelinating diseases (IDD) are a group of clinical conditions characterized by inflammation in the central nervous system leading to a damage of the myelin. These entities can be monophasic or polyphasic/chronic [1].

Case report: We present the case of a 2-year-old girl, who four days after an episode of fever at 13 months of age presented alteration of consciousness and asymmetric tetraparesis with mixed pyramidalextrapyramidal signs. The brain MRI with gadolinium showed alterations of the thalami and white matter compatible with ADEM. She was treated with high-dose methylprednisolone and intravenous immunoglobulin (IVIg) with complete recovery. Five months later, the patient presented a difficulty in walking with pyramidal signs in the lower right limb, ataxia, gaze paralysis and vertical nystagmus. The brain and spinal cord MRI highlighted multiple supra- and infratentorial white matter lesions, some of which with gd-enhancement, and a C2-C4 spinal cord lesion without gd-enhancement. She performed a CSF analysis which resulted negative, with no evidence of oligoclonal bands, anti-MOG and antiAQP4 antibodies. Systemic autoimmune screening showed ANA and p-ANCA positivity; HLA-B51 allelic variant was present. VEP and BAER showed an increased latency in both eyes and brainstem, SEP showed increased latency in lower limbs. At a cytokine assay, we found increased CSF IL-6 values. She was re-treated with steroids and IVIg with a full recovery except for a right lower limb dystonia. Since the discharge the patient has been treated with low-dose methylprednisolone and monthly IVIg cycles. At neurological examination performed 8 months later, a worsening of the right lower limb dystonia was observed. The MRI did not show any new brain or spinal cord lesion, but it emerged an arthritis of the right temporomandibular joint. In addition, at clinical evaluation a greater stiffness in the knee and ankle was evident. Although the complexity of patient's clinical picture, the clinical-radiological data of polyarthritis and the HLA-B51 positivity support the hypothesis of a Neuro-Behçet's disease. A treatment with Colchicine $(0.5$ $\mathrm{mg}$ /daily) and Methotrexate (7.5 mg/weekly) was started, and patient's neurological condition has remained stable.

Discussion: Neuro-Behçet's disease in children is uncommon, and it is rarely diagnosed before 2-years of age. The main manifestations of the disease are cranial nerve palsy or cerebral venous thrombosis, and only in few cases brain parenchyma is involved [2]. With this case, we want to remark the complexity of the management of the IDD in pediatric age. References:

1. C. Fernandez Carbonell et al. Inflammatory demyelinating diseases in children: An update. Minerva Pediatric (2013); Jun;65(3):307-23

2. I. Konè-Paut. Behcet's disease in children, an overview. Pediatric Rheumatology (2016); Feb 18;14(1):10

\section{AN INCOMPLETE GERSTMANN SYNDROME FOLLOWING A NON-DOMINANT PARIETAL STROKE: IS THIS AN END TO THE SAGA?}

\section{F. E. Pozzi, R. Clerici, S. Brajkovic, L. Beretta, M. Guidotti}

Neurology Department, Valduce Hospital (Como)

Objectives: The association of finger agnosia, chiragnosia, agraphia and acalculia has been described by Gerstmann as a syndrome resulting from left angular gyrus lesions, which would damage a common functional denominator essential across these four cognitive faculties. Heatedly debated over a century, functional imaging findings on healthy subjects recently led to interpret such syndrome as a result of the disconnection of non-overlapping, spatially contiguous but functionally unrelated white matter tracts in the dominant parietal lobe. Here we describe a patient 
carrying a lesion that would corroborate this contemporary interpretation of Gerstmann syndrome.

Materials and methods: A 84-year-old man was admitted to the Emergency Department following an acute onset of left hemiparesis. He underwent thrombolysis with excellent motor recovery. Two days later a constellation of cognitive symptoms was observed, including finger agnosia, chiragnosia, constructional apraxia, posterior alien hand, amusia, dysexecutive symptoms, unilateral sensory extinction, anosognosia. No agraphia, acalculia, apraxia, visual defects or aphasia were present. Over the course of a week some of the symptoms disappeared, with the notable exception of persistent finger agnosia and chiragnosia, and he was discharged to a rehabilitation facility.

Results: Neuropsychological tests documented the deficits encountered during neurological examinations, while brain MRI revelead ischemic lesions in the right temporal lobe, posterior insula and posterior parietal lobe. The patient was right-handed and the temporal lesion didn't result in aphasia, hence left hemispheric dominance could be inferred. In conclusion, our patient showed two of the four Gerstmann syndrome features associated with non-dominant hemispheric stroke.

Discussion and Conclusions: Only three studies have described Gerstmann syndromes following right parietal lesions, although probably related to right dominance. Our unprecedented case shows that finger gnosis and right-to-left orientation rely dramatically on the non-dominant parietal lobe contribution, thus corroborating the hypothesis that the association of symptoms described by Gerstmann shouldn't be regarded as a syndrome, being the result of a disconnection of independent white matter tracts that happen to be in close proximity to each other in the left subcortical parietal lobe. Nothing could be inferred about agraphia and acalculia, but the spatial extent of the parietal lesion suggests that the neural networks underlying these functions are limited to the dominant hemisphere. This also implies that attempts to find a Grundstörung for these four abilities could be futile, as even the pure presentation of these four arbitrarily chosen symptoms seems to constitute a spatial, but not a functional syndrome.

References:

- Rusconi E, Pinel P, Dehaene S, Kleinschmidt A. The enigma of Gerstmann's syndrome revisited: a telling tale of the vicissitudes of neuropsychology. Brain (2010);133(Pt 2):320-32

- Calvo-Romero JM, Buero-Dacal JC, Ramos-Salado JL . Síndrome de Gerstmann causado por ictus isquémico parietal derecho. Rev Neurol (2000);30(04):398-99

- Nicastro N, Tafer N, Schnider A, Di Pietro M. Gerstmann's Syndrome Associated with Right Parietal Hemorrhage and Arteriovenous Malformation. J Clin Neurol. (2017);13(3):306-07

\section{ASYMMETRIC CORE MYOPATHY ASSOCIATED WITH RYR1 PATHOGENIC VARIANT}

G. Primiano ${ }^{1}$, F. Fattori $^{2}$, C. Sancricca ${ }^{3}$, E. Bertini ${ }^{2}$, S. Servidei ${ }^{3}$

${ }^{1}$ Institute of Neurology, Catholic University of the Sacred Heart (Roma); ${ }^{2}$ Unit of Muscular and Neurodegenerative Disorders, Bambino Gesù Children's Research Hospital (Roma); ${ }^{3}$ Institute of Neurology, Agostino Gemelli IRCCS University Hospital Foundation (Roma)

Objective: Mutations in the ryanodine receptor 1 (RYR1) gene, both with recessive and dominant patterns, were associated with different clinical conditions: malignant hyperthermia susceptibility, congenital central core, congenital fiber-type disproportion, multiminicore myopathy, centronuclear myopathy, axial myopathy, King-Denborough syndrome, atypical periodic paralysis, and rhabdomyolysis. We describe an unusual phenotype of RYR1-related core myopathy in an Italian patient.

Materials and Methods: A detailed clinical characterization was performed, including laboratory investigations, electromyography, muscle MRI and muscle biopsy. Next-generation sequencing and targeted Sanger sequencing were used to identify the causative genetic defect.

Results: A 45-year-old man born to non-consanguineous parents presented a 5-year history of progressive weakness of the proximal lower limbs. He reported normal motor development and there was no family history suggestive of neuromuscular disease. Physical examination showed severe asymmetric weakness of the quadriceps muscle (MRC score $3 / 5$ on the left side and $5 / 5$ on the right), associated with a marked hypotrophy of the left side. Muscle strength of the upper extremities was normal, and there is no evidence of ptosis or external ophthalmoplegia. CK was elevated at 1,887 IU/L. Electromyogram showed a myopathic pattern in all examined muscles, with normal nerve conduction. Muscle MRI images revealed a marked asymmetric involvement of the left quadriceps, a severe fatty degeneration bilaterally in the muscles of posterior thigh compartment with relative sparing of the rectus femoris and gracilis, and showed a moderate asymmetric involvement of the gastrocnemius medialis in the lower legs. Quadriceps muscle biopsy was characterized by cores lacking oxidative enzyme stains and genetic analysis identified a pathogenic RYR1 variant in heterozygosity previously associated with malignant hyperthermia susceptibility.

Discussion and conclusions: Our findings expand the spectrum of RYR1-related disorders, including an asymmetric core myopathy.

\section{LATE-ONSET ATAXIA TELEANGIECTASIA: A CASE MIMICKING SPINAL MUSCLE ATROPHY}

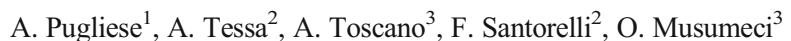

${ }^{1}$ Department of Clinical and Experimental Medicine, "G. Martino" Policlinic, University of Messina (Messina); ${ }^{2}$ Molecular Medicine, IRCCS Fondazione Stella Maris, University of Pisa (Pisa); ${ }^{3}$ Unit of Neurology and Neuromuscular Disorders, Department of Clinical and Experimental Medicine, University of Messina (Messina)

Introduction: Ataxia Teleangiectasia (AT) is an autosomal recessive disease related to AT mutated (ATM) gene mutation that is involved in the cellular response to DNA damage [1]. Classic AT presents as multisystem disorder, manifesting with progressive cerebellar ataxia, oculocutaneous telangiectasia, immunodeficiency, peripheral neuropathy and high risk for infections and malignancy leading death in the second or third decade of life [2]. A milder form of AT, characterized by a residual ATM protein activity, is recently reported.

Aim: To describe a case of variant AT with late-onset and slowly clinical progression.

Materials and methods: Our patient is a 50 year-old man, who began to complain diffuse muscle wasting and weakness with difficulty jumping, climbing stairs and staying on tiptoe at the age of 35 years old. The symptoms progressed with gait ataxia, slurred speech, head and hands' tremor. He underwent laboratory assessment, electromyography, muscle biopsy, cerebral MRI and genetic analysis.

Results: The initial clinical features of the patient raised the suspicion of Spinal Muscle Atrophy (SMA). Routine laboratory tests showed mild CK elevation (496 U/L; n.v. 28-190) and electromyography revealed fibrillation and sharp waves as spontaneous activity with denervation and reinnervation at four limbs. Genetic analysis for SMA was negative. After few years since the onset of the symptoms, neurological 
examination revealed gait ataxia with dystonic right arm posturing, slurred speech, fragmented saccades and opsoclonus, head and hands' tremor especially at left thumb, muscle atrophy and weakness at distal limbs. At finger-to-nose test, he had mild bilateral dysmetria with intentional tremor. Besides, he presented some ocular and cutaneous telangiectasia. Brain MRI evidenced moderate cerebellar atrophy. Blood laboratory tests such as autoimmunity panel, ceruloplasmin, thyroid hormones, immunoglobulins and tumor markers were normal, except for alpha-fetoprotein (101 ng/ml; n.v.0-10). Molecular analysis for ataxiarelated genes detected two ATM gene heterozygous mutations: c.6650_6657del TTAGTTTT (p.F2217fs*29) and c.8147T >C (p.V2716A).

Discussion and conclusion: We describe a case of late-onset and slowly progressive AT. The second found mutation have been already reported in literature as variant $\mathrm{AT}$, characterized by milder phenotype with predominant extrapyramidal and cerebellar signs [1]. Both patients with classic and variant AT have an increased risk of malignancy, for this reason a prompt diagnosis is fundamental to support adequately patients. References:

1. Verhagen M.M.M. et al. Clinical spectrum of ataxia-telangiectasia in adhultood. Neurology (2009) Aug 11;73(6):430-7

2. Leuzzi V. et al. Ataxia-telangiectasia. A new remitting form with a peculiar transcriptome signature. NeurolGenet (2018) Mar 27;4(2):e228

\section{TONGUE SWELLING RELATED TO CLOPIDOGREL ASSUMPTION}

R. Renna, M. Rippa, M. Napolitano, G. Alfieri, A. Ranieri, W. Di Iorio, G. Servillo, G. Maniscalco, P. Candelaresi, V. Manzo

\section{UOSC Neurology, Stroke Unit, AORN Cardarelli (Napoli)}

A 67-year-old Caucasian man presented with sudden-onset left hemiparesis. His past medical history was remarkable for hypertension and diabetes, treated with Ramipril $10 \mathrm{mg}$ and metformin $2000 \mathrm{mg}$ daily. Brain CT scan revealed an ischemic lesion in the right middle cerebral artery territory. Patient's antiplatelet therapy was switched from aspirin (100 mg daily) to clopidogrel (75 mg daily). Two days after commencement of clopidogrel patient developed painless swelling of the right hemitongue. He was successfully treated with methylprednisolone and clopidogrel was changed to aspirin ( $300 \mathrm{mg}$ daily). Clopidogrel is a widely used antiplatelet therapy. About $1 \%$ of patients have allergic or hematologic adverse reactions to clopidogrel. [1] Clopidogrel-related immunological hypersensitivity reactions are various. Cheema et al. [2] investigated 84 patients with suspected hypersensitivity reactions to clopidogrel in whom 62 cases could be confirmed. Clinical manifestation involving cutaneous symptoms can be distinguished in three different types: (1) the largest group (n. 49/62) exhibited truncal, itchy maculopapular exanthems; (2) a group of 10 patients experienced localized well-defined yet multifocal exanthems, some demonstrating a symmetrical distribution; (3) angioedema with tongue and/or lip swelling and in some cases urticaria was observed in a small number of patients (n. 3/62). Pyrexia and arthralgia were observed in two patients. The latency periods between clopidogrel intake and the onset of symptoms were on average 5 days in groups 1 and 2, and 1 day in group 3. Allergy testing showed that reactions are essentially either delayed-type cell-mediated allergic reactions or rarer allergic immediate-type reactions. The presence of crossreactivity between various P2Y12 inhibitors (ticlopidine, clopidogrel, prasugrel; ticagrelor-type) is note infrequent. In conclusion, lip or tongue swelling are exceptional reactions to clopidogrel of which the clinician must be aware.

References:

1. Lokhandwala J, Best PJ, Henry Y, Berger PB. Allergic reactions to clopidogrel and cross-reactivity to other agents. Curr Allergy Asthma Rep. (2011);11:52-7

2. Cheema AN, Mohammad A, Hong T, et al. Characterization of clopidogrel hypersensitivity reactions and management with oral steroids without clopidogrel discontinuation. J Am Coll Cardiol. (2011);58:1445-54

\section{INTRAVENOUS IMMUNOGLOBULINS (IVIGS) AS FIRST LINE THERAPY FOR IMMUNE-MEDIATED NECROTIZING MYOPATHY (IMNM): ONE YEAR FOLLOW-UP IN AN ANTI- HMGCR PATIENT}

\author{
D. Rinaldi, L. Fionda, G. Alfieri, M. Salvetti, M. Garibaldi
}

NESMOS, Sant'Andrea Hospital, Sapienza University (Roma)

Introduction: Immune-mediated necrotizing myopathies (IMNMs) are a subgroup of idiopathic inflammatory myopathy (IIM) characterized by severe and progressive proximal muscular weakness, creatin-kinase (CK) elevation and peculiar histopathological findings at muscle biopsy characterized by necrosis and regeneration of muscle fibres with minimal or absent inflammatory infiltrates. Undertreated IMNM rapidly leads to progressive fibro-fatty replacement of muscles and consequent disability. IMNMs frequently require a second or/and third line therapy to achieve the best clinical outcome. Herein we report on a 75-years-old female patient with anti-HMGCR positive IMNM successfully treated with intravenous immunoglobulin (IVIG) as first line therapy.

Case Report: The patient presented to emergency department because of progressive weakness of lower limbs for the last seven months. She suffered from cardiomyopathy, hypertension and dyslipidemia and was previously treated with statins. Laboratory exams revealed a CK elevation of $10.455 \mathrm{U} / \mathrm{I}$, and clinical examination showed severe proximal limb muscles strength. She was wheelchair bound. Electromyography showed myopathic changes with spontaneous activity and muscle biopsy was consistent with IMNM. A serum screening for principal myositis specific antibodies revealed the presence of anti-HMGCR antibodies. Muscle MRI showed mild fibro-fatty replacement in T1 sequences, with marked muscle edema/inflammation at STIR sequences. Because of age and comorbidities, IVIGs were preferred to other first line therapies and was started at $2 \mathrm{~g} / \mathrm{Kg}$ dosage admninistered in 5 days. After one month, walking was possible with the aid of a walker. A second IVIG cycle was performed after 3 months, and the patient presented a significant clinical improvement reaching walk independence. A second MRI was performed and showed marked decrease of edema. A third cycle of IVIgs was performed as maintenance therapy. At one year the patient could go upstairs and was full autonomous in activities of daily living.

Discussion and Conclusion: IVIGs represent the most effective treatment for IMNMs leading to the best clinical improvement and clinical outcome when started promptly. Even if it is still used a second or third line therapy after conventional corticosteroids and immunosuppressive agents in most centers, this report emphasizes the safety and efficacy of IVIGs as first line treatment for IMNM, supported by clinical and radiological outcome at 1 year follow-up. 


\section{CLINICAL AND NEUROPATHOLOGICAL FINDINGS IN PURE NEURITIC LEPROSY: A CLINICAL REPORT}

\author{
B. Risi ${ }^{1}$, S. Cotti Piccinelli ${ }^{1}$, T. Cavallaro ${ }^{2}$, S. Ferrari ${ }^{2}$, N. Necchini ${ }^{1}$, E. \\ Baldelli $^{1}$, A. Padovani ${ }^{1}$, M. Filosto ${ }^{1}$
}

${ }^{1}$ Center for Neuromuscular Diseases, Unit of Neurology, ASST Spedali Civili and University of Brescia (Brescia); ${ }^{2}$ UOC Neurology B, Department of Neurosciences, AOUI Verona (Verona)

Objectives: Leprous neuropathy is due to infection of nerve cells by Mycobacterium leprae and it is frequently reported in both lepromatous and tubercoloid type. We here reported clinical and neuropathological findings of a patient affected with a pure neuritic form of leprosy.

Patient and Methods: A 34-year-old Brazilian woman complained of distal sensory impairment at the four limbs for about 2 years. Neurological evaluation showed vibratory, proprioception, tactile and stocking pin-prick sensory impairment in the hands and below the knee. Ankle jerks and knee tendon reflexes were normal as well as general physical examination. No skin lesions were detected. Appropriate laboratory tests, neurophysiological examination and sural nerve biopsy were conducted in order to achieve a diagnosis.

Results: Routine laboratory tests, anti-gangliosides, anti-Borrelia Burgdorferi and anti-Treponema antibodies, immunological tests, cerebrospinal fluid examination and transthyretin gene sequencing yielded normal findings. ENG showed absence of the sural, ulnar and median SAPs. Sural nerve biopsy showed a severe damage characterized by presence of unstructured nerve bundles devoid of nerve fibers, severe inflammatory infiltration and some cells containing acid-resistant ZiehlNeelsen positive bacilli identified as Mycobacteria leprae. A treatment based on multidrug therapy was started and patient entered clinical follow-up.

Discussion: With increasing migration, cases of leprosy are becoming relatively frequent also in western countries. However, a pure neuritic form accounts only for about $5 \%$ of leprosy cases and can present with single or multiple peripheral nerve involvement. Especially in western countries, pure neuritic leprosy is a very rare and underestimate condition and only nerve biopsy can permit a correct diagnosis.

Conclusion: Since pure neuritic leprosy is a treatable disease, it must be suspected in patients from endemic areas presenting with symptoms and signs of peripheral nerve involvement, in order to start the correct therapy as quickly as possible.

\section{NEUROMUSCULAR FEATURES IN CHOREA- ACANTHOCYTOSIS: A CLINICAL REPORT}

\author{
B. Risi ${ }^{1}$, S. Cotti Piccinelli ${ }^{1}$, F. Novara ${ }^{2}$, E. Baldelli ${ }^{1}$, N. Necchini ${ }^{1}$, A. \\ Galvagni $^{1}$, R. Ciccone ${ }^{2}$, A. Padovani ${ }^{1}$, M. Filosto ${ }^{1}$
}

${ }^{1}$ Center for Neuromuscular Diseases, Unit of Neurology, ASST Spedali Civili and University of Brescia (Brescia); ${ }^{2}$ Microgenomics Laboratory (Pavia)

Objectives: Chorea-Acanthocytosis (ChAc) is a very rare autosomal recessive adult-onset neurodegenerative disorder caused by mutations in the VPS13A gene encoding for a protein called chorein which is found in various tissues throughout the body and is believed to play a role in the trafficking of proteins within cells. Core clinical features are choreiform movements affecting both trunk and extremities, orolingual dyskinesias, parkinsonism, epilepsy, behavior changes and acanthocytosis in peripheral blood smear. Some ChAc patients may develop mild clinical neuromuscular manifestations including elevated levels of creatine phosphokinase, distal muscle atrophy and weakness and areflexia. Here we report on clinical and genetic findings of a patient diagnosed with ChAc.

Patient and Methods: A 41-year-old patient complained of progressive gait impairment and dysarthria since age 38 . In the past clinical history epilepsy since age 14 and drug abuse were reported. Neurological examination showed primarily signs of neuromuscular involvement as: impaired walking on heels, weakness of thigh flexor and foot dorsal and plantar flexor muscles, upper limb-girdle and hand muscles atrophy, absent ankle jerk reflexes, hypophonia, dysarthria and dysphagia. Milder extrapyramidal signs were also noted i.e. axial and lower limb plastic rigidity, hypokinesia and hypomimia. Neurophysiological studies, muscle biopsy, brain MRI and genetic analysis were conducted to achieve a diagnosis.

Results: EMG-ENG showed widespread chronic neurogenic changes. Muscle biopsy displayed a marked non-specific myopathic pattern (variability of fiber diameters; scattered atrophic fibers; many fibers with internal nuclei; fiber splitting; one fiber necrosis; some ring and whorled fibers; rare fibers with subsarcolemmal accumulations of mitochondria and one ragged red fiber) and less prominent neurogenic changes (small fiber type grouping). Brain MRI showed atrophy and signal alterations in lenticular and caudate nuclei. Genetic study detected the homozygote frameshift mutation c. $15891595+5 \mathrm{del}$ in exon 17 of the VPS13A gene involving the splicing donor site (NP_150648.2: p.Gln529Hisfs *6).

Discussion: Chorea-Acanthocytosis is a very rare and complex autosomal recessive adult-onset neurodegenerative disorder and is difficult to diagnose. Our report shows that ChAc may present with prevalent neuromuscular clinical features due to primary myopathy and/or axonal/motor neuron chronic loss.

Conclusion: Neuromuscular involvement in association with extrapyramidal signs and epilepsy should induce the diagnostic suspicion of ChAc even in absence of significant involuntary movements.

\section{SPASMODIC DYSPHONIA INDUCED BY HYPERTHYROIDISM IN RELAPSING REMITTING MULTIPLE SCLEROSIS: A CASE REPORT}

M. Risi ${ }^{1}$, M. Esposito ${ }^{2}$, A. d'Ambrosio ${ }^{1}$, G. Bellastella ${ }^{3}$, U. Cesari ${ }^{4}$, G. Conzo $^{5}$, R. Docimo ${ }^{1}$, F. Habetswallner ${ }^{2}$, G. Tedeschi ${ }^{1}$, A. Gallo ${ }^{1}$

${ }^{1}$ I Division of Neurology, AOU Policlinico Luigi Vanvitelli, University of Campania "Luigi Vanvitelli" (Napoli); ${ }^{2}$ Clinical Neurophysiology Unit, Cardarelli Hospital (Napoli); ${ }^{3}$ Division of Endocrinology and Metabolic Diseases, Department of Advanced Medical and Surgical Sciences, University of Campania "Luigi Vanvitelli" (Napoli); ${ }^{4}$ Department of Otorhinolaryngology, University Hospital (Policlinico) Federico II (Napoli); ${ }^{5}$ Division of General and Oncologic Surgery, Department of Cardiothoracic Sciences, University of Campania "Luigi Vanvitelli" (Napoli)

Introduction: Spasmodic dysphonia (SD), a rare disorder characterized by intermittent spasm of laryngeal muscles, has been seldom reported in Multiple Sclerosis (MS) patients.

Case report: A 33 years-old Relapsing Remitting MS (RR-MS) patient was admitted to our Neurology Unit for the onset of voice breaks during speaking. He received MS diagnosis in 2018, when - because of the severity and activity of the disease - he was treated with Alemtuzumab (ALZ; first cycle on May 2018, second cycle on June 2019). During the 
monthly monitoring of ALZ, in May 2018, the patient developed a subacute Graves' disease that was poorly controlled by methimazole $10 \mathrm{mg}$ per day. At that time, his neurological examination was characterized by: 1) hyperreflexia in upper and lower limbs, 2) mild weakness in proximal muscles of left lower limb, 3) deep sensory loss in lower limbs, 4) proximal action tremor in the left arm (possibly related to homolateral rednucleus demyelinating lesion); 4) Expanded Disability Status Scale (EDSS) of 2. The latest neurological examination (November 2019) was enriched only by effortful speech with vocal tremor. Blood analysis revealed normal thyroid function tests while on $20 \mathrm{mg}$ of methimazole per day. His brain MRI showed an extensive but stable white matter lesion load (WMLL) compared to the previous MRI, without any pathological enhancement after gadolinium administration. Laryngoscopy showed frequent vocal cord spasms in adduction compatible with a SD and botulin toxin A (2.5 onabotulinumtoxin A per thyroaritenoid muscle by EMG guidance) was administered with benefit. On February 2020, given the conspicuous enlargement of the Graves-associated goiter, some swallowing and breathing difficulties appeared. The diagnostic assessment (chest x-ray, neck CT, thyroids hormones) revealed increased fT4 level and decreased TSH level with elevated AbTG, AbTPO, AbTR, with increased thyroid volumes and signs of tracheal compression. The patient underwent a surgical complete thyroidectomy followed by a hormonal replacement therapy, with complete disappearance of SD.

Discussion: In this case-report, we describe, for the first time, a SD associated to hyperthyroidism and goiter in a RRMS patient treated with Alemtuzumab. SD was efficiently treated by means of synergic effects of botulin toxin A and thyroidectomy. This report expand previous finding of botulin toxin A efficacy in SD and highlights the relevance of thyroid investigations for the correct diagnosis and treatment.

References:

- Di Stadio A, Bernitsas E, Restivo DA, Alfonsi E, Marchese-Ragona R. Spasmodic Dysphonia in Multiple Sclerosis treatment with Botulin Toxin A: A pilot study. J Voice. (2019);33(4):550-53

- Mor N, Simonyan K, Blitzer A. Central voice production and pathophysiology of spasmodic dysphonia. Laryngoscope (2018);128(1):177-83

- van Esch BF, Wegner I, Stegeman I, Grolman W. Effect of Botulinum Toxin and surgery among spasmodic Dysphonia patients. Otolaryngol Head Neck Surg. (2017);156(2):238-54

\section{IMPACT OF DIMETHYLFUMARATE ON SLEEP IN MULTIPLE SCLEROSIS PATIENTS: AN ACTIGRAPHIC STUDY}

\author{
C. Rocchi ${ }^{1}$, L. Lombardi ${ }^{2}$, V. Totaro ${ }^{1}$, M. Danni ${ }^{1}$, L. Falsetti ${ }^{3}$, G. \\ Viticchi ${ }^{1}$, M. Bartolini ${ }^{1}$, M. Silvestrini ${ }^{1}$, L. Buratti ${ }^{1}$
}

${ }^{1}$ Neurological Clinic, Marche Polytechnic University (Ancona); ${ }^{2}$ Marche Polytechnic University (Ancona); ${ }^{3}$ Internal and Subintensive Medicine, Ospedali Riuniti (Ancona)

Objective: Sleep disorders in multiple sclerosis (MS) patients are common and complex. Dimethylfumarate is an oral disease modifying drug (DMT), whose impact on sleep is not known. The aim of this study was to evaluate and characterize actigraphic patterns in MS patients treated with dimethylfumarate.

Material and Methods: Twenty relapsing remitting MS patients with low to mild disability (Expanded Disability Status Scale $<2.5$ ), aged 20$50 \mathrm{y}$, treated with dimethylfumarate for more than 6 months were enrolled. All subjects had no history of sleep disorders. Actigraphy was used to study sleep pattern in a seven-day period. Sleep quality was assessed by the Pittsburgh Sleep Quality Index (PSQI). Twenty sex-and age-matched healthy subjects served as controls.

Results: Our results showed no statistically significant differences between actigraphic patterns in MS patients treated with dimethylfumarate and healthy subjects. PSQI score was higher in MS patients but this result was not statistically significant.

Discussion: Our findings suggest that dimethylfumarate, an oral DMT with a strong persistent efficacy and a favourable benefit-risk profile, does not significantly alter sleep patterns in MS patients with no history of sleep disorders.

Conclusions: Our results suggest that dimethylfumarate does not alter sleep architecture. Given the importance of sleep in MS patients, considering the impact of DMTs on sleep could help preserve good sleep quality. Actigraphy is a simple diagnostic tool, able to support an objective measure of sleep parameters. The relative simplicity of application and low costs may allow considering its use for an adequate screening of sleep disorders in MS patients.

References:

- Lanza G, Ferri R, Bella R, Ferini-Strambi L. The impact of drugs for multiple sclerosis on sleep. Mult Scler (2017);23:5-13

- Buratti L., Iacobucci D.E., Viticchi G., Falsetti L., Lattanzi S., Pulcini A., Silvestrini M.,Sleep quality can influence the outcome of patients with multiple sclerosis. Sleep Medicine (2019);58:56-60 ISSN 1389-9457

- Montes Diaz G, Hupperts R, Fraussen J, Somers V. Dimethyl fumarate treatment in multiple sclerosis: Recent advances in clinical and immunological studies. Autoimmun Rev. 2018;17(12):1240-50

\section{NEW-ONSET REFRACTORY STATUS EPILEPTICUS (NORSE) IN POST SARS-COV-2 AUTOIMMUNE ENCEPHALITIS: A CASE REPORT}

\author{
D. Rodorigo ${ }^{1}$, F. Dono ${ }^{1}$, C. Carrarini ${ }^{1}$, M. Russo ${ }^{2}$, M. De Angelis ${ }^{2}, \mathrm{~F}$. \\ Anzellotti $^{3}$, L. Bonanni ${ }^{1}$
}

${ }^{1}$ Department of Neuroscience, Imaging and Clinical Science, University "G. D’Annunzio" (Chieti); ${ }^{2}$ Neurology Unit, "SS Annunziata" University Hospital (Chieti); ${ }^{3}$ Epilepsy Center, University “G. D’Annunzio" (Chieti)

Background: COVID-19 pandemic caused by the novel coronavirus (SARS-CoV-2) represents one of the largest emergencies that humanity has been dealing with since the last century. Several reports indicate that SARS-CoV-2 infection can be associated with acute neurological manifestations including encephalitis. Currently, little is known about the possible neurological sequelae of the infection. In this report, we describe a case of post-SARS-CoV-2 autoimmune encephalitis presenting as newonset refractory status epilepticus (NORSE).

Case presentation: On May 12th an 81-year-old man presenting persistent fever, mild dyspnea and dry coughing was admitted. Computed tomography scan of the lungs showed a ground-glass pattern. Oropharyngeal and nasopharyngeal swabs were positive for SARSCoV-2 infection.

Clinical course: The patient was empirically treated with azithromycin with resolution of the symptoms and negative swabs in 14 days. Since then, his neurological conditions started deteriorating with confusion and disorientation. After a first electroencephalogram (EEG) without evidence of epileptiform abnormalities, the patient became drowsy with Glasgow coma scale (GCS) of 5 and started to present frequent jerky myoclonic contractions of the abdomen and the right lower limb. 
Therefore, a second EEG was recorded, showing 2-2.5 Hz lateralized periodic discharges plus superimposed fast activity and leading to a diagnosis of Non-Convulsive Status Epilepticus (NCSE) with coma according to Salzburg criteria. Attempts of treatment were made using intravenous (i.v.) boluses of Lorazepam and then Levetiracetam and Perampanel, but after 48 hours myoclonic contractions reappeared. Therefore, the patient was treated with general anesthesia with midazolam and anti-seizure medication treatments with i.v. Valproate and Lacosamide, obtaining seizure control. After anesthesia interruption, recurrence of EEG abnormalities and jerks were observed, supporting the diagnosis of NORSE. Polymerase Chain Reaction (PCR) for several viruses in the cerebrospinal fluid was negative. Despite the autoimmune panel for encephalitis was negative, a diagnosis of possible autoimmune encephalitis was made according to Graus criteria. The patient started to be treated with steroids and i.v. immunoglobulins for 5 days.

Outcome: On day 32, EEG recording showed no seizure recurrence, only interictal epileptiform abnormalities. Five days later, the patient showed a GCS of 6 .

Discussion: The strict correlation among SARS-CoV-2 infection, neurological symptoms onset and negative PCR for other viruses suggests a direct causative role of the previous SARS-CoV-2 infection in the development of post-acute autoimmune response, dramatically appeared with NORSE, whose outcome is frequently fatal.

Conclusion: Our report confirms the importance of evaluating neurological sequelae in post-acute phase of SARS-CoV-2 infection.

References:

- Ellul MA, Benjamin L, Singh B, et al. Neurological associations of COVID-19 [published online ahead of print, $2020 \mathrm{Jul}$ 2]. Lancet Neurol. (2020);S1474-4422(20)30221-0

- Gaspard N, Foreman BP, Alvarez V, et al. New-onset refractory status epilepticus: Etiology, clinical features, and outcome. Neurology (2015);85(18):1604-13

\section{PRE-ICTAL MIGRAINE IN A CASE OF CERVICOCRANIAL FIBROMUSCULAR DYSPLASIA}

\author{
M. Romozzi ${ }^{1}$, E. Rollo ${ }^{1}$, P. Calabresi ${ }^{1}$, C. Vollono ${ }^{2}$ \\ ${ }^{1}$ Institute of Neurology, Catholic University of Sacred Heart (Roma); \\ ${ }^{2}$ Institute of Neurophysiopathology, Fondazione Policlinico \\ Universitario A. Gemelli IRCCS (Roma)
}

Objectives: Fibromuscular dysplasia (FMD) is a rare vascular disease that affects small- and medium-sized arteries, most commonly renal and cerebral arteries. Cerebral vessels are involved in 25-30\% of the cases [1]. We describe the case of a woman who came to our observation for incoming seizures triggered by a migraine attack.

Materials and Methods: Clinical, neuroradiologic and neurophysiological observations were performed, including CT scan, brain and spine angiographic-MRI, perfusional MRI, brain selective angiography, videoEEG and polygraphic recordings.

Results: A 46 years-old woman was admitted to our Emergency Department for incoming seizures. Ten years earlier, the patient started experiencing episodes of typical migraine systematically followed by a 2minute generalized tonic-clonic seizure, beginning about 20 to $50 \mathrm{mi}-$ nutes after the onset of headache. Antiepileptic therapy with phenobarbital and valproic acid was started. The patient continued to experience frequently focal motor seizures with secondary generalization, accompanied by transient impairment of consciousness. Few months later, the patient voluntarily discontinued the therapy. Other pharmacological treatments, alone or combined, were tried without complete control of the seizures. At the time of our examination, the patient was in post-ictal state, presenting drowsiness and confusion. The patient had a mild left hemiparesis, which progressively resolved in the following 2 hours. The CT scan performed at the Emergency Department showed no parenchymal abnormalities but revealed several vascular malformations. Brain and spine angiographic-MRI scan and Brain selective angiography were consistent with the cervicocranial FMD. Perfusional MRI scan showed a clear increased Mean Transit Time (MTT) in right temporal lobe with a concomitant reduction of the regional Cerebral Blood Volume (rCBV) when compared with contralateral homologous region. EEG showed intermittent, irregular focal theta slowing in temporal regions and occasional right sharp waves were present. Therapy was reintroduced with valproic acid and it was effective in reducing the frequency and severity of both seizures and headache attacks. The embolization of the vascular malformations could not be performed because of the tortuosity of vessels. Discussion FMD is vascular disease that involves small and medium sized arteries. In more of the $70 \%$ of the cases there is the involvement of extracranial arteries [2]. In our patient, the vascular malformations affected exclusively intracranial arteries. Numerous and complexes intracranial arterial malformation could lead a mild chronic hypoperfusion that can be a substrate that predisposes to seizures and migraine [3].

Conclusions: The close association between seizures and headache attacks permits to hypothesize an underlying common pathogenic mechanism for both of them.

References:

1. Plouin PF, Baguet JP, Thony F, et al. High prevalence of multiple arterial bed lesions in patients with fibromuscular dysplasia: the ARCADIA Registry. Hypertension (2017) Sep;70(3):652-58

2. Olin JW, Froehlich J, Gu X, Bacharach JM et al. The United States Registry for Fibromuscular Dysplasia: results in the first 447 patients. Circulation (2012);125:3182-90

3. Rogawski MA. Common pathophysiologic mechanisms in migraine and epilepsy. Arch Neurol. (2008);65(6):709-14

\section{THE MASQUERADE SYNDROME: A CASE OF VITREORETINAL LYMPHOMA MIMICKING POSTERIOR UVEITIS WITH CENTRAL NERVOUS SYSTEM INVOLVEMENT}

\author{
F. Rossato ${ }^{1}$, S. Crivellari ${ }^{1}$, A. Cagnin ${ }^{2}$, M. Corbetta ${ }^{2}$ \\ ${ }^{1}$ Neurology Clinic, Azienda Ospedaliera Universitaria of Padua \\ (Padova); ${ }^{2}$ Department of Neurosciences, University of Padua (Padova)
}

Background: Masquerade syndrome is characterized by symptoms and ophthalmological findings of chronic posterior uveitis and is caused by non-infectious diseases. Neoplastic etiology accounts for majority of cases, requiring a prompt diagnosis for prognosis modification.

Case Report: A 71-year-old lady was admitted for right hand clumsiness, blurred vision in the visual field of the right-eye, subjective loss of memory and confusion. In her past medical history she had two previous episodes of loss of sight in the left eye 9 and 6 months earlier, for which an inflammatory etiology was suspected and immunomodulant therapy with steroids was started with benefit. Previous brain MRI scans were negative in both occasions. At admission, neurological examinations revealed spatio-temporal disorientation, right hand apraxia, blurred vision (especially right superotemporal quadrant) without campimetric deficit, dyscromatopsia, optic apraxia. The ophtalmological evaluation showed 
reduced acuity in both eyes ( $\mathrm{R} 5 / 10, \mathrm{~L} 7 / 10)$, mild cellularity in the right eye, with bilateral normal fundus, nasal superior scotoma in the left eye. Fluorescin angiography revealed bilateral patches of choroidal hyperfluorescence and OCT showed bilateral focal increase of RNFL thickness. An electroretinogram resulted in a selective global rod dysfunction, without electronegative pattern. Brain MRI showed a subcortical left parietal lesion with uneven core signal with positive SWI signal, ring enhancement and marked perilesional edema, reduced rCBV and overshooting in perfusional study. Other two lesions with different imaging features (T2-w positive and without contrast enhancement) were present in the right putamen and left caudate. The FDG-PET/MR scan revealed hypermetabolism of the left parietal lesion with perilesional hypometabolism; no abnormal foci of extracerebral hypermetabolism were observed. Total body CT and mammography did not find any lesion suspicious for neoplastic origin. CSF analysis was normal. CSF and blood microbiological studies were negative for CMV, VZV, HIV1/2, TBE, B. burgdorferi, Toxoplasma, JCV, M. tuberculosis, T. pallidum. Search of MYD88 L265P mutation in CSF was negative. Vitreal cytopathology confirmed B cell proliferation.

Discussion: The causes of retinopathies mimicking a posterior uveitis are often difficult to diagnose. Vitreoretinal lymphoma is one treatable cause of masquerade syndrome and it takes an average of one year between symptoms onset and diagnosis. Most patients with vitreoretinal lymphoma will develop CNS involvement with lymphoma [1]. Since ocular symptoms were antecedent cerebral involvement, a putative diagnosis of vitreoretinal lymphoma with secondary CNS localization was made. Vitreal cytopathology and himmunoistochemistry remain the goldstandard for diagnosis this rare and treatable condition.

Reference:

1. J. S. Takhar, T. A. Doan, J. A. Gonzales Primary vitreoretinal lymphoma: empowering our clinical suspicion. Curr Opin Ophtalmology (2019);30:491-99

\section{WHOLE GENOME SEQUENCING IN A PAIR OF SIBLINGS AFFECTED WITH DUCHENNE MUSCULAR DYSTROPHY WITH DISCORDANT COGNITIVE PHENOTYPE}

D. Sabbatini ${ }^{1}$, M. Alexander ${ }^{2}$, S. Vianello ${ }^{1}$, A. Fusto ${ }^{1}$, B. Merlo ${ }^{1}$, M. Villa $^{1}$, V. Zangaro ${ }^{1}$, F. De Pascale ${ }^{3}$, G. Sorarù ${ }^{1}$, E. Pegoraro ${ }^{1}$, L. Bello ${ }^{1}$

\footnotetext{
${ }^{1}$ Neuromuscular Center, Department of Neurosciences DNS, University of Padua (Padova); ${ }^{2}$ Department of Pediatrics, University of Alabama at Birmingham (Birmingham-U.S.A.); ${ }^{3}$ Department of Biology, University of Padua (Padova)
}

Whole genome sequencing in a pair of siblings affected with Duchenne muscular dystrophy with discordant cognitive phenotype.

Aims: Central nervous system (CNS) involvement in dystrophinopathies is variable, although loosely associated with mutations in the C-terminal domains of dystrophin. We aimed to identify candidate variants involved in the modulation of CNS phenotype in dystrophinopathies, by performing whole genome sequencing (WGS) in a pair of Duchenne muscular dystrophy (DMD) siblings with discordant cognitive phenotype and filtering variants with a dedicated bioinformatic algorithm.

Materials (patients): Patient 1 was born of healthy, nonconsanguineous parents. He was diagnosed with DMD at the age of 4 years (deletion DMD exons 45-52), and lost ambulation at the age of 11. He had no speech delay nor intellectual disabilities, and came close to a University degree in Biotechnologies. He had no behavioural nor relational issues. He is currently 29 , ventilated continuously through a tracheostomy and fed by percutaneous gastrostomy. Patient 2 , his younger brother, was diagnosed with DMD due to the same mutation, and lost ambulation at the age of 12 (currently 14 y.o.). However, he presented severe speech delay, learning disabilities, and an autism-spectrum disorder (ASD) with behavioral issues requiring neuroleptics.

Methods: We performed WGS using DNA extracted from peripheral blood of Patient 1 and 2 and their parents. Structural Variants were analyzed with Breakdancer algorithm1, while indel and SNPs (single nucleotide polymorphisms) were filtered exploiting our own Python scripts in according to the following criteria: not shared between the two siblings; frequency $<5 \%$ in European population and considering function of genes involved.

Results and Discussion: WGS confirmed identical DMD deletion breakpoints in both brothers and their mother. The bioinformatic algorithm filtered a "short list" of 41 variants situated in 36 loci, reviewed individually for potential pathogenetic mechanisms and previous reports of involvement in neurodevelopmental phenotypes. The most notable variants were rs 112339619 , a G>A single SNP upstream of the ASD risk gene ANK3, previously reported in a WGS study of ASD twins 2; and rs117696080, an intronic variant in NRXN3, encoding neurexin 3, a dystrophin-associated glycoprotein (DAPG), and also identified in the same ASD twin study. Patient 2 was heterozygous for both these variants, while patient 1 had a wild-type genotype at both loci.

Conclusions: Identified variants represent putative modifiers of the cognitive phenotype of DMD. We plan to validate these findings by sequencing a larger cohort of dystrophinopathy patients with and without cognitive issues.

References:

- Fan X, Abbott TE, Larson D, Chen K. Break Dancer: Identification of genomic structural variation from paired-end read mapping. Curr Protoc Bioinforma. (2014); 45:15.6.1-11

- McKenna B, Koomar T, Vervier K, Kremsreiter J, Michaelson JJ. Whole-genome sequencing in a family with twin boys with autism and intellectual disability suggests multimodal polygenic risk. Cold Spring Harb Mol Case Stud. (2018); 4(6):a003285

\section{SLOWLY PROGRESSIVE PURE WORD DEAFNESS IN ALZHEIMER'S DISEASE: A CASE REPORT}

S. Salemme ${ }^{1}$, L. Fiondella ${ }^{2}$, C. Carbone ${ }^{2}$, G. Vinceti $^{2}$, M. Molinari ${ }^{2}$, F. Benuzzi $^{2}$, M. Malagoli ${ }^{2}$, A. Chiari ${ }^{2}$, G. Zamboni ${ }^{2}$

${ }^{1}$ Department of Biomedical, Metabolic and Neurological Sciences, University of Modena and Reggio Emilia (Modena); ${ }^{2}$ Integrated Department of Neuroscience, University of Modena and Reggio Emilia (Modena)

Objective: Pure word deafness (PWD) has been mainly described in stroke literature. Here we detail the clinical, laboratory, neuropsychological, and neuroimaging findings of a patient who presented with a slowly progressive form of PWD.

Methods: A 59-year-old right-handed man who presented to our Cognitive Neurology Clinic because of slowly progressive wordfinding difficulties underwent extensive assessment including neurological examination, comprehensive blood test panel, lumbar puncture, neuropsychological evaluation, brainstem auditory evoked potentials (BAEP), brain MRI and 18FDG-PET. Neurological examination, 
neuropsychological evaluation and bran MRI were repeated at two years from baseline.

Case description: Baseline assessment revealed difficulty in comprehending speech sounds in spite of a relatively preserved comprehension of non-speech sounds. Written comprehension and production were preserved. Language was fluent and informative but with frequent phonemic and semantic paraphasias. He could repeat single words but not non-words or sentences. He showed difficulties in the copy of the ReyOsterrieth figure. Memory, executive functions, insight and judgement were intact. BAEP were normal. Brain MRI showed bilateral atrophy in the lateral portion of the temporal and parietal lobes with relative sparing of the hippocampus and temporo-medial structures. There were no focal lesions. Brain 18FDG-PET showed glucose hypometabolism in the lateral parietal and lateral temporal lobes, more extended on the right side, where it also involved the temporal pole. Genetic testing showed E3/E3 ApoE. Lumbar puncture showed increased t-tau $(436 \mathrm{pg} / \mathrm{mL})$ and $\mathrm{p}$-tau $(79.1 \mathrm{pg} / \mathrm{mL})$, and decreased amyloid $\beta 1-42(384 \mathrm{pg} / \mathrm{mL})$ and $\beta 1-42 / \beta 1-$ 40 ratio $(0.040)$. At 2 years from baseline, neurologic examination revealed a severe speech comprehension deficit, with impaired comprehension of even the simplest orders. MMSE administered in written form was $28 / 30$, while it decreased to $11 / 30$ if administered orally. Neuropsychological assessment showed a progression of the speech sounds comprehension deficit and mild impairment of the visuospatial and deductive reasoning functions.

Discussion and Conclusion: We report a case of PWD due to Alzheimer's disease. The few cases of slowly progressive PWD reported in the literature were usually due to neurodegenerative disease of the frontotemporal dementia spectrum. To our knowledge, only one case1 was associated with $\mathrm{AD}$ pathology, as demonstrated by positive amyloid imaging. Atypical presentations of $\mathrm{AD}$ including the logopenic variant of primary progressive aphasia and posterior cortical atrophy have been largely reported and are known to occur more frequently in young subjects. The present case shows that the presence of PWD associated with predominantly temporal atrophy/hypometabolism should prompt consideration of a diagnosis of $\mathrm{AD}$.

Reference:

- S.H. Kim, M.K. Suh, S.W. Seo, et al., Pure Word Deafness in a Patient with Early-Onset Alzheimer's Disease: An Unusual Presentation. J Clin Neurol. (2011);7(4):227-30

\section{GUILLAIN BARRÈ SYNDROME CHAMELEON: GADOLINIUM MAGNETIC RESONANCE HELPS IN EARLY DIAGNOSIS}

\author{
A. Salvalaggio ${ }^{1}$, F. Castellani ${ }^{1}$, M. Anglani ${ }^{2}$, M. Campagnolo ${ }^{1}$, R. \\ Manara $^{2}$, M. Corbetta ${ }^{1}$, C. Briani ${ }^{1}$ \\ ${ }^{1}$ Neurology Unit, Department of Neuroscience, University of Padova \\ (Padova); ${ }^{2}$ Neuroradiology Unit, University Hospital of Padova (Padova)
}

Introduction: Chameleons or atypical presentations of Guillain Barré syndrome (GBS)-related disorders may be a challenge in acute clinical setting when ruling out alternative diagnosis is mandatory and when electrophysiology and cerebrospinal fluid (CSF) examination may still be negative. We report on a 38 -year-old man with BGBS in whom a brainstem stroke was initially suspected.

Methods: The patient came to our attention for numbness and motor impairment at left-side of the face, dysgeusia and weakness at right lower limb. Neurological examination, brain and spine gadolinium MR, electrophysiological study and CSF examination were performed within three days from the onset of symptoms.
Results: Neurological examination revealed hypophonia, weakness at lower limbs, prominent on the right side (3/5 MRC in proximal and distal muscles on the right, $4 / 5$ on the left), distal paresthesias at feet and left peripheral facial nerve palsy. Achilles tendon reflexes were absent. Brain MR with gadolinium revealed left V and VII cranial nerves enhancement, and spine MR showed enhancement of cauda equina roots. CSF analysis and electrophysiological study were unremarkable the day after the onset. The patient was diagnosed with GBS and treated with intravenous immunoglobulin $(2 \mathrm{~g} / \mathrm{kg})$ with prompt and complete recovery.

Discussion: The present case shows that in acute stage of atypical GBS, when electrophysiological study and CSF are still negative, MR may be crucial for confirming the clinical diagnosis. Notably, the trigeminal involvement has rarely been reported in GBS.

\section{PRIMARY DIFFUSE MENINGEAL MELANOMATOSIS: A CASE REPORT}

S. Salvatore ${ }^{1}$, M. Napolitano ${ }^{1}$, S. Barbato ${ }^{1}$, K. Longo ${ }^{1}$, R. Renna ${ }^{1}$, W. Di Iorio $^{1}$, G. Alfieri ${ }^{1}$, G. Iannuzzo ${ }^{2}$, A. Boscaino ${ }^{3}$, V. Manzo ${ }^{1}$

${ }^{1}$ Neurology and Stroke Unit, AORN Cardarelli (Napoli); ${ }^{2}$ Neurosurgery, AORN Cardarelli (Napoli); ${ }^{3}$ Pathological Anatomy, AORN Cardarelli (Napoli)

Background: Primary diffuse meningeal melanomatosis (PDMM) is a very rare neuropathological entity, defined as a primary spreading of melanocytes in the meninges sometimes associated with infiltration of the brain parenchima. Patients have variable signs and symptoms including seizures, psychiatric disturbances, cranial nerve palsies and intracranial pressure headache. The histological examination allows a certain diagnosis, recognizing polygonal neoplastic melanocytes and fibrosis of the leptomeninges. The brain computed tomography $(\mathrm{CT})$ and magnetic resonance image (MRI) show widespread thickening of the leptomeninges, with diffuse post-contrast enhancement without nodular mass. Herein we describe the case of a 44-year-old with clinical signs of endocranial hypertension, partially responsive to corticosteroids and evidence in brain stereotaxic biopsy of melanocytic encephalic pigmentation without clear evidence of malignant cells.

Case report: A 44-year old man was admitted to the hospital after two days of headaches, vomiting, progressive altered mental status with restlessness and agitation. In the last 2 years, he presented five similar episodes of unexplained aseptic meningitis, responsive to corticosteroids. The general examination was normal, in particular there were no neurocutaneous markers or other skin lesions. Ocular fundus examination revealed bilateral papilledema. MRI revealed multiple, branching, and linear high signal intensity lesions along sulcal spaces of bilateral cerebral hemispheres and spinal cord surface. Post-gadolinium T-WI brain MRI showed diffuse enhancement along the spinal subaracnoid space; on the T1-weighted image (WI), low signal intensity lesions on the T2-WI. Spinal tapping for cytological examination of cerebrospinal fluid (CSF) rule out hemorrhage and infectious diseases. The cytological examination of CSF did not isolate pathological cells. The whole body positron emission tomography CT (PET-CT) showed no abnormal findings. A stereotaxic brain biopsy was performed without complications revealing a diffuse melanocytic encephalic pigmentation without clear evidence of malignant cells. Seven days after, the patient died of the evolution of his illness.

Discussion: PDMM is rare and has a poor prognosis; it is more common in adults (fourth decade of life) and is considered to be a meningeal variant of malignant primary melanoma. PDMM can histologically mimic a variety of other lesions, including brain metastases from malignant 
melanoma in the body. The widespread growth of this neoplasm, as opposed to the localized lesions found in metastatic melanoma and the absence of malignant melanoma elsewhere in the body helps to make the diagnosis. In our case the radiological findings together with progressive neurological deterioration, strongly suggested diagnostic hypothesis of a diffuse malignant melanocytic brain neoplasm.

References:

- Bhatia R, Kataria V, Vibha D, et al. Mystery Case: Neurocutaneous melanosis with diffuse leptomeningeal malignant melanoma in an adult. Neurology (2016);86(8):e75-e79

- Smith AB, Rushing EJ, Smirniotopoulos JG. Pigmented lesions of the central nervous system: radiologic-pathologic correlation. Radiographics (2009);29(5):1503-24. doi:10.1148/rg.295095109

\section{COVID-19 INFECTION 4 MONTHS AFTER THE II CYCLE OF ALEMTUZUMAB IN A MULTIPLE SCLEROSIS PATIENT: MILDER DISEASE SYMPTOMS IN COMPARISON WITH COINFECTED RELATIVES}

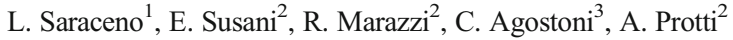

${ }^{1}$ Department of Neurology and Stroke Unit, Niguarda Cà Granda Hospital, University of Milan (Milano); ${ }^{2}$ Department of Neurosciences, Multiple Sclerosis Center, Grande Ospedale Metropolitano Niguarda Hospital (Milano); ${ }^{3}$ Department of Neurosciences, Grande Ospedale Metropolitano Niguarda Hospital (Milano)

Introduction: The Covid-19 pandemic in 2020 opened an unexpected scenario for neurologists treating Multiple Sclerosis (MS) [1]. As well as age, obesity, pulmonary diseases and other comorbidities, also immunodeficiency and, particularly, lymphopenia, could represent risk factors for infectious disease severity [2]. For this reason administration of lymphodepleting disease modifying treatments as Alemtuzumab, Cladribine and Ocrelizumab have usually been delayed or rescheduled after the high risk months for Covid-19.

Case report: In September 2018, two months after a cervical myelitis, a 28-year-old woman received the diagnosis of MS on the basis of her clinical history, magnetic resonance imaging (MRI) studies and oligoclonal bands intrathecal synthesis (McDonald 2017 criteria). Due to the following aggressive disease course, she was treated with Alemtuzumab at the end of November 2018 and twelve months later. No relapses or MRI disease activity were reported after Alemtuzumab starting. She showed clinical improvement and reduction of her Expanded Disability Status Scale (EDSS) from 2.5 to 1.0. In March 2020, four months after the second Alemtuzumab cycle, while she was leukopenic and lymphopenic (white cells 2.73x109/L; lymphocytes 630 cell/microL; CD3+ 408 cell/microL; CD19+ 119 cell $/$ microL, CD4+/CD8+0.53) her whole family was, in the same days, coinfected by Covid-19. Swabs and serological tests were positive in all four family members. Her 69-years-old father was hospitalized for two weeks due to a bilateral interstitial pneumonia causing acute respiratory failure. He had no pre-existing comorbidities. Her 67-years-old mother presented hyperpyrexia $\left(38.5^{\circ} \mathrm{C}\right)$, dysgeusia, hyposmia and widespread muscle pain for a week. Her 25-year-old partner had hyperpyrexia up to $39^{\circ} \mathrm{C}$ for 4 days, cough, chest pain, ageusia, hyposmia, widespread muscle pain and extreme fatigue. Our 30-years-old MS patient presented only mild cough, mild retrosternal chest pain, ageusia and hyposmia for 3-4 days, without even hyperpyrexia. Brain and spinal cord MRI in July 2020 showed again stable lesion load.
Conclusions: We report the case of a whole family affected by Covid19. Our MS patient presented a mild coronavirus infection despite leukopenia and lymphopenia following the second cycle of alemtuzumab. Not only she had no Covid-related complications, but it is remarkable to report that she also presented milder Covid-related signs and symptoms as compared to her coinfected relatives (father, mother, partner). It is possible that a moderate immunosoppression, as the one in our patient, could reduce the cytokine storm syndrome secondary to Covid infection. However, further studies are necessary to understand the relation between coronavirus, immune system and immunosoppressive drugs.

References:

1. M. P. Sormani, Italian Study Group on COVID-19 infection in multiple sclerosis. An Italian programme for COVID-19 infection in multiple sclerosis. Lancet Neurol. (2020) Jun;19(6):481-482. doi: 10.1016/S1474-4422(20)30147-2. Epub 2020 Apr 30. DOI: 10.1016/S1474-4422(20)30147-2

2. Fei Zhou, Ting Yu, Ronghui Du, et al. Clinical course and risk factors for mortality of adult inpatients with COVID-19 in Wuhan, China: a retrospective cohort study Lancet (2020) Mar 28;395(10229):1054-1062. doi: 10.1016/S0140-6736(20)305663. Epub 2020 Mar

\section{PRIMARY CNS VASCULITIS IN A PATIENT WITH ANTI YO POSITIVITY: PARANEOPLASTIC MANIFESTATION OR CONCOMITANT DISEASES?}

M. M. Sechi, M. Melis, V. Melas, T. Ercoli, V. Pierri, G. Cadeddu, G. Floris, M. Mascia, P. Solla, G. Defazio

Institute of Neurology, Department of Public Health and Medical Sciences, University of Cagliari (Cagliari)

Background and aims: Primary CNS angiitis (PACNS) is a rare disease (2.4 cases per 1.000 .000 person- years) with a $2: 1$ male predominance and a median age at diagnosis of 50 years. PACNS reflects characterized by infiammation and destruction of the small and medium blood vessels of the brain, spinal cord and meninges, leading to focal CNS symptoms/signs. The etiology of PACNS is unknown, but an autoimmune phatophisiology has been suggested. This case report raises the possibility that an immune response triggered by cancer cell proteins leads to PACNS, suggesting a phatogenic scenario similar to paraneoplastic syndrome $[1,2]$.

Materials and methods: A 74 y.o woman was admitted to the Neurology Department of AOU Cagliari for recurrent ischemic strokes. In the diagnostic work-up, the angiography was suggestive for primary CNS angiitis (PACNS) with a "rosary sign" aspect localized in the posterior circle (vertebral, basilar and posterior arteries), middle cerebral arteries (M1-M2 segments) and right ACA (A2 segment). An extended laboratory and radiologic screening identified high protein level (61.6 $\mathrm{mg} / \mathrm{dl}$, n.v. 8-32) and oligoclonal bands in CSF and a breast cancer with polmunar metastatic nodules. No signs of systemic infectious, autoimmune process or systemic vasculitis were found. The possibility of a paraneoplastic vasculitis was considered and investigation on paraneoplastic and onconeural markers revealed anti-yo antibodies in serum and cerebrospinal fluid.

Discussions and conclusions: Paraneoplastic vasculitis is a rare syndrome and only few cases have been described in literature (1). Actually, anti-yo antibodies against Purkinje cells are the most frequent cause of paraneoplastic cerebellar degeneration (2) and are commonly described in association with breast cancer (3). Of note, in these patients, non cerebellar signs/symptoms may also be present, including peripheral neuropathy, 
dysphagia, diplopia, and cognitive impairment [3]. To the best of our knowledge this is the first case report of CNS vasculitis possibly related to anti-yo antibodies. If confirmed by further reports, our observation may have clinical and therapeutic relevance.

References:

1. Taccone F.S. et al. Paraneoplastic vasculitis of central nervous system presenting as recurrent cryptogenic stroke. Int J Clin Oncol (2007) Apr;12(2):155-9

2. Anand $\mathrm{V}$ et al. Paraneoplastic cerebellar degeneration with anti-Yo antibodies - a review, Annals of Cl and TR. Neurology (2016) Jun 30;3(8):655-63

3. Peterson $\mathrm{K}$ et al. Paraneoplastic cerebellar degeneration. A clinical analysis of 55 anti-Yo antibody positive patients. Neurology (1992);42:1931-7

\section{TPA, STENTING AND TIROFIBAN IN A WAKE-UP STROKE DUE TO CAROTID DISSECTION: A THERAPEUTIC SUCCESSFUL APPROACH}

F. Sepe ${ }^{1}$, D. Ferrandi ${ }^{1}$, I. Gallesio ${ }^{2}$, C. Prevost $^{1}$, F. Passantino ${ }^{1}$, M. Mascolo $^{1}$, A. Gai ${ }^{1}$, R. Bottaro ${ }^{1}$, L. Agrò ${ }^{1}$, R. Demurtas ${ }^{1}$, L. Ruiz ${ }^{1}$

${ }^{1}$ Neurology, Azienda Ospedaliera SS. Antonio e Biagio e Cesare Arrigo (Alessandria); ${ }^{2}$ Radiology, Azienda Ospedaliera SS. Antonio e Biagio e Cesare Arrigo (Alessandria)

Objective: Spontaneous extracranial artery (EAD) dissection is a rare cause of stroke whose indications and technical features for recanalization remain unknown. In only a few cases reports, endovascular treatment was undertaken in the acute phase of stroke, combining IV tissue plasminogen activator (tPA), stenting and IV tirofiban administration immediately after the procedure. We report a case of woman with wake-up acute stroke and spontaneous carotid artery dissection successfully treated with this combined therapeutic approach.

Materials/Methods: A 54-year-old right-handed smoking woman, was found lying in bed at 07:00 a.m. hemiplegic to the left-hand side and with dysarthria. She was admitted to emergency department at 08:30 (NHISS 17, $\mathrm{mRs} 0$ ). At 8:49 a.m. she underwent through CT angiography: no acute ischemic lesions were detected (ASPECT 10) but a right sided EAD with good collateral compensation via leptomeningeal anastomosis between ACA/MCA was documented. Since this woman presented a wake-up stroke, with unknown time of onset, an MRI angiography was performed: an MRI diffusion/FLAIR mismatch was documented in the right cortical frontal areas. At 10:31 a.m. (door to needle 112 minutes) was treated with IV t-PA $(47 \mathrm{mg})$. Endovascular treatment using thromboaspiration followed by stenting was performed at 10:55 a.m. (door to groin 134 minutes) achieving an effective carotid recanalization (TICI 2B). 24 Hours of IV tirofiban at $0.1-0.15 \mathrm{mg} / \mathrm{kg} / \mathrm{min}$ was administered without a loading dose.

Results: Immediately after the procedure, the patient made a dramatic neurological improvement lasting until discharge (mRs 0). A CT scan was performed at 6 and 24 hours and no intracerebral hemorrhage was detected. A loading dose of aspirin ( $300 \mathrm{mg} /$ day) was administered when the tirofiban was stopped. This loading dose was followed by administration of aspirin (100 mg/day) plus clopidogrel (75 mg/day) for 3 months.

Discussion: A combined alteplase/thrombectomy/stenting/tirofiban strategy is safe and efficacious with an improved and sustained outcome. This is consistent with other studies that showed that IV tirofiban immediately after alteplase seems to be potentially more effective, reducing the risk of reocclusion and symptomatic intracranial hemorrhage, improving clinical and cognitive outcomes when compared with alteplase alone [1],[2]. Less data are available on other combined therapeutic approach [3] like this, especially in unknown time onset strokes.

Conclusions: Direct emergency angioplasty combined with alteplase/ tirofiban for wake-up stroke with extracranial carotid dissection seems to be a valid therapeutic approach, but more feasibility studies are needed. References:

1. Wei Li et al. Safety and Preliminary Efficacy of Early Tirofiban Treatment after Alteplase in Acute Ischemic Stroke Patients. Stroke (2016);47(10):2649-51 https://doi.org/10.1161/STROKEAHA. 116.014413

2. Ting Yu Yi et al. Special Endovascular Treatment for Acute Large Artery Occlusion Resulting From Atherosclerotic Disease. World Neurosurgery 103 (2017);103:65-72 https://doi.org/10.1016/j. wneu.2017.03.108

3. M. Millán et al. Safety and effectiveness of endovascular treatment of stroke with unknown time of onset. Cerebrovascular Diseases (2014); https://doi.org/10.1159/000357419.

\section{A REVERSIBLE SEVERE VITAMIN B12 DEFICENCY MIMICKING A SPORADIC CREUTZFELDT-JAKOB DISEASE}

\author{
M. Sforza, E. Bianchini
}

Neurology, Azienda Ospedaliera Sant'Andrea (Roma)

Objectives: Low vitamin B12 levels have been negatively associated with cognitive functions in older subjects. Several studies investigated the potential role of vitamin B12 supplementation to arrest or revert the cognitive decline progression. In this report we present a case of a rapidly progressive cognitive decline associated with motor-sensory deficit due to vitamin B12 deficiency mimicking a sporadic Creutzfeldt-Jakob Disease (sCJD).

Materials and methods: A 70-years-old man was referred to neuropsychiatric evaluation due to depression, behavioral changes, apathy and insomnia associated with memory loss and ataxia. The symtomps started 6 months before the visit and rapidly progressed up to complete loss of independence in the ADL and IADL. At admission, the patient was fully conscious, disoriented in time and space, partially compliant with the visit. Psychomotor retardation and attention deficit were observed. Ataxia with wide foot stance, postural instability and tendency to fall was assessed. Examination of sensibility, coordination and neuropsychiatric evaluation was impracticable due to no compliance by the patient.

Results: Lab tests demonstrated macrocitic anemia, hyperhomocysteinemia and very low vitamin B12 levels. Brain MRI showed hyperintesity of the heads of caudate nuclei and insular cortex. CSF analysis showed positity for 14-3-3 protein. RT-Quic test resulted negative. Gastric endoscopy and hystological analysis revealed signs of nonerosive chronic atrophic gastritis. Thus, supplementation therapy with Cyanocobalamin 5000 UI i.m. QW was initiated. Two months after the beginning of treatment, lab tests showed normal vitamin B12 levels. A Brain MRI demontrated the absence of the previous alterations. The neurological examination was unremarkable apart from a mild hypopallestesia and propriocetion deficit. The patient reported to be independence in ADL and IADL again.

Discussion: The presence of a positive test for 14-3-3 protein in CSF, the compatible brain MRI abnormalities and the neurological symptoms led us to the initial diagnosis of probable SCJD. Nevertheless, a cyanocobalamin supplementation therapy was started do to severe deficiency. The 
dramatic improvement of cognitive impairment, neurological deficits and neuroimaging evaluation after the supplementation therapy, excluded the diagnosis of SCJD according to the diagnostic criteria.

Conclusion: Neuropsychiatric symptoms could be the presenting feature of vitamin B12 deficiency and this clinical picture is reversible with the rapid supplementation of Vitamin B12. We suggest that vitamin B12 deficiency should be considered in the differential diagnosis of patients presenting with rapidly progressive cognitive decline.

References:

- Clarfield AM. The decreasing prevalence of reversible dementias: an updated meta-analysis. Arch Intern Med. (2003);163(18):2219-29

- Moore E, Mander A, Ames D, Carne R, Sanders K, Watters D. Cognitive impairment and vitamin B12: a review. Int Psychogeriatr. (2012);24(4):541-56

\section{AKINETIC MUTISM CAUSED BY TACROLIMUS AFTER LIVER TRANSPLANTATION: NEW INSIGHTS ON PATHOPHYSIOLOGY REVEALED BY ARTERIAL SPIN LABELING MAGNETIC RESONANCE IMAGING}

\author{
G. Smorlesi, A. Seidita, M.C. Sapere, G. Sparacia, V. Agnese, S. \\ Gruttadauria, V. Lo Re
}

ISMETT-UPMC (Istituto Mediterraneo Trapianti e Terapie ad Alta Specializzazione), University of Pittsburg Medical Center (Palermo)

Background: Neurotoxicity is one of the most common side effects of calcineurin inhibitors (CNIs). Among these, akinetic mutism is a very peculiar and fearsome major neurological complication, very rarely observed during the loading phase of the immunosuppressive regimen. Patients show a rapidly progressive slowdown of speech, and dysarthria, often evolving to mutism. Recovery always takes place with CNI withdrawal, though it is often slow, and sometimes incomplete, even after several months [1]. Quadriparesis, ataxia, and seizures can be part of the clinical scenario in more serious cases. Pathophysiology has not been clarified at all. Neuroimaging and electrophysiologic studies have often been unremarkable in previous reports [2]

Case-report: A 60-year-old right-handed woman affected with HCVrelated cirrhosis underwent an orthotopic liver transplantation. She was fully independent in ADL e IADL. Neurological examination was unremarkable, except for slight flapping tremor on upper limbs. MRI of the brain before intervention showed only bilateral pallidal hyperintensities on T2/FLAIR sequences, as commonly seen in cirrhotic patients. The sixth day after beginning tacrolimus subtle dysarthria was noticed (FK level was $11.8 \mathrm{ng} / \mathrm{ml})$. Over the following five days, the speech disorder rapidly evolved to mutism. We immediately suspended tacrolimus and started mTOR inhibitor everolimus. Despite clinical conditions worsening, with ataxic-spastic quadriparesis prevalent on right side limbs, dysphagia, and alteration of consciousness, MRI of the brain showed no acute damage. No epileptiform discharges were seen on repeated bedside electroencephalograms. Lumbar puncture showed only a modest increase in protein. We performed another MRI with arterial spin labeling (ASL) sequence, which documented a wide cortical hypoperfusion, mainly on left fronto-parietal regions. ASL is an MRI protocol that enables measurement of brain perfusion without administration of a contrast agent, tracking magnetically labelled inflowing blood [3]. At her discharge, the patient's speech was still dysarthric, with features of stuttering, effortful and monosyllabic speech, but her communication was otherwise effective. She was able to move with a walker, even if slight axial and segmental ataxia persisted. Before discharge, perfusion of the brain returned to normal on ASL sequences. Morphologic MRI was still unremarkable.

Conclusions: Our case represents a typical example of akinetic mutism following tacrolimus loading after liver transplantation. It was caused by transient cortical hypoperfusion, in the absence of any structural brain anomaly. To our knowledge this is the first report in which a reliable, noninvasive neuroimaging tool was successfully utilized to find a functional correlate of tacrolimus-related neurotoxicity.

References:

1. Bradley F., Boeve MD., et al. Dysarthria and Apraxia of Speech Associated With FK-506 (Tacrolimus). Mayo Clin Proc (1996); 71:969-72

2. Eelco F. M. Wijdicks, MD., et all. FK5 06-induced Neurotoxicity in Liver Transplantation. Ann Neurol (1994);35.498-501

3. Haller S., Zaharchuk G., et all. Arterial Spin Labeling Perfusion of the Brain: Emerging Clinical Applications. Radiology (2016) Nov;281(2):337-56

\section{A CASE OF CEREBRAL AMYLOID ANGIOPATHY-RELATED INFLAMMATION (CAA-RI) MISDIAGNOSED AS PRES}

\author{
S. Stano ${ }^{1}$, U. Scoditti ${ }^{2}$, G. Pavesi ${ }^{3}$
}

${ }^{1}$ Department of Neurology, University of Parma (Parma); ${ }^{2}$ Stroke Unit, Neuroscience Department (U.S.), University of Parma (Parma); ${ }^{3}$ Institute of Neurology, University of Parma (Parma)

Background: Cerebral amyloid angiopathy-related inflammation (CAAri) is a syndrome of reversible encephalopathy seen in a group of patients with cerebral amyloid angiopathy. CAA-ri presents with acute or subacute onset of headaches, cognitive and behavioural changes, seizures and focal neurological deficits. MRI findings include patchy or confluent $\mathrm{T} 2$ hyperintense lesions in the cortex and subcortical white matter, in addition to the presence of microbleeds on T2-weighted gradient echo sequences, the typical finding in CAA.

Case Description: We report a case of a 70 years old man admitted to our department with acute onset of confusion and disorientation. At the arrival in ER he appeared soporous and with left turned gaze. CT brain showed hypodensity and mass-like effect in left temporal lobe. A brain MRI was performed showing multifocal areas of white matter T2 hyperintensity and vasogenic edema; in the right occipital lobe, instead, minimal linear enhancement was reported in the leptomeningeal region, interpreted as possible vascular congestion. A diagnosis of probable PRES was made according to the radiologic and clinical findings. After three days there was a gradual recovery of symptoms and antihypertensive therapy as well as antiepileptic therapy was set. Brain MR was repeated after 12 days and showed a marked reduction in vasogenic edema and a complete regression of the enhancement. After 4 years the patient returned to our observation due to sudden confusional state and expressive aphasia. Brain MR performed two days after the onset showed no abnormalities and neither did EEG trace. Episode has been interpreted as epileptic recurrence. A further hospitalization was needed after two months due to sudden confusion, incongruous behavior and aphasia. Also in this case neuroimaging was normal with EEG trace showing epileptic anomalies on the right temporal region. During hospitalization the patient complained of intensive drug-responsive headache and experienced several episodes of wandering. The case was therefore rediscussed and a diagnosis of suspected CAA-ri was put forward, a 
hypothesis further supported by excellent clinical response obtained after steroid therapy.

Discussion: Our case demonstrates the importance of identifying this syndrome of reversible encephalopathy seen in a group of patients with CAA, in particular for effectiveness demonstrated by timely treatment with steroid. PRES and primary central nervous system vasculitis should be taken into account in the differential diagnosis, due to the overlapping neuroradiological features among the entities. Therefore, recent diagnostic criteria have been proposed which help distinguish CAA-ri from alternative diagnosis.

References:

- Chung KK, Anderson NE, Hutchinson D et al. Cerebral amyloid angiopathy related inflammation: three case reports and a review. $\mathrm{J}$ Neurol Neurosurg Psychiatry (2011); 82:20-26

- Kirshner HS, Bradshaw M. The Inflammatory Form of Cerebral Amyloid Angiopathy or Cerebral Amyloid Angiopathy-Related Inflammation (CAARI). Curr Neurol Neurosci Rep (2015);15:54

\section{A CASE OF NEW ONSET HEADACHE DUE TO INTRACRANIAL INTERNAL CAROTID ARTERY DISSECTION: SUSPICION OR SURPRISE}

S. Strumia, A. Patuelli, C. Leta, S. Mazzoli, M. Tassinari, S. Malagu, M. Longoni

Neurology Unit, Morgagni-Pierantoni and Bufalini Hospital (Forlì, Cesena)

Objective: Headaches with unilateral localization, high intensity and apparent time-locked recurrence may be a diagnostic challenge even if they seem to fulfill the criteria of primary headaches. We present a case of a new-onset cluster-like headache in an elderly patient with the simultaneous concurrence of an infrequent vascular lesion and a misleading clinical presentation.

Case report: A 76-year-old male patient with a previous medical history of hypertension, ischemic heart disease, thrombocytopenia, hereditary hemochromatosis and permanent atrial fibrillation was admitted to our hospital complaining of a new-onset severe right-sided headache with occipital and retroorbital localization: the pain was stabbing or pulsating, with unilateral tearing, incostantly remittent both spontaneusly and with analgesic treatment. The neurological examination did not show any pathological sign, nor trigger or painful points. Blood tests revealed the previously known thrombocytopenia and INR was in therapeutic range. During hospitalization, the pain was excruciating late at night and responsive to iv steroids or high-flux oxygen. A brain enhanced MRI and MRA excluded parenchimal lesions, cavernous thrombosis or enhancement of the middle fossa structures. We started oral steroids and Verapamil, but when a sixth nerve right palsy appeared, we planned to repeat a brain MRI looking for granulomatous tissue in the right cavernous sinus. On the 22th day from the start of the headache, the patient developed left hemiparesis. Urgent brain MRI-MRA and AngioCT revealed an ischemic stroke of the right caudate and putaminal nucleus and a dissection of the right internal carotid artery in its intracranial segment at the entrance into the cavernous sinus to the rise of the middle cerebral artery. The ischemic area underwent an hemorragic transformation with mass effect and the patient deceased.

Discussion: Headaches due to intracranial lesions mimicking primary forms can be worthwhile of description when the lesion is statistically rare and the primary headache has specific clinical characteristics. Cluster-like secondary headaches may be associated to dissection of the omolateral internal carotid artery, usually in young adults patients and in the extracranial portion up to the extra-intracranial passage. Conversely, when dissections are intracranial, descriptions of sustained headache are scanty, as ischemic or hemorragic strokes soon follow the headache. So this case may be noteworthy for the localization of carotid dissection, the age of the patient, and the long time elapsing between the start of the headache and the stroke. Because of the dynamic evolution of dissections, repeating radiologic examination may be revealing.

References:

- Edvarsson B. Symptomatic cluster headache: a review of 63 cases. SpringerPlus (2014); 3:64

- Chaves C, Estol C, Esnaola MM, Gorson K, O'Donoghue M, De Witt LD, Caplan RL. Spontaneous intracranial internal carotid artery dissection: report of 10 patients. Arch Neurol (2002) Jun;59(6):97781

- Rigamonti A, Iurlaro S, Reganati P, Zilioli A, Agostoni E. Cluster Headache and Internal Carotid Artery Dissection: Two Cases and Review of the Literature. Headache (2008) Mar;48 (3):467-70-9

\section{PSEN1 MUTATION (C.461A>G, P.TYR154CYS) ASSOCIATED WITH ALZHEIMER'S DISEASE AND SPASTIC PARAPARESIS: A CASE REPORT}

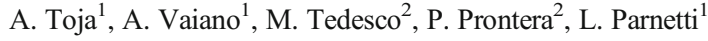 \\ ${ }^{1}$ Neurology Clinic, Department of Medicine, University of Perugia \\ (Perugia); ${ }^{2}$ Medical Genetics Unit, Hospital "Santa Maria della \\ Misericordia" (Perugia)
}

Objectives: Familial Alzheimer's disease (AD) can be caused by mutations in genes encoding for presenilin 1 (PSEN1, Chr 14), presenilin 2 (PSEN2, Chr 1), amyloid precursor protein (APP, Chr 21). Rarely mutations in PSEN1 have been identified in patients with $\mathrm{AD}$ and spastic paraparesis, suggesting the existence of PSEN1 sub-phenotype. Here we report a case of a patient carrying PSEN1 mutation affected by AD and spastic paraparesis.

Materials: A 61 years old woman came to our attention for a clinical picture characterized by progressive spastic paraparesis and cognitive decline, similar to what observed in other members of her family (father, paternal aunt and grandmother).

Methods: Patient underwent a thorough and comprehensive diagnostic assessment (brain and cervico-dorsal spine MRI, neuropsychological evaluation, cerebrospinal fluid (CSF) determination of biomarkers of amyloidosis tauopathy and neurodegeneration and genetic analysis).

Results: Brain and cervico-dorsal spine MRI did not show any significant finding; neuropsychological assessment demonstrated multiple domain cognitive deficits (clinical stage: mild dementia), CSF analysis showed a profile compatible with $\mathrm{AD}[\mathrm{A}+/ \mathrm{T}+/(\mathrm{N})+]$; genetic analysis demonstrated a heterozygous PSEN1 mutation (c.461A>G, p.Tyr154Cys).

Discussion: To the best of our knowledge, this is the first patient carrier of the p.Tyr154Cys PSEN1 mutation showing AD and spastic paraparesis. The p.Tyr154Cys mutation has been previously described only in association with $\mathrm{AD} 2$, therefore we expand the phenotypic spectrum associated with this genetic alteration, reinforcing the hypothesis that $\mathrm{AD}$ and spastic paraparesis can be part of a more wide PSEN1related disorders.

Conclusion: The cause of the phenotypic variability among patients sharing the same PSEN1 mutation has not been elucidated and it cannot 
be excluded the influence of other genetic factors. On the other hand, the collection of larger coohort of patients with PSEN1 mutation and AD/ paraparesis will help in finding the possible link between specific mutation(s)/protein domain(s) and this sub-phenotype.

References:

- Ryan NS, Nicholas JM, Weston PSJ, et al. Clinical phenotype and genetic associations in autosomal dominant familial Alzheimer's disease: a case series. Lancet Neurol. (2016);15(13):1326-35

- Hattori S, Sakuma K, Wakutani Y, et al. A novel presenilin 1 mutation $(\mathrm{Y} 154 \mathrm{~N})$ in a patient with early onset Alzheimer's disease with spastic paraparesis. Neurosci Lett. (20049;368(3):319-22

\section{LESION OF THE CORPUS CALLOSUM ASSOCIATED WITH BRITTLE DIABETES AND RENAL FAILURE}

\author{
G. Toldo, M. Carone, G. Canal, P. Rossi
}

Neurology, San Bassiano Hospital (Bassano del Grappa-VI)

Background: Mild encephalitis/encephalopathy with reversible splenial lesion (MERS) is a rare clinical-radiological entity characterized by transient magnetic resonance imaging (MRI) findings of the splenium of the corpus callosum (SCC), at first reported as a child-onset condition. Described causative factors are infections, epilepsy and antiepileptic drugs, toxic-metabolic disorders, tumors, cerebral infarctions, traumas, autoimmune encephalitis. Only few cases of MERS associated with poor diabetic control or renal failure have been reported [1].

Case Presentation: We report the case of a 50-years-old African man with brittle diabetes, nephrotic syndrome with renal failure and mild hypertension. He was hospitalized in an internal medicine ward because of fever, pneumonia, uncontrolled diabetes with psychomotor slowness and disorientation. Relevant blood exams on admission were: CRP 31,59 mg/dL, glucose $471 \mathrm{mg} / \mathrm{dL}, \mathrm{HbA} 1 \mathrm{c} 14,7 \%$, creatinine $3,12 \mathrm{mg} / \mathrm{dL}$, urea $110 \mathrm{mg} /$ dL. The emergency head CT was normal. The empiric antibiotic therapy yielded clinical improvement. One week later, he underwent a neurologic evaluation for gait ataxia, feet hypoesthesia, hypopallestesia, without cognitive impairment. NCS/EMG confirmed a severe sensory-motor polyneuropathy. EEG was normal. MRI showed a small round lesion in the SCC, hyperintense on FLAIR and DWI with reduced ADC. His metabolic disorder improved within 3 weeks. The follow-up MRI taken before discharge showed a reduction of the hyperintense signal on DWI, but also a new lesion in the genu of the corpus callosum with restriction on DWI and hypointense on $\mathrm{ADC}$, with no contrast enhancement. MRI taken after 6 months showed the previous lesion had disappeared on DWI, while two small hyperintense lesions remained on T2-FLAIR.

Discussion/Conclusion: Adult-onset MERS is an entity with nonspecific clinical symptoms [2]. Cognitive impairment is the most frequent sign. Transitory headache, fever, seizures, dysarthria have been described [3]. The reversible lesion, hyperintense on DWI and hypointense on ADC, can be also found in asymptomatic patients. Previous studies suggest that cytokine-mediated cytotoxic edema of the SCC may be a pathophysiological manifestation of MERS [1]. Patients with transient impairment of consciousness of unclear origin, especially if associated with uremic syndrome might present a callosal lesion [3]. The SCC is bloodsupplied by both the anterior and posterior pericallosal arteries and thus very sensitive to hematochemical variations, but the anterior callosal section can be also affected (type 2 MERS) [1]. Our report supports the few described MERS cases in which lesions are not completely reversible [1]. It could be that lesions associated with non-communicable diseases are less reversible due to the chronicity of the condition.
References:

1. Syuichi Tetsuka, Reversible lesion in the splenium of the corpus callosum, Brain and Behavior (2019);9(11):e01440

2. Yuan J, Yang S, Wang S, Qin W, Yang L, Hu W. Mild encephalitis/ encephalopathy with reversible splenial lesion (MERS) in adults-a case report and literature review. BMC Neurology (2017);17(1): 103

3. A. Vargas Cañas, R. Guerrero Torrealba, N. Rebolledo Villar. Clinical spectrum of callosum corpus splenium lesions: subdiagnostics of a common entity. Journal of Neurology and Stroke (2018);8(2):74-78

\section{CPT2 AND RYR1: A DOUBLE-TROUBLE CLINICAL CASE}

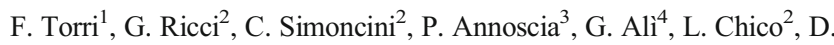 \\ Cassandrini $^{5}$, F. M. Santorelli ${ }^{6}$, G. Siciliano ${ }^{2}$
}

${ }^{1}$ Neurologic Clinic, Department of Clinical and Experimental Medicine, University of Pisa (Pisa); ${ }^{2}$ Neurologic Clinic, Department of Clinical and Experimental Medicine, AOUP (Pisa); ${ }^{3}$ Hand Surgery Division, Plastic Surgery Clinic, AOUP (Pisa); ${ }^{4}$ Anatomical Pathology Clinic, AOUP (Pisa); ${ }^{5}$ Biology Unit, IRCCS Fondazione Stella Maris (Pisa); ${ }^{6}$ Pediatric Neuropsychology Department, IRCCS Fondazione Stella Maris (Pisa)

Rhabdomyolysis is a clinical condition characterized by muscular damage, increase of myolysis indicators, myalgia, weakness, myoglobinuria, hydro-electrolitic imbalance and renal failure. Exercise, infections, toxics, trauma, dismetabolic or genetic conditions are the main causes. We present the case of our patient, a 45 years old male, with history of episodes of rhabdomyolysis following physical exertion or fever. Similar symptoms are shared by his father and brother. Notably, his parents are consanguineous. Our patient and his brother underwent muscular biopsy, showing minimal myopathic signs in his (fibers' dimension variability, with scattered atrophic fibers and mild nuclei centralization) and no pathological changes in his brother's. Genetic analysis of CPT2 gene was performed: our patient carries a homozigous mutation (c.338C $>$ T/p.S113L), while his brother is heterozygous. Moreover, in our patient a heterozygous variant in RYR1 gene of uncertain pathological meaning was found (c.10018+8G $>$ A, exon 66). CPTII is a mitochondrial longchain fatty acids oxidation enzyme. Pathologic autosomal recessive mutations are associated with three enzyme deficit patterns: the most prevalent myopathic form, characterized by rhabdomyolysis episodes following exertion, fever or fasting; infantile and neonatal forms, with severe energetic metabolism impairment, commonly lethal. RYR1 is a calcium channel involved in the muscular contraction process. Autosomal dominant mutations are associated with malignant hyperthermy and several myopathic forms, as central core, multiminicore with external ophthalmoplegia and others. The finding of the RYR1 variant in our patient, whose symptoms are probably due to the homozygous CPT2, calls for RYR1 analysis on the patient's brother and parents for further diagnostic definition.

\section{CHILDOOD-ONSET MULTIFOCAL MOTOR NEUROPATHY WITH IGM ANTIBODIES TO GANGLIOSIDES GM1: A CASE REPORT WITH POOR OUTCOME}

\author{
F. Ursitti ${ }^{1}$, S. Pro $^{2}$, G. Pruneddu ${ }^{3}$, L. Papetti ${ }^{1}$, A. Ferilli ${ }^{1}$, R. Moavero ${ }^{1}$, \\ M. Di Capua ${ }^{2}$, M. Valeriani ${ }^{1}$
}


${ }^{1}$ Child Neurology Unit, Department of Neuroscience and Neurorehabilitation, Bambino Gesù Children's Hospital IRCCS (Roma); ${ }^{2}$ Neurophysiology Unit, Department of Neuroscience and Neurorehabilitation, Bambino Gesù Children's Hospital IRCCS (Roma); ${ }^{3}$ Paediatric Neurorehabilitation Units Neuroscience and Neurorehabilitation, Bambino Gesù Children's Hospital IRCCS (Roma)

Background: Multifocal Motor Neuropathy (MMN) is a rare and slowly progressive motor demyelinating neuropathy characterized by asymmetric muscle weakness without sensory involvement. It is rarer in the pediatric population, where only 5 patients have been reported up to now. More than $80 \%$ of patients with MMN respond to treatment with intravenous immunoglobulin (IVIg), however in most cases muscle strength decreases gradually. We discuss the 3-years follow-up of a 13-year-old girl with MMN.

Materials and methods: The patient was hospitalized in May 2018 due to progressive asymmetric limb weakness without sensory involvement. Twelve months before, she had presented with left upper limb weakness that slowly deteriorated. Neurological deficit had been attributed to a car accident with traumatic spinal and brain injury. EMG showed C5-T1 denervation in left upper limb. Nerve Conduction Study (NCS) study had not been performed. Surgical exploration of left brachial plexus was performed without evidence of nerve injury. In the next months, weakness worsened with an asymmetric involvement of the lower limbs and the right upper limb. At our observation she was unable to stand up. Left harm was completely plegic and showed a distal muscle atrophy.

Results: NCS were performed and showed conduction block. Cerebrospinal Fluid Analysis was normal and serum antiganglioside GM1 IgM antibodies tested positive. A diagnosis of definite MMN was made. She underwent intravenous Methylprednisolone for 5 days followed by an oral prednisone. After 1 month she started $2 \mathrm{gr} / \mathrm{kg}$ IVIg in 3 days. Then she underwent Plasma Exchange followed by IVIG and prednisone. Finally she started Rituximab followed by IVIG every months until today. No improvement was referred.

Discussion: Our patient shared clinical and neurophysiological characteristics of the previously reported pediatric patients with MMN. Unlike previously pediatric patients, our patient showed an involvement of different cranial nerves. The poor prognosis may be explained by precocious denervation in according with axonal damage. When symptomatology started, an EMG was performed with active denervation. Unfortunately no NCS was performed to demonstrate conduction block. IVIg was performed at 12 months from clinical weakness onset. Therapeutic strategies aimed at prevention and reduction of axon loss, such as early initiation of treatment with immunosuppressive agents, should be considered in patients with MMN. Our patient did not respond to any treatment and presented poor outcome.

\section{A CASE OF REVERSIBLE THALAMIC DEMENTIA}

G. Valcamonica ${ }^{1}$, S. Lanfranconi ${ }^{1}$, I. Ghione ${ }^{1}$, D. Villa ${ }^{1}$, A. Paolucci ${ }^{2}$, N. Bresolin $^{1}$, S. Bonato ${ }^{1}$

${ }^{1}$ Stroke Unit-Neurology Department IRCCS Foundation Cà Granda, Ospedale Maggiore Policlinico, Dino Ferrari Centre, Department of Pathophysiology and Transplantation (DEPT), University of Milan (Milano); ${ }^{2}$ Neuroradiology Unit, IRCCS Foundation Ca' Granda, Ospedale Maggiore Policlinico (Milano)

Background: Bilateral thalamic lesions are uncommon and have a limited differential diagnosis that includes metabolic and toxic processes, infections, Wernicke encephalopathy, autoimmune disease, infarct of Percheron artery or other cerebrovascular disease and bilateral thalamic glioma.

Methods: Here we describe a case of a 60 years-old female admitted to our Stroke Unit for subacute onset of apathy, behavioral changes and memory loss. Symptoms started six months before and she was poorly aware of her disturbance.

Results: The patient underwent CT scan that showed a bilateral and simmetric thalamic hypodensity. Brain MRI showed bilateral thalamic oedema and signs of previous bleeding. MRA disclosed a dural arteriovenous fistula (DAVF) of the right petrosus sinus (Cognard type IV) without signs of venous thrombosis. The patient underwent successfully endovascular treatment. We started anticoagulation to prevent secondary thrombosis. Symptoms progressively improved until complete regression at two months follow-up. New cerebral MRI after treatment showed partial reduction of brain edema and complete DAVF obliteration. Thrombophilic screening revealed the presence of Lupus anticoagulant and Cardiolipin antibodies.

Conclusions: Our patient had thalamic venous hypertension caused by DAVF and subsequent retrograde flow. It is reasonable to consider diagnosis of vascular abnormalities such as DAVFs in bilateral thalamic injury. The causative factors of DAVS are still uncertain however dural sinus or venous thrombosis can contribute to their occurrence. Therefore it's important to perform thrombophilic screening to start the correct therapy and prevent other events.

\section{EPILEPTIC STATUS AFTER CAROTID STENTING: A CASE OF CEREBRAL HYPERPERFUSION SYNDROME}

G. Valcamonica, S. Lanfranconi, I. Ghione, D. Villa, N. Bresolin, S. Bonato

Stroke Unit-Neurology Department IRCCS Foundation Cà Granda, Ospedale Maggiore Policlinico, Dino Ferrari Centre, Department of Pathophysiology and Transplantation (DEPT), University of Milan (Milano)

Background: Cerebral hyperperfusion syndrome (CHS) is a rare complication of carotid surgery. Originally described as complication of endarterectomy (CEA), more recently it has also been observed after carotid stenting (CAS). The exact mechanism leading to CHS is unknown but an increased regional cerebral blood flow due to revascularization of a subocclusive stenosis seem to be the most important mechanism. Symptoms typically occur within 36 hours postoperatively for those undergoing CAS and range from headache to focal neurologic deficit and seizures. The most severe form is characterized by intracerebral haemorrhage. Preoperative risk factors include hypertension, diabetes, advanced age, female sex and severe atherosclerosis.

Methods: Here we describe a case of a 72 years-old patient admitted to Stroke Unit for ischemic stroke causing dysartria, left facial palsy and left arm weakness due to right internal carotid subocclusion. The patient underwent carotid stenting and was treated with dual antiplatelet therapy (Aspirin and Clopigogrel) and high-dose statin. Past clinical history was characterized by hypertension, obesity and dyslipidemia.

Results: On days two after stenting the patient complained drugs resistant headache and the day after she presented subcontinuos epileptic seizures poorly responsive to treatment. CT scan showed a new small hypodensity in the right motor cortex. CTA showed carotid stent patency. Patient was treated with antiepileptic drugs and anti-hypertensive therapy was successfully enhanced. Clinical features progressively improved. 
Conclusions: CHS is a rare complication of CEA and CAS. After stenting of severe carotid stenosis it's recommended an aggressive control of blood pressure keeping it within normal limits if not lower especially in patient with predisposing factors.

\section{EARLY-ONSET SEMANTIC DEMENTIA IN A PATIENT CARRYING THE RARE MAPT Q336H MUTATION}

\section{Villa, G. Rossi, I. Bizzozero, C. Boiocchi, G. Giaccone, P. Caroppo}

Neurology V and Neuropathology Unit, IRCCS Foundation "Carlo Besta” Neurological Institute (Milano)

Objectives: Semantic dementia (SD) is a neurodegenerative disorder characterized by loss of semantic knowledge of words. SD is usually sporadic and only rarely associated with mutations in FTD-ALS genes, mainly C9orf72, TARDBP and TBK1. Here, we describe the clinical and radiological characteristics of a patient with early-onset $\mathrm{SD}$, associated with the rare MAPT Q336H mutation.

Methods: The patient underwent a detailed clinical, neuropsychological and neuroimaging assessment.

Results: The patient developed difficulties in recognizing and naming objects and attention deficits at age 37. Two years after the onset, the neuropsychological assessment showed a language disorder with naming deficits and semantic paraphasias. MMSE was 25/30. Brain MRI showed atrophy in the left temporal pole. FDG-PET showed predominant hypometabolism in the left fronto-temporal region. The language disorder worsened over time. He came to our observation at age 41. During the last year he developed behavioral disorders with disinhibition, fatuity and loss of social manners. The neuropsychological evaluation showed severe language disorder with fluent speech, semantic impairment and consequent marked verbal comprehension deficit, theory of mind impairment with spared language repetition and visual-spatial abilities. MMSE was 9/30 due to severe comprehension deficits. Brain MRI showed progression of atrophy of the left temporal pole. CSF biomarkers showed isolated slight reduction of Abeta42. Genetic analysis showed a Q336H mutation in MAPT gene. Family history was negative for dementia.

Discussion and conclusion: MAPT Q336H is a rare mutation, described in just one patient with familial FTD and a clinical phenotype of behavioral variant of FTD so far [1]. The absence of familial history could indicate a de novo mutation in our case. Our patient presented a clinical phenotype of SD on the basis of current clinical criteria [2], thus widening the spectrum of phenotypes associated with this mutation. Sematic deficits are described in MAPT mutation carriers but often secondarily associated with behavioral disorders, while relatively pure SD is exceptional. Another patient with behavioral variant of FTD and a different mutation at the same codon (Q336R) has been reported [3]. In both reported cases pathological features of Pick disease have been described, suggesting a peculiar effect of the mutations at this codon on the aggregation of 3R isoforms of tau.

References:

1. Tacik P, DeTure M, Hinkle KM, et al. A Novel Tau Mutation in Exon 12, p.Q336H, Causes Hereditary Pick Disease. J Neuropathol Exp Neurol. (2015);74(11):1042-52

2. Gorno-Tempini ML, Hillis AE, Weintraub S, et al. Classification of primary progressive aphasia and its variants. Neurology (2011);76(11):1006-14

3. Pickering-Brown SM, Baker M, Nonaka T, et al. Frontotemporal dementia with Pick-type histology associated with Q336R mutation in the tau gene. Brain (2004);127(Pt 6):1415-26

\section{MYELORADICULONEURITIS ASSOCIATED WITH AUTOIMMUNE HEPATITIS: CASUALTY OR CAUSALITY?}

\author{
M. Vitiello, R. Infante, L. Mancinelli, C. Minardi, G. Napoli, M. Longoni
}

Hospital "Maurizio Bufalini", AUSL Romagna (Cesena)

Objectives: Isolated cases of neuropathy and myelitis associated with autoimmune liver disorders have been previously described. We present a patient with autoimmune hepatitis admitted to our department for subacute myeloradiculoneuritis.

Patient and methods: A 58-year-old female was admitted to our Emergency Department because of severe stabbing inter scapular pain together with numbness and weakness in left arm that progressively developed over the last ten days. Moreover, the day before she came to our attention a peripheral right facial palsy appeared. Neurological examination revealed cranial mononeuropathy, mild distal weakness in left upper limb, diminished deep tendon reflexes at both upper limbs and reduced tactile, thermal and pain perception from T2 to T10 left dermatomes.

Results: The patient was hence admitted to our ward and a complete assessment was performed. Brain MRI showed bilateral enhancement of proximal portion of the 7th cranial nerves. Spinal cord MRI revealed an area of hyper intense signal on $\mathrm{T} 2$-weighted images from $\mathrm{C} 4$ to $\mathrm{C} 7$ with gadolinium enhancement. Electroneurography (ENoG) showed diffuse absence of $\mathrm{F}$ waves. Cerebrospinal fluid (CSF) analysis revelaed lymphocytic pleiocytosis $(113 \mathrm{WBC} / \mathrm{mm} 3)$, increase of protein level $(1,98 \mathrm{~g} / \mathrm{L})$ and a mirror pattern of oligoclonal bands. CSF polymerase chain reaction (PCR) for viruses and microscopic and coltural exams for bacteria, fungi and BK yielded a negative result. Screening for viral hepatitis and human immunodeficiency virus was negative. MOG and AQ4 antibodies were undetectable. A basal autoimmune panel revealed the presence of AntiNuclear Antibodies (1:320) and Anti Mitochondrial Antibody antibodies (AMA-M2 +++, M2-3E (BPO) ++). Laboratory work-up showed increase of alanine transaminase $(128 \mathrm{U} / \mathrm{L})$ and Gamma-glutamyl transferase $(87 \mathrm{U} / \mathrm{L})$. Abdominal ultrasound and colangio pancreato-MR were normal.

Discussion: A diagnosis of myeloradiculoneuritis and autoimmune hepatitis was finally done and the patient was treated with intravenous pulse methylprednisolone ( $1 \mathrm{~g}$ /day for 5 days $)$ and IV Ig $(0,4 \mathrm{~g} / \mathrm{Kg} /$ day for 5 days). She showed improvement of numbness and of peripheral palsy. At discharge, she started a corticosteroid maintenance therapy $(1 \mathrm{mg} / \mathrm{kg})$. After three months, neurological conditions were stable. MRI showed persistence of enhancement at $\mathrm{C} 4-\mathrm{C} 5$ level whether 7 th cranial nerves were returned to normal appearance. Finally the ENoG was unchanged.

Conclusion: To our knowledge this is the first case of concomitant myelitis and radiculoneuritis recurring in a patient affected by autoimmune liver disorder. Since we excluded an infectious process or a well defined autoimmune diseases other than the underlying hepatitis, we hypothesized that the two clinical conditions were related.

References:

- Papadopoulos V, Micheli A, Nikiforidis D, Mimidis K.J Postgrad. Primary biliary cirrhosis complicated by transverse myelitis in a patient without Sjögren's syndrome. Med. (2005) JanMar;51(1):43-4

- Murata KY, Ishiguchi H, Ando R, Miwa H, Kondo T. Chronic inflammatory demyelinating polyneuropathy associated with primary biliary cirrhosis. J Clin Neurosci. (2013) Dec;20(12):1799-801 
- Luth S, Birklein F, Schramm C, Herkel J, Hennes E, MullerForell W, et al. Multiplex neuritis in a patient with autoimmune hepatitis: a case report. World J Gastroenterol. (2006);12:5396-98

\section{SPORADIC LATE-ONSET NEMALINE MYOPATHY IN A PATIENT WITH SILICONE BREAST IMPLANTS}

\author{
N. Volpi ${ }^{1}$, F. Ginanneschi ${ }^{1}$, L. De Stefano ${ }^{2}$, P. De Stefano ${ }^{3}$, A. Rossi ${ }^{1}$
}

${ }^{1}$ Department of Medical, Surgical and Neurological Sciences; Neurology-Neurophysiology Unit, University of Siena (Siena); ${ }^{2}$ Unit of Rheumatology, IRCCS Policlinico S. Matteo Foundation, University of Pavia (Pavia); ${ }^{3}$ Department of Neurology, Geneva University Hospitals (Geneva-CH)

Introduction: Myopathic disorders can occur in women undergoing silicone breast implants (SBIs) after a variable time interval. They are usually attributed to autoimmune/inflammatory pathogenic mechanisms and inserted in the context of the "Silicone implant incompatibility syndrome» (SIIS).

Case Report: A 38-year-old Italian woman underwent bilateral SBIs, twice (the first removed due to rupture). After about 3 years she presented a diffuse musculoskeletal pain, headache, dry mouth and eye, fatigue, bilateral proximal and distal muscle weakness, CPK and aldolase elevation (867 UI/L and $134 \mathrm{U} 7 \mathrm{~L}$ respectively), positive 1:5120 ANA with centromeric pattern and positive $>320$ $\mathrm{U} / \mathrm{mL}$ anti-CenpB. MRI of the clinically involved muscles revealed a widespread fatty infiltration. A muscle biopsy showed prominent myopathic changes: cytoplasmic rods (about 20\% of fibres), atrophy, occasional myonecroses, prominent myofibrillar disruption, with deposits reactive for myofibrillar proteins, as well as for MHC-I and the terminal complex of complement, cytoplasmic membrane bound vacuoles and core-like lesions. A moderate inflammatory component was present. Electron microscopy confirmed the presence of electron dense rods of varying largeness, some of which in continuity with $\mathrm{Z}$ disk, within areas of myofibrillar disarray. Genetic test ruled out a late onset genetic nemaline myopathy. EMG was unremarkable. The increase of the muscular enzymes persisted despite the immunosuppressive treatment.

Discussion: Our case is highly suggestive of a sporadic late onset nemaline myopathy (SLONM), a sporadic myopathy characterized by cytoplasmic or less frequently, intranuclear rods, described in association with MGUS or multiple myeloma, autoimmune disorders, such as Sjogren's syndrome, systemic lupus erythematosus and reported in a case of dermatomyositis. The muscular pathological features characterizing SLONM (atrophy, fibrosis, core-like lesions, myofibrillar disintegration, vacuoles, but also moderate inflammatory changes) are all detected in our patient. Our finding of MAC and HLA-ABC deposits within the disrupted sarcoplasmic districts might support an immunological origin of such myofibrillar lesions.

Conclusion: In presence of clinical and laboratory signs of chronic myopathy in SBIs patients, it is useful to perform a muscle biopsy, in order to evaluate the correct therapy and also the opportunity of prostheses removal.

\section{References:}

- E. Naddaf, M. Milone, A. Kansagra, F. Buadi, T. Taxiarchis Kourelis Sporadic lateonset nemaline myopathy. Clinical spectrum, survival, and treatment outcomes. Neurology (2019);93:e298-e305

- L.J. Schnitzler, T. Schreckenbach, A. Nadaj-Pakleza, et al. Sporadic late-onset nemaline myopathy: clinicopathological characteristics and review of 76 cases. Orphanet J. Rare Dis. (2017);12(1):86

\section{ANTI-MOG ASSOCIATED MENINGOENCEPHALOMYELITIS AND HHV-6 INFECTION IN AN IMMUNOCOMPETENT ADULT PATIENT}

\author{
N. Zoppi, S. Masciocchi, I. Volonghi, L. Poli, A. Benussi, A. Padovani
}

Neurology Unit, Department of Clinical and Experimental Sciences, University of Brescia (Brescia)

A 42-year-old male patient with unremarkable physiological and past medical history was admitted to the Neurology Unit with a clinical suspicion of acute transverse myelitis (TM). In about 24 hours he developed urinary retention, a lower thoracic sensory level and flaccid paraparesis. Routine blood laboratory showed mild lymphocytopenia and elevated inflammatory markers (ESR and CRP). We performed a lumbar puncture that showed high lymphocytes and isolation of Human Herpes Virus type 6 (HHV-6) (33851 copies/ml), with lower viral titre on serum (13414 copies/ml). Magnetic resonance showed signs of meningoencephalomyelitis with a prominent medullary involvement. In the suspicion of acute neurological involvement due to HHV-6 infection, although usually reported in immunocompromised adults, antiviral (Ganciclovir i.v.) and low dose steroid therapy were initially started, with no benefit on clinical manifestations and CSF viral copies. Meanwhile, further causes of TM were investigated and in light of the immunocompetent status of the patient and stable CSF viral copies despite ongoing therapy, a dysimmune genesis was suspected. Antiviral therapy was stopped and high dosage steroid and later immunoglobulin therapy were started with progressive clinical improvement. Antibodies against myelin-oligodendrocyte-glycoprotein (MOG) were found at high titer in serum and CSF, thus supporting our hypothesis. We further deepened the diagnostic workup through genomic analysis of HHV-6 integration in hair samples, confirming an inherited chromosomally integrated viral genome. After 20 days since admission, the patient has recovered the ability to walk independently, with residual mild hypoesthesia in lower limbs and mild urinary symptoms (EDSS 2.5). After 6 months, magnetic resonance imaging showed no signs of disease activity and the patient is clinically stable with maintenance therapy (prednisone $60 \mathrm{mg} / \mathrm{die}$ ). The clinical spectrum of anti-MOG disorders is rapidly evolving and it is important to consider in cases of widespread neurological involvement, even in the presence of neurotropic viral positivity.

References:

- Wynford-Thomas R., Jacob A., \& Tomassini V. Neurological update: MOG antibody disease. Journal of Neurology (2019);266:1280-86

- Jarius S. et al. MOG encephalomyelitis: international recommendations on diagnosis and antibody testing. J. Neuroinflammation (2018);15:134

- Flamand L. Chromosomal integration by human herpesviruses 6A and 6B. Advances in Experimental Medicine and Biology (2018);1045:209-26 
DEMENTIA AND AGING

\section{BRAIN ARCHITECTURE CHANGES ACROSS THE FTLD SPECTRUM}

F. Agosta ${ }^{1}$, C. Cividini ${ }^{1}$, S. Basaia ${ }^{1}$, E. Spinelli ${ }^{1}$, V. Castelnovo ${ }^{1}$, E. $\mathrm{Canu}^{1}$, N. Riva ${ }^{2}$, F. Caso ${ }^{3}$, G. Magnani ${ }^{3}$, M. Filippi ${ }^{4}$

${ }^{1}$ Neuroimaging Research Unit, Institute of Experimental Neurology, Division of Neuroscience, IRCCS San Raffaele Scientific Institute; Vita-Salute San Raffaele University (Milano); ${ }^{2}$ Unit of Neurorehabilitation, IRCCS San Raffaele Scientific Institute (Milano); ${ }^{3}$ Neurology Unit, IRCCS San Raffaele Scientific Institute (Milano); ${ }^{4}$ Neuroimaging Research Unit, Institute of Experimental Neurology, Division of Neuroscience; Neurology Unit and Neurophysiology Unit, IRCCS San Raffaele Scientific Institute; Vita-Salute San Raffaele University (Milano)

Objective: Motor neuron disease (MND) and the behavioral variant of frontotemporal dementia (bvFTD) lie on the same pathologic/genetic continuum. The aim of this study was to unravel distinct and shared structural MRI connectomic features of these syndromes.

Materials: $115 \mathrm{MND}$ (83 with amyotrophic lateral sclerosis [ALS] and 32 with primary lateral sclerosis), 35 bvFTD patients and 61 healthy controls underwent clinical, cognitive and MRI evaluations. According to neuropsychological testing, MND patients were classified in 79 puremotor (MNDpm) and 36 cognitive and/or behavioral impaired (MNDci/ bi - including 8 ALS-FTD). A sub-analysis was performed considering ALS patients only, classified in 54 ALSpm, 21 ALSci/bi and 8 ALSFTD.

Methods: Graph analysis and connectomics assessed global and local structural and functional topological network properties and regional structural and functional connectivity (FC).

Results: bvFTD showed altered structural and functional global network properties compared to all other groups. At the lobar level, bvFTD showed altered structural network properties within the frontotemporal and basal ganglia areas relative to all groups. Noteworthy, structural alterations in the parietal lobe discriminated bvFTD from controls and MNDpm. MND groups showed altered graph metrics within the sensorimotor and basal ganglia areas relative to controls. Focusing on ALS, structural alterations were confirmed within the same areas. Functionally, bvFTD showed altered metrics relative to controls and MND groups within frontotemporal, sensorimotor and basal ganglia areas. Regional analysis showed that widespread structural changes were observed in bvFTD relative to controls, while structural alterations within frontotemporal areas and among frontal and motor areas differentiated bvFTD from MND groups. The structural alterations in all MND subgroups encompassed connections within and among frontotemporal and sensorimotor networks and basal ganglia area, with a more widespread pattern of differences against controls. Results were confirmed in the ALS sub-analysis. Furthermore, bvFTD were characterized by reduced FC within the frontotemporal and sensorimotor networks relative to controls and all MND groups. ALS sub-analysis highlighted that ALSci/bi showed an increased FC relative to ALS-FTD within the same areas.

Discussion and Conclusions: The disruption of the structural architecture in MND phenotypes worsens in relation with the progression of cognitive deficits. Functional changes are characterized by an increase of FC in presence of exclusive motor impairment that intensifies with the occurrence of cognitive impairment in MND. The condition of comorbidity of ALS and FTD leads to a decrease in FC similar to bvFTD.
Supported by: The Italian Ministry of Health (GR-2011-02351217; GR-2013-02357415; RF-2011-02351193) and AriSLA (ConnectALS).

\section{BRAIN MRI SIGNATURES OF ATROPHY IN GENETIC FRONTOTEMPORAL LOBAR DEGENERATION}

\author{
F. Agosta ${ }^{1}$, E. Spinelli ${ }^{1}$, A. Ghirelli ${ }^{1}$, N. Riva ${ }^{2}$, S. Basaia ${ }^{1}$, C. Cividini ${ }^{1}$, \\ G. Magnani ${ }^{3}$, F. Caso ${ }^{3}$, P. Caroppo ${ }^{4}$, S. Prioni ${ }^{4}$, L. Tremolizzo ${ }^{5}$, I. \\ Appollonio $^{5}$, V. Silani ${ }^{6}$, P. Carrera ${ }^{7}$, M. Filippi ${ }^{8}$
}

${ }^{1}$ Neuroimaging Research Unit, Institute of Experimental Neurology, Division of Neuroscience, IRCCS San Raffaele Scientific Institute; Vita-Salute San Raffaele University (Milano); ${ }^{2}$ Unit of Neurorehabilitation, IRCCS San Raffaele Scientific Institute (Milano); ${ }^{3}$ Neurology Unit, IRCCS San Raffaele Scientific Institute (Milano); ${ }^{4}$ Unit of Neurology 5, Neuropathology, Fondazione IRCCS Istituto Neurologico Carlo Besta (Milano); ${ }^{5}$ Neurology Unit, "San Gerardo" Hospital and University of Milano-Bicocca (Monza); ${ }^{6}$ Department of Neurology and Laboratory of Neuroscience, IRCCS Istituto Auxologico Italiano, (Milano); ${ }^{7}$ Laboratory of Clinical Molecular Biology, IRCCS San Raffaele Scientific Institute (Milano); ${ }^{8}$ Neuroimaging Research Unit, Institute of Experimental Neurology, Division of Neuroscience Neurology Unit and Neurophysiology Unit, IRCCS San Raffaele Scientific Institute; Vita-Salute San Raffaele University (Milano)

Objectives: Genetic heterogeneity underlying different clinical presentations of the frontotemporal lobar degeneration (FTLD) spectrum hampers the identification of useful biomarkers that may be able to monitor disease progression and/or facilitate the enrolment in clinical trials. The aim of this study was to assess cortical, subcortical and cerebellar grey matter (GM) atrophy using magnetic resonance imaging (MRI) in patients affected by disorders of the FTLD spectrum with known genetic mutations.

Materials and methods: 362 patients with disorders of the FTLD spectrum referred between 2007 and 2019 were screened for known pathogenic mutations. 66 patients carrying mutations in the C9ORF72, GRN, TARDBP, SOD1, TBK1, MAPT, TREM2 or FUS genes were identified, including 44 with pure motor neuron disease (MND) and 22 with frontotemporal dementia (FTD). Patients carrying a C9ORF72 expansion were divided into two groups of C9-MND and C9-FTD. 61 patients with sporadic FTLD matched for age, sex and disease severity with genetic FTLD were also included, as well as 52 healthy controls (HC). Comprehensive clinical and neuropsychological assessments and threedimensional T1-weighted MRI sequences on a 3 Tesla scanner were obtained. First, a whole-brain voxel-based morphometry (VBM) analysis was performed. Subsequently, GM volumes of the basal ganglia, hippocampus, amygdala and cerebellar volumes were also obtained.

Results: Compared with HC, GM volume loss on VBM was generally greater and more diffuse in genetic FTD (gFTD) cases, followed by sporadic FTD (sFTD) cases and genetic MND (gMND) cases, whereas sporadic MND (sMND) showed very focal atrophy of the motor cortex. Patients carrying GRN and C9ORF72 mutations showed the most widespread cortical volume loss, whereas SOD1 and TARDBP patients were the least atrophic. Greater atrophy of the parietal cortices and thalami was found, globally, in genetic FTLD patients compared with sporadic FTLD and, particularly, in C9-MND patients compared with sMND. When assessing deep GM volumes, genetic FTLD patients showed significant volume loss compared with sporadic FTLD in the caudate nuclei and thalami. In particular, greater atrophy of the left caudate and right thalamus was found in C9MND compared with sMND. At the cerebellar level, 
greater atrophy of the right lobule VIIb could discriminate genetic FTLD from sporadic FTLD patients.

Discussion and conclusions: Our data suggest that measures of deep GM and cerebellar involvement might be useful markers of genetic FTLD, particularly C9ORF72-related disorders, regardless of the clinical presentation within the FTLD spectrum.

Supported by: Italian Ministry of Health (RF-2011-02351193; GR2011-02351217) and European Research Council (StG2016_714388_NeuroTRACK).

\section{PSYCHOLOGICAL IMPACT OF COVID-19 PANDEMIC AND LOCKDOWN ON ITALIAN CAREGIVERS OF PEOPLE WITH DEMENTIA}

\author{
M. Altieri, G. Santangelo
}

Department of Psychology, University of Campania "Luigi Vanvitelli" (Caserta)

Objective: Caregivers of people with dementia (pwD) are at risk of depression, anxiety and burden [1,2]. COVID-19 pandemic and government-imposed lockdown as a preventive measure might increase psychological symptoms in caregivers. The aim of the present study was to measure the change of psychological symptoms during quarantine/selfisolation for COVID-19 in a sample of Italian caregivers of pwD, and to investigate if resilience is associated with psychological changes in the sample.

Materials and Methods: Eighty-four caregivers of pwD completed an online survey between April 21st and May 3rd 2020, during the Italian lockdown due to COVID-19 pandemic. Participants completed the following questionnaires: 1.The Hospital Anxiety and Depression Scale (HADS) to evaluate levels of anxiety (HADS-A) and depressive symptomatology (HADS-D) before and during the lockdown; 2.The Resilience Scale for Adults (RSA) to assess levels of resilience; 3.The Caregiver Burden Inventory (CBI), to evaluate levels of caregiver burden. Multivariate analyses of variance (MANOVAs) for repeated measures was employed to evaluate (a) possible differences in scores of HADS-A and HADS-D before and during the lockdown within the whole sample, (b) to assess a possible and different psychological change due to lockdown between caregivers with high and low resilience levels (within-subjects variables: HADS-D and HADS-A pre and during lockdown; between-subjects variable: high/low resilience). Moreover, a multiple regression analysis was performed to evaluate the association between the caregiver burden during the lockdown and sociodemographic and psychological variables.

Results: The MANOVA revealed an effect of time (before and during the lockdown) in the whole group on HADS-D scores; a significant interaction between time and resilience was found on HADS-A scores, revealing that caregivers with high resilience showed a more significant increase of anxiety levels during lockdown than caregivers with low resilience. Moreover, the regression analysis revealed that caregiver burden was associated negatively with resilience scores, and positively with higher functional dependence.

Conclusions: COVID-19 pandemic and the lockdown produced psychological consequences in caregivers of pwD, with an increase of levels of depression, regardless of resilience levels. In caregivers, high resilience level had no effect on levels of anxiety and depression during the lockdown, but it was associated with lower levels of caregiver burden. All caregivers, regardless of their resilience level, should be addressed to psychological interventions to reduce levels of depression, anxiety and caregiver burden.

References:

1. Pinquart M, Sörensen S. Differences between caregivers and noncaregivers in psychological health and physical health: a meta-analysis. Psychol Aging (2003);18:250-67

2. Sallim AB, Sayampanathan AA, Cuttilan A et al. Prevalence of Mental Health Disorders Among Caregivers of Patients with Alzheimer Disease. J Am Med Dir Assoc (2015);16:1034-41

\section{CONTROL OF COURSE TEMPERATURE IN MAJOR ORTHOPEDIC SURGERY AND NEURO_TRAUMATOLOGY USING LEVOBUVICAIN FOR SPINAL ANESTHESIA IN OLD PATIENTS WITH DELIRIUM AND EVALUATION OF PRESSURE INJURIES}

\author{
B. Amarisse ${ }^{1}$, G. Zuliani ${ }^{2}$
}

${ }^{1}$ University of Ferrara, Geriatrics University of San Marino (Ferrara, San Marino); ${ }^{2}$ Department of Medical Sciences, University of Ferrara (Ferrara)

Randomised study of geriatric patients aged between $75 \pm 85$ divided into two groups with and without intraoperative heating undergoing major orthopaedic surgery. $46 \%$ of patients manifest delirium in the postoperative stage. From the study, postoperative pain is correlated to the onset of delirium in the postoperative stage. In major orthopaedic surgery in geriatric patients $A(N=25)$ aged between $75 \pm 85$ with intraoperative heating, the incidence of overall delirium is $15 \%$; delirium with a single event in the controls during the stay $40 \%$; severe delirium $5 \%$; delirium in the postoperative stage $15 \%$; delirium on discharge $5 \%$; delirium in the control stage after discharge $4 \%$. In major orthopaedic surgery in geriatric patients $B(N=25)$ aged between $75 \pm 85$ without intraoperative heating, the incidence of overall delirium is $32 \%$; delirium with a single event of delirium in the controls during the stay $50 \%$; severe delirium $12 \%$; delirium in the postoperative stage $29 \%$; delirium on discharge $13 \%$; delirium in the control stage after discharge $19 \%$. Pharmacological treatment of delirium: with intraoperative heating quetiapine $\begin{array}{llll}25 & 1 / 2 & 1\end{array}$ pill twice/day, Haloperidol 8 drops; without intraoperative heating quetiapine 251 pill twice/day and Haloperidol 1 dose i.m.; without intraoperative heating with Mini nutritional assessment low olanzapine 1 pill once/day, quetiapine 252 pills once/day, Haloperidol 1 dose i.m. Postoperative pressure sores in geriatric patients group $A(N=25$ pt.) undergoing major orthopaedic surgery with intraoperative heating is: $15 \%$ sacred pressure lesions first stage; $5 \%$ sacred pressure lesions second stage; $12 \%$ heels pressure lesions first stage; $25 \%$ heels pressure lesions second stage. Postoperative pressure sores in geriatric patients group $\mathrm{B}(\mathrm{N}=25 \mathrm{pt}$.) undergoing major orthopaedic surgery without intraoperative heating is: $8 \%$ sacred pressure lesions fourth stage; $10 \%$ sacred pressure lesions third stage; $10 \%$ heels pressure lesions third stage; $5 \%$ heels pressure lesions fourth stage.

References:

- Sessler DI Current Concepts: Mild Perioperative Hypotermia. N Engl J Med; 336: 1730-7

- B. Amarisse, V.A. Peduto Control of core temperature in major orthopaedic surgery and neuro-traumatology using levobupivacaine for spinal anaesthesia", Neurological Sciences Volume 40-October 2019 Congress of the Italian Neurological Society ABSTRACT s 132 vol. 40 Congress of the Italian Neurological Society POSTERS n. 761,762 pg. 232 -October 2019 
- B. Amarisse, V.A. Peduto Control of core temperature in major orthopaedic surgery and neuro-traumatology using levobupivacaine for spinal anaesthesia in old patiens", Neurological Sciences Volume 40-October 2019 Congress of the Italian Neurological Society ABSTRACT s 477 vol. 40 Congress of the Italian Neurological Society ORAL COMMUNICATION - October 2019

\section{CONTROL OF COURSE TEMPERATURE IN MAJOR ORTHOPEDIC SURGERY AND NEURO_TRAUMATOLOGY USING LEVOBUVICAIN FOR SPINAL ANESTHESIA IN OLD PATIENTS WITH DELIRIUM}

\author{
B. Amarisse ${ }^{1}$, A. Zurlo ${ }^{2}$ \\ ${ }^{1}$ University of Ferrara, Geriatrics University of San Marino (Ferrara, \\ San Marino); ${ }^{2}$ Department of Medical Sciences, Geriatric-Orthogeriatric \\ Unit S. Anna Hospital, University of Ferrara (Ferrara)
}

Randomised study of geriatric patients aged between $75 \pm 85$ divided into two groups with and without intraoperative heating undergoing major orthopaedic surgery. $46 \%$ of patients manifest delirium in the postoperative stage. From the study, postoperative pain is correlated to the onset of delirium in the postoperative stage. In major orthopaedic surgery in geriatric patients $(\mathrm{N}=25)$ aged between $75 \pm 85$ with intraoperative heating, the incidence of overall delirium is $15 \%$; delirium with a single event in the controls during the stay $40 \%$; severe delirium $5 \%$; delirium in the postoperative stage $15 \%$; delirium on discharge $5 \%$; delirium in the control stage after discharge $4 \%$. In major orthopaedic surgery in geriatric patients $(\mathrm{N}=25)$ aged between $75 \pm 85$ without intraoperative heating, the incidence of overall delirium is $32 \%$; delirium with a single event of delirium in the controls during the stay 50\%; severe delirium 12\%; delirium in the postoperative stage $29 \%$; delirium on discharge $13 \%$; delirium in the control stage after discharge $19 \%$. Pharmacological treatment of delirium: with intraoperative heating quetiapine $251 / 21$ pill twice/day, Haloperidol 8 drops; without intraoperative heating quetiapine 251 pill twice/day and Haloperidol 1 dose i.m.; without intraoperative heating with Mini nutritional assessment low olanzapine 1 pill once/day, quetiapine 252 pills once/day, Haloperidol 1 dose i.m. Pre-operative IADL score in geriatric patients $(\mathrm{N}=50$ pt.; $75 \pm 85)$ undergoing major orthopaedic surgery is $5 \pm 4$. Postoperative IADL score in geriatric patients group $\mathrm{A}(\mathrm{N}=25 \mathrm{pt}$.) undergoing major orthopaedic surgery with intraoperative heating is $3 \pm 2$. Postoperative IADL score in geriatric patients group $\mathrm{B}(\mathrm{N}=25 \mathrm{pt}$.) undergoing major orthopaedic surgery without intraoperative heating is $2 \pm 1$.

References:

- Sessler DI Current Concepts: Mild Perioperative Hypotermia. N Engl J Med; 336: 1730-7

- B. Amarisse, V.A. Peduto Control of core temperature in major orthopaedic surgery and neuro-traumatology using levobupivacaine for spinal anaesthesia", Neurological Sciences Volume 40-October 2019 Congress of the Italian Neurological Society ABSTRACT s 132 vol. 40 Congress of the Italian Neurological Society POSTERS n. 761,762 pg. 232 -October 2019

- B. Amarisse, V.A. Peduto Control of core temperature in major orthopaedic surgery and neuro-traumatology using levobupivacaine for spinal anaesthesia in old patiens", Neurological Sciences Volume 40-October 2019 Congress of the Italian Neurological Society ABSTRACT s 477 vol. 40 Congress of the Italian Neurological Society ORAL COMMUNICATION October 2019

\section{CONTROL OF COURSE TEMPERATURE IN MAJOR ORTHOPEDIC SURGERY AND NEURO TRAUMATOLOGY USING LEVOBUVICAIN FOR SPINAL ANESTHESIA IN OLD PATIENTS WITH DELIRIUM USING DONEZEPIL}

\author{
B. Amarisse, C. Renzini
}

University of Ferrara, Geriatrics University of San Marino (Ferrara, San Marino)

Randomised study of geriatric patients aged between $75 \pm 85$ divided into two groups with and without intraoperative heating undergoing major orthopaedic surgery. $46 \%$ of patients manifest delirium in the postoperative stage. From the study, postoperative pain is correlated to the onset of delirium in the postoperative stage. In major orthopaedic surgery in geriatric patients $(\mathrm{N}=25)$ aged between $75 \pm 85$ with intraoperative heating, the incidence of overall delirium is $15 \%$; delirium with a single event in the controls during the stay $40 \%$; severe delirium $5 \%$; delirium in the postoperative stage $15 \%$; delirium on discharge $5 \%$; delirium in the control stage after discharge $4 \%$. In major orthopaedic surgery in geriatric patients $(\mathrm{N}=25)$ aged between $75 \pm 85$ without intraoperative heating, the incidence of overall delirium is $32 \%$; delirium with a single event of delirium in the controls during the stay $50 \%$; severe delirium $12 \%$; delirium in the postoperative stage $29 \%$; delirium on discharge $13 \%$; delirium in the control stage after discharge $19 \%$. Pharmacological treatment of delirium: with intraoperative heating donezepil 51 pill day, Haloperidol 8 drops; without intraoperative heating donezepil 10 1 pill once/day and Haloperidol 1 dose i.m.; without intraoperative heating with Mini nutritional assessment low olanzapine 1 pill once/day, donezepil 101 pill once/day, Haloperidol 1 dose i.m. Pre-operative IADL score in geriatric patients $(\mathrm{N}=50$ pt.; 75 \pm 85 ) undergoing major orthopaedic surgery is $5 \pm 4$. Postoperative IADL score in geriatric patients group $\mathrm{A}(\mathrm{N}=25 \mathrm{pt}$.) undergoing major orthopaedic surgery with intraoperative heating is $3 \pm 2$. Postoperative IADL score in geriatric patients group $B(\mathrm{~N}=25$ pt.) undergoing major orthopaedic surgery without intraoperative heating is $2 \pm 1$. Assessment of the mini nutritional has been assessed in the pre and post-operative stage in major orthopaedic surgery. In the randomised study, the mini nutritional assessment has been assessed in the pre and post-operative stage with distinction in the two groups $\mathrm{A}$ and $\mathrm{B}$. In the 50 patients before the operation, the mini nutritional assessment is $23 \pm 24$, on discharge in group A with intraoperative heating the mini nutritional assessment is $21 \pm 20$; in group B without intraoperative heating, the mini nutritional assessment is $19 \pm 18$.

References:

- Sessler DI Current Concepts: Mild Perioperative Hypotermia. N Engl J Med; 336: 1730-7

- B. Amarisse, V.A. Peduto Control of core temperature in major orthopaedic surgery and neuro-traumatology using levobupivacaine for spinal anaesthesia", Neurological Sciences Volume 40-October 2019 Congress of the Italian Neurological Society ABSTRACT s 132 vol. 40 Congress of the Italian Neurological Society POSTERS n. 761,762 pg. 232-October 2019

B. Amarisse, V.A. Peduto Control of core temperature in major orthopaedic surgery and neuro-traumatology using levobupivacaine for spinal anaesthesia in old patiens", Neurological Sciences Volume 40-October 2019 Congress of the Italian Neurological Society ABSTRACT s 477 vol. 40 Congress of the Italian Neurological Society ORAL COMMUNICATION October 2019 
NIEMANN-PICK TYPE C (NPC) 1 AND NPC2 GENE VARIABILITY IN NEURODEGENERATIVE DISEASE PATIENTS WITH EVIDENCE OF AMYLOID DEPOSITION IS ASSOCIATED WITH PSYCHIATRIC PRESENTATION

\author{
A. Arighi, F. Sorrentino, M. Serpente, M. Arcaro, C. Visconte, E. \\ Rotondo, R. Vimercati, G. Fumagalli, A. Pietroboni, T. Carandini, E. \\ Scarpini, C. Fenoglio, D. Galimberti
}

\section{IRCCS Ca' Granda Foundation, University of Milan (Milano)}

Background: Mutations in Niemann-Pick Type C genes (NPC1 and NPC2), responsible for NPC in homozygous state, have been suggested to play a role as risk or disease modifying factors for neurodegenerative diseases, particularly Alzheimer's disease (AD), in light of the involvement of both NPC genes and Apolipoprotein E, the major risk factor for $\mathrm{AD}$, in cholesterol trafficking.

Methods: A targeted NGS panel was customized in order to screen NPC1 and NPC2 genes and the main genes related to neurodegenerative diseases: APP, PSEN1, PSEN2, GRN and MAPT in a cohort of 136 demented patients with Cerebrospinal Fluid (CSF) low Amyloid beta 1-42 (A $\beta$ ) levels or positive PET with $A \beta$ tracer.

Results: Seven patients were carriers of NPC variants in heterozygosis. Four of them displayed variants known to be causative for NPC (in homozygosity): NPC2 c. $441+1$ G $>$ A (2 patients with $\mathrm{AD}$ and one with Corticobasal Syndrome) and NPC2 p.V30M (one $\mathrm{AD}$ patient); two $\mathrm{AD}$ carried rare variants described in patients with neurodegenerative diseases (NPC1 p.Q241X and NPC2 p.K71R), and in one AD patient a novel variant (NPC1 $\mathrm{Y} 570 \mathrm{H})$ was found. The latter was absent in 200 non demented elderly subjects. No significant differences were observed in minor allele frequency of the other six variants in cases compared to controls. Notably, 4 of 7 patients exhibited psychiatric symptoms at onset or later.

Conclusions: The frequency of NPC1 and NPC2 heterozygous variants in patients with CSF evidence of amyloid deposition is higher than in the general population. Further studies are needed to highlight the connection between NPC, amyloid deposition and clinical presentation.

References:

- Zech M, Nübling G, Castrop F et al. Niemann-Pick C disease gene mutations and age-related neurodegenerative disorders. PloS One (2013);8(12):e82879

- Cupidi C, Frangipane F, Gallo M, et al. Role of Niemann-Pick Type C Disease Mutations in Dementia. Journal of Alzheimer's Disease (2017);55(3):1249-59

- Bauer P, Balding DJ, Klünemann HH, et al. Genetic screening for Niemann-Pick disease type $\mathrm{C}$ in adults with neurological and psychiatric symptoms: findings from the ZOOM study. Human Molecular Genetics (2013);22(21):4349-56

\section{STEPWISE CONNECTIVITY PAVES THE WAY TO REVEAL FUNCTIONAL NETWORK VULNERABILITY IN AGE-RELATED NEURODEGENERATIVE DISORDERS}

\author{
S. Basaia ${ }^{1}$, C. Cividini ${ }^{1}$, E. Spinelli ${ }^{1}$, V. Castelnovo ${ }^{1}$, M. Leocadi ${ }^{1}$, D. \\ Calderaro $^{1}$, E. Canu ${ }^{1}$, M. Filippi ${ }^{2}$, F. Agosta ${ }^{1}$
}

${ }^{1}$ Neuroimaging Research Unit, Institute of Experimental Neurology, Division of Neuroscience, IRCCS San Raffaele Scientific Institute; Vita-Salute San Raffaele University (Milano); ${ }^{2}$ Neuroimaging Research Unit, Institute of Experimental Neurology, Division of Neuroscience; Neurology Unit and Neurophysiology Unit, IRCCS San Raffaele Scientific Institute; Vita-Salute San Raffaele University (Milano)

Objective: Ageing is the main risk factor for most neurodegenerative diseases and results in complex transformations of the human brain function. The aim of this study was to investigate whether and how topological organization of the functional brain connectome changes with age using resting-state fMRI and stepwise functional connectivity (SFC) analyses.

Materials and Methods: Healthy controls were recruited and divided into two groups according to age: young (age range: $20-30$ years) and old (age range: $41-84$ years). SFC analysis is a graph-theory-based method that detects functional couplings of a seed region to other regions in the brain. SFC analysis aims to characterize regions that connect to specific seed brain areas at different levels of link-step distances. Eight wellknown hubs of the human connectome (i.e., regions highly interconnected and usually vulnerable to neurodegeneration) were selected as seeds for the present study: middle frontal gyrus, rostral anterior and posterior cingulate cortex, precuneus, inferior parietal, middle temporal and lingual gyri and pericalcarine cortex. The spatial locations of these functional cortical hubs overlap with subregions of the default mode network. Voxel-wise analyses and whole-brain two-sample t-test comparisons between groups were performed for each seed region.

Results: 138 healthy controls were recruited: 55 young (mean age: 25.44) and 83 old (mean age: 63.95). In the SFC analysis, significant differences were found between the two groups of healthy subjects. At one-link step distance, in older controls, all the seed regions displayed decreased regional-local functional connectivity with superior frontal and medial orbital frontal gyri, rostral anterior and isthmus cingulate cortex, precuneus and middle and inferior temporal gyri relative to young controls; across intermediate link-steps, a reduced connectivity was observed between all seed regions and frontal and parietal lobes. By contrast, at the first link-step distance, young controls showed lower connectivity only between few seed regions (middle frontal gyrus, anterior and posterior regions of cingulate cortex and precuneus) and precentral, paracentral and lateral occipital gyri compared to older controls. At intermediate link-step distances, increased connectivity with sensorimotor regions was found in older relative to younger controls.

Discussion and Conclusions: SFC revealed different patterns of functional network reorganization across nearly the entire human lifespan, highlighting that aging processes are characterized by increased connectivity at longer topological distances and decreased connectivity within topologically closer regions. SFC approach might have important implication providing a starting point for evaluating network disruptions in age-related neurodegenerative disorders.

Supported by: European Research Council (StG2016_714388_NeuroTRACK).

\section{PECULIAR PATTERNS OF MICROSTRUCTURAL DAMAGE IN THE FRONTAL ASLANT TRACT CHARACTERIZE DIFFERENT FORMS OF PRIMARY PROGRESSIVE APHASIA}

G. Bechi Gabrielli ${ }^{1}$, C. Marra ${ }^{2}$, C. Di Domenico ${ }^{1}$, C. Caltagirone ${ }^{3}, \mathrm{M}$. Cercignani $^{4}$, L. Serra ${ }^{1}$, M. Bozzali ${ }^{1,5}$ 
${ }^{1}$ Neuroimaging Laboratory, IRCCS Santa Lucia Foundation (Roma); ${ }^{2}$ Institute of Neurology, Catholic University (Roma); ${ }^{3}$ Department of Clinical and Behavioral Neurology, IRCCS Santa Lucia Foundation (Roma); ${ }^{4}$ Neuroimaging Laboratory, IRCCS Santa Lucia Foundation, Department of Neuroscience, Brighton and Sussex Medical School, University of Sussex (Roma, Brighton-UK); ${ }^{5}$ Department of Neuroscience "Rita Levi Montalcini", University of Torino (Torino)

Aim: The Frontal Aslant Tract (FAT) is a bundle connecting the Broca's area to the Supplementary Motor Area (SMA) and preSMA in both hemispheres [1]. This tract, which is left lateralized in right-handed subjects, is involved in planning, timing and coordination of voluntary sequential motor movements. In particular, the left FAT seems to be specialized in speech production [2]. Previous studies showed a microstructural damage of this tract in both, primary progressive aphasia (PPA) [1] and Alzheimer's disease (AD) [3]. We aimed here at exploreing in a voxel-wise fashion the microstructural integrity of the FAT in patients with PPA divided in its three different phenotypes.

Materials and Method: 20 consecutive patients with PPA were recruited and classified as suffering from nonfluent/agrammatic $(\mathrm{N}=7)$, logopenic PPA $(\mathrm{N}=8)$ or semantic PPA $(\mathrm{N}=5)$. All patients underwent MRI scanning at 3T, which included collection of diffusion imaging data used for probabilistic tractography. In each patient, we reconstructed the FAT bilaterally and quantified fractional anisotropy (FA) as measure of microstructural integrity. The statistical analysis was run by comparing, between groups, the average FA value of each tract, but also using a voxel-by-voxel approach. The latter method of analysis was run using statistical parametric mapping (SPM) 8 .

Results: There were no differences in demographic variables between the three groups. We did not find any significant difference between PPAvariants in average FA of the FAT in either hemisphere. Conversely, the voxel-wise analysis revealed reduced FA values in the portion of the left FAT near the SMA area in patients with agrammatic compared to those with semantic PPA.

Discussion: This study shows a selective pattern of microscopic damage in the left FAT of patients with agrammatic PPA patients. Patients with agrammatic were characterized by non-fluent speech, syntactic errors and speech apraxia. The peculiar localization of the additional damage we observed in agrammatic PPA patients is consistent with the functional anatomy of the left SMA, which is implicated in motor selection and execution. Additionally it accounts for the peculiar deficits in speech planning of this subgroup of PPA patients. Future studies are needed to clarify a potential use of this diffusion biomarker to identify sub-phenotypes of PPA at early clinical stages.

References:

1. Catani M, Mesulam MM, Jakobsen E, Malik F, Martersteck A, Wieneke C, Thompson CK, Thiebaut de Schotten M, Dell'Acqua F, Weintraub S, Rogalski E. A novel frontal pathway underlies verbal fluency in primary progressive aphasia. Brain (2013);136:2619-28

2. Dick A. S., Garic D., Graziano P., \& Tremblay P. The frontal aslant tract (FAT) and its role in speech, language and executive function. Cortex (2019);111:148-63

3. Serra L, Bechi Gabrielli G, Tuzzi E, SpanÃ2 B, Giulietti G, Failoni V, Marra C, Caltagirone C, Koch G, Cercignani M, Bozzali M. Damage to the Frontal Aslant Tract accounts for visuoconstructive deficits in Alzheimer's disease. J Alzheimers Dis. (2017);60(3):1015-24

\section{DISCRIMINATION OF EPISODIC MEMORY DEFICITS IN AMNESTIC AND NON-AMNESTIC FRONTOTEMPORAL DEMENTIA AND ALZHEIMER'S DISEASE}

C. Bussè, G. Zorzi, F. Fragiacomo, G. Gazzola, S. Mozzetta, A. Cagnin

Department of Neurosciences, University of Padua (Padova)

Background: Memory impairment in behavioral variant frontotemporal dementia (bvFTD) is traditionally considered to be approximately half of cases and the notion of a relatively preserved episodic memory used in the diagnostic criteria of FTD may be misinterpreted in the differential diagnosis with Alzheimer's disease (AD).

Aim: To test the cognitive profile and nature of memory deficits in patients with amnesic bvFTD (a-bvFTD) presenting with verbal memory deficits compared to non-amnestic bvFTD (na-bvFTD) and AD. Extensive cognitive testing and specific measures of Rey Auditory Verbal Learning Task (RAVLT) such as the Recency Ratio (RR) [Bruno et al. 2013] and the Recency Dominance (RD) [Kloth et al. 2019] were used.

Methods: Thirty-nine bvFTD $(n=39)$ and 63 AD patients were recruited at the Department of Neurosciences of the University of Padua (Italy). Healthy elderlies $(n=27)$ served as control group (HC). Based on the performance at the RAVLT delayed recall [as Kloth et al. 2019], bvFTD were split into amnestic (aFTD: $n=16)$ and non-amnestic (naFTD: $\mathrm{n}=22)$ subgroups. The $\mathrm{Rr}$ on trial 1 and $5(\mathrm{Rr}=$ immediate recency +1 divided by delayed recency +1 ) was computed. The Rd was also calculated for each patient by subtracting the relative primacy score (n. of recalled items on word list positions 1 and 2 divided by the overall $\mathrm{n}$. of items recalled) from the relative recency score (n. of recalled items on positions 14 and 15 divided by the overall $\mathrm{n}$. of items recalled) in learning trial 1.

Results: Patients with a-bvFTD were worse than AD only in phonological fluency $(\mathrm{p}=0.01)$ and digit span backwards $(\mathrm{p}=0.015)$. RAVLT DR did not distinguish AD from a-bvFTD ( $\mathrm{AD}=1.93 \pm 2.54$; abvFTD=1.94 $\pm 1.44 ; \mathrm{p}=1.00)$. In RAVLT- IR Trial 5, a-bvFTD patients recalled less words respect to $\mathrm{AD}(\mathrm{a}-\mathrm{bvFTD}=4.53 \pm 1.73 ; \mathrm{AD}=6.13 \pm 2.39$; $\mathrm{p}=0.010$ ) and to na-bvFTD patients (na-bvFTD=7.27 $\pm 2.80 ; \mathrm{p}=0.013$ ). Regarding serial position effects, only Primacy on 2 positions (P2) at Trial 5 reported significant differences with higher values for $\mathrm{P} 2$ in $\mathrm{AD}$ respect to a-bvFTD ( $\mathrm{a}-\mathrm{bvFTD}=0.56 \pm 0.73 ; \mathrm{AD}=1.16 \pm 0.59 ; \mathrm{p}=0.018$ ).

Conclusions: $\mathrm{AD}$ and bvFTD patients might present with comparable memory deficits in the overall immediate recalls, first four learning trials and serial position effects. However, aFTD benefited less than AD after five repeated exposure to the learning lists, suggesting that aFTD may exhibit different patterns of underlying memory processing, involving frontal and executive functions as learning strategies.

\section{BEHAVIOURAL AND PSYCHOLOGICAL EFFECTS OF COVID- 19 QUARANTINE IN PATIENTS WITH DEMENTIA}

A. Cagnin ${ }^{1}$, R. Di Lorenzo ${ }^{2}$, C. Marra ${ }^{3}$, L. Bonanni ${ }^{4}$, C. Cupidi ${ }^{5}$, V. Laganà $^{2}$, E. Rubino ${ }^{6}$, A. Vacca ${ }^{7}$, P. Provero ${ }^{8}$, V. Isella ${ }^{9}$, N. Vanacore ${ }^{10}$, F. Agosta ${ }^{11}$, I. Apollonio 9 , P. Caffarra ${ }^{12}$, I. Pettenuzzo ${ }^{1}$, R. Sambati ${ }^{13}$, D. Quaranta $^{3}$, V. Guglielmi ${ }^{3}$, G. Logroscino ${ }^{13}$, M. Filippi ${ }^{11}$, G. Tedeschi ${ }^{14}$, C. Ferrarese $^{9}$, I. Rainero ${ }^{7}$, A. Bruni ${ }^{3}$, -. COVID_19 Study Group ${ }^{15}$

${ }^{1}$ Department of Neurosciences, University of Padua (Padova); ${ }^{2}$ Regional Neurogenetic Centre, ASP Catanzaro (Lamezia Terme-CZ); ${ }^{3}$ Memory Clinic, Fondazione Policlinico Gemelli, Università IRCCS Cattolica del 
Sacro Cuore (Roma); ${ }^{4}$ Department of Neuroscience, Imaging and Clinical Sciences, University G. d'Annunzio (Chieti); ${ }^{5}$ CDCD Ospedale del Delta, AUSL Ferrara (Ferrara); ${ }^{6}$ Department of Neuroscience and Mental Health, AOU Città della Salute e della Scienza di Torino (Torino); ${ }^{7}$ Aging Brain and Memory Clinic, Department of Neuroscience, University of Torino (Torino); ${ }^{8}$ Department of Molecular Biotechnology and Health Sciences, University of Torino (Torino); ${ }^{9}$ Department of Medicine and Surgery and Milan Center for Neuroscience, University of Milano - Bicocca, and Ospedale San Gerardo (Monza); ${ }^{10}$ National Institute of Health (Roma); ${ }^{11}$ Institute of Experimental Neurology, Division of Neuroscience, Vita-Salute San Raffaele University (Milano); ${ }^{12}$ Department of Medicine and Surgery, Section of Neuroscience, University of Parma (Parma); ${ }^{13} 1$ Center for Neurodegenerative Diseases and the Aging Brain, University Aldo Moro (Bari); ${ }^{14}$ First Division of Neurology, University of Campania (Napoli); ${ }^{15}$ SINDEM - SINDEM - Siena

Background: In March 2020, the World Health Organization declared a global pandemic due to the novel coronavirus SARS-CoV-2 and several governments planned a national quarantine in order to control the virus spread. Acute psychological effects of quarantine in frail elderly subjects with special needs, such as patients with dementia, have been poorly investigated. The aim of this study was to assess modifications of neuropsychiatric symptoms during quarantine in patients with dementia and their caregivers.

Methods: This is a sub-study of a multicenter nation-wide survey. A structured telephone interview was delivered to family caregivers of patients with diagnosis of Alzheimer disease (AD), dementia with Lewy bodies (DLB), frontotemporal dementia (FTD) and vascular dementia (VD), followed regularly at 87 Italian memory clinics. Variations in behavioral and psychological symptoms (BPSD) were collected after one month since quarantine declaration and associations with disease type, severity, gender and caregiver's stress burden were analyzed.

Results: A total of 4913 caregivers participated in the survey. Increased BPSD was reported in $59.6 \%$ of patients as worsening of preexisting symptoms $(51.9 \%)$ or as new onset $(26 \%)$, and requested drug modifications in $27.6 \%$ of these cases. Irritability, apathy, agitation and anxiety were the most frequently reported worsening symptoms and sleep disorder and irritability the most frequent new symptoms. Profile of BPSD varied according to dementia type, disease severity and patients' gender. Anxiety and depression were associated with a diagnosis of $\mathrm{AD}$ (OR 1.35, CI: 1.12-1.62), mild to moderate disease severity and female gender. DLB was significantly associated with a higher risk of worsening hallucinations (OR 5.29, CI 3.66-7.64) and sleep disorder (OR 1.69, CI 1.25-2.29), FTD with wandering (OR 1.62, CI 1.12-2.35) and change of appetite (OR 1.52, CI 1.03-2.25). Stress-related symptoms were experienced by twothirds of caregivers and were associated with increased patients' neuropsychiatric burden $(\mathrm{p}<0.0001)$.

Conclusion: Quarantine induces a rapid increase of neuropsychiatric symptoms in approximately $60 \%$ of patients and in two-thirds of caregivers. Health services need to plan a post-pandemic strategy in order to address these emerging needs.

\section{RESTING-STATE ELECTROENCEPHALOGRAPHIC BIOMARKERS OF ALZHEIMER'S DISEASE}

\author{
G. Cecchetti ${ }^{1}$, F. Agosta ${ }^{1}$, S. Basaia ${ }^{1}$, C. Cividini ${ }^{1}$, M. Cursi ${ }^{2}$, R.
} Santangelo $^{3}$, F. Caso ${ }^{3}$, F. Minicucci ${ }^{2}$, G. Magnani ${ }^{3}$, M. Filippi ${ }^{4}$
${ }^{1}$ Neuroimaging Research Unit, Institute of Experimental Neurology, Division of Neuroscience, IRCCS San Raffaele Scientific Institute; Vita-Salute San Raffaele University (Milano); ${ }^{2}$ Neurophysiology Unit, IRCCS San Raffaele Scientific Institute (Milano); ${ }^{3}$ Neurology Unit, IRCCS San Raffaele Scientific Institute (Milano); ${ }^{4}$ Neuroimaging Research Unit, Institute of Experimental Neurology, Division of Neuroscience; Neurology Unit and Neurophysiology Unit, IRCCS San Raffaele Scientific Institute; Vita-Salute San Raffaele University (Milano)

Objectives: In the last decade, the challenging need for early diagnosis in Alzheimer's disease has led to the development and constant redefinition of both diagnostic and staging biomarkers. Among others, resting-state EEG shows outstanding features that make it particularly suitable for application into the study of Alzheimer's disease: non-invasiveness, speed of execution, low cost and large availability. Besides scalp power spectral analysis, recent studies have also applied different EEG source estimation algorithms that allow to disentangle the contribution of distinct cortical generators to scalp signal. In this scenario, using exact low-resolution brain electromagnetic tomography, we assessed the value of resting-state EEG cortical source biomarkers to characterize mild cognitive impairment (MCI) subjects with an Alzheimer's disease-like CSF profile, and to track neurodegeneration throughout the Alzheimer continuum. To overcome the wellknown low spatial resolution of EEG, furthermore, we applied a restingstate functional MRI-driven model of source reconstruction and tested its advantage in terms of diagnostic accuracy.

Methods: Thirty-nine patients with Alzheimer's disease dementia (ADD), 86 amnestic MCI, and 33 healthy subjects enter the EEG study. All ADD subjects, 37 out of $86 \mathrm{MCI}$ patients and a distinct group of 53 healthy controls further entered the functional MRI study. MCI subjects were divided according to the CSF phosphorylated tau $\beta$ amyloid-42 ratio (MCIpos: $\geq 0.13$, MCIneg: < 0.13). EEG lobar current densities were estimated at fixed frequencies. To combine the two imaging techniques, networks mostly affected by $\mathrm{AD}$ pathology were identified using Independent Component Analysis applied to functional MRI data. Current density analysis within MRI networks at selected frequency bands was performed. Afterwards, graph analysis was applied to EEG and functional MRI data at network level; correspondently, linear lagged connectivity and Pearson's coefficient values were entered into the connectivity matrix.

Results: ADD patients showed a widespread slowing of spectral density. MCIpos subjects showed significantly higher theta density than MCIneg (statistical evidence). Evaluating EEG sources within the MRI networks, alpha2 band distinguished MCIpos from MCIneg, ADD and healthy subjects with good accuracy. Graph analysis on EEG data showed an alteration of connectome configuration at theta frequency in ADD and MCIpos patients and a progressive disruption of connectivity at alpha2 frequency throughout the $\mathrm{AD}$ continuum.

Conclusions: Theta frequency is the earliest and most sensitive EEG marker of AD. Furthermore, the integration of EEG and functional MRI highlighted the role of alpha2 band as potential neurodegeneration biomarker.

Funding. Italian Ministry of Health (GR-2011-02351217).

\section{MATHEMATICAL MODELING REVEALS THE CORRELATES OF COGNITIVE IMPAIRMENT ACROSS THE FTLD SPECTRUM}

\author{
C. Cividini ${ }^{1}$, F. Agosta $^{1}$, S. Basaia ${ }^{1}$, E. Spinelli ${ }^{1}$, V. Castelnovo ${ }^{1}$, E.
} $\mathrm{Canu}^{1}$, N. Riva ${ }^{2}$, G. Magnani ${ }^{3}$, F. Caso ${ }^{3}$, M. Filippi ${ }^{4}$ 
${ }^{1}$ Neuroimaging Research Unit, Institute of Experimental Neurology, Division of Neuroscience, IRCCS San Raffaele Scientific Institute; Vita-Salute San Raffaele University (Milano); ${ }^{2}$ Unit of Neurorehabilitation, IRCCS San Raffaele Scientific Institute (Milano); ${ }^{3}$ Neurology Unit, IRCCS San Raffaele Scientific Institute (Milano); ${ }^{4}$ Neuroimaging Research Unit, Institute of Experimental Neurology, Division of Neuroscience; Neurology Unit and Neurophysiology Unit, IRCCS San Raffaele Scientific Institute; Vita-Salute San Raffaele University (Milano)

Objective: Patients with amyotrophic lateral sclerosis (ALS), characterized by motor system degeneration, can also develop cognitive and/or behavioural symptoms that lie on a continuum with the behavioral variant of frontotemporal dementia (bvFTD). The aim of this study was to apply mathematical modeling to unravel MRI connectomic signatures of cognitive and/or behavioral impairment in ALS patients.

Materials: Eighty-three ALS, 35 bvFTD and 61 controls underwent clinical/cognitive evaluations and MRI scan. Neuropsychological testing identified 54 ALS pure motor (ALSpm), 21 ALS with mild cognitive and/ or behavioral impairment (ALSci/bi) and 8 ALS with bvFTD (ALS-FTD).

Methods: The structural macroscale connectome of each subject was constructed. Connections linking cerebral lobes to each other were considered. The structural value of each connection for each patient was normalized relative to controls. The percentage of patients showing intra- and inter-lobe alterations was calculated considering those patients with normalized structural values below the reference value, i.e., zero. A statistical distribution analysis, intra- and inter-lobes, was performed to identify different structural patterns between bvFTD and ALSpm and to assess where ALSci/bi and ALS-FTD showed an ALS-like or a bvFTD-like pattern.

Results: Compared to ALSpm, bvFTD showed greater structural involvement of the connections within and between frontal, temporal and parietal lobes, which were altered in most bvFTD patients (92\%). On the other hand, $80 \%$ of ALSpm patients showed greater involvement of the connections between sensorimotor and basal ganglia areas, compared with bvFTD (66\%). Similar to ALSpm, ALSci/bi was characterized by a relative preservation of the connections within frontal lobe and between frontal, temporal and basal ganglia areas. Noteworthy, ALSci/bi showed an ALS-like pattern within motor areas and a bvFTD-like pattern within the parietotemporal connections. Indeed, the percentage of ALSci/bi with structural alterations in the parietotemporal areas (57\%-71\%) was increased compared to that of ALSpm (54\%-57\%). Finally, ALS-FTD showed a pattern similar to bvFTD within frontal and between frontal and sensorimotor areas, while they showed an ALSpm-like pattern within sensorimotor and basal ganglia areas.

Discussion and Conclusions: This study showed a widespread structural damage in bvFTD, particularly in the connections within frontotemporal areas and between frontal and other lobes, and a more focal structural damage within sensorimotor-basal ganglia areas in ALSpm. ALSci/bi and ALS-FTD showed an ALS-like pattern with the exception of a greater damage within the frontal and parietotemporal areas, signature of the occurrence of cognitive impairment.

Supported by. The Italian Ministry of Health (GR-2011-02351217; GR-2013-02357415; RF-2011-02351193) and AriSLA (ConnectALS).

\section{IMPACT OF DIETARY HABITS ON THE INCIDENCE OF LATE- ONSET DEPRESSION IN THE GREATAGE STUDY}

E. M. Costantini ${ }^{1,2}$, A. Daniele ${ }^{1,2}$, L. Lampignano ${ }^{3}$, M. Lozupone ${ }^{4}$, R. Donghia $^{3}$, V. Guerra ${ }^{3}$, F. D’Urso ${ }^{5}$, I. Di Gioia ${ }^{5}$, C. Piccininni ${ }^{5}$, R.

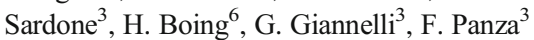

${ }^{1}$ Institute of Neurology, Catholic University of Sacred Heart (Roma); ${ }^{2}$ Fondazione Policlinico Universitario A. Gemelli IRCCS (Roma); ${ }^{3}$ National Institute of Gastroenterology "S. de Bellis", Research Hospital (Castellana Grotte-BA); ${ }^{4}$ Neurodegenerative Disease Unit, Department of Basic Medicine, Neuroscience, and Sense Organs, University of Bari Aldo Moro (Bari); ${ }^{5}$ Psychiatric Unit, Department of Clinical and Experimental Medicine, University of Foggia (Foggia); ${ }^{6}$ Department of Molecular Epidemiology, German Institute of Human Nutrition Potsdam-Rehbruecke (Nuthetal-D)

Background and Aim: The association between diet and depression has been studied in numerous studies, but little is known about the possible relationship between eating habits and late-onset depression (LOD, defined as late-age of depression onset in older people), because of LOD relationship with neurodegenerative mechanisms. In this longitudinal population-based study (from 2003 to 2018), we examined the relationship of consumption of different food groups with the incidence of LOD in a cohort from Southern Italy.

Methods: Baseline data (MICOL3 Study) were recorded from 2003 to 2006 and the follow-up data from 2013 to 2018 (GreatAGE Study) enclosed in the "Salus in Apulia Study", a public health survey funded by Italian Ministry of Health and Apulia Regional Government and conducted at IRCCS "S. De Bellis" Research Hospital, Castellana Grotte. All individuals involved in this study ( $\mathrm{n}=546,61.5 \%$ men) had the same clinical, biological and nutritional measures both at baseline and follow up. Dietary habits were evaluated through a validated food frequency questionnaire. The assessment of depressive disorders and other psychiatric disorders was obtained by the Semi-structured Clinical Diagnostic Interview for DSMIV-TR Axis I Disorders. Cox regression models were used for longitudinal analysis on associations between LOD and single food groups, with adjustment for socio-demographic, lifestyle and clinical factors.

Results: Processed meat significantly increased the risk of incident LOD [raw model hazard ratio (HR):1.02; 95\% confidence interval (CI):1.01-1.03 partially-adjusted HR:1.02; 95\% CI:1.01-1.04 and totally-adjusted HR;1.02; 95\% CI:1.01-1.03). Focusing on each single food that makes up the "processed meat" group, sausage (raw model HR:1.02; 95\% CI:1.00-1.04 and partially-adjusted HR:1.03; 95\% CI:1.01-1.05), mortadella and baked ham (raw HR: 1.03 ; 95\% CI:1.01-1.05, partially-adjusted HR:1.04; 95\% CI:1.02-1.06 and totally-adjusted HR:1.05; 95\% C.I. 1.02-1.09) and salami consumption (partially-adjusted HR:1.10; 95\% CI:1.02-1.19 and totally-adjusted HR:1.09; 95\% CI:1.01-1.18) significantly increased the risk of incident LOD.

Conclusion: Higher consumption of processed meat, characterized by a high content of salt, saturated fats and preservatives, is associated with a higher risk of incident LOD. Our findings reinforce the idea that LOD is one of the chronic diseases associated with unhealthy effects of processed meat, particularly neurodegeneration.

References

1. Quirk, S.E., Williams, L.J., O'Neil, A. et al. The association between diet quality, dietary patterns and depression in adults: a systematic review. BMC Psychiatry 13, 175 (2013). https://doi.org/10. 1186/1471-244X-13-175

2. Zhang, Y., Yang, Y., Xie, M. et al. Is meat consumption associated with depression? A meta-analysis of observational studies. BMC Psychiatry 17, 409 (2017). https://doi.org/10.1186/s12888-0171540-7

3. Morris MC, Tangney CC. Diet and Prevention of Alzheimer DiseaseDiet and Prevention of Alzheimer Disease. JAMA. 2010;303(24):2519-2520. doi:10.1001/jama.2010.844 


\section{HYPERCONNECTIVITY IN DEMENTIA IS EARLY AND FOCAL AND WANES WITH PROGRESSION}

L. Ferri ${ }^{1}$, L. Bonanni ${ }^{1}$, D. Moretti ${ }^{2}$, A. Benussi ${ }^{3}$, M. Russo ${ }^{1}$, C. Carrarini $^{1}$, F. Barbone ${ }^{1}$, D. Arnaldi ${ }^{4}$, N. Falasca ${ }^{1}$, G. Koch ${ }^{5}$, A. Cagnin $^{6}$, F. Nobili ${ }^{4}$, B. Borroni ${ }^{3}$, A. Padovani ${ }^{3}$, C. Babiloni ${ }^{8}$, M. Onofrj ${ }^{1}$, R. Franciotti ${ }^{1}$

${ }^{1}$ Department of Neuroscience, Imaging and Clinical Sciences, University G. d'Annunzio (Chieti); ${ }^{2}$ IRCCS Istituto Centro San Giovanni di Dio Fatebenefratelli, University of Brescia (Brescia); ${ }^{3}$ Neurology Unit, Department of Clinical and Experimental Sciences, University of Brescia (Brescia); ${ }^{4}$ Department of Neuroscience, Rehabilitation, Ophthalmology, Genetics, Maternal and Child Health (DINOGMI), University of Genoa (Genova); ${ }^{5}$ Non Invasive Brain Stimulation Unit/Department of Behavioral and Clinical Neurology, Santa Lucia Foundation IRCCS (Roma); ${ }^{6}$ Department of Neurosciences, University of Padua (Padova); ${ }^{8}$ Department of Physiology and Pharmacology "V. Erspamer", Sapienza University of Rome (Roma)

We investigated in a longitudinal multicentre cohort functional cortical connectivity changes along the course of Frontotemporal Dementia (FTD) and Alzheimer's Disease (AD) from the prodromal stage of the diseases. EEG was recorded in 18 FTD and 18 AD patients at the prodromal stage of dementia, at dementia onset and three years after dementia onset. 20 healthy controls (HC) underwent EEG recordings at the same time interval as the patients. Mutual Information (MI) analysis measured the strength of functional network connectivity. FTD and AD patients showed greater MI at the prodromal stage of dementia (FTD vs. HC $\mathrm{p}=2 \cdot 10-8$; AD vs. $\mathrm{HC} \mathrm{p}=4 \cdot 10-3$ ). Local connectivity was higher in left and right frontal areas of FTD $(\mathrm{p}=7 \cdot 10-5$ and $\mathrm{p}=0.03)$ and in left and right posterior areas in $\mathrm{AD}(\mathrm{l}=3 \cdot 10-5$ and $\mathrm{p}=5 \cdot 10-5)$ vs. HC. We showed cortical hyperconnectivity in the prodromal stage of dementia in areas involved in the specific pathological process of FTD (frontal regions) and $\mathrm{AD}$ (posterior regions). Hyperconnectivity disappeared during follow up, thus suggesting that it is an early electrophysiological feature of dementia, potentially useful to identify prodromal FTD and AD.

References:

- Rascovsky K, Hodges JR, Knopman D, Mendez MF, Kramer JH, Neuhaus J, et al. Sensitivity of revised diagnostic criteria for the behavioural variant of frontotemporal dementia. Brain (2011); 134: 2456-77

- Supekar K, Menon V, Rubin D, Musen M, Greicius MD. Network analysis of intrinsic functional brain connectivity in Alzheimer's disease. PLoS Comput Biol (2008); 4: e1000100

- Wang ZJ, Lee PWH, McKeown MJ. A novel segmentation, mutual information network framework for EEG analysis of motor tasks. Biomed Eng Online (2009);8:9

\section{DEMENTIA IN PATIENTS WITH PSYCHIATRIC DISORDERS: NEURODEGENERATIVE SYNDROME OR PSEUDO- DEMENTIA?}

L. Fiondella, G. Vinceti, S. Salemme, M. Tondelli, M. Molinari, A. Chiari, G. Zamboni

Integrated Neuroscience Department, AOU Modena, University of Modena (Modena)
Introduction: The term pseudo-dementia (PDEM) refers to cases that closely mimic dementia, and has been especially used to describe the cognitive impairment occurring in patients with psychiatric disorders $[1,2]$. However, neurodegenerative dementia syndromes (NDS) with atypical/behavioral presentation may also occur in patients with psychiatric disorders, making the diagnosis challenging. We studied the baseline clinical, demographic, neuropsychological features, as well as the imaging and CSF biomarkers of a cohort of patients with psychiatric disorders subsequently diagnosed with PDEM or NDS.

Methods: We retrospectively recruited patients with a history of a psychiatric disorder who had been referred to the Cognitive Neurology Clinic in Modena for assessment of behavioral/cognitive changes. Most patients had undergone neuropsychological assessment, structural MRI and FDG-PET imaging, and, in selected cases, measurement of CSF biomarkers for $\mathrm{AD}$ at baseline. We stratified them in PDEM and NDS according to the diagnosis they received at clinical follow-up. T1weighted MRI images available from a subgroup of patients were used to perform a Voxel-Based-Morphometry comparison (VBM) between PDEM, NDS and a healthy controls group (CTRL, 20 subjects). Volumes of subcortical structure were also extracted with FSL-FIRST and compared between groups.

Results: Fifty consecutive eligible patients were identified between June 2007 and December 2019, of whom 15 were diagnosed with NDS (all FTD) and 35 with PDEM. There were no baseline significant differences in demographical and CSF biomarkers data. There were no baseline significant differences in tests of verbal and visuospatial memory, WAISIV, Trial Making Test, Stroop Test and Frontal Assessment Battery. Whole-brain VBM showed that NDS patients were significantly more atrophic than CTRL in the left temporal pole and right superior temporal gyrus $(\mathrm{p}<0.05)$. PDEM patients were significantly more atrophic than CTRL in the right putamen $(\mathrm{p}<0.05)$. NDS patients were significantly more atrophic than PDEM in the left temporal pole $(p<0.05)$. As for subcortical structures, there were significant differences in the volume of the striatum between PDEM and CTRL $(p<0,05)$ and between NDS and CTRL $(\mathrm{p}<0,05)$.

Conclusions: Our study highlights a limited usefulness of neuropsychological test and CSF biomarkers in distinguishing between neurodegenerative (NDS) and pseudo-dementia (PDEM) syndromes. Structural imaging can distinguish between the two in that NDS have greater cortical atrophy. Therefore, MRI and clinical follow up are essential for a correct differential diagnosis. Interestingly, both NDS and PDEM show atrophy in striatal structures, possibly related to the effect of exposure to psychiatric treatment or to the effect of the psychiatric disease itself.

References:

1. Kiloh LG. Pseudo-dementia. Acta Psychiatr Scand. (1961);37:336-51

2. Fengqing Zhao. Pseudo-dementia: A neuropsychological review, Ann Indian Acad Neurol. (2014) Apr-Jun; 17(2): 147-54

3. Caine ED. The neuropsychology of depression: The pseudodementia syndrome. In: Grant KA, editor. Neuropsychological Assessment of Neuropsychiatric Disorders. New York: Oxford University Press; (1986):221-43

\section{LOCUS COERULEUS SEXUAL DIMORPHISM ASSESSED WITH LC-MRI IN NORMAL AGING AND ALZHEIMER'S DISEASE}

A. Galgani ${ }^{1}$, F. Lombardo ${ }^{2}$, N. Martini ${ }^{3}$, D. Della Latta ${ }^{3}$, H. Hlavata ${ }^{2}$, F. Baldacci $^{1}$, I. Ghicopulos ${ }^{1}$, N. Campese ${ }^{1}$, G. Tognoni ${ }^{1}$, R. Ceravolo ${ }^{1}$, G. Siciliano $^{1}$, F. Fornai ${ }^{4,5}$, N. Pavese ${ }^{6}$, U. Bonuccelli ${ }^{1}$, F. Sean Giorgi ${ }^{1,4}$ 
${ }^{1}$ Neurology Unit, Pisa University Hospital (Pisa); ${ }^{2}$ Magnetic Resonance Imaging and Neuroradiology Unit, "G. Monasterio" Foundation", National Research Council/Tuscany Region (Pisa); ${ }^{3}$ Deep Health Unit, Fondazione "G. Monasterio", National Research Council/Tuscany Region (Pisa); ${ }^{4}$ Department of Translational Research and of New Surgical and Medical Technologies, University of Pisa (Pisa); ${ }^{5}$ IRCCS Neuromed (Pozzilli-IS); ${ }^{6}$ Clinical Ageing Research Unit, Newcastle University (Newcastle upon Tyne-UK)

Objectives: LC is the main noradrenergic nucleus of the brain and its degeneration is an early feature of $\mathrm{AD}$ pathogenesis and has been linked to increased neuroinflammation and amyloid accumulation. Females are more at risk of developing $\mathrm{AD}$ compared to males, and several pieces of evidence support the hypothesis of LC sexual dimorphism. Therefore we evaluated LC-gender related features in normal aging and Alzheimer's Disease.

Methods: From a larger population of subjects included in a prospective study on LC-MRI, we selectred 119 subjects (70 females, 49 males), divided into three groups: AD Dementia, Mild Cognitive Impairment, and healthy controls. All subjects were submitted to neurological and neuropsychological evaluation and then underwent an MRI scan with LC-sensitive T1-weighted sequences. LC-MRI images were then processed using a semi-automatic protocol and results were expressed with the parameter VOX (i.e. number of voxels positive for LC signal).

Results: LC signal is significantly reduced in ADD when compared to $\mathrm{HC}$, while no differences were observed comparing MCI either with $\mathrm{HC}$ or ADD. Considering the whole group, VOX was significantly higher in females than in males, and similar results were obtained considering cognitively impaired subjects (ADD+MCI) and the MCI group. A slight not significant increase of VOX occurs in females HC, while no genderrelated differences were observed in the ADD group. Finally, we did not find any differences in terms of neuropsychological impairment comparing males and females, within each clinical group.

Conclusion: $\mathrm{LC}$ is impaired in subjects with ADD compared to HC. Even though females are more at risk of developing AD, we showed that in MCI and HC LC-Vox is slightly higher than in males.

Discussion: As females are characterized by higher VOX values than males, such a difference should be taken in account when performing LCMRI analysis, both in healthy subjects and in patients, in order to better interpret results of LC-MRI.

Funding sponsor: Ministero della Salute, Ricerca Finalizzata \#PE2013-02359574 (P.I.: FSG).

\section{DISCRIMINATING THE COGNITIVE PROFILE OF SUBCORTICAL DEMENTIAS: NEURODEGENERATIVE VS. CEREBROVASCULAR}

\author{
C. Gendarini ${ }^{1}$, E. Aiello ${ }^{2}$, T. Difonzo ${ }^{3}$, S. Zago $^{3}$, N. Bresolin ${ }^{1}$, M. Saetti ${ }^{1}$
}

${ }^{1}$ Department of Medical-Surgical Pathophysiology and Transplantation, IRCCS Foundation Ca' Granda Ospedale Maggiore Policlinico, University of Milan (Milano); ${ }^{2}$ School of Medicine and Surgery, University of Milano-Bicocca (Milano); ${ }^{3}$ Department of Neuroscience and Mental Health, IRCCS Foundation Ca' Granda Ospedale Maggiore Policlinico (Milano)

Objective: Neuropsychological features of subcortical dementias include attentive and executive dysfunctions, language deficits, as well as memory disturbances [1]. This clinical syndrome is believed to be caused by damages to fronto-subcortical circuits [2]. Etiologically different damages to the aforementioned circuits might give rise to different cognitive deficits. This study aimed at comparing the cognitive profile of patients with subcortical dementias of either neurodegenerative (Parkinson's disease, PD) or cerebrovascular (small vessel disease, SVD) etiology.

Materials: Cognitive performances of 110 patients diagnosed with either PD or SVD were retrospectively collected. Patients were divided into 5 groups according to the severity of cognitive impairment: PD without cognitive impairment (PD-); PD-mild cognitive impairment (PD-MCI), PD-dementia (PD-D), SVD-MCI, SVD-D. The battery included 22 clinical tests encompassing attention, executive functioning, language, memory, visuo-spatial abilities and constructional praxis.

Methods: Univariate between-group comparisons were performed by covarying age, education and disease duration in order to identify which tests were able to discriminate between different degrees of cognitive impairment in both PD and SVD groups. Selected comparisons between PD and SVD groups were similarly performed.

Results: The Trail Making Test (TMT), as well as phonemic, semantic and alternate (phonemic-semantic) verbal fluency (VF) tasks allowed to discriminate between different degrees of cognitive impairment in $\mathrm{PD}$ patients (PD- > PD-MCI; PD-MCI > PD-dementia). Almost all tests allowed to discriminate SVD-MCI from SVD-dementia patients (SVD-MCI > SVD-dementia). The vast majority of PD patients performed normally in a confrontation naming test (Boston Naming Test, BNT) and a significant difference between the 2 dementia groups was found on the BNT (PD-D > SVD-D).

Discussion: PD patients found it particularly difficult to perform tasks engaging working memory (WM) abilities. This may be due to the damaged connections between caudate nuclei and dorsolateral prefrontal cortex (an area specialized in the online monitoring and manipulating of information) that characterize PD-MCI patients' anatomical features [3]. Lexical retrieval deficit seems specific to SVD-dementia patients' cognitive profile, suggesting that the underlying subcortical pathology may be widespread and might also involve perisylvian language networks.

Conclusions: TMT and VF tasks seem to be particularly sensitive to cognitive impairment in PD patients, whereas confrontation naming tests might be able to discriminate between PD-D and SVD-D patients. The differences between the cognitive profile may be due to different underlying pathologies (localised in PD versus widespread in SVD). References:

1. Bonelli RM, Cummings JL. Frontal-subcortical dementias. Neurologist (2008);14(2):100-107

2. Tekin S, Cummings JL. Frontal-subcortical neuronal circuits and clinical neuropsychiatry: An update. J Psychosom Res. (2002);53(2):647-54

3. Hanganu A, Houde JC, Fonov VS, et al. White matter degeneration profile in the cognitive cortico-subcortical tracts in Parkinson's disease. Mov Disord. (2018);33(7):1139-11

\section{CONCORDANCE BETWEEN CLINICAL DIAGNOSIS AND AMYLOID BURDEN BIOMARKERS IN ALZHEIMER'S DISEASE AND FRONTOTEMPORAL DEMENTIA}

G. Giacomucci ${ }^{1}$, S. Mazzeo ${ }^{1}$, S. Bagnoli ${ }^{1}$, M. Casini ${ }^{1}$, S. Padiglioni ${ }^{1}$, C. Polito $^{1}$, V. Berti ${ }^{2}$, M. Carraro ${ }^{1}$, J. Balestrini ${ }^{1}$, G. Lombardi ${ }^{3}$, C. Ferrari ${ }^{1}$, A. Ingannato ${ }^{1}, \mathrm{~S}$. Sorbi ${ }^{1}$, B. Nacmias ${ }^{1}$, V. Bessi ${ }^{1}$

${ }^{1}$ Department of Neuroscience, Psychology, Drug Research and Child Health, University of Florence, Azienda Ospedaliera-Universitaria Careggi (Firenze); ${ }^{2}$ Nuclear Medicine Unit, Azienda OspedalieroUniversitaria Careggi (Firenze); ${ }^{3}$ IRCCS Fondazione Don Carlo Gnocchi (Firenze) 
Objectives: The aim of this study is to compare the accuracy of different amyloid biomarkers in patients with clinical diagnosis of Alzheimer's Disease (AD) or Frontotemporal Dementia (FTD).

Materials and methods: We considered 258 patients who referred to our center between 2010 and 2020 for evaluation of cognitive decline. All patients underwent clinical and neuropsychological assessment at baseline and neuropsychological follow-up every 12-24 months. One hundred-eighty-seven patients underwent Apolipoprotein E (ApoE) genotyping. All the patients had at least one biomarker of amyloid burden (CSF biomarkers measurement or amyloid-PET). Forty-four patients underwent both of them. Patients were rated $A \beta+$ if at least one of the amyloid biomarkers (CSF or Amyloid PET) revealed presence of $A \beta$ positivity or $A \beta$ - if none of the biomarkers revealed presence of $A \beta$ pathology. We considered patients who were received a clinical diagnosis of $\mathrm{AD}$ or of FTD. We finally included 148 patients.

Results: Of the 148 patients, 95 (64\%) were diagnosed with probable $\mathrm{AD}, 53(36 \%)$ with probable FTD. We evaluated the concordance between clinical diagnosis and single or combined $A \beta$ biomarkers. Amyloid-PET presented the highest concordance with clinical diagnosis, statistically significantly higher than CSF A $\beta 1-42(86.21 \%, 95 \%$ I.C. 8.87 vs 69.05 , IC 8.07 ) but not than CSF $A \beta 42 / 40$ ratio (78.26\% I.C. 9.73). Considering the group of patients who underwent both CFS biomarkers analysis and amyloid-PET, we evaluated the concordance among the two $\mathrm{A} \beta$ biomarkers. Concordance was very low in $\mathrm{AD}$ patients, while it was significantly higher in FTD patients $(39.13 \%$, 95\% I.C. 19.94 vs $76.92 \%, 95 \%$ I.C. 22.90). The discordance between CSF biomarkers and amyloid-PET was not influenced by demographic features. Considering $\mathrm{A} \beta+$ FTD patients, we found a trend to significance for ApoE $\varepsilon 4$ allele $(\mathrm{p}=0.065)$ which was more frequent in FTD patients with $\mathrm{A} \beta$ pathology. From a qualitative point of view, any patients with clinical diagnosis of $\mathrm{AD}$ presented both negative $\mathrm{A} \beta$ biomarkers.

Discussion: Despite diagnostic accuracy of amyloid-PET was higher than CSF A $\beta 1-42$, this significant difference was not more present when compared to CSF $A \beta 42 / 40$ ratio. When we evaluated the concordance between CSF biomarkers and amyloid-PET we found that all patients with clinical diagnosis of $\mathrm{AD}$ had at least one positive biomarker.

Conclusion: A combination of both amyloid-PET and CSF biomarkers should be performed to better characterize unclear cases of dementia. If the two biomarkers of amyloid burden are both negative, an underlying $\mathrm{AD}$ pathology could be most probably excluded.

\section{ASSESSMENT OF THE ROLE OF LOCUS COERULEUS IN ALZHEIMER'S DISEASE AND OTHER TYPES OF DEMENTIA}

F. S. Giorgi ${ }^{1,2}$, A. Galgani ${ }^{1}$, F. Lombardo ${ }^{3}$, N. Martini ${ }^{4}$, D. Della Latta ${ }^{4}$, H. Hlavata ${ }^{3}$, G. Palermo ${ }^{1}$, F. Baldacci ${ }^{1}$, I. Ghicopulos ${ }^{1}$, G. Tognoni ${ }^{1}$, G. Siciliano $^{1}$, R. Ceravolo ${ }^{1}$, F. Fornai ${ }^{2,5}$, N. Pavese ${ }^{6}$, U. Bonuccelli ${ }^{1}$

${ }^{1}$ Neurology Unit, Pisa University Hospital (Pisa); ${ }^{2}$ Department of Translational Research and of New Surgical and Medical Technologies, University of Pisa (Pisa); ${ }^{3}$ Unit "Magnetic Resonance and Neuroradiology, Fondazione "G. Monasterio", National Research Council/Tuscany Region (Pisa); ${ }^{4}$ Deep Health Unit, Fondazione "G. Monasterio", National Research Council/Tuscany Region (Pisa); ${ }^{5}$ IRCCS Neuromed (Pozzilli-IS); ${ }^{6}$ Clinical Ageing Research Unit, Newcastle University (Newcastle upon Tyne-UK)

Objectives: To evaluate the integrity of Locus Coeruleus (LC) by 3Tesla MRI in patients with dementia due to AD (ADD), in patients with other types of dementia and in MCI subjects.
Methods: From a larger population of subjects recruited prospectively at the University of Pisa Neurology Unit and Fondazione Monasterio$\mathrm{CNR}$, the present analysis was performed in 182 subjects which completed the follow up (healthy controls $(\mathrm{HC})=56 ; \mathrm{MCI} / \mathrm{ADD}=97 ; \mathrm{FTD}=11$; $\mathrm{LBD} / \mathrm{PDD}=26$ ), which were submitted to a 3 Tesla brain MRI for LC evaluation. MRI protocol, included ad-hoc neuromelanin (NM)-sensitive FSE T1-weighted sequences at the level of pons (14 contiguous slices, slice thickness $2.2 \mathrm{~mm}$, slice gap interleave; acquisition t 14,29 $\mathrm{min}$ ) to identify NM-related signal pertaining to LC. MRIs were evaluated using an ad-hoc semi-automatic software which selects Voxels whose intensity is high enough to be part of LC. An indirect measures of LC volume, based on the number of Voxels selected, is expressed by the parameter VOX. Subjects underwent full neuropsychological assessment.

Results: LC MRI analysis showed a reduced Vox in ADD compared with HC. Conversely, in FTD the estimated LC volume was comparable to HC. Vox parameter reached its lowest values In LBD/PDD and Early Onset AD (lacking APP/PSEN mutations). MCI showed an LC signal intermediate between $\mathrm{AD}$ and $\mathrm{HC}$.

Discussion: Several post-mortem studies and data obtained in experimental models of $\mathrm{AD}$ suggest the hypothesis that $\mathrm{LC}$ degeneration might precede by years the onset of $\mathrm{AD}$ and participates in its pathogenesis. In this study we confirmed that in AD there is a marked LC alteration. As expected from neuropathological and NM-MRI studies, also PDD/LBD showed LC alterations; this was not the case for FTD. MCI subjects showed an intermediate LC estimated volume reduction.

Conclusions: Through NM-sensitive MRI analysis we observed in vivo a reduction of $\mathrm{LC}$ volume in $\mathrm{AD}$, supporting a role of $\mathrm{LC}$ in $\mathrm{AD}$ pathogenesis. In MCI, a further follow up is necessary in order to evaluate whether LC alteration predicts conversion to $\mathrm{AD}$.

Funding sponsor: Ministero della Salute, Ricerca Finalizzata \#PE2013-02359574 (P.I.: FSG).

\section{AMYLOID PET: IS THERE ROOM FOR REGIONAL ANALYSIS?}

M. Grazzini ${ }^{1}$, E. Peira ${ }^{1}$, M. Pardini ${ }^{1}$, F. Sensi ${ }^{2}$, F. Massa ${ }^{1}$, M. Bauckneht $^{3}$, S. Morbelli ${ }^{3}$, D. Arnaldi ${ }^{1}$, A. Chincarini ${ }^{4}$, R. Meli ${ }^{1}$, L. Filippi ${ }^{1}$, F. Nobili ${ }^{1}$

${ }^{1}$ Dept. of Neuroscience, Rehabilitation, Ophthalmology, Genetics, Child and Maternal Health (DINOGMI), University of Genoa, INFN (Genova); ${ }^{2}$ Dept of Health Science (DISSAL), University of Genoa (Genova); ${ }^{3}$ Dept of Health Science (DISSAL), University of Genoa, IRCCS Ospedale Policlinico San Martino (Genova); ${ }^{4}$ INFN (Genova)

We investigated the role of regional amyloid burden as well as the $\mathrm{A} \beta$ spreading manner into brain regions and its implication on clinicalneuropsychological features. In this retrospective study, we retrieved from our database the clinical and cognitive characteristics, and the amy-PET images of 109 patients (age $=72.1 \pm 5.9$ years, $\mathrm{m} / \mathrm{f} 58 / 51$, MMSE $=26.7 \pm 2.7$ ) who underwent a neuropsychological assessment (TMT, RAVLT, categorical fluency, symbol digit, stroop, visuoconstruction test) and amy-PET for a clinical suspicion of AD. Each scan was visually inspected and quantified by averaging corticocerebellar SUVr and ELBA [1] metrics. An anatomo-functional pre-segmented atlas of 11 regions of interest (ROI) was used for regional quantification. Patients were grouped according to their overall $A \beta$ load and the regional correlation maps from each group were compared to the overall correlation map. The correlation was used as a measure of similarity between the different regions and a hierarchical clustering merged 
together the ROIs into a number of meta-ROIs. Regression analysis between neuropsychological assessment and the ratio of $A \beta$ load between different meta-ROIs was carried out looking for clinical implication on different $A \beta$ spreading across the meta-ROIs. The $A \beta$ uptake of the subcortical gray matter resulted independently from the cortical one, resulting poorly correlated to the other brain regions independently of the global amyloid burden. The overall brain $\mathrm{A} \beta$ load as well as the regional $\mathrm{A} \beta$ was not directly related to cognitive performance, but a significant inverse association of semantic verbal fluency was observed with a ratio of cortical/ subcortical distribution of $A \beta$. $A \beta$ accumulation affects the brain regions differently. Indeed, the variability in the covariance observed among any two brain regions reflects the differences in the levels of $A \beta$ accumulation. This observation confirms that brain regions show different vulnerability to amyloid deposition. The covariance among brain regions evolved with the global $A \beta$ levels resulting in an increased interregional correlation with the increase of global amyloid burden. Independently of the global amyloid burden, we observed poor or no correlation between the subcortical gray matter ROIs and other brain regions. However, the higher the cortical load compared to subcortical gray matter load, the lower the semantic verbal fluency performance. Currently it is not clear if the association between regional $A \beta$ load and cognition is due to a direct local effect or it represents a proxy marker of a more aggressive disease subtype. Regional $A \beta$ assessment represents an available resource on amy-PET scan with possibly clinical and prognostic implications, but it is poorly examined yet.

Reference:

1. Chincarini A., Sensi F., Reia L., Bosserth I., Morbelli S., Guerra U.P. , Frisoni G., Padovani A., Nobili F. and for the Alzheimer's Disease Neuroimaging Initiative. Standardized Uptake Value Ratio-Independent Evaluation of Brain Amyloidosis. Journal of Alzheimer's Disease (2016);54:1437-57

\section{CEREBROSPINAL FLUID TOTAL-TAU CONCENTRATION AND BRAIN HYPOMETABOLISM PREDICT PROGRESSION TO TOTAL LOSS OF LANGUAGE IN ALZHEIMER-RELATED PRIMARY PROGRESSIVE APHASIA}

S. Mazzeo ${ }^{1}$, M. Mattei ${ }^{1}$, C. Polito ${ }^{2}$, S. Bagnoli ${ }^{1}$, S. Padiglioni ${ }^{1}$, V. Berti ${ }^{2}$, G. Giacomucci ${ }^{1}$, G. Lombardi ${ }^{1}$, M. De Cristofaro ${ }^{3}$, A. Passeri ${ }^{2}$, C. Ferrari $^{1}$, B. Nacmias ${ }^{4}$, S. Sorbi ${ }^{4}$, V. Bessi ${ }^{1}$

${ }^{1}$ Department of Neuroscience, Psychology, Drug Research and Child Health, University of Florence, Azienda Ospedaliera-Universitaria Careggi (Firenze); ${ }^{2}$ Department of Biomedical, Experimental and Clinical Sciences, University of Florence, Azienda OspedalieraUniversitaria Careggi (Firenze); ${ }^{3}$ Nuclear Medicine Unit, Azienda Ospedaliero-Universitaria Careggi (Firenze); ${ }^{4}$ Department of Neuroscience, Psychology, Drug Research and Child Health, University of Florence, Azienda Ospedaliera-Universitaria Careggi, IRCCS Fondazione Don Carlo Gnocchi (Firenze)

Background and Aims: Logopenic variant of primary progressive aphasia (lvPPA) is considered an atypical presentation of Alzheimer's disease (AD). A previous work by our group suggested that also patients who had overlapping features of lvPPA and non-fluent/agrammatic PPA (nfvPPA) might be classified as PPA variant due to $\mathrm{AD}$, in line with other authors. As the disease progress, some PPA patients con present total loss of language (TLL), while other cognitive functions are still spared. We aimed to identify neuropsychological features, brain hypometabolic patterns and CFS biomarkers which could play as prognostic factors for the progression to TLL.
Methods: 23 consecutive patients, who referred to our centre for language impairment, were diagnosed with lvPPA or with overlapping features for lvPPA and nfvPPA. At baseline, all patients underwent: neuropsychological investigation, [18]F-Fludeoxyglucose-PET brain scan (FDG-PET), CSF biomarkers measurement and Apolipoprotein E (APOE) genotype analysis. Eleven patients also underwent amyloidPET brain scan. Patients have been evaluated by follow-up visit every 6 months. Brain metabolism was analyzed using an optimized and validated voxel-based SPM method.

Results: During the follow-up, 11 patients progressed to TLL and were considered as mute-PPA. 12 did not progress to mutism and were classified as non-mutePPA, with a mean follow-up time of $2.10 \pm 1.41$ years. Time of progression to mutism in mutePPA was not different from follow-up time in non-mutePPA. At baseline mutePPA made more frequent grammar errors $(\chi 2=4.10, p=0.043)$ and were more impaired in writing ( $\chi 2=5.49, \mathrm{p}=0.019)$ compared to non-mutePPA. All the patients had positive amyloid- $\beta$ biomarkers while t-tau was higher in mutePPA at baseline ( $\mathrm{p}=0.048$ ). We found a cut-off of $900 \mathrm{pg} / \mathrm{ml}$ for $\mathrm{t}$-tau to distinguish the two groups $(\mathrm{AUC}=0.76$ ). Patient with $\mathrm{t}$-tau $>900 \mathrm{pg} / \mathrm{ml}$ had different proportion of progression to mutism compared to PPA with ttau $<900 \mathrm{pg} / \mathrm{ml}(\chi 2=4.3, \mathrm{p}=0.038)$. SPM group analysis revealed that mutePPA had hypometabolism in middle and superior temporal gyrus, paracentral lobule and precuneus, while non-mutePPA had hypometabolism in inferior and middle temporal gyrus and in fusiform gyrus. DISCUSSION: there are few literature data on factors associated with different clinical course of PPA. We showed that linguistic features (grammar errors and writing impairment), CSF t-tau and brain hypometabolic pattern could predict progression to TLL in patients with Alzheimer-related PPA.

Conclusions: Identifying features associated with a more severe course of language impairment could extend our knowledge of degenerative aphasias and help clinicians in management of patients with PPA due to $\mathrm{AD}$.

References:

- Sajjadi SA, Patterson K, Nestor PJ. Logopenic, mixed, or Alzheimer-related aphasia? Neurology (2014);82:1127-31

- Funayama M, Nakagawa Y, Yamaya Y, Yoshino F, Mimura M, Kato M. Progression of logopenic variant primary progressive aphasia to apraxia and semantic memory deficits. BMC Neurology (2013);13:158

- Ferrari C, Polito C, Vannucchi S, Piaceri I, Bagnoli S, Lombardi G, Lucidi G, Berti V, Nacmias B, Sorbi S. Primary Progressive Aphasia: Natural History in an Italian Cohort. Alzheimer Dis Assoc Disord. (2019);33(1):42-6

\section{SIMILARITIES AND DIFFERENCES BETWEEN EARLY-ONSET AND LATE-ONSET DEMENTIA: A CASE-CONTROLLED STUDY}

C. Mercanile ${ }^{1}$, C. Galli ${ }^{2}$, G. Vinceti ${ }^{3}$, M. Tondelli ${ }^{2}$, L. Fiondella ${ }^{4}$, S. Salemme ${ }^{4}$, M. Costa ${ }^{2}$, M. A. Molinari ${ }^{2}$, A. Chiari ${ }^{3}$, G. Zamboni ${ }^{4}$

${ }^{1}$ Neuroscience Department, University of Modena and Reggio Emilia (Modena); ${ }^{2}$ Neuroscience Department, AUSL Modena (Modena); ${ }^{3}$ Neuroscience Department, Azienda Ospedaliero Universitaria di Modena (Modena); ${ }^{4}$ Neuroscience Department, University of Modena and Reggio, Azienda Ospedaliero Universitaria di Modena (Modena)

Introduction: Early-Onset Dementia (EOD) is defined as dementia occurring before the age of 65 years, distinct from the more common LateOnset Dementia (LOD). EOD represents an important clinical and 
psychosocial problem due to the several challenges that young patients and their families have to face. A timely diagnosis of dementia is a prerequisite to start adequate treatment, planning the future and coping with the prognosis, but previous studies have shown that the diagnosis in EOD is often delayed until the disabling symptoms are well established $[1,2]$.

Objective: We analyzed clinical, demographic, psychological, economical and social data regarding patients and caregivers from two well characterized cohorts of EOD and LOD, to test whether greater diagnostic delay in EOD also occurs in our cohorts and to identify which factors influence diagnostic delay. Methods: Patients with EOD or LOD and their caregivers were recruited in the extended network of dementia services existing in the province of Modena. Patients underwent extended clinical, imaging and neuropsychological assessment. Caregivers completed specific questionnaires to gain information on patients' symptoms, functional impairment, duration of disease, timing of diagnosis, quality of life, caregiver burden and psychological distress. The two groups were compared and multivariate regression model was used to identify the determinants of diagnostic delay.

Results: Data from 99 EOD and 73 LOD patient-caregiver dyads were examined. There were no significant differences between the two groups in the severity of cognitive impairment, disease duration, behavioural disturbances, anosognosia, cognitive reserve, caregiver distress, caregiver burden and patients quality of life. Significant differences have been found in years of education. There was a significant difference in diagnostic delay: EOD took an average of 10 months longer to be diagnosed compared to LOD. Within the EOD group, diagnostic delay was related to living alone, having a female caregiver, having had to retire because of the disease, living in cities other than Modena or Carpi and disease duration at the interview.

Conclusion: The longer time between referred onset of cognitive symptoms and diagnosis found in EOD patients relative to LOD was not associated with differences in dementia severity, but rather to living-, family- and work-related variables, suggesting that it may possibly not reflect a true diagnostic delay, rather a greater sensitivity of the family and work environment to notice the earliest cognitive changes.

References:

1. van Vliet D, de Vugt ME, Bakker C, Pijnenburg Y a. L, VernooijDassen MJFJ, Koopmans RTCM, et al. Time to diagnosis in young-onset dementia as compared with late-onset dementia. Psychol Med. (2013) Feb;43(2):423-32

2. Draper B, Cations M, White F, Trollor J, Loy C, Brodaty H, et al. Time to diagnosis in young-onset dementia and its determinants: the INSPIRED study. Int J Geriatr Psychiatry. (2016);31(11):1217-24

\section{ELECTROCORTICAL SIGNAL COMPLEXITY AS POTENTIAL BIOMARKER OF COGNITIVE DECLINE PROGRESSION FROM NORMAL AGING TO ALZHEIMER'S DISEASE THROUGH MILD COGNITIVE IMPAIRMENT}

\author{
G. Mostile, R. Terranova, A. Luca, L. Giuliano, G. Portaro, G. Donzuso, \\ C. Rascunà, C. Cicero, A. Nicoletti, M. Zappia
}

Department "G.F. Ingrassia", Section of Neurosciences, University of Catania (Catania)

Background: Pathophysiology beyond dementia is far from being understood. Non-linear analysis of EEG signal has been proposed as neurophysiological tool to assess cortical functioning in patients with cognitive impairment. We investigated changes in fractal properties of EEG signals by analyzing self-similarity of electrocortical activity through different levels of cognitive decline.

Methods: We analyzed data of patients with Alzheimer Disease ("AD"; N=24; age 68.4 \pm ) and Mild Cognitive Impairment ("MCI"; $\mathrm{N}=21$; age $65.6 \pm 9.9$ ), group-matched by age, who underwent a standardized EEG. We selected also a group of healthy controls $(\mathrm{N}=27$; age 68.8 \pm 6.2 ), age-matched with patients, with normal EEG. Power spectrum was calculated by applying a Welch's periodogram to selected electroencephalographic signal epochs using a standardized protocol. To investigate self-similarity of electrocortical activity, the power law exponent $\beta$ was computed for each recording coordinate as minus the slope of the power spectrum vs frequency of signals in a Log-Log scale.

Results: We found significant lower $\beta$ values among temporalparietal-occipital sites of recordings in MCI subjects as compared to controls, while overall significant lower $\beta$ values were observed in AD subjects as compared to controls among almost all site of recordings, except for the left frontal electrode. For each site of recording, an incremental gradient from $\mathrm{AD}$ to controls through $\mathrm{MCI}$ was observed in average $\beta$ values.

Conclusions: We found a progressive decrease in $\beta$ values from physiological conditions to dementia through MCI. Changes in fractal organization of EEG signal could represent an early electrophysiological biomarker of cognitive decline progression in $\mathrm{AD}$.

\section{COGNITIVE PROFILE OF PATIENTS WITH ESSENTIAL TREMOR: A CLINICAL AND IMAGING STUDY}

F. Novellino, V. Saccà, C. Chiriaco, G. Nicoletti, M. Salsone, R. Nisticò, A. Quattrone, M. Morelli, G. Arabia, A. Quattrone

${ }^{1}$ Institute of Bioimaging and Molecular Physiology, National Research Council (Catanzaro); ${ }^{2}$ Department of Medical and Surgical Sciences, University of Catanzaro (Catanzaro); ${ }^{3}$ Institute of Neurology, University of Catanzaro (Catanzaro); ${ }^{4}$ Neuroscience Centre, University of Catanzaro (Catanzaro)

Objective: Essential Tremor (ET) should be no longer considered as a pure motor disease, but as a heterogeneous condition that affects multiple cerebral functions such as mood and cognition. Domains particularly affected in ET are those that rely on the integrity of frontocerebellar circuits in this disease. On the other hand, recent evidences lay for a memory impairment in ET patients, with involvement of structures outside the frontocerebellar circuits, such as the hippocampus. The aim of this study was to characterize the neuropsychological profiles of patients with ET and to delineate its neuroanatomical counterpart.

Methods: Neuropsychological and MRI data were collected from 110 participants: 55 ET (mean-age 63.1 $\pm 11,14$ females) and 55 healthy controls (mean-age: $57.2 \pm 11,24$ females). Cognitive domains were categorized as follows: global cognitive status (MMSE), executive control (FAB, MCST), short- and long-term verbal memory (Digit Span Forward-DS-F, RAVLT-IR, RAVLT-DR), attention and working memory (Digit Span Backward-DS-B), verbal fluency and language comprehension (COWAT, TokenTest-TT), visuo-spatial skills (JLO-V), anxiety and depression. Each domain was evaluated individually (in order to rule out subjects with a cognitive decline), then we performed: (i)direct comparison between groups; (ii)machine learning analysis with feature selection in order to obtain the best discriminant tests between ET and controls. The tests showing differences and/or the best discriminating ability between the groups were inserted in a whole brain voxel-based morphometry (VBM) correlation model in MRI in order to evaluate the correlation 
between cognitive performances and the degree of brain atrophy in ET group without a priori hypothesis.

Results: ET patients showed scores significantly lower than healthy subjects in executive functions (MCST p:0.0016; FAB p:0.011), attention and working memory (DS-B p:0.002), and in short- and long-term verbal memory (RAVLT-IR p:0.075; RAVLT-DR p:0.011). Moreover, machine learning selected as best discriminant tests: RAVLT-DR; FAB; RAVLT-IR, COWAT and TT. The MRI analysis revealed: (i)direct correlation between tests exploring executive control (FAB), attention (DS$\mathrm{B})$ and language and areas including the left inferior and middle frontal cortices, putamen, caudate, thalamus and cerebellum $(\mathrm{p}<0.05)$; (ii)direct correlation between memory scores (RAVLT-IR; RAVLT-DR, TT) and cingulate cortex, hippocampus and thalamus.

Conclusion: Our results support the finding that cognitive impairment involving attention, memory and language are clinical features of ET. Moreover, our results demonstrate that cerebello-thalamo-cortical loop is crucial in explain damage in executive functions and attention in patients with ET. Structures outside this loop, in particular the hippocampus and the cingulate cortex contribute to the memory impairment.

References:

- Louis ED. Essential tremor. Lancet Neurol (2005);4:100-110

- Bermejo-Pareja F., Puertas-Martín V. Cognitive features of essential tremor: a review of the clinical aspects and possible mechanistic underpinnings. Tremor Other Hyperkinet Mov (N Y). (2012); 2 (pii:02-74-541-1)

- Novellino F, Vasta R, Saccà V, Nisticò R, Morelli M, Arabia G, Chiriaco C, Barbagallo G, Nicoletti G, Salsone M, Quattrone A. Hippocampal impairment in patients with Essential Tremor. Parkinsonism Relat Disord. (2020);72:56-61

\section{EFFECTS OF COVID-19 QUARANTINE ON COGNITIVE, BEHAVIORAL, AND MOTOR SYMPTOMS OF PATIENTS WITH ALZHEIMER'S DISEASE AND OTHER DEMENTIAS}

I. Rainero ${ }^{1}$, A. Bruni ${ }^{2}$, C. Marra ${ }^{3}$, A. Cagnin ${ }^{4}$, L. Bonanni ${ }^{5}$, C. Cupidi ${ }^{6}$, V. Laganà ${ }^{2}$, E. Rubino ${ }^{7}$, A. Vacca ${ }^{1}$, R. Di Lorenzo ${ }^{2}$, P. Provero ${ }^{1}$, V.

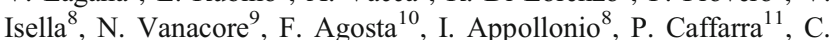
Bussè $^{4}$, R. Sambati ${ }^{12}$, D. Quaranta ${ }^{3}$, V. Guglielmi ${ }^{3}$, G. Logroscino ${ }^{12}$, M. Filippi ${ }^{10}$, G. Tedeschi ${ }^{13}$, C. Ferrarese ${ }^{8}$, a. SINdem COVID-19 Study Group $^{14}$

${ }^{1}$ Department of Neuroscience, University of Turin (Torino); ${ }^{2}$ Regional Neurogenetic Centre, ASP Catanzaro (Catanzaro); ${ }^{3}$ Memory Clinic, Polyclinic Foundation A. Gemelli, IRCCS Catholic University of the Sacred Hearth (Roma); ${ }^{4}$ Department of Neurosciences, University of Padua (Padova); ${ }^{5}$ Department of Neuroscience, Imaging and Clinical Sciences, University G. d'Annunzio (Chieti); ${ }^{6} \mathrm{CDCD}$ Ospedale del Delta, AUSL Ferrara (Ferrara); ${ }^{7}$ Department of Neuroscience and Mental Health, AOU City of Health and Science of Turin (Torino); ${ }^{8}$ Department of Medicine and Surgery, University of Milan-Bicocca (Milano); ${ }^{9}$ National Institute of Health (Roma); ${ }^{10}$ Unit of Neurology, IRCCS San Raffaele Scientific Institute, Vita-Salute San Raffaele University (Milano); ${ }^{11}$ Department of Medicine and Surgery, University of Parma (Parma);

${ }^{12}$ Department of Basic Medicine, Neuroscience and Science Organs, University of Bari Aldo Moro (Bari); ${ }^{13}$ First Division of Neurology, University of Campania Luigi Vanvitelli (Napoli); ${ }^{14}$ Sindem (Firenze)

Objectives: To control the spread of the new Coronavirus (SARS-CoV2 ), several governments planned quarantine. The effects of quarantine on patients with dementia and family caregivers are unknown. We aimed to describe the clinical changes in patients with different types of dementia through a detailed caregivers' interview. Besides, we evaluated caregivers' psychological symptoms and the impact on public health services.

Methods: Family caregivers of patients with dementia, followed at 87 Italian Dementia Centres, were interviewed 45 days after quarantine declaration. Patients with Alzheimer's Disease (AD), Dementia with Lewy Bodies (DLB), Frontotemporal Dementia (FTD), and Vascular Dementia (VD) were eligible for the study. Main outcomes were patients' changes in cognitive, behavioural, and motor symptoms. Secondary outcomes were changes in caregivers' burden, and reorganization of health services.

Results: Between April 14 and April 27, 2020, we screened 4913 patients with dementia. An acute worsening in cognitive functions $(+55.1 \%)$ and in behavioural symptoms $(+51.9 \%)$ was reported. Patients with DLB and AD showed the greatest worsening. In logistic regression analysis, previous physical independence was associated with both cognitive and behavioural worsening (odds ratio 1.85 [95\% CI 1.42 2.39]; and 1.84 [1.43 - 2.38], respectively). During the quarantine period, $25.9 \%$ of patients showed the onset of new behavioural symptoms, requiring drugs modification. A worsening in motor function was reported by $36.7 \%$ of patients. Awareness of the pandemic was negatively associated with increase in cognitive and behavioural symptoms (odds ratio 0.74 [0.65 - 0.85]; and 0.72 [0.63 - 0.82], respectively). Finally, an increase of caregiver's anxiety and depression was reported.

Discussion: Our study shows, for the first time, that quarantine is associated with an acute and dramatic worsening of clinical symptoms in patients with dementia as well as increase of caregiver's burden. Primary care physicians and health services need to implement new strategies in order to mitigate the effects of quarantine in patients with dementia.

\section{THE MY ACTIVE AND HEALTHY AGING ICT PLATFORM PREVENTS QUALITY OF LIFE DECLINE IN OLDER ADULTS: A RANDOMIZED-CONTROLLED STUDY}

\author{
I. Rainero ${ }^{1}$, M. Summers ${ }^{2}$, M. Monters ${ }^{3}$, M. Bazzani ${ }^{4}$, E. Giannouli ${ }^{5}$, G.

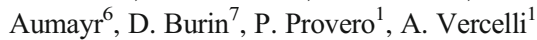

${ }^{1}$ Department of Neuroscience, University of Turin (Torino); ${ }^{2}$ School of Social Sciences, University of the Sunshine Coast (Queensland-AUS); ${ }^{3}$ Gestio Socio Sanitaria al Mediterrani, GESMED (Valencia-E); ${ }^{4}$ LINKS Foundation, Polytechnic of Turin (Torino); ${ }^{5}$ German Sport University, University of Cologne (Cologne-D); ${ }^{6}$ Department Research and Innovation, Johanniter Osterreich (Vienna-A); ${ }^{7}$ Smart Aging Research Center (SARC), Tohoku University (Sendai-J)

Objectives: Prevention of physical and cognitive decline is paramount in older adults. We evaluated the efficacy of a tailored multidomain intervention, monitored with the My-AHA platform, in reducing conversion to overt frailty and preventing decline in quality of life.

Methods: We performed a multicentre, multicultural, randomized control study. The effects of multidomain interventions on frailty parameters, quality of life, physical, cognitive and psychosocial function, nutrition and sleep were evaluated in a group of 101 prefrail older subjects and compared with 100 prefrail controls, receiving general health advice

Results: At the 12-months assessment, participants in the control group showed a decline in quality of life that was absent in the active group. In addition, active participants showed an increase in mood and nutrition function. No effect on remaining parameter was observed. 
Discussion: Our study supports the use of personalized multidomain intervention in preventing quality of life decline in older adults.

\section{RAPIDLY PROGRESSIVE DEMENTIA: NOT ONLY CREUTZFELDT-JAKOB DISEASE}

F. Rizzo, A. Introna, M. Parisi, E. D'Errico, A. Fraddosio, A. M.P. Prudenzano, I. L. Simone, M. Trojano

Department of Basic Medical Sciences, Neurosciences and Sense Organs, University of Bari (Bari)

Background: Rapidly progressive Alzheimer's Disease (rpAD) has recently been recognized as a clinical disease subtype characterized by rapidly progressive cognitive decline, short disease duration, and the possible occurrence of early focal neurological signs. These clinical features impose challenging differential diagnosis with Creutzfeldt-Jakob disease (CJD).

Case Report: A 56-years old man came to Neurology Department complaining 6-months-rapid cognitive impairment. His medical history was unremarkable except for neurosurgery intervention for cervical discectomy in 2002. His mother was diagnosed with vascular dementia. His neurological examination was significant for lack of time and space orientation, anomic-aphasia, perseveration, mild gait impairment and right harm dysmetria. His Mini-Mental State Examination (MMSE) score was $10 / 30$, neuropsychological examination showed multidomain cognitive impairment with memory, visuospatial and executive dysfunction, loss of all Activities of Daily Living (ADL 2/6) and Instrumental Activities of Daily Living (IADL 0/8). Extensive dementia work-up was performed; Serological evaluations for infectious disease such as Lyme disease, syphilis, HIV and herpes virus were negative. Autoimmunity-panel including also intracellular and surface onconeural-Abs was negative. Electroencephalogram (EEG) examinations showed diffuse theta-delta activities with periodic sharp-wave complexes (PSWC) in frontal regions. Brain Magnetic Resonance Imaging (MRI) showed diffuse but asymmetric cortical atrophia, prevalent in left hemisphere; 18fluorodeoxyglucose (FDG) positron emission tomography (PET) highlighted cortical diffuse hypometabolism prevalent in left hemisphere. Cerebral Spinal Fluid (CSF) standard examination, was unremarkable except for mild increasing protein $(55 \mathrm{mg} / \mathrm{dl})$; CSF biomarkers showed pathological increasing in total-tau [(ttau) 980] and phosphorylated-tau [(p-tau) 128], with t-tau/p-tau ratio 7.65 and mild reduction of amyloid-beta peptide $1-42$ [A $\beta 42)$ 433], with $A \beta 42 / A \beta 40$ ratio reduction [0.05]. CSF 143-3 protein assay with Wester Blot and Prion real-time quaking inducing conversion assay (RT-QuIC), genetic analysis for polymorphism codon 129 PRNP gene and other genetic mutation screening for the same gene were negative. Genetic analysis for familial $\mathrm{AD}$ is still processing. We formulated diagnosis of $\mathrm{AD}$ according to "A/T/N" system: "A+", "T+", "N+"; considering high CSF t-tau levels and rapidly progressive cognitive decline with early neurological sign we suggest rpAD.

Conclusion: Increase of CSF t-tau and p-tau levels occurs earlier in $\mathrm{rpAD}$ respect to typical $\mathrm{AD}$. According to literature approximately 8 $16 \%$ of rpAD cases have high t-tau levels whereas the same $t$-tau profile is rarely seen in typical AD. Early and massive neurodegeneration, maybe due to different neuropathological mechanism, could explain this CSF biomarkers profile and could be responsible of different clinical progression rate and disease severity, mimicking CJD.
References:

- Jack CR Jr, Bennett DA, Blennow K, et al. NIA-AA Research Framework: Toward a biological definition of Alzheimer's disease. Alzheimers Dement. (2018);14(4):535-62

- Abu-Rumeileh S, Capellari S, Parchi P. Rapidly Progressive Alzheimer's Disease: Contributions to Clinical-Pathological Definition and Diagnosis. J Alzheimers Dis. (2018);63(3):887-97

\section{CDCD (CENTRO DISTURBI COGNITIVI DEMENZE) ACTIVITIES IN SARSCOV2 PANDEMIC: TESTING A LOCAL TELEHEALTH SUPPORT}

M. Rosso, P. Ghiglione, J. Maistrelli, A. Dutto, R. Di Gesù, M.R. Bongioanni

\section{S.C. SS Annunziata Hospital ASLCN1 (Savigliano-CN)}

Introduction: The SARSCOV2 pandemic highlighted the need of new strategies for the management of persons with dementia (PWD) and the importance of care integration between hospital and home/nursing home. In these months, the most important problems to solve in this frail population have been:

- $\quad$ rapid intervention for BPSD (Behavioral and Psychological Symptoms Dementia)

- $\quad$ BPSD clinical monitoring and off label neuroleptics prescription (not included in the extension deadlines regional resolution)

- optimizing care of patients in nursing home (modified by national emergency legislation)

- $\quad$ evaluate criteria for AchEI drugs and its correct prescription (within the terms established by the extension resolution)

Materials and methods: In this emergency period, the AslCn1 Centro Disturbi Cognitivi e Demenze (CDCD) has taken several measures to ensure an efficient service to its assisted (470 PWD) through telemonitoring and telecare, including:

- daily email/phone answering from CDCD staff (8 a.m - 4 p.m.)

- bimonthly phone calls for patients taking neuroleptic drugs (using NPI-Q to trace the severity BPSD and the caregiver burden)

- $\quad$ psychological telecounseling for caregivers at risk of burden (by phone or utilizing webmeet-Webex or GoogleMeet)

- family members/caregivers medical teleconsult on request (including MMSE, ADL, IADL, GDS scales-Webex or GoogleMeet) for PWD at home

- structured interview for PWD in nursing home (filled in by physiotherapists, psychologists, general practitioner and sent by a coded email)

In this PWD subgroup we have elaborated a simple precompiled worksheet to monitor health condition and cognitive performances and to identify emerging problems in order to develop an intervention plan, to change a treatment or to provide strategies of balance.

Results: In the period March-July 2020, our CDCD received 180 phone calls and made 410 phone calls to caregivers, general practitioners, nursing home staff; 21 PWD were managed by email (on average 5 mail each). The psychologist supported 12 caregivers (10 by phone/webmeet). 
The CDCD staff managed remotely 5 nursing home resident PWD using the structured worksheet. The neurologist made 3 medical evaluation using telehealth (webmeet).

Conclusions: These early telehealth attempts ensured PWD monitoring and the prescription of the correct drugs on time. Patients, caregivers and the nursing home staff were satisfied by our service offered during this emergency period. In consideration of the users satisfaction and the latest regional resolutions* on telehealth, it is hoped that these operating methods will be improved and guaranteed in the future, especially in case of a new pandemic forecast.

\section{INCREASED OREXIN-A CONCENTRATIONS IN CEREBROSPINAL FLUID OF PATIENTS WITH BEHAVIORAL VARIANT FRONTOTEMPORAL DEMENTIA}

F. Roveta ${ }^{1}$, A. Marcinnò ${ }^{1}$, R. Cremascoli ${ }^{2}$, L. Priano ${ }^{2}$, S. Cattaldo ${ }^{2}$, E. Rubino $^{3}$, S. Boschi ${ }^{1}$, A. Grassini ${ }^{1}$, A. Mauro ${ }^{2}$, I. Rainero ${ }^{1}$

${ }^{1}$ Department of Neuroscience "Rita Levi Montalcini", University of Torino (Torino); ${ }^{2}$ Department of Neurology and Neurorehabilitation, Istituto Auxologico Italiano (Piancavallo, Verbania); ${ }^{3}$ Department of Neuroscience and Mental Health, AOU Città della Salute e della Scienza di Torino (Torino)

Objective: Behavioural variant-frontotemporal dementia (bvFTD) is the most common clinical variant of frontotemporal dementia and is characterized by insidious changes in personality and interpersonal conduct reflecting progressive disintegration of the neural circuits involved in social cognition, emotion regulation, motivation and decision making [1]. Orexins (orexin-A and orexin-B) are two neuropeptides synthesized by hypothalamic neurons that influence several CNS functions. Previous studies evaluated cerebrospinal fluid (CSF) orexin levels in Alzheimer's disease (AD), finding high level of orexin-A that correlates to AD progression, sleep fragmentation and neuropsychiatric symptoms [2]. The aim of this study was to investigate CSF orexin-A concentrations in bvFTD, evaluating correlation with clinical symptoms, in order to elucidate the role of orexins in this neurodegenerative disorder.

Materials and methods: We examined a total of 40 sporadic bvFTD patients, recruited at the Department of Neuroscience of University Hospital "Città della Salute e della Scienza" of Torino and at the Department of Neurology and Neurorehabilitation of "Ospedale S. Giuseppe", Piancavallo, Italy. Diagnosis of probable bvFTD was made according to Rascovsky Criteria [3]. As control group, CSF of 32 patients without cognitive impairment was considered. Detailed clinical history was recorded, particularly focusing on cognitive and behavioral characteristics, drugs assumption and cardiovascular risks factors. CSF orexin$\mathrm{A}$ and concentrations of $\mathrm{AD}$ biomarkers were detected in both patients and controls with commercially available ELISA kits.

Results: We found increased CSF orexin-A concentrations in bvFTD patients (mean: $0.226 \mathrm{ng} / \mathrm{mL} \pm \mathrm{SD}: 0.103$ ) compared to controls (mean: $0.139 \mathrm{ng} / \mathrm{mL} \pm \mathrm{SD}: 0.058)$, and this resulted statistically significant $(\mathrm{p}<$ 0.001). In addition, several clinical and demographic variables emerged as significantly linked to CSF orexin-A concentrations in the bvFTD group. A significant inverse correlation with MMSE $(p<0.001)$ was found and the presence of repetitive/compulsive behaviors as well as extrapyramidal signs were associated with increased CSF orexin-A concentrations.

Discussion and conclusion: We demonstrated an increase of orexin-A concentrations in cerebrospinal fluid of bvFTD patients compared to controls. Moreover, it was possible to report several associations with the clinical characteristics of the disease, reinforcing the hypothesis of a deregulation of this neuropeptide in the disease. Regarding specific neuropsychiatric manifestations, we found significant orexin-A variations when assessing for history of compulsive behaviors or extrapyramidal signs. Possible explanations are based on orexinergic physiological functions and interconnections with other neurotransmitter systems. Even if preliminary, correlations between orexin-A and cognitive status, as well as motor and psychiatric symptoms, deserves additional investigations.

References:

1. Bang J, Spina S, Miller BL. Frontotemporal dementia. Lancet (2015);386:1672-83

2. Liguori C, Mercuri N, Nuccetelli M, et al. Cerebrospinal Fluid Orexin Levels and Nocturnal Sleep Disruption in Alzheimer's Disease Patients Showing Neuropsychiatric Symptoms. J of Alzheimers Dis (2018); 66(3):993-96

3. Rascovsky K, Hodges JR, Knopman D, et al. Sensitivity of revised diagnostic criteria for the behavioural variant of frontotemporal dementia. Brain (2011);134: 2456-77

\section{EXOME SEQUENCING ANALYSIS OF AN ITALIAN FAMILY WITH LATE-ONSET ALZHEIMER'S DISEASE IDENTIFIES A NEW RARE VARIANT IN GLUTAMATE IONOTROPIC RECEPTOR NMDA TYPE SUBUNIT 2C GENE}

\author{
E. Rubino ${ }^{1}$, E. Giorgio ${ }^{2}$, S. Boschi ${ }^{3}$, A. Vacca $^{4}$, T. Pippucci ${ }^{5}$, P.
}

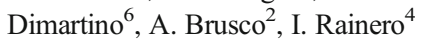

${ }^{1}$ Department of Neuroscience and Mental Health, AOU Città della Salute e della Scienza di Torino (Torino); ${ }^{2}$ Department of Medical Sciences, University of Turin (Torino); ${ }^{3}$ Department of Neuroscience, Psychology, Drug Research and Child Health, University of Florence (Firenze); ${ }^{4}$ Department of Neuroscience, University of Turin (Torino); ${ }^{5}$ Department of Medical and Surgical Sciences, University of Bologna (Bologna); ${ }^{6}$ Medical Genetics Unit, Polyclinic Sant'Orsola-Malpighi University Hospital (Bologna)

Background: Alzheimer's disease (AD) is the most common type of neurodegenerative dementia, but the cause of $\mathrm{AD}$ remained poorly understood. Many mutations in the amyloid precursor protein (APP) and presenilin 1 and 2 (PSEN1 and PSEN2) have been reported as the pathogenic causes of early-onset $\mathrm{AD}$ which accounts for up to $5 \%$ of all $\mathrm{AD}$ cases. Late-onset $\mathrm{AD}$ represents the most common form of the disease, and is associated with 28 confirmed Alzheimer's disease loci, including the APOE $\varepsilon 4$ allele with the largest risk. The aim of this study is to describe a new rare variant in the glutamate ionotropic receptor NMDA type subunit 2C (GRIN2C) in an Italian family with late-onset Alzheimer's disease.

Methods: We describe a targeted exome sequencing analysis of a large Italian kindred with late-onset $\mathrm{AD}$ with a typical clinical presentation, negative for APP, PSEN1, and PSEN2 variants. Previous analysis using NeuroX array excluded mutations in pathogenic variants linked to neurodegenerative disorders. Whole Exome Sequencing (WES) on the three affected subjects and two healthy relatives was outsourced to BGIShenzen using genomic DNA extracted from circulating leukocytes. Targeted enrichment was performed using BGI exome kit V4 (59M,100x), and captured libraries were loaded onto an BGISEQ-500 platform. Bioinformatic analysis of WES data followed a general pipeline previously described to identify single nucleotide variants and small insertions and deletions. 
Results: The c.3215 C>T (p.Ala1072Val) in GRIN2C (NM 000835) was identified and validated by Sanger sequencing. The segregation of the variant with the disease in the family was confirmed in a total of 8 affected subjects and in 6 healthy relatives.

Discussion: GRIN2C mRNA is widely expressed in the brain, and the Ala1072Val variant was considered of interest based on DANN pathogenicity scoring, allele frequency in the general population (GnomAD database), and its localization in the intracellular carboxyl-terminal domain of the protein. Indeed, genetic variants located in this specific domain have been previously associated with schizophrenia and autism spectrum disorder. Finally, the cognitive impairment of AD is closely related to synaptic plasticity, in which NMDA receptors play a key role.

Conclusion: In this study, we report for the first time a rare missense variant in GRIN2C causing late-onset Alzheimer's disease. Further studies are needed to confirm our findings in larger populations.

\section{SPECIFIC DYSFUNCTION OF THE MAGNOCELLULAR STREAM IN ALZHEIMER DISEASE SUGGESTED BY PATTERN ELECTRORETINOGRAMS AND VISUAL EVOKED POTENTIALS}

\author{
F. Sartucci ${ }^{1,2,3}$, L. Domenici ${ }^{3,4}$, G. Rutigliano ${ }^{5}$, V. Porciatti ${ }^{6}$
}

${ }^{1}$ Neurophysiopatology Unit, Department of Clinical and Experimental Medicine, Pisa University Medical School (Pisa); ${ }^{2}$ AOUP (Pisa); ${ }^{3} \mathrm{CNR}$ Neuroscience Institute (Pisa); ${ }^{4}$ Department of Biomedical Sciences and Technologies, L'Aquila University (L'Aquila); ${ }^{5}$ Psychiatry Unit, Department of Pathology, Pisa University Medical School (Pisa); ${ }^{6}$ Bascom Palmer Eye Institute (Miami-USA)

Background and Rationale: Visuo-spatial troubles represent a common feature of Alzheimer's disease (AD). The magnocellular (M) pathway has anatomo-physiological characteristic which make it more suitable for detecting form, motion and depth compared with parvocellular $(\mathrm{P})$ one. Aim of our study was to evaluate electrophysiologically visual subsystem involvement in a group of $\mathrm{AD}$ patients, using equiluminant Chromatic Pattern Electroretinograms (ChPERGs) and Chromatic Pattern Visual Evoked Potentials (ChVEPs) in attempt to detect a magnocellular deficit in this disease.

Method: Data were obtained from $15 \mathrm{AD}$ pts (9 females and 6 males, mean age + 1SD $77.6 \pm 4.01 \mathrm{yrs}$,) not yet undergoing any treatment, and from 10 age- and sex-matched healthy controls ( 5 females and 5 males, mean age + 1SD $71.3 \pm 7.2$ ). ChPERGs were recorded monocularly in response to equiluminant red-green (R-G) and blue-yellow (B-Y) stimuli, known to emphasize the contribution of parvo- and konio-cellular streams respectively, and achromatic luminance (Lum, magnocellular stream) yellow-black (Y-Bk) horizontal square gratings of $0.3 \mathrm{c} / \mathrm{deg}$ and $90 \%$ contrast $(\mathrm{K})$, reversed at $1 \mathrm{~Hz}$, displayed on a TV monitor at a viewing distance of $24 \mathrm{~cm}(59.2 * 59 \mathrm{deg}$ field). ChVEPs were recorded to onset $(300 \mathrm{~ms})$ and offset (700ms) equiluminant chromatic sinusoidal gratings of different $\mathrm{K}$ (90 and 25\%). Diagnosis was clinically and neuro-radiologically established, after having excluded other possible causes of dementia.

Results: All data were retrieved in terms of peak-amplitude and latency (for either ChPERGs and ChVEPs) and the obtained values were assessed using the Student's t-test for paired data. As expected, temporal features of ChPERGs, as well as ChVEPs, in AD patients differed from those of to luminance $\mathrm{Y}-\mathrm{Bk}$ grating in $\mathrm{AD}$ group $(\mathrm{p}<0.01)$.

Conclusions: The deficits of the responses arising from the magnocellular streams of visual processing pointed out in this study could be related or indicate a primary dysfunction of the M-pathways in Alzheimer's disease.

References:

- Sartucci F., Domenici L., Porciatti V. Commentary on "Dysfunction of the magnocellular stream in Alzheimer disease evaluated by pattern electroretinograms and visual evoked potentials". J Exp Neurol. (2020);1 (1): 17-25

- Sartucci F., Borghetti D., Bocci T., Murri L., Orsini P., Porciatti V., Origlia N., Domenici L. Dysfunction of the magnocellular stream in Alzheimer's disease evaluated by pattern electroretinograms and visual evoked potentials. Brain Res Bull. (2010);82(3-4):169-76

- Porciatti V, Sartucci F Normative data for onset VEPs to red-green and blue-yellow chromatic contrast. Clin Neurophysiol. (1999);110:772-81

\section{BRAIN METABOLISM AND AMYLOID CORRELATES IN PRECLINICAL DEMENTIA STAGES}

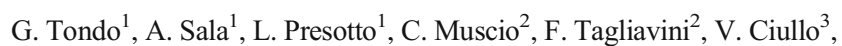
F. Piras ${ }^{3}$, S. Cappa ${ }^{4}$, G. Spalletta ${ }^{3}$, D. Perani ${ }^{1}$

${ }^{1}$ In Vivo Human Molecular and Structural Neuroimaging Unit, Division of Neuroscience, IRCCS San Raffaele Scientific Institute, Vita-Salute San Raffaele University (Milano); ${ }^{2}$ IRCCS 'Carlo Besta' (Milano); ${ }^{3}$ Laboratory of Neuropsychiatry, IRCCS Santa Lucia Foundation (Roma); ${ }^{4}$ Humanities and Life Sciences, University School for Advanced Studies (Pavia)

Objective. FDG-PET has shown high accuracy in predicting risk of progression to dementia in the prodromal stage, while the role of amyloid PET in diagnosis and prognosis is debatable [1]. Few data are available in the preclinical stages, where the role of PET imaging is not yet established. The aim of this study was to investigate brain metabolism and amyloid load in subjective cognitive complaint (SCC) and in preMild Cognitive Impairment (preMCI) conditions, and to explore the neuropsychological and behavioral correlates.

Methods: We enrolled 105 subjects (49 SCC, 56 preMCI) with full clinical evaluations. All subjects underwent a baseline FDG-PET scan, analyzed with optimized voxel-based SPM procedures [2], and 67 subjects underwent additional amyloid-PET imaging, analyzed using a validated semi-quantification method [3].

Results: The single-subject FDG-PET SPM-t maps classified subjects into six patterns: 1) normal SPM map; 2) temporal-parietal AD-like; 3) frontal-temporal FTD-like; 4) medial temporal (MT) as in tauopathies; 5) occipital DLB-like; 6) non-neurodegenerative. Notably, sixty (57\%) of the whole sample showed altered brain metabolism at SPM maps. In details, FTD-like and non-neurodegenerative patterns were the most represented (respectively $21 \%$ and $19 \%$ ), seven subjects (8\%) had hypometabolism in the MT structures, only five $(6 \%)$ showed an ADlike hypometabolism pattern, and four (3\%) showed a DLB-like pattern. Forty-five subjects $(43 \%)$ had normal brain metabolism. When considering each group with pathological FDG-PET patterns, significant higher global scores at the Neuropsychiatric Inventory in the FTD-like group, and significant lower scores in the immediate and delayed recall of the Free and cued selective reminding test in the AD-like subgroup, emerged. Among subjects who underwent amyloid-PET, seventeen (25\%) showed a pathologic amyloid burden. Normal brain FDG-PET metabolic pattern was strongly associated with normal cortical amyloid load, however, there was $15 \%$ of subjects with negative FDG PET scan and amyloid PET positivity (aging incidental amyloid). 
Discussion and conclusions: FDG-PET revealed altered brain metabolism already in SCC and pre-MCI cases. Of note, normal FDG-PET SPM maps were also frequent in this cohort and strongly associated with normal brain amyloid load. The high rate of frontal hypometabolism in SCC and pre-MCI subjects may be due to the presence of FTD neurodegenerative processes, tauopathies or to a neural dysfunctional correlate, all responsible of the increased neuropsychiatric disorders. These findings highlight the relevant role of FDG-PET in identifying early signature of neurodegeneration in preclinical stage of dementia or dysfunctional alterations. Longitudinal follow up studies are mandatory to reveal significance of these findings.

\section{References:}

1. Iaccarino L, Sala A, Perani D. Predicting long-term clinical stability in amyloid-positive subjects by FDG-PET. Ann Clin Transl Neurol. (2019) May 24;6(6):1113-20

2. Perani D, Della Rosa PA, Cerami C et al., Validation of an optimized SPM procedure for FDG-PET in dementia diagnosis in a clinical setting. Neuroimage Clin. (2014) Oct 24;6:445-54

3. Presotto L, Iaccarino L, Sala A et al., Low-dose CT for the spatial normalization of PET images: A validation procedure for amyloidPET semi-quantification. Neuroimage Clin. (2018) Jul 19;20:15360

\section{ALTERATIONS OF PRIMARY VISUAL PATHWAY IN LEWY BODY DEMENTIA: FROM RETINA TO VISUAL CORTEX}

\author{
G. Zorzi ${ }^{1}$, F. Fragiacomo ${ }^{2}$, M. Pengo ${ }^{2}$, S. Miante ${ }^{2}$, C. Bussè ${ }^{2}$, G. \\ Gazzola $^{2}$, S. Mozzetta ${ }^{2}$, F. Rossato ${ }^{2}$, S. Crivellari ${ }^{2}$, P. Gallo ${ }^{2}$, D. \\ Cecchin $^{3}$, M. Corbetta ${ }^{1}$, A. Cagnin ${ }^{1}$
}

${ }^{1}$ Department of Neuroscience, Padova Neuroscience Center, University of Padua (Padova); ${ }^{2}$ Department of Neuroscience, University of Padua (Padova); ${ }^{3}$ Nuclear Medicine, Padova Neuroscience Center, University of Padua (Padova)

Background: The visual system may be involved in several clinical features specific of dementia with Lewy bodies (DLB), such as visuospatial/ visuoperceptive impairment, visual hallucinations, and circadian dysfunction. Despite this evidence, very few data are present on the abnormalities of retinal layers in DLB and the effects of retinal changes on structural and metabolic measures of primary visual pathway regions. We therefore aim to study retinal layers abnormalities, atrophy and metabolic changes of regions along the primary visual pathway in patients with DLB through a multimodal imaging study.

Methods: Seventeen DLB patients and 30 healthy subjects (HS) (mean age $70.4 \pm 5.5$ and68.5 \pm 8.8 years respectively) underwent a full Spectral Domain retinal Optical Coherence Tomography (OCT) scan and thickness and volumes of peripapillary RNFL (pRNFL) and macular layers were analyzed using generalized estimating equation (GEE) method. All DLB patients underwent brain 18F-FDG-PET/MRI scan. T1sequences were processed using Freesurfer standard pipeline, and the anatomical Desikan-Killiany atlas was used for cortical parcellation. A partial volume correction was applied using PET-Surfer based on Symmetric Geometric Transfer Matrix (SGTM). The standardized uptake value (SUV) for each ROI, normalized with the cerebellum, was extracted. A correlation analysis between volume of pulvinar, geniculate bodies and primary visual cortex cortical thickness and 18F-FDG SUVr with OCT data was made ( $R>0.6$ were retained). Age, sex and MMSE were used as covariate nuisance factors. Results were corrected for multiple comparisons. Associations with neurological and cognitive measures were also made.

Results: DLB patients showed a thinning of retinal parafoveal ganglion cell-inner plexiform layers(GCIPL)respect to controls ( $p=0,01$ ), mostly in inferior and temporal quadrant ( $\mathrm{pFDR}=0,04$, both). pRNFL was not significantly different in DLB patients and HS; lower thickness of the retina in the temporal sector of papilla correlated with lower volumes of the lateral geniculate nuclei $(R>0.65 p=0.01)$. There were not significant correlations between OCT measures and cortical thickness and FDG metabolism of the primary visual cortex.

Conclusion: Considering the high correlation between papilla and LGN volume and the impairment of retina layers, we hypothesize a forward degeneration due the a-synuclein pathology that involved whole visual system. The uses of multimodality data could add information about the complex mechanism of degeneration, that involved not only cortical brain systems, and it could add new markers for the diagnosis in prodromal stages.

\section{CHARACTERIZATION OF PSYCHIATRIC ONSET OF NEURODEGENERATIVE DEMENTIA VERSUS LATE-ONSET PRIMARY PSYCHIATRIC DISORDERS}

G. Zorzi ${ }^{1}$, B. Roiter ${ }^{2}$, C. Bussè ${ }^{2}$, G. Gazzola ${ }^{2}$, S. Crivellari ${ }^{2}$, F. Rossato ${ }^{2}$, I. Pettenuzzo ${ }^{2}$, S. Mozzetta ${ }^{2}$, G. Perini ${ }^{3}$, G. Pigato $^{2}$, A. Cagnin ${ }^{1}$

${ }^{1}$ Department of Neuroscience, Padova Neuroscience Center, University of Padua (Padova); ${ }^{2}$ Department of Neuroscience, University of Padua (Padova); ${ }^{3}$ Casa di Cura Parco dei Tigli, Padova Neuroscience Center, University of Padua (Teolo-PD)

Background: Late-onset primary psychiatric disorder may share with psychiatric onset of neurodegenerative diseases similar clinical characteristics. Differential diagnosis id difficult leading to inaccurate diagnoses and diagnostic delays. The aim of this study was to assess clinical and cognitive fingerprint of neurodegenerative diseases presenting with psychiatric features respect to late-onset primary psychiatric disorders.

Methods: 56 patients with late-onset presentation of psychiatric disorders underwent consultation by a multidisciplinary team (psychiatrist and neurologist), a neuropsychological assessment and brain MRI. In selected cases patients had also FDG-PET $(n=26)$ and CSF examination $(n=32)$. Each patient was followed for at least 12 months.

Results: At baseline visit the following psychiatric diagnoses were made: major depression (57\%), psychosis (27), obsessive/compulsive disorder (10\%) and bipolar disorder spectrum (7\%). After diagnostic work-up 30/56 patients have been diagnosed with neurodegenerative diseases, while 26 with primary psychiatric disorder. Among neurodegenerative diseases the diagnosis of bvFTD was the most frequent $(40 \%)$ followed by DLB $30 \%$, AD $20 \%$ and other types of dementias (10\%). Presentation with psychosis was associated with a diagnosis of neurodegenerative diseases while depression was equally distributed between neurodegenerative and psychiatric diseases. As for the neuropsychological assessment, worse performances at the TMT-A test and verbal fluency could differentiate neurodegenerative from psychiatric diseases (OR 1.12 and 0.8 , respectively). bvFTD and primary psychiatric disorder had a similar cognitive profile while worse TMT-A performance was associated with a diagnosis of DLB and AD.

Conclusion: In about $50 \%$ of patients with late-onset psychiatric disorders a diagnosis of neurodegenerative disease with psychiatric presentation may be made. Late-onset psychosis and worse visual attention and verbal fluency are red flags for an underlining neurodegenerative disease. 


\section{CEREBROVASCULAR DISEASES}

\section{ADAPTIVE PERFORMANCE OF THE TUSCANY STROKE NETWORK TO THE COVID 19 OUTBREAK: A RETROSPECTIVE STUDY}

M. Baldereschi ${ }^{1}$, G. Orlandi ${ }^{2}$, F. Bellomo ${ }^{3}$, A. Chiti ${ }^{2}$, P. Nencini ${ }^{4}$, P. Palumbo $^{5}$, G. Martini ${ }^{6}$, G. Linoli ${ }^{7}$, D. Inzitari ${ }^{4}$, T. Tuscany Stroke Network $^{8}$

${ }^{1}$ Inst. of Neurosciences, Italian National Research Council CNR (Firenze); ${ }^{2}$ Stroke Unit, University of Pisa (Pisa); ${ }^{3}$ Tuscany Stroke Network, Tuscany Region (Pisa); ${ }^{4}$ Stroke Unit, University of Florence (Firenze); ${ }^{5}$ Stroke Unit, Prato Hospital (Prato); ${ }^{6}$ Stroke Unit, University of Siena (Siena); ${ }^{7}$ Stroke Unit, Arezzo Hospital (Arezzo); ${ }^{8}$ Tuscany Health System, Tuscany Region (Firenze)

Aims: The COVID 19 pandemic is impacting on acute ischemic stroke (AIS) care: fewer patients reach the hospital within the therapeutic timewindow. We aim at quantifying this impact in terms of number of treatments, as well as exploring the Tuscany Stroke Network (TSN) adaptive performance.

Materials and Methods: The TSN was implemented in 2015 and it is structured as three hub-and-spoke models throughout Tuscany. AIS patients are first taken to the nearest spoke hospital for possible t-PA treatment, assessed for eligibility to endovascular intervention, and quickly transferred to the nearest hub hospital, where appropriate. We included all patients with AIS consecutively treated in each of the 9 hospitals of the north-western region of the TSN during the first semester of 2020, using the same period from 2019 as a comparator.

Results: The network spans across $6000 \mathrm{Km} 2$ with 1,3 million inhabitans, 3260 AIS expected cases, 1 hub hospital and 8 spoke hospitals. From January 1 through June 30 2020, a total of 227 treatments were administered to AIS patients, being 238 during the same period of 2019 . In particular, there were $9 \%$ fewer t-PA treatments (194 vs 214), but $37.5 \%$ more endovascular interventions. There were also $24 \%$ fewer secondary transfers to hub. Single hospital performances varied a lot.

Discussion: The TSN could adapt very quickly to the COVID-19 outbreak. Some hospitals took over AIS patients from other overwhelmed hospitals, redirecting EMS transportations. The logistic interventions provided by the TSN counterbalanced the deleterious effects of the COVID 19 outbreak on AIS care.

Conclusions: Our data suggest both the need and the benefit of organized stroke systems. Only a well-established hospital network can adjust its organization and logistics to quickly and effectively deal with an unexpected health problem.

*TSN-Working-Group: Baldereschi, Balzi D, BaruffiMC, Bellomo F, Bertini A, Bollani E, Bracco S, Carneglia L, Caruso A, Cesari V, ChitiA, ChitiI, Colombai R, ContiA, Cosottini M, CozzaS, DeVito L, DelDotto P, DiFabrizio V, DonigagliaG, Fainardi E, Ferrini L, Fortini A, Frosini F, Galli R, Gambaccini G, Iannelli G, Inzitari D, Landini G, Laureano R, LencioniMG, Linoli G, LuchiniG, Mancuso M, MandòM, MangiaficoS, Marconi R, Marrone A, Martelli F, Martini G, Masotti L, Mazzoni M, Menichetti C,Meucci G,Nencini P, Niccolini A, Nocentini S, Orlandi G, Orsitto E, Palumbo P, Panigada G, Pennati P, PepeG, PratesiM, ProsettiD, RuggianoG, ScazzeriF, Spisni L, Spolveri S, Tassi R, Testa A, Tognarelli, Torri T, Vannini R, Vignali C, Volpi G.

References:

- R. Pop, V. Quenardelle, A. Hasiu, D. Mihoc, F. Sellal, M. H. Dugay, P. A. Lebedinsky, E. Schluck, A. LA Porta, S. Courtois, R. Gheoca,
V. Wolff and R. Beaujeux. Impact of the COVID-19 outbreak on acute stroke pathways - insights from the Alsace region in France. European Journal of Neurology (2020);10:1-5

- M Baldereschi, F Bellomo, G Orlandi, P Palumbo, G Martini, G Linoli, V Di Fabrizio, D Balzi, D Inzitari for the TSN Working Group. Successful performance of the Tuscany Stroke Network: a before-and-after study. Neurol Sci (2019);40:S67

E Bollani, A Testa, L de Toffoli, M Irolla, M Liberatori, F Maggiorelli, U Penney, G Ramacciotti, A Salzano, F Stilo, R Cecchetti, M Rossi, F Stea, G Criscione, F Scazzeri, G Meucci, M Baldereschi, F Bellomo, G Orlandi. Hyperacute stroke management before and after an integrated healthcare system introduction in Elba island-Italy. Neurol Sci (2019); 40: S167-68

\section{THE ASSOCIATION BETWEEN STROKE AND BRAIN VASCULAR LESION LOAD IN PATIENTS WITH ATRIAL FIBRILLATION ON ORAL ANTICOAGULANTS. DATA FROM STRAT-AF STUDY}

C. Barbato ${ }^{1}$, B. Formelli ${ }^{1}$, F. Galmozzi ${ }^{1}$, F. Cesari ${ }^{2}$, S. Galora ${ }^{2}$, B. Giusti $^{2}$, A. Gori ${ }^{2}$, F. Pescini ${ }^{1}$, G. Pracucci ${ }^{1}$, C. Sarti $^{1}$, S. Diciotti ${ }^{3}$, E. Fainardi ${ }^{4}$, R. Marcucci ${ }^{2}$, E. Salvadori ${ }^{1}$, A. Poggesi ${ }^{1}$

${ }^{1}$ NEUROFARBA Department, University of Florence (Firenze); ${ }^{2}$ Atherothrombotic Diseases Center, Azienda Ospedaliera Universitaria Careggi (Firenze); ${ }^{3}$ Department of Electrical, Electronic, and Information Engineering Guglielmo Marconi, University of Bologna (Cesena); ${ }^{4}$ Department of Neuroradiology, Azienda Ospedaliera Universitaria Careggi (Firenze)

Background: Oral-anticoagulants (OAC) is recommended to prevent thromboembolism in atrial fibrillation (AF)-patients with a moderate/ high stroke risk. Even if cardioembolism is the main stroke mechanism, not all cerebrovascular events in AF are due to cardioembolism. BrainMRI may provide specific clues about stroke pathogenesis. We aimed at evaluating in a cohort of AF-patients on OAC, differences in terms of cerebrovascular lesion burden comparing patients with and without stroke history.

Methods: STRAT-AF is an ongoing single-center, prospective, observational study aimed to evaluate the role of biological markers for cerebral bleeding risk stratification in AF-patients on OAC. Patients underwent a clinical, functional and neuropsychological assessments. Cerebrovascular burdens were assessed on MRI as below: 1. Markers of small vessels disease (SVD): white matter hyperintensities (modified-Fazekas scale), cerebral microbleeds (MARS scale), lacunar infarcts (LI, number), 2. Non-lacunar infarcts (N-LI, number).

Results: Among 170 patients enrolled (mean age $77.7 \pm 6.8$, Male:64\%), 38 (22\%) had previous stroke (32 ischaemic, 6 haemorrhagic). Comparing patients with and without stroke history, higher mean number of N-LI and LI were more frequent within patients who have experienced stroke (N-LI: $82 \%, \mathrm{p}<0.001$; LI:55\%, $\mathrm{p}=0.040$ ). Presence of N-LI and LI were significantly associated with stroke history in univariate (respectively $\mathrm{OR}=22.80, \mathrm{CI}-95 \%$ : 8.67-59.94, $\mathrm{p}<0.001$; $\mathrm{OR}=4.16, \mathrm{CI}-95 \%: 1.88-9.24, \mathrm{p}<0.001)$ and multivariate analysis (respectively OR=19.1, CI-95\%: 6.75-53.96, p<0.001; OR=1.46, CI95\%:1.04-2.05, $\mathrm{p}=0.030$ ) independently of age, education and gender. No differences occurred regarding other markers of SVD.

Conclusions: In our cohort of AF-patients on OAC, not only N-LI but also LI were independently associated with stroke history, suggesting that a relationship between AF and SVD may exist. 


\section{ISCHEMIC STROKE AND COVID-19: A SINGLE CENTER CASE-CONTROL RETROSPECTIVE SERIES}

J. Bottini, M. Corato, L. Fratticci, C. Mariotti D'Alessandro, M. Spinelli, L. Straffi, S. Marcheselli

Stroke Unit and Acute Neurology, Humanitas Clinical and Research Center IRCCS (Milano)

Objective: Description of a cohort of patients with acute ischemic stroke during Covid-19 outbreak at a comprehensive stroke center.

Materials and Method: Retrospective collection of data from a series of consecutive patients with ischemic stroke and PCR confirmed Covid19 infection; we compared them with two equally numerous sex-matched groups: a) consecutive patients who suffered an ischemic stroke and tested negative for Covid-19 during the pandemic period; b)consecutive patients admitted for stroke the previous year in the same period (MarchApril).

Results (average): 15 patients were identified. Covid diagnosis was at stroke time in 6 cases $(40 \%)$, afterwards 4 (26,6\%; delay 8,25 days), previously 5 (33,3\%). Age was 68 (ST 9), 78 in 2020 $(\mathrm{p}=0,015), 75$ in $2019 . \mathrm{M} / \mathrm{F}=2.75$. BMI was 29 ; risk factors: hypertension $86,7 \%$ (2019: $71,4 \%$; 2020: $60 \%$ ), diabetes $46,7 \%$ (2019: $20 \% ; 2020: 33,3 \%)$ and atrial fibrillation $26,7 \%(2019$ $20 \%$; 2020 20\%); other 53,3\% (2019: $73,33 \%$; 2020: 53,3\%). Mean NIHSS was 9.7 patients $(46,6 \%)$ received acute treatment (2019: 40\%; 2020: 60\%): iv tpa $\mathrm{n}=2$, mechanical thrombectomy $\mathrm{n}=3$, both $\mathrm{n}=2$. Last known well to ED time was 82 (2020: 305; $\mathrm{p}=0,045)$, door to scan 51, door to needle/puncture 114 minutes. Stroke etiology (TOAST): large artery 6,7\% (2019: 6,6\%; 2020: $26,6 \%$ ), small vessel 13\% (2019: 6,6\%; 2020: 6,6\%), cardioembolic 26,7\% (2019: 40\%, 2020: 26,7\%), undetermined 40\% (2019: $33,3 \%$; 2020: $40 \%)$, other $13 \%$ (0\% controls). mRS was 3 (2019: 2; 2020: 3). Two patients $(13,3 \%)$ died (2019: 6,6\%; 2020: 13,3\%). Relevant laboratory findings: CRP $6,7 \mathrm{mg} / \mathrm{dl}$ (higher compared to $2019 \mathrm{p}=0,014 ; 2020 \mathrm{p}=0,033$ ), ALT 33,46 (higher than $2019 \mathrm{p}=0,033 ; 2020 \mathrm{p}=0,046$;), LDH 317,35 U/l (higher than $2019 \mathrm{p}=0,024 ; 2020 \mathrm{p}=0,021$ ).

Discussion: In our series, stroke/Covid-19+ patients were mainly male, relatively younger, with a moderately severe stroke of prevalent undetermined etiology despite risk factors. No significant coagulation alteration was found [1]. D-dimer was elevated but not significantly. They were treated with no significant delay (a tendency to reduced door to scan time possibly because of dedicated pathways). There was a non significant tendency to a worse outcome and mortality (also in 2020 controls, probably reflecting higher NIHSS at admission and delayed presentation). Laboratory findings reflected inflammatory state and liver enzymes alterations [2]. Limitations: retrospective, small sample study.

Conclusions: Covid 19 in stroke patients poses a challenge in terms of management, pathophysiology understanding, treatment choices and outcome [3]. Further studies are needed.

References:

1. DC Hess, W Eldahshan, E. Rutkowski. COVID-19-related stroke. Translational Stroke Research (2020);11(3):322-25

2. Zhou M, Xinxin Z, Jieming Q. Coronavirus disease 2019 (COVID19): a clinical update. Frontiers of medicine (2020); 14(2):126-135

3. Markus Hugh S., M. Brainin. COVID-19 and stroke-A global World Stroke Organization perspective. International Journal of Stroke (2020);15.4:361-64
ASSESSMENT OF CEREBRAL HEMODYNAMICS IN PATIENTS WITH ACUTE STROKE AND TRANSIENT ISCHEMIC ATTACK WITH DIFFERENT ETIOPATHOGENESIS: PRELIMINARY RESULTS

\author{
N. Brunelli ${ }^{1}$, C. Altamura ${ }^{1}$, A. Fallacara ${ }^{1}$, C. Costa $^{1}$, C. Vico ${ }^{2}$, F. Vernieri ${ }^{1}$
}

${ }^{1}$ Headache and Neurosonology Unit, Neurology Unit, Campus BioMedico University of Rome (Roma); ${ }^{2}$ Neurology Unit, Campus BioMedico University of Rome (Roma)

Objectives: Cerebral hemodynamic health assessed by cerebral vasomotor reactivity (VMR), plays a fundamental role in protecting from cerebrovascular disease. An impaired VMR represents a negative prognostic factor for vascular and degenerative cognitive deterioration [1-2]. Only few studies have addressed VMR in acute phase of stroke, possibly due to methodological difficulties, reporting overall a reduced VMR in different stroke subtype [3]. We aim to evaluate VMR in patients with acute mild stroke and TIA to better understand its implication in the etiopathogenesis of different stroke (sub)types and to assess if it may represent a prognostic marker of clinical outcome.

Materials and Methods: This longitudinal observational study is started last January 2020 and is taking place at Headache and Neurosonology Unit of the Neurology Unit of Campus Bio-Medico University of Rome. We are enrolling patients admitted at our Unit with diagnosis of acute mild stroke with NIHSS $<8$, with supratentorial stroke vascular territory and with a temporal acoustic window suitable for ultrasound transit. Exclusion criteria are absence of a suitable temporal window and presence of hemodynamically significant intracranial and extracranial stenoses. We are evaluating all patients with diagnostic tests to define the etiopathogenesis according to clinical guidelines. The observation period of 12 months includes 4 assessments (T1 - 48-72h from stroke onset, T2 - 1 month follow up, T3 - 6 months follow up and T4 12 months follow up). We are performing clinical scales and cognitive tests at each time and cerebral VMR to hypercapnia using the breathholding index (BHI) with ultrasound techniques in acute phase (T1) and at 6-months follow up (T3) in all patients.

Results: From January to July 2020, 20 patients with stroke or TIA were enrolled. Of these, 12 were male and 8 female; 18 had a stroke and 2 TIA. VMR, cognitive tests (MMSE and MOCA) and NIHSS were performed at admission time, between 48-72 hours after stroke/TIA onset. NIHSS value in acute phase was also recorded. The mRS before stroke and at discharge time was recorded. 16 patients were clinically evaluated at 1 month-follow up. Only 1 patient failed to show at the 1 monthfollow-up evaluation, deciding to leave the study. 6-months follow-up evaluations are started, and we are continuing to enroll new patients.

Discussion and Conclusion: These are preliminary results about a short period of clinical observation and cerebral hemodynamics evaluation of patients with acute mild stroke or TIA admitted to our department. References:

1. Silvestrini M, Pasqualetti P, Baruffaldi R, et al. Cerebrovascular Reactivity and Cognitive Decline in Patients with Alzheimer Disease. Stroke (2006); 37:1010-15

2. Balestrini S, Perozzi C, Altamura C, Vernieri F, Luzzi S, Bartolini $\mathrm{M}$, et al. Severe carotid stenosis and impaired cerebral hemodynamics can influence cognitive deterioration. Neurology (2013); $80(23): 2145-50$

3. Cupini LM, Diomedi M, Placidi F, Silvestrini M, Giacomini P. Cerebrovascular reactivity and subcortical infarctions Arch Neurol (2001);58(4):577-81 


\section{B.U.N. TO CREATININE RATIO CAN PREDICT NEW ONSET ATRIAL FIBRILLATION IN ISCHEMIC STROKE}

\author{
M. Burattini ${ }^{1}$, E. Potente ${ }^{1}$, S. Salvemini ${ }^{1}$, L. Falsetti ${ }^{2}$, M. Bartolini ${ }^{1}$, M. \\ Silvestrini ${ }^{1}$, G. Viticchi ${ }^{1}$
}

${ }^{1}$ Neurological Clinic, Marche Polytechnic University (Ancona); ${ }^{2}$ Internal and Subintensive Medicine, Ospedali Riuniti (Ancona)

Objectives: Stroke is the leading cause of long-term disability in developed countries. Almost 25 to $30 \%$ of ischemic stroke have a heart source, especially associated to Atrial Fibrillation (AF). Previous studies have found that blood urea nitrogen (B.U.N.) to creatinine ratio (BCR) could be associated to worse outcome among patients with ischemic stroke. However, no studies have explored the relationship between BCR and AF. This study was aimed to investigate the possible relationship between BCR and $\mathrm{AF}$ in ischemic stroke patients.

Materials and Methods: We enrolled 220 patients admitted for acute ischemic stroke to the Neurological Clinic of the Ospedali Riuniti Ancona from January 2019 to December 2019. For each patient we collected age, electrolytes, serum azotemia, serum creatinine and BCR; we evaluated the occurrence of new-onset $\mathrm{AF}$, the presence of pre-existing $\mathrm{AF}$ and the presence of any type of AF. Statistical analysis was performed with SPSS 13.0 for Windows Systems.

Results: We obtained a final sample of 220 subjects, females representing $45 \%$ of the cohort, with a mean age of $70,2 \pm 12,4$ years. $58(23,6 \%)$ patients were affected by any form of AF, 33 $(15 \%)$ had pre-existing AF and $25(11,3 \%)$ had new-onset AF. We observed that patients affected by any form of AF had a significantly higher $\mathrm{BCR} 23,5[6,8]$ than patients without $\mathrm{AF}$, who had a median BCR of $21.5[7,5](p=0,029)$. Patients with pre-existing $\mathrm{AF}$ had a median $\mathrm{BCR}$ of $23[6,8]$, which resulted similar to the one of patients without AF, who had a median BCR of $22[7,5](p=0,504)$. Patients with new-onset AF had a median BCR of 25 [7], significantly higher than the BCR observed in the cohort without new-onset $\mathrm{AF}$, which was 21,5 $[7,5](p=0,023)$. ANOVA confirmed the BCR difference between the three $\mathrm{AF}$ categories (no $\mathrm{AF}$, pre-existing $\mathrm{AF}$ and new-onset AF) $(\mathrm{p}=0,001)$.

Discussion: Ischemic stroke could determine the activation of sympathetic nervous system (SNS) with the stimulation of renin-angiotensinaldosterone system (RAAS). The RAAS increases the absorption of water and sodium in the kidney tubules, causing passive reabsorption of BUN from tubules. SNS activation can also induce changes of atrial electrophysiology and induce atrial tachyarrhythmias, including AF. The relationship between $\mathrm{AF}$ and $\mathrm{BCR}$ had to be investigate by future larger study to better evidence possible consequences for people affected by ischemic stroke.

References:

- Deng L, Wang C, Qiu S, Bian H, Wang L, Li Y, Wu B, Liu M. Association between Blood Urea Nitrogen-to-creatinine Ratio and Three-Month Outcome in Patients with Acute Ischemic Stroke. Curr Neurovasc Res. (2019);16(2):166-72

- You S, Zheng D, Zhong C, Wang X, Tang W, Sheng L, Zheng C, Cao Y, Liu CF. Prognostic Significance of Blood Urea Nitrogen in Acute Ischemic Stroke. Circ J. (2018) Jan 25;82(2):572-78

- Chen PS, Chen LS, Fishbein MC, Lin SF, Nattel S. Role of the autonomic nervous system in atrial fibrillation: pathophysiology and therapy. Circ Res. (2014) Apr 25;114(9):1500-15

\section{ACUTE ISCHAEMIC STROKE TREATMENT AT AORN CARDARELLI, NAPLES}

P. Candelaresi ${ }^{1}$, G. Servillo ${ }^{1}$, G. Alfieri ${ }^{1}$, G. Ambrosanio ${ }^{2}$, W. Di Iorio ${ }^{1}$, G. Leone $^{2}$, K. Longo ${ }^{1}$, G. Maniscalco ${ }^{1}$, M. Muto ${ }^{2}$, M. Napolitano ${ }^{1}$, A. Ranieri ${ }^{1}$, R. Renna ${ }^{1}$, M. Rippa ${ }^{1}$, A. Sagnelli ${ }^{1}$, S. Salvatore ${ }^{1}$, M. Muto ${ }^{2}$, V. Manzo ${ }^{2}$

${ }^{1}$ Neurology and Stroke Unit, AORN Cardarelli (Napoli); ${ }^{2}$ Neuroradiology Unit, AORN Cardarelli (Napoli)

Introduction and Aim: Timely treatment for ischemic stroke is of paramount importance to reduce the burden on death, disability and social costs. The development of acute stroke care in the South of Italy has historically been arduous. Here we present the analysis of the first 3 years of acute ischaemic stroke treatment at AORN Cardarelli, Naples, Italy.

Materials and Methods: Data regarding acute stroke revascularization treatments since August 2017, to June 2020 are presented. We analysed: 1) number of patients treated with acute therapies; 2) pre-hospital and inhospital delays; 3) DeltaNIHSS score between admission and discharge and 3-months modified Rankin Scale score; 4) complications.

Results: AORN Cardarelli in Naples is one of the 7 stroke hubs in Campania and the largest hospital of the South of Italy. In August 2017 a local acute revascularization treatment registry was developed. The number of acute treatments grew from 39 patients in the period AugustDecember 2017 to 211 patients in the year 2018, to 229 in 2019 and settled at 147 at the end of June 2020. The percentage of treatments for unwitnessed stroke grew from $2.5 \%$ to $17 \%$. We observed a relative increase in the combined intravenous+endovascular treatments for large vessel occlusions (from $5.1 \%$ in 2017 to $41.4 \%$ in 2020), whereas the percentage of primary thrombectomies remained stable at approximately $20 \%$. The number of second referrals from peripheral hospitals grew from $5 \%$ to $28.5 \%$ of total treatments and, accordingly, the global prehospital delays significantly increased $(\mathrm{p}<0.05)$. The inhospital delays significantly decreased from the first year of treatment $(\mathrm{p}<0.05)$ but then plateaued at 80 minutes door-to-needle time and 130 minutes door-togroin time. Delta-NIHSS between admission and discharge was statistically significant $(\mathrm{p}<0.05) .46 .9 \%$ of patients was independent (mRS $0-2)$ at the 3 -months outcome, whereas $17.3 \%$ were dead. For safety measures, we observed a global $9.4 \%$ parenchymal hemorrhagic transformation, $51 \%$ of them being fatal, without any statistical difference between primary thrombectomy and intravenous+endovascular treatment ( $\mathrm{p} 0.30$ and p 0.14 , respectively)

Discussion and Conclusion: This analysis shows the increase in the number of patients treated with acute reperfusion therapies since August 2017 at AORN Cardarelli, Naples. The early and 3-months outcome are in line with literature. Interestingly, our analysis did not show a statistically significant increase in the risk for fatal and non-fatal hemorrhagic transformation between primary thrombectomy and a combined intravenous+endovascular treatment. Streamline the prehospital and inhospital stroke pathways is our principal immediate aim.

\section{THE IMPACT OF COVID-19 LOCKDOWN ON STROKE ADMISSIONS AND TREATMENTS IN CAMPANIA}

P. Candelaresi ${ }^{1}$, G. Servillo ${ }^{1}$, M. Muto ${ }^{2}$, P. Barone ${ }^{3}$, R. Napoletano ${ }^{3}$, R. Saponiero $^{4}$, V. Andreone ${ }^{5}$, V. Palma ${ }^{6}$, D. Spitaleri ${ }^{7}$, F. D'Onofrio ${ }^{7}$, G. Maniscalco $^{1}$, S. Simona ${ }^{1}$, G. Leone ${ }^{2}$, E. Capone ${ }^{2}$, C. Schettino ${ }^{3}$, D. Romano $^{4}$, G. Martusciello ${ }^{5}$, S. Miniello ${ }^{5}$, M. Mazzaferro ${ }^{6}$, S. Ascione ${ }^{6}$, V. Manzo 
${ }^{1}$ Neurology and Stroke Unit, AORN Cardarelli (Napoli); ${ }^{2}$ Neuroradiology Unit, AORN Cardarelli (Napoli); ${ }^{3}$ Neurology and Stroke Unit, AOU San Giovanni di Dio Ruggi d'Aragona (Salerno); ${ }^{4}$ Neuroradiology Unit, AOU San Giovanni di Dio Ruggi d'Aragona (Salerno); ${ }^{5}$ Neurology and Stroke Unit, AORN S. Sebastiano (Caserta); ${ }^{6}$ Neurology and Stroke Unit, ASL Napoli 1 Ospedale del Mare (Napoli); ${ }^{7}$ Neurology and Stroke Unit, AORN S. G. Moscati (Avellino)

Background and Aim: The enforcement of complete lockdown with home confinement has been necessary to limit SARS-CoV-2 contagions in Italy, one the most affected country worldwide. Simultaneously, in several Emergency Departments, a reduction in cardioand cerebrovascular presentations was noticed. This study analyses the impact of Covid-19 pandemic and lockdown on the incidence of stroke, in Campania, the most densely-populated region in Italy.

Methods: We retrospectively analysed data regarding acute stroke patients presenting at 5 Campania stroke hubs, over a 10-week period around the issue of lockdown in Italy on March 9 th.

Results: Compared to the pre-lockdown, we observed an overall reduction of $-15.4 \%$ in the number of all ischemic stroke and $-27.4 \%$ in the number of acute reperfusion treatments. Compared to the same period of 2019 , we observed a reduction of $-20.4 \%$ and $-32.6 \%$ respectively during lockdown. The time to reach medical attention was significantly longer in the lockdown phase (230 versus 155 minutes, $\mathrm{P} 0.016$ ). For patients who underwent acute reperfusion treatment we also observed longer timetoimaging ( 30 versus 40 minutes, $\mathrm{P} 0.0005$ ) and time-to-needle ( 75 versus 90 minutes $\mathrm{P}$ 0.23), but not time-to-groin.

Discussion and Conclusion: This study showed the reduction in hospital admissions for acute ischemic stroke and acute reperfusion treatments and the slowdown of stroke pathways, during the lockdown phase of Covid-19 pandemic, in Campania, the third-most-populous and the most-densely populated Italian Region. In the next future, the risk for high-grade disability and death, due to delayed or even avoided hospital presentation due to fear of contagion, may be high.

\section{MECHANICAL THROMBECTOMY IN SEVERE CEREBRAL VENOUS THROMBOSIS: OUR CASE SERIES AT NIGUARDA HOSPITAL}

\author{
A. Cascio Rizzo ${ }^{1}$, G. Giussani ${ }^{1}$, A. Gatti ${ }^{1}$, C. Motto ${ }^{1}$, A. Cervo ${ }^{2}$, G. \\ Pero $^{2}$, E. Agostoni ${ }^{1}$
}

${ }^{1}$ Neurology \& Stroke Unit, ASST Grande Ospedale Metropolitano Niguarda (Milano); ${ }^{2}$ Neuroradiology, ASST Grande Ospedale Metropolitano Niguarda (Milano)

Cerebral Venous Thrombosis (CVT) is a rare cause of stroke with higher frequency in young people ( $<40$-years of age), thrombophilia, pregnant women or receiving hormonal contraceptive therapy. Heparin is the firstline treatment, but in a few cases with increased risk of poor outcome more aggressive treatments, such as local intravenous thrombolysis or mechanical thrombectomy may be required. We present 5 cases of severe CVT with poor outcome subjected to EVT at our Neuro center from February 2019 to May 2020. Of these patients 4 (80\%) were women, median age was 49 years. Median NIHSS was 7.5, 2 patients had GCS less than 8. Baseline CT scan showed ICH in 3 patients. On cerebral angiography 3 patients $(60 \%)$ had severe multiple CVT while the other 2 had a sinus transverse and a superior sagittal sinus thrombosis respectively, complicated by ICH and midline shift. Considering factors of increased poor outcome (multiple CVT, vigilance status worsening,
ICH, midline shift) all patients underwent mechanical thrombectomy (80\% thromboaspiration plus stent retriever) with complete recanalization in 3 patients and partial in one patient. In 2 patients early reocclusion occurred. About safety profile, a new ICH occurred in 2 patients and decompressive hemicraniectomy was performed. About neurological outcome one patient died, median mRS at discharge was 1.5 in the other patients. Because of the lack of evidence, current guidelines does not provide a strong recommendation on EVT for CVT, the AHA/ASA guideline [1] recommends considering EVT only in patients who deteriorate despite anticoagulant treatment. In a systematic review of 185 cases of CVT in whom mechanical thrombectomy was performed, $84 \%$ had a good outcome (mRS 0-1) and 74\% had near to complete recanalization with a reasonable safety profile ( $10 \%$ with new or increased ICH) [2]. The TO-ACT trial [3] recently tested efficacy and safety of EVT plus standard medical care compared to standard medical care alone in patients with CVT at high risk of bad outcome. The trial didn't reach the primary endpoint but has several limitations such as small sample size, different severity of patients, technical aspects of the procedure (devices, experience of neuro-interventionalists), recruitment was stopped in 2016. Considering literature and our experience, baseline ICH seems to be a predictors of bad outcome despite EVT. Probably a better selection of patients with severe CVT and unfavorable outcome, the use of most recent available devices and techniques could show superiority of EVT when standard medical care seems to have a low likelihood of benefit.

References:

1. Saposnik G, Barinagarrementeria F, Brown RD Jr, et al; American Heart Association Stroke Council and the Council on Epidemiology and Prevention. Diagnosis and management of cerebral venous thrombosis: a statement for healthcare professionals from the American Heart Association/American Stroke Association. Stroke (2011);42(4):1158-92

2. Siddiqui FM, Dandapat S, Banerjee C et al., Mechanical thrombectomy in cerebral venous thrombosis: systematic review of 185 cases. Stroke (2015) May;46(5):1263-8

3. Coutinho JM, Zuurbier SM, Bousser MG et al. Effect of endovascular treatment with medical management vs standard care on severe cerebral venous thrombosis the TO-ACT Randomized Clinical Trial. JAMA Neurol. (2020) May 18;e201022

\section{GENDER DIFFERENCES IN CHARACTERISTICS OF INTRACEREBRAL HEMORRHAGE IN THE ELDERLY: RESULTS FROM A POPULATION-BASED STROKE REGISTRY}

\author{
G. Ceccanti, R. Ornello, E. Colangeli, C. Scarpato, E. De Matteis, F. De \\ Santis, B. Orlandi, C. Marini, F. Pistoia, G. Desideri, S. Sacco
}

Department of Neurology, University of L'Aquila (Avezzano-AQ)

Objective: To compare the characteristics and outcomes of intracerebral hemorrhage $(\mathrm{ICH})$ between men and women according to age.

Materials and Methods: We included in the present study patients residing in the district of L'Aquila suffering a first-ever ICHs between 2011 and 2018. We assessed the patients' baseline characteristics, including sex and vascular risk factors. We also assessed the severity of neurological deficit on admission (determined by the National Instituted of Health Stroke Scale [NIHSS] score), ICH volume, 30-day, and 1-year case-fatality. Those characteristics were compared between men and women in the two age groups using the chi squared or $t$ test as appropriate. 
Results: We included in the study 508 patients $(58.7 \%$ males with a mean age of $74.3 \pm 13.2$ years; $294(57.8 \%)$ patients were aged $\geq 75$ years and $214(42.2 \%)<75$ years. Men and women were equally represented $(49.7 \%$ and $50.3 \%$, respectively) in the elderly, while in younger patients men outnumbered women $(71.6 \%$ and $29.4 \%)$. In the elderly, men had higher systolic blood pressure values $(165.31 \pm 31.44 \mathrm{mmHg}$ vs 156.96 $\pm 32.51 \mathrm{mmHg} ; \mathrm{p}=0.047)$ and a lower proportion of lobar ICH $(33.1 \%$ vs $41.8 \%$; $p=0.037$ ) compared with women; the prevalence of arterial hypertension (AH), diabetes mellitus (DD), hypercholesterolemia, smoking (SA), peripheral arterial disease (PAD), atrial fibrillation (FA), and coronary heart disease (CHD) was similar in both genders. In the younger group, men had a higher prevalence of $\mathrm{AH}(74.6 \%$ vs $57.1 \%$, p< $0.05)$ and $\mathrm{DD}(28.9 \%$ vs $12.5 \%$; $\mathrm{p}=0.015)$ and a lower proportion of lobar ICH ( $28.9 \%$ vs $48.2 \%$; $\mathrm{p}=0.018$ ) compared with women, while the prevalence of hypercholesterolemia, SA, PAD, FA, and CHD was similar. The mean NIHSS score at ICH onset was higher in women than in men aged $<75$ years $(13.36$, IQR vs 9.83 , IQR; $p<0.05)$ while in elderly there were similar values according to gender.

Discussion: According to our data, vascular risk factor profile was especially unfavorable in younger men compared with women, while gender differences were less pronounced in the elderly. Lobar location of ICH was more prevalent in women than in men at any age, possibly reflecting a higher likelihood of non-hypertensive causes with respect to men.

Conclusions: According to our data, it is important to control vascular risk factors especially in young and middle-aged men as compared with women, while non-hypertensive causes should be investigated in women who are prone to lobar ICH.

\section{EXTENSION OF ENDOVASCULAR THERAPEUTIC WINDOW IN ISCHEMIC STROKE: THE CLINICAL EXPERIENCE OF NIGUARDA HOSPITAL}

\author{
G. Cecchi ${ }^{1}$, G. Giussani ${ }^{2}$, A. Cascio Rizzo ${ }^{2}$, F. Vernieri ${ }^{3}$, E. Agostoni ${ }^{2}$
}

${ }^{1}$ Multiple Sclerosis Clinical and Research Unit, Department of Systems Medicine, Tor Vergata University (Roma); ${ }^{2}$ Neurology and Stroke Unit, Niguarda Hospital (Milano); ${ }^{3}$ Headache and Neurosonology Unit, Neurology, Campus Bio-Medico (Roma)

Objective: Two trials contributed to the expansion of the therapeutic window for ischemic stroke: DEFUSE-3 and DAWN trial. The first extended time window for endovascular treatment up to 16 hours, the second one up to 24 hours. The aim of this study is to evaluate the impact of time window extension in clinical practice, analyzing the reality of the Niguarda Hospital in Milan.

Materials and methods: In this study, we retrospectively examined patients with ischemic stroke due large vessel occlusions, admitted to Niguarda's Stroke Unit and subjected to acute endovascular treatment. We collected patients from January 2017 to June 2019. We divided the patients into 2 groups: from January 2017 to March 2018 and from April 2018 to June 2019 considering that DAWN and DEFUSE-3 were published on January 2018. The main source of data in this study was the Stroke Unit Database. The primary endpoint was to evaluate mRS score after three-month in the two populations. Secondary endpoints were change in the number of treated patients, change of stroke characteristics, complications and recanalization outcome.

Results: In the first group 44 patients were treated, in the second 92 (average age was 71.9 vs 73.4). We noticed a statistically significant decrease in initial NIHSS (16 vs 10, p 0.002). Stroke in the anterior circle did not report a statistically significant variation (TACI $82 \%$ vs $87 \%$ ) same for the posterior circle (PoCI 18\% vs 13\%). We noticed an increase in M2 endovascular treatment (11\% vs 36\%). We detected a statistically significant increase of strokes with onset after 6 hours in the second group ( $9 \%$ vs $40 \%, p=0.003$ ). In the first group pre-stroke mRS was 0 in $90 \%$ of the patients and $\mathrm{mRS} 1-2$ in the remaining $10 \%$. In the second group pre-stroke mRS was 0 in $78 \%$ of the patients. We did not detect a statistically significant change by comparing the variation of $\mathrm{mRS}$ after threemonth in the two groups $(\mathrm{p}=0.932$, mRS score $0-1$ after three-month $40 \%$ vs $38 \%$ ). In the second group, we made a statistically significant increase in the use of stentrievers $(24 \%$ vs $51 \%, \mathrm{p}=0.02)$ We did not report significant changes on deaths or periprocedural complications.

Discussion: Expanding the therapeutic window increased number of treated patients leading also to the treatment of both more complex cases and more distal vessels.

Conclusion: This paper confirms the usefulness and safety of DAWN and DEFUSE-3 trials in daily clinical practice.

References:

- Nogueira R. G., Jadhav A. P., Haussen D. C., Bonafe A., Budzik R. F., Bhuva P., Jovin T. G. Thrombectomy 6 to 24 hours after stroke with a mismatch between deficit and infarct. New England Journal of Medicine (2018) Jan 4;378(1):11-21. doi.org/10.1056/ NEJMoa1706442

- Albers G. W., Lansberg M. G., Kemp S., Tsai J. P., Lavori P., Christensen S., Marks M. P. A multicenter randomized controlled trial of endovascular therapy following imaging evaluation for ischemic stroke (DEFUSE 3). International Journal of Stroke (2017) Oct;12(8):896-905 doi.org/10.1177/1747493017701147

- Adeoye O., Nyström K. V., Yavagal D. R., Luciano J., Nogueira R. G., Zorowitz R. D., Jauch E. C. Recommendations for the Establishment of Stroke Systems of Care: A 2019 Update. Stroke (2019) Jul;50(7):e187-e210 doi.org/10.1161/STR.0000000000000173

\section{ANTICOAGULATION AND THE NEUROLOGIST}

\author{
B. Censori ${ }^{1}$, T. Partziguian ${ }^{2}$, A. Giossi ${ }^{1}$, V. De Giuli ${ }^{1}$, M. Sessa ${ }^{2}$ \\ ${ }^{1}$ Neurology Unit, ASST Cremona (Cremona); ${ }^{2}$ UOC Neurology, ASST \\ Papa Giovanni XXIII (Bergamo)
}

Objective: To assess the number and type of anticoagulation (AC) cases routinely facing the neurologist working in a large tertiary hospital.

Patients and Methods: We have consecutively registered all patients admitted to Papa Giovanni XXIII Hospital from January 1st to December 31st, 2019 with active AC, or with an indication for AC identified during hospitalization (or for whom AC was seriously considered because of their main disease).

Results: We have collected 140 patients, 66 men and 74 women, with a mean \pm SD age of $76.4 \pm 11.5$ yrs. They represented $16.4 \%$ of all admissions of 2019. Of them, 124 were admitted for an acute cerebrovascular disease (CVD)(88.6\%), and 16 for non-vascular reasons (11.4\%). Among CVD, there were 101 ischemic strokes or TIAs (81.4\%), 17 intracranial hemorrhages (13.7\%), and 6 cerebral venous thromboses $(4.8 \%)$. They represented $31.5 \%$ of all admission for acute CVD. Sixty-seven patients were already under AC at admission $(47.9 \%$; $25.7 \%$ vitamin-K antagonist; $21.4 \%$ direct oral anticoagulant; $0.7 \%$ fondaparinux), 9 had recently interrupted $\mathrm{AC}(6.4 \%)$, and $64(45.7 \%)$ had an indication for AC identified during hospitalization. Reasons for AC were cardiological in 120 (85.7\%), non-cardiological in $13(9.3 \%)$, 
while 7 patients $(5.0 \%)$ had multiple acute infarcts in different vascular territories without cardioembolic conditions. Among patients taking anticoagulants at admission, thrombolysis was carried out in 1 patient $(1.5 \%)$ taking dabigatran after reversal with idarucizumab, while thrombectomy was carried out in $4(6.0 \%)$. At discharge 94 patients were under AC $(67.1 \% ; 24.3 \%$ warfarin, $37.9 \%$ direct oral anticoagulants, and $5.0 \%$ low molecular weight heparin) and 46 were not anticoagulated $(32.9 \%)$.

Conclusions: Management of anticoagulation is necessary in a substantial percentage of patients admitted to a modern Neurology ward of a tertiary care hospital, mostly because of acute cerebrovascular diseases and cardiac embolic sources. Therefore, a working knowledge of AC is a requirement for all neurologists dealing with hospitalized patients, while a deep knowledge of AC, and the many variables that influence AC treatment after a neurological event, must be systematically cultivated by vascular neurologists.

\section{PLATELET FUNCTION TESTS AFTER CAROTID STENTING DURING ENDOVASCULAR ACUTE STROKE PROCEDURES PREDICTS 90-DAYS FUNCTIONAL OUTCOME}

L. Ciolli ${ }^{1}$, N. Lelli ${ }^{2}$, F. Rosafio ${ }^{1}$, S. Maffei ${ }^{1}$, F. Sacchetti ${ }^{3}$, L. Vandelli ${ }^{1}$, M. Dell'Acqua ${ }^{1}$, S. Meletti ${ }^{4}$, A. Zini ${ }^{5}$, L. Picchetto ${ }^{1}$, G. Borzi ${ }^{1}$, R. Ricceri $^{1}$, S. Vallone ${ }^{3}$, G. Bigliardi ${ }^{1}$

${ }^{1}$ Stroke Unit, Neurology Clinic, Department of Neuroscience, Ospedale Civile di Baggiovara, Azienda Ospedaliera Universitaria di Modena (Modena); ${ }^{2}$ Laboratory of Clinical Pathology and Toxicology, Department of Laboratory Medicine, Ospedale Civile di Baggiovara, Azienda Ospedaliera Universitaria di Modena (Modena); ${ }^{3}$ Neuroradiology Unit, Department of Neuroscience, Ospedale Civile di Baggiovara, Azienda Ospedaliera Universitaria di Modena (Modena); ${ }^{4}$ Department of Biomedical, Metabolic and Neural Sciences, Sciences University of Modena and Reggio Emilia (Modena); ${ }^{5}$ IRCCS Istituto delle Scienze Neurologiche, Department of Neurology and Stroke Unit, Maggiore Hospital (Bologna)

Introduction: Many studies highlighted the role of platelet reactivity testing in predicting cardiovascular events after percutaneous coronary stenting or stroke [1,2]. High on treatment platelet reactivity has also been associated with plaque instability and thromboembolic complications during elective carotid stenting [3]. The aim of our study was to assess the role of aggregometry in predicting 90-days functional outcome in patients with ischemic stroke treated with endovascular procedures requiring emergent carotid stenting. We also evaluated the association between platelets inhibition and the risk of stent thrombosis and intracerebral hemorrhage (ICH).

Material and methods: We performed a retrospective study. We used aggregometry Multiplate ${ }^{\circledR}$ (Roche Diagnostics, Mannheim, Germany) to perform the tests. Patients were divided according to validated cutoffs in acetylsalicylic acid (ASA) and P2Y12-inhibitors responders and not responders. Age, stroke etiology, cardiovascular comorbidities, National Institute of Health Stroke Scale (NIHSS) at admission, intravenous thrombolysis, type of antiplatelets and labs finding at admission were also considered. We divided patients according to the modified Rankin Scale $(\mathrm{mRS})$ at 90 days in functionally independent ( $\mathrm{mRS} 0-2)$ and dependent (mRS 3-6).

Results: We included in the final analysis 57 patients. ASA resistance was more common in dependent patients at 90 days $(33,3 \%$ vs $6,1 \%$, p < 0.01 ) whereas P2Y12-inhibitors resistance in independent patients
(62,1\% vs 31,6\%, p: 0,04). At multivariate analysis ASA resistance was associated with poor outcome (OR: 47,74; 95\% CI, 3,71 - 614,5), together with ICH and higher glycemia, while a trend was found for improved outcome in patients with P2Y12 inhibitors resistance (OR: 4,36, 95\% CI, $0,84-22,66)$. ASA resistance was also associated with higher 90 -days mortality $(60 \%$ vs $13,5 \%, \mathrm{p}<0,01)$. Stent thrombosis was observed in 2/10 patients $(20 \%)$ with ASA resistance and 3/47 (6,4\%) without (p: $0,21)$ and in $2 / 24$ patients $(8,3 \%)$ with and without P2Y12-inhibitors resistance (p: 1). Finally, ICH occurred less frequently, without reaching statistical significance, in patients with ASA resistance $(20 \%)$ or P2Y12 inhibitors resistance $(25 \%)$ than in patients with correct platelets inhibition (40,4\%, p: 0,3 and 41,7\%, p: 0,4; respectively).

Discussion: We found a significant association between resistance to ASA and poor 90-days functional outcome and between resistance to P2Y12 inhibitors and good 90-days functional outcome.

Conclusion: Further studies will be needed, but we can speculate that ASA mediated platelets inhibition might be critical in preventing early thrombotic complications whereas P2Y12 inhibition in the hyperacute phase might increase the risk of hemorrhagic complications without reducing thrombotic events.

References:

1. Brar SS, Ten Berg J, Marcucci R, Price MJ, Valgimigli M, Kim HS, et al. Impact of platelet reactivity on clinical outcomes after percutaneous coronary intervention: A collaborative meta-analysis of individual participant data. J Am Coll Cardiol. (2011) Nov 1;58(19):1945-54

2. Fiolaki A, Katsanos AH, Kyritsis AP, Papadaki S, Kosmidou M, Moschonas IC, et al. High on treatment platelet reactivity to aspirin and clopidogrel in ischemic stroke: A systematic review and metaanalysis. J Neurol Sci. (2017) May 15;376:112-6

3. Müller-Schunk S, Linn J, Peters N, Spannagl M, Deisenberg M, Brückmann H, et al. Monitoring of clopidogrel-related platelet inhibition: Correlation of nonresponse with clinical outcome in supra-aortic stenting. Am J Neuroradio (2008) Apr;29(4):786-91

\section{PROGNOSIS OF PATIENTS WITH INTRACEREBRAL HEMORRHAGE: DATA FROM A POPULATION-BASED STROKE REGISTRY}

E. Colangeli ${ }^{1}$, F. Marrese ${ }^{1}$, R. Ornello ${ }^{1}$, I. Frattale ${ }^{1}$, C. Tiseo ${ }^{1}$, E. De Matteis $^{1}$, C. Scarpato ${ }^{1}$, C. Marini ${ }^{2}$, F. Pistoia ${ }^{1}$, F. De Santis ${ }^{3}$, B. Orlandi $^{3}$, S. Sacco ${ }^{1}$

${ }^{1}$ Department of Applied Clinical Sciences and Biotechnology, University of L'Aquila (L'Aquila); ${ }^{2}$ Department of Medicine, Public Health, Life and Environmental Sciences, University of L'Aquila (L'Aquila); ${ }^{3}$ Department of Neurology, 'SS. Filippo e Nicola' Hospital (Avezzano-AQ)

Objective: We evaluated the short and long-term outcome of patients with intracerebral hemorrhage (ICH) [1-2], with a special regard to ICH volume and hematoma expansion (HE).

Materials and Methods: We included in our registry all patients presenting with a first-ever ICH (code 431, WHO ICD-9) in 2011-2017 and resident in the L'Aquila district. The classification of HE was evaluated according to brain computerized tomography $(\mathrm{CT})$ in patients who repeated brain neuroimaging. To evaluate $\mathrm{ICH}$ volume we used the $\mathrm{ABC} / 2$ method [3].

Results: Among the 499 patients included in this study, 206 (41.3\%) were women. The mean $\pm \mathrm{SD}$ age was $74.6 \pm 13.3$ years. One hundred and eighty-one patients died at 30 day, with a case fatality rate (CFR) of 
36.3\%. At 1-year, 222 patients died, with a corresponding CFR of $44.5 \%$. The factors independently associated with 30 -day mortality were NIHSS at onset (OR 1.13, 95\% CI 1.08-1.18; $<<0.001)$, disturbance of consciousness (OR 2.56, 95\% CI 1.01-1.04; P<0.001), ICH volume (OR 1.02, 95\% CI 1.01-1.04; $\mathrm{P}<0.001$ ), and $\mathrm{mRs}$ score before ICH onset (OR 1.56, 95\% CI 1.15-2.13;P<0.001). The factors independently associated with 1 -year mortality were age (OR 1.04, 95\% CI, 1.01-1.07; $\mathrm{P}<0.001)$, NIHSS at onset (OR 1.12, 95\% CI 1.07-1.17; P<0.001), ICH volume (OR 1.02, 95\% CI 1.01-1.04; $\mathrm{P}<0.001$ ), and $\mathrm{mRs}$ score before ICH onset (OR 1.28, $95 \%$ CI 1.01-1.62; $\mathrm{P}<0.001)$. All patients had at least one brain CT. Three hundred and forty $(68.13 \%)$ of the included patients performed a second brain CT after 1 median day (IQR 1-2); the remaining 159 (31.87\%) patients performed a single brain scan because of early death. Ninetyfour patients $(27.64 \%)$ presented a HE at the second brain CT. Patients with HE had higher 30 -day $(57.4 \%$ vs $19.1 \%$; $\mathrm{P}<0.001)$ and 1 -year (69.1\% vs $29.3 \%$; $\mathrm{P}<0.001)$ CFRs as compared with those without HE. The only factors independently associated with HE was NIHSS, per point (OR 1.13, 95\% CI 1.07-1.19; $\mathrm{P}<0.001$ ). The Cox regression analysis showed that ICH volume (HR 1.04; 95\% CI, 1.01-1.06; $\mathrm{p}=0.003$ ) and NIHSS at discharge (HR per each NIHSS point 1.15; 95\% CI, 1.02-1.29; $\mathrm{p}=0.019$ ) were independent predictors of 1 -year case-fatality in patients with a second brain CT.

Discussion and Conclusions: Our study confirms a poor outcome of patients with ICH. Patients with poor outcome are those who have clinical and neuroradiological markers of more severe ICH and have early enlargement of the hematoma. Finding of therapeutic strategies which may limit hematoma enlargement may represent a tool to improve prognosis of patients with $\mathrm{ICH}$.

References:

1. Sacco S, Marini C, Toni D, Olivieri L, Carolei A. Incidence and 10year survival of intracerebral hemorrhage in a population-based registry. Stroke (2009);40(2):394-99

2. Sacco S, Ornello R, Degan D, Tiseo C, Pistoia C, Carolei A. Declining incidence of intracerebral hemorrhage over two decades in a population-based study. European Journal of Neurology (2016);23:1627-34

3. Kothari RU, Brott T, Broderick JP, et al. The ABCs of measuring intracerebral hemorrhage volumes. Stroke (1996);27(8):1304-5

\section{PREDICTION OF FUTILE MECHANICAL THROMBECTOMY FOR ACUTE ISCHEMIC STROKE IN VERY OLD PATIENTS}

\author{
F. D'Agostino ${ }^{1}$, F. Marrama ${ }^{1}$, A. Mascolo ${ }^{1}$, F. Sallustio ${ }^{1}$, A. Rocco ${ }^{1}$, V. \\ Da $\operatorname{Ros}^{2}$, R. Gandini ${ }^{2}$, M. Diomedi ${ }^{1}$
}

${ }^{1}$ Department of System Medicine, University of Rome Tor Vergata (Roma); ${ }^{2}$ Department of Biomedicine and Prevention, University of Tor Vergata (Roma)

Background: A significant impact of age on thrombectomy (MT) outcome has been proved. In spite of a clear efficacy and safety of this treatment in aged patients, a poor outcome compromises the final effect. We aimed to evaluate possible predictive factors of futile outcome after MT in patients $\geq 85$ years old to provide a useful score to predict the 3 -month outcome in this, particularly fragile population.

Methods: A retrospective analysis of an observational registry of patients aged $\geq 85$ y.o. with acute ischemic stroke due to anterior large vessel occlusion treated with MT was performed. Demographic, clinical, imaging and procedural characteristics were recorded. Main analysis was performed to evaluate clinical and imaging pre-treatment factors associated with i) 3-months mortality and ii) 3-months futile outcome (mRS $\geq$ 5 ). We created a prognostic score of futility (mRS score $\geq 5$ ) in the derivation cohort.

Results: 100 patients were identified. Thirty-nine patients (39\%) died, and in 50 patients $(50 \%)$ the treatment resulted in 3-months futile outcome. Among pre-treatment factors, occlusion site $(\mathrm{p}=0.03)$ and collateral flow $(p=0.02)$ were significantly predictive of mortality while age ( $p$ $=0.02)$, ASPECT score (0.05), admission glicemia (0.004), occlusion site $(0.01)$, onset NIHSS $(0.006)$ and collaterl flow $(0.05)$ were predictive of futile MT. All pre-treatment predictive factors were dichotomized according to their respective cut-off at ROC curve and included in the multivariate logistic regression analysis to create a new prognostic scoring system (range 0-14). The AUC was used to assess the discrimination ability of the score (AUC of 0.788 [95\% CI: $0.70-0.88$ ] to identify patients at risk for a futile outcome with a cut-off of 7. Among post-treatment factors symptomatic haemorragic infarction and $\mathrm{TICI} \leq 2 \mathrm{a}$ were associated with mortality and futility $(p=0.009$ and $p<0.001$ for mortality; $p=0.006$ and $\mathrm{p}=0.01$ for futility)

Conclusion: This prognostic score could be a useful tool for identification of very old patients with acute ischemic stroke at high risk of mortality or futile outcome despite MT. Further analysis are warranted to confirm our findings from this poorly investigated anything but small subgroup of fragile patients.

References:

- Kammersgaard LP, Jørgensen HS, Reith J, Nakayama H, Pedersen PM, Olsen TS. Short- and long-term prognosis for very old stroke patients. The Copenhagen Stroke Study. Age Ageing (2004);33:149-54

- To CY, Rajamand S, Mehra R, et al. Outcome of mechanical thrombectomy in the very elderly for the treatment of acute ischemic stroke: the real world experience. Acta Radiol Open (2015);4: 2058460115599423 CrossRef Medline

- Prabhakaran S, Ruff I, Bernstein RA. Acute stroke intervention: a systematic review. JAMA (2015);313(14):1451-62

\section{HYPERKINETIC MANIFESTATIONS IN SUPERFICIAL SIDEROSIS: EVIDENCE FOR PATHOGENIC NETWORK DISRUPTION}

A. De Mase ${ }^{1}$, G. Martusciello ${ }^{2}$, S. Miniello ${ }^{2}$, G. Piccirillo², G. Di Iasi², M. Ucciero $^{2}$, D. Saracino ${ }^{3}$, V. Andreone ${ }^{2}$

${ }^{1}$ IRCCS Istituto Neurologico Mediterraneo Neuromed (Pozzilli-IS); ${ }^{2}$ Neurology and Stroke Unit, Department of Medical Sciences, AORN Sant'Anna e San Sebastiano Hospital (Caserta); ${ }^{3}$ Sorbonne University, Paris Brain Institute (ICM) - AP-HP, Pitié-Salpêtrière Hospital (Paris-F)

Superficial siderosis (SS) of central nervous system is a rare condition characterized by hemosiderin deposition diffusely involving supratentorial and infratentorial compartments. SS usually manifests with ataxia and sensorineural hearing loss. Since the pathological findings result from deposition of iron beneath the surfaces of CNS tissues that are typically rinsed by CSF, basal ganglia are almost always spared by the degenerative process, and movement disorders are only rarely reported. We describe the case of an aged woman with apparently idiopathic SS presenting with cerebellar ataxia, hearing loss and orofacial dyskinesias, in absence of any history of previous use of antidopaminergics, ill-fitting dental devices or even edentulism. Together with some previously 
reported patients affected by SS and presenting with dystonic manifestations, our case reinforces the current hypothesis supporting a wide network disruption, rather than a direct basal ganglia damage, as the likely underlining cause of some dystonic syndromes.

References:

- Pichler M, Vemuri P, Rabinstein AA, et al. Prevalence and Natural History of Superficial Siderosis: A Population-Based Study. Stroke (2017) Dec;48(12):3210-14

- Blanchet PJ1, Abdillahi O, Beauvais C, Rompré PH, Lavigne GJ. Prevalence of spontaneous oral dyskinesia in the elderly: a reappraisal. Mov Disord. (2004) Aug;19(8):892-6

- Jochim A, Li Y, Gora-Stahlberg G, Mantel T, Berndt M, Castrop F, Dresel C, Haslinger B. Altered functional connectivity in blepharospasm/orofacial dystonia. Brain Behav. (2017) Dec 18;8(1):e00894

\section{HOSPITAL READMISSIONS AFTER INTRACEREBRAL HEMORRHAGE IN A POPULATION-BASED STROKE REGISTRY}

E. De Matteis ${ }^{1}$, R. Ornello ${ }^{1}$, C. Scarpato ${ }^{1}$, E. Colangeli ${ }^{1}$, G. Ceccanti ${ }^{1}$, B. Orlandi $^{2}$, F. De Santis ${ }^{2}$, C. Tiseo ${ }^{1}$, L. Mammarella ${ }^{3}$, C. Marini ${ }^{1}$, F. Pistoia $^{1}$, S. Sacco ${ }^{1}$

${ }^{1}$ Neuroscience Section, Department of Applied Clinical Sciences and Biotechnology, University of L'Aquila (L'Aquila); ${ }^{2}$ Department of Neurology 7051, SS Filippo and Nicola Hospital (Avezzano-AQ); ${ }^{3}$ Department of Public Health, SS Filippo and Nicola Hospital (Avezzano-AQ)

Objectives: Intracerebral hemorrhages (ICHs) are about the $10-15 \%$ of strokes and are associated to a high rate of morbidity and mortality. We aimed at assessing the proportion of hospital readmissions of patients with a first-ever ICH included in a population-based registry. We further investigated factors related to rehospitalizations, including patients' vascular risk factors and stroke severity.

Materials: We included all patients resident in the L'Aquila district, who presented a first- ever ICH from 2011 to 2017.

Methods: Patients attended a two-years follow-up to address occurrence of any rehospitalization. We compared the characteristics of patients who were readmitted to the hospital with those who did not through the $\chi 2$ and the student's $T$ tests.

Results: Out of the 498 patients who had a first-ever ICH during the study period, $181(35.3 \%)$ died within 30 days and 53 (10.4\%) had been readmitted to the hospital. The total number of hospital readmissions was 97 from 2011 to 2017. Fifty-eight $(59.8 \%)$ rehospitalizations occurred within the second year after the ICH. Specifically, 21 (21.6\%) patients reported one readmission, $7(7.2 \%)$ patients two, 3 patients three $(3.1 \%)$, one $(1 \%)$ four, and $2(2.1 \%)$ reported five readmissions. Regarding the causes of rehospitalizations, 25 (25.8\%) were due to neurological disorders, of whom $13(11.3 \%)$ were strokes (11 hemorrhagic and 2 ischemic events); 22 (22.7\%) surgical procedures unrelated to the hemorrhagic events; 7 (7.2\%) infections; 2 (2\%) neoplasms and the remaining $42.3 \%$ were due to other diseases. Among the 317 patients surviving to the ICH, those who were readmitted to the hospital were more frequently males $(41,77.3 \%)$ compared with patients who did not report rehospitalizations $(148,59.2 \%$; $\mathrm{P}=0.008)$. There were no statistical differences between the two groups in terms of comorbidities and disability burden related to the ICH.
Discussion and Conclusions: This study suggests that ICH is a condition associated with a high rate of mortality and, in case of survival, with a proportion of rehospitalizations, which is more common among males. Neurological disorders, particularly hemorrhagic strokes, are frequent causes of hospital readmission hypothesizing a patients' susceptibility to cerebrovascular disorders

\section{PREDICTORS OF FUNCTIONAL OUTCOME IN ACUTE ISCHEMIC STROKE PATIENTS ADMITTED TO NEURO- INTENSIVE CARE UNIT AFTER MECHANICAL THROMBECTOMY. A SINGLE CENTER STUDY}

\author{
M. Distefano ${ }^{1}$, S. Silva ${ }^{2}$, G. Della Marca ${ }^{1}$, G. Frisullo ${ }^{1}$, I. Valente $^{3}$, A. \\ Pedicelli $^{3}$, R. Calandrelli ${ }^{3}$, A. Caricato ${ }^{2}$, P. Calabresi ${ }^{1}$, F. Pilato $^{1}$ \\ ${ }^{1}$ Institute of Neurology, Catholic University of Sacred Heart (Roma); \\ ${ }^{2}$ Neuro-Intensive Care Unit, Catholic University of Sacred Heart \\ (Roma); ${ }^{3}$ Institute of Radiology and Neuroradiology, Catholic \\ University of Sacred Heart (Roma)
}

Objective: The aim of this study was to evaluate the profile of acute ischemic stroke (AIS) patients admitted to neuro intensive care unit (NICU) after intra-arterial mechanical thrombectomy (IAMT) to define their features and the predictors of the neurological outcome.

Materials and methods: We enrolled patients consecutively admitted to NICU for ischemic stroke due to large vessel occlusion (LVO) of the anterior cerebral circulation that underwent IAMT, either in combination with IVT (intravenous thrombolysis) or alone. A neuroimaging protocol including non-contrast-CT (NCCT) scan, CT angiography (CTA) and perfusion $\mathrm{CT}$ (PCT) were performed according to guidelines. The degree of reperfusion was defined using the mTCI scale on digital subtraction angiography and stroke severity was assessed by NIHSS. Patients were daily evaluated for dysphagia. Functional outcome was evaluated at 90 and 180 days using the $\mathrm{mRS}$ through a structured interview. A multivariate analysis was performed using a logistic regression model.

Results: 158 patients were enrolled. IVT [OR, 3.78; 95\% CI, 1.20$11.90](\mathrm{p}=.023)$ and naso-gastric tube removal [OR, 3.32; 95\% CI, 1.04$10.59](\mathrm{p}=.042)$ were associated with good outcome whereas high baseline NIHSS score [OR, .72 for each point of increase; 95\% CI, .61-.85] $(\mathrm{p}<.001)$ was a predictor of poor outcome at 3 months. Older age [OR, .95 for each year of increase; 95\% CI, .92-.99] $(\mathrm{p}=.020)]$, hemorrhagic transformation [OR, .31; 95\% CI, .11-.84] $(\mathrm{p}=.022)$, increased baseline NIHSS score [OR, .81 for each point of increase; 95\% CI, .74-.90] $(p<.001)$ were predictors of poor outcome after IAMT whereas $\mathrm{mTICI} 2 \mathrm{~b} / 3$ score was a predictor of good outcome [OR, $7.86 ; 95 \% \mathrm{CI}$, $1.65-37.39](\mathrm{p}=.010)]$ at 6 months.

Discussion: IAMT has been proven to be beneficial for most stroke patients with large vessel occlusion (LVO), but it added new issues because acute management often requires intensive care support. Recent studies analysed the effects of a non-invasive versus invasive airways management during IAMT, without producing an unequivocal conclusion. Other studies investigate how management of co-morbidities, such as hyperglycemia, hypertension, blood oxygenation, infections may influence short-term and long-term outcomes. Overall, clinical factors associated with outcome of AIS patients requiring ICU after IAMT are still poorly understood and studies in this population are lacking.

Conclusion: Our results show that acute stroke patients with LVO, who require NICU management soon after IAMT, may show specific clinical factors influencing short- and long-term neurologic 
independency. Future studies should address whether tailored therapeutic approaches may affect these factors for further improving neurological outcome.

References:

- Powers W. J., Rabinstein A. A., Ackerson T., et al. Guidelines for the Early Management of Patients With Acute Ischemic Stroke: 2019 Update to the 2018 Guidelines for the Early Management of Acute Ischemic Stroke: A Guideline for Healthcare Professionals From the American Heart Association/American Stroke Association. Stroke (2019);50:e344-e418. https://doi.org/10.1161/STR. 0000000000000211

- Venkatasubba Rao CP, Suarez JI. Management of Stroke in the Neurocritical Care Unit. Continuum (Minneap Minn) (2018);24:1658-82. https://doi.org/10.1212/CON.0000000000000670

- Zhang Y, Jia L, Fang F, et al. General Anesthesia Versus Conscious Sedation for Intracranial Mechanical Thrombectomy: A Systematic Review and Meta-analysis of Randomized Clinical Trials. J Am Heart Assoc (2019);8:e011754. https://doi.org/10.1161/JAHA.118. 011754

\section{RISK OF EARLY POST-STROKE SEIZURES FOLLOWING REPERFUSION THERAPIES: A LARGE PROPENSITY SCORE- MATCHED STUDY}

\author{
D. Ferraro ${ }^{1}$, C. Faso ${ }^{1}$, G. Giovannini ${ }^{1}$, G. Bigliardi ${ }^{2}$, G. Borzì ${ }^{2}, \mathrm{~S}$. \\ Meletti $^{1}$
}

${ }^{1}$ Department of Biomedical, Metabolic and Neurosciences, University of Modena and Reggio Emilia (Modena); ${ }^{2}$ Neurology Unit, Azienda Ospedaliero-Universitaria of Modena (Modena)

Objective: The association between intavenous tissue plasminogen activator (tPA) and/or intra-arterial treatments (IAT) and post-stroke seizures has been assessed in several studies, with discordant results. Our aim was to assess the risk of early post-stroke seizures (EPSS) in a cohort of patients treated with IAT and/or tPA compared to a cohort of untreated, propensity score-matched, stroke patients.

Methods: Data were collected from a prospective clinical registry of consecutively treated patients (2009-2018) and from case report forms of untreated patients (2002-2006). The impact of baseline variables on the risk of developing EPSS was assessed using logistic regression analysis. Propensity Score (PS) matching was carried out in order to adjust for pretreatment differences in age, sex and baseline NIHSS scores between treated and untreated patients, and to estimate the average treatment effect (ATE) on the risk of an EPSS.

Results: 3021 patients were included in the study: 1128 received tPA, 490 received IAT, or both IAT and tPA, while 1403 were untreated. EPSS were more frequent in treated $(3.1 \%)$ versus untreated $(0.6 \%)$ patients and, amongst treated patients, in patients treated with IAT $(5.9 \%)$ compared to tPA $(1.9 \%)(\mathrm{p}<0.001)$. Reperfusion therapies were associated with the risk of EPSS at multivariate logistic regression analysis (OR: 2.9; 95\%CI: 1.4-3.1) together with baseline NIHSS (OR: 1.04 95\%CI: 1.01-1.08), while small-vessel infarct significantly decreased the odds of an EPSS (OR: 0.12; 95\%CI: 0.02-0.9). Following PS matching, the adjusted ATE on the treated was a $0.06 \%$ increase in the incidence of EPSS in treated (any kind of treatment) versus untreated patients, a $2.2 \%$ increase in the incidence of EPSS in patients receiving IAT versus those receiving tPA, and a $0.03 \%$ decrease in patients receiving tPA versus untreated patients.
Conclusion: Reperfusion therapies are independent risk factors for the occurrence of EPSS. Following PS matching, only IAT, and not tPA, increase the risk of an EPSS.

\section{MANAGEMENT OF SPONTANEOUS ACUTE BASILAR ARTERY DISSECTION IN YOUNG ADULT}

\author{
L. Ferraù ${ }^{1}$, P. La Spina ${ }^{1}$, C. Casella ${ }^{1}$, M. Cotroneo ${ }^{1}$, C. Dell'Aera ${ }^{1}$, M.
} Fazio $^{1}$, F. Grillo ${ }^{1}$, G. Fiume ${ }^{1}$, A. Toscano ${ }^{2}$, R. Musolino ${ }^{1}$

${ }^{1}$ Stroke Unit, Department of Clinical and Experimental Medicine, University of Messina (Messina); ${ }^{2}$ Department of Clinical and Experimental Medicine, University of Messina (Messina)

Background: Basilar artery dissection (BAD) is one of the most uncommon causes of stroke in young and middle-age adults with high risk of mortality and morbidity. Several studies evaluated the management of the acute phase, comparing treatments, conservative versus endovascular, but the optimal management of this lesion is difficult and still remains controversial.

Case Report: Herein we described a case of 41 years-old man with a past medical history of smoking and migraine, presented to our observation with sudden onset of headache in parietal region, visual disturbances, disartria and acute ataxia. Magnetic resonance imaging showed brainstem infarction with ischemic changes in the medulla and right pons. Fluidattenuated inversion recovery (FLAIR) sequence demonstrated a hyperdense signal of basilar artery as low blood flow that may be suggestive of BAD. Computed tomography angiogram was performed approximately 24 hours after the onset of symptoms and confirmed a severe narrowing of the mid-basilar artery with lumen irregularity and a slight wall thickening on the front side, typical of vessel dissection. The patient underwent immediately to the antiplatelet therapy with clopidogrel $75 \mathrm{mg}$ and cardioaspirin $100 \mathrm{mg}$ and directed to follow-up neuroradiological checks. After about 4 months of double antiplatelets therapy, the patient had a normal neurological examination and the neuroradiological findings appeared significantly improved.

Discussion and Conclusion: BAD has a poor prognosis if not treated properly. Conservative management has been advocated in literature but the serious nature of the disease might require surgical or endovascular treatment and this depends on the risk of recurrent ischemic events and complications. [1] The medical therapy should be considered in patients with ischemic stroke without evidence of bleeding and without aneurysm formation while the intervention (surgical or endovascular) should be considered in patients with hemorrhage or aneurysms. [2] Endovascular treatment appears to be safer than neurosurgical treatment, mainly because of the difficult access to the vessel due to its anatomical position. Furthermore, endovascular reconstructive therapy with the use of appropriate stents for intracranial arteries may be the optimal strategy especially in patients with a history of recurrent stroke or without benefit from medical therapy. [3] In conclusion, we must consider the various therapeutic options for proper management of BAD to limit the risk of bleeding and thrombosis. Here we describe a case of spontaneous BAD with a good outcomes after the only antiplatelets treatment.

References:

1. Pozzati E, Andreoli A, Padovani R, et al. Dissecting aneurysms of the basilar artery. Neurosurgery (1995);36:254-58 PubMedGoogle Scholar 
2. B.M. Kim et al. Management and Clinical Outcome of Acute Basilar Artery Dissection. American Journal of Neuroradiology (2008);29 (10):1937-41

3. C. B. Patroclo et al. Endovascular treatment of a basilar artery dissecting aneurysm. Arq. Neuro-Psiquiatr. (2007);65(4):1012-4

\section{NEGATIVE CARDIOVASCULAR REACTIVITY: A DIGGER IN THE BRAIN}

P. Fiori ${ }^{1}$, G. Capaldo ${ }^{1}$, A. Corbo ${ }^{1}$, L. Iorillo ${ }^{1}$, M. Massarelli ${ }^{1}$, G. Corbo ${ }^{2}$, R. Gizzi ${ }^{3}$, C. Pelosi ${ }^{4}$, P. Savino ${ }^{4}$, F. Botticella ${ }^{5}$, C. Dragonetti ${ }^{5}$, G. Manganelli $^{5}$, S. Minichiello ${ }^{5}$, A. Morella ${ }^{5}$, V. Pellecchia ${ }^{5}$, M. Alberico $^{6}$, G. Benigni ${ }^{6}$, M. De Caro ${ }^{6}$, E. Pace ${ }^{6}$, A. Bellizzi ${ }^{4}$, G.

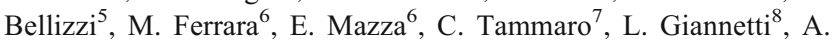
Monaco $^{1}$

${ }^{1}$ Neurology, S. Ottone Frangipane Hospital, ASL AV, University of Naples (Ariano Irpino-AV); ${ }^{2}$ Pediatrics, Policlinico (Siena); ${ }^{3}$ Physiotherapy, Criscuoli Hospital, ASL AV, University of Naples (S. Angelo dei Lombardi-AV); ${ }^{4}$ Medicine, S. Ottone Frangipane Hospital, ASL AV, University of Naples (Ariano Irpino-AV); ${ }^{5}$ Cardiology, S. Ottone Frangipane Hospital, ASL AV, University of Naples (Ariano Irpino-AV); ${ }^{6}$ Intensive Care, S. Ottone Frangipane Hospital, ASL AV, University of Naples (Ariano Irpino-AV); ${ }^{7}$ Laboratory, S. Ottone Frangipane Hospital, ASL AV, University of Naples (Ariano IrpinoAV); ${ }^{8}$ Infantile Neuropsychiatry, S. Ottone Frangipane Hospital, ASL $\mathrm{AV}$, University of Naples (Ariano Irpino-AV)

Introduction: Cardiovascular reactivity (CR) may be defined by beat indices (ratio or difference) between higher maximal or minimal heart rate on higher maximal or minimal pulse rate [1]. High heart rate predicts greater total lesion volume and higher number of micro- and macrostructure white matter lesions [2]. However, the irreversibility of ischaemic sufferance seems to be related to low CR, responsible of reduced perfusion and increased diffusion. The aim of our current study was to examine the burden of positive (PCR) and negative (NCR) CR and its correlation with cerebrovascular and cardiovascular scales and cardiac biomarkers.

Materials and Methods: One hundred and thirteen acute ischaemic syndromes (AIS: transient ischaemic attacks and minor strokes), 32 chronic cerebrovascular diseases (CCVD) and 30 other neuropsychiatric diseases (OND) were recruited. They were classified according to CHADVasc2, HAS BLED, Hachinsky scales. They underwent withdrawal for cardiac biomarkers, ECG, Holter Arterial Pressure Measurements, ECG Holter and Echocardiography, radiology. Neuropsychological evaluation was performed in a subset of patients. Correlations with the number of negative and positive $\mathrm{CR}$ events were evaluated.

Results: Number of PCR events correlated with CHADVasc2, HAS BLED, Hachinski scale, negatively, and with MMSE, positively, while inverse correlations were observed with number of NCR events. Correlations between PCR and NCR events and UPDRS were influenced by circadian rhythms, as well as those with ADLQ. Number of PCR negatively correlated with high sensitive troponin, pro-brain natriuretic peptide (pro-BNP), BNP, especially during the day compared to the night, while number of NCR events positively correlated with these parameters. In AIS affected with atrial fibrillation, number of PCR events correlated with GCS at admission, GCS at day VII, positively, and with MRS at day VII, negatively, while there were inverse correlations with number of NCR events.
Discussion and Conclusions: Our data highlight that episodes of NCR may subtly occur and progressively impair cerebrovascular and cardiovascular function during daily life, leading to chronic ischaemic sufferance. They may trigger acute events, worsen their clinical course, hinder the recovery and be harbinger of outcomes. Further studies are needed in order to prevent and reverse this pathological reflex.

References:

1. Fiori P., Capaldo G., Corbo A., Corbo G., Iorillo L., Massarelli M., Gizzi R., Pelosi C., Savino P., Bellizzi A., Botticella F., Dragonetti C., Manganelli G., Minichiello S., Morella A., Pellecchia V., Bellizzi G., Alberico M., Benigni G., De Caro M., Guerriero B., Pace E., Ferrara M., Mazza E., Tammaro C.A., Giannetti L.M. and Monaco A.: Central genesis of dysrhythmia. Neurology and Neurobiology (2020);ISSN 2613-7828 https://doi.org/10.31487/j. NNB.2020.02.02

2. Fuhrmann D., Nesbitt D., Shafto M., Rowe J.B., Price D., Gadie A., Cam-CAN, Kievit R.A.: Strong and specific associations between cardiovascular risk factors and white matter micro- and macrostructure in healthy aging. Neurobiol Aging (2019);74:46-55

\section{ASSOCIATION BETWEEN MOTOR AND COGNITIVE PERFORMANCES IN ELDERLY WITH ATRIAL FIBRILLATION: STRAT-AF STUDY}

B. Formelli ${ }^{1}$, E. Salvadori ${ }^{2}$, F. Galmozzi ${ }^{1}$, F. $\mathrm{Uda}^{1}$, C. Barbato ${ }^{1}$, E. Camilleri $^{3}$, F. Cesari ${ }^{4}$, S. Chiti ${ }^{5}$, S. Diciotti ${ }^{6}$, S. Donnini ${ }^{1}$, S. Galora ${ }^{3}$, B. Giusti $^{3}$, A. Gori ${ }^{3}$, C. Marzi ${ }^{6}$, A. Melone ${ }^{1}$, D. Mistri ${ }^{1}$, F. Pescini ${ }^{7}$, G. Pracucci $^{1}$, V. Rinnoci ${ }^{2}$, C. Sarti ${ }^{1}$, E. Fainardi ${ }^{8}$, R. Marcucci ${ }^{3}$, A. Poggesi ${ }^{1}$

${ }^{1}$ NEUROFARBA Department, Neuroscience Section, University of Florence (Firenze); ${ }^{2}$ Don Carlo Gnocchi Foundation, IRCCS Center (Firenze); ${ }^{3}$ Department of Experimental and Clinical Medicine, University of Florence (Firenze); ${ }^{4}$ Central Laboratory, Careggi University Hospital (Firenze); ${ }^{5}$ Department Health Professions, U.O. Research and Development, Careggi University Hospital (Firenze); ${ }^{6}$ Department of Electrical, Electronic, and Information Engineering 'Guglielmo Marconi', University of Bologna (Bologna); ${ }^{7}$ Stroke Unit, Careggi University Hospital (Firenze); ${ }^{8}$ Neuroradiology Unit, Department of Experimental and Clinical Biomedical Sciences, University of Florence, Careggi University Hospital (Firenze)

Objective: Growing evidence suggests a close relationship between motor and cognitive abilities, but possible common underlying mechanisms are not well established. Atrial fibrillation (AF) is associated with reduced physical performance and increased risk of cognitive decline. The study aimed to assess in a cohort of elderly AF patients: 1) the association between motor and cognitive performances and 2) the influence and potential mediating role of cerebral lesions burden.

Methods: Strat-AF is an oging prospective, observational study aimed at investigating biological markers for cerebral bleeding risk stratification in AF patients on oral anticoagulants. Consecutive patients affected by AF with age above 65 years followed at the out-patient clinic of Center of Thrombosis for oral anticoagulant therapy, and without contraindication to MR, were invited to participate in the project. Baseline protocol included: neuropsychological battery, motor assessment (Short Physical Performance Battery, SPPB, walking speed), and brain MRI used for the visual assessment of white matter hyperintensities, lacunar and nonlacunar infarcts, cerebral microbleeds, global cortical and medial temporal atrophies. 
Results and Discussion: Among the 170 patients enrolled, Mean Montreal Cognitive Assessment (MoCA) total score was 21.9 \pm 3.9 , SPPB total score $9.5 \pm 2.2$, and walking speed $0.9 \pm 0.2$. In univariate analyses, both SPPB and walking speed were significantly associated with $\operatorname{MoCA}(\mathrm{r}=.359, \mathrm{r}=.372$, respectively), Visual search $(\mathrm{r}=.361, \mathrm{r}=.322)$, Stroop ( $\mathrm{r}=-.272, \mathrm{r}=-.263)$, Short story $(\mathrm{r}=.263, \mathrm{r}=.310)$ and Semantic fluency ( $\mathrm{r}=.311, \mathrm{r}=.360)$. In multivariate models adjusted for demographics, heart failure, physical activity and either stroke history (Model 1) or neuroimaging markers (Model 2), both SPPB and walking speed were confirmed significantly associated with MoCA (Model 1: $\beta=.256$, $\beta=.236$; Model 2: $\beta=.276, \beta=.272$, respectively), Visual Search (Model 1: $\beta=.350, \beta=.313$; Model 2: $\beta=.344, \beta=.307$ ), semantic fluency (Model $1: \beta=.223, \beta=.261$ ), and short story (Model $2: \beta=.245, \beta=.273$ ).

Conclusions: In our cohort of elderly AF patients, a direct association between motor and cognitive functions consistently recurred using different evaluation of the performances, regardless of demographics, vascular risk factors and comorbidities. So far, the results did not show an evident mediating role of cerebral lesions burden within this association, but longitudinal data collection is ongoing, potentially demonstrating a causal inferences of neuroimaging data regarding the relationship between gait and cognition.

References:

- Demnitz N, Esser P, Dawes H, et al. A systematic review and metaanalysis of cross-sectional studies examining the relationship between mobility and cognition in healthy older adults. Gait Posture (2016);50:164-74

- Poggesi A, Inzitari D, Pantoni L. Atrial Fibrillation and Cognition: Epidemiological Data and Possible Mechanisms. Stroke (2015);46:3316-21

- Yogev-Seligmann G, Hausdorff JM, Giladi N. The role of executive function and attention in gait. Mov Disord. (2008);23:329-42

\section{ANGIOPLASTY AND STENTING IN PATIENTS WITH ACUTE BASILAR ARTERY OCCLUSION AND UNDERLYING ATHEROSCLEROTIC STENOSIS}

\author{
I. Francalanza ${ }^{1}$, A. Caragliano ${ }^{2}$, A. Ciacciarelli ${ }^{1}$, C. Casella ${ }^{1}$, M. \\ Cotroneo $^{1}$, C. Dell'Aera ${ }^{1}$, C. Fazio ${ }^{1}$, F. Grillo ${ }^{1}$, O. Buonomo ${ }^{2}$, G.

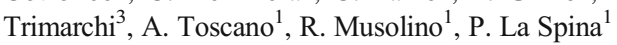

${ }^{1}$ Department of Clinical and Experimental Medicine, University of Messina (Messina); ${ }^{2}$ Biomedical Sciences and of Morphologic and Functional Images, University of Messina (Messina); ${ }^{3}$ Faculty of Medicine and Surgery, University of Messina (Messina)

Introduction: Acute ischemic stroke due to basilar artery occlusion (BAO) represents a subgroup of posterior circulation stroke, associated with high morbidity and mortality rate [1]. One of the most frequent etiologies is atherosclerotic disease [2], resulting, during endovascular treatment $(E V T)$, in high periprocedural risks and difficulty in achieve satisfactory reperfusion status [3]. The optimal treatment for these patients remains unclear, and data regarding safety and effectiveness of percutaneous transluminal angioplasty and/or stenting (PTAS) are lacking.

Aims: In this study, we aim to evaluate patients with BAO and concomitant significant vertebro-basilar (VB) stenosis, and compare patients treated with EVT only for BAO and cases underwent additional PTAS on the underlying stenosis.
Methods: We retrospectively analyzed 17 patients with BAO and significant VB stenosis treated by EVT at our stroke center. Stenosis was defined as significant if greater than $70 \%$ or hemodynamically significant. PTAS was performed in case of inadequate distal perfusion or tendency toward reocclusion after primary treatment. The following outcome parameters were collected: periprocedural complications, clinical outcome at 3 months evaluated with the modified Rankin scale (mRS), restenosis/ progression on follow-up imaging, and TIA or stroke recurrence.

Results: Of the 17 patients, in 7 cases $(41.17 \%)$ we did not perform PTAS because successful recanalization was already obtained by the firstline EVT (no-PTAS group), and $10(58.82 \%)$ underwent additional PTAS (PTAS group). Periprocedural complications occurred in 2 patients from no-PTAS group, and in one case of PTAS group. At 3 months, we observed favorable outcome (mRS $0-2$ ) in $30 \%$ of PTAS patients and in $42.85 \%$ in no-PTAS group. In a mean follow-up period of 10 months, one patient from no-PTAS group experienced a TIA and one of PTAS patients presented a stroke due to intra-stent thrombotic occlusion. At follow-up imaging, VB stenosis progression was found in 2 cases in both groups. Mortality rate at 90 days was $30 \%$ in PTAS group and $28.57 \%$ in no-PTAS group. We did not observe significant differences in terms of outcome measures, even though, at the follow-up imaging, we found a lower mean degree of stenosis in PTAS group, although not statistically significant $(\mathrm{p}=0.059)$.

Discussion and Conclusions: We presented a real-life observational study on angioplasty and/or stenting as a treatment option in the challenging scenario of BAO and underlying significant VB stenosis. Treatment decisions need to be made on a case-specific approach, dependent on individual assessment of clinical and neuro-radiological characteristics and periprocedural risks.

References:

1. Mattle HP, Arnold M, Lindsberg PJ, Schonewille WJ, Schroth G. Basilar artery occlusion. Lancet Neurol. (2011); 10(11):1002-14

2. Caplan LR, Wityk RJ, Glass TA, Tapia J, Pazdera L, Chang HM, et al. New England Medical Center Posterior Circulation registry. Ann Neurol. (2004);56(3):389-98

3. Gao F, Lo WT, Sun X, Mo DP, Ma N, Miao ZR. Combined use of mechanical thrombectomy with angioplasty and stenting for acute basilar occlusions with underlying severe intracranial vertebrobasilar stenosis: Preliminary experience from a single Chinese center. Am J Neuroradiol (2015);36(10):1947-52

\section{TRANSIENT PAROXYSMAL MANIFESTATIONS IN SPORADIC CEREBRAL AMYLOID ANGIOPATHY. A CASE SERIES AND LITERATURE REVIEW}

T. Garcea ${ }^{1}$, M. Ascoli ${ }^{1}$, E. Ferlazzo ${ }^{1}$, S. Gasparini ${ }^{1}$, G. Mastroianni ${ }^{2}$, L.

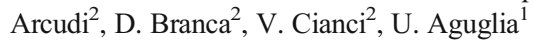

${ }^{1}$ Department of Medical and Surgical Sciences, Magna Graecia University (Catanzaro); ${ }^{2}$ Regional Epilepsy Center, Great Metropolitan Hospital (Reggio Calabria)

Introduction: Cerebral amyloid angiopathy (CAA) may present with stroke or transient paroxysmal manifestations such as seizures or amyloid spells (AS) [1]. While AS have been extensively studied in literature, less attention has been paid to seizures associated with CAA. The purpose of this study is to evaluate clinical presentation of CAA, with peculiar attention to seizures, among subjects admitted to our Neurology/Stroke Units. 
Methods: We performed a retrospective analysis on consecutive subjects admitted to the Neurology/Stroke Units of the Great Metropolitan Hospital of Reggio Calabria between 2018 and 2020, to evaluate the incidence of CAA and its clinical presentation. The diagnosis of CAA was posed according to modified Boston criteria. We also reviewed literature data on epidemiology and prognosis of seizures in CAA.

Results: Among 2252 admitted patients, CAA was diagnosed in 19 subjects $(0.7 \%)$. Of these, 10/19 (53\%) presented with stroke and 9/19 $(47 \%)$ with transient paroxysmal manifestations including seizures $(6 / 9$, $67 \%)$ and $\operatorname{AS}(3 / 9,33 \%)$. Among the 9 subjects with transient paroxysmal manifestations, 7 had superficial cortical hemorrhages and 2 had atypical lobar hemorrhage. Two subjects with superficial hemorrhage at onset later developed atypical lobar hemorrhage. Seizures were focal with bilateral tonic-clonic evolution in 6/6 subjects. Literature search retrieved 104 results, 7 papers were selected. Seizures epidemiology and prognosis in CAA are difficult to ascertain mostly due to the lack of detailed clinical and neurophysiological data.

Conclusion: CAA may present with transient paroxysmal manifestations. Seizures have not been well investigated in subjects with CAA despite representing a common manifestation of CAA at onset.

Reference:

1. G. Boulouis, A. Charidimou, S. M. Greenberg Sporadic Cerebral Amyloid Angiopathy: Pathophysiology, Neuroimaging Features, and Clinical Implications. Semin Neurol (2016);36:233-43

\section{SPONTANEOUS CAROTID ARTERY DISSECTION IN A PATIENT WITH FBN1 MUTATION. CASE REPORT}

\author{
E. Giorli ${ }^{1}$, G. Sivori ${ }^{2}$, M. Bertamino ${ }^{3}$, G. Amico ${ }^{4}$, I. Ceccherini ${ }^{4}$, A. \\ Mannironi
}

${ }^{1}$ Neurology Department, S. Andrea Hospital (La Spezia); ${ }^{2}$ Neurology Department, ASL4 Chiavarese, Lavagna Hospital (Lavagna-GE); ${ }^{3}$ Physical Medicine and Rehabilitation Unit, IRCCS Istituto Giannina Gaslini (Genova); ${ }^{4}$ Laboratory of Genetic and Genomics of Rare Disease, IRCCS Istituto Giannina Gaslini (Genova)

Background: Internal carotid artery dissection (ICAD) is an uncommon cause of stroke that typically occurs in the setting of (often minor) trauma but can also occur spontaneously. Even though ICAD accounts for only $1 \%$ to $2 \%$ of all strokes, it is responsible for $10 \%$ to $25 \%$ of strokes in young and middle-aged adults. Several arguments suggest a genetic predisposition and rarely, familiar-ICAD is associated with a known monogenic connective tissue disease, mainly vascular Ehlers-Danlos syndrome and Marfan. On the other hand, sporadic forms, even where recurrent, do not seem to have a significant prevalence of underlying monogenic disorders. We report a case of a 53 years old man, with unremarkable family history, presenting with spontaneous ICAD and harboring a recently predicted as likely pathogenic (LP) mutation in FBN1gene, with no other signs nor symptoms of Marfan syndrome.

Case report: A 53 years old man presented at the Emergency Department with clouding of the visus on the right eye and postural instability since the evening before admission. On remote pathological history he presented previous myocardial infarction on 2011 and 2017 as a result of stinging hymenoptera. He was carrier of cardiac defibrillator. In the Emergency Department the patient performed brain CT (negative for recently occurring ischemic lesions) and Angio-CT of intra and extracranial vessels which showed dissection of the left internal carotid artery at the level of the carotid siphon. Interventional neuroradiological evaluation was performed which did not indicate interventions. The patient performed an echocardiography during the recovery who showed moderate left ventricular dilation with moderate-severe systolic dysfunction. The patient could not perform brain MRI for cardiac defibrillator. The control CT brain does not show any intraparenchymal brain alteration. He was discharge with double anti-aggregation. The Angio-CT performed 2, 3 and 6 months later showed a pseudoaneurysm of the internal carotid artery on pre-cranial tract. The patient underwent NGSpanel analysis, including 127 genes associated with monogenic causes of stroke, which revealed the presence of the p.Gln117His mutation in the FBN1 gene compatible with an atypical form of Marfan syndrome.

Discussion: FBN1 encodes a member of the fibrillin family of proteins. The encoded preproprotein is proteolytically processed to generate two proteins including the extracellular matrix component fibrillin-1 and the protein hormone asprosin. Fibrillin-1 is an extracellular matrix glycoprotein that serves as a structural component of calcium-binding microfibrils. These microfibrils provide force-bearing structural support in elastic and nonelastic connective tissue throughout the body. Mutations in this gene are associated with Marfan syndrome, Familial thoracic aortic aneurysm and dissection (familial TAAD), Shprintzen-Goldberg syndrome and other conditions.

Conclusion: To our knowledge this is the first case described in literature of sporadic spontaneous cervical artery dissection carrying the likely pathogenic p.Gln117Hismutation in the FBN1 gene. Connective tissue abnormalities are reported in patients with familiar-ICAD keeping the hypothesis that systemic aberrations of the connective tissue might be implicated in the pathogenesis of the disease. However, the indication to genetic screening in sporadic forms, even when recurrent, is still debated. The use of modern NGS techniques can be helpful in such cases, being cost and time saving.

References

1. Traenka C, Kloss M, Strom T, et al. Rare genetic variants in patients with cervical artery dissection. Eur Stroke J. 2019;4(4):355-362. doi: $10.1177 / 2396987319861869$

2. Engelter ST, Traenka C, Lyrer P. Dissection of Cervical and Cerebral Arteries. Curr Neurol Neurosci Rep. 2017;17(8):59. doi:10.1007/s11910-017-0769-3

3. Henrard C, Belge H, Fastré S, et al. Cervical artery dissection: fibromuscular dysplasia versus vascular Ehlers-Danlos syndrome. Blood Press. 2019;28(2):139-143. doi:10.1080/ 08037051.2018 .1557507

4. Debette S, Markus HS. The genetics of cervical artery dissection: a systematic review. Stroke. 2009;40(6):e459-e466. doi:10.1161/ STROKEAHA.108.534669

5. Grond-Ginsbach C, Brandt T, Kloss M, et al. Next generation sequencing analysis of patients with familial cervical artery dissection. Eur Stroke J. 2017;2(2):137-143. doi: 10.1177/ 2396987317693402

\section{VASCULAR ABNORMALITIES (INTRACRANIAL STENOSIS) IN A PATIENT WITH VON RECKLINGAUSEN DISEASE (NF1): A CASE REPORT}

G. Guglielmini ${ }^{1}$, L. Pascazio $^{2}$, P. Milzi ${ }^{2}$, M. Trojano ${ }^{1}$

${ }^{1}$ Neurology, Policlinico Bari, Aldo Moro University (Bari); ${ }^{2}$ Stroke Unit, Neurology I, Department of Neurosciences and Sense, Policlinico of Bari (Bari) 
Objective: To evaluate the vascular anomalies features in a patient $(\mathrm{pt})$ with Von Recklinghausen disease (NF1).

Materials: One pt, 72 years old, affected by NF1 referred to the Emergency Room, exhibited both recurrent transient speech disorders and right brachial hyposthenia (recurrent transient ischemic attacks); neurological examination showed mild right brachial hypostenia and slight speech disorders in rapid regression.

Methods: The pt underwent brain CT/MRI-PW and CT/MR Angiography (CTA/MRA), ultrasounds, blood chemistry tests (BCTs).

Results: Brain CT and CTA highlights multiple meningiomas and subcutaneous neurofibromas, cortical atrophy, left middle cerebral artery (MCA) sub-occlusion, M1 distal and M2 tract; the MRI/PWAngiography reveals left brain frontoparietal oligoemia and confirms high grade stenosis. BCTs exhibit mild homocysteine increase and HDL at the lower limits of normal; increased fronto-central electroencephalogram theta-delta rythms are detected. Concerning other associated anomalies the pt is also affected by aortic aneurismal dilation and mild mitrotricuspid insufficiency. Supra-Aortic Ultrasonographic examination: fibrocalcific atheromasia less than $50 \%$ of the carotid axes, Transcranial Ultrasonography: high-grade stenosis of the left MCA, M1 distal and M2 tract (PSV: $800-1000 \mathrm{~cm} / \mathrm{sec}$, PDV: $400-500 \mathrm{~cm} / \mathrm{sec}$, PI: 0.67), reduced pre-stenosis flow (PSV: 64, PDV: 28, PI: 0.84), post-stenosis flow cannot be assessed because it is probably a long stenosis involving the total MCA M2 segment, nor is MCA branches insonation possible; diversion flow of the ipsilateral posterior cerebral artery is also observed.

Discussion: The pt has no vascular risk factors, except for the aforementioned and advanced age, stenosis is therefore likely to result from fibrodyspalsia and atherosclerosis, this latter being supported and strengthened by the fibro-cell proliferation typical of NF1. The pt is discharged with statins and double anti-aggregation therapy until 3month follow-up.

Conclusions: Further events could suggest alternative therapeutic strategies (PTA or PTA-stenting) to avoid ischemic stroke. The ultrasound and MRI follow-up of the long, severe stenosis, with poor or partial collateralization, will account for the stenosis natural evolution and will guide future therapeutic choices.

References:

- Timothy M. Sullivan, C. Bower, P. Gloviczki, V. Miller, D. Babovic-Vuksanovic, T. A. Macedo, A. Stanson Vascular abnormalities in patients with neurofibromatosis syndrome type I: Clinical spectrum, management, and results. Journal of Vascular Surgery (2007);46(3):475-84

- R. Conforti, A. Negro, M. Cirillo, L. Della Gatta, N. Della Vecchia, R. Capasso, A. Raucci, Lesione pseudovasculitica cerebrale associata a neurofibromatosi di von Recklinghausen. Recenti Prog Med (2013);104:450-52

\section{GENDER DIFFERENCES IN A REAL-WORLD STROKE UNIT: IS REALLY THERE A SEX GAP IN STROKE CARE?}

F. Letteri ${ }^{1}$, C. Padiglioni ${ }^{1}$, S. Mastrocola ${ }^{1}$, C. Marando $^{1}$, L. Bernetti ${ }^{1}$, E. Gallinella $^{1}$, L. Greco ${ }^{1}$, A. Mattioni ${ }^{1}$, E. Sacchini ${ }^{1}$, I. Sicilia ${ }^{1}$, P. Eusebi ${ }^{3}$, S. Ricci ${ }^{1}$, S. Cenciarelli ${ }^{1}$

${ }^{1}$ Department of Human Neurosciences, Sapienza University (Roma); ${ }^{2}$ Neurology Department, Stroke Unit, Gubbio-Gualdo Tadino and Città di Castello Hospitals, USL Umbria 1 (Città di Castello-PG); ${ }^{3}$ Health Planning Service, Department of Epidemiology, Regional Health Authority of Umbria (Perugia)
Background and objectives: A gender gap has been described in stroke care in different settings. We reviewed the problem using data from the admission register of our Stroke Unit. Our policy is to admit every suspected case of acute stroke or TIA to our Stroke Unit, without any specific age or severity based exclusion criteria.

Materials and methods: From our register, we retrieved data on 2018 and 2019 admissions. We then analyzed possible differences based on gender in age, length of stay and thrombolytic treatment. We used chisquare test for categorical variables and t-test for continuous variables.

Results: We found 388 records, of which 346 were eventually confirmed as stroke or TIA. There were 217 males and 171 females. No difference in "wrong" admissions were found between genders ( $p=0.99$ ). Among the 346 patients (194 males and 152 females), mean age was 78 , and females were significantly older than males (81 vs 76 , $\mathrm{p}<0.01)$. Mean length of stay was 9 days, with no significant difference between genders $(p=0.5)$. Fifteen patients died while in hospital ( 8 males and 7 females). There were 253 ischemic strokes, and thrombolysis was given to 25 out of 135 men and 26 out of 118 women ( $p=0.6$ ).

Discussion and conclusions: In a real-world stroke unit, sited in a first level emergency hospital, no gender differences were found in various parameters of care. When the policy is to admit each suspected stroke or TIA in the Stroke Unit (as recommended by ESO and WSO), male and female patients receive the same level and type of care.

\section{IMPACT OF PREMORBID TREATMENT ON FUNCTIONAL OUTCOMES AND COLLATERAL CIRCULATION IN ACUTE ISCHEMIC STROKE PATIENTS TREATED BY MECHANICAL THROMBECTOMY}

F. Letteri ${ }^{1}$, G. Pracucci ${ }^{2}$, V. Saia ${ }^{3}$, L. Malfatto ${ }^{4}$, M. Longo ${ }^{5}$, A. Zini ${ }^{6}$, F.

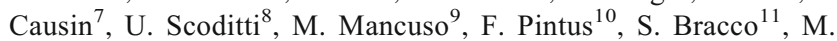
Assetta $^{12}$, M. Bergui ${ }^{13}$, C. Comelli ${ }^{14}$, S. Mangiafico ${ }^{15}$, D. Toni ${ }^{16}$

${ }^{1}$ Department of Human Neurosciences, Sapienza University (Roma); ${ }^{2}$ NEUROFARBA Department of Neuroscience Section, University of Florence (Firenze); ${ }^{3}$ Neurology and Stroke Unit, Santa Corona Hospital (Pietra Ligure-SA); ${ }^{4}$ Interventional Neuroradiology Unit, San Martino Hospital (Genova); ${ }^{5}$ Department of Biomedical Sciences and Morphological and Functional Imaging, G. Martino Hospital (Messina); ${ }^{6}$ Department of Neurology and Stroke Center, Maggiore Hospital (Bologna); ${ }^{7}$ Interventional Neuroradiology Unit, University Hospital of Padua (Padova); ${ }^{8}$ Interventional Neuroradiology Unit, Universital Hospital of Parma (Parma); ${ }^{9}$ Neuroradiology Unit, Cisanello Hospital (Pisa); ${ }^{10}$ Interventional Neuroradiology Unit, SS Annunziata Hospital (Sassari); ${ }^{11}$ Interventional Neuroradiology Unit, S. Maria delle Scotte University Hospital (Siena); ${ }^{12}$ Vascular and Interventional Radiology Unit, Civile Mazzini Hospital (Teramo); ${ }^{13}$ Interventional Neuroradiology Unit, Città della Salute e della Scienza, San Giovanni Molinette Hospital (Torino); ${ }^{14}$ Interventional Neuroradiology Unit, San Giovanni Bosco Hospital (Torino); ${ }^{15}$ Interventional Neuroradiology Unit, Careggi-University Hospital (Firenze); ${ }^{16}$ Emergency Department Stroke Unit, Department of Human Neurosciences, Sapienza University (Roma)

Background and objectives: Preliminary studies have shown a favorable clinical outcome in acute ischemic stroke patients on previous treatment with drugs commonly recommended for cerebrovascular disease prevention, such as statins, antiplatelets and anticoagulants [1,2,3] A promotion of collateral circulation has been speculated as the causal mechanism, in relation to the antithrombotic and anti-inflammatory action, exerted by 
these drugs beyond their therapeutic effect [1,2]. Aim of our study is to investigate the impact of premorbid therapy on clinical outcomes and collateral circulation in patients with acute ischemic stroke treated with mechanical thrombectomy.

Materials: We conducted an analysis of multicenter data collected in the Italian Registry of Endovascular Treatment in Acute ischemic Stroke, from 2015 to 2018.

Methods: Prestroke medications were recorded and patients were grouped on the basis of the previous treatment at the time of stroke onset, as follows: no therapy (NOT) and aspirin, anticoagulants, statin, antihypertensive or combined therapy (YES). Baseline characteristics such as age, vascular risk factors, NIHSS at admission, pre-interventional ASPECTS and Collateral Circulation Score (CCS) were evaluated. Outcome measure were baseline collateral circulation, m-TICI score and 3-month mRS score.

Results: A total of 3890 patients were included: 994 (25.5\%) on prestroke treatment with aspirin, 213 (5.4\%) with clopidogrel, 565 (14.5\%) with anticoagulants, $714(18.3 \%)$ with statins, 2067 (53.1\%) with antihypertensives, $95(2.44 \%)$ with a combined therapy (aspirin and clopidogrel). After adjustment for age, baseline NIHSS and comorbidity, higher collateral scores (CCS 2-4) were observed in patients treated with oral anticoagulants (OR 1.76, 95\% CI 1.28-2.41, $\mathrm{P}<0.001)$ and statins (OR 1.47, 95\% CI 1.10-1.95, $\mathrm{P}=0009$ ). Premorbid treatment with statins correlated with a 3-month better functional outcome (mRS 0-2: OR 1.25, 95\% CI 1.03-1.51, $\mathrm{P}=0.021$ ) and lower mortality rate (mRS 6: OR 0.71, $95 \%$ CI $0.56-0.89, \mathrm{P}=0.004)$. There were no significant correlations between premorbid treatments and $\mathrm{m}$-TICI score.

Discussion and conclusions: In acute ischemic stroke patients treated with mechanical thrombectomy, premorbid treatment with anticoagulants and statins was associated with better collateral scores, and statins pretreatment was related with 3-month favorable functional outcomes and reduced mortality.

References:

1. C. Berger, A. Stauder, F. Xia, C. Sommer, S. Schwab. Neuroprotection and glutamate attenuation by acetylsalicylic acid in temporary but not in permanent cerebral ischemia. Experimental Neurology (2007);210(2):543-48

2. J. Sargento-Freitas, J. Pagola, M. Rubiera Alan Flores et al. Preferential Effect of Premorbid Statins on Atherothrombotic Strokes through Collateral Circulation Enhancement. Eur Neurol (2012); 68: 171-176

3. Jiaying Zhu, Mengmeng Ma, Yijia Guo, Muke Zhou, Jian Guo, Li He. Pre-stroke warfarin enhancement of collateralization in acute ischemic stroke: a retrospective study. BMC Neurology (2018);18(194):2-7

\section{COST-EFFECTIVENESS OF THROMBECTOMY IN PATIENTS WITH ACUTE ISCHEMIC STROKE}

\author{
A. P. Mascolo, F. Marrama, F. D'agostino, M. Bovino, F. Sallustio, A. \\ Rocco, M. Diomedi
}

Department of Systems Medicine, University of Tor Vergata (Roma)

Introduction: Mechanical thrombectomy (MT) improves clinical outcomes in patients with acute ischemic stroke (AIS) caused by an emergent large vessel occlusion (ELVO). There are mixed results as to whether prior intravenous thrombolysis (IVT) is always necessary to subsequent MT. The primary purpose of this study was to determine whether endovascular treatment alone was superior to a combination of IVT and endovascular therapy in terms of safety and efficacy. The secondary purpose was to evaluate the investigate cost-effectiveness between these two treatment options.

Patients and methods: Four hundred-eighty-six patients with anterior circulation AIS hospitalized at the university hospital of Rome Tor Vergata, were considered. Standard safety and efficacy outcomes (symptomatic intracranial hemorrhage, successful reperfusion [mTTICI IIb/III], functional independence (mRS of 0-2) at discharge and 3 months] and mortality at 30 and 90 days) were compared between patients who underwent combined IVT and MT vs direct MT. Propensity scorematched analyses were performed. Total and mean costs for the two alternative treatment protocols were calculated. Age subgroup analysis was performed to evaluate impact on safety, efficacy and costs efficacy.

Results: After propensity score patients were assigned to each treatment group. IVT pretreatment was significantly associated with higher odds of functional independence (OR 3.53; 95\% CI 1.56-7.99) and lower mortality at 3 months $(\mathrm{p}=0.02)$. The combined treatment arm had a total mean per patient cost of $€ 11024$ and 0.132 QALYs. The direct MT arm had a total mean per patient cost of $€ 10405$ and 0.102 QALYs. The NMB resulted positive.

Conclusions: This study provides preliminary evidence that IVT pretreatment may improve outcomes in ELVO patients treated with MT. The combined treatment is also cost-effective when compared to direct MT. Since that most patients who do receive direct MT do so because of underlying contraindications to IVT, a randomized clinical trial is warranted.

References:

- Sevick, L. K. et al. Systematic Review of the Cost and CostEffectiveness of Rapid Endovascular Therapy for Acute Ischemic Stroke. Stroke (2017); 48(9):2519-26

- Steen Carlsson, K., Andsberg, G., Petersson, J. \& Norrving, B. Long-term cost-effectiveness of thrombectomy for acute ischaemic stroke in real life: An analysis based on data from the Swedish Stroke Register (Riksstroke). Int. J. Stroke (2017); 12(8):802-14

- Goyal, N. et al. Comparative safety and efficacy of combined IVT and MT with direct MT in large vessel occlusion. in Neurology (2018); 90(15):e1274-e1282

\section{SPONTANEOUS INTRAPARENCHYMAL CEREBRAL HEMORRHAGE: BIOCHEMICAL PROGNOSTIC MARKERS AND BIOMARKERS}

\author{
A. P. Mascolo ${ }^{1}$, F. Marrama ${ }^{1}$, F. D'agostino ${ }^{1}$, M. Bovino ${ }^{1}$, R. Massoud ${ }^{2}$, \\ C. Cortese ${ }^{2}$, F. Sallustio ${ }^{1}$, M. Diomedi ${ }^{1}$
}

${ }^{1}$ Department of Systems Medicine, University of Tor Vergata (Roma); ${ }^{2}$ Department of Experimental Medicine and Surgery, Tor Vergata University (Roma)

Background: The aim of the current study was to examine the prognostic value of copeptin and matrix metalloproteinase 9 (MMP-9) for acute intracerebral hemorrhage (ICH) patients.

Materials and Methods: The serum biomarkers were recorded both at admission and 24 hours later, using enzyme-linked immunosorbent assay (ELISA). The three primary outcomes recorded were mortality during admission (1), mortality at 90 days (2) and poor functional outcome at 90 days, defined by a modified Rankin scale (mRS) 0-2 (3). Associations between serum biomarkers level and 
primary outcomes were investigated in multivariable linear and logistic regression models, respectively.

Results: 42 patients were included in the study. In this group of patients showed that high copeptin values are associated with increased risk of in-hospital mortality, adjusted for confounding factors (OR 4.04 CI95\% 0.81-54, p 0.048).

Conclusion: Serum damage markers can be useful in the management of patients with cerebral haemorrhage.

References:

- Askenase M. H., Sansing L. H. Stages of the Inflammatory Response in Pathology and Tissue Repair after Intracerebral Hemorrhage. Semin Neurol (2016) Jun;36(3):288-97

- Brunswick A. S. et al. Serum biomarkers of spontaneous intracerebral hemorrhage induced secondary brain injury. J. Neurol. Sci. (2012);321:1-10

- Yu W. et al. Prognostic significance of plasma copeptin detection compared with multiple biomarkers in intracerebral hemorrhage. Clinica Chimica Acta (2014);433:174-78

\section{COGNITIVE ASSESSMENT DURING THE ACUTE PHASE OF CEREBROVASCULAR DISEASES AND AT A SIX-MONTH FOLLOW-UP}

F. Mele ${ }^{1}$, I. Cova ${ }^{1}$, F. Benzi ${ }^{2}$, F. Zerini ${ }^{2}$, L. Maggiore ${ }^{1}$, V. Cucumo ${ }^{1}$, M. Brambilla $^{1}$, A. Nicotra ${ }^{1}$, S. Rosa ${ }^{1}$, P. Bertora ${ }^{2}$, E. Salvadori ${ }^{3}$, S. Pomati ${ }^{1}$, L. Pantoni ${ }^{2}$

${ }^{1}$ Neurology Unit, "Luigi Sacco" University Hospital (Milano); ${ }^{2 " L u i g i ~}$ Sacco" Department of Biomedical and Clinical Sciences, University of Milan (Milano); ${ }^{3}$ IRCCS Don Carlo Gnocchi, Don Carlo Gnocchi Foundation (Firenze)

Aims: Cognitive impairment is a common consequence of acute cerebrovascular diseases. [1] Our aims were to assess the frequency of pre- and post-stroke cognitive impairment and the association between scores obtained at a neuropsychological screening during the acute phase and worsening of cognitive status at follow-up.

Materials and methods: As a preliminary step of a larger observational study, patients admitted to our stroke unit underwent an assessment of the pre-morbid cognitive status through the Clinical Dementia Rating (CDR) scale and were administered two neuropsychological screening tests: Clock Drawing Test (CDT), and Montreal Cognitive Assessment-Basic (MoCA-B). A neuropsychological examination was completed 6 months after the acute event, assessing the presence and severity of post-stroke cognitive impairment (PSCI).

Results: From 25 November 2018 to 25 May 2019, 50 patients were enrolled. Based on CDR score, 28 patients (56\%) had no pre-stroke cognitive impairment, 15 patients $(30 \%)$ had pre-stroke mild cognitive impairment, and 4 patients $(8 \%$ ) had pre-stroke dementia; for the remaining 3 patients $(6 \%)$ pre-stroke cognitive status was not known because no informant was available to complete CDR during hospital stay. MoCA-B was administered to 48 patients ( $96 \%$ ) and CDT to 45 patients (90\%). The mean interval $( \pm \mathrm{SD})$ between the cerebrovascular event and the neuropsychological screening was 4 days $( \pm 2)$. The mean scores $( \pm \mathrm{SD})$ were $23.6( \pm 3.8)$ for MoCA-B and $8.4( \pm 2.5)$ for CDT. At the six-month follow-up, 11 patients $(22 \%)$ did not show any cognitive impairment, 28 patients $(56 \%)$ had a mild cognitive impairment, and 11 patients $(22 \%)$ had dementia. Out of the 28 patients without pre-stroke cognitive impairment, 9 patients (32\%) remained cognitively non-impaired, 16 patients
(57\%) developed MCI, and 3 patients (11\%) developed dementia. Overall, the incidence of PSCI was $68 \%$. Among those patients who were non-demented before the acute event, the score obtained on CDT during the acute phase was significantly lower in patients who showed a worsening of the cognitive status at follow-up, compared to patients who did not worsen $(\mathrm{p}=0.003)$.

Discussion: Our study shows that pre-event cognitive impairment is a common issue in patients admitted to a stroke unit and that a significant proportion of these patients will have a worsening of their cognitive status after 6 months. Furthermore, we showed that a neuropsychological screening administered during the acute phase is informative with regard to the evolution of the cognitive status.

Reference:

1. Pendlebury ST, Rothwell PM. Prevalence, incidence, and factors associated with pre-stroke and post-stroke dementia: a systematic review and meta-analysis. Lancet Neurol. (2009);8(11):1006-18

\section{BULBAR WATERSHED ISCHEMIC STROKE}

\author{
E. Merli, M. Romoli, A. Zaniboni, A. Borghi, L. Cirillo, L. Simonetti, A. \\ Zini
}

IRCCS Institute of Neurological Sciences of Bologna (Bologna)

Objectives: We report two patients presenting with bulbar symptoms with acute onset because of an ischemic lesion. Their brain MRI studies showed a peculiar aspect, resembling a "comma" between the two main bulbar vascular territories, consisting in a watershed lesion. We highlight this neuroradiological presentation, strictly related to bulbar vascularization.

Case reports: Our first patient is a 70 years-old male, suffering from hypertension, dyslipidemia, atrial fibrillation (edoxaban) and a previous ischemic cardioembolic stroke with left posteroinferior cerebellar artery (PICA) occlusion. He attended our Emergency Department because of the acute onset of right buccal deviation, dysphagia and the worsening of dysarthria, confirmed at neurological examination. Brain CT-CT angiography showed the occlusion of left PICA and multiple atherosclerotic plaques in right vertebral (V4 segment), right internal carotid artery, and in particular in left vertebral (V0 segment), with a severe stenosis. Brain MRI revealed the presence of a peculiar left bulbar DWI+/FLAIR+ lesion in a watershed region. The second case is a 65 years-old female, presenting with hypoesthesia/dysesthesia in right trigeminal second branch territory. Personal history revealed hypertension, dyslipidemia, previous transient ischemic attacks and history of falls. Her neurological examination revealed "C" shaped hypoesthesia around the right corner of the mouth, fragmented and delayed saccades and an apraxic gait. Brain MRI showed cerebellar atrophy and a subacute ischemic right retroolivary bulbar DWI+/FLAIR+ lesion. Neck vessel ultrasound revealed bilateral $50 \%$ internal carotid stenosis and normal flow velocities in vertebral arteries. Falls were found to be related to orthostatic postural hypotension; anti-hypertensive medications were tapered off.

Discussion: In both cases MRI showed a subacute postero-lateral superior bulbar lesion. This area doesn't match to a single-vessel blood supply, but rather stands for a watershed lesion between left PICA and vertebral artery. Indeed, the upper medulla is supplied by vertebral arteries in medial and anterior areas and by PICA arteries in lateral and posterior ones. Such watershed feature on brain MRI suggests an hemodynamic genesis rather than a microvascular/thromboembolic one, leading to different therapeutic approaches. 
Conclusion: We highlight the possible presentation and typical MRI aspect of bulbar watershed lesions. This etiology should be considered in clinical reasoning as it has direct therapeutic implications.

References:

- Burger K. M. et al. Brainstem Vascular Stroke Anatomy. Neuroimaging Clinics N AM (2005) May;15(2):297-324

- A.Ortiz de Mendivil, A. Alcalá-Galiano, M. Ochoa, E. Salvador, J. M. Millán, Brainstem Stroke: Anatomy, Clinical and Radiological Findings. Seminars in Ultrasound, CT and MRI (2013) Apr;34(2):131-41

- Louis R. Caplan Caplan's Stroke: A Clinical Approach Caplan.

\section{NONCONTRAST CT MARKERS OF INTRACEREBRAL HEMORRHAGE EXPANSION AND POOR OUTCOME: A META-ANALYSIS}

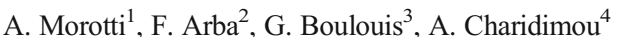

${ }^{1}$ Neurology Unit, ASST Valcamonica (Esine-BS); ${ }^{2}$ Stroke Unit, Careggi University Hospital (Firenze); ${ }^{3}$ Neuroradiology Department, Centre Hospitalier Sainte-Anne (Paris-F); ${ }^{4}$ Department of Neurology, Massachusetts General Hospital Stroke Research Center, Harvard Medical School (Boston-USA)

Objective: To provide precise estimates of the association between noncontrast CT (NCCT) markers, hematoma expansion (HE) and functional outcome in patients presenting with intracerebral hemorrhage (ICH) through a systematic review and meta-analysis.

Methods: We searched PubMed for English-written observational studies or randomized controlled trials reporting data on NCCT markers of HE and outcome in spontaneous ICH including at least 50 subjects. The outcomes of interest were HE (hematoma growth $>33 \%,>33 \%$ and/or $>6 \mathrm{~mL},>33 \%$ and/or $>12.5 \mathrm{~mL}$ ), poor functional outcome (modified Rankin Scale 3-6 or 4-6) at discharge or at 90 days, and mortality. We pooled data in randomeffects models and extracted cumulative odds ratio (OR) for each NCCT marker.

Results: We included 25 eligible studies $(n=10650)$. The following markers were associated with increased risk of $\mathrm{HE}$ and poor outcome respectively: black hole sign $(\mathrm{OR}=3.70,95 \%$ confidence interval $(\mathrm{CI})=1.42-9.64$ and $\mathrm{OR}=5.26,95 \% \mathrm{CI}=1.75-15.76)$, swirl sign $(\mathrm{OR}=3.33,95 \% \mathrm{CI}=2.42-4.60$ and $\mathrm{OR}=3.70$; $95 \% \mathrm{CI}=2.47-5.55)$, heterogeneous density ( $\mathrm{OR}=2.74$; $95 \% \mathrm{CI}=1.71-4.39$ and $\mathrm{OR}=2.80 ; 95 \% \mathrm{CI}=1.78-4.39)$, blend sign $(\mathrm{OR}=3.49 ; 95 \% \mathrm{CI}=2.20-5.55$ and $\mathrm{OR}=2.21 ; 95 \% \mathrm{CI} 1.16-4.18)$, hypodensities $(\mathrm{OR}=3.47 ; 95 \% \mathrm{CI}=2.18-5.50$ and $\mathrm{OR}=2.94$; $95 \% \mathrm{CI}=2.28-3.78)$, irregular shape $(\mathrm{OR}=2.01,95 \% \mathrm{CI}=1.27-3.19$ and $\mathrm{OR}=3.43 ; 95 \% \mathrm{CI}=2.33-5.03)$ and island $\operatorname{sign}(\mathrm{OR}=7.87,95 \%$ $\mathrm{CI}=2.17-28.47$ and $\mathrm{OR}=6.05,95 \% \mathrm{CI}=4.44-8.24$ ).

Conclusion: Our results suggest that multiple NCCT ICH shape and density features, with different effect size, are important markers for HE and clinical outcome, and may provide useful information for future randomized controlled trials.

Reference:

- Morotti A, Boulouis G, Dowlatshahi D, Li Q, Barras CD, Delcourt C, Yu Z, Zheng J, Zhou Z, Aviv RI, Shoamanesh A, Sporns PB, Rosand J, Greenberg SM, Al-Shahi Salman R, Qureshi AI, Demchuk AM, Anderson CS, Goldstein JN, Charidimou A Standards for Detecting, Interpreting, and Reporting Noncontrast
Computed Tomographic Markers of Intracerebral Hemorrhage Expansion.; International NCCT ICH Study Group. Ann Neurol. (2019) Oct;86(4):480-92

\section{PEDIATRIC ISCHEMIC STROKE: ENDOVASCULAR TREATMENT AND DIAGNOSTIC CHALLENGES}

C. Motto ${ }^{1}$, M. Piano ${ }^{2}$, P. Doneda ${ }^{3}$, S. Bergamoni ${ }^{4}$, C. Moretti ${ }^{5}$, C. de Giacomo $^{5}$, R. Vaccari $^{4}$, E. Agostoni ${ }^{1}$

${ }^{1}$ Stroke Unit, Neurology, ASST Grande Ospedale Metropolitano Niguarda (Milano); ${ }^{2}$ Interventional Neuroradiology, ASST Niguarda (Milano); ${ }^{3}$ Neuroradiology, ASST Niguarda (Milano); ${ }^{4}$ Child Neuropsychiatry, ASST Niguarda (Milano); ${ }^{5}$ Pediatry, ASST Niguarda (Milano)

Background: Although data from randomized controlled trial are lacking, reperfusion treatment may be an option for some children with acute ischemic stroke. Etiologic diagnosis remains a challenges in pediatric stroke and discriminating intracranial non inflammatory versus more common inflammatory arteriopathies may not be easy.

Methods: Case report. Endovascular treatment in a child with intracranial artery occlusion and difficult early etiologic diagnosis.

Results: A ten-year-old girl affected by Down syndrome experienced wake-up dysarthria and right hemiparesis preceded by vomiting, diarrhea and fever four days before symptom onset. No history of recent trauma was reported. Brain RM showed acute left lenticular and fronto- temporal ischemic lesions with mismatch, and non acute left fronto-parietal lesions. Angio-RM documented occlusion of distal intracranial internal carotid artery A small intracranial right carotid artery stenosis was found too. Mechanical thrombectomy was performed with internal carotid recanalization and residual vessel irregularities. No hemorrhage was observed at control RM. A complete diagnostic workup was performed and Moya-Moya syndrome was taken into account because presence of acute and non acute ischemic lesions and bilateral carotid steno-occlusion. Herpes zoster antibodies was found in cerebrospinal fluid, and inflammatory arteriopathy was diagnosed.

Discussion: Reperfusion therapy is the gold standard in acute ischemic stroke in adult. In pediatric acute ischemic stroke there are no randomized controlled trial, and only case report and small series were reported. In our case mechanical trombectomy was safe and effective, and no complications were observed. About etiologic diagnosis, the presence of acute and non acute cerebral ischemic lesions and bilateral carotid stenosis in a child with Down syndrome could suggest the diagnosis of Moya-Moya syndrome. The diagnosis of inflammatory arteriopathy was made only after a complete diagnostic workup.

Conclusion: Endovascular treatment should be considered in pediatric acute ischemic stroke with large vessel occlusion. Early etiologic diagnosis of ischemic stroke in pediatric population should be difficult, and should always include a complete workup to identify the correct etiology of the event.

\section{REDUCED ADMISSIONS FOR CEREBROVASCULAR EVENTS AND REVASCULARIZATION TREATMENTS FOR ISCHEMIC STROKE DURING COVID-19 OUTBREAK IN ITALY}

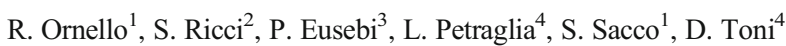


${ }^{1}$ Neuroscience Section, Department of Applied Clinical Sciences and Biotechnology, University of L'Aquila (L'Aquila); ${ }^{2}$ Neurology and Stroke Unit, Gubbio and Città di Castello Hospital, USL Umbria 1 (Perugia); ${ }^{3}$ Section of Neurology, Department of Medicine, University of Perugia (Perugia); ${ }^{4}$ Department of Human Neurosciences, Sapienza University of Rome (Roma)

Objective: We aimed to investigate the rate of hospital admissions for cerebrovascular events and of revascularization treatments for acute ischemic stroke in Italy during the Covid-19 outbreak.

Materials and Methods: The Italian Stroke Organization (ISO) performed a multicenter study involving 93 Italian Stroke Units. We collected information on hospital admissions for cerebrovascular events from March 1 to March 31, 2020 (study period) and from March 1 to March 31, 2019 (control period).

Results: Ischemic strokes decreased from 2399 in 2019 to 1810 in 2020, with a corresponding hospitalization rate ratio (HRR) of 0.75 (95\% confidence interval $[\mathrm{CI}]$ 0.71-0.80; $\mathrm{P}<0.001)$; intracerebral hemorrhages decreased from 400 to 322 (HRR 0.81; 95\% CI 0.69-0.93; P=0.004), and transient ischemic attacks decreased from 322 to 196 (HRR 0.61; 95\% CI 0.51-0.73; $\mathrm{P}<0.001)$. Hospitalizations decreased in Northern, Central, and Southern Italy. Intravenous thrombolyses decreased from $531(22.1 \%)$ in 2019 to 345 in 2020 (19.1\%; rate ratio $0.86 ; 95 \%$ CI $0.75-0.99 ; \mathrm{P}=0.032$ ), while primary endovascular procedures increased in Northern Italy (rate ratio 1.61 ; $95 \%$ CI 1.13-2.32; $\mathrm{P}=0.008)$. We found no correlation $(\mathrm{P}=0.517)$ between the HRRs for all strokes or TIA and Covid-19 incidence in the different areas.

Discussion: Limited hospitalization of the less severe patients and delays in hospital admission, due to overcharge of the emergency system by Covid-19 patients, may explain the decreased number of cerebrovascular diseases from 2019 to 2020 .

Conclusions: Hospitalizations for stroke or transient ischemic attacks across Italy were reduced during the worst period of the Covid-19 outbreak. Intravenous thrombolytic treatments also decreased, while endovascular treatments remained unchanged and even increased in the area of maximum expression of the outbreak.

\section{STRESS HYPERGLYCEMIA IS PREDICTIVE OF WORSE OUTCOME IN PATIENTS WITH ACUTE ISCHEMIC STROKE UNDERGOING INTRAVENOUS THROMBOLYSIS}

\author{
S. Pez ${ }^{1}$, G. Merlino ${ }^{2}$, C. Smeralda ${ }^{1}$, G. Gigli $^{3}$, S. Lorenzut ${ }^{2}$, A. \\ Surcinelli ${ }^{1}$, A. Marini ${ }^{1}$, M. Valente ${ }^{4}$
}

${ }^{1}$ Clinical Neurology, University Hospital (Udine); ${ }^{2}$ Clinical Neurology, Stroke Unit, Department of Neuroscience, University Hospital (Udine); ${ }^{3}$ Clinical Neurology, DMIF, University of Udine, University Hospital (Udine); ${ }^{4}$ Clinical Neurology, DAME, University of Udine, University Hospital (Udine)

Aim of the study: To investigate the possible detrimental effect of stress hyperglycemia, as measured by the glucose-to-glycated hemoglobin (HbA1c) ratio (GAR), in acute ischemic stroke (AIS) patients undergoing intravenous thrombolysis (IVT).

Materials: We retrospectively analyzed data from a prospectively collected database of patients admitted to the Udine University Hospital with AIS that were treated with IVT according to International Guidelines.

Methods: Patient's clinical, radiological and laboratoristic data were retrospectively assessed. The fasting venous blood samples within 24 hours after hospitalization were drawn after an overnight fast to measure fasting plasma glucose and $\mathrm{HbA1c}$. Stress hyperglycemia was estimated by the GAR index that was calculated using the following formula: fasting plasma glucose-(mg/dL)/HbAlc-(\%). The patients were stratified into four groups by quartiles of GAR (Q1-Q4) for further comparisons. The higher GAR index, the more severe stress hyperglycemia was considered. The following endpoints were analyzed: three-month poor outcome (defined as a modified Rankin Scale score between 3 and 6), no major neurological improvement at discharge (defined as an improvement of $>/=8$ points on the National Institute of Health Stroke Scale (NIHSS) score from baseline or a NIHSS score of 0-1 at discharge), inhospital mortality, three-month mortality, presence of symptomatic intracranial hemorrhage (SICH).

Results: AIS patients with severe stress hyperglycemia had a significantly increased risk of three-month poor outcome (OR 2.43, 95\% C.I. $=1.14-5.22$, $\mathrm{p}=0.02)$, three-month mortality (OR $2.38,95 \%$ C.I. $=1.01-5.60, \mathrm{p}=0.04)$ and SICH (OR 16.76, 95\% C.I. $=2.09-134.58, \mathrm{p}=0.008)$ after IVT.

Discussion: AIS patients undergoing IVT with hyperglycemia at admission had a worse outcome and a more frequent occurrence of SICH [1]. However, a proper interpretation of these results is complicated because underlying diabetes may act as potential confounder. Hyperglycemia may occur de novo in a high proportion of patients suffering an acute stress, such as stroke. Recently, a new index, the glucoseto-glycated hemoglobin ratio (GAR), has been developed for assessing stress hyperglycemia. While $\mathrm{HbAlc}$ reflects the baseline average glucose status over the past three months, the GAR index quantifies the extent of acute elevation in plasma glucose, i.e. stress hyperglycemia, compared to background plasma glucose levels [2]. To date, only one study used the GAR index in AIS patients and it showed that stress hyperglycemia predicted stroke recurrence and mortality in non-diabetic patients. However, only $3 \%$ of these patients were treated with IVT [3].

Conclusions: We demonstrated that stress hyperglycemia, as measured by the GAR index, is associated to worse outcome in AIS patients undergoing IVT.

References:

1. Desilles JP, Meseguer E, Labreuche J, et al. Diabetes mellitus, admission glucose, and outcomes after stroke thrombolysis: a registry and systematic review. Stroke (2013);44: 1915-23

2. Su YW, Hsu CY, Guo YW, Chen HS. Usefulness of the plasma glucose concentration-to-HbA1c ratio in predicting clinical outcomes during acute illness with extreme hyperglycaemia. Diabetes Metab (2017);43:40-47

3. Zhu B, Pan Y, Jing J, et al. Stress hyperglycemia and outcome of non-diabetic patients after acute ischemic stroke. Front Neurol (2019); 10:1003

\section{TROPONIN CHANGES IN ACUTE ISCHEMIC STROKE AS PREDICTORS OF STROKE SUBTYPE}

F. Pistoia ${ }^{1}$, C. Fattori ${ }^{2}$, A. Carolei ${ }^{1}$, S. Necozione ${ }^{3}$, R. Ornello ${ }^{1}, \mathrm{~S}$. $\mathrm{Sacco}^{1}$, C. Marini ${ }^{1}$

${ }^{1}$ Department of Applied Clinical Sciences and Biotechnology, University of L'Aquila (L'Aquila); ${ }^{2}$ Neurological Institute, University of L'Aquila (L'Aquila); ${ }^{4}$ Department of Life, Health and Environmental Sciences, University of L'Aquila (L'Aquila)

Objective: Increased troponin levels are detected in a high proportion of patients with acute stroke. The objective of this study was to investigate the presence of troponin alterations in patients with acute stroke and its possible association with stroke's etiology. 
Materials and Methods: All patients consecutively admitted to the Neurology and Stroke Unit of the San Salvatore Hospital of L'Aquila between November 2019 and May 2020, with a diagnosis of acute stroke, were included in the study. The standard comprehensive diagnostic workup for stroke, including laboratory testing, neuroimaging exams, cardiac and vascular evaluation, was performed in all the patients. Troponin levels, detected at the time of admission to the emergency department, were analyzed in order to investigate their relationship with the nature of stroke (ischemic or hemorrhagic) and, in the case of ischemic stroke, the possible association with the stroke subtype, as assessed by the TOAST (modified Trial of ORG 10172 in Acute Stroke Treatment) classification. The association between troponin levels and the presence of comorbidities (arterial hypertension, diabetes mellitus, coronary heart disease, atrial fibrillations and renal disease) was also investigated. Kruskal-Wallis test and Wilcoxon Signed-Rank Test were used for statistical analysis. A p value $<0.05$ was considered statistically significant.

Results: Forty-two patients ( 25 men and 17 women) with a diagnosis of ischemic $(n=31$; mean age $75.3 \pm 12.8)$ or hemorrhagic stroke $(n=11$; mean age $75.58 \pm 14.7)$ were prospectively included in the study. No significant differences in troponin levels were found between patients with ischemic and hemorrhagic stroke $(\mathrm{p}=0.4568)$. In the group of patients with ischemic stroke, the troponin positivity was independently associated with TOAST 1 (large artery atherosclerosis etiology) stroke subtype ( $\mathrm{p}=0.02$ ). Whether medical comorbidities were analyzed, a positive association was found between troponin levels and the presence of renal disease $(\mathrm{p}=0.006)$.

Discussion: Several studies investigated the association between the troponin levels and the etiology of stroke, in order to establish whether cardiac biomarkers may help identifying stroke etiology and mechanisms. Some studied suggested that high troponin levels predict cardioembolic or embolic strokes of unknown source subtype, whereas others highlighted the association between troponin changes and acute ischemic stroke caused by large vessel occlusion.

Conclusion: Future studies are necessary in order to confirm the possible association between the presence of positive troponin levels and the large vessel occlusion etiology for stroke. If this evidence should be confirmed, it might lead to therapeutic implications both in acute stroke care and in secondary prevention.

References:

- Yaghi S, Chang AD, Ricci BA et al. Early Elevated Troponin Levels After Ischemic Stroke Suggests a Cardioembolic Source. Stroke (2018);49:121-26

- Chang A, Ricci B, Grory BM et al. Cardiac Biomarkers Predict Large Vessel Occlusion in Patients with Ischemic Stroke. J Stroke Cerebrovasc Dis. (2019);28:1726-31

- Tomich C, Liegey JS, Sagnier S, Olindo S, Poli M, Debruxelles S, Rouanet F, Renou P, Sibon I. Contribution of routine cardiac biological markers to the etiological workup of ischemic stroke. Am J Emerg Med. (2019);37:194-98

\section{SAFETY AND EFFICACY OF TREATMENTS FOR ACUTE ISCHEMIC STROKE IN AN ELDER POPULATION: A MULTICENTER STUDY}

\author{
E. Potente ${ }^{1}$, M. Burattini ${ }^{1}$, S. Salvemini ${ }^{1}$, L. Falsetti ${ }^{2}$, M. Bartolini ${ }^{1}$, M. \\ Silvestrini $^{1}$, T. Rossi ${ }^{3}$, G. Pelliccioni ${ }^{3}$, G. Viticchi ${ }^{1}$
}

${ }^{1}$ Neurological Clinic, Marche Polytechnic University (Ancona); ${ }^{2}$ Internal and Subintensive Medicine, Ospedali Riuniti Ancona (Ancona); ${ }^{3}$ Neurology Department, INRCA Hospital (Ancona)
Introduction: Fibrynolisis with intravenous rt-PA and mechanical thrombectomy represent two well established treatments for acute ischemic stroke. Unfortunately, the larger part of randomized controlled trials has excluded or underrepresented patients over 80 years. Some metanalysis and retrospective studies suggest that fibrynolisis may have the same efficacy and safety in the elder population, while the benefits of thrombectomy appear less clear. Aim of this study was to assess efficacy and safety of fibrinolysis and thrombectomy in a population of patient $>$ 75 years with acute ischemic stroke.

Materials and Methods: We enrolled all patients admitted to two different Stroke Units (INRCA Hospital, Ancona and Ospedali Riuniti Ancona) in a period of 3 years (2017 - 2020) for acute ischemic stroke. For each patient we considered age, sex, type of treatment for acute ischemic stroke, NIHSS at admission, NIHSS at discharge, mRS at admission and $\mathrm{mRS}$ at discharge. Statistical analysis was performed with SPSS 13.0 for Windows systems.

Results: We collected 226 consecutive patients, with a mean age of $83,4( \pm 5,48)$ years. $35,8 \%$ of the subjects undergoing to fibrinolysis, $16,8 \%$ to fibrinolysis and thrombectomy, $15,0 \%$ received only thrombectomy and $32,3 \%$ remained untreated. The group with no active treatment (NAT) was associated to a significantly lower admission NIHSS in respect to fibrinolysis (3[5] versus 7[11]; $\mathrm{p}=0,0001)$, fibrinolysis and thrombectomy (16[4]; $\mathrm{p}=0,0001)$ and thrombectomy alone $(17[6] ; \mathrm{p}=0,0001)$. NAT had a significantly higher NIHSS at discharge in respect to thrombectomy $(9,50[12]$; $\mathrm{p}=0,0001)$ and fibrinolysis and thrombectomy $(12[11] ; \mathrm{p}=0,0001)$. Similarly, NAT was associated to a significantly higher $\mathrm{mRS}$ at admission when compared to fibrinolysis (2[3] versus $1[3] ; \mathrm{p}=0,006)$, fibrinolysis and thrombectomy $(0[0] ; \mathrm{p}=0,0001)$ and thrombectomy alone $(0[0] ; \mathrm{p}=0,0001)$. At discharge, mRS was similar between NAT and fibrinolysis (3[4] versus $4[3] ; \mathrm{p}=0,897)$ while thrombectomy and fibrinolysis $(5[1] ; \mathrm{p}=0,0001)$ or thrombectomy alone $(4[2] ; \mathrm{p}=0,015)$ resulted into higher $\mathrm{mRS}$ at discharge.

Discussion: Our study suggest that fibrinolysis may be considered a safe treatment in the elderly. Thrombectomy, alone or in combination with fibrinolysis, appeared more efficacious in respect to fibrinolysis alone, as it is associated with lower NIHSS at discharge, but it presents a major risk of death or significant disability.

Conclusions: Fibrinolysis and thrombectomy may be associated with a relatively good outcome in the elder population, but risks and benefits must be carefully balanced, and this is especially true for thrombectomy, for which an accurate selection of patient is needed.

References:

1. Mishra et al. Thrombolysis in very elderly people: controlled comparison of SITS International Stroke Thrombolysis Registry and Virtual International Stroke Trials Archive. British Medical Journal (2010);341:c6046

2. S. Lorenzano et al. Thrombolysis in elderly stroke patients in Italy (TESPI) trial and updated metaanalysis of randomized controlled trials. International Journal of Stroke (2019) Oct 26;1747493019884525 doi: 10.1177/1747493019884525

3. Zhao et al. Mechanical Thrombectomy for Acute Ischemic Stroke in Octogenarians: A Systematic Review and Meta-Analysis. Front. Neurol. (2020);10:1355

\section{ACUTE CAROTID STENTING IN HEMODYNAMIC STROKE}

\author{
R. Renna ${ }^{1}$, G. Leone ${ }^{2}$
}

${ }^{1}$ UOSC Neurology, Stroke Unit, AORN Cardarelli (Napoli); ${ }^{2}$ Neuroradiology Unit, AORN Cardarelli (Napoli) 
Case: A 69 years-old man was admitted for sudden-onset aphasia and weakness of the right side. Symptoms started two hours before admission. His past medical history was remarkable for hypertension and smoking. Neurological examination revealed moderate aphasia and right hemiparesis (NIHSS score 7). Brain CT scan showed no hemorrhage and no acute ischemic changes; Computed Tomography Angiography excluded any intracranial occlusion and revealed a plaque with significant stenosis in the left internal carotid artery (ICA). CT perfusion showed a large area of penumbra in ICA territory in the last meadows (hemodynamic stroke) with a small deep core. In consideration of the indication for acute carotid stenting treatment and of the high bleeding risk that would result from intravenous thrombolysis and dual antiplatelet load, primary percutaneous transluminal angioplasty and carotid stenting were performed. Patient was premedicated with oral Clopidogrel $300 \mathrm{mg}$ and intravenous Lysine-acetylsalicylate $500 \mathrm{mg}$ and underwent primary carotid stenting. Cerebral angiography showed a pre-occlusive stenosis of left ICA in absence of intracranial stenosis, thus confirming the hemodynamic origin of stroke. In heparin therapy (intravenous $3000 \mathrm{IU}$ ), a guide catheter was placed in the left common carotid. Stenosis was crossed by a Filterwire anti-embolic protection filter and pre-dilation was carried out with a $5 \times 30$ balloon on the filter guide. Carotid Wallstent was released to cover the plaque. At the end of the procedure, left ICA caliber was restored. Soon after carotid stenting neurological exam improved (NIHSS score 4). The day after neurological exam showed only a mild facial deficit (NIHSS score 1) and at carotid ultrasound the stent was patent and fully deployed. Dual antiplatelet therapy (Lysine-acetylsalicylate $100 \mathrm{mg}$ and Clopidogrel $75 \mathrm{mg}$ ) were carried on.

Discussion: In case of acute ischemic stroke due to carotid artery stenosis the optimum treatment plan is not clearly defined. Endovascular tretaments may be the optimal treatment in some cases.[1] Methods of endovascular recanalization include carotid angioplasty, stenting, or both. The use of stents requires patients to be placed on dual antiplatelet agents, which contributes to increase hemorrhagic risk. On the other side angioplasty alone may be complicated by risk of both early and long-term restenosis.[2]. Primary endovascular recanalization may be considered in acute hemodynamic stroke due to critic carotid stenosis, thus reducing hemorrhagic risk related to intravenous thrombolysis.

References:

1. O'Brien M, Chandra A. Carotid revascularization: risks and benefits. Vasc Health Risk Manag. (2014);10:403-16

2. Baudier JF, Licht PB, Røder O, Andersen PE. Endovascular treatment of severe symptomatic stenosis of the internal carotid artery: early and late outcome. Eur J Vasc Endovasc Surg Off J Eur Soc Vasc Surg. (2001);22:205-10

\section{PONATINIB-RELATED THROMBOSIS OF CERVICAL AND INTRACRANIAL ARTERIES}

R. Renna ${ }^{1}$, E. Spina ${ }^{2}$, S. Miniello ${ }^{3}$, G. Martusciello ${ }^{3}$, G. Piccirillo ${ }^{3}$, G. Di Iasi $^{3}, \mathrm{~V}$. Boemio ${ }^{3}, \mathrm{~V}$. Andreone ${ }^{3}$

${ }^{1}$ UOSC Neurology, Stroke Unit, AORN Cardarelli (Napoli); ${ }^{2}$ Department of Neuroscience, Reproductive and Odontostomatology, Federico II University (Napoli); ${ }^{3}$ Department of Neurology, AORN Sant'Anna e San Sebastiano (Caserta)

Ponatinib is a third-generation Tyrosine Kinase Inhibitor (TKI), approved as first line treatment for Chronic Myeloid Leukaemia (CML) chronic phase. In most patients CML is associated with the BCR-ABL fusion gene.[1] Ponatinib, a third generation TKI, can inhibit BCR-ABL with the gatekeeper
T315I kinase domain mutation, known to be the cause for $20 \%$ of resistant or relapsed CML cases, overcoming specific resistance to second-generation TKIs.[2,3] Here we describe a 62 year-old male patient with CML treated with Ponatinib $15 \mathrm{mg}$. His past medical history was remarkable for hypertension, treated with ramipril and furosemide. Moreover he reported previous subsequent TIAs and an ischemic stroke during Ponatinib treatment in 2018 (three months after Ponatinib initiation). At that time, stroke work-up showed normal glucose, creatinine, lipid profile and coagulation tests. MRI demonstrated an ischemic lesion in fronto-basal right area; extra and intracranial CT angiography excluded stenosis of large vessels, so ponatinib was not stopped and aspirin was initiated at the dose of $300 \mathrm{mg}$ and continued at a dose of 100 $\mathrm{mg}$. Patient was admitted 7 months later for a 3-day history of sudden onset left hemiparesis (NIHSS 4). Brain MRI showed a new acute ischemic lesion in right fronto-basal area. Angio-MRI showed bilaterally the almost total absence of signal in the intracranial tract of anterior circulation and low signal of cerebral posterior circulation. The progressive course of cervical and intracranial arteries thrombosis is well documented by Digital Subtraction Angiography (DSA) showing multiple steno-occlusions of large vessels of both anterior and posterior circulation. Due to two ischemic strokes and multiple TIAs, Ponatinib was stopped and double antiplatelet therapy was started, with no other major cerebrovascular events or TIAs in the following year. The association between cerebrovascular events and TKIs of second and third-generation has been widely described. Regarding Ponatinib the proposed mechanism is the antagonist activity on VEGF-R, thus determining endothelial dysfunction and predisposing to thromboembolic events. For these reasons, it may be used for a narrowed patient population, including adults with T315I-positive CML with a big warning box for vascular events and liver toxicity, particularly in patients with pre-existent cardiovascular risk factors. To our knowledge, this is the first case described of multiple ischemic strokes and recurrent TIAs during treatment with Ponatinib. This points out the importance of an accurate quantification of cardiovascular risk before starting the treatment and the eventual strategy of reducing the starting dose after achieving clinical or molecular response.

References:

1. Hutchison RE, Schexneider KI. Leukocytic disorders. In: McPherson RA, Pincus MR, eds. Henry's clinical diagnosis and management by laboratory methods, 22nd ed., Pennsylvania: Elsevier Saunders; (2011):616

2. Miller GD, Bruno BJ, Lim CS. Resistant mutations in CML and $\mathrm{Ph}(+)$ ALL role of Ponatinib. Biologics (2014);8:243-54

3. Molica M, Scalzulli E, Colafigli G, et al. Insights into the optimal use of ponatinib in patients with chronic phase chronic myeloid leukaemia. Ther Adv Hematol (2019);10:1-14

\section{STENTING IN ACUTE BASILAR OCCLUSION FROM VERTEBRAL V2 DISSECTION: TELESCOPING STENTS TECHNIQUE}

R. Renna ${ }^{1}$, G. Guarnieri ${ }^{2}$, V. Andreone ${ }^{3}$, M. Muto $^{2}$, G. Leone ${ }^{2}, \mathrm{~S}$. Miniello $^{3}$, G. Ambrosanio ${ }^{2}$, M. Muto ${ }^{2}$

${ }^{1}$ UOSC Neurology, Stroke Unit, AORN Cardarelli (Napoli); ${ }^{2}$ Neuroradiology Unit, AORN Cardarelli (Napoli); ${ }^{3}$ Neurology, Stroke Unit, AORN Sant'Anna e San Sebastiano (Caserta)

Background: Basilar Artery Occlusion (BAO) mortality is up to 90\%.[1] Posterior circulation tandem occlusion [proximal vertebral artery (VA) stenosis/dissection plus BAO] is rare and its management represents a challenge, since its best treatment strategy is unclear.[2] 
Case presentation: A 52 years-old male patient presented sudden loss of consciousness and right hemiparesis. Twelve hours later, he presented with binocular diplopia, left-gaze nystagmus, ataxia and right hemiparesis (NIHSS score 12). Extra-intracranial Multidetector-CT angiography showed left V2 dissection with V4 segment occlusion up to basilar artery (BA) middle segment. Right VA ended in PICA. Stroke onset contraindicated intravenous thrombolysis. Endovascular strategy was to cross V2 dissection performing a direct aspiration first-pass-technique (ADAPTtechnique). Via transfemoral access, a sheath was placed in left VA under the dissection and a microcatheter was advanced to BAO site over a guidewire twice, unsuccessfully. A "Solumbra" Technique with Solitaire-FR-stent plus microcatheter was performed without revascularization. Therefore, an acute stenting of V2 dissection followed by telescoping stents vessel reconstruction from tip BA up to left V4 segment was performed. A microcatheter was navigated distal to the V2 dissection over a guidewire. A Solitaire-AB-stent was placed into $\mathrm{V} 2$ dissection. A microcatheter was navigated distal to basilar occlusion over a guidewire. A Solitaire-AB-stent was placed from basilar tip to middle basilar segment; a Solitaire-AB-stent was deployed from middle to lower basilar segment with initial recanalization effect. An Enterprice stent was placed from lower BA to V4 segment for a complete vessel reconstruction. Angiographic control showed TICI $2 b$ restoration. Post-procedural CT scan showed no complication. Patient was admitted in ICU and woke up the day after with no neurological deficit (NIHSS score 0). Brain CT scan 24-hours later showed a small asymptomatic right cerebellar hemisphere haematoma. Brain MRI performed 3 months later showed a stable result without parenchymal lesions. Six months later, the angiographic control showed VA and BA complete recanalization (TICI 3 score).

Discussion: An aggressive endovascular management of BAO can lead to survival rates of $35-50 \%$. Endovascular approach with ADAPT-technique, Solumbra-Technique or Stent-Retriever technique may allow flow restoration.[3] In selected cases, i.e. in case of failure of standard techniques, emergent revascularization with intra-thrombus stenting placement may represent a bailouttreatment to restore blood flow, especially when occlusion is due to long extension clot or tandem lesion. This case suggests that Telescoping Stents-Technique can be considered a valid strategy with potential good outcome for posterior circulation tandem occlusions after multiple unsuccessfully conventional techniques.

References:

1. Ecker RD, Tsujiura CA, Baker CB, et al. Endovascular reconstruction of vertebral artery occlusion prior to basilar thrombectomy in a series of six patients presenting with acute symptomatic basilar thrombosis. J Neurointerv Surg. (2014);6:379-83

2. Cohen JE, Leker RR, Gomori JM, et al. Emergent revascularization of acute tandem vertebrobasilar occlusions: Endovascular Approaches and technical considerations-Confirming the role of vertebral artery ostium stenosis as a cause of vertebra basilar stroke. J Clin Neurosci. (2016);34:70-76

3. European Stroke Organisation (ESO) - European Society for Minimally Invasive Neurological Therapy (ESMINT) Guidelines on Mechanical Thrombectomy in Acute Ischemic Stroke. European Stroke Journal (2019); 4(1) 6-12

\section{ENDOVASCULAR TREATMENT FOR ACUTE BASILAR ARTERY OCCLUSION: A CASE SERIES}

F. Rizzo ${ }^{1}$, M. Petruzzellis ${ }^{1}$, D. Mezzapesa ${ }^{1}$, N. Loizzo ${ }^{1}$, M. Parisi ${ }^{1}$, G. Scaglione $^{1}$, L. Chiumarulo ${ }^{2}$, M. Savarese ${ }^{1}$, I. Simone ${ }^{1}$, M. Trojano ${ }^{1}$
${ }^{1}$ Department of Basic Medical Sciences, Neurosciences and Sense Organs, University of Bari (Bari); ${ }^{2}$ Department Neuroradiology, University of Bari (Bari)

Background and Aim: Acute basilar artery occlusion (BAO) is associated with high mortality and poor outcome. Early recanalization has been demonstrated to be the major goal of acute ischemic stroke treatment. After intravenous thrombolysis (IVT) early recanalization in BAO occur only in 13\% with high poor-outcome-rates. The efficacy and therapeutic window of mechanical thrombectomy (MT) for BAO has not yet been established.

Methods: We retrospectively analyzed seventeen BAO consecutive patients admitted to our centre from May 2018 to May 2020. At admission brain Computed Tomography (CT) scan and CT angiography (CTA) were performed in all the patients.

Discussion: Mean age was 64 years and median admission-NIHSS was 21. Five patients had proximal, four middle and nine distal BAO. In nine cases collateral flow through posterior communicating artery was observed. Six patients performed IVT prior MT, eleven patients performed direct MT due to IVT contraindication. Thromboaspiration technique was used in twelve patients, stent-retriver devices were used for two patients and Solumbra technique was performed in three patients. Successful recanalization (TICI $\geq 2 b$ ) was achieved in fifteen patients. The median onset-torecanalization-time (OTR) was 5 hours. One artery dissection and three embolization in posterior cerebral artery occurred. No symptomatic hemorrhages were observed. Despite of good recanalization rate (88\%), six patients died and only two patients had good clinical outcome ( $\mathrm{mRS} \leq 2)$ : both patients had young age, low admission-NIHSS and good collateral flow.

Conclusion: Age, admission-NIHSS, collateral flow and OTR are all predictors of good outcome. Our data confirmed admission-NIHSS, age and collateral flow as good outcome predictors. Our longer OTR, the predominantly patient selection only with CT and CTA and the lack of collateral status evaluation with standardized scores, such as posterior circulation collateral score and basilar artery on CTA (BATMAN) score, probably explain our high rate of poor clinical outcome.

References:

- van der Hoeven EJ, Schonewille WJ, Vos JA, et al. The Basilar Artery International Cooperation Study (BASICS): study protocol for a randomised controlled trial. Trials. (2013);14:200

- Liu X, Xu G, Liu Y, et al. Acute basilar artery occlusion: Endovascular Interventions versus Standard Medical Treatment (BEST) Trial-Design and protocol for a randomized, controlled, multicenter study. Int J Stroke (2017);12(7):779-85

- Alemseged F, Van der Hoeven E, Di Giuliano F, et al. Response to Late-Window Endovascular Revascularization Is Associated with Collateral Status in Basilar Artery Occlusion. Stroke (2019);STROKEAHA118023361

\section{TENECTEPLASE FOR LARGE VESSEL OCCLUSION ISCHEMIC STROKE - UNIVERSITY OF BARI REAL-LIFE EXPERIENCE}

\author{
F. Rizzo ${ }^{1}$, M. Petruzzellis ${ }^{1}$, D. Mezzapesa ${ }^{1}$, N. Loizzo $^{1}$, M. Parisi ${ }^{1}$, G. \\ Scaglione $^{1}$, L. Chiumarulo ${ }^{2}$, M. Savarese ${ }^{1}$, I. Simone ${ }^{1}$, M. Trojano ${ }^{1}$
}

${ }^{1}$ Department of Basic Medical Sciences, Neurosciences and Sense Organs, University of Bari (Bari); ${ }^{2}$ Department of Neuroradiology, University of Bari (Bari)

Background and Aim: Intravenous thrombolysis (IVT) with alteplase before mechanical thrombectomy (MT) is actually the standard of care for acute 
ischemic stroke (AIS) due to Large Vessel Occlusion (LVO). AHA/ASA 2019 Guidelines for AIS Management stated that may be reasonable choosing tenecteplase (TNK) over alteplase in patients without contraindications for IVT who are also eligible for MT. In our Stroke Center we start to use TNK for Bridging Therapy (BT) in December 2019, but during COVID-19 pandemic we decided to use TNK independently from eligibility for MT, because its workflow advantages and potentially time savings.

Methods: We retrospectively collected data of patients admitted to our Hospital from December 2019 to May 2020, who presented AIS and received TNK $0.25 \mathrm{mg} / \mathrm{Kg}$ as single IV bolus.

Results: We evaluated fifteen patients with LVO-AIS: group 1 underwent to BT, while group 2 underwent to TNK-IVT only due to Neurointerventional Radiologist decision. In group 1 there were eight patients with mean age of 72 years and median baseline NIHSS of 12 (range 4-23). Six patients had middle cerebral artery (MCA) occlusion, one internal carotid artery (ICA) T-occlusion and one tandem occlusion. Good recanalization rate (TICI $\geq 2 \mathrm{~b}$ ) was achieved in $75 \%$ of cases, with median number of passages of 1 (range 1-4); first-pass good recanalization rate was $63 \%$. Median door-to-groin and needle-to-groin times were respectively 182 minutes (range 98-294) and 81 minutes (range 20-144). Median NIHSS at discharge was 8 (range 7-18). Two patients died and four patients had good clinical outcome $(\mathrm{mRS} \leq 2)$ at 3 months. In group 2 there were seven patients with mean age of 82 years and median baseline NIHSS of 20 (range 4-26). Three patients had MCA occlusion, three ICA-T-occlusion and one tandem occlusion. Median door-to-needle time was 105 minutes (range 60-186). 24-hours Transcranial Doppler showed complete recanalization in only two patients. Median NIHSS at discharge was 18 (range 0-32). Three patients died and two patients had $\mathrm{mRS} \leq 2$ at 3 months. In both group any symptomatic intracerebral haemorrhage was reported, only two asymptomatic intracerebral haemorrhages (H1, according SITS-MOST criteria) were observed.

Conclusion: According to literature, our case series shows positive trend towards complete first-pass EVT recanalization after TNK administration for BT. TNK-IVT alone in patients with LVO-AIS achieved complete recanalization in only $28.5 \%$. Comparing to our alteplase experience, single bolus TNK did not show in-hospital delay reduction, due to data collecting during COVID-19 pandemia.

References:

- Campbell BCV, Mitchell PJ, Churilov L, Yassi N, Kleinig TJ, Dowling RJ, Yan B, Bush SJ, Dewey HM, Thijs V, et al; EXTEND-IA TNK Investigators. Tenecteplase versus alteplase before thrombectomy for ischemic stroke. N Engl J Med. (2018);378:1573-82

- Campbell BCV, Mitchell PJ, Churilov L, et al. Effect of Intravenous Tenecteplase Dose on Cerebral Reperfusion Before Thrombectomy in Patients with Large Vessel Occlusion Ischemic Stroke: The EXTEND-IA TNK Part 2 Randomized Clinical Trial. JAMA (2020); Feb 20;323(13):1257-65

- Warach SJ, Saver JL. Stroke Thrombolysis with Tenecteplase to Reduce Emergency Department Spread of Coronavirus Disease 2019 and Shortages of Alteplase. JAMA Neurol. (2020); Jul 20. doi: 10.1001/jamaneurol.2020.2396

\section{PROSPECTIVE OBSERVATIONAL STUDY OF SAFETY OF EARLY TREATMENT WITH EDOXABAN IN PATIENTS WITH ISCHAEMIC STROKE AND ATRIAL FIBRILLATION (SATES STUDY)}

I. Scala ${ }^{1}$, G. Frisullo ${ }^{2}$, G. Della Marca ${ }^{2}$, V. Brunetti ${ }^{2}$, A. Broccolini ${ }^{2}$, R. Morosetti $^{2}$, P. Profice ${ }^{3}$, S. Bellavia ${ }^{1}$, P. Calabresi ${ }^{2}$
${ }^{1}$ Neurology, Catholic University of the Sacred Heart (Roma); ${ }^{2}$ Neurology, Foundation Polyclinic University A. Gemelli (Roma); ${ }^{3}$ Neurology, Mater Olbia Hospital (Olbia)

Background and Purpose: New direct oral anticoagulants are recommended for stroke prevention in patients with non-valvular atrial fibrillation (NVAF). However, no data is available for the optimal timing for starting oral anticoagulation after a stroke or TIA. The aim of our study is to evaluate the safety of very early initiation of full dose of Edoxaban in patients with acute ischemic stroke.

Methods: In this prospective, non-randomised, single centre study, we included patients started anticoagulant treatment with Edoxaban at therapeutic dose $(60 \mathrm{mg} / \mathrm{die})$ within 72 hours from cardioembolic stroke onset. All patients underwent continuous clinical and multimodal monitoring in stroke sub-intensive care and to CT/MRI scan within $5 \pm 2$ days after the beginning of the treatment. To be enrolled into the study, patients had to be at least 18 years, a diagnosis of NVAF and acute stroke with ASPECT score $\geq 7$. Patients with acute or chronic renal failure, defined as eGFR $<50 \mathrm{ml} / \mathrm{min}$ or body weight $<60 \mathrm{~kg}$ or treated with Ciclosporina, dronedarone, eritromicina, ketoconazole or known hypersensitivity to Edoxaban or with any other contraindication to anticoagulant treatment were excluded from the study.

Results: We enrolled 50 patients, the average age was 77 years and the mean NIHSS 7.8. After 3 months we observed no intracranial bleeding, only one gastrointestinal MB which comported a temporally suspension of Edoxaban and 8 minor bleeding. We did not observe any stroke recurrence. We did not observe any symptomatic HT and the incidence of asymptomatic HT was $12 \%$ for HI-1 (small petechiae) and $8 \%$ for HI-2 (confluent petechiae) without neurological deterioration. No patient stopped the Edoxaban treatment during the follow-up period.

Discussion: Regarding the safe of treatment, no intracranial bleeding occurred in the first three months of treatment, suggesting a favourable safety profile in very acute phase of ischemic stroke. The only major bleeding was not related to the very early start of NOAC in the acute phase of stroke since the late onset of the bleeding, which occurred after 2 months from Edoxaban start, and the extracranial location. Despite the high risk of cardioembolic brain events (mean CHADsVASC score $=5.2 \pm 1.4$ ), in the three months follow-up no patient presented a recurrence of ischemic stroke or a new systemic thromboembolic event, supporting the effectiveness of Edoxaban.

Conclusion: According to our data the early initiation of Edoxaban seems to be safe in patients after a cardioembolic stroke. However, further studies are needed.

References:

- Paciaroni M, Agnelli G, Falocci N, et al. Early Recurrence and Cerebral Bleeding in Patients w ith Acute Ischemic Stroke and Atrial Fibrillation: Effect of Anticoagulation and Its Timing: The RAF Study. Stroke (2015);46(8):2175-82

- Giugliano RP, Ruff CT, Braunwald E, et al. Edoxaban versus warfarin in patients with atrial fibrillation. N Engl J Med. (2013);369(22):2093-2104

- Klijn CJ, Paciaroni M, Berge E, et al. Antithrombotic treatment for secondary prevention of stroke and other thromboembolic events in patients with stroke or transient ischemic attack and non-valvular atrial fibrillation: A European Stroke Organisation guideline. Eur Stroke J. (2019);4(3):198-223 
TRANSCRANIAL DOPPLER SONOGRAPHY IN ASYMPTOMATIC PATIENTS WITH FABRY'S DISEASE

\author{
L. Vinciguerra ${ }^{1}$, G. Lanza ${ }^{2}$, C. Vagli ${ }^{3}$, G. Zelante ${ }^{4}$, F. Fisicaro ${ }^{3}$, V.

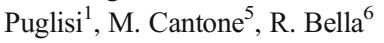

${ }^{1}$ Department of Neurology and Stroke Unit, ASST Cremona (Cremona); ${ }^{2}$ Department of Surgery and Medical-Surgical Specialties, University of Catania (Catania); ${ }^{3}$ Department of Biomedical and Biotechnological Sciences, University of Catania (Catania); ${ }^{4}$ Department of Neurology, "Giovanni Paolo II" Hospital (Ragusa); ${ }^{5}$ Department of Neurology, "Sant'Elia" Hospital (Caltanissetta); ${ }^{6}$ Department of Medical and Surgical Sciences and Advanced Technologies, University of Catania (Catania)

Background: Patients with Fabry's disease (FD) may be asymptomatic or show a spectrum of clinical manifestations, including cerebrovascular disease, mainly affecting the posterior circulation. Few and conflicting studies on cerebral blood flow (CBF) by transcranial Doppler sonography (TCD) in asymptomatic FD (aFD) subjects have been published. Our study aims to assess TCD in aFD subjects to identify any preclinical CBF change.

Methods: 30 aFD subjects were consecutively recruited and compared to 28 healthy controls. Brain magnetic resonance imaging was normal in all participants. TCD was used to study blood flow velocity and indices of resistance of intracranial arteries from the middle cerebral artery (MCA), bilaterally, and from the basilar artery (BA). Cerebral vasomotor reactivity (CVR) was also evaluated from MCA.

Results: No difference was found between groups for MCA parameters of $\mathrm{CBF}$ and CVR. Compared to controls, a higher mean blood flow velocity and a lower resistance index from BA were observed in FD subjects. No correlation was found between any BA-derived TCD parameter and the level of lyso-globotriaosylceramide.

Conclusions: aFD subjects show evidence of altered CBF in the posterior circulation. Preclinical detection of neurovascular involvement in FD might allow appropriate management and prevention of future cerebrovascular complications and disability.

\section{THE INFLUENCE OF SEX AND OVERWEIGHT ON ISCHEMIC STROKE SEVERITY}

G. Viticchi ${ }^{1}$, L. Falsetti ${ }^{2}$, M. Burattini ${ }^{1}$, E. Potente $^{1}$, M. Bartolini ${ }^{1}$, L. Buratti $^{1}$, M. Silvestrini ${ }^{1}$

${ }^{1}$ Neurological Clinic, Marche Polytechnic University (Ancona); ${ }^{2}$ Internal and Subintensive Medicine, Ospedali Riuniti (Ancona)

Background and Purpose: The role of overweight and obesity has not been fully assessed in the clinical evolution of ischemic stroke. Moreover, we did not have conclusive data about the impact of gender on the stroke in-came and out-came: women seem to present a worse outcome, often related to a higher mean age, more serious symptoms at onset and delayed treatments Aim of this study was to evaluate the possible correlations among overweight, gender and ischemic stroke severity and functional outcomes.

Methods: All consecutive patients admitted for an acute non-lacunar ischemic stroke at Stroke unit of Neurological Clinic of the University Hospital of Ancona, over a one-year period, were retrospectively considered. According to the body mass index (BMI) value, patients were classified in three categories: normal (healthy weight, BMI $>18.5 \mathrm{~kg}$ / $\mathrm{m} 2$ and $\leq 25 \mathrm{~kg} / \mathrm{m} 2$ ); overweight (BMI $>25 \mathrm{~kg} / \mathrm{m} 2$ and $\leq 30 \mathrm{~kg} / \mathrm{m} 2$ ); obese patients (BMI $>30 \mathrm{~kg} / \mathrm{m} 2$ ). Only metabolically unhealthy subjects were selected. The relationship between sex, BMI and clinical variables (National Institutes of Health Stroke Scale (NIHSS) at the admission and modified Rankin scale (mRS) scores at the end of hospitalization) was analysed with ANOVA and multivariate models.

Results: 180 metabolically unhealthy subjects affected by stroke were included. We observed a significantly increasing trend in the NIHSS along with the increase of the BMI category $(\mathrm{p}=0.030)$ among females and a significantly decreasing trend in the NIHSS along with the increase of the BMI category $(\mathrm{p}=0.040$ ) among men. We observed a significantly increasing trend in the $\mathrm{mRS}$ score with the increase in the BMI category $(p=0.001)$ among females and a non-significant decreasing trend in the mRS score at discharge along with the increase of BMI category ( $\mathrm{p}=0.290$ for trend).

Conclusion: Our study shows an opposite trend between the two sexes on severity of stroke at onset and at discharge in a population of metabolically unhealthy subjects. BMI could be considered a useful assessment measure to stratify patients according to the severity and risk of an unfavourable outcome of ischemic non-lacunar stroke. Our data show worse outcomes among women and suggest the need of a gendertailored therapy on stroke.

References:

- Falsetti L, Viticchi G, Buratti L, Balucani C, Marra AM, Silvestrini M. From head to toe: Sex and gender differences in the treatment of ischemic cerebral disease. Pharmacol Res (2017);121:240-50

- Ovbiagele B, Bath PM, Cotton D, Vinisko R, Diener HC. Obesity and recurrent vascular risk after a recent ischemic stroke. Stroke (2011);42:3397-3402

- Viticchi G, Falsetti L, Buratti L, Luzzi S, Bartolini M, Acciarri MC, et al. Metabolic syndrome and cerebrovascular impairment in Alzheimer's disease. Int J Geriatr Psychiatry (2015);30:1164-70

\section{VASCULAR EHLERS-DANLOS SYNDROME: A CHALLENGING RARE CAUSE OF STROKE WITH THERAPEUTIC IMPLICATIONS}

\author{
A. Zauli ${ }^{1}$, G. Reale ${ }^{2}$, G. Della Marca ${ }^{1}$, P. Caliandro ${ }^{2}$
}

${ }^{1}$ Institute of Neurology, Catholic University of Sacred Hearth (Roma); ${ }^{2}$ Neurology Unit, Agostino Gemelli University Hospital (Roma)

Objectives: Stroke in young adults is uncommon, but its incidence is increasing. Alongside modifiable risk factors, drug abuse, patent foramen ovale and arterial cervical dissection, many strokes are due to genetic cause.

Materials: A 32-year-old female without previous significant medical history was admitted to the Emergency Department with recent onset (6 hours) of acute dysphagia and partial paralysis of right lower face. She reported severe neck pain in the previous days. The neurological examination also showed a mild dysarthria and dysphonia, along with hypoesthesia to temperature and pain of the left side of the face and the upper left arm and a Horner's syndrome of the right eye.

Methods: A brain and neck CT-angiography was performed, showing an occlusive dissection of the right vertebral artery. Brain MRI revealed a posterior right bulbar area of hyperintensity in diffusion-weighted image (DWI) sequences, which was compatible with a recent ischemic lesion in the ipsilateral vertebral artery territory. She was then started on 
antiplatelet medication with aspirin. A thorough physical examination revealed a joint's hyperlaxity involving the knee, the elbow, the wrist and the interphalangeal joints bilaterally. The patient also had a particular facial appearance with prominent eyes and thin face and nose. These physical characteristics, combined with the vertebral artery dissection and focal aneurysms of both the right and left internal carotid arteries, rose the suspicion of Vascular Ehlers-Danlos syndrome (vEDS).

Results: A blood test was carried out for DNA analysis, which resulted positive for the c.2060G $>\mathrm{T}$ (p.Gly687Val) mutation in one allele of the COL3A1 gene confirming the diagnosis. Aspirin was then suspended after 90 days from ischemia because the risk of bleeding in vEDS patients overweighs the potential benefit of a long lasting anti-thrombotic therapy.

Discussion: Ehlers-Danlos Syndrome Type IV, or vEDS, is an autosomal dominant disorder caused by mutations of the COL3A1 gene which result in a defect in type III collagen synthesis. In $50 \%$ of cases, the mutation is de novo and patients do not report any familiar history. Vascular complications of vEDS are typically arterial ruptures, but arterial dissections are also frequently reported.

Conclusions: The peculiar appearance of our patient, together with the etiology of her stroke, led us to the hypothesis of an underlying collagenopathy and eventually to the diagnosis of this rare but lifethreatening disease. This is especially important because this condition contraindicates prolonged antithrombotic therapy which is the standard secondary prevention in post stroke patients.

References:

- Bhatt N, Malik AM, Chaturvedi S. Stroke in young adults: Five new things. Neurol Clin Pract. (2018);8(6):501-06

- Pepin M, Schwarze U, Superti-Furga A, Byers PH. Clinical and genetic features of Ehlers-Danlos syndrome type IV, the vascular type. N Engl J Med. (2000);342(10):673

- Malfait F, Francomano C, Byers P, et al. The 2017 international classification of the Ehlers-Danlos syndromes. Am J Med Genet C Semin Med Genet. (2017);175(1):8-26

\section{DEGENERATIVE DISEASES}

\section{CHARACTERIZATION OF A PARKINSON'S DISEASE SALIVARY FINGERPRINT USING RAMAN SPECTROSCOPY AND MULTIVARIATE ANALYSIS}

\author{
C. Carlomagno, M. Meloni, S. Picciolini, A. Gualerzi, P. Banfi, M. \\ Bedoni
}

Fondazione Don Gnocchi, IRCCS Santa Maria Nascente (Milano)

Objectives: The main objective of the study regards the identification and the interpretation of a salivary Raman fingerprint able to assess the onset of the Parkinson's Disease (PD), characterizing the differences between the spectra obtained from Healthy Subjects (CTRL) and patients affected by Alzheimer's Disease (AD). Raman Spectroscopy (RS) is able to provide a fast, detailed and highly sensitive spectrum of a specific biofluid, which contains all the information regarding the concentration, mutation, presence and interactions of all the contained biochemical molecules including proteins, nucleic acids, lipids, hormones and metabolites [1]. The Raman analysis provides datasets with a huge quantity of biological data that can be used to create and to train a classification model using machine learning and Multivariate Analysis (MVA), increasing in this way the discriminant ability of the methodology [2].
Materials: All the materials were used as purchased. The Raman microscope Aramis (Horiba, France) was used for the analysis of saliva deposited on commercially available aluminum. Saliva was collected using Salivette (Sarstedt, Germany).

Methods: Saliva analysis was performed following the procedure described by Carlomagno et al [3]. All the statistical and MVA analysis were performed using Origin (OriginLab, USA). 24 PD, 10 AD and 36 CTRL were recruited. Inclusion and exclusion criteria were selected following the procedures reported in [3].

Results: After the analysis of the three experimental groups (PD, AD, CTRL), a Raman fingerprint for each condition was created, highlighting differences in the spectral portions attributed to proteins, lipids and molecules related to damages of radical oxygen species. Analyzing the data with the MVA approach, we created and trained a classification model (leave-one-out cross-validation) able to discriminate the spectra with an associated error-rate of the $1.46 \%$ and with related accuracy, precision, sensitivity and specificity of $97-98 \%$.

Discussion: RS represents a fast, sensitive (10-15 M) and label-free technique, which requires almost no-sample preparation for the analysis. The development of a RS diagnostic technique could represent a powerful instrument for those pathologies with a really difficult and timeconsuming diagnostic procedure, also for the symptoms shared with other neurological diseases. Moreover, the created classification model could improve the discriminative ability of the system, providing hidden clustering information on the patient's stratification.

Conclusions: In conclusion, we identified an easy collectable pathological signature, able to discriminate the onset of different neurological disorders with related sensitivity and specificity higher than $96 \%$.

References:

1. Devitt G, Howard K, Mudher A, Mahajan S. Raman Spectroscopy: An Emerging Tool in Neurodegenerative Disease Research and Diagnosis. ACS Chem Neurosci. (2018);9(3):404-20

2. Gautam R, Vanga S, Ariese F, Umapathy S. Review of multidimensional data processing approaches for Raman and infrared spectroscopy. EPJ Tech Instrum. (2015);2(1):8

3. Carlomagno C, Banfi PI, Gualerzi A, et al. Human salivary Raman fingerprint as biomarker for the diagnosis of Amyotrophic Lateral Sclerosis. Sci Rep. (2020);10(1):10175

\section{THE ITALIAN DEMENTIA WITH LEWY BODIES STUDY GROUP (DLB-SINDEM): A MULTICENTER SURVEY ON THE ACCURACY AND THE PREVALENCE OF DLB DIAGNOSIS}

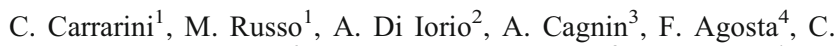
Babiloni $^{5}$, B. Borroni ${ }^{6}$, M. Bozzali $^{7}$, A. Bruni ${ }^{8}$, M. Filippi $^{4}$, D. Galimberti $^{9}$, R. Monastero ${ }^{10}$, C. Muscio ${ }^{11}$, L. Parnetti ${ }^{12}$, D. Perani ${ }^{13}$, L. Serra $^{7}$, V. Silani ${ }^{14}$, P. Tiraboschi ${ }^{11}$, M. Onofrj ${ }^{1}$, A. Padovani ${ }^{6}$, L. Bonanni ${ }^{1}$

${ }^{1}$ Department of Neuroscience, Imaging and Clinical Sciences, University G. d'Annunzio of Chieti-Pescara (Chieti); ${ }^{2}$ Department of Medicine and Sciences of Aging, University G. d'Annunzio of Chieti-Pescara (Chieti); ${ }^{3}$ Department of Neurosciences, University of Padua (Padova); ${ }^{4}$ Division of Neuroscience, Neuroimaging Research Unit, and Department of Neurology, Institute of Experimental Neurology, San Raffaele Scientific Institute (Milano); ${ }^{5}$ Department of Physiology and Pharmacology, University of Rome "La Sapienza" (Roma); ${ }^{6}$ Neurology Unit, Department of Clinical and Experimental Sciences, University of Brescia (Brescia); ${ }^{7}$ Neuroimaging Laboratory, IRCCS Santa Lucia 
Foundation (Roma); ${ }^{8}$ Regional Neurogenetic Centre, ASP CZ (Lamezia Terme-CZ); ${ }^{9}$ Neurology Unit, Department of Pathophysiology and Transplantation, Ca' Granda Foundation, IRCCS University of Milan (Milano); ${ }^{10}$ Department of Experimental Biomedicine and Clinical Neuroscience (BioNeC), University of Palermo (Palermo); ${ }^{11}$ Division of Neurology V and Neuropathology, IRCCS Foundation "Carlo Besta" Neurological Institute (Milano); ${ }^{12}$ Center for Memory Disturbances, University of Perugia (Perugia); ${ }^{13}$ Division of Neuroscience, Nuclear Medicine Unit, San Raffaele Hospital, San Raffaele Scientific Institute, Vita- Salute San Raffaele University (Milano); ${ }^{14}$ Department of Neurology-Stroke Unit and Laboratory of Neuroscience, IRCCS Auxologic Institute (Milano)

Objectives: Dementia with Lewy bodies (DLB) is the second most common neurological cause of dementia after Alzheimer's disease (AD). However, the accuracy of the clinical diagnosis tends to be not satisfactory, because clinical features of DLB may overlap with AD and other forms of dementia. The recent research stream is focusing on the identification of early biomarkers which could help to diagnose properly DLB. The aim of the present study is to re-evaluate the prevalence and the accuracy of DLB diagnosis.

Materials and Methods: In this cross-sectional observational study, two questionnaires, Lewy Body Composite Risk Score (LBCRS) and Assessment Toolkit for Dementia with Lewy Bodies (AT-DLB), were distributed to 135 Cognitive Decline and Dementia Centers (CDCD). We asked to administer the two questionnaires to all patients referred in the following three months, independently of the potential clinical diagnosis.

Results: 1876 patients from twenty-one Italian centers, which joined the survey, were recruited. $75(4 \%)$ patients were diagnosed as DLB, whereas $229(12 \%)$ received a diagnosis of AD. $178(10 \%)$ and 79 (4\%) patients suffered from Vascular Dementia (VaD) and behavioral variant of Frontotemporal Dementia (FTD), respectively. 50 (3\%) patients were considered as reversible causes of cognitive impairment (Non Deg), whereas 1265 (67\%) patients received a diagnosis of unidentified neurodegenerative condition (Deg NDD). After LBCRS and ATDLB administration, 63 DLB patients were classified as LBCRS positive, whereas 12 DLB patients as LBCRS negative. Differently, 14 DLB patients were AT-DLB negative, 7 were AT-DLB possible, and 54 were AT-DLB probable. Of 1801 patients with a different clinical diagnosis, 449 resulted LBCRS positive, 531 were AT-DLB possible, and 268 were AT-DLB probable. Even though a mild lower sensitivity (SE), criteria for AT-DLB probable classification reached better specificity (SP) and positive and negative predictive values (PPV and NPV) in comparison with LBCRS positive ones in all comparisons. SE, SP, PPV and NPV of ATDLB possible criteria resulted very unsatisfying.

Discussion: Currently DLB diagnosis, based on clinical criteria, seems to be insufficiently accurate to differentiate DLB from other forms of dementia, such as mixed dementia, which may be wrongly classified as DLB, since different clinical phenotypes may overlap and neurodegenerative diseases are not neuropathologically confirmed.

Conclusions: Therefore, great efforts have recently been placed on the necessity to identify more specific and sensitive diagnostic markers in order to recognize a prodromal DLB stage and to administer timely pharmacological and management interventions.

References:

- Bonanni L, Cagnin A, Agosta F, et al. The Italian dementia with Lewy bodies study group (DLB-SINdem): toward a standardization of clinical procedures and multicenter cohort studies design. Neurol Sci. (2017);38(1):83-91

- Thomas, A. J. Taylor, J. P. McKeith, I. , Bamford, C. , Burn, D. , Allan, L. , O'Brien, J. Development of assessment toolkits for improving the diagnosis of the Lewy body dementias: feasibility study within the DIAMOND Lewy study. Int J Geriatr Psychiatry (2017);32:1280-1304

- Galvin JE. Improving the clinical detection of Lewy body dementia with the Lewy body composite risk score. Alzheimers Dement (Amst). (2015);1(3):316-24

\section{ASYMMETRICAL COCHLEAR DYSFUNCTION AS A LATERALIZED NON-MOTOR FEATURE IN PARKINSON'S DISEASE}

\author{
R. Cerroni ${ }^{1}$, A. Viziano ${ }^{2}$, A. Moleti ${ }^{3}$, R. Sisto $^{4}$, N. Mercuri ${ }^{1}$, A. Stefani ${ }^{1}$, \\ M. Pierantozzi ${ }^{1}$
}

${ }^{1}$ Parkinson Centre, Department of System Medicine, University of Rome Tor Vergata (Roma); ${ }^{2}$ Department of Clinical and Translational Medicine, Department of Physic, University of Rome "Tor Vergata" (Roma); ${ }^{3}$ Department of Physics, University of Rome Tor Vergata (Roma); ${ }^{4}$ INAIL Research, Department of Occupational and Environmental Medicine, Epidemiology and Hygiene (Roma)

The aim of our study was to investigate the occurence of hearing loss in Parkinson's disease (PD), it's possible lateralization and its possible correlation with motor/non-motor symptoms. We enrolled PD patients without history of hearing loss, noise trauma, head injury, ear surgery or other otological/labyrinthine disorders, without others neurological/psychiatric diseases, in agreement with the study design. Exclusion criteria were all conditions that could influence hearing function. We also enrolled a healthy controls group. Pure tone audiometry (PTA) and distortion product otoacoustic emissions (DPOAEs) were used as outcome variables. Patients and controls underwent neurological and audiological examination. Staging and motor impairment were assessed through H\&Y and MDS-UPDRS III respectively. Audiological examination consisted of PTA and DPOAEs. We enrolled 86 patients and 50 controls. PD patients showed significantly lower hearing levels than controls; moreover, our data suggest a significant asymmetry in cochlear function, since DPOAE responses seem to be worse in the ear ipsilateral to the most affected motor side. Our research confirms the presence of impairment of hearing function in PD patients [1-2], but, as novelties, shows a lateralization of hearing deficit. Both peripheral and central mechanisms can be hypothesized to explain the asymmetric cochlear damage in PD; our results seem to suggest a dysfunction involving hearing regulation systems. The ear dopaminergic system, residing in lateral olivocochlear bundle, modulates auditory nerve discharges; here a dopamine depletion has been shown to decrease auditory function. However, the more severe cochlear dysfunction ipsilateral to patients' more affected motor side, may represent the expression of a greater degeneration in contralateral brainstem structures, involving other neurotransmitters, notably cholinergic neurons. The medial olivocochlear bundle, prevalently cholinergic, modulates outer hairy cells, receiving input from both cochlear nuclei in the brainstem. It could play a major role with respect to dopamine loss in the asymmetry of cochlear dysfunction in PD; so, the auditory dysfunction in PD may be ascribed to a contralateral brainstem dysfunction. these findings are consistent with studies that show an impairment of Brainstem-Auditory-EvokedPotentials contralateral to the most affected motor side [3]. Our preliminary data showing asymmetry in DPOAE responses suggest that the impairment of cochlear function could represent a lateralized non-motor feature in PD. Further investigations will be necessary, but we may find ourselves faced with the first asymmetric non-motor feature concerning bilateral sensory systems. 


\section{References:}

1. Vitale C, Marcelli V, Allocca R, et al. Hearing impairment in Parkinson's disease: expanding the nonmotor phenotype. Mov Disord. (2012);27(12):1530-35

2. Pisani V, Sisto R, Moleti A, et al. An investigation of hearing impairment in de-novo Parkinson's disease patients: A preliminary study. Park Relat Disord. (2015);21(8):987-91

3. Shalash AS, Hassan DM, Elrassas HH et al. Auditory- and Vestibular-Evoked Potentials Correlate with Motor and NonMotor Features of Parkinson's Disease. Front Neurol. (2017) Feb 27; 8:55

\section{CLEARANCE OF BETA-AMYLOID OLIGOMERS BY PERIPHERAL MONOCYTES: FURTHER OBSERVATIONS REGARDING THE MODULATORY ROLE OF DONEPEZIL}

A. Karantzoulis ${ }^{1}$, E. Conti $^{1}$, M. Slongo ${ }^{1}$, V. Rodriguez-Menendez ${ }^{2}$, M. Mauri $^{3}$, F. Da Re ${ }^{1}$, A. Aliprandi ${ }^{4}$, P. Basilico ${ }^{4}$, A. Salmaggi ${ }^{4}$, I. Appollonio $^{1}$, C. Ferrarese ${ }^{1}$, L. Tremolizzo ${ }^{1}$

${ }^{1}$ School of Medicine and Surgery, Neurology Unit, San Gerardo Hospital and NeuroMi, University of Milano-Bicocca (Monza); ${ }^{2}$ Experimental Neurology Unit, School of Medicine and Surgery, University of Milano-Bicocca (Monza); ${ }^{3}$ School of Medicine and Surgery, University of Milano-Bicocca (Monza); ${ }^{4}$ Neurology Unit, "Manzoni" Hospital (Lecco)

Object: Beta-amyloid (Abeta) aggregates are currently considered as the key culprit driving Alzheimer's disease (AD) degeneration, in part through the initiation of an inflammatory cascade. Peripheral monocytes can migrate into the CNS and participate to the clearance of Abeta oligomers. Donepezil is an anti-Alzheimer drug whose mechanism of action still remains largely elusive. Donepezil has the capacity to interact with monocyte $\alpha 7$-nicotinic acetylcholine receptors $(\alpha 7 \mathrm{nAChR})$, which, in turn, are involved in Abeta phagocytosis. We hypothesised that Donepezil binding to the $\alpha 7 \mathrm{nAChR}$ could modulate Abeta phagocytosis driven by two receptors, the $\alpha 7 \mathrm{nAChR}$ itself and TREM 2 .

Materials and Methods: The monocytic cell line U937 was differentiated with phorbol 12-myristate 13-acetate (PMA) for $72 \mathrm{~h}$ and treated for $24 \mathrm{~h}$ with $125 \mathrm{pM}$ Abeta42 oligomers or MLA ( $\alpha 7 \mathrm{nAChR}$ inhibitor) and/or pre-treated with Donepezil. Nicotine was used as a positive control. Western blot and qRT-PCR were performed. Confocal microscopy analyses with fluorescent Abeta were performed to assess phagocytosis. Moreover, $n=20$ AD patients $(n=10$ treated with Donepezil and $n=10$ without any treatment) and $n=10$ controls were recruited from our Neurology unit for assessing TREM2 mRNA levels in PBMC.

Results: In U937 cells, Abeta 125 pM was able to significantly reduce TREM2 levels, while co-administration of Donepezil significantly restored TREM2 levels; nicely, this action was blocked by the addiction of MLA. Confocal microscopy studies demonstrated that Abeta internalization was increased following Donepezil treatment and that this increase was reverted by MLA. The study in patients and controls showed a decrease of TREM2 mRNA levels in untreated AD with respect to controls. On the other hand, TREM2 levels were higher in those AD patients taking Donepezil.

Discussion: The interaction between Donepezil and the $\alpha 7 \mathrm{nAChR}$ seems to be clearly modulating Abeta phagocytosis, sustained by either, the direct involvement of the $\alpha 7 \mathrm{nAChR}$ itself, or, indirectly, by the increase of TREM2 levels.
Conclusion: Our data suggest that Donepezil may modulate the response against Abeta, possibly further strengthening the idea underlying the treatment of patients with anticholinesterase inhibitors.

\section{CSF HEMOGLOBIN CUT-OFFS TO QUANTIFY BLOOD CONTAMINATION: RELEVANCE FOR $\alpha$-SYNUCLEIN MEASUREMENT}

\author{
S. Paciotti ${ }^{1}$, G. Bellomo ${ }^{1}$, F. Paolini Paoletti ${ }^{1}$, M. Petricciuolo ${ }^{1}$, D. \\ Chiasserini $^{2}$, L. Parnetti ${ }^{1}$ \\ ${ }^{1}$ Laboratory of Clinical Neurochemistry, Department of Medicine, \\ University of Perugia (Perugia); ${ }^{2}$ Department of Experimental \\ Medicine, University of Perugia (Perugia)
}

Aims: Cerebrospinal fluid $\alpha$-synuclein (CSF $\alpha$-syn) represents a possible biomarker in Parkinson's disease (PD) diagnosis [1]. Since red blood cells (RBC) are the major source of $\alpha$-syn in blood, blood contamination can introduce a bias in cohort studies assessing the diagnostic value of CSF $\alpha$ syn. To date, CSF samples with a red blood cells (RBC) count $>50 \mathrm{RBC} /$ $\mu \mathrm{L}$ or hemoglobin $(\mathrm{Hb})$ concentration $>200 \mathrm{ng} / \mathrm{mL}$ are excluded from biomarker studies [2]. However, these cut-offs were not defined applying systematic methods, and ad hoc studies designed to determinate the real impact of blood contamination on CSF $\alpha$-syn concentration are still missing. Here, we evaluated the effect of blood contamination in $348 \mathrm{CSF}$ samples from a cohort of 42 patients with different neurological conditions, following a robust systematic procedure.

Methods: We simulated blood contamination by controlled spiking of CSF samples with fresh whole blood collected from the same patient and serially diluted to $800,400,200,100,75$, 50, 25, 5, $0 \mathrm{RBC} / \mu \mathrm{L}$. CSF $\mathrm{Hb}$ and $\alpha$-syn levels were measured using ELISA kits.

Results: The average $\mathrm{Hb}$ concentration ranged from $18 \pm 49 \mathrm{ng} / \mathrm{mL}$ (mean \pm SD) in no spiked CSF samples, to $20536 \pm 7146 \mathrm{ng} / \mathrm{mL}$ for the highest spike of $800 \mathrm{RBC} / \mu \mathrm{L}$. $\alpha$-syn concentration increased in a dose dependent manner in spiked CSF samples ranging from $1936 \pm 636 \mathrm{pg} /$ $\mathrm{mL}$ in neat $\mathrm{CSF}$, to $4817 \pm 1456 \mathrm{pg} / \mathrm{mL}(+149 \%$ variation) in samples containing $800 \mathrm{RBC} / \mu \mathrm{L}$. Using ROC analysis, we established different cut-offs for discriminating samples with $\alpha$-syn level above 5\%,10\% and $20 \%$ variation, corresponding to a $\mathrm{Hb}$ (RBC) concentration of $1569 \mathrm{ng} /$ $\mathrm{mL}(37 \mathrm{RBC} / \mu \mathrm{L}), 2082 \mathrm{ng} / \mathrm{mL}(62 \mathrm{RBC} / \mu \mathrm{L})$ and $3118 \mathrm{ng} / \mathrm{mL}(87 \mathrm{RBC} /$ $\mu \mathrm{L})$, respectively.

Discussion: Blood contamination can significantly alter the composition of the CSF proteome, potentially introducing systematic biases also in $\alpha$-syn CSF measurements. As consistent data show that in PD patients CSF total $\alpha$-syn is lower compared to controls (10-20\% $\alpha$-syn decrease in $\mathrm{PD}$ with respect to controls) [3], CSF samples which contain a Hb concentration $>1569 \mathrm{ng} / \mathrm{mL}$ ( $5 \% \alpha$-syn change), or more than $37 \mathrm{RBC} / \mu \mathrm{L}$, should be excluded for analysis.

Conclusions: Our data show the high impact of CSF blood contamination on CSF $\alpha$-syn levels, highlighting the measurement of $\mathrm{Hb}$ concentration as mandatory for assessing CSF $\alpha$-syn. The thresholds we calculated are useful to classify CSF samples for blood contamination, considering as reliable only those showing a $\mathrm{Hb}$ concentration $<1569 \mathrm{ng} / \mathrm{mL}$.

References:

1. Parnetti L, Gaetani L, Eusebi P, Paciotti S, Hansson O, El-Agnaf O, et al. CSF and blood biomarkers for Parkinson's disease. Lancet Neurol. (2019);18:573-86 
2. Mollenhauer B, Batrla R, El-Agnaf O, Galasko DR, Lashuel HA, Merchant KM, et al. A user's guide for $\alpha$-synuclein biomarker studies in biological fluids: Perianalytical considerations. Mov Disord. (2017);32:1117-30

3. Eusebi P, Giannandrea D, Biscetti L, Abraha I, Chiasserini D, Orso $\mathrm{M}$, et al. Diagnostic utility of cerebrospinal fluid $\alpha$-synuclein in Parkinson's disease: A systematic review and meta-analysis. Mov Disord. (2017);32:1389-400

\section{HOW TO PREVENT ALZHEIMER'S DISEASE THROUGH TRANSPINAL DIRECT CURRENT STIMULATION}

\author{
F. Pisano ${ }^{1}$, C. Caltagirone ${ }^{2}$, F. Satriano ${ }^{2}$, R. Perri ${ }^{2}$, L. Fadda ${ }^{2}$, P. \\ Marangolo ${ }^{1}$
}

${ }^{1}$ Department of Humanities Studies, University Federico II (Napoli); ${ }^{2}$ IRCSS Santa Lucia Foundation (Roma)

Objective: Several studies have already shown that transcranial direct current stimulation (tDCS) is efficacious in improving cognitive abilities in $\mathrm{AD}$ patients, in particular, their memory performance [1]. More recently, a growing body of literature has pointed out that, from the early stage of the disease, one of the most impaired ability in $\mathrm{AD}$ patients is executive functions processing, such as a deficit in verbal reasoning, problem solving and in the ability to cope with novelty [2]. These deficits strongly interfere with daily life activities by worsening the quality of life of $\mathrm{AD}$ individuals. Given that sensorimotor processes act as a basis for cognitive activities related to executive functions [3], in the present study, we wanted to investigate if enhancing activity into the spinal sensorimotor pathways through transpinal direct current stimulation (tsDCS), would improve executive functions processing in a group of AD patients.

Methods: In a randomized-double blind design, sixteen $\mathrm{AD}$ patients (Mild (N=8) to Moderate Dementia $(\mathrm{N}=8)$ ) underwent different cognitive trainings combined with tsDCS. During the treatment, each subject received tsDCS (20 min, $2 \mathrm{~mA}$ ) over the thoracic vertebrae (IX-X vertebrae) in two different conditions: (1) anodal, and (2) sham while performing three computerized tasks: alertness, selective attention and executive functions. Each experimental condition was run in ten consecutive daily sessions over two weeks (Monday to Friday, weekend off, Monday to Friday). In all patients, neuropsychological assessment was collected before (T0), at the end (T10) and one month after the end of each treatment condition (anodal vs. sham).

Results: After anodal tsDCS, a greater improvement in all tasks related to executive functions compared to sham was found. More importantly, the follow-up testing revealed that these effects lasted over one month after the intervention and generalized to the different neuropsychological tests, including the attentional domain.

Conclusion: These findings suggest, for the first time, that tsDCS combined with cognitive treatments is efficacious for $A D$ patients. We hypothesize that anodal tsDCS, through the spinal sensorimotor pathways, has exerted functional neural changes into the cortex and, in particular, into the prefrontal region, leading to an improvement of executive functions. Since the spinal cord is directly connected, through its ascendant tracts, with different cortical regions, we believe that spinal stimulation might result more efficacious than tDCS since it might enhance activity into a network of cortical regions promoting compensatory strategies also in the case of neurodegenerative diseases.
References:

1. Chang CH, Lane HY, Lin CH, Brain Stimulation in Alzheimer's Disease, Front Psychiatry, (2018);9:201

2. Guarino A, Favieri F, Boncompagni I, Agostini F, Cantone M, Casagrande M, Executive Functions in Alzheimer Disease: A Systematic Review, Front Aging Neurosci. (2019);10: 437

3. Gottwald JM, Achermann S, Marciszko C, Lindskog M, Gredebäck G. An Embodied Account of Early Executive-Function Development. Psychol Sci. (2016);27:1600-10

\section{INCIPIENT CHRONIC TRAUMATIC ENCEPHALOPATHY IN ACTIVE AMERICAN FOOTBALL PLAYERS: NEUROPSYCHOLOGICAL ASSESSMENT AND BRAIN PERFUSION MEASURES}

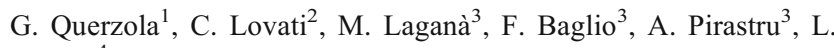 \\ Pantoni $^{4}$
}

${ }^{1}$ Luigi Sacco Hospital, Neurology Unit, University of Milan (Milano); ${ }^{2}$ Luigi Sacco Hospital, ASST FBF Sacco (Milano); ${ }^{3}$ Don Gnocchi, University of Milan (Milano); ${ }^{4}$ 'Luigi Sacco' Department of Biomedical and Clinical Sciences, University of Milan (Milano)

Background and aims: Chronic traumatic encephalopathy (CTE) is a degenerative disease caused by repetitive head impacts and traumatic brain injury (TBI), such as those occurring in American football. CTE is defined by the deposition of paired helical filament tau in the brain. Because CTE can be definitely diagnosed only post-mortem, it would be important to have possible clinical and radiological correlates of CTE and TBI. The aims of this study were 1) to investigate the possible relationship between the neuropsychological profile of active American football players and the traumatic load;2) to study if traumatic brain injury associated with American Football activity is associated with a specific cerebral perfusion pattern; 3) and if this perfusion pattern correlates with neuropsychological performances.

Methods: In 20 American football players [mean age $27.3 \pm 8.5$, all males], we evaluated sport and medical history, the traumatic load and possible reported symptoms using the TraQ (Trauma Questionnaire), cognitive performances with seven neuropsychological tests (NPST), and brain perfusion using arterial spin labeling (ASL) MRI.

Results: Despite all cognitive performances were within normal ranges, we found different cognitive performances between American football players stratified according to their field position and career length. Offensive and defensive linemen players were found to have poorer cognitive performances in executive functions, verbal competence, and visual abilities. Similarly, a career $>7$ years was associated with poorer performances. American football players had a statistically significant hypoperfusion on cerebral blood flow (CBF) maps in sensorymotor areas, specifically pre and postcentral gyri, in comparison with healthy controls. Poorer neuropsychological performances correlated with lower perfusion in specific brain areas.

Conclusions: We clinically confirmed that CTE in American football players is driven by the field position. The decrease in cognitive performances is correlated with cerebral hypoperfusion in specific areas.

References:

- Alosco M. L., Stein T. D., Tripodis Y., et al. Association of White Matter Rarefaction, Arteriolosclerosis, and Tau with Dementia in Chronic Traumatic Encephalopathy. JAMA Neurology (2019) August 5; doi: 10.1001/jamaneurol.2019.2244 
- Montenigro P. H., Baugh C. M., Daneshvar D. H., Mez J., Budson A. E., Au R., et al. Clinical subtypes of chronic traumatic encephalopathy: literature review and proposed research diagnostic criteria for traumatic encephalopathy syndrome - Review: discusses historical examples of CTE, proposes research diagnostic criteria for CTE. Alzheimer's Res Ther. (2014) Sep 24;6(5):68

- Querzola G. et al. A semi-quantitative sport-specific assessment of recurrent traumatic brain injury: the TraQ questionnaire and its application in American Football. Neurol Sci (2019); 40:1909-1915 DOI 10.1007/s10072-019-03853-Z

\section{NEUROTRANSMITTER IMBALANCE WITHIN THE VENTROMEDIAL PREFRONTAL CORTEX IS LINKED TO SOMATIC SYMPTOM DISORDER IN PARKINSON'S DISEASE AT THE ONSET OF MOTOR SYMPTOMS}

M. Russo ${ }^{1}$, C. Carrarini ${ }^{1}$, S. Delli Pizzi ${ }^{1}$, R. Franciotti ${ }^{1}$, A. Ferretti ${ }^{1}$, R.

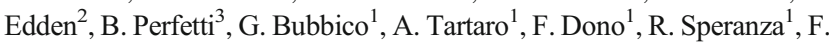
Calvarese $^{4}$, A. Thomas ${ }^{1}$, M. Onofrj ${ }^{1}$, L. Bonanni ${ }^{1}$

${ }^{1}$ Department of Neuroscience, Imaging and Clinical Sciences, University G. d'Annunzio of Chieti-Pescara (Chieti); ${ }^{2}$ Russell H. Morgan Department of Radiology, The Johns Hopkins University School of Medicine (Baltimore-USA); ${ }^{3}$ Parkinson's Disease and Movement Disorder Unit, IRCCS San Camillo Hospital Foundation (Venezia Lido); ${ }^{4}$ Department of Psychological Sciences, Health, and the Territory, University "G. d'Annunzio" of Chieti-Pescara (Chieti)

Objectives: Parkinson's Disease (PD) is associated with a broad spectrum of non-motor symptoms. Among these, somatic symptom disorder can antedate the onset of motor signs and it is associated with the subsequent development of dementia in PD. Despite the neural substrates underlining somatic symptom disorder remain unclear, growing evidences hint ventromedial prefrontal cortex (vmPFC) involvement. Interestingly, recent studies suggested that the vmPFC neurotransmission plays a central role for limbic system activity, emotion regulation and autonomic response modulation.

Materials and Methods: We enrolled fourteen PD patients with somatic symptom disorder (s-PD), fourteen PD patients without somatic symptom disorder (ns-PD) and fifteen age-matched healthy subjects. S-PD and ns-PD were at the onset of motor symptoms. Before the inclusion in the study, all patients underwent computerized tomography/magnetic resonance imaging (MRI) and DAT scan. At enrollment, all patients performed Unified Parkinson's Disease Rating Scale III (UPDRS-III), Hoehn and Yahr scale and a neuropsychological assessment. We investigated by proton magnetic resonance spectroscopy (1H-MRS), the Î3aminobutyric acid (GABA) and Glx (glutamate+glutamine) contents within vmPFC in the three groups of patients. Total creatine $(\mathrm{tCr})$ was used as internal reference. Age, gender and grey matter within 1H-MRS voxel were included as covariate.

Results: The vmPFC GABA/tCr content in the s-PD patients was significantly higher as compared with ns-PD patients and controls. No difference was found in $\mathrm{Glx} / \mathrm{tCr}$ amount among groups.

Discussion: We posit a central role of GABA-ergic neurotransmission within $\mathrm{VmPFC}$ in patients at the early stage of PD presenting with somatic symptoms. We hypothesize that the GABA-ergic imbalance within vmPFC could trigger functional alteration in the amygdala and insula, leading to loss of emotional control and exaggerated expectancy of somatosensory perception.
Conclusions: Hence, a close link between vmPFC GABA-ergic imbalance and the presence somatic symptom disorder in PD could open new insights in the symptom pathophysiology and in the development of new pharmacological targets.

References:

- Franciotti R, Delli Pizzi S, Russo M, et al. Somatic symptoms disorders in Parkinson's disease are related to default mode and salience network dysfunction. Neuroimage Clin. (2019);23:101932

- Delli Pizzi S, Padulo C, Brancucci A, Bubbico G, Edden RA, Ferretti A, Franciotti R, Manippa V, Marzoli D, Onofrj M, Sepede G, Tartaro A, Tommasi L, Puglisi-Allegra S, Bonanni L. GABA content within the ventromedial prefrontal cortex is related to trait anxiety. Soc Cogn Affect Neurosci. (2016) May;11(5):758-66

- Stone J, Zeman A, Simonotto E, Meyer M, Azuma R, Flett S, Sharpe M. FMRI in patients with motor conversion symptoms and controls with simulated weakness. Psychosom Med. (2007) Dec;69(9):961-9

\section{PARKINSON'S DISEASE FLUCTUATIONS TREATMENT PATHWAY (PD-FPA): AN INTERIM ANALYSIS OF A MULTICENTER ITALIAN RETROSPECTIVE OBSERVATIONAL STUDY}

F. Stocchi ${ }^{1}$, A. Tessitore ${ }^{2}$, M. Zappia ${ }^{3}$, U. Bonuccelli ${ }^{4}$, P. Barone ${ }^{5}$, A. Padovani $^{6}$, N. Modugno ${ }^{7}$, L. Lopiano ${ }^{8}$, F. Radicati $^{1}$

${ }^{1}$ Center for Parkinson Disease, University and IRCCS San Raffaele Pisana (Roma); ${ }^{2}$ Department of Advanced Medical and Surgery Sciences, University of Campania "Luigi Vanvitelli" (Napoli); ${ }^{3}$ Department G.F. Ingrassia, University of Catania (Catania); ${ }^{4}$ Department of Clinical and Experimental Medicine, Neurology Unit, University of Pisa (Pisa); ${ }^{5}$ Center for Neurodegenerative Diseases (CEMAND), Department of Medicine, Neuroscience Section, University of Salerno (Salerno); ${ }^{6}$ Center for Neuromuscular Diseases, Unit of Neurology, ASST Spedali Civili and University of Brescia (Brescia); ${ }^{7}$ Neurology Unit, IRCCS Neuromed (Pozzilli-IS); ${ }^{8}$ Department of Neuroscience, University of Torino, Azienda Ospedaliero-Universitaria Città della Salute e della Scienza di Torino (Torino)

Introduction: The therapeutic pathway of Parkinson's disease (PD) is rather complex. $80-100 \%$ of patients develop fluctuations after 10 years of levodopa. Real-life data monitoring fluctuations are limited in literature, especially related to Italy.

Objective: To describe patient and disease characteristics, fluctuations, motor and non-motor symptoms of an Italian PD population with 10-15 years PD diagnosis.

Materials and Methods: This retrospective observational multicentre cohort study was conducted in 8 Italian centers. In each center, the first 50 consecutive patients with 10-15 years PD diagnosis and at least 2-years history of fluctuations were enrolled. The retrospective data collected refers to two different timepoints before enrollment visit ( $\mathrm{Vi}$ - index data), approximately 2 (T2) and 1 (T1) year earlier. During Vi the status of the disease by clinician's judgment, the "wearing off" status by the WOQ-19 (Wearing-Off Questionnaire 19-items) and motor and non-motor symptoms by MDS-UPDRS (MDS-Unified Parkinson's Disease Rating Scale) and PDQ-39 (Parkinson's Disease Questionnaire 39-items) were assessed. Information on fluctuations was collected at Vi, T1 and T2.

Results: The analysis included 181 patients (male: 61\%). The mean age at enrollment was $68.4 \pm 9.4$ years and at diagnosis was $56.4 \pm 9.2$ 
years. The mean age at first fluctuation was $63.4 \pm 10.5$ years. The questionnaires were completed for 102 patients $(56.4 \%) .84 \%$ of patients was in stage 2 or 3 of the Hoehn and Yahr scale. WOQ-19 identified 74 patients $(72.5 \%)$ that had WO-related symptoms. $56.6 \%$ of the symptoms improved or disappeared after taking levodopa. No significant difference was found between $\mathrm{Vi}, \mathrm{T} 1$ and $\mathrm{T} 2$ regarding characteristic of fluctuations, in fact around $70 \%$ of patients had "wearing off". Mild or moderate "depressed mood" was found in $30 \%$ of patients. $34 \%$ of patients had mild or moderate anxious feelings, while $21 \%$ stated that apathy interfered mildly or moderately with social activities and interactions. $41 \%$ of patients reported mild or moderate "sleep problems". Mild or moderate constipation problems was found in $46 \%$ of patients. $48 \%$ of patients showed mild or moderate fatigue. Nearly half of patients reported mild or moderate problems in speaking $(40 \%)$, dressing $(41 \%)$, walking and balance $(47 \%)$ or freezing (44\%). Around $33 \%$ of patients spent between $26 \%$ and $75 \%$ of waking day with dyskinesias and $40 \%$ of patients spent the same time in OFF state. The PDQ-39 mean score was 33.3 \pm 14.0 .

Conclusions: Fluctuations, non-motor and motor manifestations have to be carefully addressed throughout the course of the PD because of their impact on patients' daily live.

\section{MOTOR NEURON DISEASES}

\section{UNDERSTANDING PHONEMIC FLUENCY IN MOTOR NEURON DISEASE: INSIGHTS FROM DISTRIBUTIONAL SEMANTIC MODELS}

\author{
E. N. Aiello ${ }^{1}$, C. Barattieri Di San Pietro ${ }^{2}$, G. Mora ${ }^{3}$, D. Pain ${ }^{3}$, M. \\ Marelli $^{2}$
}

${ }^{1}$ School of Medicine and Surgery, University of Milano-Bicocca (Milano); ${ }^{2}$ Department of Psychology, University of Milano-Bicocca (Milano); ${ }^{3}$ IRCCS Milano, Istituti Clinici Scientifici Maugeri (Milano)

Aims: In motor neuron disease (MND) patients, deficits in semanticbased strategies during phonemic verbal fluency (PVF) tasks have been reported via qualitative scoring methods [1]. However, these findings have not been replicated via quantitative approaches. Distributional semantic (DS) models of language [2] can provide insights into both linguistic (lexical-semantic integrity) and extra-linguistic components of PVF via quantitative and replicable measures of semantic similarity between words. The present study aimed at investigating cognitive underpinnings of PVF tasks in MND patients via DS models.

Materials: PFV outputs from 23 MND patients (20 with amyotrophic lateral sclerosis and 3 with primary lateral sclerosis) and 23 age-, sex- and education-matched healthy controls (HC) were collected. F-A-S version of the PVF task was administered. If either severely dysarthric or anarthric, patients performed the task in written form; in this case, execution times were doubled.

Methods: Beside the standard outcome (total number of words), DS models were adopted to compute: the size of cluster (SC) of semanticallyrelated words; the number of switches from one cluster to another; overall semantic coherence of the verbal output. Between-group comparisons were performed via both linear and generalized linear models, controlling for the number of words.

Results: Patients' mean age and education in years was $60.7 \pm 14.6$ and $12.7 \pm 3.4$, respectively; 13 were male. Mean disease duration in months was $30.7 \pm 25.3$, whereas mean Amyotrophic Lateral Sclerosis Functional Rating Scale - Revised score was $31 \pm 8.6 ; 8$ patients showed bulbar signs at recruitment. No between-group differences were found with regard to the number of words. At a descriptive level, patients systematically tended to make more switches. When compared to HCs, patients produced significantly smaller clusters, and their PVF output was significantly less coherent. A significant correlation was found between the SC and coherence, especially for patients.

Discussion: When performing PVF tasks, MND patients may tend to follow a semantic-based strategy to a lesser extent than HCs - this suggesting subtle lexical-semantic deficits in patients. On the contrary, HCs might retrieve letter-cued words within a semantic cluster until it is exhausted before switching to another one. Semantic coherence of PFV outputs might represent a previously-uninvestigated [1] lexical-semantic outcome that is able to discriminate MND patients from HCs.

Conclusions: DS model-based comparisons between MND patients' and HCs' PVF outputs are able to reveal fine-grained differences that might not be captured if the number of words produced is regarded as the sole outcome. References:

1. Lepow L., Van Sweringen J., Strutt A.M., Jawaid A., MacAdam C., Harati Y., Schulz P.E., York M.K. Frontal and temporal lobe involvement on verbal fluency measures in amyotrophic lateral sclerosis. Journal of Clinical and Experimental Neuropsychology (2010); 32:913-922

2. Mikolov T., Sutskever I., Chen K., Corrado G. S., Dean J. Distributed representations of words and phrases and their compositionality. Proceedings of the 26th International Conference on Neural Information Processing Systems (2013);2:3111-19. Curran Associates Inc.: Red Hook, New York, USA

\section{VERB NAMING TESTS FOR DETECTING COGNITIVE CHANGES IN AMYOTROPHIC LATERAL SCLEROSIS}

\author{
E. N. Aiello ${ }^{1}$, C. Luzzatti ${ }^{2}$, D. Pain ${ }^{3}$, M. Gallucci ${ }^{2}$, K. Marinou ${ }^{3}$, R. \\ Sideri $^{3}$, G. Mora $^{3}$
}

${ }^{1}$ School of Medicine and Surgery, University of Milano-Bicocca (Milano); ${ }^{2}$ Department of Psychology, University of Milano-Bicocca (Milano); ${ }^{3}$ IRCCS Milano, Istituti Clinici Scientifici Maugeri (Milano)

Aims: Deficits of verb processing have been frequently reported in amyotrophic lateral sclerosis (ALS) [1]. Neurofunctional evidence suggests that motor areas are involved in verb processing [2], thus raising the issue whether verb deficit can reflect cognitive changes due to cortical reorganization in ALS patients. Since current guidelines for cognitive assessment in ALS suggest that verb processing tests should be included [1], the present study aimed at investigating their relevance to clinical neuropsychological practice in this population, as well as their relation with clinical features.

Materials: Twenty-seven consecutive ALS patients were compared to 26 healthy controls (HC) matched for age and education on a verbnaming (VN) test [3] by controlling for executive functioning measures (phonemic and alternate verbal fluency). Patients underwent cognitive screening via the Edinburgh Cognitive and Behavioral ALS Screen (ECAS). Functional outcome (ALS Functional Rating Scale - Revised; ALSFRS-R), disease duration, site of onset and presence of bulbar signs were taken into account.

Methods: Clinical judgments on patients' cognitive performance were provided via the Equivalent Scores (ES) method. An executive functioning index (EFI) was computed from fluency tasks. Between-group comparisons were performed via generalized linear models. Associations of interest were assessed via either Pearson's or Spearman's coefficient. Missing values were excluded pairwise. 
Results: Patients performed worse than HCs on VN, even when controlling for the EFI. Seven patients $(25.9 \%)$ were borderline/defective $(E S=1 / 0)$ on VN. Fluency tasks did not discriminate patients from HCs. Among the patients with no missing scores $(\mathrm{N}=17), 4$ were defective/ borderline on $\mathrm{VN}$, one was borderline on ECAS-Fluency sub-test, whereas none on fluency tasks, ECAS-total and other ALS-specific scores. VN performance was related to ECAS total and ALS-specific scores, as well as to fluency tasks. VN performance was inversely related to ALSFRS-R total scores and not to other clinical characteristics.

Discussion: ALS patients can show deficits on VN that are not fully accounted for by executive dysfunction. $\mathrm{VN}$ tests might be able to discriminate between HCs and ALS patients that are normal at cognitive screening and fluency tasks. VN tests might be predictive towards both global cognition and language/executive functioning of ALS patients.

Conclusions: VN might represent a sensitive test for detecting cognitive changes in ALS patients.

References:

1. Strong M. J., Abhrahams S., Goldstein L. H., Woolley S., McLaughlin P., Snowden J., Mioshi E., Roberts-South A., Benatar M., Hortobagyi T., Rosenfeld J., Silani V., Ince P. G., Turner M. R. Amyotrophic lateral sclerosis-frontotemporal spectrum disorder (ALS-FTSD): revised diagnostic criteria. Amyotrophic Lateral Sclerosis and Frontotemporal Degeneration (2017);18:153-4

2. De Zubicaray G., Arciuli J., McMahon K. Putting an "end" to the motor cortex representations of action words. Journal of Cognitive Neuroscience (2013);25:1957-74

3. Crepaldi D., Aggujaro S., Arduino L.S., Zonca G., Ghirardi G., Inzaghi M.G., Colombo M., Chierchia G., Luzzatti C. Noun-verb dissociation in aphasia: The role of imageability and functional locus of the lesion. Neuropsychologia (2006);44:73-89

\section{STAPEDIAL REFLEX: A NOVEL BIOMARKER OF EARLY BULBAR INVOLVEMENT IN AMYOTROPHIC LATERAL SCLEROSIS PATIENTS}

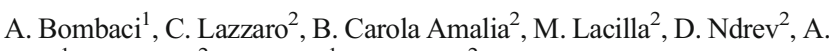

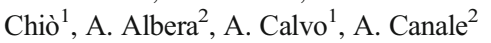 \\ ${ }^{1}$ "Rita Levi Montalcini" Department of Neuroscience, University of Turin \\ (Torino); ${ }^{2}$ ENT Unit, Department of Surgical Sciences, University of \\ Turin (Torino)
}

Background and aim: Amyotrophic lateral sclerosis (ALS) is a neuromuscular progressive disorder, characterised by limb and bulbar muscle wasting and weakness. $30 \%$ of patients present a bulbar onset, while $70 \%$ a spinal outbreak, although most of them develop bulbar impairment later on. Due to the lack of an early biomarker of bulbar involvement, we chose to evaluate the role of stapedial reflex (SR) in order to predict preclinical bulbar impairment in ALS.

Materials and methods: We enrolled 36 ALS patients. We assessed revised-ALS functional-rating-scale and SR for a total of 4 visits. We established the presence of SR, Acoustic Reflex Latency Test (ARLT) and SR's Decay. Patients who had not developed bulbar signs at 4th visit continued follow-up up to fifteen months. Data were analysed by using Mann-Whitney $\mathrm{U}$ test, Friedman test, and Cox regression analysis.

Results: We observed that SR's Decay at 500 and $1000 \mathrm{~Hz}$ is the first parameter of SR to get altered in all ALS patients before the development of bulbar impairment. 28 patients developed bulbar impairment during the study. We highlighted a correlation between the progression rate of disease and both time of SR's Decay alteration and time of bulbar impairment from disease onset. 4 patients who did not develop bulbar impairment had a progression rate lower than the other ones $(\mathrm{p}<0.05)$.

Discussion and conclusions: This study shows that stapedial reflex Decay test could be a sensitive measure for detecting pre-symptomatic bulbar involvement in ALS and could represent a simple, non-invasive and useful biomarker of disease progression.

References:

- Chiò, Adriano, and Bryan J. Traynor. "Biomarkers for ALS-in Search of the Promised Land." Nature Reviews Neurology (2015);11(2):72-4

- Canale, Andrea et al. "Acoustic Reflex Patterns in Amyotrophic Lateral Sclerosis." European Archives of Oto-Rhino-Laryngology (2017);274(2):679-83

- Yamane, M., and Y. Nomura. "Analysis of Stapedial Reflex in Neuromuscular Diseases.” ORL (1984);46(2):84-96

\section{THE BABIŃSKI SIGN: FROM “TOES PHENOMENON” TO "GREAT TOE PHENOMENON"}

F. Brigo

Department of Neurology, Hospital of Merano (SABES-ASDAA) (Merano-BZ)

Objectives: The Babinski sign (BS) is one of the most important signs in clinical neurology. It refers to the extension (dorsiflexion) of the great toe following stimulation of the foot sole. I traced back the accounts of this sign made by Joseph Francois Félix Babiński (1857-1932) and other influential neurologists of the 19th Century to evaluate whether and how its description changed over time.

Materials and methods: Analysis of articles by Babiński and other neurologists of the 19th Century providing descriptions of the BS.

Results: In his first description (1869), Babiński did not report the part of the sole of the foot that was stimulated, and did not mention the movement of the great toe, but of all toes [1]. In 1898, he recognized that the extension is predominating in the great toe or the first two toes. In 1903, André Léri (1875-1930) - who had studied under Babinski - still wrote about toes extension (in the plural), although he mentioned the existence of a reflex of the great toe in abduction. The same year, Gheorghe Marinesco (1863-1938) wrote that «the toes phenomenon [phénomène des orteils] consists, according to Babinski, in a movement of extension which is more evident at the level of the great toe, whereas the other toes extend a little, remain immobile, or even flex». In 1903 Babiński completed his initial description, by drawing attention to the abduction of the toes ("the fan sign", "signe de l'éventail") [2]. In 1917, Babiński provided a comprehensive description of the sign: «the reflex movement of toes following stimulation of the foot sole is usually inverted: the toes and particularly the great toe instead of flexing extend on the metatarsus (phenomenon of the toes). The extension of the great toe is often associated with the abduction of the other toes (the fan sign)» [3].

Discussion: Although initially Babiński described the sign emphasizing the extension of all toes ("phénomène des orteils", "toes phenomenon"), later on - probably recognizing that extensions of all toes not always occur - he narrowed the definition by drawing attention to the extension of the big toe, and the abduction of the 
other toes. The BS was not described in its complete form from the very beginning, but it took some time for Babiński to fully realize that what characterized the sign was the extension of the great toe alone, and not of all toes.

References:

1. Babinski J. Sur le réflexe cutané plantaire dans certaines affections organiques du systeme nerveux central. C R Soc Biol (1896);48:207-08

2. Babinski J. De l'abduction des orteils. Rev Neurol (1903);11:72829

3. Babinski J, Froment J. Hystérie-pithiatisme et troubles nerveux d'ordre réflexe en neurologie de guerre. Paris: Masson et Cie, (1917)

\section{THE INTERPLAY AMONG EDUCATION, BRAIN METABOLISM, AND COGNITIVE IMPAIRMENT SUGGESTS A ROLE OF COGNITIVE RESERVE IN AMYOTROPHIC LATERAL SCLEROSIS}

A. Canosa ${ }^{1}$, F. Palumbo ${ }^{1}$, B. Iazzolino ${ }^{1}$, L. Peotta ${ }^{1}$, F. Di Pede ${ }^{1}$, U. Manera $^{1}$, R. Vasta ${ }^{1}$, M. Grassano ${ }^{1}$, L. Solero ${ }^{1}$, V. Arena ${ }^{2}$, C. Moglia ${ }^{1}$, M. Pagani ${ }^{3}$, A. Calvo ${ }^{1}$, A. Chiò ${ }^{1}$

${ }^{1}$ ALS Centre, "Rita Levi Montalcini" Department of Neuroscience, University of Turin (Torino); ${ }^{2}$ Positron Emission Tomography Centre, AFFIDEA-IRMET S.p.A. (Torino); ${ }^{3}$ Institute of Cognitive Sciences and Technologies, C.N.R. (Roma)

Aim: To test the Cognitive Reserve (CR) hypothesis in ALS.

Methods: We enrolled 111 ALS patients. We used years of education as CR proxy, 18F-FDG-PET to assess brain lesion load, and the ECAS to measure cognitive functioning. Education was regressed out against whole brain metabolism, including age, sex, site of onset, ALSFRS-R, and ECAS score as covariates. Metabolic clusters showing a significant correlation were used as seed regions in an interregional correlation analysis (IRCA) to identify regions whose metabolism was correlated with that of the seed clusters.

Results: We found a negative correlation between brain metabolism and education in right anterior cingulate and bilateral medial frontal gyrus. In the IRCA the metabolism of the medial frontal cluster positively correlated with that of frontotemporal regions (right>left), bilateral caudate nuclei, and right insula, and negatively correlated with that of corticospinal tracts, cerebellum and pons.

Discussion: Our results are in agreement with the CR hypothesis, since higher education is associated with lower metabolism in frontal regions, independently from the level of cognitive decline.

\section{AMYOTROPHIC LATERAL SCLEROSIS AND OXIDATIVE STRESS: A DOUBLE-BLIND, PLACEBO-CONTROLLED, RANDOMIZED, CLINICAL TRIAL AFTER CURCUMIN SUPPLEMENTATION}

L. Chico ${ }^{1}$, E. Schirinzi ${ }^{1}$, A. Govoni ${ }^{1}$, C. Simoncini ${ }^{1}$, F. Bianchi ${ }^{1}, \mathrm{~S}$. Piazza $^{2}$, D. Orsucci ${ }^{3}$, A. Petrucci ${ }^{4}$, A. Pellegrinetti ${ }^{5}$, M. Maluccio ${ }^{6}$, M. Polzella $^{7}$, G. Siciliano ${ }^{1}$
${ }^{1}$ Department of Clinical and Experimental Medicine, Neurological Clinic, University of Pisa (Pisa); ${ }^{2}$ Unit of Neurology, Apuane Hospital (Massa); ${ }^{3}$ Unit of Neurology, San Luca Hospital (Lucca); ${ }^{4}$ Center of Neuromuscular and Neurological Rare Diseases, S. Camillo Forlanini Hospital (Roma); ${ }^{5}$ Unit of Neurology, Ospedali Riuniti (Livorno); ${ }^{6}$ Unit of Neurology, Felice Lotti Hospital (Pontedera-PI); ${ }^{7}$ Laboratories Aliveda (Crespina Lorenzana-PI)

Objective: To evaluate the efficacy of curcumin oral supplementation (1500 mg/day, Alibrain Advanced) in patients with amyotrophic lateral sclerosis (ALS).

Methods: Patients were randomized to receive, for 6 months, a treatment with curcumin (Curcumin Group, $\mathrm{CG}, \mathrm{n}=9$ ) or placebo (Placebo Group, PG, n=8). Both clinical/anthropometric parameters, such as ALSfunctional rating scale (ALS-FRS), medical research council (MRC), weight, body mass index (BMI) and oxidative stress biomarkers, including oxidative protein products (AOPPs), ferric reducing ability (FRAP), total thiols (t-SH), were evaluated before (T0), after 3 months (T1) and 6 months (T2) of curcumin or placebo treatment. Comparison considering inter-group $(\mathrm{CG}>\mathrm{PG})$ single evaluation times, and intra-group ( $\mathrm{CG}$ or $\mathrm{PG})$, between times $(\mathrm{T} 2>\mathrm{T} 1>\mathrm{T} 0)$, evaluations were carried out.

Results: Over the entire study no change was observed in the clinical and anthropometric parameters after curcumin administration. The intragroup analysis showed a significant increase of AOPP levels in PG at $\mathrm{T} 1>\mathrm{T} 0(\mathrm{p}=0.01)$ that was not observed in CG in which AOPP levels remained unchanged over time $(\mathrm{T} 2>\mathrm{T} 1>\mathrm{T} 0, \mathrm{p}=\mathrm{ns})$. In $\mathrm{CG}$, with respect to PG, FRAP increased after curcumin administration both at $\mathrm{T} 1>\mathrm{T} 0$ (p $<0.001)$ and at $\mathrm{T} 2>\mathrm{T} 1(\mathrm{p}<0.01)$, as well as t-SH levels were significantly increased at $\mathrm{T} 1>\mathrm{T} 0(\mathrm{p}=0.02)$, with a positive trend also after 6 months of curcumin administration ( $\mathrm{T} 2>\mathrm{T} 0, \mathrm{p}=0.06)$. The inter-group analysis (CG compared to PG) revealed lower AOPP levels, at T1 ( $\mathrm{p}=0.02)$ and T2 (not significant, $\mathrm{p}=0.07)$, as well as significant higher FRAP and t-SH levels both at $\mathrm{T} 1$ and $\mathrm{T} 2(0.01<\mathrm{p}<0.001)$.

\section{PATTERNS OF OCULOMOTOR DYSFUNCTIONS IN PATIENTS WITH AMYOTROPHIC LATERAL SCLEROSIS}

\author{
C. Chisari, S. Lo Fermo, E. D'Amico, S. Toscano, S. Arena, F. Patti, M. \\ Zappia
}

GF Ingrassia, Section of Neurosciences, University of Catania (Catania)

Objective: Although the ocular motor system was traditionally believed to be spared in ALS, several abnormalities in ocular motility have been described. However, patterns of oculomotor dysfunction in ALS have not been fully established. Our aim was to identify eye movements abnormalities in patients with early-diagnosed ALS.

Materials: This retrospective study screened all ALS patients referring to our Neurology Clinic of the University of Catania in the period between 15th December 2018 and 30th April 2020. Patients were categorized according to onset pattern into bulbar onset or spinal onset. Twenty sex- and age-matched controls were also enrolled.

Methods: Eye movements were recorded using an infrared-emitting video-based eye tracker (EyeLink 1000 Plus $®$ ). Saccades were evaluated using a visual target jumping at $0.25 \mathrm{~Hz}$ with amplitude ranging 20 degrees/ang horizontally and 10 degrees/ang vertically. Mean peakvelocity (pvel), mean latency, percentage of hypometric and dysmetric saccades for all directions were calculated. Smooth pursuit task consisted of a dot moving at $0.2 \mathrm{~Hz}$ both horizontally and vertically. Gain and catch-up saccades (CUS) rate were calculated. For fixation task, 
consisting of one spot sited in the centre for 100 seconds, we evaluated square wave intrusion (SWI) rate and the mean SWI amplitude (AmpSWI).

Results: Out of 31 patients screened, 29 (mean age $64.3 \pm 12.9$ years; $16[55.3 \%]$ males) fulfilled the Awaji criteria for ALS and were enrolled. Twenty (69\%) patients had spinal onset and $9(31 \%)$ had a bulbar onset. The mean disease duration was $13.6 \pm 9.9$ months. ALS patients showed a significant increase in saccadic latency for each direction compared to controls ( $321.6 \pm 24.8$ vs $271.1 \pm 11.8 \mathrm{msec}, \mathrm{p}<0.01)$, while no differences in terms of pvel were observed. ALS patients also exhibit a higher percentage of hypometric $(43.4 \pm 9.6$ vs $26.1 \pm 5.9, \mathrm{p}<0.05)$ and dysmetric saccades $(34.1 \pm 11.2$ vs $8.5 \pm 4.6, \mathrm{p}<0.001)$ compared to controls. Smooth pursuit recording showed a lower gain $(0.68 \pm 0.16$ vs 0.91 $\pm 0.09, \mathrm{p}<0.01)$ in ALS than controls. Bulbar-onset patients showed a lower pvel, a higher percentage of dysmetric saccades and a higher Amp-SWI compared to spinal-onset.

Discussion: Our results confirmed that eye motility could be affected in ALS. In particular, ALS patients showed longer saccade latencies, saccadic hypometria and dysmetria, and abnormal smooth pursuits.

Conclusion: Our data may provide useful insights into the pathogenesis of the ALS confirming the involvement other extramotor areas including the cerebellum and frontal areas. Oculomotor abnormalities may represent a biomarker of neurodegeneration beyond motor neurons in ALS patients, especially in those with bulbar-onset.

References:

- Averbuch-Heller L, Helmchen C, Horn AK, Leigh RJ, ButtnerEnnerver JA. Slow vertical saccades in motor neuron disease: correlation of structure and function. Ann Neurol (1998);44:641-48

- Donaghy C, Thurtell MJ, Pioro EP, Gibson JM, Leigh RJ. Eye movements in amyotrophic lateral sclerosis and its mimics: a review with illustrative cases. J Neurol Neurosurg Psychiatry (2011);82:110-16

- Bong-Hui Kanga, Jae-Il Kimb Young-Min Lima Kwang-Kuk Kima. Abnormal Oculomotor Functions in Amyotrophic Lateral Sclerosis. J Clin Neurol (2018);14(4):464-71

\section{COGNITIVE RESERVE INFLUENCES THE CLINICAL EXPRESSION OF AMYOTROPHIC LATERAL SCLEROSIS}

\author{
M. Consonni, E. Dalla Bella, E. Bersano, A. Telesca, G. Lauria \\ IRCCS Foundation "Carlo Besta" Neurological Institute (Milano)
}

Objective: Long-term life experiences, such as education, occupational attainment, leisure activities and bilingualism, have been considered proxies of cognitive reserve (CR) [1]. In neurodegenerative disease, CR is considered as a modulator associated with more favourable cognitive trajectory and motor functions [2,3]. Our study first sought to investigate the role of CR on cognitive and motor involvement in a large cohort of incident patients with amyotrophic lateral sclerosis (ALS).

Methods: Assessments of cognition and clinical and demographic information were obtained in 101 incident patients with ALS. CR was measured considering years of education, level of occupation, amount of leisure activities and bilingualism. Correlation and regression analyses were performed to test the influence of $\mathrm{CR}$ on the clinical expression of ALS.

Results: We found that all proxies of CR were positively associated with executive function, verbal fluency and memory domains. Motor impairment was inversely related to educational level and occupational attainment. Regression analyses documented the positive predictive value of CR in cognitive performances in all patients and the predictive role of $\mathrm{CR}$ in modulating the motor functional disabilities in patients with bulbaronset.

Discussion: We confirmed that CR mediates the extent of cognitive decline and found that it is also associated with better functional status in patients with bulbar onset. Our findings are in keeping with previous studies in neurodegenerative diseases suggesting that CR is an environmental factor moderately modulating the loss of function caused by progressive brain degeneration also in ALS. Investigating premorbid longterm life experiences could be important to have a comprehensive picture of the reserve that might enrich the resilience to progressive brain damage in ALS.

Conclusion: Our results suggest that, in ALS, the concept of reserve should encompass cognitive and motor domains.

References:

1. Stern Y. Cognitive reserve. Neuropsychologia 2009; 47:2015-2028 Fratiglioni L, Wang HX. Brain reserve hypothesis in dementia. J Alzheimers Dis (2007); 12:11-22

2. Perani D, Farsad M, Ballarini T, Lubian F, Malpetti M, Fracchetti A, Magnani G, March A, Abutalebi J. The impact of bilingualism on brain reserve and metabolic connectivity in Alzheimer's dementia. Proc Natl Acad Sci U S A (2017);114:1690-95

\section{NEURAL ORGANOIDS FOR DISEASE MODELING AND THE DEVELOPMENT OF NEW THERAPEUTICS IN C9ORF72 ALS}

G. Costamagna, F. Biella, M. Taiana, S. Ghezzi, I. Faravelli, N. Bresolin, G. Comi, M. Nizzardo, S. Corti

Department of Pathophysiology and Transplantation, University of Milan (Milano)

Background and aims: Amyotrophic lateral sclerosis (ALS) is a devastating neurodegenerative disease. C9orf72 repeat expansion is the most frequent genetic cause of ALS (C9ALS) in Europe and North America. Partially owing to an incomplete understanding of disease etiopathogenesis, disease-modifying therapies in C9ALS still lack. A better insight into C9ALS pathomechanisms in reliable models is fundamental for developing new therapeutics. Here, we aim to model C9ALS pathology in 3D human neural organoids.

Material and Methods: We differentiated iPSCs from C9ALS patients and healthy controls fibroblasts using a free-floating 3D-culture method. We generated early cerebral-like organoids (COs) using standard methods and ventral spinal-cord like organoids (vSCOs) with a modified protocol inducing neural caudalization and ventralization. Then, we treated C9ALS COs and vSCOs with morpholino antisense oligonucleotides (MO) binding the C9Orf72 hexanucleotide repeat expansion. Finally, we evaluated the differentiation of organoids at different time points with immunohistochemical and qPCR analysis.

Results: We obtained control and C9ALS COs and vSCOs organoids displaying different co-existing neuronal subpopulations. COs exhibited progenitor, forebrain, and immature post-mitotic neuronal markers; vSCOs expressed progenitor, pan-neuronal markers, ventro/caudal and motor neuron markers. Also, C9ALS organoids presented increased dipeptide repeat proteins (DPRs) levels compared to controls. C9ALS organoids dissociated into single cells showed pBRCA1 and $\gamma \mathrm{H} 2 \mathrm{AX}$ foci, markers of DNA damage associated with C9orf72 expansion, and 
increased tp53, involved in DNA-repair pathways. In addition, dissociated cells exhibited TDP-43. MO-treated organoids showed reduced C9orf72 protein production. Preliminary results on gene expression analysis using qPCR reported differential expression of genes involved in DNA damage response in MO treated C9ALS organoids.

Discussion and Conclusions: Here, we generated COs and SCOs that show different neuronal and motoneuron precursors, express neuropathological hallmarks of C9ALS, and recapitulate some disrupted mechanisms involved in C9ALS pathogenesis. In addition, MO may reduce the level of $\mathrm{C} 9$ orf72 protein isoforms in our model. Neural organoids are a valuable iPSC-based technology used to recapitulate different neurodegenerative disorders affecting the brain, including Huntington disease, Alzheimer disease, and frontotemporal dementia [1]. In addition, organoid-based models of the neuromuscular junction have helped to reproduce aspects of myasthenia gravis in a dish [2]. In parallel, recent technical improvements have prompted the generation of spinal cord-like organoids to model spinal muscular atrophy in vitro [3]. Altogether, these findings suggest that neural organoids represent an innovative in vitro system and a valuable platform for modeling aspects of C9ALS pathology, studying C9ALS pathomechanisms, and potentially developing new treatments in vitro.

References:

1. Faravelli I, Costamagna G, Tamanini S, Corti S. Back to the origins: Human brain organoids to investigate neurodegeneration. Brain Res. (2020);1727:146561. doi:10.1016/j.brainres.2019.146561

2. Faustino Martins J-M, Fischer C, Urzi A, et al. Self-Organizing 3D Human Trunk Neuromuscular Organoids. Cell Stem Cell. (2020);26(2):172-186.e6. doi:10.1016/j.stem.2019.12.007

3. Hor JH, Soh ES-Y, Tan LY, et al. Cell cycle inhibitors protect motor neurons in an organoid model of Spinal Muscular Atrophy. Cell Death Dis. (2018);9(11):1100. doi:10.1038/ s41419-018-1081-0

\section{COVID-19 AND AMYOTROPHIC LATERAL SCLEROSIS: AN OBSERVATIONAL STUDY}

S. Cotti Piccinelli ${ }^{1}$, C. Lunetta ${ }^{2}$, N. Ticozzi ${ }^{3}$, N. Necchini ${ }^{1}$, B. Risi ${ }^{1}$, E. Baldelli $^{1}$, V. Sansone ${ }^{2}$, V. Silani ${ }^{3}$, A. Padovani ${ }^{1}$, M. Filosto ${ }^{1}$

${ }^{1}$ ASST Spedali Civili of Brescia, University of Brescia (Brescia); ${ }^{2} \mathrm{Nemo}$ Clinical Center for Neuromuscular Diseases, Fondazione Serena Onlus (Milano); ${ }^{3}$ U.O. Department of Neurology, Stroke Unit and Laboratory of Neuroscience, IRCCS Istituto Auxologico Italiano (Milano)

Objectives: Patients affected with Amyotrophic Lateral Sclerosis (ALS) represent a fragile population due to progressive muscle weakness and respiratory failure. Objective of this study was to evaluate the impact of COVID-19 in an ALS population living in Lombardy, the Italian region with the highest number of COVID-19 positive patients.

Methods: A specific questionnaire was administered by phone to 233 ALS patients over the age of 18 diagnosed according to revised El Escorial Criteria. ALSFRS-R score, ventilatory status and ATS dyspnea grading scale were assessed in order to evaluate the clinical status pre- and during outbreak.

Results: $16.7 \%$ patients developed signs and symptoms likely due to COVID-19 and, out of these, $12.8 \%$ died during outbreak. Symptomatic patients had a lower ALSFRS-R score (25.44 versus 30.24; p $<0.05)$ and poorer ventilatory status than asymptomatic patients at baseline. Deceased patients were older and had a lower
ALSFRS-R score than those who survived $(73,60+/-6,84$ versus $61,94+/-11,47 ; 19,80+/-8,67$ versus $25,82+/-11,01 ; \mathrm{p}<0.05)$. In the surviving patients ATS score worsened $(0.21 \mathrm{vs} 0.07 ; \mathrm{p}<0.05)$ without any significant changes in ALSFRS- R score compared to asymptomatic patients.

Discussion: ALS patients have a high risk to develop symptoms related to COVID-19, which is higher in patients who have a lower baseline ALSFRS-R score and NIV $<10$ hours. Lower ALSFRS-R score and older age at baseline are negative prognostic factors quoad vitam. As expected, symptomatic patients present a more rapid deterioration of the respiratory state.

Conclusions: Our study showed that, despite the lockdown imposed early by the Italian authorities, a significant number among our ALS patients developed symptoms suggestive for COVID-19. Accurate guidelines in using NIV during COVID-19 infection as well as a strict compliance with the recommendations delivered by scientific societies and authorities are highly needed.

\section{ELECTROMYOGRAPHY AS A STAGING TOOL AND MARKER OF PROGNOSIS IN AMYOTROPHIC LATERAL SCLEROSIS}

\author{
M. A. R. Daviddi, U. Manera, M. Torrieri, A. Canosa, A. Bombaci, M. \\ Grassano, C. Moglia, A. Chiò, A. Calvo
}

"Rita Levi Montalcini" Department of Neuroscience, University of Turin (Torino)

Aims: ALS is a neurodegenerative disease, affecting both upper and lower motor neurons. The diagnosis of ALS is based on clinical criteria supported by EMG, which investigates spontaneous activity, motor unit potential and the voluntary maximum recruitment pattern. The first aim of this paper is to assess, at diagnosis, some of the main ALS electromyographic findings and compare them to King's staging system, MiToS and NBRI. The second aim is to stratify patients' prognosis on the basis of electromyographic findings.

Materials and Methods: Electromyographic data of 7 muscle districts in 394 patients (2012-2018), belonging to the Piemonte and Valle d'Aosta Registry for Amyotrophic Lateral Sclerosis (PARALS), were recorded. We selected the EMG examination performed at diagnosis and compared it with the nearest ALSFRS-r scale.

Results: The increase of MiToS, King's and NBRI systems stage is correlated with a higher percentage of muscle districts with alterated voluntary maximum recruitment pattern. Fib/PSW are predominant in distal muscle districts; fasciculations are mostly recorded in upper limbs rather than lower limbs (chi-squared test $\mathrm{p}<0,001$ ). Fasciculations in the upper limbs (right brachial biceps $\mathrm{p}=0,009$, right deltoid $\mathrm{p}=0,006$, left brachial biceps $p=0,006$, left deltoid $p=0,003$ ), have been used to stratify patients, belonging to the same clinical stage, in groups characterized by different prognosis.

Discussion: The level of disability at the time of EMG examination is correlated to the alteration of voluntary maximum recruitment pattern, rather than spontaneous activity. Different features of EMG spontaneous activity (Fib/PSW vs fasciculations) have distinct distribution in muscle districts, maybe underlying different neuropathological mechanisms. Fib/ PSW are frequently found in distal muscle districts: this may be explained by early distal denervation in ALS onset. Fasciculations are often observed in upper limbs, possibly correlating to wider cortical projection of upper limb muscles [1]. Fasciculations has been correlated not only to a lower motor neuron disease, but also to an increased cortical excitability [2]. Frequent fasciculations in specific muscle 
districts are correlated, in patients in the same disease stage, with poorer prognosis. Our data suggest that the presence of spontaneous activity in the proximal districts of upper limbs could be linked to a faster involvement of the respiratory muscles. This hypothesis is consistent with previous data [3].

Conclusion: Needle EMG is a useful tool in motor neuron diseases, as it can be used to highlight different neuropathological characteristics in ALS patients cohorts with different prognosis.

References:

1. De Carvalho, Mamede, Kiernan, M. C., \& Swash, M. Fasciculation in amyotrophic lateral sclerosis: Origin and pathophysiological relevance. In Journal of Neurology, Neurosurgery and Psychiatry (2017) Sep;88(9):773-779. https://doi.org/10.1136/jnnp-2017315574

2. Kleine, B. U., Stegeman, D. F., Schelhaas, H. J., \& Zwarts, M. J. Firing pattern of fasciculations in ALS: Evidence for axonal and neuronal origin. Neurology (2008) Jan 29;70(5):353-9. https://doi. org/10.1212/01.wnl.0000300559.14806.2a

3. Zhang H, Zhang S, Zhang N, Fan D. The presence of spontaneous EMG activity in sternocleidomastoid is associated with ventilatory dysfunction in ALS. Neurophysiol Clin. (2016);46(2):145-148. doi:10.1016/j.neucli.2016.02.002

\section{TELEHEALTH APPROACH FOR AMYOTROPHIC LATERAL SCLEROSIS PATIENTS: THE EXPERIENCE DURING COVID- 19 PANDEMIC}

\author{
F. De Marchi ${ }^{1}$, M. Sarnelli ${ }^{2}$, M. Serioli ${ }^{3}$, I. De Marchi ${ }^{2}$, E. Zani ${ }^{4}$, N. \\ Bottone $^{4}, \mathrm{~S}$. Ambrosini ${ }^{5}$, R. Garone ${ }^{6}$, R. Cantello ${ }^{2}$, L. Mazzini ${ }^{2}$
}

\begin{abstract}
${ }^{1}$ Department of Neurology, University of Piemonte Orientale (Novara); ${ }^{2}$ Department of Neurology and ALS Centre, University of Piemonte Orientale (Novara); ${ }^{3}$ Department Dietetic and Clinical Nutrition, University of Piemonte Orientale (Novara); ${ }^{4}$ Healthy Reply, Business Unit, Santer Reply S.p.A (Torino); ${ }^{5}$ Consoft Sistemi (Torino); ${ }^{6}$ Health Management, Maggiore della Carità Hospital (Novara)
\end{abstract}

Background and objective: During disease course, specialized multidisciplinary ALS care has been shown to extend survival and improve patients and caregiver's quality of life, supporting by a coordinated interprofessional care able to manage the complex needs of patients. However, the management and monitoring of neurological patients suddenly had to quickly change due to Coronavirus disease-19 pandemic and telemedicine has proven effective for patient evaluation, allowing health care professionals to evaluate patients in remote locations using telecommunications technology. Here we report the experience with telemedicine of a Tertiary ALS Center in an Italian geographical area with high infectious risk during COVID-19 pandemic,

Methods: Patients with ALS were evaluated in telemedicine by a multidisciplinary team including: 1) neurologist - functional evaluation (ALSFRS-R), monitoring of intercurrent events, and drug prescriptions; 2) dietician - diet monitoring (collection of weight, BMI, arm circumference, caloric intake and baseline, type of diet, use of supplements and thickeners); 3) psychologist - psychological assessment and support; 4) physiotherapist - functional status, pain management and device prescription). The telemedicine approach was performed using the online platform "IoMT Connected Care Platform (Ticuro Reply)", where we generated a personal account for each healthcare provider and patient/ caregiver.
Results: 19 patients were enrolled. Firstly, all patients reported a positive perception of talking face-to-face with healthcare professionals and were satisfied on how the team understood their problems during telemonitoring. During a video televisit, there was a change in the patient's medication regimen in 11/19 patients; $2 / 19$ required urgent pneumological evaluation and started NIV; 9/16 patients required devices prescription. The mean monthly decline of ALSFRS-R for patients before televisit was 0.88 (SD 1.17), and during televisit of 0.49 (SD 0.75). Body weight and daily caloric content remain stable during observational period. From psychological point of view, reduction in HADS scores, which means a reduction in anxiety and depression level, is observed. For ALSAQ-40, we found a stability in total score and each subscore.

Discussion: Our study our study highlights the important support provided by telemedicine during the COVID-19 pandemic. We positively reproduced the standard multidisciplinary approach currently used with ALS patients, trying to stabilize the functional and metabolic status and improving the psychological one. Future directions for telehealth in ALS field include a personalized telemedicine program according to the patient needs, which may change both in terms of televisit frequency and healthcare providers involved.

References:

- Haulman A, Geronimo A, Chahwala A, Simmons Z. The use of telehealth to enhance care in ALS and other neuromuscular disorders. Muscle Nerve (2020);61(6):682-91

- Andrews JA, Berry JD, Baloh RH, et al. Amyotrophic Lateral Sclerosis Care and Research in the USA During the COVID-19 Pandemic: Challenges and Opportunities. Muscle Nerve (2020) Aug;62(2):182-86

- Capozzo R, Zoccolella S, Musio M, Barone R, Accogli M, Logroscino G. Telemedicine is a useful tool to deliver care to patients with Amyotrophic Lateral Sclerosis during COVID-19 pandemic: results from Southern Italy. Amyotroph Lateral Scler Front Degener. (2020):1-7

\section{HUMAN NEURAL ORGANOIDS TO INVESTIGATE SPINAL MUSCULAR ATROPHY PATHOLOGY AND THERAPY}

I. Faravelli ${ }^{1}$, P. Rinchetti ${ }^{1}$, S. Mancinelli ${ }^{2}$, L. Mapelli ${ }^{3}$, G. Forotti ${ }^{1}$, M. Rizzuti $^{1}$, C. Cordiglieri ${ }^{4}$, H. Moulton ${ }^{5}$, E. D'Angelo ${ }^{3}$, N. Bresolin ${ }^{1}$, G. Comi $^{1}$, S. Lodato ${ }^{2}$, M. Nizzardo ${ }^{1}$, S. Corti ${ }^{1}$

${ }^{1}$ Department of Pathophysiology and Transplantation, IRCCS Foundation $\mathrm{Ca}$ ' Granda Ospedale Maggiore Policlinico (Milano); ${ }^{2}$ Developmental Neurobiology Lab, Humanitas Research Hospital (Pieve Emanuele-MI); ${ }^{3}$ Neurophysiology Unit, Department of Brain and Behavioral Sciences, University of Pavia (Pavia); ${ }^{4}$ Istituto Nazionale Genetica Medica (Milano); ${ }^{5}$ Biomedical Sciences, College of Veterinary Medicine, Oregon State University (Corvallis-USA)

Background: Spinal muscular atrophy (SMA) is a neuromuscular disease and the leading cause of genetic death during infancy. The optimization of available treatments and the study of complementary approaches requires a deep knowledge of SMN pathophysiology in reliable models. We exploited a novel model of SMA pathology, using 3D human neural organoids that can be obtained from induced pluripotent stem cell (iPSCs) differentiated with a free-floating 3D culture method. Cerebral and ventral spinal cord-like organoids present interdependent neural regions reproducing the integrated connection of the central nervous system. To test 
our model for therapeutic screening, we exploited an optimized novel antisense oligonucleotide (ASO) that dramatically enhanced SMN levels.

Material and Methods: We generated iPSCs starting from SMA patients and healthy controls and developed cerebral organoids, using an already published protocol [1]. Derived organoids gave rise to an early cortex-like formation containing progenitor cells and more mature neural subtypes. Exploiting a novel modified differentiation method based on small molecules to promote caudalization and ventralization, we also derived ventral spinal cord-like organoids. Immunohistochemical and molecular analyses were used to test the organoid differentiation state. Calcium assay and electrophysiological studies were also performed to investigate circuit function and activity.

Results: Preliminary results showed that SMA organoids exhibited a significant alteration in their neurofilament elongation, suggesting that lack of SMN caused defects of axonal metabolism. Interestingly, electrophysiological recording showed that control organoids had higher basal spike frequency compared to the affected ones, but SMA organoids were more responsive to the glutamatergic stimuli. A major limitation in the use of ASOs for SMA therapy is represented by their limited tissues distribution. One potential strategy is the use of peptides to increase ASO biodistribution and effectiveness. In this context, we tested a novel ASO compound, suited to modulate SMN2 splicing and increasing SMN level. Treatment of SMA ventral spinal cord-like organoids rescued SMN levels and main pathological features.

Conclusions: Even though SMA is a genetic disorder, few studies have analyzed SMA tissues from prenatal stages of the disease, when SMN is particularly required, to determine whether SMN deficiency impairs neural development. SMA organoids can be exploited to reproduce the key features of the early central nervous system to unravel disease mechanisms. In this work, we report the generation of cerebral and ventral spinal cord-like organoids from human iPSCs derived from healthy and SMA subjects as potential platform to investigate pathogenetic features and test new therapeutic strategies.

Reference:

1. Lancaster MA, Knoblich JA. Generation of cerebral organoids from human pluripotent stem cells. Nat Protoc. (2014);9(10):2329-2340. doi: $10.1038 /$ nprot. 2014.158

\section{MUTATIONS IN GBA GENE INCREASE RISK FOR COGNITIVE IMPAIRMENT AND FTD IN ALS PATIENTS}

M. Grassano ${ }^{1}$, A. Calvo ${ }^{1}$, A. Canosa ${ }^{1}$, U. Manera ${ }^{1}$, R. Vasta ${ }^{1}$, M.

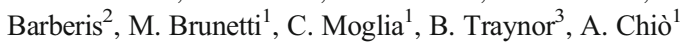

${ }^{1}$ Department of Neuroscience, University of Turin (Torino); ${ }^{2}$ Medical Genetics, City of Health and Science University Hospital (Torino); ${ }^{3}$ Laboratory of Neurogenetics, National Institute of Aging (BetehsdaUSA)

Background: Mutations in the GBA gene are important risk factors for Parkinson disease (PD) and are associated with greater cognitive impairment and risk of dementia in PD patients. It is also known that variants in GBA gene exhibit pleiotropic effects in PD-related disorders.

Objective: In this study, we tested the hypothesis that GBA genotype may influence the risk of cognitive symptoms in ALS.

Methods: The study population consisted of 600 patients identified through the Piemonte and Valle d'Aosta Register for ALS (PARALS) incident in the 2007-2015 period. Patients' cognitive status was classified according to the revised ALS-FTD Consensus Criteria. Variants in GBA gene were identified through whole-genome sequencing. All patients were previously screened for mutations in ALS-related genes and C9ORF72 hexanucleotide repeated expansions. The association between a clinical diagnosis of cognitive impairment and GBA mutation status was assessed in mixed logistic regression models. The analysis was adjusted for age in years, age at onset of ALS, gender, stage at diagnosis, onset phenotype and C9orf72 mutation status.

Results: We observed 11 GBA mutations in our cohort. We focused on the three variants (p.E326K, p.T369M, p.N370S) that are established risk factor for cognitive impairment in neurodegenerative disorders. We identified 16 ALS patients who were heterozygous carriers of neurological/severe GBA mutations. The prevalence of cognitive impairment in GBA carriers was $68.75 \%$. GBA mutation status was associated with clinical diagnosis of cognitive impairment or FTD (OR $=5.8,95 \%$ CI $1.7-19.9, \mathrm{p}=0.005)$. The association persisted in the multivariate analysis $(\mathrm{OR}=6.2,95 \% \mathrm{CI} 1.3-29.0, \mathrm{p}=0.021)$.

Discussion: We found that carriers of GBA pathogenic mutations had an increased risk of presenting with cognitive dysfunction. Our results suggest that GBA alteration may play a broader role in neurodegenerative diseases and is a genetic modifier of ALS phenotype.

Conclusions: In this study, we examined the association of GBA variants with cognitive impairment in a population-based ALS cohort. Our findings provide evidence that GBA variants are associated with cognitive decline in ALS patients and account for some of the clinical heterogeneity observed in ALS.

\section{ROLE OF HNRNPA1 IN AN ITALIAN ALS POPULATION- BASED COHORT}

M. Grassano ${ }^{1}$, A. Calvo ${ }^{1}$, A. Canosa ${ }^{1}$, U. Manera ${ }^{1}$, R. Vasta $^{1}$, C.

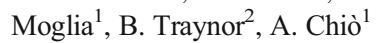

${ }^{1}$ Department of Neuroscience, University of Turin (Torino); ${ }^{2}$ Laboratory of Neurogenetics, National Institute of Aging (Bethesda-USA)

Objective: To assess the role of heterogeneous nuclear ribonucleoproteins (hnRNP) in amyotrophic lateral sclerosis (ALS), we examined hnRNPA1 rare variants in an ALS cohort from Italy. We sought to determine the prevalence of HNRNPA1 mutations and their associated clinical phenotype.

Background: Mutations in the prion-like domain of the gene encoding the hnRNPA1 protein have been implied in ALS pathogenesis and previously reported in ALS patients. However, evidence for their role in ALS is still inconclusive.

Design/Methods: We identified variants in hnRNPA1 gene through whole-genome sequencing of 957 individuals with sporadic and familial ALS, of the Piemonte ALS Register (PARALS) and 677 control subjects. We performed a gene-based rare variants analysis and then assessed the clinical characteristics of the patients who carry the candidate diseaseassociated variants.

Results: We detect an enrichment of hnRNPA1 rare variants in ALS patients (p-value 0.034$)$. We found $5(0.5 \%)$ individuals carrying 5 distinct nonsynonymous SNV that were absent in the control population. Four of these variants are located in the prion-like domain of the gene: the variant c.C666G (p.F222L) in exon 6 and the variants c.G824T (p.G275V), c.C876G (p.N292K) and G883A (p.G295R) in exon 8. All cases were apparently sporadic. Limb onset occurred in all five patents and none of them showed cognitive impairment. Mean age of onset was 58.0 years. The mean rate of decline for ALS-FRS was 0.37 points/ months; two patients showed a late involvement of bulbar (29.0 months) and respiratory functions (39.5 months). hnRNPA1 variants were 
associated with a longer survival (HR 13.7, 95\% C.I. 4.78-39.5, p < $0.001)$ than non-mutated ALS patients from the PARALS.

Conclusions: Our data demonstrate that hnRNPA1 is a low frequency cause of disease in our cohort. Mutations in hnRNPA1 define a relatively uniform slow-progressive subset of ALS.

\section{COGNITIVE AND BEHAVIORAL PROFILE OF C90RF72 ALS PATIENTS: A NEUROPSYCHOLOGICAL DOMAIN PERSPECTIVE}

\author{
B. Iazzolino ${ }^{1}$, L. Peotta ${ }^{1}$, J. Zucchetti ${ }^{1}$, A. Canosa ${ }^{1}$, U. Manera ${ }^{1}$, F. \\ Palumbo $^{1}$, M. Brunetti ${ }^{1}$, M. Barberis ${ }^{2}$, L. Sbaiz ${ }^{2}$, C. Moglia ${ }^{1}$, A. \\ Calvo $^{1}$, A. Chiò ${ }^{1}$
}

${ }^{1}$ ALS Centre, Department of Neuroscience "Rita Levi Montalcini", University of Torino (Torino); ${ }^{2}$ Medical Genetic Unit, Azienda Ospedaliero-Universitaria Città della Salute e della Scienza of Torino (Torino)

Objectives: The aim of this study was to determine whether ALS patients with C9orf72 expansion showed a different impairment of cognitive and behavioural domains compared to patients without C9orf72 expansion (a) at the same degree of cognitive and behavioral deficit, classified according to the revised ALS-FTD Consensus Criteria; and (b) at the same level of motor impairment, classified according to King's staging system. Materials: the study population includes 741 ALS patients who were consecutively seen at the Turin ALS expert center in the period 20102018. ALS patients underwent a battery of neuropsychological tests (encompassing executive function, memory, visuospatial function, language and behavioural dysfunction) selected according to the ALS-FTD Consensus Criteria. ALS Functional Rating Scale revised (ALSFRS-R), Kings' staging and genetic analysis at time of cognitive testing were collected for all patients.

Methods: Comparisons were performed on age-, sex-, and education-corrected scores. Since all cognitive tests had not a normal distribution, the Mann-Whitney $U$ test was used for comparisons. First, we compared the results of cognitive tests of ALSC9+ vs ALSC9- patients for each cognitive and behavioral level, merging the intermediate cognitive categories. Second, comparisons were performed for each King's stage, independently from their level of cognitive impairment. Third, we compared the number of ALSC9+ vs. ALSC9- cases who showed an impairment in each neuropsychological domain.

Results: ALSC9+ patients had significant lower scores in tests exploring executive functions, and verbal memory both when classified as cognitively normal and when diagnosed in the intermediate cognitive categories. Considering the clinical perspective, ALSC9+ patients showed significantly lower scores compared to ALSC9- patients at King's stage 1 and 3 in almost all the examined neuropsychological domains, while at King's stage 2 ALSC9+ patients were more severely affected only in the verbal memory domain. Behavioral function was comparably impaired in the two cohorts.

Discussion: In our cohort we found that ALSC9+ patients show a different neuropsychological profile compared to ALSC9- ones, being more impaired in executive functions and verbal memory domains at all King's stages. Verbal memory emerged as a particularly vulnerable function in ALSC9+, with worse performances even when patients were still classified as cognitively normal. This could imply in ALSC9+ patients a 'cognitive' presymptomatic/subclinical condition characterized by lower performances at specific cognitive tasks when motor symptoms are already present.

Conclusions: ALSC9+ patients show worse performances at several neuropsychological domains compared to ALSC9-, already at cognitively normal level and initial clinical Kings' staging.

References:

- Chiò A, Moglia C, Canosa A, et al. Cognitive impairment across ALS clinical stages in a population-based cohort. Neurology (2019); 93:e984-e994

- Mahoney CJ, Downey LE, Ridgway GR, et al. Longitudinal neuroimaging and neuropsychological profiles of frontotemporal dementia with C9ORF72 expansions. Alzheimers Res Ther (2012); 4:41

- Strong MJ, Abrahams S, Goldstein LH, et al. Amyotrophic lateral sclerosis - frontotemporal spectrum disorder (ALS-FTD): Revised diagnostic criteria. Amyotroph Lateral Scler Frontotemporal Degener 2017;18:153-174.

\section{ARTERIAL BLOOD GAS ANALYSIS (ABG) IN AMYOTROPHIC LATERAL SCLEROSIS: CARBONATE (HCO3-) ELEVATION ALONE AS A MARKER OF EARLY RESPIRATORY FAILURE}

U. Manera $^{1}$, M. Torrieri ${ }^{1}$, C. Moglia ${ }^{1}$, M. Viglione ${ }^{1}$, M. Daviddi ${ }^{1}$, E. Matteoni $^{1}$, L. Solero ${ }^{1}$, F. Palumbo ${ }^{1}$, R. Vasta ${ }^{1}$, A. Canosa ${ }^{10}$, F. D'Ovidio $^{11}$, F. Ribolla ${ }^{2}$, M. Bellochia ${ }^{2}$, L. Focaraccio ${ }^{3}$, A. Mattei ${ }^{2}$, G. Mora $^{4}$, A. Calvo ${ }^{1}$, A. Chiò ${ }^{1}$

${ }^{1}$ ALS Centre, "Rita Levi Montalcini" Department of Neuroscience, University of Turin (Torino); ${ }^{2}$ Pneumology Unit, AOU Città della Salute e della Scienza of Turin (Torino); ${ }^{3}$ Pneumology Unit, ASL of Turin (Torino); ${ }^{4}$ ALS Center, Istituti Clinici Scientifici Maugeri, IRCCS (Milano)

Objective: To determine correspondence between main arterial blood gases $(\mathrm{ABG})$ parameters (carbon dioxide, $\mathrm{pCO} 2$, blood carbonate, HCO3-, and standard base excess, SBE) and forced vital capacity (FVC\%), and their ability to predict non-invasive mechanical ventilation (NIMV) usage and survival in ALS patients.

Materials and Methods: We collected ABGs and pulmonary function tests (PFTs) performed on ALS patients from the Turin ALS Centre, diagnosed from 2000 to 2015 . Clinical data were collected using registry data. pCO2, $\mathrm{HCO} 3-$, and $\mathrm{SBE}$ were compared with $\mathrm{FVC} \%$ and clinical data, both singularly and combined, to stratify patients on the basis of significant cut-offs. Their ability to predict NIMV start at 3 months and overall survival was assessed, adjusting for age, sex, clinical phenotypes, and progression rate.

Results: 488 patients with paired ABGs, PFTs, and ALSFRS-r score were collected. Single ABG parameters showed a significant correlation with $\mathrm{FVC} \%$ values. A significant decline in $\mathrm{FVC} \%$ values was recorded for a pCO2 cut-off of $42 \mathrm{mmHg}$, for every $\mathrm{HCO} 3$ - cut-off above 26 $\mathrm{mmol} / \mathrm{L}$, and every SBE cut-off above $2 \mathrm{mmol} / \mathrm{L}$. Each of the above mentioned ABGs cut-offs turned out to be the most sensitive in predicting NIMV start at 3 months. ABGs measurements were predictive of death/ tracheostomy only in patients who did not undergo NIMV. Patients with normal $\mathrm{pCO} 2$ and $\mathrm{HCO} 3$ - values at $\mathrm{ABG}$ survived longer than patients with normal $\mathrm{pCO} 2$ and increased $\mathrm{HCO} 3-(1.39$ years, IQR $1.23-1.55$ vs 0.87 years, IQR $0.73-1.02 ; \mathrm{p}<0.001)$ and patients with increased $\mathrm{pCO} 2$ and $\mathrm{HCO} 3$ - levels $(0.75$ years, IQR $0.60-0.90 ; \mathrm{p}<0.001$. 
Discussion: Our data indicate that the use of current guidelines for NIMV initiation could lead to a delayed treatment of respiratory failure in ALS patients [1,2]. HCO3- elevation alone without significant $\mathrm{pCO} 2$ elevation occurred in about $25 \%$ of our patients, being a day-time marker of nocturnal hypoventilation $(\mathrm{NH})$ [3]. After excluding any metabolic cause, we suggest that ALS patients could benefit from NIMV also in presence of an isolated increase of blood $\mathrm{HCO} 3-/ \mathrm{SBE}$ levels.

Conclusions: ABG is a sensitive substitute for other PFTs in ALS patients that should be used both in clinical and experimental settings, especially considering $\mathrm{HCO} 3-, \mathrm{SBE}$ and $\mathrm{pCO} 2$ as early predictor of respiratory failure.

References:

1. Andersen PM, Abrahams S, Borasio GD, et al. EFNS guidelines on the clinical management of amyotrophic lateral sclerosis (MALS)revised report of an EFNS task force. Eur J Neurol (2012); 19: 36075

2. Miller RG, Jackson CE, Kasarskis EJ, England JD, Forshew D, Johnston W, Kalra S, Katz JS, Mitsumoto H, Rosenfeld J, Shoesmith C, Strong MJ, Woolley SC; Quality Standards Subcommittee of the American Academy of Neurology. Practice parameter update: the care of the patient with amyotrophic lateral sclerosis: drug, nutritional, and respiratory therapies (an evidencebased review): report of the Quality Standards Subcommittee of the American Academy of Neurology. Neurology (2009) Oct 13;73(15):1218-26

3. Boentert M, Glatz C, Helmle C, Okegwo A, Young P. Prevalence of sleep apnoea and capnographic detection of nocturnal hypoventilation in amyotrophic lateral sclerosis. J Neurol Neurosurg Psychiatry (2018) Apr;89(4):418-24

\section{A NOVEL INTRONIC FUS MUTATION IN A FAMILIAL ALS CASE: EFFECTS ON PROTEIN EXPRESSION IN PERIPHERAL BLOOD MONONUCLEAR CELLS}

E. Matteoni ${ }^{1}$, A. Calvo ${ }^{1}$, C. Moglia ${ }^{2}$, M. Grassano ${ }^{1}$, M. Barberis ${ }^{1}$, M. Brunetti $^{1}$, A. Lomartire ${ }^{1}$, G. De Marco ${ }^{1}$, A. Chio' ${ }^{13}$, A. Canosa ${ }^{1}$

\footnotetext{
1 "Rita Levi Montalcini" Department of Neuroscience, University of Turin (Torino); ${ }^{2}$ Rita Levi Montalcini' Department of Neuroscience, ALS Centre, University of Turin (Torino); "Rita Levi Montalcini" Department of Neuroscience, Azienda Ospedaliero-Universitaria Città della Salute e della Scienza of Turin (Torino)
}

Objectives: We report a familial ALS case carrying a novel heterozygous FUS mutation in the acceptor splice site of intron 14 (c.1542 -1 g>t). We aimed at evaluating its impact on protein expression in Peripheral Blood Mononuclear Cells (PBMC), to investigate the underlying pathomechanisms.

Materials and Methods: PBMC were isolated for total RNA extraction and analysis (cDNA synthesis, sequencing and one-step RT-PCR analysis), Western Immunoblot (WI), and Immunofluorescence (IF). Four antibodies were used for WI: one against the $\mathrm{N}$-terminal portion, one against the epitope 400-450 (used also for IF), one recognizing an unknown epitope at the $\mathrm{C}$-terminus and one against the $\mathrm{C}$-terminal end of the protein (epitope 500-526). The first three antibodies were expected to detect both the wild-type and the mutated protein. The fourth was expected to detect only the wild type one.

Results: The proband developed head drop at the age of 42 . He showed a 18-month disease course. His mother died from ALS. He was negative for SOD1, TARDBO, FUS and C9ORF72 mutations, but showed a mutation in the acceptor splice site of intron 14 (c.1542 -1 g>t) of FUS. The cDNA analysis revealed the skipping of exon 15 and a premature stop codon at the position c.228 (in the 3'-UTR) due to the mutation. The RT-PCR analysis revealed reduced FUS mRNA by more than half in the proband compared to a healthy control (HC) and an ALS patient without genetic mutations (wtALS). For all the four antibodies used for WI, the intensity of FUS band in the proband was $30-50 \%$ compared to the one detected in the HC and wtALS. The antibody recognizing the epitope 500-526 did not detect any decrement in the intensity of FUS band as compared to the other three antibodies-IF revealed no differences among the HC, the proband and the wtALS.

Discussion: The reduction by half or more in FUS mRNA and protein in PBMC suggests that the truncated protein is absent, probably due to nonsense mediated decay (NMD), a pathway preventing the translation of mRNA in presence of a premature stop codon. These findings arise the possibility of a loss of function as a possible pathomechanism in this case. Nevertheless, the aberrant protein could be expressed and show aggregation-prone behavior in the nervous tissue.

\section{PREDICTORS OF SELF-PERCEIVED HEALTH WORSENING OVER COVID-19 EMERGENCY IN ALS}

G. $\mathrm{Meo}^{1}$, C. Cabona ${ }^{2}$, P. Ferraro ${ }^{3}$, L. Roccatagliata ${ }^{3}$, A. Schenone ${ }^{1}, \mathrm{M}$. Inglese $^{1}$, F. Villani ${ }^{2}$, C. Caponnetto ${ }^{1}$

${ }^{1}$ Department of Neurology, Ospedale Policlinico San Martino, IRCCS (Genova); ${ }^{2}$ Department of Neurophysiology, Ospedale Policlinico San Martino, IRCCS (Genova); ${ }^{3}$ Department of Neuroradiology, Ospedale Policlinico San Martino, IRCCS (Genova)

Introduction: Determinants of self-perceived health status over COVID19 emergency in ALS patients are still unknown.

Methods: A questionnaire investigating healthcare changes and the possible presence of self-perceived anxiety, depression, and clinical worsening was administered to ALS patients currently followed at San Martino Hospital. Regression analyses were applied to identify predictors of self-perceived health status.

Results: $35.08 \%$ of ALS patients reported anxiety symptoms, $36.84 \%$ depressive symptoms, and $35.08 \%$ a clinical worsening. Significant predictors of anxiety symptoms severity were female gender, greater motor impairment, more aggressive disease course, and healthcare changes. The only predictor of depressive symptoms severity was a more aggressive disease course. Significant predictors of clinical worsening were longer disease duration and healthcare changes.

Discussion: Healthcare changes over COVID-19 emergency exerted a significant impact on self-perceived health status in ALS patients, particularly in those with more advanced disease. These findings have potential to improve disease-oriented management in the next phase.

References:

- Lai et al. Severe Acute Respiratory Syndrome Coronavirus 2 (SARS-CoV-2) and Coronavirus disease-2019 (COVID-19): The Epidemic and the Challenges. Int J Antimicrob Agents. (2020) Mar;55(3):105924

- Onder et al. Case-Fatality rate and characteristics of patients dying in relation to COVID-19 in Italy. JAMA. (2020);323(18):1775-76 


\section{CORTICOFUGAL SPREAD IN AMYOTROPHIC LATERAL SCLEROSIS PATIENTS WITH PREDOMINANT UPPER MOTOR NEURONS IMPAIRMENT: A STATISTICAL MEDIATION ANALYSIS ON PRIMARY MOTOR CORTEX AND CORTICOSPINAL TRACT}

G. Milella, D. Mezzapesa, E. D'Errico, A. Fraddosio, A. Introna, G. Scaglione, A. Morea, M. Ucci, I. Simone

Department of Basic Medical Sciences, Neurosciences and Sense Organs, University of Bari (Bari)

Introduction: Amyotrophic lateral sclerosis (ALS) is a progressive and fatal neurodegenerative disorder involving both upper (UMN) and lower motor neurons (LMN). Several theories have been proposed about its progressive spreading pattern: it could follow an anterograde degeneration [1], or it could start as a distal axonopathy and extend through a dying back mechanism [2].

Aim: To test the hypothesis of a corticofugal or a centripetal spreading by exploring if primary motor cortex thinning mediates corticospinal tracts (CSTs) damage in ALS patients.

Methods: Structural weighted-T1 and Diffusion Tensor Imaging (DTI) sequences were obtained from 25 ALS patients and 25 healthy controls (HC), matched for sex and age. Cortical reconstruction was performed using FreeSurfer image analysis. CSTs were reconstructed along motor seeds regions using TRACULA software. Mean Fractional Anisotropy (FA) of bilateral CSTs were extracted and compared between the two groups using a General Linear Model with age and gender as covariates. CSTs were projected to their motor cortical end-regions, where cortical thickness (CT) was calculated. A statistical mediation analysis was performed to assess the indirect effects of the independent variable (ALS patients or HC group) on the dependent variable (mean FA of CSTs) through the independent variable's effect on a mediating variable (CT) [3].

Results: Applying the 50\% of Penn Upper Motor Neuron Score (a standardized measure of UMN signs in ALS) as cut-off to stratify patients, $80 \%$ of them showed predominant UMN impairment. ALS patients showed reduced FA of bilateral CSTs compared to HC (left: $\mathrm{p}<0.0001$ and right: $\mathrm{p}=0.037$ ). Statistical mediation analyses demonstrated that reduced FA in bilateral CSTs was mediated by reduced CT in motor area (estimated indirect effect, left hemisphere: $b=-0,0077,95 \%$ confidence interval $(\mathrm{CI}):-0,0185,-0,0003$; right: $b=-0,005,95 \%$ CI: $0,0110,-0,0007)$.

Discussion: In our ALS cohort predominantly characterized by UMN impairment, we showed that bilater primary motor cortex thinning acts as mediating factor for the reduced FA of corresponding CSTs, suggesting an anterograde degeneration hypothesis. The biological basis could be a cortical hyperexcitability or a pathological protein misfolding which spreads along the axon in a prion-like way. In order to understand if the spread of pathology is ever corticofugal or centripetal, this MRI multimodal approach could be applied in different ALS phenotypes, such as patients with predominant LMN impairment, or with sport-related physical exercise, how it was demonstrated in soccer players.

References:

1. Eisen A, Kim S, Pant B. Amyotrophic lateral sclerosis (ALS): a phylogenetic disease of the corticomotoneuron? Muscle Nerve (1992);15(2):219-24

2. Fischer LR, Culver DG, Tennant P, et al. Amyotrophic lateral sclerosis is a distal axonopathy: evidence in mice and man. ExpNeurol. (2004);185(2):232-40
3. Valeri L, Vanderweele TJ. Mediation analysis allowing for exposure-mediator interactions and causal interpretation: theoretical assumptions and implementation with SAS and SPSS macros [published correction appears in Psychol Methods (2013) Dec;18(4):474. PsycholMethods.

\section{TELEMEDICINE FOR ALS DURING COVID-19 PANDEMIC: AN ITALIAN ALS REFERRAL CENTER EXPERIENCE}

C. Moglia, R. Vasta, F. D'Ovidio, F. DiPede, F. DeMattei, S. Cabras, L. Peotta, B. Iazzolino, S. Giusiano, U. Manera, F. Palumbo, A. Bombaci, M. Torrieri, A. Ilardi, E. Mastro, M. Arcari, L. Solero, M. Grassano, M. Daviddi, E. Matteoni, P. Salamone, G. Fuda, A. Canosa, A. Chio', A. Calvo

ALS Centre, Rita Levi Montalcini Dept of Neuroscience, University of Torino (Torino)

Objective: To describe the telemedicine experience of an Italian ALS tertiary Center during COVID-19 pandemic.

Methods: All visits scheduled between 6th March and 6th April 2020 were considered for telemedicine. Additionally, the Center maintained the possibility for patients to phone call specialists. A few days after each contact, patients and caregivers were surveyed for assessing their satisfaction with the service received.

Results: A total of 144 controls were scheduled during the study period and consisted mostly of neurological or psychological visits $(139,96.5 \%)$. One hundred thirty-nine $(96.5 \%)$ visits was performed as telemedicine and mostly via phone call $(112,80.6 \%)$. Three $(2.1 \%)$ visits were considered as urgent and maintained as outpatient care. Patients were globally stable with respect to the previous visit (median $\Delta$ ALSFRSr total score 1, IQR 0-4). Many requests of contact were addressed at getting information about the scheduled visits or examinations $(45,43.3 \%)$. Globally, patients and caregivers were satisfied of the telemedicine service. However, the majority $(85,65.9 \%)$ would globally prefer a face-to-face visit.

Conclusions: We were able to rapidly adapt our assistance to the social restrictions due to COVID-19 pandemic. Such organization was fully appreciated by our patients and caregivers and could be a solution for the future, also regardless of the COVID-19 spreading.

\section{KING'S RATE, A NEW PROGNOSTIC BIOMARKER IN AMYOTROPHIC LATERAL SCLEROSIS?} A. Morea, A. Introna, G. Scaglione, G. Milella, M. Ucci, A. Fraddosio, E.
D'Errico, I. Simone

Department of Basic Medical Sciences, Sense Organs and Neurosciences, Neurology Unit, University of Bari "Aldo Moro" (Bari)

Background and Objectives: Recent interest was given to measures of functional staging in ALS, and the development of clinically applicable prognostic biomarkers is very important to patient management and recruitment in clinical trials. Our aim is to evaluate the prognostic value of a new marker of disease progression in Amyotrophic Lateral Sclerosis, based on King staging, namely the "King's rate".

Materials: A total of 656 patients ALS patients referred to the Centre of Motoneuron Diseases of University of Bari were recruited from 
January 1998 to February 2020. At first observation the following variables were: age, sex, site of onset, ALSFRS-r, disease duration from symptom onset, progression rate, time to generalization if present at first visit, and score of King's staging, retrospectively assigned. The King's rate was calculated as: "( 0 - King's staging at time of first visit $) /$ duration from onset to first visit expressed in months)"

Statistical Analysis: Primary endpoint of the study was the overall survival (OS), a composite time variables (death or tracheostomy as events). Patients who did not reach the endpoints were censored at February 2020. King's rate was correlated with progression rate, and OS. Receiver operating curve (ROC) was used to calculate an optimal cut-off of King's rate to discriminate patients with different survival. Time to event analysis was performed using the Kaplan-Meier method. Cox proportional hazard model was used to identify indipendent predictors of survival.

Results: King's rate correlated negatively with progression rate $(\mathrm{p}<$ $0.001)$ and positively with OS $(\mathrm{p}<0.001)$. Using ROC analysis, the King's rate was a valid predictor of survival (AUC 0.654; $p<0.001$, C.F.95\% 0.60-0.71, sensitivity $65 \%$ and specificity $37 \%$ ) Patients with lower value of King's rate had shorter survival than the higher King's rate group $(\mathrm{p}<0.001)$. In a time-to-event analysis shorter King rate and higher progression rate were both valid predictors of shorter survival (HR 0.22 and 1.2 , respectively $\mathrm{p}<0.0001$ )

Discussion: King's rate may be considered a valid predictive biomarker of survival for ALS, as well as the progression rate. Although these parameters have the same prognostic power, King's rate is based on more objective measures (such as forced vital capacity, sniff nasal inspiratory pressure test) and reflects the directionality of King's stages that include the death event. On the other hand, the progression rate is mainly based on ALSFRS-r score, reflecting its limits, indeed the death event could occur with any score of this scale.

References:

- Julie Labra, Parvathi Menon, Karen Byth, Shea Morrison, Steve Vucic Rate of disease progression: a prognostic biomarker in ALS. J Neurol Neurosurg Psychiatry (2016) Jun;87(6):628-32

- R. Tortelli, M. Copetti, F. Panza, A. Fontana, R. Cortese, R. Capozzo, A. Introna, E. D'Errico, S. Zoccolella, S. Arcuti, D. Seripa, I. L. Simone, G. Logroscino. Time to generalization and prediction of survival in patients with amyotrophic lateral sclerosis: a retrospective observational study. Eur J Neurol. (2016) Jun;23(6):1117-25

\section{SOCIAL COGNITION IN AMYOTROPHIC LATERAL SCLEROSIS: A POPULATION-BASED STUDY}

F. Palumbo, B. Iazzolino, L. Peotta, A. Canosa, R. Vasta, U. Manera, C. Moglia, A. Chio', A. Calvo

ALS Centre, Rita Levi Montalcini Department of Neuroscience, University of Torino (Torino)

Objective: To assess Social Cognition (SC) functions in Amyotrophic Lateral Sclerosis (ALS) patients at diagnosis. Secondly, to estimate a possible relationship with severity of motor symptoms and motor phenotype.

Background: Social Cognition (SC), particularly Emotion Recognition (ER) and Theory of Mind (ToM), has been intensively studied in the last decades in several neurodegenerative diseases, including Amyotrophic Lateral Sclerosis. Most studies report an impairment both in ER a ToM in ALS patients, however, results are still controversial because of high motor and cognitive phenotypical variability and remarkable heterogeneity in assessing tasks used.

Methods: We included 52 ALS patients attending the ALS Center of Turin University Hospital between May 2019 and July 2020. All patients underwent Neuropsychological assessment and SC assessment. This was conducted through Ekman 60 faces test (ET), Reading Mind in Eyes task (RMET), Story-Based Empathy task-Emotion Attribution (SET-EA) and Intention Attribution (SET-IA). Both SET-IA and SET-EA were compared to a control condition of Causal Inference (SET-CI).

Results: Subdivision of patients into Cognitive Categories (according to Strong revised criteria, 2017) is shown as follows: 25 cognitively normal (ALS-CN), 13 with cognitive impairment (ALSci), 4 with behavioural impairment (ALSbi), 7 with cognitive and behavioural impairment (ALScbi), 3 with dementia. Not demented patients with some degree of cognitive or behavioural impairment (ALSci-bi-cbi) performance were significantly worse compared to CN patients in RMET ( $p$ 0.003), SETEA $(p<0.001)$ and SET-IA $(\mathrm{p}<0.001)$, as expected. However, also CN patients showed deficit in at least one SC test (10 out of 25 ). Specifically among them, 4 showed deficit in SET-EA. Moreover, out of 4 patients with c9orf72 mutation defined as $\mathrm{CN}$ at diagnosis, 3 showed selective deficit in ER. Multiple linear regression analysis showed significant correlation between SET-IA score and ALSFRS-total score (p 0.012).

Discussion: Our findings supports the hypothesis that ALS-CN patients may show deficit in SC functions, in particular in Emotion Attribution (Affective Theory of Mind), since the early stage of disease. Moreover, it supports the hypothesis of a parallel worsening of cognitive and motor performance in Intention Attribution (Cognitive Theory of Mind) show Social Cognition impairment in ALS patients since the early stage of disease and also in patients classified as cognitively normal.

\section{THE CHARACTERISTICS OF COGNITIVE IMPAIRMENT IN ALS PATIENTS DEPEND ON THE LATERALIZATION OF MOTOR DAMAGE}

L. Peotta ${ }^{1}$, U. Manera ${ }^{1}$, B. Iazzolino ${ }^{1}$, A. Canosa ${ }^{1}$, R. Vasta ${ }^{1}, F$. Palumbo $^{1}$, M. Torrieri ${ }^{1}$, L. Solero ${ }^{1}$, M. Daviddi ${ }^{1}$, M. Grassano ${ }^{1}$, C. Moglia $^{1}$, M. Pagani ${ }^{2}$, A. Chiò ${ }^{1}$

${ }^{1}$ Department of Neuroscience "Rita Levi Montalcini", University of Turin (Torino); ${ }^{2}$ Institute of Cognitive Sciences and Technology, CNR (Roma)

Objectives: Cognitive features of patients with Amyotrophic Lateral Sclerosis (ALS) have never been specifically analyzed according to the lateralization of motor impairment: in the present study we investigated the cognitive performances of ALS patients to describe the relationship between motor and cognitive dysfunction, according to site and side of disease onset.

Materials: Six-hundred and nine ALS patients underwent a comprehensive neuropsychological evaluation at diagnosis in Turin ALS Centre (MMSE, FAB, TMT A-B, Digit Span Forward and Backward, FAS, CAT, RAVLT, BSRT, ROCFT, WCST, CPM47). Demographic and clinical data at the time of onset were collected too, encompassing side and site of onset, hand-dominance, age at onset, years of education and gender.

Methods: Z- scores of neuropsychological tests were calculated and adjusted for age, gender and education. Cognitive performances of patients, grouped by side and site of onset, as well as clinical and 
demographic data, were statistically compared using $\chi 2$ test, KruskallWallis test and Mann Whitney U test.

Results: Bulbar patients and bilateral spinal onset patients (Sbil) were generally characterized by lower cognitive performances in most neuropsychological tests, when compared to patients with lateralized onset (right-side spinal onset, Sri and left-side spinal onset, Sle). Left-side spinal onset patients (Sle) performed significantly better than right-side (Sri) at verbal memory tasks (RAVLT and BSRT). Digit Span Backward and visual memory tasks (ROCFT) median z-scores were significantly higher in Sri patients when compared to bulbar/Sbil patients, while verbal memory tasks (RAVLT and BRST) were higher in Sle patients.

Discussion: Spinal patients with symmetric motor impairment (Sbil) showed significant worse cognitive performance than those with lateralized damage, and appeared to have a cognitive dysfunction similar to bulbar patients. Moreover, we found a strong lateralisation in neuropsychological tests for asymmetric spinal onset patients, reflecting hemispheric functional lateralization of language and visuospatial abilities. Specifically, Sle's left hemisphere relative sparing could justify their better performance at verbal memory tasks, while Sri's right hemisphere relative sparing could explain their better performances at visuospatial abilities and working memory tasks.

Conclusions: Our findings point out that cognitive features of ALS patients are related to motor impairment and follow hemispheric lateralisation, suggesting a possible disease spreading or simultaneous degeneration of highly interconnected frontal and precentral neurons [1]. This is keeping with previous studies which found a direct relationship between lateralized motor and cognitive features $[2,3]$.

References:

1. Chiò A, Pagani M, Agosta F, et al. Neuroimaging in amyotrophic lateral sclerosis: insights into structural and functional changes. Lancet Neurol (2014);13(12):1228-40 doi:10.1016/S14744422(14)70167-X

2. Williams LN, Seignourel P, Crucian GP, et al. Laterality, region, and type of motor dysfunction correlate with cognitive impairment in Parkinson's disease. Mov Disord (2007); 22(1):141-5

3. Kang SH, Park YH, Lee D, et al. The Cortical Neuroanatomy Related to Specific Neuropsychological Deficits in Alzheimer's Continuum. Dement Neurocogn Disord. (2019);18(3):77-95

\section{EPIDEMIOLOGY OF AMYOTROPHIC LATERAL SCLEROSIS IN SARDINIA, 2015 -2019}

V. Pierri, G. Primicerio, G. Cadeddu, E. Bullita, F. Pili, G. Borghero, G. Defazio

Department of Medical Sciences and Public Health and Neurology Unit, AOU Cagliari, University of Cagliari (Cagliari)

Objectives: Incidence and prevalence of amyotrophic lateral sclerosis (ALS) were investigated in a large population from Southern Sardinian, insular Italy (over 900000 population).

Materials and Methods: Incidence rates were computed for the time interval 2015 -2019 whereas prevalence was calculated on December 31 2018 (prevalence day). All ALS patients in the study area were retrospectively included.

Results: The ALS crude incidence from 2015-2019 was $3.97 \mathrm{x}$ 100000 population / year (95\% CIs: $3.44-4.58), 4.83$ in men and 3.15 in women. Onset occurred most often at the age of $70-74$ in both men and women. In the study area, incidence rates varied between $3.04 \mathrm{x}$ 100000 population / year (95\% CIs:1.9 - 4.7) in the Campidano area to 5.1 x 100000 population / year (95\% CIs: 3.3 - 7.5) in Sulcis-Iglesiente area. On December 31, 2018, the ALS crude prevalence (without taking into account tracheostomy) was 8.73 per 100,000 (95\% CIs: 7.03 10.83), 11.07 in men and 6.47 in women. Over the study period $76.6 \%$ of ALS patients of the study area had a spinal onset, while $23.4 \%$ had a bulbar variant. The correspondent incidence rates were 3.04 per 100,000/ year (95\% CIs: 2.58 - 3.58) for spinal onset ALS and 0.93 per 100,000/ year (95\% CIs: $0.69-1.25$ ) for bulbar onset ALS. Prevalence was 7.13 per 100,000 (95\% CIs: $5.62-9.06)$ for the spinal form and 1.6 per 100,000 (95 \% CIs: $0.97-2.63$ ) for the bulbar variant.

Discussion and Conclusions: The population-based incidence and prevalence data from Sardinia indicates that in this area ALS frequency is in the upper part of the worldwide range of variability. [1,2,3]

References:

1. Lu Xu, Tanxin Liu, Lili Liu et al. Global variation in prevalence and incidence of amyotrophic lateral sclerosis: a systematic review and meta-analysis. Journal of Neurology (2020);267:944-53

2. B. Marin, F. Boumèdiene, G. Logroscino et al. Variation in worldwide incidence of amyotrophic lateral sclerosis: a meta-analysis. International Journal of Epidemiology (2017):57-74

3. M. Pugliatti, L. D. Parish, P. Cossu et al. Amyotrophic lateral sclerosis in Sardinia, insular Italy, 1995-2009. J Neurol (2013) Feb;260(2):5729

\section{VALIDATION OF THE ITALIAN VERSION OF SELF- ADMINISTERED ALSFRS-R SCALE}

L. Solero, U. Manera, S. Cabras, M. Daviddi, R. Vasta, M. Torrieri, F. Palumbo, A. Bombaci, M. Grassano, L. Peotta, B. Iazzolino, A. Canosa, A. Calvo, A. Chio', C. Moglia

"Rita Levi Montalcini" Neuroscience Department, AOU Città della Salute e della Scienza, Molinette Hospital (Torino)

Objectives: To validate and assess the reliability of the Italian version of self-administered ALSFRS-R, considering patients' clinical and cognitive features and caregiver's help.

Methods: From 27th March 2020 to 5th May 2020, we recruited ALS patients during regular telemedicine follow-up assessments performed by the Turin ALS Centre due to lockdown measures. All patients met the revised El Escorial Criteria for defined, probable and probable-laboratory supported ALS. By analysing the results of 70 paired self-administered vs standard telephone-administered ALSFRS-R, we calculated overall score, single items scores, ALSFRS-R domain scores, King's and MiToS stage inter-rater agreement and reliability using different validated methods. We created the Italian version of self-administered ALSFRS-R following ENCALS recommendation.

Results: Seventy patients (43 females, 61.4\%) accepted to participate in the study. Correlation between the two scales was 0.94 and no systematic directional bias was found. Intraclass correlation coefficient (ICC) was very high $(>0.90)$ for the vast majority of the considered classification criteria, especially King's total score (0.96) and MiToS score (0.94). The overall ICC for ALSFRS-R did not differ according to gender or cognition, while it was slightly higher when patients answered the ALSFRS-R with the help of the caregiver (0.91 vs 0.95$)$; patients who needed 
caregiver assistance in filling in the ALSFRS-R assessment were significantly older, had a more aggressive disease phenotype, higher motor impairment and/or impaired cognition.

Discussion: In this study, we confirmed that the self-assessed ALSFRS-R score is a valid and reliable tool to monitor disease burden and progression. Moreover, we showed that its reliability increases with the possibility to complete the assessment together with a caregiver, since ALS patients are frequently affected by cognitive impairment even in the early stages of disease.

Conclusions: Online self-administered ALSFRS-R scale is a valid tool to stratify ALS patients into clinical stages and to implement telemedicine monitoring.

References:

- Montes J, Levy G, Albert S, Kaufmann P, Buchsbaum R, Gordon $\mathrm{PH}$, Mitsumoto H. Development and evaluation of a selfadministered version of the ALSFRS-R. Neurology (2006);67(7):1294-6

- Bakker LA, Schroeder CD, Tan HHG, Vugts SMAG, van Eijk RPA, van Es MA, Visser-Meily JMA, van den Berg LH. Development and assessment of the inter-rater and intra-rater reproducibility of a selfadministration version of the ALSFRS-R. J Neurol Neurosurg Psychiatry (2020);91(1):75-81

- Beeldman E, Govaarts R, de Visser M, Klein Twennaar M, van der Kooi AJ, van den Berg LH, Veldink JH, Pijnenburg YAL, de Haan RJ, Schmand BA, et al. Progression of cognitive and behavioural impairment in early amyotrophic lateral sclerosis. J Neurol Neurosurg Psychiatry (2020);91(7):779-80

\section{RANDOMIZED CLINICAL TRIALS IN AMYOTROPHIC LATERAL SCLEROSIS: CURRENT ISSUES AND POSSIBLE SOLUTIONS}

\author{
M. C. Torrieri, U. Manera, R. Vasta, A. Canosa, C. Moglia, A. Chiò, A. \\ Calvo
}

Department of Neurosciences "Rita Levi Montalcini", University of Turin (Torino)

Aims: To compute mortality rates of amyotrophic lateral sclerosis (ALS) patients during a simulation of a randomized clinical trial (RCT), depending on forced vital capacity (FVC) and time interval between disease onset and trial entry, to analyze which prognostic factors should be considered for recruitment.

Materials: We selected the first spirometry of ALS patients included in the PARALS Registry, diagnosed from 1995 to 2016, performed during 4 time intervals from disease onset: 6-12 months (group 1; 453 patients); 12-18 months (group 2; 405 patients); 18-24 months (group 3; 272 patients); 24-36 months (group 4; 263 patients). The date of spirometry corresponded to trial entry.

Methods: For each group mortality rates were computed at $3,6,9,12,18,24$ months from trial entry. Risk for death/tracheostomy was computed using Cox proportional hazards models, adjusted for the most relevant prognostic factors. Patients were further stratified in normal (NPs, $\triangle$ ALSFRS $<1.1$ ) and fast progressors (FPs, $\triangle$ ALSFRS $\geq 1.1$ ). Median survival from trial entry was calculated depending on $\mathrm{FVC} \%$ value at recruitment. Finally patients were considered together and the first spirometry for each patient was included: Cox proportional hazard models were used to investigate if risk factors were different between NPs and FPs.
Results: As the time interval from disease onset increased, patients were younger, the number of women raised, median FVC\% values and the proportion of FPs included decreased. Risk for death/ tracheostomy increased at reducing FVC\% values $(\mathrm{p}<0.001)$. Mortality rates decreased extending time interval onset-recruitment, probably due to the progressive reduction of FPs included. FPs represented the $21.1 \%$ of all patients with a risk for death/tracheostomy increased by $53.8 \%$. The only prognostic factors for FPs were FVC\% and NIMV usage (HR 0.991, $\mathrm{p}<0.001$ and HR 0.618, $\mathrm{p}=0.001$, respectively). Median survival was above 1 year for FPs with $\mathrm{FVC} \%$ $>80.0 \%$ and for NPs with $\mathrm{FVC} \%>60 \%$.

Discussion: Success rate of RCTs for finding a treatment for ALS is unacceptably low, also due to the absence of a homogeneous set of inclusion criteria and to a high mortality rate of included patients. The new guidelines for RCTs suggest to use broader inclusion criteria and to stratify patients for the most relevant prognostic factors. Our data strongly confirm the importance of using prognostic categories, mostly depending on disease progression rate and pulmonary function.

Conclusions: Stratifying patients in prognostic categories is essential for RCTs. Trial entry for FPs should be accelerated increasing the chance of having a good pulmonary function.

References:

- Mora JS, Genge A, Chiò A, et al. Masitinib as an add-on therapy to riluzole in patients with amyotrophic lateral sclerosis: a randomized clinical trial. Amyotroph Lateral Scler Frontotemporal Degener. (2020);21(1-2):5-14. doi:10.1080/21678421.2019.1632346

- U.S. Department of Health and Human Services Food and Drug Administration. Amyotrophic Lateral Sclerosis: Developing Drugs for Treatment Guidance for Industry. September 2019. Available at: https://www.fda.gov/media/130964/download

- Van den Berg LH, Sorenson E, Gronseth G, et al. Revised Airlie House consensus guidelines for design and implementation of ALS clinical trials. Neurology (2019);92(14):e1610-e1623. doi:10.1212/ WNL.0000000000007242

\section{TARGETED SEQUENCING PANELS IN ITALIAN ALS PATIENTS SUPPORT DIFFERENT ETIOLOGIES IN THE ALS/ FTD CONTINUUM}

V. Vacchiano ${ }^{1}$, A. Bartoletti-Stella ${ }^{2}$, S. De Pasqua ${ }^{3}$, G. Mengozzi $^{2}$, D. De Biase $^{4}$, I. Bartolomei ${ }^{2}$, P. Avoni ${ }^{1}$, R. Giovanni ${ }^{1}$, P. Parchi ${ }^{5}$, V. Donadio ${ }^{2}$, A. Chiò ${ }^{6}$, A. Pession ${ }^{4}$, F. Oppi ${ }^{2}$, F. Salvi ${ }^{2}$, R. Liguori ${ }^{1}$, S. Capellari ${ }^{1}$

${ }^{1}$ Deparment of Biomedical and Neuromotor Sciences of Bologna, University of Bologna, IRCSS Institute of Neurological Sciences of Bologna (Bologna); ${ }^{2}$ IRCSS Institute of Neurological Sciences of Bologna (Bologna); ${ }^{3}$ Deparment of Biomedical and Neuromotor Sciences of Bologna, University of Bologna (Bologna); ${ }^{4}$ Department of Pharmacy and Biotechnology, Molecular Diagnostic Unit, University of Bologna (Bologna); ${ }^{5}$ Department of Experimental Diagnostic and Specialty Medicine, IRCSS Institute of Neurological Sciences of Bologna (Bologna); ${ }^{6}$ Rita Levi Montalcini Department of Neuroscience, University of Turin (Torino)

Background: $5-10 \%$ of patients with amyotrophic lateral sclerosis (ALS) have a positive family history (fALS). More than 30 genes have been identified in association with ALS/Frontotemporal dementia (FTD) spectrum. However, mutations in four major genes (C9orf72, SOD1, FUS, TARDBP) account for $60 \%-70 \%$ of fALS [1]. Recent studies have 
highlighted the role of genetic risk factors even in sporadic patients (sALS), in which the inheritability would represent about $21 \%$ [2]. We aimed to assess the contribution to the pathogenesis of major and rare ALS related genes in an Italian cohort.

Methods: 330 ALS patients (63 years, range 27-87) were analyzed for ALS related genes by direct sequencing or Next Generation Sequencing multigene panels. Regarding the family history, we differentiated patients with a positive family history for ALS (fALS-ALS) from patients with a family history for other neurodegenerative diseases (fALS-ND), i.e. dementia and parkinsonism.

Results: $9.09 \%$ of our patients were fALS-ALS, while $16.36 \%$ were fALS-ND, accounting for a total of $25.45 \%$ defined as fALS. 55 patients $(16.67 \%)$ presented a pathogenic or probably pathogenic genetic abnormality in one of the four major genes. Mutation frequency was $41.67 \%$ in fALS and $8.13 \%$ in sALS. Frequencies were significantly different between fALS-ALS and fALS-ND patients $(76.7 \%$ vs. $24.1 \%, \mathrm{p}<0.0001)$. The repeated expansion of hexanucleotide in the C9orf72 gene was the most frequent genetic abnormality (7.27\% of all cases, $21.43 \%$ of fALSALS and $18.52 \%$ of fALS-ND cases), followed by mutations in SOD1 (overall $4.85 \%, 26.67 \%$ of fALS-ALS), FUS (overall $2.12 \%, 10 \%$ fALSALS and $1.85 \%$ fALS-ND) and TARDBP (2.42 of all cases, $13.33 \%$ of fALS-ALS and $1.85 \%$ of fALS-ND) genes. We also identified 27 different variants in genes rarely involved in ALS and FTD/ALS spectrum. Of these, eight were not previously reported, and considered potentially pathogenic based on the in silico prediction of pathogenicity. SQSTM1 (3.38\%), and CHMP2B (1.93\%) were the most frequent genes. Together, rare FTD/ALS genes accounted for $12 \%$ of our population. fALS-ND patients were more likely to carry a mutation in rare FTD/ALS genes (20\%) as compared to fALS-ALS patients $(4.1 \%)$.

Discussion and conclusion: In our cohort, the frequency mutation of major and rare ALS genes was partially consistent with previous findings [3]. Interestingly, variant distribution showed a clustering of ALS major genes variants in fALS-ALS patients, while FTD/ALS related genes variants were grouped into fALS-ND cases. These data pointed out that family history may be considered in order to choose a genetic screening protocol.

References:

1. Corcia P, Couratier P, Blasco H, et al. Genetics of amyotrophic lateral sclerosis. Rev Neurol (Paris). (2017);173(5):254-62

2. Keller MF, Ferrucci L, Singleton AB, et al. Genome-wide analysis of the heritability of amyotrophic lateral sclerosis. JAMA Neurol. (2014);71(9):1123-34

3. Lamp M, Origone P, Geroldi A, et al. Twenty years of molecular analyses in amyotrophic lateral sclerosis: genetic landscape of Italian patients. Neurobiol Aging (2018);66:179.e5-179.e16

\section{NECK FLEXORS WEAKNESS AT DIAGNOSIS PREDICTS RESPIRATORY IMPAIRMENT IN AMYOTROPHIC LATERAL SCLEROSIS}

\author{
R. Vasta, M. Torrieri, F. D'ovidio, A. Circiello, F. De Mattei, U. Manera, \\ A. Canosa, A. Calvo, A. Chio', C. Moglia
}

ALS Center, Department of Neuroscience, University of Turin (Torino)

Objectives: Neck flexors weakness at diagnosis has been previously suggested to influence prognosis in Amyotrophic Lateral Sclerosis (ALS) [13]. Here, we aimed to assess the prognostic role of neck muscles weakness at diagnosis with respect to survival and respiratory impairment using a large cohort of ALS patients.
Methods: We conducted a retrospective cohort study. All ALS patients seen in the Turin ALS Center from 2007 to 2014 were included. Muscles strength at diagnosis was evaluated using the Medical Research Council (MRC) scale. Survival was considered as time from diagnosis to death or tracheostomy; time to respiratory impairment was considered as the interval from diagnosis to the first event among ALSFRSr 10 item $<4$, forced vital capacity $<70 \%$, start of non-invasive ventilation or tracheostomy. Time from diagnosis to dysarthria, dysphagia and walking impairment were considered as secondary outcomes. Cox proportional Hazard regression models adjusted by sex, age at diagnosis, diagnostic delay, onset site, genetics status and other muscles groups MRC scores were used to assess the prognostic role of neck muscles.

Results: A total of 370 patients were included in the study. Fifty-nine (15.9\%) patients showed neck flexors weakness at diagnosis; MRC values were mostly concord for neck extensors. Neck flexors were the only muscles able to predict survival (HR 0.49 , 95\% CI $0.28-0.86$; $\mathrm{p}=0.01$ ). Furthermore, neck flexors normal strength decreased the risk of respiratory impairment $(\mathrm{HR}=0.46,95 \%$ CI $0.22-0.96 ; \mathrm{p}=0.04)$ but did not influence any secondary outcomes.

Discussion: Neck flexors weakness at diagnosis predicts survival and respiratory impairment in ALS. Since analyses were adjusted for other muscles groups, this result does not reflect an overall poor strength, but suggests a specific mechanism of neck flexors in influencing ALS patients' respiratory functionality. Furthermore, the relationship was shown to be specific to respiratory impairment rather than to a general worsening in muscle strength. Based on these results, it can be hypothesized that neck flexors weakness predicts respiratory impairment and eventually death in part because of their recruitment as accessory respiratory muscles.

Conclusions: Neck flexors weakness at diagnosis should be included among ALS prognostic factors. We believe this result could be valuable for both planning of patients' interventions and clinical trials' design. References:

1. Nakamura R, Atsuta N, Watanabe H, Hirakawa A, Watanabe H, Ito $\mathrm{M}$, et al. Neck weakness is a potent prognostic factor in sporadic amyotrophic lateral sclerosis patients. J Neurol Neurosurg Psychiatry (2013) Dec;84(12):1365-71

2. Pinto S, de Carvalho M. Motor responses of the sternocleidomastoid muscle in patients with amyotrophic lateral sclerosis. Muscle Nerve (2008) Oct;38(4):1312-7

3. Yu C-J, Wang L, Mao S-L, Zhang Y, Song L-L, Cai L-Y, et al. The clinical assessment of amyotrophic lateral sclerosis patients' prognosis by ZNF512B gene, neck flexor muscle power score and body mass index (BMI). BMC Neurol. (2018) Dec 19;18(1):211

\section{MANAGEMENT OF PATIENTS WITH MOTONEURON DISEASE IN BERGAMO PROVINCE AT THE TIME OF CORONA VIRUS EMERGENCY}

\author{
M. Vedovello, V. Bonito, A. Biemmi, M. Sessa
}

Neurology Unit, ASST Papa Giovanni XXIII (Bergamo)

Introduction: Bergamo province has been the worst affected italian area by the COVID-19 pandemic. Since the end of February 2020 our hospital has been the reference point for severe cases of COVID-19 with 500 out of 780 beds dedicated to the management of this condition [1]. From March the 8th out patients clinics and "one day admissions" appointments were cancelled. Nine neurologists were deployed to the COVID-19 Units. 
Objective: To evaluate the management and the clinical outcome of Motor Neuron Disease (MND) patients during the pandemic peak from March to May 2020.

Results: In 50 out of 120 patients referring to our centre, clinical assessment trough telephone calls was carried out. 13 reported from February to April symptoms consistent with viral pneumonia, a half of whom associated with fever. None had radiological investigation nor SARS CoV-2 tests. No admission to hospital was carried out for non invasive ventilatory support, tracheostomy or percutaneous endoscopic gastrostomy (compared respectively to 3, 2 and 4 of the same period of 2019). We could not obtain any advance care planning disposition (compared to 3 of the same period of 2019). Only three patients were admitted to the hospital at the Emergency Unit: two turned out to have acute respiratory failure caused by SARS CoV-2. One patient died in the COVID-19 Unit, one was rapidly released from hospital. Between January and May 2020 the deaths were 18 out of 120 ( 6 in care homes) compared to 11 out of 111 (4 in care homes) of 2019; only one death was attributable to COVID-19. In the emergency period only one patient died in a care home for acute respiratory failure with non-infectious etiology.

Discussion: The deployment of the majority of the resources for COVID-19 brought the care of MND patients to a complete halt. That stresses the shortage of resources for chronic neurological patients, specially from a territorial point of view. From our data we cannot draw conclusions on incidence and outcome of SARS CoV-2 infection in our patients, however our impression is that these were not more susceptible than the general population even in the care homes, where the reported overall mortality in the Bergamo province was as high as $35 \%$. The implementation of remote-medical-care can lead to a more safe rational and efficient management of MND patients. On top of that a greater financial commitment on territorial medicine as well as the institution of a multidisciplinary team dedicated to chronic neurological patients are desiderable.

Reference:

1. Fagiuoli S. Lorini FL, Remuzzi G. Adaptations and Lessons in the Province of Bergamo. N Eng J of Med. (2020) May 21;382(21):e71

\section{CEREBROSPINAL FLUID PHOSPHORYLATED NEUROFILAMENT HEAVY CHAIN AND CHITOTRIOSIDASE IN PRIMARY LATERAL SCLEROSIS}

F. Verde ${ }^{1}$, G. Zaina ${ }^{1}$, C. Bodio $^{2}$, M. Borghi ${ }^{1}$, D. Soranna ${ }^{2}$, S. Peverelli ${ }^{2}$, N. Ticozzi ${ }^{1}$, C. Morelli ${ }^{2}$, A. Doretti ${ }^{2}$, S. Messina ${ }^{2}$, L. Maderna ${ }^{2}$, C. Colombrita $^{2}$, V. Gumina ${ }^{2}$, C. Tiloca ${ }^{2}$, P. Meroni ${ }^{1}$, A. Zambon ${ }^{1}$, A. Ratti $^{1}$, V. Silani ${ }^{1}$

${ }^{1}$ Istituto Auxologico Italiano IRCCS, University of Milan (Milano); ${ }^{2}$ Istituto Auxologico Italiano IRCCS (Milan)

Objectives: To evaluate the two ALS CSF biomarkers phosphorylated neurofilament heavy chain (pNFH) and chitotriosidase (Chit1) reflecting axonal degeneration and microglial inflammation, respectively - as biomarkers for primary lateral sclerosis (PLS), with special reference to the differential diagnosis between PLS and ALS. PLS is a rare upper motor neuron disease with slower progression and longer survival compared to ALS. Diagnostic differentiation between PLS and ALS is crucial but sometimes difficult, especially in the early phases, resulting in delayed or wrong diagnosis.

Materials: We included patients evaluated at our Institute between 2010 and 2019. Among our large consecutive series of PLS patients (n $=52$ ), we had to focus on those 10 (males $[\mathrm{M}]=5$, females $[\mathrm{F}]=5)$ who had undergone a lumbar puncture and whose CSF was stored in our biobank. The other categories were ALS patients $(\mathrm{n}=28 ; \mathrm{M}=16, \mathrm{~F}=$ 12) and patients with neurological complaints but without neurodegenerative diseases (neurological controls, $\mathrm{NCs} ; \mathrm{n}=30 ; \mathrm{M}=13, \mathrm{~F}=17$ ).

Methods: CSF levels of pNFH and Chit1 were measured with commercially available ELISA assays.

Results: Both pNFH and Chit1 showed a gradient with high levels in ALS, intermediate levels in PLS, and low levels in NCs. pNFH enabled good discrimination between ALS and NCs (area under the ROC curve [AUC], 0.996) and between PLS and NCs (AUC, 0.933), also showing promise in distinguishing ALS from PLS (AUC, 0.771). Chit1 had slightly lower AUCs (ALS vs NCs: 0.981; PLS vs NCs: 0.848; ALS vs PLS: 0.740), but the differences between the AUCs of the two biomarkers were not statistically significant. The combination of $\mathrm{pNFH}$ and Chit 1 was not superior to either biomarker alone for differentiating between ALS and PLS (AUC, 0.790). Moreover, pNFH correlated with disease progression rate $(\mathrm{PR})$ in ALS $(r=0.529)$ and in MND (ALS + PLS; $r=0.620)$, while Chit1 correlated with PR in MND $(r=0.504)$. Finally, pNFH levels were negatively associated with survival in ALS $(\mathrm{HR}=4.42)$.

Discussion: In PLS, both pNFH and Chit1 have intermediate levels between ALS and NCs, probably reflecting a lower intensity of axonal degeneration and microglial inflammation compared to ALS. This supports use of pNFH and conceivably Chit1 for distinguishing PLS from NCs, but also, more importantly, for the differential diagnosis between PLS and ALS.

Conclusions: pNFH and possibly Chit1 deserve consideration for use as diagnostic biomarkers for PLS.

References:

- Steinacker P, Feneberg E, Weishaupt J, Brettschneider J, et al. Neurofilaments in the Diagnosis of Motoneuron Diseases: A Prospective Study on 455 Patients. J Neurol Neurosurg Psychiatry (2016);87:12-20

- Thompson AG, Gray E, Bampton A, Raciborska D, Talbot K, Turner MR. CSF chitinase proteins in amyotrophic lateral sclerosis. J Neurol Neurosurg Psychiatry (2019);90:1215-20

- Turner MR, Barohn RJ, Corcia P, Fink JK, et al. Delegates of the 2nd International PLS Conference, Mitsumoto H. Primary lateral sclerosis: consensus diagnostic criteria. J Neurol Neurosurg Psychiatry (2020);91:373-77

\section{NEUROMUSCULAR DISEASES}

\section{TACKLING OXALIPLATIN INDUCED PERIPHERAL NEUROTOXICITY: FROM BED TO BENCH SIDE AND BACK}

P. Alberti, E. Ballarini, A. Canta, A. Chiorazzi, G. Fumagalli, A. Malacrida, L. Monza, E. Pozzi, C. Meregalli, P. Marmiroli, G. Cavaletti

Experimental Neurology Unit, University of Milano-Bicocca (Monza)

Objectives: Oxaliplatin (OHP) induced peripheral neurotoxicity (OIPN), a potentially persistent side effect with no treatment, is not only characterized by a sensory, length-dependent, chronic polyneuropathy: acute manifestations occur nearly in all OHP treated patients and they have a striking resemblance with genetic channelopathies causing axonal hyperexcitability; they last 24-72 hours after each OHP administration. Even though they are transient and never dose-limiting, they mirror a state of axonal hyperexcitability that leads to cellular stress and neuronal damage; acute OIPN predisposes to chronic OIPN, therefore, acute OIPN is a 
target for preventing chronic OIPN. We aimed to devise a mouse model able to reproduce both phenomena to ensure a translational approach in finding a cure for OIPN.

Methods: Balb/c mice were treated with OHP $7 \mathrm{mg} / \mathrm{Kg}$, iv, once a week per 8 weeks (1qw8ws), and compared with a vehicle treated group $(n=8$ each). Acute neurotoxicity was assessed with nerve excitability testing (NET) soon after the 1st administration. Chronic neurotoxicity was assessed with nerve conduction studies (NCS), behavioural tests and neuropathology (IENF and caudal nerve morphometry). To demonstrate the acute changes induced by OHP at each administration, despite transient, were able to determine a pathological depolarization in neurons we measured NCX2 levels (western blot [WB]) on DRG pool at the end of treatment.

Results: After the 1st administration changes at NET were compatible con acute neurotoxicity (recovery cycle was matching alterations known from clinical practice). NCS, behavioural tests and neuropathology at the end of 2 months of treatment showed a significant difference between the 2 groups, demonstrating an axonal, sensory, polyneuropathy had ensued in OHP group. NCX2 WB analysis showed a significant decrease in OHP group $(\mathrm{p}<0.001)$

Discussion: NET allowed us to demonstrate acute OIPN had ensued since the 1st administration and end of treatment outcome measures confirmed the schedule was able to induce all morphological and functional changes typical of chronic OIPN. Notably, NCX2 downregulation seen on DRG pool allowed us to confirm that our model was able to induce a relevant depolarization in neurons: a relevant - non physiological - depolarization is, in fact, known to induce $\mathrm{NCX} 2$ downregulation.

Conclusion: Our schedule, $7 \mathrm{mg} / \mathrm{Kg}$, iv, 1qw8ws, was able to induce all phenomena known from clinical practice and confirmed the presence of a sustained, despite transient, depolarization in neurons, making our model comprehensive of OHP induced neuronal alterations in patients. Therefore, our model can be suggested as ideal to test translationally new neuroprotectants against OIPN.

\section{LONG-TERM FOLLOW-UP OF THE PHASE 1 START TRIAL OF ONASEMNOGENE ABEPARVOVEC GENE THERAPY IN SPINAL MUSCULAR ATROPHY TYPE 1}

M. Bernat Fuertes ${ }^{1}$, J. R. Mendell ${ }^{2}$, R. Shell ${ }^{3}$, K. J. Lehman ${ }^{4}$, M. $\mathrm{McColly}^{4}$, L. P. Lowes ${ }^{2}$, L. N. Alfano ${ }^{4}$, N. F. Miller ${ }^{4}$, M. A. Iammarino $^{4}$, K. Church ${ }^{4}$, S. P. Reyna ${ }^{1}$, H. Ouyang ${ }^{1}$, E. Kernbauer ${ }^{1}$, S. Joshi $^{1}$, M. Meriggioli ${ }^{1}$, A. Kleyn ${ }^{1}$, S. Al-Zaidy ${ }^{5}$

${ }^{1}$ AveXis, Inc. United States (Bannockburn-USA); ${ }^{2}$ Center for Gene Therapy, Nationwide Children's Hospital; Department of Pediatrics, Ohio State University; Department of Neurology, Ohio State University (Columbus-USA); ${ }^{3}$ Department of Pediatrics, Ohio State University; Department of Neurology, Ohio State University; Section of Pulmonary Medicine, Nationwide Children's Hospital (Columbus-USA); ${ }^{4}$ Center for Gene Therapy, Nationwide Children's Hospital (Columbus-USA); ${ }^{5} \mathrm{Al}-$ Zaidy and Associates, LLC (Columbus-USA)

Targets: Onasemnogene abeparvovec (formerly AVXS-101) is designed to address the genetic root cause of spinal muscular atrophy type 1 (SMA1). In the phase 1 trial (START; NCT02122952), patients who received a one-time proposed therapeutic dose infusion $(n=12)$ demonstrated significantly improved outcomes versus untreated natural history. Here, we evaluate long-term safety in patients previously treated in START and long-term efficacy in patients from both Cohorts. START patients could electively enroll into a LTFU study (NCT03421977).
Methods: Primary objective: long-term safety. Patients have annual visits (5 years) followed by annual phone contact (additional 10 years). Assessments include medical history/record review, physical examination, clinical laboratory evaluation, pulmonary assessments, and milestone maintenance.

Results: As of 31 Dec 2019, 13 patients (low dose, n=3; therapeutic dose, $n=10$ ) were enrolled. The oldest patients were aged 6.2 (low dose) and 5.6 (therapeutic dose) years. Serious adverse events (AEs) were reported in 8/13 patients; however, no serious AEs were considered related to treatment or lead to study discontinuation supporting a favorable riskbenefit profile. All patients who received the therapeutic dose have survived and are free of permanent ventilation (mean [range] age at last datacut: 4.8 [4.3-5.6] years; mean [range] time since dosing: 4.5 [4.15.2] years). These patients have either maintained all previously attained milestones or gained new milestones; 2 patients have newly achieved standing with assistance while not receiving concomitant survival motor neuron 2 protein (SMN2) upregulating therapy at any point. Of the 10 enrolled patients who received therapeutic dose, 6 did not require regular, daily respiratory support more than 4 years after dosing. Additionally, 6 have never received concomitant SMN2 upregulating therapy.

Discussion: Onasemnogene abeparvovec demonstrated sustained and durable efficacy up to 5.2 years (therapeutic dose cohort) from initial dosing, in patients up to 5.6 years of age. No AEs of special interest have been reported to date, specifically none associated with liver enzyme elevations, hematology values, new malignancies or autoimmune disorders.

Conclusions: Onasemnogene abeparvovec shows a favorable riskbenefit profile, and continues to demonstrate efficacy with new milestone developments.

\section{ONASEMNOGENE ABEPARVOVEC GENE THERAPY IN PRESYMPTOMATIC SPINAL MUSCULAR ATROPHY: SPR1NT STUDY UPDATE}

M. Bernat Fuertes ${ }^{1}$, K. A. Strauss ${ }^{2}$, M. A. Farrar ${ }^{3}$, K. J. Swoboda ${ }^{4}$, K. Saito $^{5}$, C. A. Chiriboga ${ }^{6}$, S. T. Iannaccone ${ }^{7}$, J. M. Krueger ${ }^{8}$, J. M. Kwon ${ }^{9}$, H. J. McMillan ${ }^{10}$, L. Servais ${ }^{11}$, J. R. Mendell ${ }^{12}$, J. Parsons ${ }^{13}$, M. Scoto ${ }^{14}$, P. B. Shieh ${ }^{15}$, C. Zaidman ${ }^{16}$, M. Schultz ${ }^{1}$, S. P. Reyna ${ }^{1}$, S. Kavanagh ${ }^{1}$, D. Chand $^{1}$, S. Tauscher-Wisniewski ${ }^{1}$, B. E. McGill ${ }^{1}$, F. Muntoni ${ }^{14}$

${ }^{1}$ AveXis, Inc., (Bannockburn-USA); ${ }^{2}$ Clinic for Special Children (Strasburg-USA), ${ }^{3}$ Department of Neurology, Sydney Children's Hospital Network, UNSW Sydney, Sydney Children's Hospital Network; (Randwick; Sydney-AUS); ${ }^{4}$ Department of Neurology, Massachusetts General Hospital (Boston-USA); ${ }^{5}$ Institute of Medical Genetics, Tokyo Women's Medical University (Tokyo-J); ${ }^{6}$ Division of Pediatric Neurology, Columbia University Medical Center (New YorkUSA); ${ }^{7}$ Department of Pediatrics, University of Texas Southwestern Medical Center (Dallas-USA); ${ }^{8}$ Department of Neurology, Helen DeVos Children's Hospital (Grand Rapids-USA); ${ }^{9}$ Department of Neurology, University of Wisconsin School of Medicine and Public Health, (Madison-USA); ${ }^{10}$ Department of Pediatrics, Children's Hospital of Eastern Ontario, (Ottawa-CDN); ${ }^{11}$ Department of Pediatrics, MDUK Oxford Neuromuscular Center (Oxford-UK); ${ }^{12}$ Center for Gene Therapy, Nationwide Children's Hospital, Department of Pediatrics, Ohio State University, Department of Neurology, Ohio State University, Nationwide Children's Hospital; Ohio State University (Columbus-USA); ${ }^{13}$ Department of Pediatrics, University of Colorado School of Medicine, (Aurora-USA); ${ }^{14}$ The Dubowitz Neuromuscular Centre, University College London, Great Ormond Street Institute of Child Health (London-UK); ${ }^{15}$ Department of 
Neurology, David Geffen School of Medicine at UCLA (Los AngelesUSA); ${ }^{16}$ Department of Neurology, Division of Pediatric Neurology, Washington University School of Medicine (St. Louis-USA)

Targets: Spinal muscular atrophy (SMA) causes loss of motor/respiratory function due to survival of motor neuron 1 (SMN1) gene deletion/mutation. Copies of a similar gene (SMN2) modify disease severity. Here, we assess the safety and efficacy of onasemnogene abeparvovec (formerly AVXS-101) in presymptomatic SMA patients.

Methods: SPR1NT (NCT03505099) is an ongoing multicenter, openlabel, phase 3 study. Asymptomatic patients expected to develop SMA $(2-3 \mathrm{SMN} 2$, aged $\leq 6$ weeks) receive a one-time IV infusion and are assessed through 18/24 (2/3SMN2) months. Primary outcomes: sitting for $\geq 30$ seconds $(2 \mathrm{SMN} 2) /$ standing unassisted for $\geq 3$ seconds (3SMN2). Secondary outcomes: survival and independent feeding with normal weight (2SMN2); independent walking (3SMN2). Exploratory outcome: motor function improvement (CHOP INTEND [2SMN2]). Safety outcomes: incidence of adverse events (AEs)/serious AEs.

Results: As of 31 Dec 2019, 30 patients were dosed (2SMN2/3SMN2/ $4 \mathrm{SMN} 2, \mathrm{n}=14 / 15 / 1)$ and enrollment is complete. Mean age at dosing (days, range): 2SMN2, 20.6 (8.0-34.0); 3SMN2, 28.7 (9.0-43.0). Mean age at last follow-up visit (months, range): 2SMN2, 11.2 (6.0-18.6); 3SMN2, 9.7 (3.3-15.1). All patients are alive did not use ventilatory support as of last visit. Among 2SMN2 patients, all have achieved CHOP INTEND scores of $\geq 50$, which exceeds the maximal score observed in untreated patients; 8 sat (all within the World Health Organization (WHO) 1st-99th percentile; range: 5.7-11.8 months); 4 stood and walked alone (range: $12.2-18.3$ months). Among 3SMN2 patients, 4 stood alone (9.5-12.4 months); 3 walked alone (12.2-15.1 months). Remaining patients in both cohorts who have not achieved these milestones have not yet passed the normal age range for attaining these milestones. All patients are able to maintain weight without need for feeding support, and most remained within the appropriate, healthy weight range and are thriving. $30 / 30$ patients experienced $\geq 1 \mathrm{AE}$; treatment-related AEs and AEs of special interest were reported in 17/ 30 patients and 26/30 patients had elevations in liver function tests that resolved with prednisolone. All serious AEs (6/30 patients) resolved and were considered unrelated to treatment. Reported AEs were manageable and consistent with the known safety profile of onasemnogene abeparvovec.

Discussion: Prompt diagnosis and early treatment intervention may minimize motor neuron loss and maximize patient benefit.

Conclusions: Presymptomatic SMA patients dosed with onasemnogene abeparvovec continued to meet primary endpoints (WHO motor milestones), demonstrating a clinically significant therapeutic benefit. Presymptomatic treatment enables achievement of ageappropriate motor milestones.

\section{A RARE CASE OF GUILLAIN-BARRÉ SYNDROME ASSOCIATED WITH CAVITARY PULMONARY LESION}

\author{
M. Cantone ${ }^{1}$, F. Borrata ${ }^{2}$, L. Iurato $^{1}$, I. D. Mazzù ${ }^{1}$, C. Occhipinti ${ }^{1}$, M. G. \\ Randisi ${ }^{1}$, A. Terminella ${ }^{2}$, M. M. Vecchio ${ }^{1}$
}

${ }^{1}$ Department of Neurology, Sant'Elia Hospital, ASP (Caltanissetta); ${ }^{2}$ Thoracic Surgery, Department of Cardiac, Thoracic and Vascular Surgery, University Hospital Policlinico "G. Rodolico (San Marco of Catania-CT)

Guillain-Barré Syndrome (GBS) is an acute neuropathy characterized by ascending muscle weakness and areflexia and it is the most common cause of acute flaccid paralysis. In most cases the onset is secondary to respiratory or gastrointestinal infections [1,2] and its pathogenesis is related to the auto-antibodies that bind to epitopes of the peripheral nervous system. We describe the case of GBS associated with an atypical lung cavitary lesion.

Clinical case: A 37-year-old man presented after a two-weeks history of progressive distal sensitive deficit and slight paresis with difficulty walking. The neurological examination revealed an atassic gait, decreased distal muscle strength with $4 / 5$ power in limbs and absent tendon reflexes. In the suspicious of GBS the patient performed complete blood examination, lumbar puncture, neurography, brain and spinal magnetic resonance imaging (MRI). The cerebrospinal fluid presented albumino-cytological dissociation and it was negative for neurotrophic viral or bacterial antigen. Neurography showed the absence of the $\mathrm{F}$ waves and bilateral radicular involvement at lower extremities. The MRI showed enhancement of the extra special tract of the lumbar nerve roots. Chest X-ray showed a cavitary lesion in the left lung and Computed Tomography (CT) confirmed the presence of the cavitary lesion in the left upper lobe $(60 \times 48 \times 35 \mathrm{~mm})$. Nasopharyngeal-swab for SARS-CoV-2 (COVID19), Mantoux reaction, Ida test, autoimmune and HIV screens were negative.

Results and discussion: The patient was referred to Thoracic Surgery were a flexible fiberoptic video-bronchoscopy was performed. Biopsies of the lesion and bronchoalveolar lavage (BAL) were taken during the procedure. Phatologic exam was negative for malignancy. BAL culture showed the presence of Pseudomonas Aeruginosa. The patient was initially treated with intravenous immunoglobulin with moderate clinical recovery and later treated with Ciprofloxacin as indicated in the antibiogram. The patient was well at 3-month follow-up and chest CTscan demonstrated sub-total resolution of the cavitary lesion. To date, several studies has shown evidence for Campylobacter Jejuni, Haemophilus influenzae, Cytomegalovirus, and Epstein-Barr virus to be causative infections in GBS patients [1,2]. However, there are no known cases of GBS associated with this atypical cavitary lung lesion extending the broad pathological spectrum of GBS.

References

1. Yuki N. Infectious origins of, and molecular mimicry in, GuillainBarre and Fisher syndromes. Lancet Infect Dis (2001);1:29-37

2. Koga M, Gilbert M, Li J, Koike S, Takahashi M, Furukawa K, Hirata K, Yuki N. Antecedent infections in Fisher syndrome: a common pathogenesis of molecular mimicry. Neurology (2005);64:1605-11

\section{CLINICAL AND MOLECULAR CHARACTERIZATION IN AN ITALIAN COHORT OF POLG MUTATED MITOCHONDRIAL PATIENTS}

L. Caumo ${ }^{1}$, L. Bello ${ }^{1}$, C. Discardi ${ }^{1}$, P. Riguzzi $^{1}$, V. Zangaro ${ }^{1}$, M. Valentino $^{2}$, L. Caporali ${ }^{3}$, G. Sorarù ${ }^{1}$, R. Manara ${ }^{4}$, V. Carelli ${ }^{2}$, E. Pegoraro ${ }^{1}$

${ }^{1}$ Department of Neurosciences, University of Padua (Padova); ${ }^{2}$ Department of Biomedical and Neuromotor Sciences (DIBINEM), University of Bologna (Bologna); ${ }^{3}$ IRCCS Institute of Neurological Sciences of Bologna, Bellaria Hospital (Bologna); ${ }^{4}$ Neuroradiology Unit, Department of Neurosciences, University of Padua (Padova)

Aims: POLG-disease is a rare genetically determined mitochondrial disease. It is caused by mutations of the nuclear POLG gene which 
affect the function of the DNA polymerase of mitochondria damaging mitochondrial replication and leading to cellular and tissue dysfunction and, thus, to a multi-systemic disease. The aim of this study is to evaluate the clinical, neurophysiological, neuroimaging and muscle histolopathological features in a subset of mitochondrial patients harbouring POLG gene mutations in order to allow a prompt diagnosis and widen the clinical spectrum of POLG-related diseases.

Methods: This study was designed as an observational crosssectional study of clinical, neuroimaging, histopathological, and molecular features in POLG-related diseases. Patients were identified through screening of the database of the Neuromuscular Center of the University of Padova. Ten patients belonging to six families were identified.

Results: Based on the disease progression, the presenting organ system, and the combination of symptoms we identified four major clinical syndromes: PEO (progressive external ophthalmoplegia) in 3/7, MNGIE-like (mitochondrial neurogastrointestinal encephalomyopathy) in $3 / 7$, ataxia neuropathy spectrum (MIRAS and SANDO) plus dystonia in $1 / 7$ and myoclonus. Of the 7 index cases only two PEO patients have the classical phenotype associated to POLG mutations, where all the others differ in significant clinical and diagnostic aspects. Two twins brothers present with a MNGIElike phenotype but at a difference from other reports also showed a severe leukoencephalopathy and demyelinating neuropathy. One twin manifested a Wernicke encephalopathy that rapidly resolved with thiamine supplementation. Muscle biopsy showed only in two PEO cases the typical features of a mitochondrial disease (RRFs, COX-negative/SDH-positive fibers), while in the other not specific abnormalities pointing to a mitochondrial problem were detected. We identified 11 different POLG mutations, including 3 novel variants (Y233D, R853G, R597Q) and 8 previously reported mutations (A467T, W748S in cis with E1143G and S28C, P587L in cis with T251I, G517V, A143V). Segregation analysis in two families allowed the identification of POLG silent allele in the heterozygous status.

Discussion and Conclusions: Our study add to the clinical heterogeneity of the POLG-related disease and underlies the difficulties in genotype-phenotype correlations. The correct combination of clinical observation, ancillary test, muscle biopsy and neuroimaging may lead to the suspicion of a mitochondrial or POLG-related disease, but only the identification of POLG mutations poses the final diagnosis but opens also a Pandora box given the difficulties to demonstrate the pathogenetic role of the variants identified.

\section{THE THIAMINE-DEFICIENCY ACUTE NEUROMUSCULAR INVOLVEMENT DILEMMA}

\section{R. D'Angelo ${ }^{1}$, E. Merli $^{2}$, G. Asioli ${ }^{2}$, M. Guarino $^{1}$, R. Rinaldi $^{1}$}

${ }^{1}$ IRCCS Institute of Neurological Sciences of Bologna, UOC Metropolitan Neurology Clinic NEUROMET, Neurology AOU S.Orsola-Malpighi, University of Bologna (Bologna); ${ }^{2}$ Department of Biomedical and Neuromotor Sciences, University of Bologna (Bologna)

Objective: Thiamine-deficiency (TD) may occur in various clinical contexts including dietary imbalance, chronic alcoholism, postgastrectomy and hyperemesis. Clinical manifestations encompass central nervous system (Wernicke-Korsakoff-syndrome, WKS), peripheral nervous system (dry-beriberi, DB) and cardiovascular (wet-beriberi) dysfunction. DB classically presents with a symmetrical distal sensory/motor peripheral neuropathy, nevertheless few cases of proximal neuromuscular phenotypes have been reported. Regarding this last presentation, neither the primarily affected tissue (proximal nerves or muscles) nor the pathogenic mechanism are ascertain.

Materials and Methods: Patient-1 is a 27-year-old African female refugee, 14-weeks pregnant, admitted for irrepressible hyperemesis, general weakness and confusion, with concomitant hyperparathyroidism and hyperthyroidism. Patient-2 is a 43-year-old EasternEuropean woman with moderate alcohol consumption and liver cirrhosis, admitted for severe ascites, jaundice, and pulmonary infection in intensive-care. After two days, she complained of progressive severe lower-limb weakness.

Results: Patient-1 was confused and slow-reactive; we observed wide-jerk nystagmus, proximal lower-limb hyposthenia with quadriceps myalgias and Lasegue sign bilaterally. Brain-MRI revealed diencephalon FLAIR-hyperintensities (III ventricle, thalami, periaqueductal area) consistent with WKS. Serial EMG progressively showed acute denervation in lower-limb muscles (proximal $>>$ distal) with transitory mild deterioration of nerve conduction studies (NCS). CK was always normal. Muscle-MRI revealed slight oedema of the quadriceps. Parental thiamine supplementation (pTS) led to short-term improvement of alertness and nystagmus but not of neuromuscular impairment. EMG at 3months follow-up showed initial re-innervation signs. Patient-2 showed proximal lower-limb hyposthenia and tactile hypoesthesia with absent tendon reflexes. CK and spine-MRI were normal. CSF examination revealed albumin-cytologic dissociation. EMG showed acute denervation in all lower-limb muscles (proximal $>>$ distal) with normal NCS. Intravenous immunoglobulins and pTS did not lead to any short-term improvement, while at 9-months follow-up she partially recovered standing and walking abilities. EMG revealed reduction of denervation with neurogenic changes of lower-limb muscle motor units.

Discussion: Thiamine has an important role in metabolism of carbohydrates, branched-chain amino-acids and pentose-phosphate pathway. Moreover, it regulates nerve functions probably by synthesis of glutamate and $\gamma$-aminobutyric acids, myelin sheath maintenance and membrane ion channels fixing. The impairment of these processes is likely to induce neurological clinical features (WKS, DB) related to TD Concerning proximal neuromuscular involvement, the pathogenesis is unclear and literature observations gave different interpretations (myogenic or neurogenic damage). Our findings suggest proximal non-lenght dependent neuropathic dysfunction, less responsive to pTS.

Conclusions: DB consists of both symmetrical peripheral neuropathy (common) and proximal amyotrophy (rare). In a TD scenario, diagnostic approach and prognostic counselling can be correctly formulated.

References:

- Shible AA, Ramadurai D, Gergen D, Reynolds PM. Dry Beriberi Due to Thiamine Deficiency Associated with Peripheral Neuropathy and Wernicke's Encephalopathy Mimicking Guillain-Barré syndrome: A Case Report and Review of the Literature. Am J Case Rep (2019); 20:330-334; DOI: 10.12659/AJCR.914051

- Ba A. Metabolic and Structural Role of Thiamine in Nervous Tissues. Cell Mol Neurobiol (2008); 28:923-931; DOI 10.1007/ s10571-008-9297-7

- Koike H, Watanabe H, Inukai A, Iijima M, Mori K, Hattori N, Sobue G. Myopathy in thiamine deficiency: analysis of a case. J Neurol Sci (2006); 249:175-179. doi:10.1016/j.jns.2006.06.016 


\section{DEVELOPMENT OF AN ALGORITHM TO SUPPORT THE DIFFERENTIAL DIAGNOSTIC PROCESS IN PERIPHERAL NEUROPATHIES: ALADDIN PROJECT}

C. Demichelis ${ }^{1}$, M. Garnero ${ }^{1}$, G. Mennella ${ }^{2}$, A. Assini ${ }^{3}$, F. Centanaro ${ }^{4}$, A. Beronio ${ }^{5}$, A. Granata ${ }^{6}$, L. Ruiz ${ }^{7}$, E. Rota $^{8}$, D. Faga ${ }^{9}$, G. Ribizzi ${ }^{10}$, E. Arena $^{11}$, M. Mascolo ${ }^{12}$, L. Agro' ${ }^{12}$, E. Giorli ${ }^{5}$, P. Romani ${ }^{13}$, P. Scotto ${ }^{9}$, A. Mannironi $^{5}$, M. Del Sette ${ }^{3}$, F. Bandini ${ }^{4}$, T. Tassinari ${ }^{6}$, C. Serrati ${ }^{1}$, A. Schenone $e^{2}$

${ }^{1}$ U.O.C. Neurology, ASL1 (Imperia); ${ }^{2}$ Department of Neuroscience, Rehabilitation, Ophthalmology, Genetics, Maternal and Child Health, University of Genoa (Genova); ${ }^{3}$ U.O.C. Neurology, Ente Ospedaliero Ospedali Galliera (Genova); ${ }^{4}$ S.S.D. Neurophysiology, ASL2 (Savona); ${ }^{5}$ U.O.C. Neurology, Sant'Andrea Hospital (La Spezia); ${ }^{6}$ U.O.C. Neurology, ASL2 (Pietra Ligure-SA); ${ }^{7}$ U.O.C. Neurology, Azienda Ospedaliera SS. Antonio e Biagio e Cesare Arrigo (Alessandria); ${ }^{8}$ U.O.C. Neurology, San Giacomo Hospital (Novi Ligure-AL); ${ }^{9}$ U.O.C. Neurology, Villa Scassi Hospital (Genova); ${ }^{10}$ U.O.C. Neurology, IRCCS Policlinico San Martino (Genova); ${ }^{11}$ U.O.C. Neurology, ASL2 (Savona); ${ }^{12}$ U.O.C. Neurology, Azienda Ospedaliera SS. Antonio e Biagio e Cesare Arrigo (Alessandria); ${ }^{13}$ U.O.C. Neurology, ASL4 (Lavagna-GE)

The scope of our work is to elaborate an algorithm, which can be a support to neurologists in the diagnostic process of peripheral neuropathies. The algorithm can be easily used also be neurologist not specialized in peripheral nerves diseases and should help in rapidly achieve a correct diagnosis and, as a consequence, an appropriate treatment. To elaborate the algorithm, data were collected from the most recent and updated scientific literature for different types of peripheral neuropathies that most often can be included in the differential diagnosis of each other. Neuropathies included in the algorithm are: chronic inflammatory demyelinating polyradiculoneuropathy (CIDP), anti-MAG neuropathy, multifocal motor neuropathy (MMN), Familial amyloidotic polyneuropathy (FAP), AL amyloidosis neuropathy, POEMS syndrome, diabetic neuropathy, the most common variants of hereditary neuropathies (CMT), vasculitic neuropathies and paraneoplastic forms. For each of them, suggestive signs and symptoms have been identified; characteristics of the neurophysiological study, CSF examination, MRI data, blood chemistry and any associated systemic signs were collected. These data were then entered into a computer algorithm thanks to which each type of neuropathy corresponds to a set of signs, symptoms and instrumental tests. A web application was therefore created within which the neurologist can select, from a list of numerous data divided by categories (e.g. signs, symptoms, blood tests, CSF examination, MRI..), those reported by his patient. At the end of the compilation of the web page, based on the data entered, the diagnostic suggestions appear, in order from most to least probable. Once the most probable suggestion has been selected, it is also possible to view a card with advice on any further investigations to be proposed to the patient for diagnostic confirmation and possible therapeutic strategies. The algorithm created at the moment has been tested on 50 cases with a known diagnosis and in almost all cases neurologists and algorithm diagnosis matched (95\%). The second step of our work is to validate the algorithm retrospectively by testing it on 200 cases whose diagnosis is already known. Once validated it will be used prospectively as a real support to the differential diagnosis process. In the field of peripheral neuropathies, the rapid achievement of a correct diagnosis has become always more important considering the high number of increasingly available therapies. In this way, our web application can become a useful tool.

\section{HIGH RESOLUTION ULTRASONOGRAPHY IN DIFFERENTIATING HEREDITARY TRANSTHYRETIN AMYLOIDOSIS WITH POLYNEUROPATHY FROM CIDP}

G. Di Pietro ${ }^{1}$, L. Leonardi ${ }^{2}$, L. Fionda ${ }^{2}$, M. Garibaldi ${ }^{2}$, G. Di Stefano ${ }^{1}$, E.

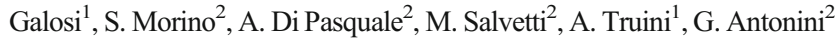

${ }^{1}$ Department of Human Neurosciences, Sapienza University (Roma); ${ }^{2}$ Department of Neuroscience Mental Health and Sensory Organs (NESMOS), Faculty of Medicine and Psychology, Neuromuscular and Rare Disease Centre, Sant'Andrea Hospital, Sapienza University of Rome (Roma)

Introduction: Hereditary transthyretin amyloidosis with polyneuropathy (ATTRV-PN) is a rare autosomal dominant disease. In endemic areas it presents as an early onset length-dependent sensory-motor neuropathy with autonomic impairment. Late onset ATTRv-PN in non-endemic areas is frequently associated with a later onset, large fiber involvement with mild autonomic involvement and no family history [1]. In this setting, clinical, neurophysiological and laboratory findings may prompt other diagnosis, being chronic inflammatory demyelinating polyneuropathy (CIDP) the most frequently reported [2]. We aimed to investigate the utility of nerve high resolution ultrasonography (HRUS) as a diagnostic tool to differentiate ATTRv-PN from CIDP.

Methods: We enrolled patients diagnosed with ATTRv-PN and CIDP at the Neuromuscular and Rare Disease Center of Sant' Andrea Hospital, Rome from February 2015 to February 2020. We included 11 treatmentnaive patients ( 8 males, 3 females) with genetically confirmed late onset ATTRv-PN and 25 CIDP patients (17 males, 8 females). For each patient we collected clinical data (including MRC-80) and in the peripheral nerve ultrasound investigation we evaluated 10 nerve sites, both quantitatively and qualitatively.

Results: ATTRv-PN patients had a shorter disease duration $(\mathrm{p}=0.043)$ and comparable MRC-80 than patients with CIDP. CIDP patients showed a higher number of affected nerve segments at non entrapment points than ATTRv-PN patients $(\mathrm{p}=0.002)$. Cross sectional areas of median nerve at the axilla $(\mathrm{p}=0.002), \operatorname{arm}(\mathrm{p}=0.004)$ and forearm $(\mathrm{p}=0.001)$; ulnar nerve at the forearm $(p=0.038)$, peroneal nerve at popliteal fossa $(p<0.001)$ were significantly higher in CIDP. Frequency of brachial plexus abnormality was higher in patients with CIDP $(p<0.001)$. ROC analysis was performed to recognize nerve scanning points with the best diagnostic yield between the two groups. Median nerve at axilla ( $\mathrm{AUC}=0.72$ ), arm (AUC=0.71) and forearm (AUC=0.74); ulnar nerve at the forearm (AUC $=0.65$ ) and peroneal nerve at the popliteal fossa $(\mathrm{AUC}=0.76$ ), presence of brachial plexus abnormalities ( $\mathrm{AUC}=0.82$ ) were best suitable to distinguish the two neuropathies.

Discussion: Although HRUS alterations at the axilla and arm may be surprising in a distal symmetrical neuropathy, several studies suggested that distal fiber loss in ATTRv-PN may be the consequence of proximal nerve involvement [3]. CIDP patient had a higher number of affected sites at non-entrapment points and frequency of brachial plexus abnormalities than ATTRv-PN.

Conclusions: Although ATTRv-PN is not associated with a univocal ultrasonographic pattern, HRUS can be used to better differentiate ATTRv-PN from CIDP, thus improving diagnostic accuracy and prompt specific treatment.

References:

1. Yukio Ando, Teresa Coelho, John L Berk, Márcia Waddington Cruz, Bo-Göran Ericzon, Shu-ichi Ikeda, W David Lewis, Laura Obici, Violaine Planté-Bordeneuve, Claudio Rapezzi, Gerard Said, 
Fabrizio Salvi, Guideline of transthyretin-related hereditary amyloidosis for clinicians, Orphanet J Rare Dis. (2013) Feb 20;8:31

2. Arman Çakar, Hacer Durmuş-Tekçe, Yeşim Parman, Familial Amyloid Polyneuropathy, Noro Psikiyatr Ars. (2019) May 6;56(2):150-56

3. N Hanyu, S Ikeda, A Nakadai, N Yanagisawa, H C Powell, Peripheral nerve pathological findings in familial amyloid polyneuropathy: a correlative study of proximal sciatic nerve and sural nerve lesions Case Reports Ann Neurol. (1989) Apr;25(4):340-50

\section{DISTRIBUTION PATTERN OF SERUM MATRIX METALLOPROTEINASES-2 AND-9 AND TISSUE INHIBITORS IN PATIENTS WITH GENERALIZED MYASTHENIA GRAVIS}

\author{
V. Di Stefano ${ }^{1}$, G. Cosentino ${ }^{2}$, C. Tubiolo ${ }^{1}$, A. Gagliardo ${ }^{1}$, R. Lo Presti ${ }^{3}$, \\ M. Montana ${ }^{3}$, G. Caimi ${ }^{3}$, B. Fierro ${ }^{1}$, F. Brighina ${ }^{1}$
}

${ }^{1}$ Department of Biomedicine, Neuroscience and advanced Diagnostic (BIND), University of Palermo (Palermo); ${ }^{2}$ Department of Brain and Behavioral Sciences, University of Pavia (Pavia)

Background: Metalloproteinases (MMPs) are a heterogeneous group of endopeptidases with high affinity for different components of the extracellular matrix involved in the autoimmune response [1]. We evaluated serum levels of MMP-2, MMP-9 and their inhibitors TIMP-1 and -2 in a cohort of patients with generalized myasthenia gravis (MG) to test their potential usefulness as a biomarker for disease activity.

Methods: We collected the serum of patients with generalized MG and healthy controls matched for age and sex from the general population. The severity of the disease was assessed by the Osserman classification. Plasma concentrations of MMP-2, MMP-9, TIMP-1 and TIMP-2, were determined using the ELISA technique.

Results: We enrolled 14 generalized MG patients (7 males, 57.2 \pm 8.6 years) and 13 healthy subjects ( 5 males, $49.5 \pm 4.7$ years). The MMP-9 concentration was higher in the MG group compared to controls, differently to MMP-2 concentration that resulted reduced. The TIMP-1 concentration was lower in the MG group compared to controls, while TIMP2 concentration did not differ between patients and controls. Patients with more severe disease were younger and presented a higher MMP-2 serum level compared to patients in the less symptomatic stages.

Conclusions: We observed an increased serum MMP-9 concentration, together with the reduction of TIMP-1 and MMP-2 levels in MG patients compared to controls. Patients with more severe symptoms presented an increased level of MMP-2 and MMP-2/TIMP-2 ratio. We hypothesize that MMP-9 could be involved in all phases of the disease, while MMP-2 might be associated with a higher risk of deterioration $[2,3]$.

References:

1. Hopps E, Lo Presti R, Montana M, Canino B, Averna MR, Caimi G. Study of the correlations among some parameters of the oxidative status, gelatinases, and their inhibitors in a group of subjects with metabolic syndrome. Mediators Inflamm. (2014);2014

2. Hurnaus S, Mueller-Felber W, Pongratz D, Schoser BGH. Serum levels of matrix metalloproteinases- 2 and -9 and their tissue inhibitors in inflammatory neuromuscular disorders. Eur Neurol. (2006) Aug;55(4):204-8

3. Helgeland G, Petzold A, Luckman SP, Gilhus NE, Plant GT, Romi FR. Matrix metalloproteinases in myasthenia gravis. Eur Neurol. (2011) Jan;65(1):53-8

\section{PREVALENCE AND RELEVANCE OF DIABETES MELLITUS IN CHRONIC INFLAMMATORY DEMYELINATING POLYNEUROPATHY}

P. E. Doneddu ${ }^{1}$, D. Cocito $^{2}$, F. Manganelli ${ }^{3}$, R. Fazio ${ }^{4}$, C. Briani ${ }^{5}, \mathrm{M}$. Filosto $^{6}$, L. Benedetti ${ }^{7}$, E. Bianchi ${ }^{8}$, S. Jann ${ }^{9}$, A. Mazzeo ${ }^{10}$, G. Antonini $^{11}$, G. Cosentino ${ }^{12}$, G. Marfia ${ }^{13}$, A. Cortese ${ }^{12}$, A. Clerici ${ }^{14}, \mathrm{M}$. Carpo $^{15}$, A. Schenone ${ }^{16}$, G. Siciliano ${ }^{17}$, M. Luigetti ${ }^{18}$, G. Lauria ${ }^{19}$, T. Rosso $^{20}$, G. Cavaletti ${ }^{21}$, E. Beghi ${ }^{8}$, G. Liberatore ${ }^{1}$, L. Santorio ${ }^{3}$, E. Spina $^{3}$, E. Peci ${ }^{2}$, S. Tronci ${ }^{4}$, M. Ruiz ${ }^{5}$, S. Cotti-Piccinelli ${ }^{6}$, E. Verrengia ${ }^{9}$, L. Gentile ${ }^{10}$, L. Leonardi ${ }^{11}$, G. Mataluni ${ }^{13}$, E. Nobile-Orazio ${ }^{1}$

${ }^{1}$ Neuromuscular and Neuroimmunology Service, IRCCS Humanitas Clinical and Research Institute (Milano); ${ }^{2}$ Department of Neuroscience, University of Turin (Torino); ${ }^{3}$ Department of Neuroscience, Reproductive Sciences and Odontostomatology, University of Naples Federico II (Napoli); ${ }^{4}$ Department of Neurology, San Raffaele Scientific Institute (Milano); ${ }^{5}$ Department of Neuroscience, University of Padua (Padova); ${ }^{6}$ Department of Neurology, University of Brescia (Brescia); ${ }^{7}$ Department of Neurology, IRCCS Ospedale Policlinico San Martino (Genova); ${ }^{8}$ Mario Negri Institute of Pharmacological Research (Milano); ${ }^{9}$ Department of Neuroscience, Niguarda Ca Granda Hospital (Milano); ${ }^{10}$ Department of Clinical and Experimental Medicine, University of Messina (Messina); ${ }^{11}$ Unit of Neuromuscular Diseases, Sapienza University of Rome (Roma); ${ }^{12}$ Department of Neurology, IRCCS Mondino Foundation (Pavia); ${ }^{13}$ Dysimmune Neuropathies Unit, Tor Vergata University of Rome (Roma); ${ }^{14}$ Neurology Unit, Circolo \& Macchi Foundation Hospital (Varese); ${ }^{15}$ Neurology Unit, ASST Bergamo Ovest- Ospedale Treviglio (Treviglio-BG); ${ }^{16}$ Department of Neuroscience, University of Genoa (Genova); ${ }^{17}$ Neurology Unit, University of Pisa (Pisa); ${ }^{18}$ Department of Neuroscience, Catholic University of the Sacred Heart (Roma); ${ }^{19}$ Department of Neuroalgology, IRCCS Carlo Besta Neurological Institute (Milano); ${ }^{20}$ Department of Neurology, ULSS2 Marca Trevigiana (Treviso); ${ }^{21}$ Department of Neurology, University of Milano Bicocca (Monza)

Objectives: To determine the prevalence and relevance of diabetes mellitus (DM) in chronic inflammatory demyelinating polyradiculoneuropathy (CIDP).

Materials: We used a structured questionnaire to collect information on DM from 393 CIDP patients fulfilling the EFNS/PNS criteria included in the Italian CIDP database.

Methods: Frequency of DM in CIDP was compared with the estimated age- and gender-specific prevalence rates of DM in Italy. Fulfillment of the recently proposed diagnostic criteria of CIDP for the patients with DM was assessed.

Results: DM was significantly more frequent in CIDP patients than expected in the general Italian population (SPR 1.66; 95\% CI 1.31-2.07). Mean score of the diagnostic criteria for CIDP in DM among our patients with CIDP-DM was 12 (range 1-18; $\mathrm{SD} \pm 3.6$ ), with only one patient with a score below 2 points. Mean age of onset of $\mathrm{DM}$ was 51 years $(\mathrm{SD} \pm 13)$, and $51 \%$ of the patients with DM were treated with oral antidiabetic therapy alone, $12.5 \%$ with insulin therapy alone, and $34 \%$ were not receiving therapy, suggesting a higher frequency of type 2 DM compared type 1. Patients with DM had an older age at symptoms onset, more frequent signs of autonomic impairment, increased CSF proteins levels, higher disability by RODS and INCAT, worse QoL, and a less frequent response to treatment compared to patients without.

Conclusion: DM is more frequent in CIDP than in the general population and type $2 \mathrm{DM}$ seems to be the most frequently associated type. DM seems to have an impact on disease severity and treatment response. 


\section{SMALL FIBERS DAMAGE FEATURES IN LATE-ONSET TRANSTHYRETIN FAMILIAL AMYLOID POLYNEUROPATHY: A CLINICAL, NEUROPHYSIOLOGICAL AND SKIN BIOPSY STUDY}

P. Falco, E. Galosi, A. Fasolino, L. Leonardi, G. Di Pietro, G. Di Stefano, C. Leone, A. Di Lionardo, S. La Cesa, E. Sgro, G. Antonini, A. Truini

Human Neuroscience, La Sapienza University (Roma)

Transthyretin familial amyloid polyneuropathy (TTR-FAP) is a rare inherited rapidly progressive axonal polyneuropathy. Despite great heterogeneity regarding disease severity and clinical presentation between different ethnic groups, small fibers damage predominance in clinical picture has traditionally been considered a diagnostic clue. Our clinical, neurophysiological and skin biopsy study aims at characterizing small sensory and autonomic fibers involvement in an Italian cohort of late-onset TTR-FAP patients. We enrolled 20 patients with a genetically confirmed diagnosis of TTR-FAP and 7 asymptomatic TTR mutations carriers. Each individual underwent clinical examination, DN4, NPSI, SFN-SIQ questionnaires, a structured interview on autonomic symptoms, nerve conduction study and skin biopsy with PGP9.5 immunostaining from distal calf and proximal thigh. Intraepidermal (IENFD) and piloerector muscle (PMNFD) nerve fibers density quantification and sweat gland innervation semi-quantitative evaluation (GNFD) were performed on skin samples from patients and 20 healthy controls. Distal IENFD positively correlated with PMNFD ( $\mathrm{r}=0.6)$, GNFD $(\mathrm{r}=0.8)$ and sural SAP amplitude $(r=0.7)$. All variables negatively correlated with disease stage and duration. Asymptomatic carriers showed a selective distal IENFD $(p=0.01)$ and PMNFD $(p=0.02)$ reduction respect to healthy subjects, while symptomatic patients showed since early stages (PND1) sural SAP amplitude reduction $(\mathrm{p}=0.001)$ and all skin biopsy parameters impairment (distal and proximal IENFD, PMNFD, GNFD). Distal IENFD reduction showed a positive correlation with pinprick hypoesthesia and neuropathic pain presence, while PMNFD and GNFD didn't correlate with autonomic symptoms reports. Our findings show that sub-clinical small fibers involvement characterizes asymptomatic TTR-FAP stages. Symptomatic patients show since early disease stages a parallel involvement of large and small fibers, with both small sensory and autonomic fibers reduction at skin biopsy. While sensory small fibers involvement clinically manifests through pain hypoesthesia and neuropathic pain, autonomic fibers impairment often goes unnoticed, being scarcely symptomatic.

References:

- Masuda T, Ueda M, Suenaga G, Misumi Y, Tasaki M, Izaki A, Yanagisawa $\mathrm{Y}$, Inoue $\mathrm{Y}$, Motokawa $\mathrm{H}$, Matsumoto $\mathrm{S}$, Mizukami M, Arimura A, Deguchi T, Nishio Y, Yamashita T, Inomata Y, Obayashi K, Ando Y. Early skin denervation in hereditary and iatrogenic transthyretin amyloid neuropathy. Neurology (2017) Jun 6;88(23):2192-97

- F. Escolano-Lozano, A. P. Barreiros, F. Birklein, C. Geber. Transthyretin familial amyloid polyneuropathy (TTR-FAP): Parameters for early diagnosis. Brain and Behaviour (2017); 8(1):e00889

- C., Andrade. A peculiar form of peripheral neuropathy. Familial atypical generalized amyloidosis with special involvement of the peripheral nerves. Brain (1952); 75:408-27

\section{MUSCLE MRI FINDINGS AND CORRELATION WITH CLINICAL AND IMMUNOLOGICAL PARAMETERS IN A COHORT OF IMNM PATIENTS}

L. Fionda ${ }^{1}$, F. Vanoli ${ }^{1}$, L. Leonardi ${ }^{1}$, J. Alonso Perez ${ }^{2}$, J. Diaz Manera ${ }^{2}$, G. Merlonghi ${ }^{1}$, S. Morino ${ }^{1}$, G. Alfieri ${ }^{1}$, A. Lauletta ${ }^{1}$, G. Antonini ${ }^{1}$, M. Garibaldi $^{1}$

${ }^{1}$ Sant'Andrea Hospital, NESMOS Department, Sapienza University (Roma);

${ }^{2}$ Neurology Department, Hospital de la Santa Creu i Sant Pau (Barcelona-E)

Introduction: Immune-mediated necrotising myopathies (IMNMs) are characterized by progressive and severe muscle impairment, frequently associated to fatty replacement of muscular tissue that leads to a permanent deficit [1]. The aim of this study is to detect, through a whole body muscle MRI, the presence and the evolution of inflammation and muscular damage, and if there is a correlation with clinical and immunological aspects, and comparing response to different therapeutic strategies.

Materials and Methods: Twenty-two patients were included. Age, disease duration, timing and type of therapy performed with respect to the moment of MRI acquisitions, were recorded for each patient. Muscle imaging was examined using T1-TSE and STIR weighted sequences. A total of 39 muscles of lower limbs and 20 of scapular girdle and arm were analyzed. The presence of fatty infiltration was evaluated according to Fischer's semi-quantitative scale. The presence of edema/inflammation of muscles was established on STIR sequences.

Results: At the moment of MRI acquisition, 8 anti-HMGCR, 7 antiSRP and 7 seronegative IMNM were aged $60,1 \pm 17,3$. Eight patients were without treatment, and disease duration was $5,8 \pm 7,8$ years. Eleven patients underwent a second muscle MRI, after a median time of 15 months. Eleven patients needed an association therapy with immunosuppressors, while twelve underwent IVIG. Fourteen patients did not recover completely muscle strength. Age at onset, disease duration and antiSRP positivity were associated with a more severe muscle involvement. Most affected muscles, according to T1-wheigted images, were lumbar paravertebral, gluteal, adductors and hamstring groups. Muscle edema /inflammation distribution seems to be a predictor of fatty replacement overtime. Finally, despite the small size of our sample, IVIG therapy seems to show the best outcome in terms of reduction of inflammation and prevention of muscular fatty replacement overtime.

Discussion and Conclusion: Although IMNMs are frequently characterized by a clinical relapsing pattern, especially in SRP+ patients, muscle impairment is progressive and severe and there could be a fatty replacement of muscular tissue that leads to a permanent muscular deficit that seems to involve mostly pelvic muscles and adductors [2]. In fact, with respect to other IIM, for these patients combined therapies or other treatment strategies are more frequently required [3]. Our preliminary results show that MRI could be a sensitive biomarker of inflammation and disease progression in these patients. Pelvifemoral muscles are the most frequently involved, and different therapies could have a role in the progression of the disease.

References:

1. Dalakas MC. Inflammatory muscle diseases. N Engl J Med. (2015);373:393-4

2. Zheng Y, Liu L, Wang L, Xiao J, Wang Z, Lv H, Zhang W, Yuan $Y$. Magnetic resonance imaging changes of thigh muscles in myopathy with antibodies to signal recognition particle. Rheumatology (2015) 54:1017-24

3. Allenbach Y, Benveniste O. Peculiar clinicopathological features of immune-mediated necrotizing myopathies. Curr Opin Rheumatol. (2018) Nov;30(6):655-63 


\section{DIAGNOSTIC CHALLENGES IN ACUTE-ONSET BINOCULAR DIPLOPIA: A RETROSPECTIVE STUDY IN NEUROLOGICAL UNITS}

G. Fisco, E. Cerulli Irelli, B. Orlando, G. Di Pietro, F. Barone, A. Francesco, A. Morano, A. Giallonardo, C. Di Bonaventura

Department of Human Neurosciences, Sapienza University of Rome (Roma)

Objective: To investigate the aetiology of acute-onset binocular diplopia in neurological units and to identify the key diagnostic procedures in this setting.

Materials and Method: We retrospectively reviewed data from patients hospitalized for binocular diplopia at Neurology Department of Sapienza University of Rome, from 2010 to 2020. Subjects presenting to emergency department for binocular diplopia were included in the study. Clinicodemographic data, neurological examination and diagnostic work-up (brain $\mathrm{CT}$, brain MRI, angiography, lumbar puncture, electroneurography and electromyography) were reviewed in each patient. Diplopia plus was defined as diplopia associated with other neurological symptoms.

Results: One-hundred-and-twenty patients were included in the study and the median age was 62.5 years (IQR 44-72). According to the clinical presentation, $73.3 \%$ of the patients were admitted for isolated diplopia, whereas $26.7 \%$ of them displayed diplopia plus. Among the latter, the most frequent associated neurological symptoms were weakness/sensory impairment in 18/32 subjects and disequilibrium in 10/32. According to the extrinsic ocular motility impairment, patients were divided in relation to single (92/120 subjects) or multiple (28/120) nerve involvement. Etiology of diplopia remained unknown in 39/120 patients. The most common diagnoses at discharge were the following: diabetic ophtalmoparesis in $16 \%$ of the subjects, stroke in $16 \%$, multiple sclerosis in $11,1 \%$, Myasthenia Gravis in $9,9 \%$, cerebrospinal fluid hypo/hypertension in $9,9 \%$, aneurysm in $8,6 \%$, Miller-Fisher syndrome in 7,4\% and tumors in 7,4\%. Unknown-etiology patients did not significantly differ in terms of medical comorbidities and demographical characteristics with those with a defined etiology, except for a previous history of thyroid disease $(\mathrm{p}=0.05)$. Single oculomotor involvement remains more often undiagnosed compared with multiple oculomotor nerves impairment $(\mathrm{p}=0.049)$. In the overall population, the most useful diagnostic test was brain MRI in $31.7 \%$ of the subjects. Brain MRI diagnostic yield was significantly different according to the presence or not of neurological symptoms other than diplopia (isolated diplopia $18 \%$ vs diplopia plus $64 \%, \mathrm{p}<0.001)$ and to the involvement of single or multiple oculomotor nerves $(\mathrm{p}=0.009)$. Brain $\mathrm{CT}$ was diagnostic in $0 / 47$ patients with isolated diplopia.

Discussion: Brain MRI was confirmed to be the best diagnostic tool in our cohort,[1] however no diagnostic procedure alone was found to be pivotal in the setting of binocular diplopia and a combination of diagnostic procedures is usually needed to establish a diagnosis.

Conclusions: Even if diplopia remained undiagnosed in almost one third of the patients a thorough diagnostic work-up should be performed considering that it may herald life-threatening conditions.[2]

References:

1. Nazerian P, Vanni S, Tarocchi C, et al. Causes of diplopia in the emergency department: diagnostic accuracy of clinical assessment and of head computed tomography. Eur J Emerg Med. (2014);21(2):118-124

2. Merino P, Fuentes D, Gómez de Liaño P, Ordóñez MA. Binocular diplopia in a tertiary hospital: Aetiology, diagnosis and treatment. Diplopía binocular en un hospital terciario: etiología, diagnóstico y tratamiento. Arch Soc Esp Oftalmol. (2017);92(12):565-70

\section{REDEFINING MUSCLE INVOLVEMENT IN MYOTONIC DYSTROPHY TYPE1 (DM1): A MUSCLE MRI STUDY IN A LARGE COHORT OF PATIENTS}

M. Garibaldi ${ }^{1}$, T. Nicoletti ${ }^{2}$, L. Fionda ${ }^{1}$, E. Bucci ${ }^{1}$, T. Tartaglione ${ }^{3}$, G.

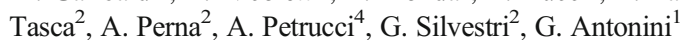

${ }^{1}$ Neuromuscular and Rare Disease Center, Department of Neuroscience, Mental Health and Sensory Organs (NESMOS), SAPIENZA University, Sant'Andrea Hospital (Roma); ${ }^{2}$ Fondazione Policlinico Universitario "A. Gemelli" IRCCS, Università Cattolica del Sacro Cuore (Roma); ${ }^{3}$ Department of Radiology, Istituto Dermopatico dell'Immacolata, IRCCS (Roma); ${ }^{4}$ Neurology Unit, San Camillo-Forlanini Hospital (Roma)

Objectives: To investigate the muscle involvement in a large cohort of DM1 patients and to search a correlation with clinical and genetic data.

Material and Methods: 135 genetically confirmed DM1 patients underwent whole body muscle MRI. T1 and STIR sequences were analysed in 32 muscles of lower limbs and 17 of neck-scapular girdle, bilaterally. Fibro-fatty replacement, muscle atrophy and inflammation were evaluated using 3 different scales.

Results and Discussion: The most frequent pattern of muscle involvement showed that multifidus, gluteus minimus, distal quadriceps and medial gastrocnemius were the most affected muscles. Most of patients showed muscle atrophy in cervical muscles regardless of fibro-fatty replacement. More than $80 \%$ of patients had STIR positive muscles. A subset of patients $(\sim 10 \%)$ showed a very-mild and diffuse muscle involvement without typical pattern, regardless of age, CTG repeats, or years of disease. The overall severity of muscle involvement (fibro fatty replacement) correlated with clinical severity, but not with genetic background. A variable degree of muscle involvement was also detected in milder- asymptomatic patients (MIRS 1-2) suggesting the high sensitivity of MRI as a biomarker also for the milder spectrum of disease.

Conclusions: As observed in other myopathies, a characteristic pattern of fibro-fatty replacement exist for DM1, even if a subset of patients do not show this pattern. STIR positive muscles consistent with muscle edema/inflammation as well as the observation of T1-negative atrophic muscles suggest other pathophysiological mechanisms of muscle weakness in DM1 beyond the fibro-fatty replacement.

\section{RNAI THERAPEUTIC PATISIRAN IN HATTR AMYLOIDOSIS: TOLERABILITY AND MANAGEMENT FROM TWO CENTRE EXPERIENCE}

L. Gentile ${ }^{1}$, M. Russo ${ }^{1}$, M. Luigetti ${ }^{3,4}$, G. Bisogni ${ }^{2}$, A. Di Paolantonio ${ }^{3,4}$, A. Romano ${ }^{2,3}$, V. Guglielmino ${ }^{3,4}$, M. Sabatelli ${ }^{2,3}$, A. Toscano ${ }^{1}$, G. Vita ${ }^{1}$, A. Mazzeo ${ }^{1}$

${ }^{1}$ Unit of Neurology and Neuromuscular Diseases, Department of Clinical and Experimental Medicine, University of Messina (Messina); ${ }^{2}$ Centro Clinico NEMO adulti (Roma); ${ }^{3}$ Catholic University of the Sacred Heart (Roma); ${ }^{4}$ Fondazione Policlinico Universitario A. Gemelli IRCCS, Neurology Unit (Roma)

Introduction: Hereditary transthyretin amyloidosis (hATTR) is a severe disease characterized by progressive axonal sensory-motor polyneuropathy with autonomic dysfunction. Heart, kidneys and eyes involvement are common too. Patisiran is a si-RNA able to degrade the m-RNA strand from TTR gene, reducing the synthesis of the mutated 
protein. A phase 3 trial demonstrated the improvement of symptomatology, measured by the modified Neuropathy Impairment Score+7 (NIS+7) and Norfolk Quality of Life Diabetic Neuropathy score (QOL-DN), without significant adverse events except for mild infusion site reactions.

Materials and methods: Since 2014, we have treated with patisiran 23 patients. Among them, ten harboured Phe64Leu mutation, five Glu89Gln, four Val30Met, two Thr49Ala, one Val122Ile and one Ala109Ser. 4/23 patients were also in treatment with tafamidis. 19/23 were male. Age at the first infusion ranged between 48 and 78 years. Seven patients were at stage II and sixteen in stage I of the disease, according to FAP staging. All patients were evaluated by NIS scale, QOL-DN score and Sudoscan at baseline and every 6 months.

Results: We observed a stabilization of clinical parameters during the therapy with mild improvements in some cases. We reported two side effects secondary to the use of premedication drugs: hyperglycemia in one patient (maximum value: $361 \mathrm{mg} / \mathrm{dL}$ ) and hypertension in two patients (maximum systolic value: $180 \mathrm{mmHg}$; maximum diastolic value: $125 \mathrm{mmHg}$ ). Three patients temporarily discontinued the therapy because of hospitalizations respectively for severe anaemia, fever, and diarrhoea. All the patients started again the therapy after their discharge. We also reported three death: two patients presented a sudden death, probably of cardiac origin; one patient died as complication of severe dehydration. It is highly unlikely that these episodes are related to patisiran infusion.

Conclusions: Clinical stabilization was observed during patisiran therapy. Adverse events were correlated mainly to the use of premedical drugs.

\section{LONG-TERM OUTCOME AFTER THERAPY AND PREDICTIVE ROLE OF SERUM VEGF LEVELS ON CLINICAL DETERIORATION IN PATIENTS WITH POEMS SYNDROME}

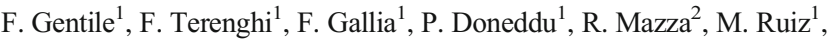 \\ A. Nozza ${ }^{3}$, E. Nobile-Orazio ${ }^{4}$
}

${ }^{1}$ Neuromuscular and Neuroimmunology Service, IRCCS Humanitas Clinical and Research Institute (Milano); ${ }^{2}$ Department of Medical Oncology and Haematology, Humanitas Cancer Centre, Humanitas Clinical and Research Hospital IRCCS (Milano); ${ }^{3}$ Unit of Internal Medicine, Oncology, Hospital of Vigevano (Pavia); ${ }^{4}$ Neuromuscular and Neuroimmunology Service, Department of Medical Biotechnology and Translational Medicine, Milan University, Humanitas Clinical and Research Hospital IRCCS (Milano)

Objectives: Therapeutic options for POEMS syndrome show high effectiveness in the short-term, but only few studies assessed their long-term efficacy. Serum VEGF level is a useful diagnostic marker for POEMS, but its role on disease monitoring after therapeutic response is less clear. We evaluated the long-term outcome after therapy in patients with POEMS and the value of serial VEGF monitoring in the prediction of clinical relapses.

Materials and Methods: We retrospectively reviewed the records of 31 patients (median age at symptoms onset 56 years, range 40-68; M:F 2:1) diagnosed with POEMS syndrome in our institute between 2004 and 2018. Clinical assessment including serial VEGF measurement and response to therapies were collected. We defined treatment efficacy as an improvement in at least two disease parameters (neurologic, clinical, VEGF, hematologic). Progression-free survival (PFS) outcomes were calculated. The relationship between serum VEGF (sVEGF) levels and clinical relapses was also investigated.
Findings: The majority of patients underwent autologous stem cell transplant (ASCT, 43\%), followed by radiotherapy (RT, 24\%), lenalidomide (18\%), and low-dose alkylators (15\%). Improvement after therapy was observed in $76 \%$ of the patients with a median PFS of 60 months. There was no significant difference in the initial response and PFS among groups. During follow-up, 21/31 patients had at least 1 relapse (10 had one relapse, 6 had two relapses, 5 had three relapses). When serially examined, an increase of sVEGF of $>1500 \mathrm{ng} / \mathrm{ml}$ was observed within 18 months before clinical deterioration in $57 \%$ of relapses. Most common rescue therapy was lenalidomide, which maintained a high response rate $(92 \%)$ with a subsequent median PFS of 47 months.

Conclusions: Despite the efficacy of available therapies, the majority of patients relapsed at some point after therapy and in most of them VEGF predicted clinical worsening leading to treatment adjustment. Lenalidomide showed high efficacy both as first line therapy and after relapse.

\section{HIGHER MUSCLE DAMAGE IN PATIENTS WITH ANTI-MI2- POSITIVE DERMATOMYOSITIS: A SINGLE CENTRE RETROSPECTIVE COHORT}

\author{
F. Girolamo ${ }^{1}$, M. Fornaro ${ }^{2}$, M. Giannini ${ }^{3}$, A. Amati ${ }^{1}$, A. Lia ${ }^{1}$, M.

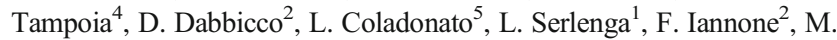 \\ Trojano $^{1}$
}

${ }^{1}$ Department of Basic Medical Sciences, Neurosciences and Sense Organs, University of Bari School of Medicine (Bari); ${ }^{2}$ Department of Emergency and Organ Transplantation, University of Bari School of Medicine (Bari); ${ }^{3}$ Unité d'Explorations Fonctionnelles Musculaires, Hôpitaux Universitaires de Strasbourg (Strasbourg-F); ${ }^{4}$ Patology Clinic, SS. Annunziata Hospital (Taranto); ${ }^{5}$ Reumatology, Policlinico Universitario of Bari (Bari)

Aim: To investigate clinical and histopathological hallmarks in our retrospective cohort adult Dermatomyositis (DM) patients positive and negative for Myositis Specific Autoantibodies (MSAs) considering that clinical and histopathological spectrum could differ according to autoantibody status.

Material and Methods: Clinical data of 56 adult DM patients were collected. Their serum samples were tested using line immunoassays methods for MSAs and Myositis Associated Autoantibodies (MAAs): 14 patients were anti-Mi2 + and 42 anti-Mi2 negative. Histomorphometric quantitative and semiquantitative analyses were carried out on 26 diagnostic muscle biopsy samples (12 anti-Mi2+, 4 antiMDA,+ 1 anti-TIF $1 \gamma+, 3$ anti-SAE1/2+, 1 anti-NXP2+, and 5 anti-MSA/ MAA-negative).

Results: Anti-Mi2+ DM patients had the highest levels of serum creatine kinase at onset $(4653 \pm 5041 \mathrm{UI} / \mathrm{L}$ vs $809 \pm 1337 \mathrm{UI} / \mathrm{L}, \mathrm{p}<0.001)$ and lower prevalence of interstitial lung disease (ILD) (2/14 (14.3\%) vs 16/42 (38.1\%), $\mathrm{p}=0.18)$. Multivariate analysis showed that age at onset (HR 1.07; 95\% CI 1.02-1.13) and rapidly progressive ILD (HR 30.8; 95\% CI 5.6-169.7) correlated with a poor prognosis in the entire cohort. Higher prevalences of necrotic/degenerating myofibers with sarcoplasmic deposition of membrane attack complex, of endomysial macrophage number and of perifascicular atrophy score were observed in anti-Mi2+ DM patients.

Conclusion: Our data confirm that anti-Mi2+ patients represent a specific DM subset with higher muscle damage, sarcoplasmic deposition of complement, and endomysial macrophages infiltration as histological hallmarks. 
References:

- Dos Passos Carvalho MIC, Shinjo SK. Frequency and clinical relevance of anti-Mi-2 autoantibody in adult Brazilian patients with dermatomyositis. Adv Rheumatol (2019); 59:27

- Pinal-Fernandez I, Mecoli CA, Casal-Dominguez M, Pak K, Hosono $\mathrm{Y}$, Huapaya J, et al. More prominent muscle involvement in patients with dermatomyositis with anti-Mi2 autoantibodies. Neurology (2019);93:e1768-e1777

- Hengstman GJ, Vree Egberts WT, Seelig HP, Lundberg IE, Moutsopoulos HM, Doria A,et al. Clinical characteristics of patients with myositis and autoantibodies to different fragments of the Mi-2 beta antigen. Ann Rheum Dis (2006);65:242-45

\section{PERIVASCULAR M1 MACROPHAGES EXPRESSING VEGF AND SDF1 PROMOTE ANGIOGENESIS IN ANTI-HMGCR IMMUNE MEDIATE NECROTIZING MYOPATHY}

F. Girolamo ${ }^{1}$, M. Fornaro ${ }^{2}$, M. Giannini ${ }^{3}$, L. Coladonato ${ }^{4}$, A. Amati ${ }^{1}$, A.

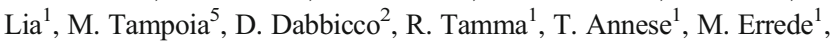
D. Virgintino ${ }^{1}$, D. Ribatti ${ }^{1}$, L. Serlenga ${ }^{1}$, F. Iannone ${ }^{2}$, M. Trojano $^{1}$

\begin{abstract}
${ }^{1}$ Department of Basic Medical Sciences, Neurosciences and Sense Organs, University of Bari School of Medicine (Bari); ${ }^{2}$ Department of Emergency and Organ Transplantation, University of Bari School of Medicine (Bari); ${ }^{3}$ Unité d'Explorations Fonctionnelles Musculaires, Hôpitaux Universitaires de Strasbourg (Sstrasbourg-F); ${ }^{4}$ Reumatology, Policlinico Universitario of Bari (Bari); ${ }^{5}$ Patology Clinic, SS. Annunziata Hospital (Taranto)
\end{abstract}

Aim: Few data are available concerning the phenotype of macrophages in Idiopathic Inflammatory Myopathies (IIMs). Besides their role in innate immunity, macrophages are also involved in tissue regeneration and angiogenesis promotion.

Materials and methods: The density and distribution of the subsets of macrophages, capillary and regenerating myofibres were investigated in human muscle samples from diagnostic biopsies of patients affected by IIMs [6 anti-3-hydroxy-3-methylglutaryl coenzyme A reductase (anti-HMGCR) Immune Mediated Necrotizing Myopathy (IMNM), 3 anti-signal recognition particle (anti-SRP) IMNM, 6 seronegative IMNM, 6 adult Dermatomyositis (DM), 6 Polymyositis (PM), 5 PM with mitochondrial pathology (PM-Mito), 6 sporadic Inclusion Body Myositis (sIBM), 6 Scleromyositis, 6 AntiSynthetase Syndrome with Myositis (ASS), 3 control samples from mitochondrial myopathy (MM), and 6 control samples from agematched, asymptomatic, hyperCK level individuals].

Results: Endomysial capillary density and capillary/fiber ratio were higher than the control group in HMGCR+ IMNM and MM, but lower in DM, PM, Scleromyositis, and sIBM. M1 macrophages were preferentially associated with endomysial microvessels in HMGCR+ IMNM and their density significantly correlated with microvessel density. Most of M1 macrophages expressed Vascular Endothelial Growth Factor AA (VEGF) and Stromal Cell-Derived Factor 1, which likely promote angiogenesis acting on microvascular cells. Additional VEGF was also immunolocalized in myofibres specifically in HMGCR+ IMNM.

Discussion: Our findings show that a reciprocal cross-talk among myofibres, infiltrating macrophages, and microvascular cells occur.

Conclusion: A restorative role for macrophages in modulating the muscle regeneration potential and angiogenesis is suggested.

\section{References:}

- Chung T., Christopher-Stine L., Paik J. J., Corse A., Mammen, A. L. The composition of cellular infiltrates in anti-HMG-CoA reductaseassociated myopathy. Muscle Nerve (2015);52:189-95

- Preusse C., Goebel H. H., Held J., Wengert O., Scheibe F., Irlbacher K., Koch A., Heppner F. L., Stenzel W. Immune-mediated necrotizing myopathy is characterized by a specific Th1-M1 polarized immune profile. Am J Pathol. (2012);181:2161-71

- Rigamonti E., Zordan P., Sciorati C., Rovere-Querini P., Brunelli S. Macrophage plasticity in skeletal muscle repair. Biomed Res Int. (2014);560629

\section{MYASTHENIC CRISIS IN TYPE 3 AUTOIMMUNE POLYENDOCRINE SYNDROME: A CASE REPORT}

\author{
E. Iori, A. Ariatti, M. Mazzoli, G. Galassi
}

Department of Biomedical, Metabolic and Neural Sciences, University Hospital of Modena (Modena)

Introduction: Autoimmune Polyendocrine Syndromes (APS) are characterized by activation of the immune system against multiple epitopes in endocrine and non-endocrine organs. The disease is classified, based on the involved organs, as APS1 (Addison's disease, hypoparathyroidism and chronic mucocutaneous candidiasis), APS2 (Addison's disease, autoimmune thyroid disease and type 1 diabetes) and APS3 (autoimmune thyroiditis and at least another autoimmune disease). Myasthenia gravis (MG) is one of the non-endocrine autoimmune diseases that can be present in APS.

Case report: A 55-year-old woman, with history of insulinedependent diabetes from childhood and of allergic asthma since age 40 , developed bilateral uveitis. She underwent extensive diagnostic work-up for assessment of sarcoidosis, but, despite the presence of elevation of Angiotensin-converting enzyme (ACE) and compatible alterations at chest CT, she didn't meet the definite diagnostic criteria for sarcoidosis. A positivity of anti-thyroid peroxidase antibodies suggested a Hashimoto thyroiditis and diagnosis of APS3 was made. One year later, the patient was admitted to our Neurological clinic for dropped head, dysphagia, dysphonia, dyspnoea and muscle fatigability. Electrophysiological findings and presence of anti-AchR antibodies were consistent with diagnosis of MG and therapy with pyridostigmine and intravenous immunoglobulin was started. Some days later, she exhibited respiratory arrest and was intubated and treated with a course of plasmapheresis, with improvement. In consideration of the difficulty to set up a steroid therapy because of her insufficient glycemic control, immunosuppressive therapy with rituximab was started. A year later she is in good neurological condition, with just minimal fatigability. During hospitalization, a large screening for autoimmune diseases was performed. Positivity for anti-GAD, anti-insuline, antinuclear (ANA), anti-anticardiolipin and anti-beta2GPI autoantibodies, without alteration of lupus anticoagulant (LAC) and coagulation parameters, was found. There were no alterations consistent with adrenocortical or parathyroids dysfunction. The diagnosis of APS3 was confirmed.

Discussion: We present a case of APS3 with both endocrine (diabetes and thyroiditis) and non-endocrine (myasthenia gravis) diseases. Our patient also presented clinical and laboratory elements suggestive for concurrent autoimmune conditions (sarcoidosis, antiphospholipid syndrome and autoimmune hepatitis). 
Conclusions: APS are syndromes characterized by multiple endocrine and non-endocrine autoimmune diseases, which can lead to potentially lethal conditions, such as myasthenic crisis. Different autoimmune disorders can occur with many years of delay after the antecedent, so a regular follow up is important.

References:

- Husebye ES, Anderson MS, Kämpe O. Autoimmune Polyendocrine Syndromes. N Engl J Med (2018);378:1132-41. DOI: 10.1056/ NEJMra1713301

- Cutolo M. Autoimmune polyendocrine syndromes. Review Autoimmun Rev (2014) Feb;13(2):85-9. DOI: 10.1016/ j.autrev.2013.07.006

- Gobaru M, Ashida K, Yoshinobu S et al. Human Leukocyte Antigen (HLA) Subtype Dependent Development of Myasthenia Gravis, Type-1 Diabetes Mellitus, and Hashimoto Disease: A Case Report of Autoimmune Polyendocrine Syndrome Type 3. Am J Case Rep, (2019); 20: 1709-1714 DOI: 10.12659/AJCR.918996

\section{TIME-COURSE OF RESPIRATORY DECLINE IN TYPE 1 MYOTONIC DYSTROPHY: A LONGITUDINAL 18 YEAR EXPERIENCE FROM A NEUROMUSCULAR CLINIC}

\author{
E. Iori ${ }^{1}$, M. Mazzoli ${ }^{1}$, A. Ariatti ${ }^{1}$, A. Marchioni ${ }^{2}$, G. Galassi ${ }^{1}$, V. \\ Agnoletto $^{3}$
}

${ }^{1}$ Department of Biomedical, Metabolic and Neural Sciences, University Hospital (Modena); ${ }^{2}$ Respiratory Unit, University Hospital (Modena); ${ }^{3}$ Cardiology Unit, University Hospital (Modena)

Introduction: The relationship between CTG repeat length, natural history and time-course of respiratory functional decline in DM1 is still not clear. Furthermore, the role of central respiratory drive dysfunction is not established yet.

Methods: The study included 33 DM1 patients with confirmed abnormal CTG expansion of DMPK gene referred to our Clinic between 2000 and 2018 and followed up for 18 years. Trinucleotide repeat year scores, reflecting increasing disease duration and severity of mutation, were determined. Participants were assessed with Muscular Impairment Rating Scale (MIRS) and pulmonary function tests, such as forced vital capacity (FVC), forced expiratory volume in $1 \mathrm{sec}$ (FEV1) and FEV1/FVC (Tiffeneau index), performed at the time of enrollment and at every annual follow-up visit. The impact on MIRS and NIV requirement (planned by pneumologist in presence of symptoms suggestive of chronic respiratory insufficiency plus at least FVC less than $50 \%$ of predicted in seated position) as outcomes at fixed times (i.e. baseline, 24, 36, 48, 72 and 96 months) were evaluated using a logistic regression, survival and variance analysis.

Results: NIV was initiated in 12 patients $(36.4 \%)$ at a median age of 50.5 years (Range 33-54, IQR 12.5; mean $48.0 \pm 9.41$ ). Median time from diagnosis to NIV was 195 months (Range 24-553, IQR 200 ; mean $214.9 \pm 130.1$ ). FVC values showed a significant worsening between baseline and last follow up $(\mathrm{p}=0.003)$ and were lower at baseline in patient who underwent NIV $(\mathrm{p}<0.001)$. Linear regression suggested cumulative effect of age and trinucletide repeat size at baseline and last follow up on FVC values. MIRS scores inversely related with NIV need, meaning that respiratory impairment is linked to neuromuscular weakness. FVC was a reliable indicator of respiratory decline and of progression of neurological impairment assessed with MIRS in the logistic regression model $(p=0.02)$.
Conclusion: Severity of muscular impairment, CTG expansion size and age predict respiratory impairment in DM1. FVC resulted an independent predictor of outcome in DM1.

References:

- Hawkins AM, Hawkins CL, Abdul Razak K, et al. Respiratory dysfunction in myotonic dystrophy type 1: A systematic review. Neuromuscul Disord (2019);29:198-212. DOI:10.1016/ j.nmd.2018.12.002

- Mazzoli M, Ariatti A, Garuti GC et al. Predictors of respiratory decline in myotonic dystrophy type 1 (DM1): a longitudinal cohort study. Acta Neurologica Belgica. (2020) Jul. DOI: 10.1007/s13760020-01425-Z

- Vivekananda U, Turner C. A model to predict ventilator requirement in myotonic dystrophy type 1. Muscle Nerve (2019);59:683-687. DOI: $10.1002 /$ mus.26471

\section{EFGARTIGIMOD IN CHRONIC INFLAMMATORY DEMYELINATING POLYNEUROPATHY: ADHERE PHASE 2 TRIAL DESIGN}

G. Lauria Pinter ${ }^{1}$, G. Siciliano ${ }^{2}$, H. Haard ${ }^{3}$, W. Parys ${ }^{4}$, P. Ulrichts ${ }^{3}$, A. Guglietta $^{5}$, E. Hofman ${ }^{5}$, S. Schmidt ${ }^{4}$, A. Tse ${ }^{5}$

${ }^{1}$ IRCCS Foundation Neurological Institute Carlo Besta, Department of Biomedical and Clinical Sciences "Luigi Sacco", University of Milan (Milano); ${ }^{2}$ Department of Clinical and Experimental Medicine, University of Pisa (Pisa); Argenx BV (Ghent-B); ${ }^{4}$ Medical, Argenx BV (Ghent- B); ${ }^{5}$ Clinical Development, Argenx BV (Ghent-B)

Introduction: Chronic inflammatory demyelinating polyneuropathy (CIDP) is an immune-mediated disorder presumably associated with pathologic $\mathrm{IgG}$ antibodies. Neonatal Fc receptor (FcRn) central to $\mathrm{IgG}$ homeostasis is responsible for the long half-life of IgG. Efgartigimod, a human IgG1 antibody Fc-fragment, outcompetes endogenous IgG binding, prevents IgG recycling, and reduces IgG and autoantibody levels. Efgartigimod is currently tested in phase 2 and 3 trials in other autoimmune conditions.

Material: Efgartigimod SC, a co-formulation with ENHANZE® technology (rHuPH20 Hyaluronidase) or Placebo

Methods: This multicenter, randomized, double-blind, placebocontrolled Phase 2 study investigates efficacy, safety and tolerability of efgartigimod PH20 SC in approximately 130 newly-diagnosed CIPD patients or those with active CIDP withdrawn from standard of care during a 13-week run-in period. Treatment dependence is measured by Inflammatory Neuropathy Cause and Treatment Disability (INCAT), Inflammatory-Rasch-built Overall Disability Scale (I-RODS), and grip strength. Following a $<12$-week open-label phase of weekly efgartigimod PH20 SC (Stage A), responders enter a 48-week randomized phase of weekly treatment vs. placebo (Stage B), followed by a separate open label extension study. Efgartigimod PH20 SC is a new subcutaneous preparation co-formulated with the permeation enhancer rHuPH20 which helps to increases dispersion and absorption of efgartigimod. Approximately $6 \mathrm{ml}$ is administered weekly as a single $\mathrm{SC}$ injection over $\sim 1.5$ minutes).

Results: Primary efficacy analysis will be based on relapses (adjusted INCAT of $\geq 1$ point). The study endpoint is defined as 88 relapse events in Stage B. Additional efficacy, safety and quality of life endpoints are included. 
Discussion: While the pathogenesis of CIDP is not fully understood, nerve-reactive $\mathrm{IgG}$ is found in CIDP patients. In vitro and passive transfer studies show pathogenic potential of IgG and CIDP patients show clinical response to IVIg or plasma exchange. Thus, IgG may play a key role in CIDP, and FcRn inhibition (IgG reduction) with efgartigimod is a rational approach.

Conclusion: The study is being conducted in North America, Europe and Asia. Recruitment of the study is on-going and Italian centers are participating in the study. An interim analysis is planned after 30 patients have completed stage A.

\section{DISTINGUISHING FEATURES OF ACUTE- AND CHRONIC- ONSET CHRONIC INFLAMMATORY DEMYELINATING POLYRADICULONEUROPATHY}

G. Liberatore ${ }^{1}$, F. Manganelli ${ }^{2}$, D. Cocito $^{3}$, R. Fazio ${ }^{4}$, C. Briani ${ }^{5}$, M. Filosto $^{6}$, L. Benedetti ${ }^{7}$, A. Mazzeo ${ }^{8}$, G. Antonini ${ }^{9}$, G. Cosentino ${ }^{10}, \mathrm{~S}$. Jann $^{11}$, A. Cortese ${ }^{12}$, G. Marfia ${ }^{13}$, A. Clerici ${ }^{14}$, G. Siciliano ${ }^{15}, \mathrm{M}$. Carpo $^{16}$, M. Luigetti ${ }^{17}$, G. Lauria ${ }^{18}$, T. Rosso ${ }^{19}$, G. Cavaletti ${ }^{20}$, P. Doneddu $^{1}$, L. Santoro ${ }^{2}$, E. Peci ${ }^{3}$, S. Tronci ${ }^{4}$, M. Ruiz ${ }^{5}$, S. Cotti Piccinelli $^{6}$, A. Schenone ${ }^{21}$, L. Leonardi ${ }^{9}$, A. Toscano ${ }^{8}$, G. Mataluni ${ }^{13}$, E. Spina ${ }^{2}$, L. Gentile $^{8}$, E. Nobile Orazio ${ }^{1}$

${ }^{1}$ Neuromuscular and Neuroimmunology Service, IRCCS Humanitas Clinical and Research Institute (Rozzano-MI); ${ }^{2}$ Department of Neuroscience, Reproductive Sciences and Odontostomatology, University of Naples 'Federico II' (Napoli); ${ }^{3}$ Presidio Sanitario Major, Istituti Clinici Scientifici Maugeri (Torino); ${ }^{4}$ Division of Neuroscience, Department of Neurology, Institute of Experimental Neurology (INSPE), San Raffaele Scientific Institute (Milano); ${ }^{5}$ Neurology Unit, Department of Neuroscience, University of Padua (Padova); ${ }^{6}$ Center for Neuromuscular Diseases and Neuropathies, Unit of Neurology, ASST 'Spedali Civili', University of Brescia (Brescia); ${ }^{7}$ Neurology Unit, Sant'Andrea Hospital (La Spezia); ${ }^{8}$ Department of Clinical and Experimental Medicine, Unit of Neurology, University of Messina (Messina); ${ }^{9}$ Unit of Neuromuscular Diseases, Department of Neurology Mental Health and Sensory Organs (NESMOS), Faculty of Medicine and Psychology, 'Sapienza' University of Rome, Sant'Andrea Hospital (Roma); ${ }^{10}$ Department of Experimental BioMedicine and Clinical Neurosciences (BioNeC), University of Palermo (Palermo); ${ }^{11}$ Department of Neuroscience, Niguarda Ca' Granda Hospital (Milano);

${ }^{12}$ Neurology Unit, IRCCS Foundation C. Mondino National Neurological Institute (Pavia); ${ }^{13}$ Dysimmune Neuropathies Unit, Department of Systems Medicine, Tor Vergata University of Rome (Roma); ${ }^{14}$ Neurology Unit, Circolo \& Macchi Foundation Hospital, Insubria University, DBSV (Varese); ${ }^{15}$ Neurology Unit, Department of Clinical and Experimental Medicine, University of Pisa (Pisa); ${ }^{16}$ Neurology Unit, ASST Bergamo Ovest-Ospedale Treviglio (Treviglio-BG); ${ }^{17}$ Fondazione Policlinico Universitario A. Gemelli IRCCS, Neurology Unit, University Cattolica del Sacro Cuore (Roma); ${ }^{18}$ Unit of Neuroalgology, IRCCS Foundation 'Carlo Besta' Neurological Institute (Milano); ${ }^{19}$ Neurology Unit-Castelfranco Veneto, ULSS2 Marca Trevigiana (Treviso); ${ }^{20}$ School of Medicine and Surgery and Experimental Neurology Unit, University of Milano-Bicocca (Monza);

${ }^{21}$ Department of Neuroscience, Rehabilitation, Ophthalmology, Genetics, Maternal and Child Health, University of Genoa and IRCCS AOU San Martino-IST (Genova)

Objective: Clinical presentation of Chronic Inflammatory Demyelinating Polyradiculoneuropathy (CIDP) can be very heterogeneous and, up to
$16 \%$ of cases, patients can have an acute onset similar to Guillain-Barré Syndrome (GBS). Little is known on the clinical, laboratory and neurophysiologic features distinguishing patients with A-CIDP from those with a chronic-onset (C-CIDP).

Methods: We implemented a multicentric web-based database to collect data from patients with CIDP followed throughout Italy to determine the frequency and characteristic of CIDP and its variants, the diagnostic criteria used for their diagnosis, and their response to therapy. We defined as acute onset-CIDP (A-CIDP) a neuropathy that was initially diagnosed as GBS but that continued to progress or relapsed after more than two months from disease onset.

Results: By February 2020 we included 437 patients with a confirmed diagnosis of typical CIDP and variants. In 42 patients (10\%) there was an acute onset of CIDP, followed by a relapsing or progressive course in $68 \%$ and $32 \%$ of cases, respectively (55\% and $45 \%$ in typical CIDP). Comparing clinical features between the two groups, patients with ACIDP were younger at onset ( 45.2 vs 50.3 ys, $\mathrm{p}=0.017$ ), had a worse INCAT score at enrolment ( $3.2 \mathrm{vs} 2.5, \mathrm{p}=0.048)$, a more frequent involvement of strength $(100 \%$ vs $90 \%, \mathrm{p}=0.014)$ and cranial nerve $(50 \%$ vs $17 \%, \mathrm{p}<0.001$ ); as to therapy, A-CIDP had a higher response to overall therapies compared to C-CIDP ( $97 \%$ vs $82 \%, p=0.027$ ). As to supportive criteria, A-CIDP had a more frequent protein elevation at CSF exam ( $91 \%$ vs $65 \%, \mathrm{p}=0.019)$ with higher median value $(111 \mathrm{vs} 88 \mathrm{mg} / \mathrm{dL})$, but no statistically significant differences emerged from electrodiagnostic test. Interestingly, among tested patients, A-CIDP had a more frequently positivity for paranodal antibodies anti-CNTN-1 and NF-155 (9.5\% vs $2.5 \%, \mathrm{p}=0.021$ ).

Discussion: Some distinguishing features are present in A-CIDP patients and may reflect some overlap between CIDP and GBS.

\section{OPEN-LABEL EVALUATION OF ETEPLIRSEN IN PATIENTS WITH DUCHENNE MUSCULAR DYSTROPHY AMENABLE TO EXON 51 SKIPPING: PROMOVI TRIAL}

C. McDonald ${ }^{1}$, P. Shieh ${ }^{2}$, H. Abdel-Hamid ${ }^{3}$, A. Connolly ${ }^{4}$, N. Khan ${ }^{5}$, E.

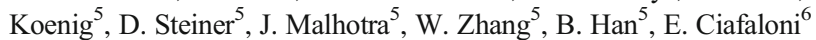

${ }^{1}$ Physical Medicine and Rehabilitation Pediatrics, University of California Davis Health (Sacramento-USA); ${ }^{2}$ David Geffen School of Medicine, UCLA (Los Angeles-USA); ${ }^{3}$ Children's Hospital of Pittsburgh, UPMC (Pittsburgh-USA); ${ }^{4}$ St. Louis Children's Hospital, Washington University School of Medicine (St. Louis-USA); ${ }^{5}$ Research and Development, Sarepta Therapeutics Inc. (Cambridge-USA); ${ }^{6}$ School of Medicine and Dentistry, University of Rochester (Rochester-USA)

Targets: Eteplirsen received accelerated FDA approval for treatment of patients with Duchenne muscular dystrophy (DMD) mutations amenable to exon 51 skipping. We report results from PROMOVI, a Phase 3, openlabel study evaluating eteplirsen in boys with DMD amenable to exon 51 skipping therapy.

Materials/Methods: Males aged 7-16 years inclusive, with confirmed DMD and frame-shift mutations amenable to treatment by exon 51 skipping, received eteplirsen IV $30 \mathrm{mg} / \mathrm{kg} / \mathrm{week}$ for 96 weeks. An untreated cohort of boys with DMD not amenable to exon 51 skipping was also enrolled. Safety was assessed in patients who had received up to 96 weeks of treatment. Efficacy outcomes assessed over 96 weeks included dystrophin protein quantification, ambulatory function (loss of ambulation, ability to rise, and North Star Ambulatory Assessment), and pulmonary function (percent predicted forced vital capacity). 
Results: 78/79 patients received eteplirsen and completed 96 weeks of treatment. Recruitment and retention of patients in the untreated group failed as only 13/30 patients completed the study. The most frequently reported adverse events (AEs) were headache, vomiting, and cough. The majority of AEs were mild to moderate and considered unrelated to eteplirsen. There were no treatment discontinuations due to an AE. Eteplirsen-treated patients showed a mean increase in dystrophin over baseline by Western blot. Comparison with the untreated cohort was confounded by withdrawals and considered inappropriate because of recently demonstrated differences in disease trajectories of the mismatched genotypes included. Comparisons of ambulatory and pulmonary function to reported natural history data suggest slowing of disease progression with eteplirsen treatment.

Discussion: Overall, 96 weeks of once-weekly eteplirsen IV appeared to be well tolerated. Dystrophin production and ambulatory and pulmonary measures suggest a positive treatment effect of eteplirsen as seen in previous studies.

Conclusions: PROMOVI contributes to the growing body of evidence for efficacy of eteplirsen, and confirms evidence of a favourable longterm safety profile, with generally mild to moderate AEs and no discontinuations due to AEs.

\section{LONG-TERM SAFETY AND EFFICACY OF GOLODIRSEN IN MALE PATIENTS WITH DUCHENNE MUSCULAR DYSTROPHY AMENABLE TO EXON 53 SKIPPING}

F. Muntoni ${ }^{1}$, L. Servais ${ }^{2}$, V. Straub ${ }^{3}$, M. Guglieri ${ }^{3}$, A. Dugar ${ }^{4}$, M. Whalen-Kielback $^{4}$, D. Steiner ${ }^{4}$, E. Koenig ${ }^{4}$, T. Feng ${ }^{4}$, B. Han ${ }^{4}$, X. Wang $^{4}$, E. Mercuri ${ }^{5}$

${ }^{1}$ Dubowitz Neuromuscular Centre, UCL Institute of Child Health and Great Ormond Street Hospital for Children (London-UK); ${ }^{2}$ Institute IMotion, Hôpital Armand-Trousseau (Paris-F); ${ }^{3}$ Newcastle Hospitals NHS Foundation Trust, Newcastle University John Walton Muscular Dystrophy Research Centre (Newcastle upon Tyne-UK) ${ }^{4}$ Research and Development, Sarepta Therapeutics, Inc. (Cambridge-USA); ${ }^{5}$ Paediatric Neurology and Centro Clinico Nemo, Catholic University and Policlinico Gemelli, Fondazione Policlinico Universitario Agostino Gemelli IRCSS (Roma)

Targets: Golodirsen is a synthetic phosphorodiamidate morpholino antisense oligomer designed to restore the mRNA reading frame in patients with Duchenne muscular dystrophy (DMD) gene mutations amenable to exon 53 skipping. We report the safety and efficacy of long-term golodirsen treatment in this first-in-human, Phase 1/2, 2-part, multicentre trial.

Materials/Methods: Twelve patients from part 1 (randomised, 12 week dose-titration phase; golodirsen, $n=8$; placebo, $n=4$ ) plus 13 additional patients with genotypically confirmed DMD amenable to exon 53 skipping therapy were included in part 2 (open label). All 25 patients received continuous, open-label treatment with golodirsen IV $30 \mathrm{mg} / \mathrm{kg} /$ week until Week 168. Safety and measures of pulmonary function (percent predicted forced vital capacity) and motor/muscle function (6-minute walk test, time to rise from floor, performance of upper limb, and muscle MRI) were assessed.

Results: Golodirsen-treated patients had a median age of 8 years at recruitment. Median golodirsen exposure was 168 weeks and safety data were collected up to 189 weeks. All golodirsen-treated patients experienced $\geq 1$ treatment-emergent adverse event (TEAE), including rhinitis, nasopharyngitis, headache, and proteinuria. Overall, 12 patients $(48 \%)$ had mild TEAEs, 8 (32\%) had moderate TEAEs, and $5(20 \%)$ had severe TEAEs. All severe TEAEs were nonserious and considered unrelated to golodirsen. There were no deaths, and no patients discontinued treatment due to an AE. Golodirsen increased exon 53 skipping by 29 -fold, and increased dystrophin protein production by 16 -fold at Week 48 . Motor, muscle, and respiratory function were evaluated over 144 weeks; assessment of functional data in golodirsen-treated patients vs matched natural history cohorts will be presented.

Discussion: Long-term treatment with golodirsen demonstrated an acceptable safety profile and was well tolerated.

Conclusions: These safety data, and data on dystrophin protein expression at 48 weeks, support golodirsen as a treatment option in patients with DMD amenable to exon 53 skipping.

\section{NEUROFILAMENT LIGHT CHAIN AS A POSSIBLE BIOMARKER IN A COHORT OF 30 SMA 3 PATIENTS UNDERGOING NUSINERSEN TREATMENT}

\author{
G. Musso ${ }^{1}$, V. Bozzoni ${ }^{1}$, L. Caumo ${ }^{1}$, L. Bello ${ }^{1}$, C. Cosma $^{2}$, G. Sorarù ${ }^{1}$, \\ M. Plebani ${ }^{3}$, E. Pegoraro ${ }^{1}$
}

${ }^{1}$ Department of Neuroscience, University of Padua (Padova); ${ }^{2}$ Department of Laboratory Medicine, University-Hospital of Padua (Padova); ${ }^{3}$ Department of Medicine-DIMED, University of Padua (Padova)

Aims: The recent approval of nusinersen as the first modifying therapy for Spinal Muscular Atrophy (SMA) urges the need of reliable outcome measures. This study aims to investigate the role of neurofilaments light chain (NfL) as disease and treatment-response biomarker in a cohort of adult SMA type 3 patients.

Materials: 30 SMA type 3 patients were recruited at the Neuromuscular Center of Padua Hospital, where nusinersen treatment was administered from February 2018 to September 2019 in a loading phase (L1 baseline, L2 day 14, L3 day 28, and L4 day 63) and a maintenance phase (M1, M2 and M3, every four months). Cerebrospinal fluid (CSF) samples were collected at each administration; NfL was tested at each time point, additional neurodegeneration biomarkers total tau ( $t-$ $\mathrm{Tau}$ ) and phosphorylated tau (p-Tau) proteins were tested at time points L1 and L3.

Methods: NfL concentration was determined with a commercial enzyme-linked immunosorbent assay (ELISA) kit (UmanDiagnostics, Umea, Sweden). T-Tau and pTau were measured with an automated Chemiluminescent Enzyme Immunoassay (CLEIA) analyzer (LUMIPULSE G600 II by Fujirebio, Japan). Neuromuscular outcomes were tested at L1, L4, M1, M2 and M3 with appropriated validated motor scales (HFMSE, RULM, 6MWT, MRC and NSAA). Statistical analyses were performed as appropriate with $\mathrm{R}$ v.3.5.3.

Results: Baseline CSF NfL, t-Tau and p-Tau levels were overall included in the reference ranges for healthy donors provided by testing assay manufacturers. Correlation was found between baseline $\log [\mathrm{NfL}]$ and age $(\mathrm{r}=0.70, \mathrm{p}<0.0001)$ and between $\log [\mathrm{t}-\mathrm{Tau}]$ and $\log [\mathrm{p}-\mathrm{Tau}]$ with $\log [\mathrm{NfL}](\mathrm{r}=0.57, \mathrm{p}=0.0014$ and $\mathrm{r}=0.45$, $\mathrm{p}=0.013$ ) at $\mathrm{L} 1$, but not at L3, although a slight significant increase was found in $\mathrm{t}-\mathrm{Tau}(\mathrm{p}=0.016)$ and $\mathrm{p}$-Tau $(\mathrm{p}=0.004)$ at L3. NfL significantly increased in loading phase until L3 (mean increase $274.1 \mathrm{pg} / \mathrm{ml}$ ). From L4 NfL, although higher than baseline, started 
to decrease and no difference was found with baseline at M1, M2 and M3. No correlation was found between NfL and motor scores at each time point.

Discussion: Our study partially reinforces recently published results in similar patients $[1,2,3]$ adding insights on NfL dynamic during the first month of treatment. Neurodegenerative biomarkers might inadequately relate to long disease duration.

Conclusions: Results of shorter time points in our cohort might suggest a putative limited axonal remodelling, although a transient side effect of lumbar puncture might not be excluded.

References:

1. Wurster C. D. et al. Neurochemical markers in CSF of adolescent and adult SMA patients undergoing nusinersen treatment. Ther Adv Neurol Disord (2019);12:1-8

2. Wurster C. D. et al. Neurofilament light chain in serum of adolescent and adult SMA patients under treatment with nusinersen. $\mathrm{J}$ Neurol (2020);267:36-44

3. Faravelli I. et al. Nusinersen treatment and cerebrospinal fluid neurofilaments: An explorative study on Spinal Muscular Atrophy type 3 patients. J Cell Mol Med (2020);24: 3034-39

\section{DELETION IN HSPB\& GENE LEADS TO DILATED CARDIOMYOPATHY WITH IMPAIRED AUTOPHAGY WORSENED BY IMMUNOSUPPRESSIVE DRUGS}

\author{
I. Orologio, G. Bruno, F. Napolitano, M. Melone, S. Sampaolo
}

Department of Advanced Medical and Surgical Sciences, Second Division of Neurology, University of Campania Luigi Vanvitelli (Napoli)

Background and aims: Mutations in genes encoding for small heat shock proteins such as HSPB8, were reported to cause distal hereditary motor neuropathies (dHMN) and distal rimmed vacuoles myopathy. We characterize a new clinical phenotype in a family with a novel mutation in HSPB8 gene and we support the role of peripheral blood smear (PBS) as a simple tool for screening in myopathies with impaired autophagy.

Methods: The proband, a 52 years old man, complained about distal muscle weakness, persistent hyperCkemia and a severe dilated cardiomyopathy. Familial history revealed a sister with mild cardiomyopathy and his mother deceased for heart failure. A complete neuromuscular protocol was carried out, including whole exome sequencing (WES). Three months later he was hospitalized for receiving heart transplant and started treatment with cyclosporine.

Results: WES identified a novel heterozygous deletion in HSPB8 (c.266del/p.Pro89HisfsTer12), also present in his affected sister. PBS disclosed PAS-positive vacuoles in lymphocytes as well as muscle biopsy. Immunofluorescence study conducted using LC3 and P62 confirmed an impaired autophagy on muscle and PBS both. After one-year follow-up, neurological examination revealed a severe muscle weakness including axial and proximal districts with dropped head.

Conclusion: We expanded the phenotypic spectrum of HSPB8 mutations describing for the first time a familial cardiomyopathy related to a deletion in this gene. Moreover, it is well known that HSPB8 silencing exhibited blocking effects on the autophagosome-lysosome fusion and we suppose that cyclosporine could have worsened muscle weakness due to his effect on autophagic flux.
QUALITY OF LIFE AND DISABILITY IN MYASTHENIA GRAVIS SUBTYPES

\author{
F. Pasqualin ${ }^{1}$, S. Guidoni ${ }^{1}$, M. Ermani ${ }^{2}$, E. Pegoraro $^{2}$, D. Bonifati ${ }^{1}$ \\ ${ }^{1}$ Neurology, Ca Foncello Hospital (Treviso); ${ }^{2}$ Neurosciences, University \\ of Padua (Padova)
}

Objective: To compare disability and quality of life in different subtypes of myasthenia gravis (MG) for individualizing management and goals of MG patients.

Materials and Methods: Questionnaires about quality of life (QOL15) and disability (MGDIS) were administered to 100 consecutive MG patients at last follow-up visit. At the same time clinical status was measured with INCB-MG and ADL scales. The ADL was filled according to the original instructions with the medical asking the questions and noting the answers in the form. The Italian validated version of this score was used. Outcome to treatment was evaluated with MGFA-PIS and MGSTI scores.

Results: The 100 patients included were classified as follows: 12 ocular, 22 early onset (EOMG), 41 late onset (LOMG), 12 thymoma-associated, 6 anti-MuSK and 7 double seronegative (SN). MGDIS score was on average $31,2 \pm 10,5$, (range 20-65). No differences between subgroups and no correlation with age or disease duration were found. Global QOL-15 score did not differ among subgroups. Item 12 related to difficulties in walking was significantly different in LOMG compared to EOMG and antiMusk subgroups. No significant difference in the global ADL score emerged among subgroups but SN subtype had more difficulties in swallowing and more frequent diplopia in comparison with both late- and early-onset MG subtypes. Regarding clinical status measured with INCB score only facial weakness differed between subgroups $(p=0,029)$. A slight inverse correlation between respiratory district and duration of the disease was found $(R=-0,26, p<=0,008)$. Comparing the different scores and questionnaires, we found a high correlation between MGDIS and QOL15, and low between them and INCB-MG.

Discussion and conclusions: In our study quality of life and disability were similar in different MG subtypes. The homogeneous results among different types of MG can be attributed to the self-administration of the questionnaire. This makes patients evaluate their own disability and quality of life, perceiving them in comparison with their own expectations, influenced by age and basal condition of the individual. INCB-MG has confirmed to be a good tool for clinical evaluation of MG patient. The slight correlation between self-administrated scores and medical related ones suggest the usefulness of including all of them in patient's follow up in order to have a global description of the status of the patient. Our study confirms the efficiency of the scores in describing different aspect of the disease.

\section{RESPONSE TO TREATMENT AND OUTCOME IN LATE ONSET MYASTHENIA GRAVIS}

\author{
F. Pasqualin ${ }^{1}$, S. Guidoni ${ }^{1}$, M. Ermani ${ }^{2}$, E. Pegoraro ${ }^{2}$, D. Bonifati ${ }^{1}$ \\ ${ }^{1}$ Neurology, Ca Foncello Hospital (Treviso); ${ }^{2}$ Neurosciences, University \\ of Padua (Padova)
}


Objectives: Recently different subtypes of myasthenia gravis (MG) have been described. They differ for clinical features and pathogenesis but the prognosis and response to treatment is less clear. The aim of the study was to evaluate treatment, outcome and side effects in early versus late onset MG (LOMG).

Methods: We reviewed the clinical records of $208 \mathrm{MG}$ patients attending Myasthenic out-patient clinic at the Ca' Foncello, Hospital, Treviso. Age at onset, severity of the disease at onset and maximum severity using MGFA classification, diagnostic criteria, serology, clinical features and comorbidities, treatment and adverse effects were recorded. Outcome at the last follow-up was evaluated with MGSTI and MGPIS scales. MGSTI is a new scale that identifies 6 subgroups of patients on the basis of clinical remission or minimal manifestations together with the amount of immunotherapy.

Results: The 208 patients were classified as follow: 36 ocular MG, 40 early onset MG (EOMG), 72 LOMG, 25 thymoma-associated, 14 antiMuSK and 21 double seronegative. Similar positive outcome was achieved in early versus late subgroups. We found pharmacological remission and minimal manifestations at the MGFA-PIS in the 95\% and $94,4 \%$ of EOMG and LOMG respectively but in LOMG a lower dose of immunosuppressors $(\mathrm{MGSTI}<2)$ was required compared to EOMG $(p=0,048)$. Severe side effects were present in a small percentage of patients in both groups but diabetes was more frequent in LOMG vs EOMG $(2,2 \%$ vs $5 \%, \mathrm{p}=0.017)$.

Discussion and Conclusions: The goal of care in MG is to achieve maximal clinical benefit with lower dose of immunosuppressants. In our study response to treatment and outcome were evaluated with the MGSTI score, a new score that needs to be validated in everyday clinical practice and observational studies. In our study it showed to be reliable and with higher sensibility than MGFA-PIS in picking up differences between subgroups. It seems particularly important to consider the burden of immunotherapy when subgroups with similar clinical features but different age are compared. Despite LOMG has more comorbidities that might interfere with treatment and outcome, therapeutic management does not seem to differ between EOMG and LOMG, with similar positive outcome. LOMG group seems to require lower doses of medication to control symptoms. In EOMG side effects such as cataract, diabetes and osteoporosis were similar to LOMG but more frequent compared to the agematched population.

\section{THYMECTOMY IN LATE ONSET MYASTHENIA GRAVIS: THE ROLE OF THYMIC ABNORMALITY AT CHEST CT}

\author{
F. Pasqualin ${ }^{1}$, S. Guidoni ${ }^{1}$, M. Ermani ${ }^{2}$, E. Pegoraro ${ }^{2}$, D. Bonifati ${ }^{1}$
}

${ }^{1}$ Neurology, Ca Foncello Hospital (Treviso); ${ }^{2}$ Neurosciences, University of Padua (Padova)

Objective: The aim of the study was to evaluate if thymic imaging might identify people with late onset myasthenia gravis (LOMG) and thymic hyperplasia. These subgroups of patients may benefit from thymectomy as well as early onset myasthenia gravis (EOMG).

Materials and Methods: We reviewed the clinical records of patients with MG attending Myasthenic out-patient clinic at the Ca' Foncello, Hospital, Treviso. Age at onset, severity of the disease at onset and maximum severity using MGFA classification, diagnostic criteria, serology, clinical features and comorbidities, treatment and adverse effects, and outcome at the last follow-up were recorded. Absence or presence of thymic tissue at the chest radiology was correlated with MG subgroups and age at onset. In the patients who underwent thymectomy, thymic histology was recorded.

Results: Detailed thymic radiology was available for $194 \mathrm{MG}$ patients. 120 patients did not show any thymic abnormality on imaging of whom $50,8 \%$ were LOMG and 5,8\% EOMG. 52 belonged to other subtypes. Five patients without thymic abnormality underwent thymectomy, Hyperplasia was found in 2 (1 EOMG) and atrophy in 3 patients (none LOMG). 74 patients $(38,4 \%)$ had a thymic abnormality and 67 underwent thymectomy. The histology was thymoma in $25(37,3 \%)$ and hyperplasia in 42 patients $(62,7 \%)$. In hyperplasia subgroup there was higher prevalence of EOMG (78,6\% vs 9,5\%). The four LOMG patients with hyperplasia had an age of 50,51,51 and 66 years respectively.

Discussion: Thymectomy is part of MG treatment mainly in EOMG with thymic hyperplasia but clear criteria to propose intervention are lacking. Recently a randomized trial has demonstrated its efficacy in non-thymomatous MG. Age as inclusion criteria was extended from 60 to 65 years due to slow recruitment. No radiological criteria were used. We evaluated if thymic abnormality on imaging might identify hyperplasia in older patients. In our study 20 patients had LOMG and thymic abnormality. Leaving out thymomas four patients had a histological diagnosis of hyperplasia. Three of them were just older than 50 years while he fourth patient had an age at onset of 66 years. These data suggest that 50 years is an appropriate age to distinguish the two forms but chest imaging may help to identify patients with a late form but an "early" pathogenesis that can benefit from thymectomy as well.

Conclusions: The presence of thymic abnormality on imaging should be considered when thymectomy is proposed in LOMG.

\section{FABRY DISEASE AND SKIN ASPECTS}

\section{F. Pietrocarlo}

\section{ASL Latina, Sapienza University (Roma)}

Objectives: Find a therapy for the angiocheratoma in Fabry Disease.

Material: Patient 65 years old with vascular lesion of white matter, hearth and also skin.

Method: E.O. vascular lesion of the skin also on the genital region. It is a genetic disease (lisosomal).

Result: Every type of drug is symptomatic; it is possible to use migalastat. There is an improvement of the clinic conditions.

Discussion: Migalastat or not migalastat?

Conclusion: Drugs not always give a complete effect.

\section{GNE MYOPATHY IN AN ITALIAN COHORT OF PATIENTS: LONG-TERM FOLLOW-UP AND CLINICAL FEATURES}

\author{
A. Pugliese ${ }^{1}$, C. Bonanno ${ }^{1}$, G. Nicocia ${ }^{1}$, A. Lupica ${ }^{1}$, S. Messina ${ }^{1}, \mathrm{G}$. \\ Vita $^{2}$, G. Vita ${ }^{1}$, A. Toscano ${ }^{1}$, C. Rodolico ${ }^{1}$ \\ ${ }^{1}$ Department of Clinical and Experimental Medicine, University of \\ Messina (Messina), ${ }^{2}$ Nemo Sud Clinical Centre, University of Messina \\ (Messina)
}

Background: GNE myopathy is a rare genetic disorder due to mutations in the GNE gene, which encodes for the key enzyme of sialic acid 
biosynthesis [1]. At disease onset, GNE patients present distal lower limb muscles weakness that slowly progresses with the involvement of other lower and upper extremities muscles [2].

Aim: To describe our cohort of patients affected by GNE myopathy, undergone clinical and laboratory follow-up for a mean time of 10 years. Patients and methods: We report 13 patients ( $9 / 13$ women and 4/13 men) affected by GNE myopathy. The diagnoses were based on clinical evaluation, EMG, muscle MRI/CT, muscle biopsy and genetic analysis.

Results: Our patients have an average age of 44 years old. The onset of symptoms was between the second and third decade of life. In the early stage, every patient complained difficulty in walking caused by foot drop. Since the onset of symptoms, 10 patients are now wheelchair-bound and quadriceps is typically spared in each patient, as commonly described for GNE myopathy. Only one patient developed respiratory failure requiring non-invasive ventilation. EMG study revealed a myopathic pattern in all patients. Muscle biopsy showed atrophic fibers with "rimmed" vacuoles. All our patients are genetically characterized: $4 / 13$ present a homozygous mutation in GNE gene, 9/13 a compound heterozygous one.

Conclusions: GNE myopathy is a very rare muscle disorder, probably under-diagnosed because of the large group of diseases manifesting with foot drop. Although that, it's necessary to suspect it in case of distal and bilateral lower limb muscles weakness with quadriceps sparing. Several aspects of this myopathy are still unclear and there is no approved therapy, but novel therapeutic strategies continue to be explored [2].

References:

1. Carrillo N. et al. GNE Myopathy: Etiology, Diagnosis, and Therapeutic Challenges. Neurotherapeutics (2018);15:900-14

2. Nishino I. et al. GNE Myopathy: current update and future therapy. J Neurol Neurosurg Psychiatry (2015) April; 86(4): 385-92

\section{LONG-TERM FOLLOW-UP IN A LARGE COHORT OF LATE ONSET POMPE DISEASE (LOPD) PATIENTS: A SINGLE CENTRE EXPERIENCE}

\author{
A. Pugliese, G. Nicocia, G. Tavilla, A. Ciranni, R. Oteri, C. Rodolico, O. \\ Musumeci, A. Toscano
}

Department of Clinical and Experimental Medicine, Neurology and Neuromuscular Disorders Unit, University of Messina (Messina)

Background: Pompe disease is a metabolic disorder, caused by deficiency of lysosomal acid $\alpha$-glucosidase (GAA) that is involved in glycogen degradation. It may occur in infantile and late onset (LOPD) forms. LOPD presents as a multisystem disease, mainly characterized by proximal limb-girdle myopathy with respiratory failure [1]. Since 2006, an Enzyme Replacement Therapy (ERT) is available with lack of data on long-term efficacy [2]

Aim: To evaluate clinical, laboratory and therapeutic data in a large cohort of LOPD patients diagnosed and followed in our ERN-NMD Centre.

Patients and methods: In the last 30 years, we have diagnosed and followed up 46 LOPD patients by neurological, respiratory and muscle MRI studies, also with functional tests as 6MWT, GSGC and respiratory tests. Muscle biopsy and GAA assay activity in skeletal muscle were performed in 41 patients. Since 2008, we have treated 38/46 patients with ERT.

Results: $52 \%$ of patients manifested at onset proximal/ axial muscle weakness, $44 \%$ hyperCKemia, myalgia and exercise intolerance, $4 \%$ some atypical features (i.e. eyelid ptosis, rigid spine). At the time of diagnosis, 43/46 patients presented elevated CK values (range 200-2000 UI/l). In 26/41 muscle biopsy revealed vacuolar myopathy with increased glycogen; GAA residual activity was markedly reduced (range $0.3 \%$ - $30 \%$ ). Since 2008,38 patients started ERT and have been followed for a variable period (follow up range 112 years). During the course of ERT, we registered a significant worsening at $6 \mathrm{MWT}$ in $46 \%$ of patients, while $21 \%$ improved in motor performances and $32 \%$ remained stable. We observed worsening in respiratory function in $50 \%$ of patients.

Conclusions: Our study confirms that in LOPD patient's clinical presentations and laboratory results are heterogeneous. Long-term results on treated patients evidenced variable responses to therapy, showing in some patients' stability or positive results but overtime almost $50 \%$ of the patients show a deterioration of muscle and respiratory parameters.

References:

1. Toscano A. et al. Multisystem late onset Pompe disease (LOPD): an update on clinical aspects.Ann Transl Med. (2019) Jul;7(13):284

2. Schoser B. et al. Survival and long-term outcomes in late-onset Pompe disease following alglucosidase alfa treatment: a systematic review and meta-analysis. J Neurol. (2017) Apr;264(4):621-30

\section{NOVEL GAA MUTATION IN A PATIENT WITH LATE ONSET POMPE DISEASE: A CASE REPORT}

\author{
L. Rapisarda, S. Barone, F. Fortunato, I. Sammarra, A. Gambardella, P. \\ Valentino
}

Institute of Neurology, Department of Medical and Surgical Sciences, Magna Graecia University (Catanzaro)

Introduction: Pompe disease (PD) is an autosomal recessive disorder caused by deficiency of the lysosomal glycogen-hydrolyzing enzyme acid alpha-glucosidase (GAA), resulting in glycogen accumulation primarily in skeletal, cardiac and smooth muscle. Generally, it manifests in childhood, but it may present in adulthood typically with more subtle manifestations [1].

Case description: A 53-years-old man presented proximal limb-girdle and truncal weakness and exercise intolerance. These symptoms started about ten years ago, with a slow progressive worsening. In the same period the patient was diagnosed a cardiopathy with severe aortic valvular insufficiency and ascending aorta dilation. For this reason, he underwent to aortic valve and ascending aorta replacement. Moreover, he developed a ventilatory insufficiency mixed type and started ventilatory therapy with continuous positive airway pressure (CPAP) device. Neurological examination evidenced an anserine gait, marked hypotrophy of shoulder and pelvic girdle and proximal muscular weakness, positive Gower's maneuver, lower limbs hyporeflexia and normal sensitivity. Serum biochemical test were unremarkable except for elevated creatine kinase (806 UI/L). Electrophysiological test revealed normal nerve conduction study, absence of spontaneous muscular activity, early recruitment of proximal limb muscles. Quantitative motor unit action potential (MUAP) analysis showed MUAPs with low amplitude, short duration and polyphasic. Acid alphaglucosidase activity measured through dry blood spot (DBS) assays showed a slight reduction of enzymatic activity $(<6 \mathrm{nmol} / \mathrm{h} / \mathrm{ml})$. Genetic analysis of GAA gene revealed the presence of two mutation in heterozygous state: G370S (c.1108G >A) in exon 7 and E689K (c.2065G>A) in exon 15. In addition, it has been performed Multiplex Ligation Probe Amplification (MLPA) analysis which did not evidence any deletion or duplication.

Discussion and conclusion: We described the case of a middle-age man with a chronic myopathy, with cardiac and respiratory involvement. This led us to investigate him for PD. Genetic analysis displayed two different mutation in heterozygous state. It has already been reported that E689K (c.2065G $>$ A) substitution does not appear to affect GAA activity 
directly, but it might exert a modifying influence if it was present on an allele bearing a deleterious mutation [2]. Particularly this mutation in cis with $c .1726 \mathrm{G}>\mathrm{A}$ mutation is associated with pseudodeficiency of GAA [3]. G370S (c.1108G $>A$ ) mutation has never been described. We speculate that similarly to the known mutation c. $1726 \mathrm{G}>\mathrm{A}$, this may exert a deleterious effect of enzymatic activity and cause a pseudodeficiency of GAA. Further studies are needed to ascertain the effect of this novel mutation.

References:

1. Chun J et al. The emerging phenotype of late-onset Pompe disease: A systematic literature review. Molecular Genetics and Metabolism (2017); 120(3):163-72

2. Huie ML et al. Identification of an E689K substitution as the molecular basis of the human acid alpha-glucosidase type 4 allozyme (GAA*4). Ann Hum Genet (1996);60(5):365-8

3. Peruzzo P, Pavan E Dardis A. Molecular genetics of Pompe disease: a comprehensive overview. Ann Transl Med (2019);7(13):278

\section{NEUROMUSCULAR DISEASES IN SARSCOV2 PANDEMIC SETTING: CRITICAL ASPECTS AND FUTURE OPPORTUNITIES. TUSCANY EXPERIENCE (SINTON-COVID STUDY GROUP)}

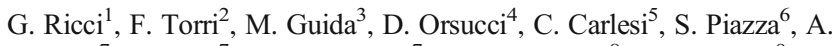
Barilaro $^{7}$, S. Mata' ${ }^{7}$, M. Del Mastio ${ }^{7}$, A. Pellegrinetti ${ }^{8}$, E. Venturini ${ }^{9}$, M. Maluccio $^{10}$, M. Cincotta ${ }^{11}$, M. Briccoli ${ }^{12}$, F. Giannini ${ }^{13}$, M. Dotti ${ }^{14}$, M. Mancuso $^{2}$, G. Siciliano ${ }^{2}$

${ }^{1}$ Department of Clinical and Experimental Medicine, University of Pisa (Pisa); ${ }^{2}$ Neurology, University of Pisa (Pisa); ${ }^{3}$ Neurology, Cisanello Hospital (Pisa); ${ }^{4}$ Neurology, S. Luca Hospital (Lucca); ${ }^{5}$ Neurology, Versilia Hospital (Camaiore-LU); ${ }^{6}$ Neurology, NOA Hospital (Massa); ${ }^{7}$ Neurology 2, Careggi Hospital (Firenze); ${ }^{8}$ Neurology, Civile Hospital (Livorno); ${ }^{9}$ Neurology and Neurophysiopathology, Hospital of Arezzo (Arezzo); ${ }^{10}$ Neurology, Lotti Hospital (Pontedera-PI); ${ }^{11} \mathrm{SOC}$ Neurology, S. Giovanni di Dio Hospital (Firenze); ${ }^{12}$ Neurology and Neurophysiology, S. Stefano Hospital (Prato); ${ }^{13}$ Neurology and Neurophysiology Clinic, University of Siena (Siena); ${ }^{14}$ Department of Medicine, Surgery and Neuroscience, University of Siena (Siena)

Objectives: We aimed at evaluating the effect of COVID emergency in the management of patients with neuromuscular disorders (NMD). These diseases put together count for a wide and heterogeneous group of illnesses that, given their variable effects on respiratory, cardiac and motor functions but also on social and care-giving aspects, represent a peculiar management matter in the SarsCoV2 pandemic scenario, in the acute as in the chronic disease setting.

Materials and Methods: The Tuscan Neurology Operative Group, referring to the Italian Society of Neurology (Società Italiana di Neurologia, SIN), SINTON, developed a multi-center survey for Tuscan Neurology Units, in order to evaluate new critical aspects in NMD patient's management during Covid 19. This is a cross-sectional study based on a multicentric survey comparing the March-April 2020 period with the same period of 2019. Twelve Neurology Units in Tuscany participate. Questions were asked about management of outpatients visits, means of communication between Centers and patients, management of emergencies, organization of scheduled intra-hospital therapies, continuity of extra-hospital services as physio kinesitherapy, nurse home assistance and multidisciplinary evaluations.
Results and discussion: The most evident information is about the reduction of about $70 \%$ of the visits in the 2020 period compared with 2019; this was partly due to the forced stop imposed to regular scheduled visits and partly to cancelation of appointments by patients; as a result, non-urgent visits and diagnostics were rescheduled. This not only caused difficulties in evaluating patients but also led to an overload of visits in the following months. Emergency evaluations, on the other hand, did not significantly change in number compared to the previous year. The majority of Centers activated a telemedicine service, with telephone contacts between neurologists. This made it possible to safely reschedule visits of stable, chronic patients without losing sight of their global conditions, and to permit a safe access to the Clinic to urgent and more fragile patients that needed to be evaluated in the pandemic period.

Conclusion: The COVID period experience made it clear that, although not sufficient for detailed and accurate patient's follow-up, televisits and other e-health tools may serve as a useful mean of patient's management in peculiar scenarios, and they should be ameliorated and made more accessible to patients themselves in order to improve communication with clinicians.

References:

- Guidon AC, Amato AA. COVID-19 and neuromuscular disorders. Neurology (2020) Jun 2;94(22):959-969. doi: 10.1212/ WNL.0000000000009566. Epub 2020 Apr 13. PMID: 32284362

- Solé G, Salort-Campana E, Pereon Y, Stojkovic T, Wahbi K, Cintas P, Adams D, Laforet P, Tiffreau V, Desguerre I, Pisella LI, Molon A, Attarian S. Guidance for the care of neuromuscular patients during the COVID-19 pandemic outbreak from the French Rare Health Care for Neuromuscular Diseases Network. FILNEMUS COVID19 study group. Rev Neurol (Paris). (2020) Jun;176(6):507-15

- Di Stefano V, Battaglia G, Giustino V, Gagliardo A, D'Aleo M, Giannini O, Palma A, Brighina F. Significant reduction of physical activity in patients with neuromuscular disease during COVID-19 pandemic: the long-term consequences of quarantine. J Neurol (2020) Jul 13;1-7

\section{PROPOSAL OF A NEW CLINICAL PROTOCOL TO STUDY ENDURANCE AND FATIGABILITY IN ADULT SMA PATIENTS IN EVALUATING THE OUTCOME TO TREATMENT}

\author{
G. Ricci ${ }^{1}$, A. Govoni ${ }^{1}$, A. Borelli ${ }^{1}$, I. Bortone ${ }^{2}$, L. Manca ${ }^{1}$, G. Siciliano ${ }^{1}$
}

${ }^{1}$ Department of Clinical and Experimental Medicine, University of Pisa (Pisa); ${ }^{2}$ Department of Basic Medicine, Neuroscience, and Sense Organs, University of Bari (Bari)

Objective: The wide phenotypic spectrum of patients with SMA-2 and -3 needs to define criteria for eligibility to new treatments and to identify sensitive and specific outcome measures. We aimed to define a new comprehensive clinical protocol in adult SMA patients, named Endurance and Fatigability Scale (EFS), useful to evaluate and characterize motor performance, exercise endurance and fatigue with a quantitative tool, in addition to the standard motor evaluation commonly used in clinical practice and trials.

Materials and Methods: Validity and reliability of FES has been assessed in a subset of 10 adult patients.

Results: The EFS includes several sections with different motor tests in order to investigate fatigability both at upper and lower limbs, such as: Endurance in keeping up a weight, - Exhaustible exercise of proximal upper limbs with repetitive tasks, - HandGrip endurance with repetitive maximal 
isometric contraction, -Repeated nine-hole peg test (r9HPT), -Open and close hands test, -Digital dexterity repetitive test, -Repetitive TUG test.

Discussion: The development of comprehensive set of endurance items is a pivotal step to address fatigability in patients with SMA.

References:

- Dunaway Young S, Montes J, Kramer SS, Podwika B, Rao AK, De Vivo DC. Perceived Fatigue in Spinal Muscular Atrophy: A Pilot Study. J Neuromuscul Dis. (2019);6(1):109-17

- Montes J, Dunaway Young S, Mazzone ES, et al. Nusinersen improves walking distance and reduces fatigue in later-onset spinal muscular atrophy. Muscle Nerve (2019) Oct;60(4):40914

- Montes J, McDermott MP, Martens WB, et al. Six-Minute Walk Test demonstrates motor fatigue in spinal muscular atrophy. Muscle Study Group and the Pediatric Neuromuscular Clinical Research Network. Neurology (2010) Mar 9;74(10):833-8

\section{ANTI-MAG NEUROPATHY OR CIDP: DIAGNOSIS BEYOND ANTIBODIES}

M. Ruiz ${ }^{1}$, P. Doneddu ${ }^{1}$, F. Manganelli ${ }^{2}$, L. Benedetti ${ }^{3}$, G. Liberatore ${ }^{1}$, E. Nobile-Orazio $^{1}$

${ }^{1}$ Neuromuscular and Neuroimmunology Service, Humanitas Clinical Institute, Milan University (Rozzano-MI); ${ }^{2}$ Department of Neurosciences, Reproductive and Odontostomatological Sciences, University Federico II of Naples (Napoli); ${ }^{3}$ Ospedale Policlinico San Martino IRCCS, University of Genoa (Genova)

Neuropathy with anti-myelin-associated glycoprotein (MAG) IgM antibodies (anti-MAG neuropathy) is a paraproteinemic demyelinating polyneuropathy characterized by sensory ataxia, tremor, slow progression and abnormally increased distal latencies. In the EFNS/PNS Guideline for chronic inflammatory demyelinating polyradiculoneuropathy (CIDP) the presence of these antibodies excludes the diagnosis of CIDP even if some patients with typical CIDP may have these antibodies. Discrimination between antiMAG neuropathy and CIDP with anti-MAG IgM may have relevant therapeutic implications. We developed a diagnostic score based on supportive (+) and unsupportive (-) clinical $(7$ points + and 5 points -), electrophysiological (4+ and 4-) and laboratory (4+) parameters to distinguish CIDP with anti-MAG IgM from anti-MAG neuropathy. On a scale of 15 points, we determined a cut-off of 10 points to distinguish anti-MAG neuropathy from CIDP. We applied it to: (i) 46 patients with anti-MAG neuropathy from our center, (ii) 32 patients with distal acquired demyelinating symmetric (DADS) neuropathy from the Italian CIDP database, and (iii) 17 patients with a clinical diagnosis of CIDP but with high anti-MAG monoclonal IgM from the Italian CIDP database. The scores in patients with anti-MAG neuropathy ranged from 10 to 15 , while patients with DADS ranged from -1 to 8 with a statistically significant difference between the two groups $(\mathrm{P}<0.0001)$. Using this score, 6/17 patients with a clinical diagnosis of CIDP and anti-MAG antibodies were defined to have anti-MAG neuropathy while eleven maintained a diagnosis of CIDP. We created a diagnostic tool that combines clinical, biochemical and electrophysiological parameters that enable structured approach and can quantitatively provide the likelihood of the diagnosis of MAG neuropathy. This diagnostic approach may help to best categorize patients with clinical CIDP pattern and associated anti-MAG antibodies. Patients with a score of 9 or lower should probably be diagnosed as CIDP, independently from the Buhlmann (BTU) titers, and immunomodulatory therapies such as steroids and intravenous immunoglobulin should be tried.

The Italian CIDP Study Group

References:

- Magy L, Kaboré R, Mathis S, et al. Heterogeneity of Polyneuropathy Associated with Anti-MAG Antibodies. J Immunol Res. (2015);2015:450391. doi:10.1155/2015/450391

- Svahn J, Petiot P, Antoine JC, et al. Anti-MAG antibodies in 202 patients: clinicopathological and therapeutic features. J Neurol Neurosurg Psychiatry. (2018);89(5):499-505. doi:10.1136/jnnp2017-316715

- Lupu VD, Mora CA, Dambrosia J, Meer J, Dalakas M, Floeter MK. Terminal latency index in neuropathy with antibodies against myelin-associated glycoproteins. Muscle Nerve. (2007);35(2):196202. doi:10.1002/mus. 20678

\section{HEREDITARY AMYLOID TRANSTHYRETIN AMYLOIDOSIS: HOW HAS THE LIFE OF PATIENTS CHANGED IN THE PAST 30 YEARS?}

M. Russo, L. Gentile, A. Toscano, G. Vita, A. Mazzeo

Unit of Neurology and Neuromuscular Disorders, Department of Clinical and Experimental Medicine, University of Messina (Messina)

Hereditary amyloid transthyretin (ATTRv) amyloidosis with polyneuropathy, is an autosomal dominant rare disease of adult onset, affecting the sensorimotor and autonomic function along with other organs (heart, gastrointestinal tract, eyes, kidney). Early-onset of the disease, defined as $\leq 50$ years of age at symptom onset is common in Portugal and in endemic foci in Japan while late-onset cases are predominant in other nations. Once polyneuropathy has started its progression is rapid and evolves into three successive stages: in the first patients have a sensory polyneuropathy that leads to difficulty walking without assistance; in the second there is a sensorimotor polyneuropathy and assistance for walking is required; in the last stage the patients are wheelchair bound or bedridden. Death occurs on average 12 years after the onset of the disease. The management of the disease is sensationally improved in the last three-decade, various diseasemodifying therapies have been developed, moreover, the awareness of being in front of a multisystem pathology that requires a multidisciplinary approach, has also improved the management of symptoms. In parallel, the increased knowledge and attention to the disease also reduced the diagnostic delay, favouring an earlier start of treatment. In this report, we compare how the diagnosis, the clinical progression, and the treatment of patients with ATTRv has evolved in the last three decades in our department, which is the regional reference centre for the diagnosis and treatment of genetic amyloidosis in Sicily.

\section{CAPTURING THE PATIENT-REPORTED IMPACT OF MYASTHENIA GRAVIS IN THE REAL-WORLD SETTING USING A SMARTPHONE APPLICATION}

F. Saccà ${ }^{1}$, P. Assouly ${ }^{2}$, K. Claeys ${ }^{3}$, R. Mantegazza ${ }^{4}$, E. Bagshaw ${ }^{6}$, H.

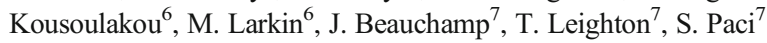


${ }^{1}$ NSRO Department, Federico II University (Napoli); ${ }^{2}$ Association des Myasthéniques Isolés et Solidaires (La Chapelle-en-Serval-F); ${ }^{3}$ Department of Neurology, University Hospitals Leuven (Leuven-B); ${ }^{4}$ Department of Neuroimmunology and Neuromuscular Diseases, Fondazione IRCCS, Istituto ${ }^{5}$ Neurologico Carlo Besta (Milano), ${ }^{6}$ Vitaccess Ltd. (London-UK); ${ }^{7}$ Argenx (Ghent-B)

Background: Myasthenia gravis (MG) is a chronic autoimmune disease, causing debilitating muscle weakness. Patient-reported data are crucial to improve knowledge about the burden of this condition and patients' unmet needs.

Aims: To design a patient-centric real-world study to allow people with MG to provide data about disease burden using an application on their smartphones.

Materials and methods: The study methodology and application were co-developed with patients, patient advocacy groups (PAGs), and disease experts (neurologists, researchers), who also reviewed and validated the protocol. Health economists and statisticians were also consulted. Clinicaltrials.gov: NCT04176211

Results: We will conduct a two-year, prospective, observational study of 2,000 people with MG, $\geq 18$ years, any gender, resident in the US, Japan, Germany, France, the UK, Italy, Spain, Canada, and Belgium. Participants will use the application to check eligibility, provide consent and contribute data. They will complete a personal profile about demographics, MG characteristics and previous care; a monthly event tracker about recent treatments, healthcare visits, adverse events and productivity losses; and a monthly selection of validated patient-reported outcomes tools about MG symptoms, functioning and quality of life. To promote engagement, participants will receive in-app reminders to contribute data, acknowledgement for received data, and updates on study findings. The application will also support participants by providing clinician-validated information about MG and its management.

Discussion and conclusions: Collecting data from people with MG via a smartphone application should enable a rich and granular dataset to be generated, to help researchers and clinicians understand the real-world impact of the condition and the unmet needs of patients.

\section{ULTRASOUND CHANGES OF VAGUS NERVE IN DIABETES NEUROPATHY: A POSSIBLE MARCKER OF AUTONOMIC NEUROPATHY}

\author{
F. Sartucci ${ }^{1}$, M. Tomassini ${ }^{1}$, M. Santin ${ }^{1}$, T. Bocci ${ }^{2}$, E. Gianetti ${ }^{3}$, P. \\ Marchetti $^{3}$
}

${ }^{1}$ Neurophysiopatology Unit, Department of Clinical and Experimental Medicine, Pisa University Medical School, University of Pisa (Pisa);

2"Aldo Ravelli" Center for Neurotechnology and Experimental Brain Therapeutics, Department of Health Sciences, University of Milano (Milano); ${ }^{3}$ S.D. Endocrinology and Metabolism of Organ and Cell Transplants, Department of General Surgery, University of Pisa (Pisa)

Background and Rationale: Somatic diabetic demyelinating distal sensory-motor polyneuropathy (D-DSP) represents a very common complication in diabetes mellitus (DM); autonomic neuropathy may be part of the disease and leads to high morbidity and mortality. Vagus Nerve (VN) is the longest autonomic nerve and commonly parasympathetic involvement precedes sympathetic symptoms. Aims of the present study was to assess sonographic changes of the $\mathrm{VN}$ in DM patients in cases with or without autonomic symptoms and neuropathy.
Methods: The VN was scanned on the left side in 20 healthy volunteers and 61 patients $(32 \mathrm{~m}, 29 \mathrm{f})$ with DM in the axial plane at the lateral neck. The acquisition of ultrasound images was performed using a 19MHz probe with an Esaote MyLab Gamma device; cross-sectional area (CSA), perimeter, echogenicity and echo structure were considered.

Results: Mean cross-sectional area of the vagus nerve was significantly smaller $(3.13 \mathrm{~mm} 2 ; \mathrm{SD}=1.54)$ in patients with diabetes compared with controls $(5.8 \mathrm{~mm} 2, \mathrm{DS}=1.2)$ and did not correlate with disease duration and parameters of cardiovagal functions.

Conclusions: Our data prove a degree of vagus nerve atrophy in DM patients. This finding may have relevance in the diagnosis and management of diabetic neuropathy, overally on parasympathetic involvement, even if further investigations are needed to confirm the relevance of the findings.

References:

- Sartucci F., Tomassini M., Santin M., Bocci T., Gianettti E., Marchetti P. Ultrasound changes of vagus nerve in diabetes neuropathy. Abstracts ISPNI 8th Annual Meeting, Trieste (2019)

- Giovagnorio F, Martinoli C. Sonography of the cervical vagus nerve: normal appearance and abnormal findings. AJR Am J Roentgenol (2001); 176:745-9

- Tawfik EA, Walker FO, Cartwright MS, El-Hilaly RA. Diagnostic Ultrasound of the Vagus Nerve in Patients with Diabetes. J Neuroimaging (2017);27(6): 589-93

\section{IMPACT OF LOCKDOWN BY COVID INFECTION ON FATIGUE AND PAIN PERCEPTION IN HEREDITARY MOTOR AND SENSORY NEUROPATHY PATIENTS}

\author{
E. Schirinzi, A. Govoni, L. Fantozzi, E. Merico, R. Calabrese, G. Ricci, \\ G. Siciliano
}

Neurological Clinic, Department of Clinical and Experimental Medicine, University of Pisa (Pisa)

In hereditary motor and sensory neuropathy (HMSN) fatigue and neuropathic pain are relevant symptoms which influence the quality of life of the patients. However, the quarantine, due to the imposed social limitations and its emotional impact, could be considered an important stressor to get worse the symptoms. Aim of the study was to evaluate the burden of fatigue and neuropathic pain during lockdown due to COVID 19 pandemia in a group of patients with diagnosis of HMSN, in relation to the clinical disease severity, quality of life and physical activity. 19 patients ( 8 men and 11 women; mean age 47.7 years), with genetic diagnosis of HMSN and classified for disease severity according to Charcot-Marie Tooth Neuropathy Score (CMTNS), underwent to a clinimetric evaluation by phone interview. In particular, we assessed fatigue burden by means of fatigue severity scale (FSS) and of neuropathic pain by means of NRS and DN4 questionnaires, physical activity intensity, considered as weekly hours of physical exercise, quality of life by means of SF36 scale. We evaluated changes from the 2 months immediately before lockdown (from 1th January to 9th March 2020) to the 2 months of quarantine (from 10 th March to 3th May 2020). All patients had electrophysiological parameters stable in the examination within 3 months respect to the previous last year. 14 patients presented a FSS score $\geq 4.6$, statistically higher compared to the preCOVID period, independently by age and sex. 13 patients presented a DN4 score $\geq 4 / 10$. No significant difference concerning gender or 
CMT subtype were found, while mean age, disease duration and pain intensity, according to NRS score, were statistically greater respect to the group with $\mathrm{DN} 4<4$; although no differences were found comparing data in pre and post-COVID period, the pain was significantly related to fatigue, severity degree of illness and a significant worse perception of mental health, while the global perception of physical health status remained stable, evaluated by SF36 Health Survey. No significant reduction was observed for physical activity intensity that was nevertheless significantly related to higher pain and fatigue perception. Fatigue and neuropathic pain are frequent symptoms in patients with HMSN, associated with an increased disease severity and an impaired quality of life. The lockdown, probably influencing the patient's mood, could have contributed to exacerbate the disease symptoms perception. A more prolonged follow up should be useful to confirm the impact of social and emotive factors on the disease course perception.

\section{MUSCLE MRI OF UPPER GIRDLE IN GNE MYOPATHY}

\author{
E. Torchia ${ }^{1}$, M. Lucchini ${ }^{1}$, S. Bortolani ${ }^{1}$, M. Monforte ${ }^{2}$, M. Mirabella ${ }^{2}$, \\ E. Ricci ${ }^{2}$, G. Tasca ${ }^{2}$ \\ ${ }^{1}$ Università Cattolica del Sacro Cuore, Fondazione Policlinico \\ Universitario A. Gemelli IRCCS (Roma); ${ }^{2}$ Neurology, Fondazione \\ Policlinico Universitario A. Gemelli IRCCS (Roma)
}

Background: GNE myopathy (GNEM) is a rare autosomal recessive adult-onset myopathy presenting with distal weakness and atrophy of lower limb (LL) muscles typically sparing the quadriceps. Muscle MRI pattern has been described in the LL with early involvement of biceps femoris short head and distal leg muscles and sparing of the vasti. Upper girdle involvement has never been investigated so far.

Objectives: To explore upper girdle involvement in GNEM patients with muscle MRI; to compare the severity of involvement with LL; to validate the LL pattern in unpublished patients.

Materials and Methods: We analyzed muscle MRI of the shoulder girdle in 6 GNEM patients. Fourteen muscles were examined on each side and degree of fatty replacement was scored using a 4-point scale. In four of these patients a LL scan was also available. Two additional unpublished patient were also studied by means of LL MRI only.

Results: The most common feature was fatty replacement of subscapularis muscle (5/6 patients). $3 / 6$ patients also had moderatesevere involvement of serratus anterior. All patients presented a more severe muscle involvement in LL. One patient had normal upper girdle scan despite extensive alterations in the LL. The MRI pattern in LL was confirmed also in the unpublished patients.

Discussion and Conclusions: Scapular girdle muscles are relatively spared, even in the advanced stage of GNEM, with the exception of subscapularis and serratus anterior muscles. We confirm the previously published pattern in the LL, with the additional observation of lumbar paraspinal involvement.

\section{ANTI-HMGCR NECROTIZING MYOPATHY: CLINICO- PATHOLOGICAL, BIOCHEMICAL AND RADIOLOGICAL CHARACTERIZATION IN A SINGLE-CENTER EXPERIENCE}

D. Velardo ${ }^{1}$, L. Andreoli ${ }^{1}$, E. Abati ${ }^{1}$, G. Costamagna ${ }^{1}$, D. Gagliardi ${ }^{1}$, I. Faravelli $^{1}$, E. Mauri ${ }^{1}$, R. Brusa ${ }^{1}$, M. Meneri ${ }^{1}$, F. Magri ${ }^{1}$, P. Ciscato ${ }^{1}$, M. Sciacco $^{1}$, C. Cinnante ${ }^{2}$, S. Corti ${ }^{1}$, N. Bresolin ${ }^{1}$, G. Comi ${ }^{1}$
${ }^{1}$ IRCCS Foundation Ca' Granda Ospedale Maggiore Policlinico, Department of Neuroscience, University of Milan (Milano); ${ }^{2}$ IRCCS Foundation Ca' Granda Ospedale Maggiore Policlinico, Neuroradiology Unit, University of Milan (Milano)

Anti-HMGCR myopathy is a rare disease, associated with an aggressive clinical course and potentially responsive to immunosuppressive therapy. Although originally observed in statin-exposed patients, it has been increasingly diagnosed in statin-naïve patients as well. New promising therapies are emerging, more tailored thanks to a better knowledge of pathogenetic mechanisms. Based on these considerations, a study has been designed with the aim of identifying and describing patients with compatible clinical pictures starting from 2014 to the end of 2019. The disease has been diagnosed through serological methods in 16 patients, of which 10 had been treated with statins and 6 had never been exposed to these medications. All of them have been clinically evaluated and followed in time and response to therapy has been comprehensively assessed, up to the present day. The study consists of a first part where patient's clinical, biochemical and histological features are thoroughly described in the attempt of broadening the knowledge about this disease and identify its peculiar characteristics and potential biomarkers of damage and therapeutic response. A second part deals more specifically with muscle MRI images, obtained at different time points, in order to identify muscle involvement and monitor response to treatment. This aims to evaluate musculoskeletal imaging as a useful, although not yet validated, tool in guiding and modulating treatment. We believe early diagnosis with comprehensive identification of both clinical and pathological features to be paramount in order to initiate a timely and appropriate treatment regimen. Besides, we believe that the use of multiple tools including muscle MRI in the monitoring phase should be implemented to provide steady grounds to therapeutic choices.

References:

- Mohassel P, Mammen AL. Anti-HMGCR Myopathy. J Neuromuscul Dis. (2018);5(1):11-20

- Allenbach Y, Mammen AL, Benveniste O, et al. 224th ENMC International Workshop: Clinico-sero-pathological classification of immune-mediated necrotizing myopathies Zandvoort, The Netherlands, 14-16 October 2016. Neuromuscul Disord. (2018);28(1):87-99

- Meyer A, Troyanov Y, Drouin J, et al. Statin-induced anti-HMGCR myopathy: successful therapeutic strategies for corticosteroid-free remission in 55 patients. Arthritis Res Ther. (2020);22(1):5

\section{LONG-TERM FUNCTIONAL CHANGES IN BECKER MUSCULAR DYSTROPHY}

M. Villa, S. Mastellaro, L. Caumo, P. Riguzzi, V. Zangaro, D. Sabbatini, A. Fusto, B. Merlo, S. Vianello, L. Bello, E. Pegoraro

Department of Neurosciences, University of Padua (Padova)

Aims of the study: The purpose of the study was to provide a quantitative description of functional changes over time in BMD patients, describing their correlation with age and mutation groups.

Material and methods: we applied linear mixed models (LMM) to longitudinally collected functional data regarding 6 Minute Walk Test (6MWT), North Star Ambulatory Assessment (NSAA), and Timed Function Tests (TFTs): run/walk $10 \mathrm{~m}$ (TRUN), rise from the floor 
(TRISE), climb 4 standard steps (TCLIMB), collected in the BMD cohort followed at our Center from 2012 to 2020.

Results and discussion: We collected functional data from 97 BMD patients, 77 aged 18 or older, average age $30.8 \pm 17.4$ years. Average follow-up was $4.2 \pm 2.6$ years (maximum 8 years), with $4.9 \pm 2.4$ evaluations (maximum 9), for a total of 480 evaluations. The most frequent mutations were del 45-48 ( $\mathrm{n}=16)$, del 45-47 ( $=14)$, del $48(\mathrm{n}=12)$, del 45-51 ( $\mathrm{n}=8)$, and del 45-55 $(\mathrm{n}=5)$. All outcomes significantly decreased with age in adult patients: NSAA $-0.67 \pm 0.05$ points/year, $\mathrm{p}<$ $0.0001 ; 6 \mathrm{MWT}-2.3 \pm 0.8 \mathrm{~m} /$ year, $\mathrm{p}=0.0062$; TRUN $-0.036 \pm 0.008$ $\mathrm{m} / \mathrm{s}^{*}$ year, $\mathrm{p}<0.0001$; TRISE $-0.007 \pm 0.002 \mathrm{~s}-1 /$ year; and TCLIMB $0.034 \pm 0.006 \mathrm{steps} / \mathrm{s}^{*}$ year, $\mathrm{p}<0.0001$. Functional measures did not decrease with age in several mutation groups, including deletions ending on exon 51 and exon 48.

Conclusion: We confirm genotype/phenotype correlations and provide mutation-specific, quantitative natural history data to be applied to BMD clinical trial design.

\section{MOLECULAR AND CLINICAL CHARACTERIZATION IN ASYMPTOMATIC AND SYMPTOMATIC DMD CARRIERS}

\author{
V. Zangaro ${ }^{1}$, P. Riguzzi ${ }^{1}$, B. Merlo ${ }^{1}$, S. Vianello ${ }^{1}$, R. Bariani ${ }^{2}$, L. \\ Lamagna $^{1}$, B. Bauce ${ }^{2}$, G. Sorarù ${ }^{1}$, L. Bello $^{1}$, E. Pegoraro ${ }^{1}$ \\ ${ }^{1}$ Department of Neurosciences, University of Padua (Padova); ${ }^{2}$ Cardiac, \\ Thoracic, and Vascular Sciences, University of Padua (Padova)
}

Objectives: To determine the molecular and clinical characteristics of a cohort of asymptomatic and symptomatic Duchenne muscular dystrophy (DMD) carriers.

Materials and methods: Seventy-two female DMD carriers were enrolled (average age 46.3+- 14.0 years): 50 were asymptomatic and 22 showed muscle weakness or reported myalgias. Disease endpoints were collected (age at loss of ambulation, age at loss of ability to raise from the floor, age at cardiomyopathy onset). Muscle strength was assessed by MRC scale in proximal, distal and axial muscles. Transthoracic echocardiogram was done to assess heart function. Muscle biopsies, when available, were used to assess dystrophin amount by Western Blot analysis. Blood samples were collected to evaluate CK levels and for DNA analyses. Pattern of $\mathrm{X}$ inactivation was determined by methylation sensitive restriction enzyme digestion. The polymorphic CAG repeat in the first exon of the androgen receptor was used to identify the two X chromosome alleles. Genotyping at selected gene modifiers (SPP1, LTBP4, CD40, ACTN3 haplotypes) was done.

Results: The clinical criteria for a manifesting carrier diagnosis were consistently elevated serum CK (above 1,000 IU/1) and myopathic histopathology. DMD gene mutation in our cohort included 43 multiple exons deletion, 20 nonsense, 7 multiple exons duplication, and 2 complex DMD gene rearrangement. Among the 22 symptomatic carriers muscle weakness was moderate/severe in 18/22 and 3 of this lost ambulation at a mean age of 54 years. In 4 patients no muscle weakness was detected but myalgias were reported. We found a good correlation between extent of dystrophin deficiency, skewed X-inactivation pattern, serum CK, and clinical phenotype in the symptomatic carriers. These correlations did not hold up in the asymptomatic carriers. Seven of the 22 symptomatic carriers showed a dilated cardiomyopathy and two died for heart failure at age 65 and 71 years. LTBP4 genotyping showed a better cardiac profile in the IAMM haplotype DMD carriers.

Discussion and conclusions: Accurate genotype/phenotype correlations in manifesting carriers of Duchenne dystrophy have been limited by the ability to accurately quantitate the degree of $\mathrm{X}$ inactivation and dystrophin content of muscle. Cardiac evaluation is foremost important in DMD symptomatic and asymptomatic carriers in a proactive therapy assessment. Genotyping at modifier loci may help to define an at risk haplotype in DMD carriers.

\section{A CASE OF SLOWLY PROGRESSIVE HMGCR MYOPATHY NOT EXPOSED TO STATINS}

\author{
A. Zuppa ${ }^{1}$, G. $\mathrm{Meo}^{1}$, F. Germano ${ }^{1}$, C. Gemelli ${ }^{1}$, M. Infantino ${ }^{2}$, M. Manfredi $^{2}$,

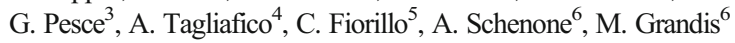

${ }^{1}$ Department of Neuroscience, Rehabilitation, Ophthalmology, Genetics, Maternal and Child Health, University of Genoa (Genova); ${ }^{2}$ Immunology and Allergy Laboratory, San Giovanni di Dio Hospital, Azienda USL Toscana Centro (Firenze); ${ }^{3}$ Autoimmunity Laboratory DiMI, University of Genoa (Genova); ${ }^{4}$ Department of Health Sciences (DISSAL), University of Genoa (Genova); ${ }^{5}$ Paediatric Neurology and Neuromuscular Disorders Unit, Department of Neurosciences, Rehabilitation, Ophthalmology, Genetics, Maternal and Child Health, University of Genoa, Istituto Giannina Gaslini (Genova); ${ }^{6}$ Department of Neuroscience, Rehabilitation, Ophthalmology, Genetics, Maternal and Child Health, IRCCS Ospedale Policlinico San Martino, University of Genoa (Genoa)

Introduction: Anti-3-hydroxy-3-methyl-glutaryl-coenzyme A reductase (HMGCR) myopathy is a rare, disabling, auto-immune necrotizing myopathy, usually described in elderly adults after statin exposure, with an acute and sub-acute course. Recently, different anti-HMGCR myopathy phenotypes have been described, including a chronic myopathy resembling limb-girdle muscular dystrophy (LGMD), usually occurring in younger patients unexposed to statins $[1,2]$.

Object: To describe a case of atypical clinical presentation of HMGCR-myopathy with chronic progressive course in a woman never treated with statins.

Results: Herein we report a case of 66-year-old woman, without statin exposure or familiar history of neuromuscular disease, that in 2017 developed a progressive proximal right lower limb weakness, exercise-induced myalgia associated to hyperCKemia $(6000 \mathrm{CU} / \mathrm{ml}$ vs $20 \mathrm{CU} / \mathrm{ml}$ normal value). The Needle electromyography (EMG) showed signs of muscle primary impairment. She underwent extended laboratory tests (thyroid function, neoplastic markers, autoimmunity exams) and radiological examinations that excluded the more frequent acquired myopathies. During the following two years, she presented a slowly progressive worsening in proximal muscles strength (Medical Research Council sum scale for muscle strength, MRC-SS = 50; normal value 60) associated to increasing CPK elevation (>11.127 U/L), therefore she was admitted to our neurological department. Thigh MRI (on 1.5 Tesla) showed signs of mild degree atrophy in the proximal tract of the left small gluteal muscle without contrast enhancement. We decided to investigate the presence of antibodies anti-HMGCR that, unexpectedly, were detected high titres $(210 \mathrm{CU} / \mathrm{mL}$; negative values: $\leq 20 \mathrm{CU} / \mathrm{mL}$ ). Muscle biopsy of right quadriceps was performed and confirmed a necrotizing myopathy with a high number of necrotic muscular fibers, with only mild focal cellular infiltration. We decided to treat our patient with intravenous methylprednisone $500 \mathrm{mg} /$ day for three days, followed by oral steroid maintenance therapy (prednisone $50 \mathrm{mg} /$ day) and repeated cycles of intravenous Immunoglobulin $2 \mathrm{~g} / \mathrm{kg}$ for 5 days. Despite a short follow-up, the patient presented a rapid improvement of myalgia, initial muscle weakness (MRC-SS=52) and CK levels (5875 U/L) improvement. 
Conclusion: The Anti-HMGCR myopathy represents a heterogeneous spectrum of myopathies with acute or chronic progressive course affecting different ages and occurring in patients receiving statins or not. The diagnosis of progressive slowly anti-HMGR myopathy is critical because patients may respond to immunotherapy.

References:

1. Arouche-Delaperche L, Allenbach Y, Amelin D, Preusse C, Mouly $\mathrm{V}$, Mauhin W, et al. Pathogenic role of anti-signal recognition protein and anti-3-Hydroxy-3-methylglutaryl-CoA reductase antibodies in necrotizing myopathies: Myofiber atrophy and impairment of muscle regeneration in necrotizing autoimmune myopathies. Annals of Neurology (2017);81:538-48. doi:10.1002/ana.24902

2. Mohassel P, Landon-Cardinal O, Foley AR, Donkervoort S, Pak KS, Wahl C, et al. Anti-HMGCR myopathy may resemble limb-girdle muscular dystrophy. Neurology - Neuroimmunology Neuroinflammation (2018);6. doi:10.1212/nxi.0000000000000523

\section{MOVEMENT DISORDERS}

\section{FUNCTIONAL CONNECTIVITY AS AN EARLY MARKER OF INDICATION FOR DEEP BRAIN STIMULATION TREATMENT IN PARKINSON'S DISEASE}

L. Albano ${ }^{1}$, F. $\operatorname{Agosta}^{1}$, S. Basaia ${ }^{1}$, C. Cividini ${ }^{1}$, T. Stojkovic ${ }^{2}$, I. Stankovic $^{2}$, A. Tomic ${ }^{2}$, E. Stefanova ${ }^{2}$, V. Markovic ${ }^{2}$, V. Kostic ${ }^{2}$, M. Filippi $^{3}$

${ }^{1}$ Neuroimaging Research Unit, Institute of Experimental Neurology, Division of Neuroscience, IRCCS San Raffaele Scientific Institute; Vita-Salute San Raffaele University (Milano); ${ }^{2}$ Clinic of Neurology, Faculty of Medicine, University of Belgrade (Belgrade-SRB); ${ }^{3}$ Neuroimaging Research Unit, Institute of Experimental Neurology, Division of Neuroscience; Neurology Unit and Neurophysiology Unit, IRCCS San Raffaele Scientific Institute; Vita-Salute San Raffaele University (Milano)

Objective: Deep brain stimulation (DBS) has been established as a highly-effective therapy for Parkinson's disease (PD) patients. This study is aimed at identifying early neuroimaging predictive biomarkers of the need for DBS use in patients with PD.

Materials: Longitudinal prospective clinical, cognitive and restingstate magnetic resonance imaging (RS fMRI) data of a wide cohort of patients affected by idiopathic PD, at different stages of disease, were retrospectively analyzed. PD population was divided in two groups: 1) 21 patients eligible for DBS (PD-DBS) over 48 months according to the evidence-based clinical criteria, and 2) 105 patients who did not meet the criteria to undergo DBS surgery (PD-noDBS). 60 age- and sex-matched controls were also enrolled.

Methods: Brain network functional alterations were assessed in PD groups and healthy controls at baseline. Four-year trends of progression of brain network changes were compared between PD groups using multiple serial RS fMRI scans. Furthermore, correlations between fMRI measures and both clinical and cognitive data were tested at baseline and over time. Analysis were adjusted for age, sex, disease duration and levodopa equivalent daily dose changes.

Results: PD-DBS patients were characterized by significant longer disease duration and more severe motor signs/symptoms than PDnoDBS cases at baseline; cognitive features were similar between the two groups. Lobar network analysis showed a significant higher mean nodal strength, local efficiency and clustering coefficient of the occipital areas in PD-DBS relative to both controls and PD-noDBS at baseline. These results were then confirmed by regional analysis. A significant increased functional connectivity (FC) between frontal and basal ganglia networks was found in PD-noDBS compared to both controls and PDDBS patients at baseline. Referring to longitudinal analysis, PD-DBS patients showed a progressive decreased FC within occipital and between occipital and parietal networks compared to PD-noDBS (stable over time). Progressive decreased FC between frontal and basal ganglia networks occurred in PD-noDBS relative to PD-DBS (stable over time). Functional alterations within occipital network were positively related to tremor only in PD-DBS at baseline and over time.

Discussion and Conclusions: These findings revealed different FC patterns in PD patients eligible or not for DBS. RS fMRI analysis might represent an early biomarker that help clinicians to establish the correct indication for DBS in PD patients.

Funding. Ministry of Education, Science, and Technological Development of the Republic of Serbia [grant number \#175090]; Italian Ministry of Health [grant number \# RF-2018-12366746].

\section{CLINICAL AND KINEMATIC FEATURES OF TREMOR IN PATIENTS WITH VALPROATE INDUCED TREMOR}

L. Angelini ${ }^{1}$, G. Paparella ${ }^{2}$, A. De Biase ${ }^{1}$, A. Cannavacciuolo ${ }^{1}$, D. Colella ${ }^{1}$, A. Giallonardo ${ }^{1}$, C. Di Bonaventura ${ }^{1}$, A. Berardelli ${ }^{1}$, M. Bologna ${ }^{1}$

${ }^{1}$ Department of Human Neuroscience, Sapienza University of Rome (Roma); ${ }^{2}$ IRCCS Neuromed (Pozzilli-IS)

Objectives: Tremor is a frequent movement disorder and it can be induced by medications, including those used for the treatment of epilepsy, such as Valproate. Clinical and neurophysiological features of Valproateinduced tremor, however, are still under-investigated. We here performed a detailed clinical and kinematic assessment of Valproate-induced tremor. In this condition, we tested for possible correlations between demographic and clinical data and kinematic features. Results in Valproate-induced tremor were compared with those collected in a large sample of patients with essential tremor.

Materials: In tremor assessment, Fahn-Tolosa-Marin Tremor Rating Scale total score and the subscores for each part $(\mathrm{A}-\mathrm{C})$ of the scale were considered. Kinematic recordings of tremor were performed using an optoelectronic system and tremor analysis was performed using dedicated software to determine the tremor magnitude $\left(\mathrm{GRMS}^{\wedge} 2\right)$ and the dominant frequency peak $(\mathrm{Hz})$.

Method: Sixteen Valproate-induced tremor patients and 93 essential tremor patients were enrolled. All participants underwent a neurological examination and a standardized video recording. Patients underwent an objective assessment of postural, kinetic, and rest tremor of the upper limbs and head tremor through kinematic analysis. Non-parametric tests were used for the statistical comparisons of the two groups.

Results: The clinical evaluation showed head, voice, and lower limb involvement and occurrence of rest tremor in a significant proportion of patients with Valproate-induced tremor, overall higher than in patients with essential tremor thus resulting in the higher tremor rating scores in the former group. The kinematic analysis showed a substantial variability within the group of patients with Valproate-induced tremor in terms of magnitude and frequency of postural and rest tremor of the upper limbs and head tremor as well as for the kinematic data of kinetic tremor. Also, kinematic data in VIT significantly overlapped with those recorded in ET.

Discussion: The study represents a comprehensive tremor characterization in patients with Valproate-induced tremor through the combination 
of clinical and kinematic assessment in the same sample of patients. Topography of tremor, rather than tremor severity, distinguishes Valproate-induced from essential tremor, possibly indicating that Valproate diffusely interferes with the cerebellar function.

Conclusions: Our results may have implications for a better understanding of tremor pathophysiology in different disease entities. Together with clinical examination, the kinematic assessment of tremor may help clinicians in diagnostic processes.

References:

- Alonso-Juarez M, Baizabal-Carvallo JF. Distinguishing features between valproate-induced tremor and essential tremor. Acta Neurol Scand (2018); 138: 177-81

- Mehndiratta MM, Satyawani M, Gupta S, Khwaja GA. Clinical and surface EMG characteristics of valproate induced tremors. Electromyogr Clin Neurophysiol (2005);45: 177-82

- Morgan JC, Kurek JA, Davis JL, Sethi KD. Insights into Pathophysiology from Medication-induced Tremor [Internet]. 1 2017 [cited 2019 Dec 2] Available from: https://tremorjournal.org/ index.php/tremor/article/view/442.

\section{MHEALTH FOR MOVEMENT DISORDERS: ANALYSIS OF STRENGTHS AND PITFALLS}

C. A. Artusi ${ }^{1}$, G. Imbalzano ${ }^{1}$, A. Sturchio ${ }^{2}$, A. Pilotto ${ }^{3}$, E. Montanaro ${ }^{1}$, A. Padovani $^{3}$, W. Maetzler ${ }^{4}$, A. Espay ${ }^{2}$, L. Lopiano ${ }^{1}$

${ }^{1}$ Department of Neuroscience 'Rita Levi Montalcini', University of Torino (Torino); ${ }^{2}$ Gardner Family Center for Parkinson's disease and Movement Disorders, Department of Neurology, University of Cincinnati (Cincinnati-USA); ${ }^{3}$ Department of Clinical and Experimental Sciences, University of Brescia (Brescia); ${ }^{4}$ Department of Neurology, Christian-Albrechts-University of Kiel (Kiel-D)

Objectives: We aim to analyze the use of mHealth in ongoing clinical trials in movement disorders. Exploratorily we also analyzed the constructs of interest for Parkinson's disease (PD) which can be obtained by a smartphone/tablet and a single motion sensor worn at the belt.

Materials and Methods: We performed a systematic search of the ClinicalTrials.gov database to find and include all phase 2, 3, or 4 clinical trials for movement disorders using mHealth, defined as wearable or portable devices capturing data in a frequent or continuous, and minimally invasive way. The search was conducted in January 2020 using the following keywords: Parkinson's disease, dystonia, tremor, chorea, and movement disorders. The outcome measures of each trial were evaluated for the presence of devices. No limitations in dates were applied except for trials concluded before or without update after 2018. For the exploratory analysis of validated and published algorithms demonstrating to reliably assess PD motor and non-motor constructs of interest we reviewed PubMed to January 2020.

Results: A total of $29 \%(\mathrm{n}=54 / 184)$ registered trials were using mHealth, mainly in PD and essential tremor (59.3\% and 27.8\%). In most cases, mHealth were used in phase- 2 trials $(83.3 \%)$ as secondary outcome measures (59.3\%). Only five phase-3 trials, representing $9.3 \%$ of the total, used mHealth ( 1 as primary outcome measure, 3 as secondary, 1 as tertiary). Only $3.7 \%(n=2 / 54)$ of all trials used mHealth for measuring both motor and non-motor symptoms, and $23.1 \%(n=12 / 52)$ used mHealth for unsupervised, ecologic outcomes. For the exploratory analysis, we found that PD patients wearing an inertial sensor at the trunk while using a smartphone or tablet can be monitored with validated algorithms for: tremor, bradykinesia, reaction time, dyskinesia, postural transitions, balance, gait, falls risk, freezing of gait (FoG), upper limb freezing, writing quality, turning, physical activity (number of steps), and sleep quality in a supervised setting; dyskinesia, FoG, postural transition, turning, gait, physical activity, and falls risk in a home-like environment; and sleep quality in an unsupervised setting.

Discussion and Conclusion: Our findings suggest that mHealth remain largely relegated to phase-2 trials for secondary or tertiary outcome measures. Efforts toward greater alignment of mHealth with patient-centered outcomes and development of a universal, common-language platform to synchronize data from one or more devices will assist future efforts toward the integration of mHealth into clinical trials.

\section{CORRELATION BETWEEN QUALITY OF LIFE, COGNITION, MOTOR AND NON-MOTOR SYMPTOMS IN PATIENTS WITH PARKINSON'S DISEASE L-DOPA NAÏVE: A PROSPECTIVE STUDY}

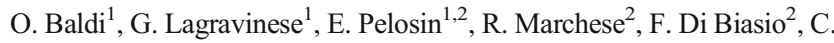
Ogliastro $^{1}$, G. Abbruzzese ${ }^{1}$, L. Avanzino ${ }^{2,3}$

${ }^{1}$ Department of Neuroscience, Rehabilitation, Ophthalmology, Genetics, Maternal and Child Health (DiNOGMI), University of Genoa (Genova); ${ }^{2}$ IRCCS Polyclinic San Martino Hospital (Genova); ${ }^{3}$ Department of Experimental Medicine, Section of Human Physiology, University of Genoa (Genova)

Introduction: It is well known that the overall burden of non-motor symptoms (NMSs) in parkinsonian fluctuating and non-fluctuating patients is a more significant contributor to the quality of life $(\mathrm{QoL})$ than motor symptoms $[1,2]$. Some studies have also shown that there is an effect of dopaminergic medication on NMSs in late-stage Parkinson's Disease (PD) [3].

Objectives: The aim of the present study is to assess which are the best predictors of QoL in early-stage PD patients who still do not assume Ldopa and whether L-dopa therapy would impact QoL by acting on motor and non-motor symptoms.

Methods: PD patients in Hoehn and Yahr stage I-II who had never taken L-dopa are recruited for this study. Health-related QoL is measured with the 39-item PD Questionnaire (PDQ-39). Subjects complete the MDS UPDRS Parts I-IV as well as scales to assess anxiety (HAM-A), depression (HAM-D), apathy (AES), cognition (MOCA, TMT-A, TMTB, FAB), autonomic dysfunction (Scopa-AUT). NMSs are assessed by using the modified version of the Non-Motor Symptoms Scale (NMSS). At baseline visit we used univariate analyses to select clinical predictors. In this prospective study, patients will be followed for two years with evaluations every 6 months.

Results: So far 17 PD patients (11 males, mean age 66,7 $\pm 9,4)$ were included in this study and performed baseline evaluation. QoL significantly correlated with NMSs $(\mathrm{p}<0.001)$. A significant correlation was found between PDQ-39 and UPDRS I $(\mathrm{p}<0.001)$ and UPDRS II ( $\mathrm{p}=0.018)$, but not with UPDRS III $(\mathrm{p}=0.22)$. PDQ-39 correlated also with HAM-A $(\mathrm{p}=0.005)$ and HAM-D $(\mathrm{p}=0.008)$ scores. There was no significant correlation between PDQ-39 and cognitive functions ( $\mathrm{p}$ always $>0.05$ ).

Conclusions: NMSs are the ones that mostly affect QoL in our small cohort of early-stage PD patients. Prospective evaluations will help in understanding whether L-dopa therapy would influence QoL by acting also on NMSs. 


\section{References:}

1. Barone P, Antonini A, Colosimo C, Marconi R, Morgante L, Avarello TP, Bottacchi E, Cannas A, Ceravolo G, Ceravolo R, Cicarelli G, Gaglio RM, Giglia RM, Iemolo F, Manfredi M, Meco G, Nicoletti A, Pederzoli M, Petrone A, Pisani A, Pontieri FE, Quatrale R, Ramat S, Scala R, Volpe G, Zappulla S, Bentivoglio AR, Stocchi F, Trianni G, Dotto PD; PRIAMO study group. The PRIAMO study: A multicenter assessment of nonmotor symptoms and their impact on quality of life in Parkinson's disease. Mov Disord. (2009) Aug 15;24(11):1641-9

2. Skorvanek M, Rosenberger J, Minar M, Grofik M, Han V, Groothoff JW, Valkovic P, Gdovinova Z, van Dijk JP. Relationship between the non-motor items of the MDS-UPDRS and Quality of Life in patients with Parkinson's disease. J Neurol Sci. (2015);353(1-2):87-91. doi: 10.1016/j.jns.2015.04.013. Epub 2015 Apr 17

3. Rosqvist K, Odin P, Hagell P, Iwarsson S, Nilsson MH, Storch A. Dopaminergic Effect on Non-Motor Symptoms in Late Stage Parkinson's Disease. J Parkinsons Dis. (2018);8(3):409-420. doi: 10.3233/JPD-181380. Erratum in: J Parkinsons Dis. 2019;9(2):447-448

\section{CLINICAL FEATURES AND THERAPEUTIC MANAGEMENT OF PAROXYSMAL DYSKINESIAS THROUGHOUT PREGNANCY}

\author{
R. Bovenzi, M. Pierantozzi, N. Mercuri, A. Pisani, A. Stefani, T. Schirinzi
}

Department of System Medicine, University of Rome Tor Vergata (Roma)

Objective: Parosysmal dyskinesias (PxDs) are a group of rare hyperkinetic movement disorders, mostly occurring in young individuals [1]. As antiepleptic drugs, which are teratogen, are the main type of treatment of PxD, an emerging issue is the management of pregnancy in these patients. As well, PxD clinical course during pregnancy is almost unknown.

Materials: Clinical features and therapeutic management of PxDs during pregnancy have been delineated from the retrospective analysis of an unreported patient with PRRT2-related PxD and other cases already described in existing literature.

Methods: To collect all pregnant patients suffering with PxD, an indepth literature search using the Medline database was performed. Keywords "Paroxysmal Dyskinesias" plus "pregnancy", "PRRT2" plus "pregnancy", "MR-1" plus "pregnancy" and "movement disorders" plus "pregnancy" were used. In addition, a patient assessed by the authors was included. A descriptive statistical analysis of data collected was performed.

Results: A cohort of 19 PxD pregnant patients has been collected, five of them with defined genetic diagnosis. Up to $70 \%$ (all of whom genetically confirmed) presented amelioration of symptoms during pregnancy. In our novel case, antidyskinetic therapy withdrawal (lamotrigine) led to a safe pregnancy outcome.

Discussion: The most described movement disorders in pregnancy are restless leg syndrome and chorea gravidarum. Pregnancy has been reported to exacerbate pre-existing hyperkinetic movement disorders such as hemimasticatory spasms and dystonia, and recently cases of dystonia gravidarum have been reported [2]. Evidence on primary PxD and pregnancy is instead scarce and controversial. Our study revealed that PxDs substantially improve during pregnancy. A possible cause for the pregnancy-related amelioration of symptoms can be human chorionic gonadotropin (hCG), whose levels significantly increase in the first days of pregnancy [3]. Relevance of hormones in clinical course of PxD is also highlighted by the occurrence of transient worsening with menses.

Conclusions: PxDs face with an improvement of clinical course during pregnancy, suggesting a role for hormonal fluctuations in pathophysiology of movement disorders. While this finding may support therapy discontinuation during pregnancy, the overall knowledge in this field remains still anecdotal and scarce, thus requiring specific studies.

References:

1. Garone G, Capuano A, Travaglini L, Graziola F, Stregapede F, Zanni G, et al. Clinical and Genetic Overview of Paroxysmal Movement Disorders and Episodic Ataxias. International journal of molecular sciences. (2020);21(10):3603

2. Lim ECH, Seet RCS, Wilder-Smith E, Ong BKC. Dystonia gravidarum: A new entity? Mov Disord. (2006);21(1):69-70

3. Madeja M, Kurlemann G. Ceasing of movement-disorder attacks immediately after the onset of pregnancy: Possible effect of human chorionic gonadotropin. Lancet. (1999);354(9180):745

\section{EVALUATION OF IMAGING BIOMARKERS FOR RISK OF DEMENTIA PROGRESSION IN AN EARLY-STAGE PD COHORT}

\author{
D. Calandrella ${ }^{1}$, G. Carli ${ }^{2,3}$, P. Amami ${ }^{1}$, G. D'este ${ }^{2}$, G. Vanoli ${ }^{4}$, A. \\ Albanese $^{1}$, D. Perani ${ }^{2,3,4}$
}

${ }^{1}$ Department of Neurology, IRCCS Humanitas Research Hospital (Rozzano-MI); ${ }^{2}$ School of Psychology, Vita-Salute San Raffaele University (Milano); ${ }^{3}$ In Vivo Human Molecular and Structural Neuroimaging Unit, Division of Neuroscience, IRCCS San Raffaele Scientific Institute (Milano); ${ }^{4}$ Nuclear Medicine Unit, San Raffaele Hospital (Milano)

Objective: Severe cardiac sympathetic denervation at ${ }^{123}$ iodinemetaiodobenzylguanidine (MIBG)-SPECT and posterior brain hypometabolism at ${ }^{18}$ fluorodeoxyglucose (FDG)-PET are biomarkers potentially able to predict progression to dementia [1]. Combining different functional imaging techniques may be key to clinical diagnosis [2]. We investigated the correlation between FDG-PET and MIBG-SPECT measures, using a multimodal neuroimaging approach in a cohort of patients with clinically definite $\mathrm{PD}$.

Materials: Within the frame of the CARIPLO progression PD study, we included 34 patients in early- stage PD and performed FDG-PET and planar myocardial MIBG exams.

Methods: We evaluated the pattern of FDG-PET hypometabolism at single-subject level by using an optimized FDG-PET statistical parametric mapping (SPM) analysis [3]. Early and delayed heart-to-mediastinum (e-H/M; 1-H/M) ratios, as well as the washout rate (WR) were calculated in each patient. Then, we investigated the relationship between FDG-PET metabolism and MIBG-SPECT parameters using two different approaches, namely a voxel-based and a ROI-based approaches.

Results: FDG-PET maps revealed different brain metabolic patterns in the PD cohort: 7 patients showed no brain hypometabolism or heterogeneity in brain hypometabolism involving motor and premotor regions, somatosensory cortex, and frontal cortex, and at subcortical level the globus pallidus and putamen (PD-typical pattern); 17 patients presented hypometabolism in occipito-temporo-parietal regions (DLBlike pattern). Analysis of planar MIBG images revealed 
pathological values of e-H/M ratio $(\mathrm{N}=19), 1-\mathrm{H} / \mathrm{M}$ ratio $(\mathrm{N}=17)$ and WR $(\mathrm{N}=21)$. PD sub-groups divided according to the hypometabolism patterns (PD-typical and DLB-like) differed in $\mathrm{e}-\mathrm{H} / \mathrm{M}$ and $1-/ \mathrm{M}$ ratio, as well as, WR values. Patients with DLB-like pattern showed greater myocardial sympathetic denervation compared to PD-typical patients. Voxel-based and ROIsbased approaches demonstrated a strong relationship between severe myocardial sympathetic denervation and reduced metabolism in occipito-temporo-parietal regions.

Discussion: We present the first data from the ongoing progression study, suggesting that early stage PD can be associated with different brain metabolic patterns and variable cardiac sympathetic denervation. These heterogeneities of metabolic pattern and cardiac sympathetic denervation may reflect distinct pathophysiological events due to underlying synucleinopathy. If confirmed, vulnerability of the occipital cortex, associated with a severe cardiac sympathetic denervation, might identify patients with high risk of progression towards dementia.

Conclusion: FDG-PET SPM at the single-subject level together with MIBG measures represent useful in vivo biomarkers for clinical risk stratification in early PD stage. Longitudinal studies are mandatory to confirm possible disease evolutions.

Supported by CARIPLO grant 2014-0832 to AA. References

1. Kim, J. S., Shim, Y. S., Song, I. U., Yoo, J. Y., Kim, H. T., Kim, Y. I., \& Lee, K. S. Cardiac sympathetic denervation and its association with cognitive deficits in Parkinson's disease. Parkinsonism \& Related Disorders (2009);15(9):706-08

2. Albanese A, Cocco A, Milani P, Lalli S, Palladini G. Parkinsonism and dysautonomia: Multiple system atrophy? Parkinsonism Relat Disord, (2020, in press). DOI: 10.1016/j.parkreldis.2019.05.005

3. Perani, D., Della Rosa, P. A., Cerami, C., Gallivanone, F., Fallanca, F., Vanoli, E. G., ... \& Garibotto, V. Validation of an optimized SPM procedure for FDG-PET in dementia diagnosis in a clinical setting. NeuroImage: Clinical (2014);6:445-54

\section{KINEMATICS FROM VIDEO: QUANTIFYING BRADYKINESIA FOR THE VIRTUAL AGE}

A. Cannavacciuolo ${ }^{1}$, L. Marsili ${ }^{2}$, J. S. Guerrero Cristancho ${ }^{3}$, N. R. Calvo Ariza $^{3}$, G. Paparella ${ }^{4}$, J. R. Orozco-Arroyave ${ }^{3}$, A. J. Espay ${ }^{2}$, M. Bologna,

${ }^{1}$ Department of Human Neurosciences, Sapienza University of Rome (Roma); ${ }^{2}$ Gardner Family Center for Parkinson's Disease and Movement Disorders, Department of Neurology, University of Cincinnati (Cincinnati-USA); ${ }^{3}$ GITA Lab, Faculty of Engineering, University of Antioquia UdeA (Medellín-CO); ${ }^{4}$ IRCCS Neuromed (Pozzilli-IS)

Objective: Bradykinesia is a cardinal feature of parkinsonism. The current gold standard for quantitative bradykinesia assessment is an in-person kinematic analysis with dedicated equipment, software, and trained personnel, precluding the large-scale applicability of this technique.

Materials: A computer vision technology (DeepLabCut - http:// www.mousemotorlab.org/deeplabcut) can be adapted to quantify bradykinesia from smartphone video recordings. We sought to assess the extent to which bradykinesia measures using computer vision technology measures correlates with those obtained with laboratory-based kinematic.
Methods: Twelve idiopathic Parkinson's disease (PD) patients OFF dopaminergic medications underwent kinematic recording and analysis of repetitive finger tapping (15 seconds, more affected side) using an optoelectronic system (Smart, BTS, Milan, Italy). Movement parameters included number of taps, speed, amplitude, decrement, and rhythm. During kinematic recording, patients were videotaped with a smartphone. The video-recordings were then tracked on a frame-by-frame basis with DeepLabCut in a blinded fashion. Pearson product moment correlation coefficient was used to test the correlation between the movement parameters of the kinematic analysis and DeepLabCut.

Results: There was a high correlation between measures obtained through kinematic techniques and computer vision technology for number of taps, speed, amplitude, and decrement ( $\mathrm{r}$ ranging from 0.60 to 0.91 ; all $\mathrm{p}$-values $<0.05)$ and a trend for rhythm $(\mathrm{r}=0.46$; $\mathrm{p}=0.13)$.

Conclusion: Contactless motion analysis on smartphone video recording provides accurate measures of movement parameters and may be deployed to quantify bradykinesia from remote locations.

\section{NEW MOLECULAR INSIGHTS IN THE DEVELOPMENT OF LONG-TERM MOTOR COMPLICATIONS IN PARKINSON'S DISEASE: POTENTIAL ROLE OF CD4+ T LYMPHOCYTES TRANSCRIPTION FACTORS}

\author{
E. Contaldi ${ }^{1}$, L. Magistrelli ${ }^{1}$, A. Milner ${ }^{1}$, M. Versino ${ }^{2}$, B. Minafra ${ }^{3}$, M. \\ Cosentino $^{4}$, F. Marino ${ }^{4}$, C. Comi $^{1}$ \\ ${ }^{1}$ Movement Disorders Centre, Neurology Unit, Department of \\ Translational Medicine, University of Eastern Piedmont (Novara); \\ ${ }^{2}$ Center for Research in Neuroscience, University of Insubria (Varese); \\ ${ }^{3}$ Center for Research in Neurodegenerative Diseases, "C. Mondino" \\ National Neurological Institute (Pavia); ${ }^{4}$ Center of Research in Medical \\ Pharmacology, University of Insubria (Varese)
}

Background: Management of motor complications represents a major challenge in long-term treatment of Parkinson's Disease (PD) patients. In this context, the role of peripheral adaptive immunity may provide new insights, since neuroinflammatory mechanisms have been proved crucial in the disease $[1,2]$. The aim of this study was therefore to analyze transcription factors genes involved in CD4+ T cells development (Th1, Th2, Th17 and Treg) in order to uncover specific molecular signatures in patients with and without motor complications.

Methods: mRNA levels of the transcription factor genes TBX21, STAT1, STAT3, STAT4, STAT6, RORC, GATA3, FOXP3, and NR4A2 were measured in CD4+ T cells from 40 PD patients, divided into two groups according to motor complications (wearing-off, delayedON, no-ON, random ON-OFF, peak-dose or diphasic dyskinesias) and matched for age, sex, years of disease, years of antiparkinsonian and Levodopa treatment, age at onset, Hoehn and Yahr stage, UPDRS part III score, antiparkinsonian therapies and Levodopa Equivalent Daily Dose. Also 40 age and sex matched healthy controls were enrolled.

Results: Compared to healthy controls, PD patients had higher levels of STAT1, GATA3, STAT6 and FOXP3. In addition, STAT1 and NR4A2 were significantly higher in patients without motor complications $(\mathrm{p}=0.004 ; \mathrm{p}=0.003)$ whereas STAT6 levels were greater in fluctuators $(\mathrm{p}=0.04)$, thus suggesting the role of Th1/Treg/Th2 interplay. Also ROC curve analysis confirmed STAT1 and NR4A2 (respectively involved in Th1 and Treg differentiation) as feasible biomarkers in identifying patients without motor complications ( $\mathrm{AUC}=0.76, \mathrm{p}=0.005,95 \%$ CI $0.59-0.92 ; \mathrm{AUC}=0.75, \mathrm{p}=0.007,95 \%$ CI $0.58-0.90$ ) and they both showed good positive likelihood ratios $(12 ; 5.5)$ and specificity values 
(95\%; 90\%). Moreover, STAT1 and NR4A2 mRNA levels were reliable predictors for the absence of motor fluctuations in a binary logistic regression model with odds ratios of 3.070 (95\% CI 1.325-7.11, $\mathrm{p}=0.009)$ and 2.412 (95\% CI 1.28-4.54, $\mathrm{p}=0.006)$.

Conclusions: Though exploratory, these results shed light to the relevance of peripheral immune system in long-term motor complications, thus unraveling the role of CD4+ T lymphocytes as potential therapeutic targets in PD management.

References:

1. Kustrimovic N., et al. Parkinson's disease patients have a complex phenotypic and functional Th1 bias: cross-sectional studies of CD4+ Th1/Th2/T17 and Treg in drug-naïve and drug-treated patients. J. Neuroinflammation (2018);15:205. https://doi.org/10. 1186/s12974-018-1248-8

2. Boi L., et al. Immunomodulatory drugs alleviate 1-dopa-induced dyskinesia in a rat model of Parkinson's disease. Mov. Disord. Off. J. Mov. Disord. Soc. (2019);34:1818-30

\section{ATYPICAL PHENOTYPE ASSOCIATED TO A NEW DOMINANT MUTATION OF THE MITOCHONDRIAL GENE TWINKLE}

F. Cortese ${ }^{1}$, R. Carrozzo ${ }^{2}$, D. Verrigni ${ }^{2}$, F. Fattori ${ }^{2}$, L. De Giglio ${ }^{1}$, M. Altavista $^{3}$, V. Moschella ${ }^{3}$, E. Pennisi ${ }^{1}$

${ }^{1}$ Rare Neurological Diseases Center, Neurology Unit, San Filippo Neri Hospital (Roma); ${ }^{2}$ Unit of Muscular and Neurodegenerative Disorders, Laboratory of Molecular Medicine, Bambino Gesù Children's Hospital IRCCS (Roma); ${ }^{3}$ Movement Disorders Center, Neurology Unit, San Filippo Neri Hospital (Roma)

Objectives: We describe a patient with a peculiar phenotype characterized by extrapyramidal and endocrine system involvement, carrying a novel heterozygous mutation in the Twinkle gene, a mitochondrial DNA helicase.

Materials and methods: A 74 years-old female presented with myalgias (without strength reduction) and symmetrical extrapyramidal symptoms, including bradikinesia, gait disturbance (in small steps with instability), amimia, apathy, dysarthria and dysprosody. Blood test were normal except for mild hyperckemia (362 U/1). She also presented elevated testosterone with alopecia and suffered from hypothyroidism, hypertension, and ischemic heart disease. No family history of neurological diseases was reported. The patient underwent brain and muscular MRI, brain DaTscan, EMG, muscle biopsy and genetic examinations trough NGS, using the TruSightONE Sequencing Panel (Illumina) containing 4813 genes.

Results: Brain DaTscan showed a mild bilateral (slightly asymmetrical) reduction in the uptake of the radioactive tracer in the posterior putamen. Brain MRI evidenced calcifications of basal ganglia, mild cerebellar atrophy and vascular ischemic leukoencephalopathy. Muscular MRI evidenced atrophy with adipose tissue substitution in the paravertebral lumbar muscles and a mild involvement of the semimembranosus muscles bilaterally. EMG/ENG reported a mild myopathic pattern, while nerve conductions were normal. A muscle biopsy in the deltoid muscle evidenced modest not specific morphological changes and an intense staining of rare muscular fibers with SDH. The patient started a therapy with Levodopa/Benserazide, reporting no significant benefits. Genetic tests demonstrated the presence of a heterozygous variant in the gene Twinkle (mtDNA Helicase): c. 1061G >A (p.Arg354His). Notably, a different mutation in the same locus $(\mathrm{c} .1061 \mathrm{G}>\mathrm{C})$ was reported in a Chinese family with progressive external ophtalmoplegia (PEO) [1].

Discussion: Heterozygous Twinkle mutations, causing dysfunctions in mitochondrial DNA duplication, are known to cause a mitochondrial myopathy mainly characterized by a progressive external ophtalmoplegia (PEO). In the last decade, few cases of ophtalmoplegia associated with extrapyramidal disturbances have been reported [2]. Indeed, Twinkle is important for mitochondrial integrity and its mutations were shown to lead to substantia nigra degeneration in mice [3]. To our knowledge, this is the first case of extrapyramidal syndrome associated to Twinkle mutation, without a PEO phenotype.

Conclusions: Our data contribute to expand our knowledge on the phenotypic spectrum of Twinkle mutations. Interestingly, in our patient the onset of the symptoms is in the adult age, posing problems in the differential diagnosis. This clinical case suggests to consider genetic testing for Twinkle gene in a selected group of patients affected by atypical parkinsonism.

References:

1. Ji K, Liu K, Lin P, Wen B, Luo YB, Zhao Y, Yan C. Twinkle mutations in two Chinese families with autosomal dominant progressive external ophthalmoplegia. Neurol Sci. (2014) Mar;35(3):443-8

2. Kiferle L, Orsucci D, Mancuso M, Lo Gerfo A, Petrozzi L, Siciliano G, Ceravolo R, Bonuccelli U. Twinkle mutation in an Italian family with external progressive ophthalmoplegia and parkinsonism: a case report and an update on the state of art. Neurosci Lett. (2013) Nov 27;556:1-4

3. Song L, McMackin M, Nguyen A, Cortopassi G. Parkin deficiency accelerates consequences of mitochondrial DNA deletions and Parkinsonism. Neurobiol Dis. (2017) Apr;100:30-38

\section{THE PATHOPHYSIOLOGICAL CORRELATES OF PARKINSON'S DISEASE CLINICAL SUBTYPES}

M. Costanzo ${ }^{1}$, D. Belvisi ${ }^{2}$, A. Fabbrini ${ }^{2}$, M. De Bartolo ${ }^{2}$, N. Manzo ${ }^{2}$, G. Fabbrini $^{1,2}$, G. Defazio ${ }^{3}$, A. Conte ${ }^{1,2}$, A. Berardelli ${ }^{1,2}$

${ }^{1}$ Department of Human Neurosciences, University of Rome "La Sapienza" (Roma); ${ }^{2}$ IRCCS Neuromed (Pozzilli-IS); ${ }^{3}$ Department of Medical Sciences and Public Health, University of Cagliari (Cagliari)

Objectives: Possible pathophysiological mechanisms underlying Parkinson's disease (PD) clinical subtypes are unknown. The aim of this study is to identify pathophysiological correlates of PD subtypes by using neurophysiological techniques.

Methods: One-hundred de novo PD patients participated. We collected patient demographic and clinical data, which were used to perform a hierarchical cluster analysis. The neurophysiological assessment tested primary motor cortex excitability and plasticity using transcranial magnetic stimulation. To evaluate motor performance, we performed a kinematic analysis of fast index finger abduction. To investigate sensory function and sensorimotor mechanisms, we measured the somatosensory temporal discrimination threshold at rest and during movement, respectively.

Results: Hierarchical cluster analysis identified two clinical clusters. Cluster I ("mild motor-predominant") included patients who had milder motor and non-motor symptom severity than Cluster II patients, who had a combination of severe motor and non-motor manifestations. ("diffuse 
malignant"). We observed that the diffuse malignant subtype had increased cortical excitability and reduced plasticity when compared with the mild motor-predominant subtype. Kinematic analysis of motor performance demonstrated that the diffuse malignant subtype was significantly slower than the mild motor-predominant subtype. Conversely, we did not observe any significant differences in sensory function or sensorimotor integration between the two PD subtypes.

Conclusion De novo PD subtypes showed different patterns of motor system dysfunction, while sensory function and sensorimotor integration mechanisms did not differ between subtypes. Our findings suggest that the subtyping of PD patients is not a mere clinical classification but reflects different pathophysiological mechanisms. Neurophysiological parameters may represent promising biomarkers to evaluate PD subtypes and their progression.

References:

- Nutt JG. Motor subtype in Parkinson's disease: Different disorders or different stages of disease?: Motor Subtypes of PD. Movement Disorders (2016);31(7):957-61

- Erro R, Picillo M, Vitale C, et al. Clinical clusters and dopaminergic dysfunction in de-novo Parkinson disease. Parkinsonism \& Related Disorders (2016);28:137-40

- Fereshtehnejad S-M, Zeighami Y, Dagher A, Postuma RB. Clinical criteria for subtyping Parkinson's disease: biomarkers and longitudinal progression. Brain (2017);140(7):1959-76

\section{INTRINSIC BRAIN FUNCTIONAL CONNECTIVITY PREDICTS TREATMENT-RELATED MOTOR COMPLICATIONS IN DRUG- NAIVE PARKINSON'S DISEASE PATIENTS}

\author{
R. De Micco ${ }^{1}$, F. Di Nardo ${ }^{1}$, M. Siciliano ${ }^{1}$, G. Caiazzo ${ }^{1}$, F. Esposito ${ }^{2}$, G. \\ Tedeschi $^{1}$, A. Tessitore ${ }^{1}$
}

${ }^{1}$ Department of Advanced Medical and Surgical Sciences, University of Campania "Luigi Vanvitelli" (Napoli); ${ }^{2}$ Department of Medicine, Surgery and Dentistry, Scuola Medica Salernitana, University of Salerno (Baronissi-SA)

Objectives: Dopamine replacement therapy (DRT) is the most effective treatment for patients with PD. However, chronic DRT may be complicated by the evolution of motor complications, (i.e. motor response fluctuations and levodopa-induced dyskinesias) which develop progressively over the disease course. Risk factors include long treatment duration, high initial dose of levodopa, young age at onset, female sex, but yet these factors alone cannot predict whether an individual patient will develop such treatment-related complications over time. Using resting-state functional MRI, we investigated intrinsic brain networks connectivity at baseline in a cohort of drug-naïve Parkinson's disease (PD) patients which successively developed treatment-related motor complications over a 4years follow-up period compared with patients who did not.

Materials: Baseline 3Tesla MRI images of 88 drug-naïve PD patients and 20 healthy controls (HC) were analyzed. After the baseline assessments, all patients started DRT and were followed for an observation period lasting a maximum of 4 years. At the end of the observation period, 35 patients have developed treatment-related motor complications (PDComp) whereas 53 had not (PD-No-Comp).

Methods: Single-subject and group-level independent component analysis was used to investigate functional connectivity differences within the major motor and extra-motor resting state networks. Additionally, a region-of-interest analysis was performed within the basal ganglia. Sex and age were run as covariates. Multivariate regression analyses were used to investigate baseline predictors of motor complications development.

Results: At baseline, an increased connectivity within the defaultmode and the frontoparietal networks as well as within the basal ganglia were detected in PD-Comp patients compared with PD-no-Comp. Functional connectivity changes at the disease onset showed to be an independent predictor of motor complications at 4-year follow-up.

Conclusions: Our findings demonstrated that specific functional connectivity changes may characterize drug-naïve PD patients more prone to develop treatment-related complications while under DRT. involving crucial striatal projections. We hypothesize that these findings may reflect the presence of early differences within the dopaminergic pathways and might predict development of motor complications over time.

\section{A COMPARATIVE STUDY OF IMMUNOFLUORESCENCE STAINING AND SEEDING ACTIVITY BY RT-QUIC OF SKIN PATHOLOGICAL $\alpha$-SYNUCLEIN FOR THE IN VIVO DIAGNOSIS OF SYNUCLEINOPATHIES}

V. Donadio ${ }^{1}$, Z. Wang ${ }^{2}$, A. Incensi ${ }^{3}$, G. Rizzo ${ }^{1}$, E. Fileccia ${ }^{3}$, V. Vacchiano $^{3}$, S. Capellari ${ }^{1}$, M. Magnani ${ }^{3}$, C. Scaglione ${ }^{3}$, M. Stanzani Maserati $^{3}$, P. Avoni ${ }^{3}$, Q. Zou ${ }^{2}$, R. Liguori ${ }^{1}$

${ }^{1}$ IRCCS Istituto delle Scienze Neurologiche di Bologna, University of Bologna (Bologna); ${ }^{2}$ Departments of Pathology and Neurology, Case Western Reserve University School of Medicine (Cleveland-USA); ${ }^{3}$ IRCCS Istituto delle Scienze Neurologiche di Bologna, AUSL Bologna (Bologna)

Background and Aims: The premortem diagnosis of synucleinopathies lacks a specific biomarker. RT-QuIC and immunofluorescence (IF) have provided promising data in the last years although exploring different aspects of pathological $\alpha$-synuclein ( $\alpha$-syn). This study is aimed at ascertaining: 1 ) the reproducibility of IF; and subsequently 2 ) the in vivo diagnostic accuracy of IF and RT-QuIC in a large cohort of patients with synucleinopathies.

Materials and Methods: We prospectively recruited 90 patients fulfilling clinical and instrumental diagnostic criteria for all clinical variants that belong to $\alpha$-synucleinopathies (SOPs) and non-synucleinopathies (nonSOPs mainly including Alzheimer disease, tauopathies, and vascular parkinsonism or dementia). The reproducibility of IF was ascertained in 21 patients: 9 SOPs and 12 non-SOPs. Then IF and RT-QuIC were compared in an additional 69 patients: 31 with SOPs and 38 with non-SOPs. Twenty-four patients with mainly peripheral neuropathies were used as controls for the comparative study. Patients underwent skin biopsy for IF and RT-QuIC whereas CSF was performed only in patients who underwent lumbar puncture for diagnostic purposes. IF and RT-QuIC analysis were made blinded to the clinical diagnosis.

Results: Recruited patients and controls were matched for age and disease duration. IF showed reproducible results between two pairs of neighbouring skin samples in all 29 sites analysed. Furthermore, both IF and RT-QuIC showed high sensitivity and specificity in discriminating SOPs from non-SOPs and controls but IF presented the highest diagnostic accuracy. IF presented a good level of agreement with RT-QuIC in SOPs patients although the agreement was lower with skin RT-QuIC mainly due to positive skin sample findings in a few patients with non-SOPs.

Discussion and conclusions: Our data showed that: 1) both IF and RTQuIC showed a high diagnostic accuracy although IF displayed the better 
value as well as an optimal reproducibility; 2) they presented a good level of agreement in SOPs supporting the use of a less invasive tests such as skin IF or RT-QuIC instead of CSF RT-QuIC as diagnostic tool for synucleinopathies.

\section{CORTICO-SUBCORTICAL FINDINGS IN IDIOPATHIC REM SLEEP BEHAVIOR DISORDER (IRBD) AND PARKINSON'S DISEASE: A VBM STUDY}

G. Donzuso ${ }^{1}$, R. Squillaci ${ }^{1}$, L. Giuliano ${ }^{1}$, E. Cicero ${ }^{1}$, A. Luca ${ }^{1}$, G. Mostile $^{1}$, S. Palmucci ${ }^{2}$, M. Zappia ${ }^{1}$, A. Nicoletti ${ }^{1}$

${ }^{1}$ Department "GF Ingrassia", Section Neuroscience, University of Catania (Catania); ${ }^{2}$ Radiodiagnostic and Radiotherapy Unit, University of Catania (Catania)

Objectives: Idiopathic rapid eye movement (REM) sleep behavior disorder (iRBD) is a parasomnia characterized by the loss of physiological atonia with abnormal behavior during REM sleep. RBD could be the prodromal manifestation of neurodegenerative diseases, such as Parkinson's disease (PD). Neuroimaging studies showed the presence of structural and functional abnormalities involving cortical and subcortical regions in RBD.

Materials and methods: Patients with iRBD, diagnosed based on thorough clinical interviews and VPSG, were recruited. Patients with PD according to the UK Brain Bank criteria were recruited from the PaCoS cohort, evaluated with the RBD Single-Question Screen and then divided into PD with RBD (PD-RBD+) and PD without RBD (PD-RBD-). A group of healthy controls (HC) were also recruited. Each subject underwent a 3D T1-weighted brain MRI.

Results: Sixteen patients with iRBD were enrolled, 13 PD-RBD, 17 PD patients and 30 age- and sex-matched HC. VBM analysis revealed a pattern of grey matter atrophy (GM) with a gradient from HC to PD$\mathrm{RBD}$, involving left and right middle temporal gyrus and right cerebellum. An inverse pattern, showing GM increase, was found in right caudate body, left midbrain and left middle frontal gyrus $(\mathrm{p}<0.05 \mathrm{FWE})$.

Discussion: We found the reduction of GM density in temporal areas and cerebellum with a decreasing pattern of atrophy from PD-RBD to $\mathrm{HC}$, and the presence of increased GM volume in basal ganglia structures, together with the involvement of midbrain, with an inverse pattern, from PD-RBD to HC. Previous findings showed the involvement of cortical and subcortical structures. Thus, these regions seemed to be involved in REM sleep mechanisms, but also exhibited an overlap with early stage of PD.

Conclusion: This pattern of GM abnormalities could suggest the presence of a neurobiological link connecting RBD to a-synucleinopathies and could be used as a predicting biomarker of PD.

References:

- Haruo Hanyua, Yuichi Inoue, Hirofumi Sakurai, Hidekazu Kanetaka, Masaki Nakamura, Tomoyuki Miyamoto, Taeko Sasai, Toshihiko Iwamoto. Voxel-based magnetic resonance imaging study of structural brain changes in patients with idiopathic REM sleep behavior disorder. Parkinsonism and Related Disorders (2012);18;136-39

- Rahayel S, Gaubert M, Postuma RB, Montplaisir J, Carrier J, Monchi O, Rémillard-Pelchat D, Bourgouin PA, Panisset M, Chouinard S, Joubert S, Gagnon JF. Brain atrophy in Parkinson's disease with polysomnography-confirmed REM sleep behavior disorder. Sleep. (2019);11;42(6):Zsz062
- J. Heller, N. Brcina, I. Dogan, F. Holtbernd, S. Romanzetti, J. B. Schulz, J. Schiefer, K. Reetz. Brain imaging findings in idiopathic REM sleep behavior disorder (RBD) - A systematic review on potential biomarkers for neurodegeneration. Sleep Medicine Reviews (2017);34;23-33

\section{THE PHENOMENOLOGY OF IDIOPATHIC NON-TASK- SPECIFIC UPPER LIMB DYSTONIA}

T. Ercoli ${ }^{1}$, R. Erro ${ }^{2}$, R. Pellicciari ${ }^{3}$, M. Mascia ${ }^{1}$, G. Fabbrini ${ }^{4}$, A. Albanese $^{5}$, S. Lalli ${ }^{5}$, R. Eleopra ${ }^{6}$, P. Barone ${ }^{2}$, R. Marchese ${ }^{7}$, R. Ceravolo $^{8}$, C. Scaglione ${ }^{9}$, R. Liguori ${ }^{9}$, M. Esposito ${ }^{10}$, A. Bentivoglio ${ }^{11}$, L. Bertolasi ${ }^{12}$, M. Altavista ${ }^{13}$, F. Bono ${ }^{14}$, A. Pisani ${ }^{15}$, P. Girlanda ${ }^{16}$, P. Cimino $^{1}$, G. Ferrazzano ${ }^{4}$, G. Devigili ${ }^{6}$, S. Scannapieco ${ }^{2}$, F. Di Biasio ${ }^{7}$, S. Mazzucchi $^{8}$, F. Habetswallner ${ }^{10}$, M. Petracca ${ }^{11}$, C. Zivelonghi ${ }^{12}$, L. Polidori $^{13}$, L. Manzo ${ }^{14}$, G. Di Lazzaro ${ }^{15}$, C. Terranova ${ }^{16}$, M. Cotelli ${ }^{17}$, A. Castagna ${ }^{18}$, B. Minafra $^{19}, \mathrm{~S}$. Misceo ${ }^{20}$, L. Magistrelli $^{21}$, M. Zibetti ${ }^{22}$, G. Cossu $^{23}$, M. Coletti Moja ${ }^{24}$, A. Berardelli ${ }^{4}$, G. Defazio ${ }^{1}$

${ }^{1}$ Department of Medical Sciences and Public Health, University of Cagliari (Cagliari); ${ }^{2}$ Department of Medicine, Surgery and Dentistry "Scuola Medica Salernitana", University of Salerno (Baronissi-SA); ${ }^{3}$ Department of Basic Science, Neuroscience and Sense Organs, Aldo Moro University of Bari (Bari); ${ }^{4}$ Department of Human Neurosciences, Sapienza University of Rome (Roma); ${ }^{5}$ Department of Neurology, IRCCS Istituto Clinico Humanitas (Milano); ${ }^{6}$ Neurology 1 , Carlo Besta Neurological Institute (Milano); ${ }^{7}$ IRCCS Ospedale Policlinico San Martino (Genova); ${ }^{8}$ Department of Clinical and Experimental Medicine, University of Pisa (Pisa); ${ }^{9}$ IRCCS Institute of Neurological Sciences, University of Bologna (Bologna); ${ }^{10} \mathrm{Clinical}$ Neurophysiology Unit, Cardarelli Hospital (Napoli); ${ }^{11}$ Institute of Neurology, University Cattolica del Sacro Cuore (Roma); ${ }^{12}$ Neurologic Unit, University Hospital (Verona); ${ }^{13}$ Neurology Unit, San Filippo Neri Hospital ASL Roma 1 (Roma); ${ }^{14}$ Center for Botulinum Toxin Therapy, Neurologic Unit, A.O.U. Mater Domini (Catanzaro); ${ }^{15}$ Department of Systems Medicine, University of Rome Tor Vergata (Roma); ${ }^{16}$ Department of Clinical and Experimental Medicine, University of Messina (Messina);

${ }^{17}$ Neurology Unit, ASST Valcamonica (Esine-BS); ${ }^{18}$ IRCCS Fondazione Don Carlo Gnocchi (Milano); ${ }^{19}$ Parkinson's Disease and Movement Disorders Unit, IRCCS Mondino Foundation (Pavia); ${ }^{20}$ Neurologic Unit, San Paolo Hospital (Bari); ${ }^{21}$ Movement Disorders Centre, University of Piemonte Orientale (Novara); ${ }^{22}$ Department of Neuroscience 'Rita Levi Montalcini', University of Turin (Torino); ${ }^{23}$ Neurology Service and Stroke Unit, Department of Neuroscience, AO Brotzu (Cagliari); ${ }^{24}$ Neurology Unit, Umberto I Hospital (Torino)

Objective: Idiopathic adult-onset upper limb dystonia (ULD) is characterized by the presence of abnormal postures and patterned movements involving the upper limbs [1]. An important feature of ULD is its task-specificity, meaning that dystonia manifests only during the execution of specific motor activities (e.g. writer's cramp [WC]). However, it's been reported that task-specific ULD may lose its specificity over time and thus become a non-task-specific dystonia. ULD may also begin as a non-task-specific dystonia that appears during different types of voluntary movement or even at rest. The aim of our study is to describe the clinical and demographic features of idiopathic upper limb dystonia, and the possible differences between task-specific dystonia and non-task-specific one.

Methods: In this study, adult patients with idiopathic upper limb dystonia, were enrolled from the Italian Dystonia Registry [2]. Among the 
patients with focal upper limb dystonia, dystonia spread was estimated by survival analysis.

Results: We identified 182 patients with upper limb dystonia from the Italian Dystonia Registry. Task-specific dystonia was present in $38.5 \%$ of enrolled cases. Women predominated among non-task-specific patients, whereas men predominated in the task-specific group. Peak age of upper limb dystonia onset was in the sixth decade in the non-task-specific group and in the fourth decade in the task-specific group. In both groups, upper limb dystonia started as focal dystonia or as part of a segmental dystonia. Segmental onset was more frequent among non-task-specific patients, whereas focal onset predominated among task-specific patients. Dystonic action tremor was more frequent among non-task-specific patients. No significant differences between groups emerged in terms of sensory trick frequency, rest tremor, or family history of dystonia. Dystonia spread was greater in the non-task-specific group.

Conclusion: Novel information from our study therefore introduces the possibility that non-task-specific ULD and task-specific ULD (namely WC) may be considered two stages of the same condition or even distinct clinical subtypes. However, it remains to be determined whether the clinical differences between non-task-specific ULD and WC also reflect pathophysiological differences.

References:

1. Albanese A, Bhatia K, Bressman SB, et al. Phenomenology and classification of dystonia: A consensus update. Mov Disord. (2013);28(7):863-73

2. Defazio G, Esposito M, Abbruzzese G, et al. The Italian Dystonia Registry: rationale, design and preliminary findings. Neurol Sci. (2017);38(5):819-25

\section{THE IMPACT OF A FRAILTY ON PARKINSON'S DISEASE MOTOR AND NON-MOTOR SYMPTOMS: A CROSS- SECTIONAL STUDY}

M. Giangrosso, D. Belvisi, M. Canevelli, M. Costanzo, A. Fabbrini, G. Bruno, A. Berardelli, G. Fabbrini

Department of Human Neurosciences, Sapienza University of Rome (Roma)

Objective: Frailty is an age-related state of increased risk that reflects multisystem physiological changes that influence the clinical expression and disease progression of neurodegenerative disorders. The aim of the present study was to clarify the role of frailty in Parkinson's disease (PD), by administering the frailty index (FI).

Materials and Methods: We consecutively enrolled 150 patients affected by PD and 146 age- and sex-matched healthy controls. We administered a FI questionnaire specifically designed for PD which was generated following a standard procedure computing 50 age-related multidimensional deficits (including signs, symptoms, activities of daily living, instrumental activities of daily living, laboratory abnormalities, and comorbidities). The severity of the disease was evaluated by using Hoen and Yahr scale. Motor symptoms and complications were evaluated by using means of the International Parkinson and Movement Disorder SocietyUnified Parkinson's Disease Rating Scale II, III and IV. Non-motor symptoms were evaluated by means of the Non-Motor Symptoms assessment scale for PD, MDS-UPDRS part I and Montreal cognitive assessment.

Results: FI scores were significantly higher in patients than controls. In both patients and controls, FI score was significantly associated with age. On the base of the median value of FI we identified patients with high
FI score and patient with low FI score. Patients with high FI score displayed more severe motor and non-motor symptoms, including cognitive impairment, than those with low FI score. FI scores correlated with non-motor symptoms severity. A linear regression model showed a significant association between FI score and motor symptoms severity.

Discussion: Our findings suggest that PD patients have an increased status of frailty and that frailty significantly influences the phenotypic manifestations and the clinical spectrum of the disease. The complex set of pathophysiological mechanisms underlying frailty, such as metabolic changes, energy imbalance, oxidative stress, chronic inflammation, and immune activation may exert a direct effect on the central nervous system, thus worsening dopaminergic and non-dopaminergic pathway dysfunction, with a consequent increase in the clinical expression of parkinsonian symptoms. Frailty, therefore, may act as a mediator between multisystem pathological involvement and clinical expression of PD symptoms.

Conclusion: FI is a useful instrument to measure frailty in PD. Frailty may influence parkinsonian motor and non-motor symptoms severity, including the presence of cognitive impairment. Future longitudinal studies will clarify whether frailty is a predictor of disease progression in PD. References:

- Clegg A, Young J, Iliffe S, Rikkert MO, Rockwood K. Frailty in elderly people. Lancet (2013); 381: 752-62

- Walston J, Hadley EC, Ferrucci L, Guralnik JM, Newman AB, Studenski SA, Ershler WB, Harris T, Fried LP. Research agenda for frailty in older adults: toward a better understanding of physiology and etiology: summary from the American Geriatrics Society/ National Institute on Aging Research Conference on Frailty in Older Adults. J Am Geriatr Soc (2006); 54: 991-1001

- Morley JE, Vellas B, van Kan GA, Anker SD, Bauer JM, Bernabei R, Cesari M, Chumlea WC, Doehner W, Evans J, Fried LP, Guralnik JM, Katz PR, Malmstrom TK, McCarter RJ, Gutierrez Robledo LM, Rockwood K, von Haehling S, Vandewoude MF, Walston J. Frailty consensus: a call to action. J Am Med Dir Assoc (2013);14:392-7

\section{EFFECTS OF AGING ON STRIATAL [123I]FP-CIT BINDING AND INFLUENCE OF CAUDATE DAT IMAGING ON NEUROPSYCHOLOGICAL PERFORMANCES AND COGNITIVE OUTCOME IN A COHORT OF DE NOVO PARKINSON'S DISEASE PATIENTS}

\author{
S. Giannoni ${ }^{1}$, G. Palermo ${ }^{1}$, D. Frosini ${ }^{1}$, T. Depalo ${ }^{2}$, D. Volterrani ${ }^{2}$, G. \\ Siciliano $^{1}$, U. Bonuccelli ${ }^{1}$, R. Ceravolo ${ }^{1}$
}

${ }^{1}$ Department of Clinical and Experimental Medicine, Neurology Unit, University of Pisa (Pisa); ${ }^{2}$ Department of Clinical and Experimental Medicine, Regional Center of Nuclear Medicine, University of Pisa (Pisa)

Introduction: Cognitive impairment is a possible complication in Parkinson's disease (PD), even in early stages, with dementia occurring in the majority of patients with advanced PD. Previous studies have shown that the latency of dementia decreases with increasing age at onset of PD. Even if the role of caudate in cognitive performances has been amply demonstrated, nevertheless studies about predictive role of striatal dopaminergic dysfunction on cognitive complications have shown heterogeneous results.

Objectives: To study age-related differences in dopamine transporter (DAT) availability in de novo PD patients by [123I]FP-CIT SPECT, investigate the relationship between DAT availability and neuropsychological performances at baseline, and determine the effect of striatal dopamine depletion on cognitive prognosis. 
Methods: We retrospectively studied 129 patients with early PD who underwent [123I]FP-CIT SPECT and detailed neuropsychometric tests at baseline. Mean caudate and putamen SBR were obtained for each patient to assess the spatial patterns of striatal dopamine depletion. Linear regression analyses were used to investigate the relationship between such baseline SPECT measures and age of PD onset as well as possible independent associations with neuropsychological scores at baseline. Finally, we performed Cox regression analysis to evaluate the relationship between baseline [123I]FP-CIT-SPECT binding ratios and cognitive complications, using MCI and dementia as end-point.

Results: As expected, [123I]FP-CIT-SPECT binding ratios showed significant correlations with age both for the caudate $(B=-0.025$; $\mathrm{p}=0.013)$ and for the putamen $(\mathrm{B}=-0.013 ; \mathrm{p}=0.044)$, demonstrating significantly lower mean uptake values in elderly onset patients, but with a slope of the regression more marked for the caudate. After adjusting for age, we found a significant positive correlation of caudate uptake with MMSE $(\mathrm{B}=0.35 ; \mathrm{p}=0.042)$ and Rey Complex Figure scores (copy: $p<0.001$; immediate recall: $p=0.008$; delayed recall: $p=0.004$ ). In the other neuropsychological tests there was a correlation between lower scores and lower caudate uptake but this does not reach the levels of statistical significance. Instead, putaminal uptake had no evident specific relationship with neuropsychological scores. Successively, using the survival analysis, we found a significant association between caudate [123I]FP-CIT uptake values and the onset of MCI [HR: 0.490; $\mathrm{p}=0.004$ ] as well as the development of dementia [HR: 0.469; $\mathrm{p}=0.025]$.

Conclusion: Our findings confirm a different age effects of [123I]FPCIT binding in the striatal subregions of de novo PD patients. The agerelated decline in caudate dopaminergic dysfunction might represent a critical determinant of cognitive performances at baseline and could be an important predictor for long-term cognitive outcome.

References:

- Lee CS, Kim SJ, Oh SJ, et al. Uneven age effects of [18F]FP-CIT binding in the striatum of Parkinson's disease. Ann Nucl Med (2014);28:874-79

- Li H, Hirano S, Furukawa S, et al. The Relationship Between the Striatal Dopaminergic Neuronal and Cognitive Function With Aging. Front Aging Neurosci (2020);12:1-10

- Yousaf T, Pagano G, Niccolini F, Politis M Predicting cognitive decline with non-clinical markers in Parkinson's disease (PRECODE-2). J Neurol (2019);266:1203-10

\section{DRIVING MOTOR RHYTHMS MODULATES BRADYKINESIA AND CORTICAL EXCITABILITY IN PARKINSON'S DISEASE}

\author{
A. Guerra, M. Bologna, D. Colella, M. Giangrosso, A. Cannavacciuolo, \\ G. Paparella, A. Suppa, A. Berardelli
}

Department of Human Neurosciences, Sapienza University of Rome (Roma)

Objective: Beta $(\beta)$ and gamma $(\gamma)$ are the main resonant rhythms of M1 in humans. In patients with Parkinson's Disease (PD), $\beta$ and $\gamma$ oscillatory activities are altered within the basal ganglia, and this abnormality contributes to the pathophysiology of motor symptoms, including bradykinesia. However, whether cortical $\beta$ and $\gamma$ oscillations influence bradykinesia in PD is still unclear. Transcranial alternating current stimulation (tACS) is a neurophysiological technique that entrains cortical rhythms, transiently increasing their power. In this study, we tested whether driving $\beta$ and $\gamma$ rhythms of the primary motor cortex (M1) by using tACS modulates voluntary movements abnormalities and M1 excitability in patients with PD.

Material: tACS was delivered through conductive rubber electrodes enclosed in saline-soaked sponges through a BrainSTIM (EMS). Kinematic recordings were made by using an optoelectronic system (SMART motion system, BTS Engineering). Transcranial magnetic stimulation (TMS) was carried out by using MAGSTIM 200 and a standard figure-of-eight $70 \mathrm{~mm}$ coil delivering monophasic pulses.

Methods: Kinematic features of repetitive finger movement were recorded during $\beta$-, $\gamma$ - and sham-tACS, delivered in a random order over M1. In the same stimulation conditions, we also recorded motor evoked potentials (MEPs) elicited by single and paired-pulse TMS (short-interval intracortical inhibition - SICI, and short-latency afferent inhibition - SAI). Patients were studied OFF and ON therapy, in two different randomized sessions. A group of healthy subjects (HS) was also tested.

Results: Patients showed various movement abnormalities compared to HS. SICI was higher (reduced inhibition) in patients than in HS. In patients, $\beta$-tACS deteriorated movement velocity, while $\gamma$-tACS improved movement amplitude. Also, $\beta$-tACS decreased (reduced inhibition) both SICI and SAI, whereas $\gamma$-tACS decreased SICI. The effects of tACS were independent of the medication status (OFF or ON). In PD, OFF state, the degree of modulation of SICI during tACS correlated with tACS-induced changes in movement velocity and amplitude.

Discussion: In PD, specific bradykinesia features and intracortical excitability measures of M1 are modulated by $\beta$ - and $\gamma$-tACS. Our findings support the evidence for an anti-kinetic role of cortical $\beta$ and a prokinetic role of cortical $\gamma$ oscillations in PD. Furthermore, our data suggest that GABA-A-ergic interneuronal activity, as tested by SICI, plays a relevant role in $\beta$ - and $\gamma$-induced modulation of bradykinesia.

Conclusion: $\beta$ and $\gamma$ oscillations at the M1 level contribute to the pathophysiology of bradykinesia in $\mathrm{PD}$, and changes in intracortical GABA-A-ergic activity modulate their effect.

\section{PLASMA CYSTATIN C CORRELATES WITH PLASMA NFL LEVELS AND PREDICTS DISEASE PROGRESSION IN PARKINSON'S DISEASE}

A. Imarisio ${ }^{1}$, A. Pilotto ${ }^{1,2}$, E. Garrafa ${ }^{3}$, F. Conforti ${ }^{1}$, A. Scalvini ${ }^{1}$, S. Masciocchi $^{1}$, S. Nocivelli ${ }^{1}$, R. Grasso ${ }^{1}$, R. Turrone ${ }^{1}$, S. Gipponi ${ }^{1}$, E. Cottini $^{1}$, B. Borroni ${ }^{1}$, M. Rizzetti ${ }^{2}$, M. Pizzi ${ }^{4}$, A. Bellucci ${ }^{4}$, L. Bonanni $^{5}$, H. Zetterberg ${ }^{6}$, N. Ashton ${ }^{7}$, A. Hye ${ }^{8}$, A. Padovani ${ }^{1}$

${ }^{1}$ Neurology Unit, Department of Clinical and Experimental Sciences, University of Brescia (Brescia); ${ }^{2}$ FERB Onlus, S. Isidoro Hospital (Trescore Balneario-BG); ${ }^{3}$ Department of Biochemical Chemistry, Department of Molecular and Translational Medicine, University of Brescia (Brescia); ${ }^{4}$ Division of Pharmacology, Department of Molecular and Translational Medicine, University of Brescia (Brescia); ${ }^{5}$ Department of Neuroscience Imaging and Clinical Sciences, University G. d'Annunzio of Chieti-Pescara (Chieti); ${ }^{6}$ Department of Psychiatry and Neurochemistry, Institute of Neuroscience and Physiology, The Sahlgrenska Academy; Clinical Neurochemistry Laboratory; Department of Neurodegenerative Disease; UK Dementia Research Institute at UCL, University of Gothenburg (Gothenburg-S, Molndal-S, London-UK); ${ }^{7}$ Department of Psychiatry and Neurochemistry, Institute of Neuroscience and Physiology, The Sahlgrenska AcademyWallenberg Centre for Molecular and Translational Medicine, Department of Psychiatry and Neurochemistry, Institute of Neuroscience and University of Gothenburg, Maurice Wohl Clinical Neuroscience Institute; South London \& Maudsley NHS Foundation (Gothenburg-S, 
London-UK); ${ }^{8}$ King's College London, Institute of Psychiatry, Psychology \& Neuroscience; NIHR Biomedical Research Centre for Mental Health \& Biomedical Research Unit for Dementia; Maurice Wohl Clinical Neuroscience Institute; South London \& Maudsley NHS Foundation (London-UK)

Introduction: Recent reports have shown peripheral immune system activation in Parkinson's disease (PD) and dementia with Lewy bodies (DLB). Both conditions have been associated with pro-inflammatory serum profile that might impact on disease progression over time. Plasma neurofibrillary light chain (NfL) is a marker of axonal damage in neurodegenerative diseases.

Objective: To evaluate the different distribution of peripheral markers in PD and DLB and their relationship with motor and non-motor symptoms, neurodegenerative markers and clinical progression over time.

Methods: Plasma IL6, CRP, cystatin C and NfL were assessed in a longitudinal study including 68 non demented PD and 24 DLB patients who underwent an extensive motor and cognitive assessment at baseline and after 2 years of follow-up. The association with disease severity was evaluated in a multilinear model adjusted for the effect of age, sex and disease duration.

Results: DLB patients exhibited higher levels of plasma IL6 ( $p=$ $0.050)$ and cystatin $\mathrm{C}(\mathrm{p}=0.022)$ compared to PD. In PD, IL6 correlated with disease duration $(r=0.315, p=0.009)$ and with Cumulative Illness Rating Scale - Severity Score (CIRS-S) $(r=0.46, p=0.000)$, whereas Cystatin $\mathrm{C}$ correlated with age $(\mathrm{r}=0.313, \mathrm{p}=0.005)$ and dysautonomic impairment $(p=0.017)$. Plasma NfL correlated with cystatin $C(r=0.269$, $\mathrm{p}=0.033$ ), but not with CRP and IL6 levels. In DLB, we didn't find any association between peripheral markers and cognitive/behavioral abnormalities or disease progression. In PD, a multivariate model showed that patients with higher Cystatin levels exhibited faster motor and cognitive progression at two years of follow-up $(\mathrm{p}=0.030$ and $\mathrm{p}=0.046$, respectively).

Conclusions: Our study found evidences of a pro-inflammatory immune system activation in DLB compared to PD patients. In PD, Cystatin $\mathrm{C}$ was associated with major non-motor symptoms, higher NfL levels and a remarkably faster motor and cognitive progression. Further studies are needed in order to understand the mechanism underlying the role of Cystatin $\mathrm{C}$ as potential modulator of disease progression in $\mathrm{PD}$.

\section{EFFECTS OF LOW FREQUENCY SUBTHALAMIC STIMULATION ON EVENT-RELATED POTENTIALS IN PARKINSON DISEASE}

G. Imbalzano, A. Romagnolo, M. Zibetti, M. Lenzi, S. Vighetti, C. Pongmala, C. Artusi, E. Montanaro, M. Rizzone, L. Lopiano

Department of Neuroscience "Rita Levi Montalcini", University of Turin (Torino)

Objective: Low frequency subthalamic Deep-Brain-Stimulation (STNDBS) $(60-80 \mathrm{~Hz})$ improve axial symptoms in Parkinson's disease (PD), and preliminary evidences show its beneficial effect on the cognitive component of motor planning. We sought to assess if different stimulation frequencies in STN-DBS may induce modifications in the configuration of the $\mathrm{P} 300$ component of cortical event-related auditory potentials (ERPs), a reliable index of attentive cognitive functions.

Materials and Methods: Inclusion criteria were at least 3 months of follow-up since surgery, stable stimulation parameters, and stable doses of dopaminergic treatment for at least four weeks prior to enrolment. Exclusion criteria were dementia, presence of other neurological illnesses and previous psychotic episode. ERPs recordings was conducted after at least 12 hours of dopaminergic therapy withdrawal, using a standard oddball auditory paradigm with STN-DBS at $60 \mathrm{~Hz}, 80 \mathrm{~Hz}, 130 \mathrm{~Hz}$, and OFF-stimulation, applied in a randomized double-blind sequence. ERPs analysis considered the peak amplitude and latency of the P300 components at midline electrode positions ( $\mathrm{Fz}, \mathrm{Cz}, \mathrm{Pz})$.

Results: The cohort consisted of $12 \mathrm{PD}$ patients treated with bilateral STN-DBS. P300 latency over $\mathrm{Cz}$ and Pz electrodes significantly increased with STN-DBS at $130 \mathrm{~Hz}$ compared to OFF-stimulation. P300 latency was also significantly increased, though to a lesser degree, over Pz electrode with stimulation at $80 \mathrm{~Hz}$. No significant P300 latency modifications were detected at $60 \mathrm{~Hz}$ stimulation compared to OFF-stimulation condition. P300 amplitude did not change significantly for any of the stimulation conditions tested.

Discussion: We found a gradient of progressively increased P300 latency from OFF-stimulation condition to higher stimulation frequencies. LFS, and in particular $60 \mathrm{~Hz}$ stimulation, was associated with lower values of P300 latency, not significantly different from the OFFstimulation condition. Although P300 amplitude values showed a gradient of progressive reduction with the increase of stimulation frequencies, the modifications did not reach the statistical threshold. Based on hypothesis that LFS of STN, by enhancing the alpha/low beta-band $(11-15 \mathrm{~Hz})$ oscillations implicated in cortico-striato-pallidal-STN pathways relevant for the top-down control of movements, our observation of a milder interference of LFS on P300 latencies suggest a possible positive effect of LFS on the cognitive components of complex movement in PD patients.

Conclusions: Low frequency STN-DBS is associated with minor modifications of P300 latency compared to conventional stimulation at $130 \mathrm{~Hz}$, possibly suggesting that 60 and $80 \mathrm{~Hz}$ may have less interference with attentive and cognitive processes involved in the top-down control of movement in PD patients.

References:

- Zibetti M, Moro E, Krishna V, Sammartino F, Picillo M, Munhoz $\mathrm{RP}$, et al. Low-frequency Subthalamic Stimulation in Parkinson's Disease: Long-term Outcome and Predictors. Brain Stimul (2016);9:774-79

- Seer C, Lange F, Georgiev D, Jahanshahi M, Kopp B. Event-related potentials and cognition in Parkinson's disease: An integrative review. Neurosci Biobehav Rev (2016);71:691-714

- Gerschlager W, Bloem BR, Alesch F, Lang W, Deecke L, Cunnington R. Bilateral subthalamic nucleus stimulation does not improve prolonged P300 latencies in Parkinson's disease. J Neurol (2001);248:285-89

\section{EMOTIONAL STATE MODULATES GAIT TERMINATION DIFFERENTLY IN PD PATIENTS WITH OR WITHOUT FREEZING OF GAIT}

T. Lencioni ${ }^{1,2}$, M. Meloni $^{1}$, T. Bowman ${ }^{1}$, A. Marzegan ${ }^{1}$, A. Castagna ${ }^{1}$, C. Cosentino ${ }^{2}$, M. Putzolu ${ }^{2}$, M. Ferrarin ${ }^{1}$, E. Pelosin ${ }^{2,3}$

${ }^{1}$ IRCCS Don Carlo Gnocchi Foundation (Milano); ${ }^{2}$ Department of Neuroscience, Rehabilitation, Ophthalmology, Genetics and Maternal Child Health, University of Genoa (Genova); ${ }^{3}$ IRCCS Policlinico San Martino (Genova) 
Objectives: To investigate whether gait termination characteristics are influenced by auditory-emotional stimuli in patients with Parkinson's Disease (PD), with or without freezing of gait (FOG).

Materials: Fifteen patients with PD (Hoehn\&Yahr 2-3, 7 without FOG(-) and 8 with FOG(+)) and eleven elders (ELD).

Methods: The experiment took place during the "on" state. During the experimental protocol PD and ELD were required to walk unconstrained in the movement analysis laboratory while wearing Bluetooth headphones, markers and two footswitches under the heels. Fifteen sounds that we usually hear/feel in our life were chosen as emotional stimuli (ES) and a neutral voice saying 'Stop' as baseline stimulus (BS). Participants were asked to stop with both feet on the ground as soon as they hear one of the sounds. Then, subjects had to restart walking whenever they felt to. The optoelectronic system recorded the 3D coordinates of both heel markers on the right and left foot. The auditory stimuli were delivered in a random sequence. The reaction time (RT) was calculated as the difference between the instant with both feet still (i.e. velocity of both heel coordinates below threshold for $20 \mathrm{~ms}$ ) and the instant of sound start. Changes in RT (CRT) were expressed as \% of baseline RT: ([RTES -RTBS]/RTBS) x 100. CRT index was analyzed with a repeated measures ANOVA (Group: FOG+/FOG-/ELD, Emotional stimuli: Pleasant $(\mathrm{P}) /$ Unpleasant $(\mathrm{U}))$. $\mathrm{P}$ values $<0.05$ were considered statistically significant.

Results: ANOVA revealed a significant effect of the main factor Group ( $\mathrm{p}=0.01$, CRT [\%] mean (95\%CI): FOG+, -18 (-29/-5); FOG-, $10(-2 / 23)$; HS, $-3(-14 / 6))$. Bonferroni post-hoc test revealed a significant difference between FOG+ and FOG- $(\mathrm{P}=0.009)$.

Discussion: Compared to baseline condition, $\mathrm{PD} \mathrm{FOG+} \mathrm{were} \mathrm{faster} \mathrm{in}$ stopping than PD FOG- under the effect of emotional stimuli. In PD FOG+ the motor reactions to emotional stimuli could occur rapidly in a reflex-like manner due to widespread connection between amygdala and striatum [1]. The limbic input to the striatum could be exacerbated by a breakdown in the frontoparietal attentional top-down control network (FPN) over the emotional responses [2]. It is possible that a reduced ability of the FPN to exert top-down control over the amygdala in PD FOG+ may result in shorter reaction times in response to auditory-emotional stimuli.

Conclusions: Our observations confirmed a dysfunction of top-down cortical control processes over emotional subcortical systems in PD FOG+. References:

1. Marchand WR Cortico-basal ganglia circuitry: a review of key research and implications for functional connectivity studies of mood and anxiety disorders. Brain Struct Funct (2010); 215:73-96

2. Rohr CS, Dreyer FR, Aderka IM, Margulies DS, Frisch S, Villringer A, Okon-Singer H, Individual differences in common factors of emotional traits and executive functions predict functional connectivity of the amygdala. Neuroimage (2015);120:154-63

\section{A SIMPLE, LOW-COST, SMARTPHONE-BASED GAIT ANALYSIS. PRELIMINARY RESULTS IN HEALTHY SUBJECTS}

\author{
M. Lo Giudice ${ }^{1}$, G. Varone ${ }^{1}$, M. Ascoli ${ }^{1}$, S. Gasparini ${ }^{1}$, V. Cianci ${ }^{2}$, G. \\ Mastroianni $^{2}$, V. Dattola ${ }^{2}$, T. Garcea ${ }^{1}$, S. Neri ${ }^{1}$, C. Lobianco ${ }^{1}$, C. \\ Ieracitano $^{3}$, F. C. Morabito ${ }^{3}$, E. Ferlazzo ${ }^{1}$, U. Aguglia ${ }^{1}$
}

${ }^{1}$ Department of Medical and Surgical Sciences, Magna Græcia University (Catanzaro); ${ }^{2}$ Regional Epilepsy Center and Neurology Unit, Great Metropolitan Hospital (Reggio Calabria); ${ }^{3}$ DICEAM Department, Mediterranean University of Reggio Calabria (Reggio Calabria)
Introduction: Gait analysis is usually performed by mean of complex baropodometric examinations. We used a simplified, low-cost, smartphone-based gait analysis to encourage patients to constantly monitor their gait and to report possible critical issues to the neurologist. We here present normative data from heathy subjects.

Materials and methods: 50 healthy subjects were asked to wear a smartphone placed on the left wing, held by an elastic band, and to perform a standardized path. The free, open source, android "Phyphox" app acquired data from accelerometer during gait. The recorded data were transferred to a PC and elaborated in both Phyton and MATLAB languages. For each step, we extracted six features (min, max, mean, kurtosis, skewness and energy) of linear acceleration of the 3 axes ( $x=$ longitudinal, $y=$ transversal, $z=$ vertical) obtaining the normative data from our sample.

Results: The following data were recorded: X-axis: min was 3.51 ( $\mathrm{SD} \pm 1.62$ ), max was 4.90 ( $\mathrm{SD} \pm 2.34$ ), mean was 0.12 (SD \pm 0.32 ), kurtosis was 3.03 ( $\mathrm{SD} \pm 1.02$ ), skewness was 0.40 (SD \pm 0.65 ), energy was 14.86 ( $\mathrm{SD} \pm 5.27$ ). $Y$-axis: min was -3.89 (SD $\pm 1.95)$, max was $6.11(\mathrm{SD} \pm 2.45)$, mean was $0.37(\mathrm{SD} \pm 0.36)$, kurtosis was 2.85 ( $\mathrm{SD} \pm 0.91)$, skewness was 0.41 ( $\mathrm{SD} \pm 0.53)$, energy was $18.21(\mathrm{SD} \pm 5.84)$. Z-axis: $\min$ was $-5.07(\mathrm{SD} \pm 1.62)$, $\max$ was 3.67 ( $\mathrm{SD} \pm 2.36$ ), mean was -0.20 ( $\mathrm{SD} \pm 0.32$ ), kurtosis was 2.96 $(\mathrm{SD} \pm 1.02)$, skewness was $-0.51 \pm(\mathrm{SD} \pm 0.65)$, energy was 15.31 $(\mathrm{SD} \pm 5.27$ ).

Conclusion: We provide normative results of smartphone-based, low cost gait analysis. Studies evaluating patients with neurological gait disturbances are ongoing.

\section{GENDER MAY INFLUENCE SIDE OF THE MOTOR SYNDROME AND COGNITION IN IDIOPATHIC PARKINSON'S DISEASE}

M. R. Lo Monaco ${ }^{1}$, R. Liperoti ${ }^{2}$, D. Fusco ${ }^{3}$, E. Di Stasio ${ }^{4}$, A. Tondinelli ${ }^{3}$, A. Maino ${ }^{5}$, C. Cipriani ${ }^{3}$, A. Bentivoglio ${ }^{5}$, C. Siveri ${ }^{6}$

${ }^{1}$ Department of Geriatrics, Neurosciences and Orthopedics, Catholic University of the Sacred Heart (Roma); ${ }^{2}$ Institute of Internal Medicine and Geriatrics, Catholic University of the Sacred Heart (Roma); ${ }^{3}$ Department of Geriatrics, Neurosciences and Orthopedics, Fondazione Policlinico Universitario A. Gemelli, IRCCS (Roma); ${ }^{4}$ Institute of Biochemistry and Clinical Biochemistry, Catholic University of the Sacred Heart (Roma); ${ }^{5}$ Institute of Neurology, Catholic University of the Sacred Heart (Roma); ${ }^{6}$ Department of Psychology, Catholic University of the Sacred Heart (Milano)

Background and objective: The side of motor symptoms in Parkinson's disease (PD) would predict clinical and demographic variables and types of cognitive decline. However, the available data on these issues are inconsistent. The present study aimed to explore if the side of the motor symptoms might predict demographic and clinical variables and type of neuropsychological disorder.

Design, methods and results: Ninety-seven non-demented, righthanded participants with asymmetric PD were recruited. Sixty had left-sided and thirty-seven right-sided motor symptoms. The two subgroups had comparable age, onset, disease duration, and severity of the neurological picture; nonetheless, left-sided participants had the lower scores at the functional scale (Schwab and England). Males prevailed on females (67\% vs. $33 \%$ ); gender, however, was not equally represented in the two subgroups with a significant prevalence of males compared to females in the left-sided participants $(77 \%$ vs. $23 \%)$, while males and females were equally 
distributed in the right-sided participants (51\% vs. $49 \%)$. The two groups did not differ in any neuropsychological task score, but the female gender predicted better performance in the naming task.

Conclusion: Based on studies on the laterality of functional connectivity density, we interpreted the results assuming that the greater lateralization and prevalent rightward lateralization in males might be associated with a lower possibility of compensation and higher sensitivity of the right hemisphere to the effect of neurodegeneration compared to females, consistent with both the higher prevalence of PD in males and a higher probability of right hemisphere damage.

\section{References:}

- R. Djaldetti, I. Ziv, E. Melamed, The mystery of motor asymmetry in Parkinson's disease. Lancet Neurol (2006). doi:10.1016/S14744422(06)70549-X

- R.P. Munhoz, A.J. Espay, F. Morgante, J.Y. Li, H.A. Teive, E. Dunn, E. Gallin, I. Litvan, Long-duration Parkinson's disease: Role of lateralization of motor features. Park. Relat. Disord (2013). doi:10.1016/j.parkreldis.2012.07.008

- D. Tomasi, N.D. Volkow, Language network: segregation, laterality and connectivity. Mol. Psychiatry (2012). doi:10.1038/mp.2012.99

\section{PHONEMIC VERBAL FLUENCY AND MIDBRAIN ATROPHY IN PROGRESSIVE SUPRANUCLEAR PALSY}

A. Luca, A. Nicoletti, G. Donzuso, C. Terravecchia, C. Cicero, C. D'Agate, C. Rascunà, R. Manna, G. Mostile, M. Zappia

Department GF Ingrassia, University of Catania (Catania)

Background: The neuropsychological profile of PSP patients is mainly characterized by executive dysfunction. Aims of the present study were: to assess which specific test evaluating the executive functioning is more frequently impaired; to evaluate possible correlations between executive dysfunction and midbrain atrophy.

Methods: PSP patients who had undergone a neuropsychological battery particularly assessing executive functioning with the Frontal Assessment Battery (FAB), the phonemic verbal fluency F-A-S, the Raven's Progressive Colored Matrix (RPCM) and the Stroop word colors test (time and errors) were enrolled in the study. A group of PD patients matched by age, sex, education and global cognitive status was selected. All the enrolled patients also underwent a volumetric T1-3D brain MRI.

Results: Thirty-five PSP patients (22 men and 13 women; mean age $69.2 \pm 7.7$ ) and 35 PD patients (22 men and 13 women; mean age 68.2 \pm 7.4 ) were enrolled. A significantly greater impairment in the phonemic verbal fluency F-A-S was recorded in PSP (16.7 \pm 7.9 ) than PD patients $(23.4 \pm 8.7$; p-value $<0.001)$. PSP patients obtained a significant lower score at the FAB total score (13.7 \pm 3.4$)$ than PD patients (11.5 \pm 3.8 ; p-value 0.013$)$. At the brain MRI analysis, midbrain area was significantly lower in PSP patient $(83.9 \pm 20.1)$ than in PD patients $(134.5 \pm 19.9$; p-value $<0.001)$. In PSP patients, a statistically significant positive correlation between the phonemic verbal fluency F-A-S and the midbrain area ( $\mathrm{r}=0.421$; p-value: 0.028$)$ was found.

Conclusions: Our findings suggest that the phonemic verbal fluency is the most frequently impaired executive function in PSP patients and is strongly correlated to midbrain atrophy.

\section{NIVOLUMAB-INDUCED PARKINSONISM: A CASE REPORT}

M. C. Mantovan ${ }^{1}$, M. Ravagnan ${ }^{2}$, T. Scaravilli ${ }^{2}$, E. Cagliari ${ }^{3}$, C. La Monaca ${ }^{3}$, M. Cazzagon ${ }^{3}$, S. Grandesso ${ }^{4}$, F. Oniga ${ }^{5}$, R. Carnuccio ${ }^{5}$, R. Quatrale $^{2}$

${ }^{1}$ Department of Neurological Sciences, Ospedale dell'Angelo, AULSS3 Serenissima (Venezia); ${ }^{2}$ Department of Neurology, AULSS3 Serenissima (Mestre-VE); ${ }^{3}$ Department of Neuroradiology, AULSS3 Serenissima (Mestre-VE); ${ }^{4}$ Clinical Pathology, AULSS3 Serenissima (Dolo-VE); ${ }^{5}$ Department of Oncology, AULSS3 Serenissima (MestreVE)

Objective: To describe a case of Nivolumab-associated atypical Parkinsonism. Nivolumab is a checkpoint inhibiting immunotheraputic agent prescribed for the treatment of resistant cancers. Many immunerelated adverse effects have been described, but central nervous system (CNS) toxicities are rarely reported.

Materials and Methods: We present a 68-year-old woman (B.F.) with history of pulmonary squamous cell carcinoma. She had been treated with Nivolumab from September 2018 till April 2019 (240 mg every 15 days). She developed progressive severe parkinsonism, weakness, confusion, sleep disturbances, hyposmia. In April 2019 part I, II, III of the Unified Parkinson's Disease Rating Scale (UPDRS), brief neuropsychological examination (ENB-2), Mini Mental State (MMSE) and Frontal Assessment Battery (FAB), brain magnetic resonance imaging (MRI), electrophysiological study and lumbar puncture (LP), vascular screenings (ECG, ECG holter, Doppler carotid ultrasound study, echocardiogram) were performed. After stopping Nivolumab, a follow-up (UPDRS, ENB-2, brain MRI) was perfomed in October 2019.

Results: In April 2019 B.F. presented with severe parkinsonism (UPDRS 45) and cognitive deficits including attention/executive, visuospatial, and language functions, agnosia and apraxia (ENB-2 score 39, MMSE 24.27, FAB 9.3). Brain MRI showed multiple hyperintense T2 flair lesions in white matter primary in the parietal lobes and in basal ganglia. None of these lesions were enhanced following contrast administration. No signs of neuropathy were found. Cerebral spinal fluid was normal and all tests were negative for infectious pathogens including polymerase chain reaction for JCV. Parkinsonism and cognition improved after stopping Nivolumab for 6 months. Motor and non-motor symptoms as sleep disturbances and hyposmia significantly improved. She scored 15 on the UPDRS, 42 on the ENB-2, 25.27 on the MMSE, 11.3 on the FAB. Brain MRI didn't changes.

Discussion: These findings were suggestive of a parkinsonism associated to a demyelinating encephalitis immunotherapy-induced. The diagnosis was assumed after temporal association with the initiation of Nivolumab and after excluding other causes.

Conclusions: Autoimmune encephalitis should be considered as possible adverse Nivolumab-related effect. Drug-induced parkinsonism is the second most common cause of parkinsonism after Parkinson's disease and their distinction has crucial implications in terms of management and prognosis. Nivolumab should be included into drugs that can cause partially reversible parkinsonism.

References:

- Zubair Zafar, Carrie Volgerm Tamer Hudali, Mukul Bhattari. Nivolumab-Associated Acute Demyelinating Encehhalitis: A case Report and Literature Review. Clinical Medicine and Research, (2019);17(1-2):29-33 
- F. Brigo, R. Erro, A. Marangi, K. Bhatia, M. Tinazzi. Differentiating drug-induced parkinsonism from Parkinson's disease: An update on non-motor symptoms and investigations. Parkinsonism and Related Disorders (2014);20:808-14

\section{PLASMA NFL CORRELATES WITH WIDESPREAD EXTRASTRIATAL MONAMINERGIC DEFICITS IN EARLY PARKINSON'S DISEASE}

S. Masciocchi ${ }^{1}$, A. Pilotto ${ }^{1}$, M. Toffali ${ }^{1}$, S. Nocivelli ${ }^{1}$, A. Imarisio ${ }^{1}$, E. Premi $^{1}$, F. Schiano di Cola ${ }^{1}$, A. Scalvini ${ }^{1}$, B. Paghera ${ }^{2}$, L. Bonanni ${ }^{3}$, Z. Zetterberg $^{4}$, N. Ashton ${ }^{4}$, A. Hye ${ }^{4}$, A. Padovani ${ }^{1}$

${ }^{1}$ Department of Clinical and Experimental Sciences, University of Brescia (Brescia); ${ }^{2}$ Nuclear Medicine Unit, University of Brescia (Brescia); ${ }^{3}$ Department of Neuroscience Imaging and Clinical Sciences, University G. d'Annunzio of Chieti-Pescara (Chieti); ${ }^{4}$ Institute of Psychiatry, Psychology and Neuroscience, Maurice Wohl Clinical Neuroscience Institute, King's College London, Institute of Psychiatry, Psychology and Neuroscience, Maurice Wohl Clinical Neuroscience Institute, King's College London (London-UK)

Background and aims: Peripheral NfL has been related to clinical progression in Parkinson's disease (PD). The aim of this study was to investigate the relationship between striatal and extrastriatal 123IFP-CIT SPECT monoaminergic projections and plasma neurofilament light chain $(\mathrm{NfL})$ in patients with recent diagnosis of PD.

Methods: Consecutive patients with suspected PD underwent 123IFP-CIT SPECT imaging, motor and cognitive assessment and blood sampling. Plasma NfL levels were quantified by Single molecule array (Simoa; Quanterix). 123I-FP-CIT SPECT binding in nigrostriatal and extrastriatal regions of interest (ROI) was calculated in each patient from spatially normalized images. The relationship between NfL plasma levels and 123I-FP-CIT was evaluated by ROI analyses and whole-brain linear regression model adjusting for the effects of age of onset, sex, disease duration and motor functions (UPDRS). A covariance analysis provided the correlates of local and long-distance regions related to higher peripheral NfL levels.

Results: Forty-two patients with suspected parkinsonism entered the study and twenty-eight patients with established PD at follow-up underwent imaging analyses. Higher NfL plasma levels correlated with lower 123I-FP-CIT SPECT binding in several extrastriatal regions, especially anterior cingulate and temporal lobe $(\mathrm{p}<0,001)$ without significant nigrostriatal binding differences. Covariance patterns revealed a widespread monoaminergic depletion in PD patients with high NfL levels, including frontal and parietal lobes.

Conclusion: Our data showed for the first time that peripheral NfL is associated with widespread extrastriatal monoaminergic deficits in PD patients. This suggest a strong relationship between NfL and cortical function and pathology in $\mathrm{PD}$, pointing out NfL's role as early marker of motor and cognitive progression.

\section{A GAIT DATA-DRIVEN APPROACH TO IDENTIFY DIFFERENT CLINICAL SUBTYPES OF PARKINSON'S DISEASE: A PROPOSAL FOR A NEW CLASSIFICATION}

G. Mostile ${ }^{1}$, G. Portaro ${ }^{1}$, V. Dibilio ${ }^{2}$, R. Terranova ${ }^{1}$, P. Cunsolo ${ }^{1}$, F. Contrafatto $^{1}$, G. Donzuso ${ }^{1}$, C. Rascunà ${ }^{1}$, C. Cicero ${ }^{1}$, A. Luca ${ }^{1}$, A. Nicoletti ${ }^{1}$, M. Zappia ${ }^{1}$
${ }^{1}$ Department "G.F. Ingrassia", Section of Neurosciences, University of Catania (Catania); ${ }^{2}$ Centro Spoke A. O. Cannizzaro, IRCCS Centro Neurolesi Bonino Pulejo (Messina, Catania)

Background: Gait disorders are characteristics of Parkinson's Disease (PD). Spatio-temporal and kinematic parameters can be routinely quantified by gait analysis. Numerous attempts have been made to identify different clinical subtypes with poor agreement and temporal inconsistency. The principal aim of this study was to identify different clinical subtypes based on cluster analysis of gait parameters applied to a cohort of PD patients.

Methods: We retrospectively analyzed data of PD patients who underwent gait analysis. They all performed ten trials walking at their self-selected speed along a six-m walkway during their "on" pharmacological state if treated. A non-hierarchical cluster analysis using k-means method was performed using average values of forty selected spatiotemporal and kinematic parameters for the optimum solution based on the Calinski-Harabasz criterion.

Results: We enrolled thirty-nine patients. Three different subtypes were identified by cluster analysis: a first subtype (A) including the majority of enrolled subjects; a second subtype (B) characterized by pronounced instability, with prominent reduced stance phase, cadence and step length as well as enlarged step width as compared to the other groups; a third phenotype (C) with significant kinematic modifications consisting in pronounced hip flexion-extension and pelvic tilt while walking compared to A and B. No differences were detected in terms of age, disease duration and severity, treatment and cognitive profile among the three identified groups.

Conclusions: A gait data-driven approach may be adopted to practically categorize PD patients in different clinical subtypes. This could be helpful to personalize rehabilitative programs since earlier stages of disease.

\section{EXPECTED AND UNEXPECTED ACUTE EFFECTS ON MOTILITY AND BALANCE IN DE NOVO PARKINSON'S DISEASE PATIENTS DUE TO A STANDARD DOSE OF L- DOPA. SUBCLINICAL INSTRUMENTAL EVIDENCES}

G. Mostile, A. Nicoletti, G. Portaro, R. Terranova, P. Cunsolo, F. Contrafatto, G. Donzuso, C. Rascunà, C. Cicero, A. Luca, M. Zappia

Department "G.F. Ingrassia", Section of Neurosciences, University of Catania (Catania)

Background: Gait impairments are a hallmark of Parkinson's disease (PD). Although patients benefit from L-dopa therapy, its acute effect on gait is poorly understood. This study investigates the acute effects of Ldopa on balance and motility in patients with de novo Parkinson's disease (PD) using an instrumental approach.

Methods: We studied twenty subjects newly diagnosed as clinically probable PD. All patients underwent a standardized acute L-dopa challenge test. Gait assessment was carried out both at baseline and at pharmacologic peak. For each section, subjects performed the Timed Up and Go (TUG) test wearing an inertial sensor. Conventional kinematic parameters processed by the system together with parameters from non-linear multifractal analysis of raw motion data were obtained.

Results: A common trend of improvement on medication was observed for most sensorial parameters. A subgroup of fourteen patients was identified based on short-duration response magnitude with a greater clinically detectable motor response. In these patients, L-dopa effect 
results in unexpected accelerations during postural changes, possibly reflecting instability. Multifractal analysis of motion signals revealed an opposite behavior as expected by the normalization effect of the drug in the rotational tasks.

Discussion: Balance and motility processes may respond differently to L-dopa in PD, also in an early stage of disease. Patients with a greater acute motor response may present worse postural control when on medication. L-dopa may sub-clinically worse rotational tasks, requiring an instrumental monitoring for treatment optimization.

\section{ALPHA-SYNUCLEIN HETEROMERS WITH BETA-AMYLOID AND TAU IN THE DIFFERENTIAL DIAGNOSIS OF LEWY BODY DEMENTIAS, ALZHEIMER'S DISEASE AND HEALTHY CONTROLS}

G. Palermo ${ }^{1}$, L. Giampietri ${ }^{2}$, S. Daniele ${ }^{3}$, R. Piccarducci ${ }^{3}$, D. Frosini ${ }^{2}$, G. Tognoni $^{2}$, U. Bonuccelli ${ }^{2}$, C. Martini ${ }^{3}$, F. Baldacci ${ }^{2}$, R. Ceravolo ${ }^{2}$

${ }^{1}$ Unit of Neurology, Department of Clinical and Experimental Medicine, University of Pisa (Pisa); ${ }^{2}$ Department of Clinical and Experimental Medicine, University of Pisa (Pisa); ${ }^{3}$ Department of Pharmacy, University of Pisa (Pisa)

Background: Lewy Body Dementias (LBDs) are heterogeneous disorders in which Alzheimer disease (AD) related pathologies (tau and betaamyloid $[A \beta]$ ) are an additional common feature thought to contribute to the cognitive decline. Similarly, $\geq 60 \%$ of AD cases have Lewy-type synucleinopathy pathology. $\alpha$-syn, tau and $\mathrm{A} \beta$ have been shown to interact each other or with other "pathological proteins" to form toxic heteroaggregates. Red blood cells (RBC) contains the majority of $\alpha$ synuclein ( $\alpha$-syn) in blood, representing an interesting model for studying the peripheral pathological alterations proved in neurodegeneration.

Objective: To investigate the diagnostic value of total $\alpha$-syn, betaamyloid (A $\beta 1-42)$, tau and their heteroaggregates in RBC of Lewy Body Dementias (LBDs) and Alzheimer's disease (AD) patients compared to healthy controls (HC).

Methods: By the use of enzyme-linked immunosorbent assays, RBC levels of total $\alpha$-syn, $A \beta 1-42$, tau and their heteroaggregates $(\alpha$-syn/ $\mathrm{A} \beta 1-42$ and $\alpha$-syn/tau) were measured in 27 subjects with LBDs (PDD, $\mathrm{n}=17$; DLB, $\mathrm{n}=10$ ), 51 AD patients (AD dementia, $\mathrm{n}=37$, prodromal $\mathrm{AD}, \mathrm{n}=14$ ) and $60 \mathrm{HC}$

Results: Compared with $\mathrm{HC}$, total tau and $\alpha$-syn concentrations as well as $\alpha$-syn/tau heterodimers were significantly lower in LBDs $(\mathrm{p}=0.009, \mathrm{p}=0.009$ and $\mathrm{p}<0.001$ respectively) and AD patients $(\mathrm{p}=0.011, \mathrm{p}=0.003$ and $\mathrm{p}<0.001$, respectively) whereas $\alpha$-syn $/ \mathrm{A} \beta 1-42$ levels were only significantly lower in the AD dementia group $(\mathrm{p}<0.001) . \mathrm{RBC} \alpha$-syn/tau heterodimers had the higher diagnostic accuracy for differentiating patients with LBDs vs HC (AUROC $=0.80$ ). Additionally, RBC total tau had a fair diagnostic accuracy (AUROC $=0.73$ ) in differentiating between the LBD group and HC, whereas AUC values indicated only poor diagnostic accuracy for RBC total $\alpha$-syn

Conclusion: RBC $\alpha$-syn heteromers may be useful for differentiating between neurodegenerative dementias (LBDs and $\mathrm{AD}$ ) and HC. In particular, RBC $\alpha$-syn/tau heterodimers have demonstrated good diagnostic accuracy for differentiating LBDs from HC. However, they are not consistently different between LBD and AD. Our findings also support the notion that $\alpha$-syn, $A \beta 1-42$, and tau interact in vivo to promote the aggregation and accumulation of each other presumably accelerating cognitive dysfunction.

\section{UPPER LIMB CARDIOPULMONARY EXERCISE TEST FOR MITOCHONDRIAL FUNCTION EVALUATION IN FRIEDRICH ATAXIA}

C. Pane ${ }^{1}$, A. Salzano ${ }^{2}$, A. Trinchillo ${ }^{1}$, C. Del Prete ${ }^{1}$, C. Casali $^{3}$, C. Marcotulli $^{3}$, G. Defazio ${ }^{4}$, V. Guardasole ${ }^{2}$, R. Vastarella ${ }^{2}$, F. Giallauria ${ }^{2}$, G. Puorro ${ }^{1}$, A. Marsili ${ }^{1}$, G. De Michele ${ }^{1}$, A. Filla ${ }^{1}$, A. Cittadini ${ }^{2}$, F. Saccà

${ }^{1}$ Department of Neurosciences Reproductive and Odontostomatological Sciences, Federico II University (Napoli); ${ }^{2}$ Department of Translational Medical Sciences, Federico II University (Napoli); ${ }^{3}$ Department of Medical and Surgical Sciences and Biotechnologies, Sapienza University of Rome (Latina); ${ }^{4}$ Department of Medical Sciences and Public Health, University of Cagliari (Cagliari)

Objective: In Friedreich Ataxia (FRDA) primary endpoints for phase IIb trials, or secondary functional endpoints are currently missing. Studies underline the alteration of mitochondrial function as the neurodegenerative pathological mechanism of FRDA. Aim of our study was to explore the feasibility of upper limbs cardiopulmonary exercise testing (CPET) to test mitochondrial function in FRDA patients and compare the results with a sex, age and Body Mass Index (BMI) matched cohort of Healthy Controls (HC).

Methods: CPET was performed using an upper limbs cycle ergometer (Ergoselect 400, Blitz, Germany) on fasting subjects. Subjects followed a ramp protocol of $10 \mathrm{~W} / \mathrm{min}$. Respiratory gas exchange measurements were obtained with a commercially available system (Vmax 29C; Yorba Linda, CA, USA). Peak oxygen uptake (VO2max) was recorded at the mean value of $\mathrm{VO} 2$ during the last $20 \mathrm{sec}$ of the test. The ventilatory anaerobic threshold (AT) was detected by the use of the V-slope method. We performed echocardiography with an ultrasound system equipped with a $2.5 \mathrm{MHz}$ multifrequency transducer for complete M-mode, two-dimensional, Doppler and Tissue Doppler Imaging analyses. Continuous variables were compared between groups using an unpaired $\mathrm{t}$ test, categorical variable was compared using a chisquare test. Correlation was performed using the Parson's correlation coefficient.

Results: We studied 55 FRDA and 54 healthy matched controls (HC). Age $(35.3 \pm 13.8$ vs $32.1 \pm 10.5 ; \mathrm{p}=0.186)$, gender $(\mathrm{M}: \mathrm{F} 29: 27$ vs 27:27; $\mathrm{p}=0.851)$, and BMI $(23.1 \pm 4.6$ vs $23.5 \pm 3.5 ; \mathrm{p}=0.557)$ did not differ between groups. VO2 max showed a significant $31 \%$ reduction in FRDA patients compared to $\mathrm{HC}(15.2 \pm 5.7$ vs $22.0 \pm 6.1 \mathrm{~mL} / \mathrm{Kg} / \mathrm{min} ; \mathrm{p}<0.001)$, and AT-VO2 a $36 \%$ reduction $(\mathrm{p}<0.001)$. Peak workload was reduced by $41 \%$ in FRDA $(42.9 \pm 12.5$ vs $73.1 \pm 21.2$ watts; $p<0.001)$. In FRDA patients, VO2max inversely correlated with the SARA score, disease duration, and 9HPT performance, and directly correlated with ADL. The AT occurred at $48 \%$ of peak workload time in FRDA patients and at $85 \%$ in $\mathrm{HC}(\mathrm{p}<0.001)$. In $\mathrm{HC}$, time at AT correlated with $\mathrm{O} 2$ consumption $(\mathrm{R}=.586 ; \mathrm{p}<0.001)$ and workload $(\mathrm{R}=.610 ; \mathrm{p}<0.001)$, but did not in FRDA where AT was time dependent. VE/VCO2 slope was higher in FRDA (33.0 \pm 5.4 vs $27.1 \pm 4.9$; $\mathrm{p}<0.001)$.

Conclusions: Upper limb CPET is useful in the assessment of exercise tolerance and in the determination of functional severity of mitochondrial oxidative defect in patients with FRDA. CPET is an ideal functional endpoint for phase II and III trials as it can give an in-depth view of mitochondrial fitness through a simple, non-invasive, and safe exercise test. 


\section{ANATOMICAL AND NEUROCHEMICAL BASES OF THEORY OF MIND IN DE NOVO PARKINSON'S DISEASE}

M. Pardini ${ }^{1}$, B. Orso ${ }^{1}$, D. Arnaldi ${ }^{1}$, F. Famà ${ }^{1}$, N. Girtler ${ }^{1}$, A. Brugnolo ${ }^{1}$, E. Doglione ${ }^{1}$, L. Filippi ${ }^{1}$, F. Massa ${ }^{1}$, E. Peira ${ }^{2}$, M. Bauckneht ${ }^{3}$, S. Morbelli ${ }^{3}$, F. Nobili ${ }^{4}$

${ }^{1}$ Department of Neuroscience, Rehabilitation, Ophthalmology, Genetics, Maternal and Child Health, University of Genoa (Genova); ${ }^{2}$ Department of Neuroscience, Rehabilitation, Ophthalmology, Genetics, Maternal and Child Health, University of Genoa and National Institute of Nuclear Physics (INFN) (Genova); ${ }^{3}$ Department of Health Science, University of Genoa and Policlinico S. Martino (Genova); ${ }^{4}$ Department of Neuroscience, Rehabilitation, Ophthalmology, Genetics, Maternal and Child Health, University of Genoa and Policlinico S. Martino (Genova)

Objective: Theory of mind (ToM) deficit is a frequent finding in subjects with neurological and psychiatric conditions. While a number of brain regions play a role in ToM, to date the contribution of the diffuse projection systems is less understood. Our aim was thus to explore the topographical and neurochemical bases of ToM using multi-tracer molecular imaging and quantitative electroencephalography (qEEG).

Materials and Methods: We recruited a group of 30 drug-naïve, de novo Parkinson 's Disease (PD) patients (mean age $73.39 \pm 8.93$ years, 11 females) and for ToM evaluation, an age-matched group of 60 healthy controls. ToM was assessed using the "Reading the Mind in the Eyes Task" (RMET) [1], while general cognition with was assessed with the MMSE. We acquired FDG-PET images (as a marker of regional neurodegeneration), I-123 Ioflupane Single Photon Emission Computed Tomography (123 I-FP-CIT-SPECT, as a marker of dopaminergic impairment in the basal ganglia and in the cortex and as a proxy marker of serotoninergic deafferentation in the thalamus [2]), and qEEG recordings (using the Theta/ Alpha power ratio as marker of cholinergic deafferentation).

Results: PD subjects presented with a significantly worse RMET score compared to 60 controls $(20.7 \pm 5.5$ vs. $27.5 \pm 3.0 \mathrm{p}=0.001)$ while there was no difference between the two groups in age, education or MMSE. The voxelwise analysis of total RMET score and regional metabolism showed a positive correlation in the superior temporal gyrus and in the insula. Among the proxy markers of dopaminergic degeneration, serotoninergic and cholinergic deafferentation, ToM presented only an inverse correlation with 123 I-FPCIT thalamic specific binding ratio (SBR) values -a proxy serotoninergic marker- which remained significant after correction for FDG metabolism in the areas associated with ToM. On the other hand, MMSE only correlated with qEEG posterior Theta/Alpha power.

Discussion and conclusion: Using a multi-modal and multi-tracer molecular imaging approach and quantitative EEG we were able to point to an independent contribution of insula and superior temporal gyrus degeneration and of the serotoninergic system on ToM abilities in de novo PD patients. These findings point to the presence of a specific cortical and neurochemical signature of ToM in PD and suggest possible therapeutic targets to treat social cognition deficits in this population.

References:

1. Baron-Cohen S., Wheelwright S., Hill J., Raste Y., Plumb I. The "Reading the Mind in the Eyes" Test revised version: a study with normal adults, and adults with Asperger syndrome or highfunctioning autism. The Journal of Child Psychology and Psychiatry and Allied Disciplines (2001);42(2):241-51

2. Roselli F. et al. Midbrain SERT in degenerative parkinsonisms: A 123I-FP-CIT SPECT study. Movement Disorders (2010);25(12):1853-59
FUNCTIONAL MRI CONNECTIVITY OF THE PRIMARY MOTOR CORTEX IN FUNCTIONAL DYSTONIA PATIENTS

\author{
N. Piramide ${ }^{1}$, E. Sarasso ${ }^{2}$, A. Tomic ${ }^{3}$, E. Canu ${ }^{1}$, I. Petrovic ${ }^{3}$, M. Svetel ${ }^{3}$,
} S. Basaia ${ }^{1}$, N. Dragasevic Miskovic ${ }^{3}$, V. Kostic ${ }^{3}$, M. Filippi ${ }^{4}$, F. Agosta $^{1}$

${ }^{1}$ Institute of Experimental Neurology, Division of Neuroscience, Scientific Institute and University "Vita-Salute" San Raffaele, University "Vita-Salute" San Raffaele (Milano); ${ }^{2}$ Neuroimaging Research Unit, Institute of Experimental Neurology, Division of Neuroscience, IRCCS San Raffaele Scientific Institute; Vita-Salute San Raffaele University (Milano); ${ }^{3}$ Clinic of Neurology, Faculty of Medicine, University of Belgrade (Belgrade-SRB); ${ }^{4}$ Neuroimaging Research Unit, Institute of Experimental Neurology, Division of Neuroscience; Neurology Unit and Neurophysiology Unit, IRCCS San Raffaele Scientific Institute; Vita-Salute San Raffaele University (Milano)

Objectives: To explore the functional connectivity (FC) of the primary motor (M1) cortex in functional dystonia (FD) patients relative to healthy controls, with a focus on different clinical phenotypes.

Materials: Forty FD patients (12 fixed [FixFD]; 28 mobile [MobFD]) and 43 healthy controls (14 young FixFD-age-matched [yHC]; 29 old MobFD-age-matched [oHC]) were recruited.

Methods: FD patients and controls underwent resting state fMRI. A seed-based FC analysis was performed using bilateral M1 as regions of interest.

Results: Compared to controls, FD patients showed reduced FC between left M1 and left dorsal anterior cingulate cortex, and between right M1 and left M1, premotor/supplementary motor area (SMA), dorsal posterior cingulate cortex (PCC), and bilateral precuneus. Relative to $\mathrm{yHC}$, FixFD patients showed reduced FC between M1 and precuneus bilaterally. Compared to oHC, MobFD patients revealed reduced FC between right M1 and left M1, premotor/SMA, dorsal-PCC, bilateral primary sensory cortices and parieto-occipital areas, and increased $\mathrm{FC}$ of right $\mathrm{M} 1$ with right associative visual cortex and bilateral ventral-PCC. FixFD patients, relative to MobFD, showed lower FC between the right M1 and right associative visual area, and bilateral precuneus and ventralPCC.

Discussion: Our study confirm previous findings in the whole FD group, suggesting a disconnection between motor areas and regions involved in sense of agency (precuneus), emotional regulation and inhibitory function (cingulate cortex). In addition, our results point toward different FC changes in the two FD phenotypes, with FixFD patients having abnormalities mainly in areas related to sense of agency, while MobFD in regions involved in sensorimotor functions (reduced FC) and emotional processing (increased FC).

Conclusions: The different FC alterations we found in the two groups of FD patients sustained the presence of FixFD and MobFD clinical phenotypes, supporting previous clinical and structural MRI findings.

Funding. Ministry of Education, Science, and Technological Development of the Republic of Serbia (project \#175090).

\section{LONGITUDINAL STRUCTURAL BRAIN ALTERATIONS IN PARKINSON'S DISEASE PATIENTS WITH FREEZING OF GAIT}

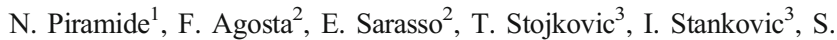

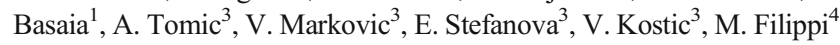


${ }^{1}$ Institute of Experimental Neurology, Division of Neuroscience, Scientific Institute and University "Vita-Salute" San Raffaele, University "Vita-Salute" San Raffaele (Milano); ${ }^{2}$ Neuroimaging Research Unit, Institute of Experimental Neurology, Division of Neuroscience, IRCCS San Raffaele Scientific Institute; Vita-Salute San Raffaele University (Milano); ${ }^{3}$ Clinic of Neurology, Faculty of Medicine, University of Belgrade (Belgrade-SRB); ${ }^{4}$ Neuroimaging Research Unit, Institute of Experimental Neurology, Division of Neuroscience; Neurology Unit and Neurophysiology Unit, IRCCS San Raffaele Scientific Institute; Vita-Salute San Raffaele University (Milano)

Objectives: The main objective of this study is to investigate the cortical and subcortical grey matter (GM) changes in Parkinson's disease (PD) patients with freezing of gait (FoG) over one and two years of follow-up. We also investigated GM features of PD patients developing FoG (PDFoG-converters) within two years.

Materials: Thirty PD-FoG and 11 PD-FoG-converters patients were recruited.

Methods: Patients underwent 3D T1-weighted magnetic resonance imaging. Whole brain cortical thickness and volumes of basal ganglia, thalamus, nucleus accumbens, amygdala and hippocampus were assessed. Paired $t$ test corrected for multiple comparisons using the Bonferroni procedure at $\mathrm{p}<0.05$ were used to assess GM changes over one and two years of follow-up in PDFoG and PD-FoG-converters patients.

Results: Both PD-FoG and PD-FoG-converters patients showed no cortical thickness changes over time. The analysis of GM volumes showed that PD-FoG patients presented decreased volume of the right putamen between baseline and one year and reduced volume of left pallidum, right thalamus and hippocampus between one and two years of follow-up. Moreover, PDFoG-converters showed GM atrophy accumulation in the right putamen and hippocampus between baseline and two years and of the right putamen between one and two years of follow-up.

Discussion: These findings suggested that PD-FoG-converters are characterized by an accumulation of GM atrophy in the putamen and hippocampus, which probably predispose to FoG development. PDFoG patients continued to accumulate GM atrophy in these areas together with pallidum and thalamus that are progressively involved as the FoG symptoms worsened.

Conclusions: PD-FoG and PD-FoG-converters are characterized by a progressive accumulation of GM atrophy in areas involved both in motor and cognitive functions such as basal ganglia and hippocampus. These findings support the hypothesis concerning the problematic interplay between motor and cognitive circuits in the underlying mechanisms of FoG in PD.

Funding. This work was supported by the Ministry of Education, Science, and Technological Development of the Republic of Serbia [grant number \#175090] and the Italian Ministry of Health [grant number \# RF2018-12366746].

\section{ASSESSMENT OF COGNITIVE FUNCTIONS IN PATIENTS UNDERGOING MAGNETIC RESONANCE IMAGING-GUIDED FOCUSED ULTRASOUND (MRGFUS) VENTRAL INTERMEDIATE NUCLEUS (VIM) THALAMOTOMY}

\author{
F. Pistoia ${ }^{1}$, C. Marini ${ }^{2}$, G. Saporito ${ }^{1}$, D. Cerone ${ }^{3}$, A. Catalucci $^{4}$, F. \\ Bruno $^{4}$, C. Masciocchi ${ }^{1}$, S. Sacco ${ }^{1}$, T. Russo ${ }^{3}$, P. Sucapane ${ }^{3}$
}

${ }^{1}$ Department of Biotechnological and Applied Clinical Sciences, University of L'Aquila (L'Aquila); ${ }^{2}$ Department of Medicine, Health and Environment Sciences, University of L'Aquila (L'Aquila);
${ }^{3}$ Neurology Unit, San Salvatore Hospital (L'Aquila); ${ }^{4}$ Radiology Department, San Salvatore Hospital (L'Aquila)

Objective: Magnetic resonance imaging-guided focused ultrasound (MRgFUS) ventral intermediate nucleus (Vim) thalamotomy is a safe and effective treatment for medically refractory essential tremor (ET) and Parkinson's Disease (PD)-related tremor. To date, no relevant cognitive dysfunctions have been reported in association with the treatment. The objective of this study was to investigate cognitive functions, before and after the procedure, in a cohort of patients undergoing MRgFUS-Vim thalamotomy.

Materials and Methods: Seventeen patients (14 men and 3 women, mean age 71.07 \pm 9.6 ) with a diagnosis of ET ( $n=6$; mean age 78.0 \pm 6.7$)$ or PD-related tremor $(\mathrm{n}=11$; mean age $65.8 \pm 8.2)$ were prospectively investigated before (T0) and 2 months following successful treatment for tremor (T1), through a comprehensive neuropsychological screening (Montreal Cognitive Assessment, Frontal Assessment Battery, Verbal and Semantic fluency Test, Mini-mental state examination, Rey 15-Item Test, Raven's progressive Matrices, Beck Depression Inventory and Hamilton Anxiety Rating Scale). A paired t-test was chosen to determine differences between preoperative and postoperative scores. Statistical analyses were performed using IBM SPSS version 19 (IBM Corp.). A p value $<0.05$ was considered statistically significant.

Results: No relevant alterations in cognitive functioning were found between preoperative and postoperative scores across all neuropsychological tests with the exception of the Beck Depression Inventory scale, whose scores resulted significantly improved in patients after MRgFUSVim thalamotomy $(\mathrm{p}=0.005)$.

Discussion: The possible cognitive impairment associated with MRgFUS-Vim thalamotomy is the object of a wide debate and investigation. Cognitive domains, which are at risk of being impaired, include verbal fluency, verbal memory, and language after a left-sided lesion, and visual-spatial abilities after a right-sided lesion. This prompts caution about the possible use and safety of bilateral MRgFUS-Vim thalamotomy for bilateral medically refractory and highly disabling tremor. Our findings do not suggest the presence of relevant cognitive dysfunctions arising up to two months after unilateral procedure. On the other hand, a significant decrease in Beck Depression Inventory scores two months after MRgFUS-Vim thalamotomy may indirectly suggest an improvement of quality of life in patients.

Conclusion: Future studies are necessary to confirm the absence of cognitive dysfunctions associated with unilateral MRgFUS-Vim thalamotomy. A longer neuropsychological follow-up at 6-months and 1-year is essential in order to confirm such data and to endorse the effectiveness and safety of MRgFUS-Vim thalamotomy, as a treatment for ET and PD-related essential tremor not interfering with cognitive balance and stability. This may pave the way for additional research about the possible use of bilateral MRgFUS-Vim thalamotomy.

References:

- Bruno F, Catalucci A, Arrigoni F, Sucapane P, Cerone D, Cerrone P, Ricci A, Marini C, Masciocchi C. An experience-based review of HIFU in functional interventional neuroradiology: transcranial MRgFUS thalamotomy for treatment of tremor. Radiol Med (2020);125:877-86

- Jung NY, Park CK, Chang WS, Jung HH, Chang JW. Effects on cognition and quality of life with unilateral magnetic resonanceguided focused ultrasound thalamotomy for essential tremor. Neurosurg Focus (2018);44:E8

- Meng Y, Lipsman N. Editorial. Tremor, thalamotomy, and cognition. Neurosurg Focus (2018);44:E9 


\section{CLINICAL EVALUATION OF SWITCHING TO LITHIOFOR (LITHIUM SULPHATE SLOW-RELEASE, LI-SR, TABLETS) FROM CARBOLITHIUM (LITHIUM CARBONATE IMMEDIATE-RELEASE, LI-IR, CAPSULES) IN BIPOLAR PATIENTS, POORLY TOLERANT TO LITHIUM IMMEDIATE- RELEASE TREATMENT}

M. Pompili ${ }^{1}$, L. Dell'Osso ${ }^{2}$, E. Bondi ${ }^{3}$, M. Amore ${ }^{4}$, A. Fagiolini ${ }^{5}$, P. Iazzetta $^{6}$, F. Pelacchi ${ }^{7}$, M. Gorini ${ }^{7}$, D. Pierucci ${ }^{7}$, E. Quarchioni ${ }^{8}$, E. Salvatori ${ }^{7}$, A. Comandini ${ }^{7}$, A. Cattaneo ${ }^{7}$

${ }^{1}$ Complex Operational Unit Psychiatry, Sant'Andrea UniversityHospital, University of Rome La Sapienza (Roma); ${ }^{2}$ Department of Clinical and Experimental Medicine, University of Pisa (Pisa); ${ }^{3}$ Psychiatric Service Diagnosis and Care, Papa Giovanni XXIII Hospital (Bergamo); ${ }^{4}$ University Psychiatric Clinic, Institute of Rehabilitation and Care for Scientific Character San Martino Polyclinic Hospital (Genova); ${ }^{5}$ Psychiatry, University-Hospital of Siena (Siena); ${ }^{6}$ Functional Unit Mental Health Adults, San Giovanni di Dio Hospital (Orbetello-GR); ${ }^{7}$ Global Medical Department, Angelini Pharma S.p.A. (Roma); ${ }^{8}$ Biometry Department, Angelini Pharma S.p.A. (Roma)

Objectives: Lithium is a well-established treatment for Bipolar Disorder (BD). Lithium therapy may cause side effects such as tremor, that can reduce treatment compliance. The development of prolonged-release (PR) formulations of lithium was aimed to improve treatment adherence minimizing the risk of side effects correlated to peak plasma levels observed following the administration of immediate-release (IR) formulations $[1,2]$. The primary objective of the study was the assessment of change in lithium-induced tremor when switching from IR to PR formulation, after 1 week of treatment. The secondary objectives were the comparison between IR and PR formulations relatively to: tremor (up to 12 weeks), polyuria and polydipsia (UKU scale); maniac and depressive symptoms (YOUNG, MADRS scales); patient satisfaction with treatment (TSQM); quality of life (Q-LES_Q-SF); CGI (Clinical Global Impression); incidence of adverse events.

Materials: This was a randomized, parallel group, blinded-assessor, multicenter, prospective study, including Type I/II BD patients, 18-65 years old, being treated with Carbolithium ${ }^{\circledR}$, showing low tolerability in terms of tremor. The study foresaw a total of 7 visits within a 3month treatment period.

Methods: The primary endpoint was analyzed in the m-ITT population using a Fisher's exact test at a statistical significance of 0.05 (twosided). The secondary endpoints tremor and polyuria/polydipsia were analyzed using the Fisher's exact test. The changes from baseline on the MADRS and YMRS total scores and for each sub-scale of the TSQM were assessed using an analysis of covariance (ANCOVA) or ANOVA model, as appropriate. The quality of life and CGI were analyzed by descriptive statistics.

Results: Despite fewer than planned patients were enrolled in the study, the analysis of the primary endpoint showed a significant improvement of tremor when switching from IR to $P R$ formulation ( $P=0.0006)$. The difference between groups remained significant at weeks 4 and 12 . Concerning the secondary endpoints, there were no significant differences over time between the two groups except for TSQM convenience $(\mathrm{P}=0.0012)$. A greater proportion of patients were judged as improved in the lithium PR group at weeks 1 and 12 (blinded assessor's CGI).

Discussion: This is the first study showing a reduction of lithiuminduced tremor when switching from IR to PR formulation. The study results, however, should be interpreted with caution and long-term clinical observation from larger patients' cohorts may be needed.
Conclusions: The results of this clinical trial confirmed the benefits in terms of tolerability of PR lithium therapy compared to lithium IR in BD treatment.

References:

1. Grandjean EM, Aubry JM. Lithium: updated human knowledge using an evidence-based approach: Part I: Clinical efficacy in bipolar disorder. CNS Drugs (2009);23:225-40

2. Grandjean EM, Aubry JM. Lithium: updated human knowledge using an evidence-based approach. Part II: Clinical pharmacology and therapeutic monitoring. CNS Drugs (2009);23: 331-49

\section{A NEW MRI MEASURE TO EARLY DIFFERENTIATE PARKINSONISM IN CLINICAL PRACTICE: AN INTERNATIONAL STUDY}

A. Quattrone ${ }^{1}$, A. Antonini ${ }^{2}$, D. Vaillancourt ${ }^{3}$, K. Seppi ${ }^{4}$, R. Ceravolo ${ }^{5}$, A. Strafella ${ }^{6}$, M. Morelli ${ }^{7}$, S. Nigro ${ }^{1}$, B. Vescio ${ }^{8}$, M. Bianco ${ }^{9}$, R. Vasta ${ }^{1}$, P. Arcuri ${ }^{10}$, L. Weis ${ }^{11}$, E. Fiorenzato ${ }^{11}$, R. Biundo ${ }^{11}$, R. Burciu ${ }^{12}$, F. Krismer $^{12}$, N. McFarland ${ }^{13}$, C. Mueller ${ }^{4}$, E. Gizewski ${ }^{14}$, M. Cosottini ${ }^{15}$, E. Del Prete ${ }^{5}$, S. Mazzucchi ${ }^{5}$, A. Quattrone ${ }^{1}$

${ }^{1}$ Neuroscience Research Center, University Magna Graecia (Catanzaro); ${ }^{2}$ Department of Neuroscience, University of Padua (Padova); ${ }^{3}$ Department of Applied Physiology and Kinesiology, University of Florida (Gainesville-USA); ${ }^{4}$ Department of Neurology, Medical University Innsbruck (Innsbruck-A); ${ }^{5}$ Department of Clinical and Experimental Medicine, Unit of Neurology, University of Pisa (Pisa); ${ }^{6}$ Krembil Research Institute, University of Toronto (Toronto -CDN); ${ }^{7}$ Institute of Neurology, Department of Medical and Surgical Sciences, University Magna Graecia (Catanzaro); ${ }^{8}$ Biotecnomed S.C.aR.L (Catanzaro); ${ }^{9}$ Department of Health Sciences, University Magna Graecia (Catanzaro); ${ }^{10}$ Department of Radiology, Pugliese-Ciaccio Hospital (Catanzaro); ${ }^{11}$ IRCCS San Camillo Hospital (Venezia);

${ }^{12}$ Department of Kinesiology and Applied Physiology, University of Delaware (Newark-USA); ${ }^{13}$ Department of Neurology and Biomedical Engineering, University of Florida (Gainesville-USA); ${ }^{14}$ Neuroimaging Core Facility, Medical University Innsbruck (Innsbruck-A); ${ }^{15}$ Department of Translational Research and New Technologies, University of Pisa (Pisa)

Objective: Enlargement of the third ventricle ( $3 \mathrm{rdV}$ ) has been reported in atypical parkinsonisms, but not in Parkinson's disease (PD)[1]. We investigated whether the measurement of $3 \mathrm{rdV}$ width could be a biomarker to distinguish PD from progressive supranuclear palsy (PSP).

Methods: We assessed a new MR T1-weighted measurement (3rdV width/internal skull diameter, $3 \mathrm{rdV} / \mathrm{ID}$ ) in a training cohort of 268 participants (98 PD, 73 PSP, 98 controls from our centre), and in a testing cohort of 291 participants ( 82 de novo PD and 133 controls from the Parkinson's Progression Markers Initiative [2], and 76 early-stage PSP from an international research group)[3]. In both cohorts, PD diagnosis was confirmed after 4-year follow-up. Diagnostic performance of the $3 \mathrm{rdV} / \mathrm{ID}$ was assessed using receiver operating characteristic curve (ROC) with bootstrapping. To maximize the generalizability and reproducibility of the results, data from the training cohort were validated in the testing cohort.

Results: In both cohorts, 3rdV/ID values did not differ between PD and controls but were significantly lower in PD than in PSP patients $(\mathrm{p}<0.0001)$. In PD, 3rdV/ID values did not change significantly between baseline and follow-up evaluation. ROC analysis accurately differentiated PD from PSP in the training cohort (AUC: 0.94, 95\% CI 91.1-97.6) and 
this finding was confirmed in the testing cohort (AUC: $0.91,95 \% \mathrm{CI}$ 87.0-97.0), validating the generalizability of the results.

Conclusion: Our study provides a new validated, reliable MRI biomarker for the early differentiation of PD and PSP. The simplicity and generalizability of this biomarker make it suitable for routine clinical practice worldwide.

\section{References:}

1. Quattrone A, Morelli M, Nigro S, et al. A new MR imaging index for differentiation of progressive supranuclear palsy-parkinsonism from Parkinson's disease. Parkinsonism Relat Disord. (2018); 54: 3-8

2. Parkinson Progression Marker Initiative. The Parkinson Progression Marker Initiative (PPMI). Prog Neurobiol. (2011); 95: 629-35

3. Nigro S, Antonini A, Vaillancourt DE, et al. Automated MRI Classification in Progressive Supranuclear Palsy: A Large International Cohort Study. Mov Disord. (2020);24. doi: $10.1002 / \mathrm{mds} .28007$

\section{REDUCED STRIATAL DAT UPTAKE NORMALIZES AFTER SHUNT IN NORMAL PRESSURE HYDROCEPHALUS}

\author{
A. Quattrone ${ }^{1}$, A. Sarica ${ }^{2}$, M. Quarantelli ${ }^{3}$, P. Arcuri ${ }^{4}$, A. Mechelli ${ }^{5}$, D. \\ La Torre $^{6}$, M. Vaccaro $^{7}$, G. Cascini ${ }^{8}$, A. Quattrone $^{1}$
}

\begin{abstract}
${ }^{1}$ Neuroscience Research Center, University Magna Graecia (Catanzaro); ${ }^{2}$ Department of Medical and Surgical Sciences Neuroscience Centre, University Magna Graecia (Catanzaro); ${ }^{3}$ Institute of Biostructure and Bioimaging, National Research Council (Napoli); ${ }^{4}$ Department of Radiology, Pugliese-Ciaccio Hospital (Catanzaro); ${ }^{5}$ Institute of Neurology, University Magna Graecia (Catanzaro); ${ }^{6}$ Institute of Neurosurgery, University Magna Graecia (Catanzaro); ${ }^{7}$ Institute of Neurology, Department of Medical and Surgical Sciences, University Magna Graecia (Catanzaro); ${ }^{8}$ Department of Nuclear Medicine, University Magna Graecia (Catanzaro)
\end{abstract}

Objective: A reduced striatal DAT uptake has been reported in patients with idiophatic normal pressure hydrocephalus (iNPH), [1] but the mechanisms underlying this finding are not fully understood, and it is not yet clear whether this damage might be caused by the enlarged ventricular system, or if INPH patients might have comorbidity with parkinsonism. In this pilot study, we evaluated the effect of shunt surgery on the reduced striatal DAT uptake in six definite iNPH patients, hypothesizing that a secondary damage due to a compressive mechanism could revert after surgery, while a neurodegenerative process would not be influenced by the shunt.

Methods: Six definite iNPH patients [2] with abnormal pre-shunt 123I-FP-CIT-SPECT (DAT-SPECT) and 10 control subjects were consecutively enrolled in the study. All patients underwent clinical examination, brain 3T MRI and DAT-SPECT before (T0), and three (T1) and six months (T2) after shunt surgery. The control subjects underwent MRI and DAT-SPECT at T0. Automated volumetry was performed using Freesurfer to define the regions of interest (ROI) of caudate, putamen, occipital cortex and lateral ventricles on MR images. DAT-SPECT images were co-registered to the MR 3D T1-weighted images with SPM12 and the correction of partial volume effect (PVE) was performed using the Müller-Gärtner's algorithm as implemented in PVELab. [3] The striatal uptake values were then compared between iNPH and controls, and among the different timepoints in iNPH patients.

Results: Five out of 6 iNPH patients had bilateral parkinsonism with a slight clinical prevalence in the right side and lower DAT uptake values in the left striatum (most affected side, MAS). At baseline (T0), the striatal uptake of the MAS was significantly lower in iNPH patients than in controls $(\mathrm{p}=0.007)$, while it normalized at $\mathrm{T} 1$ and $\mathrm{T} 2$. In $\mathrm{iNPH}$, the lateral ventricle volume of MAS decreased at T1 and T2 compared with T0 but values remained significantly higher than controls. A negative correlation was found between the MAS striatal uptake and the lateral ventricle volume of the same side $(\mathrm{r}=-0.8, \mathrm{p}=0.001)$.

Discussion: Our study provides the first evidence that the striatal dopaminergic damage observed in iNPH normalized after shunt surgery, suggesting that it may be due to the compressive effect of the enlarged ventricular system on basal ganglia circuits rather than to a comorbidity with parkinsonism. These findings provide a new insight on the mechanisms underpinning striatal dopaminergic damage in iNPH and can positively impact the clinical care of patients with this disease.

References:

1. Broggi M, Redaelli V, Tringali G, et al. Normal Pressure Hydrocephalus and Parkinsonism: Preliminary Data on Neurosurgical and Neurological Treatment. World Neurosurg (2016); 90: 348-356. doi: 10.1016/j.wneu.2016.03.004

2. Mori E, Ishikawa M, Kato T, et al. Guidelines for management of idiopathic normal pressure hydrocephalus: second edition. Neurol Med Chir (Tokyo) (2012);52:775-809. doi: 10.2176/nmc.52.775

3. Quarantelli M, Berkouk K, Prinster A, et al. Integrated software for the analysis of brain PET/SPECT studies with partial-volume-effect correction. J Nucl Med (2004);45:192201

\section{PARKINSON AND PARKINSONISMS IN OUTPATIENTS VISITED IN MEDICAL CLINIC AND PATIENTS VISITED HOME}

\section{F. Rodolico}

Neurology, ASP 203 Catania, P.T.A. Giarre (Giarre-CT)

The aim of this study was to screen outpatients and patients visited at home in a catchment area of 96000 citizens all the patients were affected with Parkinson's disease, Parkinsonism or Essential Tremor. We analyzed the disability parameters between outpatient clinic and home patient, with particular attention to patient with Parkinsonism. This Patient had a very long period waiting for a correct Diagnosis and had particular therapeutical needs. Therapy problem with neuroleptic hypersensitivity, Dementia Problem, hallucinations, bedsores, particular nursery needs. In the time period front Sept. 2018 and Sept. 2019 We have visited 117 patients, 58 male. We visited in outpatient clinic 87 patient and 30 home; 44 outpatients with Parkinson's clinic ( 24 male) medium age 71,86 yrs (H. \& Y. 2,68); 12 patient home with Parkinson's (4 male), medium age 78,62 (H.\& Y. 4,18). Patient affected with Parkinsonism were 32 (22 men); 14 in outpatient clinic, medium age 74,66(H.\& Y.2,82) 18 patients home, medium age77,74; (H.\& Y. 4,28); 29 patients affected with Essential Tremor (14 male)of whom 9 patient had a tremor interfering with the activities of daily living; we have not seen Essential Tremor patients home. Home visit is very important and contact with the specialist to explain to the relatives what is happening to the patient. Very important is too the prognosis and assistance for the last wills and the care giver strain heavier in home patients. The main finding of our study is the significative 
difference in disability and the complexity of management between outpatient and the more disabled home patients.

Reference:

- C. G. Goetz, W. Poewe, O. Rascol, C. Sampaio et al. Movement Disorder Society Task Force Report on the Hoehn and Yahr Staging Scale: Status and Raccomandations. Movement Disorders (2004);19:1020-28

\section{BRAIN STRUCTURAL ALTERATIONS IN PATIENTS WITH GCH1 MUTATIONS ASSOCIATED DOPA-RESPONSIVE DYSTONIA}

E. Sarasso ${ }^{1}$, A. Tomić ${ }^{2}$, N. Kresojević ${ }^{2}$, S. Basaia ${ }^{1}$, M. Svetel ${ }^{2}$, M. Copetti $^{3}$, V. Kostić ${ }^{2}$, M. Filippi ${ }^{4}$, F. Agosta ${ }^{1}$

${ }^{1}$ Neuroimaging Research Unit, Institute of Experimental Neurology, Division of Neuroscience, IRCCS San Raffaele Scientific Institute (Milano); ${ }^{2}$ Clinic of Neurology, Faculty of Medicine, University of Belgrade (Belgrade-SRB); ${ }^{3}$ Unit of Biostatistics, Fondazione IRCCS Casa Sollievo della Sofferenza (San Giovanni Rotondo-FG); ${ }^{4}$ Neuroimaging Research Unit, Institute of Experimental Neurology, Division of Neuroscience; Neurology Unit and Neurophysiology Unit, IRCCS San Raffaele Scientific Institute; Vita-Salute San Raffaele University (Milano)

Objectives: Lack of changes in neuropathological and in majority of dopaminergic imaging studies in patients with GCH1-related DOPA-responsive dystonia (DRD) suggested that DRD is a neurotransmitter rather than a neurodegenerative disorder. The aim of this study is to evaluate patterns of cortical thickness, basal ganglia volumes, and white matter (WM) microstructural alterations in genetically-proven DRD patients relative to healthy controls by using structural magnetic resonance imaging (MRI).

Materials: Nine unrelated, clinically manifesting DRD patients and 37 healthy controls were included.

Methods: MRI (1.5 T) measurement of cortical thickness, volumetry, and white matter (WM) abnormalities (tract-based spatial statistics) was obtained in patients and controls.

Results: DRD patients relative to controls had cortical thinning of the right primary motor cortex and bilateral associative sensorimotor areas including right supramarginal, left temporal and bilateral posterior cingulate cortices, an increased volume of the right basal ganglia, and a widespread right lateralized WM damage, involving the main sensorimotor and association tracts.

Discussion: Our findings showed that DRD patients had atrophy of the right M1that might lead to aberrant motor output. Together, DRD subjects showed cortical thinning and WM damage of associative brain regions controlling different levels of sensorimotor processing, supporting the presence of a complex multi-level structural alterations of the sensorimotor integration and control of movement in DRD patients.

Conclusions: Significant morphological changes were observed in DRD patients when compared to healthy controls that might reflect primary brain vulnerability to $\mathrm{GCH} 1$ mutation or the loss of compensatory mechanisms due to a long disease duration and dopamine deficiency.

Funding. Ministry of Education, Science, and Technological Development of the Republic of Serbia (project \#175090).

\section{BRAIN STRUCTURAL CHANGES IN FOCAL DYSTONIA - WHAT ABOUT TASK SPECIFICITY? A MULTIMODAL MRI STUDY}

E. Sarasso ${ }^{1}$, F. Agosta ${ }^{1}$, A. Tomić ${ }^{2}$, M. Svetel ${ }^{2}$, N. Kresojević ${ }^{2}$, A. Fontana $^{3}$, E. Canu ${ }^{4}$, I. Petrović ${ }^{2}$, V. Kostić ${ }^{2}$, M. Filippi ${ }^{5}$

${ }^{1}$ Neuroimaging Research Unit, Institute of Experimental Neurology, Division of Neuroscience, IRCCS San Raffaele Scientific Institute; Vita-Salute San Raffaele University (Milano); ${ }^{2}$ Clinic of Neurology, Faculty of Medicine, University of Belgrade (Belgrade-SRB); ${ }^{3}$ Unit of Biostatistics, Fondazione IRCCS Casa Sollievo della Sofferenza (San Giovanni Rotondo-FG); ${ }^{4}$ Neuroimaging Research Unit, Institute of Experimental Neurology, Division of Neuroscience, IRCCS San Raffaele Scientific Institute (Milano); ${ }^{4}$ Neuroimaging Research Unit, Institute of Experimental Neurology, Division of Neuroscience; Neurology Unit and Neurophysiology Unit, IRCCS San Raffaele Scientific Institute; Vita-Salute San Raffaele University (Milano)

Objective: The neural basis of task-specificity in dystonia is still poorly understood. This study investigated grey and white matter brain alterations in patients with task-specific and non-task-specific dystonia.

Methods: Thirty-six patients with task-specific dystonia (spasmodic dysphonia, writer's cramp), 61 patients with non-task-specific dystonia (blepharospasm, cervical dystonia), and 83 healthy controls underwent 3D T1-weighted and diffusion tensor magnetic resonance imaging. Whole brain cortical thickness and voxel-based morphometry, volumes of basal ganglia, thalamus, nucleus accumbens, amygdala and hippocampus, and white matter damage were assessed. ANOVA models were used to compare MRI measures between groups, adjusting for age and botulinum toxin treatment.

Results: The comparison between focal dystonia patients showed cortical thickness and grey matter volume differences (i.e., decreased in nontask-specific dystonia, increased in task-specific dystonia) in frontal, parietal, temporal and occipital cortical regions, basal ganglia, thalamus, hippocampus and amygdala. Cerebellar atrophy was found in non-task-specific dystonia patients relative to controls. White matter damage was more severe and widespread in taskspecific relative to non-task-specific dystonia cases. Task-specific dystonia patients receiving botulinum toxin, relative to non-treated cases, had cortical thickening, mainly in the temporal lobe, and increased grey matter volume in fronto-parietal, temporal and occipital regions. Non-task-specific dystonia patients experiencing pain showed cortical thickening of areas involved in pain inhibitory mechanisms.

Conclusions and Discussion: Task-specific and non-task-specific dystonia are characterised by opposite alterations of the main cortical and subcortical sensorimotor and cognitive-controlling brain structures, suggesting the possible presence of different pathophysiological and/or compensatory mechanisms underlying the complexity of the two clinical phenotypes of focal dystonia.

Funding. Ministry of Education, Science, and Technological Development of the Republic of Serbia (project \#175090).

\section{SEX-SPECIFIC WHOLE-BRAIN NETWORK TOPOLOGIC ORGANIZATION IN DRUG NAÏVE PARKINSON'S DISEASE PATIENTS}

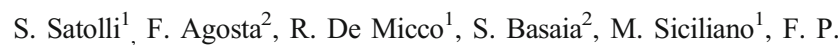

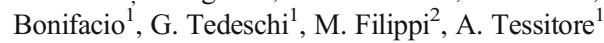


${ }^{1}$ Department of Advanced Medical and Surgical Sciences, University of Campania "Luigi Vanvitelli" (Napoli); ${ }^{2}$ Neuroimaging Research Unit, Institute of Experimental Neurology, Division of Neuroscience, San Raffaele Scientific Institute, Vita-Salute San Raffaele University (Milano)

Objectives: Male sex is a prominent risk factor for developing Parkinson's disease (PD). Conversely, as the disease progresses female PD patients seem to be at higher risk to develop treatment-related motor complications. Compelling evidence suggests that a gender-specific pattern and functioning within the nigro-striatal dopaminergic pathway may underlie these differences. The aim of our study is to investigate the potential sex-difference effect on the whole-brain network topologic organization in a large cohort of drug-naïve PD patients using resting-state functional MRI (rs-fMRI) and its correlation with baseline and longitudinal clinical features.

Materials: 3T MRI images of 147 drug-naïve PD patients (85/62 male/female), and 38 age- and sex-matched controls (20/18 male/female) were acquired.

Methods: Graph analysis and connectomics were used to assess global and local topological network properties and regional functional connectivity (FC) in female PD patients compared to males. Multivariate linear and logistic regressions investigated whether functional imaging data at baseline were predictors of clinical outcome over a 3-year period.

Results: At baseline, no clinical and demographical difference was detected between male and female PD patients. At 3-year follow-up, female PD patients were presenting with more likely to present treatment-related motor complications, especially dyskinesia. At baseline, female PD patients showed a preserved global functional brain architecture compared to controls. Male PD patients showed altered functional topological properties within the basal ganglia network compared to female PD patients. No FC differences were detected between male and female controls. Functional connectivity changes within the basal ganglia at baseline showed to be correlated with risk of motor complications at follow-up.

Discussion: Our findings revealed the presence of a disease-related, sex-specific functional architecture within the basal ganglia in a large cohort of early PD patients.

Conclusions: We hypothesize that these findings may be related to the presence of different gender-specific nigrostriatal dopaminergic pathways and might be potentially used to predict disease progression over time.

\section{STRIATAL ATROPHY AND HYPOMETABOLISM ASSOCIATED WITH DRUG-RESISTANT NON-KETOTIC HYPERGLYCEMIC CHOREA-BALLISM}

\section{P. G. Scamarcia ${ }^{1}$, F. Agosta ${ }^{1}$, N. Anzalone ${ }^{2}$, M. Volonté ${ }^{3}$, M. Filippi $^{4}$}

${ }^{1}$ Neuroimaging Research Unit, Institute of Experimental Neurology, Division of Neuroscience, IRCCS San Raffaele Scientific Institute; Vita-Salute San Raffaele University (Milano); ${ }^{2}$ Department of Neuroradiology, IRCCS San Raffaele Scientific Institute; Vita-Salute San Raffaele University (Milano); ${ }^{3}$ Neurology Unit, IRCCS San Raffaele Scientific Institute (Milano); ${ }^{4}$ Neuroimaging Research Unit, Institute of Experimental Neurology, Division of Neuroscience; Neurology Unit and Neurophysiology Unit, IRCCS San Raffaele Scientific Institute; Vita-Salute San Raffaele University (Milano)

Objective: To describe a unique case of non-ketotik hyperglycemic chorea-ballism $(\mathrm{NKHCB})$ in a patient with evidence of caudate and putaminal atrophy, documented with a quantitative approach, 4 months after symptoms onset.

Materials: Description of a case of a 56-years old woman, known for type 2 Diabetes Mellitus (DM) with poor glycemic control, who experienced the sudden onset of involuntary choreoatetosic and ballic movements located in the left limbs. The patient was diagnosed with NKHCB, even involuntary movements persisted 4-months after symptoms onset.

Methods: The patient underwent two magnetic resonance imaging (MRI) scans, at baseline and after 4-months from symptoms onset. Using a thresholding segmentation technique, the caudate nucleus head, putamen and lateral ventricle of each side at each time point volumes were computed and compared. At the 4-months time point, the patient underwent also a fluorine-18 fluorodeoxyglucose (FDG) Positron Emission Tomography (PET) scan.

Results: MRI study initially showed T1-weigthed hyperintensity of the head of the right caudate nucleus and putamen with a corresponding hyperintensity in T2-weighted and Fluid Attenuated Inversion Recovery (FLAIR) scans. The follow-up scan showed the resolution of the signal abnormalities and highlighted the atrophic evolution of right head of the caudate nucleus and putamen, with a slight enlargement of the right lateral ventricle. These findings were confirmed by MRI volumes quantification. The FDG-PET study showed an intense hypometabolism of the right caudate nucleus head and putamen.

Discussion: NKHCB is a rare movement disorder associated with poorly controlled type 2 diabetes [1], characterized by the triad unilateral hemicorea-hemiballism, contralateral striatum hypertintensity on T1weighted MRI, and symptoms improvement and radiological resolution after blood glucose correction [2,3]. This case demonstrates a permanent structural modification of grey matter nuclei usually involved in NKHCB that occurs with a relatively short-term timing, suggesting that the nature of this disorder might not be always transitory.

Conclusions: We described a unique case of a NKHCB that showed focal striatal atrophy after the signal alteration improvement in a 4-month follow-up MRI scan, associated with an initial poor response to glucosecontrol and anti-chorea treatment. We observed an irreversible alteration in a syndrome classically considered as transitory from the clinical and radiological point of view.

References:

1. Chua C-B, Sun C-K, Hsu C-W, Tai Y-C, Liang C-Y, Tsai I-T. Diabetic striatopathy: clinical presentations, controversy, pathogenesis, treatments, and outcomes. Sci Rep. (2020) Jan 31;10(1):1594

2. Lai PH, Tien RD, Chang MH, Teng MM, Yang CF, Pan HB, et al. Chorea-ballismus with nonketotic hyperglycemia in primary diabetes mellitus. AJNR Am J Neuroradiol. (1996) Jul;17(6):1057-64

3. Chang K-H, Tsou J-C, Chen S-T, Ro L-S, Lyu R-K, Chang H-S, et al. Temporal features of magnetic resonance imaging and spectroscopy in non-ketotic hyperglycemic chorea-ballism patients. Eur J Neurol. (2010) Apr;17(4):589-93

\section{THE ROLE OF WHITE MATTER HYPERINTENSITIES IN PARKINSON'S DISEASE PROGRESSION AND OUTCOME}

\author{
P. G. Scamarcia ${ }^{1}$, F. Agosta ${ }^{1}$, E. Spinelli ${ }^{1}$, T. Stojković ${ }^{2}$, I. Stankovic ${ }^{2}$, V. \\ Markovic $^{2}$, I. Petrović ${ }^{2}$, E. Stefanova ${ }^{2}$, V. Kostic ${ }^{2}$, M. Filippi ${ }^{3}$
}

${ }^{1}$ Neuroimaging Research Unit, Institute of Experimental Neurology, Division of Neuroscience, IRCCS San Raffaele Scientific Institute; Vita-Salute San Raffaele University (Milano); ${ }^{2}$ Clinic of Neurology, 
Faculty of Medicine, University of Belgrade (Belgrade-SRB); ${ }^{3}$ Neuroimaging Research Unit, Institute of Experimental Neurology, Division of Neuroscience; Neurology Unit and Neurophysiology Unit, IRCCS San Raffaele Scientific Institute; Vita-Salute San Raffaele University (Milano)

Objective: To investigate the influence of white matter (WM) damage on the longitudinal evolution of motor and non-motor symptoms and the clinical outcome in Parkinson's disease (PD).

Materials: 154 PD patients underwent clinical assessment, comprehensive cognitive evaluation and Magnetic Resonance Imaging (MRI) scan at baseline and clinical and neuropsychological follow-up once a year up to 48 months.

Methods: White matter hyperintensities (WMH) were identified on T2-weighted scans and WMH total volume was computed for each scan at baseline. PD patients were divided in subgroups with low (lowH, $\leq$ 25 th quartile, $n=45$ ), intermediate (mediumH, between 25 th and 75 th quartile, $n=77$ ) and high (highH, $\geq 75$ th quartile, $n=32$ ) WMH burden according to WMH total volume. Analysis of variance (ANOVA) was used to compare groups at baseline and age-corrected linear regression models for longitudinal data, applied to define differences in longitudinal changes of clinical and neuropsychological variables between groups. The influence of WMH burden on the progression to Hoehn \& Yahr $(\mathrm{H} \& \mathrm{Y}) \geq 3$ and dementia was investigated with Kaplan-Meier estimator analysis (KM).

Results: Subjects in PD highH were the oldest $(\mathrm{p}<0.001)$ and showed significantly lower scores in Mini Mental State Examination and Addenbrooke's Cognitive Examination compared with lowH, while no significant differences were detected in sex, education, motor impairment, Levodopa Equivalent Daily Dose, as well as individual cognitive domains. Longitudinally, the highH group showed a significant worsening of all considered variables $(\mathrm{p}<0.001)$ compared to lowH and mediumH, independent of the effect of age. The KM analysis showed lower rates of progression to dementia $(p=0.03)$ and to $H \& Y$ score $\geq 3(p=0.02)$ in the lowH group.

Discussion: WMH have an important role in cognitive impairment onset in normal brain ageing in the general population [1], while the relationship with clinical picture and disease progression in PD is still controversial $[2,3]$. Our study showed that higher WMH volumes are associated with a worse progression of both motor and non-motor symptoms, independently from age. Moreover, PD patients with high WMH volumes are more likely to progress to dementia and to advanced disease stages in the following 4 years.

Conclusions: Our results suggest an important role of WMH burden in PD progression and that MRI might provide a sensitive biomarker to identify patients with higher risk of disease evolution.

Funding: Ministry of Education and Science Republic of Serbia (Grant \#175090).

References:

1. Mohamad Habes, Guray Erus, Jon B Toledo, et al. White matter hyperintensities and imaging patterns of brain ageing in the general population. Brain (2016) Apr;139(Pt 4):1164-79

2. Pozorski V, Oh JM, Okonkwo O, et al. Cross-sectional and longitudinal associations between total and regional white matter hyperintensity volume and cognitive and motor function in Parkinson's disease. NeuroImage Clinical (2019);23:101870

3. Sunwoo MK, Jeon S, Ham JH, et al. The burden of white matter hyperintensities is a predictor of progressive mild cognitive impairment in patients with Parkinson's disease. European Journal of Neurology (2014);21:922-e950

\section{AMYLOID- $\beta$-42/NEUROGRANIN RATIO IS A POTENTIAL INDEX FOR COGNITIVE IMPAIRMENT IN PATIENTS WITH PARKINSON'S DISEASE}

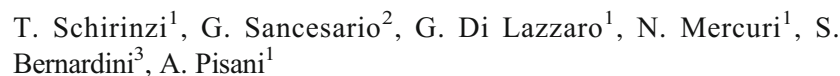

${ }^{1}$ Department of Systems Medicine, University of Roma Tor Vergata (Roma); ${ }^{2}$ Experimental Neurosciences, IRCCS Fondazione Santa Lucia (Roma); ${ }^{3}$ Department of Experimental Medicine and Surgery, University of Roma Tor Vergata (Roma)

Objective: To assess a panel of CSF synaptopathy-related biomarkers, including the novel neurogranin/amyloid- $\beta-42(\mathrm{~A} \beta 42 / \mathrm{NG})$ ratio, in a cohort of patients with Parkinson's disease (PD), to establish possible associations with the cognitive level and other clinical parameters.

Materials: 30 PD patients (age $=64.48 \pm 9.05$ years, $44 \%$ female) and 30 controls (subjects with non-neurodegenerative conditions; age $=65.04$ \pm 11.03 years, $66 \%$ female) undergoing CSF sampling and standardized clinical evaluation.

Methods: CSF levels of NG, A $\beta 42, A \beta 40$, total and phosphorylated tau, and $\mathrm{A} \beta 42 / \mathrm{NG}$ ratio were measured in patients and controls. In both the groups, the cognitive level was tested by the adjusted MMSE and the presence of comorbidities scored by the Charlson Comorbidity Index. PD patients were further assessed through UPDRS III, Hoehn and Yahr scale, calculation of the levodopa equivalent daily dose. Group differences were tested, adjusting for sex and age. Relationships among clinical and biochemical parameters were explored either by simple models or using age and sex as covariates. ROC analysis was performed to estimate accuracy of biomarkers in distinguishing the cognitive status ("normal"=adjusted MMSE $>25$ vs “impaired"=adjusted MMSE $\leq 25$ ).

Results: NG and $\mathrm{A} \beta 42$ were significantly reduced in $\mathrm{PD}$, with higher NG levels in those patients with worse cognition. In PD, the A $\beta 42 / \mathrm{NG}$ ratio showed a direct correlation with MMSE, independently from age and sex, and differentiated cognitively impaired patients with $92 \%$ sensitivity and $71.4 \%$ specificity, accuracy higher than NG alone. No correlations instead emerged with motor disturbances or therapy.

Discussion: Synaptopathy is critical in pathophysiology of PD. CSF levels of NG and $A \beta 42$ are considered markers of synaptic dysfunction in neurodegenerative diseases. Specifically, NG is postsynaptic proteins, whose CSF levels directly reflect more severe cognitive decline in patients with Alzheimer's disease (AD) and MCI. A $\beta 42$ operates instead at presynaptic level, playing a role in the pathogenesis of $\mathrm{AD}$ and $\mathrm{PD}$-dementia. While data on CSF A $\beta 42$ in PD are abundant and solid, those about NG are very scarce. Moreover, these two markers in combination as $\mathrm{A} \beta 42 / \mathrm{NG}$ ratio has never been tested before, whereas it may constitute a global index of synaptic dysfunction, with potential relevance in PD. Indeed, we found that the $\mathrm{A} \beta 42 / \mathrm{NG}$ ratio exhibits an accuracy to discriminate $\mathrm{PD}$ patients with cognitive impairment higher than NG alone.

Conclusions: The novel A $\beta 42 / \mathrm{NG}$ ratio couples either presynaptic or postsynaptic markers of synaptic dysfunction, representing a potential global index of synaptopathy, useful to track cognitive functions in PD.

\section{THE EFFECTS OF THE HD GENE: COMPARISONS BETWEEN PATIENTS WITH THE SAME 41 CAG TRIPLET AND TYPICAL OR TARDIVE AGE AT ONSET}

M. Solito ${ }^{1}$, S. Di Tella ${ }^{2}$, P. Zinzi ${ }^{1}$, M. Lo Monaco ${ }^{3}$, E. Cammareri $^{4}$, A. Bentivoglio $^{1}$, M. Silveri ${ }^{51}$ 
${ }^{1}$ Fondazione Policlinico Universitario A. Gemelli IRCCS, Department of Neurosciences, University Cattolica del Sacro Cuore (Roma); ${ }^{2}$ Fondazione Don Carlo Gnocchi IRCCS (Milano); ${ }^{3}$ Geriatric DayHospital and Movement Disorders Unit, Fondazione Policlinico Universitario A. Gemelli IRCCS (Roma); ${ }^{4}$ Department of Neurosciences, University Cattolica del Sacro Cuore (Roma); ${ }^{5}$ Department of Psychology, University Cattolica del Sacro Cuore (Milano)

Objectives: Huntington's disease (HD) is a genetic neurodegenerative disorder characterised by progressive motor, cognitive and behavioural symptoms. The expansion in CAG repeat number in HTT gene causes HD typically occurring in mid adult life. CAG repetitions $\geq 40$ are fully penetrant and negatively correlate with age of onset, but there is a wide variability as triplets number accounts for max $70 \%$ of the variation [1-2]. We aimed to examine clinical, behavioural and cognitive characteristics of HD patients with same low CAG repeat length and typical or tardive onset to better define their phenotype [3]

Materials and Methods: From Periodic Dataset of Enroll-HD (PDS4) comprising data from $>15.000$ participants from all over the world we obtained baseline data of 4893 HD European subjects. We selected 593 patients with 41 CAG: 360 with common onset (CoHD) and 233 with late onset $\geq 60$ years (LoHD). The two groups were compared for socio-demographic, clinical and neuropsychological characteristics.

Results: CoHD and LoHD did not differ for gender nor for smaller allele and were currently mean aged respectively $60.1 \pm 7.6$ years and 70.6 \pm 4.7 with mean disease duration shorter in the LoHD group (8.1 \pm 5.8 vs $6.4 \pm 3.8, \mathrm{p}<0.001)$. Phenotypical differences evidenced in motor and functional performance with worse scores in LoHD group (all pvalues $<0.001)$. In psychiatric anamnesis of $\mathrm{CoHD}$ patients was more often reported depression $(p=0.005)$ and suicidal ideation/behaviour $(\mathrm{p}=0.001)$. Current depression/anxiety score was higher in CoHD group $(\mathrm{p}=0.005)$ while no differences evidenced in apathy, irritability, psychosis, and obsessive/compulsive behaviours. Neuropsychological outcomes evidenced no significant differences in general cognitive status on MMSE and in most of the scales examining subcortical executive domain once possible confounding variables considered: education, age, disease duration. Significant differences were found only in Stroop Interference Test $(\mathrm{p}=0.023)$ and in Trail Making Test Part $\mathrm{B}(\mathrm{p}=0.020)$ with worse performance for LoHD group.

Discussion: CoHD and LoHD show similar cognitive deterioration pattern, while motor and functional performance is worse in tardive onset group. LoHD with unknown or missing inheritance, whose diagnosis was often the first into the family, were about $30 \%$ confirming literature. On psychiatric side the LoHD group showed less behavioural onset, lower anxiety and depression scores and less suicidal and depression history.

Conclusions: Our data suggest that characterise endophenotypes may help in delineate diagnostic and prognostic pathways providing indication for clinical management in typical and tardive onset. Further studies will be needed to investigate possible protective and /or precipitating factors may influence age of onset and phenotypical differences.

References:

1. Wexler NS, Lorimer J, Porter J, et al. Venezuelan kindreds reveal that genetic and environmental factors modulate Huntington's disease age of onset. Proc Natl Acad Sci U S A. (2004) Mar 9;101(10):3498-503

2. Oosterloo M, Bijlsma EK, van Kuijk SM, Minkels F, de DieSmulders CE. Clinical and genetic characteristics of late-onset Huntington's disease. Parkinsonism Relat Disord. (2019);61:101105. doi:10.1016/j.parkreldis.2018.11.009
3. Capiluppi E, Romano L, Rebora P, et al. Late-onset Huntington's disease with 40-42 CAG expansion. Neurol Sci. (2020);41(4):869876. doi:10.1007/s10072-019-04177-8

\section{A SYNTHETIC KINEMATIC INDEX OF TRUNK DISPLACEMENT CONVEYING THE OVERALL MOTOR CONDITION IN PARKINSON'S DISEASE}

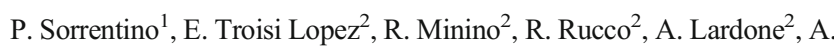 \\ Carotenuto $^{3}$, V. Manzo ${ }^{3}$, M. Liparoti ${ }^{2}$, G. Sorrentino ${ }^{2}$
}

${ }^{1}$ Institut de Neuroscience des Systemès, Aix-Marseille University (Marseille-F); ${ }^{2}$ Department of Motor Sciences, Parthenope University (Napoli); ${ }^{3}$ Department of Neurology, Cardarelli Hospital (Napoli)

Objectives: Parkinson's disease (PD) is a neurodegenerative disorder that occurs with both motor and non-motor symptoms. Gait alterations and balance impairment strongly contribute to an increased risk of falling [1]. The diagnosis of PD is mainly based on clinical criteria. An objective way to analyze movement might help to overcome such limitations. Threedimensional gait analysis (3D-GA) is a quantitative method regarded as the gold standard for movement evaluation [2]. Under an evolutionary perspective, the trunk and the upper girdle play a crucial role in maintaining the balance A key aspect facilitating the stability is that the pectoral girdle should not be oscillating too much as compared to the center of mass (COM) [2]. The aim of our study is to find an objective biomechanical index to synthetically convey the effect of the complex motor impairment in PD on stability. We introduced a new index, which quantifies the trunk displacement (TDI) in relation to the COM.

Materials: We recruited twenty-three PD patients and twenty-three healthy controls, matched for age, gender, and education.

Methods: All the participants underwent 3D gait analysis. The PD patients were acquired before and after a subclinical L-DOPA intake, and each acquisition was preceded by an UPDRS-III evaluation [3]. The TDI values and the gait parameters (e.g. speed, stride length, stance time, the variability of gait, joint range of motion, etc.) were extracted from the kinematic data. To test the sensitivity of the TDI, we compared the PD patients in both off and on condition. Finally, to investigate the inherent characteristics of the new measure, we correlated the TDI values with both gait parameters and UDPRS-III scores.

Results: The TDI was able to point out a statistical difference between PD patients in on and off-state ( $\mathrm{p}=0.004)$, showing high trunk displacement in patients before L-DOPA intake. Any other gait parameter failed to show such a difference. Moreover, the TDI correlated with gait parameters, representative of velocity and stability of gait. Finally, TDI showed a positive correlation with the UPDRS-III $(\mathrm{r}=0.538 ; \mathrm{p}<0.001)$, expression of the clinical motor impairment.

Discussion: Our results indicate that the TDI may be considered a synthetic and sensitive measure that reflects the overall motor condition in PD. Specifically, the correlations with the gait parameters provide the TDI with a stability significance, while the correlation with the UPDRSIII highlight its clinical relevance.

Conclusions: TDI may offer an improvement in assessing the motor impairment in $\mathrm{PD}$.

References:

1. Gökçal E, Gur VE, Selvitop R, Yildiz GB, Asil T. A. Motor and non-motor symptoms in Parkinson's disease: effects on quality of life. Noro Arch Psikiytr. (2017);54(2):143 
2. van Mastrigt NM, Celie K, Mieremet AL, Ruifrok ACC, Geradts Z. Critical review of the use and scientific basis of forensic gait analysis. Forensic Sci Res. (2018);3(3):183-93

3. Jo S. Hypothetical neural control of human bipedal walking with voluntary modulation. Med Biol Eng Comput. (2008);46(2):17993

\section{DEEP BRAIN STIMULATION IN PARKINSON'S DISEASE PATIENTS AND NEUROTOXIC RODENT'S MODELS: END OF THE SYNERGY?}

\author{
A. Stefani ${ }^{1}$, R. Cerroni ${ }^{2}$, N. Mercuri ${ }^{2}$, S. Galati ${ }^{3}$
}

${ }^{1}$ Dept System Medicine, University of Tor Vergata (Roma); ${ }^{2} \mathrm{UOC}$ Neurology, Policlinico Tor Vergata (Roma); ${ }^{3}$ Neurocenter Southern Switzerland, University of Svizzera Italiana (Lugano-CH)

Objectives: In the eighties, the emerging evidence of a tight circuitry governing the mammalian basal ganglia did open out the chance to locally modulate the firing pattern in the subthalamic nucleus (STN) and internal globus pallidus (GPi). Indeed, the history of deep brain stimulation (DBS) for Parkinson disease (PD) represented a paradigmatic crosstalk between mammalian disease models (either in non-human primates, NHP, or rodents) and clinical evidence in humans. The high frequency stimulation (HFS) into the STN of MPTP-treated primates promoted significant amelioration. An analogous strategy relieved tremor and hypokinetic parameters in PD patients. In addition, the 6hydroxydopamine (6-OHDA) rodent model has mastered decades of research, contributing in understanding the PD pathology. However, this paper is discussing the modern efficiency of this routine neurotoxic model, when routinely used to understand mechanisms and efficacy of STNDBS in PD patients. Are most of experimental models still useful, nowadays, for electrical-mediated targeting of PD signs?

Methods: A critical, extensive revision of the literature was done. Focusing, in particular, on results achieved (or presumed to) in rodent's models.

Results: Some findings collected following 6-OHDA, promoted dogmatic visions, as the wrong contention that suppression of STN glutamate was the key therapeutic player. Instead, changes of glutamate release are negligible, in humans, during transition to ON-state. Besides, the imbalance of basal ganglia endogenous band frequencies, the beta $(\beta)$ band increase and the cortical-basal ganglia synchronization, undisputedly shared by models and PD patients, does not govern the whole spectrum of non-motor PD signs, difficult to investigate in rodents. Further, the tonic release of dopamine, inferred during HFS in rodents, was not replicated in humans. Finally, neurotoxic rodent's models describes a "pure" dopamine depletion sparing pathways crucial in parkinsonian phenotypes, i.e. noradrenergic and cholinergic ones.

Discussion: Our study emphasizes possible pitfalls of 6-OHDA models, if intended to provide insights for the present, and fast developing DBS techniques (such as adaptive stimulation). Basic scientists are strongly invited to share their projects with expert clinicians, if their intent is not simply to publish robust evidence, but also to transfer results into meaningful products.

Conclusions: Although the utilization of neurotoxic models is still providing major advancements, we stress the opportunity to rejuvenate the experimental approaches. Strategy may include a refreshment of "in vivo" recording approaches; recordings extension towards motor thalamus; smart use of transgenic mice, adaptation of closed-loop device for small animals.

\section{THE ROLE OF ARTIFICIAL INTELLIGENCE FOR AN ACCURATE INTERPRETATION OF 18F-DOPA PET/CT IN THE DIAGNOSIS OF PARKINSON'S DISEASE}

L. Strada ${ }^{1}$, A. Piccardo ${ }^{2}$, R. Cappuccio ${ }^{3}$, G. Bottoni ${ }^{1}$, A. Cirone ${ }^{4}, \mathrm{~S}$. Righi $^{4}$, M. Ugolini ${ }^{1}$, P. Bianchi ${ }^{5}$, P. Bertolaccini ${ }^{5}$, M. Massollo ${ }^{1}$, A. Castaldi $^{6}$, L. Mazzella ${ }^{7}$, A. Cistaro ${ }^{1}$, M. Del Sette ${ }^{1}$

${ }^{1}$ Neurology Unit, E.O. Ospedali Galliera (Genova); ${ }^{2}$ Nuclear Medicine Unit, E.O. Ospedali Galliera (Genova); ${ }^{3}$ I.N.F.N., I.N.F.N. Pisa Section (Pisa); ${ }^{4}$ Medical Physics Department, E.O. Ospedali Galliera (Genova); ${ }^{5}$ Department of Nuclear Medicine, Azienda USL Toscana NordOvest (Massa Carrara); ${ }^{6}$ Neuroradiology Unit, E.O. Ospedali Galliera (Genova); ${ }^{7}$ Department of Neurology, ASL3 di Genova (Genova);

Aim: To test the role of 3D Convolutional Neural Network (CNN) in order to evaluate brain 18F-DOPA PET scan and to identify patients with movement disorders due to neurodegenerative disease. We also tested the robustness of this method of analysis comparing it with the regional analysis of the affected striatum using region-to-reference (striatal-to-occipital) ratio (SOR).

Materials and methods: We analysed all patients who underwent $18 \mathrm{~F}-$ DOPA PET/CT from 2016 to 2018. Two expert examiners interpreted $\mathrm{PET} / \mathrm{CT}$ images and then classified them into positive and negative. All patients with PET findings qualitatively doubtful or not concordant with clinical diagnosis were excluded. At least two years of follow-up and an ascertained clinical diagnosis was required and was used as standard of truth. We developed a $3 \mathrm{D} \mathrm{CNN}$ in order to accurately evaluate 18F-DOPA PET/CT and refine the imaging diagnosis of movement disorder. This system needed a training process by using PET images of about $2 / 3$ of patients and then a testing process including the remaining patients.

Results: Ninety-eight patients were enrolled. Forty-three with clinical diagnosis of Parkinson's disease or other neurodegenerative disease causing nigrostriatal degeneration, and 55 with other causes of parkinsonism and/or tremor. We trained the network over 250 epochs for each PET scan and the model showed a very good convergence of error and accuracy. After the training including 69 patients, we used the best-weights set to make a prediction on the gold standard. Then, in the test subgroup including 29 patients, the diagnostic performance of 3D CNN was calculated. Sensitivity, specificity, negative predictive value, positive predictive value and accuracy were $100 \%, 89 \%, 100 \%, 85 \%$ and $93 \%$ respectively. Thus, 3D CNN incorrectly interpreted as positive 2 negative $18 \mathrm{~F}$ DOPA PET/CT scans. When we compared the 3D CNN with the regional analysis of the affected striatum by using SOR, we found that the two false positive patients showed SOR values $5^{\circ}$ percentile of the distribution of the controls.

Discussion: We showed that the deep learning-based brain $18 \mathrm{~F}$ DOPA PET interpretation system could accurately determine dopaminergic degeneration and refine diagnosis. To our knowledge, this is the first study regarding the application of artificial intelligence to brain $18 \mathrm{~F}$ DOPA PET/CT in order to identify patients affected by PD.

Conclusions: After proper training, 3D CNNs is able to correctly interpret 18F-DOPA PET images revealing patients affected by Parkinson's disease.

References:

- Morbelli S, Esposito G, Arbizu J, et al. EANM practice guideline/ SNMMI procedure standard for dopaminergic imaging in Parkinsonian syndromes 1.0 [published online ahead of print, 2020 May 9]. Eur J Nucl Med Mol Imaging (2020) 
- Choi H, Ha S, Im HJ, Paek SH, Lee DS. Refining diagnosis of Parkinson's disease with deep learning-based interpretation of dopamine transporter imaging. Neuroimage Clin. (2017) Sep 10;16:58694

- Marinelli L, Piccardo A, Mori L, et al. Orbitofrontal (18) F-DOPA Uptake and Movement Preparation in Parkinson's Disease. Parkinsons Dis. (2015);2015:180940

\section{FOCUSED ULTRASOUND BILATERAL THALAMOTOMY FOR MEDICALLY REFRACTORY ESSENTIAL TREMOR}

P. Sucapane ${ }^{1}$, F. Pistoia ${ }^{2}$, G. Saporito ${ }^{2}$, D. Cerone ${ }^{1}$, T. Russo ${ }^{1}$, A. Catalucci $^{3}$, F. Bruno ${ }^{3}$, S. Sacco ${ }^{2}$, C. Masciocchi ${ }^{2}$, C. Marini ${ }^{4}$

${ }^{1}$ Neurology Unit, San Salvatore Hospital (L'Aquila); ${ }^{2}$ Department of Applied Clinical Sciences and Biotechnology, University of L'Aquila (L'Aquila); ${ }^{3}$ Radiology Department, San Salvatore Hospital (L'Aquila); ${ }^{4}$ Department of Medicine, Health and Environment Sciences, University of L'Aquila (L'Aquila)

Objective: Unilateral magnetic resonance imaging-guided focused ultrasound (MRgFUS) ventral intermediate nucleus (Vim) thalamotomy is a well-established treatment for medication-refractory essential tremor (ET). Scant data are available about the efficacy and safety of bilateral MRgFUS-Vim thalamotomy. Here we report the case of a patient treated with a bilateral procedure.

Materials and Methods: A 65-years old man suffered from bilateral and disabling essential tremor whose onset had been 7-years earlier, in the absence of other symptoms or neurological signs. Previous treatments (beta-blockers, trazodone, primidone and topiramate) had failed in the management of tremor, which was classified as medically refractory. Tremor was particularly disabling for the patient, as interfering with main daily-living activities, such as feeding, drinking and writing. The patient reported frustration about the presence of tremor, which caused discomfort, embarrassment and social withdrawal.

Results: On March 2018 the patient underwent MRgFUS-left-Vim thalamotomy leading to a stable improvement of right-arm tremor, scored through the Fahn-Tolosa-Marin scale, without side effects or complications. Two years later, following patient's specific request and signed informed consent, contralateral MRgFUS-Vim thalamotomy was performed, resulting in marked improvement of the left arm tremor without side effects or complications. Neuropsychological functions were investigated before and after each single treatment through a comprehensive neuropsychological test battery (Digit Span Forward, Digit Span Backward, Rey 15-Item Test, Rey-Osterrieth complex figure test, Raven's progressive Matrices, verbal and semantic fluency tests, Token Test, Trail Making Tests, Symbol Digit test, Frontal Assessment Battery, Beck Depression Inventory Scale, Hamilton Anxiety Scale, Basic and Instrumental activities of daily living assessment) and no dysfunctions were detected. On neurological exam, the patient did not show any neurological deficits also including ataxia and dysarthria.

Discussion: While a large amount of data is available about the outcomes of unilateral MRgFUS-Vim thalamotomy, few experiences have been reported about the safety and effectiveness of bilateral approaches. In our case, the second procedure was widely time-delayed, in order to carefully monitor neurological and neuropsychological conditions and to assure patient's safety. Dysarthria and gait instability, which are the complications most commonly associated with bilateral thalamotomy, were not observed in the patient, who was completely satisfied about the treatment, that improved his daily performances and quality of life.
Conclusions: Further experiences are needed to evaluate the usefulness and safety of staged bilateral MRgFUS-Vim thalamotomy for medically refractory bilateral disabling tremor, especially when patients are not eligible for other treatments.

References:

- Alshaikh J and Fishman PS. Revisiting bilateral thalamotomy for tremor. Clinical Neurology and Neurosurgery (2017);158:103-07

- Bruno F, Catalucci A, Arrigoni F, Sucapane P, Cerone D, Cerrone P, Ricci A, Marini C, Masciocchi C. An experience-based review of HIFU in functional interventional neuroradiology: transcranial MRgFUS thalamotomy for treatment of tremor. Radiol Med (2020);125:877-86

- Walters H, Shah BB. Focused Ultrasound and Other Lesioning Therapies in Movement Disorders. Curr Neurol Neurosci Rep. (2019);19:66

\section{CLINICAL CORRELATES OF FUNCTIONAL MOTOR DISORDERS: AN ITALIAN MULTICENTRE STUDY}

M. Tinazzi ${ }^{1}$, F. Morgante ${ }^{2,3}$, E. Marcuzzo ${ }^{1}$, R. Erro ${ }^{4}$, P. Barone ${ }^{4}$, R. Ceravolo $^{5}$, S. Mazzucchi ${ }^{5}$, A. Pilotto ${ }^{6,7}$, A. Padovani ${ }^{6}$, L. M. Romito ${ }^{8}$, R. Eleopra ${ }^{8}$, M. Zappia ${ }^{9}$, A. Nicoletti ${ }^{9}$, C. Dallocchio ${ }^{10}$, C. Arbasino ${ }^{10}$, F. Bono $^{11}$, A. Pascarella ${ }^{11}$, B. Demartini ${ }^{12}$, O. Gambini $^{12}$, N. Modugno $^{13}$, E. Olivola ${ }^{13}$, V. Di Stefano ${ }^{14}$, A. Albanese ${ }^{15}$, G. Ferrazzano ${ }^{16}$, A. Tessitore $^{17}$, M. Zibetti ${ }^{18}$, G. Calandra-Buonaura ${ }^{19,20}$, M. Petracca ${ }^{21}$, M. Esposito $^{22,23}$, A. Pisani ${ }^{24}$, P. Manganotti ${ }^{25}$, F. Stocchi ${ }^{26}$, M. Coletti $\mathrm{Moja}^{27}$, A. Antonini ${ }^{28}$, G. Defazio ${ }^{29}$, C. Geroin ${ }^{1}$

${ }^{1}$ Neurology Unit, Movement Disorders Division, Department of Neurosciences, Biomedicine and Movement Sciences, University of Verona (Verona); ${ }^{2}$ Neurosciences Research Centre, Molecular and Clinical Sciences Neurosciences Research Centre, Molecular and Clinical Sciences Research Institute, St George's University of London (London-UK); ${ }^{3}$ Department of Experimental and Clinical Medicine, University of Messina (Messina); ${ }^{4}$ Center for Neurodegenerative Diseases (CEMAND), Department of Medicine, Surgery and Dentistry, Scuola Medica Salernitana, University of Salerno (Baronissi-SA); ${ }^{5}$ Neurology Unit, Department of Clinical and Experimental Medicine, University of Pisa (Pisa); ${ }^{6}$ Department of Clinical and Experimental Sciences, University of Brescia (Brescia); ${ }^{7}$ FERB Onlus, Ospedale S. Isidoro (Trescore Balneario$\mathrm{BG}) ;{ }^{8}$ Parkinson and Movement Disorders Unit, Fondazione IRCCS Istituto Neurologico Carlo Besta (Milano); ${ }^{9}$ Department G.F. Ingrassia, Section of Neurosciences, University of Catania (Catania); ${ }^{10}$ Department of Medical Area, Neurology Unit, ASST Pavia (Pavia); ${ }^{11}$ Botulinum Toxin Center, Neurology Unit, A.O.U. Mater Domini (Catanzaro); ${ }^{12}$ Aldo Ravelli Research Center for Neurotechnology and Experimental Brain Therapeutics, Department of Health Sciences, University of Milan (Milano); ${ }^{13}$ IRCCS Neuromed (Pozzilli-IS); ${ }^{14}$ Department of Neuroscience, Imaging and Clinical Sciences, University G. d'Annunzio (Chieti); ${ }^{15}$ Department of Neurology, Humanitas Research Hospital (Rozzano-MI); ${ }^{16}$ Department of Human Neurosciences, La Sapienza University (Roma); ${ }^{17}$ Department of Advanced Medical and Surgery Sciences, University of Campania "Luigi Vanvitelli" (Napoli); ${ }^{18}$ Department of Neuroscience Rita Levi Montalcini, University of Turin (Torino); ${ }^{19}$ Department of Biomedical and Neuromotor Sciences, University of Bologna (Bologna); ${ }^{20}$ IRCCS Institute of Neurological Sciences of Bologna (Bologna);

${ }^{21}$ Movement Disorder Unit, Fondazione Policlinico Universitario A. 
Gemelli IRCCS (Roma); ${ }^{22}$ Clinical Neurophysiology Unit, Cardarelli Hospital (Napoli); ${ }^{23}$ Department of Neurosciences, Reproductive and Odontostomatological Sciences, University of Naples Federico II (Napoli); ${ }^{24}$ Department of Systems Medicine, University of Rome Tor Vergata (Roma); ${ }^{25}$ Clinical Neurology Unit, Department of Medical, Surgical and Health Services, University of Trieste (Trieste); ${ }^{26}$ University and Institute of Research and Medical Care San Raffaele (Roma); ${ }^{27}$ Mauriziano Hospital Umberto I (Torino); ${ }^{28}$ Movement Disorders Unit, Department of Neuroscience, University of Padua (Padova); ${ }^{29}$ Department of Medical Sciences and Public Health, University of Cagliari (Cagliari)

\section{Co-investigators. Italian Registry of Functional Motor Disorders (IRFMDs) Study Group.}

- Elisabetta Zanolin, Department of Public Health and Community Medicine, University and Hospital Trust of Verona, Verona

- Mara Zenorin, Neurology Unit, Movement Disorders Division, Department of Neurosciences, Biomedicine and Movement Sciences, University of Verona, Verona

- Elena Antelmi, Neurology Unit, Movement Disorders Division, Department of Neurosciences, Biomedicine and Movement Sciences, University of Verona, Verona

- Sofia Cuoco, Center for Neurodegenerative Diseases (CEMAND) Department of Medicine, Surgery and Dentistry - Scuola Medica Salernitana, University of Salerno, Baronissi (SA)

- Sara Scannapieco, Center for Neurodegenerative Diseases (CEMAND) Department of Medicine, Surgery and Dentistry Scuola Medica Salernitana, University of Salerno, Baronissi (SA)

- Daniela Frosini, Neurology Unit, Department of Clinical and Experimental Medicine, University of Pisa, Pisa

- Eleonora Del Prete, Neurology Unit, Department of Clinical and Experimental Medicine, University of Pisa, Pisa.

- Andrea Scalvini, Department of Clinical and Experimental Sciences, University of Brescia, Brescia

- Ferb Onlus, Ospedale S. Isidoro, Trescore Balneario, Bergamo

- Alberto Imariso, Department of Clinical and Experimental Sciences, University of Brescia, Brescia

- Antonio Emanuele Elia, Parkinson and Movement Disorders Unit, Fondazione IRCCS Istituto Neurologico Carlo Besta, Milano

- Nico Golfrè Andreasi, MD

Parkinson and Movement Disorders Unit, Fondazione IRCCS Istituto Neurologico Carlo Besta, Milano

- Giovanni Mostile, Department G.F. Ingrassia, Section of Neurosciences, University of Catania, Catania

- Antonina Luca, Department G.F. Ingrassia, Section of Neurosciences, University of Catania, Catania

- Cinzia Femiano, IRCCS Neuromed, Pozzilli

- Giada Ricciardo Rizzo, IRCCS Neuromed, Pozzilli

- Angela Matinella, Department of Medical Area, Neurology Unit, ASST Pavia, Pavia

- Gabriele Bellavia, Department of Medical Area, Neurology Unit, ASST Pavia, Pavia

- Giulio Demonte, Botulinum Toxin Center, Neurology Unit A.O.U. Mater Domini, Catanzaro

- Lucia Manzo, Botulinum Toxin Center, Neurology Unit A.O.U. Mater Domini, Catanzaro
- Alberto Priori Aldo Ravelli Research Center For Neurotechnology and Experimental Brain Therapeutics, Department of Health Sciences, University of Milan, Milano

- Marco Onofrj, Department of Neuroscience, Imaging and Clinical Sciences -University G. D'Annunzio, Chieti-Pescara

- Stefania Lalli, Humanitas Research Hospital, Rozzano, Milano

- Giovanni Fabbrini, Department of Human Neurosciences, La Sapienza, University of Rome, Roma

- IRCCS Neuromed, Pozzilli

- Rosa De Micco, Department of Advanced Medical and Surgery Sciences, University of Campania - Luigi Vanvitelli, Napoli

- Margherita Fabbri, Department of Neuroscience - Rita Levi Montalcini, University of Turin, Torino

- Instituto De Medicina Molecular, Faculdade De Medicina, Universidade De Lisboa, Lisbon (P)

- Vincenzo Mastrangelo, Department of Biomedical and Neuromotor Sciences, University of Bologna, Bologna

- Anna Rita Bentivoglio, Institute of Neurology, Movement Disorder Research Center, Università Cattolica del Sacro Cuore; Movement Disorder Unit, Fondazione Policlinico Universitario A. Gemelli IRCCS, Roma

- Giulia Di Lazzaro, Department Systems Medicine, University of Rome Tor Vergata, Roma

- Giulia Bellavista, Clinical Neurology Unit, Department of Medical, Surgical and Health Services, University of Trieste

Objective:1) to describe the clinical manifestations of FMDs, including non-motor symptoms and occurrence of other functional neurological disorders (FND);2) to report the frequency of isolated and combined FMDs and their relationship with demographic and clinical variables.

Methods: For this multicentre, observational study, we enrolled consecutive outpatients with a definite diagnosis of FMDs attending 25 tertiary movement disorders centres in Italy. Each subject underwent detailed clinical evaluation with definition of phenotype, number of FMD (isolated, combined) and assessment of associated neurological and psychiatric symptoms.

Results: Out of 410 FMDs (71\% females; mean age $47 \pm 16.1$ years) the most common phenotypes were weakness and tremor. People with FMDs had higher educational level than general population, and frequent non-motor symptoms, especially anxiety, fatigue and pain. Almost half of FMDs patients had associated other FND, such as sensory symptoms, non-epileptic seizures and visual symptoms. Subjects with combined FMDs showed a higher burden of non-motor symptoms and more frequent other FND. Multivariate regression analysis showed that diagnosis of combined FMDs was more likely to be delivered by a movement disorders neurologist. Also, FMDs duration, pain, insomnia, a diagnosis of somatoform disease and treatment with antipsychotic were all significantly associated to combined FMDs.

Conclusions: Our findings highlight the need for multidimensional assessment in patients with FMDs, given the high frequency of nonmotor symptoms and other FND, especially in patients with combined FMDs.

\section{References:}

- Edwards MJ, Bhatia KP. Functional (psychogenic) movement disorders: merging mind and brain. Lancet Neurol. (2012); 11:250-60

- Espay AJ, Aybek S, Carson A, Edwards MJ, Goldstein LH, Hallett M, LaFaver K, LaFrance WC Jr, Lang AE, Nicholson T, Nielsen G, Reuber M, Voon V, Stone J, Morgante F. Current Concepts in Diagnosis and Treatment of Functional Neurological Disorders. JAMA Neurol. (2018); 75:1132-41 


\section{THE C-TERMINAL CROSSLINKED TELOPEPTIDE OF TYPE I COLLAGEN (CTX-I) AS A CARDIOMYOPATHY BIOMARKER IN FRIEDREICH ATAXIA PATIENTS}

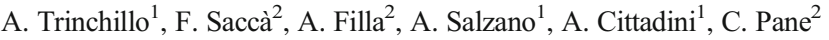 \\ ${ }^{1}$ Internal Medicine, "Federico II" University (Napoli); ${ }^{2}$ Neurology, \\ Federico II University (Napoli)
}

Objectives: Cardiomyopathy (CM), usually hypertrophic, occurs often in Friedreich Ataxia (FRDA) and is the predominant cause of death. In CM, cardiac extracellular matrix is predominantly composed of collagen type I and III. Their terminal pro-peptides are cleaved and released into blood. The carboxyterminal pro-peptide of type I collagen (PICP) is a marker of the synthesis. Serum PICP undergoes a spontaneous denaturation into the C-terminal crosslinked telopeptide of type I collagen (CTX-I). The aim of this study is to find an early biomarker able to predict CM in FRDA patients, before it was clinically evident, to implement therapeutic strategies to prevent it.

Materials and Methods: We measured serum CTX-I as a marker of cardiac fibrosis in FRDA patients compared to healthy controls (HC) and evaluated the correlation between CTX-I serum level and echocardiographic, clinical and biological variables. Blood samples were taken from 24 FRDA and 19 HC. CTX-I was measured with ELISA technique. CTX-I levels were correlated to morphological cardiac changes established with Echocardiography (EcoCG); clinical features using the Scale for the Assessment and Rating of Ataxia (SARA) and biochemical markers.

Results: CTX-I Mean \pm SD were $43.8 \pm 38.6 \mu \mathrm{g} / \mathrm{L}$ in FRDA and 18.2 \pm 8.3 in $\mathrm{HC}$ (mean difference $+25.7 \mu \mathrm{g} / \mathrm{L} ; \mathrm{CI} 95 \%+6.1,+45.2 ; \mathrm{p}=0.007$ ). Bivariate correlation analysis found a correlation between CTX-I value and TT3 ( $R=0.883 ; p>0.001)$. To better identify independent predictors of CTX-I levels, we attempted to create a multivariate linear regression model using CTX-I as dependent variable. In the first biochemical and clinical model we found that independent predictors were (intercept -64): disease duration (coeff. $-2.34 ; \mathrm{p}=0.032$ ), TT3 (coeff. 127.17; $\mathrm{p}=0.013$ ). In the echocardiographic model we found the following predictors (intercept $=-841.02$ ): relative wall thickness (coeff. 1629; $\mathrm{p}=0.028$ ), posterior left ventricular wall thickness (coeff. $-90.29 ; \mathrm{p}=0.016$ ), left ventricular end diastolic diameter (coeff 19.12; $p=0.015$ ), ejection fraction (coeff. 352.75; $\mathrm{p}=0.005$ ).

Discussion and Conclusions: Biomarkers that reflect CM state are urgently needed to define patients at risk in order to improve the management of the disease and develop endpoints useful for clinical trials. In our study we report a higher CTX-I levels in FRDA patients compared to HC. Its relationship with echocardiographic, clinical and biological variables makes it a new available marker useful to predict cardiological evolution in FRDA. CTX-I is an easy to measure biomarker and could be a promising tool for a comprehensive $\mathrm{CM}$ evaluation.

References:

- Querejeta R, Varo N, López B, et al. Serum carboxyterminal propeptide procollagen type $\mathrm{I}$ is a marker of myocardial fibrosis in hypertensive heart disease. Circulation (2000);101:1729-35

- Weidemann F, Rummey C, Bijnens B, et al. The heart in Friedreich ataxia: definition of cardiomyopathy, disease severity, and correlation with neurological symptoms. Circulation (2012) Apr 3;125(13):1626-34

- Raman SV, Phatak K, Hoyle JC, Pennell ML, McCarthy B, Tran T, et al. Impaired myocardial perfusion reserve and fibrosis in Friedreich ataxia: a mitochondrial cardiomyopathy with metabolic syndrome. Eur Heart J (2011);32(5):561-7

\section{IMPACT OF HEALTHCARE ORGANIZATIONAL CHANGES IN THE FIRST THREE MONTHS OF COVID-19 EPIDEMIC ON PEOPLE WITH PARKINSON'S DISEASE OR PARKINSONISM: THE PARKLINK BOLOGNA COHORT, ITALY (IOCC-PARK)}

L. Vignatelli ${ }^{1}$, C. Zenesini ${ }^{1}$, L. Belotti ${ }^{1}$, E. Baldin ${ }^{1}$, G. CalandraBuonaura $^{1}$, P. Cortelli ${ }^{1}$, C. Descovich ${ }^{2}$, G. Giannini ${ }^{1}$, M. Guarino ${ }^{1}$, C. Scaglione $^{1}$, S. Trombetti ${ }^{2}$, R. D'Alessandro ${ }^{1}$, F. Nonino ${ }^{1}$, x. on behalf of ParkLink Bologna group

${ }^{1}$ IRCCS Institute of Neurological Sciences of Bologna, AUSL of Bologna (Bologna); ${ }^{2}$ Clinical Governance, AUSL of Bologna (Bologna)

Objective: Emilia-Romagna (ER) ranked as third Italian region as number of COVID-19 reported cases. Up to June 2, 2020, 5,036 cases were reported in Bologna (metropolitan area: population about $800,000)$. To limit crowding in healthcare facilities, during the lockdown period (March 9 through May 18, 2020) the Local Healthcare Trust of Bologna (LHTB) cancelled all scheduled non-urgent outpatient appointments (with telemedicine visit as replacement, in selected cases). Through such abrupt rearrangement, the COVID-19 pandemic may have remarkably impacted on healthcare systems, hampering the management of patients with chronic diseases with possible worsening of their health status. People with Parkinson's disease (PD) and Parkinsonian syndromes (PS) require tailored follow-up and supportive care in dedicated healthcare settings. We aim to compare clinical and organisational healthcare outcomes in PD and PS patients with a matched general population cohort during the lockdown and the same period from 2017 to 2019 within the LHTB.

Materials: Clinically confirmed PD or PS recruited since 2016 (LHTB ParkLink cohort) through a record linkage system (ParkLink Bologna) and an anonymous matched LHTB general population cohort, alive in 2019.

Method: Historical cohort design comparing the ParkLink cohort with the control cohort matched (ratio 1:10) for age, sex, Charlson Index and residence, recruited through administrative databases. Data of the month of March 2017, 2018 and 2019 were compared with March 2020. Outcomes: any hospital admission, any urgent hospital admission, disease-specific hospital admission (major injuries, infections, gastrointestinal events, thromboembolic events), any urgent outpatient visit, any urgent test.

Results: In both cohorts (ParkLink N=972 subjects, 696 PD, 276 PS; control $\mathrm{N}=9,507) 58 \%$ were male and mean age was 76 yrs (SD 9). Compared to March 2017-2019, in March-2020 the mean number of any urgent outpatient visit and of any urgent test were reduced in both cohorts (visits: ParkLink -39/1,000; control -18/1,000; tests: -56/1,000; 60/1,000). In March 2017 to 2019 the mean number of any hospital admission was 26/1,000 in both cohorts while in March 2020 it was 37/ 1,000 in the ParkLink cohort (11/1,000 increase) and 25/1,000 in the control cohort (1/1,000 decrease). Urgent admissions increased in both cohorts ( $+14 / 1,000$ ParkLink, $+4 / 1,000$ control cohort) as well as diseasespecific admissions for major injuries, thrombotic events, infections, gastro-intestinal events or hypotension $(+11 / 1,000$ ParkLink cohort, $+4 /$ 1,000 control cohort).

Discussion: During the first month of the COVID-19 lockdown we observed an increase of hospital admissions (overall, urgent and diseasespecific) among people with PD/PS in comparison with a matched population and with historical data. 
Conclusion: The restraint of healthcare provision through the cancellation of non-urgent outpatient appointments may have worsened clinical and process outcomes of PD/PS patients, with more frequent resort to urgent hospitalization.

\section{WEARABLE TECHNOLOGIES FOR BALANCE ASSESSMENT IN NEUROLOGICAL DISORDERS: A COMPREHENSIVE UPDATE}

A. Zampogna ${ }^{1}$, I. Mileti ${ }^{2}$, F. Asci ${ }^{1}$, A. Manoni ${ }^{3}$, E. Palermo ${ }^{2}$, F. Irrera ${ }^{3}$, A. Suppa ${ }^{4}$

${ }^{1}$ Department of Human Neurosciences, Sapienza University (Roma); ${ }^{2}$ Department of Mechanical and Aerospace Engineering, Sapienza University (Roma); ${ }^{3}$ Department of Information Engineering, Electronics and Telecommunications, Sapienza University (Roma); ${ }^{4}$ Department of Human Neurosciences/IRCCS Neuromed, Sapienza University (Roma)

Rationale/Objectives: Balance impairment is one of the major factors leading to falls in patients with neurological disorders. The assessment of balance commonly consists of clinical and laboratory examinations respectively based on standardised scales and posturography. However, these approaches suffer from several limitations affecting the sensitivity and ecological value of balance assessment. Recently, wearable technologies, including multimodal wireless sensors, have been increasingly used to quantitatively and unobtrusively assess balance in neurological disorders. We aim to provide a comprehensive and updated discussion concerning the evaluation of balance through wearable technologies in patients with neurological disorders.

Material/methods: We performed literature research of studies published by July 31 th, 2020 on several electronic databases, examining balance through wearable technologies in patients with Alzheimer's disease, Parkinson's disease (PD), multiple sclerosis, Huntington's disease, cerebellar ataxia, stroke, traumatic brain injury, neuropathies, and vestibular disorders.

Results: Most studies have applied inertial devices (accelerometers and gyroscopes), even integrated in common tablet computers and smartphones, whereas only a few have adopted wireless sEMG sensors for balance assessment in neurological disorders. Based on the static or dynamic postural task performed, authors have used 1 or multiple devices placed on the upper and lower limbs, head, trunk, and waist. Filtered acceleration signals through inertial sensors have shown increased postural sway in patients with neurological disorders, whereas sEMG measures have demonstrated impaired anticipatory postural adjustments and muscle synergies in PD patients. Inertial measures have even identified subclinical abnormalities correlating with the amount of clinical disability in specific neurological disorders. Finally, several authors have agreed on the moderate or strong correlation between the performance of wireless sensors and standardised laboratory instrumentations (i.e. force plates and infrared optical systems). All these studies have involved supervised laboratory settings.

Discussion/Conclusions: Wearable technologies are sensitive tools to measure balance, but it is unclear whether sensor-based measures of balance may discriminate various neurological disorders. Proper cost and energy-benefit analysis, as well as the efforts for patients and caregivers, should be always considered when addressing the issue of type, number and placement of wireless sensors for balance assessment. Although a great experience in the use of wireless sensors for balance assessment has been achieved in the laboratory setting, much still needs to be done for the technological migration of wearables to the domestic unsupervised environment. This migration involves several challenges, also including ethical issues, but would open multiple valuable prospects, such as the implementation of teleneurology and telerehabilitation approaches.

References:

- Zampogna A, Mileti I, Palermo E, Celletti C, Paoloni M, Manoni A, Mazzetta I, Dalla Costa G, Pérez-López C, Camerota F, Leocani L, Cabestany J, Irrera F, Suppa A. Fifteen Years of Wireless Sensors for Balance Assessment in Neurological Disorders. Sensors (Basel) (2020); 20:3247. doi: 10.3390/s20113247

- Stolze H, Klebe S, Zechlin C, Baecker C, Friege L, Deuschl G. Falls in frequent neurological diseases - prevalence, risk factors and aetiology. J. Neurol. (2004);251:79-84. doi: 10.1007/s00415-0040276-8

- Dorsey ER, Glidden AM, Holloway MR, Birbeck GL, Schwamm LH. Teleneurology and mobile technologies: the future of neurological care. Nat Rev Neurol. (2018);14:285-297. doi: 10.1038/ nrneurol.2018.31

\section{EPILEPSY}

\section{TWO-DIMENSIONAL CONVOLUTIONAL NETWORK ANALYSIS FOR INTERICTAL EEG DISCRIMINATION BETWEEN SUBJECTS WITH EPILEPTIC SEIZURES AND PNES: PRELIMINARY RESULTS}

M. Ascoli ${ }^{1}$, G. Varone ${ }^{1}$, M. Lo Giudice ${ }^{1}$, S. Gasparini ${ }^{1}$, V. Cianci ${ }^{2}$, G.

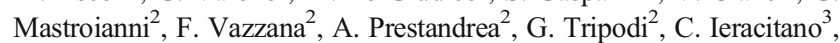
C. Morabito ${ }^{3}$, U. Aguglia $^{1}$, E. Ferlazzo ${ }^{1}$

${ }^{1}$ Department of Medical and Surgical Sciences, Magna Grecia University (Catanzaro); ${ }^{2}$ Regional Epilepsy Center, Great Metropolitan Hospital (Reggio Calabria); ${ }^{3}$ DICEAM Department, Mediterranean University of Reggio Calabria (Reggio Calabria)

Objective: The differential diagnosis between epileptic seizures (ES) and psychogenic nonepileptic seizures (PNES) may be difficult, due to the lack of reliable and distinctive clinical features. Interictal EEG may also be normal in patients with ES. The gold standard for diagnosis of PNES is the video-EEG recording of a typical episode but this may be difficult to obtain and poses ethical problems. Innovative tools such as non-linear EEG analysis with the use of neural networks could provide important diagnostic support.

Materials and Methods: 18 patients with ES ( 12 males, 6 females) and 18 patients with video-EEG recorded PNES ( 2 males, 16 females). All included subjects had normal interictal EEG. None was taking psychotropic drugs. A two-dimensional convolutional neural network (2DCNN) scheme was utilized to classify the two categories of subjects (ES vs. PNES). The proposed architecture performs a feature engineering step (EEG time-frequency transformation and extraction of multiple features) and a classification step with a 2D-CNN.

Results: The developed 2D-CNN classified the EEG recordings of ES vs. PNES subjects with a fair reliability. Average accuracy was $91.25 \%$ (SD 11.39), average precision 93.75\% (SD 16.53), F-measure $89.88 \%$ (SD 14.20) and recall $90.63 \%$ (SD 17.40\%).

Discussion and Conclusions: These preliminary data on a small sample of subjects encourage the use of these innovative diagnostic techniques. EEG-based neural network learning are promising tools for accurate differential diagnosis between ES and PNES. 


\section{STATINS IN PRIMARY PREVENTION OF POST-STROKE SEIZURES AND EPILEPSY: A SYSTEMATIC REVIEW}

\author{
F. Brigo, F. Rinaldi, B. Nucera, R. Nardone
}

Department of Neurology, Hospital of Merano (SABES-ASDAA) (Merano-BZ)

Objectives: Cerebrovascular disease is the most common cause of seizures in adults and the elderly. So far, no drug is recommended as primary prevention of acute symptomatic post-stroke seizures (ASPSS) or poststroke epilepsy (PSE). This systematic review aimed to evaluate the association between the use of statins after stroke and the risk of developing ASPSS or PSE following cerebral infarct or hemorrhage (primary prevention).

Materials and methods: We included studies evaluating the poststroke use of statins as primary prevention of ASPSS or PSE, irrespective of stroke type. We excluded uncontrolled studies and studies with prestroke statin use. The main outcome included the occurrence of ASPSS or PSE and the effect of statins by type and dose. The odds ratios (ORs) or hazard ratios (HR) with 95\% confidence intervals (CIs) were used as the measures of association between treatment and outcome.

Results: Four studies were included. One study showed a reduced risk of ASPSS after ischemic stroke (OR 0.25; 95\% CI: 0.10-0.59; $\mathrm{p}=0.0016$ ). Three studies consistently reported a reduced risk of PSE after ischemic stroke, and one study a reduced risk of PSE after hemorrhagic stroke (HR 0.62 ; $95 \%$ CI: 0.42-0.90; $\mathrm{p}=0.01$ ).

Discussion and conclusions: Data from the literature suggest an association between statin use and a reduced risk of ASPSS after ischemic stroke, and a reduced risk of PSE after ischemic and hemorrhagic stroke. Although the certainty of the evidence is low, these findings appear promising and worthy of further investigation.

\section{WORKING RESTRICTIONS AND DISABILITY BENEFITS IN PATIENTS WITH PSYCHOGENIC NONEPILEPTIC SEIZURES: AN ITALIAN SURVEY}

F. Brigo, F. Rinaldi, B. Nucera, R. Nardone

Department of Neurology, Hospital of Merano (SABES-ASDAA) (Merano-BZ)

Objectives: To investigate the opinions and attitudes of Italian healthcare providers managing patients with psychogenic non-epileptic seizures (PNES) on working restrictions and disability benefits eligibility.

Materials and methods: Online survey of neurologists/ psychiatrists from Italy, members of the Italian Chapter of the International League Against Epilepsy (LICE), as part of an international study on this topic.

Results: 89 physicians participated in the study (62.9\% women; age: $47 \pm 12$ years; $97.8 \%$ neurologists; years in clinical practice: median: 15 , range: $0.5-54$ years). $52.8 \%$ of participants reported having seen and managed 20 to 100 PNES patients in their lifetime; $27 \%$ less than 20 ; $11.2 \%$ between 100 and 200; and $9 \%$ more than 200 patients. A minority (14.6\%) of responders thought that all patients with PNES should be counselled to pursue any type of work they would like, without any working restriction. Among them, 50\% reported to not be aware of any scientific evidence that patients with PNES are at increased risk of difficulties with any work. $12.4 \%$ of participants reported that patients with PNES should be counselled to avoid performing any type of work as long as they have active PNES. Among responders, the length of time being seizure-free before being considered for work permission varied between 3 (21.9\%), 6 (28.1\%), and 12 months (18.8\%). 46.1\% of participants thought that patients with PNES should be qualified for disability benefits; among them, 95.5\% thought that PNES patients should receive these benefits lifelong.

Discussion and conclusions: Italian neurologists and psychiatrists have different attitudes and opinions towards working restrictions and disability benefits for patients with PNES. Further studies should investigate the reasons for these differences, and propose solutions to avoid discrimination and unequal access to employment and disability benefits.

\section{EPILEPSY COURSE DURING COVID-19 PANDEMIC IN THREE ITALIAN EPILEPSY CENTERS}

C. Cabona ${ }^{1}$, F. Deleo $^{2}$, L. Marinelli ${ }^{1,3}$, D. Audenino ${ }^{4}$, D. Arnaldi ${ }^{3}$, F. Rossi $^{5}$, R. Di Giacomo ${ }^{2}$, C. Buffoni ${ }^{6}$, G. J. Rosa ${ }^{7}$, G. Didato ${ }^{2}$, E. Arboscello $^{5}$, M. de Curtis ${ }^{2}$, F. Villani ${ }^{1}$

${ }^{1}$ Division of Clinical Neurophysiology and Epilepsy Center, IRCCS San Martino Hospital (Genova); ${ }^{2}$ Epilepsy Unit, Fondazione IRCCS Neurologic Institute C. Besta (Milano); ${ }^{3}$ Dept. of Neuroscience (DINOGMI), Univesity of Genoa (Genova); ${ }^{4}$ Neurology Unit, E.O. Galliera Hospital (Genova); ${ }^{5}$ Emergency Dept., IRCCS San Martino Hospital (Genova); ${ }^{6}$ Anesthesia and Intensive Care, IRCCS San Martino Hospital (Genova); ${ }^{7}$ Epilepsy Center, AOU Sant'Anna di Cona (Ferrara)

During epidemic outbreaks, epilepsy course can be modified by different physical and psychological stressors and, most importantly, by irregular therapy intake. The effect of COVID-19 and quarantine isolation on the course of epilepsy and on incidence of new-onset seizures is still unclear. With the aim of managing epilepsy in quarantined patients, three Italian Epilepsy Centers set up telephone consultations using a semi-structured interview, allowing a prospective collection of data on seizure course and other seizure-related problems during pandemic. The collected data on seizure course were compared with the analogous period of 2019. The level of patients' concern relating to the COVID-19 pandemic was also assessed using a numeric rating scale. To address the effect of pandemic COVID-19 on seizure incidence, data collection included the number of consultations for first seizures, relapse seizures, and status epilepticus (SE) in the emergency department of one of the participating centers. Clinical telephone interviews suggest the absence of quarantine effect on epilepsy course in our cohort. No differences in incidence of emergency consultations for seizures over a two-month period were also observed compared to a control period. As demonstrated in other infective outbreaks, good AED supplying, precise information and reassurance are the most important factors in chronic conditions to minimize psychological and physical stress, and to avoid unplanned treatment interruptions.

\section{CENOBAMATE IS A NOVEL ANTI-EPILEPTIC DRUG WITH A UNIQUE, DUAL, COMPLEMENTARY MECHANISMS OF ACTION}

G. Camattari ${ }^{1}$, E. Alvarez-Baron ${ }^{2}$, R. Roeloffs ${ }^{3}$, I. Lombardo ${ }^{2}$

${ }^{1}$ Arvelle Therapeutics Netherlands BV, Italian Branch (Milano); ${ }^{2}$ Arvelle Therapeutics International $\mathrm{GmbH}(\mathrm{Zug}-\mathrm{CH}) ;{ }^{3}$ Arvelle Therapeutics Inc. (New York-USA) 
Objectives: Cenobamate is a novel antiseizure medication (ASM) recently approved in the United States. However, its mechanism of action (MoA) has been only partially described. Here we present data supporting cenobamate's dual MoA of increasing $\mathrm{GABA}_{\mathrm{A}}$-receptor-mediated inhibitory currents and preferentially blocking persistent sodium excitatory currents.

Materials: Relative activities on human $\mathrm{GABA}_{\mathrm{A}}$ receptor subtypes were studied on six human $\mathrm{GABA}_{\mathrm{A}}$ ion channel subtypes expressed in heterologous cells. Potentiation of GABA-induced currents and effects on both phasic/tonic $\mathrm{GABA}_{\mathrm{A}}$ currents were assessed in rat hippocampal CA3 neurons, dentate gyrus granule cells (DGGC), and mouse/rat hippocampal CA1 neurons.

Methods: Cenobamate was tested in models of radioligand binding displacement to assess its binding on $\mathrm{GABA}_{\mathrm{A}}$ receptors. Conventional whole-cell patch clamp assays were used to obtain electrophysiological recordings.

Results: Cenobamate enhanced the current induced by $1 \mu \mathrm{M}$ GABA in a concentration-dependent manner, demonstrating positive modulation of $\mathrm{GABA}_{\mathrm{A}}$ receptors. Enhancement of $\mathrm{GABA}_{\mathrm{A}}$ receptor-mediated inhibitory currents occurred in both the phasic and tonic modalities in rodent hippocampal neurons. In addition to its modulation of several properties of voltage-gated $\mathrm{Na}+$ channels, cenobamate acts as a preferential $\mathrm{I}_{\mathrm{NaP}}$ inhibitor in neuronal voltage-gated $\mathrm{Na}+$ channels to exert its anti-epileptic efficacy.

Discussion: Most current ASMs either decrease neuronal excitation or increase neuronal inhibition. Cenobamate impacts both: it acts as a positive allosteric modulator of the $\mathrm{GABA}_{\mathrm{A}}$ receptor, binding to a site distinct from benzodiazepines, and preferentially blocks persistent sodium currents, enhancing the inactivated state of voltage-gated sodium channels.

Conclusion: The complementary mechanism of action of cenobamate might be a key contributor to the high rates of responders shown during the placebo-controlled clinical trials.

\section{ELECTROENCEPHALOGRAPHIC PATTERNS IN THE EVOLUTION OF CREUTZFELDT-JACOB DISEASE: A RETROSPECTIVE ANALYSIS}

\author{
A. Castelli, F. Placidi, C. Liguori, A. Pagano, L. Mari, N. Manfredi, N. \\ Mercuri, F. Izzi
}

Epilepsy Unit, University of Rome "Tor Vergata" (Roma)

Objectives: Creutzfeldt-Jacob disease (CJD) is a spongiform encephalopathy most commonly presenting as subacute cognitive impairment. Although electroencephalographic features, especially in the early stages of the disease, may not be specific, periodic sharp wave complexes (PSWC) are accounted among the major diagnostic criteria of CJD. The aim of the analysis is to describe the evolution of electroencephalographic features along the course of disease in a cohort of patients, as well as their relationship with the main clinical sub-phenotypes.

Methods: We conducted a retrospective study on patients diagnosed with CJD from 2015 to 2020 at the University Policlinic of Rome "Tor Vergata". We evaluated EEG, neuroradiological features, clinical presentation and disease duration.

Results: The analysis included 13 patients ( 7 women, 6 men, mean age $69 \pm 9.1$ years). Ten patients were diagnosed with sporadic CJD (sCJD, 2 with classical, 3 with cognitive, 3 with ataxic and 2 with psychiatric presentation); 3 patients had familial prion disease (fCJD, 2 with V210I, 1 with V180I mutation). At disease onset, EEG showed typical PSWC (either generalized or lateralized) in $6 / 13$ patients ( 2 patients with classical sCJD, 1 patient with the cognitive variant, 1 patient with ataxic presentation, and 2 patients with fCJD); non-periodic triphasic waves were identified in $3 / 13$, focal slowing in $3 / 13$, only one patient showed unremarkable EEG. At $6.5 \pm 0.7$ weeks follow-up, all patients presented triphasic waves at EEG (periodic in 8/12, not periodic in 4/12). Typical MRI alterations (cortical and/or basal ganglia DWI signal restriction) were found in 9/11 patients during diagnostic work-up and all patients presented such changes during follow-up. The average survival time was $8.5 \pm 10.9$ months, and it was significantly lower in the 6 patients with earlier PSWC occurrence, compared to patients without PSWC at the first EEG ( $2.5 \pm 0.83$ vs $17.5 \pm 13.4$ months; $\mathrm{p}<.05)$.

Conclusions: the typical PSWC pattern may not be present in the early stage of the disease, especially in non-classical variant of sCJD; earlier occurrence is associated with reduced survival time. No association between EEG pattern and radiological features were found.

References:

- Wieser HG, Schindler K, Zumsteg D. EEG in Creutzfeldt-Jakob disease. Clin Neurophysiol. (2006);117(5):935-51. doi:10.1016/ j.clinph.2005.12

- Ayyappan S, Seneviratne U. Electroencephalographic changes in sporadic Creutzfeldt-Jakob disease and correlation with clinical stages: a retrospective analysis. J Clin Neurophysiol. (2014);31(6):586-93. doi:10.1097/WNP.0000000000000097

- Cambier DM, Kantarci K, Worrell GA, Westmoreland BF, Aksamit AJ. Lateralized and focal clinical, EEG, and FLAIR MRI abnormalities in Creutzfeldt-Jakob disease. Clin Neurophysiol. (2003);114(9):1724-28. doi:10.1016/s1388-2457(03)00109-3

\section{RECURRENT EYELID MYOCLONIA WITH ABSENCE STATUS EPILEPTICUS IN A FEMALE PATIENT WITH A NOVEL NEXMIF MUTATION}

M. C. Cioclu ${ }^{1}$, A. Coppola ${ }^{2}$, A. Vaudano ${ }^{3}$, G. Giovannini ${ }^{1}$, S. Krithika ${ }^{4}$, M. Iacomino ${ }^{5}$, R. Michelucci ${ }^{6}$, F. Zara ${ }^{5}$, S. Sisodiya ${ }^{4}$, S. Meletti ${ }^{1}$

${ }^{1}$ Department of Biomedical, Metabolic and Neurosciences, University of Modena and Reggio Emilia, (Modena); ${ }^{2}$ Epilepsy Center, Department of Neuroscience, Odontostomatological and Reproductive Sciences, Federico II University (Napoli); ${ }^{3}$ Department of Neurology, University Hospital (Modena); ${ }^{4}$ Department of Clinical and Experimental Epilepsy, UCL Queen Square Institute of Neurology (London-UK); ${ }^{5}$ Laboratory of Neurogenetics and Neuroscience, IRRCS Gaslini Institute (Genova); ${ }^{6}$ IRCCS Institute of Neurological Sciences, Department of Neurology, Bellaria Hospital (Bologna)

Objectives: To describe the case of a female patient with a novel NEXMIF gene mutation and a peculiar clinical and electroencephalographic picture.

Materials: The patient underwent an extensive metabolic and genetic screening (epilepsy gene panel and whole exome sequencing), besides prolonged interictal and ictal video EEG recording, MRI and EEG-fMRI coregistration.

Methods: A continuous video EEG was recorded during ictal and interictal phases; the patient underwent a full metabolic screening; neuroimaging as well as an EEG-fMRI were performed, evaluating the patient's cortical response to eye-closure respect with healthy controls, according to a previously published protocol. First an epilepsy gene panel and subsequently a whole exome sequencing was completed in the patient and her parents. 
Results: The patient showed mildly delayed developmental milestones and a borderline intellectual functioning (IQ 75). Drug-resistant epilepsy developed from 9 years of age in the form of recurrent episodes of prolonged NCSE, characterized by mydriasis, eyelid myoclonia and reduced responsiveness to environmental stimuli. The EEGs recorded during NCSE showed a continuous, diffuse, poli-spike and wave discharge, worsened by eye closure, hyperventilation and by intermittent photic stimulation. Characteristically eye closure induces the epileptic anomalies while these tend to reduce or disappear when the patient is asked to open her eyes/stare or to perform a mental task. EEG-fMRI during the interictal period revealed an altered network related to eyeclosure when compared to healthy controls. Whole exome sequencing identified a novel de novo mutation ([c.2171del]) in the NEXMIF gene, confirmed by Sanger sequencing and classified as likely pathogenic.

Discussion: NEXMIF (neurite extension and migration factor), previously known as KIAA2022, is a gene located on the X chromosome (Xq13.3) that encodes for a nuclear protein with a key role in neurodevelopment. Mutations in this gene are recognized as a cause of $\mathrm{X}$-linked intellectual disability and autism in male patients and, more recently, as a cause of intellectual disability and refractory epilepsy in females. We report another case of a female patient, with a novel NEXMIF mutation, drug-resistant epilepsy and recurrent non convulsive status epilepticus (NCSE) episodes.

Conclusion: We describe a novel NEXMIF mutation in a female patient with drug-resistant epilepsy characterized by recurrent episodes of eyelid myoclonia with absence SE, that may expand the phenotypic spectrum of this condition.

References:

- de Lange IM, Helbig KL, Weckhuysen S, et alDe novo mutations of KIAA2022 in females cause intellectual disability and intractable epilepsy. Journal of Medical Genetics (2016);53:850-58

- Gilbert J., Man H. Y. (2016). The X-linked autism protein KIAA2022/ KIDLIA regulates neurite outgrowth via $\mathrm{N}$-cadherin and deltacatenin signaling. eNeuro 2016 Oct 28;3(5):ENEURO.0238-16.2016

- Vaudano AE, Ruggieri A, Avanzini P, Gessaroli G, Cantalupo G, Coppola A, Sisodiya SM, and Meletti S. Photosensitive epilepsy is associated with reduced inhibition of alpha rhythm generating networks. Brain (2017);140:981-97

\section{ABNORMAL CORTICAL AND SUBCORTICAL STRUCTURE IN JUVENILE MYOCLONIC EPILEPSY DEMONSTRATED WITH ADVANCED MRI ANALYSIS}

G. Demonte ${ }^{1}$, E. Fratto ${ }^{1}$, I. Sammarra ${ }^{1}$, L. Rapisarda ${ }^{1}$, F. Fortunato ${ }^{1}$, M. Caligiuri $^{2}$, M. Trimboli ${ }^{1}$, A. Labate ${ }^{1}$, A. Gambardella ${ }^{1}$

${ }^{1}$ Institute of Neurology, "Magna Graecia" University (Catanzaro); ${ }^{2}$ Neuroscience Research Center, "Magna Graecia" University (Catanzaro)

Introduction: Genetic generalized epilepsies (GGEs) are traditionally defined as a group of heterogeneous epileptic conditions characterized by generalized spike and wave discharges on electroencephalography (EEG) occurring at over $2.5 \mathrm{~Hz}$ with normal intellect and brain imaging, for which complex genetic inheritance is highly suspected [1]. Juvenile myoclonic epilepsy (JME) is one of the four subsyndromes recognized in GGEs. This clinical condition typically occurs during adolescence and presents with prominent myoclonic seizures, which can be intermixed with generalized tonic-clonic seizures and even absence seizures. The aim of this study was to investigate accurately the presence of brain structural abnormalities in a group of patients with JME using advanced MRI analysis.

Materials and methods: We enrolled 16 consecutive patients $(9$ women, mean age: $30.2+10.4$ years) with JME, and 16 age and sex matched healthy controls. All subjects underwent MRI using standard protocol that included T1-3D TFE (Turbo Field Echo) images with $1 \mathrm{~mm}$ thickness, in order to measure surface and thickness of several cortical Regions of Interest (ROIs) and volume of several subcortical ROIs. The volumes of deep subcortical GM structures were extracted and vertex-wise shape analysis was performed using standard Freesurfer analysis.

Results: Our study analysed 158 quantitative parameters. Notably, 13/ 158 variables presented significative differences between the two groups. In patients with JME, we found bilateral volumetric reduction of thalami, putamina, nuclei caudati and nuclei accumbentes, associated with a bilateral increase of volume in globi pallidi. Moreover, it was found an increased surface of left frontal pole, a decreased surface of right pars opercularis and a reduced thickness of left medial orbitofrontal cortex.

Discussion and Conclusion: These findings indicate a structural cortical and subcortical abnormality in JME, with main involvement of mesiofrontal structures. We believe that these structural abnormalities are involved in the epileptogenetic mechanisms underlying JME. Previous studies already illustrated dysregulation of fronto-thalamic network, that is considered a causative mechanism underlying seizures, due to the absence of thalamic inhibitory inputs towards cortical structures [2]. Our data also showed significative volumetric differences in basal ganglia, whose modulatory role on cortical discharge has been recently advocated [3]. In this way, our findings expand the knowledge of JME and open new perspectives about the potential correlation among structural abnormalities, different genetic profiles and epileptogenesis in patients affected.

References:

1. Mullen SA, Berkovic SF, the ILAE Genetics Commission Genetic generalized epilepsies. Epilepsia (2018);59:1148-53

2. O’Muircheartaigh J., Vollmar C., Barker G.J. et al. Abnormal thalamocortical structural and functional connectivity in juvenile myoclonic epilepsy. Brain (2012);135:3635-44

3. Vuong J., Devergnas A. The role of the basal ganglia in the control of seizure. J. Neural Transm. (2018);125(Suppl.3):531-45

\section{LATERALIZED PERIODIC DISCHARGES IN APHASIC STATUS EPILEPTICUS: TREATMENT STRATEGIES AND CLINICAL OUTCOMES}

F. Dono ${ }^{1}$, D. Rodorigo ${ }^{1}$, C. Carrarini ${ }^{1}$, M. Russo ${ }^{1}$, L. Bonanni ${ }^{1}, \mathrm{~F}$. Anzellotti ${ }^{2}$, C. Vollono ${ }^{3}$

${ }^{1}$ Department of Neuroscience, Imaging and Clinical Science, University "G. D'Annunzio" (Chieti); ${ }^{2}$ Epilepsy Center, "SS Annunziata" University Hospital (Chieti); ${ }^{3}$ Department of Geriatrics, Neurosciences \& Orthopedics Unit of Neurophysiopathology and Sleep Medicine, IRCCS Policlinico Universitario Agostino Gemelli Catholic University (Roma)

Purpose: Language disturbances can be found in various pathological acute conditions that involve the dominant frontal and temporal lobes. The occurrence of prolonged aphasia as the only manifestation of focal status epilepticus is rarely described and only a few cases have been 
documented. Several EEG patterns have been associated with Aphasic Status Epilepticus (ASE) including Lateralized Periodic Discharges (LPDs). The LPDs pattern usually correlates with structural lesions of cortical or subcortical areas triggered by pathological conditions such as acute stroke, brain tumors, infections, traumas, or metabolic diseases. The origin of LPDs is controversial as well as whether they correlate with either ictal or inter-ictal patterns.

Method: In the study, we describe ten cases of ASE associated with LPDs. ASE was defined according to the Rosenbaum's criteria modified by Grimes \& Guberman. All these patients underwent 21 derivations EEG recordings in line with the 10-20 international system, 3T Magnetic Resonance Imaging (MRI) of the brain and were tested with Aphasia Rapid Test (ART). A systematic review of the literature was performed by searching the terms "Aphasic Status Epilepticus" and "Lateralized Periodic Discharges" on PubMed, Medline, Scopus, and Google Scholar. The search returned 10 articles and 17 cases that were the object of analysis.

Results: Our data indicate a strong electro-clinical correlation between ASE and LPDs. The most consistent features of the correlation are 1) higher frequency of LPDs and 2) the presence of "plus modifier". High doses of antiepileptic drug (AEDs) have been associated with better outcomes compared to general anesthesia. Time to recovery was estimated to be 6.75 days after treatment introduction.

Conclusions: Taking into consideration focal SE in patients presenting aphasia and LPDs is of critical importance. High doses of AEDs for 7 days is recommended and associated with good clinical outcomes.

References:

- L.J. Hirsch et al. American Clinical Neurophysiology Society's Standarized Critical Care EEG Terminology: 2012 version. J. of Clin. Neurophy. (2013);30(1):1-27

- M. Leitinger et al. Salzburg Consensus Criteria for Non-Convulsive Status Epilepticus- approach to clinical application. Epilepsy and Behac (2015); 49:158-63

- A. R. Ruiz et al. Association of periodic and Rythmic Electroencephalographic Patterns with Seizure in Critically Ill Patients. JAMA Neurology (2017); 74(2):181-188

\section{EPILEPTIC SEIZURES IN A COHORT OF MULTIPLE SCLEROSIS PATIENTS: EPIPHENOMENON OR CASUAL FACTOR?}

G. Falcicchio, P. Iaffaldano, L. Bollo, T. Francavilla, N. Pilolli, A. Alicino, M. Trojano, A. La Neve

Department of Basic Medical Sciences, Neurosciences and Sense Organs, University of Bari Aldo Moro (Bari)

Background: The prevalence of epileptic seizures (ESs) in multiple sclerosis (MS) patients is higher than in general population, even though the association between these two chronic conditions is not fully understood.

Materials and methods: We retrospectively identified and described patients with both MS and ESs seen between 2018 and 2020 at the Epilepsy Centre of the University of Bari.

Results: Of the 1,800 patients followed by our Centre 17 individuals (F/M 3:1) with at least one ES and defined MS were identified. Median age was 31 years (range 1-52) at first seizure and 27 years (range 1-40) at MS onset. 10 of these 17 subjects (59\%) met the criteria for epilepsy diagnosis. The remaining 7 patients $(41 \%)$ experienced ESs with clinical and instrumental characteristics resembling acute symptomatic seizures (ASSs). The most common MS subtype was relapsing-remitting (RRMS) in 15 patients $(88 \%)$. In 11 cases $(64 \%)$ seizures followed MS onset with a mean time interval of 10 years (range 1-19); 3 patients (18\% of cases) presented seizures as the first clinical manifestation of MS; in 3 cases (18\%) ESs preceded MS onset with a mean time interval of 13 years (range 9-22). The most frequent seizure type was focal to bilateral tonic-clonic ( 9 patients), followed by focal with impaired awareness ( 8 cases). 14 patients $(82 \%)$ assumed anti-epileptic treatment with levetiracetam as the most used drug in mono or polytherapy. 13 patients $(76 \%)$ were seizure-free for a median value of 8 years at last follow-up (range 0 15). Only 4 patients (24\%) were drug resistant.

Discussion: In our cohort, the seizure recurrence after the first crisis did not seem to depend on the MS subtype, which was RRMS at that time. In the majority of cases MS onset preceded the ESs, indicating that the crisis may be the consequence of a chronic brain insult. Focal seizures were the most frequent type supporting the fact that cortical lesions of MS may play a role in precipitating ESs.

Conclusion: Every ES experienced by an MS patient should be categorized as ASS or unprovoked seizure. Although the identification of an ASS requires a brain imaging showing active lesions or a suggestive electroencephalogram, this diagnosis has a better prognosis than the epilepsy one.

References:

- Schorner A., Weissert R. Patients with Epileptic Seizures and Multiple Sclerosis in a Multiple Sclerosis Center in Southern Germany Between 2003-2015. Front. Neurol. (2019); 10:613

- R. Uribe-San-Martìn et al. Prevalence of epilepsy in a cohort of patients with multiple sclerosis. Seizure (2014);23:81-83

- Allen et al. Association between multiple sclerosis and epilepsy: large population-based record-linkage studies. BMC Neurology (2013);13:189

\section{PARIETO-PREMOTOR FUNCTIONAL CONNECTIVITY DURING PARIETAL LOBE SEIZURES WITH MOTOR SEMIOLOGY}

\author{
D. Fonti ${ }^{1}$, S. Lagarde ${ }^{2}$, W. Aboubakr ${ }^{3}$, F. Pizzo ${ }^{2}$, G. Defazio ${ }^{4}$, M. \\ Puligheddu ${ }^{4}$, F. Bartolomei ${ }^{2}$
}

${ }^{1}$ Department of Biomedical Sciences, University of Cagliari (Cagliari); ${ }^{2}$ Epileptology Department, Timone Hospital, Inst Neurosci Syst, AixMarseille University, APHM, INSERM (Marseille-F); ${ }^{3}$ Inst Neurosci Syst, Aix-Marseille University, INSERM (Marseille-F); ${ }^{4}$ Department of Medical Sciences and Public Health, Neurology Section, University of Cagliari (Cagliari)

Background: Parietal lobe epilepsies (PLE) are rare, but probably underestimated due to misleading clinical and electrical features, attributed to high connectivity of the parietal cortex with other cortices [1]. Actually, some of the clinical manifestations observed in parietal lobe seizures (PLS), as like motor signs, are often due to the extension of discharges to extra-parietal areas. The main aim of this work was to explore the relation between motor semiology and functional connectivity (FC) changes during parietal lobe seizures.

Materials and methods: We retrospectively selected patients affected by drug-resistant epilepsy who underwent Stereoelectroencephalography (SEEG) for pre-surgical evaluation at the epileptology department of the Timone Hospital, Marseille, France. All had a seizure onset zone (SOZ) in the parietal cortex. SOZ was defined visually and quantified by the epileptogenicity index (EI) method [2]. For each patient included in the study it was selectioned one representative spontaneous seizure recorded during video-SEEG monitoring. According to ictal semiology it has been created two groups of seizures: "motor seizures" and "non-motor 
seizures". Interdependencies between bipolar SEEG signals were estimated by pairwise nonlinear regression analysis based on the $\mathrm{h}^{2}$ coefficient computed using the Anywave software [3]. For studing the FC changes between parietal, frontal and temporal regions, for each patient, z- score values of 16 cortico-cortical interaction have been obtained comparing $\mathrm{h}^{2}$ coefficients of pre-ictal, seizure onset and discharges propagation periods. Estimation statistics was used for assessing statistical differences between "motor" and "non-motor seizures" values.

Results: We included 22 patients, 13 with "motor seizures" and 9 with "non-motor seizures". Z-scores computed between the onset and the propagation of discharges were significantly higher in "motor seizures", between lateral pre-motor area and precuneus $(\mathrm{p}=0,01)$, lateral pre-motor area and superior parietal lobule $(\mathrm{p}=0.03)$ and between inferior parietal lobule and supplementary motor area (SMA) $(\mathrm{p}=0.04)$.

Conclusions: Our study show that ictal FC is differently modified according to the presence (or the absence) of motor semiology in PLS, in particular "motor" PLS show an increased connectivity between parietal and premotor areas during the propagation of discharges. The parietal lobe, overall in its posterior part, is anatomically and functionally largely connected with lateral pre-motor area in motor planification network, as well as with SMA and temporal areas. Our results indicate that preferential routes of coupling are responsible of the prominent motor presentation during PLS propagation, a presentation that can be misleading for localization.

References:

1. Ristić, A. J., Alexopoulos, A. V., So, N., et al. Parietal lobe epilepsy: The great imitator among focal epilepsies. Epileptic Disorders (2012);14(1):22-31

2. Bartolomei F, Chauvel P., \& Wendling F. Epileptogenicity of brain structures in human temporal lobe epilepsy: A quantified study from intracerebral EEG. Brain (2008); 131(7): 1818-30

3. Colombet, B., Woodman, M., Badier, J. M., Bénar, C. G. AnyWave: A cross-platform and modular software for visualizing and processing electrophysiological signals. Journal of Neuroscience Methods (2015);242:118-26

\section{PERAMPANEL AS FIRST ADD-ON CHOICE ON THE TREATMENT OF MESIAL TEMPORAL LOBE EPILEPSY: AN OBSERVATIONAL REAL-LIFE STUDY}

\author{
F. Fortunato ${ }^{1}$, A. Labate ${ }^{1}$, A. Giugno ${ }^{1}$, M. Caligiuri ${ }^{2}$, A. Gambardella ${ }^{1}$ \\ ${ }^{1}$ Institute of Neurology, Magna Graecia University (Catanzaro); \\ ${ }^{2}$ Neuroscience Research Center, Magna Graecia University (Catanzaro)
}

Aim: To evaluate the efficacy of perampanel (PER) [1-2] in patients with a diagnosis of mesial temporal lobe epilepsy (MTLE) taking PER as first add-on option due to inefficacy of first antiepileptic drug (AED), rather than late add-on choice. [3]

Materials and Methods: 37 MTLE patients aged $\geq 12$ years were recruited consecutively with a minimum duration of follow up of one year and intermediate follow up of 3 months. Patients were divided into two groups: 20/37 taking PER as first add-on due to inefficacy of first AED (first group) and 17/37 taking PER as late add-on due to inefficacy of $\geq$ two AEDs (second group). Efficacy, retention rate and safety were evaluated.

Results: At three months, the $70 \%$ of the first group had a reduction $>50 \%$ of seizure frequency, with six patients becoming also seizure free, while in the second group only the $23.5 \%$ had a reduction $>50 \%$ of seizure frequency and none became seizure free $(\mathrm{p}=0.005)$. Six patients of first group were also switched to a monotherapy of PER and five out of six remained seizure free at 12 months. At one year of follow up efficacy of PER was $70 \%$ for the first group, while only of $29.4 \%$ for the second group $(\mathrm{p}=0.014)$. Retention rate of first group at three months and one year was $85 \%$, while for the second group was, respectively, $82.3 \%$ and $64.7 \%$.

Discussion: We found a very good efficacy of this drug in a specific epileptic syndrome such as MTLE, further supporting the effectiveness of PER in resistant epilepsy mainly TLE with hippocampal sclerosis, the subtype with the higher rates of drug-resistant seizures. The current work further confirms and reinforce that efficacy of PER was significantly higher when PER was used as first option in patients that failed the first AED rather than after many AEDs.

Conclusion: PER was significantly successful and tolerated in MTLE patients when used as first add-on option rather than as late add-on.

References:

1. Hanada T, Hashizume Y, Tokuhara N, Takenaka O, Kohmura N, Ogasawara A, et al. Perampanel: a novel, orally active, noncompetitive AMPA-receptor antagonist that reduces seizure activity in rodent models of epilepsy. Epilepsia (2011);52:1331-40. doi: 10.1111/j.1528-1167.2011.03109

2. Rogawski MA, Hanada T. Preclinical pharmacology of perampanel, a selective non- competitive AMPA receptor antagonist. Acta Neurol Scand Suppl (2013):19-24. doi: 10.1111/ ane. 12100

3. Kim JH, Kim DW, Lee SK, Seo DW, Lee JW, Park HJ et al. First add-on perampanel for focal-onset seizures: an open-label, prospective study. Acta Neurol Scand. (2020) Feb;141(2):132-140. doi: 10.1111/ane.13197. Epub 2019 Dec 4

\section{MILD CASE OF UNVERRICHT-LUNDBORG DISEASE PRESENTING AS JUVENILE MYOCLONIC EPILEPSY}

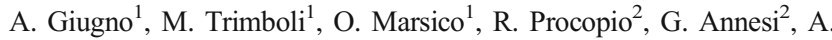 \\ Labate $^{1}$, A. Gambardella ${ }^{1}$
}

${ }^{1}$ Institute of Neurology, Magna Graecia University (Catanzaro); ${ }^{2}$ Institute of Molecular Bioimaging and Physiology, National Research Council, Section of Germaneto (Catanzaro)

Objectives: Juvenile myoclonic epilepsy (JME) and UnverrichtLundborg disease (ULD) are distinct entities, but they share several clinical features: onset usually between 6 and 15 years of age, photosensitivity, myoclonus and generalized tonic-clonic seizures (GTCs). The gene for ULD is the cystatin B (CSTB) gene on chromosome 21q22.3, coding for an inhibitor of cysteine proteases. The most common mutation is a dodecamer repeat, although rare point mutations have also been described1-2. We wish to report a JME phenotype in a small Italian family with ULD.

Materials: A 27-year-old man had one generalized tonic-clonic seizure during sleep at the age of 13 years, and onset of myoclonic jerks on awakening at around the same time, which are well-controlled with valproic acid. He carries a clinical diagnosis of JME. He is now seizure free under valproate. His 37-year-old sister was diagnosed clinically with ULD, with onset of action myoclonus, GTCs, photosensitivity and ataxia, around the age of 12 years. She is treated by clonazepam, valproate, levetiracetam and perampanel. There was no cognitive impairment. The parents were known to be consanguineous. 
Methods: An extensive clinical and laboratory investigation was carried out. Screening for mutations in the CSTB gene was performed in the two patients and their parents.

Results: In the proband, EEG-polygraphic recording revealed generalized spike and waves (GSWs) and photo-paroxysmal-response (PPR), with no myoclonus. Somatosensory evoked potentials (SSEPs) were unremarkable, C-reflex was absent. In her sister, EEG-polygraphic study showed GSWs, marked PPR, involuntary, stimulus and action activated myoclonic jerks. We also depicted giant SSEPs and C-reflex bilaterally. Brain MRI at 3T was normal in both patients. Clinical and EEG evaluations were normal in their parents. In both siblings, screening of CSTB gene detected similar (60 to 68 ) homozygous dodecamer expansions.

Discussion: We have identified a CSTB dodecamer expansion as the genetic cause in a patient with definite JME phenotype. Although ULD is often confused with JME in the early stages of the disease [1-2], it is exceptional to find patients with ULD around age 30 who are as well controlled and high functioning as this patient. It remains unclear the reason why similar size of CSTB dodecamer expansions are associated with extremely heterogenous phenotypes.

Conclusions: Our findings emphasize the mild end of the phenotypic spectrum of CSTB mutations and confirm the phenotypic heterogeneity of ULD. The screening of the CSTB gene should be considered in patients with JME and evidence of recessive transmission.

References:

1. Kälviäinen R., Khyuppenen J., Koskenkorva P., Eriksson K., Vanninen R., Mervaala E. Clinical picture of EPM1-UnverrichtLundborg disease. Epilepsia (2008);49(4):549-56

2. Mumoli L., Tarantino P., Michelucci R., Bianchi A., Labate A., Franceschetti S., Marini C., Striano P., Gagliardi M., Ferlazzo E., Sofia V., Pennese L., Annesi G., Aguglia U., Guerrini R., Zara F., Gambardella A., and on behalf of the Genetic Commission, Italian League Against Epilepsy No evidence of a role for cystatin B gene in juvenile myoclonic epilepsy. Epilepsia (2015); 56(4): e40-e43

\section{NEUROPSYCHOLOGICAL PROFILE OF PATIENTS WITH STRUCTURAL AND MRI-NEGATIVE FOCAL EPILEPSY}

\author{
B. Kassabian, S. Facchini
}

Neurological Clinic, University of Padua (Padova)

Objectives: In literature, data regarding the variables related to cognitive deficits in epilepsy are conflicting and unconclusive [1,2]. Our study evaluates the correlation between anatomical and electro-clinical variables, and the neuropsychological profile of patients with focal epilepsy.

Methods: A retrospective study on 23 patients aged $>18$ years (average age 32.7) with focal epilepsy. Exclusion criteria were: psychogenic seizures, severe psychiatric comorbidities, intellectual disability, epilepsy surgery. The patients underwent neuropsychological assessment, which consisted of a battery of tests proposed by the Italian league aginst epilepsy (L.I.C.E.) [3], neurological examination, EEG/videoEEG and brain MRI.

Results: The duration of disease is directly related to the Stroop Test $(\mathrm{R}=0.51 ; \mathrm{p}<0.05)$ and inversely related to the inverse Digit Span $(\mathrm{R}=-$ $0.48 ; \mathrm{p}<0.05)$. The time for the Stroop Test is related to the age of onset $(\mathrm{R}=-0.81 ; \mathrm{p}<0.05)$. Drug-resistant patients perform worse in the Rey Auditory Verbal Learning Test $(U=29 ; p<0.05)$ and in the delayed recall $(U=29 ; p<0.05)$, in phonemic $(U=28.5 ; p<0.05)$ and semantic $(U=25 ; p$ $<0.05)$ fluences, in the forward $(U=27 ; p<0.05)$ and backward $(U=32.5$; $\mathrm{p}<0.05)$ Corsi block-tapping Test, in the forward $(\mathrm{U}=24.5 ; \mathrm{p}$ $<0.05)$ and backward $(\mathrm{U}=31.5 ; \mathrm{p}<0.05)$ Digit Span, in the Memory with interference $(U=28 ; p<0.05)$, TMT-A $(U=25.5 ; p$ $<0.05)$ and Cipher $(U=23.5 ; p<0.05)$. The group with structural damage to MRI has a worse performance in the Rey Auditory Verbal Learning Test $(U=14.5 ; \mathrm{p}<0.05)$ and in the Semantic Fluence $(U=30.5 ; \mathrm{p}<0.05)$.

Discussion: Our data confirmed the role of drug-resistance on cognitive performances, with a correlation between a long disease duration with high frequency of crisis (focal or generalized) and a worse performance in all the tests. Contrarily to other studies, we found a worse cognitive performance in patients with structural epilepsy.

Conclusions: In our sample, longer disease duration, early onset and drug resistance are associated with poorer cognitive performances. Patients with structural MRI lesions have a worse cognitive performance than those with negative MRI. A wider sample will allow a better definition of the results.

References:

1. Castro et al. Low prevalence but high specificity of materialspecific memory impairment in epilepsy associated with hippocampal sclerosis. Epilepsia (2013);54(10):1735-42. https://doi. org/10.1111/epi.12343

2. Ozkaraet al. Memory in patients with drug-responsive mesial temporal lobe epilepsy and hippocampal sclerosis. Epilepsia (2004);45(11):1392-96. https://doi.org/10.1111/j.0013-9580. 2004.23304.x

3. https://www.lice.it/LICE_ita/gruppi/neuropsicologia/attivita.php? idpadre $=19$

\section{LATE-ONSET EPILEPSY WITH UNKNOWN ETIOLOGY: A PILOT STUDY ON NEUROPSYCHOLOGICAL PROFILE, CEREBROSPINAL FLUID BIOMARKERS, AND QUANTITATIVE EEG CHARACTERISTICS}

E. Nardi Cesarini ${ }^{1}$, C. Babiloni ${ }^{2}$, N. Salvadori ${ }^{1}$, L. Farotti ${ }^{1}$, C. Del Perci ${ }^{2}$, M. Pascarelli ${ }^{3}$, G. Noce ${ }^{4}$, R. Lizio ${ }^{4}$, F. Da Re ${ }^{5}$, V. Isella ${ }^{5}$, L. Tremolizzo ${ }^{5}$, C. Calvello ${ }^{1}$, M. Romoli ${ }^{6}$, J. DiFrancesco $^{5}$, L. Parnetti ${ }^{1}$, C. Costa $^{1}$

${ }^{1}$ Neurology Clinic, University of Perugia, S. Maria della Misericordia Hospital (Perugia); ${ }^{2}$ Department of Physiology and Pharmacology "Vittorio Erspamer", Sapienza University of Rome (Roma); ${ }^{3}$ Institute of Research and Medical Care, (IRCCS) OASI (Troina-EN); ${ }^{4}$ Institute of Research and Medical Care, (IRCCS) SDN (Napoli); ${ }^{5}$ Department of Neurology, San Gerardo Hospital, Milan Center for Neuroscience, School of Medicine and Surgery, University of Milano-Bicocca (Monza); "Neurology Unit, Rimini "Infermi” Hospital, AUSL Romagna (Rimini)

Introduction: Despite epilepsy has been associated with cognitive decline, neuropsychological, neurobiological and neurophysiological features in patients with late-onset epilepsy of unknown etiology (LOEU) are still unknown. This cross-sectional study aims at investigating neuropsychological profile, cerebrospinal fluid (CSF) biomarkers of Alzheimer's disease (AD), and resting-state quantitative electroencephalographic (qEEG) cortical rhythms in LOEU patients with mild cognitive impairment (LOEU-MCI) and with normal cognition (LOEU-CN), compared to non-epileptic MCI (NE-MCI) and cognitively normal (CN) controls.

Methods: Consecutive patients in two clinical Units diagnosed with LOEU-CN (19), LOEU-MCI (27), and NE-MCI (21) were enrolled, and 
compared to age and sex-matched cognitively normal subjects $(\mathrm{CN}, 11)$. Patients underwent standardized comprehensive neuropsychological evaluation and CSF core AD biomarkers assessment (i.e., CSF A $\beta 42$, phospho-tau and total tau, classified through $\mathrm{A} / \mathrm{T} /(\mathrm{N})$ system). Recordings of resting-state eyes-closed electroencephalographic (EEG) rhythms were collected and cortical source estimation of delta $(<4 \mathrm{~Hz})$ to gamma $(>30 \mathrm{~Hz})$ bands with exact Low Resolution Electromagnetic Tomography (eLORETA) was performed.

Results: Most of LOEU patients had MCI status at seizure onset (59\%). Patients with LOEU-MCI performed significantly worse on measures of global cognition, visuo-spatial abilities and executive functions compared to NE-MCI patients $(\mathrm{p}<0.05)$. Regarding MCI subtype, multiple-domain $\mathrm{MCI}$ was 3-fold more frequent in the LOEU-MCI than in the NE-MCI patients (OR 3.14, 95\%CI 0.93-10.58, p=0.06). CSF A 342 levels were lower in the LOEU-MCI compared with the LOEU$\mathrm{CN}$ group. Finally, parietal and occipital sources of alpha $(8-12 \mathrm{~Hz})$ rhythms were less active in the LOEU-MCI than NE-MCI and CN groups, and the opposite was true for frontal and temporal cortical delta sources.

Discussion: MCI status was relatively frequent in LOEU patients, involved multiple cognitive domains, and might have been driven by amyloidosis according to CSF biomarkers. LOEU-MCI status was associated with abnormalities in cortical sources of EEG rhythms related to quiet vigilance. Future longitudinal studies should cross-validate our findings and test the predictive value of CSF and EEG variables.

References:

- Sen A., Capelli V., Husain M. Cognition and dementia in older patients with epilepsy. Brain (2018);141:1592-1608

- Costa C., Romoli M., Liguori C., Farotti L., Eusebi P., Bedetti C., Siliquini S., Nardi Cesarini E., Romigi A., Mercuri N.B., Parnetti L., Calabresi P. Alzheimer's disease and late-onset epilepsy of unknown origin: two faces of beta amyloid pathology. Neurobiol Aging. (2019);73:61-67

- Babiloni C., Carducci F., Lizio R., Vecchio F., Baglieri A., Bernardini S., Cavedo E., Bozzao A., Buttinelli C., Esposito F., Giubilei F., Guizzaro A., Marino S., Montella P., Quattrocchi C.C., Redolfi A., Soricelli A., Tedeschi G., Ferri R., Rossi-Fedele G., Ursini F., Scrascia F., Vernieri F., Pedersen T.J., Hardemark H.G., Rossini P.M., Frisoni G.B. Resting state cortical electroencephalographic rhythms are related to gray matter volume in subjects mild cognitive impairment and Alzheimer's disease. Hum Brain Mapp (2013) Jun;34(6):1427-46

\section{PREDICTIVE FACTORS OF STATUS EPILEPTICUS AND ITS RECURRENCE: A MULTICENTER, LONG FOLLOW-UP COHORT STUDY}

S. Neri ${ }^{1}$, S. Gasparini ${ }^{1}$, E. Ferlazzo ${ }^{1}$, G. Gigli ${ }^{2}$, G. Pauletto ${ }^{3}$, L. Bilo ${ }^{4}$, C. Lettieri $^{2}$, A. Labate ${ }^{1}$, F. Fortunato ${ }^{1}$, A. D'Aniello ${ }^{5}$, G. Di Gennaro ${ }^{5}$, G. D'Orsi $^{6}$, F. Dono ${ }^{7}$, P. Versace ${ }^{8}$, E. Africa ${ }^{8}$, A. Porcelli ${ }^{8}$, L. Frosina ${ }^{8}$, A. Armentano $^{8}$, S. Cavalli ${ }^{1}$, V. Cianci ${ }^{9}$, M. Ascoli ${ }^{1}$, G. Mastroianni ${ }^{9}$, T. Garcea $^{1}$, A. Gambardella ${ }^{1}$, U. Aguglia ${ }^{1}$

\footnotetext{
${ }^{1}$ Medical and Surgical Sciences Department, School of Medicine, Magna Graecia University (Catanzaro); ${ }^{2}$ Neurology Unit, Department of Medicine, University of Udine Medical School (Udine); ${ }^{3}$ Department of Neurosciences, Santa Maria della Misericordia University Hospital (Udine); ${ }^{4}$ Epilepsy Center, University Federico II (Napoli); ${ }^{5}$ IRCCS Neuromed (Pozzilli-IS); ${ }^{6}$ Epilepsy Center, Clinic of Nervous System
}

Diseases, Riuniti Hospital, University of Foggia (Foggia); ${ }^{7}$ Department of Neuroscience, Imaging and Clinical Sciences, University G. d'Annunzio of Chieti-Pescara (Chieti); ${ }^{8}$ Neuroradiology Unit, Great Metropolitan Hospital (Reggio Calabria); ${ }^{9}$ Regional Epilepsy Centre, Great Metropolitan Hospital (Reggio Calabria)

Objective: Status epilepticus (SE) is associated with high short and longterm morbidity and mortality, for which the predictors are poorly defined. This multicenter study aims at identifying the factors associated with the occurrence of SE and the predictors of its recurrence after a first SE.

Methods: We enrolled 468 patients from 11 Italian Centers with at least one SE that were consecutively observed at our center in the period going from 1983 to 2020 (median follow-up 3,16 years); in addition, a randomized selection of 1192 patients with epilepsy diagnosed without SE history was enrolled approximately with a 3.1 ratio. Different clinicaldemographic variables were evaluated and were then included in a univariate/multivariate logistic regression model.

Results: The occurrence of SE was independently correlated with age at onset of SE ( $p<0.001$; OR 1.027; 95\% CI 1.019-1.035), disease duration ( $p$ $<0.001$; OR 1.018; 95\% CI 1.008-1.027); absence of known etiology (p $<0.001$; OR 0.312 ; $95 \%$ CI $0.208-0.470$ ) and number of anti-epileptic drugs taken at the last observation ( $\mathrm{p}=0.03$; OR 1.29 ; 95\% CI 1.03-1.62). Interestingly, the recurrence of SE was negatively correlated to its onset in an acute symptomatic context ( $\mathrm{p}=0.019$; OR 0.22 ; 95\% CI 0.063-0.785).

Discussion and Conclusions: Late onset, long disease duration and the presence of a known etiology predict the occurrence of SE in a multicentric cohort of patients. The occurrence of the first SE in an acute symptomatic context reduces the risk of recurrence.

\section{HOW CEREBRAL CORTEX PROTECTS ITSELF FROM INTERICTAL SPIKES: THE ALPHA/BETA INHIBITION MECHANISM}

G. Pellegrino ${ }^{1}$, E. Kobayashi ${ }^{2}$

${ }^{1}$ S. Camillo IRCCS Hospital and Research Center (Venezia); ${ }^{2}$ Montreal Neurological Institute, McGill University (Montreal-CDN)

Objective: Interactions between interictal epileptiform discharges (IEDs) and distant cortical regions subserve potential effects on cognition of patients with focal epilepsy. We hypothesize that 'healthy' brain areas at a distance from the epileptic focus may respond to the interference of IEDs by generating inhibitory alpha and beta oscillations. We predict that more prominent alpha-beta oscillations can be found in patients with less impaired neurocognitive profile.

Methods: We performed a source imaging magnetoencephalography (MEG) study, including 41 focal epilepsy patients: 21 with frontal lobe epilepsy (FLE) and 20 with mesial temporal lobe epilepsy (TLE). We investigated the effect of anterior (i.e., frontal and temporal) IEDs on the oscillatory pattern over posterior head regions. We compared cortical oscillations $(5-80 \mathrm{~Hz})$ temporally linked to 3,749 IEDs (1,945 frontal and 1,803 temporal) versus an equal number of IED-free segments. We correlated results from IED triggered oscillations to global neurocognitive performance.

Results: Only frontal IEDs triggered alpha-beta oscillations over posterior head regions. IEDs with higher amplitude triggered alpha-beta oscillations of higher magnitude. The intensity of posterior head region alpha-beta oscillations significantly correlated with a better neuropsychological profile. 
Discussion and Conclusions: Our study demonstrated that cerebral cortex protects itself from IEDs with generation of inhibitory alpha-beta oscillations at distant cortical regions. The association of more prominent (i.e., long lasting, higher frequency) oscillations with a better cognitive status suggests that this mechanism might play a role in determining the cognitive resilience in patients with FLE.

\section{PREDICTORS OF SURVIVAL IN LAFORA DISEASE: PROGNOSTIC SYSTEMATIC REVIEW AND INDIVIDUAL PARTICIPANT DATA META-ANALYSIS}

\author{
F. Pondrelli ${ }^{1}$, L. Muccioli ${ }^{1}$, L. Licchetta ${ }^{1,2}$, B. Mostacci ${ }^{2}$, C. Zenesini ${ }^{2}$, L. \\ Vignatelli $^{2}$, P. Tinuper ${ }^{1,2}$, F. Bisulli ${ }^{1,2}$
}

${ }^{1}$ DIBINEM Department of Biomedical and Neuromotor Sciences, University of Bologna (Bologna); ${ }^{2}$ IRCCS Institute of Neurological Sciences, Bellaria Hospital (Bologna)

Objective: Lafora disease (LD) is a rare autosomal recessive disorder characterized by myoclonus, epilepsy and mental deterioration. Due to the rarity of the disease, LD natural history has been described only in case reports and small series of patients to date. Here we present a systematic review of all the available cases reported in literature, with the aim to better define LD course and possibly enucleate prognostic factors, in view of the release of specific therapies in the next future.

Materials and Methods: According to PRISMA guidelines, two independent reviewers extracted relevant data from articles selected by using PubMed/MEDLINE and Embase databases. Disagreements were resolved by discussion and, if still unresolved, by a third reviewer. We included in statistical analysis only genetically confirmed LD cases. We performed Kaplan-Meier Estimate to calculate the cumulative timedependent probability to death (or loss of autonomy), univariate and multivariate Cox regression analyses in order to study the association between time to death (or loss of autonomy) and prognostic factors.

Results: of 2091 citations, 70 studies with a total of 262 cases $(232$ families) were identified. Mean age at disease onset was $13,8 \pm 3,5$ years. EPM2A was mutated in 92 families (39,7\%), while EPM2B in $140(60,3 \%)$. Mean disease duration in 64 deceased cases was $8,1 \pm 5,6$ years $(9,5 \pm 9$ years in $21 \mathrm{EPM} 2 \mathrm{~A}$ cases and $7,5 \pm 3,2$ years in $43 \mathrm{EPM} 2 \mathrm{~B}$ cases). Loss of physical/mental autonomy (grade 3 of LD disability scale) occurred after a mean of $6,4 \pm 5,6$ years from onset $(4,8 \pm 4,9$ years for 20 EPM2A cases and 7,1 $\pm 5,7$ years for 45 EPM2B cases). We found no statistically significative differences in terms of overall survival or rate of progression as regards type of mutated gene. Conversely, age at onset $<18$ years, onset with cognitive symptoms and geographic origin from developing countries have been enucleated as negative prognostic factors.

Discussion and Conclusions: Disease duration in LD seems to vary widely even between cases with the same altered gene, suggesting that mutation type could play a major role. Interestingly, we found that averagely $50 \%$ of the patients died within 10 years from onset, while the reminder showed a longer disease duration, in contrast to what is classically reported in literature. Even if a prospective study is still needed to further characterize the disease, here we have described for the first time a large LD cohort and we hypothesized clinical prognostic factors, potentially useful to evaluate outcomes of upcoming therapeutic protocols.

References:

- Franceschetti S et al. Clinical and genetic findings in 26 italian patients with Lafora disease. Epilepsia (2006);47:640-3
- Turnbull J et al. Lafora disease. Epileptic Disorders (2016); 8(Suppl 2):38-62

\section{A RARE CASE OF ALTERNATING HEMIPLEGIA OF CHILDHOOD}

\author{
R. Renna ${ }^{1}$, F. Pagliuca ${ }^{2}$, M. Pagliuca ${ }^{2}$ \\ ${ }^{1}$ UOSC Neurology, Stroke Unit, AORN Cardarelli (Napoli); \\ ${ }^{2}$ Neurological Clinic, L. Vanvitelli University (Napoli); ${ }^{3}$ Centro \\ Emicenter (Casavatore-NA)
}

Background: Alternating Hemiplegia of Childhood (AHC) is a rare neurodevelopmental disease characterized by episodes of hemiplegia and paroxysmal disorders (i.e. abnormal eye-movements, episodes of dystonia, seizures), developmental delay and mental retardation. It is caused by mutations in ATP1A3 gene, encoding the $\alpha 3$-subunit of $\mathrm{Na}+\mathrm{K}+$-ATPase. Mutations in ATP1A2 gene have been reported in less than $1 \%$ of patients.

Case presentation: The patient was born in 1979 from unrelated parents. His familiar history was negative for neurological diseases. Patient's history began with febrile seizures and episodes of alternating hemiplegia lasting from 30 minutes to few hours occurred from 18 months to 4 years of age. Serial electroencephalograms in that period documented epileptic paroxysms with spikes and spike-waves in the central regions of the right hemisphere or in both hemispheres independently, with prevalence on the right side. Electroencephalograms performed at the age of 5 and 6 years documented generalized spike-wave complexes at $3 \mathrm{~Hz}$. Carbamazepine and clobazam were started with benefit. When he was 13 years-old, antiepileptic drugs (AEDs) were reduced and then stopped, as the patient was seizure-free, the episodes of hemiplegia were resolved and electroencephalograms did not show any epileptiform abnormalities. In the next years he presented only occasional brief episodes of hemiplegia. Since patient was 33 years-old, he presented recurrent episodes of NonConvulsive Status Epilepticus (NCSE) and migraine. At neurological exam patient presented mild intellectual disability, no involuntary/ paroxysmal movements, no focal deficit. Brain MRI was normal. AEDs (levetiracetam, carbamazepine and clobazam) were introduced. NCSE episodes resolved after therapy reintroduction, while sporadic episodes of migraine persisted and there were not further episodes of hemiplegia. Genetic study revealed normal karyotype. Whole-exome sequencing revealed a pathogenic missense variant in ATP1A2 gene, that was absent in parents and then de novo in the proband.

Conclusion: AHC, a rare syndrome first reported in 1971, is characterized by early onset of episodic hemi- or quadriplegia.[1] Hemiplegic episodes develop before 18 months of age and involve either side of the body or shifting from one side to the other during the same episode with a period of bilateral weakness.[2] Epileptic seizures are reported in sporadic patients.[2-3] An association with migraine has also been reported. AHC is primarily caused by mutations in ATP1A3 gene, being the ATP1A2 gene involved very rarely. Our case enlarges the clinical phenotype of AHC and underlines the efficacy of AEDs on seizures and hemiplegic episodes, but not on migraine, suggesting different underlying mechanisms.

References:

1. Verret S, Steele JC. Alternating hemiplegia in childhood: a report of eight patients with complicated migraine beginning in infancy. Pediatrics (1971);47:675-80

2. Aicardi J, Bourgeois M, Goutieres F. Alternating hemiplegia of childhood: clinical findings and diagnostic. In: Anderman F, 
Aicardi J, Vigevano F, eds. Alternating hemiplegia of childhood. New York: Raven Press, (1995):3-18

3. Silver K, Andermann F. Alternating hemiplegia of childhood: the natural history of the disorder in a group of 10 patients. In: Anderman F, Aicardi J, Vigevano F. Alternating hemiplegia of childhood. New York: Raven Press, (1995):19-28

\section{FOCAL (LEFT TEMPORAL) NON CONVULSIVE STATUS EPILEPTICUS (NCSE) IN A PATIENT WITH CEREBRAL AMYLOID ANGIOPATHY-RELATED INFLAMMATION (CAARI)}

\author{
R. Rizzi, M. Russo, G. Salomone, J. Rossi, R. Pascarella, M. Zedde, F. \\ Valzania
}

Neurology, ASMN Reggio Emilia, IRCCS AUSL RE (Reggio Emilia)

Purpose: Cerebral Amyloid Angiopathy-Related Inflammation (CAARI) is a recently recognized and probably underestimated encephalopathy seen in a subgroup of patients with Cerebral Amyloid Angiopathy (CAA) in which, the amyloid deposition is accompanied by inflammation or edema. We describe a 64-year-old, right-handed man who develops stroke-suggestive acute Wernicke's aphasia, but later revealed to be symptomatic of focal non convulsive status epilepticus (NCSE), related to a condition of cortical inflammatory infiltration and edema involving the left temporal lobe until the temporo-parietal and temporo-occipital junction, hippocampus and the posterior thalamus included, mimicking autoimmune encephalitis.

Materials and methods: We investigated our patient with brain CT, CT angio and MRI scans, EEG recordings, whole body including brain F-18 FDG PET, neuropsychological evaluation, CSF examination, neuronal (targeting intracellular onconeural antigens and against synaptic receptors and other neuronal cell surface proteins) and anti-beta-Amyloid autoantibodies research, APOE genotyping.

Results: We detected a condition of focal NCSE (EEG: left temporoparietal periodic discharges with a frequency $0.5-1 \mathrm{~Hz}$ ). Brain $\mathrm{CT}$ and angio TC were normal. MRI showed: widespread cortical thickening and hyperintensity in $\mathrm{T} 2$ of the left temporal lobe until the temporoparietal and temporo-occipital junction, hippocampus and the posterior thalamus included; multiple lobar microbleeds. FDG PET displayed left lateral temporal hypometabolism. CSF analysis demonstrated increased protein level with oligoclonal bands in liquor and serum without pleiocitosis and negative search for neoplastic cells and neurotropic viruses including HSV, JCV, SARS-Cov2. The neuronal surface and intracellular autoantibodies research were negative. CSF biomarkers testing showed decrease beta-Amiloyd with elevated tau and P-tau levels. The patient had the apolipoprotein E4/E4 genotype. In progress anti-BetaAmyloid autoantibodies research. We treated our patient with clobazam $30 \mathrm{mg}$ and lacosamide $500 \mathrm{mg} / \mathrm{die}$ in association with metilprednisolone $1 \mathrm{~g} /$ die iv for 5 days followed by oral prednisone $75 \mathrm{mg} /$ die slowly tapered. Resolution of NCSE was documented electroencephalographically within 10 days with improvement of aphasic disorder (without complete recovery) and reduction of the left temporal altered MRI signal area.

Conclusions: Focal NCSE can be a presentation of different clinical conditions. In our case, the features and evolution of MRI, with a better definition of cortical involvement and improvement of damage caused by prolonged seizure activity, together with the negativity for all tested autoantibodies, guided us from the initial suspicion of autoimmune encephalitis to CAARI. Therefore, in case of focal NCSE, CAARI to be considered in the differential diagnosis among the autoimmune encephalopathies. References:
1) Howard S. Kirshner \& Michael Bradshaw, The Inflammatory Form of Cerebral Amyloid Angiopathy or Cerebral Amyloid Angiopathy-Related Inflammation (CAARI), Curr Neurol Neurosci Rep (2015);15(54):1-6

2) Carlo Salvarani et al. Imaging Findings of Cerebral Amyloid Angiopathy, Ab-Related Angiitis (ABRA), and Cerebral Amyloid Angiopathy-Related Inflammation, Medicine (2016);95(20):1-7

3) Auriel E, Charidimou A, Gurol ME, et al., Validation of clinicoradiological criteria for the diagnosis of cerebral amyloid angiopathy-related inflammation. JAMA Neurol. (2016);73(2):197-202

\section{MANAGEMENT AND TREATMENT OF STATUS EPILEPTICUS: A RETROSPECTIVE STUDY}

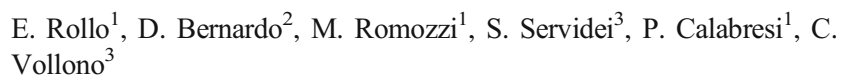

${ }^{1}$ Institute of Neurology, Catholic University of the Sacred Heart (Roma); ${ }^{2}$ Centro Clinico NEMO, Fondazione Policlinico Universitario Agostino Gemelli IRCCS (Roma); ${ }^{3}$ Unit of Neurophysiopathology, Fondazione Policlinico Universitario Agostino Gemelli IRCCS (Roma)

Objectives: Status Epilepticus (SE) is the second most frequent neurological emergency [1]. The aim of the present retrospective study was to determine responsiveness to therapy in a large number of SE episodes treated in our hospital.

Materials: A total of 179 episodes in 143 patients (aged 18 to 97 years old) were assessed. Patient's demographic information and epilepsy history were collected. Data about treatment, complications and outcome were available for all of them.

Methods: We retrospectively assessed the management of SE episodes in our hospital over a 6-year period. The primary outcome was the identification of a drug superiority among all those used, considering as effective the last drug introduced before seizure cessation. The secondary outcome was the identification of possible predictive factors of drug response. Patients were differentiated into two groups ('Anesth + ' and 'Anesth -'), according to Anesthetics administration or not. Clinical, neuroradiological, neurophysiological, Status Epilepticus Severity Score (STESS), Glasgow Coma Scale (GCS) and total number of AEDs used for each SE were compared between groups. A descriptive statistics analysis was conducted. Fisher's Exact Test, contingency table and ChiSquare Test were used to compare drugs effectiveness.

Results: The overall response rates were $54.54 \%, 37.73 \%, 26.26 \%$ and $21.62 \%$ for phenytoin, valproate, levetiracetam and benzodiazepines respectively. Anesthetics, administered in $50.83 \%$ of cases (91/179), stopped SE in $60.43 \%$ of treated episodes even if associated with worse outcome. Comparing the 'Anesth+' and 'Anesth-' groups, age ( $\mathrm{p}>0.05)$, GCS ( $>0.01)$ and total number of AEDs used in each SE $(p>0.01)$ were statistically different; no difference of efficacy was found for what concerns the first drug used in the two groups. In the 'Anesth+' group, all the AEDs were more effective than benzodiazepines ( $p>0.01$ ); comparison between phenytoin and valproate did not reach significant difference. Conversely, phenytoin showed higher efficacy compared to levetiracetam $(p<0.05)$. Finally, in the 'Anesth-' group, there was no difference regarding efficacy between AEDs but phenytoin showed the higher response rate.

Discussion: A recent randomized blinded trial showed similar efficacy and safety for phenytoin, valproate, and levetiracetam for the treatment of 
refractory status epilepticus [2]. In our study the use of such drugs was more effective than benzodiazepines in resolving SE, confirming benzodiazepines inefficacy in subsiding refractory SE. However, in our cohort of SE episodes phenytoin showed higher efficacy compared to levetiracetam.

Conclusions: Our data confirms effectiveness and tolerability of second-line drugs for SE treatment. Their early use may possibly reduce the need for anaesthetics.

References:

1. Hesdorffer DC, Logroscino G, Cascino G, Annegers JF, Hauser WA. Incidence of status epilepticus in Rochester Minnesota, 19651984. Neurology (1998);50:735-41

2. Kapur J, Elm J, Chamberlain JM et al. Randomized Trial of Three Anticonvulsant Medications for Status Epilepticus, New England Journal of Medicine (2019);381(22): 2103-13

\section{JUVENILE MYOCLONIC EPILEPSY PHENOTYPE DUE TO THALAMIC INVOLVEMENT IN WILSON DISEASE}

\author{
J. Rossi ${ }^{1}$, F. Cavallieri ${ }^{2}$, G. Giovannini ${ }^{1}$, A. Vaudano ${ }^{1}$, F. Ferrara ${ }^{3}$, F. \\ Benuzzi $^{1}$, S. Contardi ${ }^{4}$, A. Pietrangelo ${ }^{5}$, E. Corradini ${ }^{5}$, S. Meletti ${ }^{1}$
}

\begin{abstract}
${ }^{1}$ Department of Biomedical, Metabolic, and Neural Sciences, University of Modena and Reggio Emilia (Modena); ${ }^{2}$ Neuromotor \& Rehabilitation Department, Neurology Unit, Azienda USL-IRCCS of Reggio Emilia (Reggio Emilia); ${ }^{3}$ Internal Medicine Unit and Centre for Hemochromatosis and Metabolic Liver Diseases, Policlinico - Azienda Ospedaliero Universitaria di Modena (Modena); ${ }^{4}$ IRCCS Istituto di Scienze Neurologiche di Bologna, Ospedale Maggiore (Bologna); ${ }^{5}$ Department of Medical and Surgical Sciences, University of Modena and Reggio Emilia (Modena)
\end{abstract}

Objectives: Wilson disease (WD) is an autosomal recessive inherited disorder due to mutation of ATP7B, resulting in pathological accumulation of copper in many organs and tissues, leading to liver disease and/or neuropsychiatric disturbances. Frequent neurologic symptoms include movement disorders, frequently associated with dysphagia, dysarthria, and drooling. Seizures are reported in $4.2-7.7 \%$ of patients. There is no evidence about any correlation between Wilson disease and specific epileptic phenotypes, especially Myoclonic Epilepsy (ME).

Materials and methods: A 40-years-old woman with a previous diagnosis of Juvenile Myoclonic Epilepsy (JME) from the adolescence, developed hepatic failure during her first pregnancy. A hepatic biopsy showed an accumulation of copper in the liver, confirming the diagnosis of Wilson disease. A brain MRI showed bilateral thalamic hyperintensities. The patient underwent a more in-depth study using multimodal structural and functional MRI. Specifically, the functional connectivity fMRI study demonstrated at rest an altered pattern of connectivity of the supplementary motor area (SMA) with the rest of the brain.

Discussion and conclusion: In our case, the thalamic involvement due to Wilson disease may support the role of the thalamus in the pathogenesis of a JME phenotype. The altered functional connectivity of the SMA further supports the involvement of the thalamo-frontal network in the JME pathogenesis. Recent computational analyses of multimodal MRIs indeed have disclosed focal brain abnormalities, particularly in the thalamus and supplementary motor area in JME patients, supporting the notion that JME may represent a model of a "network" epilepsy. This is also the first case of Wilson disease associated with a specific epileptic syndrome phenotype.
References:

- P. Ferenci Pathophysiology and Clinical features of Wilson Disease. Metabolic Brain Disease (2004);19:229-39

- L.K. Prashanth, S. Sinha, A.B. Taly, A. Mahadevan, M.K. Vasudev, S. K. Shankar. Spectrum of epilepsy in Wilson's disease with electroencephalographic, MR imaging and pathological correlates. Journal of the Neurological Sciences (2010):44-51

- Kim JH. Grey and white matter alterations in juvenile myoclonic epilepsy: a comprehensive review. J Epilepsy Res. (2017); 7:77-88

\section{IMPAIRED EMBODIED COGNITION IN PATIENTS WITH MESIAL TEMPORAL LOBE EPILEPSY}

I. Sammarra ${ }^{1}$, R. Dalla Volta ${ }^{2}$, O. Marsico ${ }^{1}$, G. Demonte ${ }^{1}$, M. Trimboli ${ }^{1}$, A. Labate ${ }^{1}$, A. Gambardella ${ }^{1}$

${ }^{1}$ Institute of Neurology, Magna Graecia University (Catanzaro); ${ }^{2}$ Department of Medical and Surgical Sciences, Magna Graecia University (Catanzaro)

Aims: Sensorimotor systems, involved in the experiences, are recruited in attributing meaning to words, configuring the so-called embodied cognition [1]. Our aim is to assess whether the processing of graspable objects and their nouns is impaired in patients with mild mesial temporal lobe epilepsy (mMTLE).

Material: Five patients ( 3 women; mean age: $31.4 \pm 9.66$ years) with mMTLE (mean age at onset: $26.4 \pm 9.56$ years) and 10 matched controls were enrolled. In all subjects, a 3 T MRI and a neuropsychological examination were performed, showing no abnormalities. At the time of study, $2 / 5$ patients were taking antiepileptic drug. None had history of language deficits.

Methods: Forty Italian nouns of natural objects and 40 pseudowords (obtained by substituting one consonant and one vowel in two distinct syllables) as well as 40 digital color photos depicting natural objects and 40 scrambled imaged (created by applying distorting graphic filters) were used as stimuli. Twenty nouns and 20 photos were referred to natural graspable objects. The remaining twenty of each type of stimuli were related to natural non-graspable ones. The subjects had to respond when the stimulus concerned a real object and to abstain when it was meaningless (go-no go paradigm) [2]. Mean value of reaction times (RTs) and of errors' number were calculated for each combination of Object Graspability and Stimulus Type.

Results: RTs below $130 \mathrm{~ms}$ or above $1000 \mathrm{~ms}$ were excluded from analysis. In control group, the slower RTs were recorded for stimuli related to graspable objects $(606 \pm 103 \mathrm{~ms})$ compared to non-graspable ones $(566 \pm 109 \mathrm{~ms})$. The mMTLE group had faster RTs for nouns referred to graspable objects $(509 \pm 107 \mathrm{~ms})$ compared to those non-graspable (499 $\pm 98 \mathrm{~ms}$ ). Delayed RTs were recorded for photos concerned graspable objects compared to non-graspable ones both in controls (graspable: $616 \pm 98 \mathrm{~ms}$; non-graspable: $557 \pm 111 \mathrm{~ms}$ ) and in epileptic patients (graspable: $598 \pm 54 \mathrm{~ms}$; non-graspable: $525 \pm 70 \mathrm{~ms}$ ). Mean error number was greater in mMTLE group $(3 \pm 3)$ compared to controls $(2 \pm 1)$.

Discussion: Patients did not show the typical slower RTs related to graspable objects expressed by nouns, appearing less accurate to perform the task. The average of errors was higher in this group. No difference was recorded in RTs for graspable and non-graspable objects' photos between two groups. These findings would indicate that mMTLE patients have troubles in processing graspable objects and their nouns. 
Conclusions: These results give evidence that embodied cognition is impaired in MMTLE patients, which may be indicative of a dysfunction in fronto-parietal sensorimotor systems.

References:

1. Buccino G, Colage'I, Gobbi N, Nicola G, Bonaccorso G. Grounding Meaning in Experience: A Broad Perspective on Embodied Language. Neurosci Biobehav Rev. (2016); 69:69-78

2. Buccino G, Dalla Volta R, Arabia G, Morelli M, Chiriaco C, Lupo A, Silipo F, Quattrone A. Processing graspable object images and their nouns is impaired in Parkinson's disease patients. Cortex (2017);100:32-39

\section{ELECTROENCEPHALOGRAPHIC QUANTITATIVE (QEEG) MODIFICATIONS DURING OVARIAN CYCLE IN PATIENTS WITH CATAMENIAL C1-PATTERN TEMPORAL LOBE EPILEPSY (TLE): A PILOT STUDY}

G. Scorrano ${ }^{1}$, F. Dono ${ }^{1}$, C. Carrarini ${ }^{1}$, M. Russo ${ }^{1}$, M. Di Pietro ${ }^{1}$, M. Onofrj $^{1}$, L. Bonanni ${ }^{2}$, C. Vollono ${ }^{3}$, F. Anzellotti ${ }^{4}$

${ }^{1}$ Department of Neuroscience, Imaging and Clinical Science, University "G. D'Annunzio" (Chieti); ${ }^{2}$ Department of Geriatrics, Neurosciences \& Orthopedics, Unit of Neurophysiopathology and Sleep ${ }^{3}$ Medicine, IRCCS Policlinico Universitario Agostino Gemelli, Catholic University (Roma); ${ }^{4}$ Epilepsy Center, "SS Annunziata" University Hospital (Chieti)

Purpose: The term "catamenial epilepsy" refers to cyclic seizure exacerbation in relation to the ovarian cycle. There are three recognized patterns: perimenstrual $(\mathrm{C} 1)$, peri-ovulatory $(\mathrm{C} 2)$ and entire luteal phase in anovulatory cycles (C3). The prevalence of catamenial epilepsy is estimated to be $70 \%$ in women with epilepsy. Temporal lobe epilepsy (TLE) represents the most common form of epilepsy in the adulthood. Temporal lobe can define a direct influence on the function of the hypothalamic-pituitary axis which is involved in the regulation, production and secretion of sexual steroids. Vice versa, sex steroids can modulate brain activity. The aim of this study is to evaluate the hormone-induced quantitative electroencephalographic (qEEG) modifications in patients with catamenial C1-pattern TLE.

Method: Thirty-five women with diagnosis of TLE were enrolled. All patients presented regular ovary cycle ( $28 \pm 5$ days). According to the presence of catamenial C1-pattern, patients were divided into 2 subgroups: catamenial TLE (17 patients), non-catamenial TLE (18 patients). Each patient underwent a 30-minute 21-electrods EEG recording during menstrual, follicular and luteal phase of the ovarian cycle. A total of 90 epochs of 2 s-long per patient were processed by a specific software (BrainVision Analyzer) performing a fast fourier transform (FFT) on each second of EEG acquisition. The mean power spectrum was divided automatically into four frequency bands: delta $(1-3.9 \mathrm{~Hz})$, theta $(4-7.9 \mathrm{~Hz})$, alpha $(8-12 \mathrm{~Hz})$ and beta $(13-18 \mathrm{~Hz})$.

Result: In the temporal regions, alpha mean power spectrum was significantly lower whereas theta mean power spectrum was significantly higher during menstrual phase compared to follicular and luteal phase.

Conclusion: The reduction of alpha rhythm during $M$ phase can explain the tendency in seizure exacerbation in C1 TLE patients. This qEEG finding can represent a biomarker of $\mathrm{C} 1$ pattern, supporting diagnosis, and aiming to differentiate it from other catamenial patterns.

References:

- Herzog AG. Catamenial epilepsy: Update on prevalence, pathophysiology and treatment from the findings of the NIH Progesterone Treatment Trial. Seizure (2015);28:18-25
- Foldvary-Schaefer N, Falcone T. Catamenial epilepsy: pathophysiology, diagnosis, and management. Neurology (2003);61(6 Suppl 2):S2-S15

\section{EFFICACY AND SAFETY OF CENOBAMATE IN EUROPEAN EPILEPSY PATIENTS WITH UNCONTROLLED FOCAL ONSET SEIZURES}

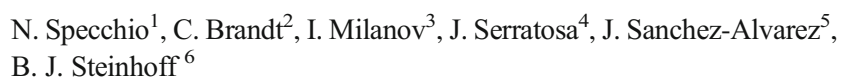

${ }^{1}$ Division of Neurology, Bambino Gesù Children's Hospital IRCCS (Roma); ${ }^{2}$ Department of General Epileptology, Bethel Epilepsy Centre, Mara Hospital (Bielefeld-D); ${ }^{3}$ Neurology Clinic, Medical University of Sofia (Sofia-BG); ${ }^{4}$ Epilepsy Unit, Department of Neurology, Fundación Jiménez Díaz (Madrid-E); ${ }^{5}$ Neurology Service, Hospital Clinico Universitario San Cecilio (Granada-E); ${ }^{6}$ Department for Adults, Kork Epilepsy Center (Kehl-Kork-D)

Objective: There is a need for more effective antiseizure medications (ASMs) since approximately $40 \%$ of patients do not achieve seizure freedom despite treatment with 2 ASMs. Here, we present the results of cenobamate, a novel ASM, in European epilepsy patients with uncontrolled focal onset seizures (FOS).

Materials: Patients were assigned to once-daily adjuvant cenobamate $100 \mathrm{mg}, 200 \mathrm{mg}, 400 \mathrm{mg}$, or placebo. There was a 6 -week titration and a 12-week maintenance phase.

Methods: This was a post-hoc analysis of an international, doubleblind, placebo-controlled trial that enrolled adults with uncontrolled FOS treated with concomitant 1-3 ASMs. Primary European endpoint was responder rate $(\geq 50 \%$ reduction in seizure frequency from baseline) in the maintenance phase; prespecified secondary included seizure freedom (maintenance phase). Safety and tolerability were assessed.

Results: In Europe, 250 patients were enrolled. Median disease duration ranged from $21-28$ years. Responder rates ( $\geq 50 \%$ reduction) during the maintenance phase were $42 \% / 52 \% / 63 \%$ for $100 \mathrm{mg} / 200 \mathrm{mg} / 400 \mathrm{mg}$ cenobamate vs $31 \%$ for placebo. Seizure freedom occurred in $4 \% / 15 \%$ $25 \%$ in patients receiving $100 \mathrm{mg} / 200 \mathrm{mg} / 400 \mathrm{mg}$ vs $2 \%$ for placebo. Overall, the most common AEs $(\geq 10 \%)$ were somnolence, dizziness, headache, fatigue, and diplopia. Efficacy and safety were consistent with the overall study population.

Discussion: Complete seizure control is the ultimate goal of therapy, but the probability of achieving seizure-freedom diminished sharply with each treatment failed. Adjunctive treatment with cenobamate showed significantly higher percentage of responders compared with placebo, including $100 \%$ responders.

Conclusion: Cenobamate is a novel ASM with the potential of improving outcomes for FOS patients with uncontrolled epilepsy. Results in European epilepsy patients were consistent with the overall patient population.

\section{SAFETY OF CENOBAMATE AS ADJUNCTIVE TREATMENT FOR UNCONTROLLED FOCAL SEIZURES: RESULTS FROM A LARGE, INTERNATIONAL, OPEN-LABEL SAFETY STUDY}

N. Specchio ${ }^{1}$, J. Sanchez-Alvarez ${ }^{2}$, D. Fabo ${ }^{3}$, E. Santamarina ${ }^{4}$, I. Milanov $^{5}$, Y. Winter ${ }^{6}$, C. Brandt ${ }^{7}$ 
${ }^{1}$ Division of Neurology, Bambino Gesù Children's Hospital IRCCS (Roma); ${ }^{2}$ Neurology Service, Hospital Clinico Universitario San Cecilio (Granada-E); ${ }^{3}$ National Institute of Clinical Neuroscience Budapest-H); ${ }^{4}$ Epilepsy Unit, Department of Neurology, Vall d'Hebron University Hospital (Barcelona-E); ${ }^{5}$ Neurology Clinic, Medical University of Sofia (Sofia-BG); ${ }^{6}$ Mainz Comprehensive Epilepsy Center, Johannes Gutenberg-University (Mainz-D); ${ }^{7}$ Department of General Epileptology, Bethel Epilepsy Centre, Mara Hospital (Bielefeld-D)

Objective: Cenobamate is a novel antiseizure medication (ASM) with a unique, complementary, dual mechanism of action, which has shown a significant seizure frequency reduction, including seizure freedom, in 2 well-controlled studies. Among the first 953 adults exposed to cenobamate, three confirmed cases of drug reaction with eosinophilia and systemic symptoms (DRESS) occurred. This study was designed to assess whether a slower titration and lower starting dose would reduce the incidence of DRESS.

Materials: Increasing daily doses of cenobamate were administered $(12.5,25,50,100,150$, and $200 \mathrm{mg})$ at 2-week intervals. Further increases to a maximum dose of $400 \mathrm{mg}$ /day by increments every other week were allowed.

Methods: This ongoing, open-label study (NCT 02535091) enrolled epilepsy patients 18-70 years old with uncontrolled focal onset seizures taking stable doses of 1-3 ASMs. A key objective was to assess the rate of DRESS after 6 months. Hypersensitivity reactions were reviewed monthly. This analysis used a data cutoff of April 23, 2018.

Results: 1,339 patients were dosed (2,192 patients/year; July 2019 data cut-off). No cases of DRESS occurred. The most frequent AEs (incidence $\geq 10 \%$ ) were somnolence (30.8\%), dizziness (26.8\%), fatigue $(18.8 \%)$ and headache $(15.5 \%)$. Serious AEs occurred in $14.2 \%$ of patients, severe AEs in $10.2 \%$ and TEAEs leading to discontinuation in $13.1 \%$.

Discussion: Long-term treatment with adjunctive cenobamate was generally safe and well tolerated, with the most common TEAEs being CNS-related. No cases of DRESS were identified in 1340 patients exposed to cenobamate initiated at $12.5 \mathrm{mg}$ and titrated every 2 weeks.

Conclusions: This study shows preliminary evidence that reducing the starting dose and slowing the titration rate of cenobamate to $2 \mathrm{w}$ intervals might mitigate the risk of DRESS.

\section{SEIZURES AND AUTOIMMUNITY: WHICH PATIENTS WITH AUTOIMMUNE ENCEPHALITIS AND SEIZURES SHOULD BE CONSIDERED EPILEPTIC?}

\section{Tontini, C. Mutti, C. Bandoni, I. Florindo, L. Zinno \\ Neurosciences Unit, Department of Medicine and Surgery, University of Parma (Parma)}

Objectives: Seizures are among the most common manifestations of autoimmune encephalitis (AE). However, the extent to which the activation of the immune system truly plays a role in epileptogenesis has not been clarified yet. To shed light on the relationship between epilepsy and AE, the International League Against Epilepsy (ILAE) has recently proposed to differentiate two diagnostic entities: acute symptomatic seizures secondary to AE, and autoimmune-associated epilepsy; only the latter is linked with an enduring predisposition to seizures. Our aim has been to explore the prevalence and clinical characteristics of seizures associated to AE. We tried to classify collected cases with respect to the recently proposed ILAE classification.
Materials and methods: We have retrospectively collected data regarding adult patients with diagnosis of AE admitted to the A.O.U. of Parma between 01/15/2015 and 10/15/2019: among the 13 patients collected, 10 of them $(77 \%)$ suffered from epileptic manifestations and were included in the survey. We collated clinical and electroencephalographic data during the acute phase of the disease and at the 3-12-months follow-up.

Results: Among the 10 patients, different types of antibodies were found: against intracellular agents (anti-Yo, anti-Ma2/Ta), against synaptic receptor (anti-NMDA) and against ion-channels (anti-LGI1). We also found autoantibody-negative $\mathrm{AE}(5 / 10,50 \%)$ and a case of Hashimoto encephalopathy. All the patients were treated with immunotherapy and antiepileptic drugs (AEDs). At the 3-months follow-up, the vast majority of patients was seizure-free with a significant EEG improvement; 2 of them were still assuming immunotherapy; 4 patients started AEDs withdrawal. One patient with antibody-negative EA was still suffering from focal seizure, and he kept assuming immunotherapy and AEDs. A second follow-up at 12 months was performed for 6 patients: 5 out of 6 were seizure-free, so one patient stopped AEDs, while the patient with limbic AE clinically worsened and required addition to a second-line immunotherapy.

Discussion and conclusion: According to the new ILAE classification, one of the patient in our cohort was affected by acute symptomatic seizures secondary to AE. Another patient, showing limbic AE, was affected by autoimmune-associated epilepsy, requiring both AEDs and immunotherapy to achieve seizure control. The other 8 cases are seizure-free but hadn't discontinued medical therapy, so they cannot be classified yet. Further studies are required to explore the prognosis of patients with $\mathrm{AE}$ that show seizures during the acute phase of the disease. New studies would help in supporting the clinician work-up, avoiding over-treatment and limiting the social implication of the diagnosis.

References:

- Steriade C, Britton J, Dale R C, Gadoth A, Irani S R, Linnoila J, McKeon A, Shao X, Venegas V, Bien C G. Acute symptomatic seizures secondary to autoimmune encephalitis and autoimmuneassociated epilepsy: Conceptual definitions. Epilepsia (2020) Jul;61(7):1341-1351. Doi: 10.1111/epi.16571

- F. Graus, M. J Titulaer, R. Balu. A clinical approach to diagnosis of autoimmune encephalitis. Lancet Neurol. (2016) Apr; 15(4): 391404. Doi: 10.1016/S1474-4422(15)00401-9

\section{EEG SEIZURE ONSET PATTERNS IN STATUS EPILEPTICUS}

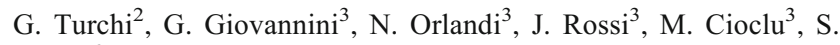
Meletti $^{3}$

${ }^{1}$ Neurology Department, University of Modena and Reggio Emilia (Modena); ${ }^{2}$ Neurology Department, Azienda Ospedaliera-Universitaria of Modena (Modena); ${ }^{3}$ Dept of Biomedical, Metabolic, and Neural Sciences, University of Modena and Reggio-Emilia (Modena)

Introduction: Seizure-onset (SOn) patterns have been studied especially in isolated seizures in epilepsy surgery candidates. EEG studies characterizing the SOn patterns in status epilepticus (SE) are lacking. We aim to classify the different SOn patterns during SE and to evaluate any differences in terms of mortality, morbidity and treatment response across different SOn patterns.

Materials and methods: Consecutive EEG recorded from adult patients admitted for focal SE, from January 2015 to August 2019 were 
reviewed. Five SOn patterns were identified (in accordance with Tanaka et al. 2018): (1) paroxysmal rhythmic slow activity at $<13 \mathrm{~Hz}$; (2) paroxysmal rhythmic fast activity at $\geq 13 \mathrm{~Hz}$; (3) repetitive epileptiform discharge; (4) suppression of background activity to $\leq 10 \mu \mathrm{V}$; and (5) artifacts. For each patient 1 to 5 seizures were analyzed, and each seizure's duration was registered.

Results: 307 seizures were analyzed in 100 consecutive patients/SE episodes (mean age $70 \mathrm{yrs}$ ); the most frequent SOn pattern was pattern 3 (39 patients) followed by pattern 1 (34 patients) and pattern 2 (14 patients); pattern 4 and 5 were less frequently observed ( 1 and 3 patients respectively). Nine patients presented with multiple SOn patterns. Seizures with SOn pattern 3 showed longest duration $(\mathrm{p}<0.05)$. No statistical difference in demographics, SE etiology, semeiology and treatment response was observed among the different SOn, while a higher 28-day mortality was observed in SOn pattern 3 ( $p=0.02$; HR 3.00; 95\% CI $1.13-7.97)$.

Conclusions: This is the first EEG study on scalp seizure onset patterns in status epilepticus. In SE the pattern 3, characterized by repetitive epileptiform discharges, was the most frequent, with the longest mean seizures duration, and associated to highest short-term mortality. Analysis of SOn patterns could improve our understanding on SE mechanism and could become a useful EEG biomarker.

References:

- Trinka E, Cock H, Hesdorffer D, Rossetti A O, Scheffer I E, Shinnar S, Shorvon S, and Lowenstein D H. A definition and classification of status epilepticus - Report of the ILAE Task Force on Classification of Status Epilepticus. Epilepsia (2015);56(10):1515-23

- Tanaka H, Khoo HM, Dubeau F, Gotman J. Association between scalp and intracerebral electroencephalographic seizure-onset patterns: A study in different lesional pathological substrates. Epilepsia (2018); 59:420-30

- Ferrari-Marinho T, Perucca P, Dubeau F, Gotman J. Intracranial EEG seizure onset- patterns correlate with high-frequency oscillations in patients with drug-resistant epilepsy. Epilepsy Res. (2016) Nov; 127:200-06

\section{HEADACHES}

\section{LOCKING DOWN CGRP PATHWAY DURING COVID-19 PANDEMIC LOCKDOWN: THE PANDEMIG STUDY}

C. Altamura ${ }^{1}$, S. Cevoli ${ }^{2}$, C. Aurilia ${ }^{3}$, G. Egeo ${ }^{3}$, L. Fofi $^{3}$, P. Torelli ${ }^{4}$, N. Brunelli $^{1}$, G. Pietrangeli ${ }^{5}$, V. Favoni ${ }^{2}$, A. Fallacara ${ }^{1}$, U. Pensato ${ }^{5}$, P. Barbanti $^{3}$, F. Vernieri ${ }^{1}$

${ }^{1}$ Headache and Neurosonology Unit, Campus Bio-Medico University of Rome (Roma); ${ }^{2}$ Neurology, IRCCS Istituto delle Scienze Neurologiche di Bologna (Bologna); ${ }^{3}$ Headache and Pain Unit, IRCCS San Raffaele Pisana (Roma); ${ }^{4}$ Department of Medicine and Surgery, University of Parma (Parma); ${ }^{5}$ Department of Biomedical and NeuroMotor Sciences (DIBINEM), University of Bologna (Bologna)

Objectives: The COVID-19 pandemic and the subsequent lockdown came as a storm disrupting people's everyday life. This study aimed at observing whether the COVID-19 related lockdown influenced migraine frequency and disability in migraine patients on therapy with monoclonal antibodies inhibiting the CGRP pathway.

Methods: In this longitudinal observational cohort study, 147 consecutive patients receiving monthly administration of Erenumab or Galcanezumab were enrolled in four Italian Headache Centers. All patients filled a questionnaire concerning working and household settings, recent flu symptoms or COVID-19 diagnosis, and any family loss due to COVID-19 infection. Monthly migraine days (MMDs), monthly painkiller intake (MPI), and Hit-6 disability relating to the first month of lockdown imposition (T-lock) and the month before (T-free) were also collected.

Results: From T-free to T-lock, the cohort displayed a reduction in MMDs (from 10.5 \pm 7.6 to $9.8 \pm 7.6, p=.024$ ) and Hit-6 scores (from 59.3 \pm 8.3 to $57.8 \pm 8.8, \mathrm{p}=.009$ ), while MPI resulted unchanged (from 11.6 \pm 11.5 to $11.1 \pm 11.7$; $\mathrm{p}=.114$ ). MMDs, MPI, and HIT- 6 variations from T-free to T-lock did not differ according to work settings or household. Patients beyond the first three months of therapy presented less often a reduction in MMDs ( $\mathrm{p}=.006)$ and men reduced MPI more frequently than women $(\mathrm{p}=.005)$.

Conclusions: Our study observed that the lockdown impact on everyday life did not affect the migraine load in patients receiving monoclonal antibodies inhibiting the CGRP pathway. Patients in the first months of therapy experienced a greater improvement according to drug pharmacokinetics, while women more frequently needed rescue medications, possibly indicating presenteeism.

\section{MIGRAINEURS PSYCHOLOGICAL TRAITS DO NOT INFLUENCE RESPONSE TO ERENUMAB}

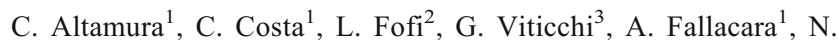
Brunelli $^{1}$, G. Egeo ${ }^{2}$, C. Aurilia ${ }^{2}$, S. Migliore ${ }^{4}$, P. Barbanti ${ }^{2}$, M. Silvestrini $^{3}$, F. Vernieri ${ }^{1}$

${ }^{1}$ Headache and Neurosonology Unit, Campus Bio-Medico University of Rome (Roma); ${ }^{2}$ Headache and Pain Unit, IRCCS San Raffaele Pisana (Roma); ${ }^{3}$ Neurological Clinic, Marche Polytechnic University (Ancona); ${ }^{4}$ Huntington and Rare Diseases Unit, Fondazione IRCCS Casa Sollievo della Sofferenza Research Hospital (San Giovanni Rotondo-FG)

Objectives: Psychopathological aspects have been implied in many facets of migraine. However, it is still a matter of debate if there is any association between the psychological traits of migraine patients and the response to prophylactic treatments. This study aimed at assessing if Erenumab action on migraine prevention may be influenced by patients' psychological traits.

Methods: Before starting the treatment, all subjects receiving Erenumab upon clinical indication filled the following psychological questionnaires: Beck Depression Inventory-II (BDI-II), The Barratt Impulsiveness Scale (BIS-11), Toronto Alexithymia Scale (TAS-20), The Difficulties in Emotion Regulation Scale (DERS), and the StateTrait Anxiety Inventory-Y (STAI-Y). Monthly migraine days (MMDs), monthly painkiller intake (MPI), and HIT-6 scores were collected at each Erenumab injection. The primary endpoint was to observe if patients achieving $\geq 50 \%$ or $\geq 75 \%$ MMDs responder rates after three months of therapy (i.e. Responders) presented different scores at psychological evaluation scales compared with non-Responders.

Results: We enrolled 68 patients (49.38 SD 10.9; $73.6 \%$ of female). Of these, $80.9 \%$ of subjects were affected by chronic migraine $(\mathrm{CM})$ and $65.2 \%$ presented had also $\mathrm{MOH}$. The psychological scales at baseline resulted as follows: BDI-II 8.0 (18 IQr), BIS-11 50.0 (12 IQr), TAS-20 48.0 (15 IQr), DERS 83.0 (24 IQr), STAI-Y 47.0 (8). At baseline, enrolled patients presented 18 MMDs (14 IQr) and 16 MPI (14 IQr), while Hit-6 score was 66 (7 IQr). At baseline, patients with $\mathrm{MOH}$ presented higher scores of BDI-II [p =.032, 17.5 (21IQr) vs 5 (15 IQr)]. Psychological scale scores did not differ between episodic migraine 
(EM) and CM, however, the low number of EM patients may have concealed a disparity. After three months of therapy, MMDs [9.5 (9 IQr)], MPI [10 (10IQr)], and HIT-6 score [66 (10 IQr)] were significantly reduced (consistently $\mathrm{p}<.0001$ ). The responders were $42.3 \%$ while $15.4 \%$ were super-responders. The results of the psychological tests did not differ between $50 \%$ or $75 \%$ responders and non-responders (table). Moreover, no interaction was observed between responders or superresponders and positive history for psychiatric disorder or $\mathrm{MOH}$ at baseline ( $\mathrm{p}$ consistently $>.1$ ).

Conclusions: The results of our study showed that although perceived symptoms of depression (BDI-II scores) differently characterize patients with or without $\mathrm{MOH}$, migraineurs psychological traits seem not to influence response to Erenumab.

\section{A PROSPECTIVE ITALIAN MIGRAINE REGISTRY (I-GRAINE- NEW) TO INCREASE DISEASE AWARENESS, TREATMENT AND OUTCOME}

\author{
P. Barbanti ${ }^{1}$, L. Fofi ${ }^{1}$, L. Hollander ${ }^{2}$, G. Egeo ${ }^{1}$, C. Aurilia ${ }^{1}$, G.

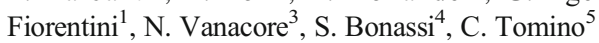

\begin{abstract}
${ }^{1}$ Headache and Pain Unit, IRCCS San Raffaele Pisana (Roma); ${ }^{2}$ Scientific Direction, CD Pharma Group (Milano); ${ }^{3}$ National Center for Disease Prevention and Health Promotion, National Institute of Health (Roma); ${ }^{4}$ Unit of Clinical and Molecular Epidemiology, IRCCS San Raffaele Pisana; San Raffaele University (Roma); ${ }^{5}$ Scientific Direction, IRCCS San Raffaele Pisana; San Raffaele University (Roma)
\end{abstract}

Background: Migraine is the leading cause of disability in people under 50 but remains underdiagnosed, underrecognized and undertreated $\mathrm{A}$ comprehensive appraisal of the full clinical, social and economic complexity of the disease is a fundamental prerequisite for its proper management. One significant step forward is the development of a dedicated migraine registry which may represent a valuable platform for the collection of relevant clinical, biological and epidemiological information about the disease, with potential benefits to patients, health professionals and organizations.

Objectives: To describe the establishment of the Italian Migraine Registry (I-GRAINE-NEW), aimed to provide information on the natural history of migraine, generate epidemiological, clinical and social data, and to assess how does patient management impact on disease evolution and outcome.

Design and methods: I-GRAINE-NEW is an observational, prospective, Italian Migraine Registry comprising of 46 headache centers, coordinated by the IRCCS/University San Raffaele, Rome, and supervised by a scientific committee. I-GRAINE-NEW will include $10 \%$ of all the adult patients affected by episodic or chronic migraine visited at each center, selected according to the "systematic random" method and will last at least 5 years. After signing an informed consent, patients will be evaluated by face-to-face interviews using a detailed ( 200 items), semi-structured questionnaire assessing socio-demographic factors, lifestyle, medical history, migraine characteristics and disability, patient's journey and healthcare resource use. A subgroup of 6000 incident migraine patients referred for a first outpatient visit, will be considered for a retrospectiveprospective cohort study - lasting24 months - aimed at analysing more in-depth ( $\sim 500$ items questionnaire) clinical endophenotypes, treatments and the detailed history of migraine onset and evolution. A Contract Research Organization will supervise and support all the clinical operation and provide the centralization and the management of data stored in the electronic case report forms. All procedures will by compliant with the current European privacy policy for the protection of personal patient's data (GDPR 2016/697).

Results and conclusions: The I-GRAINE registry is expected to shed light on migraine unmet needs, define the endophenotypes, and improve clinical management, resulting in increased disease awareness, better healthcare resource allocation, and reduced economic burden.

References:

Vanacore N, Cevoli S, Torelli P, Aurilia C, Egeo G, Fofi L, Grazzi L, Bussone G, Manzoni GC, Cortelli P, Barbanti P. A proposal for a national registry on chronic migraines. J Headache Pain. (2015) Dec;16(Suppl 1):A40. doi: 10.1186/1129-2377-16-S1-A40

- Barbanti P, Fofi L, Cevoli S, Torelli P, Aurilia C, Egeo G, Grazzi L, D'Amico D, Manzoni GC, Cortelli P, Infarinato F, Vanacore N. Establishment of an Italian chronic migraine database: a multicenter pilot study. Neurol Sci. (2018) May;39(5):933-937. doi: 10.1007/ s10072-018-3280-y. Epub 2018 Feb 22

- Barbanti P, Fofi L, Grazzi L, Torelli P, Vernieri F, Cevoli S, Vanacore N. The Italian chROnic migraiNe (IRON) Registry: a report from the first 340 patients. Neurology (2019);92 (S15):P 3.10-004

\section{AN ITALIAN LONG-TERM (9-MONTHS), REAL-LIFE, MULTICENTER ( $N=15$ ), STUDY WITH ERENUMAB IN HIGH- FREQUENCY EPISODIC AND CHRONIC MIGRAINE: THE EARLY II (ERENUMAB IN REAL LIFE IN ITALY) STUDY}

P. Barbanti ${ }^{1}$, C. Aurilia ${ }^{1}$, G. Egeo ${ }^{1}$, L. Fofi ${ }^{1}$, C. Costa $^{2}$, N. Brunelli ${ }^{2}$, A. Fallacara $^{2}$, S. Cevoli ${ }^{3}$, G. Pierangeli ${ }^{3}$, V. Favoni ${ }^{3}$, B. Colombo ${ }^{4}$, M. Filippi $^{4}$, R. Messina ${ }^{4}$, F. Frediani ${ }^{5}$, P. Di Fiore ${ }^{5}$, P. Torelli ${ }^{6}$, F. Bono ${ }^{7}$,

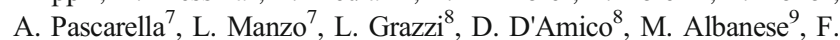
D'Onofrio $^{10}$, C. Finocchi ${ }^{11}$, A. Salerno ${ }^{12}$, B. Mercuri ${ }^{12}$, M. Zucco ${ }^{13}$, L. Di Clemente $^{13}$, M. Trimboli ${ }^{14}$, A. Carnevale ${ }^{15}$

${ }^{1}$ Headache and Pain Unit, IRCCS San Raffaele (Roma); ${ }^{2}$ Headache and Neurosonology Unit, Policlinico Universitario Campus Bio-Medico (Roma); ${ }^{3}$ Headache Unit, IRCCS Istituto delle Scienze Neurologiche (Bologna); ${ }^{4}$ Neurology Unit, IRCCS San Raffaele Scientific Institute, Vita-Salute San Raffaele University (Milano); ${ }^{5}$ Headache Center, ASST Santi Paolo Carlo (Milano); ${ }^{6}$ Department of Medicine and Surgery, Headache Center, Unit of Neurology, University of Parma, Azienda Ospedaliero-Universitaria of Parma (Parma) ${ }^{7}$ Center for Headache and Intracranial Pressure Disorders, Neurology Unit, A.O.U. Mater Domini (Catanzaro); ${ }^{8}$ Neuroalgology Unit, Headache Center, Fondazione IRCCS Istituto Neurologico (Milano); ${ }^{9}$ Headache Center, Policlinico Tor Vergata (Roma); ${ }^{10}$ Headache Center, Institute of Neurology, San G. Moscati Hospital (Avellino); ${ }^{11}$ Headache Center, IRCCS Ospedale Policlinico San Martino (Genova); ${ }^{12}$ Headache Center, San Giovanni Addolorata Hospital (Roma); ${ }^{13}$ Headache Center, San Camillo-Forlanini Hospital (Roma); ${ }^{14}$ Headache Center, San Carlo Hospital (Potenza); ${ }^{15}$ Headache Center, San Filippo Neri Hospital (Roma)

Objective: To assess the long-term (9-month) effectiveness, safety and tolerability of erenumab in high-frequency episodic migraine (HFEM) and chronic migraine $(\mathrm{CM})$ in real-life, trying to identify responsiveness predictors.

Background: In the EARLY I study [1], we documented the effectiveness, tolerability and safety of a 12-week erenumab treatment in HFEM and $\mathrm{CM}$ in real life. 
Methods: This is a multicenter, prospective, cohort, real-life study. All eligible consecutive patients with HFEM or CM seen from 20/12/2018 to 31/12/2019 in 15 Italian Headache Centers were treated with erenumab $70 \mathrm{mg}$ subcutaneously every 28 days. Erenumab was increased to $140 \mathrm{mg}$ in non-responders and in responders with efficacy wear-off. We evaluated changes in monthly migraine days (MMDs: primary endpoint), monthly analgesic intake (MAI), pain intensity (Visual Analogue Scale, VAS), headache impact test (HIT-6), $>50 \%,>75 \%$ and $100 \%$ responder rates, tolerability and safety at week36 compared to baseline.

Results: We treated 673 migraine patients $(\mathrm{HFEM}=173, \mathrm{CM}=500)$ with $>1$ erenumab $70 \mathrm{mg}$ dose. Mean prior therapeutic failures were 3.9 in HFEM and 5.3 in CM. One-hundred-thirty-five were treated for $>36$ weeks and considered for statistical analysis. At week 36, erenumab decreased MMDs by $4.5 \pm 4.1$ days in patients with HFEM and by $12.3 \pm$ 7.8 days in those with CM compared to baseline. At week 36, VAS score was reduced by $1.9 \pm 2.4$, HIT score by $11.8 \pm 10.0$ and median MAI from 12.0 (interquartile range [IQR] 10.0-14.0) to 5.0 (IQR 2.7-8.0) in HFEM, while in CM, VAS was reduced by $2.7 \pm 2.4$, HIT by $13.7 \pm 9.3$ and median MAI from 21.0 (IQR 15.0-30.0) to 7.0 (IQR 4.0-12.0). At week $36,>50 \%$ responders were $55.0 \%$ for HFEM and $66.3 \%$ for CM, $>75 \%$ responders were $17.5 \%$ and $38.9 \%$ and $100 \%$ responders $7.5 \%$ and $4.2 \%$, respectively. Adverse events were uncommon (13.1\%), mild or moderate, constipation being the most frequent one $(9.1 \%)$. Eenumab dose was increased to $140 \mathrm{mg}$ in 239 patients (35.5\%). Ten patients discontinued the study due to ineffectiveness and 24 for adverse events. Erenumab responsiveness was positively associated with allodynia (OR: 8.17 95\%CI: 1.89-35.38; $\mathrm{p}=0.005$ ) in HFEM, and with baseline migraine frequency (OR: 1.13, 95\% CI: $1.04-$ 1.22; $\mathrm{p}=0.003$ ) and unilateral autonomic symptoms (OR: 2.81, 95\%CI: $1.08-7.3 ; \mathrm{p}=0.034)$ in CM.

Conclusions: Erenumab is effective, safe and well tolerated in longterm migraine treatment in real-life. Easily obtainable clinical features may help in predicting patient's responsiveness.

Reference:

1. Barbanti P. et al. The First, Italian, Multicenter, Real-life Study with Erenumab in the Prevention of High Frequency Episodic and Chronic Migraine. Neurology (2020) April 14; 94 (15 Suppl) $\# 1.004$

\section{ARTIFICIAL INTELLIGENCE FOR MEDICATION OVERUSE PREDICTION IN MIGRAINE}

\author{
P. Barbanti ${ }^{1}$, P. Ferroni ${ }^{2}$, F. Zanzotto ${ }^{2}$, M. Scarpato ${ }^{2}$, A. Spila ${ }^{2}$, L. Fofi ${ }^{1}$, \\ G. Egeo ${ }^{1}$, C. Aurilia ${ }^{1}$, A. Rullo ${ }^{2}$, R. Palmirotta ${ }^{3}$, F. Guadagni ${ }^{2}$
}

\begin{abstract}
${ }^{1}$ Department of Neurological, Motor and Sensorial Sciences, IRCCS San Raffaele Pisana (Roma); ${ }^{2}$ Research Center BioDAT Laboratory, IRCCS San Raffaele Pisana (Roma); ${ }^{3}$ Department of Biomedical Sciences and Human Oncology, University of Bari (Bari)

Objectives: Predicting medication overuse (MO) is a compelling challenge in migraine clinical management. Artificial intelligence (AI) with machine learning (ML) has shown great potential in building automatic predictors for migraine classification, but detectors for $\mathrm{MO}$ are still in their infancy. Thus, a proof-of-concept study was designed to investigate the performance of a novel ML-based approach to derive a decision support system (DSS) for MO risk assessment in migraine.
\end{abstract}

Patients: The performance of a customized ML-based DSS combining Support Vector Machines and Random Optimization (RO-MO) was investigated using real world data from a large $(\mathrm{N}=777)$, well clinical characterized migraine outpatient population, detailing lifestyle, behavioral, socio-demographic factors as well as clinical and biochemical data.

Methods: RO-MO was used to produce predictive discriminators (RO-MO-x) yielding the best classification performance over a training $(\mathrm{N}=543$ ) (3-fold cross validation) and test $(\mathrm{N}=234)$ set, randomly divided. Group clustering was performed according to the attributes clinical significance. ROC curve, Bayesian analysis and logistic regression were used to estimate MO probability.

Results: $21 \%$ (162 of 777) of the enrolled patients reported the presence of MO which had lasted for at least 2 years. Using this dataset we derived a set of predictors with discriminatory power for MO. The best four were incorporated into the final ML-based DSS and risk evaluation on a five-level stratification was performed. Of interest, all four predictors were not only clinically plausible, but also had a complementary configuration of weights for grouped variables. ROC analysis resulted in a cstatistic of 0.83 with a sensitivity and specificity of 0.69 and 0.87 , respectively, and an accuracy of 0.87 when $\mathrm{MO}$ was predicted by at least three RO-MO models. Logistic regression analysis further confirmed that the ML-based DSS could effectively distinguish between MO and non-MO with ORs of 5.72 (95\% CI: 1.56-20.9) and 21.0 (95\% CI: 8.5-52) for patients classified as probably ( 3 predictors positive), or definitely at risk of MO (4 predictors positive), respectively.

Discussion: These results demonstrate that an AI-based approach can be useful in performing an automated prediction for $\mathrm{MO}$ in migraine, with an accuracy higher than that reported for currently available tools [e.g. computerized headache assessment tool (CHAT) for MO, accuracy $82.7 \%$, holding the potential for improving model precision.

Conclusions: A combination of ML and RO represents a valuable approach to MO prediction in migraine and a useful tool to help neurologist to advise people from an early stage and tailor treatment based on individual risk.

References:

- Palmirotta R, Barbanti P, Ludovici G, Egeo G, Aurilia C, et al. Establishment of a biorepository for migraine research: the experience of Interinstitutional Multidisciplinary BioBank (BioBIM). Neurol Sci (2013);34:1659-63

- Ferroni, P, Zanzotto, F.M, Scarpato, N, Riondino, S, Nanni, U, et al. Risk assessment for venous thromboembolism in chemotherapy treated ambulatory cancer patients: a precision medicine approach. Med Decis Making (2017);37:234-42

- Maizels M, Wolfe WJ An expert system for headache diagnosis: the Computerized Headache Assessment tool (CHAT). Headache (2008);48:72-8

\section{COPING WITH COVID-19 PANDEMIC IN A NEUROLOGICAL OUTPATIENT CENTER: RESULTS FROM THE IMPLEMENTATION OF A TELEMEDICINE SERVICE}

\author{
V. Caponnetto ${ }^{1}$, I. Frattale ${ }^{1}$, R. Ornello ${ }^{1}$, L. Lancia ${ }^{2}$, F. Ferrara ${ }^{3}$, S. Sacco ${ }^{1}$ \\ ${ }^{1}$ Department of Neurology; Department of Applied Clinical Sciences and \\ Biotechnology, Avezzano Hospital; University of L'Aquila (Avezzano- \\ AQ, L'Aquila); ${ }^{2}$ Nursing Section, Department of Health, Life, and \\ Environmental Sciences, University of L'Aquila (L'Aquila); ${ }^{3}$ ALTEMS: \\ High School of Economics and Management of Health Systems, Catholic \\ University of Sacred Hearth (Roma)
}

Background: During coronavirus disease (COVID-19) pandemic, most of low-complex healthcare not related to the health emergency were suspended. In our neurological outpatient Center, the need to restore visits 
has been growing since early phase- 2 . However, procedures related to infection containment imply longer time for in-person visits compared to the past, making it difficult to meet patients' requests.

Aim: Evaluate a new method to perform control visits at our neurological outpatient Center.

Methods: We implemented a protocol to perform tele-consultations for patients who had a first in-person access to our center and needed a control visit. We performed tele-visits with a free-use and widespread platform in compliance with UE Regulation 2016/679 (GDPR). Patients had a first contact with a research nurse to collect relevant clinical documents, check ongoing treatments, and assess patients' needs; the following day patients had a tele-contact with a clinician who performed the medical visit. We collected data about sociodemographic characteristics of the patients and their distance from our Center, device used and audiovideo connection quality, duration of tele-visits, cases' clinical complexity, need to be followed-up in-person, difficulty in interaction and document sharing, and patients' satisfaction.

Results: From May to July 2020, we involved 41 patients, mostly female $(85.4 \%)$, with a median age of 51 (IQR: 39-60) years. Patients' median distance from our Center was 102 (IQR: 67-128) km. To perform tele-visits, patients used: personal computer (58.5\%), smartphone (31.7\%), and tablet (9.8\%). Median duration of tele-visits was 10 (IQR: 8-12) minutes and the audio-video connection quality was high almost always (95.1\% tele-visits). Clinical complexity of the cases was scarce for $80.5 \%$ of the visits, median for $14.6 \%$ of the visits and high for $4.9 \%$ of the visits, while thirteen $(31.7 \%)$ cases required to be followed-up inperson. Interacting with patients was very easy in $78.0 \%$ tele-visits, quite difficult in $12.2 \%$ tele-visits, and very difficult in $9.8 \%$ tele-visits. Thirtyone $(79.5 \%)$ patients declared to have had no difficulty in sharing documents, while $20.5 \%$ of them had low difficulty. Almost all the patients were highly satisfied about the service (95.1\%).

Discussion and Conclusion: During phase-2 of COVID-19 pandemic, telemedicine allowed to control low-complexity cases and perform clinical inspections, avoided patients to reach the hospital, allowed to increase visits performed, ensured easy interaction, and determined high satisfaction. After COVID-19 pandemic, telemedicine should still be used for control visits, allowing to save time, avoid patients' difficulties and costs, and face future challenges.

\section{EFFICACY OF GREATER OCCIPITAL NERVE BLOCK IN PATIENTS WITH CERVICOGENIC HEADACHE: A SYSTEMATIC REVIEW}

\author{
V. Caponnetto ${ }^{1}$, R. Ornello ${ }^{1}$, I. Frattale ${ }^{1}$, C. Di Felice ${ }^{2}$, L. Lancia ${ }^{3}$, S. \\ Sacco $^{1}$
}

${ }^{1}$ Department of Neurology; Department of Applied Clinical Sciences and Biotechnology, Avezzano Hospital, University of L'Aquila (AvezzanoAQ, L'Aquila); ${ }^{2}$ Department of Applied Clinical Sciences and Biotechnology, University of L'Aquila (L'Aquila); ${ }^{3}$ Nursing Section, Department of Health, Life, and Environmental Sciences, University of L'Aquila (L'Aquila)

Background: Cervicogenic headache $(\mathrm{CGH})$ is a secondary headache disorder caused by a lesion or disorder of the cervical spine or soft tissues of the neck, with a worldwide prevalence of $2.2-4.1 \%$. Few evidencebased pharmacological treatments of $\mathrm{CGH}$ are available. However, greater occipital nerve blocks (GONBs) performed through suboccipital injections of a mixture of steroids and/or local anesthetics are considered an effective and safe treatment option. GH.

Aim: To summarize the effectiveness and safety of GONBs in treating

Methods: We conducted a systematic review searching Pubmed and Web of Science in June 2020, combining the keywords 'greater occipital nerve' and 'cervicogenic headache'. We included original studies that reported clinical outcomes of patients suffering from $\mathrm{CGH}$ who underwent GONB through suboccipital injection.

Results: Out of 132 retrieved studies, 6 reported relevant information and were included. The included studies were mostly observational, published from 1998 to 2019, and involved 116 patients with CGH. In almost all the studies, authors performed unilateral blockade of the greater occipital nerve. In three studies, authors injected only a local anesthetic, while in the other three authors administered both local anesthetic and steroid. Timepoints for injections repetition and outcome evaluation widely varied among the studies (from 1 week to 9 months). All the studies reported a significant reduction in pain in almost all cases: in 4 studies mean pain reduction ranged from -8.2 (at 2 weeks after the first block) to -0.1 (at 1 month after the third block); one study documented $66.6 \%$ reduction of pain intensity and another study documented a significant median reduction of pain intensity at 3 months (decreased from 5.5 to 2.3 ) and not at 9 months. One out of two studies documented a significant reduction of pain frequency at each timepoint compared to baseline. Single studies evaluated duration of analgesia, quantification of painkillers, and quality of life measures, documenting a duration of analgesia of 6 months after one procedure at baseline, significant reduction in painkiller intake, and a significant improvement in quality of life measures. Three studies reported minor adverse events in some cases, such as increased pain during injection day, temporary numbness, local sensitivity and pain.

Discussion and Conclusion: The few available observational studies suggest that GONBs are effective and safe in treating CGH, also when performed only with a local anesthetic. Larger randomized trials are needed to confirm efficacy and establish the optimal treatment protocol, especially regarding administration timepoints.

References:

- Barmherzig, R., Kingston, W. Occipital neuralgia and cervicogenic headache: diagnosis and management. Current Neurology and Neuroscience Reports (2019);19(5):20

- Headache Classification Committee of the International Headache Society (IHS) The International Classification of Headache Disorders, 3rd edition. Cephalalgia (2018);38(1):1-211

- Knackstedt H, Bansevicius D, Aaseth K, Grande RB, Lundqvist C, Russel MB. Cervicogenic headache in the general population: the Akershus study of chronic headache. Cephalalgia (2010);30(12):1468-76

\section{HEADACHE-RELATED FUNCTIONAL DISABILITY ACCURATELY EVALUATED THOUGH A NEW DIARY: A PILOT STUDY AND FUTURE PERSPECTIVES}

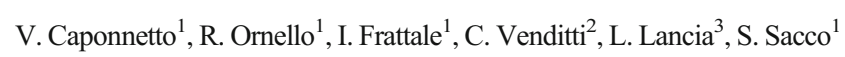

${ }^{1}$ Department of Neurology; Department of Applied Clinical Sciences and Biotechnology, Avezzano Hospital, University of L'Aquila (AvezzanoAQ, L'Aquila); ${ }^{2}$ Department of Applied Clinical Sciences and Biotechnology, University of L'Aquila (L'Aquila); ${ }^{3}$ Nursing Section, Department of Health, Life, and Environmental Sciences, University of L'Aquila (L'Aquila)

Background: Measurement of headache-related functional disability (HRFD) is a relevant area of interest in headache medicine for clinical 
and research purposes and has also been recently considered by legislative initiatives. Therefore, accurate measurement of HRFD represents a cornerstone of for appropriate management of patients. However, most of the available instruments to evaluate HRFD depend on patients' recall.

Aim: We aimed at comparing objective (diary-based) and perceived reporting of HRFD.

Methods: We conducted an observational pilot study implementing a headache diary where patients reported headache impact on daily life through a three-level scale, describing their functionality as preserved (D0), impaired (D1, i.e. 'presenteeism' at work, school, or home), or totally impaired (D2, i.e. 'absenteeism' at work, school, or home). From January 2020, during follow-up visits at our Center, we prospectively collected data reported on the diaries for one month and we asked patients to fill the 'Six-item Headache Impact Test' (HIT-6). We descriptively compared the distribution of the median days that patients spent in D0, $\mathrm{D} 1$, and D2 condition within each 'headache-impact' category as revealed through HIT-6 scale.

Results: We collected data from 35 migraine patients, mostly female $(88.6 \%)$, with a median age of 50 (range 19-76) years. Most of them had chronic migraine $(\mathrm{n}=29,82.9 \%)$ and almost all $(91.4 \%)$ were under preventative treatments for migraine. Patients reported 8 median migraine days (range 0-3) and a median HIT-6 score of 60 (range 36-87) classifying headache impact as minimum for $25.7 \%$ patients, mild for $8.6 \%$ patients, moderate for $11.4 \%$ patients, and severe for $54.3 \%$ patients. In median, migraineurs reporting minimum impact spent 2 days in D0 (IQR: 2-5), 0 in D1 (IQR: 0-1), and 0 in D2; migraineurs reporting mild impact spent 4 days in D0 (IQR: 0-18), 2 in D1 (IQR: 0-2), and 0 in D2 (IQR: 0-2); migraineurs reporting moderate impact spent 3 days in D0 (IQR: 2-4), 7 in D1 (IQR: 4-9), and 0 in D2; migraineurs reporting severe impact spent 2 days in D0 (IQR: 0-10), 1 in D1 (IQR: 0-4), and 2 in D2 (IQR: 0-8).

Conclusion: Preliminary data collected through D0-D1-D2 classification agreed with HIT-6 classification. However, the new diary would allow to evaluate monthly HRFD without recall bias, to assess patients' condition more objectively, and to avoid administering questionnaires, saving time during clinical visits. Therefore, it is reasonable to conduct larger research with the instrument to accurately identify patients with high HRFD.

References:

- Steiner T. J., Jensen R., Katsarava Z., Linde M., MacGregor E. A., Osipova V., Paemeleire K., Olesen J., Peters M., Martelletti P. Aids to management of headache disorders in primary care (2nd edition): on behalf of the European Headache Federation and Lifting The Burden: the Global Campaign against Headache. The Journal of Headache and Pain, (2019):20(1): 57. doi: 10.1186/s10194-0180899-2

- Steiner T. J., Stovner Lars J., Vos Theo, Jensen R., Katsarava Z. Migraine is first cause of disability in under 50s: will health politicians now take notice? The journal of headache and pain, (2018);19(1):17-17. doi: 10.1186/s10194-018-0846-2

- Public Law n. 81/2020. Disposizioni per il riconoscimento della cefalea primaria cronica come malattia sociale. (20G00100) (GU n.188/28-7-2020)

\section{ACUTE MYOCARDIAL INFARCTION IN A PATIENT WITH CHRONIC MIGRAINE AND TRIPTAN OVERUSE TREATED WITH ERENUMAB FOR MIGRAINE PREVENTION: A CASE REPORT}

\author{
I. Cetta, R. Messina, B. Colombo, M. Filippi
}

Neurology Unit, IRCCS San Raffaele Scientific Institute (Milano)

Background: Triptans induce vasoconstriction of arteries, raising concerns about triptan use in patients with vascular risk factors. Calcitoningene related peptide (CGRP) acts as a potent vasodilator. Whether the impairment of CGRP-mediated vasodilatory mechanisms might interact with triptan-induced vasoconstriction is unknown.

Clinical case: We present the case of a 55-year-old man with chronic migraine and triptan overuse treated with erenumab for migraine prevention who developed an acute myocardial infarction. For his migraine attacks, the patient has been taking sumatriptan $6 \mathrm{mg}$ injection or almotriptan $12.5 \mathrm{mg}$ tablets at least once a day for the last 10 years. He had tried several preventives in the past without any benefit. The patient had a history of myocardial infarction, after which he was recommended to stop taking triptans. Although our recommendation, the patient continued taking triptans for his migraine. In March 2019, in agreement with his cardiologist, we started erenumab $70 \mathrm{mg}$ with a slight reduction in the severity of the headache pain. The monthly subcutaneous injections were well tolerated, and no side effects were reported. In the absence of treatment response in terms of migraine frequency reduction, the dosage of erenumab was increased to $140 \mathrm{mg}$ after three months. During the fifth moth of treatment with erenumab, few minutes after having subcutaneous sumatriptan $6 \mathrm{mg}$ injection, the patient had sudden onset of chest pain described as pressure localized to the retrosternal area and radiated to the right arm. Shortly afterwards, the patient presented to the emergency department: his initial 12-leads electrocardiogram showed signs compatible with inferior ST-segment elevated myocardial infarction, associated with atrial fibrillation. Cardiac enzymes showed a spike of troponin of $2490 \mathrm{pg} / \mathrm{ml}$. The patient underwent coronary angiography showing severe constriction of the left coronary (70-90\%) and complete thrombotic occlusion of the distal tract of the right coronary. Intracoronary fibrinolysis was performed, with success. The clinical course was stable, and the patient was released from the hospital a week later.

Conclusions: Caution should be paid when using treatments targeting the CGRP pathway in migraine patients with triptan overuse and preexisting cardiovascular risk factors.

\section{CONVERSION FROM CHRONIC TO EPISODIC MIGRAINE IN PATIENTS TREATED WITH ERENUMAB: REAL-LIFE DATA FROM AN ITALIAN REGION}

E. Colangeli ${ }^{1}$, R. Ornello ${ }^{1}$, A. Casalena ${ }^{2}$, I. Frattale $^{1}$, V. Caponnetto ${ }^{1}$, A. Gabriele $^{3}$, G. Affaitati ${ }^{4}$, M. Giamberardino ${ }^{4}$, M. Assetta ${ }^{2}$, M. Maddestra ${ }^{5}$,

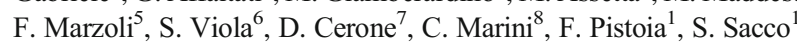

${ }^{1}$ Department of Applied Clinical Sciences and Biotechnology, University of L'Aquila (L'Aquila); ${ }^{2}$ Department of Neurology, 'G. Mazzini' Hospital (Teramo); ${ }^{3}$ Neurology Service, 'SS. Annunziata' Hospital (SulmonaAQ); ${ }^{4}$ Department of Medicine and Science of Aging, 'G. D'Annunzio' University (Chieti); ${ }^{5}$ Department of Neurology, 'F. Renzetti' Hospital (Lanciano-CH); ' Department of Neurology, 'S. Pio da Pietrelcina' Hospital (Vasto-CH); ${ }^{7}$ Department of Neurology, 'S. Salvatore' Hospital (L'Aquila); ${ }^{8}$ Department of Medicine, Public Health, Life and Environmental Sciences, University of L'Aquila (L'Aquila)

Objective: Most patients treated with erenumab in clinical practice have chronic migraine $(\mathrm{CM})$. We assessed the rate and possible predictors of conversion from $\mathrm{CM}$ to episodic migraine (EM) in a real-life study. 
Materials and Methods: We performed a subgroup analysis of patients treated with erenumab from January 2019 to February 2020 in the Abruzzo region, central Italy. Treatment was provided according to current clinical practice. For the purpose of the present study, we included patients fulfilling the definition of $\mathrm{CM}$ for the three months preceding erenumab treatment and with at least 6 months of follow-up after treatment. We assessed the rate of conversion to EM from baseline to Months 4-6 of treatment and during each month of treatment. To test the clinical validity of conversion to EM, we also assessed the decrease in monthly headache days (MHDs), acute medication days, and median headache intensity on a Numerical Rating Scale (NRS).

Results: We included in our study 91 patients with CM. At Months 46, 62 patients $(68.1 \%)$ converted from CM to EM; the proportion of converters increased from Month 1 to Month 5. Median MHDs decreased from 26.5 (IQR 20-30) to 7.5 (IQR 5-16; P<0.001) compared with baseline in the overall group, from 25 (interquartile range [IQR] 20-30) to 6 (IQR 3-8; $\mathrm{P}<0.001$ ); median acute medication days decreased from 21 (IQR 16-30) to 6 (IQR 3-10; $\mathrm{P}<0.001$ ); median NRS decreased from 8 (IQR 7-9) to 6 (IQR 4-7; P<0.001). Significant decreases were found both in converters and in non-converters. We found no significant predictors of conversion to EM among the patients' baseline characteristics.

Discussion and Conclusions: In our study, two thirds of patients with CM converted to EM during 6 months of treatment with erenumab. MHDs, acute medication use, and headache intensity decreased regardless of conversion from CM to EM.

\section{DOES REMOTE WORKING (SMARTWORKING) HAVE AN IMPACT ON HEADACHE FREQUENCY? RESULTS FROM A WORKPLACE SURVEY}

\author{
B. Colombo ${ }^{1}$, M. Filippi ${ }^{1}$, E. Grillo ${ }^{2}$, E. Paruccini ${ }^{2}$, L. Chiodo ${ }^{2}$, \\ M. Bartezaghi ${ }^{2}$
}

${ }^{1}$ Department of Neurology, San Raffaele Scientific Hospital (Milano); ${ }^{2}$ Novartis Farma S.p.A (Origgio-VA)

Objectives: COVID-19 emergency has had a profound impact on everyone's life. During lockdown, many companies have offered their employees the opportunity to work from home. Smart-working entails important modifications in habits and lifestyle; factors like the possible increase in hours spent on PC may lead to changes in the course of headache. The objective of this survey was to understand the impact of smart-working on headache frequency in Novartis employees.

Materials and methods: In May, after $\geq 2$ months of smart-working, a 34 -question anonymous survey was delivered through email to 2,210 employees. Only those who usually had headaches or started to experience them during the smart-working period were asked to complete the questionnaire. Questions were about demographics, headache history and characteristics, change in habits, changes in monthly headache days (MHD) during smart-working versus the 3 months preceding lockdown (baseline period).

Results: 141 employees (106 F; 35 M) completed the survey. Mean age was 43.7 years (range 26-75). 18.4\% had headache onset less than one year before taking the survey, $48.9 \%>10$ years before. $47.5 \%$ of respondents had not received a diagnosis yet, while $26.3 \%, 17.7 \%$, and $4.9 \%$ were diagnosed with migraine, tension-type headache, and cluster headache $(\mathrm{CH})$, respectively. Mean baseline MHD was 3.1. Work intensification was reported by $74.1 \%$ of respondents and significant changes in the following habits were observed: number of daily hours spent on PC ( 5.3 before vs 8.0 after), hours spent sitting ( 5.3 vs 8.6 ), hours spent driving ( 2.98 vs 0.23 ), hours dedicated to physical activity (3.11 vs 2.64 ), food intake (28.3\% reported an increase in quantities, $32.6 \%$ consumption of healthier meals). $62.4 \%$ of respondents reported an increase in headache frequency. Mean MHD increase vs baseline during the smart-working period was 1.83 days (3.00 days and 1.96 days for $\mathrm{CH}$ and tension-type headache, respectively, $\mathrm{p}<0.01 ; 0.86$ and 0.65 days for migraine w/wo aura, respectively, $\mathrm{p}>0.05$ ). $72.8 \%$ of patients who reported an increase in the number/intensity of working hours experienced a worsening in headache frequency versus $28.5 \%$ of those reporting a decrease in working activities. The only factor significantly associated with headache frequency increase was change in hours of sleep (Spearman coefficient $-0.20831 ; \mathrm{p}=0.0139)$. $\mathrm{CH}$ patients worsened significantly more than migraine patients did $(\mathrm{p}=0.0153)$.

Discussion and conclusions: Smart-working was associated with an increase in MHD that was more marked in $\mathrm{CH}$ patients. A larger sample size may be required to detect correlations between change in habits and MHD frequency.

\section{HEADACHE AND LITERATURE: INTERRELATIONSHIPS ON CREATIVITY}

\section{B. Colombo, M. Filippi}

Headache Center, IRCCS San Raffaele Hospital, Vita Salute University (Milano)

Objective: To focus on characters with headache in poems, stories and novels. To analyze how migraine and other headaches are described by novelists over the last centuries.

Material: An accurate search on library database for headache and migraine in Italian and International writers production.

Results: Literary accounts of migraine and headaches are both related to men and women. Headache symptoms are used both as a specific plot device or as a narrative device. The examination of works of several authors (Austen, Simenon, Pessoa, Woolf, Charlotte Bronte, Bulgakov and others) gives insights into the possibility that headache is something familiar to them (directly affected or experienced by family members). It is noteworthy that in many cases art is imitating life, the author's experience of headache prompts the writing of peculiar scene as Pilate's headaches in the Master and Margarita, according to Bulgakov's migraine. It was the same for Lewis Carroll's experience on migraine. It is possible that the depiction of Alice's curious experience of contracting and expanding in size in Alice in Wonderland may reflect a migraine visual aura. A different point of view came from Maugham who, as a medical student, had the opportunity to observe patients. This was the source of material for the novel Liza of Lambeth, with detailed headache descriptions. The same was true for Chekhov. As a doctor he was familiar with headache, and a number of his characters in several plays are reported to be suffering from migraine. Woolf wrote about the difficulty to explain what migraine is. She said: let a sufferer try to describe a pain in his head to a doctor and the language at once runs dry. Nevertheless, picturesque and detailed migraine description outline some characters of Auster (Mr Vertigo), McEwan (Atonement), Mann (Doctor Faustus). In Italian Literature, Sciascia (in the story: La morte di Stalin), D'Annunzio (Il piacere) and Tabucchi (Tristano muore) described migraine as an important trait of some main characters. Several empirical therapies were mentioned by many authors, I.e. dental exctractions and strong tea in Austen's Sandition and Mansfield Park or glass balls in Marquez's 100 years of solitude. 
Discussion: In literature, migraine is described as an obstacle/barrier for relationships, and associated with a sense of despair, loneliness and melancholy.

Conclusion: We confirm the negative connotation for migraine in literature, regardless of historical period, author's nationality, sex and age.

\section{THALAMO-CORTICAL NETWORK ACTIVITY IN SUBGROUPS OF MIGRAINE WITH AURA PATIENTS}

G. Coppola ${ }^{1}$, A. Di Renzo ${ }^{2}$, E. Tinelli ${ }^{3}$, B. Petolicchio ${ }^{3}$, V. Parisi ${ }^{2}$, M.

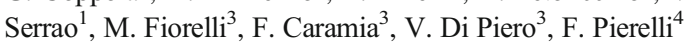

${ }^{1}$ Department of Medico-Surgical Sciences and Biotechnologies, Sapienza University of Rome Polo Pontino (Latina); ${ }^{2}$ Research Unit of Neurophysiology of Vision and Neurophthalmology, IRCCS Fondazione Bietti (Roma); ${ }^{3}$ Department of Human Neurosciences, Sapienza University of Rome (Roma); ${ }^{4}$ Headache Clinic, IRCCS Neuromed (Pozzilli-IS)

Aims: We were aimed to study resting-state functional connectivity (FC) between brain networks and its relationship with the microstructure of the thalamus in migraine patients with pure visual auras (MA), and in patients with complex neurological auras (MA+), i.e. with the addition of at least one of sensory and language symptoms.

Methods: 3T MRI data from 20 patients with MA and 15 with MA+ were collected and compared with data from 19 healthy controls (HCs). We collected resting-state data among independent component networks. Diffusivity metrics values of bilateral thalami were calculated and correlated with resting-state ICs Z-scores.

Results: As compared to HCs, both patients with MA and MA+ showed disrupted FC between the default mode network (DMN) and the right dorsal attention system (DAS). MA+ subgroup of patients showed lower microstructural metrics than those of both HCs and MA, and peculiar correlation with the strength of DMN. Despite the microstructural metrics of MA patients did not differ from those of HCs, they did not show the same correlations with the strength of DAS than HCs.

Conclusions: Present findings suggest that clinical heterogeneity of migraine with aura MRI profiles is associated with common and specific morpho-functional features of the nodes of the thalamo-cortical network.

\section{SPINAL SENSITIZATION AND CIRCULATING LEVELS OF ENDOCANNABINOID AND NON-ENDOCANNABINOID LIPID MEDIATORS DURING EXPERIMENTALLY-INDUCED MIGRAINE ATTACKS}

R. De Icco ${ }^{1}$, R. Greco ${ }^{2}$, C. Demartini ${ }^{2}$, A. Zanaboni ${ }^{3}$, A. Reggiani ${ }^{4}$, N. Realini $^{4}$, M. Allena ${ }^{5}$, D. Martinelli ${ }^{1}$, V. Grillo ${ }^{5}$, G. Sances ${ }^{5}$, C. Tassorelli ${ }^{1}$

${ }^{1}$ Headache Science Centre, IRCCS Mondino Foundation, Department of Brain and Behavioral Sciences, University of Pavia (Pavia); ${ }^{2}$ Laboratory of Neurophysiology of Integrative Autonomic Systems, IRCCS Mondino Foundation (Pavia); ${ }^{3}$ Laboratory of Neurophysiology of Integrative Autonomic Systems, IRCCS Mondino Foundation, Department of Brain and Behavioral Sciences, University of Pavia (Pavia); ${ }^{4}$ Department of Drug Discovery and Development, Istituto Italiano di Tecnologia (Genova); ${ }^{5}$ Headache Science Centre, IRCCS Mondino Foundation (Pavia)
Migraine subjects experience a derangement of the nociceptive system control as the disease progresses. The endocannabinoids system may modulate the nociceptive pathways. Here, we evaluated the single stimulus threshold (RTh) and the temporal summation threshold (TST) of the nociceptive withdrawal reflex (NWR) together with anandamide (AEA) and palmitoylethanolamide (PEA) circulating levels in patients with episodic migraine that were exposed to glyceryl trinitrate (GTN). We enrolled 24 patients (33.0 \pm 8.1 years, 22 female) and 19 healthy controls (HC - 29.5 $\pm 9.3,15$ female). The NWR and AEA and PEA plasma levels were recorded at baseline, and then at 30 (T30), 60 (T60) and 120 (T120) minutes after GTN administration ( $0.9 \mathrm{mg}$ sublingual). The subjects were observed until 180 minutes after GTN administration. The GTN test qualified as positive (MIG+ group) for subjects that developed a migraine-like headache according to criteria for experimentally-induced migraine. In all other cases, the test was considered as negative (MIGgroup). In subjects with a positive test within the 180 minutes of observation, the NWR and AEA and PEA plasma levels were also recorded at the onset of the migraine-like headache (T-MIG), and after 1 hour (TMIG-1h). Sixteen migraine patients $(66.7 \%)$ qualified for positive provocation test. Among these, 13 patients developed a migraine-like headache within the 180 minutes of observation, and so completed the additional described time-points. AEA levels significantly increased in all subjects ( $\mathrm{p}=0.035$ vs baseline), without differences between groups. In contrast, PEA levels significantly increased only in migraine patients $(p=0.007)$, specifically at T120 $(p=0.026)$. We confirmed the GTNinduced spinal sensitization in migraine patients, detectable as a decrease of RTh, and TST ( $\mathrm{p}=0.001$ and 0.016 , respectively). For all the described results, we did not find significant differences between MIG+ and MIGgroups. We did not find significant correlations between endocannabinoids plasma levels and neurophysiological variables at any time-points. Regarding the analysis performed at migraine onset in a subgroup of patients as previously described, we confirmed a significant increase of PEA plasma levels in these patients $(\mathrm{p}=0.035)$, specifically at T-MIG- $1 \mathrm{~h}$ ( $p=0.028$ vs. baseline). RTh and TST were significantly reduced in when recorded at T-MIG and T-MIG- $1 \mathrm{~h}$ time points $(\mathrm{p}=0.035$, and $\mathrm{p}=0.001$, respectively). The present study suggested a that the endogenous release of PEA could exert an analgesic and anti-inflammatory role in the ictal phase of episodic migraine patients. These observations encourage to study the possible role of ES and FAAH inhibition as a novel therapeutic target.

\section{EARLY OUTCOMES OF MIGRAINE AFTER ERENUMAB DISCONTINUATION: DATA FROM A REAL-LIFE SETTING}

\author{
E. De Matteis ${ }^{1}$, G. Affaitati ${ }^{2}$, I. Frattale ${ }^{1}$, V. Caponnetto ${ }^{1}$, F. Pistoia ${ }^{1}$, M. \\ Giamberardino $^{2}$, S. Sacco ${ }^{1}$, R. Ornello ${ }^{1}$
}

${ }^{1}$ Neuroscience Section, Department of Applied Clinical Sciences and Biotechnology, University of L'Aquila (L'Aquila); ${ }^{2}$ Department of Medicine and Science of Aging, 'G. D’Annunzio' University (Chieti)

Objectives: This study aimed at assessing the consequences of erenumab discontinuation after a 12-month treatment in patients who had a continuous positive response to the drug for the last 6 months of treatment. Monoclonal antibodies targeting Calcitonin gene-related peptide, including erenumab, are migraine-specific preventive treatments, whose longterm effectiveness has still to be evaluated in real-life settings.

Materials: We evaluated the short-term outcomes after erenumab withdrawal in patients with migraine enrolled in a real-life multicenter register of migraineurs treated with erenumab. All patients had received monthly 
erenumab for 12 months and attended a follow-up of two months after drug discontinuation. We only included patients who reported less than half of baseline migraine days for at least the last 6 months of treatment.

Methods: Outcomes were reduction in monthly migraine days, acute medications days, and pain intensity on a 0-10 Numerical Rating Scale.

Results: All 24 recruited patients registered a reduction in monthly migraine days from a median value of 23 (IQR 12.25-30) at baseline to 3.5 (interquartile range (IQR) $2-5 ; \mathrm{P}=0.001$ ) at the 12 th month. One month after treatment discontinuation, we observed an increase in monthly migraine days up to 7.5 (IQR 5-18.7; P=0.001 vs the 12th month and 0.001 vs baseline). Similarly, Numerical Rating Scale and acute medications days reduced during the 12-month treatment and slightly increased one month after treatment discontinuation, although staying lower than baseline. Eight patients resumed erenumab achieving good results soon after the new course. The remaining 16 patients had a sustained response two months after treatment withdrawal.

Discussion and Conclusions: Erenumab discontinuation after a 12month treatment led to a rapid increase in median monthly migraine days, acute medications days, and Numerical Rating Scale in some patients, who might benefit from prolonged duration of treatment; on the other hand, some patients showed long-lasting benefits. Larger studies might define the optimal treatment duration according to patients' characteristics.

\section{CLINICAL RESPONSE TO ONABOTULINUMTOXIN A AND CGRP MONOCLONAL ANTIBODIES IN MEDICATION OVERUSE HEADACHE AND CHRONIC MIGRAINE}

\section{Di Cesare ${ }^{1}$, F. Schiano Di Cola ${ }^{2}$, S. Caratozzolo ${ }^{1}$, P. Liberini $^{3}$, R. $\mathrm{Rao}^{3}$, A. Padovani ${ }^{1}$}

${ }^{1}$ Neurology Unit, Department of Neurological Sciences and Vision, University and Spedali Civili (Brescia); ${ }^{2}$ Neurology Unit, Clinical and Experimental Sciences Department, University and Spedali Civili (Brescia); ${ }^{3}$ Neurology Unit, Department of Neurological Sciences and Vision, Spedali Civili (Brescia)

Background: Aim of the present study was to compare clinical response to Onabotulinumtoxin A and monoclonal antibodies targeting CGRP in patients with chronic migraine $(\mathrm{CM})$ versus patients with chronic migraine complicated by medication overuse $(\mathrm{CM}$ and $\mathrm{MOH})$ at baseline and following 3 and 6 months of treatment.

Materials and Methods: The present study was conducted at the Headache Centre of Spedali Civili of Brescia. Sixty-seven consecutive CM patients were enrolled, of whom 44 complicated by concomitant medication overuse. Mean age was 48 yrs (SD 10.6) and a mean disease duration of $10.3 \mathrm{yrs}$ (SD 6.6). At baseline mean MIDAS score was 79.4 (SD 72.4). Thirty-five patients were on Onabotulinumtoxin A, whereas thirty-two on monoclonal antibodies targeting CGRP (nine on Galcanezumab, twenty-three on Erenumab). Only ten out of 44 patients with MO underwent an inpatient withdrawal treatment. At baseline, the mean reported headache-days frequency was 21.4 (SD 6.5) per month, while the mean number of severe headache-days of 9.3 (SD 6.6) and a mean monthly medication intake of 23 (SD 19.4).

Results: A statistically significant reduction for all patients regardless of the diagnosis and prophylatic treatment, from baseline to 3 and 6 months of treatment in total headaches days $(p<0.0001)$, high intensity headaches' days $(\mathrm{p}<0.0001)$, MIDAS score $(\mathrm{p}=0.01)$ and analgesics consumption $(\mathrm{p}<0.0001)$ per month was found. The two groups compared (CM and $\mathrm{CM}$ with $\mathrm{MOH}$ ) differed in terms of total headache days $(p=0.004)$, medication intake $(p=0.005)$ and MIDAS score $(p=0.04)$ only at baseline. At baseline, patients with $\mathrm{CM}$ and $\mathrm{MOH}$ reported a higher number of headache days (23.9 versus 18.03), analgesics consumption (27.7 versus 14.04) and MIDAS score (81.3 versus 77.5$)$ compared to patients with chronic migraine. No differences were found at 3 and 6 months of treatment, even when comparing the type of prophylaxis (Onabotulinumtoxin A versus CGRP monoclonal antibodies).

Conclusion: Both groups of patients shared the same response, regardless of the diagnosis. Most importantly, 34 out of 44 patients with $\mathrm{MOH}$ had the same response as the chronic migraine group without attending a withdrawal treatment. However, the number of patients enrolled is small; also, since we've evaluated the treatment response within six months and not further, conclusions are partial. A continuation of the study is needed in order to have more unquestionable data. Nevertheless, this finding could be useful for clinicians when managing migraine overuse headache in order to start with a prophylactic treatment without withdrawal.

\section{THE IMPACT OF SOCIAL DISTANCING ON THE LIFESTYLE OF PATIENTS WITH MIGRAINE: RESULTS FROM A MULTICENTER CROSS-SECTIONAL STUDY}

V. Di Stefano ${ }^{1}$, R. Ornello ${ }^{2}$, A. Torrente ${ }^{1}$, E. Illuminato ${ }^{3}$, V. Caponnetto ${ }^{2}$, I. Frattale ${ }^{2}$, R. Golini ${ }^{4}$, C. Di Felice ${ }^{4}$, F. Graziano ${ }^{1}$, M. Caccamo ${ }^{1}$, D. Ventimiglia $^{1}$, G. Matarazzo ${ }^{2}$, F. Armetta ${ }^{3}$, A. Gagliardo ${ }^{1}$, A. Firenze ${ }^{3}$,

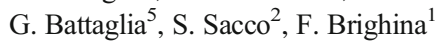

${ }^{1}$ Department of Biomedicine, Neuroscience and Advanced Diagnostic (BIND), University of Palermo (Palermo); ${ }^{2}$ Department of Applied Clinical Sciences and Biotechnology, University of L'Aquila (L'Aquila); ${ }^{3}$ Department of Health Promotion, Mother and Child Care, Internal Medicine and Medical Specialties "G. D'Alessandro", University of Palermo (Palermo); ${ }^{4}$ Department of Internal Medicine, Public Health, Life and Environmental Sciences, University of L'Aquila (L'Aquila); ${ }^{5}$ Department of Psychology, Educational Science and Human Movement, University of Palermo (Palermo)

Background: Social distancing was the measure taken by governments to control the rapid spread of COVID-19 $[1,2]$. This restriction resulted in a sudden change in people's lifestyle, leading to an increase in sedentary behaviors, change in eating habits and sleep-wake cycle [3]. Hence, the aim of this study was to estimate the levels of physical activity (PA), alterations in eating habits and the presence of sleep disturbances among patients with migraine before and during the last week of quarantine.

Methods: A total of 261 patients affected by migraine completed a detailed interview comprehensive of three parts: 1 ) an adapted version of the IPAQ-SF, which measured PA as energy expenditure (MET-minute/ week); 2) a questionnaire that measured the frequency of intake of the main foods; 3 ) the insomnia severity index (ISI) questionnaire regarding the presence of sleep disorders. Participants were enrolled at the "Headache Clinic" of Palermo and Avezzano. The Mann-Whitney U and the Kruskal-Wallis rank-sum tests were used for statistical analyses.

Results: 186 patients (71.3\%) had chronic migraine; 227 patients $(87.0 \%)$ were female; the mean age of patients was $44.5 \pm 12.3$ years. During social distancing, 72 patients $(28 \%)$ reported a headache worsening, $86(33 \%)$ an improvement, and 103 (39\%) a stable headache frequency.

We observed a significant decrease of the mean total amount of weekly PA levels during COVID-19 quarantine ( -183 METs; $p=0.008)$ in the whole sample and a specific decrease in walking (while increasing the sitting time) in each group ( -197 mean METs; $\mathrm{p}<0.001)$. Moreover, patients who reported a worsening of headache during social distancing presented a significant increase in the time spent with computers $(+0.8$ mean hours; $\mathrm{p}<0.001$ ) compared to patients who have improved. No clear association were found depending on eating habits, but a significant 
difference was reported regarding sleep disorders. In fact, patients who presented a stable or worsening headache reported an overall increase on ISI scores during social distancing $(+2.0$ mean points, $\mathrm{p}=0.006$, and +1.0 mean points, $\mathrm{p}=0.007)$ compared with patients that reported an improvement in migraine ( +0.6 mean points; $\mathrm{p}=0.351)$ during social distancing.

Discussion: Our study confirmed that the measures to contain the spread of COVID-19 have affected the practice of PA levels and sleep quality among patients with migraine.

Conclusions: Our experience indirectly suggests that PA and sleep quality should be assessed in migraine sufferers to find strategies for an improvement in quality of life.

References:

1. Velavan TP, Meyer CG. The COVID-19 epidemic. Tropical Medicine and International Health. (2020); 25(3):278-80

2. Bersano A, Pantoni L. On being a neurologist in Italy at the time of the COVID-19 outbreak. Neurology (2020); 94(21):905-06

3. Di Stefano V, Battaglia G, Giustino V, et al. Significant reduction of physical activity in patients with neuromuscular disease during COVID-19 pandemic: the long-term consequences of quarantine [published online ahead of print, 2020 Jul 13]. J Neurol. (2020);1-7. doi:10.1007/s00415-020-10064-6.

\section{A SINGLE-BLIND, RANDOMIZED, CONTROLLED STUDY ON THE EFFICACY OF OSTEOPATHIC MANIPULATIVE TREATMENT IN HIGH-FREQUENCY MIGRAINE (THE REHAB PROJECT)}

L. Fofi ${ }^{1}$, F. Ciallella ${ }^{2}$, F. Scoppa ${ }^{3}$, S. Fantacci Rauber ${ }^{2}$, L. Pacini ${ }^{2}$, G. Egeo $^{1}$, C. Aurilia ${ }^{1}$, S. Proietti ${ }^{4}$, S. Bonassi ${ }^{5}$, P. Barbanti ${ }^{1}$

${ }^{1}$ Headache and Pain Unit, IRCCS San Raffaele (Roma); ${ }^{2}$ Osteopathy School, Chinesis IFOP (Roma); ${ }^{3}$ Masters' Degree Course in Posturology, Faculty of Medicine and Dental Surgery, Sapienza University of Rome; Osteopathy School, Chinesis IFOP (Roma); ${ }^{4}$ Scientific Direction, IRCCS San Raffaele (Roma); ${ }^{5}$ Department of Human Sciences and Quality of Life Promotion of San Raffaele University, Unit of Clinical and Molecular Epidemiology, IRCCS San Raffaele (Roma)

Objective: To assess the efficacy of osteopathic manipulative treatment (OMT) for the prevention of high-frequency episodic migraine (EM).

Material: This is a 2-arm, single blind, randomized, 24 -week, shamcontrolled study. Consecutive adults affected by high frequency (8-14 days/month) EM were prospectively enrolled.

Method: Patients were randomly treated with sham-OMT for 12 weeks followed by real-OMT for other 12 weeks (L-OMT group) or real-OMT for 24 weeks (H-OMT group). Each OMT session lasted 45 minutes and was performed every 2 weeks by board-certified osteopaths. Neurologists and patients were blinded about sham; the allocation of randomization was done by the osteopaths. Monthly migraine days (MMD), monthly analgesic intake (MAI), pain intensity (VAS), migraine disability (MIDAS), quality of life (SF-36), and osteopathic assessment (Zink, Spring and cranial dysfunction test) were evaluated at baseline, after 6 treatments (week 12) and after 12 treatments (week 24) by both neurologists and osteopaths. OMTs' techniques did not follow a pre-determined protocol but were established according to an individual, specific, need-based treatment approach. In group L-OMT, sham-OMT mimicked the real-OMT treatment but involved different, clinically silent body's areas.

Results: 50 patients were enrolled in the study. Their demographic and clinical characteristics were comparable in both OMT group. 5 patients randomized to group L-OMT discontinued for low compliance. At week $12, \mathrm{MMD}$ and MAI were significantly reduced in both OMT groups $[\mathrm{H}-$ OMT: -1.6 day/month ( $\mathrm{p}=0.029),-2.18$ analgesics $(\mathrm{p}=0.003)$; L-OMT: -1 day/month $(\mathrm{p}=0.029),-1.8$ analgesic $(\mathrm{p}=0.012)]$ compared to baseline, whereas MIDAS score was reduced only in H-OMT (-27.2). SF36 score did not vary in both groups. A significant normalization of Zink test ( $\mathrm{p}=$ $0.001)$, Spring test $(p=0.025)$ and cranial dysfunction test $(p=0.003)$ was observed only in H-OMT. At week 24, both H-OMT and L-OMT induced a reduction in MMD [-3.65 days/month $(\mathrm{p}<0.001) ;-4.23$ days/month $(\mathrm{p}$ $<0.001)]$, MAI [-3.95 $(\mathrm{p}<0.001) ;-4.12(\mathrm{p}<0.001)]$, VAS score $[-2.35$ $(\mathrm{p}=0.001) ;-2.12(\mathrm{p}=0.003)]$, and MIDAS score [-26.4 $(\mathrm{p}=0.041)$ and $31.9(\mathrm{p}=0.041)$ ], compared to baseline. SF-36 score did not vary in both groups. Zink $(\mathrm{p}=0.025)$ and cranial dysfunction tests $(\mathrm{p}=0.001)$ improved in L-OMT; cranial dysfunction test further improved in H-OMT $(\mathrm{p}=0.014)$.

Discussion: This is the first prospective trial on OMT in highfrequency EM aimed to evaluating the modification of migraine and osteopathic parameters. We document that both L-OMT and H-OMT induce a progressive reduction of migraine frequency, intensity, analgesic use and disability and an improvement of body balance and adaptive skills. L-OMT seems more effective than H-OMT.

Conclusions: OMT could represent a useful complementary therapy for migraine prevention. Our findings need to be confirmed in larger clinical trials.

References:

- Cerritelli F, Lacorte E, Ruffini N, Vanacore N. Osteopathy for primary headache patients: a systematic review. J Pain Res. (2017) Mar 14;10:601-611. doi: 10.2147/JPR.S130501. eCollection 2017. PMID: 28352200

- Cerritelli F, Ginevri L, Messi G, Caprari E, Di Vincenzo M, Renzetti C, Cozzolino V, Barlafante G, Foschi N, Provinciali L. Clinical effectiveness of osteopathic treatment in chronic migraine: 3Armed randomized controlled trial. Complement Ther Med. (2015) Apr;23(2):149-56. doi: 10.1016/j.ctim.2015.01.011. Epub 2015 Jan 21. PMID: 25847552

- Voigt K, Liebnitzky J, Burmeister U, Sihvonen-Riemenschneider H, Beck M, Voigt R, Bergmann A. Efficacy of osteopathic manipulative treatment of female patients with migraine: results of a randomized controlled trial. J Altern Complement Med. (2011) Mar;17(3):225-30. doi: 10.1089/acm.2009.0673. Epub 2011 Mar 8. PMID: 21385086

\section{POSITIVE EFFECT OF TRIPTANS PREDICT EFFECTIVENESS OF ERENUMAB FOR MIGRAINE PREVENTION: A REAL-LIFE EXPERIENCE}

I. Frattale ${ }^{1}$, R. Ornello ${ }^{1}$, V. Caponnetto ${ }^{1}$, A. Casalena ${ }^{2}$, M. Assetta ${ }^{2}$, M. Maddestra $^{3}$, F. Marzoli ${ }^{3}$, G. Affaitati ${ }^{4}$, M. Giamberardino ${ }^{4}$, S. Viola ${ }^{5}$, A. Gabriele $^{6}$, F. Pistoia ${ }^{1}$, D. Cerone ${ }^{7}$, C. Marini ${ }^{8}$, S. Sacco ${ }^{1}$

${ }^{1}$ Department of Applied Clinical Sciences and Biotechnology, University of L'Aquila (L'Aquila); ${ }^{2}$ Department of Neurology, G. Mazzini Hospital (Teramo); ${ }^{3}$ Department of Neurology, F. Renzetti Hospital (Lanciano$\mathrm{CH}) ;{ }^{4}$ Department of Medicine and Science of Aging, G. D'Annunzio University (Chieti); ${ }^{5}$ Department of Neurology, S. Pio da Pietrelcina Hospital (Vasto-CH); ${ }^{6}$ Neurology Service, SS. Annunziata Hospital (Sulmona-AQ); ${ }^{7}$ Department of Neurology, S. Salvatore Hospital (L'Aquila); ${ }^{8}$ Department of Life, Health and Environmental Sciences, University of L'Aquila (L'Aquila)

Objectives: Triptans are migraine-specific acute drugs that act on serotonin receptors and indirectly inhibit the release of calcitonin gene- 
related peptide (CGRP)(Negro). Monoclonal antibodies linking the CGRP or its receptor, including erenumab, also act on a migrainespecific pathway (Tiseo); to date, there are no predictors of their efficacy. We assessed whether previous response to triptans predicts the later efficacy of erenumab. We also assessed whether the loss of efficacy of triptans over time predicts erenumab ineffectiveness and whether erenumab treatment improves the efficacy of triptans.

Materials: Patients referred to the Headache Centers of the Abruzzo region from January 2019 to March 2020 and treated with erenumab were lincluded in this study. We collected information about use of triptans at any time before starting erenumab treatment. Patients were classified in triptan users and nonusers. Triptan users were classified as 'responders' if they were headache-free within 2 hours after treating at least three migraine attacks with one triptan. Patients who had a $\geq 50 \%$ reduction in monthly migraine days compared with baseline between the 4th and the 6th month of treatment were classified as 'erenumab responders' (Goadsby), while the remaining patients were classified as 'erenumab non-responders'.

Methods: Each patient compiled an online survey in April 2020 about the efficacy of the six triptans available in Italy at any time before and after starting treatment with erenumab. Response to triptans was correlated with that to erenumab treatment.

Results: One hundred and five patients compiled the survey. Ninetyone patients $(86.6 \%)$ had taken at least one triptan in their life; $73(80.2 \%)$ of them were classified as 'triptan responders' and $18(19.8 \%)$ as triptan non-responders. Forty-six (63.0\%) triptan responders reported a loss of efficacy of triptans over time. The proportion of erenumab responders was higher among triptan responders than among triptan nonresponders $(69.9 \%$ vs $38.9 \%$; $\mathrm{P}=0.014)$. Patients reporting a loss of efficacy of triptans over time had a proportion of erenumab responders similar to that of patients not reporting any loss of efficacy of triptans $(60.9 \%$ vs $66.7 \% ; \mathrm{P}=0.565)$. Nineteen $(32.8 \%)$ erenumab responders and 10 $(30.3 \%)$ erenumab non-responders reported an improvement of triptan effectiveness after starting erenumab treatment.

Discussion: According to our results, a history of response to triptans is associated with a possible later response to erenumab, even when triptans had lost their efficacy over time.

Conclusions: The response to triptans can predict response to targeted preventative treatments. An improved response to triptans might be an additional parameter to test the efficacy of preventive drugs.

References:

- Negro A, Lionetto L, D'Alonzo L, Casolla B, Marsibilio F, Vignaroli G, Simmaco M, Martelletti P.Pharmacokinetic evaluation of almotriptan for the treatment of migraines. Expert Opin Drug Metab Toxicol. (2013);9(5):637-44

- Tiseo C, Ornello R, Pistoia F and Sacco S.How to integrate monoclonal antibodies targeting the calcitonin gene-related peptide or its receptor in daily clinical practice. J Headache Pain, (2019); 6;20(1):49

- Goadsby PJ, Reuter U, Hallström Y, Broessner G, Bonner JH, Zhang F, Sapra S, Picard H, Mikol DD, Lenz RA. A Controlled Trial of Erenumab for Episodic Migraine. N Engl J Med, (2017); $377: 2123-32$

\section{TWELVE-MONTHS REAL-LIFE DATA ON PATIENTS TREATED WITH ERENUMAB IN THE ABRUZZO REGION}

I. Frattale $^{1}$, R. Ornello ${ }^{1}$, V. Caponnetto ${ }^{1}$, A. Casalena ${ }^{2}$, M. Assetta ${ }^{2}$, M. Maddestra $^{3}$, F. Marzoli ${ }^{3}$, G. Affaitati ${ }^{4}$, M. Giamberardino ${ }^{4}$, S. Viola ${ }^{5}$, A.

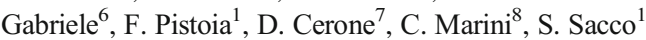

${ }^{1}$ Department of Applied Clinical Sciences and Biotechnology, University of L'Aquila (L'Aquila); ${ }^{2}$ Department of Neurology, G. Mazzini Hospital (Teramo); ${ }^{3}$ Department of Neurology, F. Renzetti Hospital (Lanciano$\mathrm{CH}) ;{ }^{4}$ Department of Medicine and Science of Aging, G. D'Annunzio University (Chieti); ${ }^{5}$ Department of Neurology, S. Pio da Pietrelcina Hospital (Vasto-CH); ${ }^{6}$ Neurology Service, SS. Annunziata Hospital (Sulmona-AQ); ${ }^{7}$ Department of Neurology, S. Salvatore Hospital (L'Aquila); ${ }^{8}$ Department of Life, Health and Environmental Sciences, University of L'Aquila (L'Aquila)

Objectives: Erenumab, a fully human monoclonal antibody against the CGRP receptor (Tiseo), is the first approved as specific migrainetreatment (European Medicine Agency), We aimed to assess the sustained efficacy and safety of erenumab over 12 months in a real-life multicenter study.

Materials: We performed an observational study on patients referred to Headache Centers in the Abruzzo region, Italy, who started treatment with erenumab in 2019 and concluded a 12-month treatment.

Methods: Each patient compiled a migraine diary during the 12month treatment with erenumab. We evaluated MMD (Monthly migraine days), days of acute medication use, a $50 \%$ or $75 \%$ reduction in MMD compared with baseline, and adverse events over four 3-month periods. We also reported the proportion of patients discontinuing treatment for ineffectiveness.

Results: Forty-nine patient were included, mostly female $(87.8 \%)$, with a mean age of $47.7 \pm 10.4$ years. Thirty-six patients (73.5\%) had chronic migraine, while $29(59.2 \%)$ had medication overuse. Two (4\%) patients discontinued treatment with erenumab because of adverse events and $13(26.5 \%)$ because of ineffectiveness. Twenty-two $(44.9 \%)$ patients had a $50 \%$ reduction in MMD during the first trimester, $31(63.3 \%)$ during the second, $31(63.3 \%)$ during the third, and $33(67.3 \%)$ during the fourth, while the corresponding numbers and proportions for the $75 \%$ response were 13 $(26.5 \%),, 20(40.8 \%), 20(40.8 \%)$, and $17(34.7 \%)$, respectively. Among the 31 patients reporting a $50 \%$ response during the second trimester, $27(87.1 \%)$ maintained the response during the third and $30(96.8 \%)$ during the fourth trimester; among the 20 patients reporting a $75 \%$ response during the second trimester, $17(85.0 \%)$ maintained the response during the third and $13(65.0 \%)$ during the fourth trimester. $\mathrm{MOH}$ resolved in $17(58.6 \%)$ patients during the first trimester, $24(82.8 \%)$ during the second, $23(79.3 \%)$ during the third, and $24(82.8 \%)$ during the fourth trimester. Twenty $(40.8 \%)$ patients has adverse events, all reported since the first trimester of treatment, the most common being constipation (30.6\%).

Discussion: Our data confirm the evidence of open-label extensions of the available trials, suggesting that the benefit obtained by two trimesters of erenumab treatment is maintained up to 12 months, in the absence of increased risk of adverse events.

Conclusions: Our multicenter study showed the sustained efficacy and safety of erenumab over 12 months in difficult-to-treat patients with migraine and multiple preventive failures.

References:

- Tiseo C, Ornello R, Pistoia F, Sacco S. How to integrate monoclonal antibodies targeting the calcitonin gene-related peptide or its receptor in daily clinical practice. The Journal of Headache and Pain (2019);20:49

- European Medicine Agency. Aimovig, INN-erenumab. https:// www.ema.europa.eu/en/documents/product-information/aimovigepar-productinformation_en.pdf. (2019) Accessed 25 Nov 2019 


\section{ANY LESSONS FROM ANTI-CGRP MONOCLONAL ANTIBODIES IN CLUSTER HEADACHE?}

\section{Giani, A. Proietti Cecchini, M. Leone}

Neuroalgology Unit, Foundation IRCCS Carlo Besta Neurological Institute (Milano)

Objectives: Many observations suggested Calcitonin Gene Related Peptide (CGRP) as a key molecule in the pathophysiology of $\mathrm{CH}$ : CGRP increases in the ipsilateral-to-the pain jugular vein during $\mathrm{CH}$ attacks and decreases when pain improves after acute treatments. Moreover, infusion of CGRP can induce $\mathrm{CH}$ attacks in $\mathrm{CH}$ patients. On these bases monoclonal antibodies (MoAb) against CGRP have been tested in the prophylaxis of cluster headache $(\mathrm{CH})$. Aim of the present work is to examine results from these studies and reconsider the role of CGRP in $\mathrm{CH}$.

Methods: Four randomized clinical trials have been conducted in $\mathrm{CH}$, two in episodic $\mathrm{CH}(\mathrm{ECH})$ and two in chronic $\mathrm{CH}(\mathrm{CCH})$. In the galcanezumab studies a total of $343 \mathrm{CH}$ patients (episodic and chronic) were randomized and treated; 166 received galcanezumab (49 ECH, 117 $\mathrm{CCH})$ and 177 were assigned placebo $(57 \mathrm{ECH}$ and $120 \mathrm{CCH})[1,2]$. The two fremanezumab studies (episodic and chronic $\mathrm{CH}$ ) were prematurely interrupted.

Results: In ECH galcanezumab proved effective in reducing the frequency of attacks [1]. The drug did not improve $\mathrm{CCH}$ [2]. Both fremanezumab studies (episodic and chronic $\mathrm{CH}$ ) were prematurely interrupted after the pre-specified interim futility analysis concluded that the primary endpoint, reduction in weekly cluster attacks, would not be achieved. It has been proposed that the different results in ECH could be due to different study design. As expected, in the ECH trials both active and placebo groups had a favourable outcome at the end of the first month due to the natural ending of cluster periods [1]. In the galcanezumab study the endpoint was set at week 3 while in the fremanezumab study it was at week 4 . In the $\mathrm{ECH}$ trial galcanezumab had a therapeutic gain over placebo of nearly 10 attacks less in 3 weeks. The number needed to treat (NNT) to obtain a $50 \%$ reduction of headache frequency at week 3 was $5.6[3]$.

Discussion: The high cost imposes to carefully estimate costeffectiveness of the drug over current treatments. Galcanezumab could be considered as a valid option in cases of treatment failure or when facing safety or tolerability issues. In addition, studies aimed to identify responders to the drug are highly desirable. Both galcanezumab and fremanezumab did not improve chronic $\mathrm{CH}$ $(\mathrm{CCH})$ : this and other observations suggest a different role of CGRP in the two conditions.

Conclusions: The role of CGRP needs to be carefully re-examined in $\mathrm{CH}$, particularly in the chronic form.

References:

1. Goadsby PJ, Dodick DW, Leone M, et al. Trial of galcanezumab in prevention of episodic cluster headache. N Engl J Med (2019); 381: $132-41$

2. Dodick DW, Goadsby PJ, Lucas C, et al. Phase 3 randomized, placebo-controlled study of galcanezumab in patients with chronic cluster headache: Results from 3-month double-blind treatment. Cephalalgia (2020); 033310242090532

3. Giani L, Proietti Cecchini A, Leone M. Galcanezumab for the prevention of Cluster Headache. Expert Opin Biol Ther (2020); Aug 4;1-9

\section{CHRONIC HIV EXPOSURE AND HEADACHE IN SUB- SAHARAN AFRICA: A PILOT STUDY}

\author{
L. Giani ${ }^{1}$, M. Mwazangati ${ }^{2}$, D. Uluduz ${ }^{3}$, T. Șașmaz ${ }^{4}$, M. Kamponda ${ }^{2}$, V. \\ Tamba Tolno ${ }^{2}$, G. Guidotti ${ }^{2}$, M. Marazzi ${ }^{2}$, T. Steiner ${ }^{5}$, M. Leone $^{1}$
}

${ }^{1}$ Neuroalgology Unit, Foundation IRCCS Carlo Besta Neurological Institute (Milano); ${ }^{2}$ DREAM Program, Community of S. Egidio (Blantyre-ZA) ${ }^{3}$ Neurology Department, Cerrahpasa School of Medicine (Istanbul-TR); ${ }^{4}$ Department of Public Health, Mersin University School of Medicine (Mersin-TR); ${ }^{5}$ Lifting The Burden, the Global Campaign against Headache (London-UK)

Objectives: Primary headache disorders in sub-Saharan Africa (SSA) have been found to be at least as prevalent as in high-income western countries [1]. In SSA about 26 million people are HIV+. These patients are living longer thanks to improved access to antiretroviral treatments but continuously exposed to a virologically suppressed HIV infection: non-AIDS complications including neurological disorders are increasing as HIV increases the risk to develop stroke and epilepsy, both having relevant associations with headache. Data about headache in the HIV+ population are scarce. The aim of this pilot study was to make a first estimate of headache prevalence and burdens attributable to headache in a HIV+ population in a SSA country.

Materials: The study was conducted in Malawi, a SSA country with 18.5 million population where medical activities are usually performed by non-medical health workers due to lack of doctors. The study was conducted in collaboration with the DREAM (Disease Relief through Excellent and Advanced Means) program, active in 12 SSA countries (since 2005 in Malawi) to provide health care for HIV/AIDS and noncommunicable diseases (NCDs) [2].

Methods: Trained personnel at the DREAM Centre in Blantyre [3] administered a structured questionnaire to consecutively attending HIV+ patients aged 6-65 years who had been followed for at least 1 year under ART.

Results: Among the 500 participants (mean age 34.2years, $71.8 \%$ female) viral load was undetectable in $84.0 \%$. The overall 1-year prevalence of any headache was $80.4 \%$. In the month preceding interview $80.6 \%$ subjects had at least one headache, with a mean of $5.5 \pm 5.5$ headache days/month and mean headache duration of $9.5 \pm 20.8$ hours. The estimated proportion of time spent with headache over the last month was $4.7 \%$. Analgesics were used by $77.4 \%$. Days of work lost because of headache averaged $0.5 \pm 1.9$ days/month, ie $2.3 \%$ of all workdays were lost to headache. Headache yesterday was reported by $16.4 \% ; 4.0 \%$ of the whole sample were completely disabled (3.8\%partially disabled). Considering yesterday was a typical day, our findings indicate that one in six HIV+ patients would have headache on any particular day, and almost half of these would be at least partially disabled as a consequence.

Discussion: This is the first study investigating headache occurrence and its burden in a HIV+ population in a SSA country.

Conclusions: The results show that headache is an underreported health problem among HIV+ patients and call for further studies to understand the relationship between continuous HIV exposure and headache.

References:

1. Zebenigus M, Tekle-Haimanot R, Worku DK, Thomas H, Steiner TJ. The prevalence of primary headache disorders in Ethiopia. J Headache Pain (2016); 17: 110. doi: 10.1186/s10194-016-0704-z

2. DREAM Community of Sant'Egidio. In: DREAM. https://dream. santegidio.org/?lang=en/. Accessed 30 Jul 2020 
3. Leone M, Palombi L, Guidotti G, Ciccacci F, Lunghi R, Orlando S, Nurja MA, Sangare MH, Marazzi CM. What headache services in sub-Saharan Africa? The DREAM program as possible model. Cephalalgia (2019); 39: 1339-40

\section{DRUG RESPONSIVENESS IN CLUSTER HEADACHE: VERAPAMIL. RESULTS FROM THE CLUSTER HEADACHE DATABASE}

\section{Giani, A. Proietti Cecchini, M. Leone}

Neuroalgology Unit, Foundation IRCCS Carlo Besta Neurological Institute (Milano)

Objectives: Verapamil is the drug of first choice for the preventive treatment of cluster headache $(\mathrm{CH})$ [1]. In this study we retrospectively assessed the response to verapamil to evaluate its real-life effectiveness in a wide $\mathrm{CH}$ population.

Materials: Clinical records of 542 patients diagnosed as trigeminal autonomic cephalalgias (TACs) at our Centre from 2011 were retrospectively investigated. Clinical data were extracted from a dedicated database at our Institution. Patients with a definite diagnosis of $\mathrm{CH}$ according to the International Headache Society diagnostic criteria [2] last visited from $2014 / 01 / 01$ to $2019 / 05 / 31$ were included.

Methods: Response was evaluated for the most recent trial of the drug. Change in headache frequency was considered to evaluate the drug response: "Good" when reduction was $\geq 50 \%$; "Partial": $<50 \%$ reduction and continuing treatment; "No": no change or worsening; "Unknown": response was not clearly reported; "Tolerance": no present improvement, but with a previous successful trial or recurrence while continuing therapy. Diagnosis (chronic or episodic $\mathrm{CH}$ ), sex, disease length was considered as possible modifying factors We report descriptive statistics, Chisquare tests for different distribution of response between diagnostic and gender groups, One-way ANOVA test for disease duration between response groups. Differences were considered significant for $\mathrm{p}<.05$.

Results: A total of $330 \mathrm{CH}$ patients were included, $39 \%(\mathrm{~N}=130)$ chronic $(\mathrm{CCH}), 61 \%(\mathrm{~N}=200)$ episodic $(\mathrm{ECH}), 18 \%(\mathrm{~N}=60)$ females, mean age 45.8 years, mean disease duration 14.4 years. Out of 330 subjects, $75 \%(\mathrm{~N}=245)$ had tried verapamil $(15 \%$ female, $46 \%$ chronic $\mathrm{CH})$; 45 patients with an unknown response were excluded from further analysis. Verapamil improved $\mathrm{CH}$ in $80 \%(\mathrm{~N}=160)$ of the patients: response was good in $21 \%(\mathrm{~N}=42)$, partial in $28.5 \%(\mathrm{~N}=57)$, transient in $31.5 \%$ $(\mathrm{N}=63) ; 19 \%(\mathrm{~N}=38)$ never responded to verapamil. Sex didn't affect the response. Patients with chronic $\mathrm{CH}$ had a greater risk to develop tolerance vs ECH (41\% vs $21 \%$; OR 2.6; $95 \%$ CI 1.4 - 4.8; p 0.004). The mean disease length was similar in $\mathrm{ECH}$ and $\mathrm{CCH}$ (16.6 vs 15 years, $\mathrm{p} 0.3)$ and among response groups ("good" 11.9, "partial" 17.3, "no" 17.4, "tolerance" 14.8 years; $p$ 0.56).

Discussion: In our sample, verapamil produced sustained improvement in about $50 \%$ of $\mathrm{CH}$ patients. A high proportion (about one third) of patients develop tolerance to verapamil: a better understanding of the mechanisms leading to tolerance is necessary.

Conclusions: These results highlight the urgent need to expand the therapeutic armamentarium against this invalidating condition.

References:

1. May A, Leone M, Áfra J, Linde M, Sándor PS, Evers S, Goadsby PJ. EFNS guidelines on the treatment of cluster headache and other trigeminal-autonomic cephalalgias. Eur. J. Neurol. (2006);13:1066-77
2. Headache Classification Committee of the International Headache Society (IHS). The International Classification of Headache Disorders, 3rd edition. Cephalalgia (2018);38:1-211. doi: $10.1177 / 0333102417738202$ RISK FOR DEVELOPING CHRONIC CLUSTER HEADACHE:
SURVIVAL ANALYSIS AND POSSIBLE PREDICTIVE FACTORS

\author{
L. Giani, A. Proietti Cecchini, M. Leone
}

Neuroalgology Unit, Foundation IRCCS Carlo Besta Neurological Institute (Milano)

Objectives: Patients with episodic cluster headache $(\mathrm{CH})$ can develop a chronic unremitting course with a huge impact on their lives [1]. The probability and mechanisms of this transformation are unclear [2]. We aimed to estimate the long-term risk of developing chronic $\mathrm{CH}(\mathrm{CCH})$ after an episodic onset using survival analysis. Next, we questioned if sex and age at onset of $\mathrm{CH}$ have any influence on the process.

Materials: Data was collected from clinical documentation of 539 patients with a diagnosis of $\mathrm{CH}$ visited at our Institute between 1/1/ 2011 and 19/3/2020.

Methods: The following were extracted: diagnosis at last observation, sex, age at onset of disease, disease course, year of onset of the chronic form. The possible disease courses were: episodic throughout all the disease duration $(\mathrm{ECH})$; $\mathrm{CCH}$ developed after episodic onset (secondary $\mathrm{CCH}, \mathrm{sCCH}$ ); chronic since onset (primary $\mathrm{CCH}, \mathrm{pCCH}$ ); Unknown, if disease length less than 1 year - too short to diagnose episodic or chronic $\mathrm{CH}$ - or the clinical course not clear. Subjects with pCCH or Unknown course, or with missing data were excluded. The Kaplan-Meier method was used to examine the time before the development of sCCH ("survival") at 5, 10, 20, 30 years in the whole population. The Log-Rank Test was used to compare the survival between sexes at 5, 10, 20 years and between groups defined by decades of age at onset at 5 years.

Results: ECH were 357 (66.2\%), $\mathrm{CCH}$ were 182 (33.8\%); 449 $(83.3 \%)$ were male. $\mathrm{CH}$ courses were: $\mathrm{ECH} 334$ (62\%), pCCH 82 (15.2\%), sCCH 97 (18\%), Unknown 26 (4.8\%). Cases suitable for survival analysis were 349 (261 $\mathrm{ECH}$ and $88 \mathrm{sCCH})$. The cumulative proportion developing $\mathrm{sCCH}$ was $9.3 \%, 18.1 \%, 24.5 \%$, $36 \%$ at $5,10,20,30$ years respectively. Patients with a later onset of $\mathrm{CH}$ had a higher risk of becoming chronic within 5 years (p 0.015 for overall comparison, p 0.05 for linear trend). Sex may have an influence in the long-term, but didn't reach statistical significance $(\mathrm{p}=0.7$ at 5 years, $\mathrm{p}=0.09$ at 20 years).

Discussion: Overall more than one third of ECH patients shift to $\mathrm{sCCH}$. Age at onset influences the transformation from $\mathrm{ECH}$ to $\mathrm{sCCH}$.

Conclusions: The provided estimated risk of developing $\mathrm{CCH}$ may be of help to inform patients. Further clinical research on wider samples may disclose other factors influencing the transformation from $\mathrm{ECH}$ to $\mathrm{CCH}$.

References:

1. D’Amico D, Rigamonti A, Solari A, Leone M, Usai S, Grazzi L, Bussone \& G, Bussone G. Health-related quality of life in patients with cluster headache during active periods. Cephalalgia (2002);22:818-21

2. Torelli P, Manzoni GC. What predicts evolution from episodic to chronic cluster headache? Curr Pain Headache Rep (2002);6:6570. doi: 10.1007/s11916-002-0026-5 


\section{MINDFULNESS AS AN ADD-ON TREATMENT FOR PATIENTS WITH CHRONIC MIGRAINE AND MEDICATION OVERUSE: A PRELIMINARY ANALYSIS}

\author{
L. Grazzi ${ }^{1}$, E. Sansone ${ }^{2}$, A. Raggi ${ }^{2}$, M. Leonardi ${ }^{2}$, D. D'amico ${ }^{1}$
}

${ }^{1}$ Neuroalgology Unit, Headache Center, Fondazione IRCCS Istituto Neurologico Carlo Besta (Milano); ${ }^{2}$ Neurology, Public Health and Disability Unit, Fondazione IRCCS Istituto Neurologico Carlo Besta (Milano)

Objective: Chronic migraine (CM) is a disabling condition often associated with medication overuse. This condition involves psychological and social problems and it is difficult to manage. The joint use of pharmacological and non-pharmacological techniques is desirable to evaluate the pathology in a bio-psycho-social perspective and improve the trend of clinical indices. In recent years, various types of non-pharmacological treatments have given encouraging results and Mindfulness in particular helps patients to better manage their pain. Our aim is to test the use of Mindfulness as a therapy in addition to usual pharmacological prophylaxis.

Materials: We collected data at baseline and 3 months follow-up. The frequency of headaches and the use of medications were collected through diary. The level of disability with the Migraine disability assessment (MIDAS), the impact with the Headache Impact Test (HIT-6) and people's awareness of their inner state with the Mindfulness Awareness Attention Scale (MAAS).

Methods: Patients, enrolled with the diagnosis of $\mathrm{CM}$ and $\mathrm{MOH}$ according to the ICHD 3rd edition criteria, are randomized into one of two groups: TaU Group i.e. treatment as usual (withdrawal, psychoeducation, pharmacological prophylaxis) and MIND Group (TaU program plus 6 weekly sessions of 45 minutes of Mindfulness Therapy). The sessions include exercises and meditations to be done in small groups under the supervision of a psychologist and home assignments. Means and standard deviations were used to represent data. Non-parametric repeated measures tests were used to analyze data.

Results: 76 patients (36 MIND Group and 40 TaU Group) completed the 3 months follow up. Data reveal an improvement in headache frequency (approximately 11-13 days reduction) and drug consumption (approximately 26-29 intakes reduction) in both groups. A similar trend in other endpoints, namely disability and impact levels, and $50 \%$ responder rate, was observed, with significant results in both groups, and more evident changes in the MIND Group than in the TaU Group.

Discussion: Our results show that patients receiving Mindfulness as an add on to TaU seem to have a better short-term trend than patients in the TAU group in terms of clinical outcome in particular on headache frequency and medication intake; moreover, they seem to have a stronger improvement in disability and impact.

Conclusions: Our results provide initial support for the beneficial effect of mindfulness-based treatment in the management of $\mathrm{CM}-\mathrm{MOH}$. Further data are needed to confirm whether the improvement herein described will be maintained over time.

\section{NEUROPHYSIOLOGICAL CHANGES AND CLINICAL OUTCOME IN A COHORT OF MIGRAINE PATIENTS TREATED WITH ERENUMAB}

A. Laporta, S. Quitadamo, G. Libro, E. Vecchio, M. Delussi, K. Ricci, M. de Tommaso
Department of Basic Medical Sciences, Neurosciences and Sense Organs, University of Bari (Bari)

Background: Migraine is a neurological pain syndrome that causes a negative impact on the patients' quality of life. Its treatment is frequently complicated by the failure of preventive therapies. Adverse events and limited effectiveness associated with these treatments highlight the need for an alternative approach [1]. Recent studies reveal the role of Calcitonin Gene Related Peptide (CGRP) in migraine pathogenesis [2]. Erenumab is a fully human monoclonal antibody blocking the CGRP receptors, recently approved for the treatment of drug resistant migraine. Symptomatic drugs and preventive treatments modulate laser evoked potentials (LEP) amplitude and habituation in migraine patients [3].

Objectives: To evaluate the effect of single dose of Erenumab $70 \mathrm{mg}$ on LEP and correlate the neurophysiological changes with clinical outcome on migraine frequency, intensity and allodynia after 6 monthly doses.

Methods: We included migraine patients not responding to at least three preventive drugs belonging to the tertiary Headache Centre of Applied Neurophysiology and Pain Unit of Bari Policlinico General Hospital from September to November 2019. All clinical and therapeutic data of these patients were collected. LEPs were recorded before, 1hour and 15 days after Erenumab $70 \mathrm{mg}$ injection. LEPs were recorded by 61 scalp electrodes and for LEP stimulation, cutaneous heat stimuli were delivered by a $\mathrm{CO} 2$ laser. ANOVA analysis was performed including these covariates: percent rate of change of headache frequency, visual analogue scale (VAS) score and allodynia after 6 months treatment. For the topographical analysis of statistical changes of LEP amplitudes performed a point by point ANOVA analysis at $256 \mathrm{~Hz}$ resolution, with condition T0 vs T1 vs T2 as within factor.

Results: Statistical analysis shows a significant decrease of the N1 wave on the left temporal and central frontal regions, and N2 wave on bilateral temporo-parietal regions. The $t$ test confirmed a significant amplitude reduction in $\mathrm{T} 2$ condition for the $\mathrm{N} 1$ and $\mathrm{N} 2$ waves, as compared to the basal $\mathrm{T} 0$ condition. In the $\mathrm{T} 1$ condition emerged a slight decrease of the N2 wave on the prefrontal regions. After six months of Erenumab treatment, patients showed a significant improvement of headache frequency, intensity and allodynia. No correlation between neurophysiological changes and clinical data emerged.

Conclusions: Enerumab exerts an inhibiting effect on cutaneous adelta fibers in the facial district, acting on the CGRP receptors widely represented in the trigeminal system.

References:

1. M. Delussi, E. Vecchio, S. G. Quitadamo, G. Libro, M. de Tommaso Failure of Preventive Treatments in Migraine: an Observational Retrospective Study in a Tertiary Headache Center BMC Neurol (2020);20;256. https://doi.org/10.1186/s12883-020-01839-5

2. S. Lyengar, K. Johnson, M. Ossipov, S. Aurora.CGRP and the Trigeminal System in Migraine The Journal of Head and Face Pain (2019);59:659-681

3. de Tommaso M, Losito L, Libro G, et al. Effects of symptomatic treatments on cutaneous hyperalgesia and laser evoked potentials during migraine attack. Cephalalgia (2005);25(5):359368.doi:10.1111/j.1468-2982.2004.00866.x

\section{IMPLEMENTING THE TRAINING TOOL FOR MANAGERS: THE ROLE OF EMPLOYMENT IN THE CARE PATHWAY OF NEUROLOGICAL PATIENTS}

\author{
M. Leonardi, M. Eigenmann, C. Scaratti, F. Silvaggi
}


Neurology, Public Health and Disability Unit, Foundation IRCCS Neurological Institute Carlo Besta (Milano)

Objective: The number of people with chronic diseases (CDs) is dramatically increasing with significant social and economic implications for the employment sector. CDs can bring to have poorer employment prospects and to early retirement. This regards in particular people with psychiatric and neurological conditions such as migraine, multiple sclerosis and stroke. Therefore, it is fundamental to redesign the role of the workplace, developing employers' and employees' skills in facing such challenge. Misinformation on the work ability of people with CDs, can result in stigma and discriminatory attitudes.

Material: The EU Joint Action "Chrodis Plus: Implementing good practices for Chronic Disease", has a specific work package on employment and chronic diseases that has developed a Training Tool for managers based on a biopsychosocial approach to health, targeting human functioning, and chronic diseases commonalities.

Methods: A pilot testing of such tool, conducted in 7 organizations from 5 European countries, has been developed to test and ensure the feasibility and usability of the tool in the workplace.

Results: Results show that the Training Tool helps managers to increase their own and others awareness on the benefits of a good management of CDs in the workplace, reducing discrimination, opening the way for increased employment, and improving the quality of life of people with CDs. The training sections deal with inclusiveness, workability and strategies for helping people with CDs to stay or return to work.

Discussion: Results suggest the Chrodis Plus Checklist on environmental inclusiveness, which results feasible to screen areas of the organization, which need actions for a better inclusion of all employees. Composed of 40 items, it has been evaluated as an effective first step towards the generation of new ideas for action.

Conclusion: Targeting managers for promoting inclusion and participation of all employees in the labor market through the implementation of this training tool, will have numerous benefits: employees affected by chronic conditions will have the possibility to include employment in their care pathway; the organization as a whole will benefit from the inclusion and achieved work ability of all its employees.

\section{ONE-YEAR ERENUMAB EFFECTIVENESS IN A HIGH FREQUENCY AND CHRONIC MIGRAINE COHORT: A PATIENT-PERCEIVED SATISFACTION ANALYSIS}

D. Mascarella, V. Favoni, E. Matteo, U. Pensato, G. Asioli, E. Merli, C. Calabrò, A. Pascazio, P. Cortelli, G. Pierangeli, S. Cevoli

IRCCS ISNB Institute of Neurological Science of Bologna, University of Bologna (Bologna)

Objectives: We aimed to assess patient-perceived satisfaction regarding one-year therapy with the Anti-CGRP-R monoclonal antibody Erenumab in a cohort of high-frequency episodic migraine (HFEM) and Chronic Migraine (CM) patients.

Materials: This observational study includes patients who completed a prospective, monocentric, real-life cohort study conducted in the Headache Centre of the IRCCS-Istituto delle Scienze Neurologiche di Bologna, between May 2019 and July 2020. Inclusion criteria were the following: 1) patients with HFEM (8-14 headache days/month) or CM ( $\geq 15$ headache days/month) with or without Medication Overuse. All eligible patients received a maximum of one-year treatment with Erenumab once every 4 weeks. Erenumab dose was $70 \mathrm{mg}$ for the first two months; then, according to the reduction in monthly migraine days (MMDs), we decided whether to continue with Erenumab $70 \mathrm{mg}$ or to switch to Erenumab $140 \mathrm{mg}$.

Methods: All patients that completed one-year treatment with Erenumab, were interviewed. We evaluated patient-perceived satisfaction in three domains: Headache Frequency Reduction Satisfaction (HFRS), Headache Intensity Reduction Satisfaction (HIRS) and Overall Satisfaction (OS). All patients were asked to express their satisfaction on a subjective numerical scale from 1 (absolutely unsatisfactory) to 10 (completely satisfactory) for each of the three domains mentioned above.

Results: 159 patients joined the study. In July 2020, 39 of the 159 patients completed one year of treatment. 26 (67\%, 14 females, 12 males) accepted the interview. 24 out of 26 patients $(92 \%)$ rated their HFRS $\geq 6$ and total mean HFRS value was $7.66( \pm 1.64)$. Mean value for HIRS was $7.8( \pm 1.21)$ and 25 of the 26 patients $(96 \%)$ experienced a subjective reduction in headache intensity (rate $\geq 6$ ). We observed an Overall Satisfaction in $100 \%$ of the cohort ( 26 out of 26 rated $\geq 6$ ), with a mean rating of $7.73( \pm 1.30)$ ) 2 patients were unsatisfied with HFRS, 1 patient was unsatisfied with HIRS. None of the patients reported unsatisfaction in more than one of the three items.

Discussion: These results demonstrate a long-term satisfaction with Erenumab treatment in terms of reduction of both Headache Frequency and Headache Intensity, even in a cohort of patient with HFEM and CM. Furthermore, levels of satisfaction among the three domains were comparable. However, the exclusion of the few non-responder patients after 3 months of treatment is a limit of this study.

Conclusion: We assessed a high level of patient-perceived satisfaction in all domains evaluated after one-year treatment with Erenumab.

\section{VESSEL-WALL MRI STUDY IN PRIMARY HEADACHES}

\author{
E. Merli, A. Rustici, V. Favoni, G. Pierangeli, P. Cortelli, S. Cevoli, L. \\ Cirillo
}

IRCCS Institute of Neurological Sciences of Bologna (Bologna)

Objectives: To highlight possible vassal alterations during migrainous attack or cluster period using Vessel-wall MRI study (VW-MRI).

Materials and Methods: We enrolled 8 patients with migraine without aura (1 M, 7 F; mean age 43 years) and 8 patients (7 M, 1 F; mean age 46 years) with episodic cluster headache. They underwent VW-MRI study outside the pain or during migrainous attack/cluster period. Our protocol includes high resolution (HR) VW-MRI with HR T1-weighted (T1-w) black-blood (BB) vessel wall sequence before and after intravenous administration of gadolinium. BB MRI sequences are utilized with 3dimensional (3D) pre- and post-contrast T1-weighted sequences to characterize the vessel wall and demonstrate pathological vessel wall enhancement as a marker of inflammation.

Results: No vassal alterations were registered both during and outside the migrainous attack/cluster period. We excluded any characteristic vassal wall enhancement of the two types of headache and the possibility to correlate enhancement patterns to clinical characteristics of headache (eg. side, intensity, pulsatility of pain).

Discussion: Computed tomography angiography and MR angiography are able to define luminal characteristics (such as sac size, location, and morphology) but not structural wall changes $[1,2]$. VW-MRI has a high sensitivity and negative predictor factor for vassal instability. VW-MRI enhancement can detect in-vivo vassal inflammation [2,3]. It is diffusely used in clinical practice to investigate cases of possible reversible cerebral 
vasoconstriction syndrome, primary angioitis of central nervous system, systemic vasculitis with central nervous system involvement and intracranial aneurysms [1]. VW-MRI enhancement has a very high sensitivity $(95.0 \%)$, specificity $(62.7 \%)$, positive predictive value $(55.8 \%)$, and negative predictive value $(96.2 \%)$ in identifying unstable aneurysms [3]. The lack of wall enhancement is a strong predictor of aneurysm stability as it has a negative predictive factor of $96 \%$ (CI: 92.8 - 98.0) [3]. Our results show that VW-MRI study in primary headaches in negative for any alteration both during and outside the pain. This data confirms its high negative predictive factor for vassal instability and secondary headaches.

Conclusion: VW-MRI study is negative in patients with primary headaches, outside and during migrainous attack/cluster period. VWMRI is a useful tool in distinguishing primary to secondary primary headaches. It may play a role in diagnostic and therapeutic decisionmaking in complex doubtful cases of patients affected by migraine or cluster headache presenting with an attack with atypical characteristics.

References:

1. D.M. Mandell, M. Mossa,Basha, Y. Qiao, C.P. Hess, F. Hui, C. Matouk, M.H. Johnson, M.J.A.P. Daemen, A. Vossough, M. Edjlali, D. Saloner, S.A. Ansari, B.A. Wasserman, D.J. Mikulis, Intracranial Vessel Wall MRI: Principles and Expert Consensus Recommendations of the American Society of Neuroradiology, American Journal of Neuroradiology (2017);38 (2): 218-229; DOI: 10.3174/ajnr.A4893

2. Larsen N, von der Brelie C, Trick D, Riedel CH, Lindner T, Madjidyar J, Jansen O, Synowitz M, Flüh C, Vessel Wall Enhancement in Unruptured Intracranial Aneurysms: An Indicator for Higher Risk of Rupture? High-Resolution MR Imaging and Correlated Histologic Findings, AJNR Am J Neuroradiol. (2018) Sep; 39(9):1617-1621. doi: 10.3174/ ajnr.A5731. Epub 2018 Jul 19

3. Texakalidis P, Hilditch CA, Lehman V, Lanzino G, Pereira VM, Brinjikji W, Vessel Wall Imaging of Intracranial Aneurysms: Systematic Review and Meta-analysis, World Neurosurg (2018) Sep; 117:453-458.e1. doi: 10.1016/j.wneu.2018.06.008. Epub 2018 Jun 12

\section{RESTING STATE FUNCTIONAL CONNECTIVITY CHANGES OF THE HYPOTHALAMUS IN MIGRAINE PATIENTS: A CROSS- SECTIONAL AND LONGITUDINAL STUDY}

\author{
R. Messina ${ }^{1}$, P. Valsasina ${ }^{1}$, P. Misci ${ }^{1}$, M. Filippi ${ }^{2}$, M. Rocca $^{3}$
}

${ }^{1}$ Neuroimaging Research Unit, Institute of Experimental Neurology, Division of Neuroscience, IRCCS San Raffaele Scientific Institute (Milano); ${ }^{2}$ Neuroimaging Research Unit, Institute of Experimental Neurology, Division of Neuroscience; Neurology Unit and Neurophysiology Unit, IRCCS San Raffaele Scientific Institute; VitaSalute San Raffaele University (Milano); ${ }^{3}$ Neuroimaging Research Unit, Institute of Experimental Neurology, Division of Neuroscience; and Neurology Unit, IRCCS San Raffaele Scientific Institute (Milano)

Objective: Previous studies support the role of the hypothalamus in the early and interictal phase of the migraine attack. The aim of our study was to explore cross-sectional and longitudinal resting state functional connectivity (RS FC) changes of the hypothalamus in patients with migraine.

Materials: Using a 3.0 Tesla scanner, RS functional magnetic resonance imaging (MRI) and 3D T1-weighted scans were acquired from 92 headache-free episodic migraine patients and 73 controls. Twenty-three migraineurs and 23 controls were reexamined after 4 years.
Methods: Maps of hypothalamic RS FC were obtained for each subject using a seed-region correlation approach. A whole-brain voxel-wise analysis was performed to assess hypothalamic RS FC abnormalities between groups using the general linear model and theory of gaussian fields, as implemented in SPM12. The correlation between functional MRI abnormalities and patients' clinical characteristics was also investigated.

Results: At baseline, compared to controls, migraine patients showed a decreased RS FC between the right and left hypothalamus and the right cerebellum, left parahippocampus and bilateral orbitofrontal cortex (OFC). The left hypothalamus had also a decreased RS FC with the left middle frontal gyrus. While, the right hypothalamus had a decreased RS FC with the right inferior temporal gyrus, lingual gyrus and left calcarine cortex. At baseline, the decreased RS FC between the right hypothalamus and the ipsilateral lingual gyrus correlated with higher migraine attack frequency ( $\mathrm{r}=-0.4, \mathrm{p}<0.05$, FWE corrected). After 4 years, migraine patients developed an increased FC between the hypothalamus and the OFC, bilaterally, while RS FC between the right hypothalamus and the ipsilateral lingual gyrus decreased. RS FC between the right hypothalamus and the ipsilateral OFC correlated with lower migraine attack frequency at year 4 ( $r=-0.6, \mathrm{p}<0.001$, uncorrected $)$.

Discussion: During the interictal phase, the hypothalamus modulates the activity of pain and visual processing areas in migraine patients. The recurrent experience of migraine attacks might disrupt the functional interaction between the hypothalamus and high-order visual processing areas. An increased RS FC between the hypothalamus and brain areas belonging to the descending pain-inhibitory pathway might reduce migraine attack frequency over time.

Conclusions: RS FC of the hypothalamus changes dynamically in migraine patients. Longitudinal assessment of the functional interaction between the hypothalamus and the other brain areas might elucidate its role in migraine pathophysiology and its influence on disease activity.

\section{TRACKING THE EVOLUTION OF NON-HEADACHE SYMPTOMS THROUGH THE MIGRAINE ATTACK}

\author{
R. Messina ${ }^{1}$, I. Cetta ${ }^{1}$, B. Colombo ${ }^{1}$, M. Filippi ${ }^{2}$
}

${ }^{1}$ Neurology Unit, IRCCS San Raffaele Scientific Institute (Milano); ${ }^{2}$ Neuroimaging Research Unit, Institute of Experimental Neurology, Division of Neuroscience; Neurology Unit and Neurophysiology Unit, IRCCS San Raffaele Scientific Institute; Vita-Salute San Raffaele University (Milano)

Objective: Previous studies have highlighted non-headache symptoms associated with migraine occurring during the prodrome or postdrome phase. However, non-headache symptoms can start before the onset of the pain, persist during the headache phase, and can go on after migraine headache resolution. The aim of this study was to track the evolution of non-headache symptoms through the different phases of the migraine attack.

Materials: One hundred and eight migraine patients ( 81 females, mean age: 47 years) were enrolled. Patients were asked to recall retrospectively whether non-headache symptoms occurred during the prodrome, headache and postdrome phase of their migraine attacks.

Methods: The Cochran's Q test was performed to assess any significant differences in the frequency of non-headache symptoms across the three phases of the migraine attack. The co-occurrence of symptoms 
during the different phases was tested using Cohen's and Fleiss' kappa $(\mathrm{k})$ with $95 \%$ confidence intervals $(\mathrm{CI})$.

Results: The frequency of most non-headache symptoms was significantly different throughout the three phases of the migraine attack, being higher during the headache phase than during the prodrome and postdrome phase. The rate of thirst and food craving did not significantly change over the migraine attack. The symptoms with the highest co-occurrence through all three phases were food craving ( $\mathrm{k}=0.35,95 \%$ CI $0.34-0.35)$, neck stiffness $(\mathrm{k}=0.34,95 \%$ CI $0.33-0.34)$ and thirst $(\mathrm{k}=0.34,95 \%$ CI $0.33-0.34)$. If thirst occurs during the headache phase it is highly likely that it will persist during the postdrome phase $(\mathrm{k}=0.59,95 \%$ CI $0.59-0.60)$, while if neck stiffness occurs during the prodrome phase it will probably occur also during the headache phase $(\mathrm{k}=0.46$, 95\% CI 0.46-0.47). We also found that if yawning is present during the prodrome phase it is highly likely that it will occur also during the postdrome phase $(\mathrm{k}=0.47,95 \%$ CI $0.46-0-47)$.

Discussion: Although cognitive, mood and homeostatic changes are characteristics of the prodrome and postdrome phase, migraine patients most often recall having these symptoms in association to the headache pain. There is evidence showing that thirst, food craving and neck stiffness can be mediated by the hypothalamus. Thirst, food craving and neck stiffness occurred throughout all three phases, suggesting a constant involvement of the hypothalamus during the migraine attack.

Conclusions: The phases of the migraine attack do not occur in a linear sequential order. Non-headache symptoms can start during any phase of the migraine attack and be present during all phases.

\section{EFFICACY AND SAFETY OF GREATER OCCIPITAL NERVE BLOCK FOR THE TREATMENT OF CLUSTER HEADACHE: A SYSTEMATIC REVIEW AND META-ANALYSIS}

\author{
R. Ornello ${ }^{1}$, G. Lambru ${ }^{2}$, V. Caponnetto ${ }^{3}$, I. Frattale ${ }^{4}$, C. Di Felice ${ }^{3}$, F. \\ Pistoia $^{4}$, S. Sacco ${ }^{4}$
}

${ }^{1}$ Neuroscience Section, Department of Applied Clinical Sciences and Biotechnology, University of L'Aquila (L'Aquila); ${ }^{2}$ Headache Centre, Guy's and St Thomas' NHS Foundation Trust (London-UK); ${ }^{3}$ Department of Internal Medicine, Public Health, Life and Environmental Sciences, University of L'Aquila (L'Aquila); ${ }^{4}$ Department of Applied Clinical Sciences and Biotechnology, University of L'Aquila (L'Aquila)

Objectives: We aimed to summarize the evidence of efficacy and safety of greater occipital nerve blocks (GONBs) in $\mathrm{CH}$.

Materials and Methods: We included papers indexed in PubMed and Web of Science from the beginning of indexing to May 5, 2020. We included both observational and randomized studies referring to patients with episodic and/or chronic $\mathrm{CH}$.

Results: We identified 12 studies on 365 patients. Five studies (two randomized controlled trials) could be included in the meta-analyses. The pooled proportion of pain-free subjects at one month was $50 \%$ (95\% CI $24-76 \%$ ) with considerable heterogeneity ( $\mathrm{I} 2=88 \% ; \mathrm{P}<0.01)$. The pooled relative risk ratio of pain freedom at one month in active versus control groups in the two included randomized controlled trials was $4.86(95 \%$ CI, 1.35-17.55) without statistical heterogeneity ( $\mathrm{I} 2=0 \% ; \mathrm{P}=0.39)$. Three studies showed decreased attack intensity, frequency, and duration after GONBs. The studies reported mild and transient adverse events.

Discussion: The treatment of cluster headache $(\mathrm{CH})$ is challenging in view of the few evidence-based treatments.
Conclusions: Despite several limitations and considerable heterogeneity, the available data support the efficacy and safety of GONBs for the treatment of $\mathrm{CH}$. Further large randomized trials are needed to confirm efficacy and establish optimal treatment protocols.

\section{HOW LONG IS IT WORTH CONTINUING CHRONIC MIGRAINE TREATMENT WITH ONABOTULINUMTOXIN A BEFORE DECLARING TREATMENT FAILURE? INSIGHTS FROM REAL-LIFE MULTICENTER DATA}

R. Ornello ${ }^{1}$, I. Frattale ${ }^{2}$, A. Negro ${ }^{3}$, P. Martelletti ${ }^{3}$, A. Alpuente ${ }^{4}$, P. PozoRosich $^{4}$, A. Miscio ${ }^{5}$, A. Santoro ${ }^{5}$, L. Grazzi ${ }^{6}$, S. Cevoli ${ }^{7}$, N. Brunelli ${ }^{8}$, F. Vernieri $^{8}$, C. Baraldi ${ }^{9}$, S. Guerzoni ${ }^{9}$, M. Russo ${ }^{10}$, P. Torelli ${ }^{10}$, K. Kamm $^{11}$, R. Ruscheweyh ${ }^{11}$, M. Straburzynski ${ }^{12}$, A. Gryglas-Dworak ${ }^{13}$, E. Filatova ${ }^{14}$, N. Latysheva ${ }^{14}$, S. Sacco ${ }^{2}$

${ }^{1}$ Neuroscience Section, Department of Applied Clinical Sciences and Biotechnology, University of L'Aquila (L'Aquila); ${ }^{2}$ Department of Applied Clinical Sciences and Biotechnology, University of L'Aquila (L'Aquila); ${ }^{3}$ Headache Center, Sant'Andrea Hospital, University of Rome "Sapienza" (Roma), ${ }^{4}$ Headache Unit, Neurology Department, University Hospital Vall d'Hebròn (Barcelona-E); ${ }^{5}$ Department of Neurology, Casa Sollievo della Sofferenza (San Giovanni RotondoFG); ${ }^{6}$ Department of Clinical Neuroscience, Headache Unit, C. Besta Neurological Institute and Foundation (Milano); ${ }^{7}$ Neurologic Clinic, IRCCS Istituto delle Scienze Neurologiche di Bologna (Bologna); ${ }^{8}$ Neurology Unit, Campus Bio-Medico University (Roma); ${ }^{9}$ Medical Toxicology, Headache and Drug Abuse Centre, University of Modena and Reggio Emilia (Modena); ${ }^{10}$ Department of Clinical and Experimental Medicine, Parma Headache Centre, University of Parma (Parma); ${ }^{11}$ Deèartment of Neurology, Ludwig-Maximilians-University Hospital (Munich-D); ${ }^{12}$ Headache Clinic, Terapia Neurologiczna Samodzielni (Warsaw-PL); ${ }^{13}$ Department of Neurology, Gromkovski Voivodship Hospital (Wroclaw-PL); ${ }^{14}$ Department of Neurology, Institute for Postgraduate Education, Sechenov First Moscow State Medical University (Moscow-RUS)

Objectives: We aimed to provide real-life data to establish the reasonable duration of treatment with BT-A before declaring its failure.

Materials and Methods: We performed a retrospective re-analysis on real-life prospectively collected in 12 European headache centers from 2012 to 2020; data refer to the first three BT-A cycles administered to patients or equivalent follow-up. We defined patients as 'complete responders' if reporting a $\geq 50 \%$ reduction in monthly headache days compared with the three months before starting BT-A, 'partial responders' if reporting a $30-49 \%$ reduction in monthly headache days, and 'non-responders' if reporting a $<30 \%$ reduction in monthly headache days or stopping the treatment earlier than the third cycle. We also assessed the patients' baseline characteristics to identify factors associated to complete response during the the third cycle.

Results: We included 1800 patients, $82.5 \%$ female, with a mean age of $47.1 \pm 11.8$ years and a mean migraine duration of $31.4 \pm 12.5$ years. During the first cycle , 522 (29.0\%) patients were complete responders, $349(19.4 \%)$ partial responders, and 929 (51.6\%) non-responders; the corresponding data were 670 (37.2\%), $409(22.7 \%)$, and $721(40.1 \%)$ respectively during the second cycle, and 880 (48.9\%), $214(11.9 \%)$, and $706(39.2 \%)$ respectively during the third cycle. Multivariate analyses showed that complete (OR 14.16; 95\% CI 8.44-25.74; $\mathrm{P}<0.001)$ or partial response (OR 5.78; 95\% CI 2.81-11.88; $\mathrm{P}<0.001)$ during the second $\mathrm{BT}$ A cycle were the only factors independently associated with complete 
response during the third cycle. Of note, only 44 (7.9\%) of the 556 patients not responding to the first two cycles achieved a complete response to the third.

Discussion: Onabotulinumtoxin A (BT-A) is the first approved treatment for chronic migraine (CM). Current guidelines suggest continuing BT-A treatment for 6-9 months before declaring treatment failure, as many patients develop a late response to BT-A. However, it is unclear whether two or three administrations are the optimal length to assess BTA response.

Conclusions: According to our data, a relevant proportion of patients not responding to the first cycle of BT-A responds to the second and the third cycle. It is reasonable to continue treatment in all patients until the second cycle and to proceed to the third cycle in those who have $\geq 30 \%$ reduction in monthly headache days.

\section{ADDITIONAL VITAMIN D THERAPY IN CLUSTER HEADACHE}

\section{Pizza ${ }^{1}$, S. Montella ${ }^{2}$, A. Silvestro ${ }^{2}$, D. Cassano ${ }^{3}$, M. Lerza ${ }^{4}$, M.} Gargiulo $^{4}$, V. Busillo ${ }^{4}$, E. Iorio ${ }^{5}$

${ }^{1}$ Neurology Division, San Luca Hospital (Vallo della Lucania SA); ${ }^{2}$ Neurology Unit, ASL Salerno (Vallo della Lucania-SA); ${ }^{3} 60$ District, ASL Salerno (Nocera Inferiore-SA); ${ }^{4}$ Neurology Unit, ASL Salerno (Eboli-SA); ${ }^{5}$ International Oxidative Center (Salerno)

Introduction: Recently numerous evidences have shown a possible pathophysiological and therapeutics relationship between vitamin D and migraine. On the other hand, there is no evidence for cluster headache and vitamin D. In a poster presentation, efficacy of vitamin D therapy was reported in a patient with cluster headache. Vitamin D deficiency was also demonstrated in a series of $28 \mathrm{CH}$ patients compared with migraine and healthy controls.

Objective: We describe a 38-year-old young man with cluster headache treated with additional vitamin D therapy.

Case: We observed a 38-year-old young man with episodic cluster headache (ICHD II criteria) patients belonging to our headache centre, in San Luca Hospital, Vallo Della Lucania. For about 20 years suffering from episodic cluster headache in the period NovemberMarch almost every year with a frequency of 2-4 attacks per day. The patient has practiced drug therapy with corticosteroids, verapamil, carbolithium over the years. The patient was administered a daily dose by sublingual spray ( 4 sprays, about $0.7 \mathrm{ml}$ ) of: vitamin D3 $25 \mathrm{mcg}$ (1000 IU) $500 \% \mathrm{VNR}$, vitamin $\mathrm{K} 260 \mathrm{mcg} 80 \% \mathrm{VNR}$; in addition to its basic therapy with verapamil $360 \mathrm{mg} /$ day and amitriptyline $50 \mathrm{mg} /$ day. The frequency of attacks at the control at 1,2 months decreased by 60 and $90 \%$ respectively with the disappearance of the headache after 3 months with a clear improvement over the clusters of the previous years. The basal value (November) of vitamin $\mathrm{D}$, measured by MicroVue 25-OH Vitamin D EIA, was $13.4 \mathrm{ng} / \mathrm{ml}$ while the final control (April) was $36.8 \mathrm{ng} / \mathrm{ml}$.

Conclusion: In the case we observed, we highlighted a low basal value of vitamin D which at the end of the treatment (6 months) completely returned to normal values. At the same time, the additional therapy with vitamin D resulted in a significant improvement of the headache compared to the trend of the episodes of the previous years, highlighting a faster resolution of the attacks and a shorter duration of the cluster. This observation also on the basis of the reports in the literature calls for a controlled study of both baseline values and additional therapy with vitamin D in patients with cluster headache.

\section{References:}

- Nowaczewska M., Wichinsky M., Osinski S., Kazmierczak H. The role of vitamin $\mathrm{D}$ in primary headache - from potential mechanism to treatment. Nutrients (2020);12:2431-17; doi:10.3390/ nu12010243

- Peter B. A. survey of cluster headache $(\mathrm{CH})$ sufferers using vitamin $\mathrm{D} 3$ as a $\mathrm{CH}$ preventative. Poster presented of the 66th Annual meeting of American Academy of Neurology Philadelphia, April 26May (2014)

- S. Straube, R. Andrew Moore, S. Derry, HJ McQuay Vitamin D and chronic pain. Pain (2009);141(1-2):10-13

\section{MIGRAINE AND VITAMIN D SERUM LEVEL}

V. Pizza ${ }^{1}$, S. Montella ${ }^{2}$, A. Silvestro ${ }^{2}$, P. Delli Santi ${ }^{3}$, D. Cassano ${ }^{4}$, M. Lerza $^{5}$, M. Gargiulo ${ }^{5}$, V. Busillo ${ }^{5}$, E. Iorio ${ }^{6}$

${ }^{1}$ Neurology Division, San Luca Hospital (Vallo della Lucania -SA); ${ }^{2}$ Neurology Unit, ASL Salerno (Vallo della Lucania-SA); ${ }^{3}$ Laboratory Service, ASL Salerno (Vallo della Lucania-SA); ${ }^{4} 60$ District, ASL Salerno (Nocera Inferiore-SA); ${ }^{5}$ Neurology Unit, ASL Salerno (EboliSA); ${ }^{6}$ International Oxidative Center (Salerno)

Introduction: Vitamin D is associated with chronic pain disorder, depression and several neurological disorders. Recently numerous evidences have shown a possible pathophysiological and therapeutics relationship between vitamin $\mathrm{D}$ and migraine.

Objective: Our aim is to determine $25(\mathrm{OH}) \mathrm{D}$ levels in our migraineurs patients and in a healthy control group.

Material and Methods: At our Headache Centre (S.Luca Hospital, Vallo della Lucania, SA,Italy) we collected 25(OH)D level in 100 consecutive migraineurs without aura (ICHD-II criteria) with mean age of $37.5 \pm 13.7$ years ( 78 womens and 22 mens with mean age $37 \pm 14.7$ and 37 \pm 14.7 years respectly) e 100 healthy controls with mean age of $37.1 \pm 7.8$ $(\mathrm{p}=\mathrm{ns})$ years $(70$ womens and 30 males with mean age was $37.1 \pm 21.9$ and $37.2 \pm 6.5$ years). 25 -Hydroxy vitamin D $[25(\mathrm{OH}) \mathrm{D}]$ serum levels (cut-off $<30 \mathrm{ng} / \mathrm{ml}$ ) were measured by MicroVueTM 25-OH Vitamin D EIA Kit (Quidel, San Diego, CA, US). The mean disease duration was 8.5 \pm 7.3 (womens $8.1 \pm 7.6$ and mens $9.7 \pm 6.9$ ) years. Statistical evaluation was performed by t-test $(\mathrm{p}<0.05)$.

Results: There was no statistically significant age difference $(\mathrm{p}>0.05)$ between patients and controls and between the two sexes in their respective groups. There is also no statistically significant difference in disease duration between the two sexes. The value serum levels 25-OH Vitamin $\mathrm{D}$ in patients was $15.1 \pm 5.4 \mathrm{ng} / \mathrm{ml}$ (womens $15.3 \pm 5.2$; mens $13.2 \pm 3.7$ ) while in health controls was $17.4 \pm 7.8 \mathrm{ng} / \mathrm{ml}$ (womens $17.1 \pm 0.9$; mens $18.3 \pm 6.2)$. There was a significant difference $(\mathrm{p}<0.05)$ between patients and controls for the vitamin $\mathrm{D}$ values (in both groups insufficient) and also between the values of the respective 2 sexes (W/M), in particular highly significant $(\mathrm{p}<0.001)$ in the comparison between the males. Furthermore, there was no statistically significant difference in vitamin $\mathrm{D}$ values $(\mathrm{p}<0.05)$ between the two sexes in their respective groups.

Conclusion: Vitamin D insufficiency is a frequent finding in clinical practice. In our study in all the subjects studied (migraine and controls) we found insufficient values of vitamin $\mathrm{D}$, but statistically significantly lower in the group of migraine. Neverthless we cannot postulate a definite relationship between vitamin D deficiency and migraine. We suggest that this conclusion needs to be supported with randomised clinical studies containing a larger number of samples and controls. Such an observation 
could suggest vitamin D supplementation in these patients in order to reduce migraine attacks.

References:

- Eyles DW, Burne TH, McGrath JJ. Vitamin D, effects on brain development, adult brain function and the links between low levels of vitamin D and neuropsychiatric disease. Front Neuroendocrinol (2013); 34:47-64

- S. Straube, R Andrew Moore, S Derry and HJ McQuay: Vitamin D and chronic pain. Pain (2009);141(1-2):10-13

- Nowaczewska M., Wichinsky M., Osinski S., Kazmierczak H. The role of vitamin $\mathrm{D}$ in primary headache - from potential mechanism to treatment. Nutrients (2020);12:2431-17; doi:10.3390/nu12010243

\section{HAEMODYNAMIC ACTIVITY CHARACTERIZATION OF RESTING STATE NETWORKS BY FRACTAL ANALYSIS IN CHRONIC MIGRAINE}

\author{
C. Porcaro ${ }^{1}$, A. Di Renzo ${ }^{2}$, E. Tinelli ${ }^{3}$, G. Di Lorenzo ${ }^{4}$, V. Parisi ${ }^{2}$, F. \\ Caramia $^{3}$, M. Fiorelli ${ }^{3}$, V. Di Piero ${ }^{3}$, F. Pierelli ${ }^{5}$, G. Coppola ${ }^{5}$
}

${ }^{1}$ ISTC, CNR (Roma); ${ }^{2}$ IRCCS Fondazione Bietti (Roma); ${ }^{3}$ Department of Human Neurosciences, Sapienza University of Rome (Roma); ${ }^{4}$ Laboratory of Psychophysiology and Cognitive Neuroscience, University of Rome Tor Vergata (Roma); ${ }^{5}$ Department of MedicoSurgical Sciences and Biotechnologies, Sapienza University of Rome Polo Pontino (Latina)

Objectives: We aimed to investigate how fractal analysis, a measure of complexity derived from chaos theory, of the blood oxygenation level dependent (BOLD) activity at rest can be used to differentiate Resting State Networks (RSNs) in chronic migraine (CM) respect to healthy controls (HC).

Materials: Resting-state functional Magnetic Resonance Imaging (rsfMRI) data were collected from 20 untreated CM without history of medication overuse and $20 \mathrm{HC}$

Methods: After data pre-processing, rs-fMRI data of all participants as a concatenated groups ( $\mathrm{HC}$ and $\mathrm{CM}$ ) were analysed using spatial independent component analysis (ICA) as implemented in the Group ICA of fMRI Toolbox (GIFT; http://trendscenter.org/software/gift/) to decompose the data into functional networks that exhibited a unique time course profile. Subject-specific spatial maps and time courses were obtained using the back-reconstruction approach (GICA) within GIFT toolbox. Finally, in both groups, we estimated the Higuchi's Fractal Dimension (HFD, (Higuchi, 1988)).

Results: CM showed higher HFD values within dorsal attention system (DAS) and the anterior part of default-mode network (DMN), and lower FD values within the posterior DMN compared to HC.

Discussion: Despite the fact that linear methods are predominantly used in characterizing brain oscillations in both healthy and pathological conditions, linear analysis may not be suitable to describe the irregular and non-periodic patterns recorded by electrophysiological and neuroimaging techniques [1]. Recently, in fact, have been demonstrated that in many cases brain signals considered as belonging to a frequency-defined class of brain rhythms do not represent sustained oscillations, but rather brief bouts of activity that are repeated intermittently (i.e., non-rhythmic). Recognition that physiological time series contain "hidden information" that might be captured by non-linear methods such as fractal analysis, may provide crucial and so far overlooked physiological information in healthy and pathological conditions [2]. To this end, we characterised the specific BOLD signature of each RSN, using HFD that has advantages over classical linear methods that are best suited to conditions where the analysed signals are stationary.

Conclusions: Here, we probe that HFD can be used to estimate complexity within networks activity extracted from the MRI at rest using an independent component analysis. The application of this analysis on $\mathrm{CM}$ patients demonstrated that an aberrant increase in complexity within the DAS and anterior DMN and a lower complexity within the posterior DMN compared to HC. This abnormal pattern of FD within DAS and DMN may reflect disruption of cognitive control of head pain.

References:

1. Cole, S.R., Voytek, B. Brain Oscillations and the Importance of Waveform Shape. Trends Cogn. Sci. (2017);21:137-49

2. Goldberger, A.L., Amaral, L.A.N., Hausdorff, J.M., Ivanov, P.C., Peng, C.K., Stanley, H.E. Fractal dynamics in physiology: Alterations with disease and aging. Proc. Natl. Acad. Sci. U. S. A. (2002);99:2466-72

3. Higuchi, T. Approach to an irregular time series on the basis of the fractal theory. Phys. D Nonlinear Phenom. (1988) https://doi.org/ $10.1016 / 0167-2789(88) 90081-4$

\section{BENEFIT-RISK ASSESSMENT OF GALCANEZUMAB VERSUS PLACEBO FOR THE TREATMENT OF EPISODIC AND CHRONIC MIGRAINE: RESULTS FROM EVOLVE-1, EVOLVE- 2, AND REGAIN CLINICAL TRIALS}

D. Ruff ${ }^{1}$, A. Tockhorn-Heidenreich ${ }^{1}$, S. Foster ${ }^{1}$, R. Nichols ${ }^{1}$, V. Stauffer ${ }^{1}$, S. Zanigni ${ }^{2}$

${ }^{1}$ Eli Lilly and Company (Indianapolis-USA); ${ }^{2}$ Eli-Lilly Italy S.p.A. (Sesto Fiorentino-FI)

Target: To evaluate the benefit-risk profile of galcanezumab (GMB) versus placebo $(\mathrm{PBO})$ for the treatment of episodic (EM) and chronic migraine $(\mathrm{CM})$ in adults.

Materials and Methods: Data from three pivotal phase 3 trials of GMB were used to estimate response rates (RR), a clinically meaningful outcome for decision makers, based on monthly migraine headache day (MHD) reductions in patients with EM (EVOLVE-1 and EVOLVE-2; 6-month treatment duration) and patients with CM (REGAIN; 3-month treatment duration). Corresponding numbers needed to treat (NNT) for one patient to benefit from drug and numbers needed to harm $(\mathrm{NNH})$ for one patient to be harmed by drug based on discontinuation due to adverse events (DCAE) were estimated for each trial separately.

Results and Discussion: For EVOLVE-1, NNT were 5 and 6 with GMB 120 and $240 \mathrm{mg}$, respectively, at 30\% RR, 5 for both doses of GMB at $50 \%$ RR, and 6 for both doses at $75 \%$ RR; EVOLVE-2: 5 for both doses of GMB at 30\% and 50\% RR, and 7 and 6 with GMB 120 and $240 \mathrm{mg}$, respectively, at 75\% RR; REGAIN: 8 for both doses of GMB at $30 \%$ RR, 9 for both doses at 50\% RR, and 40 and 24 with GMB 120 and $240 \mathrm{mg}$, respectively, at $75 \% \mathrm{RR}$. In all trials, the proportion of patients achieving these RRs was significantly higher in patients treated with GMB $120 \mathrm{mg}$ and $240 \mathrm{mg}$ versus those treated with PBO. Corresponding NNTs were similar across trials (5 to 9$)$ with the exception of those obtained for $\geq 75 \%$ RR in REGAIN ( 24 to 40 ). Across all trials, NNH based on DCAE were 92 and 57 (EVOLVE-1), 213 and 46 (EVOLVE-2), and not relevant as higher risk with placebo (NR) and 295 (REGAIN) with GMB $120 \mathrm{mg}$ and $240 \mathrm{mg}$, respectively. 
Conclusion: In all trials, GMB showed a favorable benefit-risk profile versus PBO based on low NNTs for RR and higher NNH for DCAE. Higher NNTs observed in patients with CM may be due to higher disease burden (higher baseline MHD and greater disability) of these patients and shorter treatment duration ( 3 vs. 6 months).

\section{HEADACHE AND HYPERTENSION; A CLOSE RELATIONSHIP AFTER KIDNEY TRANSPLANTATION}

\author{
S. Salvemini ${ }^{1}$, M. Burattini ${ }^{1}$, E. Potente ${ }^{1}$, L. Falsetti $^{2}$, M. Silvestrini ${ }^{1}$, M. \\ Bartolini $^{1}$, G. Viticchi ${ }^{1}$
}

${ }^{1}$ Neurological Clinic, Marche Polytechnic University (Ancona); ${ }^{2}$ Internal and Subintensive Medicine, Ospedali Riuniti Ancona (Ancona)

Objectives: Between 70 and $90 \%$ of transplant patients suffer of high blood pressure. It is well established that blood pressure control plays a fundamental role in the transplanted organ's as well as the patient's survival. Aim of this study was to evaluate, in a population of migraine patients submitted to kidney transplant, if anti-hypertensive drugs can be significantly associated with an improvement of headache symptoms. Moreover, we want to assess if some classes of antihypertensive drugs could determine a better headache control than others.

Materials and Methods: In a one-year period, we enrolled all patients undergone to kidney transplantation in the previous five years. For each patient we collected demographic (age and gender) and clinical data (diagnosis of migraine, type of kidney disease and dialysis before transplant). The types of antihypertensive drugs used (beta-blockers (BB), angiotensinconverting-enzyme inhibitors (ACEi), angiotensin-receptorblockers (ARB), calcium channel blockers (CCB)) were collected as categorical variables and compared with chi-squared test. The statistical analysis was performed with SPSS 13.0 for Windows operating systems.

Results: A final sample of 110 patients was obtained. In $38.2 \%$ (42 patients) a migraine with or without aura was diagnosed. In this migraine group antihypertensive drug use was associated with a greater reduction of migraine attacks $(59.5 \%, 25$ patients) compared to no-use $(11,9 \%, 5$ patients; $\mathrm{p}=0.022$ ). When we directly compared different classes of antihypertensives, a significant headache improvement could be associated with the use of BB as opposed to ACEi and ARB ( $p=0.039)$. The use of CCB showed a notable improvement as well, compared to ACEi and $\operatorname{ARB}(\mathrm{p}=0.046)$.

Discussion: Anti-hypertensive drugs use seems associated with an improvement of migraine symptoms; in particular BB and $\mathrm{CCB}$ have been correlated with a stronger improvement of symptoms than ACEi and ARB. These results appeared in line with the literature data which highlight how ACEi and ARB are less effective as opposed to other migraine prophylaxis therapies. On the other side, $\mathrm{BB}$ and $\mathrm{CCB}$ were usually employed in migraine prophylaxis.

Conclusion: The superiority of $\mathrm{BB}$ and $\mathrm{CCB}$ to ACEi or ARB shows that there is a probability of post-transplant headaches with migraine characteristics having the same etiopathogenesis and therapy-induced improvement as migraine in patients who have not undergone transplantation.

References:

- Maggioni F., Mantovan M.C., Rigotti P. et al. Headache in kidney
transplantation. J Headache Pain (2009);10:455-60
- Viticchi G., Falsetti L., Buratti L. et al. Headache and kidney transplantation: an intriguing relationship. Neurol Sci (2019);40:199-200

\section{EFFICACY OF ERENUMAB 140MG SUBCUTANEOUS INJECTION IN PREVENTIVE THERAPY OF CYCLIC VOMITING SYNDROME: A CASE REPORT OF AN ADULT PATIENT}

\author{
P. Scatena, R. Renie', S. Salvetti, G. Sette
}

NESMOS Department, Faculty of Medicine and Psychology, Sapienza University of Roma (Roma)

Background: Cyclic vomiting syndrome (CVS) is a chronic debilitating illness functional gastrointestinal disorder characterized by stereotypic episodic bouts of uncontrollable vomiting and migraine separated by periods of relative wellness. These attacks are often accompanied by other symptoms including nausea, abdominal pain, headaches, photo, phonophobia, and several autonomic symptoms similar to those observed in migraine headache. The etiology of CVS is unknown, but it shares similar characteristics to migraine. The prevalence of migraine headaches is $36.5 \%$ in adult CVS patients. Furthermore, prevalence of a family history of migraines is $56 \%$ in adult CVS patients. Though not fully understood, this association suggests a common pathophysiological mechanism with a diverse array of symptoms caused by a more generalized underlying central nervous system disorder. This hypothesis describes CVS as a variant of migraine. Current management strategies include abortive and prophylactic migraine medication therapies that reduction or remission of CVS symptoms in more than $70 \%$ of patients. CGRP release during cortical spreading depression has been identified as an important factor required for sustaining a migraine and pharmacological inhibition of the CGRP-receptor system shows therapeutic promise in preventing migraines.

Aims: Evaluation of Erenumab® benefit in CVS therapy.

Materials and methods: We report a case of a 54-year-old man with 6 years history of recurrent severe episodes of stereotypic nausea, vomiting, and abdominal pain, related-anorexia and serious migraine. Episodes lasted less than 1 week in duration, with three or more of these episodes/year. The events were unresponsive to main acute and preventive therapies. So many times was therefore hospitalized. Laboratory examinations, gastroenteroscopy and brain magnetic resonance imaging all failed to reveal abnormalities that would explain his symptoms. The diagnosis for CVS is based on Rome criteria in 2016. The patient was subjected to a subcutaneous injection of Erenumab ${ }^{\circledR} 140 \mathrm{mg}$ every 4 weeks for 1 year. The patient improved significantly benefit since the beginning of therapy and since the first month has no longer had crisis. After two months from the suspension of the therapy the patient has resumed the same crises of CVS.

Conclusions: In this single case, we observed that, Erenumab therapy should be an effective therapeutic method for preventing recurrence migraine-vomiting episodes in CVS.

References:

- R. Burstein, R. Noseda, D. Borsook. Migraine: multiple processes, complex pathophysiology. Journal of Neuroscience (2015);35.17:6619-29

- C. Prakash et al. Similarities in cyclic vomiting syndrome across age groups. The American journal of gastroenterology (2001);96.3:68488 


\section{EVALUATION OF 1-YEAR ERENUMAB THERAPY IMPACT ON QUALITY OF LIFE, MOOD, ANXIETY AND SLEEP IN HIGH FREQUENCY MIGRAINEURS: A DATA ANALISIS}

\section{P. Scatena, R. Renie', M. Salvetti, G. Sette}

NESMOS Department, Faculty of Medicine and Psychology, Sapienza University of Roma (Roma)

Background: Migraine is a painful and disabling condition. Many studies demonstrating that migraine, mood, anxiety and sleep disorders are comorbid with each other with increased migraine-related disability in such patients. Erenumab ${ }^{\circledR}$ (Novartis) is a novel specific treatment strategy for the prevention of migraine targeting the CGRP receptor.

Aims: This study aims to identify how Erenumab® treating migraine improves quality of life.

Materials and methods: Thirty patients ( 28 women, 2 men, mean age 40.5 \pm 3.8 ) with high frequency migraine and not responsive to $\geq 3$ preventive medications, were subjected to a subcutaneous injection of Erenumab ${ }^{\circledR}$ every 4 weeks for 1 year. The first three months at a dosage of $70 \mathrm{mg}$ and then, for partial benefit, at a dosage a $140 \mathrm{mg} / \mathrm{month}$. All patients were submitted to the following psychometric scales once a month: HIT-6; MIDAS; MsQOL; PSQI; BDI; STAI-Y; TAS-20. Clinical endpoints also included: VAS; number of headache days and number of migraine attacks per month; number of painkiller and time to achieve pain relief. The data were analyzed using twoway ANOVA test, a Bonferroni for the differences at each "time-point", the Greenhouse-Geisser correction. To evaluate the linear regression quality, the rho coefficient was used.

Results: At T0, the mean number of headaches/month was $12.10 \pm$ 3.07 and following the 3 th injection, it was reduced to $4.1 \pm 2.11$ (p < 0.001 ). A significant correlation was found between the reduction of the monthly days of migraine headache and the use of painkillers. Significant improvement were observed from T0 to T12, on STAI X-2 (T0: 50.18 $\pm 5.47 ;$ T12: 39.45 $\pm 1.52 ; \mathrm{P}<.05$ ), HIT-6 (T0: 69 \pm 2.20 ; T12: 48.27 $\pm 2.24 ; \mathrm{P}<.05$ ), and BDI (T0: $29 \pm 2.65 ; \mathrm{T} 12: 5 \pm 1.5 ; \mathrm{P}<.05$ ) PSQI (T0: $10 \pm 2.10 ; \mathrm{T} 12: 3 \pm 2.10 ; \mathrm{P}<.05)$. Improvement of Depression and anxiety score were highly correlated with each other and TAS-20 $(\mathrm{r}=0.485, \mathrm{p}=0.001)$ and all its subscales in turn. This data is linked to improvements in MsQOL.

Conclusions: Erenumab ${ }^{\circledR}$ administered at a monthly dose of $140 \mathrm{mg}$, in a period of 12 months, have significative migraine preventive effects, reduces anxiety, sleep disturbances and improves mood and quality of life in high frequency migraine patients.

References:

- Dresler T. An update on behavioral treatments in migraine - current knowledge and future options. Expert Rev Neurother. (2017);17(11):1059-1068

- Garland SG et al. Erenumab: A First-in-Class Monoclonal Antibody for Migraine Prevention. Ann Pharmacother. (2019);53(9):933-939

\section{THE PERSPECTIVE OF THE SPEDALI CIVILI DI BRESCIA HEADACHE CENTRE DURING THE COVID-19 PANDEMIC}

F. Schiano di Cola, S. Caratozzolo, M. Di Cesare, P. Liberini, R. Rao, A. Padovani

Neurology Unit, University and Spedali Civili, Clinical and Experimental Sciences Department, University of Brescia (Brescia)
Background: To prevent and control for COVID-19 infection, outpatient evaluations during the COVID-19 pandemic have undergone severe restrictions. In particular, throughout the Italian lockdown period, routine outpatient evaluations were cancelled and patients contacted by telephone in order to assess whether the visit could be rescheduled or upgraded to an urgent neurological evaluation.

Materials and Methods: The present study was conducted at the Headache Centre of the ASST Spedali Civili Brescia. Due to COVID19 pandemic no routine outpatient evaluation was allowed during the period March 2nd - May 11th. Two hundred and seven patients had been scheduled in this time period at our centre. Each patient was contacted by telephone a few days prior the scheduled visit in order to assess and manage the patient accordingly.

Results: Out of two hundred and seven outpatient evaluations, eightyone were first-time visits whereas one hundred and twenty-six were control visits, of whom ninety (fourty-five chronic migraine patients every four weeks) related to monoclonal antibodies collection and twenty to onabotulinumtoxin A administration. Out of eighty-one first-time visits, only seven were deemed urgent, requiring a formal neurological inhospital evaluation. The remaining sixty-three (eleven patients could not be contacted as no telephone number was available) first-time visits were all postponed, in agreement with the patients, who all considered their evaluation not urgent, given the overall in-hospital situation due to the COVID-19 pandemic. Of the forty five patients on monoclonal antibodies all but four did not attend the monthly collection, three due to COVID-19 infection and one due to personal fear of coming to the hospital. No onabotulinumtoxin A administration was performed during the pandemic, with all patients reporting stable conditions since the last treatment cycle.

Discussion: In our experience at the Headache Centre of one of the most COVID-19 affected hospital in Northern Italy, two main considerations can be drawn. The first one regards the actual pertinence of many, if not most, first-time visits at our Headache Centre which should be restricted to severe/urgent cases. The second regards chronic migraine and how this disease severely affects people lives, considering that all but one patient on monoclonal antibodies would rather take the risk to reach a COVID-19 hospital than miss the opportunity of a "migraine-free month".

\section{ERENUMAB EFFICACY ON COMORBID CLUSTER HEADACHE IN PATIENTS WITH MIGRAINE: A REAL-WORD CASE SERIES}

\author{
F. Scotto di Clemente, M. Silvestro, A. Tessitore, G. Tedeschi, A. Russo
}

Headache Center, Department of Medical, Surgical, Neurological, Metabolic and Aging Sciences, University of Campania Luigi Vanvitelli (Napoli)

Background: Monoclonal antibodies (mAbs) against CGRP or its receptor have emerged as effective and well tolerated preventive medications for migraine. The key role played by CGRP has been recently demonstrated also in the pathophysiology of cluster headache $(\mathrm{CH})$, paving the way for studies aimed to investigate the effectiveness of $\mathrm{mABs}$ targeting CGRP also in $\mathrm{CH}$. However, no trials have been conducted so far to test the efficacy and tolerability of Erenumab as $\mathrm{CH}$ preventive treatment.

Case series: We describe the cases of five patients with both migraine and $\mathrm{CH}$ with previous failures of preventive treatments. All patients were treated with monthly Erenumab (70 or $140 \mathrm{mg}$ ) showing good results not only on migraine but also on $\mathrm{CH}$ attacks frequency and intensity. Improvements of both intensity and frequency of $\mathrm{CH}$ attacks occurred only after at least 3 months of treatment, with monthly Erenumab $140 \mathrm{mg}$ 
suggesting that longer treatment and higher doses are needed in $\mathrm{CH}$ in comparison to migraine.

Discussion and conclusion: Our findings support the efficacy and tolerability of monthly Erenumab $140 \mathrm{mg}$ as preventive treatment in patients suffering from both migraine without aura and $\mathrm{CH}$. We speculate that Erenumab could represent a low-risk alternative for $\mathrm{CH}$ patients (with or without comorbid migraine) who did not tolerated common $\mathrm{CH}$ preventatives therapies or for whom the therapies were not successful. Certainly, randomized trials are needed to confirm these observations and we hope that our data, showing a delayed therapeutic effect only with the highest dose of Erenumab (140 mg/month), can be taken into account in designing future trials.

References:

- May A, Schwedt TJ, Magis D, Pozo-Rosich P, Evers S, Wang SJ. Cluster headache. Nature Review Dis Primers (2018); 1;4:18006

- Hoffmann J, May A. Diagnosis, pathophysiology, and management of cluster headache. Lancet Neurol. (2018);17(1):75-83

- Robbins MS, Starling AJ, Pringsheim TM, Becker WJ, Schwedt TJ. Treatment of Cluster Headache: The American Headache Society Evidence-Based Guidelines. Headache (2016);56(7):1093-1106

\section{CAN CRANIOMETRIC ANALYSIS PLAY A ROLE OF IN CLUSTER HEADACHE DIAGNOSIS? A PILOT EXPLORATORY TC-3D BASED STUDY}

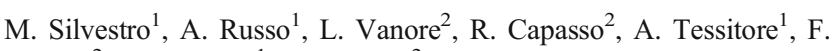 \\ Caranci $^{2}$, G. Tedeschi ${ }^{1}$, R. Conforti ${ }^{2}$ \\ ${ }^{1}$ Department of Advanced Medical and Surgical Sciences, University of \\ Campania Luigi Vanvitelli (Napoli); ${ }^{2}$ Department of Radiology, \\ University of Campania Luigi Vanvitelli (Napoli)
}

Background: The impression of being able to discriminate patients suffering from cluster headache based on some facial, somatic or behavioral traits is a common clinical experience for any neurologist dealing with headaches. Since the first pioneering observations of Graham in the 1970s which reported the so-called "leonine face" in cluster headache patients, only few studies have investigated cranial and facial features of these patients by different non-standardized approaches and reporting conflicting results.

Aim: We aim to investigate whether abnormalities in craniometric measurements could characterize cluster headache patients and represent reliable and reproducible diagnostic biomarkers able to discriminate cluster headache patients from healthy controls.

Methods: Tridimensional brain Computed Tomography images were obtained 24 cluster headache patients and in 24 matched healthy controls. Then, several craniometric measurements were obtained to verify possible morphological variations of neurocranium or splanchnocranium. Finally, a logistic regression analysis was used to investigate baseline imaging predictors of cluster headache.

Results: Cluster headache patients exhibited statistically significant higher frontal bone height $(\mathrm{p}=0.02)$ and facial width $(\mathrm{p}=0.003)$ when compared with healthy controls. Other neurocranium or splanchnocranium measurements did not show significant differences between cluster headache patients and healthy controls. Logistic regression analysis indicated that the model, containing the index obtained by multiplying higher frontal bone height and facial width was statistically significant (LR $\chi 2=13.88 ; \mathrm{p}<0.0002$; Log likelihood: -26.33 ; R2 di Mc Fadden: 0.21). The analysis of the ROC curve related to logistic regression showed an AUC of 0.7986. Finally, the Youden test identified a cut-off value of the index of 155.5 with a sensibility of $71 \%$ and a specificity of $96 \%$.

Discussion: Although the clinical features of the cluster headache attacks are so peculiar, the diagnosis is often challenging as witnessed by frequent misdiagnosis and delay. In the present study we found abnormal craniometric measures when compared cluster headache patients with healthy controls. Specifically, higher frontal bone height and facial width were two parameters able to distinguish cluster headache patients from healthy controls with a good sensitivity and a very high specificity.

Conclusion: The craniometric evaluation by means of brain 3D-TC may represent a widespread, noninvasive and accurate tool to overcome the risk of incorrect diagnosis and reduce the burden related to diagnostic lateness of a very disabling and excruciating pain such as cluster headache.

\section{References:}

- Hoffmann J, May A. Diagnosis, pathophysiology, and management of cluster headache. Lancet Neurol. (2018);17(1):75-83

- Graham JR, Rogado AZ, Rahman M, Gramer IV. Some physical, physiological and psychological characteristics of patients with cluster headache. In: Cochrane AL ed Background to Migraine. London; Heinemann (1970): 38-51

- Afra J, Proitetti A, Schoenen J. Craniometric measures in cluster headache patients. Cephalalgia (1998);18:143-45

\section{MULTIDIMENSIONAL ASSESSMENT OF THE EFFECTS OF ERENUMAB IN CHRONIC MIGRAINE PATIENTS WITH PREVIOUS UNSUCCESSFUL PREVENTIVE TREATMENTS: A COMPREHENSIVE REAL-WORLD EXPERIENCE}

\author{
M. Silvestro, A. Russo, F. Scotto di Clemente, F. Trojsi, A. Bisecco, S. \\ Bonavita, A. Tessitore, G. Tedeschi
}

Department of Advanced Medical and Surgical Sciences, University of Campania Luigi Vanvitelli (Napoli)

Background: Erenumab was safe and effective in clinical trials for the prevention of migraine. However, real-life data are still lacking. Here we report the clinical experience from an Italian real-world setting using erenumab in patients with chronic migraine experiencing previous unsuccessful preventive treatments.

Methods: Seventy patients with chronic migraine and failure to $\geq 4$ migraine preventive medication classes initially received monthly erenumab $70 \mathrm{mg}$ s.c. Patients without a clinically meaningful improvement, considered as a $>30 \%$ reduction in headache days per month, after $\geq 3$ months of therapy switched to monthly erenumab $140 \mathrm{mg}$. At the first administration and after 3 and 6 months, patients underwent extensive interviews to assess clinical parameters of disease severity and migrainerelated disability and impact, and validated questionnaires to explore depression/anxiety, sleep, and quality of life (QoL). Finally, the Pain Catastrophizing Scale, Allodynia Symptom Checklist-12 and MIGraine attacks-Subjective COGnitive impairments scale (MIG-SCOG) were administered.

Results: $70 \%$ of patients were "responders" after the third administration of erenumab $70 \mathrm{mg}$, whereas $30 \%$ switched to erenumab $140 \mathrm{mg}$; $29 \%$ (6 pts) responded after the sixth administration. The headache-day frequency was reduced from $21.1 \pm 0.7$ to $11.4 \pm 0.9$ days after the third administration $(\mathrm{p}<0.001)$ and to $8.9 \pm 0.7$ days after the sixth administration $(\mathrm{p}<0.001) .53 \%$ and $70 \%$ of patients, respectively, showed a 
reduction of $\geq 50 \%$ of headache days/month after the third and the sixth administrations. Also improved were headache pain severity, migrainerelated disability, and impact on daily living, QoL, pain catastrophizing and allodynia (all $\mathrm{p}<0.001$ ), quality of sleep, symptoms of depression or anxiety $(\mathrm{p}<0.05)$ but not MIG-SCOG. There were no new adverse event signals.

Conclusion: These real-world data support monthly erenumab 70 or $140 \mathrm{mg}$ s.c. as a safe and effective preventive treatment to reduce headache frequency and severity in chronic migraine patients experiencing previous unsuccessful preventive treatments.

References:

- Dodick DW CGRP ligand and receptor monoclonal antibodies for migraine prevention: Evidence review and clinical implications. Cephalalgia (2019);39:445-58

- Barbanti P, Aurilia C, Egeo G, Fofi L Erenumab: From scientific evidence to clinical practice-the first Italian real-life data. Neurol Sci. (2019);40:177-79

- Ornello R, Casalena A, Frattale I, Gabriele A, Affaitati G, Giamberardino MA et al. Real-life data on the efficacy and safety of erenumab in the Abruzzo region, central Italy. J Headache Pain (2020);21:32

\section{MANAGEMENT OF CEREBRAL VENOUS THROMBOSIS IN SPONTANEOUS INTRACRANIAL HYPOTENSION}

M. Trimboli ${ }^{1}$, E. Ferrante ${ }^{2}$

${ }^{1}$ Institute of Neurology, AOU Mater Domini, Department of Medical and Surgical Sciences, Magna Græcia University (Catanzaro); ${ }^{2}$ Neurology Department, Alto Vicentino Hospital, AULSS 7 Pedemontana (Santorso-VI)

Background: The occurrence of cerebral venous thrombosis (CVT) in patients with spontaneous intracranial hypotension $(\mathrm{SIH})$ raises difficult practical questions regarding the management of the 2 conditions. The first-line therapy for CVT is anticoagulation (AC); however, its potential benefit in SIH/CVT patients, especially if complicated by subdual fluid collection, must be carefully evaluated taking account of the intracranial haemorrhage risk. Venous system recanalization and good prognosis in $\mathrm{SIH} / \mathrm{CVT}$ patients treated with epidural blood patch (EBP), the main treatment option for $\mathrm{SIH}$, have been described.

Methods: We reviewed our cases of SIH complicated by CVT among a cohort of over $400 \mathrm{SIH}$ patients observed and treated during the last years. We also revised the cases reported in the literature, and summarized and analyzed all clinical/radiological data of patients with $\mathrm{SIH}$ and CVT.

Results: Eight (2\%) out of 400 patients suffering with SIH, were also diagnosed with CVT. Seven (87\%) out of eight patients had orthostatic headache, three of them experienced a change in their headache pattern over the SIH course. One patient reported non-postural headache. Six out of eight patients received both AC and EBP treatments. Two patients were treated using only AC or EBP. A bilateral subdural hematoma enlargement after 1 month of $\mathrm{AC}$ was observed. Complete CVT recanalization after treatment was observed in three patients, including two with multiple CVT at baseline; partial CVT recanalization was detected in two patients. Three patients had no CVT recanalization. After 6-48 months' follow-up patients were still asymptomatic.

Conclusions: The use of AC therapy should be weighed against the risk and should be monitored carefully if initiated. Effective and prompt EBP, even without AC therapy, could lead to a good prognosis.
References:

- Ferrante E, Trimboli M, Rubino F. Spontaneous intracranial hypotension: review and expert opinion. Acta Neurol Belg. (2020) Feb;120(1):9-18

- Schievink WI, Maya MM. Cerebral Venous Thrombosis in Spontaneous Intracranial Hypotension. Headache (2008); 48:1511-19

- Zhang D, Wang. J, Zhang Q, et al. Cerebral Venous Thrombosis in Spontaneous Intracranial Hypotension: A Report on 4 Cases and a Review of the Literature. Headache (2018);58(8): 1244-55

\section{ERENUMAB IN A COHORT OF DIFFICULT-TO-TREAT SUBJECTS WITH CHRONIC MIGRAINE WITH/WITHOUT MEDICATION OVERUSE HEADACHE: REAL LIFE EXPERIENCE IN A TERTIARY HEADACHE CENTER}

\author{
G. Vaghi ${ }^{1}$, R. De Icco ${ }^{1}$, G. Fiamingo ${ }^{1}$, E. Guaschino ${ }^{2}$, S. Bottiroli ${ }^{2,3}$, V.

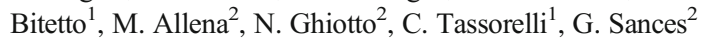

${ }^{1}$ Headache Science Center, Department of Brain and Behavioral Sciences, IRCCS Mondino Foundation, University of Pavia (Pavia); ${ }^{2}$ Headache Science Center, IRCCS Mondino Foundation (Pavia); ${ }^{3}$ Faculty of Law, Giustino Fortunato University (Benevento)

Purpose: To present real-life long-term clinical data on effectiveness and tolerability of the anti-CGRP monoclonal antibody erenumab in subjects with chronic migraine (CM) with/without medication overuse.

Materials: Subjects completed the following questionnaires at baseline and every three months: MIDAS, HIT-6, ASC-12 (allodynia) and EQ-5D-5L. They were also instructed to fill in daily a headache diary for the entire period.

Methods: Erenumab (70 or 140-mg dose) was administered monthly to 82 patients (F59, M23, mean age: $49.5+9.9 \mathrm{SD}$ ) who had already failed at least two preventive therapies. The majority of patients $(91 \%)$ had undergone prior detoxification treatments. We collected changes in monthly migraine days, acute medication intake and related disability, reporting here the data about the first 9 months of treatment. Statistical analysis was conducted using ANOVA for repeated measures and subsequent post-hoc tests.

Results: All clinical parameters showed a significant improvement already at mont1 $(\mathrm{T} 1)(\mathrm{p}<0.001)$ : monthly migraine days (baseline: 22.1+5.8, T1: $12.2+8.5$ ), monthly acute medication doses (baseline: $34.9+30.9 ; \mathrm{T} 1: 14.2+18.3$ ) and days of drug intake (baseline: $19.1+8.1$; $\mathrm{T} 1: 9.1+7.4)$. The effectiveness persisted over time and actually tended to become more marked: at $\mathrm{T} 9$ the number of migraine days was down to $9.04+7.9$ ). The percentage of patients who manifested a pattern reversal from chronic to episodic migraine was $50 \%$ at $\mathrm{T} 1,61 \%$ at $\mathrm{T} 3,65 \%$ at $\mathrm{T} 6$, $72 \%$ at T9. Disability and health status improved accordingly $(\mathrm{p}<0.001$ for all questionnaires at all time points). Only mild side effects were reported, mainly tolerable and transient local skin reactions, asthenia and worsening or new onset of constipation.

Discussion: Clinical improvement was detected already after one month of treatment and it was maintained over the long-term observation period. The treatment was well tolerated over time and none of our patients interrupted it because of side-effects.

Conclusions: These findings provide a long-term real-life experience with erenumab in a cohort of difficult-to-treat chronic migraineurs, 
showing marked improvement in multiple indicators of effectiveness which is maintained over time - and a good tolerability profile.

Disclosures of potential conflicts of interest: GV, GF, RDI, EG, SB, $\mathrm{VB}$, and MA, have no conflicts of interest to declare. CT received honoraria for the participation in advisory boards or for oral presentations from: Allergan, Electrocore, Eli-Lilly, Novartis and Teva. CT has no ownership interest and does not own stocks of any pharmaceutical company. GS received honoraria for the participation in advisory boards or for oral presentations from: Eli-Lilly, Novartis.

\section{KETOGENIC DIET IN PEDIATRIC PATIENTS WITH CHRONIC MIGRAINE}

M. Valeriani ${ }^{1}$, D. Elia ${ }^{2}$, C. Dionisi Vici ${ }^{3}$, L. Papetti ${ }^{1}$, M. Ferilli ${ }^{1}$, F.

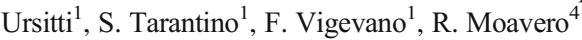

${ }^{1}$ Neurology Unit, Bambino Gesù Hospital (Roma); ${ }^{2}$ Artificial Nutrition, Bambino Gesù Hospital (Roma); ${ }^{3}$ Methabolic Diseases, Bambino Gesù Hospital (Roma); ${ }^{4}$ Infantile Neuropsychiatry, Tor Vergata University (Roma)

Objectives: Aim of this study was to evaluate efficacy, safety and feasibility of ketogenic diet $(\mathrm{KD})$ in children and adolescents with chronic migraine (CM).

Materials and Methods: We prospectively enrolled patients aged 1018 years with $\mathrm{CM}$ unresponsive to previous prophylactic treatments or that refused other therapies. A biochemical screening was performed to exclude inborn errors of metabolism. KD was then introduced at 1:1 ratio. Biochemical analysis was repeated at 1 week, 1 and 3 months of treatment. Daily ketones and glucose measurements at home were required.

Results: We enrolled 16 patients $(3 \mathrm{M}, 13 \mathrm{~F})$ to start KD. Among them 8 decided not to initiate KD mainly due to concerns about dietary restrictions and difficult compliance. Therefore, KD was initiated in 8 patients (1M, $7 \mathrm{~F}$ - age 11-18 years). In 3 patients KD was withdrawn in the first 3 months of treatment due to inefficacy. In one case KD determined a partial benefit, but the patient had an onset of bowel inflammatory disease and KD was withdrawn. In 2 patients, after an initial response headache appeared again, and although at a lower frequency and intensity than before $\mathrm{KD}$, both patients decided to stop KD due to unsatisfactory pros/cons ratio after 4 and 5 months of treatment. One patient was lost to follow-up, and one patient is still under treatment after 4 months, with reduction of headache frequency.

Discussion: In our headache Center we experienced difficulties in enrolling patients for $\mathrm{KD}$, mainly due to concerns about palatibility. In patients enrolled we observed a partial efficacy in $50 \%$ of cases. However, benefits were transient in 2 of them, and were not considered sufficient to justify such a dietary regimen according to our patients.

Conclusion: According to our experience, although $\mathrm{KD}$ has a potential to treat chronic migraine, it requires a significant effort to patients and their families, making it a hardly feasible option in childhood and adolescence.

\section{PRIMARY STABBING HEADACHE IN CHILDREN AND ADOLESCENTS: IS IT A “MIGRAINE PRECURSOR"?}

M. Valeriani ${ }^{1}$, F. Ursitti ${ }^{1}$, M. Ferilli ${ }^{1}$, R. Moavero $^{2}$, S. Tarantino ${ }^{1}$, G. Sforza $^{2}$, C. Ruscitto ${ }^{2}$, F. Vigevano ${ }^{1}$, L. Papetti ${ }^{1}$
${ }^{1}$ Neurology Unit, Bambino Gesù Hospital (Roma); ${ }^{2}$ Infantile Neuropsychiatry, Tor Vergata University (Roma)

Objective: In pediatric age, primary stabbing headache (PSH) is an uncommon, but not rare, primary headache. The aims of our prospective study were to describe the clinical characteristics of the pediatric PSH and to investigate whether in young subjects PSH is related to more common primary headaches.

Materials and Methods: Nineteen consecutive patients with PSH, diagnosed according to the ICHD-III criteria, were recruited. There were 13 girls and 6 boys, aged from 4 to 16 years (mean age: $9.9 \pm 3.4$ years).

Results: In our patients, pain had different locations, but it usually involved the bilateral fronto-temporal region. Four patients failed to identify a precise pain location. Stabs were very short, usually lasting less than 1 minute. Only in one patient, each attack included several stabs and lasted around 20 minutes. Pain intensity was usually judged to be mild to moderate. Strong pain intensity was referred only by 2 patients. Eight out of 19 patients presented with associated symptoms, such as photophobia (5), phonophobia (6), and nausea (3). Migraine was associated with PSH in 5 patients and tension-type headache (TTH) in one. Episodic syndromes which may be associated with migraine, such as infantile colic, motion sickness, limb pain, recurrent abdominal pain, and vertigos, were referred by 13 patients.

Discussion and Conclusions: In our pediatric case series, PSH clinical features were very similar to those described in adulthood. However, while in adults PSH is frequently associated with migraine and TTH (Hagler et al., 2014), only 32\% of our young patients referred another primary headache. It is noteworthy that around $70 \%$ of our PSH patients had a clinical history of episodic syndromes. These elements suggest that in pediatric age PSH can represent an age-related phenotype of the migrainous syndrome which will turn later into a more typical migraine. Longitudinal studies in which pediatric PSH patients are followed for several years will be needed to confirm this hypothesis.

\section{EFFECTIVENESS, SAFETY AND TOLERABILITY OF GALCANEZUMAB IN A REAL LIFE SETTING IN PATIENTS WITH MIGRAINE IN ITALY (THE GARLIT STUDY)}

F. Vernieri ${ }^{1}$, C. Altamura ${ }^{1}$, C. Aurilia ${ }^{2}$, N. Brunelli ${ }^{1}$, G. Egeo ${ }^{2}$, L. Fofi ${ }^{2}$, C. Costa $^{1}$, A. Fallacara ${ }^{1}$, V. Favoni ${ }^{3}$, G. Pierangeli ${ }^{3}$, M. Aguggia ${ }^{4}$, D. Bertuzzo $^{4}$, M. Albanese ${ }^{5}$, F. Frediani ${ }^{6}$, P. Di Fiore ${ }^{6}$, S. Cevoli ${ }^{3}$, P. Barbanti $^{2}$

${ }^{1}$ Headache and Neurosonology Unit, Campus Bio-Medico University Hospital (Roma); ${ }^{2}$ Headache and Pain Unit, IRCCS San Raffaele Pisana (Roma); ${ }^{3}$ UOC Neuromet Clinic, IRCCS Institute of the Neurological Sciences di Bologna (Bologna); ${ }^{4}$ SOC Neurology and Stroke Unit, Cardinal Massaia Hospital (Asti); ${ }^{5}$ Neurological Clinic, Tor Vergata University Hospital (Roma); ${ }^{6}$ Headache Centre, S. Carlo Borromeo Hospital (Milano)

Objective: Aim of the study was to observe the effectiveness, safety and tolerability of Galcanezumab in migraine patients treated in a real-life setting at 6 Italian headache centers. Galcanezumab is a humanized monoclonal antibody binding to calcitonin gene-related peptide (CGRP). The efficacy and safety of galcanezumab have been demonstrated in 3 Phase III clinical trials, EVOLVE-1 and EVOLVE-2 in episodic migraine (EM) and REGAIN in chronic migraine (CM).

Methods: In this open, multicenter, prospective real-life study, patients were treated with a first loading dose of $240 \mathrm{mg}$ of galcanezumab 
and then with galcanezumab $120 \mathrm{mg}$ monthly. The primary endpoint was to observe the change in monthly migraine days (MMDs) at the end of month 3 compared to baseline. Secondary endpoints included changes in monthly painkillers intake (MPI), Visual Analogue Scale (VAS), Headache Impact Test (HIT-6) and MIgraine Disability Assessing Scale (MIDAS) scores at the end of month 3 compared to baseline. Moreover, $50 \%, 75 \%$ and $100 \%$ responder rates were calculated for EM and CM groups at month 1,2 and 3 .

Results: Sixty-six $(85,3 \%$ female, aged $48.2 \pm 10.6)$ completed 3 months of therapy and were included; of these, at baseline $73.1 \%$ were affected by CM, $26.9 \%$ by EM. Patients with $\mathrm{CM}$ at baseline presented 20.0 (7 IQr) MMDs, 20.0 (17 IQr) month painkillers (MPs), VAS = 8.0 (1 IQr), HIT-6 = 67.5 (7 IQr) and MIDAS = 71.0 (35 IQr). Patients with EM at baseline presented 11.5 (4 IQr) MMDs, 12.0 (5 IQr) MPs, VAS = $8.0(1$ IQr), HIT-6 = 64.5 (3 IQr) and MIDAS = 46.50 (53 IQr). After 3 months of therapy patients presented a significant decrease in MMDs (CM: 8.5, 10 IQr; EM: 3.0, 2 IQr p<.0001), MPI (CM: 7.5 MPs, 6 IQr; EM: 5.0 MPs, 6 IQr p<.0001), VAS (CM:6.5, 9 IQr; p<.0001; EM: 6.0, 3 IQr p $=.001$ ), HIT-6 (CM: 59.0, 13 IQr; EM: 55.0, 9 IQr p<.0001) and MIDAS (CM: 67.5.0, 99 IQr; $p$ >.05 EM: 7.0, 59 IQr $p=.038$ ). It is worth noting that the decrease in MMDs, MPI and HIT-6 was largely significant just after one month of therapy $(\mathrm{p}<.0001)$ both in CM and in EM. After three months $50 \%$ responder rate was observed in $75.5 \%$ of CM patients and in $77.7 \%$ of EM. Among EM $33.3 \%$ were $75 \%$ responders after three months of treatment, and $32.6 \%$ among $\mathrm{CM}$. At three months only one CM patient was free from attack (100\% responder). Finally, patients neither presented serious adverse event nor treatment emerging adverse event to stop the treatment; $9.8 \%$ presented minor side effects (i.e. itching, dizziness) in at least one month of therapy, $7.3 \%$ in at least two months of therapy and $2.4 \%$ in three months of therapy.

Conclusions: Galcanezumab appears effective, safe, and welltolerated for the preventive treatment of CM and EM also in a real-life setting.

References:

- Stauffer VL, Dodick DW, Zhang Q, et al. Evaluation of Galcanezumab for the Prevention of Episodic Migraine: The EVOLVE-1 Randomized Clinical Trial. JAMA Neurol. (2018);75(9):1080-88

- Skljarevski V, Matharu M, Millen BA, et al. Efficacy and safety of galcanezumab for the prevention of episodic migraine: Results of the EVOLVE-2 Phase 3 randomized controlled clinical trial. Cephalalgia. (2018);38(8):1442-54

- Detke HC, Goadsby PJ, Wang S, et al. Galcanezumab in chronic migraine: The randomized, double-blind, placebo-controlled REGAIN study. Neurology (2018);91(24):e2211-e2221

\section{POOR SLEEP QUALITY IN PATIENTS AFFECTED BY MIGRAINE: A POPULATION STUDY}

G. Viticchi ${ }^{1}$, C. Altamura ${ }^{2}$, L. Falsetti ${ }^{3}$, S. Salvemini ${ }^{1}$, F. Polidoro ${ }^{1}$, A. Silvestrini $^{1}$, L. Buratti ${ }^{1}$, F. Vernieri ${ }^{2}$, M. Bartolini ${ }^{1}$

${ }^{1}$ Neurological Clinic, Marche Polytechnic University (Ancona); ${ }^{2}$ Neurology Department, Headache and Neurosonology Unit, Campus Bio-Medico University of Rome (Roma); ${ }^{3}$ Internal and Subintesive Medicine, Ospedali Riuniti (Ancona)

Objectives: Several studies described a narrow relationship between sleep quality and migraine severity, and an alteration in the night sleeping could easily induce a migraine attack on waking up. Several sleep disturbances can influence headache frequency, in particular insomnia, OSAS, bruxism or restless legs syndrome. Furthermore, some drugs employed for migraine prophylaxis could bring changes in circadian rhythms. Aim of this study is to evaluate, adopting a validated tool (the Pittsburgh Sleep Quality Index), the quality and the possible alterations of night sleeping in a population of migraine patients without any previous prophylaxis treatment for headache. We also investigated a possible relationship between sleep quality and migraine severity and frequency.

Methods: We enrolled for this study all the patients arrived at two Headache Centers, the Headache Center of Ospedali Riuniti di Ancona and the Headache Unit of Campus Bio-Medico University of Rome, during a six-month period. We submitted each patient with a first migraine diagnosis to two scales: the Pittsburgh Sleep Quality Index (PSQI) to obtain an assessment of the sleep quality and the Migraine Disability Assessment (MIDAS) questionnaire to evaluate the impact of headache on life-quality. Statistical analysis was performed with SPSS 13.0 for Windows systems.

Results: We obtained a final cohort of 35 consecutive patients. Patients had a median of 6.48[8] crises in the previous month and a median of 18[24] years of illness. Median of MIDAS score was 20[41], while Pittsburg score had a median of 4[5] points. In the overall cohort, 16 subjects $(45.7 \%)$ had a Pittsburg score $>5$ points. The best-fitting relationship between Pittsburg and MIDAS scores was an exponential trendline (Rsq=0.204; $p=0.011$ ). Patients with a Pittsburg score $\leq 5$ had a significantly lower median MIDAS score (11[35]) than subjects with a Pittsburg score $>5$ (28[56]; $\mathrm{p}=0.040)$.

Discussion: Patients with a greater impairment of PSQI score presented a most relevant migraine attack severity. Our data confirmed the narrow correlation between sleep quality and migraine impact. A tailored therapy should be considered for patients with both migraine and a poor sleep quality, to ensure a better quality of life and to finally reduce the number of drugs assumed.

References:

- Vgontzas A, Pavlović JM. Sleep Disorders and Migraine: Review of Literature and Potential Pathophysiology Mechanisms. Headache (2018);58:1030-39

- Viticchi G, Falsetti L, Paolucci M, et al. Influence of chronotype on migraine characteristics. Neurol Sci (2019);40:1841-48

- Viticchi G, Falsetti L, Bartolini M, et al. Migraine: incorrect selfmanagement for a disabling disease. Neurol Int (2018);10:7510

\section{EFFICACY OF GALCANEZUMAB IN PATIENTS WITH HIGH- FREQUENCY EPISODIC OR CHRONIC MIGRAINE AND 3 PREVIOUS PREVENTIVE TREATMENT FAILURES: A SUBGROUP ANALYSIS FROM THE CONQUER STUDY}

M. Weatherall ${ }^{1}$, M. Matharu ${ }^{2}$, A. Pain ${ }^{3}$, A. Tockhorn-Heidenreich ${ }^{3}$, M. Paget $^{3}$, G. Dell'Agnello ${ }^{3}$, G. Lambru ${ }^{4}$, S. Zanigni ${ }^{5}$

${ }^{1}$ Department of Neurology, Buckinghamshire Healthcare NHS Trust, Stoke Mandeville Hospital (Aylesbury-UK); ${ }^{2}$ Headache and Facial Pain Group, UCL Queen Square Institute of Neurology and The National Hospital for Neurology and Neurosurgery (London-UK); ${ }^{3}$ Eli Lilly and Company (Indianapolis-USA); ${ }^{4}$ Headache Centre, Guy's and St Thomas' NHS Foundation Trust and Wolfson CARD, King's College London (London-UK); ${ }^{5}$ Eli-Lilly Italy S.p.A. (Sesto Fiorentino-FI)

Target: Is galcanezumab efficacious in patients with high-frequency episodic migraine (HFEM) or chronic migraine (CM), and a prior history of failure with $\geq 3$ preventive treatments? 
Materials and Methods: CONQUER (Phase3, multicentre, randomised-controlled trial) assessed the efficacy and safety/tolerability of galcanezumab in treatment-resistant migraine. Patients (18-75 years) with 2-4 migraine prevention medication category failures in the past 10 years were randomised 1:1 to placebo and galcanezumab $120 \mathrm{mg} / \mathrm{month}$ (240-mg loading dose). We evaluated a subgroup of patients with HFEM ( $<15$ headache days/30-day period, 8-14 are migraine headache) or CM ( $\geq 15$ headache days/30-day period, migraine headache: $\geq 8$ ), and $\geq 3$ prior preventive medication category failures. Evaluated endpoints included change in monthly migraine headache days and proportion of patients achieving $\geq 50 \%$ and $\geq 75 \%$ reduction in monthly migraine headache days across the 3-month double-blind period. Migraine-Specific Quality of Life (MSQ) Role Function-Restrictive (RF-R) and Migraine Disability Assessment (MIDAS) were assessed at Month3.

Results and Discussion: 166 patients (36\% of CONQUER patients; HFEM=80, CM=86) were included in baseline analyses. Baseline characteristics were not statistically different between treatment groups. Following treatment, there were statistically significant differences between placebo and galcanezumab in mean change from baseline in monthly migraine headache days (placebo vs galcanezumab: baseline mean [SD]: 15.38 [5.61] vs 14.66 [5.23]; least squares [LS] mean change [SE] from baseline: -1.54 [0.75] vs -5.85 [0.68]; LS mean change difference [SE] vs placebo: -4.31 [0.78]; $\mathrm{p}<0.01$ ), $\geq 50 \%$ response (estimated rate [SE]: $11.3 \%$ [3.0] vs 39.4\% [4.2]; odds ratio (95\% CI) vs placebo: 5.07 [2.55, 10.10]; $\mathrm{p}<0.01$ ), $\geq 75 \%$ response (estimated rate [SE]: $3.9 \%$ [1.9] vs $17.1 \%$ [3.3] ]; odds ratio (95\% CI) vs placebo: $5.05[1.69,15.06] ; \mathrm{p}<0.01)$, MSQ RF-R score (baseline mean [SD]: 41.56 [20.48] vs 44.05 [17.73]; least squares [LS] mean change [SE] from baseline: -10.14 [2.84] vs 26.46 [2.60]; LS mean change difference [SE] vs placebo: 16.32 [3.12]; $\mathrm{p}<0.01$ )and MIDAS score (baseline mean [SD]: 60.77 [57.29] vs 53.23 [49.47]; least squares [LS] mean change [SE] from baseline: -2.09 [6.77] vs -27.43 [6.30]; LS mean change difference $[\mathrm{SE}]$ vs placebo: $25.34 ; \mathrm{p}<0.01$ ).

Conclusion: Galcanezumab significantly improved key efficacy endpoints versus placebo in patients with HFEM or CM who had previously failed $\geq 3$ preventive treatment categories.

\section{NEUROCOVID}

\section{COVID-19 ASSOCIATED GUILLAIN-BARRÉ SYNDROME: STILL UNSOLVED QUESTIONS}

F. Aruta ${ }^{1}$, A. Iovino ${ }^{1}$, S. Tozza ${ }^{1}$, D. Severi ${ }^{1}$, M. Bellofatto ${ }^{1}$, E. Spina ${ }^{1}$, P.

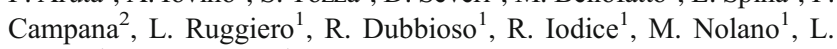
Santoro $^{1}$, F. Manganelli ${ }^{1}$

${ }^{1}$ Department of Neuroscience, Reproductive Sciences and Odontostomatology, University of Naples Federico II (Napoli); ${ }^{2}$ Department of Translational Medicine, University of Naples Federico II'(Napoli)

Aims: To describe a new case of GBS associated to coronavirus disease 2019 (COVID-19) and to discuss the features in light of the literature review.

Materials: Evaluation of a GBS patient features and his relationship with SARS-CoV-2 infection.

Methods: A 47-years-old female patient was referred to the emergency department for lower limb weakness rapidly evolving to a severe flaccid paraparesis over the previous 3 days. At admission, she complained of dyspnea and underwent nasopharyngeal swab and chest CT. Five days later the patient developed respiratory failure and was intubated and transferred to intensive care unit (ICU). Neurophysiological study was performed only after discharge from ICU and complete resolution of SARS-CoV-2 infection.

Results: Nasopharyngeal swab was positive for SARS-CoV-2 and chest CT scan showed interstitial pneumonia typical of COVID-19. Neurophysiological findings revealed a motor-sensory axonal neuropathy.

Discussion: Our case report is consistent with an axonal GBS and the temporal co-incidence of neurological and pulmonary symptoms prompts toward considering this GBS as a para-infectious complication of COVID-19.

Conclusions: This stimulates the current debate whether COVID-19 associated GBS is a para- or post- infectious disease as well it is an axonal or demyelinating neuropathy.

References:

- Román GC, Reis J, Spencer PS, Buguet A, Öztürk S, Wasay M; World Federation of Neurology Environmental Neurology Specialty Group. COVID-19 international neurological registries. Lancet Neurol (2020);414:116884

- Gigli GL, Bax F, Marini A, Pellitteri G, Scalise A, Surcinelli A, Valente M. Guillain-Barré syndrome in the COVID-19 era: just an occasional cluster? J Neurol (2020);19:1-3

- Sedaghat Z, Karimi N. Guillain Barre syndrome associated with COVID-19 infection: A case report. J Clin Neurosci (2020);76:233-235

\section{VIRTUAL VISITS FOR CHRONIC NEUROLOGIC DISORDERS DURING COVID-19 PANDEMIC}

D.M. Bonifati, I. Rosellini, S. Guidoni, A. Palmieri, F. Giopato, A. Ghazaryan, M. Fuccaro, A. Terrin, R. Vitaliani, M. Rigoni, M. Vianello

Unit of Neurology, Ca' Foncello Hospital (Treviso)

Aim: Covid-19 pandemic has boosted telemedicine in medical clinical practice. Telemedicine has been used in neurology mainly as telestroke to bring trombolysis in rural and underserved areas. Experiences in the management of chronic neurological disorders are limited and scattered. The aim of the study was to evaluate feasibility and efficacy of virtual visit for chronic neurological disorders during lockdown due to Covid-19 pandemic.

Materials and Methods: All patients scheduled for a visit during the lockdown period were contacted, virtual visit was proposed and consent obtained. The case manager scheduled the appointment and collected any useful documentation. Google-meet was used to perform virtual visit. During the virtual visit neurological examination was performed. We recorded the demographic and clinical characteristics of the patients as well as the aim of the visit and the given suggestions. After the virtual visit the patients could fill an online satisfaction survey.

Results: 169 virtual visits were performed for the following diseases: myasthenia gravis (43 visits), multiple sclerosis (79), epilepsia (11), headache (7) and Parkinson disease (28). The patients were 63 male and 105 female with a mean age of 53.5 yrs (range 17-91). During virtual visit we were able to obtain a good neurological examination. 42 patients filled the satisfaction questionnaire. All patients were satisfied about virtual visit (20 patients very, 11 enough, 11 just), 40 out of 43 patients found virtual visit a good alternative even if all patients consider it useful in the followup of the disease if combine with traditional face-to-face visit. Only 3 patients found medical prescription more difficult to understand. Most of 
the patients ( $86 \%$ ) found doctor-patients relationship similar between the two type of visit (for 5 patients it was better while only 1 patient found the relationship worst.

Discussion and Conclusion: Chronic neurological disorders are an important part of neurology clinical practice. These patients needs regular control not only to perform neurological examination but often to check blood or neuroradiological exams. We demonstrated feasibility and efficacy of virtual visit in the management of a large group of patients with common chronic neurological disorders. Virtual visit may be part of the management of these patients but setting and aims should be clarified. All patients were satisfied with virtual visit and suitable in the follow-up when combine with traditional face-to-face visit. Medical suggestions and patient-doctor relationship don't seem different between the two types of visit.

\section{ACUTE INFLAMMATORY DEMYELINATING POLYNEURITIS IN ASSOCIATION WITH AN ASYMPTOMATIC INFECTION BY SARS-COV-2}

\author{
M. Bracaglia ${ }^{1}$, I. Naldi ${ }^{1}$, A. Govoni ${ }^{2}$, D. Brillanti Ventura ${ }^{3}$, L. Sani ${ }^{1}$, P. \\ De Massis
}

${ }^{1}$ Neurology Unit, Medical Oncological Department, S. Maria Della Scaletta Hospital (Imola-BO); ${ }^{2}$ Internal Medicine, Medical Oncological Department, S. Maria Della Scaletta Hospital (Imola-BO); ${ }^{3}$ Montecatone Rehabilitation Institute (Imola-BO)

Objectives: After the recognition of COVID-19 disease, caused by the SARS-CoV-2, several reports refer to neurological symptoms in such patients [1], including Guillain-Barré Syndrome (GBS) [2,3]. We describe a case of acute demyelinating polyneuritis in association with asymptomatic SARS-CoV-2 infection.

Materials and Methods: A 66-years old Maroccan woman, resident in the Bologna area, was admitted to Imola Hospital on March 15th, 2020, for severe hyposthenia in lower limbs, mild in fist clenching, with distal tingling sensation, tendon reflexes abolished and pronounced lumbar pain for about eight days. She was unable to walk, had difficulty in swallowing and speeching. Vital signs were normal, medical history was negative. We hypothesized GBS and performed nerve conduction studies (NCS) and cerebrospinal fluid (CSF) analysis.

Results: CSF confirmed our hypothesis (protein content $245 \mathrm{mg}$ / $\mathrm{dl}$, cells $13 / \mathrm{mmc}$, polymorphonucleate $61.5 \%$ ) and NCS were consistent with demyelinating acute polyneuropahty (prolonged motor distal latencies, reduce cMAP amplitudes due to temporal dispersion, reduced SAPs amplitude). Microbiologic testing on CSF and serum was negative. Anti-ganglioside antibodies were negative. Blood analysis showed elevated CPK (461 U/L, normal<145), CRP $(5.65 \mathrm{mg} / \mathrm{dl}$, normal<0.5), lymphocytopenia $(0 \cdot 68 \times 109 / \mathrm{L}$, normal 1·10-4), mild increase of LDH (284 U/L, normal <248), GOT and GPT (549 and $547 \mathrm{U} / \mathrm{L}$, normal <35), similarly to COVID-19 patients laboratory profile. Considering the rapid spread of this infection in our region, we decided to perform a RT-PCR for SARSCoV-2 on nasopharyngeal swab, which resulted positive. We found elevation of Interleukin $6(11 \mathrm{pg} / \mathrm{mL}$, normal $<5 \cdot 9)$, also associated with Covid-19 disease. She was transferred to Covid-19 department and received a five-days course of intravenous immune globulin (IvIg), she never developed respiratory symptoms or fever, thoracic CT scan was normal. After IvIg, she significantly improved in all limbs, while facial diplegia has developed. After two weeks, two nasopharyngeal swabs, 24 hours apart, resulted negative.
Discussion and Conclusions: To our knowledge this is the first case of GBS in patient with asymptomatic COVID-19 and laboratory tests consistent with SARS-COV-2 infection. The association between COVID19 and GBS has recently been described both as parainfectious [2] and as post-infective event [3], suggesting a mechanism of molecular mimicry or a systemic inflammation triggered by the virus. Interesting in our case a patient asymptomatic for COVID-19 develops neurological impairment as unique clinical event. Unfortunately, we couldn't perform serological test or CSF PCR for COVID-19. We believe this association may not be a coincidence and underlines the importance of looking for neurological impairment in COVID-19, also for respiratory function worsening independently from pneumonitis.

References:

1. Huang C, Wang Y, Li X et al. Clinical features of patients infected with 2019 novel coronavirus in Wuhan, China. Lancet (2020) Feb 15;395(10223):497-506. doi: 10.1016/S01406736(20)30183-5. Epub 2020 Jan 24. Erratum in Lancet 2020 Jan 30

2. Zhao H, Shen D, Zhou H, Liu J, Chen S. Guillain-Barré syndrome associated with SARS-CoV-2 infection: causality or coincidence? Lancet Neurol. (2020) May; 19(5):383-384.doi: 10.1016/S14744422(20)30109-5. Epub 2020 Apr 1

3. Toscano G, Palmerini F, Ravaglia S et al. Guillain-Barrè Syndrome Associated with Sars-CoV-2. N Engl J Med. (2020) Apr 17; doi: 10.1056/NEJMc2009191

\section{COGNITIVE AND BEHAVIORIAL FEATURES OF COVID-19 PATIENTS IN POST-ACUTE PHASE}

E. Canu ${ }^{1}$, F. Agosta $^{1}$, A. Barbieri ${ }^{2}$, P. Bernasconi ${ }^{2}$, G. Cecchetti ${ }^{3}$, M. Vabanesi $^{3}$, V. Castelnovo ${ }^{1}$, G. Giacalone ${ }^{2}$, M. Falautano ${ }^{2}$, P. RovereQuerini $^{4}$, L. Roveri ${ }^{2}$, M. Filippi ${ }^{5}$

${ }^{1}$ Neuroimaging Research Unit, Institute of Experimental Neurology, Division of Neuroscience, IRCCS San Raffaele Scientific Institute; Vita-Salute San Raffaele University (Milano); ${ }^{2}$ Neurology Unit, IRCCS San Raffaele Scientific Institute (Milano); ${ }^{3}$ Neurology Unit and Neurophysiology Unit, IRCCS San Raffaele Scientific Institute (Milano); ${ }^{4}$ Department of Immunology, Transplantation and Infectious Diseases, IRCCS San Raffaele Scientific Institute; Vita-Salute San Raffaele University (Milano); ${ }^{5}$ Neuroimaging Research Unit, Institute of Experimental Neurology, Division of Neuroscience; Neurology Unit and Neurophysiology Unit, IRCCS San Raffaele Scientific Institute; Vita-Salute San Raffaele University (Milano)

Objective: To explore cognitive and behavioural features within two months from hospital discharge in a cohort of patients with confirmed COVID-19.

Materials: We enrolled 49 patients (age-range: $28-80$ years; $73 \%$ men) that were previously admitted at the San Raffaele Hospital due to confirmed COVID-19 (clinical symptoms with positive RT-PCR test from a nasopharyngeal/throat swab). Within two months from hospital discharge, all patients underwent a comprehensive neuropsychological assessment investigating the main cognitive functions. A clinical interview was also performed to investigate the presence of mood alterations and/or features of post-traumatic stress disorder (PTSD) at the time of the visit.

Methods: The total sample was split based on age (yC-19, age $<50$ : $\mathrm{N}=8,37 \%$ men; $\mathrm{mC}-19$, age range 50-64: $\mathrm{N}=21,76 \%$ men; oC-19, age $>65: \mathrm{N}=20,85 \%$ men) and the frequencies of cognitive and 
behavioural alterations, with respect to the normative data, were reported for the total sample and for each sub-group. In all patients, correlations were tested between neuropsychological scores and the severity of acutephase respiratory symptoms at the time of hospital admission.

Results: At the time of the visit, 5 (10\%) patients (1 yC-19, 4 mC-19) presented with depressive symptoms and 9 (18\%) (3 yC-19, 4 mC-9, 2 oC-19) reported PTSD features. $45 \%$ of the total sample showed executive dysfunctions (i.e., frontal dysfunctions and verbal working memory) and difficulties during encoding of new verbal material; $30 \%$ showed visuospatial difficulties, and 25\% long-term verbal and nonverbal memory problems. No patients showed language disturbances. Specifically, the $\mathrm{yC}-19$ group showed the most severe profile with 60 to $75 \%$ patients showing executive dysfunctions and encoding difficulties, $50 \%$ pure visuospatial dysfunctions and $40 \%$ primary long-term memory problems. The $\mathrm{mC}-19$ group showed primary executive $(>40 \%)$ and visuospatial (25\%) dysfunctions. Finally, $40 \%$ of the oC-19 group showed executive dysfunctions, $30 \%$ poor visuospatial abilities, and $25 \%$ long-term memory problems. The total sample showed a negative relationship between frontal executive performances and the severity of acute-phase respiratory symptoms at the hospital admission $(\mathrm{R}=-0.347 ; \mathrm{p}<0.01)$.

Discussion and Conclusions: This study showed that cognitive and behavioural alterations are associated with COVID-19 infection within two months from hospital discharge. The cognitive and behavioural symptoms were more severe in the youngest group. Whether these alterations are directly linked with the infection itself or with its related consequences is still to be determined, as well as whether they are reversible or part of a neurodegenerative process.

\section{COVID-19 DOES NOT REDUCE STROKE-RELATED EMERGENCIES}

S. Caproni ${ }^{1}$, F. Galletti ${ }^{1}$, D. Costanti ${ }^{1}$, E. Montinaro ${ }^{1}$, G. Barresi ${ }^{1}$, A. Riva $^{1}$, C. Chinigioli ${ }^{1}$, G. Parisi ${ }^{2}$, C. Colosimo ${ }^{1}$

${ }^{1}$ Department of Neuroscience, "S. Maria" University Hospital (Terni); ${ }^{2}$ Emergency Department, "S. Maria" University Hospital (Terni)

Objective: On the 8th March 2020, the Italian Government ordered a nationwide travel ban, quarantine and lockdown to limit the spread of severe acute respiratory syndrome coronavirus 2 (SARS-CoV-2)-induced disease (COVID-19). The burden of COVID-19 epidemic has been very severe, with more than 247,000 cases and over 35,000 deaths among Italian residents by the end of July 2020. Although in some Northern Italian regions a reduction of the number of stroke cases has been reported, this observation was not widely confirmed.

Materials and Methods: We collected data on the neurological consultations and admissions from the Emergency Department between 08:00 AM on 26th March and 02:00 PM on 27th March 2020, and we compared these results with similar periods (two working days) in November 2018 and 2019, to determine whether there was any reduction in acute neurological events.

Results: Although there has been an overall reduction in the total number of admissions to the Emergency Department during the COVID-19 outbreak in the Umbria region, our data indicate that the percentage of patients requiring an acute neurological consultation remained stable (11.1\% vs. $10 \%$ in 2018 and $12.6 \%$ in 2019). Furthermore, despite the fall in the overall number of hospital admissions ( $16.7 \%$ vs $22.3 \%$ in 2018 and $30.5 \%$ in 2019), admissions to the neurology ward increased if compared with the previous two years (50\% vs. $7.7 \%$ in 2018 and $9.5 \%$ in 2019).
Discussion: According to these data, the COVID-19 epidemic has not significantly altered the incidence of strokes or the prompt admission to a comprehensive stroke centre in our central Italian region. A possible explanation for the discrepancy between our data and those reported by other Italian centres may be the significantly lower prevalence of COVID-19 infection in our region than in other regions, particularly those in northern Italy. Whatever the explanation for these discrepant findings in Italy, we would like to stress the need for the establishment of stroke networks and care systems able to deliver high-quality emergency stroke care at any given time, including times of crisis, particularly when and where the number of comprehensive stroke centres needs to be reduced for any reason

Conclusions: COVID-19 does not reduce stroke-related emergencies. We should, therefore, be prepared to deal with the consequences of any COVID-19 outbreak on neurological care that may result from the increased prevalence of patients with this virus according to specific protocols and the best scientific evidence.

Reference:

- Caproni S, Di Schino C, Costantini F, Parisi G, Colosimo C. Letter to the Editor: "COVID-19 does not reduce stroke-related emergencies". Acta Neurol Belg. (2020) Jul 17:1-2

\section{HETEROGENEOUS NEUROIMAGING FEATURES OF NEURO- COVID: A CASE SERIES}

S. Caproni ${ }^{1}$, F. Galletti ${ }^{1}$, D. Costanti ${ }^{1}$, E. Montinaro ${ }^{1}$, G. Barresi ${ }^{1}$, A. Riva $^{1}$, C. Chinigioli ${ }^{1}$, M. Principi ${ }^{2}$, C. Colosimo ${ }^{1}$

${ }^{1}$ Department of Neuroscience, "S. Maria" University Hospital (Terni); ${ }^{2}$ Department of Radiology, "S. Maria" University Hospital (Terni)

Objective: Severe Acute Respiratory Syndrome Coronavirus 2 (SARS-CoV-2)-induced disease (COVID-19) is determining a global pandemic, affecting at the end of July 2020 more than 17 million people, with almost 700 thousands of deaths. The involvement of the nervous system in patients suffering from COVID-19 has been well documented, with a complex and variable clinical picture (Neuro-COVID). In particular, encephalitis, seizures, encephalopathy, Guillain-Barré syndrome (GBS), anosmia, neuromuscular disorders, and demyelinating diseases have been reported. The main putative mechanisms of Neuro-COVID are the direct SARS-CoV-2 nervous system invasion and a systemic dysimmune reaction ("cytokines storm"). Neuro-COVID patients admitted to Intensive Care Units (ICU) because of respiratory failure can present severe central nervous system manifestations, that can seriously affect their prognosis. In this case series, we report the various clinical and neuroimaging pictures of Neuro-COVID patients admitted to our Hospital.

Materials and Methods: The clinical and neuroimaging pictures of certificated COVID patients, admitted to Emergency Department and then to ICU have been reported and showing specific neurological complications or manifestations, as case series, with particular attention to the imaging characteristics. All patients were taken into care until discharge or death.

Results: We report 4 Neuro-COVID cases (all males, mean age 76 years old): a hemorrhagic/necrotizing encephalitis, a dysimmune encephalopathy, a large vessel embolic stroke with associated with a severe myocardial infarction treated with mechanical thrombectomy, and an ischemic stroke (partial anterior circulation) treated with intravenous thrombolysis. 
Discussion: In this case series we report the main neuroimaging pictures observed in Neuro-COVID, characterized by different pathophysiological mechanisms. Direct SARS-CoV-2 brain invasion can trigger a severe hemorrhagic/necrotizing encephalitis, complicated by immunological and coagulative reaction. On the other hand, dysimmune reaction inducted by Neuro-COVID can attempt the brain, in particular gray matter, mimicking a paraneoplastic mechanism. Ischemic and hemorrhagic manifestations have been well documented in Neuro-COVID; one patient of our was affected by a massive systemic coagulopathy, presenting both myocardial and brain ischemia; since the stroke time of onset was unknown (estimated from 6 to 12 hours), he was treated by mechanical thrombectomy, with good reperfusion but poor clinical outcome. The last patient suffered from a partial anterior circulation syndrome and was treated by intravenous thrombolysis; at the 24 hours control CT scan a large contralateral hemorrhage was detected, with poor clinical outcome.

Conclusions: Neuro-COVID can be associated with several, serious neuroimaging pictures, characterized by specific clinical severity and outcome.

\section{WAS COVID-19 ALREADY THERE? CLINICAL FEATURES OF PATIENTS WITH ACUTE ISCHAEMIC STROKE JUST BEFORE THE ITALIAN INDEX CASE}

T. Carandini ${ }^{1}$, A. Pietroboni ${ }^{1}$, S. Lanfranconi ${ }^{1}$, A. Novella ${ }^{2}$, A. Arighi ${ }^{1}$, E. Abati $^{1}$, R. Brusa ${ }^{1}$, G. Costamagna ${ }^{1}$, G. Lazzeri ${ }^{1}$, E. Mauri ${ }^{1}$, M. Pozzato $^{1}$, L. Sacchi ${ }^{1}$, G. Valcamonica ${ }^{1}$, D. Villa ${ }^{1}$, S. Bonato ${ }^{1}$, G. Comi $^{1}$, N. Bresolin ${ }^{1}$, D. Galimberti ${ }^{1}$, E. Scarpini ${ }^{1}$, A. Nobili ${ }^{2}$

${ }^{1}$ Fondazione IRCCS Ca' Granda Ospedale Maggiore Polilclinico, University of Milan (Milano); ${ }^{2}$ Istituto di Ricerche Farmacologiche Mario Negri IRCCS, University of Milan (Milano)

Objectives: Since the end of February 2020, Italy has suffered one of the first and most severe outbreaks of coronavirus disease-2019 (COVID-19) [1]. However, what happened just before the Italian index case has not yet been investigated. To answer this question, we evaluated the potential impact of COVID-19 on the clinical features of a cohort of neurological inpatients admitted right before the Italian index case, as compared to the same period of the previous year.

Methods: Demographic, clinical, treatment, and laboratory data were extracted from medical records. Data collected included all inpatients that had been admitted to the Neurology and Stroke Units of the Ospedale Maggiore Policlinico, Milan, Italy, from December 15th, 2018, to February 20th, 2019, and from December 15th, 2019, to February 20th, 2020.

Results: Of 248 patients, 97 subjects $(39.1 \%)$ were admitted for an acute cerebrovascular event: 46 in the 2018/2019 period (mean[SD] age, 72.3 [15.6] years; 22 men [47.8\%]), and 51 in the 2019/2020 interval (mean[SD] age, 72.8[12.4] years; 24 men [47.1\%]). The number of cryptogenic strokes has increased during the 2019-2020 year, as compared with the previous year $(30[58.8 \%]$ vs $18[39.1 \%], \mathrm{p}=0.05)$. These patients had a longer hospitalization (mean[SD] day, 15.7[10.5] days vs mean[SD] day, 11.7[7.2] days, $\mathrm{p}=0.03$ ) and more frequent cerebrovascular complications $(9[30.0 \%]$ vs $2[11.1 \%])$, but presented a lower incidence of cardio-cerebral risk factors $(18[60.0 \%]$ vs $14[77.8 \%])$. Fortythree out of 51 total strokes in the 2019/2020 interval were then contacted on the phone. Only 11 had undergone swab for SARS-CoV2, but all cryptogenic strokes tested resulted positive (6 of 6), while none of the negatives was a cryptogenic stroke.
Discussion: Current evidence suggests that COVID-19 may predispose to thrombotic disease due to different mechanisms [2]. Cerebrovascular complications have been frequently reported in patients with either asymptomatic or early-stages of COVID-19, also in subjects with lower cardio-cerebrovascular risk factors. The higher incidence of cryptogenic stroke may be associated with a transient hypercoagulable state, leading to thrombi formation in the cerebral vessels. Consistently, we observed a significant increase in the number of cryptogenic strokes for no apparent reason in the 2019/2020 interval, as compared to the previous year. In our cohort, cryptogenic subtypes were about $60 \%$ of all strokes in the 2019/2020 interval, compared to the literature data, in which cryptogenic strokes account for 30\%-40\% of ischemic strokes [3].

Conclusions: Right before the Italian index case, an increase in cryptogenic strokes has occurred, possibly due to the concomitant COVID-19. References:

1. Protezione Civile: Coronavirus. http://www.protezionecivile.gov.it/ attivita-rischi/rischiosanitario/emergenze/coronavirus

2. Bikdeli B, Madhavan MV, Jimenez D, Chuich T, Dreyfus I, Driggin E, et al. COVID-19 and Thrombotic or Thromboembolic Disease: Implications for Prevention, Antithrombotic Therapy, and Follow-up J Am Coll Cardiol. (2020);75(23):2950-73

3. Yaghi S, Bernstein RA, Passman R, Okin PM, Furie KL. Cryptogenic Stroke: Research and Practice. Circ Res. (2017);120(3):527-40

\section{COVID-19 AND MANAGEMENT OF ADVANCED THERAPIES IN PARKINSON'S DISEASE; DO ALL THERAPIES HAVE THE SAME NEEDS?}

\author{
R. Cerroni, N. Mercuri, S. Scalise, M. Pierantozzi, A. Stefani
}

Parkinson Centre, Department of System Medicine, University of Rome Tor Vergata (Roma)

COVID-19 emergency forced our Parkinson Centre to organize different modalities of health care [1]. The purpose of this study was to try to overcome SARS-CoV-2 outbreak difficulties by alternatively obtaining clinical information from our PD patients in advanced therapy, to promote targeted intervention. From March 8th to June 3rd, forced to cancel scheduled visits, we produced and administered a specific survey, to identify critical issues in PD patients in device related therapy (DrT). The interview was structured in two parts: the first one investigated about symptoms and any COVID-19 related diagnostic process, the presence of epidemic-related concerns and precautionary measures taken by patients; the second part evaluated the presence of motor/non-motor worsening, and the impact of the SARS-CoV-2 outbreak on DrTs. Using both the classic phone-call and the smartphone video-call on smartphone, we administered the survey, studying the possible clinical deterioration requiring a device adjustment, the possibility of remote intervention and if COVID-19 emergency changed patients' relationship with device. We interviewed 21 patients treated with Deep Brain Stimulation (DBS), 32 patients on Levodopa-Carbidopa Intestinal Gel (LCIG) therapy and two patients in continuous subcutaneous apomorphine infusion (CSAI). Our survey suggests that there are substantial differences between different DrTs. Amongst the 32 patients under LCIG, only 4 required an active intervention on the pump consisting of a dosage modulation, successfully performed using a smartphone video-call with the help of caregiver. No urgency was found in two patients under apomorphine. Conversely, DBS patients presented greater difficulties (6 out of 21). Two required a large increase in Levodopa daily-dosage, in absence of significant worsening 
of motor performance as judged by smartphone video-call. Two patients developed an anxiety disorder, which could not be resolved by remote intervention, requiring a dedicated access to clinic in a clean dedicated path. Two patients, dissatisfied with their motor condition, independently increased the levodopa intake, with the unmasking of dyskinesias, otherwise controlled until then. LCIG patients maintained good confidence in DrT; nevertheless, DBS patients, while acknowledging that without DBS they would be worsened, accrued a greater mistrust towards their therapy. DBS therapy requires special attention during crisis. Developing dedicated clinical pathways for patients with DBS is extremely important, considering possible psychopathological vulnerability and the relevance of placebo/ nocebo-effect. It's necessary to implement telemedicine tools that allow large-scale remote intervention on DBS patients [2], knowingly taking into account that PD patients in DrT are not a homogeneous cohort.

References:

1. Schirinzi T, Cerroni R, Di Lazzaro G, et al. Self-reported needs of patients with Parkinson's disease during COVID-19 emergency in Italy. Neurol Sci. (2020) Jun; 41(6):1373-75

2. Seritan AL, Heiry M, Iosif AM, Dodge M, Ostrem JL. Telepsychiatry for patients with movement disorders: a feasibility and patient satisfaction study. J Clin Mov Disord (2019);6:1

\section{THE POTENTIAL IMPACT OF ENHANCED HYGIENIC MEASURES DURING THE COVID-19 OUTBREAK ON HOSPITAL-ACQUIRED INFECTIONS: A CASE-CONTROL STUDY IN NEUROLOGICAL UNITS}

E. Cerulli Irelli ${ }^{1}$, B. Orlando ${ }^{1}$, E. Cocchi ${ }^{2}$, F. Fattapposta ${ }^{1}$, V. Di Piero ${ }^{1}$, D. Toni ${ }^{1}$, M. Ciardi ${ }^{2}$, A. Giallonardo ${ }^{1}$, G. Fabbrini ${ }^{1}$, A. Berardelli ${ }^{1}$, C. Di Bonaventura $^{1}$

\section{${ }^{1}$ Department of Human Neurosciences, Sapienza University of Rome (Roma); ${ }^{2}$ Department of Public Health and Pediatrics, University of Turin (Torino)}

Objective: Hospital-acquired infections (HAIs) are frequent complications among acute patients hospitalized in neurological units, especially among those hospitalized for stroke.[1] During the COVID-19 outbreak, greater attention to hygienic measures, including a significant increase in the use of personal protective equipment (PPE), has been observed among healthcare workers.[2] With this background, we aimed to analyze possible differences in terms of HAIs and antibiotic prescriptions between neurological patients hospitalized during the COVID-19 outbreak in 2020 and those hospitalized during the same period in 2019.

Materials: A cohort of patients hospitalized in neurological and stroke unit from March 8, 2020 and discharged prior to May 31, 2020 were included in the study and compared with patients hospitalized during the same period in 2019. Demographic and clinical data were extracted from electronic medical records and retrospectively reviewed.

Methods: This is a retrospective single-center case-control study. The primary outcome was to evaluate differences in terms of HAIs and antibiotic prescriptions between 2020 and 2019. Multivariable logistic and Poisson regression models were used to adjust for possible confounders.

Results: A total of 319 patients were included in the study ( $n=103$ in $2020, n=216$ in 2019). Among patients hospitalized in 2019, the incidence of HAIs was $31.5 \%$ (95\% confidence interval (CI): 0.25-0.38), compared with $23.3 \%$ (95\% CI: $0.15-0.32)$ in $2020(\mathrm{p}=0.12)$. Multivariable logistic regression showed that hospitalization during 2020 was independently associated with a lower risk of HAIs (odds ratio:
0.34, 95\% CI: 0.16-0.71, p=0.004). Poisson regression models showed that hospitalization during 2020 was also independently associated with both a lower number of HAIs (relative risk (RR): $0.56,95 \%$ CI: 0.38 $0.81, \mathrm{p}=0.01$ ) and a lower number of prescribed antibiotics per patient (RR: 0.66, 95\% CI: 0.49-0.87, $\mathrm{p}=0.02$ ).

Discussion: HAIs were less common during the 2020 COVID-19 outbreak as compared with the same period in 2019, likely due to enhanced hygienic measures, including the increased use of PPE among healthcare workers. To the best of our knowledge, this is the first study providing evidences regarding the impact of stricter hygienic measures on HAIs.

Conclusion: Our study provides evidences supporting the needing to extend the use of stricter hygienic measures even after the COVID-19 outbreak. Our finding are particularly relevant when considering the burden of HAI in terms of mortality and public spending, especially among neurological patients.

References:

1. Emsley HC, Hopkins SJ. Acute ischaemic stroke and infection: recent and emerging concepts. Lancet Neurol. (2008);7(4):341353. doi:10.1016/S1474-4422(08)70061-9

2. World Health Organization. (2020). Rational use of personal protective equipment for coronavirus disease (COVID-19) and considerations during severe shortages: interim guidance. Avalaible at: https://apps.who.int/iris/handle/10665/331695

\section{TELEMEDICINE FOR PARKINSONISM: A TWO-STEP MODEL BASED ON THE COVID-19 EXPERIENCE IN MILAN, ITALY}

\author{
R. Cilia ${ }^{1}$, F. Mancini ${ }^{2}$, S. Bonvegna ${ }^{1}$, G. Straccia $^{3}$, N. Golfre' Andreasi ${ }^{1}$, \\ A. Elia ${ }^{1}$, L. Romito $^{1}$, G. Devigili ${ }^{1}$, B. Bloem ${ }^{4}$, R. Eleopra ${ }^{1}$ \\ ${ }^{1}$ Department of Clinical Neurosciences, Foundation IRCCS Neurological \\ Institute Carlo Besta (Milano); ${ }^{2}$ Department of Neurology and \\ Laboratory of Neuroscience, IRCCS Istituto Auxologico Italiano \\ (Milano); ${ }^{3}$ Department of Medical Sciences and Advanced Surgery, \\ University of Campania (Napoli); ${ }^{4}$ Department of Neurology, Radboud \\ University Nijmegen Medical Centre, Donders Institute for Brain, \\ Cognition and Behaviour (Nijmegen-NL)
}

Aims: Whereas the initial response strategy to COVID-19 aimed to contain its spread, policy makers gradually lifted lockdown restrictions moving to the mitigation phase. To avoid a second peak in incidence, we propose a two-step telehealth model for patients with parkinsonism.

Methods: The first step involves a forward triage performed by a readily accessible case manager, who can solve many medical problems remotely by using predefined semi-structured interview and creating an online dossier, which is available for patients, neurologist and the multidisciplinary care team. Whenever a subsequent input by a medical specialist is needed, the second step consists of remote assessment by a neurologist/geriatrician.

Results: First step: from March 12th to May 14th, 2020, the case manager managed 2021 interactions with 525 patients throughout Italy, of whom 398 patients required to be managed by the case manager. Among these patients, $62 \%$ were fully managed by the case manager, while $38 \%$ required subsequent referral to a multidisciplinary team. Only one third (36.4\%) of referred patients needed a neurologist; the remaining patients required physiotherapists (35.8\%), psychologists (16\%), and other professionals. Second step: from March 30th to May 14th, 2020, videoconsultations with experienced neurologists became $71.4 \%$ of the total 
number of outpatient assessments. Out of the 174 scheduled visits, $54.6 \%$ were replaced by video-consultation, $23.6 \%$ were cancelled (mostly due to lockdown restrictions, COVID-19-related symptoms, or mild/stable PD), only $21.8 \%$ of patients needed in-person visit.

Discussion: We propose a two-step model to optimize the management of patients with parkinsonism. Patients contact the case manager, who can directly manage the problem, or refer to the multidisciplinary team. Whenever referral to a neurologist is needed, the case manager assigns a 4-level code triage, where greater urgency would lead to reduced time-to-assessment. Code assignment is based on two gateway questions: does this change increase the risk of morbidity in the shortterm? Has functional independence changed? Accordingly, we hypothesize four scenarios: (a) white-code: non-urgent; (b) green-code: non urgent but needing video-consultation; (c) yellow-code: timely video consultation to solve urgent issues and to decide whether in-person visit is needed; (d) red-code: life-threatening event needing hospitalization. Before the outbreak, it has been proposed that in-person visits might become the "Option B" for frail patients with chronic disease.[1] Our successful experience is in line with previous randomized controlled trials on patients with parkinsonism suggesting that similar health outcomes may be reached using telehealth compared with in-person visits.[2,3]

References:

1. Duffy S, Lee TH. In-person health care as option B. N Engl J Med. (2018);378(2):104-06

2. Flodgren G, Rachas A, Farmer AJ, Inzitari M, Shepperd S. Interactive telemedicine: effects on professional practice and health care outcomes. Cochrane Database Syst Rev. (2015);2015(9):CD002098

3. Beck CA, Beran DB, Biglan KM, et al. National randomized controlled trial of virtual house calls for Parkinson disease. Neurology (2017);89(11):1152-61

\section{NEUROLOGICAL FEATURES IN SARS-COV-2 INFECTED PATIENTS WITH SMELL AND TASTE DISORDER}

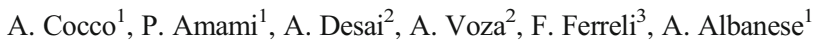 \\ ${ }^{1}$ Neurology Unit, Humanitas Research Hospital (Rozzano-MI); \\ ${ }^{2}$ Emergency Department, Humanitas Research Hospital (Rozzano-MI); \\ ${ }^{3}$ Otolaryngology Unit, Humanitas Research Hospital (Rozzano-MI)
}

Objective: It has been postulated that new-onset of smell and taste disorders (STD) in SARS-CoV-2 infected patients may indicate brain involvement. Ability to invade the central nervous system (CNS) through the olfactory neuroepithelium has been demonstrated for previous strains of coronavirus [1]. CNS involvement is supported by the observation of MRI abnormalities in the olfactory bulb of some patients with SARSCoV-2 infection and STD [2]. We studied a cohort of SARS-CoV-2 infected patients with new-onset STD.

Materials and methods: We assessed patients with documented SARS-CoV-2 infection and no concomitant CNS involvement. Patients were surveyed for smell/taste dysfunction and assessed for a predefined list of neurological symptoms. Categorical variables were expressed as frequencies $(\%)$, continuous variables as means $( \pm \mathrm{SD})$. For between group comparisons, the Fisher exact test was used for categorical variables and the T-test for continuous variables. The statistical level was set at .05 .

Results: The cohort included 105 infected patients. Their mean age was 56.2 years; $40 \%$ were women. Any altered sense of smell or taste was reported by 78 patients $(74.3 \%$; $95 \%$ CI, $64.8-82.3)$. Overall, $88 \%$ of women had STD compared to $65 \%$ of men. Disease severity, as evidenced by the clinical management at home or in hospital, was unrelated to STD occurrence. In approximately half of the patients smell/taste dysfunction recovered within 20 days. Patients with new-onset STD had a significantly higher prevalence of mild neurological symptoms compared to patients without STD. Symptoms with higher prevalence in SARSCoV-2 patients with STD included: headache, balance impairment, dysphonia, dizziness, mild confusion, sensory and visual disturbances.

Discussion: These data suggest a possible mild and transient CNS involvement in patients with SARS-CoV-2 infection. The reported neurological symptoms were mild and not suggestive of anatomically defined CNS lesions, at variance with a direct nervous system lesioning documented in a minority of patients with SARS-CoV-2 infection [3]. The underlying pathophysiology of neurological manifestations in SARSCoV-2 remains to be fully determined. SARS-CoV-2 infection is a systemic disease where mild neurological signs may be overlooked when the clinical picture is dominated by respiratory symptoms or in patients who are isolated at domicile. These results must be interpreted with caution, due to unavoidable study limitations. We suggest, however, that consideration be given to performing detailed neurological assessment in patients with SARS-CoV-2 infection and new-onset STD.

References:

1. Dubé M, Le Coupanec A, Wong AHM, Rini JM, Desforges M, Talbot PJ. Axonal Transport Enables Neuron-to-Neuron Propagation of Human Coronavirus OC43. J Virol. (2018);92(17):e00404-18. Published (2018) Aug 16

2. Politi LS, Salsano E, Grimaldi M. Magnetic Resonance Imaging Alteration of the Brain in a Patient with Coronavirus Disease 2019 (COVID-19) and Anosmia. JAMA Neurol. Published online May 29, (2020)

3. Whittaker A, Anson M, Harky A. Neurological Manifestations of COVID-19: A systematic review and current update. Acta Neurol Scand. (2020);142(1):14-22

\section{SELECTIVE CRANIAL MULTINEURITIS IN SEVERE COVID-19 PNEUMONIA: TWO CASES AND LITERATURE REVIEW}

\author{
R. De Gennaro, E. Gastaldo, C. Tamborino, M. Baraldo, N. Casula, R. \\ Quatrale
}

Dell'Angelo Hospital, AULSS3 Serenissima (Mestre-VE)

Objective: To report two cases of cranial multineuritis after severe acute respiratory syndrome caused by Coronavirus -2 .

Methods: Patients data were obtained from medical records of the clinical chart from Ospedale dell'Angelo Hospital, Venice - Italy.

Results: The first patient is a 42 years-old male patients who developed, 10 days after the resolution of Coronavirus- 2 pneumonia and intensive care unit period with hyperactive delirium, a cranial multineuritis with asymmetric distribution (bilateral hypoglossus involvement and right Claude Bernard Horner syndrome). No albumino-cytologic dissociation was found in cerebro spinal fluid, severe bilateral denervation in hypoglossus nerve, with normal EMG of other cranial muscles, blink reflex, and normal cerebral MRI also with gadolinium. He presented a striking improvement after intravenous human immunoglobulin therapy. The second case is a 67 years old male patient who developed a cranial neuritis (left hypoglossus paresis), with dyslalia and deglutition difficulties. He had cerebrospinal fluid abnormalities (albumin-cytologic 
dissociation), no involvement of the ninth and tenth cranial nerves, no other neurologic involvement.

Discussion: Cranial neuritis is a possible neurological manifestation of Coronavirus 2 pneumonia. Aetiology is not clear: the hypothesis of direct neuroinvasion, through olfactory nasopharyngeal termination and medulla dissemination is the most frequent one. The hypothesis of immunomediated genesis is suggested by the immunoglobulin therapy response.

Conclusion: Neurological involvement of Coronavirus 2 pneumonia patients is frequent. No valid biomarkers are available for understanding the aetiology nor pathogenetic mechanism, but every patient needs a careful neurologic examination because immunomodulatory therapy can be useful and safe.

References:

- S. J. Pleasure, A. J. Green, S. A. Josephson The Spectrum of Neurologic Disease in the Severe Acute Respiratory Syndrome Coronavirus 2 Pandemic Infection Neurologists Move to the Frontlines. JAMA Neurology Published online April 10, 2020.

- Pleasure SJ, Green AJ, Josephson SA. The Spectrum of Neurologic Disease in the Severe Acute Respiratory Syndrome Coronavirus 2 Pandemic Infection: Neurologists Move to the Frontlines [published online ahead of print, 2020 Apr 10]

- Ling Mao, Huijuan Jin, Mengdie Wang, Yu Hu, Shengcai Chen, Quanwei He, Jiang Chang, Candong Hong, Yifan Zhou, David Wang, Xiaoping Miao, Yanan Li, Bo Hu. Neurologic Manifestations of Hospitalized Patients with Coronavirus Disease 2019 in Wuhan, China. JAMA Neurol 2020 Apr 10;e201127. doi: 10.1001/jamaneurol.2020.1127

- M. Padroni, V. Mastrangelo, G. M. Asioli, L. Pavolucci, S. Abu Rumeileh, M. G. Piscaglia, P. Querzani, C. Callegarini, M. Foschi. Guillain Barré syndrome following COVID 19: new infection, old complication? Journal of Neurology https://doi.org/10.1007/ s00415-020-09849-6

\section{ACUTE DISSEMINATED ENCEPHALOMYELITIS (ADEM) IN COVID-19: A FEASIBLE APPROACH}

V. De Giuli ${ }^{1}$, C. Campana ${ }^{1}$, A. Giossi ${ }^{1}$, C. Ambrosi ${ }^{2}$, G. Maccabelli ${ }^{2}$, F. Baglivo $^{3}$, B. Censori ${ }^{1}$, C. Costanzi ${ }^{1}$

${ }^{1}$ Department of Neurology, ASST Cremona (Cremona); ${ }^{2}$ Department of Neuroradiology, ASST Cremona (Cremona); ${ }^{3}$ Department of Pneumology, ASST Cremona (Cremona)

Introduction: Neurologic involvement was confirmed in the infection of other coronavirus such as SARS-CoV and MERS-CoV and neurological complication of novel coronavirus SARS-CoV-2 infection have been increasingly reported, involving both central and peripheral nervous systems.

Case Report: An obese 66-year-old man was admitted at hospital for dyspnea and respiratory failure. No focal or cognitive neurological symptoms were described. Chest-CT showed the presence of bilateral ground glass areas and nasopharynx swab sample confirmed SARS-CoV-2 infection. He was intubated and tracheotomy was performed. Patient was treated with hydroxychloroquine, lopinavir/ritonavir, piperacillin/tazobactam, methylprednisolone. Interleukin-6 receptor-antibody, tocilizumab, was administered after 45 days, because of low response to treatments. The day after tocilizumab he was extubated and upon awakening he showed neurologic symptoms rapidly worsening: right hemiparesis, alterated consciousness, psychomotor agitation and left hemianopsia. Brain-MRI revealed focal oval hyperintensities in T2- weighted images bilaterally in the pons, in the right superior cerebellar peduncle, right internal capsule, corpus callosum and deep periventricular frontal white matter, with restricted diffusion and irregular, peripheral enhancement of some of them. These features were most compatible with acute disseminated encephalomyelitis. CSF analysis revealed normal WBC count and mildly elevated protein count. CSF SARS-Cov-2 RTPCR assay was negative as other RT-PCR for neurotropic viruses. AntiAQP4, anti-MOG and oligoclonal bands were negative. He was treated with high-dose-methylprednisolone intravenously and tapering oral prednisone for 5 weeks. He clinically improved during the first week; followup MRI showed volumetric reduction of lesions, reduced hyperintensity on DW-images and no gadolinium enhancement.

Discussion: ADEM is a rare CNS autoimmune demyelinating disorder thought to be the result of inflammation triggered by an environmental stimulus. Although the concept of inflammatory storms remains controversial, it has been generally recognized that immune-mediated inflammation plays an important role in the pathogenesis of COVID-19. In murine models, a human coronavirus has been found to cause a chronic demyelinating condition resembling multiple sclerosis. Our patient was treated with methylprednisolone and tocilizumab, which was established as effective in various autoimmune diseases, owing to the crucial role of IL-6 in stimulating both B and T-cells. A recent study reported a powerful disease modulation by tocilizumab in rituximab-refractory neuromyelitis optica. Even though brain MRI showed enhancing active lesions after tocilizumab, we thought its positive effect on the cytokine cascade could still be helpful in the CNS. In conclusion this case provides additional support to the hypothesis that even SARS-CoV2 may be a trigger factor of CNS demyelinating disease in humans.

References:

- L. Mao, H. Jin, M. Wang, et al. Neurologic Manifestations of Hospitalized Patients with Coronavirus Disease 2019 in Wuhan, China. JAMA Neurol. Published online April 10, 2020. doi:10.1001/jamaneurol.2020.1127

- D. Pohl, G. Alper, K. Van Haren, et al. Acute disseminated encephalomyelitis: updates on an inflammatory CNS syndrome. Neurology (2016); 87:S38-S45

- H. Hayward-Koennecke, M. Reindl, R. Martin, S. Schippling. Tocilizumab treatment in severe recurrent anti-MOG-associated optic neuritis. Neurology (2019); 92(16):765-67

\section{CoV2 SARS EXPERIENCE IN THE SOUTH OF ITALY}

P. Fiori ${ }^{1}$, G. Capaldo ${ }^{1}$, A. Corbo ${ }^{1}$, I. Iorillo ${ }^{1}$, M. Massarelli ${ }^{1}$, D. Cataldo ${ }^{2}$, A. Cavalli ${ }^{2}$, G. Ciano ${ }^{2}$, C. Cocca ${ }^{2}$, R. D'Amato ${ }^{2}$, F. Lo Calzo ${ }^{2}$, L.

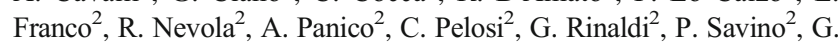
Bianchino $^{3}$, A. Di Gianni ${ }^{3}$, P. Gualdiero ${ }^{3}$, G. Manganelli ${ }^{3}$, S. Minichiello $^{3}$, V. Pellecchia ${ }^{3}$, M. Alberico ${ }^{4}$, G. Benigni ${ }^{4}$, T. Damiano, ${\text { A. } \text { Iuorio }^{4} \text {, E. Pace }}^{4}$, B. Brogna ${ }^{5}$, M. Grappone ${ }^{6}$, M. Ferrara ${ }^{4}$, E. Mazza ${ }^{5}$, C. Tammaro ${ }^{6}$, A. Monaco ${ }^{1}$, G. Bellizzi ${ }^{3}$, A. Bellizzi ${ }^{2}$

${ }^{1}$ Neurology, S. Ottone Frangipane Hospital, ASL AV, University of Naples (Ariano Irpino-AV); ${ }^{2}$ Medicine, S. Ottone Frangipane Hospital, ASL AV, University of Naples (Ariano Irpino-AV); ${ }^{3}$ Cardiology, S. Ottone Frangipane Hospital, ASL AV, University of Naples (Ariano Irpino-AV); ${ }^{4}$ Intensive Care, S. Ottone Frangipane Hospital, ASL AV, University of Naples (Ariano Irpino-AV); ${ }^{5}$ Radiology, S. Ottone Frangipane Hospital, ASL AV, University of Naples (Ariano IrpinoAV); ${ }^{6}$ Laboratory, S. Ottone Frangipane Hospital, ASL AV, University of Naples (Ariano Irpino-AV) 
Introduction and aim: Coronaviruses $(\mathrm{CoV})$ were described as mite agents, causing benign upper respiratory tract and intestinal infections. Asian outbreaks of CoV-related Severe-Acute- Respiratory-Syndrome (SARS) and Middle-East-Respiratory-Syndrome (MERS) pointed out the risk of diffusion. CoV2 SARS threatened health care and economical steady state all over the world. Many questions on virulence have still not found answers. We report our experience in the Cov2 Unit of a southern community hospital.

Materials and Methods: Sixty patients came to our observation.

Results: Clinical manifestations were mainly represented by dyspnea. The majority of elderly patients presented speech disturbs and confusion, because of hypoxic encephalopathy in interstitial, often accompanied by lobar pneumonia. They had absolute lymphopenia with neutrophilia. Oxygen was administered through nasal cannula, Venturi mask. In a minority of patients, Non-Invasive-Positive-Pressure-Ventilation (NIPV) was necessary. Rarely, it was followed by Invasive-OxygenTherapy (IOT). Mortality was around $15 \%$ in $>80,5 \%$ in $65-80,1,7 \%$ in $35-65,0 \%$ in $<35$ years old patients.

Discussion and Conclusions: Although the incidence of CoV2 SARS was high, the mortality was inferior compared to the north of Italy. This was explained by low level of environmental pollution, early diagnosis and therapy. Respiratory exercises were recommended. In elderly ones, comorbidities negatively interfered with recovery. Blood cell counts may help in discriminating immune-depressed, highly-contagious from immune-competent, low-contagious patients, in order to isolate the former and to study herd immunity in the latter. The negativity of rapid test may include false negative. Oropharyngeal swab detects the presence of viral RNA, not alive virus. Its negativity does not exclude ongoing infection. The diagnosis must be confirmed by radiological examinations. Bronchoaspiration/lavage may reveal low respiratory infection, but they are invasive. An alternative may be represented by plasma biomarkers of epithelial and endothelial injury. In elderly patients IOT is a difficult task because of individual heterogeneity of pathological findings. Weaning from IOT is even more arduous. Ventilator-associated-lung-injury seems to be related to the pathological process rather than to applied techniques. Alveolar epithelial and endothelial damage, immune-dysregulation, intravascular coagulation, neuro-muscular and cardiological dysfunction may lead to fibrosis, irreversible pulmonary failure, death. Viral load and mutagenesis rate are expression of contagion spreading in high density population, especially in case of extreme social and economic gap, poor environmental resources, conflicts, migrations, climate changes and environmental disasters. The most powerful strategy is health education. It is mandatory not to disappoint trust on health care. Protective devices must be smartly used, because they are not ecologically disposable.

\section{THE EFFECTS OF COVID-19 AND QUARANTINE MEASURES ON THE LIFESTYLES AND MENTAL HEALTH OF PEOPLE OVER 60 AT INCREASED RISK OF DEMENTIA}

\section{F. Franchini, B. Filiputti, A. Martone, S. Sannino, S. Di Santo}

Department of Clinic and Behavioral Neurology, IRCCS Santa Lucia Foundation (Roma)

Aims: The lockdown strategies adopted to limit the spread of COVID-19 infection may lead to adopting unhealthy lifestyles which may impact on the mental well-being and future risk of dementia. Older adults with mild cognitive impairment (MCI) or subjective cognitive decline (SCD) may suffer important mental health consequences from measures of quarantine and confinement. The study aimed to explore the effects of COVID-19 and quarantine measures on lifestyles and mental health of the elderly at increased risk of dementia.

Methods: 126 community-dwelling seniors with MCI or SCD were phone-interviewed and assessed with questions regarding variables related to COVID-19 pandemic, lifestyle changes, and scales validated for the assessment of depression, anxiety, and apathy.

Results: The sample included $55.6 \%$ of patients with MCI and 56 people with SCD. Over $1 / 3$ of the sample reduced their physical activity and nearly $70 \%$ reported an increase in idle time. Adherence to the Mediterranean diet decreased in almost $1 / 3$ of respondents and over $35 \%$ reported weight gain. Social activities were abolished and 1/6 of participants also decreased productive and mental-stimulating activities. $19.8 \%$ were depressed, $9.5 \%$ anxious and $9.5 \%$ apathetic.

Discussion: A significant association existed between depression and living alone or having a poor relation with cohabitants and between anxiety and SCD, cold or flu symptoms, and reduction in productive leisure activities.

Conclusions: Seniors with SCD and MCI underwent lifestyle changes that are potentially harmful to their future cognitive decline, even if, except for leisure activities, they do not appear to be cross-sectionally associated with psychiatric symptoms.

References:

- Santini ZI, Jose PE, York Cornwell E, Koyanagi A, Nielsen L, Hinrichsen C, Meilstrup C, Madsen KR, Koushede V Social disconnectedness, perceived isolation, and symptoms of depression and anxiety among older Americans (NSHAP): a longitudinal mediation analysis. Lancet Public Heal. (2020);5:e62-e70

- Armitage R, Nellums LB COVID-19 and the consequences of isolating the elderly. Lancet (2020) Public Heal

- Li JQ, Tan L, Wang HF, Tan MS, Tan L, Xu W, Zhao QF, Wang J, Jiang T, Yu JT Risk factors for predicting progression from mild cognitive impairment to Alzheimer's disease: A systematic review and meta-analysis of cohort studies. J. Neurol. Neurosurg. Psychiatry (2016);87:476-84

\section{IMPACT OF COVID-19 ON PATIENTS WITH NEUROMUSCULAR DISORDERS IN LOMBARDY AREA, ITALY}

D. Gagliardi ${ }^{1}$, G. Costamagna ${ }^{1}$, E. Abati ${ }^{1}$, E. Mauri ${ }^{2}$, R. Brusa ${ }^{2}$, F. Magri $^{2}$, M. Meneri ${ }^{2}$, D. Velardo ${ }^{2}$, M. Sciacco ${ }^{3}$, N. Bresolin ${ }^{4}$, S. Corti ${ }^{4}$, G. $\mathrm{Comi}^{5}$

${ }^{1}$ IRCCS Foundation Ca' Granda Ospedale Maggiore Policlinico di Milano, University of Milan (Milano); ${ }^{2}$ Neurology Unit, IRCCS Foundation Ca' Granda Policlinico di Milano (Milano); ${ }^{3}$ Neuromuscular and Rare Disease Unit, IRCCS Foundation Ca' Granda Policlinico di Milano (Milano); ${ }^{4}$ Neurology Unit, IRCCS Foundation Ca' Granda Ospedale Maggiore Policlinico di Milano, University of Milan (Milano); ${ }^{5}$ Neuromuscular and Rare Disease Unit, IRCCS Foundation Ca' Granda Ospedale Maggiore Policlinico di Milano, University of Milan (Milano)

Background and Aims: The coronavirus disease-2019 (COVID-19) pandemic has forced life changes and has limited access to care for patients with chronic diseases. Among these, individuals presenting impaired respiratory function or receiving immunosuppressive treatments might show a higher vulnerability to COVID-19 infection or a severe disease course. We investigated how the pandemic has affected patients with 
neuromuscular disorders (NMD) in Lombardy - a COVID-19 high-incidence area.

Material and Methods: We conducted a cross-sectional phone-based survey study on a sample of 300 NMD patients followed up at our clinic in Milan, Italy. Patients were asked about comorbidities, health condition regarding the underlying NMD and quality of life (QoL) before and during the COVID-19 pandemic. The presence of symptoms suggestive of COVID-19 infection and confirmed cases were also evaluated.

Results: We collected 205 responses. 53 (25.9\%) patients reported a subjective worsening of the underlying NMD. Comparing pre-pandemic and pandemic time frames, every NMD subgroup reported a perceived worsening of the underlying NMD. Overall, negative changes in each item (appetite, sleep, mood, job, social relationships, pain) of QoL were reported during COVID-19 pandemic. 79 (38.5\%) patients reported at least one symptom ascribable to COVID-19 infection. Among the 10 patients tested with nasopharyngeal swabs in our cohort, 6 resulted positive and 3 died from respiratory failure, including 2 patients on corticosteroid/immunosuppressive therapy.

Discussion and Conclusions: COVID-19 pandemic had an overall subjective negative impact on access to visits and ancillary services and QoL of our NMD patients. Clinical course and outcome of COVID-19 in NMD patients are highly variable and the role of immunosuppression needs to be further investigated.

\section{ASYMPTOMATIC PERIPHERAL NEUROPATHY IN PATIENTS WITH SARS-COV-2 INFECTION}

\author{
I. Gandoglia ${ }^{1}$, A. Assini ${ }^{1}$, K. Rikani ${ }^{1}$, E. Pontali ${ }^{2}$, G. Siri ${ }^{3}$, M. Del Sette ${ }^{1}$ \\ ${ }^{1}$ Neurology Unit, Galliera Hospital (Genova); ${ }^{2}$ Infectious Diseases Unit, \\ Galliera Hospital (Genova); ${ }^{3}$ Unit of Biostatistics, Scientific Directorate, \\ Galliera Hospital (Genova)
}

Background: Several neurological complications related to SARS-CoV-2 infection have been recently reported. The involvement of peripheral nervous system (PNS) in SARS-CoV-2 infection consists in the development of immune mediated neuropathies such as Guillain-Barrè Syndrome and other variants [1]. In this study we assessed possible asymptomatic abnormalities in peripheral nerves conduction during acute phase of SARS-CoV-2 infection and their possible correlation with blood circulating markers of inflammation or vascular damage. The aim of this study was to understand the impact of COVID-19 on PNS and to identify early alterations possibly associated to the development of symptomatic neuropathies.

Material and Methods: We performed the electroneurographic study of the lower limbs and blood tests in 17 patients affected by COVID19. The study was conducted at two time points: the first one was within the first 7 days after hospital admission (T0) and the second one after $30 \pm 10$ days (T1). We excluded patients with symptoms or risk factors for peripheral neuropathies and patients with documented lumbosacral root pathologies.

Results: Electroneurographic changes were found at least on one nerve at $\mathrm{T} 0$ in 10 out of 17 patients recruited (58.8\%), consisting of axonal or demyelinating changes. Electroneurography at T1 showed significant improvement in 6 out of 10 patients. Patients with normal electrophysiological study at T0 showed significant improvement of electrophysiological parameters at T1. The improvements in electroneurographic data were significantly correlated with the trend of laboratory parameters, in particular with fibrinogen, D-Dimer, ferritin, C Reactive protein and lymphocytes. None of the 17 enrolled patients developed symptoms or signs of full-blown peripheral neuropathy over time.

Conclusions: Our study shows that asymptomatic alterations of the peripheral nervous system can be found during the acute phase of SARSCoV-2 infection. It also demonstrates that these alterations significantly improve after 20-40 days from the acute phase of infection and that the improvement correlates significantly with the trend of laboratory parameters. Further studies are needed to evaluate possible long-term neurological complications and the predictive value of subclinical damage of PNS in the acute phase of infection

Reference:

1. Ellul, M. A. et al. Neurological associations of COVID-19. The Lancet Neurology (2020) Jul 2;S1474-4422(20)30221-0

\section{SARS-COV-2 AND STROKE CHARACTERISTICS: THE EXPERIENCE OF THE STROKE STUDY GROUP IN LIGURIA REGION}

I. Gandoglia ${ }^{1}$, F. Bandini ${ }^{2}$, C. Serrati ${ }^{3}$, C. Finocchi ${ }^{4}$, T. Tassinari ${ }^{5}$, P. Scotto $^{6}$, G. Trivelli ${ }^{7}$, A. Mannironi ${ }^{8}$, C. Stilo ${ }^{6}$, M. Del Sette ${ }^{1}$

${ }^{1}$ Neurology Unit, Galliera Hospital (Genova); ${ }^{2}$ Department of Neurology, San Paolo Hospital (Savona); ${ }^{3}$ Department of Neurology, Hospital of Imperia (Imperia); ${ }^{4}$ Stroke Unit, Ospedale Policlinico San Martino (Genova); ${ }^{5}$ Stroke Unit, Department of Neurology, Santa Corona Hospital (Pietra Ligure-SA); ${ }^{6}$ Neurology Unit, Villa Scassi Hospital (Genova); ${ }^{7}$ Neurology Unit, Lavagna Hospital (Lavagna-GE); ${ }^{8}$ Neurology Unit, Sant'Andrea Hospital (La Spezia)

Background: Stroke is reported as a consequence of SARS-CoV-2 infection [1]. We report features and clinical characteristics of patients with stroke and COVID19 in Liguria (Italy).

Material and Methods: We conducted a multicenter observational study on features of acute ischemic strokes (AIS) and intracranial hemorrhages (ICH) among patients who accessed 8 hospitals in Liguria during Italian lockdown. For each enrolled patient, we reported demographics, clinical characteristics, stroke severity measured by National Institute of Health stroke scale (NIHSS), and stroke subtype identified by the TOAST criteria.

Results: $\mathrm{N}=388$ patients were enrolled during from March 8 th to May 17th 2020: 333/388(85\%) patients had an AIS and 55/388(15\%) had an ICH. Twenty-six out of $388(6,7 \%)$ patients had COVID19 at hospital admission: $24 / 333(7,2 \%)$ of patients affected by AIS and $2 / 55(3,6 \%)$ of patients affected by ICH. Mean age of non-COVID19 patients was $77 \pm 12$ years (range: $20-94$ ), mean age of patients with COVID19 was $81,5 \pm 9,7$ years (range: $60-96$ ) ( $\mathrm{P}=0.08)$, only $4 / 26(15,4 \%)$ were under 70 . Among non-COVID19 patients $184 /$ $362(50 \%)$ were females; among COVID19 patients $8 / 26(30,7 \%)$ were females $(\mathrm{P}=0.06)$. Eight out of $26(30,7 \%)$ patients died for respiratory complications due to COVID19. Fifty-two out of 362(14,3\%) not infected patients died for complications $(\mathrm{P}=0.01)$. Data on stroke subtype were available for 21/24 patients with COVID19: $3 / 21(14,3 \%)$ had an atherothrombotic stroke, $7 / 21(33.3 \%)$ had cardioembolic event, $8 / 21(38 \%)$ had a lacunar stroke and 3/21(14,3\%) had an embolic stroke of undetermined source. Twenty-four out of 26(92,3\%) COVID19 patients with AIS or ICH had other risk factors for cerebrovascular diseases, $2 / 26(7,8 \%)$ had not any previous clinical history. Three out of $24(12,5 \%)$ COVID19 patients with AIS were treated by both intravenous thrombolysis and mechanical thrombectomy, and 1/24 underwent only mechanical thrombectomy. The mean NHISS of patients affected by COVID19 at the 
discharge was not significantly different from that one of non-COVID19 patients $(3,7 \pm 4,4$ vs $3,9 \pm 4,9 \mathrm{P}=0,6)$.

Conclusions: This study is comprehensive of all stroke cases in Liguria accessed to hospitals during Italian lockdown. Although in the cohort of patients with COVID19 there was a trend in having higher percentage of males and a higher mean age, the two studied cohorts did not show any significant difference in demographics and cerebrovascular risk factors indicating that in population of Liguria, SARS-CoV2 did not work as a trigger for cerebrovascular diseases. Moreover, we observed that SARS$\mathrm{CoV} 2$ infection increased the risk of dying but it did not impact on the final stroke outcome in term of disability in survived patients.

Reference:

1. Ellul, M. A. et al. Neurological associations of COVID-19. The Lancet Neurology (2020) Jul 2;S1474-4422(20)30221-0

\section{COVID-19 AND NEUROIMMUNOLOGY: A CASE OF GUILLAIN BARRĖ SYNDROME ASSOCIATED WITH CNS VASCULITIS IN A PATIENT WITH CORONAVIRUS DISEASE}

\author{
A. Innocenti ${ }^{1}$, C. Motto ${ }^{1}$, D. Facchetti ${ }^{1}$, G. Gelosa ${ }^{1}$, M. Brioschi ${ }^{1}$, D. \\ Dalla Costa $^{2}$, E. Agostoni ${ }^{1}$
}

${ }^{1}$ Neurology and Stroke Unit, ASST Grande Ospedale Metropolitano Niguarda (Milano); ${ }^{2} \mathrm{SC}$ Rehabilitation Medicine and Neurorehabilitation, ASST Grande Ospedale Metropolitano Niguarda (Milano)

Objectives: Through an emblematic clinical case, we highlight how coronavirus- 2 infection can induce a disimmune response and immunomediated diseases both in central and peripheral nervous system.

Materials and Methods: Introduction SARS-CoV-2 infection could be associated with neurological complications including among others stroke, acute nerve diseases, encephalitis. Damage within Central Nervous System (CNS) or Peripheral Nervous System (PNS) could be caused directly by the virus or by the immune-response to infection. Indeed, the coronavirus- 2 infection is associated with elevated expression of cytokine and inflammatory pathways. Activation of pro-inflammatory pathways can damage the nervous system in different manner, such as by inducing a pro-thrombotic pattern as in ischemia or by direct inflammation of brain as in encephalitis. Interestingly, in some cases the mechanism is indirect and immune-mediated. This immunomediated mechanism with both CNS and PNS involvement is evident in our clinical case.

Results/Clinical Case: A 62-year-old man was admitted to ER for fever and cough, and diagnosis of coronavirus-2 pneumonia was made. After an initial respiratory improvement, the patient worsened with progressive sensory deterioration and areflexic tetraparesis. A brain MRI showed two subcortical lesions with diffusion restriction suggestive of stroke. Considering symptoms, an electromyography and CSF analysis were also performed. Electromyography revealed a demyelinating polyneuropathy with increased proteins in CSF: Guillain-Barrè Syndrome (GBS) was diagnosed and patient was treated with intravenous immunoglobulin. The patient also underwent a control brain MRI showing small T2-FLAIR hyperintensities with restricted diffusivity in left occipital and parietal cortex; the multiple lesions were interpreted as vasculitic rather than embolic. A third MRI the following month was stable, without restricted diffusion. Patient had a good recovery after rehabilitation.

Discussion: GBS is an immune-mediated disease: although mechanisms for coronavirus PNS disease are not well understood, viral infection could probably cause an immune-response and a pro-inflammatory state that result in dysimmune diseases. The mechanisms of CNS vascular disease coronavirus related are probably more complex. The main mechanism is linked to a proinflammatory state with activation of thrombotic pathways and microvascular damage. Stroke can result from other mechanisms: among these, immune-response and pro-inflammatory status related to coronavirus can result in a vasculitic process. In our case, also CNS involvement had a probably dysimmune cause, with ischemic lesions of probable vasculitic origin, mainly posterior, with a monophasic course. The excellent response to immunoglobulin is consistent with this hypothesis.

Conclusion: COVID-19 can cause a dysimmune response involving both PNS (GBS) and CNS (CNS vasculitis).

Reference:

- Ellul MA et al. Neurological associations of COVID-19. Lancet Neurol. (2020);S1474-4422(20):30221-0

\section{COVID-19 PANDEMIC IN LOMBARDY: RISK PERCEPTION AND COPING STRATEGIES OF AGING PEOPLE}

M. Leonardi ${ }^{1}$, C. Toppo ${ }^{1}$, F. Magnani ${ }^{1}$, R. Romano ${ }^{2}$, C. Facchini ${ }^{3}$, R. Campioni $^{2}$, E. Brambilla ${ }^{2}$, E. Guastafierro ${ }^{1}$

${ }^{1}$ Neurology, Public Health and Disability Unit, Fondazione IRCSS Istituto Neurologico Carlo Besta (Milano); ${ }^{2}$ AUSER Regionale Lombardia (Milano); ${ }^{3}$ Associazione Nestore, University of Milano Bicocca (Milano)

Objectives: During the COVID-19 pandemic in Italy, lockdown and other measures were settled to limit the spread of contagion. As emerged from previous studies, vulnerable populations, especially aging people, are at higher risk of contracting the virus and of isolation due to lockdown, thus it is important to know their needs and how they face the situation to plan appropriate intervention strategies. The present study aimed to investigate risk perception and coping strategies in older adults during the COVID19 pandemic in Italy.

Materials: 514 people over 65 years, residing in the Lombardy region of Italy, were administered with a structured online interview collecting socio-demographic information, sources of information used, daily actions undertaken to avoid the contagion, risk perception related to COVID-19 and other threats, and coping strategies.

Methods: We explored whether there was any difference between the perceived risk of contracting the COVID-19 and the other treats, such as common flu or cancer. Furthermore, we checked the possible correlation of the risk perception associated with COVID-19 and both sources of information and daily actions undertaken to avoid the contagion. Finally, to assess which strategy was mainly used by the sample, we compared the avoidant and approaching coping strategies through Wilcoxon signed-rank test.

Results: Risk perception related to COVID-19 was significantly lower than the perceived risk associated with other threats, and it was correlated to the number of sources of information used. Most of the sample relied on avoidant coping strategies with a tendency to accept the situation by avoiding stressors more than rebelling to the situation with approaching strategies.

Discussion: Participants put into action most of the possible behaviors aimed to avoid contracting the COVID-19, showing a tendency in using avoidance strategies. This confirmed that the choice of the best strategy to use depends on the features of the situation a person must face. Regarding 
risk perception, it is necessary to take into consideration its link with the perception of control: the lockdown condition lets older adults to feel safer and less vulnerable to the virus.

Conclusions: During an emergency, it is particularly important to collect the aging people' perceptions and needs, considering that, in addition to being exposed to higher health risks, they are more at risk of being disadvantaged and marginalized. Providing appropriate information and exploring the attitudes of aging people is crucial for policymakers and public health officials to plan tailored intervention and supporting strategies.

\section{THE IMPACT OF LOCKDOWN DURING SARS-COV-2 OUTBREAK ON BEHAVIORAL AND PSYCHOLOGICAL SYMPTOMS OF DEMENTIA}

\author{
A. Manini ${ }^{1}$, M. Brambilla ${ }^{2}$, L. Maggiore ${ }^{2}$, S. Pomati $^{2}$, L. Pantoni ${ }^{1}$
}

${ }^{1}$ Stroke and Dementia Lab, "Luigi Sacco" Department of Biomedical and Clinical Sciences, University of Milan (Milano); ${ }^{2}$ Center for Cognitive Disorders and Dementias (CDCD), Neurology Unit, "Luigi Sacco" Hospital (Milano)

Aims: On February 21, 2020, the first Italian case of SARS-CoV-2 infection (Covid-19) was reported. As SARS-CoV-2 rapidly spread throughout Italy and the Italian healthcare system risked collapsing, the government adopted restrictive limitations and declared nationwide lockdown on March 9, which lasted until May 4, producing dramatic consequences on people lives. The aim of our study was to assess the impact of prolonged quarantine on behavioral and psychological symptoms of dementia (BPSD), and to explore the role of specific factors in the development or worsening of neuropsychiatric disturbances in demented patients.

Materials: Between April 30 and June 8, 2020, we interviewed with a semi-structured telephone-based questionnaire the caregivers of the community-dwelling adults affected by dementia who had a follow-up visit at the Center for Cognitive Disorders and Dementias of the "Luigi Sacco" Hospital scheduled from March 9 to May 15 and cancelled due to lockdown.

Methods: Changes in accommodation, living arrangements and lifestyle habits due to lockdown measures, access to emergency care services, development of clinical symptoms, and patients BPSD, including the Neuropsychiatric Inventory (NPI), were assessed. Statistical analysis was performed, including non-parametric test to compare differences between NPI scores over time, and logistic regression models to explore the impact of different factors on BPSD worsening.

Results: During the above reported period, 109 follow-up visits were scheduled and cancelled. A total of 94/109 caregivers of patients completed the interview. During the observation period (from March 9 to May 15), 4/94 patients were admitted to hospital because of Covid-19 infection. Apathy, irritability, agitation and aggression, and depression were the most common neuropsychiatric symptoms experienced by patients both at baseline and during Covid-19 pandemic. Changes in total NPI score and caregiver distress score between baseline and during lockdown, although statistically significant, were modest. Logistic regression models failed to determine predictors of change in total NPI score and total caregiver distress score.

Discussion: This is one of the first studies to investigate the presence of BPSD during SARS-CoV-2 outbreak and related nationwide lockdown, showing only slight, likely not clinically relevant, differences in BPSD burden, concerning mostly agitation and aggression, anxiety, apathy and indifference, and irritability.
Conclusions: The changes detected in neuropsychiatric symptoms were overall modest and of unclear clinical relevance, suggesting that people with dementia present a fairly large resilience, and that BPSD are not strongly influenced by quarantine and changes in daily life, at least when they are limited in time.

\section{EXTRA-PULMONARY OUTCOMES AFTER SARS-CoV-2: A CASE REPORT}

\author{
R. Pagani, A. Robustelli, A. Previtera
}

DISS, San Paolo Hospital, Rehabilitation Unit, University of Milan (Milano)

COVID-19 may result in pneumonia and Acute Respiratory Distress Syndrome (ARDS). The possible sequelae may include a spectrum of functional impairments, mostly deriving from peripheral and central nervous system involvement [1]. Early recognition of these conditions may lead to earlier treatment and consequently improve the recovery. In this report, we describe a patient with extra-pulmonary sequelae after a Severe Acute Respiratory Syndrome Coronavirus (SARS-CoV-2) infection.

Case Report: A 64-years-old gentleman was admitted to the intensive care unit (ICU) for acute hypoxemic respiratory failure leading to invasive mechanical ventilation. He had a history of hypertension, chronic atrial fibrillation and ischemic stroke. SARS-CoV-2 infection was confirmed. After two weeks, he was transferred to a Sub-ICU. At admission, he presented mild disorientation, hyposmia, hoarseness, dysphagia, diffuse muscle wasting and weakness, and hyporeflexia of lower limbs. He complained of fatigue and mood deflection. Nerve conduction studies and electromyography findings were consistent with Critical Illness Polyneuromyopathy (CIPNM) [2] and left peroneal nerve palsy. The tracheostomy tube was removed. He received nutritional supplements and underwent a rehabilitation program aimed at recovery of swallowing, standing and walking. Furthermore, he benefited from psychological support.

Discussion: This patient presented clinical symptoms and electromyographic findings compatible with Intensive Care Unit-Acquired Weakness (ICUAW) [3]. Muscle atrophy from disuse, CIPNM and the hypercatabolic state contribute in various proportions to ICUAW. Thus, early recognition of this condition may lead to a tailored rehabilitative treatment. Dysphagia is a common condition after invasive mechanical ventilation, and it may be associated with adverse clinical outcomes; it becomes crucial to evaluate swallowing to include specific rehabilitation exercises and proper selection of food consistencies. Nutritional supplementation may improve muscular trophism and enhance recovery of physical and cognitive functions. Cognitive impairment after ARDS may occur with different degrees of severity and may affect a wide variety of cognitive domains. In this case, we observed a transient confusional state, although cognitive abilities were preserved at subsequent assessments. Emotional distress is frequent in ARDS survivors, including mood disturbances, like depressive and non-specific anxiety phenomena and psychological support has proven to be helpful.

Conclusion: This case highlights the wide spectrum of clinical outcomes after SARS-CoV-2 infection, which may often include extrapulmonary sequelae. Therefore, the clinical evaluation of COVID-19 patients should not focus on respiratory function alone. All patients should undergo a thorough and early assessment of physical, neurocognitive and emotional functions since a prompt recognition of impairment may prevent long-term disabilities. 


\section{References:}

1. Mao L, Jin H, Wang M, et al. Neurologic Manifestations of Hospitalized Patients with Coronavirus Disease 2019 in Wuhan, China. JAMA Neurol. Published online April 10, (2020). doi:10.1001/jamaneurol.2020.1127

2. Pleasure SJ, Green AJ, Josephson SA. The Spectrum of Neurologic Disease in the Severe Acute Respiratory Syndrome Coronavirus 2 Pandemic Infection: Neurologists Move to the Frontlines. JAMA Neurol. Published online April 10, (2020). doi:10.1001/ jamaneurol.2020.1065

3. Shepherd S, Batra A, Lerner DP. Review of Critical Illness Myopathy and Neuropathy. Neurohospitalist (2017);7(1):41-48. doi:10.1177/1941874416663279

\section{NEUROLOGICAL INVOLVEMENT IN ELDERLY PATIENTS RECOVERED IN HEALTH RESIDENCE ASSISTED (RSA) DURING THE PANDEMY FROM NEW CORONAVIRUS 2019}

\author{
M. Perini ${ }^{1}$, S. Vernocchi ${ }^{2}$, P. Manfroi ${ }^{3}$, S. Ipsaro Passione ${ }^{3}$ \\ ${ }^{1}$ Humanitas Institute (Milano); ${ }^{2}$ Internal Medicine, Abbiategrasso \\ Hospitals (Abbiategrasso-MI); ${ }^{3}$ Associazione Interstudi Europeo, \\ Ostrava University (Chiasso-CH)
}

The involvement of the respiratory system with the development of interstitial pneumonia, the evolution in ARDS and respiratory failure are the most known causes of death in Covid-19 patients. In patients with preexisting neurological problems, death can occur due to the prevalent neurological involvement (neurocovid) this often occurs in RSA (Assisted Healthcare Residence), in elderly patients who have perhaps passed the respiratory cytokine phase. We performed consecutively, from 18 April to 18 May 2020, pulmonary ultrasound to detect interstitial pneumonia on 106 patients hosted by four RSA in Lombardy, with respiratory symptoms from COVID 19 Brain CT was performed in patients with neurological problems. A total of 106 patients were evaluated of which: 34 men and 62 women, aged between 63 and 102, average 85.98. As of $31 / 106,29.25 \%$ of the patients died. Causes of death 11 patients whose cause of death can be attributed mainly to COVID-19: 10 patients, $9.43 \%$ died of respiratory failure in COVID-19 pneumonia for no other apparent reason. $\bullet 1$ patient died from disseminated intravascular coagulation related to the cytokine storm. For the other 20 patients, COVID-19 pneumonia represents only an aggravating factor of more severe diseases that led to death: $\bullet 5$ patients, $4.72 \%$ for cardiovascular or SCC decompensation, including 1 SCC patient with high range and two low range with septic state; $\bullet 3$ patients, $2.83 \%$ for complications of dysphagia and ab-ingestis pneumonia coexisting with covid-19, of these patients, 2 were fed and hydrated by means of a gastric nose tube (SNG); at least 4 other patients had both neurological problems and dysphagia. 12 patients, $11.32 \%$ died of neurological causes with worsening slumber, lethargy and irreversible coma, of these 5 also had pneumonia ab ingestis. Of the 8 patients who died for neurological reasons without ab-ingestis, the symptoms detected were changes in the state of alertness for 7 patients, with lethargy and sleepiness up to how, in the absence of focal deficits, one patient instead presented with right hemiparesis.

Discussion: The exit occurred in $11.32 \%$ of our patients for neurological causes, perhaps neurocovid. They were not investigated with instrumental investigations such as MRI or CSF analysis, important tests for the diagnosis but, given the pre-existing neurological conditions of the patients and the severe prognosis, it was decided not to proceed.

\section{COVID-19 IN CLADRIBINE-TREATED PATIENTS WITH RELAPSING-REMITTING MULTIPLE SCLEROSIS: A MONOCENTRIC EXPERIENCE}

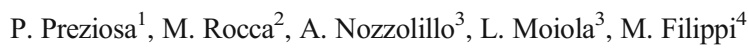

${ }^{1}$ Neuroimaging Research Unit, Institute of Experimental Neurology, Division of Neuroscience IRCCS San Raffaele Scientific Institute, VitaSalute San Raffaele University (Milano); ${ }^{2}$ Neuroimaging Research Unit, Institute of Experimental Neurology, Division of Neuroscience; and Neurology Unit, IRCCS San Raffaele Scientific Institute (Milano); ${ }^{3}$ Neurology Unit, IRCCS San Raffaele Scientific Institute (Milano); ${ }^{4}$ Neuroimaging Research Unit, Institute of Experimental Neurology, Division of Neuroscience; Neurology Unit and Neurophysiology Unit, IRCCS San Raffaele Scientific Institute; Vita-Salute San Raffaele University (Milano)

Objectives: Cladribine significantly reduces disease activity and disability progression in relapsing-remitting multiple sclerosis (RRMS) through a selective but transient depletion of lymphocyte subsets. The SARS-COV2 outbreak has raised several concerns regarding cladribine use for RRMS patients. However, the influence of cladribine on the risk of developing SARS-COV-2 infection and complications has not been investigated yet. Here, we evaluated the prevalence and clinical features of COVID-19 disease among RRMS patients treated with cladribine.

Materials and Methods: Fifty-six RRMS patients treated with cladribine in our centre (female $=39$; mean [standard deviation] age $=33.8$ [8.1] years; median [interquartile range] Expanded Disability Status Scale $[\mathrm{EDSS}]=1.5[1.0-2.0]$, disease duration $[\mathrm{DD}]=5.2[2.14-$ 11.1] years, treatment duration=1.15 [0.9-1.5] years) were asked if they had developed manifestations suggestive of SARS-COV-2 infection up to June 30th 2020 , independently from nasal/pharyngeal swabs and/or serology results. Their detailed demographic, clinical, and paraclinical characteristics were collected.

Results: At June 30th 2020, nasal/pharyngeal swabs have been found positive in $0.94 \%$ of the Lombardy population. Since the pandemic start, 2/56 (3.6\%) RRMS treated with cladribine complained a symptomatology suggestive of COVID-19 disease, with a prevalence similar to that of the whole MS population of our center $(84 / 2950,2.8 \%)$. The first patient was a 30 -year-old male with RRMS ( $\mathrm{DD}=1.2$ years, $\mathrm{EDSS}=1.5$ ) and no comorbidities. He started cladribine on January 10th 2020. One week later, he developed fever $\left(<37.5^{\circ}\right)$, ageusia, cough, fatigue, sputum production, sore throat, nasal congestion, shortness of breath without desaturation and conjunctivitis. The second patient is a 39-year-old female with RRMS ( $\mathrm{DD}=13.2$ years, $\mathrm{EDSS}=3.5$ ), and no comorbidities. She started cladribine on February 13th 2020 and underwent the second week of the first treatment course from March 5th 2020. On March 30th, she developed fever $\left(<37.8^{\circ}\right)$, anosmia, ageusia, cough, fatigue, and bone/joint pain. Serology for SARS-COV-2 performed in May 2020 was positive. For both patients, blood examinations performed before and after COVID-19 disease were within normal limits. Both patients stayed at home, telephone-monitored by their neurologists, and completely recovered within 15 days.

Discussion: Only a minority RRMS patients receiving cladribine developed a mild and self-limiting COVID-19 disease. In our cohort, this occurred only in two RRMS patients within a few weeks from the treatment course and the possible nadir of selective immunosuppression. Both patients recovered completely.

Conclusions: Cladribine administration seems to be safe also in the setting of the COVID-19 pandemic. A mild COVID-19 disease could occur in the transient phase of immunosuppression after drug administration. 


\section{PARA-INFECTIOUS IMMUNE-MEDIATED ENCEPHALOMYELORADICULITIS IN A PATIENT AFFECTED BY COVID-19}

\author{
G. Primiano, F. Madia, R. Iorio, B. Merico, G. Masi, S. Servidei
}

Department of Neuroscience, Catholic University of the Sacred Heart (Roma)

Objective: The neurological manifestations in COVID-19 mostly fit in one of the following categories: 1) encephalopathy with normal MRI 2) inflammatory CNS syndrome mostly with the aspect of ADEM 3) ischaemic stroke 4) peripheral neurological disorders (mostly GBS) [1]. However, the pathogenic correlation with SARS-CoV-2 is still poorly understood. We tried to define the mechanism leading to acute encephalomyeloradiculitis in a COVID-19 patient.

Patient: A 77-year-old male affected by COVID-19 was admitted in ICU for severe respiratory distress. 18 days after admission the patient acutely developed symmetrical flaccid paraplegia with lower limb areflexia. He was also disoriented and agitated complaining of visual hallucinations despite a withdrawal from sedation. C-reactive protein $(168.5 \mathrm{mg} / \mathrm{L}$; n.v.<5), IL6 (82.6 ng/L; n.v.<4.4), and D-dimer (7228 $\mathrm{ng} / \mathrm{ml} ;$ n.v. $<500$ ) were increased.

Results: EEG revealed diffuse bilateral frontal slowing and EMG demonstrated extensive denervation in both lower limbs consistent with a severe, symmetric process affecting anterior horn cells or motor axons. MRI revealed bilateral symmetric lesions in the caudal portion of the medulla with restricted diffusion, multiple focal T2 hyperintensities and scattered microbleeds in the subcortical white matter, and T1 weighted images showed contrast-enhancement of the intradural trait of the roots of cauda equina. CSF was normal, without oligoclonal bands, and the analysis of neurotropic viruses was negative. CSF RT-PCR for SARS-CoV-2 was also negative. Patient's serum was tested for neural-specific autoantibodies $(\mathrm{Ab})$ by indirect immunofluorescence assays (IFA) on frozen sections of mouse cerebellum, kidney and stomach. IFA revealed a prevalent immunoreactivity of the granular layer and the Purkinje cells layer of the cerebellum. Immunoblot for Ab specific to onconeural antigens and cell-based assays for NMDAR-IgG, LGI1-IgG, Caspr2IgG, AMPAR-IgG, GABABR-IgG, DPPX-IgG was negative. The patient was put on therapy with intravenous methylprednisolone $120 \mathrm{mg} /$ day and intravenous immune globulins. After 10 days of treatment, a follow-up MRI revealed improvement of the lesions in the medulla and a mild increase of the subcortical hyperintensities.

Discussion and conclusion: There are three points of interest in this patient: peculiar clinical aspects, as the development of acute pure motor axonal neuropathy; unusual MRI findings with symmetric involvement of medulla in absence of other demyelinating features; the demonstration of at least two different pathogenic mechanisms that work together but independently: brain microvascular damage that progressed despite the therapy and an immune-mediated process with an immunostaining pattern consistent with an Ab binding a yet uncharacterized neural antigen in the contest of the hyperinflammation syndrome.

Reference:

1. Paterson RW, Brown RL, Benjamin L, et al. The emerging spectrum of COVID-19 neurology: clinical, radiological and laboratory findings [published online ahead of print, $2020 \mathrm{Jul}$ 8]. Brain (2020);awaa240. doi:10.1093/brain/awaa240
NEUROLOGICAL COMORBIDITY AFFECTS COVID-19 SEVERITY

A. Romagnolo ${ }^{1}$, R. Balestrino ${ }^{1}$, G. Imbalzano ${ }^{1}$, G. Ciccone $^{2}, \mathrm{~F}$. Riccardini $^{3}$, C. Artusi ${ }^{1}$, M. Bozzali ${ }^{1}$, B. Ferrero ${ }^{1}$, E. Montalenti ${ }^{1}$, E. Montanaro $^{1}$, M. Rizzone ${ }^{1}$, G. Vaula ${ }^{1}$, M. Zibetti ${ }^{1}$, L. Lopiano ${ }^{1}$

${ }^{1}$ Department of Neuroscience "Rita Levi Montalcini", University of Turin (Torino); ${ }^{2}$ Section of Clinical Epidemiology, Center for Epidemiology and Oncologic Prevention (CPO Piemonte), Città della Salute e della Scienza (Torino); ${ }^{3}$ Front Line P.S. P.O. Molinette, Department of Medical Sciences, Città della Salute e della Scienza (Torino)

Objective: Neurological symptoms of COVID-19 patients have been recently described [1]. However, no comprehensive data have been reported on pre-existing neurological comorbidities and COVID-19. This study aims at evaluating the prevalence of neurological comorbidities, and their possible association with COVID-19 severity.

Methods: In this retrospective, single center cohort study, we analyzed the clinical charts of all consecutive patients admitted to the Emergency Room (ER) of the Città della Salute e della Scienza di Torino Hospital between the 3rd March 2020 and the 14th April 2020, and diagnosed with COVID-19. Data on neurological and nonneurological diseases were extracted, as well as data on demographic characteristics and on severity degree of COVID-19. The prevalence of neurological comorbidities was calculated, and multivariate binary logistic regression analyses were used to estimate the association between neurological diseases and COVID-19 severity. Moreover, the type of discharge from ER was evaluated to assess patients' severity.

Results: We analyzed data of 344 patients. Neurological comorbidities accounted for $22.4 \%$ of cases, with cerebrovascular diseases and cognitive impairment being the most frequent. Neurological comorbidity resulted independently associated with severe COVID-19 (OR: 2.305; $\mathrm{p}=0.012$ ), as well as male gender $(p=0.001)$, older age $(p=0.001)$, neoplastic diseases $(p=0.039)$, and arterial hypertension $(\mathrm{p}=0.045)$. When neurological comorbidity was associated with non-neurological comorbidities, the OR of severe COVID-19 rose to $7.394(\mathrm{p}=0.005)$. Neurological patients, in particular cerebrovascular and cognitively impaired ones, were more frequently hospitalized and received more mechanical respiratory support indication.

Conclusions: Neurological comorbidities represent an independent determinant of COVID-19 severity, deserving a thorough evaluation since the earliest phases of infection. The vulnerability of patients affected by neurological diseases should suggest a greater attention in targeting this population for proactive viral screening.

Reference:

- Mao L, Jin H, Wang M et al. Neurologic Manifestations of Hospitalized Patients with Coronavirus Disease 2019 in Wuhan, China. JAMA Neurol (2020);77:1-9

\section{SUBJECTIVE COGNITIVE IMPAIRMENTS IN RECOVERED COVID-19 PATIENTS}

G. Salvato ${ }^{1}$, F. Valentini ${ }^{2}$, G. Palmas ${ }^{2}$, A. Bollani ${ }^{2}$, S. Basilico ${ }^{2}$, E. Agostoni $^{3}$, G. Bottini ${ }^{2}$ 
${ }^{1}$ Department of Brain and Behavioral Sciences, University of Pavia (Pavia); ${ }^{2}$ Cognitive Neuropsychology Centre, ASST Grande Ospedale Metropolitano Niguarda (Milano); ${ }^{3}$ Neurology and Stroke Unit, ASST Grande Ospedale Metropolitano Niguarda (Milano)

Objectives: Severe acute respiratory syndrome coronavirus 2 (SARSCoV-2), the cause of coronavirus disease 2019 (Covid-19), is characterized by an acute pneumonic process with extensive radiologic opacity and, on autopsy, diffuse alveolar damage, inflammatory infiltrates, and microvascular thrombosis. It is now increasingly recognized that Covid19 also affects the nervous system [1]. Here we aimed at exploring the subjectively cognitive sequelae of Covid-19 in patients who recovered from the illness.

Materials: In literature, subjective cognitive impairments have been associated with structural and functional alterations of the brain [2]. We created an ad hoc self-administered neuropsychological questionnaire investigating the subjective presence of disorders involving attention, memory, gnosis, praxis, orientation in time and space, and executive functions.

Method: We performed an analysis of the frequency of subjective cognitive impairments in the different cognitive domains. We also correlated the time of the illness (days) with the most frequently referred cognitive impairments. We screened a cohort of patients admitted for Covid-19 at the ASST "Grande Ospedale Metropolitano" Niguarda in Milano. The subjective cognitive impairments were assessed 47.6 (SD:17.4) days after virological recovery. Patients with previous neurological disorders were excluded from the sample.

Results: Of the 172 patients who were hospitalized for Covid-19, $110(64 \%)$ were males. The total sample had a mean age of 58.7 years (SD:13.8). We found that COVID-19 survivors subjectively reported cognitive difficulties involving memory $(37.8 \%)$, orientation in time and space $(7 \%)$, language $(24.4 \%)$, praxis $(2.9 \%)$, attention $(31.4 \%)$, executive functions $(4.1 \%)$. None of the patients subjectively reported agnosia. Interestingly, patients with a longer COVID-19 duration have more probability of perceiving an episodic memory deficit.

Discussion: Covid-19 may directly or indirectly affect the nervous system. Its widespread effect may cause subjective cognitive impairments also one month after the virological recovery. The subjective cognitive impairments mainly involved memory and attention. The referred neuropsychological loss could be compatible with the well-known vascular and/or anoxic phenomena associated with Covid-19.

Conclusions: This preliminary study shed new light on the possible subjective cognitive sequelae in recovered Covid-19 patients. A comprehensive neuropsychological assessment is required to objectively verify the referred cognitive impairment perception and the persistence of neuropsychological disorders. The neuropsychological assessment will also include scales to identify affective disorders. These symptoms appear to be very relevant in patients with COVID-19, considering the exceptional situation related to the pandemic.

References:

1. A. Gupta, M. V. Madhavan, K. Sehgal, N. Nair, S. Mahajan, T. S. Sehrawat, B. Bikdeli et al. Extrapulmonary manifestations of COVID-19. Nat Med (2020) Jul;26(7):1017-32

2. F. Jessen, Re. E. Amariglio, R. F. Buckley, W. M. van der Flier, Y. Han, J. Luis Molinuevo, L. Rabin et al. The characterisation of subjective cognitive decline. Lancet Neurol (2020) Mar;19(3):271-78

\section{LOW MOLECULAR WEIGHT HEPARIN IN COVID-19 PATIENTS PREVENTS DELIRIUM AND SHORTENS HOSPITALIZATION}

R. Speranza ${ }^{1}$, D. D'Ardes ${ }^{2}$, C. Carrarini ${ }^{1}$, M. Russo ${ }^{1}$, F. Dono ${ }^{1}$, A. Digiovanni $^{1}$, G. Martinotti ${ }^{1}$, A. Di Iorio ${ }^{2}$, M. Onofrj ${ }^{1}$, F. Cipollone ${ }^{2}$, L. Bonanni $^{1}$

${ }^{1}$ Department of Neuroscience, Imaging and Clinical Sciences, University G. d'Annunzio of Chieti-Pescara (Chieti); ${ }^{2}$ Department of Medicine and Aging Sciences, University G. d'Annunzio of Chieti-Pescara (Chieti)

Objectives: The virus that is causing the current coronavirus outbreak has been named 'severe acute respiratory syndrome coronavirus 2' (SARSCoV-2). The most common symptoms of Covid-19 are fever, fatigue, and dry cough. In the most severe cases, the infection may cause pneumonia and severe acute respiratory syndrome. Delirium is a well-recognised complication of respiratory illness in older adults and in hospitalized patients it is a strong independent predictor of mortality, increased hospital length of stay (LOS), cognitive impairment, cost of care, and subsequent hospitalizations. Recent studies indicate that $20-30 \%$ of COVID19 patients present with or develop delirium during their hospitalization. The aim of this study was to analyze the possible association between delirium onset and demographic and clinical factors in COVID-19 patients.

Materials and Methods: We monitored COVID-19 patients with moderate symptoms referred to COVID-19 Unit of SS. Annunziata Hospital, Chieti, Italy and followed until the complete disease remission. All patients were assessed for clinical signs of delirium and performed two brief questionnaires, the Assessment test for delirium $\&$ cognitive impairment (4AT) and the Confusion Assessment Method for the Intensive Care Unit (CAM-ICU), in order to evaluate the presence of delirium.

Results: 14 out of 56 patients admitted to our COVID-19 Unit experienced delirium during the hospitalization. We found a strong association between the use of Low molecular weight heparin (LMWH) and absence of delirium, independently of comorbidities and age. LMWHprescription was the only statistically significant predictor of delirium prevention. LOS was longer in patients untreated with LMWH independently of age and CAM score.

Discussion: We tried to explain these results analyzing the pathophysiology of delirium. It is suspected that systemic inflammation, which commonly characterizes critical illness, stimulates a process of deranged coagulation, whose end product is microvascular damage and thrombosis in vital organs, including the brain, contributing to the development and maintenance of delirium.

Conclusions: Hence, the use of LMWH, by preventing the development of thrombosis subsequent to the inflammatory process, may help preventing the occurrence of delirium in COVID-19 patients, with consequent possible reduction of LOS and sequelae.

References:

- Shane O'Hanlon, Sharon K Inouye. Delirium: a missing piece in the COVID-19 pandemic puzzle, Age and Ageing Epub 06 May (2020)

- McNeil JB, Hughes CG, Girard T, et al. Plasma biomarkers of inflammation, coagulation, and brain injury as predictors of delirium duration in older hospitalized patients. PLoS One (2019);14:e0226412

- Girard TD, Ware LB, Bernard GR, et al. Associations of markers of inflammation and coagulation with delirium during critical illness. Intensive Care Med (2012);38:1965-73 


\section{PROSPECTIVE EEG EVALUATION IN PATIENTS WITH RECENT COVID-19 AND COGNITIVE DISTURBANCES}

M. Vabanesi ${ }^{1}$, F. Agosta ${ }^{2}$, G. Cecchetti ${ }^{3}$, E. Canu ${ }^{2}$, G. Fanelli ${ }^{4}$, A. Barbieri $^{1}$, P. Bernasconi ${ }^{1}$, S. Lazzarin ${ }^{3}$, G. Giacalone ${ }^{1}$, M. Falautano ${ }^{1}$, P. Rovere-Querini ${ }^{5}$, F. Minicucci ${ }^{4}$, L. Roveri $^{1}$, M. Filippi ${ }^{6}$

${ }^{1}$ Neurology Unit, IRCCS San Raffaele Scientific Institute (Milano); ${ }^{2}$ Neuroimaging Research Unit, Institute of Experimental Neurology, Division of Neuroscience, IRCCS San Raffaele Scientific Institute; Vita-Salute San Raffaele University (Milano); ${ }^{3}$ Neurology Unit; Neurophysiology Unit; and Neuroimaging Research Unit, Institute of Experimental Neurology, Division of Neuroscience, IRCCS San Raffaele Scientific Institute (Milano); ${ }^{4}$ Neurophysiology Unit, IRCCS San Raffaele Scientific Institute (Milano); ${ }^{5}$ Department of Immunology, Transplantation and Infectious Diseases, IRCCS San Raffaele Scientific Institute; Vita-Salute San Raffaele University (Milano); ${ }^{6}$ Neuroimaging Research Unit, Institute of Experimental Neurology, Division of Neuroscience; Neurology Unit and Neurophysiology Unit, IRCCS San Raffaele Scientific Institute; Vita-Salute San Raffaele University (Milano);

Objectives: To assess EEG findings and their clinical and neuropsychological correlates in patients with recent COVID-19.

Materials: We enrolled 55 adult patients with recent COVID-19, good recovery (functional independence) and objective and/or self-reported cognitive disturbances, observed at follow-up neurological evaluation performed within 2 months after discharge. Same-day basal EEG and neuropsychological testing battery were performed. Past and recent medical history was recorded.

Methods: EEG were assessed for background activity, presence/ location of focal slowing, epileptiform abnormalities and seizures. Individual alpha frequency (IAF) was calculated according to [1]. Correlations with neuropsychological and clinical variables were assessed using linear/logistic regression, controlling for age/sex/ education.

Results: We evaluated 55 patients $(70.9 \%$ males, mean age $60.5 \pm 13.3$ years); 13/55 (23.6\%) had needed ventilatory support (12 NIV, 1 intubation). Mean adjusted Montreal Cognitive Assessment (MoCA) score was 21.3 \pm 2.8 . 14 patients had symptoms suggestive of reactive depression and/or post-traumatic stress disorder (PTSD). Modest EEG backgroundactivity alteration was observed in $2 / 55$ (3.6\%) patients; pathologic drowsiness in 3/55 (5.5\%). Prevailing fronto-temporal focal slow-wave activity (SWA) was observed in 6/55 (10.9\%) patients. Brief $(<5 \mathrm{sec})$ anterior theta/alpha activity sequences were recorded in 10/55 (18.2\%) subjects. No epileptiform/seizure discharges were recorded. Mean IAF was $9.7 \pm 1.2 \mathrm{~Hz}$. $41 / 55$ (74.5\%) patients had experienced mild neurological symptoms during acute-phase COVID-19, namely headache (29.0\%), hyposmia/hypogeusia $(45.4 \%)$, confusion/disorientation (25.4\%). Only confusion/disorientation was associated with EEG parameters, in particular with anterior theta/alpha activity (OR:1.32 [95\%C.I. 1.03-1.69], $\mathrm{p}=0.03$ ). IAF did not correlate with acute-phase respiratory severity scores or neuropsychological findings. Presence of anterior thetaalpha activity was independently associated with lower executivefunction scores at frontal assessment battery (OR:0.93 [95\%C.I. 0.90 0.97], $\mathrm{p}=0.001$ ) and symbol-digit modalities test (OR:0.99 [95\%C.I. $0.98-1.00], \mathrm{p}=0.064$ ). Executive-function impairment was not associated with background-activity alteration or SWA.

Discussion: This is the first study describing EEG features in a prospective adult-patient cohort with recent COVID-19 and objective and/or selfreported cognitive disturbances. We frequently observed anterior theta- alpha activity, often associated with history of acute-phase confusion/ disorientation and with lower scores at frontal-lobe cognitive tests at follow-up. Differently from our acute-phase study [2], EEG data recorded at 2-month follow-up did not correlate with respiratory infection severity scores; however, we observed an association with history of confusion/ disorientation, a commonly reported COVID-19-associated symptom [3].

Conclusion: EEG alterations after COVID-19 might be at least partially independent of acute infection severity and suggest instead a link with ongoing neuro-psychiatric symptoms. Further follow-up data is needed to confirm the reversibility and/or evolution of our findings. References:

1. Caso F, Cursi M, Magnani G, et al. Quantitative EEG and LORETA: valuable tools in discerning FTD from AD? Neurobiol Aging (2012);33(10):2343-2356. doi:10.1016/ j.neurobiolaging.2011.12.011

2. Cecchetti G, Vabanesi M, Chieffo R, et al. Cerebral involvement in COVID-19 is associated with metabolic and coagulation derangements: an EEG study [published online ahead of print, 2020 Jun 15]. J Neurol. (2020);1-5. doi:10.1007/s00415-020-09958-2

3. Varatharaj A, Thomas N, Ellul MA, et al. Neurological and neuropsychiatric complications of COVID-19 in 153 patients: a UK-wide surveillance study [published online ahead of print, 2020 Jun 25]. Lancet Psych. (2020);1-8. doi:10.1016/ S2215-0366(20)30287-X

\section{RISK OF HOSPITALIZATION AND DEATH FOR COVID-19 IN PEOPLE WITH PARKINSON'S DISEASE OR PARKINSONISM: THE PARKLINK BOLOGNA COHORT, ITALY (RHOC-PARK)}

L. Vignatelli ${ }^{1}$, C. Zenesini ${ }^{1}$, L. Belotti ${ }^{1}$, E. Baldin ${ }^{1}$, G. CalandraBuonaura $^{1}$, P. Cortelli ${ }^{1}$, C. Descovich ${ }^{2}$, G. Giannini ${ }^{1}$, M. Guarino ${ }^{1}$, C. Scaglione $^{1}$, S. Trombetti ${ }^{2}$, R. D'Alessandro ${ }^{1}$, F. Nonino ${ }^{1}$, x. on behalf of ParkLink Bologna ${ }^{1}$

${ }^{1}$ IRCCS Institute of Neurological Sciences of Bologna, AUSL Bologna (Bologna); ${ }^{2}$ Clinical Governance, AUSL Bologna (Bologna)

Objective: Emilia-Romagna (ER) ranked as third Italian region as number of COVID-19 reported cases $(29,670$ as for July 31, 2020 [1]). COVID19-related risk and mortality rates are higher among fragile populations such as the elderly affected by chronic conditions. Persons with Parkinson's disease (PD) and Parkinsonian syndromes (PS) show a paradigmatic pattern of frailty, being elderly and often with multiple agerelated comorbidities, including cognitive impairment. However, it is unknown whether people with PD/PS have an increased risk for COVID-19. We report preliminary results on the risk of hospital admission and death for COVID-19 in a cohort of patients with PD/PS compared to a matched population cohort, during the SARS-COVID-2 epidemic bout in ER.

Materials: Clinically confirmed PD or PS (ParkLink cohort) recruited through a record linkage system (ParkLink Bologna) and a matched general population cohort, alive in 2019 in the area of the Local Health Trust of Bologna (LHTB) (population about 800,000). ParkLink Bologna is a project linking PD/PS clinical diagnosis to administrative data within the LHTB. Since 2016, about 20 neurologists (within the Italian NHS or in private practices) have been involved in the project.

Method: Historical cohort design comparing the ParkLink cohort with a population-based control cohort matched (ratio 1:10) for age, sex, Charlson Index and residence, recruited through administrative databases. Outcomes: hospital admission rate for Covid-19 (ICD9cm codes 079.82 
and 480.3) and in-hospital death from February 1 to May 31, 2020. Cox regression analysis estimated the Hazard Ratio (HR) with 95\% confidence interval (CI) associating Covid-19 risk and in-hospital death to the presence of $\mathrm{PD} / \mathrm{PS}$.

Results: In both cohorts (ParkLink N=972 subjects, 696 PD, 276 PS; control $\mathrm{N}=9,507$ subjects) $58 \%$ were male and mean age was 76 (SD 9). The first COVID-19 patient was admitted on February 27, 2020. Hospital admissions for COVID-19 were $9(0.9 \%)$ in the ParkLink cohort and $66(0.7 \%)$ in the control cohort. The HR for admission was 1.3 (95\% CI 0.7-2.7, p=0.42). Overall, 29 (39\%) patients died while in hospital: 4 (44\%) among those with PD/PS and $25(38 \%)$ among controls; HR for death was 1.3 (95\% CI $0.4-$ 3.6, $\mathrm{p}=0.67)$.

Discussion: During the SARS-COVID-2 epidemic bout in ER, we did not observe significant differences of risk of COVID-19 hospital admission and death between a population-based PD/PS cohort and a control population with the same age and comorbidities.

Conclusion: Our preliminary data suggest that having PD/PS is not per-se a risk factor for COVID-19 and COVID-19-related death.

Reference:

- https://protezionecivile.regione.emilia-romagna.it/notizie/attualita/ coronavirus-aggiornamento-31-luglio

\section{SUBCLINICAL MYOPATHIC CHANGES IN COVID 19}

D. Villa ${ }^{1,2}$, G. Ardolino ${ }^{1}$, L. Borellini ${ }^{1}$, F. Cogiamanian ${ }^{1}$, M. Vergari ${ }^{1}$, V. Savojardo $^{3}$, F. Peyvandi ${ }^{2,4}$, S. Barbieri ${ }^{1}$

${ }^{1}$ Department of Neurophysiology, Foundation Ca' Granda Ospedale Maggiore Policlinico (Milano); ${ }^{2}$ Department of Pathophysiology and Transplantation, University of Milan (Milano); ${ }^{3}$ Department of Emergency Medicine, IRCCS Foundation Ca' Granda Ospedale Maggiore Policlinico (Milano); ${ }^{4}$ Angelo Bianchi Bonomi Hemophilia and Thrombosis Center, Department of Medicine and Medical Specialities, University of Milan, IRCCS Maggiore Hospital, Mangiagalli and Regina Elena Foundation (Milano)

Background: Coronavirus disease 2019 (Covid 19) is associated to neuromuscular symptoms in up to $10.7 \%$ of hospitalized patients [1]. Nevertheless, the extent of muscular involvement in infected subjects has never been assessed with neurophysiological investigations.

Methods: Over a 3-week period - from April 30 through May 20, 2020 - a total of 70 patients were hospitalized in the Internal Medicine Ward of the Fondazione IRCCS Ca' Granda Ospedale Maggiore Policlinico in Milan, Italy. After excluding patients who underwent invasive ventilation and steroid treatment, 12 patients were evaluated. The Covid-19 diagnosis was confirmed by 2 positive nasopharyngeal swabs performed before and after our evaluation. All patients were asymptomatic for muscular involvement

Results: While nerve conduction studies were unremarkable, needle electromyography showed myopathic changes in 6 out of 12 subjects. Clinical features and laboratory findings did not show relevant differences between the two groups.

Conclusion: Our data show that in SARS-CoV-2 infection muscular involvement occurs despite the absence of clinical signs or symptoms and should be considered part of the disease spectrum. The application of muscle biopsy to unravel the mechanisms of myofiber damage on tissue specimen could help to clarify the pathogenesis and the treatment response of coronavirus-mediated injury.
Reference:

1. Mao L, Jin H, Wang M, et al. Neurologic Manifestations of Hospitalized Patients With Coronavirus Disease 2019 in Wuhan, China [published online ahead of print, 2020 Apr 10]. JAMA Neurol. (2020);e201127. doi:10.1001/jamaneurol.2020.1127

\section{NEUROGENETICS}

\section{NEUROFIBROMATOSIS OUTPATIENT CLINIC POPULATION}

\author{
E. Agazzi, M. Sessa
}

USC Neurology, ASST-PG23 (Bergamo)

Purpose: We report the incidence, demographics, and clinical manifestations of neurofibromatosis type 1 among a population-based cohort of patients.

Methods: The medical records of all patients diagnosed as having neurofibromatosis type 1 from January 2015 through December 31, 2019 were retrospectively reviewed.

Results: Forty seven patients came to our out-patient clinic, some of them already diagnosed as having neurofibromatosis type 1 and some being diagnosed at our center, during the 5-year period, yielding an incidence of 1 per 100,000 individuals. The mean age at diagnosis was 14 years. A de novo mutation rate was of $46 \%$. During a mean follow-up of 5 years (café-au-lait macules were diagnosed in $95 \%$ individuals, neurofibromas in $40 \%$ of patients and skeletal anomalies in $20 \%$. Three individuals were diagnosed as having glioma of the central nervous system at a mean age of 14 years. Only $1(2 \%)$ patient was diagnosed as having malignant nerve sheath tumor.

Conclusions: The prevalence of de novo mutation rate of neurofibromatosis type 1, café-au-lait macules, neurofibromas and skeletal abnormalities are similar to prior reports.

\section{LEBER'S HEREDITARY OPTIC NEUROPATHY IN WOMEN}

G. Amore ${ }^{1}$, M. Romagnoli ${ }^{2}$, M. Carbonelli ${ }^{2}$, L. Caporali ${ }^{2}$, R. Karanjia ${ }^{3}$, A. Sadun ${ }^{4}$ C. La Morgia ${ }^{1}$, V. Carelli ${ }^{1}$

${ }^{1}$ DIBINEM Department of Biochemical and Neuromotor Sciences, University of Bologna (Bologna); ${ }^{2}$ IRCCS Istituto Scienze Neurologiche di Bologna, Bellaria Hospital (Bologna); ${ }^{3}$ Ottawa Eye Institute, University of Ottawa (Ottawa-CDN); ${ }^{4}$ Doheny Eye Center, Department of Ophthalmology, University of California Los Angeles (Los Angeles-USA)

Background: Leber's Hereditary Optic Neuropathy (LHON) is a mitochondrial disorder due to mtDNA mutations, characterized by selective degeneration of retinal ganglion cells with significant gender bias and incomplete penetrance. We aimed at refining the characteristics of LHON in women.

Methods: We prospectively administered a questionnaire designed ad hoc for the study about hormonal/toxic factors and comorbidities to 150 females carrying LHON mutation, 39 affected and 111 unaffected. We evaluated age at onset in 294 patients and the neuro-ophthalmological phenotype in a subgroup of 92 , comparing females and males. We also determined the disease penetrance in 2779 LHON-mutation carriers from 127 families, stratified by gender and mutations. 
Results: Unaffected women had higher frequency and number of pregnancies $(83 \%$ vs $61 \%, \mathrm{p}=0.004)$. Among affected women, $23 \%$ developed the disease after menopause. The affected group showed heavier alcohol consumption $(\mathrm{p}=0.08)$ and smoking habit $(38 \%$ vs $19 \%, \mathrm{p}=0.02)$. We found high rates of Multiple Sclerosis in the affected group (10\%) and of migraine (55\%) considering all LHON women compared to general population. Compared to males, females showed older onset of disease $(52 \pm 20$ vs $45 \pm 17, p=0.002)$ apparently due to three peaks of incidence, respectively at 18 (typical), 40 (environmentally-triggered) and after 50 years (menopause). The neuro-ophthalmogical evaluation disclosed a longer time interval between eyes and worse visual acuity in the first 5 years of disease in females compared to males $(\mathrm{p}<0.01)$. General penetrance of disease was 15\% (increasing from mutation 14484/ND6 to $11778 / \mathrm{ND} 4$ and 3460/ND1), with female to male ratio of 1:5.

Discussion: Our results support the protective role of oestrogens, confirm alcohol and smoke as risk factors for LHON and point to a possible association of LHON with Multiple Sclerosis and migraine in women. Mean age at onset in females is significantly higher comparing to males due to cases affected after menopause, while neuro-ophthalmological phenotype is comparable. Finally, we also refined the previously reported disease penetrance, gender and mutation distribution in our large cohort of LHON carriers, confirming also the less evident gender gap for the 3640/ND1 mutation.

References:

- Giordano C., Montopoli M., Perli E., Orlandi M., Fantin M., RossCisneros F.N., Caparrotta L., Martinuzzi A., Ragazzi E., Ghelli A., Sadun A.A., d'Amati G., Carelli V. Oestrogens ameliorate mitochondrial dysfunction in Leber's hereditary optic neuropathy. Brain (2011);134 (Pt 1):220-34

- Carelli V., d'Adamo P., Valentino M.L., La Morgia C., RossCisneros F.N., Caporali L., Maresca A., Loguercio Polosa P., Barboni P., De Negri A., Sadun F., Karanjia R., Salomao S.R., Berezovsky A., Chicani F., Moraes M., Moraes Filho M., Belfort Jr., R., Sadun A.A. Parsing the differences in affected with LHON: genetic versus environmental triggers of disease conversion. Brain (2016); Mar; 139:e17

\section{A NOVEL DE NOVO ATP1A2 MUTATION IN THE PROBAND OF A FAMILY WITH HEMIPLEGIC MIGRAINE ASSOCIATED WITH FEBRILE SEIZURES}

\author{
P. Banfi ${ }^{1}$, A. Clemenzi ${ }^{1}$, V. Rebecchi ${ }^{1}$, V. Mariani ${ }^{1}$, T. Damawandi \\ Payam $^{2}$, M. Versino ${ }^{3}$
}

\footnotetext{
${ }^{1}$ Neurology and Stroke Unit, Circolo Hospital ASST Settelaghi (Varese); ${ }^{2}$ Bicocca University Milan (Milano); ${ }^{3}$ Neurology Unit, ASST SettelaghiInsubria University-DMC (Varese)
}

Introduction: Familial Hemiplegic Migraine (FHM) is an autosomal dominantly inherited form of migraine with aura characterized by the occurrence of transient hemiparesis during the aura. Mutations in four genes have been identified in FHM, from which CACNA1A (FHM type 1) and SCN1A (FHM type 3) code for neuronal voltage-gated calcium or sodium channels, respectively, while ATP1A2 (FHM type 2) encodes the $\alpha_{2}$ isoform of the $\mathrm{Na}^{+}, \mathrm{K}^{+}$-ATPase's catalytic subunit. FHM2 frequently share comorbidity with other neurological disorders, such as febrile seizures and epilepsy.

Case Report: The proband is a 39-year-old-man with a history of febrile seizures at the age of 18 months; he experiences attacks of hemiplegic migraine since the age of 27 . The proband's mother and two maternal uncles have episodes of hemiplegic migraine lasting from their adolescence; a maternal cousin has a history of febrile seizures. The proband's attacks are stereotyped: right-side sensory symptoms, speech disturbances and hemiparesis lasting up to 1 hour and followed by left-side pulsating headache lasting up to 1day accompanied by nausea, vomiting and occasionally fever. Peri-ictal EEG shows slowing over the left temporal lobe; magnetic resonance imaging of the brain and follow-up neurological examination are normal.

Genetic Analysis: Direct sequencing of all exons and flanking intronic regions of ATP1A2 (NM_000702), CACNA1A (LRG_7t1-NM 001127221), SCN1A (NM_001202435) has been performed in the proband. Mutation analysis of ATP1A2 gene has identified an heterozygous novel missense mutation: the c. $1843 \mathrm{G}>\mathrm{A}$ mutation predicting the amino acid replacement glycine to arginine on position 615 (p.Gly615Arg); no mutations have been identified in CACNA1A and SCN1A genes.

Discussion: The migraine episodes in our family share the features of FHM with hemiparesis as a part of the aura in association with other reversible cortical symptoms including paresthesias and language impairment; the diagnosis was made before the genetic testing. The genetic variant is not present in the population database. The cooccurrence of febrile seizures and migraine has also been reported in families with FHM [1]; in some families, FHM cosegregated with benign familial infantile seizures [2] and epileptic seizures occurring independently from the migraine attacks have been reported in families with FHM2 [3].

Conclusions: FHM linked ATP1A2 mutation involves dysfunction of ion transportion and epilepsy is part of the phenotypic spectrum. It may be worthwhile to screen patients with a combination of epilepsy and migraine and a positive family history of either migraine or epilepsy for mutations in the ATP1A2 gene.

References:

1. De Vries B, Stam A H, Kirkpatrick M et al Familial hemiplegic migraine is associated with febrile seizures in an FHM2 family with a novel de novo ATP1A2 mutation. Epilepsia, (2009); 50:2503-04

2. Vanmolkot K.R, Kors E.E. Hottenga J.J. et al. Novel mutations in the Na,K-ATPase pump gene ATP1A2 associated with familial hemiplegic migraine and benign familial infantile convulsions. Ann. Neurol. (2003);54:360-66

3. Deprez L, Weckhuysen S, Peeters K et al. Epilepsy as part of the phenotype associated with ATP1A2 mutations. Epilepsia (2008);49:500-08

\section{A PSEUDOXANTHOMA ELASTICUM FAMILY WITH CLINICAL AND GENETIC HETEROGENEITY: MTHFR AND SERPINE1 VARIANTS AS POSSIBLE DISEASE MODIFIERS FACTORS IN DEVELOPING CEREBROVASCULAR COMPLICATIONS}

\author{
G. Bruno ${ }^{1}$, M. Ritelli ${ }^{2}$, L. Cipriano ${ }^{1}$, A. Di Pietro ${ }^{1}$, M. Colombi $^{2}$, G. Puoti ${ }^{1}$
}

${ }^{1}$ Department of Advanced Medical and Surgical Sciences, Second Division of Neurology, University of Campania "Luigi Vanvitelli" (Napoli); ${ }^{2}$ Division of Biology and Genetics, Department of Molecular and Translational Medicine, University of Brescia (Brescia)

Objectives: Pseudoxanthoma elasticum (PXE) is a rare autosomal recessive connective tissue disorder caused by mutations in the ABCC6 gene. The phenotypic spectrum of PXE is highly variable and includes principally three major features: skin lesions, eye and other vascular manifestations. Cerebral involvement is less common, mainly due to small vessels disease. To date about 400 different PXE-associated pathogenic variants in ABCC6 are described, however, no genotype-phenotype correlations have emerged so far. 
Materials and Methods: Herein, we report clinical and molecular findings of a large PXE family from Southern Italy carrying two different ABCC6 variants, R518Q and V1285M, with prominent intra-familial clinical and genetic variability. In order to investigate why some individuals of our PXE family had more significant vascular manifestations, also including ischemic stroke, we searched for classic pro-thrombotic gene variants.

Results: We found that members carrying SERPINE1 $(4 \mathrm{G} / 5 \mathrm{G})$ and MTHFR (C677T) variants had more severe vascular complications, in particular homozygousity $4 \mathrm{G} / 4 \mathrm{G}$ of SERPINE1 was present in patients with ischemic stroke.

Discussion: We speculate that the SERPINE14G/4G genotype could represent an additional risk factor for developing ischemic stroke in PXE, which add together with already known predisposing conditions including high blood pressure, hypercholesterolemia, diabetes, high Hcy levels and APOE variants.

Conclusion: Besides conventional risk factors for stroke, PXE patients might be investigated for both MHTFR and SERPINE1 genetic variants in order to adopt an appropriate therapeutic approach. Finally, PXE patients should be adequately screened for cerebral vasculopathy, even more if familial history is suggestive of brain complications.

References:

- Kranenburg G, Baas AF, de Jong PA, et al. The prevalence of pseudoxanthoma elasticum: Revised estimations based on genotyping in a high vascular risk cohort. Eur J Med Genet. (2019);62(2):90-92

- Van den Berg JS, Hennekam RC, Cruysberg JR, et al. Prevalence of symptomatic intracranial aneurysm and ischemic stroke in pseudoxanthoma elasticum. Cerebrovasc Dis. (2000);10(4):315-19

- Boraldi F, Costa S, Rabacchi C, Ciani M, Vanakker O, Quaglino D. Can APOE and MTHFR polymorphisms have an influence on the severity of cardiovascular manifestations in Italian Pseudoxanthoma elasticum affected patients? Mol Genet Metab Rep. (2014);1:477482. Published 2014 Nov 16.

\section{MULTIDIMENSIONAL NEUROPSYCHIATRIC EVALUATION IN AN ITALIAN COHORT OF GAUCHER DISEASE 1 PATIENTS: A PROSPECTIVE STUDY}

E. Cerulli Irelli ${ }^{1}$, G. Tessari ${ }^{2}$, R. Turchetta ${ }^{3}$, S. Ferracuti ${ }^{4}$, A. Giallonardo $^{1}$, R. Plateroti ${ }^{3}$, F. Atturo ${ }^{3}$, L. Cardarelli ${ }^{4}$, D. De Benedittis ${ }^{4}$, M. Nebbioso ${ }^{3}$, T. Di Pippo ${ }^{4}$, P. Mancini ${ }^{3}$, F. Caramia ${ }^{1}$, C. Di Bonaventura $^{1}$, F. Giona ${ }^{4}$

${ }^{1}$ Department of Human Neurosciences, Sapienza University of Rome (Roma); ${ }^{2}$ Department of Neuropsychology, Sapienza, University of Rome (Roma); ${ }^{3}$ Department of Sense Organs, Sapienza, University of Rome (Roma); ${ }^{4}$ Department of Translational and Precision Medicine, Sapienza, University of Rome (Roma)

Objective: Gaucher Disease type 1 (GD1) has been classically described as the non-neuronopathic variant of GD and a protective role of N370s mutation against neurological impairment had been hypothesized.[1] However, clinical and subclinical neurological abnormalities have been repeatedly identified among GD1 patients during the past years.[2,3] Nevertheless, data from a comprehensive multidimensional approach in these patients are still lacking. The aim of our study was to systematically evaluate neuropsychiatric and sensorineural abnormalities among GD1 patients.

Materials and methods: In this prospective observational study we consecutively enrolled 22 GD patients (19 GD1, 3 GD3) and data concerning GD1 subjects are hereby reported. Neurological evaluation included Unified Parkinson's Disease Rating scale part III, Epworth Sleepiness scale and an extensive neuropsychological assessment; neurophysiological evaluations include Electroencephalography, Visual Evoked Potentials (VEP), Brainstem Auditory Evoked Potential. All patients underwent $3 \mathrm{~T}$ brain MRI, audiometry and Spectral Domain Optical Coherence Tomography (SD-OCT). All examinations were performed at baseline and will be repeated after 12 and 24 months. Preliminary results concerning the baseline evaluation are reported.

Results: Nineteen GD1 patients with a median age at diagnosis of 20 years (range 1-46.5) and a median age at study entry of 44.5 years (range 17-68), were enrolled. Parkinsonian motor signs were found in $9 / 19$ patients: among them $6 / 9$ had isolated bradykinesia, whereas $3 / 9$ had a combination of rigidity and bradykinesia. A slowing/increased latency of saccades was observed in 2/19 patients. Excessive daytime sleepiness (EDS) was found in $8 / 19$ patients and 4/19 subjects showed cognitive impairment (especially attention, language, memory). Neuropsychiatric evaluation displayed some psychiatric aspects, such as anxiety, depression and somatic concerns, in 12/22 patients. Audiometric evaluation revealed slight/moderate sensorineural hearing loss in 13/19 subjects. VEP showed an increased latency of N100 component in $5 / 19$ patients. The SD-OCT examination showed impairment in retinal nerve fiber layers in 6/19 subjects. Qualitative brain MRI assessment was unremarkable. Overall, all GD1 patients showed at least a neuropsychiatric or sensorineural impairment independently of the presence of N370S mutation.

Discussion: A wide spectrum of neuropsychiatric and sensorineural abnormalities were found in all tested patients, highlighting that the neurological involvement is common among GD1 patients. N370S status did not impact the likelihood to develop neurological abnormalities. Finally, the high prevalence of EDS suggests a possible underlying sleep disorder which should be further investigated in future studies.

Conclusion: Our study showed that a multidisciplinary approach including a detailed neuropsychiatric evaluation is useful in evaluating GD1 patients

References:

1. Stirnemann J, Belmatoug N, Camou F, et al. A Review of Gaucher Disease Pathophysiology, Clinical Presentation and Treatments. Int JMol Sci.(2017);18(2):441. doi:10.3390/ijms18020441

2. Capablo JL, Saenz de Cabezón A, Fraile J, et al. Neurological evaluation of patients with Gaucher disease diagnosed as type 1 . J Neurol Neurosurg Psychiatry (2008);79(2):219-222. doi: $10.1136 /$ jnnp.2006.111518

3. Giona F, Di Bonaventura C, Mancini P, et al. Multidisciplinary Study Based on Clinical, Electrophysiological and Psychological Evaluations Combined with Advanced Neuroimaging in Gaucher Disease Patients. Blood. (2019);134(Supplement_1):2185. doi:10.1182/blood-2019-128143

\section{MACULAR DEGENERATION AS A DISTINCTIVE FEATURE OF SPINOCEREBELLAR ATAXIA TYPE 1}

\author{
E. Cioffi ${ }^{1}$, L. Ziccardi ${ }^{2}$, V. Parisi ${ }^{2}$, M. Serrao ${ }^{3}$, C. Casali $^{3}$
}

${ }^{1}$ Department of Human Neurosciences, Sapienza University of Rome (Roma); ${ }^{2}$ IRCCS Bietti Foundation (Roma); ${ }^{3}$ Department of Medical and Surgical Sciences and Biotechnologies, Sapienza University of Rome (Latina) 
Introduction: Spinocerebellar ataxia type 1 (SCA1) is an autosomal dominant, neurodegenerative disease, caused by CAG repeat expansion in the ATXN1 gene. In some patients, it has been noted loss of vision due to inconstant fundoscopic macular abnormalities [1]. Macular degeneration has been reported in few recent case reports [2]. This is the first multimodal analysis of retinal morphology and function in a series of SCA1 patients.

Materials and Methods: Nine patients with genetically confirmed SCA1 aged 36-68 years, six symptomatic patients (SARA Score 8-29) and three presymptomatic, underwent complete ophthalmological examination. Visual acuity (LogMAR scale - 0.0-1.0), morphology and function of the retinal structure (in particular the macula) were assessed. Morphology was studied by means of the macular volume of Outer Retina (mvOR) and optical coherence tomography (OCT), along with fundoscopy. External retinal function was studied with Full-field (Flash and Flicker) Electroretinogram (ERG), while function of macular region was assessed with Multifocal ERG (mfERG), which explores macular and perifoveal region into radians from the center [3]. Visual pathways were assessed through Visual Evoked Potentials (VEPs).

Results: Only two subjects (SARA $>16$ ) complained of visual loss (LogMAR 0.3 and 0.2). Fundoscopy revealed slight and aspecific macular abnormalities in 3 patients, and OCT showed loss of mvOR in 2 patients. $\mathrm{mfERG}$ was abnormal in the first radian $\left(0-2,5^{\circ}\right)$ in all patients, but was normal in regions outside the fovea at $5-20^{\circ}$. Flash and flicker ERG were also normal as well as VEPs.

Discussion: Flicker and flash ERG showed normal function of rods and cones in the external layers of the retina. Conversely, we observed altered function of the foveal region (primarily bipolar cells) in all patients via $\mathrm{mfERG}$, including presymptomatic subjects. Functional alteration can be detected before morphologic abnormalities, as in other forms of macular degeneration. Patients with clinical visual impairment had more severe advanced disease.

Conclusions: In patients with SCA1, with or without visual impairment, macular degeneration is an important, early and almost constant feature. Often not visible at fundoscopy or OCT, it can be reliably detected with mfERG, which has greater diagnostic sensitivity compared to morphological tests. Complete visual function assessment should be part of clinical evaluation in SCA1 patients, in order to detect early macular changes even in presymptomatic subjects. Visual function parameters could be used for monitoring progression in early stages of the disease and as endpoints for future clinical trials.

References:

1. P. Lebranchu et al. Maculopathy and Spinocerebellar Ataxia Type 1: A New Association? Journal of Neuro-Ophthalmology (2013);33:225-31

2. Koji M. Nishiguchi, Toshiaki Abe et al. Macular degeneration as a common cause of visual loss in spinocerebellar ataxia type 1 (SCA1) patients. Ophthalmic Genetics (2018);40(1): 49-53

3. A. G. Robson et al. ISCEV guide to visual electrodiagnostic procedures. Doc Ophthalmol (2018);136:1-26

\section{LEBER'S HEREDITARY OPTIC NEUROPATHY AND CONCOMITANT GLAUCOMA CAN BE ASSOCIATED WITH ATYPICAL FINDINGS: A CASE REPORT}

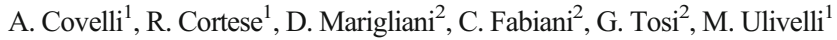

${ }^{1}$ Department of Medicine, Surgery and Neuroscience, University of Siena (Siena); ${ }^{2}$ Ophthalmology Unit, Department of Medicine, Surgery and Neuroscience, University of Siena (Siena)
Although rare, Leber's hereditary optic neuropathy (LHON) may occur in association with glaucoma, leading to an increase in its susceptibility. The raised intraocular pressure may trigger LHON conversion from dormant to active disease, leading to vision loss. We describe a case of the concomitant occurrence of the two diseases and atypical presentation. A 51-year-old man with no significant past medical history presented with sudden painless visual loss in the left eye (LE). On examination, he showed reduced visual acuity (20/200) and increased intraocular pressure $(24 \mathrm{mmHg})$ in $\mathrm{LE}$, while right eye (RE) was normal. MRI showed T2 hyperintensity in the left optic nerve with gadolinium enhancement, and hyperintense signals of the left optic nerve in the intraconal space on diffusion-weighted imaging (DWI). Brain and cervical cord MRI were unremarkable. The patient was treated with intravenous methylprednisolone and topical agents for glaucoma with no recovery. Three months later he developed vision decrease in the right eye (RE), associated with increased intraocular pressure (41 $\mathrm{mmHg}$ ). Repeat MRI scan showed bilateral T2 hyperintensity in the retrobulbar segment of the optic nerves and optic tracts, with no gadolinium enhancement. There was bilateral diffuse retinal nerve fiber layer thinning on optical coherence tomography. A cerebrospinal fluid (CSF) examination showed hyperproteinorrachia and one oligoclonal band (OCB) not located in the alkaline $\mathrm{PH}$ region. He was investigated to exclude a range of alternative autoimmune inflammatory aetiologies, of which none were identified. The genetic test revealed an $\mathrm{m} 11778 \mathrm{G}>\mathrm{A}$ mtDNA mutation, which is pathognomonic for LHON. He was treated with a further course of intravenous methylprednisolone, followed by plasma exchange and Yaglaser Iridotomy, with a gradual mild improvement of the visual acuity in both eyes after 1 month. We describe a LHON patient with severe, bilateral, sequential visual loss and glaucoma, associated with atypical findings (i.e. involvement of the anterior optic nerve, contrast enhancement and DWI alteration in the acute phase, and one OCB in the CSF). Therefore, LHON should be considered in patients with vision loss with poor visual recovery, even in the presence of another ocular diagnosis and atypical findings.

References:

- Souto, Fernanda Maria Silveira et al. Differentiating Leber Hereditary Optic Neuropathy from Normal-Tension Glaucoma. Neuro-ophthalmology (Aeolus Press) (2017);41(2):99-102

- Filatov, Asia et al. Leber Hereditary Optic Neuropathy: Case Report and Literature Review. Cureus (2020);12(4):e7745

- Lin, Yun-Hsuan et al. "Juvenile open-angle Glaucoma associated with Leber's hereditary optic neuropathy: a case report and literature review.” BMC Ophthalmology (2018);18(1): 323

\section{HETEROZYGOUS NIEMANN-PICK TYPE C 2 MUTATION IN A YOUNG WOMAN WITH MILD COGNITIVE IMPAIRMENT, SOMATOFORM DISTURBANCES, AND SUPRANUCLEAR GAZE PALSY}

\author{
G. Gazzola ${ }^{1}$, S. Mozzetta ${ }^{1}$, M. Carecchio ${ }^{1}$, C. Bussè $^{2}$, M. Cassina ${ }^{3}$, A. \\ Dardis $^{4}$, A. Cagnin ${ }^{1}$
}

${ }^{1}$ Department of Neurosciences, University of Padua (Padova); ${ }^{2}$ Department of Neurosciences, Psychology, Pharmacology and Child Health NEUROFARBA, University of Florence (Firenze); ${ }^{3}$ Department of Woman and Child Health, University of Padua (Padova); ${ }^{4}$ Department of Laboratory Medicine, Hospital "Santa Maria della Misericordia" (Udine)

Background: Niemann-Pick disease type C (NPC) is a fatal, autosomal recessive lipidosis characterized by lysosomal accumulation of unesterified cholesterol and multiple neurological symptoms, such as vertical supranuclear ophthalmoplegia, progressive ataxia and dementia, 
caused by homozygous mutations of NPC 1 or NPC 2 genes. Recently, studies have ascertained a various prevalence of heterozygous genotype in neurodegenerative syndromes.

Aim: We present the case of a patient carrying heterozygous NPC 2 mutation, affected by mild vertical supranuclear gaze palsy, mild cognitive impairment, anxiety disorder with somatoform disturbances.

Case report: A 52 years old Caucasian woman, came to our attention for mild cognitive impairment characterized by difficulties of attention and anterograde memory. In the past medical history, she reported migraine with sensitive symptoms, three stroke-like episodes occurred with left hemiparesis, with no brain MRI ischemic lesions. She also reported a 2year history of brief, stereotyped episodes characterized by loss of consciousness, dysarthria, ocular gaze deviation. At first visit in the Memory Clinic the neurological examination revealed mild limitation of vertical gaze and symptoms of discomfort with gaze movements (increased rate of blinking, periocular muscle myokymia, impersistence). Neuropsychological assessment revealed a mild impairment of attentive and visual-spatial function, mild deficit of anxiety disorder with somatoform disturbances. Brain MRI was normal. EEG showed focal slow activity and spikes in bilateral temporo-parieto-occipital regions. We performed brain FDGPET/MRI, demonstrating a moderate hypometabolism of both parietal lobes. Amyloid PET with F-florbetapir was negative and CSF analysis showed normal amyloid 1-42, total tau e p-tau proteins levels. Genetic analysis discovered NPC 2 heterozygous mutation (c. $441+1 \mathrm{G}>\mathrm{A}$ ) and analysis of fibroblasts on skin biopsy showed modification of mRNA slicing and positive filipin test. Plasmatic oxysterols levels were normal.

Conclusions: A pathological role of heterozygous NPC2 mutations has been recently suggested in a few patients with cognitive decline and psychiatric symptoms. Further studies are necessary to understand the potential benefit of specific therapy for patient carrying NPC mutations with neurological symptoms, regardless of diagnosis of NPC2 disease.

Reference:

- Cupidi C, Frangipane F, Gallo M, Clodomiro A, Colao R, Bernardi L, Anfossi M, Conidi ME, Vasso F, Curcio SA, Mirabelli M, Smirne N, Torchia G, Muraca MG, Puccio G, Di Lorenzo R, Zampieri S, Romanello M, Dardis A, Maletta RG, Bruni AC. Role of NiemannPick Type C Disease Mutations in Dementia. J Alzheimers Dis. (2017);55(3):1249-59

\section{A MUTATION IN A NOVEL LYSOSOMAL GENE CAUSES ADULT-ONSET GENERALIZED DYSTONIA IN AN ITALIAN PATIENT}

E. Monfrini ${ }^{1}$, F. Cogiamanian ${ }^{2}$, S. Salani ${ }^{1}$, L. Straniero ${ }^{3}$, A. Bordoni ${ }^{1}$, M. Aureli $^{4}$, M. Garbellini ${ }^{1}$, L. Borellini ${ }^{2}$, E. Carsana ${ }^{4}$, S. Corti ${ }^{1}$, G. Comi ${ }^{1}$, N. Bresolin ${ }^{1}$, S. Duga ${ }^{3}$, A. Di Fonzo

${ }^{1}$ Foundation IRCCS Ca' Granda Ospedale Maggiore Policlinico, Neurology Unit, Dino Ferrari Center, Neuroscience Section, Department of Pathophysiology and Transplantation, University of Milan (Milano); ${ }^{2}$ Neuropathophysiology Unit, Fondazione IRCCS Ca' Granda Ospedale Maggiore Policlinico, Fondazione IRCCS Ca' Granda Ospedale Maggiore Policlinico (Milano); ${ }^{3}$ Humanitas Clinical and Research Center, IRCCS, Department of Biomedical Sciences, Humanitas University (Rozzano-MI, Milano); ${ }^{4}$ Department of Medical Biotechnology and Translational Medicine, University of Milano (Milano)

Objectives: Our aim was to find the genetic cause of adult-onset generalized dystonia in an Italian male patient.
Materials and Methods: A movement disorders specialist performed a neurological examination of the proband. The subject underwent brain MRI and neurophysiological studies. Blood samples were collected. Skin biopsy was obtained from the proband through a punch on the ventral part of the left forearm. Whole-exome sequencing (WES) was performed on genomic DNA of the patient. Functional studies (immunoblotting, enzymatic activities, and electron microscopy) were conducted on patientderived fibroblasts to prove mutation pathogenicity.

Results: The family of the proband lived in a very small village in Southern Italy for many generations. The proband was born at term after an uneventful pregnancy. All the stages of psychomotor development were normal. Reportedly, from the age of 30 years, the proband developed involuntary dystonic movements, initially affecting the right limbs. After 5 years from disease onset, dystonia became generalized, involving the trunk, limbs, neck (torticollis), and vocal cords (until complete anarthria). Brain MRI displayed atrophy of basal ganglia and marked symmetrical hypointensity in $\mathrm{T} 2$ - and $\mathrm{T} 2 *$-weighted sequences in substantia nigra, red nucleus, nigrostriatal projections, and globus pallidus. The suspected consanguinity of the parents suggested a homozygous mutation as the cause of the disease. WES was performed on the proband. No pathogenic mutations were found in known disease genes for inherited dystonias and NBIA. A filtering analysis for rare (allele frequency $<0.5 \%$ ) homozygous variants with protein impact was performed. Only one candidate variant in a gene involved in lysosomal and autophagic functions was identified. Segregation analysis in the family was consistent with autosomal recessive inheritance. Functional studies on patient-derived fibroblasts showed a striking defect of lysosomal and autophagic function.

Discussion and Conclusion: The advent of NGS provided an impressive step forward in the identification of the genetic causes of inherited dystonias, leading to the description of many novel genes in the last ten years. The lysosomal pathway has been recently associated with the pathogenesis of several forms of inherited dystonias, such as in VPS16- and VAC14-related diseases and in some neurodegenerations with brain iron accumulations (i.e. Kufor-Rakeb and BPAN). This work represents the first association of a mutation in a novel lysosomal gene with a form of adult-onset generalized dystonia and provides strong in vitro evidence of mutation pathogenicity. The identification of this novel gene confirms the important role of lysosomes in dystonia pathogenesis.

\section{THE ITALIAN FACE OF SCA21 / SCA-TMEM240}

\author{
V. Riso ${ }^{1}$, G. Silvestri ${ }^{2}$, A. Perna ${ }^{2}$, S. Rossi ${ }^{2}$, T. Nicoletti ${ }^{2}$, V. Leuzzi ${ }^{3}$, S.
} Galosi $^{3}$, C. Casali ${ }^{4}$, F. Santorelli ${ }^{5}$, D. Galatolo ${ }^{5}$

${ }^{1}$ Neurology, Catholic University of the Sacred Heart (Roma); ${ }^{2}$ Neurology, Fondazione Policlinico Universitario A. Gemelli IRCSS (Roma); ${ }^{3}$ Neuroscience Department, Sapienza University (Roma); ${ }^{4}$ Neurorehabilitation, Sapienza University Polo Pontino (Roma); ${ }^{5}$ Molecular Medicine, IRCCS Fondazione Stella Maris (Pisa)

Introduction and aim: Spinocerebellar Ataxia 21 is a rare autosomal dominant neurodegenerative disorder caused by mutations in the TMEM240 gene. To date, SCA21 has been reported only in a limited number of families worldwide. Here we describe clinical and molecular findings in 5 additional SCA2 1 patients from 4 unrelated families, diagnosed through a Next-Generation Sequencing (NGS)based molecular screening on a large cohort of degenerative and congenital ataxias collected in Italy through a multicentre collaborative effort. 
Materials and Methods: We screened by target resequencing panel a cohort of 393 patients with ataxia of unknown aetiology. Following the identification of heterozygous pathogenic TMEM240 variants, an indepth phenotyping of the novel SCA21 patients was performed.

Results and discussion: Five novel SCA21 patients from 4 unrelated families, three of Italian and one of Libyan origin were identified, all carriers of previously reported TMEM240 mutations. The clinical spectrum of our SCA21 cohort includes both adult-onset, slowly progressive cerebellar ataxias associated with cognitive impairment resembling Cerebellar Cognitive Affective Syndrome (CCAS) and early-onset forms also associated with cognitive delay and neuropsychiatric features. None of our patients exhibited signs of extrapyramidal involvement. The so termed "recurrent" c.509C $>$ T (p.Pro170Leu) mutation was detected in two out of four families, corroborating its role as hot-spot.

Conclusion: Our results confirm that SCA21 is present also in Italy, suggesting that it might be not so rare as previously thought. The phenotype of new SCA21 cases points out to identify slowly progressive cerebellar ataxia, cognitive and psychiatric symptoms as the most typical clinical features associated with mutations in the TMEM240.

References:

- Delplanque J, Devos D, Huin V, et al. TMEM240 mutations cause spinocerebellar ataxia 21 with mental retardation and severe cognitive impairment. Brain (2014);137:2657-63

- Traschütz A, van Gaalen J, Oosterloo M et al. The movement disorder spectrum of SCA21 (ATX-TMEM240): 3 novel families and systematic review of the literature. Parkinsonism Relat Disord. (2019) May;62:215-20

- Braga-Neto P, Pedroso J, Barsottini O, Schmahmann JD. Cognition in SCA21 Reflects Developmental and Adult Onset Cerebellar Cognitive Affective Syndrome. Brain (2015) Jul;138(Pt 7):e364

\section{"EYE OF TIGER SIGN" MIMIC IN PATIENTS WITH SPASTIC PARAPLEGIA GENE 7 (SPG7) MUTATIONS}

\author{
G. Rizzo ${ }^{1}$, C. Tonon ${ }^{1}$, L. Gramegna ${ }^{1}$, M. Bassi ${ }^{2}$, R. Lodi $^{1}$, R. Liguori ${ }^{1}$
}

${ }^{1}$ Department of Biomedical and Neuromotor Sciences, University of Bologna (Bologna); ${ }^{2}$ Laboratory of Molecular Biology, Scientific Institute IRCCS E. Medea (Bosisio Parini-LC)

Background and aims: Mutations in spastic paraplegia gene 7 (SPG7) are associated with a variable phenotype, including spastic paraplegia, cerebellar ataxia, ophthalmoplegia, cognitive impairment, neuropathy, optic atrophy, deafness, parkinsonism and epilepsy [1]. SPG7 encodes paraplegin, which assembles with the homologous subunit AFG3L2 into a mitochondrial matrix quality control protease complex [2]. Recently, four siblings with two novel compound heterozygous variants in the AFG3L2 gene has been reported to have an eye-of-the-tiger (EOT)-like sign on MRI [3]. Given the close link between AFG3L2 and paraplegin, we search for a similar MRI sign in five unrelated SPG7 patients.

Materials and Methods: Patient 1: a 44-year-old woman with spastic paraplegia and cerebellar signs from the age of 30 . Genetic testing revealed the homozygous mutation c.1616delC (p.Val540Cysfs*51), in the SPG7 gene. Patient 2: a 33-year-old man with spastic paraplegia, ataxia and mild signs of myopathy from the age of 31 . Genetic testing revealed the homozygous mutation c.1729G >A (p.Gly577Ser). Patient 3: a 72-year-old man with spastic paraplegia and mild cerebellar signs from the age of 52. Genetic testing revealed the homozygous mutation c. $1529 \mathrm{C}>\mathrm{T}$ (p.Ala510Val). Patient 4: a 52-year-old man with spastic paraplegia, sensorineural hearing loss and mild motor polyneuropathy from the age of 47 . Genetic testing revealed a compound heterozygous mutation, c.1616delC (p.Val540Cfs*51) and c.1045G>A (p.Gly349Ser). Patient 5: a 73-year-old man with cerebellar ataxia, pyramidal signs and ophthalmoplegia from the age of 62 . Genetic testing revealed the homozygous mutation c. 1529C $>\mathrm{T}$ (p.Ala510Val). All SPG7 variants reported are known mutations except p.Gly577Ser in patient 2.1 This variant is predicted to be pathogenic by all bioinformatics tools used and reported as likely pathogenic by ClinVar. All patients underwent brain 3T-MRI.

Results: MRI disclosed mild to moderate cerebellar atrophy in all patients except patient 4 . Focusing on the basal ganglia, a bilateral T2weigthed central hyperintense signal in the pallida was clearly visible in the patients 1 and 2, associated with a mild surrounding hypointensity in patient 1. For this MRI feature, mutations in PANK2, FLT, PLA2G6, FA2H and MPAN were previously excluded in patient 1 .

Discussion and Conclusions: Two out of our five patients carrying SPG7 mutation had the same "EOT sign" mimic on MRI reported for the patients with AFG3L2 mutation. This sign could indicate a specific mitochondrial structure dysfunction and be useful for the diagnostic workup of patients with mutations in SPG7/AFG3L2. Future studies on larger samples can add further information about the possible association with specific mutations.

References:

1. De la Casa-Fages B, Fernández-Eulate G, Gamez J, et al. Parkinsonism and spastic paraplegia type 7: Expanding the spectrum of mitochondrial Parkinsonism. Mov Disord. (2019); 34: $1547-61$

2. Atorino L, Silvestri L, Koppen M, et al. Loss of m-AAA protease in mitochondria causes complex I deficiency and increased sensitivity to oxidative stress in hereditary spastic paraplegia. J Cell Biol (2003); 163: 777-87

3. Calandra CR, Buda G, Vishnopolska SA, et al. Spastic ataxia with eye-of-the-tiger-like sign in 4 siblings due to novel compound heterozygous AFG3L2 mutation. Parkinsonism Relat Disord. (2020);73:52-4

\section{A CLINICAL AND EPIDEMIOLOGICAL PREVALENCE STUDY ON FRIEDREICH'S ATAXIA IN LATIUM, ITALY}

S. Romano ${ }^{1}$, I. Bacigalupo ${ }^{2}$, E. Bertini ${ }^{3}$, G. $\operatorname{Vasco}^{4}$, G. Silvestri ${ }^{5}$, A. Perna $^{6}$, A. Petrucci ${ }^{7}$, R. Massa ${ }^{8}$, E. Frezza ${ }^{8}$, C. Romano ${ }^{1}$, M. Salvetti ${ }^{9}$, G. Ristori ${ }^{10}$, N. Vanacore ${ }^{2}$, C. Casali $^{11}$

${ }^{1}$ Department of Neurosciences, Mental Health and Sensory Organs (NESMOS), University of Rome "Sapienza" (Roma); ${ }^{2}$ National Centre for Disease Prevention and Health Promotion, National Institute of Health (Roma); ${ }^{3}$ Unit of Neuromuscular and Neurodegenerative Disorders, Bambino Gesu' Children's Hospital, IRCCS (Roma); ${ }^{4}$ Unit of Neurorehabilitation, Bambino Gesu' Children's Hospital, IRCCS (Roma); ${ }^{5}$ UOC Neurology/Institute of Neurology, Dept of Neuroscience, Fondazione Policlinico Universitario A. Gemelli IRCSS/ Catholic University of Sacred Heart (Roma); ${ }^{6}$ Institute of Neurology, Dept of Neuroscience, Catholic University of Sacred Heart (Roma); ${ }^{7}$ Neuromuscular and Neurological Rare Diseases Center, S. CamilloForlanini Hospital (Roma); ${ }^{8}$ Unit of Neuromuscular Disorders, Department of Systems Medicine, Tor Vergata University of Rome (Roma); ${ }^{9}$ Department of Neurosciences, Mental Health and Sensory 
Organs (NESMOS), University of Rome "Sapienza", S. Andrea Hospital/ IRCCS Istituto Neurologico Mediterraneo (INM) Neuromed (Roma, Pozzilli-IS); ${ }^{10}$ Department of Neurosciences, Mental Health and Sensory Organs (NESMOS), University of Rome "Sapienza", S. Andrea Hospital (Roma); ${ }^{11}$ Department of Medical Surgical Sciences and Biotechnologies (DSBMC), Polo Pontino-University of Rome "Sapienza" (Latina)

Background: The epidemiological studies of FRDA in Italy were performed in small regions or single parts of region, with a prevalence ranging from 1.1 to 2.1 per 100,000 . This large variability may be due to the rarity of the condition and possible differences in the population age and sex structure. Our aims were to estimate the FRDA prevalence in a highly populated region of Italy, and to define the patients' molecular and clinical characteristics by a population-based approach.

Methods: For the point-prevalence study we considered patients belonging to families with a molecular diagnosis of FRDA and resident in Latium on 1 January 2019. The crude prevalence of FRDA, specific for age and sex, was calculated, assuming a Poisson distribution and standardizing for age with the direct method and using the Italian population at 1 January 2019. Moreover, we investigated possible correlations among patients' genetic profile, symptoms and age of onset.

Results: We identified 63 FRDA patients; the crude prevalence for total, males and females were 1.07 (95\% confidence interval [CI] 0.81-1.34), 0.81 (95\% CI 0.48-1.14) and 1.32 (95\% CI 0.911.72 ), per 100,000 , respectively. The distribution of FRDA patients by three age-at-onset resulted in three groups (early-onset EOFA $73 \%$; late-onset LOFA $11.1 \%$; very late-onset VLOFA $15.9 \%$ ). The repeat number of the shorter allele showed a biased distribution between the EOFA, LOFA, and VLOFA ( $\mathrm{p}=0.001)$ and was correlated with age at onset $(\mathrm{r}=-0.522 ; \mathrm{p}=0.001)$. In EOFA patients there was a significant excess of scoliosis and cardiomyopathy $(\mathrm{p}=0.001)$. Significant differences between the three age-at-onset groups emerged for the scale for the assessment and rating of ataxia (SARA; $\mathrm{p}=0.001)$ and disease duration $(\mathrm{p}=0.045)$. An annual rate of progression, operationally defined as the ratio between SARA score and years of disease, was $1.21 \pm 0.71$. It correlated with the short allele $(\mathrm{r}=0.282, \mathrm{p}=0.04)$ and the age prevalence $(\mathrm{r}=-0.388$, $\mathrm{p}=0.003$ ). To determine the contribution of age prevalence, sex, length of shorter allele, disease duration and SARA score, we performed a multivariate regression analysis that gave an R2 value of $45.3 \%$

Conclusions: We estimated the age-standardized prevalence and the sex and age distribution of FRDA in Latium, also according to a clinical classification (EOFA, LOFA, VLOFA). This standardization allows to compare the prevalence reported in different geographic areas when using the same reference population. It is also helpful to monitor a populationbased disease prevalence over time in cohort studies, and/or for developing disease registry.

References:

- Leone M, Brignolio F, Rosso MG, et al. Friedreich's ataxia: a descriptive epidemiological study in an Italian population. Clin Genet (1990);38:161-9

- Reetz K, Dogan I, Hilgers RD, et al. Progression characteristics of the European Friedreich's Ataxia Consortium for Translational Studies (EFACTS): a 2 years cohort study. Lancet Neurol. (2016);15(13):1346-54

- Patel M, Isaacs CJ, Seyer L, et al. Progression of Friedreich ataxia: quantitative characterization over 5 years. Ann Clin Transl Neurol. (2016);3(9):684-94

\section{PRELIMINARY DATA FROM A LARGE ITALIAN COHORT OF PATIENTS AFFECTED BY HEREDITARY SPASTIC PARAPLEGIA TYPE 4 DUE TO SPAST MUTATIONS}

S. Rossi ${ }^{1}$, F. Santorelli ${ }^{2}$, G. Silvestri ${ }^{1}$, V. Riso ${ }^{1}$, L. Ulgheri ${ }^{3}$, C. Cereda ${ }^{4}$, L. Diamanti ${ }^{5}$, M. Plumari ${ }^{6}$, B. Dal Fabbro ${ }^{5}$, G. Straccia ${ }^{7}$, I. Orologio ${ }^{7}$, C. Dato $^{8}$, A. Filla ${ }^{9}$, C. Criscuolo ${ }^{9}$, M. Dotti ${ }^{10}$, A. Mignarri ${ }^{10}$, L. Orsi ${ }^{11}$, M. $\mathrm{D}^{\prime}$ Angelo ${ }^{12}, \mathrm{M}$. Bassi ${ }^{13}$, A. Martinuzzi ${ }^{14}$, C. Stefan $^{15}$, M. Scarlato ${ }^{16}$, O. Musumeci $^{17}$, E. Bertini ${ }^{18}$, F. Nicita ${ }^{18}$, R. Massa ${ }^{19}$, R. Liguori ${ }^{20}$, G. Rizzo $^{20}$, M. Seri ${ }^{21}$, S. Romano ${ }^{22}$, A. Di Muzio ${ }^{23}$, A. Petrucci ${ }^{24}$, G. Vazza $^{25}$, E. Pegoraro ${ }^{26}$, A. Orlacchio ${ }^{27}$, M. Melone ${ }^{7}$, C. Casali ${ }^{28}$

${ }^{1}$ Institute of Neurology, Catholic University of the Sacred Heart (Roma); ${ }^{2}$ Molecular Medicine, IRCCS Fondazione Stella Maris (Pisa); ${ }^{3}$ Department of Biomedical Sciences, Clinical Genetics Service, Azienda Ospedaliero-Universitaria (Sassari); ${ }^{4}$ Genomic and postGenomic Center, IRCCS Mondino Foundation (Pavia); ${ }^{5} \mathrm{C}$. Mondino National Neurological Institute, IRCCS Mondino Foundation (Pavia); ${ }^{6}$ Unit of Medical Genetics and Neurogenetics, Fondazione IRCCS Istituto Neurologico Carlo Besta (Milano); ${ }^{7}$ Department of Medical Sciences and Advanced Surgery, University of Campania (Napoli); ${ }^{8}$ Neurology Unit, IRCCS Policlinico San Donato (Milano); ${ }^{9}$ Department of Neurosciences and Reproductive and Odontostomatological Sciences, University of Naples (Napoli);

${ }^{10}$ Department of Medicine, Surgery and Neurosciences, Medical School, University of Siena (Siena); ${ }^{11}$ Neurologic Division 1, Department of Neuroscience and Mental Health, Città della Salute e della Scienza di Torino (Torino); ${ }^{12}$ Neuromuscular Unit, Scientific Institute IRCCS E. Medea (Lecco); ${ }^{13}$ Laboratory of Molecular Biology, Scientific Institute IRCCS E. Medea (Lecco); ${ }^{14}$ Conegliano Research Center, Scientific Institute IRCCS E. Medea (Treviso); ${ }^{15}$ Acquired Neuropsychological Disease Rehabilitation Unit, Scientific Institute IRCCS E. Medea (Treviso); ${ }^{16}$ Institute of Experimental Neurology (InSpe), Division of Neuroscience, IRCCS Ospedale San Raffaele (Milano); ${ }^{17}$ Department of Clinical and Experimental Medicine, University of Messina (Messina); ${ }^{18}$ Unit of Neuromuscular \& Neurodegenerative Disorders, Dept of Neurosciences \& Neurorehabilitation, Bambino Gesù Children's Research Hospital (Roma);

${ }^{19}$ Neuromuscular Diseases Unit, Department of Systems Medicine, University of Rome Tor Vergata (Roma); ${ }^{20}$ Department of Biomedical and Neuromotor Sciences, University of Bologna (Bologna); ${ }^{21}$ Medical Genetics Unit, Sant'Orsola-Malpighi University Hospital (Bologna); ${ }^{22}$ Centre for Experimental Neurological Therapies, Department of Neurosciences, Mental Health and Sensory Organs, Sapienza University (Roma); ${ }^{23}$ Center for Neuromuscular Disease, Center for Excellence on Aging, Gabriele D'Annunzio University Foundation (Chieti); ${ }^{24}$ Center of Neuromuscular and Neurological Rare Diseases, S. Camillo Forlanini Hospital (Roma); ${ }^{25}$ Department of Biology, University of Padua (Padova); ${ }^{26}$ Neuromuscular Center, University of Padua (Padova); ${ }^{27}$ Department of Surgery and Biomedical Sciences, University of Perugia (Perugia); ${ }^{28}$ Department of Medical and Surgical Sciences and Biotechnologies, University of Rome Sapienza (Latina)

Aim: Hereditary spastic paraplegias (HSPs) are rare, inherited neurological disorders, characterized by degeneration of the corticospinal tracts and dorsal columns, whose prominent clinical feature is represented by spastic gait. SPG4, caused by heterozygous mutations of the SPAST gene, represents the most common form. Herein, we present findings from a large cohort of Italian SPG4 patients from 22 Italian Centers, aiming to define the clinical spectrum of SPG4 in Italy, and find any significant genotype-phenotype correlations. 
Materials and methods: We retrospectively collected clinical and molecular data from 729 patients (421 males, 57.8\%) from 320 families. Family history for SPG4, age at the examination, age at onset (AAO) were gathered from medical records. Disease severity was assessed by SPATAX disability and SPRS scale. Numerical and categorical variables were analyzed by Mann-Whitney and Chi-squared test, respectively.

Results: Overall, penetrance of SPG4 was 0.96, being higher in males ( 0.97 vs $0.93, p=0.16)$. Mean $\mathrm{AOO}(\mathrm{n}=639)$ was $34.47 \pm 17.48$ years, with a bimodal distribution (first and third-fifth decades). Comparing males with females, males showed more frequently spastic gait $(96.9 .4 \%, 376 /$ 388 vs $90.2 \%, 248 / 275, \mathrm{p}<0.01)$ with a higher SPRS score $(19.56 \pm 12.56$ vs $16.18 \pm 12.51, \mathrm{p}=0.014)$, and were more frequently assuming myorelaxant drugs $(70.1 \%, 199 / 284$ vs $59.3 \%, 121 / 204, \mathrm{p}=0.01)$. Overall, 200 different SPAST mutations were recorded, the missense ones being the most common $(286 / 711,40.2 \%)$. Compared to truncating mutations, patients with missense mutations had a shorter disease duration $(10.15 \pm 12.25$ vs $14.80 \pm 14.37$ years, $\mathrm{p}<0.01)$, a higher SPATAX score $(3.43 \pm 1.77$ vs $3.02 \pm 1.47, \mathrm{p}<0.01)$, and more frequently intellectual deficiency $(13.5 \%, 35 / 259$ vs $15 / 335,4.5 \%, \mathrm{p}<0.01)$. On the other hand, patients with truncating mutations showed more frequently spasticity at rest $(64.1 \%, 236 / 368$ vs $44.9 \%, 118 / 263, \mathrm{p}<0.01)$, lower limbs (LL) proximal weakness $(61.7 \%, 224 / 363$ vs $44.1 \%, 116 / 263, \mathrm{p}<0.01)$, urinary disturbances $(51.3 \%, 181 / 353$ vs $34.4 \%, 90 / 262, \mathrm{p}<0.01)$, polyneuropathy $(10.3 \%, 27 / 263$ vs $2.0 \%, 4 / 200, \mathrm{p}<0.01)$, cerebral atrophy $(55.7 \%, 34 / 61$ vs $17.1 \%, 7 / 41, \mathrm{p}<0.01)$, and dementia $(9.5 \%, 32 / 338$ vs $2.3 \%, 6 / 256, \mathrm{p}<0.01)$.

Discussion: In accordance with the work of Perodi et al., we found a higher penetrance in males, and, in patients with missense mutations, more frequently intellectual disability. On the contrary, we documented a more severe motor involvement in males, and a more severe disability along with a shorter disease duration in patients carrying missense mutations. Moreover, we found more frequently LL involvement, cerebral atrophy, dementia, and polyneuropathy in patients with truncating mutations.

Conclusions: Differences found in our cohort may be explained by the different genetic background as well as by the retrospective nature of the study. This wide multicenter study allowed to define both phenotypic and genotypic spectrum of SPG4 in Italy, creating a network that will be fundamental to ensure participation of patients to clinical trials.

This work was funded by A.I. Vi.P.S. Onlus. *Equal contribution. References:

- Sparodi L, Rydning SL, Tallaksen C, Durr A. Spastic Paraplegia 4. In: Adam MP, Ardinger HH, Pagon RA, et al., eds. GeneReviews®. Seattle (WA): University of Washington, Seattle; (1993)

- Fink JK. Hereditary spastic paraplegia: clinico-pathologic features and emerging molecular mechanisms. Acta Neuropathol. (2013);126(3):307-328. doi:10.1007/s00401-013-1115-8

- Parodi L, Fenu S, Barbier M, et al. Spastic paraplegia due to SPAST mutations is modified by the underlying mutation and sex. Brain (2018);141(12):3331-3342. doi:10.1093/brain/ awy 285

\section{IDENTIFICATION OF 11 NOVEL SUSCEPTIBILITY GENES AND MIRNA-TARGET GENE INTERACTIONS ASSOCIATED WITH PARKINSON DISEASE: EVIDENCE OF GENETIC AND EPIGENETIC NETWORKS}

C. Strafella ${ }^{1}$, V. Caputo $^{1}$, A. Termine ${ }^{2}$, F. Assogna ${ }^{3}$, C. Pellicano ${ }^{3}$, F. Pontieri $^{4}$, L. Macchiusi ${ }^{3}$, G. Minozzi ${ }^{5}$, S. Gambardella ${ }^{6,7}$, D. Centonze ${ }^{7}$, P. Bossù ${ }^{8}$, G. Spalletta ${ }^{3}$, C. Caltagirone ${ }^{9}$, E. Giardina ${ }^{10}$, R. Cascella $^{11}$
${ }^{1}$ Genomic Medicine Laboratory; Department of Biomedicine and Prevention, IRCCS Santa Lucia Foundation; Tor Vergata University (Roma); ${ }^{2}$ Genomic Medicine Laboratory, IRCCS Santa Lucia Foundation (Roma); ${ }^{3}$ Laboratory of Neuropsychiatry, Department of Clinical and Behavioral Neurology, IRCCS Santa Lucia Foundation (Roma); ${ }^{4}$ Department of Neuroscience, Mental Health and Sensory Organs, "Sapienza" University of Rome (Roma); ${ }^{5}$ Department of Veterinary Medicine, University of Milan (Milano); ${ }^{6}$ Department of Biomolecular Sciences, University of Urbino "Carlo Bo" (Urbino); ${ }^{7}$ Neuromed Institute IRCCS (Pozzilli-IS); ${ }^{8}$ Laboratory of Experimental Neuropsychobiology, Department of Clinical and Behavioral Neurology, IRCCS Santa Lucia Foundation (Roma); ${ }^{9}$ Department of Clinical and Behavioral Neurology, IRCCS Santa Lucia Foundation (Roma); ${ }^{10}$ UILDM Lazio ONLUS Foundation; Department of Biomedicine and Prevention, IRCCS Santa Lucia Foundation; Tor Vergata University (Roma); ${ }^{11}$ Genomic Medicine Laboratory; Department of Biomedical Sciences, IRCCS Santa Lucia Foundation; Catholic University Our Lady of Good Counsel (Roma, Tirana-AL)

Objectives: The present study investigated the association of 120 genetic variants with the susceptibility to idiopathic Parkinson Disease (PD) in order to identify novel biomarkers and therapeutic targets.

Materials: The study cohort included 342 Italian patients affected by idiopathic PD recruited from the Laboratory of Neuropsychiatry of IRCCS Santa Lucia Foundation (Rome) and IRCCS Neuromed (Pozzilli). A blood sample was taken from patients, in order to perform DNA extraction. As control group, 503 samples representative of the European general population were utilized.

Methods: The recruited patients were subjected to a genotyping analysis of 120 SNPs selected by literature and bioinformatic approach. Noncoding variants were largely preferred as their contribution to disease susceptibility may provide additional knowledge concerning the regulatory gene networks and the biological pathways underlying PD. The genotyping analysis of the DNA samples was conducted by OpenArray Real-Time PCR on Quant Studio 12K Flex Real Time PCR System and Taqman Genotyper Software. Statistical and bioinformatic analysis were performed to assess the association with PD and identify network of genes and miRNAs interactions, which could be useful to identify potential biomarkers and therapeutic targets.

Results: The genetic analysis reported 26 associated-SNPs and 11 novel susceptibility genes for PD. A network of susceptibility genes (APOE, CLU, IL6, IL7R, IL12B, INPP5D, MAPK1, MEF2C, MIF, TNFSF14) and miR-499a, miR-196a2 and miR-29a were identified as new therapeutic targets. In addition, we obtained 12 significant eQTL variants distributed in multiple brain regions and 15 epistatic interactions among the associated SNPs, which may contribute to PD susceptibility by altering gene expression in disease-relevant tissues.

Discussion: The present study identified 11 novel susceptibility genes for PD, which may alter multiple signalling pathways critically involved in cellular homeostasis and dopaminergic neurons wiring. The study of miRNA-target genes networks highlighted miR-499a, miR-196a2 and miR-29a as three new therapeutic targets, which may modulate several neuroinflammatory and neurodegenerative mechanisms underlying PD physiopathology. Moreover, the study described a network of interconnected genes, which may act as upstream regulators in the modulation of biological pathways relevant to $\mathrm{PD}$, including oxidative stress, immuno-inflammatory response, synaptogenesis and HGMB1 signalling. Particular attention should be deserved to IL-6 as a possible treatment option for PD since its modulation may indirectly regulate the network of interconnected genes and their related-pathways. 
Conclusions: In conclusion, the identified networks of interconnected genes and miRNA-target gene interactions may be helpful for developing therapeutic or drug repurposing strategies for the treatment of PD.

References:

- Strafella C., Caputo V., Galota M.R., Zampatti S., Marella G., Mauriello S., Cascella R., Giardina E. Application of Precision Medicine in Neurodegenerative Diseases. Front Neurol (2018);9:701

- Nalls MA, Blauwendraat C, Vallerga CL, Heilbron K, Bandres-Ciga $\mathrm{S}$, Chang D et al. Identification of novel risk loci, causal insights, and heritable risk for Parkinson's disease: a meta-analysis of genomewide association studies. Lancet Neurol. (2019);18:1091-102

- Blauwendraat C, Nalls MA, Singleton AB. The genetic architecture of Parkinson's disease. Lancet Neurol. (2020);19:170-8

\section{NEUROIMAGING}

\section{TRANSIENT BRAIN MAGNETIC RESONANCE CHANGES AFTER STATUS EPILEPTICUS AND SINGLE SEIZURES: A DIAGNOSTIC CHALLENGE}

\author{
A. M. Alicino, D. Mezzapesa, M. Savarese, A. La Neve, I. Simone, M. \\ Trojano
}

Department of Neurology, Policlinico of Bari/University of Bari (Bari)

Background: Transient Magnetic Resonance Imaging (MRI) changes are described not exclusively in Status Epilectus (SE), but even after a single seizure. These are due to cytotoxic edema, caused by ictal depolarization leading to the massive influx of sodium and calcium ions and potassium efflux, resulting in a transient breakdown of the $\mathrm{Na} / \mathrm{K}$-ATPase and vasogenic edema, related to the breakdown of the blood-brain-barrier and ictal hyperperfusion. We describe three cases of diagnostically challenging transient MRI cerebral alteration.

Case series: A 68-years-man with cerebrovascular risk factors, came to our attention after an episode of behavior arrest followed by a tonicclonic seizure. The neurological examination revealed ideomotor slowness, left hemianopia and left arm hyposthenia. Brain MRI was performed, showing a right thalamus diffusion-weighted-imaging (DWI) restriction compatible with an acute ischemic area and a right parietaloccipital chronic ischemic area. Therefore, we started acetylsalicylic acid and clopidogrel therapy. The day after he was asymptomatic. A new MRI did not confirm the thalamic lesion. A 76-years-woman with a previous history of ischemic stroke, was admitted to our department after an episode of aphasia. In the emergency room a tonic-clonic seizure occurred and an electroencephalogram showed a SE with paroxysms from left temporal areas. Antiepileptic therapy (lacosamide and levetiracetam) was started. Brain MRI evidenced chronic ischemic areas and a left thalamic DWI restriction and FLAIR hyperintensity, with no abnormal ADC signal. Acyclovir therapy was started but lumbar puncture excluded encephalitis. A new MRI did not evidence thalamic abnormalities. An 81years-woman with a probable cognitive decline came to our attention after an episode of dysphasia. MRI revealed a right lobe hemorrhagic lesion, a right temporal-mesial DWI restriction and T2 hyperintensity, a cerebellar ischemic lesion with mild hemorrhagic infarction. An electroencephalogram was performed after psychomotor agitation episodes and showed electrical seizure starting from right temporal-parietal-occipital region. Clinicians decided to perform a lumbar puncture (unremarkable) but did not begin any empiric therapy. A new MRI did not confirm the temporal-mesial lesion excluding the previous hypothesis of encephalitis/ vasculitis.

Discussion and conclusion: Peri-ictal DWI or FLAIR abnormalities often involve temporal-mesial lobe and thalamus. Thalamus is part of a circuit for the initiation and spread of seizures. Transient MRI cerebral alterations due to seizures or SE may imitate other pathological entities: it is important to recognize them to avoid unnecessary and invasive diagnostic procedures or inappropriate therapies, as occurred in our cases. An MRI check-up could ensure the transient and benign nature of these lesions. References:

- A.M. Katramados, D. Burdette, S.C. Patel, L.R. Schultz, S. Gaddam, P.D. Mitsias Periictal diffusion abnormalities of the thalamus in partial status epilepticus Epilepsia (2009);50: 265-275

- J.A. Kim, J.I. Chung, P.H. Yoon, D.I. Kim, T.S. Chung, E.J. Kim, E.K. Jeong Transient MR signal changes in patients with generalized tonicoclonic seizure or status epilepticus: periictal diffusionweighted imaging AJNR Am. J. Neuroradiol. (2001);22:1149-60

- Hong KS, Cho YJ, Lee SK, Jeong SW, Kim WK, Oh EJ. Diffusion changes suggesting predominant vasogenic oedema during partial status epilepticus. Seizure 2004;13(5):317-321. doi:10.1016/j.seizure.2003.08.004

\section{BRAIN HYPOMETABOLISM AND MICROGLIA ACTIVATION IN MILD COGNITIVE IMPAIRMENT: A COMBINED FDG AND PK11195 PET STUDY}

\author{
C. Boccalini ${ }^{1}$, G. Tondo ${ }^{1}$, S. Caminiti ${ }^{1}$, L. Presotto ${ }^{2}$, D. Perani ${ }^{1}$
}

${ }^{1}$ In Vivo Human Molecular and Structural Neuroimaging Unit, Division of Neuroscience, IRCCS San Raffaele Scientific Institute, Milan, VitaSalute San Raffaele University (Milano); ${ }^{2}$ Nuclear Medicine Unit, San Raffaele Hospital (Milano)

Objective: Mild Cognitive Impairment (MCI) is a transitional condition between normal cognitive function and dementia. Once a diagnosis of MCI is made, it is essential to establish the etiology of the cognitive decline. PET scan with [18F]fluorodeoxyglucose ([18F]FDG-PET) reveals brain hypometabolism patterns in distinct dementia conditions, since MCI phase and before manifest brain atrophy occurs. Activated microglia could be part of the pathogenetic processes leading to neuronal dysfunction/hypometabolism and neurodegeneration. Using [11C]-(R)PK11195 and [18F]FDG PET, we in vivo investigated the presence of microglial activation, and whether a relationship exists with brain hypometabolism, a biomarker of neurodegeneration in MCI.

Materials: We examined 8 subjects fulfilling the Petersen criteria for MCI diagnosis [1]. All subjects underwent both [18F]FDG PET and [11C]-(R)-PK11195 PET.

Methods: [18F]FDG PET brain glucose metabolism was assessed at the single-subject level using an optimized and validated SPM procedure [2]. [11C]-(R)-PK11195 binding potentials (BPs) were estimated adopting a receptor parametric mapping procedure and the images were analysed using the curve distance clustering algorithm (CDCA)[3]. We evaluated 1. the amount of spatial overlap between hypometabolism and [11C]-(R)-PK11195 BPs maps throughout the Dice similarity coefficient; 2. the correlation between [18F]FDG brain metabolism and [11C]-(R)PK11195 BPs using Pearson's correlation at subject level; 3. whether the relationship between neuroinflammation and hypometabolism was due to the spatial overlap between the two signals, by means of linear regression analysis. 
Results: Single-subject [18F]FDG PET SPM analysis revealed heterogeneous hypometabolism patterns, as expected in MCI with different underlying aetiologies. The most frequent hypometabolic pattern involved temporalparietal cortex, bilaterally, as in typical Alzheimer disease. [11C]-(R)PK11195 PET single subject analysis showed several clusters of significant microglia activation, which inversely correlated with the regional brain metabolism. A spatial concordance with regional hypometabolism was evident in all MCI subjects, and it was proportional to the strength of the betweensignals correlation coefficient $(\beta=-0.804 ; \mathrm{p}=0.016$ ).

Discussion: Previous PET studies investigating neuroinflammation in MCI subjects reported conflicting results, with presence or lack of increased regional microglial activation. In our study, using an advanced algorithm, we found microglia activation in all MCI subjects, with spatial concordance and inverse correlation with brain glucose hypometabolism, suggesting that activated microglia may contribute to neurodegeneration and synaptic dysfunction.

Conclusion: These findings have important implications in highlighting the local immune activity in the neurodegenerative processes from the prodromal phases, and in considering anti-inflammatory therapies.

References:

1. R.C. Petersen, R.O. Roberts, D.S. Knopman, B.F. Boeve, Y.E. Geda, R.J. Ivnik, G.E. Smith, C.R. Jack, Mild cognitive impairment: ten years later, Arch. Neurol. (2009);66:1447-55

2. D. Perani, P. Anthony, D. Rosa, C. Cerami, F. Gallivanone, F. Fallanca, E. Giovanna, A. Panzacchi, F. Nobili, S. Pappatà, A. Marcone, V. Garibotto, I. Castiglioni, G. Magnani, S.F. Cappa, L. Gianolli, E. Consortium, Validation of an optimized SPM procedure for FDG-PET in dementia diagnosis in a clinical setting, NeuroImage Clin. (2014);6:445-54

3. L. Presotto, L. Iaccarino, V. Bettinardi, L. Gianolli, D. Perani, An automated clustering algorithm for reference region extraction of brain 11 C-PK11195 studies, in: 2015 IEEE Nucl. Sci. Symp. Med. Imaging Conf., IEEE, (2015):1-3

\section{GENDER-RELATED VULNERABILITY OF DOPAMINERGIC NEURAL NETWORKS IN PARKINSON'S DISEASE}

\author{
C. Boccalini ${ }^{1}$, G. Carli $^{1}$, A. Pilotto ${ }^{2}$, A. Padovani ${ }^{3}$, D. Perani ${ }^{1}$
}

${ }^{1}$ In vivo human molecular and structural neuroimaging Unit, Division of Neuroscience, IRCCS San Raffaele Scientific Institute, Vita-Salute San Raffaele University (Milano); ${ }^{2}$ Neurology Unit, Department of Clinical and Experimental Sciences, University of Brescia (Brescia); ${ }^{3}$ Parkinson's Disease Rehabilitation Centre, FERB ONLUS S. Isidoro Hospital (Trescore Balneario- BG)

Objectives: In Parkinson's disease (PD) neurodegeneration of dopaminergic systems leads to motor and non-motor abnormalities. Sex might influence the clinical phenotypes and progression of PD [1]. Molecular imaging data have only addressed nigrostriatal dopamine system that appears more preserved in women. Evidence on sex/gender differences in the mesolimbic dopaminergic system is still lacking. We aimed at assessing PD gender differences in both the dopaminergic pathways, by using a metabolic connectivity approach. This approach is based on the evidence of a significant coupling between neurotransmission impairment and alteration of metabolic networks.

Materials: We included 34 idiopathic PD patients and 60 age-matched healthy controls for comparison.
Methods: The molecular architecture of both the dopaminergic networks were estimated throughout partial correlation analyses using FDGPET metabolic data (threshold set at $\mathrm{p}<0.01$, corrected for Bonferroni multiple comparisons). In order to quantify the severity of metabolic connectivity alterations in PD males and females, we calculated the percentage of altered metabolic connections of each networks. We performed a gender comparison by means of chi-squared test.

Results: Male patients were characterized by a widespread altered connectivity of nigro-striato-cortical network and a spared reconfiguration of the mesolimbic pathway. On the other hand, PD females showed greater changes of mesolimbic network and only partially reconfiguration of the nigro-striato-cortical one. The comparison between PD males and females showed that the metabolic connectivity alteration of nigro-striatocortical network was significantly more severe in PD males $(\mathrm{X} 2=$ $27.8571, \mathrm{p}<0.00001)$ and the metabolic connectivity alteration of the mesolimbic network was significantly more severe in PD females $(\mathrm{X} 2=$ 87.8938, $\mathrm{p}<0.00001)$.

Discussion: Our findings add remarkable knowledge on the neurobiology of gender differences in PD with the identification of selective neural vulnerabilities of the dopaminergic networks in male and female subjects. These results highlight a males' vulnerability of dopaminergic nigro-striato-cortical system, since females show less alterations in these networks probably due to oestrogens neuroprotection effects [1]. On the other hand, the mesolimbic pathway, known to be linked to psychiatric symptoms [2], is more affected in females compared to males, according to the fact that psychiatric symptoms are more likely to occur in women [3]. The gender differences here obtained exploring brain system metabolic connectivity, might be at the intersection between biological and sociodemographic factors.

Conclusion: Gender differences in PD should be considered for a more comprehensive picture which may influence treatments and the targeting of modifiable risk factors.

References:

1. S. Cerri, L. Mus, F. Blandini, Parkinson's Disease in Women and Men: What's the Difference?, J. Parkinsons. Dis. (2019);1-15

2. A. Castrioto, S. Thobois, S. Carnicella, A. Maillet, P. Krack, Emotional manifestations of PD: Neurobiological basis, Mov. Disord. (2016);31:1103-13

3. P. Martinez-Martin, C.F. Pecurariu, P. Odin, J.J. Van Hilten, A. Antonini, J.M. Rojo-Abuin, V. Borges, C. Trenkwalder, D. Aarsland, D.J. Brooks, Gender-related differences in the burden of non-motor symptoms in Parkinson's disease. J. Neurol. (2012);259:1639-47

\section{MRI-BASED CLUSTERING OF MULTIPLE SCLEROSIS PATIENTS IN THE PERSPECTIVE OF PERSONALIZED MEDICINE}

\author{
R. Bonacchi ${ }^{1}$, M. Rocca ${ }^{1}$, A. Meani ${ }^{1}$, E. Pagani ${ }^{1}$, M. Filippi ${ }^{2}$
}

${ }^{1}$ Neuroimaging Research Unit, Institute of Experimental Neurology, Division of Neuroscience, IRCCS San Raffaele Scientific Institute (Milano); ${ }^{2}$ Neuroimaging Research Unit, Institute of Experimental Neurology, Division of Neuroscience; Neurology Unit and Neurophysiology Unit, IRCCS San Raffaele Scientific Institute; VitaSalute San Raffaele University (Milano)

Aims: Multiple sclerosis (MS) patients have heterogeneous clinical manifestations, natural history, and treatment response, due to heterogeneous 
underlying pathophysiological differences. We aimed to find clusters of MS patients with homogeneous underlying pathophysiology, as determined by advanced MRI techniques.

Materials: One-hundred-and-fifteen MS (57 relapsing-remitting, 12 primary- and 46 secondary-progressive) patients, and 44 age- and sexmatched healthy controls (HC) were included in the study.

Methods: MRI acquisition was performed on a 3T scan, including brain and cervical cord pulse sequences for assessing lesions, atrophy, and microstructural damage (with diffusion-tensor metrics). A complete neurological assessment, with rating of Expanded Disability Status Scale (EDSS) was also performed. Clusters of MS patients were identified with hierarchical clustering on age- and sex-adjusted MRI variables.

Results: Five clusters of MS patients were identified: "early"; "intermediate-cord", "intermediate-cortical", "intermediate-late-lesion"; and "late". "Early" patients showed similar MRI metrics vs HC (except lesions), low EDSS and short disease duration. "Intermediate" groups had altered MRI metrics, higher EDSS and longer disease duration, compared to "early" $(\mathrm{p}<0.01)$. "Intermediate-cord" patients were characterized by high cord T2-lesion volume (LV) ( $<<0.001$ vs all but "late" groups), and "intermediate-cortical" by low cortical thickness ( $p<0.001$ vs all but "intermediate-late-lesion" and "late" groups). "Intermediate-late-lesion" patients showed higher brain T2-LV and deep grey matter (GM) atrophy, but also a longer disease duration, compared to all but "late" groups $(p<0.01)$. "Late" patients had higher EDSS and disease duration, compared to "intermediate-cord" and "intermediate-cortical" $(\mathrm{p}<0.01)$; and worst corticospinal-tract diffusion-tensor metrics and cord/brain atrophy $(\mathrm{p}<0.01$ vs all). "Intermediate-cord" patients could be divided into 2 groups with similar disease duration characterized by different cord GM atrophy and cortical thickness ( $\mathrm{p}<0.01)$, the more impaired one including mostly progressive phenotypes and higher EDSS.

Discussion: MRI-based clustering of MS patients is feasible. Cervical cord lesions, cortical GM atrophy, and white matter lesions may be the main contributors to disability. "Intermediate-cord" patients may be the best target to study neuroprotective and regenerative strategies.

Conclusions: MRI-based clustering of MS patients contributes to better characterize disease heterogeneity and in the future it may be useful for personalized medicine.

Funding: Partially supported by Fondazione Italiana Sclerosi Multipla (FISM/2018/R/16).

\section{IMPROVING RELIABILITY OF DIFFUSION-DERIVED AND QUANTITATIVE SUSCEPTIBILITY MAPPING BRAIN MEASURES IN MULTI-CENTER STUDIES}

M. Bozzali ${ }^{1,2}$, G. Giulietti ${ }^{2,3}$, A. Nigri ${ }^{4}$, C. Ambrosi ${ }^{5}$, L. Mascaro ${ }^{5}$, R. Gasparotti $^{6}$, C. Muscio $^{7}$, M. G. Bruzzone ${ }^{4}$

${ }^{1}$ Department of Neuroscience 'Rita Levi Montalcini', University of Torino (Torino); ${ }^{2}$ Neuroimaging Laboratory, IRCCS Santa Lucia Foundation (Roma); ${ }^{3}$ Nuclear Medicine Unit, Division of Neuroscience, San Raffaele Hospital (Milano); ${ }^{4}$ Radiology Department, IRCCS Carlo Besta Neurological Institute (Milano); ${ }^{5}$ Department of Diagnostic Imaging, ASST Spedali Civili Hospital (Brescia); ${ }^{6}$ Neuroradiology Unit, University of Brescia (Brescia); ${ }^{7}$ Division of Neurology V, Neuropathology, IRCCS Carlo Besta Neurological Institute (Milano)

Background: To reach the sample size needed for obtaining good statistical power and generalizability of MRI results, it is often necessary to acquire data from multiple sites using different MRI scanners.
Considerable non-biological variability of measurements is due to MR scanners and acquisition protocols. Using Combat algorithm [refl], we harmonized diffusion-derived and quantitative susceptibility mapping (QSM) measures from different centers, comparing data reliability using intraclass correlation coefficient (ICC).

Methods: Two-shell diffusion-weighted and multi-echo gradient-echo (GRE) sequences were acquired from 3 different centers (IRCCS C. Besta, Milan; IRCCS Santa Lucia, Rome; ASST Spedali Civili Hospital, Brescia) using 2 types of MRI scanners (Philips Achieva 3T and Siemens Skyra 3T), within the Italian Alzheimer's Disease Project (AD-NET). Each center collected brain images from 5 healthy subjects (15 in total) who underwent MRI scanning twice (test/retest) at about oneweek interval ( 30 scans in total). Diffusion-weighted data were processed to derive fractional anisotropy (FA), mean diffusivity (MD), orientation dispersion (ODI) and neurite density (NDI) indexes, while multi-echo GRE images were processed to obtain susceptibility maps (QSM). All 5 groups of 30 derived maps were spatially normalized to MNI space and then separately harmonized using Combat algorithm to remove unwanted (due to scanner) inter-site variability. Inter-site reliability (reflecting the degree of correlation and agreement between measurements) was assessed using the mean ICC value (two-way random-effect model) in gray (GM) and white matter (WM) separately for each derived map, before and after Combat application. Note: ICC ranges between 0 and 1 , with values closer to 1 representing stronger reliability (ICC $\leq 0.40$ : poor reliability; ICC $\leq 0.41-0.60$ : moderate reliability; ICC $\leq 0.61-0.80$ : good reliability; ICC $\geq 0.81$ : excellent reliability).

Results: We found a consistent increase of ICC in all data filtered with Combat, in both GM and WM tissue. Particularly impressive is the gain obtained for the mean ICC value relative to MD maps, which increased from 0.19 to 0.58 in the WM and from 0.42 to 0.69 in the GM.

Conclusions: Our results strongly indicate that Combat algorithm is able to detect and remove most of data variability due to scanners, preserving the biological information in diffusion-derived and susceptibility measures. In light of these findings, we recommend the harmonization of these kinds of data when collected in multi-center studies or with different scanners before running any statistical analyses.

Reference:

- JP Fortin, DP, Birkan Tunç, T Watanabe, MA Elliott, K Ruparel, DR Roalf, TD Satterthwaite, RC Gur, RE Gur, RT Schultz, R Verma, RT Shinohara. Harmonization of Multi-Site Diffusion Tensor Imaging Data. Neuroimage (2017) Nov 1;161:149-70

\section{THE RELATIONSHIP AMONG LEPTOMENINGEAL ENHANCEMENT CLINICAL, RADIOLOGICAL ACTIVITY AND CEREBROSPINAL FLUID MARKERS}

N. Bruschi ${ }^{1}$, C. Lapucci $^{1}$, M. Cellerino ${ }^{1}$, G. Boffa ${ }^{1}$, E. Sbragia ${ }^{1}$, E.

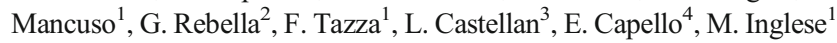

${ }^{1}$ Department of Neuroscience, Rehabilitation, Ophthalmology, Genetics, Maternal and Child Health (DINOGMI), University of Genoa (Genova); ${ }^{2}$ Department of Neuroradiology, University of Genoa (Genova); ${ }^{3}$ Department of Neuroradiology; IRCSS Hospital San Martino, University of Genoa (Genova); ${ }^{4}$ IRCSS Hospital San Martino, University of Genoa (Genova)

Background: Meningeal inflammation is a progressively recognized finding in multiple sclerosis (MS). The real prevalence of leptomeningeal 
enhancement (LME) in different stages of MS and its association with neurodegeneration is still a matter of debate.

Objective: To assess the in-vivo prevalence of meningeal inflammation in relapsing-remitting MS (RRMS) and to evaluate its association with clinical/radiological activity and cerebrospinal fluid (CSF) markers.

Methods: This is an ongoing observational study. LME was assessed by two blinded neurologists with a 3D 1x1x1 mm FluidAttenuated-Inversion-Recovery (FLAIR) acquired 20 minutes post gadolinium (TR $6000 \mathrm{~ms}$; TE $356 \mathrm{~ms}$ ) LME was defined as signal intensity within the subarachnoid space greater than that of brain parenchyma and brighter on postcontrast scans. MRI activity was defined as at least 1 gadolinium enhancing and/or new/enlarging T2 lesions. Differences in terms of clinical, radiological, CSF characteristics between patients with and without LME were tested with ANOVA, chi square and binary logistic regression analysis as appropriate.

Results: 38 RRMS patients were included in the analysis: [65,2\% female, mean age $37,8 \pm 10.1$ years mean disease duration $10,1 \pm 9.2$ years, median Expanded-Disability-Status-Scale (EDSS) $2(0-6,5)]$. $78,3 \%$ of patients had MRI activity in the previous 2 years: among them $17,4 \%$ had uniquely radiological activity and $60,9 \%$ had clinical relapses (experiencing disease progression in 17,4\% of cases), $26.1 \%$ had ongoing MRI activity. LME was found in $37 \%$ of patients, median number 1 (range 1-3). No difference in EDSS, age and disease duration were found between patients with or without nodules. LME prevalence was higher in patients with previous MRI activity $(\mathrm{P}=0.047)$. Multivariable models adjusted for baseline EDSS exploring predictive value of clinical progression, previous MRI activity, ongoing MRI activity show that previous MRI activity was the only variable associated with LME $(\mathrm{p}=0.002)$. CSF parameters had no predictive value for LME development nor any association was found between presence of oligoclonal bands and LME.

Discussion: Our preliminary data suggest that LME is associated with previous disease activity but not with ongoing MRI activity. Therefore, this aspect of the process of autoimmunity compartmentalization may start early in the disease course, its extent may be related to the amount of precedent inflammatory activity and it would persist even in absence of MRI disease activity.

Conclusions: LME was found in a discrete proportion of RRMS patients and it was associated with previous radiological activity. A prospective clinical evaluation is needed in order to assess the prognostic value of our findings.

References:

- Zurawski J., Lassmann H. \& Bakshi R. Use of Magnetic Resonance Imaging to Visualize Leptomeningeal Inflammation in Patients with Multiple Sclerosis. JAMA Neurol. (2017); 74(1):100-109

- Ighani M. et al. No association between cortical lesions and leptomeningeal enhancement on 7-Tesla MRI in multiple sclerosis. Mult. Scler. J. (2020);26:165-176

- Absinta M. et al. Gadolinium-based MRI characterization of leptomeningeal inflammation in multiple sclerosis. Neurology (2015);85:18-28

\section{MR T2-RELAXATION TIME AS AN INDIRECT MEASURE OF BRAIN WATER ACCUMULATION IN NEUROMYELITIS OPTICA SPECTRUM DISORDERS}

\author{
L. Cacciaguerra ${ }^{1}$, M. Rocca ${ }^{2}$, E. Pagani ${ }^{1}$, M. Radaelli ${ }^{3}$, S. Mesaros ${ }^{4}$, V. \\ Martinelli $^{3}$, J. Ivanović ${ }^{4}$, M. Filippi ${ }^{5}$
}

${ }^{1}$ Neuroimaging Research Unit, Institute of Experimental Neurology, Division of Neuroscience, IRCCS San Raffaele Scientific Institute (Milano); ${ }^{2}$ Neuroimaging Research Unit, Institute of Experimental Neurology, Division of Neuroscience; and Neurology Unit, IRCCS San Raffaele Scientific Institute (Milano); ${ }^{3}$ Neurology Unit, IRCCS San Raffaele Scientific Institute (Milano); ${ }^{4}$ Clinic of Neurology, Faculty of Medicine, University of Belgrade (Belgrade-SRB); ${ }^{5}$ Neuroimaging Research Unit, Institute of Experimental Neurology, Division of Neuroscience; Neurology Unit and Neurophysiology Unit, IRCCS San Raffaele Scientific Institute; Vita-Salute San Raffaele University (Milano)

Objectives: To provide an indirect estimation of brain water content in neuromyelitis optica spectrum disorders (NMOSD) by measuring T2relaxation time (T2rt) and to assess whether it differs in patients having a short-term relapse.

Materials: In this multicenter study, brain dual echo turbo spin echo MR images, were obtained from 77 AQP4-positive NMOSD and 84 healthy controls (HC).

Methods: T2rt was calculated from brain dual echo turbo spin echo images assuming a mono exponential decay. T2rt maps of normal appearing white matter (NAWM), gray matter (GM) and basal ganglia were obtained from. Short-term relapses were defined as those occurring within one month before or after MRI scan. Differences between NMOSD and HC were assessed with age-, sex- and site-adjusted linear models. ROC analyses were run to identify discriminators between stable and short-term relapsing patients.

Results: NMOSD patients and $\mathrm{HC}$ had similar ages. Compared to HC, T2rt was increased in the GM (103 vs $97 \mathrm{~ms}$ ), NAWM ( $88 \mathrm{vs} 84 \mathrm{~ms}$ ) and putamen ( $75 \mathrm{vs} 72 \mathrm{~ms}$ ) of NMOSD patients ( $<0.001$ for all). Short-term relapses occurred in 20/77 (26\%) of patients. According to ROC analysis, T2rt cutoffs of $87 \mathrm{~ms}$ in the NAWM, $87 \mathrm{~ms}$ in the thalamus and $88 \mathrm{~ms}$ in the caudatus were able to discriminate between short-term relapsing and stable patients with good accuracy ( $\mathrm{AUC}=0.70,0.76$ and 0.79 respectively, $\mathrm{p} \leq 0.027$ ).

Discussion: One of the main unsolved issues in the clinical management of NMOSD is the lack of biomarkers predicting short-term relapses. In physiological conditions, the blood brain barrier (BBB) protects the CNS from water unbalance, with aquaporin-4 (AQP4) water channels on astrocytes podocytes being the main regulator of water influx and efflux. In NMOSD, BBB integrity might be threatened by the presence of antibodies targeting AQP4 water channels and triggering complementmediated astrocytes damage.

Conclusions: NMOSD patients had increased T2rt values, in line with the hypothesis of subclinical water accumulation in this disorder. The burden of T2rt alterations might be useful for identifying those patients with incipient or recent relapses.

\section{IN VIVO IMAGING OF MICROGLIA ACTIVATION IN CEREBRAL AMYLOID ANGIOPATHY: A MULTIMODAL NEUROIMAGING STUDY}

\author{
S. P. Caminiti ${ }^{1}$, F. Piazza ${ }^{2}$, L. Presotto ${ }^{3}$, J. Di Francesco ${ }^{4}$, M. Zedde ${ }^{5}$, A.

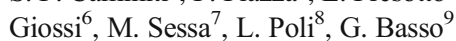 \\ ${ }^{1}$ In Vivo Human Molecular and Structural Neuroimaging Unit, Division \\ of Neuroscience, IRCCS San Raffaele Scientific Institute (Milano); \\ ${ }^{2} \mathrm{CAA}$ and $\mathrm{AD}$ Translational research and Biomarkers Laboratory, \\ School of Medicine and Surgery, University of Milano-Bicocca \\ (Monza); ${ }^{3}$ Nuclear Medicine Unit, San Raffaele Hospital (Milano); \\ ${ }^{4}$ Department of Neurology, San Gerardo Hospital, and University of
}


Milano-Bicocca (Monza); ${ }^{5}$ Neurology Unit, Stroke Unit, Arcispedale Santa Maria Nuova - IRCCS (Reggio Emilia); ${ }^{6}$ Neurology Unit, Azienda Socio-Sanitaria Territoriale di Cremona (Cremona); ${ }^{7}$ Neurology Unit, Ospedale Papa Giovanni XXIII (Bergamo); ${ }^{8}$ Neurology Unit, Department of Neurological and Vision Sciences, ASST Spedali Civili (Brescia); ${ }^{9}$ School of Medicine and Surgery, University of Milano-Bicocca (Milano)

Objectives: Cerebral Amyloid Angiopathy-related inflammation (CAAri) is an autoimmune encephalopathy presumably targeting cerebrovascular deposited $\beta$-amyloid in cortical and leptomeningeal arteries, arterioles, and capillaries $\beta$-amyloid accumulations within the brain. In the current study, we aim to describe, for the first time in vivo, TSPO activity/neuroinflammatory responses in a group of CAA-ri patients, also considering patients' disease course. TSPO is a mitochondrial protein which is overexpressed by activated microglia and is considered a biomarker of neuroinflammation.

Materials: With a multimodal PET/MRI neuroimaging approach, we studied a case series of five patients with CAA-ri diagnosis supported by clinical, radiological, and CSF assessments [1,2].

Methods: We investigated in vivo TSPO activity with 11C-PK11195PET ( 3 patients out of five were acquired twice during the disease course, both at disease presentation and after corticosteroids therapy at clinical remission). All patients underwent 18F-FDG-PET, to assess brain metabolism and an extensive MRI protocol, including T1, T2*/SWI, DWI, to assess white matter lesions and hemorrhagic lesions, including microbleeds, cortical superficial siderosis and intracerebral hemorrhage.

Results: The patterns of brain hypometabolism showed different topography in each of the presented cases. None of them showed an Alzheimer's disease-related hypometabolism pattern or suggestive for other neurodegenerative condition. 11C-PK11195-PET single subject analysis showed several clusters of significant microglia activation, mostly located in the occipital lobe. 11C-PK11195-PET binding potential (BP) peak-signals co-localized with MRI evidences of acute hemorrhagic/inflammatory lesions on both FLAIR and DWI. The subjects studied twice showed decreased 11C-PK11195-PET BP after therapy in the remission phase.

Discussion: Although the present study includes only few cases, this is the first assessment in vivo of the presence of activated microglia in CAA-ri. The results show some variability in the amount of microglia activation among the patients, mostly related to the presence of acute lesions (macro and microhemorragic) in each case. Of note, we did not find any massive neuroinflammatory responses in the brain areas showing siderosis or chronic white matter lesions. This might suggest that in the acute inflammatory phase of CAA-ri the role of microglia is more related to a reactive response to the acute cerebrovascular damage, likely with reparatory and phagocytic activity.

Conclusions: Future studies are needed in order to better clarify the nature of neuroinflammatory responses associated with CAA-ri, also in relation to the disease course and therapy.

References:

1. A. Eitan, A Charidimou, M. E Gurol, J Ni, ES. Van Etten, S Martinez-Ramirez, G Boulouis et al. Validation of clinicoradiological criteria for the diagnosis of cerebral amyloid angiopathy-related inflammation." JAMA Neurology (2016);73(2):197-202

2. Piazza F, SM. Greenberg, M Savoiardo, M Gardinetti, L Chiapparini, I Raicher, R Nitrini et al. Anti-amyloid $\beta$ autoantibodies in cerebral amyloid angiopathy-related inflammation: Implications for amyloid-modifying therapies. Annals of Neurology (2013); 73(4):449-58

\section{INTERHEMISPHERIC FUNCTIONAL CONNECTIVITY CORRELATES OF UPPER-LIMB MOTOR PERFORMANCE IN MULTIPLE SCLEROSIS PATIENTS WITH DIFFERENT CLINICAL PHENOTYPES}

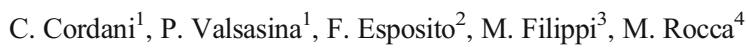

${ }^{1}$ Neuroimaging Research Unit, Institute of Experimental Neurology, Division of Neuroscience, IRCCS San Raffaele Scientific Institute (Milano); ${ }^{2}$ Neurology Unit, IRCCS San Raffaele Scientific Institute (Milano); ${ }^{3}$ Neuroimaging Research Unit, Institute of Experimental Neurology, Division of Neuroscience; Neurology Unit and Neurophysiology Unit, IRCCS San Raffaele Scientific Institute; VitaSalute San Raffaele University (Milano); ${ }^{4}$ Neuroimaging Research Unit, Institute of Experimental Neurology, Division of Neuroscience; and Neurology Unit, IRCCS San Raffaele Scientific Institute; VitaSalute San Raffaele University (Milano)

Aims: Using voxel-mirrored homotopic connectivity (VMHC), we investigated in a large cohort of multiple sclerosis (MS) patients: i) abnormalities of inter-hemispheric resting state (RS) functional connectivity (FC) according to MS clinical phenotype; ii) correlations between altered VMHC and measures of manual dexterity, as well as global clinical disability.

Materials: One-hundred and twenty-one right-handed healthy controls (HC) and $340 \mathrm{MS}$ patients (234 relapsing-remitting [RR] and 106 progressive $[\mathrm{P}] \mathrm{MS}$ ) underwent a manual-dexterity assessment, using NineHole-Peg Test (9HPT) and finger-tapping (FT). In MS, clinical disability was rated with Expanded Disability Status Scale (EDSS) score. 3T brain 3D T1-weighted and RS fMRI scans were also acquired.

Methods: VMHC analysis was performed on RS MRI scans to produce maps of homotopic RS FC in all study subjects. SPM and voxelwise general linear models were used to investigate between-group differences of VMHC and correlations with clinical measures.

Results: Compared to HC, RRMS patients showed significantly increased VMHC in occipital, thalamic, hippocampal, superior temporal and insular regions. Conversely, decreased VMHC was observed in cerebellar, middle temporal, superior and middle frontal regions, as well as in the precunei, cingulate cortices and supplementary-motor areas (SMAs). A similar VMHC increase was observed in PMS vs HC while VMHC reductions were detected in frontal, sensorimotor, parietal and cerebellar regions. These reductions were significant also between PMS and RRMS. In RRMS patients, worse motor scores correlated with increased VMHC in thalamic and insular regions, and with decreased VMHC in SMAs, cingulate and frontal cortices, and small clusters in cerebellar lobules (rrange $=-0.35 / 0.25$, p-range $<0.001 / 0.05)$. EDSS was associated with increased thalamic $(\mathrm{r}=0.30, \mathrm{p}<0.001)$ and decreased cerebellar VMHC $(\mathrm{r}=-$ $0.15, \mathrm{p}=0.02$ ). In PMS, worse left-9HPT and right-FT scores correlated with a widespread decrease of VMHC in parietal and cerebellar regions (r-range $=0.19 / 0.22$, p-range $=<0.01 / 0.05)$. Conversely, a higher EDSS score was correlated with increased VMHC in thalamic $(r=0.19$, $\mathrm{p}=0.05)$ and inferior frontal $(\mathrm{r}=0.21, \mathrm{p}=0.03)$ regions.

Discussion: While increased interhemispheric RS FC in thalamic regions contributed to explain global clinical disability in both RRMS and PMS, upper-limb motor dysfunction was associated with a complex pattern of increased and decreased VMHC in different regions, with a major involvement of the cerebellum in patients with PMS.

Conclusions: Distinct VMHC abnormalities characterized different MS clinical phenotypes and contributed to explain upper-limb motor deficits and global clinical disability. A closer relationship between altered VMHC and upper-limb motor impairment was found in RRMS than in PMS. 


\section{ATROPHY OF DIFFERENT CORTICAL AND SUBCORTICAL COMPARTMENTS CONTRIBUTES TO EXPLAIN CLINICAL DISABILITY IN PATIENTS WITH MS: A MULTICENTER STUDY}

\author{
M. Hidalgo de la Cruz ${ }^{1}$, M. Rocca ${ }^{2}$, P. Valsasina ${ }^{1}$, C. Gobbi ${ }^{3}$, A. Gallo ${ }^{4}$, \\ C. Zecca $^{3}$, A. Bisecco ${ }^{4}$, M. Filippi ${ }^{5}$
}

${ }^{1}$ Neuroimaging Research Unit, Institute of Experimental Neurology, Division of Neuroscience, San Raffaele Scientific Institute, Vita-Salute San Raffaele University (Milano); ${ }^{2}$ Neuroimaging Research Unit, Institute of Experimental Neurology, Division of Neuroscience; Neurology Unit, IRCCS San Raffaele Scientific Institute (Milano); ${ }^{3}$ Multiple Sclerosis Center, Department of Neurology, Neurocenter of Southern Switzerland, Civic Hospital (Lugano-CH); ${ }^{4}$ Department of Advanced Medical and Surgical Sciences, and 3T MRI Center, University of Campania "Luigi Vanvitelli" (Napoli); ${ }^{5}$ Neuroimaging Research Unit, Institute of Experimental Neurology, Division of Neuroscience; Neurology Unit and Neurophysiology Unit, IRCCS San Raffaele Scientific Institute; Vita-Salute San Raffaele University (Milano)

Objectives: To perform a multiparametric assessment of cortical, deep grey matter (DGM), cerebellar and cervical cord atrophy to characterize multiple sclerosis (MS) phenotypes and to explain patients' disability.

Materials: 3T brain and cervical cord T2- and 3D T1-weigthed images were acquired from $198 \mathrm{MS}$ patients (139 relapsing-remitting [RR] MS, 59 progressive $[\mathrm{P}] \mathrm{MS}$ ) and 67 healthy controls $(\mathrm{HC})$ at three European sites.

Methods: Cortical thickness (CTh), DGM volumes, cerebellar volumes and cervical cord cross-sectional area (CSA) were compared between MS patients and $\mathrm{HC}$ and across clinical phenotypes. In patients, sex-, age-, and site-corrected stepwise linear regression models investigated the association of brain and cord lesion burden and cortical, DGM, cerebellar and cervical cord atrophy with clinical disability.

Results: Compared to HC, MS patients had widespread atrophy in all cortical lobes, DGM nuclei and cerebellar lobules, as well as reduced cord CSA. Similar results were observed in RRMS patients vs HC, except for the left superior parietal lobule and left frontal pole $(\mathrm{p}=$ range from $<0.001$ to 0.04 ). In PMS patients, additional cortical atrophy vs RRMS was identified in all investigated lobes ( $\mathrm{p}=$ range from $<0.001$ to 0.03 ), except for selected cingulate, parietal and occipital regions. At the univariate analysis, in MS patients higher disability was associated with more severe cortical, DGM, cerebellar and cervical cord atrophy $(\mathrm{p}=$ range $<0.00$ 0.047 ). The multivariate model retained cerebellar and cervical cord atrophy as significant predictors of higher EDSS score $(\mathrm{R} 2=0.45, \mathrm{p}<0.001)$ as well as of pyramidal ( $R 2=0.42, p<0.001)$, sensory $(R 2=0.28, p<0.001)$ and cerebellar $(\mathrm{R} 2=0.50, \mathrm{p}<0.001)$ functional system scores.

Discussion: In this study, we used a multiparametric approach to identify atrophy in cortical (mainly in sensorimotor, attention and executive cortices), subcortical, cerebellar, and cervical spinal cord regions which identified the main MS phenotypes. Atrophy within all cortical, subcortical, cerebellar and cervical cord regions correlated with more severe clinical disability in MS. However, atrophy within the cerebellum and the cervical spinal cord were independently and relevantly associated with more severe clinical disability, mainly in the pyramidal, sensory, and cerebellar domains. Thus, it seems reasonable to hypothesize that MSrelated neurodegenerative damage within the cerebellum and the cervical spinal cord play a crucial role in generating more severe sensorimotor clinical disability.

Conclusions: Abnormalities of regional CTh, DGM volume, volume of the cerebellar lobules and cervical cord CSA characterized the main
MS clinical phenotypes. In addition, atrophy within the cerebellum and cervical cord was crucial for explaining clinical disability, mainly within sensorimotor domains.

\section{RESTING STATE FUNCTIONAL CONNECTIVITY CORRELATES OF EXECUTIVE FUNCTION IN PATIENTS WITH MULTIPLE SCLEROSIS}

O. Marchesi ${ }^{1}$, P. Valsasina ${ }^{1}$, L. Conti ${ }^{1}$, D. Mistri ${ }^{1}$, P. Preziosa ${ }^{2}$, M. Filippi $^{3}$, M. Rocca ${ }^{2}$

${ }^{1}$ Neuroimaging Research Unit, Institute of Experimental Neurology, Division of Neuroscience, IRCCS San Raffaele Scientific Institute (Milano); ${ }^{2}$ Neuroimaging Research Unit, Institute of Experimental Neurology, Division of Neuroscience; and Neurology Unit - IRCCS San Raffaele Scientific Institute (Milano); ${ }^{3}$ Neuroimaging Research Unit, Institute of Experimental Neurology, Division of Neuroscience; Neurology Unit and Neurophysiology Unit, IRCCS San Raffaele Scientific Institute; Vita-Salute San Raffaele University (Milano)

Objectives: Aim of this study was to investigate changes of resting state (RS) functional connectivity (FC) in patients with multiple sclerosis (MS) and their correlation with neuropsychological measures related to executive function (EF).

Materials: High-resolution T1-weighted and RS functional MRI (fMRI) scans were acquired at 3.0 Tesla from 116 right-handed MS patients and 65 matched healthy controls (HC). All subjects underwent a neuropsychological evaluation, including the computerized version of the Wisconsin Card Sorting Test (WCST), a multidimensional EF assessment. MS patients also underwent a clinical evaluation, with rating of clinical disability using the expanded disability status scale (EDSS) score.

Methods: RS FC was assessed using a seed-voxel correlation analysis. Seed regions relevant for EF were derived from previous literature and included the left inferior parietal sulcus (IPS), the left frontal pole (FP) and the right cerebellum (Crus I and II). SPM and voxel-wise models were used to compare RS FC between MS patients and HC within the identified networks. Then, associations between RS FC and age- and education-corrected WCST scores and EDSS were evaluated.

Results: Twenty-five (21.5\%) MS patients failed at the WCST. Compared to HC, MS patients showed significantly decreased RS FC of the left IPS with bilateral middle frontal, left middle temporal and left cerebellar regions, as well as increased RS FC of the left IPS with bilateral thalami. MS patients also exhibited decreased RS FC between the left FP and superior parietal regions. Finally, a widespread RS FC decrease was found in MS vs HC between the right Crus I/II and bilateral cerebellar regions and fronto-parietal cortices. Significantly increased RS FC was also detected between the right Crus I/II and the bilateral orbitofrontal cortex. In MS patients, significantly increased RS FC between the right Crus I/II regions and the orbitofrontal cortex was associated with a better performance at the WCST ( $r=$ range 0.19 to 0.27 , $\mathrm{p}=$ range $0.03-0.003$ ). Conversely, decreased fronto-cerebellar and parieto-cerebellar RS FC was correlated with a higher EDSS score ( $r=$ range -0.19 to -0.35 , $\mathrm{p}=$ range $0.03-<0.001)$.

Discussion: In a MS group relatively spared by clinically relevant EF deficits, increased strength of functional connections in brain networks known to be related to EF was detected. The association between increased RS FC and better WCST scores suggests a compensatory role of detected RS FC abnormalities.

Conclusion: In MS, abnormally high RS FC between cerebellar and orbitofrontal regions may contribute to preserve $\mathrm{EF}$. 


\section{A METABOLIC IMAGING SIGNATURE OF BEHAVIOURAL FRONTOTEMPORAL DEMENTIA RESPECT TO PSYCHIATRIC PHENOCOPY SYNDROME}

\author{
S. Mozzetta ${ }^{1}$, I. Pettinuzzo ${ }^{1}$, G. Zorzi ${ }^{1}$, C. Campi ${ }^{2}$, S. Pompanin ${ }^{1}$, F. \\ Fragiacomo $^{1}$, G. Gazzola ${ }^{1}$, G. Pigato ${ }^{1}$, D. Cecchin ${ }^{3}$, A. Cagnin ${ }^{1}$ \\ ${ }^{1}$ Department of Neurosciences, University of Padua (Padova); \\ ${ }^{2}$ Department of Mathematics, University of Padua (Padova); \\ ${ }^{3}$ Department of Nuclear Medicine, University of Padua (Padova)
}

Patients with primary psychiatric disorders may present with a frontallobe syndrome meeting the diagnostic criteria of possible behavioural variant frontotemporal dementia (bvFTD) but without clear neuroimaging (MRI/FDG-PET) alterations, a condition named phenocopy syndrome of bvFTD (bvFTD-PS). A few studies pointed out that 20-30\% of psychiatric bvFTD-PS patients could have an abnormal FDG-PET imaging with hypometabolism in frontal and/or temporal regions.

Aim: We aimed at finding the spatial pattern of glucose hypometabolism specifically related to neurodegenerative bvFTD respect to late-onset psychiatric conditions with frontal lobe syndrome.

Methods: Thirty-seven patients with probable/definite bvFTD (mean age $63+/-8.7$ years) and 20 patients with psychiatric diseases (mean age $63+/-7.9$ years) meeting the current criteria for bvFTDPS were evaluated with an extensive cognitive and behavioural scales and 18-FDG-PET/ MRI brain scan. 3T-T1-MPRAGE MRI and a PET-FDG data were coacquired for all subjects. T1-sequences were processed using Freesurfer standard pipeline, and the cortical surface/subcortical regions were divided in more than 100 ROIs. Output of this segmentation was applied to PET images and standardized uptake value (SUV) for each ROI, normalized with the brainstem, was extracted. Data driven analysis of FDGSUVr values was conducted using Principal Component Analysis (PCA).

Results: The two groups were comparable as for disease duration, age at onset, impairment of executive function and total burden of behavioural disorders. The mean MMSE score was lower in bvFTD than in psychiatric diseases $(22.3$ vs $25 / 30, p=0.05)$. On qualitative FDG-PET study, $9 / 20$ psychiatric patients have abnormal images with two patterns: anterior cingulate and superior parietal hypometabolism. PCA revealed a cluster of regions explaining the $45 \%$ of variance. The regions contributing more to this component, and being more hypometabolic in the bvFTD group, belong to the bilateral ventrolateral prefrontal cortex (caudal and rostral middle frontal, pars opercularis and triangularis), orbitofrontal cortex (lateral orbital), and caudate. The SUVr values of these regions were able to cluster the two groups with a few patients misclassified: 11/37 bvFTD and 4/20 bvFTD-PS. Among the 11 misclassified bvFTD, 4 have c9orf expansion and 2 have bvFTD plus MND, while 2 have temporal pole hypometabolism.

Discussion and conclusions: Data driven PCA analysis revealed a spatial pattern of cortical and subcortical regions useful in differentiating patients with neurodegenerative bvFTD from patients with psychiatric conditions mimicking bvFTD. Gene mutations or association with MND may drive a different distribution of glucose hypometabolism. A few psychiatric patients showed a intriguing superior parietal hypometabolism.

\section{CORTICAL ABNORMALITIES IN PEDIATRIC PATIENTS WITH MIGRAINE WITHOUT AURA: ANALYSIS OF THE MORPHOLOGY OF THE GIRIFICATION AND CORTICAL THICKNESS}

L. Papetti ${ }^{1}$, A. Guarnera ${ }^{2}$, F. Bottino ${ }^{2}$, A. Napolitano ${ }^{2}$, D. Longo ${ }^{2}$, M. Valeriani ${ }^{1}$
${ }^{1}$ Neuroscience, Bambino Gesù Children Hospital (Roma); ${ }^{2}$ Neuroradiology, Bambino Gesù Children Hospital (Roma);

Objective: Verify the presence of anomalies of the morphology of the gyration in pediatric patients with migraine without aura and identify the clinical-radiological correlations.

Materials and Methods: A search was made in the Picture Archiving Communications Systems (PACS) using the terms "headache" and "migraine" between 01/01/2018 and 31/01/2020. A neurologist examined the patients' medical records (Pcs) to confirm the diagnosis of migraine without aura and to exclude psycho-physical comorbidities. A radiologist assessed MRI tests to rule out the presence of accidental findings, gross morphological and signal abnormalities. The estimate of the cortical thickness and the morphology of the gyration was carried out on the 3D T1 MPRAGE sequence without contrast medium of $48 \mathrm{Pcs}$ and 26 controls. The statistical permutational analysis for linear models (1000 permutations) was carried out to assess the significance of the results obtained.

Results: Statistically significant difference in cortical thickness between migraine patients and bilaterally healthy controls was demonstrated at the level of the convolutions: upper frontal; pre and post center; girdle; precuneus; wedge; calcarine fissure and superior parietal lobule ( $\mathrm{p}>$ 0.001); in the right half-brain at the level of the convolutions: upper, middle and lower front; upper and middle thunderstorm; supramarginal and insula $(\mathrm{p}<0.05)$.

Discussion and Conclusion: A difference in the cortical thickness of migraine patients has been demonstrated at the level of cortical areas involved in the networks of nociception and pain processing, and executive functions. Since the thickness of the cortex is a variable parameter over time and related to the pathology, the demonstration of anomalies in pediatric patients confirms the need for early diagnosis and personalized treatment. Reference:

- Schwedt TJ, Berisha V, Chong CD. Temporal lobe cortical thickness correlations differentiate the migraine brain from the healthy brain. PLoS One (2015) Feb 13;10(2):e0116687. doi: 10.1371/ journal.pone.0116687.

\section{UNRAVELING DEEP GRAY MATTER ATROPHY, IRON AND MYELIN CHANGES IN MULTIPLE SCLEROSIS}

M. Petracca ${ }^{1}$, P. Pontillo ${ }^{2}$, S. Monti ${ }^{3}$, M. Quarantelli ${ }^{3}$, C. Criscuolo ${ }^{1}$, R. Lanzillo $^{1}$, E. Tedeschi ${ }^{4}$, A. Elefante ${ }^{4}$, V. Brescia Morra ${ }^{1}$, S. Cocozza ${ }^{4}$, G. Palma $^{3}$, A. Brunetti ${ }^{4}$

${ }^{1}$ Department of Neurosciences and Reproductive and Odontostomatological Sciences, University of Naples Federico II (Napoli); ${ }^{2}$ Department of Advanced Biomedical Sciences, University of Naples Federico II (Napoli); ${ }^{3}$ Institute of Biostructure and Bioimaging, National Research Council (Napoli); ${ }^{4}$ Department of Advanced Biomedical Sciences, University of Naples Federico II (Napoli)

Objective: To disentangle the contribution of atrophy, iron and myelin changes to deep GM (DGM) damage in MS, simultaneously exploring their relationship with clinical disability through the application of quantitative susceptibility mapping (QSM) and longitudinal relaxation rate (R1) relaxometry.

Material and Methods: In this cross-sectional study, 91 patients and 55 healthy controls were imaged with 3T MRI to compute QSMs and R1 maps, from which iron and myelin concentration maps were estimated by applying an external model. Modifications of DGM iron and myelin (mean concentration-dependent from atrophy and total contentindependent from atrophy) were investigated at both global and regional 
levels. Significantly altered MRI features were tested as disability predictors in hierarchical linear regression models.

Results: Compared to controls, MS patients showed reduced thalamic $(p<0.001)$ and increased pallidal $(p<0.001)$ iron concentrations. No differences emerged regarding total myelin or iron content in the basal ganglia, while actual iron depletion was found in the thalamus $(\mathrm{p}<0.001)$. At the voxel-based analysis, patients showed increased iron concentration in the basal ganglia $(p \leq 0.001)$ and reduced iron and myelin local content in thalamic posteromedial regions $(\mathrm{p} \leq 0.004)$, corresponding to reduced iron and myelin content in the pulvinar $(\mathrm{p} \leq 0.001)$ at the subnuclei analysis. Thalamic volume $(B=-0.341, p=0.02)$, iron concentration $(B=-$ $0.379, \mathrm{p}=0.005)$ and content $(\mathrm{B}=-0.406, \mathrm{p}=0.009)$ significantly predicted disability, as well as pulvinar iron $(\mathrm{B}=-0.415, \mathrm{p}=0.003)$ and myelin $(\mathrm{B}=-$ $0.406, \mathrm{p}=0.02$ ) content, independently of atrophy.

Discussion and Conclusion: Quantitative MRI suggests an atrophyrelated iron increase within the basal ganglia of MS patients, along with an absolute reduction of thalamic iron and myelin, which correlates with disability. Atrophy-independent depletions of thalamic iron and myelin may represent clinically relevant, sensitive markers of subcortical GM damage.

\section{NEURITE DENSITY EXPLAINS CORTICAL T1-/T2-WEIGHTED RATIO IN MULTIPLE SCLEROSIS}

P. Preziosa ${ }^{1}$, M. Rocca ${ }^{2}$, P. Bouman ${ }^{3}$, S. Kiljan ${ }^{3}$, M. Steenwijk ${ }^{3}$, P. Pouwels $^{4}$, J. Geurts ${ }^{3}$, L. Jonkman ${ }^{3}$, M. Filippi ${ }^{5}$

${ }^{1}$ Neuroimaging Research Unit, Institute of Experimental Neurology, Division of Neuroscience; IRCCS San Raffaele Scientific Institute; Vita-Salute San Raffaele University (Milano); ${ }^{2}$ Neuroimaging Research Unit, Institute of Experimental Neurology, Division of Neuroscience; and Neurology Unit, IRCCS San Raffaele Scientific Institute (Milano); ${ }^{3}$ Department of Anatomy \& Neurosciences, Amsterdam UMC, Location VU University Medical Center (Amsterdam-NL); ${ }^{5}$ Neuroimaging Research Unit, Institute of Experimental Neurology, Division of Neuroscience; Neurology Unit and Neurophysiology Unit, IRCCS San Raffaele Scientific Institute; Vita-Salute San Raffaele University (Milano)

Objectives: Cortical damage is clinically relevant in multiple sclerosis (MS), however reliable MRI markers for its monitoring are still an unmet need. Ratio of T1-weighted (T1w) and T2-weighted (T2w) sequences (i.e., T1w/T2w-ratio) has been suggested as a feasible MRI measure to assess cortical abnormalities in patients with MS (PwMS), but its histopathological substrate has yet to be definitively elucidated. We aimed to define the histopathological substrate of $\mathrm{T} 1 \mathrm{w} / \mathrm{T} 2 \mathrm{w}$-ratio in normalappearing and demyelinated cortices of PwMS by performing a combined post-mortem MRI and histopathology study.

Materials: Fifteen PwMS and ten age- and sex-matched non-neurological controls (nNC) underwent post-mortem in situ 3T MRI with 3D $\mathrm{T} 1 \mathrm{w}$ and $\mathrm{T} 2 \mathrm{w}$ sequences, followed by brain dissection.

Methods: One hundred and five paraffin embedded tissue blocks (49 from PwMS, 56 from nNC) were collected from 6 standardized cortical regions. Tissue regions were matched to $\mathrm{T} 1 \mathrm{w} / \mathrm{T} 2 \mathrm{w}$-ratio maps to obtain regional cortical $\mathrm{T} 1 \mathrm{w} / \mathrm{T} 2 \mathrm{w}$-ratio. Using immunohistochemistry and silver staining, cortical density of myelin, microglia, astrocytes and neurites, and density and volume of neurons and glial cells were evaluated. Correlates of $\mathrm{T} 1 \mathrm{w} / \mathrm{T} 2 \mathrm{w}$-ratio alterations with histological markers were assessed through linear mixed-effects models.
Results: Twenty-six cortical lesions (85\% subpial) were found in 24/ $49(51 \%)$ cortical regions from PwMS and none in nNC. Compared to nNC's cortex, both PwMS' normal-appearing and demyelinated cortices had a significantly lower T1w/T2w-ratio ( $\mathrm{p}=0.045$ and 0.001 , respectively). In PwMS, demyelinated cortex showed a significant lower T1w/T2wratio compared to normal-appearing cortex ( $\mathrm{p}=0.007)$. In PwMS, neurite density was significantly lower in both normal-appearing and demyelinated cortices compared to $\mathrm{nNC}(\mathrm{p}=0.041$ and 0.001 , respective$1 \mathrm{y})$, and in demyelinated vs. normal-appearing cortex $(\mathrm{p}=0.048)$. Demyelinated cortex showed also significant lower myelin density compared to normal-appearing cortex in both $\mathrm{nNC}$ and PwMS $(\mathrm{p}<0.001$, for both). Regarding the pathological substrate, $\mathrm{T} 1 \mathrm{w} / \mathrm{T} 2 \mathrm{w}$-ratio was positively associated with neurite density $(\beta=3.464 \times 10-2, p=0.004)$, whereas only a trend for myelin density was found $(\beta=0.198 \times 10-2, \mathrm{p}=0.082)$.

Discussion: Both demyelination and neurite loss were found in the cortex of PwMS. By evaluating several histopathological markers in $\mathrm{nNC}$ and PwMS (in normal-appearing and demyelinated cortices), T1w/T2wratio was found to be sensitive to MS cortical damage and more specific to neurite than myelin density.

Conclusions: T1w/T2w-ratio could be useful to investigate cortical damage in MS.

\section{RELEVANCE OF NODDI TO CHARACTERISE IN VIVO THE MICROSTRUCTURAL ABNORMALITIES OF MULTIPLE SCLEROSIS CORTEX AND CORTICAL LESIONS: A 3T STUDY}

\author{
P. Preziosa ${ }^{1}$, E. Pagani ${ }^{1}$, R. Bonacchi ${ }^{2}$, L. Cacciaguerra ${ }^{2}$, M. Filippi ${ }^{3}$, M. \\ Rocca $^{2}$
}

${ }^{1}$ Neuroimaging Research Unit, Institute of Experimental Neurology, Division of Neuroscience, IRCCS San Raffaele Scientific Institute (Milano); ${ }^{2}$ Neuroimaging Research Unit, Institute of Experimental Neurology, Division of Neuroscience; and Neurology Unit, IRCCS San Raffaele Scientific Institute; Vita-Salute San Raffaele University (Milano); ${ }^{3}$ Neuroimaging Research Unit, Institute of Experimental Neurology, Division of Neuroscience; Neurology Unit and Neurophysiology Unit, IRCCS San Raffaele Scientific Institute; VitaSalute San Raffaele University (Milano)

Objectives: In multiple sclerosis (MS), cortical damage is a relevant predictor of clinical disability, but MRI measures more specific to the different cortical pathological processes are needed. Neurite orientation dispersion and density imaging (NODDI) model is a multi-compartment diffusion model to better evaluate the complexity of brain microarchitecture. Using NODDI, we characterized the microstructural abnormalities of normal-appearing cortex (NA-cortex) and cortical lesions (CLs) and their relations with disease phenotypes and clinical disability in a relatively large cohort of MS patients.

Materials: Brain 3D T1-weighted, FLAIR, double inversion recovery (DIR) and diffusion-weighted (DW) sequences were acquired from 164 MS patients (94 relapsing-remitting [RR], 70 progressive [P] MS) and 51 age- and sex-matched healthy controls (HC).

Methods: We segmented the cortex from 3D T1-weighted sequence and CLs were quantified on DIR. CLs and NA-cortex masks were transformed into DW space. CLs were then expanded to include the entire cortical portion affected by demyelination. Using NODDI model, intracellular volume fraction (ICV_f), representing neurite density, extracellular volume fraction (ECV f) and orientation dispersion index (ODI), reflecting neurite orientation variability, were assessed in NA-cortex and CLs. Between-group comparisons were performed using linear models. 
Correlations with clinical and structural MRI measures were also assessed.

Results: 112 (68.3\%) MS patients had $\geq 1$ CL. MS NA-cortex had a significant lower ICV f vs HC NA-cortex $(p=0.001)$. CLs showed a significant increased ECV f $(p<0.001)$ and decreased ICV $f$ and ODI compared to NA-cortex of both $\mathrm{HC}(\mathrm{p}<0.001)$ and MS patients ( $\mathrm{p}=0.035$ and $<0.001$ ). Compared to RRMS, PMS had a significant decreased NA-cortex ICV $\mathrm{f}(\mathrm{p}=0.024)$. Higher number and volume of CLs $(p<0.001)$ were found in PMS vs RRMS, without microstructural differences. In MS patients, NA-cortex ICV_f, ECV f and ODI were significantly correlated with disease duration, EDSS, white matter lesion volumes, CL volumes and whole brain and gray matter atrophy ( $\mathrm{r}$ from -0.37 to $0.71, \mathrm{p}$ from $<0.001$ to 0.048 ).

Discussion: A significant neurite loss occurs in MS NA-cortex, being more severe in patients with longer disease duration, higher disability and progressive phenotypes. CLs show a further reduction of neurite density, together with an increased extracellular space, possibly related to inflammation and gliosis, and a reduced ODI suggestive of increased tissue coherence and simplification of dendritic arborisation and neurite complexity.

Conclusions: NODDI is a reliable and clinically relevant method to investigate in vivo the heterogeneous pathological processes affecting MS cortex.

This study is supported by a senior research fellowship Fondazione Italiana Sclerosi Multipla - cod. 2019/BS/009 and financed or cofinanced with the ' 5 per mille' public funding.

\section{DIFFERENTIAL INVOLVEMENT OF CORPUS CALLOSUM IN IDIOPATHIC NORMAL PRESSURE HYDROCEPHALUS AND ITS MIMICS: A DTI STUDY}

A. Quattrone ${ }^{1}$, M. Caligiuri ${ }^{1}$, A. Sarica ${ }^{2}$, M. Quarantelli ${ }^{3}$, P. Arcuri ${ }^{4}$, A. Mechelli ${ }^{5}$, D. La Torre ${ }^{6}$, M. Vaccaro ${ }^{5}$, G. Cascini ${ }^{7}$, A. Quattrone ${ }^{1}$

${ }^{1}$ Neuroscience Research Center, Magna Graecia University (Catanzaro); ${ }^{2}$ Department of Medical and Surgical Sciences, Neuroscience Centre, Magna Graecia University (Catanzaro); ${ }^{3}$ Institute of Biostructure and Bioimaging, National Research Council (Napoli); ${ }^{4}$ Department of Radiology, Pugliese-Ciaccio Hospital (Catanzaro); ${ }^{5}$ Department of Neurology, Magna Graecia University (Catanzaro); ${ }^{6}$ Department of Neurosurgery, Magna Graecia University (Catanzaro); ${ }^{7}$ Department of Nuclear Medicine, Magna Graecia University (Catanzaro)

Objective: Corpus callosum thinning and microstructural alterations have been reported in idiopathic normal pressure hydrocephalus (iNPH) [1]. The objective of this study was to investigate changes in the principal diffusion direction (PDD) orientation of callosal fibers in iNPH, in progressive supranuclear palsy (PSP) and in Alzheimer's Disease (AD).

Methods: Twenty-three patients with iNPH, 27 with PSP, 25 with AD and 14 control subjects were enrolled in this study. All patients underwent brain 3T MRI including a diffusion-weighted sequence. PDD maps were obtained from diffusion-weighted MRI data, and were subsequently decomposed in polar coordinates (theta and phi angles) at each voxel. A mask of the corpus callosum was then used to limit the analysis to voxels belonging to the callosal bundle. A voxel was considered altered if both theta and phi values exceeded the mean \pm 2.5 standard deviation calculated over the control subjects. Then, a histogram of the altered voxels along the latero-lateral axis of the corpus callosum was calculated for each subject, and subsequently averaged over each patient group.
Results: We observed a different topographical distribution of altered voxels in the corpus callosum across diseases. iNPH patients showed alterations of the PDD in the medial regions of the corpus callosum, while PSP patients showed alterations in lateral portions of this structure. Patients with $\mathrm{AD}$, showed a mixed pattern, with alterations overlapping PSP- and NPH-specific regions.

Discussion: These findings suggest that the corpus callosum is differently affected in patients with NPH, PSP or AD. The topographical distribution of PDD alterations of callosal fibers may help differentiate iNPH from $\mathrm{AD}$ and PSP.

Reference:

1. Siasios I, Kapsalaki EZ, Fountas KN, et al. The role of diffusion tensor imaging and fractional anisotropy in the evaluation of patients with idiopathic normal pressure hydrocephalus: a literature review. Neurosurg. Focus (2016);41:E12. doi: 10.3171/ 2016.6.FOCUS16192

\section{MICROSTRUCTURAL CHANGES OF NORMAL-APPEARING WHITE MATTER IN SLEEP APNEA ARE REVERSIBLE AFTER TREATMENT}

M. Salsone ${ }^{1}$, M. Caligiuri ${ }^{2}$, V. Castronovo ${ }^{3}$, N. Canessa $^{4}$, A. Quattrone ${ }^{5}$, S. Marelli ${ }^{3}$, A. Quattrone ${ }^{2}$, L. Ferini-Strambi ${ }^{6}$

${ }^{1}$ Institute of Molecular Bioimaging and Physiology, National Research Council, Magna Graecia University (Catanzaro); ${ }^{2}$ Neuroscience Center, University "Magna Graecia" (Catanzaro); ${ }^{3}$ Sleep Disorders Center, San Raffaele Scientific Institute (Milano); ${ }^{4} \mathrm{NEtS}$ Center, Scuola Universitaria Superiore IUSS (Pavia); ${ }^{5}$ Institute of Neurology, University "Magna Graecia" (Catanzaro); "Division of Neuroscience, Vita-Salute San Raffaele University (Milano)

Objective: Visually appreciable white matter (WM) changes were described in Obstructive Sleep Apnea (OSA), yet few data exist on the involvement of the silent WM abnormalities. This study aimed to investigate the microstructural integrity of normal appearing white matter (NAWM) in OSA patients before and after continuous positive airway pressure (CPAP) treatment, using a neuroimaging approach.

Methods: Magnetic resonance imaging (MRI) data were acquired from a total of 15 severe never-treated OSA patients. Diffusion tensor imaging (DTI) and Tract-based spatial statistics (TBSS) were performed to assess the microstructural NAWM changes. In order to assess the therapy efficacy, OSA patients underwent MRI evaluations at two timepoints at baseline and after 3 months of treatment (follow-up).

Results: CPAP-treatment significantly increased fractional anisotropy in NAWM of brainstem, corpus callosum and bilateral internal capsule of OSA patients at follow-up compared to baseline ( $\mathrm{p}$ $<0.05$ TFCE-corrected). Moreover, OSA patients showed increases of axial diffusivity in the major tracts of the right hemisphere $(p<$ 0.05 TFCE-corrected) after CPAP-treatment. A significant negative correlation was found between FA of the corona radiata, corpus callosum, internal capsule, limbic structures and neuropsychological scores at follow-up evaluation.

Conclusions: Our results demonstrate that DTI metrics of NAWM in major tracts such as the corpus callosum and the internal capsule were significantly increased after CPAP-treatment, as potential beneficial effect of ventilatory therapy. The recovery of NAWM damage might relate to the improvement of neurocognitive profile and suggest that nonclearly-visible WM alterations may contribute to the physiopathology of OSA-related cognitive impairment. 


\section{SUGGESTIVE NEUROIMAGING CRITERIA OF HIPPOCAMPAL DEVELOPMENTAL ABNORMALITIES IN PATIENTS WITH TEMPORAL LOBE EPILEPSY AND IN HEALTHY CONTROLS}

\author{
I. Sammarra ${ }^{1}$, M. Caligiuri ${ }^{2}$, M. Trimboli ${ }^{1}$, G. Demonte $^{1}$, L. Rapisarda ${ }^{1}$, \\ A. Labate ${ }^{1}$, A. Gambardella ${ }^{1}$
}

${ }^{1}$ Institute of Neurology, Magna Graecia University (Catanzaro); ${ }^{2}$ Neuroscience Research Center, Magna Graecia University (Catanzaro)

Aims: To evaluate the frequency of qualitative features for Hippocampal Developmental Abnormalities (HiDeA) diagnosis on magnetic resonance imaging (MRI) in mesial temporal lobe epilepsy (MTLE) patients and healthy controls, highlighting which were more sensitive and specific to the epileptic syndrome.

Material: One-hundred and eighty-seven patients with MTLE and 93 age- and sex-matched controls underwent a 3 Tesla MRI. A Harmonized Neuroimaging of Epilepsy Structural Sequences (HARNESS-MRI) protocol was used [1], including a 3D T1-weighted spoiled gradient recall echo (sagittal acquisition, FOV $=256 \mathrm{~mm}$, repetition time $[\mathrm{TR}] /$ echo time $[\mathrm{TE}]=3.7 / 9.2 \mathrm{~ms}$, flip angle $=12^{\circ}$, isotropic voxel $=1 \times 1 \times 1 \mathrm{~mm} 3$ ).

Methods: Images were visually inspected by two expert raters, instructed to assess the presence of eight criteria for HiDeA identification [2] [3]: 1) medial positioning of hippocampus; 2) round, globular shape and vertical orientation of hippocampus; 3 ) flattened temporal horn; 4) misplaced fimbria on the dorsolateral edge of Ammon's horn; collateral sulcus 5) deep and verticalized or 6) protruding into a flattened temporal horn; 7) reduction of parahippocampal gyrus upper horizontal portion adjacent to hippocampal fissure; 8) thickened subiculum. Presence of HiDeA was recorded if at least 3 out of 8 criteria were present. After evaluating the prevalence of HiDeA in MTLE and controls, we assessed the frequency of each sign. Then, we classified differences in type or number of HiDeA diagnostic features, calculating their sensitivity and specificity. Fisher's exact test was used to assess statistical significance.

Results: Inter-rater agreement between the two experts was $98 \%$. HiDeA was detected in 36/187 MTLE cases (19.25\%) and in 8/93 $(8.6 \%)$ controls. Among all visual criteria here considered, HCS showed higher sensitivity both in MTLE group (91\%). HCP, thickened subiculum (HCD) and reduction of parahippocampal gyrus' upper horizontal portion (HCTH) signs demonstrated a 100\% specificity. In healthy controls, HCS confirmed to have highest sensitivity $(100 \%)$, while HCP showed the highest specificity $(98.8 \%)$ All these criteria were statistically associated with HiDeA. An association was not found among signs of HiDeA and treatment responsiveness.

Discussion: Some qualitative criteria, evaluated before quantitative measures, are highly indicative of HiDeA diagnosis. In MTLE patients, the presence of HCTH, HCP or HCD signs might help HiDeA identification, considering their specificity of $100 \%$. Among all criteria assessed, HCTH was the best marker of HiDeA in epilepsy, showing a very high sensitivity and specificity.

Conclusions: The identification of sensitive and, more importantly, specific criteria of HiDeA could be helpful to make a more confident visual diagnosis.

References:

1. Bernasconi A, Cendes F, Theodore WH, Gill RS, Koepp MJ, Hogan RE, et al. Recommendations for the use of structural magnetic resonance imaging in the care of patients with epilepsy: A consensus report from the International League Against Epilepsy Neuroimaging Task Force. Epilepsia (2019);60(6):1054-68
2. Barsi P, Kenéz J, Solymosi D, Kulin Á, Halász P, Rasonyi G, et al. Hippocampal malrotation with normal corpus callosum: a new entity? Neuroradiology (2000);42:339-45

3. Bernasconi N, Kinay D, Andermann F, Antel S, Bernasconi A. Analysis of shape and positioning of the hippocampal formation: an MRI study in patients with partial epilepsy and healthy controls. Brain (2005);128:2442-52

\section{HIPPOCAMPAL RATIO AND ITS ASSOCIATION WITH MEMORY PERFORMANCES IN ALZHEIMER'S DISEASE}

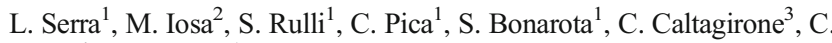 \\ Marra $^{4}$, M. Bozzali ${ }^{1,5}$
}

${ }^{1}$ Neuroimaging Laboratory, Fondazione Santa Lucia IRCCS (Roma); ${ }^{2}$ SMART Laboratory, Fondazione Santa Lucia IRCCS (Roma); ${ }^{3}$ Laboratory of Clinical and Behavioural Neurology, Fondazione Santa Lucia IRCCS (Roma); ${ }^{4}$ Institute of Neurology, Catholic University of Sacred Heart (Roma); ${ }^{5}$ Department of Neuroscience, University of Torino (Torino)

Objectives: The hippocampus plays a fundamental role in consolidation of information from short- to long-term memory [1]. Hippocampal atrophy is typically observed in patients with typical Alzheimer's disease (AD) [2]. Aim of this study was to assess whether the proportions of hippocampal subparts change over the transition from normal aging to AD.

Materials and Method: A cohort of 152 participants, 60 with a diagnosis of probable $\mathrm{AD}, 35$ with a diagnosis of amnestic mild cognitive impairment (a-MCI), and 57 healthy subjects (18 young-HSy, and 39 elderly-HSe) underwent an extensive neuropsychological battery and MRI at 3T. T1-weighted volumes were collected from each subject and used for the manual segmentation of the hippocampus in head (HH), body (HB) and tail (HT). Changes in hippocampal subparts were assessed by calculating ratios in length and volume (HR-line and HR-volume respectively) using the following formula: $(\mathrm{HB}+\mathrm{HH}+\mathrm{HT}) / \mathrm{HB}$. A series of ANOVAs were used to assess between group differences in HRvolume and HR-line. Correlations and multiple regressions were used to assess associations between memory performances and HR-volume and HR-line.

Results: Reduced volumes of HB were found in HSe compared to HSy, as well as in a-MCI compared to HSe. AD patients showed reduced volumes in every hippocampal subpart when compared to both a-MCI and HSe. We found that bilateral HR-volumes in HSy was significantly reduced in $\mathrm{HSe}$, and between $\mathrm{AD}$ to a-MCI patients; we also found significant reduction in the bilateral HR-line comparing HSe to both a-MCI and $\mathrm{AD}$ patients and comparing a-MCI to AD patients. Significant correlations were found in AD patients between the right HR-volume and scores obtained at the visuospatial memory test. In HSe individuals correlations were found between verbal episodic memory scores and both, the left HRvolume and the right HR-line. Multiple regressions confirmed associations between memory performances and HR changes in both $\mathrm{AD}$ patients and HSe individuals

Discussion: This study highlights changes in the hippocampal subparts that occur over the transitional stage from normal aging to $\mathrm{AD}$ and account for the decline in specific memory performances. In HS, HRs approached the so called golden value of 1.618. Interestingly, this is believed to be the perfect proportion of many biological structures to ameliorate their functioning [3]. 
Conclusions: Our results show a non-uniform hippocampal atrophy occurring in the transition to $\mathrm{AD}$, which firstly involves the HB.

References:

1. Serra L, Bozzali M, Fadda L, De Simone MS, Bruschini M, Perri R, Caltagirone C, Carlesimo GA. The role of hippocampus in the retrieval of autobiographical memories in patients with amnestic Mild Cognitive Impairment due to Alzheimer's disease. J Neuropsychol. (2020) Mar;14(1):46-68

2. Serra L, Petrosini L, Salaris A, Pica L, Bruschini M, Di Domenico C, Caltagirone C, Marra C, Bozzali M. Testing for the Myth of Cognitive Reserve: Are the Static and Dynamic Cognitive Reserve Indexes a Representation of Different Reserve Warehouses? J Alzheimers Dis. (2019);72(1):111-26

3. Ozturk S, Yalta K, Yetkin E. Golden ratio: A subtle regulator in our body and cardiovascular system? Int J Cardiol. (2016) Nov 15;223:143-45

\section{LONGITUDINAL PATTERNS OF ATROPHY IN CLINICAL VARIANTS OF FRONTOTEMPORAL LOBAR DEGENERATION}

\author{
E. G. Spinelli ${ }^{1}$, S. Basaia ${ }^{2}$, C. Cividini ${ }^{3}$, N. Riva ${ }^{4}$, G. Magnani ${ }^{5}$, F. Caso ${ }^{5}$, \\ P. Caroppo ${ }^{6}$, S. Prioni ${ }^{6}$, L. Tremolizzo ${ }^{7}$, I. Appollonio ${ }^{7}$, V. Silani ${ }^{8}$, P. \\ Carrera $^{9}$, M. Filippi ${ }^{10}$, F. Agosta $^{3}$
}

${ }^{1}$ Institute of Experimental Neurology, Division of Neuroscience, Scientific Institute and University "Vita-Salute" San Raffaele, University "Vita-Salute" San Raffaele (Milano); ${ }^{2}$ Neuroimaging Research Unit, Institute of Experimental Neurology, Division of Neuroscience, IRCCS San Raffaele Scientific Institute (Milano); ${ }^{3}$ Neuroimaging Research Unit, Institute of Experimental Neurology, Division of Neuroscience, IRCCS San Raffaele Scientific Institute; Vita-Salute San Raffaele University (Milano); ${ }^{4}$ Unit of Neurorehabilitation, IRCCS San Raffaele Scientific Institute (Milano); ${ }^{5}$ Unit of Neurology, IRCCS San Raffaele Scientific Institute (Milano); ${ }^{6}$ Unit of Neurology 5, Neuropathology, Fondazione IRCCS Istituto Neurologico Carlo Besta (Milano); ${ }^{7}$ Neurology Unit, "San Gerardo" Hospital and University of Milano-Bicocca (Monza); ${ }^{8}$ Department of Neurology and Laboratory of Neuroscience, IRCCS Istituto Auxologico Italiano (Milano); ${ }^{9}$ Laboratory of Clinical Molecular Biology, IRCCS San Raffaele Scientific Institute (Milano); ${ }^{10}$ Neuroimaging Research Unit, Institute of Experimental Neurology, Division of Neuroscience and Unit of Neurology, IRCCS San Raffaele Scientific Institute; Vita-Salute San Raffaele University (Milano)

Objectives: The progression of frontotemporal lobar degeneration (FTLD) is accompanied by a widespread disruption of grey matter (GM) that extends well beyond frontal and temporal cortical regions. The aim of this study was to assess longitudinal patterns of atrophy shown by magnetic resonance imaging (MRI) in the cortical and subcortical GM of patients affected by different clinical variants of the FTLD spectrum.

Materials and methods: 59 patients with presentations of the FTLD spectrum, in the absence of known pathogenic mutations, underwent longitudinal MRI evaluations on a 3T scanner at 6-month intervals for a maximum of 1 year. Patient groups included 26 with behavioral variant of frontotemporal dementia (bvFTD), 10 non-fluent/agrammatic variant of primary progressive aphasia (nfvPPA), 12 semantic variant of PPA (svPPA), and 11 motor neuron disease (MND). 33 age- and sexmatched healthy controls (HC) underwent the same longitudinal MRI protocol as FTLD patients. Three-dimensional T1-weighted MRI sequences from all timepoints of each subject were analyzed to assess the longitudinal evolution of GM atrophy in patients, compared with normal aging represented by HC. A whole-brain voxel-based morphometry
(VBM) analysis using the Computational Anatomy Toolbox in SPM12 was performed. ANOVA models with a time-by-group interaction were used to assess differences in longitudinal atrophy in patients compared with HC. The threshold of significance was set at $p<0.05$, Family-Wise Error corrected for multiple comparisons.

Results: At baseline, severe diffuse atrophy of frontotemporal cortical regions and basal ganglia was found in bvFTD, nfvPPA and svPPA groups, whereas MND did not show significant GM atrophy, compared with HC. At 6-month follow-up, bvFTD and PPA groups showed significant progression of atrophy in the putamen (bvFTD, nfvPPA and svPPA), caudate (nfvPPA and svPPA), thalamus (svPPA) and hippocampus (nfvPPA), bilaterally. At 12-month follow-up, similar patterns of atrophy progression were found, with the additional involvement of insular cortical regions in nfvPPA and thalami in bvFTD and svPPA. No significant progression of atrophy was found in MND patients, compared with HC.

Discussion and conclusions: Our data suggest that atrophy of subcortical GM closely reflects the progression of neurodegeneration in FTLD cases with cognitive impairment, in contrast with a plateau that has been reached in frontotemporal cortical atrophy. Particularly, measures of atrophy of the basal ganglia and thalami hold the promise to provide in vivo biomarkers of disease progression in the behavioral and linguistic presentations of frontotemporal dementia.

Supported by: European Research Council (StG2016_714388_NeuroTRACK).

\section{NEUROIMMUNOLOGY AND NEUROINFECTIVOLOGY}

\section{FIRST TWO CASES OF MYELITIS ASSOCIATED WITH CSF SARS-COV2 ANTIBODIES}

\author{
E. Agazzi, G. Camera, R. Riva, M. Sessa
}

USC Neurology, ASST-PG23 (Bergamo)

Objectives: To demonstrate the correlation between oIgG antibodies to SARS-CoV2 in the CSF and transverse myelitis post-COVID-19 infection.

Materials: Patient 1 healthy 62-year-old man presented acute back pain radiating to lower limbs, correlated to sneeze, followed by sensory changes, weakness, and constipation. Four days before he had fever SARS-CoV2 nasopharyngeal swab and thoracic CT scan were negative, Covid-19 G (IgG) antibodies to SARS-CoV2 (148 AU/ml) positive. Legs motor weakness progressively worsening in few days, predominant on left side and diminished sensation below the T11 sensory dermatome. The tendon reflexes were normal bilaterally at upper limbs and absent at lower limbs, with normal cutaneous plantar reflex. Patient 2: a 66-year-old man presented with a slowly progressive spastic paraparesis and numbness involving both lower extremities over the past month after fever, anosmia and ageusia 45 days before. Two weeks later, walking disturbances started with independent walking impossible in few days. On admission he showed paraparesis, lower limbs distal marked hypopallestesia and touch hypoestesia, soles paresthesia, brisk tendon reflexes, bilateral distal clonus and normal cutaneous plantar reflex. A spastic paraparetic gait was observed. Serology testing revealed positive IgG antibodies to Covid-19 (88,6 AU/ml) and chest CT scan had fibrotic alterations.

Method and Results: Patient 1 CSF analysis showed only hyperproteinorrachia $(118 \mathrm{mg} / \mathrm{dl}$; nv $15-45), \operatorname{IgG}(208 \mathrm{mg} / \mathrm{L} ; \mathrm{nv}<34)$ and $\operatorname{IgM}(17,9 \mathrm{mg} / \mathrm{dl} ; \mathrm{nv}<1,3)$, and the presence of CSF type 1 policlonal bands. CSF RT-PCR for bacteria, common neurotropic virus and SARS$\mathrm{CoV} 2$ were negative. IgG anti-SARS-CoV-2 were detected in the CSF, markedly positive Reduction of maximal voluntary activity was evident 
at the EMG. SEP and MEP showed a bilateral medullar subtotal block for the lower limbs). MRI of the spinal cord was unremarkable. Brain MRI was normal. Methylprednisolone, immunoglobulins and plasma exchange were administered. The patient regained almost normal motor power with persisting mild sensory deficits at discharge. Patient $2 \mathrm{CSF}$ was positive for IgG anti-SARS-CoV2. The EMG confirmed a reduction of the maximal voluntary recruitment. SEP and MEP showed a significant slowdown in the central conduction time of the motor and sensitive responses for the lower limbs. MRI of the spine demonstrated C5-C6 light stenosis. Brain MRI was normal. High-dose intravenous steroids and plasma exchange were administered at discharge normal strength, no sensory deficits were present, paretic gait persisted.

Discussion: Sharp sensitive level, sparing of upper limbs, pyramidal signs along with neurophysiological data pointing out bilateral medullar subtotal block of conduction of the long motor and sensitive pathway for the lower limbs, exclusion of pathological peripheral neurophysiological signs, temporal correlation with post-SARS-CoV2 clinical symptoms, positive SARS-CoV2 blood and CSF antibodies sustained a strong SARS-CoV2 related myelitis hypothesis.

Conclusions: We report for the first time in the literature the presence of IgG antibodies to SARS-CoV2 in the CSF of two patients with transverse myelitis post-COVID-19 infection. Which could be an indirect sign of tropism for the central nervous system and the underlying inflammatory state

References:

- Huang C, Wang Y, Li X, et al. Clinical features of patients infected with 2019 novel coronavirus in Wuhan, China. Lancet (2020);395(10223): 497-506. doi:10.1016/s0140-6736(20)30183-5

- WHO. Virtual press conference on COVID-19-11 March 2020. https://www.who.int/docs/default-source/coronaviruse/transcripts/ who-audio-emergencies-coronavirus-press-conference-full-andfinal-11mar2020.pdf?sfvrsn=cb432bb3_2

- Saglietto A, D’Ascenzo F, Biondi Zoccai G, De Ferrari GM. COVID-19 in Europe: the Italian lesson. Lancet (2020);395(10230):1110-11. doi: 10.1016/S0140-6736(20)30690-5

\section{NEUROTOXICITY-RELATED TO CART-CELL THERAPY: PROPOSED PROTOCOL AND PRELIMINARY DATA FROM BOLOGNA}

\author{
G. Amore ${ }^{1}$, U. Pensato ${ }^{1}$, R. Rinaldi ${ }^{2}$, S. Mondini ${ }^{2}$, F. Rondelli ${ }^{2}$, R. \\ D'Angelo $^{2}$, M. Nicodemo ${ }^{2}$, L. Sambati ${ }^{1,2}$, R. Santoro ${ }^{2}$, C. Di Mauro ${ }^{1}$, \\ E. Matteo ${ }^{1}$, B. Casadei $^{3}$, F. De Felice ${ }^{3}$, I. Defrancesco ${ }^{4}$, C. Pellegrini ${ }^{3}$, \\ P. Zinzani ${ }^{3}$, P. Tacchetti ${ }^{3}$, E. Zamagni ${ }^{3}$, F. Bonifazi ${ }^{3}$, E. Pierucci $^{5}$, M. \\ $\mathrm{Cavo}^{3}$, P. Cortelli ${ }^{1,2}$, M. Guarino ${ }^{2}$
}

${ }^{1}$ DIBINEM Department of Biochemical and Neuromotor Sciences, University of Bologna (Bologna); ${ }^{2}$ UOC Metropolitan Neurology Clinic NEUROMET, IRCCS Institute of Neurological Sciences Bologna (Bologna); ${ }^{3}$ Institute of Hematology Seragnoli, Department of Experimental, Diagnostic and Specialty Medicine, University of Bologna (Bologna); ${ }^{4}$ Department of Molecular Medicine, University of Pavia (Pavia); ${ }^{5}$ Anesthesia and Intensive Care Medicine, Azienda ospedaliera Sant'Orsola-Malpighi (Bologna)

Aims: Chimeric antigen receptor (CAR) T-cells therapy is an effective treatment for haematological malignancies. However, it is associated with cytokine release syndrome (CRS) and immune effector cell-associated neurotoxicity syndrome (ICANS) in up to $77 \%$. ICANS is a neuropsychiatric syndrome (ranging from confusion, aphasia and seizures to severe encephalopathy) still not fully investigated. We present our neurological protocol to diagnose and treat ICANS and preliminary data collected over one-year experience.

Methods: From September 2019, at Haematological Seragnoli Institute (Bologna), patients affected by refractory B-cells lymphoma (DLBCL) or multiple myeloma (MM) were candidates to CAR-T therapy. Patients were screened through a comprehensive neurological investigation (neurological examination, EEG, brain MRI, electroneurography, neuropsychological tests) one month before infusion in order to exclude CNS involvement. Prophylactic anti-epileptic treatment (Levetiracetam) was started 15 days prior treatment. From the day of CAR-T infusion, patients underwent every other day serial neurological examinations, including EEG and cognitive evaluation, to monitor development of neurotoxicity. In the presence of ICANS, daily neurological evaluation, neuroimaging, CSF analysis and cerebral 18F-PET were performed. Treatment was based on ICANS severity and consisted in high-doses IV corticosteroids, variably associated with anti-IL6 and anti-IL1 monoclonal antibodies, in selected cases whenever severe CRS was detected. Follow-up was scheduled at day 60 and 365.

Results: Twelve patients (9/12 DLBCL, 3/12 MM; 7/12 females) were treated (mean age: $59 \pm 7$ years). Brain MRI, EEG, electromyography and neuropsychological testing were substantially normal at screening. After CAR-T infusion, nine patients developed CRS: six mild, two severe, mean duration was $5,1 \pm 2,3$ days. Five patients ( $3 / 5$ women) with DLBCL developed ICANS: three mild and two severe, mean duration was $9,2 \pm 4$ days. In four patients ICANS was preceded by CRS. Earliest symptoms included ideo-motor slowing and reduced speech fluency; dysgraphia followed by a rapid evolution over few hours to a severe encephalopathy occurred in 2/5 cases. Except one patient, brain MRI was unremarkable. EEG showed predominant frontal slowing in all cases. Four patients were treated with IV steroids at increasing dosage. One patient developed steroid refractory neurotoxicity with multifocal brain oedema, eventually dying at day 16 , whereas other cases completely recovered.

Conclusions: We report the first Italian data on ICANS, confirming its high incidence and severity. Our neurological protocol with frequent clinical/instrumental assessment was essential in order to recognize early signs of neurotoxicity in CAR-T and promptly start appropriate treatment. Interestingly, we observed frontal electroclinical abnormalities as earliest features of ICANS in all patients.

References:

- Lee DW, Santomasso BD, Locke FL, et al. ASTCT Consensus Grading for Cytokine Release Syndrome and Neurologic Toxicity Associated with Immune Effector Cells. Biol Blood Marrow Transplant (2019);25:625-38

- Rubin DB, Danish HH, Ali AB, et al. Neurological toxicities associated with chimeric antigen receptor T-cell therapy. Brain (2019);142:1334-48

- Santomasso BD, Park JH, Salloum D, et al. Clinical and Biological Correlates of Neurotoxicity Associated with CAR T-cell Therapy in Patients with B-cell Acute Lymphoblastic Leukemia. Cancer Discov (2018);8:958-71

\section{DIFFERENTIAL DIAGNOSIS IN SUSPECTED PARANEOPLASTIC NEUROLOGICAL SYNDROMES: A POPULATION-BASED STUDY}

F. Bax ${ }^{1}$, G. Gigli ${ }^{1}$, M. Valente ${ }^{1}$, A. Marini ${ }^{1}$, A. Bernardini ${ }^{1}$, A. Vogrig ${ }^{2}$ 
${ }^{1}$ Clinical Neurology, Udine University Hospital (Udine); ${ }^{2}$ French Reference Center for Paraneoplastic Neurological Syndromes, Lyon University Hospital (Lyon-F)

Objectives: To categorize and describe the differential diagnosis spectrum of paraneoplastic neurological syndromes (PNS) and to evaluate the appropriateness of antibody-testing request based on initial clinical presentation

Materials and methods: A 9-year (2009-2017) population-based epidemiological study of PNS was performed in the Friuli-Venezia Giulia region (Italy). All patients undergoing search for neuronal antibodies indicative of PNS were screened and PNS diagnosis was defined according to the 2004 Euronetwork criteria. Data on alternative diagnoses were collected and categorized according to either central or peripheral nervous system involvement. When etiology was found, a subsequent etiological classification was performed.

Results: 661 patients with alternative diagnosis were included in the study, $335(50 \%)$ were males and mean age was 62.4 years $( \pm 17.7) .393$ patients (59.4\%) had central nervous system (CNS) involvement, 218 $(33 \%)$ had peripheral nervous system (PerNS) while $50(7.6 \%)$ had extra nervous system involvement. In patients with CNS involvement the most frequent etiologies were neurodegenerative $(97 ; 36.6 \%)$, autoimmune $(47 ; 17.8 \%)$ and vascular $(34 ; 12.9 \%)$. In patients with PerNS involvement the most frequent etiologies were autoimmune $(51 ; 44.3 \%)$, toxic $(28 ; 24.3 \%)$ and vascular $(14 ; 12.2 \%)$.

Discussion and conclusions: Many different diseases can mimic PNS, including treatable disorders that require prompt identification. Diseases with CNS involvement are the most frequent differential diagnosis encountered and a real-life characterization of PNS differential diagnosis spectrum may be used to build a clinical-based diagnostic algorithm to approach suspected PNS.

\section{COVID-19 PATHOPHYSIOLOGY: LINKS WITH MULTIPLE SCLEROSIS AND OTHER AUTOIMMUNE CONDITIONS}

G. Bellucci ${ }^{1}$, C. Ballerini ${ }^{1}$, R. Mechelli ${ }^{2}$, R. Bigi ${ }^{1}$, V. Rinaldi ${ }^{1}$, R. Reniè ${ }^{1}$, M. Buscarinu ${ }^{1}$, S. Baranzini ${ }^{3}$, L. Madireddy ${ }^{3}$, G. Matarese ${ }^{4}$, M. Salvetti ${ }^{1}$, G. Ristori ${ }^{1}$

${ }^{1}$ Centre for Experimental Neurological Therapies (CENTERS), Department of Neurosciences, Mental Health and Sensory Organs, Sapienza University of Rome (Roma); ${ }^{2}$ IRCCS San Raffaele Pisana, San Raffaele Roma Open University (Roma); ${ }^{3}$ Department of Neurology, Weill Institute for Neurosciences, University of CaliforniaSan Francisco (San Francisco-USA); ${ }^{4}$ Department of Molecular Medicine and Medical Biotechnologies, Federico II University of Naples (Napoli)

Background and Objectives: Severe coronavirus disease 2019 (COVID19) is associated with multiple comorbidities [1] and is characterized by an auto-aggressive inflammatory state leading to massive collateral damage, to whom may contribute an impaired type I IFN response [2]. Here we applied an omics approach to investigate the molecular interactions between SARS-CoV-2 and host, and how they translate into disease pathophysiology. We also performed a drug repurposing screening to identify potential therapeutic strategies.

Methods: We matched all the proteins known to interact with human coronaviruses and other respiratory viruses with lists of genes associated with autoimmune diseases and comorbidities linked to worse COVID-19 course. We then selected the genes included in the statistically significant intersection between SARS-CoV-2 network and disease associated gene sets, identifying a meta-interactome. We analyzed the meta-interactome genes expression in samples derived from lungs of infected humans, and their regulation by IFN- $\beta$. Finally, we exploited an integrated biomedical database to target the network's most critical nodes with clinically actionable molecules.

Results: We found a significant enrichment of SARS-CoV-2 interactors in immunological pathways and a strong association with autoimmunity (including multiple sclerosis - MS) and three prognostically relevant conditions (type 2 diabetes, coronary artery diseases, asthma), that present more independent physiopathological subnetworks. We observed a reduced expression of meta-interactome genes in human lungs after SARS-CoV-2 infection, and a regulatory potential of type I interferons. We also highlighted multiple repurposable drugs to tailor the therapeutic strategies.

Discussion and conclusions: Our data suggest the presence of two mechanisms underlying COVID-19 pathophysiology: one is SARSCoV-2-specific, and under the influence of a host's genetic background that is akin to that of various autoimmune conditions; the other is influenced by the presence of other diseases that synergize with the virus in a non-specific way as they do with many other microbial and non-microbial diseases. The regulatory potential of type I IFN on SARS-CoV-2 metainteractome genes is in line with the encouraging results of a COVID-19 trial with interferon $\beta-1 b$, a pivotal disease-modifying therapy for MS [3]. Our study also provides information on virus-host interactions that may increase the probability of success in repurposing and development of new treatments for COVID-19.

References:

1. S. Richardson, J.S. Hirsch, M. Narasimhan, J.M. Crawford, T. McGinn, K.W. Davidson, D.P. Barnaby, L.B. Becker, J.D. Chelico, S.L. Cohen, J. Cookingham, K. Coppa, M.A. Diefenbach, A.J. Dominello, J. Duer-Hefele, L. Falzon, J. Gitlin, N. Hajizadeh, T.G. Harvin, D.A. Hirschwerk, E.J. Kim, Z.M. Kozel, L.M. Marrast, J.N. Mogavero, G.A. Osorio, M. Qiu, T.P. Zanos, Presenting Characteristics, Comorbidities, and Outcomes Among 5700 Patients Hospitalized With COVID-19 in the New York City Area, Jama. (2020) Apr 22;323(20):2052-59

2. D. Blanco-Melo, B.E. Nilsson-Payant, W.C. Liu, S. Uhl, D. Hoagland, R. Møller, T.X. Jordan, K. Oishi, M. Panis, D. Sachs, T.T. Wang, R.E. Schwartz, J.K. Lim, R.A. Albrecht, B.R. tenOever, Imbalanced Host Response to SARS-CoV-2 Drives Development of COVID-19, Cell. 181 (2020);1036-1045.e9. https://doi.org/10.1016/j.cell.2020.04.026

3. I.F. Hung, K. Lung, E.Y. Tso, R. Liu, T.W. Chung, M. Chu, Y. Ng, J. Lo, J. Chan, A.R. Tam, H. Shum, V. Chan, A.K. Wu, K. Sin, W. Leung, W. Law, D.C. Lung, S. Sin, P. Yeung, C.C. Yip, R.R. Zhang, A.Y. Fung, E.Y. Yan, K. Leung, J.D. Ip, A.W. Chu, W. Chan, A.C. Ng, R. Lee, K. Fung, A. Yeung, T. Wu, J.W. Chan, W. Yan, W. Chan, J.F. Chan, A.K. Lie, O.T. Tsang, Articles Triple combination of interferon beta- $1 \mathrm{~b}$, lopinavir - ritonavir , and ribavirin in the treatment of patients admitted to hospital with COVID19: an open-label, randomised, phase 2 trial. Lancet (2020) May 30;395(10238):1695-1704

A SCORE BASED ON CLINICAL AND NEUROIMAGING
CHARACTERISTICS TO PREDICT AQP-4 IGG POSITIVITY IN
PATIENTS WITH LONGITUDINALLY EXTENSIVE
TRANSVERSE MYELITIS

L. Campetella, G. Spagni, C. Papi, R. Iorio

Institute of Neurology, Catholic University of the Sacred Heart (Roma) 
Background: Neuromyelitis Optica Spectrum Disorders (NMOSD) are autoimmune astrocytopathies that most commonly involve the optic nerve and the spinal cord. Immunoglobulin $\mathrm{G}(\mathrm{IgG})$ autoantibodies specific to the aquaporin- 4 water channel (AQP4) are detected in around $75 \%$ of NMOSD patients. Longitudinally extensive transverse myelitis (LETM) is a characteristic manifestation of NMOSD and usually leads to severe disability. Bright Spotty Lesions (BSLs) were recently described as a relatively specific MRI feature of AQP4-IgG positive patients. Early recognition of AQP4-IgG positive LETM is critical to initiate appropriate treatment to prevent relapses and limit neurological disability.

Objective: To develop a grading score that predicts AQP-4 IgG positivity in patients with a first episode of LETM.

Materials and Methods: We retrospectively analyzed the clinical data of 44 patients with a first episode of LETM admitted to the Neurology Department of our Institution between 2012 and 2019. All patients were tested for AQP4-IgG by cell-based assay. Available MRIs were reviewed for the presence of BSLs on axial T2-weighted images. ROC curves were generated to compare the sensitivity and specificity of different scores and cut-offs.

Results: Twenty-seven patients (61\%) were AQP4-IgG positive. Female sex was predominant in the AQP4-IgG+ group (20/27 patients, $74 \%$, v. 6/17 patients, 35\%). Female sex (OR 4.16; CI 1.02-16.91; $\mathrm{p}=0.0426$ ) and lesions involving the cervical cord (OR 5.14; CI 1.1423.14; $\mathrm{p}=0.02834$ ) were significantly associated with AQP4-IgG positivity. Area postrema syndrome, tonics spasms, neurogenic pruritus and autoimmune comorbidity were more frequent in the AQP4-IgG+ group. The following neuroimaging features were more frequent in $\mathrm{AQP} 4-\mathrm{IgG}+$ patients: lesion length $\geq 4$ segments, involvement of the medulla or cervical cord, hypointense lesions on T1-weighted images, gadolinium contrast enhancement and central gray matter involvement. BSLs were present in 6 out of 12 patients, of which 5 were AQP4-IgG positive. The clinical and neuroimaging characteristics were included in a 9-item, 13-point AQP4-IgG positive Myelitis (AIM) score. A score of $\geq 4$ predicts AQP4-IgG positivity with a sensitivity of $81 \%$ and specificity of $88 \%$. When BSLs were added to the score, sensibility and specificity raised to $100 \%$.

Conclusions: The AIM score predicts AQP4-IgG positivity in patients with LETM with good sensitivity and specificity. Further prospective studies on larger populations are needed to validate it and to assess inter- and intra-rater reliability.

References:

- Iorio R., Damato V., Mirabella M. et al. Distinctive clinical and neuroimaging characteristics of longitudinally extensive transverse myelitis associated with aquaporin-4 autoantibodies. J Neurol (2013);260:2396-2402. https://doi.org/10.1007/s00415-013-6997-9

- Trebst C., Raab P., Voss E. et al. Longitudinal extensive transverse myelitis-it's not all neuromyelitis optica. Nat Rev Neurol (2011);7:688-98. https://doi.org/10.1038/nrneurol.2011.176

- Iorio R; Pittock SJ. Neuromyelitis optica and the evolving spectrum of autoimmune aquaporin- 4 channelopathies. Clin Exp Neuroimmunol. (2014);5:175-187. doi: 10.1111/cen3.12103

\section{SPHINGOSINE 1-PHOSPHATE RECEPTOR MODULATORS AS A POTENTIAL TREATMENT OPTION IN COVID-19 INDUCED ACUTE RESPIRATORY DISTRESS SYNDROME: MECHANISTIC INSIGHTS AND BENEFIT-RISK ASSESSMENT}

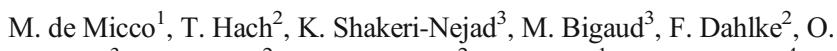
Petricoul $^{3}$, G. Graham ${ }^{2}$, D. Piani-Meier ${ }^{2}$, R. Turrini ${ }^{1}$, V. Brinkmann ${ }^{4}$, F. Nicoletti ${ }^{5}$
${ }^{1}$ Novartis Farma S.p.A (Origgio-VA); ${ }^{2}$ Novartis Pharma AG (Basel-CH); ${ }^{3}$ Novartis Institutes for Biomedical Research (Basel-CH); ${ }^{4}$ Independent Consultant (Freiburg-D); ${ }^{5}$ Department of Physiology and Pharmacology, University Sapienza of Rome (Roma)

Objectives: Coronavirus disease 2019 (COVID-19) is a viral infection caused by a newly emergent coronavirus, SARS-CoV-2, primarily affecting the respiratory tract. Maladjusted immune responses, e.g. cytokine release syndrome, may result in immunopathology and acute respiratory distress syndrome (ARDS). Sphingosine-1phosphate (S1P), a bioactive lipid mediator, is crucial in maintaining endothelial cell chemotaxis and barrier integrity. An industry-independent clinical study is currently underway in China investigating the efficacy of oral fingolimod $0.5 \mathrm{mg}$ (a non selective S1P receptor modulator) taken once-daily, for three consecutive days in patients with COVID-19.

Methods: Here we review the potential mechanisms by which fingolimod may regulate the inflammatory response to SARSCoV-2 and assess the potential benefit-risk of short-term treatment with fingolimod in patients with COVID-19 experiencing ARDS.

Results: The key hypotheses through which beneficial effects manifest are (1) attenuation of cytokine release via activation of serine/ threonine protein phosphatase 2A (PP2A); (2) inhibition of Th17mediated pathway; and (3) enhancement of the pulmonary endothelial barrier via c-Abl tyrosine kinase pathway.

Discussion: The short-term intervention with fingolimod might rapidly attenuate maladjusted immune responses while sparing memory immune responses and thus has relatively low risk of infections. Any potential effects on heart rate and cardiac rhythm could be managed under the intensive care treatment setting.

Conclusions: S1P receptor modulators, such as fingolimod, may represent a potential treatment option to ameliorate immune responses against SARS-CoV-2 and merit further investigation following careful benefit-risk evaluation in this setting.

This study has been first presented at the 6th Congress of the European Academy of Neurology (virtual, May 23-26 2020).

References:

1. Yan-Rong Guo, Qing-Dong Cao, Zhong-Si Hong, Yuan-Yang Tan, Shou-Deng Chen, Hong-Jun Jin, Kai-Sen Tan, De-Yun Wang \& Yan Yan. The origin, transmission and clinical therapies on coronavirus disease 2019 (COVID-19) outbreak - an update on the status. Military Medical Research (2020);7(11)

2. Dawei Wang, MD; Bo Hu, MD; Chang Hu, MD; Fangfang Zhu, MD; Xing Liu, MD; Jing Zhang, MD; Binbin Wang, MD; Hui Xiang, MD; Zhenshun Cheng, MD; Yong Xiong, MD; Yan Zhao, MD; Yirong Li, MD; Xinghuan Wang, MD; Zhiyong Peng, MD. Clinical Characteristics of 138 Hospitalized Patients With 2019 Novel Coronavirus-Infected Pneumonia in Wuhan, China. JAMA (2020);323(11):1061-69

3. S. Uhlig, Y. Yang. Sphingolipids in Acute Lung Injury. Handbook of Experimental Pharmacology (2013);(216):227-46

\section{FAMILIAL ANTI-LGI1 ENCEPHALITIS IN TWO SISTERS}

\author{
G. De Stefano, A. Morano, F. Pauri, F. Fattapposta, M. Faedda, P. \\ Pulitano
}

Department of Human Neurosciences, Sapienza University (Roma) 
Introduction and Objective: Anti-leucine-rich glioma-inactivated protein 1 (LGI1) encephalitis is the second most common type of limbic encephalitis, with distinctive clinical features and serological biomarkers. [1] Despite the pivotal role of autoimmunity in the pathogenesis of this disease [1], recent evidence from genotyping studies [2] raises the hypothesis that also genetic factors - especially human leukocyte antigen (HLA) alleles - may significantly contribute.

Materials and Methods: We describe the case of a 76-year-old, previously healthy woman attending our outpatient clinic for the subacute onset of mental status alteration associated with neuropsychiatric symptoms such as delusions and behavioural changes. Four years earlier, her sister also presented with abrupt confusion and muscle spasms and was eventually diagnosed with anti-LGI1 encephalitis. Our patient's neurological examination showed temporal disorientation, memory impairment, emotional lability, anosmia and involuntary movements involving the face and the arms, suggestive for faciobrachial dystonic seizures. Routine laboratory tests and cerebrospinal fluid (CSF) analysis were performed, along with neuroimaging studies.

Results: Blood tests showed mild hyponatremia. The brain MRI scan documented T2-hyperintensity and swelling of the medial right temporal lobe. Routine CSF analysis was within the normal range, but screening for neuronal autoantibodies disclosed the presence of anti-LGI antibodies in both the CSF and the serum. A total body CT scan showed no evidence of malignancies, and the patient received steroids and intravenous immunoglobulin, with major clinical improvement. HLA typing showed the presence of HLADRB $1 * 07$ allele. Further genetic studies are currently ongoing in both sisters.

Discussion: This is the first reported case of familial anti-LGI1 encephalitis occurring in two siblings, and further supports the association between anti-LGI1 encephalitis and HLA-DRB $1 * 07$ [3], suggesting that genetic factors involved in the immune response regulation might play a crucial role in these emerging diseases. Our case also highlights that family history should be accurately investigated when limbic encephalitis is suspected.

References:

1. Graus F, Titulaer MJ, Balu R, et al. A clinical approach to diagnosis of autoimmune encephalitis. Lancet Neurol. (2016);15(4):391-404. doi:10.1016/S1474-4422(15)00401-9

2. Mueller SH, Färber A, Prüss H, et al. Genetic predisposition in antiLGI1 and anti-NMDA receptor encephalitis. Ann Neurol. (2018);83(4):863-69

3. van Sonderen A, Roelen DL, Stoop JA, et al. Anti-LGI1 encephalitis is strongly associated with HLA-DR7 and HLA-DRB4. Ann Neurol. (2017);81(2):193-98

\section{SEVERE REACTIVATION OF MULTIPLE SCLEROSIS AFTER COMBINED VACCINATION FOR MEASLES - MUMPS - RUBELLA (MMR) AND DIPHTERIA-TETANUS-PERTUSSIS (DTP)}

G. Di Mauro ${ }^{1}$, D. Landi ${ }^{1}$, C.G. Nicoletti ${ }^{1}$, G. Cola ${ }^{1}$, G. Mataluni ${ }^{1}$, M. Iannetta $^{2}$, N.B. Mercuri ${ }^{3}$, G. Marfia ${ }^{1,4}$

${ }^{1}$ Multiple Sclerosis Clinical and Research Unit, Department of Systems Medicine, Tor Vergata University (Roma); ${ }^{2}$ Infectious Diseases Clinic, University Hospital "Tor Vergata" (Roma); ${ }^{3}$ Neurology Unit, Department of Systems Medicine, Tor Vergata University (Roma); ${ }^{4}$ Unit of Neurology and Neurorehabilitation, IRCCS Neuromed (Pozzilli-IS)

Objective: Vaccination is an emerging best practice in patients with recent diagnosis of MS in order to prevent later infectious complications during immunosuppressive treatments. Safety of vaccines with respect to MS relapse is still debated. We report a case of severe reactivation of multiple sclerosis (MS) after combined vaccination.

Materials and methods, results: A 25 years-old female patient was diagnosed with Relapsing - Remitting MS in August 2019 following recent onset of right hemisoma paresthesia and dysesthesia and finding of several typical gadolinium enhancing and non-enhancing demyelinating lesion on brain and spinal cord MRI. In order to stratify infectious risk and in consideration of pregnancy intention, a complete infective screening was conducted. She was found negative for measles-mumps-rubella (MMR) antibodies, so an appropriate vaccination plan was made before starting disease modifying therapy; patient got MMR plus diphteria tetanus - pertussis (DTP) vaccination 40 days after the first relapse. Two weeks later she complained new onset of headache and subjective and objective right sensory-motor syndrome (EDSS 3.5). Infections were ruled out through blood and CSF analysis. Brain and spine MRI scan showed a massive left periventricular white matter demyelinating lesion, with irregular and peripheral enhancement, surrounded by white matter edema causing left lateral ventricular compression and minimal midline shift. Partial clinical recovery (EDSS 2) was observed after Methylprednisolone 1g/die, Mannitol and Furosemide treatment. Natalizumab was started with full disease control.

Discussion: This case highlights the potential role of vaccination in triggering MS relapse under special circumstances. However, it remains unclear if the risk of reactivation depends on the burden or the type of vaccines or on ongoing pre-existing patient's inflammatory status or on a synergic effect of all those factors.

Conclusion: We acknowledge the importance of vaccination in MS patients, nevertheless there is a need to define the optimal therapeutic window and vaccination protocol in order to secure patients' safety.

Reference:

Zrzavy T, Kollaritsch H, Rommer PS, et al. Vaccination in Multiple Sclerosis: Friend or Foe?. Front Immunol. (2019);10:1883.

Published 2019 Aug 7. doi:10.3389/fimmu.2019.01883

\section{CLINICAL AND NEUROIMAGING CHARACTERISTICS OF MOG AUTOIMMUNITY IN CHILDREN WITH ACQUIRED DEMYELINATING SYNDROMES}

M. A. N. Ferilli ${ }^{1}$, M. Valeriani ${ }^{1}$, C. Papi $^{2}$, L. Papetti ${ }^{1}$, C. Ruscitto ${ }^{3}$, L.

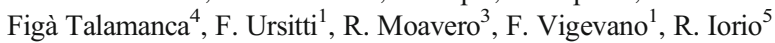

${ }^{1}$ Department of Neuroscience, Bambino Gesù Children's Hospital, IRCCS (Roma); ${ }^{2}$ Department of Neuroscience, University Cattolica del Sacro Cuore (Roma); ${ }^{3}$ Child Neurology Unit, Systems Medicine Department, Tor Vergata University Hospital of Rome (Roma); ${ }^{4}$ Imaging Department, Bambino Gesù Children's Hospital, IRCCS (Roma); ${ }^{5}$ Department of Neuroscience, Fondazione Policlinico Universitario A. Gemelli IRCCS (Roma)

Objective: Aim of this study was to describe the clinical and neuroimaging features and the long-term outcome of children with acquired demyelinating syndromes (ADS) of the central nervous system (CNS) associated with MOG-IgG.

Methods: The clinical and neuroradiological features of children with ADS of the CNS admitted between January 2014 and December 2019 were evaluated. All patients underwent brain and spinal cord magnetic resonance imaging (MRI), lumbar puncture for cerebrospinal fluid (CSF) analysis and MOG-IgG and AQP4-IgG testing. 
Results: Forty-eight pediatric patients with ADS of the CNS were recruited. MOG-IgG were detected in 11/48 (25\%) patients. Patients with MOG-IgG had the following clinical diagnoses: encephalomyelitis (EM) 8/11 (73\%); optic neuritis (ON) 2/11 8 (18\%); transverse myelitis (TM) $1 / 11(9 \%)$. Patients negative for MOG-IgG were diagnosed with Multiple Sclerosis (MS) $(n=15)$, EM $(n=7)$, ON $(n=7)$, neuromyelitis optica spectrum disorders (NMOSD) $(n=5), T M(n=2)$ and encephalitis $(n=1)$. MOG-IgG positive patients were younger at disease onset compared with children negative for MOG-IgG. They more frequently experienced encephalopathy and epileptic seizures. EM and inflammatory lesions involving optic nerves on MRI imaging were more frequent in MOG-IgG positive patients. None of the patients with MOG-IgG became persistently seronegative during the follow-up, although a decrease in MOG-IgG titer was observed. Patients with MOG-IgG showed a good response to therapy and only two patients presented relapses during follow-up.

Conclusion: $\mathrm{EM}$ and $\mathrm{ON}$ are the most frequent clinical presentations of MOG autoimmunity in pediatric patients. Epilepsy can occur in positive patients. MOG-IgG seropositivity tends to persist, although a decrease in antibody titer is observed during the follow-up. This study supports the distinction of MOG autoimmune oligodendrocytopathy as a unique disease entity, with clinical features different from MS and AQP4-IgG-positive NMOSD.

\section{DIAGNOSTIC AND THERAPEUTIC CHALLENGES IN MOG ANTIBODY DISEASE: A CASE REPORT}

M. P. Giannoccaro ${ }^{1}$, C. La Morgia ${ }^{1}$, M. Gastaldi ${ }^{2}$, M. Foschini ${ }^{3}$, G. Asioli $^{4}$, P. Avoni ${ }^{1}$, G. Rizzo ${ }^{1}$, V. Donadio ${ }^{5}$, D. Franciotta ${ }^{2}$, R. Liguori ${ }^{1}$

${ }^{1}$ IRCCS Institute of Neurological Sciences of Bologna, University of Bologna (Bologna); ${ }^{2}$ IRCCS C. Mondino National Neurological Institute, Neuroimmunology Laboratory (Pavia); ${ }^{3}$ Department of Biomedical and Neuromotor Sciences, Unit of Anatomic Pathology at Bellaria Hospital, University of Bologna (Bologna); ${ }^{4}$ Department of Biomedical and Neuromotor Sciences, University of Bologna (Bologna); ${ }^{5}$ IRCCS Istituto delle Scienze Neurologiche di Bologna, AUSL di Bologna (Bologna)

Objectives: Myelin oligodendrocyte glycoprotein antibodies (MOG-Abs) are associated with clinical presentations as acute disseminated encephalomyelitis, optic neuritis (ON), myelitis, and brainstem encephalitis, usually with a relatively benign course.

Case report: A 34-year-old man developed sudden bilateral visual loss, two weeks after a flu-like syndrome. Ophthalmologic examinations revealed severely reduced visual acuity (OD, counting fingers, OS, light perception), relative afferent pupillary defect, bilateral optic disc edema reflected by increased retinal nerve fiber layer (RNFL) thickness and prolonged visual evoked potentials latency. Brain MRI was normal; CSF analysis showed blood-brain-barrier dysfunction and negative oligoclonal bands. Serum AQ4- and MOG-Ab were negative. He was diagnosed with bilateral $\mathrm{ON}$ and treated with a course of intravenous methylprednisolone therapy (IVMP) and discharged. Four days after steroids suspension, he developed agitation, confusion and somatic illusion, followed by right hemiparesis, hemi-hypoesthesia and non-fluent aphasia. Brain MRI showed multiple T2-hyperintense white matter lesions, with restricted diffusion, in the brainstem, thalamo-capsular-corona radiata region, and parietal gyrus, without gadolinium enhancement. $\mathrm{He}$ was restarted on IVMP followed by oral prednisolone and 3 courses of plasmapheresis. Despite hemisyndrome's improvement, a new brain MRI showed an increment of the white matter lesions and hyperintensity of the optic nerves, extending to the chiasma, with left optic nerve's enhancement. CSF analysis showed pleocytosis; MOG- and AQ4-Ab in CSF and serum retested negative. Rituximab was started but withdrawn after the first infusion due to increased liver enzyme levels. Due to the radiological progression despite immunotherapy, a brain biopsy was performed. Neuropathology showed demyelination and perivascular and parenchymal cell infiltrates consisting mainly of astrocytes, CD68-positive macrophages and CD4-positive T-cells with very rare CD8 positive T-cells and no CD20-positive B-cells. Repetition of antibody testing on the first serum sample using a live cell-based assay showed positivity for MOG$\mathrm{Ab}$. The patient was started on IV immunoglobulin and mycophenolate with progressive reduction of the brain MRI lesions and no further relapse. Last ophthalmological evaluation confirmed a severe visual loss and bilateral optic disc pallor.

Conclusions: Most often, MOG-Abs are associated with a relatively benign course and a good response to steroids. Although MOG ON attacks have been shown to permanently damage the optic nerve, the majority of patients fully or partially recover visual function [1-2]. Our patient presented a severe disease course without visual recovery and profound optic atrophy despite high doses of steroids, outlining the necessity for early identification and aggressive treatment to prevent longterm sequelae.

References:

1. Jarius S, Ruprecht K, et al. MOG-IgG in NMO and related disorders: a multicenter study of 50 patients. Part 2: Epidemiology, clinical presentation, radiological and laboratory features, treatment responses, and long-term outcome. J Neuroinflammation. (2016);13(1):280

2. Pache F, Zimmermann H, et al. MOG-IgG in NMO and related disorders: a multicenter study of 50 patients. Part 4: Afferent visual system damage after optic neuritis in MOG-IgG-seropositive versus AQP4-IgG-seropositive patients. J Neuroinflammation (2016);13(1):282

\section{UNDIAGNOSED AND MISDIAGNOSED: THREE NEW CASES OF IGLON5-ASSOCIATED ENCEPHALITIS}

\author{
M. P. Giannoccaro ${ }^{1}$, V. Mastrangelo ${ }^{2}$, F. Pizza ${ }^{1}$, G. Plazzi ${ }^{1}$, C. La \\ Morgia $^{1}$, P. Avoni ${ }^{1}$, R. Liguori ${ }^{1}$
}

${ }^{1}$ IRCCS Institute of Neurological Sciences of Bologna, University of Bologna (Bologna); ${ }^{2}$ Department of Biomedical and Neuromotor Sciences, University of Bologna (Bologna)

IgLON5 antibodies (IgLON5-Ab) are associated with a syndrome characterised by complex sleep disorders, bulbar symptoms, gait abnormalities and oculomotor problems. Due to this variegate presentation, a misdiagnosis could be frequent. We report three patients initially misdiagnosed with seronegative myasthenia.

Cases presentation: Case1. A 60-years-old man developed apathy, sleep disorders, dysphonia and dysphagia. He started pyridostigmine without benefit. Subsequently, he developed gait instability, hands tremor, sialorrhea and bladder dysfunction. The examination, at age 66, showed mild hypomimia, dysarthria, dysphagia, nystagmus, action and postural tremor, hyperreflexia and ataxic gait. Case 2. A 71-year-old woman was referred for suspected myasthenia. Two years prior, she had developed walking difficulties, dysphagia, non-fluctuant ptosis, dyspnea and obstructive sleep apnoea (OSA) requiring nocturnal continuous positive airway pressure, disturbed sleep. Examination revealed bilateral non-fatigable ptosis, horizontal gaze restriction, mild lower limb weakness with absent reflexes, unsteady gait. Case 3. A 71-years-old 
woman developed gait instability, fluctuating ptosis and dysphagia along with a sleep disorder characterised by apneas, somniloquy and RBD. One year later, she was admitted for an episode of acute-on-chronic respiratory failure. Examination showed mild fatigable ptosis and unsteady gait.

Results: Brain MRI and CSF analysis were unremarkable. Repetitive nerve stimulation was altered in case 3; single fibre-EMG showed increased jitter and block in case 2. AchR and Musk antibodies were negative. IgLON5-Ab were positive in serum and CSF. Videopolysomnography showed poorly structured N2 and normal N3 sleep, limb movements and vocalisations, OSA, snoring and stridor in patient 1 and severe periodic limb movements and occasional desaturations, despite positive airway pressure treatment, in case 2 . Response to immunotherapy was variable. Case 1 was treated with plasma exchange and IV immunoglobulin (IVIg) without clear benefit. However, an improvement occurred after rituximab. Case 2 was treated with oral corticosteroids without improvement. A course of IVIg in association with high dose corticosteroids improved the ptosis, but the patient died for acute respiratory failure. Case 3 was treated with IVIg with partial benefit.

Conclusion: We reported three IgLON5-disease cases with myasthenia mimic features. Our cases outline the delay in identifying this disorder and the role of sleep investigations in pointing towards the correct diagnosis. Both neurodegenerative and immunological mechanisms are involved in the pathogenesis of the disease, and a delay in treatment can likely impact on the prognosis. Our patients showed a variable response to immunotherapy but support the possible utility of second-line therapies in patients not responding to first-line interventions.

\section{STIFF PERSON SPECTRUM DISORDER - A DIAGNOSTIC AND THERAPEUTIC CHALLENGE}

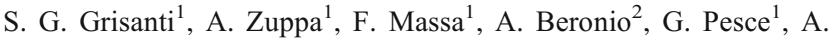 \\ Uccelli $^{3}$, A. Schenone ${ }^{3}$, L. Benedetti ${ }^{3}$
}

${ }^{1}$ Department of Neuroscience, Rehabilitation, Ophthalmology, Genetics, Maternal and Child Health (DINOGMI), University of Genoa (Genova); ${ }^{2}$ Department of Neurology, S. Andrea Hospital (La Spezia); ${ }^{3}$ Department of Neurology, University of Genoa (Genova)

Background: Stiff-person syndrome (SPS) is characterized by muscle rigidity and painful spasms occurring spontaneously or triggered by diverse stimuli. Other forms, such as stiff-limb syndrome (SLS) and progressive encephalomyelitis with rigidity and myoclonus (PERM), are considered within the spectrum of SPS. SPS is a difficult disease to diagnose due to the heterogenicity of presentation and the limited specificity of diagnostic tests. For example, $33 \%$ of cases are seronegative, OCBs can be detected in CSF only in $60 \%$ of patients and EMG shows abnormalities in about $72 \%$ of cases. Moreover, there isn't a standard consensus about the adequate therapy. Here, we present the case series of two different center.

Methods: We collected the data about clinical presentation, CSF, MRI, EMG and antibody essay of all the patients with SPS admitted to IRCCS San Martino in Genoa and Ospedale Sant'Andrea in La Spezia between 2005 and 2019. Patients were treated with symptomatic therapies (BDZ), first-line immunomodulatory therapies (corticosteroids, IVIG) or secondline immunomodulatory therapies (azatoprine and rituximab).

Results: We analyzed the data of 8 patients ( 5 female, mean age 53 years), 5 classified as SLS, 2 as classical-SPS and one as PERM. In addiction to spasm and stiffness, $75 \%$ of patients had psychiatric symptoms, $37.5 \%$ had urinary disorders and $50 \%$ had taste and smell alterations. We found OCBs in CSF in $25 \%$ of samples. $25 \%$ of EMG showed typical abnormalities. $35 \%$ of sera lacked specific antibodies. MRI of brain and spinal cord were unremarkable, except in a patient with PERM in which MRI showed contrast enhancement of cauda equina. Only one patient found full benefit from BZD administrated alone, one from BZD plus low doses of corticosteroids, and two from the coadministration of azathioprine. Two patients showed partial benefit with BZD and lower doses of steroids. Two patients didn't improve after different therapies. The three patients treated with rituximab didn't show any benefit.

Conclusions: We found the same percentage of seronegative-SPS reported in literature, but EMG and CSF analyses were less useful in the diagnosis of SPS. We observed some clinical features (e.g. smell and taste alterations) in classical-SPS and SLS and not only in PERM. Then, we confirmed the efficacy of BDZ and the lack of response to rituximab, as previously reported in literature. More studies about predictive response to treatment are needed to clarify these findings.

\section{NEUROSARCOIDOSIS: A SINGLE CENTER EXPERIENCE IN 203 CONSECUTIVE PATIENTS}

\author{
B. Labella ${ }^{1}$, F. Cinetto ${ }^{2}$, R. Manara ${ }^{3}$, R. Scarpa ${ }^{2}$, E. Pegoraro ${ }^{1}$, P. Gallo ${ }^{1}$, \\ C. Agostini ${ }^{2}$, M. Corbetta ${ }^{1}$, C. Briani ${ }^{1}$
}

${ }^{1}$ Department of Neurosciences, Neurology Unit, University of Padua (Padova); ${ }^{2}$ Department of Medicine, University of Padua (Padova); ${ }^{3}$ Radiology, University of Padua (Padova)

Sarcoidosis is a systemic inflammatory disease of unknown etiology characterized by non-necrotizing epithelioid granulomas affecting mainly skin, lungs and lymph nodes. Neurological involvement is rare, occurring in $5-15 \%$ of patients, either isolated or associated with other systemic features of sarcoidosis. The objective of our study was to assess the prevalence, the main clinical features, and the most useful investigations for neurosarcoidosis diagnostic workup in 203 consecutive patients with sarcoidosis followed at the Centre for Rare Diseases of the Treviso and Padua Hospital since 1998. For each patient clinical, instrumental and laboratory data were collected. Organ involvement was assessed according to the World Association of Sarcoidosis and Other Granulomatours Disorders (WASOG) criteria.

Results: Nineteen patients $(9.4 \%$ of the total sample) with neurosarcoidosis were identified, 4 women $(21.1 \%)$ and 15 men $(78.9 \%)$. The median age at diagnosis was $44.27( \pm 12.15)$ years. Neurosarcoidosis was detected at disease onset in $15 / 19(78.9 \%)$ patients. Focal lesions (12/19) and cranial neuropathies (2 optic neuritis, 3 facial nerve palsy, 2 eighth nerve involvement), were the most frequent manifestations, followed by myelitis, hydrocephalus, vasculitis, radiculopathy, myopathy and meningitis. CSF analysis was performed in 14/19 (73.68\%) patients and pleocytosis (12/14) and hyperproteinorrachia (11/ 14) were the most common alterations). Brain and spine magnetic resonance with gadolinium was the gold standard to detect neurosarcoidosis lesions. Twelve patients also underwent 18 -fluorodeoxyglucose positron emission tomography, in order to assess systemic involvement and detect possible sites of biopsy. Biopsy was performed in the affected neurological site only in three patients (thalamus, temporal area and muscle). When compared with patients without neurological involvement, patients with neurosarcoidosis presented more frequently acute disease onset and longer diagnostic delay. With regard to organ localizations, patients with neurosarcoidosis had more extra-thoracic lymph node and glandular (parotid/salivary) involvement. All patients with neurosarcoidosis were treated with corticosteroids at disease onset, but then required a steroid- 
sparing therapy, mainly immunosuppressants (16/19), with 6 patients also undergoing anti-TNF $\alpha$ drugs as third-line treatment. Therapy response was good, although seven $(36.84 \%)$ patients with neurosarcoidosis had a neurological relapse. In conclusions, neurological involvement in sarcoidosis is uncommon but not negligible, and an early diagnosis is crucial for timely therapy and better outcome.

\section{ASSESSING THE ROLE OF A TISSUE-BASED ASSAY IN THE DIAGNOSTIC ALGORITHM OF AUTOIMMUNE ENCEPHALOPATHIES}

G. Masi, G. Spagni, A. Evoli, L. Campetella, G. Monte, C. Papi, E. Sabatelli, R. Iorio

Institute of Neurology, Policlinico Universitario A. Gemelli IRCCS, Catholic University of the Sacred Heart (Roma)

Background: The diagnosis of autoimmune encephalopathies (AE) relies on clinical, laboratory and imaging features. In this regard, the detection of neural autoantibodies (NAb) is important, as it may lead to the identification of an occult cancer and to the prompt initiation of immunotherapy. NAb screening methods vary consistently among centers, and the role of immunochemical assays on a substrate of murine brain sections (tissue-based assays, TBA) is debated. The aim of this study is to evaluate the sensitivity and specificity of TBA for AE diagnosis.

Methods: We retrospectively evaluated patients suspected of having $\mathrm{AE}$, whose samples were sent to our laboratory for NAb screening from December 2012 to June 2020. Patients' serum and cerebrospinal fluid (CSF) were tested by indirect immunofluorescence on a composite substrate of mouse brain, kidney and stomach, cell-based assays and immunoblots. Demographic and clinical data were obtained by chart review.

Results: From 403 patients initially recruited, clinical data were available for 164 patients, who are the focus of this study. The median age at disease onset was 52 years (range $0-88$ ) and $84(51 \%)$ were women. For 111 patients both serum and CSF were tested, while in 18 and 35 cases respectively either serum or CSF was available. TBA showed an IgGspecific immunoreactivity restricted to the nervous system in 89 patients $(54 \%)$. Of them, 79 patients (89\%) were finally diagnosed with $\mathrm{AE}$, whereas in 10 patients $(11 \%)$ other conditions were ascertained. Notably, eight patients had herpes simplex virus encephalitis (HSVE), one infectious meningoencephalitis and one stroke. Among patients with a negative TBA, 10/75 (13\%) were diagnosed with AE. In the remaining cases, viral encephalitis was diagnosed in $25 / 75$ patients (33\%), epilepsy in $9 / 75(12 \%)$ and brain tumor in $6 / 75(8 \%)$, followed by other, less frequent diseases. TBA showed $89 \%$ sensitivity and $88 \%$ specificity for detecting patients with $\mathrm{AE}$, with a positive predictive value of $89 \%$ and a negative predictive value of $88 \%$.

Discussion and Conclusions: TBA showed good sensitivity and specificity for the diagnosis of $\mathrm{AE}$ and can be considered a valuable screening method in the diagnostic algorithm of AE. Extensive microbiological investigation should never be dismissed, however, since a positive TBA does not rule out the possibility of an infectious disease, as in the case of HSVE.

References:

- Iorio R, Damato V, Spagni G, et al. Clinical characteristics and outcome of patients with autoimmune encephalitis: clues for paraneoplastic aetiology. European Journal of Neurology (2020) Jun 19

- Dalmau J, Graus F. Antibody-Mediated Encephalitis. Ropper AH, editor. New England Journal of Medicine (2018);378:840-51
- Ricken G, Schwaiger C, De Simoni D, et al. Detection Methods for Autoantibodies in Suspected Autoimmune Encephalitis. Frontiers in Neurology (2018) Oct 10;9:841

\section{FDG-PET UNVEILS THE COURSE OF PARANEOPLASTIC CEREBELLAR DEGENERATION: A SEMIQUANTITATIVE ANALYSIS}

\author{
F. Massa ${ }^{1}$, L. Filippi ${ }^{1}$, L. Benedetti ${ }^{1}$, S. Morbelli ${ }^{2}$, F. Nobili ${ }^{1}$
}

${ }^{1}$ Department of Neuroscience, Rehabilitation, Ophthalmology, Genetics, Maternal and Child Health (DINOGMI), University of Genoa (Genova); ${ }^{2}$ Department of Health Sciences (DISSAL), University of Genoa (Genova)

Background: In paraneoplastic cerebellar degeneration (PCD) the standard diagnostic workup might be inconclusive, especially in seronegative subtypes. Brain 18F-FDG PET is an accurate supportive diagnostic tool in immune-mediated disorders, but findings in PCD are controversial. Semiquantitative analysis of brain 18F-FDGPET has been shown to be of value in assisting visual assessment in several conditions and has been mainly applied to disclose regional hypometabolism. Though, semiquantitative data on regional hypermetabolism and even more longitudinal changes during the PCD course are lacking.

Materials and Methods: Herein, we describe a seronegative PCD associated with small cell lung cancer in which we performed brain $18 \mathrm{~F}$ FDG PET standard visual assessment and also semiquantitative analysis both in acute and post-treatment remission stages compared to a healthy control group.

Results: With respect to visual analysis, the semiquantitative analysis allowed to identify in the acute stage a more widespread hypermetabolism both in cerebellar and in some extracerebellar subcortical structures, likely underpinned by inflammation and mostly related to motor impairment. Also, hypometabolism in frontal areas, associated with behavior disorder was highlighted. During the motor-remission stage cerebellar hypermetabolism disappeared whereas cortical hypometabolism widespread, paralleling affective and cognitive disorders.

Conclusions: 18F-FDG PET assisted by semiquantitative analysis accurately disclosed the longitudinal pathological changes of brain metabolism occurring in the different phases of PCD and paralleling clinical impairment and response to treatment.

References:

- Ducray F, Demarquay G, Graus F, et al. Seronegative paraneoplastic cerebellar degeneration: the PNS Euronetwork experience. Eur J Neurol (2014);21:731-35

- Clapp AJ, Hunt CH, Johnson GB, et al. Semiquantitative analysis of brain metabolism in patients with paraneoplastic neurologic syndromes. Clin Nucl Med (2013);38:241-47

- Hoche F, Guell X, Vangel MG, et al. The cerebellar cognitive affective/Schmahmann syndrome scale. Brain (2018);141:248-70

\section{RITUXIMAB IN IGM ANTI-MYELIN-ASSOCIATED GLYCOPROTEIN NEUROPATHY: NEUROPHYSIOLOGICAL EVALUATION IN A 24 MONTHS FOLLOW UP}

M. Parisi ${ }^{1}$, B. Ferrero ${ }^{1}$, D. Bertuzzo ${ }^{2}$, A. Di Liberto ${ }^{3}$, I. Dogliotti ${ }^{4}$, G. Benevolo $^{5}$, L. Orsucci ${ }^{5}$, S. Ferrero ${ }^{4}$, L. Lopiano ${ }^{1}$ 
${ }^{1}$ Department of Neuroscience "Rita Levi Montalcini", University of Turin (Torino); ${ }^{2}$ Department of Neurology, Cardinal Massaia Hospital (Asti); ${ }^{3}$ Department of Neurology, San Giovanni Bosco Hospital (Torino); ${ }^{4}$ Division of Hematology 1, City of Health and Science University Hospital of Turin/University of Turin (Torino); ${ }^{5}$ Division of Hematology 2, City of Health and Science University Hospital of Turin (Torino)

Aims: We present data from 16 patients evaluated in a 24-months follow up, affected by IgM-MGUS (19\%) or Waldenstrom Macroglobulinemia $(81 \%)$ with anti-myelin-associated glycoprotein antibody demyelinating neuropathy (IgM anti-MAG) who underwent therapy with Rituximab $375 \mathrm{mg} / \mathrm{m} 2$, in order to define clinical and neurophysiological predictors of efficacy and criteria for patients selection.

Materials: In this longitudinal prospective study, conducted in collaboration with the Hematologic Department, sixteen patients with IgM antiMAG demyelinating neuropathy and never undergone immunomodulatory drugs were selected for rituximab therapy based on mSMART Mayo Stratification of Macroglobulinemia and Risk Adapted Therapy 2016 guidelines. They underwent neurological, hematologic (MYD88 assay), and neurophysiological evaluation at baseline, 6, 12 and 24 months after treatment.

Methods: For the assessment of the polyneuropathy, in terms of clinical severity and disability, we used validated scales such as MRC, mISS and INCAT, and QOL-scale. For the neurophysiological study we performed motor and sensory nerve conduction study, including the study of F wave, at four limbs. The Terminal Latency Index was calculated for median and ulnar nerves and a value $<0,25$ was considered significative for distal demyelination. Statistical analysis was made comparing distal latency, CMAP amplitude, conduction velocity and potential duration of each nerve both sides using the Wilcoxon non-parametric paired test.

Results: The analysis at 12 and 24 months from therapy showed lack of changes in MRC, mISS or INCAT, but a significant change in QOLscale. We observed, at the analysis of neurophysiological data, statistically significant improvement at 12 months $(\mathrm{p}<0,05)$ in ulnar motor distal latency $(\mathrm{p}<0,01)$ calculated on all patients and in median SAP amplitude and conduction velocity $(\mathrm{p}<0,05)$.

Discussion: Rituximab is a chimeric anti-CD20 antigen monoclonal antibody that induces depletion of peripheral B-cells and presumably reduces pathogenic monoclonal IgM protein and anti-MAG antibody titers. Two randomized trials comparing rituximab to placebo failed to demonstrate improvement on the primary end points. Based on our sample's baseline characteristics, as previously reported, response to rituximab seems to be associated with short duration and less severity of disease. In literature, few neurophysiological data are present in relation to rituximab therapy in anti-MAG neuropathic patients. We observed significant improvement in few nerve conduction parameters, no correlations with MRC, mISS and INCAT, but significant change in QOLscores at 12 and 24 months.

Conclusions: We suggest that some nerve conduction parameters, could be sensitive enough to measure improvement after rituximab treatment in anti-MAG neuropathy.

References:

- Svahn J, Petiot P, Antoine J. The Francophone anti-MAG cohort Group, et al Anti-MAG antibodies in 202 patients: clinicopathological and therapeutic features. Journal of Neurology, Neurosurgery \& Psychiatry (2018);89:499-505

- Dalakas MC, Rakocevic G, Salajegheh M, et al. Placebo-controlled trial of rituximab in IgM anti-myelin-associated glycoprotein antibody demyelinating neuropathy. Ann Neurol. (2009);65(3):286-93
- Léger JM, Viala K, Nicolas G, et al. Placebo-controlled trial of rituximab in IgM anti-myelin-associated glycoprotein neuropathy. Neurology (2013);80(24):2217-25

\section{A CASE OF RAPIDLY PROGRESSIVE POLINEUROPATHY WITH VENTILATORY FAILURE. AN ATYPICAL CIDP OR A DIFFERENT DISEASE WITH ANTIBODY-MEDIATED PATHOGENESIS?}

\author{
E. Sabatelli ${ }^{1}$, A. Conte ${ }^{1}$, K. Patanella ${ }^{1}$, G. Bisogni ${ }^{1}$, E. Meleo ${ }^{2}$, A. \\ Romano $^{1}$, R. Iorio ${ }^{1}$
}

${ }^{1}$ Neurology, Cattolica del Sacro Cuore (Roma); ${ }^{2}$ Pneumology, Cattolica del Sacro Cuore (Roma)

Background: Chronic inflammatory demyelinating polyneuropathy (CIDP) is an immune-mediated neuropathy with a reported prevalence as high as 8.9 per 100,0001. Although macrophage-induced demyelination is considered pivotal to the pathogenesis of CIDP, recent studies have indicated the presence of distinctive mechanisms initiated by autoantibodies against nodal/paranodal junction proteins. These disease subsets differ in terms of clinical presentation, pathological features, pathophysiological mechanisms and therapeutic implications. Severe respiratory muscle paralysis and ventilatory failure is rare, but has recently been described in patients of CIDP. [2,3]

Case Presentation: a 78-year-old man, with a history of multiple surgeries for lumbar and cervical stenosis, diabetes mellitus type II, in march 2018 presented with gradual onset of weakness and muscle atrophy in the distal lower limbs, with subsequent involvement of the upper limbs. Over the following year he lost the ability to walk. He also experienced respiratory failure requiring ventilatory support since november 2019. On our evaluation in november 2019 the patient presented moderate-severe strength deficit with disto-prossimal gradient in his limbs with distal numbness. EMG/ENG showed a diffuse demyelinating and axonal sensorimotor polyneuropathy, with signs of neurogenic injury and ongoing denervation; brain and spinal cord MRI were unremarkable except for a mild lumbar stenosis. Spirometry showed a chronic ventilatory failure with restrictive pattern (FVC 53\%). Indirect immunofluorescence assay on mouse brain sections revealed an IgG-specific immunoreactivity restricted to the cerebellum, hippocampus, thalamus, cerebral cortex and myoenteric plexus. Onconeural antibodies (anti-Hu, Anti-Yo, Anti-Ri, Anti-Amfifisina, Anti-CV2, Anti- Ma-2/Ta) were negative. Total-body CT scan showed no abnormalities. The patient was treated with multiple cycles IVIG and oral steroids, without benefit. Because of worsening symptoms in february 2020 he was treated with two cycles $(1 \mathrm{~g} /$ cycle) of Rituximab, with clinical improvement.

Discussion and conclusions: The case has some aspects that differ from the CIDP spectrum. First of all, the neuropathy evolved with a rapid progressive course. The presence of respiratory failure, not correlated to extent of deficits, and the central nervous system staining on the IFA, arise the suspicion of a central origin of the ipoventilation. This element and the good response to Rituximab may suggest an antibody-mediated pathogenesis.

References:

1. Laughlin RS, Dyck PJ, Melton LJ 3rd, Leibson C, Ransom J, Dyck PJ. Incidence and prevalence of CIDP and the association of diabetes mellitus. Neurology (2009) Jul 7;73(1):39-45. doi: 10.1212/ WNL.0b013e3181aaea47. PMID: 19564582; PMCID: PMC2707109 
2. Henderson RD, Sandroni P, Wijdicks EF. Chronic inflammatory demyelinating polyneuropathy and respiratory failure. J Neurol. (2005) Oct;252(10):1235-7. doi: 10.1007/s00415-005-0848-2. Epub 2005 Jun 13. PMID: 15940387

3. Polat M, Tosun A, Serdaroğlu G, Cağlayan E, Karapinar B, Gökben S, Tekgül H. Chronic inflammatory demyelinating polyradiculopathy: an atypical pediatric case complicated with phrenic nerve palsy. Turk J Pediatr. (2007) Apr-Jun;49(2):210-4. PMID: 17907525

\section{EPIDEMIOLOGY OF NEURONAL-SURFACE ANTIBODY- MEDIATED AUTOIMMUNE ENCEPHALITIS AND ANTIBODY- BASED DIAGNOSTICS: A 13-YEAR SINGLE-CENTER EXPERIENCE}

L. Zuliani ${ }^{1}$, P. De Gaspari ${ }^{2}$, S. Marangoni ${ }^{3}$, I. Rosellini ${ }^{4}$, M. Nosadini ${ }^{5}$, V. De Riva ${ }^{1}$, E. Galloni ${ }^{1}$, S. Sartori ${ }^{5}$, F. Perini ${ }^{1}$, M. Zoccarato ${ }^{6}$, B. Giometto $^{3}$

\begin{abstract}
${ }^{1}$ Department of Neurology, San Bortolo Hospital, Azienda ULSS8 Berica (Vicenza); ${ }^{2}$ Neuroimmunology Group, Pediatric Research Institute Città della Speranza (Padova); ${ }^{3}$ Department of Neurology, Santa Chiara Hospital (Trento); ${ }^{4}$ Department of Neurology, Azienda ULSS2 Marca Trevigiana (Treviso); ${ }^{5}$ Department of Women and Children Health, Paediatric Neurology and Neurophysiology Unit, University of Padua (Padova); ${ }^{6}$ O.S.A. Department of Neurology, Azienda Ospedale University of Padua (Padova)
\end{abstract}

Background: Autoimmune Encephalitis (AEs) and specifically Neuronal Surface Antibody (NSAb)-associated AE (NSAE) are rare diseases generating rapidly increasing interest in the last 15 years. However, epidemiological data are scarce and depend on different antibody-based diagnostic approaches.

Objective: To review the 13-year biobank-data collection by our Neuroimmunology Service and provide the incidence of NSAE in Trento and Treviso provinces over a 5-year period.

Methods: In 2005 the Neuroimmunology Service for Anti-Neuronal antibodies based in Padova and subsequently in Treviso started collecting samples to be tested for NSAbs. Samples were tested for neuronal antibodies at the referring clinicians request and according to the availability of assays for novel neural antigens. The epidemiological analysis included all NSAbpositive adult patients with a confirmed diagnosis of AE (according to Graus 2016 criteria), residing in the catchment areas of Treviso and Trento (global population of approx. 1,400,000) between July 2013 and June 2018, and prospectively followed up. During this 5-year period all samples from adult cases from the two provinces with a possible $\mathrm{AE}$ were tested by our service in accordance with the standard diagnostic approach recommended by the Italian Association of Neuroimmunology (AINI). Positive samples were independently retested for confirmation by Vicenza Laboratory of Neurobiology. Published studies documenting epidemiological data on NSAE including case series reporting rates and frequency of antibodybased diagnosis of NSAE were reviewed and compared.

Results: Between 2005 and 2018, our Neuroimmunology Service diagnosed 159 patients with serum and/or CSF testing positive for wellcharacterized Anti-Neuronal Antibodies (NAb): NAb with an intracellular target (i.e. onconeural antibodies and GAD65) $=48$ patients; Neuronal Surface Antibodies (NSAb) $=111$ patients. The most common NSAbs were: LGI1 (46 patients), NMDAR (42) and CASPR2 (18). Other NSAbs included: AMPAR (2), GABAbR (2) and GlyR (2). During the 5-year study period (July 2013 - June 2018), 12 cases of confirmed NSAE in Treviso and Trento provinces tested positive for NSAb (Lgil $=6$, NMDAR $=3$, CASPR2 $=1$, GABAbR $=1$, GlyR $=1$ ). The estimated incidence of NSAE in the adult population was about 0.2 per 100,000 .

Conclusions: Lgil-NSAE is the most frequent neuronal antibodyassociated form of AE among adults. NSAE are confirmed to be rare diseases in line with published data. Published data on the incidence of NSAE are heterogeneous and largely dependent on classification, diagnostic approach, availability of assays, and clinical and laboratory expertise. Larger multicentric epidemiological studies are warranted.

References:

Zuliani L, Nosadini M, Gastaldi M et al. Management of antibodymediated autoimmune encephalitis in adults and children: literature review and consensus-based practical recommendations. Neurol Sci. (2019) Oct;40(10):2017-30

- Zuliani L, Zoccarato M, Gastaldi M et al. Diagnostics of autoimmune encephalitis associated with antibodies against neuronal surface antigens. Neurol Sci. (2017) Oct;38(Suppl 2):225-29

- Dubey AD, Pittock SJ, Kelly CR et al. Autoimmune encephalitis epidemiology and a comparison to infectious encephalitis. Ann Neurol (2018);83(1):166-77

\section{NEURO-ONCOLOGY}

\section{A STROKE ONSET OF LHERMITTE-DUCLOS DISEASE. CASE REPORT AND A COMPREHENSIVE LITERATURE REVIEW}

\author{
F. Barbato ${ }^{1}$, F. Gabriele ${ }^{2}$, B. Romano ${ }^{2}$, L. Fraioli ${ }^{2}$, M. Carello ${ }^{2}$ \\ ${ }^{1}$ Neurology, Catholic University of Sacred Heart (Napoli); ${ }^{2}$ UNAI, San \\ Raffaele (Cassino-FR)
}

Introduction: Lhermitte-Duclos disease is characterized by the presence of a hamartomatous lesion located in cerebellum. It is a rare disease, only 302 cases having been reported in the literature with greater prevalence in the age group between 30 and 50 years [1]. A key point is the possibility of association with Cowden syndrome [1].

Case Report: We describe a case of a 51-year-old lady presented with left limb hyposthenia, dysarthria and unconsciousness that began abruptely. She was hospitalized immediately in view of a possible thrombolytic treatment and underwent a CT scan. Although there was a hypodense area in the left cerebellar hemisphere, no fibrinolytic therapy was administrated due to the presence of microhaemorrhagic spots. For the presence of mass effect an external ventricular derivation (DVE) was placed. Later a new neurological evaluation documented a bilateral cerebellar syndrome, dysarthria and papilledema. Therefore, she was subjected to brain MRI with gadolinium and a diagnosis was made. All tests were negative for Cowden's syndrome. Subsequent interviews with family members and the patient, a slowly evolving cerebellar syndrome associated with headache has been documented since 2009. A genetic investigation was requested for PTEN.

Discussion: So we did a review of literature, and we identified 138 articles, and, of these, most were case reports (79 articles), and 2 articles were case series. So far, there are about 302 cases published in the literature at today (2 reports are Italian). In this report cerebral ischemia was considered for the patient. Although the radiological picture showed a totally anomalous bilateral involvement as a vascular territory, the subsequent anamnestic connection with family members also suggested an evolution of cerebellar symptoms starting from 2009. The definitive treatment of LDD is surgical resection [2]. The case study of Jiang et al, the 
most numerous so far, has shown that if surgical resection is possible, usually in over $50 \%$ of cases, there is no recurrence or that the risk that arises is very low. In this case complete resection of the tumor is difficult due to the impossibility of clearly defining its margins [3].

Conclusion: Lhermitte-Duclos disease is considered a rare cause of progressive headache, associated with cerebellar symptoms. The T2weightened MRI, which can demonstrate the classical "tiger-striped" pattern, is the preferential exam. While tumor resection is recommended for all symptomatic patients, a conservative management may be considered for incidentally diagnosed asymptomatic patients.

References:

1. Ideta, Paschoalino, Oliveira, dos Santos, Sanz, Oliveira de Amorim. Lhermitte-Duclos Disease and Cowden Syndrome: A Case Report and Literature Review. Arquivos Brasileiros de Neurochirurgia: Brazilian Neurosurgery (2019);38(04):319-23. DOI: 10.1055/s0039-1693682

2. Barone F, Noubari BA, Torrisi A, Lanzafame S, Tropea R, Mancuso P. Lhermitte Duclos disease and Cowden disease: clinical, pathological and neuroimaging study of a case. J Neurosurg Sci. (2000);44(4):234-37

3. Jiang T, Wang J, Du J, et al. Lhermitte-Duclos Disease (Dysplastic Gangliocytoma of the Cerebellum) and Cowden Syndrome: Clinical Experience from a Single Institution with Long-Term Follow- Up. World Neurosurg (2017);104:398-406

\section{GLIOBLASTOMA MIMICKING TEMPORAL ENCEPHALITIS}

\section{A. G. L. Botturi, P. Gaviani, E. Lamperti, G. Simonetti, A. Silvani}

Neuro-oncology, Istituto Neurologico C. Besta (Milano)

Introduction: Glioblastoma (GBL) is a malignant tumor with a short clinical medical history and poor prognosis. Classical presentation rise by direct mass effect with focal neural and cognitive deficits. Also, there are effects due to increased intracranial pressure and epilepsy. A minority of patients have atypical presentations with relevant psychiatric symptoms. The management of these patients is complicated, especially if the images are not eloquent, and the histological diagnosis is not proven. Differential diagnosis includes temporal autoimmune encephalitis or cooccurrence of viral coinfection (herpetic type).

Case Report: A 69 years old male patient developed memory impairment, subsequently personality change and mood deflection, so that it was sent to the psychiatrist specialist. Magnetic Resonance Imaging (MRI) showed a hyperintense signal in T2-weighted images of mesial temporal structures with bilateral localizations and without pathological contrast enhancement. CSF analysis did not reveal specific alterations, but only a slight increase in anti GluR3 antibodies. Polymerase Chain Reaction (PCR) was negative for the Herpes virus. EEG was not pathognomonic. The patient has been treated first with steroids, which led to clinical worsening. A high dose of immunoglobulin slightly improved cognitive deficits but did not affect the neuroradiological data. After 3 months of the neuroradiological diagnosis, the patients developed a status epilepticus. The subsequent MRI showed a swelling of the temporal lobe and the presence of signal alteration at the level of the thalamus and the right capsula. Brain Positron Emission Tomography (PET) with FDG did not show hypermetabolism. Patients underwent a biopsy, which showed the presence of a GBL.

Discussion: The patient had a peculiar presentation of GBL with predominant psychiatric symptoms. The lesion was diffused but with a little tendency to grow. The neurological picture mimicked temporal encephalitis or a viral coinfection. Moreover, the patient presented several elements that disagreed with each other: non-typical lesion morphology, negativity for herpetic viruses, and antibodies for autoimmune encephalitis. Furthermore, brain PET showed no hypercaptation.

Conclusion: A biopsy should accompany inconclusive tests for encephalitis because a GBL could be hidden.

References:

- Tai-Seung N, Kang-Ho C, Myeong-Kyu K, Ki-Hyun C. Glioblastoma Mimicking Herpes Simplex Encephalitis. J Korean Neurosurg Soc. (2011) Aug; 50(2): 119-22

- Vogrig A, Joubert B, Ducray F et al. Glioblastoma as differential diagnosis of autoimmune encephalitis. J Neurol. (2018) Mar;265(3):669-77

\section{PANCREATIC MENINGIOMA METASTASES IN PATIENTS WITH LOCOREGIONAL RECURRENCE AND EXTRACRANIAL METASTASIS}

\section{A. G. L. Botturi, P. Gaviani, E. Lamperti, G. Simonetti, A. Silvani}

Neuro-oncology, Istituto Neurologico Carlo Besta (Milano)

Introduction: Meningiomas (MG) are the most frequent brain tumor; (in the USA, about 36\%). According to the World Health Organization (WHO) MG can be classified into 3 grades (benign, atypical, anaplastic/malignant). Generally, they have a favorable prognosis. However, about $1-2 \%$ of $M G$ exhibit malignant behavior, with locoregional recurrence (in a percentage between 65 and 100\%) and remote diffusion. Extracranial MG metastases are considered a rare event. The most frequently affected sites are lung, bones, spine, liver, exceptional lymph nodes, cute, muscle. Histological grading can only partially predict the risk of local recurrence. According to a recent review, more than $50 \%$ of MG with distant metastasis originate from tumors classified as a benign or intermediate grade.

Case Report: A 43-year-old male patient underwent brain falcine parasagittal MG removal, ivolving inferior sagittal sinus. Histological diagnosis (DI) was grade I transitional meningioma focally infiltrating the dura meninges (Ki67 1\%). Two locoregional recurrences 2 and 5 years after their onset were treated with CyberKnife. Seven years after the first diagnosis, a computed tomography (CT) of the chest showed a pulmonary mass in the left apex, a lesion of the VII ipsilateral rib, and pancreatic nodulations. The total-body positron emission tomography (TB-PET) exam confirmed the locations. He underwent costal resection with DI = spindle cell proliferation and transitional and fibrous growth patterns with immunophenotype EMA +, progesterone +, S 100-CD34cytokeratins AE1AE3 negative. MIBI 5\% mitosis 4 / 10HPF. The needle aspiration of pancreatic lesions showed localization of MG.

Discussion: Our patient with a history of recurring disease showed a peculiar localization of extracranial metastases. According to previous literature, histological grading does not explain the extremely aggressive behavior of the meningioma. The dissemination pathways considered responsible for dissemination are the passage at the level of the right atrium into the inferior vena cava; paravertebral plexus, the lymphatic pathway; the cerebral spinal fluid. The patient was suffered for thoracic localization, and surgical and radiation treatment has been attempted to keep them under control.

Conclusion: Meningioma extracranial metastases are increasingly reported in the literature. However, there is still no precise protocols to detect and treat them. A histological registry could be useful to stratify the most at-risk patients and to be able to carry out oncological surveillance and provide indications for guidelines.

References:

- Dolecek T A, Propp J M, Stroup N E, Kruchko C. CBTRUS statistical report: primary brain and central nervous system tumors 
diagnosed in the United States in 2005-2009. Neuro Oncol. (2012) Nov;14 Suppl 5(Suppl 5):v1-49

- Louis D N, Perry A, Reifenberger G et al. The 2016 World Health Organization Classification of Tumors of the Central Nervous System: a summary. Acta Neuropathol. (2016) Jun;131(6):803-20

- Surov A, Gottschling S, Bolz J et al. Distant metastases in meningioma: an underestimated problem. J Neurooncol. (2013) May;112(3):323-7

\section{LIQUID BIOPSY OF THE CEREBROSPINAL FLUID IN A COHORT OF GLIOBLASTOMA PATIENTS}

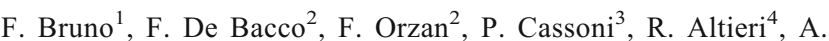 \\ Melcarne $^{4}$, D. Garbossa ${ }^{4}$, C. Boccaccio ${ }^{2}$, R. Soffietti ${ }^{1}$, R. Rudà $^{1}$
}

\begin{abstract}
${ }^{1}$ Dept. of Neuro-Oncology, City of Health and Science Hospital (Torino); ${ }^{2}$ Laboratory of Cancer Stem Cell Research, Candiolo Cancer Institute, FPO-IRCCS (Candiolo-TO); ${ }^{3}$ Pathology Unit, Department of Medical Science, University and City of Health and Science Hospital (Torino); ${ }^{4}$ Neurosurgical Unit, Department of Neuroscience, University and City of Health and Science Hospital (Torino)
\end{abstract}

Introduction: Liquid biopsy by cerebrospinal fluid (CSF) can be useful to identify circulating tumour DNA (ctDNA). The aim of our study is to assess the effectiveness of liquid biopsy of the CSF in detecting ctDNA which mirrors the same genetic profile of the tumour, and to investigate the clinical and radiological aspects influencing the availability of CSF ctDNA.

Material and Method: Tumour tissue and CSF samples of 13 GBM patients undergoing surgery was collected. CSF was withdrawn from the proximity of the tumour surface before the excision. DNA extracted from tissue was analysed by qPCR to identify genetic alterations such as copy number variations (EGFR, PDGFRA, CDK4, MDM2, CDKN2A), and point mutations (TP53, PTEN, IDH, NRAS, PI3K1, pTERT). CtDNA extracted from CSF was analysed by droplet digital PCR to assess the presence of the alterations found in the matching tissue. Both contrastenhanced (CE) and FLAIR volumes of the lesions were measured in the MRI

Results: From June 2016 to February 2020 we prospectively collected 13 GBM patients. Median age was 73 years. All lesions showed CE at the MRI; other radiological findings included necrosis $(84.6 \%)$, oedema (76.9\%), cortical, ventricular or meningeal involvement $(76.9 \%, 30.8 \%$, and $15.4 \%$ respectively). Median volumes of CE and FLAIR lesions were 28.6 and $25.5 \mathrm{~cm} 3$, with a median FLAIR/CE ratio of 72.9. Surgery was subtotal $(<95 \%)$ in all patients. All GBM tissues were tested for the following alterations: EGFR, PDGFRA, CDK4, MDM2, CDKN2A; 76.9\% were tested for TP53, PTEN, and IDH mutations; $38.5 \%$ for NRAS and pTERT mutations; $30.8 \%$ for PI3KR1 mutation. MGMT methylation was assessed in 12 cases $(92.3 \%)$ and found in $7(58.3 \%)$. Median CSF volume, ctDNA quantity and concentration were $0.45 \mathrm{~mL}$, $59.64 \mathrm{ng}$, and $0.42 \mathrm{ng} / \mu \mathrm{L}$, respectively. Processable DNA was found in 11 CSF specimens $(84.6 \%)$, in 8 of which $(61.5 \%)$ it carried the same alteration expressed by the matched tissue, while in 3 cases $(23.1 \%)$ it showed a different genetic profile; finally, in 2 cases it was not possible to detect any circulating DNA in the CSF. Preliminary data on 13 patients suggest that the ctDNA concentration in the CSF could be related to the FLAIR/CE volume ratio $(\mathrm{p}=0.02)$.

Conclusions: Our study confirms that liquid biopsy of CSF can detect ctDNA carrying the same molecular profile harboured in the tumour. Therefore, it seems to be an accurate method to identify markers useful for the diagnosis and the monitoring of the disease. Additionally, our ongoing study is trying to demonstrate a potential correlation between radiological features of the tumour and availability of ctDNA in CSF.

References:

- Wang Y, Springer S, Zhang M, et al. Detection of tumor-derived DNA in cerebrospinal fluid of patients with primary tumors of the brain and spinal cord. Proc Natl Acad Sci USA. (2015);112(31):9704. doi:10.1073/pnas.1511694112

- De Mattos-Arruda L, Mayor R, Ng CKY, et al. Cerebrospinal fluidderived circulating tumour DNA better represents the genomic alterations of brain tumours than plasma. Nature Communications. (2015);6(1):8839. doi:10.1038/ncomms9839

- Miller AM, Shah RH, Pentsova EI, et al. Tracking tumour evolution in glioma through liquid biopsies of cerebrospinal fluid. Nature (2019);565(7741):654-658. doi:10.1038/s41586-019-0882-3

\section{COGNITIVE FUNCTIONING IN ADULT MEDULLOBLASTOMA}

\author{
A. Carpaneto, F. Franchino, A. Pellerino, F. Bruno, F. Mo, E. Muscolino, \\ R. Rudà, R. Soffietti
}

Department of Neuro-Oncology, University and City of Health and Science Hospital (Torino)

Impaired neurocognitive functions as consequence of treatments are wellestablished in pediatric cases of medulloblastoma, while its frequency and features are quite unknown in adult cases. In this study, cognition was evaluated in long-term survivors ( $>5$ years follow up without disease recurrence) with a range interval from first line treatments of 5-21 years (median 10 years). All subjects were in follow-up at the time of the neuropsychological evaluation, which took place in conjunction with the six-monthly control visit. Ten patients were recruited between January and July 2019 for the analysis. Six males and 4 females with an age range of 25-53. Neurocognitive functioning was assessed using an ad hoc battery, administered by a neuropsychologist. The overall duration of the evaluation was approximately 30 minutes. Investigated domains are: verbal long-term memory; verbal and spatial working memory; executive functions; sustained visual attention, ideo-motor speed, spatial research; and lexical verbal fluency. Furthermore, health-related quality of life was evaluated at the same time of cognitive evaluation. All neurocognitive scores were corrected for age and schooling and compared to normative data. Descriptive statistics was used for data analysis to observe neurocognitive functioning and quality of life of these patients. In our study qualitative and quantitative results emerging from cognitive assessment are quite heterogeneous, but revealed a conserved clinical picture overall and demonstrated major affections in executive functions, verbal and spatial working memory. Cognitive deficits were found mostly (at least two domains under the cut-off) in four patients, but this fact appeared independent from socio-demographic variables. These global cognitive findings are in line with data reported in some recent studies on adult population. Unless lower cognitive performances QoL seemed, in our cohort, well preserved. Overall the neurocognitive evalutation suggests that after years of diagnosis and therapies for adult medulloblastoma cognitive functions are relatively preserved in most of patients. Surely specific neuropsychological and QoL assessment tools target to specific domains and prospective assessment from diagnosis and before any treatment, could be crucial in these patients to correctly classify and understand cognitive assessment. Limitations of this analysis are the retrospective nature and small number of cases, however the long follow up and data taken from real life correlate with other results from previous studies 
and underline the need of prospective multicentric trials to better identify patients who might profit from individualized therapy regimens.

Reference:

- R.A. Harrison, S. R. Kesler, J. M. Johnson, M. Penas-Prado, C. M. Sullaway, J. S. Wefel. Neurocognitive dysfunction in adult cerebellar medulloblastoma. Psycho-oncology (2019);28:131-38

\section{INCIDENCE 2016-2019 OF PRIMARY CNS TUMORS IN SOUTHERN SARDINIA}

\author{
S. Dagostino ${ }^{1}$, G. Piga ${ }^{2}$, V. Pierri ${ }^{2}$, F. Bruder ${ }^{3}$, P. Cappai ${ }^{4}$, C. Conti ${ }^{4}$, C.

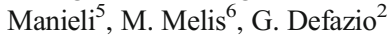 \\ ${ }^{1}$ Emergency Department, Neurology Department, AOU Cagliari, \\ University of Cagliari (Cagliari); ${ }^{2}$ Department of Medical Sciences and \\ Public Health, Institute of Neurology, University of Cagliari and AOU \\ Cagliari (Cagliari); ${ }^{3}$ Department of Medical Oncology, Hospital "A. \\ Businco" (Cagliari); ${ }^{4}$ Department of Neurosurgery, Hospital "G. \\ Brotzu" (Cagliari); ${ }^{5}$ Service of Pathological Anatomy, Hospital "G. \\ Brotzu" (Cagliari); 'Department of Neurology, Hospital "G. Brotzu" \\ (Cagliari)
}

Objective: To date there is no established Cancer Registry recording case patients from Southern Sardinia. This study aims to assess the incidence of Central Nervous System Neoplasms in the population of South Sardinia, over a four-year span.

Materials and Methods: Information was obtained from clinical records of patients diagnosed with a primary CNS Tumor at the Departments of Neurology, Neurosurgery, Pathology and Neuroncology of southern Sardinian hospitals during 2016-2019. The study area included Cagliari Metropolitan Area and South Sardinia province. The latter area included 3 different subareas (Sulcis - Iglesiente, Campidano, Sarrabus). Demographics of Southern Sardinia general population were retrieved from Italian National Institute of Statistics. Incidence rates (x 105 population/year) were calculated in the years 2016-2019, and compared with the correspondent rates from the Italian Association of Medical Oncology yearly epidemiologic report [1] and the American CBTRUS statistical reports [2]. To this aim, tumors were classified according with the WHO 2016 Classification of Tumors of the Central Nervous System and grouped in two categories, non-malignant tumors (meningiomas, pituitary adenomas, schwannomas) and malignant tumors (diffuse astrocytic and oligodendrial tumours, ependymal tumours, Choroid plexus tumours, tumors of the pineal region, embryonal tumours, tumors of the sellar region, lymphomas, Mesenchymal tumours).

Results: In the study area, 476 patients were diagnosed with CNS Tumors during 2016-2019. The overall incidence was 15.14x105/year. Incidence rates were 7.19x105/year for primary non-malignant CNS Neoplasms, and $7.95 \times 105 /$ year for primary malignant CNS neoplasms. Diagnosis of malignant tumors was supported by histologic examination in $85.2 \%$ of cases.

Discussion and Conclusions: Our findings would suggest a lower incidence of non-malignant tumors in Southern Sardinia than in the USA (15.81 x 105/year. This was probably due to incomplete ascertainment of meningiomas in our setting. The incidence of malignant CNS Tumors tend to be higher than in the USA $(7,95$ vs. 6,84$)$ but lower than the expected 2017-2019 incidence of the correspondent tumors in the Italian population (10.1X105/year).

References:

1. AIOM, AIRTUM I numeri del cancro in Italia - 2017, 2018, 2019

2. Ostrom QT, Cioffi G, Gittleman H, et al. CBTRUS Statistical Report: Primary Brain and Other Central Nervous System
Tumors Diagnosed in the United States in 2012-2016. Neuro Oncol. (2019);21(Suppl 5):v1-v100. doi:10.1093/neuonc/ noz150

\section{CLINICO-RADIOLOGICAL, MOLECULAR AND THERAPEUTIC FEATURES OF MEDULLOBLASTOMA SUBGROUPS OF THE ADULT: RESULTS FROM AN INSTITUTIONAL SERIES}

\author{
F. Franchino ${ }^{1}$, V. Interno ${ }^{1}$, F. Roveta ${ }^{1}$, E. Pronello ${ }^{1}, \mathrm{~F}_{\text {. Bruno }}{ }^{1}, \mathrm{~F} . \mathrm{Mo}^{1}$, \\ A. Pellerino ${ }^{1}$, I. Morra ${ }^{2}$, C. Mantovani ${ }^{3}$, R. Ruda ${ }^{1}$, R. Soffietti ${ }^{1}$
}

${ }^{1}$ Department of Neuroscience, division of Neuro-Oncology, University and City of Health and Science Hospital of Turin (Torino); ${ }^{2}$ Department of Anatomo Pathology, University and City of Health and Science Hospital of Turin (Torino); ${ }^{3}$ Department of Radiotherapy, University and City of Health and Science Hospital of Turin (Torino)

Introduction: Medulloblastoma is a highly malignant, embryonal and cerebellar tumor of the childhood. In adults it is rare and showed quite distinct clinical, histopathological, molecular and treatment response features.

Patients and Methods: We retrospectively included 44 adults (17-48 years), with a histological diagnosis of medulloblastoma. In 23 of them immunohistochemistry identified the molecular subgroups and in this sub-population we described the main demographic, diagnostic and therapeutic data, calculating PFS (progression-free-survival) and OS (overall survival).

Results: At molecular characterization 17 patients were SHH and 6 non-WNT/non-SHH (5 group 4 and 1 group 3); no WNT subgroup was found. In SHH subgroup prevail the classic histology in $82 \%$ (14 patients), with only 2 cases of nodular/desmoplatic and 1 anaplastic variant, while in group 4 there were 3 classic and 2 desmoplastic/nodular histology (60\% and $40 \%$ respectively); patient of group 3 showed a classic variant. In all subgroups we observed a male prevalence. Median age at diagnosis was higher in SHH (32 years) and group 3 (48 years) than in group 4 (23 years). Prevalent onset symptoms, primary tumor site and MRI images were similar in all subgroups (lateral and paravermian cerebellar localization of inhomogeneous contrast-enhanced lesions), only $1 \mathrm{SHH}$ showed infra and supratentorial bilateral localizations. Clinical or radiological signs of meningeal involvement at diagnosis prevail in SHH subgroup (9/17: 53\%) compared to group 4 (1/5: $20 \%)$. Ten SHH patients underwent a STR (subtotal resection) (59\%), 6 a GTR (gross-total resection) (35\%) and 1 only a biopsy (6\%) while in group 4 three patients underwent a GTR and 2 STR $(60 \%$ and $40 \%$ respectively); in group 3 a STR was made due to diffuse meningeal involvement. Patients classified as High risk were 11/17 in SHH $(65 \%)$ and $1 / 5(20 \%)$ in group 4 . Group 3 patient was classified as an HR. All patients underwent craniospinal irradiation associated in $10 / 17 \mathrm{SHH}$ cases, in $4 / 5$ group 4 cases and in group 3 patient with chemotherapy. Eight SHH patients (47\%) demonstrated a PD (mainly with local and meningeal involvement: 5/8) with a median time of 29 months, while only 1 patient of group 4 showed a PD after 97 months. Median OS were 5.8, 7.8 and 1.5 years in SHH, group 4 and group 3 patients, respectively.

Conclusions: Adult medulloblastoma molecular subclassification could help to better understand clinical and treatment response characteristics defining tailored therapies in different prognostic subgroups, also considering the availability of target therapy, such as vismodegib in SHH-subgroup. 


\section{References:}

- Atalar B, Ozsahin M, Call J, et al. Treatment outcome and prognostic factors for adult patients with medulloblastoma: the Rare Cancer Network (RCN) experience. Radiother Oncol (2018); 127(1):96-102

- Thompson EM, Hielscher T, Bouffet E, et al. Prognostic value of medulloblastoma extent of resection after accounting for molecular subgroup: a retrospective integrated clinical and molecular analysis. Lancet Oncol (2016);17(4):484-95

- Liu X, Ding C, Tan W, Zhang A Medulloblastoma: molecular understanding, treatment evolution, and new developments. Pharmacol Ther (2020);210:107516. https://doi.org/10.1016/j.pharmthera. 2020.10751

\section{DETERMINANTS OF WORKABILITY IN PATIENTS WITH BRAIN TUMOR AND BREAST CANCER: PRELIMINARY RESULTS FROM THE LOMBARDY REGION PROSPECTIVE STUDY}

M. Leonardi ${ }^{1}$, F. Tagliavini ${ }^{2}$, A. Silvani ${ }^{3}$, E. Lamperti ${ }^{3}$, S. Schiavolin ${ }^{1}$, G. Apolone $^{2}$, S. Di Cosimo ${ }^{4}$, S. Folli ${ }^{5}$, F. Silvaggi ${ }^{1}$

${ }^{1}$ Neurology, Public Health and Disability Unit, Neurological Institute C. Besta IRCCS Foundation (Milano); ${ }^{2}$ Scientific Direction, Neurological Institute C. Besta IRCCS Foundation (Milano); ${ }^{3}$ Neuro-Oncology Unit, Neurological Institute C. Besta IRCCS Foundation (Milano); ${ }^{4}$ Department of Molecular, National Cancer Institute of Milan (Milano); ${ }^{5}$ Department of General Surgery 3, National Cancer Institute of Milan (Milano)

Objectives: An increasing number of cancer survivors return to work following surgery reporting consistent limitations at work due to their health conditions. Patients often see return to work as a mark of complete recovery and of regaining a normal life. The aim of the study is to examine changes in work ability scores in patients with brain cancers and patients with breast cancer at 6 and 12 months after surgery and to assess the factors related to their workability. Some studies show that low work ability in oncology patients is influenced by the duration of disease and by treatment with radiation and chemotherapy We present the methodology and preliminary result of a study funded by Lombardy Region, that started in September 2019 and will finish in February 2021.

Materials: 7 questionnaires and a semi-structured interview were used to obtain information on fatigue, social support, resilience, cognitive difficulties, disability.

Method: An observational prospective study on patients of all workingages, undergoing brain or breast surgery in the period between March 2019 and February 2020 has been done. The sample is composed of 27 patients with brain cancer (glioma II and III) and 40 patients with breast cancer enrolled after surgery. Patients had to be between 18 and 65 years, in paid employment at time of surgery and Italian mother-tongue. They have been consecutively recruited in national National Cancer Institute and National Neurological Institute Besta in Milan. The study has been carried out with the approval of the Institutes' ethical committees. Questionnaires were distributed at 6 and 12 months after surgery. The evaluations consist of online questionnaires sent to both groups of patients via email. The methodology include also interviews to $20 \%$ of sample.

Results: Preliminary results show a relationship between high scores of resilience and high scores of work ability. Low scores of fatigue are associated to high scores of workability.
Conclusions: The measure of workability allows to take appropriate actions to prevent declining capacity. Occupational health professionals can help patients to continue working and extend their professional lives as much as possible with programs ad hoc. Future studies should take into account the differences between self-employed versus employees, the differences in working in small medium or large enterprises, and the impact of cognitive deficits on work-related activities.

\section{THE EVALUATION OF PATIENTS' FUNCTIONING TO IMPROVE OUTCOME PREDICTION IN NEUROSURGERY}

\author{
M. Leonardi ${ }^{1}$, P. Ferroli ${ }^{2}$, M. Broggi ${ }^{2}$, S. Schiavolin ${ }^{1}$
}

${ }^{1}$ Neurology, Public Health and Disability Unit, Neurological Institute C. Besta IRCCS Foundation (Milano); ${ }^{2}$ Department of Neurosurgery, Neurological Institute C. Besta IRCCS Foundation (Milano)

Objectives: The outcome assessment and the identification of prognostic factors in brain tumor surgery are essential for therapeutic decisions. An agreement on which factors are more significant for outcome prediction does not exist and the influence of psychological and environmental factors on patients' perception of their health status is not usually investigated. In addition to clinical outcome evaluation performed by neurosurgeons, patients' perspective and the evaluation of their functioning are becoming more and more important in therapeutic decisions with a consequent increase of the use of patients reported outcome measures (PROMs). As reported in previous studies, patients and neurosurgeons can assess surgical outcomes differently. Our aim is to present a study on outcome predictors in neurosurgery using a protocol evaluating patients' functioning and to show some preoperative results.

Material: We are evaluating disability with WHODAS-12, quality of life with EORTC QLQ, lifestyle, resilience with the RS-14, social support with the OSS-3, mood state with the HADS, personality traits with the TIPI.

Results: 58 patients, 22 with glioma and 36 with meningioma, have been enrolled so far. The preliminary preoperative mean scores are WHODAS-12 $=24.0 \pm 19.7$; HADS (emotional distress) $=12.7 \pm 7.3$; OSS$3=11$; RS-14=77.4; EORTC QLQ= $57.7 \pm 26.3$ and the most frequent personality type is conscientiousness.

Discussion: The holistic approach of our study is providing a more complete patients' profile taking into account also patients' functioning. Personalized information allow to better predict the postoperative course on the basis of patients' specific needs as well as of their personality and environment. In fact, the same diagnosis or clinical condition might have different effects on daily life and could be experienced differently by patients.

Conclusion: This study is an example of the application of the biopsychosocial model on which ICF is based that could be used also in other surgical settings and with other pathologies. Furthermore, from our data we will develop a simple and easy to use questionnaire to routinely investigate patient's functioning during a preoperative evaluation.

\section{PATTERNS OF CARE IN 335 PATIENTS WITH SURGICALLY RESECTED BRAIN METASTASES FROM DIFFERENT SOLID TUMORS: A RESTROSPECTIVE SINGLE INSTITUTIONAL ANALYSIS}

F. Mo $^{1}$, V. Internò ${ }^{1}$, L. Salvati ${ }^{2}$, F. Franchino ${ }^{1}$, A. Pellerino ${ }^{1}$, L. Bertero ${ }^{3}$, D. Garbossa ${ }^{2}$, C. Mantovani ${ }^{4}$, R. Rudà ${ }^{1}$, R. Soffietti ${ }^{1}$ 
${ }^{1}$ Clinical Neuro-Oncology Unit, City of Health and Science and University of Turin (Torino); ${ }^{2}$ Neurosurgery Unit, City of Health and Science and University of Turin (Torino); ${ }^{3}$ Pathology Unit, City of Health and Science and University of Turin (Torino); ${ }^{4}$ Radiotherapy Unit, City of Health and Science and University of Turin (Torino)

Aim: Evaluating clinical features and outcome of patients with surgically resected brain metastases $(\mathrm{BrM})$ from different solid tumors particularly focusing on frequence and characteristics of seizures. Moreover, a secondary objective is investigating the potential correlation between seizure and different BrM's molecular profiles.

Materials and methods: We included patients with surgically resected BrM from solid tumors. We explored histology of primary tumor and $\mathrm{BrM}$, their molecular profile (when available), karnofsky performance status (KPS) before surgery, presence of seizures and their characteristics, number ( 1 vs $>1$ ) and site (infra vs supratentorial) of BrM, the timing of BrM's diagnosis (synchronous vs metachronous), visceral metastatic disease stadiation at time of BrM's diagnosis (absent, stable, progression), overall survival (OS) both from primary tumor and BrM diagnosis.

Results: We collected 335 patients with brain metastases, who underwent surgical procedure at our Center from 2006 to 2019 (139 NSCLC, 79 Breast Cancers, 32 Colorectal Cancers, 25 melanoma, 23 urinary tract tumors, 15 gynaecological tumors, 9 gastroesophageal tumors, 4 endocrine tumors, 4 HCC, 2 mesotelioma, 2 head and neck tumors and 1 soft tissue cancer). KPS was $\geq 70$ in 267 patients (80\%), 218 patients $(65 \%)$ had single BrM and $212(63 \%)$ had a supratentorial location while $78(23 \%)$ had an infratentorial location and $45(14 \%)$ both infra and supratentorial. Seizures were diagnosed in $35 \%$ of patients, the majority of which $(65 \%)$ occurred at BrM diagnosis. In 106 patients $(32 \%)$ BrM's diagnosis was synchronous with primary tumor diagnosis while the majority of patients (229) developed BrM after months from primary tumor's diagnosis. In 134 patients (40\%) visceral metastatic disease was absent or stable at the time of BrM onset. mOS from primary tumor diagnosis was 32 months, while mOS from BrM diagnosis was 7 months.

Discussion: Our survival findings are similar to those reported in literature, further analysis will be performed to analyse molecular profile of BrM and to establish a potential correlation among molecular and clinical paramethers in respect to survival, both from primary tumor and $\mathrm{BrM}$ diagnosis. In our casistic, the percentage of patients in which occurred seizures was slightly higher if compared to the data available in literature, expecially for patients with metastatic ovaric cancer $(80 \%)$, melanoma (44\%), breast cancer (42\%) and colorectal cancer (37,5\%).

Conclusion: Further data are needed to complete our interesting results, especially those concerning the prevalence of seizures in patients with BrM.

References:

- KJ Stelzer, Epidemiology and prognosis of brain metastases. Surg Neurol Int. (2013);4(Suppl 4): S192-S202

- R Rudà, F Mo, A Pellerino, Epilepsy in brain metastasis: an emerging entity. Curr Treat Options Neurol (2020);8;22(2):6

\section{ANTICANCER PROPERTIES OF THE ANTIPSYCHOTIC DRUG CHLORPROMAZINE AND ITS SYNERGISM WITH TEMOZOLOMIDE IN RESTRAINING GLIOBLASTOMA PROLIFERATION}

A. Pace ${ }^{1}$, S. Matteoni ${ }^{2}$, P. Matarrese ${ }^{3}$, B. Ascione ${ }^{3}$, M. Buccarelli ${ }^{4}$, L. Ricci-Vitiani $^{4}$, R. Pallini ${ }^{5}$, V. Villani ${ }^{1}$, M. Paggi ${ }^{2}$, C. Abruzzese ${ }^{2}$
${ }^{1}$ Neuroncology Unit, IRCCS Regina Elena Cancer Institute (Roma); ${ }^{2}$ Cellular Networks and Molecular Therapeutic Targets, Proteomics Unit, IRCCS - Regina Elena National Cancer Institute (Roma); ${ }^{3}$ Center for Gender Specific Medicine, Oncology Unit, Istituto Superiore di Sanità (Roma); ${ }^{4}$ Department of Hematology, Oncology and Molecular Medicine, Istituto Superiore di Sanità (Roma); ${ }^{5}$ Institute of Neurosurgery, Catholic University School of Medicine, Fondazione Policlinico Universitario A. Gemelli IRCCS (Roma)

The extremely poor prognosis of patients affected by glioblastoma multiforme (GBM, glioblastoma) prompts the search for new and more effective therapies. In this regard, drug repurposing, when scientifically supported, can represent a safe, swift and inexpensive way to bring novel pharmacological approaches from bench to bedside. Chlorpromazine, a medication in use since six decades for the therapy of psychiatric disorders, shows in vitro several features that make it eligible for repositioning in GBM therapy. In our experimentation on six GBM cell lines, three of which growing as patient-derived neurospheres and displaying stem-like properties, chlorpromazine inhibited cell viability in an apoptosis-independent way, induced polyploidy, reduced cloning efficiency as well as neurosphere formation and downregulated the expression of stemness genes. Notably, we found that chlorpromazine synergized with temozolomide, the first-line therapeutic in GBM patients, in reducing cell viability and strongly cooperated in reducing cloning efficiency and inducing cell death in vitro for all the GBM cell lines assayed. With these assumptions, we started a Phase II clinical trial on GBM patients by adding chlorpromazine to temozolomide in the adjuvant phase of the standard first-line therapeutic protocol. We are confident that our in vitro results on the effects of chlorpromazine on GBM cells, especially in combination with temozolomide, would be replicated in a clinical setting, thus providing an effective, fast and inexpensive therapeutic approach for these patients.

\section{COPING STRATEGIES IN MALIGNANT GLIOMAS AND THEIR CAREGIVERS}

\author{
A. Pace, S. Ieraci, V. Villani, D. Benincasa, L. Guariglia
}

Neuroncology Unit, IRCCS Regina Elena Cancer Institute (Roma)

Introduction: Patients diagnosed with glioma have, despite increased treatment options, a limited survival. Fear about death, rapid physical decline, and desire for information about the disease engender the need, both for patients and caregivers, of adaptive strategies to deal with the disease trajectory. According to Lazarus coping can be defined as "constantly changing cognitive and behavioural efforts to menage specific external or internal demands that are exceeding the resources of a person". Coping may be organized in five categories: fighting spirit, avoiding spirit, anxious preoccupation, helpless-hopeless and fatalism. In this study, we investigated factors that may affect coping strategies (quality of life, psychological distress, disease awareness) in patients with glioma and in their main caregivers.

Methods: A prospective study on 24 dyads of patients affected by malignant glioma and their care-givers was conducted at Regina Elena Cancer Institute of Rome between may 2016 and July 2018. Styles of coping of patients and caregivers were evaluated using the Mini-Mental Adjustment to Cancer (Mini-MAC) scale (Grassi 2005). The questionnaire is composed by 29 questions relating to the five coping strategies. The Mini-MAC has five domains: Fighting Spirit (FS 4 items); Helpless- 
Hopeless (HH 8 items); Anxious Preoccupation (AP 8 items); Fatalism (FA 5 items); Cognitive Avoidance, (CA 4 items).

Results: At baseline, patients reported higher score in the domain of Fighting Spirit (mean 3.23) and Cognitive Avoidance (mean 3.05). At individual level most frequent coping strategy resulted Cognitive Avoidance (18 patients, 75\%) and Fighting Spirit (17 patients, 70\%). A longitudinal evaluation was possible only in 8 patients and their caregivers. At recurrence, patients showed higher score in the domain of Fighting Spirit $(60 \%)$ and Fatalism (60\%). Avoidance coping style was present in $50 \%$ of patients. Analyzing the interconnection between patient's and caregivers, coping with a fighting spirit or with helpless-hopeless in the patient was significantly correlated with the same style of coping in the caregiver $(\mathrm{Rho}=0.539$, $\mathrm{p}$-value $=0.007$ and $\mathrm{Rho}=0.448, \mathrm{p}$-value $=0.028$, respectively $)$.

Conclusions: Our results confirm that coping is not a persistent dimension of personality, but can change depending on the situation. In addition, patients' and caregivers' reactions could be different, although a mutual influence was present, according to a dyadic model.

\section{TRAJECTORY OF DISEASE AND PATTERN OF CARE IN THE LAST 2 MONTHS OF LIFE IN BRAIN TUMORS IN THE LAZIO REGION: ANALYSIS OF A COHORT OF 3045 PATIENTS IN THE LAST 10 YEARS}

\author{
A. Pace ${ }^{1}$, V. Belleudi ${ }^{2}$, L. Pinarelli ${ }^{2}$, V. Villani ${ }^{1}$, F. Poggi ${ }^{2}$, D. \\ Benincasa $^{1}$, M. Davoli $^{2}$
}

${ }^{1}$ Neuroncology Unit, IRCCS Regina Elena Cancer Institute (Roma); ${ }^{2}$ Department of Epidemiology, Lazio Regional Health Service (Roma)

The final days and weeks of life in Brain Tumor (BT) patients present special challenges and often palliative care approach is under utilyzed. Several studies reported that BT in the last months of life receive frequent inappropriate hospital readmission and Emergency Room accesses as result of bad quality of end of life care. Palliative care is focused on relief of suffering for those with serious and advanced illnesses, and delivery of coordinated, continuous, and comprehensive end of life care for patients and their families. Early integration of palliative care has been demonstrated to improve the quality of care in advanced stage of disease and quality of death in cancer patients. With the aim to evaluate pattern of treatment and the rate of hospital readmission in the last months of life we retrospectively analyzed a consecutive serie of patients discharged after a diagnosis of BT.

Methods: Data regarding the number of hospital readmissions and treatment received in the last two months of life were retrospectively analyzed from hospital discharge records stored in the database of the regional Health Service. All patients discharged with a diagnosis of BT (ICD-9 191.*) since 1/1/2010 until 31/12/2019, with age $>18$ years, residents in the Lazio region, were included in this study. Patients with a diagnosis of BT in the previous 5 years were excluded.

Results: 6672 patients were identified in the period of analysis and 3045 , responding to the inclusion criteria and death before 31/12/2019 were included in the study (median age $=67 \mathrm{y} ; \mathrm{M} 1700, \mathrm{~F} 1345$ ). In the last 60 days of life $42,6 \%$ of patients received an hospital readmission $(4,6 \%$ in intensive care) and $37,9 \%$ had an ER access. $24,5 \%$ received chemotherapy and 12,1 radiotherapy. In the last 30 days before death $33 \%$ of patients were readmitted in hospital (3,7 in intensive care) and 24,2\% were admitted in ER. Also, $11,7 \%$ of patients received chemotherapy and $6 \%$ radiotherapy.

Discussion: Strategies to improve continuity of care and decrease rehospitalization are becoming increasingly important both for improving the quality of care of BT patients and for reducing futile treatments and the economic cost of the health care system. Patients who are approaching the end of their life need high-quality treatment and care that support them to live as well as possible until they die, and to die with dignity.

\section{NERATINIB FOR TREATMENT OF LEPTOMENINGEAL METASTASES FROM HER2-POSITIVE BREAST CANCER IN EXTENDED ACCESS PROGRAM: PRELIMINARY RESULTS}

\author{
A. Pellerino, R. Palmiero, F. Mo, F. Bruno, E. Muscolino, F. Franchino, \\ R. Rudà, R. Soffietti
}

Dept Neuro-Oncology, University and City of Health and Science Hospital (Torino)

Introduction: Leptomeningeal metastases (LM) occurs in 5\% of human epidermal growth factor receptor 2 (HER2) breast cancer (BC) with a poor overall survival (OS) of 3 months. Neratinib is an oral, irreversible tyrosine kinase pan-inhibitor that was approved by FDA for the treatment of HER2-enriched BC, who completed a prior adjuvant trastuzumabbased therapy. The aim of the study was to evaluate the activity of neratinib in LM from HER2-positive BC after the failure of multiple lines of treatment, including trastuzumab.

Patients and methods: Inclusion criteria were as follows: age $\geq 18$ years; histological diagnosis of primary HER2-positive BC; newlydiagnosed LM according to LANO criteria [1]; KPS $\geq 60$ at the time of diagnosis of LM; coexistence of BM that have or not received WBRT or radiosurgery; systemic disease with a life expectancy of at least 3 months; concomitant drugs, including capecitabine, trastuzumab, T-DM1, pertuzumab, and hormone therapy were allowed, with the exclusion of lapatinib or other investigational agents. Neratinib was administered $240 \mathrm{mg}$ daily continuously. Primary endpoint was the OS after the diagnosis of LM. Secondary endpoints were progression-free survival (PFS) following the diagnosis of LM, neurological benefit, radiological response rate, and tolerability.

Results: From January 2018 to July 2020, 7 patients with LM have been enrolled. Median age at the time of diagnosis of LM was 44 years (95\%CI 36-59) with a median KPS of 80 (95\%CI 60-90). Median time since LM onset from the diagnosis of primary BC was 55 months $(95 \% \mathrm{CI}$ 26-166), and patients underwent a median number of adjuvant treatments before LM of 3 (95\%CI 2-5). Two patients developed LM alone, and other 5 had LM associated with multiple BM. Six-months and 1-year OS were $57.1 \%$ and $28.6 \%$, respectively, with a median OS of 6 months (95\% CI 3-13*). Median PFS was 4 months (95\% CI 2-6) after the start of treatment. A neurological improvement was reported in $2 / 7$ patients (28.6\%), while in other $3 / 7$ patients $(42.8 \%$ ) was achieved a neurological stabilization lasting for a median time of 5 months (95\%CI 2-19). The best radiological response was SD in $4 / 7$ patients $(57.1 \%$ ), while no CR or PR were achieved according to LANO and RANO criteria $[1,2]$, respectively. Grade III-IV adverse events were not reported, and 2 patients only had mild diarrhea correlated with neratinib.

Conclusions: This is the first study that shows that neratinib might be a safe and effective treatment in LM from heavily pretreated HER2positive $\mathrm{BC}$

References:

1. Chamberlain M, Junck L, Brandsma D, et al. Leptomeningeal metastases: a RANO proposal for response criteria, Neuro Oncol. (2017);19(4):484-92

2. Lin NU, Lee EQ, Aoyama H, et al. Response Assessment in NeuroOncology (RANO) group, Lancet Oncol. (2015);16(6):e270-78 


\section{ISOLATED RICHTER'S SYNDROME OF THE CENTRAL NERVOUS SYSTEM: CASE REPORT WITH MYD88 L265P MUTATION EVALUATION AND LITERATURE REVIEW}

\author{
E. Pronello ${ }^{1}$, F. $\mathrm{Mo}^{2}$, D. Gottardi ${ }^{3}$, R. Palmiero ${ }^{4}$, L. Bertero ${ }^{5}, \mathrm{M}$. \\ Lanotte $^{6}$, M. Ferrio ${ }^{7}$, R. Rudà ${ }^{2}$, R. Soffietti ${ }^{2}$
}

${ }^{1}$ Department of Neurooncology, University of Turin (Torino); ${ }^{2}$ Division of Neuro-Oncology, University and City of Health and Science Hospital (Torino); ${ }^{3}$ Division of Hematology, A.O.U. S. Giovanni Battista A.O. Mauriziano-Umberto I (Torino); ${ }^{4}$ Division of Neuro-Oncology, University and City of Health and Science Hospital (Torino); ${ }^{5}$ Pathology Unit, Department of Medical Sciences, University and City of Health and Science Hospital (Torino); ${ }^{6}$ Division of Functional Neurosurgery, Department of Neuroscience, University and City of Health and Science Hospital (Torino); ${ }^{7}$ Division of Neuroradiology, Department of Neuroscience, University and City of Health and Science Hospital (Torino)

Objective: Richter syndrome (RS) is defined as the transformation of a B-cell chronic lymphocytic leukemia (CLL) into an aggressive lymphoma and is a rapidly progressive disease, usually chemotherapy resistant. The isolated Central Nervous System (CNS) involvement in RS has been described in few cases of the literature only. To our knowledge, this is the first case in whom the MyD88 mutation in a CNS-RS was investigated and found positive.

Case report: A 74 years-old male was referred to our hospital because of leukocytosis in 2009 and was diagnosed with CLL. A watch and wait strategy were performed. In 2013 he progressed to an advance stage and received treatment with Chlorambucil and Rituximab, achieving an objective response. On March 2015 the patient relapsed, received Bendamustine and Rituximab with a reduction of infiltration of B-CLL and started a close follow-up. Three years later he developed neurological signs and an MRI showed an intense and homogenous enhancing lesion, suspicious for either a primary central nervous system lymphoma or a CNS localization of CLL (CNS-RS). A stereotactic biopsy was performed and the histological diagnosis confirmed a CNS-RS. A polychemotherapy regimen with high-dose Methotrexate and Rituximab was started, but he rapidly progressed and died 4 months after RS diagnosis.

Discussion and conclusion: CNS-RS is a very rare and aggressive disease. The diagnosis of a CNS-RS requires a complete pathological and molecular study. An optimal treatment is not available. The MyD88 mutation could be an option for future targeted therapies.

References:

- Xu L, Song JC, Sun XH, Gao ZF, Lv L, Zhu J. Richter's syndrome of the central nervous system diagnosed concurrently with chronic lymphocytic leukaemia: A case report and literature review. Medicine (Baltimore). (2018);97(41):e12701

- Lee JH, Jeong H, Choi JW, Oh H, Kim YS. Clinicopathologic significance of MYD88 L265P mutation in diffuse large B-cell lymphoma: a meta-analysis. Sci Rep. (2017);7(1):1785

- Mao Z, Quintanilla-Martinez L, Raffeld M, et al. IgVH mutational status and clonality analysis of Richter's transformation: diffuse large B-cell lymphoma and Hodgkin lymphoma in association with B-cell chronic lymphocytic leukemia (B-CLL) represent 2 different pathways of disease evolution. Am J Surg Pathol (2007);31:160514

\section{EXTRACRANIAL METASTASES IN SECONDARY GLIOBLASTOMA MULTIFORME}

J. Rossi ${ }^{1}$, L. Giaccherini ${ }^{2}$, F. Cavallieri ${ }^{3}$, M. Napoli ${ }^{4}$, C. Moratti ${ }^{4}$, E. Froio $^{5}$, S. Serra ${ }^{5}$, A. Fraternali ${ }^{6}$, R. Ghadirpour ${ }^{7}$, S. Cozzi ${ }^{2}$, P.

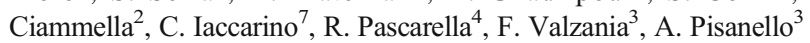

${ }^{1}$ Department of Biomedical, Metabolic, and Neural Sciences, University of Modena and Reggio Emilia (Modena); ${ }^{2}$ Radiation Oncology Unit, Oncology Department and Advanced Technologies, AUSL-IRCCS of Reggio Emilia (Reggio Emilia); ${ }^{3}$ Neuromotor and Rehabilitation Department, AUSL-IRCCS of Reggio Emilia (Reggio Emilia); ${ }^{4}$ Neuroradiology Service, Department of Diagnostic Imaging and Laboratory Medicine, AUSL-IRCCS of Reggio Emilia (Reggio Emilia); ${ }^{5}$ Pathological Anatomy Service, Oncology Department and Advanced Technologies, AUSL-IRCCS of Reggio Emilia (Reggio Emilia); ${ }^{6}$ Nuclear Medicine Service, Oncology Department and Advanced Technologies, AUSL-IRCCS of Reggio Emilia (Reggio Emilia); ${ }^{7}$ Neurosurgery Unit, Neuromotor and Rehabilitation Department, AUSL-IRCCS of Reggio Emilia (Reggio Emilia)

Objectives: Glioblastoma (GBM) is known for its devastating intracranial infiltration and its unfavorable prognosis. GBM metastases are rare and this is putatively attributed to the short overall survival and the lack of a favorable environment for an extracranial spreading of tumor cells. Metastases usually occur after primary GBM, while extracranial involvement from secondary GBM is extremely rare.

Materials and methods: A 29-year-old woman underwent subtotal surgical removal of a right frontal WHO grade II Astrocytoma in June 2015. The lesion remained stable at the follow-up until October 2017, when she underwent new brain surgery for locoregional recurrence. Histological investigations confirmed a WHO Grade II Astrocytoma (MGMT promoter methylated, IDH1-mutated). Five months after the second surgery the patient presented a sudden clinical worsening and a new Brain MRI documented a massive recurrence of the right frontal lesion with evident signs of grading change. A third surgery revealed a WHO grade IV (MGMT promoter methylated, IDH1-mutated). In September 2018, a right cervical lymph node swelling appeared: a needle biopsy was performed and confirmed the presence of an extracranial metastatic localization of GBM which was successfully treated with locoregional radiotherapy. At the same time, the patient complained of the appearance of severe diffuse drug-resistant arthralgia. A whole-body 18F-FDG PET/CT scan showed multiple increased 18F-FDG uptake areas involving ilium bilaterally and the proximal third of the femurs, scapula, and humeral head bilaterally, some ribs, some vertebrae, and the sacrum. None of the $18 \mathrm{~F}-\mathrm{FDG}$ uptake areas correlated with significant structural alteration on a CT scan. Pelvis MRI demonstrated signal alteration areas compatible with GBM metastatic bone infiltration. A CT guided biopsy was performed, and the histopathological examination confirmed the presence of secondary bone localizations of GBM. In the face of this diffusion, brain-MRI documented stability of the brain disease for 14 months, and no clinical or radiological signs of progression were discovered in the irradiated lymph node site for approximately 1-year. About 18 months after the appearance of the first metastatic site, the patient was hospitalized in a Hospice where she died at the age of 33years.

Discussion and conclusions: Despite a favorable biomolecular pattern, grade II Astrocytoma may evolve to a GBM and rarely lead to a secondary metastatic diffusion. Nonspecific signs and symptoms, like local tumefactions or bone and joint pain, should never be underestimated, as they may be suggestive for a systemic diffusion of the GBM. 


\section{References:}

- Elena A, Melina C, Raffaele N, et al. Extraneural metastases in glioblastoma patients: two cases with YKL-40-positive glioblastomas and a meta-analysis of the literature. Neurosurg Rev. (2016);39(1):37-46

- Cunha MLV da, Maldaun MVC. Metastasis from glioblastoma multiforme: a meta-analysis. Rev Assoc Med Bras. (2019);65(3):424-33

- Cervio A, Piedimonte F, Salaberry J, et al. Bone metastases from secondary glioblastoma multiforme: A case report. J Neurooncol. (2001);52(2):141-48

\section{THE ROLE OF BODY MASS INDEX AS RISK FACTORS RELATED TO PICC COMPLICATIONS IN NEURO ONCOLOGICAL PATIENTS}

\author{
G. Simonetti ${ }^{1}$, P. Gaviani ${ }^{1}$, A. Sommariva ${ }^{2}$, M. Listrani ${ }^{3}$, E. Anghileri ${ }^{1}$, \\ A. Botturi ${ }^{1}$, E. Lamperti ${ }^{1}$, A. Silvani ${ }^{1}$
}

${ }^{1}$ Neurooncology Department, Fondazione IRCCS Istituto Neurologico Carlo Besta (Milano); ${ }^{2}$ Department of Neuro-anesthesia, Fondazione IRCCS Istituto Neurologico Carlo Besta (Milano); ${ }^{3}$ Department of Neuro-radiology, Fondazione IRCCS Istituto Neurologico Carlo Besta (Milano)

Introduction: The use of central venous catheter with peripheral insertion (PICC) has increased rapidly in recent years particularly in cancer patients. However, the benefit provided by the use of the device may occasionally be affected by severe complications. An observational study conducted at our Institute, showed a significant increase of thromboembolic risk in neuro-oncological patients compared to general cancer population ( $16.3 \%$ vs $6.7 \%$ respectively). For this reason, it is essential to identify independent risk factors in order to avoid preventable harm.

Materials and Methods: Neuro-oncological patients with PICC that developed a thromboembolic event were retrospectively analyzed. We evaluated clinical data, BMI, KPS, steroid therapies and anticoagulants at the time of positioning of the PICC and at the onset of the thrombotic event.

Results: Overall, 80 neurooncological patients have been analyzed. The most frequent histology was represented by glioblastoma, followed by anaplastic astrocytoma, primary central nervous system lymphoma, grade II glioma. Among these, $15 \%$ patients (75\% males, 25 females, median age 49.5) developed thromboembolic events within a median time of 30.5 days (range 5-140) after PICC implantation. The median KPS was 90 (range 80-100), and the median ECOG was 1 (range 02). $67 \%$ of patients had a median BMI $>28$ (range 26-37,56). $16.6 \%$ of patients were at first diagnoses, $41.6 \%$ were at the first recurrence, and the remaining patients were at the second or third recurrence. $17 \%$ had a malpositioning that required up to 3 insertion attempts. No patients were on anticoagulant therapy but all were treated with a median dose of $5.5 \mathrm{mg}$ of steroids (range 3,5-12), stable between PICC insertion and onset of adverse event.

Discussion and conclusion: $67 \%$ of patients who developed a thromboembolic event had BMI $>28$ at the time of picc insertion, suggesting a possible trend towards a risk of developing thromboembolic events in overweight/obese patients. Other independent risk factors for PICC associated complications seems to be amount of previous chemotherapies administered: $83 \%$ of patients who developed thrombotic complications had already undergone at least one line of chemotherapy before PICC placement, suggesting this condition as a possible risk factor for the onset of the event. Also failed positioning attempts could damage vascular endothelium, contributing to the onset of thrombotic complications. An accurate anamnesis and a physical evaluation with particular attention to the presence of malnutrition could help in the in the early identification of independent risk factors that could farther negatively influence the outcome of neuro- oncological patients.

\section{WHO GRADE II AND III MENINGIOMA: A LARGE MONO- INSTITUTIONAL RETROSPECTIVE SERIES}

\author{
G. Simonetti ${ }^{1}$, P. Gaviani ${ }^{1}$, I. Tramacere ${ }^{2}$, M. Farinotti ${ }^{3}$, F. Legnani ${ }^{4}$, V.
} Pinzi $^{5}$, B. Pollo ${ }^{6}$, A. Botturi ${ }^{1}$, E. Lamperti ${ }^{1}$, A. Silvani ${ }^{1}$

${ }^{1}$ Department of Neurooncology, Fondazione IRCCS Istituto Neurologico Carlo Besta (Milano); ${ }^{2}$ Department of Research and Clinical Development, Scientific Directorate, Fondazione IRCCS Istituto Neurologico Carlo Besta (Milano); ${ }^{3}$ Unit of Neuroepidemiology, Fondazione IRCCS Istituto Neurologico Carlo Besta (Milano); ${ }^{4}$ Department of Neurosurgery, Fondazione IRCCS Istituto Neurologico Carlo Besta (Milano); ${ }^{5}$ Department of Radiotherapy, Fondazione IRCCS Istituto Neurologico Carlo Besta (Milano); ${ }^{6}$ Neuropathology Unit, Fondazione IRCCS Istituto Neurologico Carlo Besta (Milano)

Objective: Meningiomas are usually considered benign lesions, however a part of them can show very aggressive behavior with tendency to metastasize. This subgroup is known as high-grade meningiomas (HGM). Due to the rarity of the disease, effective medical treatments are lacking, especially at the time of recurrence. We aim to describe the clinical, radiological and molecular features of a large population of HGM diagnosed between 2010 and 2018. The secondary aim was to evaluate survival (PFS) and overall survival (OS).

Materials and Methods: We collected clinical and survival data from primitive WHO grade II and III meningioma patients treated at Fondazione IRCCS Istituto Neurologico Carlo Besta from January 2010 to December 2018. Records were collected on a web-based platform (Microsoft Excel) that was customized for this study. The database, contained epidemiological, diagnostic (radiological and histological/molecular), surgical, therapeutic and recurrence information, as well as survival data.

Results: 183 patients (105 females and 78 males), with median age of 58 years (25-88), were included; 168 were atypical, 12 anaplastic, 3 rhabdoid. Overall, m-PFS was 4.2 years, and m-OS was 10.3 years. Gross-total resection had a 5-year survival rate of $95 \%$ compared with subtotal $/$ partial resection ( $86 \%$ and $67 \%)(\mathrm{p}=0.002)$. Higher expression of Ki-67/MIB-1 seems associated with higher risk of death (HR:1.06 with 95\% CI, 1.00-1.12, $\mathrm{p}=0.03$ ). No statistically significant differences were seen in survival between the group managed with a wait-and-see strategy vs the group treated with RT while a difference on PFS was seen (4.1 years vs 5.2 years $\mathrm{p}=0.03$ ). After second recurrence, the most employed treatments were systemic therapies with a very limited effect on disease control.

Discussion and Conclusions: Data confirmed the aggressive behavior of HGM. The extent of resection seems to correlate with a favorable outcome regardless histological subtypes. The role of RT remains controversial, with no statistically significant impact on OS but a possible role on PFS. At relapse, no chemotherapies are able to achieve disease control and recurrent HGM remains. 


\section{NEUROCOGNITIVE EFFECTS OF STEREOTACTIC RADIATION THERAPY IN CANCER PATIENTS WITH MULTIPLE BRAIN METASTASES}

\author{
A. Tanzilli ${ }^{1}$, L. Marucci ${ }^{2}$, A. Farneti ${ }^{2}$, G. Sanguineti ${ }^{2}$, A. Pace ${ }^{1}$, V. \\ Villani ${ }^{1}$
}

${ }^{1}$ Neuroncology Unit, I.R.C.C.S. Regina Elena National Cancer Institute (Roma); ${ }^{2}$ Radiotherapy Unit, I.R.C.C.S. Regina Elena National Cancer Institute (Roma)

Background: Literature data report the presence of secondary brain metastases in $20-40 \%$ of cancer patients [1]. Since overall survival in these patients has increased in the last decade, it is important to treat brain metastases minimizing neurocognitive toxicity [2]. Stereotactic radiation therapy approach has imposed itself as standard treatment because of its reduced neurotoxicity. Several studies confirm the efficacy of stereotactic radiation therapy in patients with 1-3 metastases, but neurocognitive effects in patients with 4 or more metastases have not been exhaustively studied [3]. The aim of this study is to longitudinally investigate neurocognitive effects of stereotactic radiation therapy in patients with multiple brain metastases.

Methods: An extended neuropsychological battery of standardized tests, tapping on 6 cognitive domains, was administered to each patient to assess neurocognitive status at baseline (before radiotherapy) at 3, 6, and 9 months post treatment. Domains evaluated were: verbal learning; short and long-term memory; executive functions; attention and visuo-constructional abilities. We furtherly collected demographic and clinical data: age at diagnosis, education, number of metastases

Results: We assessed 15 patients with brain metastases at baseline with median age at diagnosis of 68 years (range 49-80). 5 patients $(33 \%)$ presented cognitive deficit in at least one domain (median 0 ; range $0-1$ ), and 10 (66\%) showed no cognitive impairment. Neuropsychological deficits affected executive functions $(n=2)$, long term memory $(n=2)$ and visuo-constructional abilities $(n=1)$. Out of the 15 patients 6 underwent a three months follow-up test session and no patients showed deficits. In particular, three patients improved their cognitive status, since their memory and constructional problems regressed. 4 patients underwent a 6 months test session, showing stable and preserved cognitive status.

Conclusions: Our preliminary results suggest that stereotactic radiation therapy is not associated with central neurotoxicity.

References:

1. Kim S. H., Weil R. J., Chao S. T., Toms S. A., Angelov L., Vogelbaum M. A., ... \& Barnett G. H. Stereotactic radiosurgical treatment of brain metastases in older patients. Cancer: Interdisciplinary International Journal of the American Cancer Society (2008); 113(4):834-840

2. Kondziolka D., Martin J. J., Flickinger J. C., Friedland D. M., Brufsky A. M., Baar J., ... \& Lunsford L. D. Long-term survivors after gamma knife radiosurgery for brain metastases. Cancer: Interdisciplinary International Journal of the American Cancer Society (2005); 104(12): 2784-91

3. Yamamoto M., Serizawa T., Shuto T., Akabane A., Higuchi Y., Kawagishi J., ... \& Nagano O. Stereotactic radiosurgery for patients with multiple brain metastases (JLGK0901): a multi-institutional prospective observational study. The Lancet Oncology (2014);15(4):387-95

\section{NEUROCOGNITIVE REHABILITATION IN A HETEROGENEOUS SAMPLE OF CANCER PATIENTS}

\author{
A. Tanzilli, V. Villani, A. Pace
}

Neuro-Oncology Unit, I.R.C.C.S. Regina Elena National Cancer Institute (Roma)

Background: Neurocognitive impairment is one of the most debilitating symptoms in different subsets of cancer survivors [1]. Its etiology is not fully understood, but many reports suggest a multifactorial origin, as neurocognitive impairment can be direct or indirect consequence of tumor and anti-tumor treatments [2]. Cancer itself may alter the hypothalamic-pituitary-adrenal axis functioning, worsen microvascular circulation or induce neurotoxic injuries [3]. Anti-cancer treatments may interfere with neurocognitive functioning by producing brain inflammation or oxidative stress and symptoms can be tightened by demographical and clinical conditions. Nevertheless, contrasting cancer-related neuropsychological dysfunctions is mandatory and cognitive rehabilitation is considered a first-line intervention. In the present study we aimed at investigating the effect of cognitive training in different types of cancer patients.

Methods: In this retrospective study, each patient underwent pre and post treatment neuropsychological assessments describing 7 cognitive domains: verbal learning (VL); short and long-term memory (STM, LTM); executive functioning (FE); abstract reasoning (AR); attention (ATT) and language (L). A computer-based rehabilitation program (E.R.I.C.A. giuntipsychometrics) was administered for two months according to the cognitive deficits detected. We also collected demographic and clinical data.

Results: We assessed 24 cancer patients affected by glioma, breast and lung tumor (13 female) [median age: 49,5 (range 3370) median education: 13 (range 8-18)]. All of them had undergone surgery and post-operative treatments (CT and RT). At baseline evaluation most of the patients presented with deficit in LTM (11), STM (8) and ATT (7). At follow-up assessment, [median time of 6 months (range5-7)], most represented deficits concerned LTM (9), STM (6) and ATT (6). 17 patients (89\%) with radiological response to treatments (stable or partial response) did not worsen cognitive status. In particular, $11(58 \%)$ of them improved their neurocognitive performance and 7 (37\%) did not show any deficit. Patients with disease progression instead, worsened neurocognitive status and 2 patients showed deterioration despite a stable disease condition.

Conclusions: Our results underline the central role of disease course on neurocognitive deficits in cancer patients. Nevertheless, cognitive training may help to improve neuropsychological status in patients with disease control, thus supporting a better quality of life.

References:

1. Von Ah, D. Cognitive changes associated with cancer and cancer treatment: state of the science. Clinical Journal of Oncology Nursing, (2015);19(1)

2. Bender C. M., \& Thelen B. D. Cancer and cognitive changes: the complexity of the problem. In Seminars in Oncology Nursing (2013);29(4):232-37

3. WB Saunders, Ahles T. A., Root J. C., \& Ryan E. L. Cancer-and cancer treatment-associated cognitive change: An update on the state of the science. Journal of Clinical Oncology (2012);30(30):3675 


\section{IDENTIFICATION OF POTENTIAL TARGET THERAPY IN GLIOMA PATIENTS WITH TWO DIFFERENT PANEL FOR NGS: PRELIMINARY RESULTS}

\author{
V. Villani ${ }^{1}$, B. Casini ${ }^{2}$, A. Di Benedetto ${ }^{2}$, C. Ercolani ${ }^{2}$, P. Falbo ${ }^{3}$, G. \\ Lanzetta $^{3}$, A. Pace ${ }^{1}$, M. Carosi ${ }^{2}$
}

${ }^{1}$ Neuroncology Unit, IRCSS Istituto Regina Elena (Roma); ${ }^{2}$ Pathology Unit, IRCSS Istituto Regina Elena (Roma); ${ }^{3}$ Oncology Unit, INI (Grottaferrata-RM)

Background: Gliomas are the most common primary brain tumours accounting for approximately $70 \%$ of all neoplasms of central nervous system (CNS). Current standard treatments consist of surgical resection followed by radiotherapy and/or chemotherapy. However, the prognosis of CNS tumours is still very poor, especially for glioblastoma. Molecularly targeted therapies using receptor inhibitors, small molecules or monoclonal antibodies are routinely applied in oncology. However, in neuroncology the treatment is not yet customized due to the failure to identify actionable mutations. Our aim was to establish the frequency of mutations in glioma and to detect potential molecular targets.

Methods: We analyzed the glioma tissue at diagnosis through the next generation sequencing (NGS) method performed on DNA extracted from conventional histological samples (tDNA) of glial fixed, formalin-fixed and paraffin-embedded (FFPE) neoplasms. First, we used a 50-gene NGS panel in 93 patients with glioblastoma $(n=54)$ or other glioma types $(n=39)$. More recently, we used a 50-gene Focus panel for the presence of point variants and fusion genes or variations in the number of gene copies in another smaller group of 10 patients.

Results: The 50-gene NGS panel detected at least one mutation in 64 gliomas, including IDH1 $(\mathrm{n}=30)$, TP53 $(\mathrm{n}=21)$, EGFR $(n=8)$, PIK3 $(n=8)$. In the remaining cases, the other genes were wild-type. Seven patients were discarded from the analysis since the NGS was not valid. Longer overall survival (OS) and progression-free survival (PFS) were observed in the presence of IDH1 mutation $(p<0.001)$ and TP53 mutation versus wild-type $(\mathrm{p}<0.05)$. A multivariate analysis confirmed that IDH1 and TP53 were two significant prognostic factors. Shorter PFS and OS were also found in patients having all the 50 wild-type genes when compared with those with at least one (whatever) NGS-panel mutation $(\mathrm{p}<0.005)$. The Focus panel detected mutations in 4 out of 10 patients, as follows: PIK3 $(n=1), \operatorname{CDK} 4(n=1), \operatorname{BRAF}(n=1)$, RET $(n=1)$. In these 4 patients, we gave indication to alpesilib, abemaciclib cobimetinib and patients with RET fusion was enrolled in phase 1 protocol, respectively. However, only the patient with BRAF+ cervical glioma and RET fusion started treatment, whereas in the other patients the deteriorated clinical conditions contraindicated the treatment initiation.

Conclusion: These preliminary results not only confirm the prognostic role of IDH1 and TP53 mutations in brain gliomas, but also indicate the importance of the precision medicine in glioma.

Reference:

- Reifenberger G, Wirsching HG, Knobbe-Thomsen CB, Weller M. Advances in the molecular genetics of gliomas - implications for classification and therapy. Nat Rev Clin Oncol. (2017) Jul;14(7):434-452. doi: 10.1038/nrclinonc.2016.204. Epub 2016 Dec 29
CLINICAL NEUROPHYSIOLOGY

DESCRIPTIVE STUDY ON THE CORRELATION BETWEEN VISUAL AND AUDITORY EVOKED POTENTIALS ASSOCIATED WITH VISUAL AND AUDITORY P3OOS USING WAVELET ANALYSIS OF INDIVIDUAL TRACKS IN NORMAL, MCI AND SDAT SUBJECTS

\author{
G. Aiello, G. Toti
}

Neuropsicophysiology Clinic (Bernareggio- MB)

Introduction: The discovery of electroencephalography, made in animals by Caton and in humans by Berger, a century ago, has profoundly changed the vision of the structures and functions of the central nervous system. This work will present the results of the visual and auditory evoked potentials, associated with event related potentials (ERPs) followed by wavelet analysis of each individual trace. The analyses were done in a small sample of subjects: normal, MCI (minimal cognitive impairment) and DAT (dementia Alzheimer type).

Materials and methods: The subjects were randomly selected from the authors' database using the keywords: normal subjects, MCI, SDAT, have been selected: 28 SDAT; 21 MCI; 25 normal, of both sexes and aged between 18 yrs and 75 yrs.

Results: The data show an altered functioning of the visual and auditory systems highlighted by the wavelet analysis, with the following organized gradient: NORMAL better than MCI better than SDAT. Visual and Auditory P300 also have higher amplitude values in NORMAL $>$ MCI $>$ SDAT, latency SDAT $>>$ MCI $>>$ NORMAL

Conclusion: The results of this study show a good correlation between perceptual abnormalities at latency, amplitude of VEPs and AEPs and visual and auditory P300, supported by wavelet analysis that highlights an altered frequency composition that justifies the worsening of cognitive functions in the three classes of subjects. This data can help us in the development of new types of treatments with: NEUROFEEDBACK (NFB) and transcranic magnetic stimulation (TMS).

References:

Todd C. Handy Event Related Potentials (A Methods Handbook) MIT PRESS (2005)

- E. Basar Brain Function and Oscillation Springer VV I AND II (1999)

\section{ELECTROENCEPHALOGRAPHY, MULTILEVEL ANALYSIS AND THEIR CORRELATIONS. METHODOLOGICAL AND CLINICAL ASPECTS}

G. Aiello, S. Merati

Neuropsicophysiology Clinic (Bernareggio-MB)

Introduction: The first EEG recording dates back to 1875, when Caton recorded brain electrical activity in the monkey and dog. 50 years later in 1924, H. Berger recorded the brain bioelectric activity with the discovery of alpha and beta rhythms. The recording of the EEG over time has evolved, with the acquisition of new and powerful computational tools, which have brought out aspects of the track that are not visible to simple visual inspection. These include a special attention to the experience of 
the authors of the work: 1. Analysis with frequency maps; 2. Frequency coherence analysis; 3 . Nonlinear dynamic analysis.

Materials and methods: The authors developed a series of algorithms, already known in the literature, to correlate them with each other in order to obtain a verifiable system for both clinical definition and therapy. The methods described are: a. EEG recording with mean reference; $b$. analysis with frequency maps c. Calculating coherence in the 4 frequency bands defined in the maps $d$. Nonlinear dynamic analysis with calculation of the following parameters: delay, embedding dimension, correlation dimension, large Lyapunov exponent

Conclusions: This data set shows the possibility of integrating the different EEG signal analysis algorithms in a clinically meaningful way, thus allowing us to verify possible changes in relation to the different therapies. In this presentation we will describe the different electrophysiological parameters in relation to normal subjects, with minimal cognitive impairment. The data highlight a clear difference between normal and MCI subjects, confirming the clinical usefulness of the parameters described.

References:

- H. Kantz, T. Schreiber Non Linear Time Series Analysis Cambridge (2002)

- Fish \& Spehlmann's EEG Primer Elsevier (2008)

- G. Aiello Neuropsicofisiologia Clinica Centro Scientifico Editore (2003)

\section{MACHINE-LEARNING ANALYSIS OF VOICE SAMPLES RECORDED THROUGH SMARTPHONES: THE COMBINED EFFECT OF AGEING AND GENDER}

\author{
F. Asci ${ }^{1}$, G. Costantini ${ }^{2}$, P. Di Leo ${ }^{2}$, A. Zampogna ${ }^{1}$, G. Ruoppolo ${ }^{3}$, A. \\ Berardelli $^{4}$, G. Saggio ${ }^{2}$, A. Suppa ${ }^{4}$
}

${ }^{1}$ Department of Human Neurosciences, Sapienza, University of Rome (Roma); ${ }^{2}$ Department of Electronic Engineering, University of Rome Tor Vergata (Roma); ${ }^{3}$ Department of Sense Organs, Otorhinolaryngology Section, Sapienza University of Rome (Roma); ${ }^{4}$ Department of Human Neurosciences and IRCCS Neuromed, Sapienza University of Rome and Neuromed Institute (Roma, Pozzilli-IS)

Objectives: Experimental studies using qualitative or quantitative analysis have demonstrated that the human voice progressively worsens with ageing [1]. These studies, however, have mostly focussed on specific voice features without examining their dynamic interaction [2]. To examine the complexity of age-related changes in voice, more advanced techniques based on machine learning have been recently applied to voice recordings but only in a laboratory setting. We here recorded voice samples in a large cohort of healthy subjects. To improve the ecological value of our analysis, we collected voice samples directly at home using smartphones.

Materials and Methods: 138 younger adults (65 men, age range: 1530 ) and 123 older adults ( 47 men, age range: $40-85$ ) produced a sustained emission of a vowel or a sentence. The recorded voice samples underwent a machine learning analysis through a support vector machine [3].

Results: The machine learning analysis of voice samples from both speech tasks discriminated between younger and older adults, and between males and females, with high statistical accuracy.

Discussion and Conclusions: By recording voice samples through a smartphone in an ecological setting, we demonstrated the combined effect of age and gender on voice. Our machine learning analysis would offer a new tool to assess the effect of ageing on voice.
References:

1. Mueller P. The Aging Voice. Semin Speech Lang (1997);18:159 69, doi:10.1055/s-2008-1064070

2. Goy H., Fernandes D.N., Pichora-Fuller M.K., van Lieshout P. Normative Voice Data for Younger and Older Adults. Journal of Voice (2013);27:545-55, doi:10.1016/j.jvoice.2013.03.002

3. Suppa A., Asci F., Saggio G., Marsili L., Casali D., Zarezadeh Z., Ruoppolo G., Berardelli A., Costantini G. Voice analysis in adductor spasmodic dysphonia: Objective diagnosis and response to botulinum toxin. Parkinsonism Relat. Disord. (2020);73:23-30, doi:10.1016/j.parkreldis.2020.03.012

\section{MOTOR DYSFUNCTION IN MILD COGNITIVE IMPAIRMENT AS TESTED BY KINEMATIC ANALYSIS AND TRANSCRANIAL MAGNETIC STIMULATION}

D. Colella ${ }^{1}$, A. Guerra ${ }^{2}$, G. Paparella ${ }^{2}$, E. Cioffi ${ }^{1}$, A. Di Vita ${ }^{3}$, A. Trebbastoni $^{1}$, A. Berardelli ${ }^{1,2}$, M. Bologna ${ }^{1,2}$

${ }^{1}$ Department of Human Neuroscience, Sapienza University of Rome (Roma); ${ }^{2}$ IRCCS Neuromed (Pozzilli-IS); ${ }^{3}$ Department of Psychology, Sapienza University of Rome (Roma)

Objectives: Previous studies have demonstrated voluntary movement alterations as well as motor cortex excitability and plasticity changes in patients with mild cognitive impairment (MCI). To investigate the pathophysiology of movement abnormalities in MCI, we tested possible relationships between abnormalities of finger tapping movements and primary motor cortex (M1) alterations in patients.

Materials: Clinical evaluation included the motor section (part III) of the Movement Disorder Society-sponsored revision of the Unified Parkinson's Disease Rating Scale (UPDRS-III), the Montreal Cognitive Assessment (MoCA), the Frontal Assessment Battery (FAB), and the Beck Depression Inventory (BDI-II). Repetitive finger tapping was recorded using an optoelectronic motion system (SMART motion system, BTS Engineering). Transcranial Magnetic Stimulation (TMS) was performed by using a MAGSTIM 200 (Magstim Company Limited) connected to a standard figure- of-eight $70 \mathrm{~mm}$ coil delivering monophasic pulses.

Methods: Fourteen amnestic MCI (aMCI) patients and 16 healthy controls were studied. Cognitive assessment was performed using clinical scales. The motor task consisted of 15 seconds of finger tapping, performed at the maximal voluntary rate. At the end of the kinematic recordings, TMS was used to test M1 excitability including the input/output curve of motor evoked potentials, intracortical inhibition, and shortlatency afferent inhibition. M1 plasticity was probed by theta burst stimulation. We investigated correlations between movement abnormalities, clinical scores, and cortical neurophysiological parameters.

Results: MCI patients showed altered rhythm but no other movement abnormalities. Cortical excitability measures were normal in patients, whereas plasticity was reduced. Movement rhythm abnormalities correlated with frontal dysfunction scores.

Discussion: These findings demonstrate that altered movement rhythm represents the only motor abnormality in MCI patients, and is independent of M1 excitability and plasticity parameters. Thus, altered M1 plasticity does not necessarily constitute a pathophysiological substrate of motor impairment in MCI. Instead, altered movement rhythm could reflect dysfunction in frontal areas by providing evidence of a negative correlation between movement rhythm and FAB scores, i.e. 
the less rhythmic the movement, the greater the cognitive impairment in executive functions, located in the frontal lobes.

Conclusion: Our study in MCI patients demonstrated abnormal voluntary movement and plasticity changes, with no correlation between the two. Altered rhythm correlated with frontal dysfunction. Our results suggest that altered rhythm in fine voluntary movements, as assessed by kinematic techniques, may be an early motor feature in patients with cognitive decline, and contribute to the understanding of pathophysiological mechanisms of motor impairment in MCI.

References:

- Rabinowitz I, Lavner Y. Association between Finger Tapping, Attention, Memory, and Cognitive Diagnosis in Elderly Patients. Percept Mot Skills (2014);119:259-78

- Bologna M, Guerra A, Colella D, Cioffi E, Paparella G, Di Vita A, D'Antonio F, Trebbastoni A, Berardelli A. Bradykinesia in Alzheimer's disease and its neurophysiological substrates. Clin Neurophysiol (2020);131:850-8

- Di Lorenzo F, Motta C, Casula EP, Bonnì S, Assogna M, Caltagirone C, Martorana A, Koch G. LTP-like cortical plasticity predicts conversion to dementia in patients with memory impairment. Brain Stimulation (2020);13:1175-82

\section{MOTOR EVOKED POTENTIALS ELICITED AT THE OPTIMUM PHASE OF BETA CORTICAL OSCILLATIONS HAVE HIGHER AMPLITUDE, SHORTER ONSET LATENCY AND LOWER VARIABILITY}

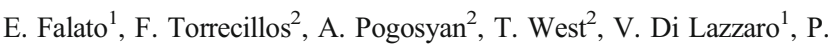 \\ Brown $^{2}$
}

${ }^{1}$ Unit of Neurology, Neurophysiology, Neurobiology, Department of Medicine, Campus Bio-Medico University of Rome (Roma); ${ }^{2}$ Nuffield Department of Clinical Neurosciences, John Radcliffe Hospital, University of Oxford, Medical Research Council Brain Network Dynamics Unit, University of Oxford (Oxford-UK)

Introduction: Brain oscillations involve rhythmic fluctuations of neuronal excitability. Transcranial magnetic stimulation (TMS) inputs delivered at the excitable phase of beta oscillations in the motor cortex are known to lead to muscle responses of increased amplitude.

Aim: We investigated if the phase of motor cortex rhythmic oscillations in the beta band also correlates with Motor Evoked Potentials (MEPs) response variability - expressed as coefficient of variation - and MEPs onset latency.

Methods: We delivered single-pulse TMS to the motor cortex of healthy human volunteers (10 females and 7 males) with a figure of 8-coil and a posterior-to-anterior orientation, during electroencephalography (EEG) recordings made at rest. We recorded MEPs from the right first dorsal interosseous muscle. A second experimental session was performed on 7 subjects to confirm and further characterize our results, with a larger number of trials, higher time resolution and an optimized experimental protocol. Phase-dependency was assessed by circular-linear correlation between MEPs Amplitude, MEPs latency, and the EEG beta phase at the time of the TMS pulses.

Results: TMS pulses delivered at the optimum phase of motor cortex beta oscillations elicited MEPs with significantly shorter onset latency and lower coefficient of variation. Latency modulation was stronger at TMS intensities near to the resting motor threshold.
Discussion and Conclusions: TMS inputs aligned to the optimal phase of the beta EEG oscillations in the motor cortex enjoy transmission amplitude gain and may also benefit from a less variable and more rapid transmission at subsequent synapses. All told, our data show that the motor system is dynamic even at rest and that the phase of neural beta oscillations recorded in the motor cortex may impact corticospinal communication.

This work has been published:DOI 10.1523/JNEUROSCI.195319.2019. *joint first Authorship

References:

- Fries P. A mechanism for cognitive dynamics: neuronal communication through neuronal coherence. Trends Cogn Sci (2005);9:47480

- van Elswijk G, Maij F, Schoffelen JM, Overeem S, Stegeman DF, Fries P Corticospinal beta-band synchronization entails rhythmic gain modulation. J Neurosci (2010);30:4481-88

- Khademi F, Royter V, Gharabaghi A. Distinct Beta-band Oscillatory Circuits Underlie Corticospinal Gain Modulation. Cereb Cortex (2018);28(4):1502-15

\section{CHANGES IN RECRUITMENT PROPERTIES OF THE CORTICOMOTOR PATHWAY AFTER EXERCISE TRAINING IN PARKINSON DISEASE}

\author{
F. Ginanneschi, A. Rossi
}

Department of Medical, Surgical and Neurological Sciences; NeurologyNeurophysiology Unit, University of Siena (Siena)

Objective: A critical node of the functional circuits impacted in Parkinson disease (PD) is the primary motor cortex (M1), which plays an important role in transforming the abnormally patterned neuronal activity in PD, into motor signs. In present paper we verified whether 8 weeks of specialized upper limbs exercise training influenced the recruitment properties of the corticomotor pathway in response to transcranial magnetic stimulation (TMS).

Methods: We recruited 7 PD subjects. Before exercise training, we performed the input-output (I-O) curve by TMS and recording from ADM muscle, peripheral nerve stimulation of the ulnar nerve, the $\mathrm{H}$ reflex and clinical assessment by the UPDRS part-III motor examination. The exercise protocol consisted in 16 sessions, twice a week. We utilized the Angel Wing's device. After 2-months exercise training, the protocol was repeated. Paired T test and Boltzmann sigmoidal function were used for statistic.

Results: All patients reported a clinical improvement, testified by a statistically significant improvement of the UPDRS III mean score at the end of the exercise training: $2.12 \pm 0.9$ pre training and $0.62 \pm 0.58$ post training $(\mathrm{p}<0001)$. Maximal $\mathrm{H}$ reflex amplitude, peripheral nerve stimulation and threshold of TMS stimulation did not changed. The plateau value of the I-O curve post training was significantly higher than pre exercise ( $\mathrm{p}=0.009$ ); conversely, both the values of V50 and slope of the relationship were unchanged.

Discussion: Our data suggest that an exercise training paradigm influences motor cortical excitability in PD Distinct cortical neuron subtypes may be restored by the exercise training, in particular the motoneurons (MNs) activated at higher TMS stimulation intensity, i.e. those that contribute to the plateau of the I-O curve. The higher plateau value may mirror a firing rates restoration and greater tendency for synchronization of the corticospinal discharges with increasing stimulus strength. The 
higher UPDRS motor score associated with higher plateau of the I-O curve showed post training, must be discussed also on the basis of myofibers modifications characteristic of the PD. The clinical improvement showed in our patients, may depend upon a restoration of the recruitment and firing rate of the large motor unit, i.e. those necessary to perform rapid and strong movements (type 2 myofibers), which in turn is caused by excitability changes of the MNs activated at higher intensity by TMS.

Conclusion: M1 functional activity might behaves abnormally in PD, and, in turn, could alter Mns/motor units behavior. These anomalies may be reversed by an exercise paradigm.

References:

- Messa LV, Ginanneschi F, Momi D, Monti L, Battisti C, Cioncoloni D, Pucci B, Santarnecchi E, Rossi A.Functional and Brain Activation Changes Following Specialized Upper-Limb Exercise in Parkinson's Disease. Front Hum Neurosci. (2019);13:350

- Glendinning DS, Enoka RM. Motor unit behavior in Parkinson's disease. Phys Ther. (1994);74(1):61-70

\section{TAGGING EEG FEATURES WITHIN EXAM REPORTS TO QUICKLY GENERATE A DATABASE FOR RESEARCH PURPOSES}

\author{
L. Marinelli ${ }^{1}$, C. Cabona ${ }^{2}$, I. Pappalardo ${ }^{2}$, D. Arnaldi ${ }^{1}$, F. Nobili ${ }^{1}$, F. \\ Villani $^{2}$
}

${ }^{1}$ DINOGMI, University of Genoa (Genova); ${ }^{2}$ Department of Neuroscience, IRCCS Ospedale Policlinico San Martino (Genova)

Objectives: A script that automatically extracts information from EEG reports following pre-defined tags may accelerate data collection and sorting for research on neurological conditions associated with peculiar EEG findings.

Materials and methods: The EEG report template used by Nicolet NicVue Patient Management Software (Natus Medical Incorporated) is written in HTML language; during report generation it is processed and edited by Microsoft Word, becoming a MHTML (MIME encapsulated aggregate HTML) document. Including a list of tags (labels) describing EEG features allows quick selection of appropriate tags during report editing. The generated MHTML files are consequently parsed by a custom script written in PHP language, able to extract the tags from each report and sort them in a table in comma separated value format, easily imported by spreadsheet, database and statistical software. The script called "EEG Report Parser" has been published as free and open source software under GNU-GPL license on Gitlab. We present a convenient sample of 437 EEG recording performed at the Division of Clinical Neurophysiology of the IRCCS Ospedale Policlinico San Martino (Genova) between June 1st and July 15th, 2020. Tags reflected EEG background (attenuation, suppression, $5-6 \mathrm{~Hz}$ frequency, $7-8 \mathrm{~Hz}$ frequency, no gradient, asymmetric, discontinuous, unreactive, brain death), epileptiform activity (generalized, focal, multifocal, ictal, interictal, spike\&wave, SE, NCSE), periodic discharges (LPD, BIPD, GPD, SIRPID, burst-suppression, triphasic waves), aetiology (anoxic, lesional, TBI, encephalitis, dementia, prion disease) and other features (normal variants, focal slowing, rhythmic delta, extreme delta brush).

Results: Tags selection during report preparation required only a few additional seconds. Among the 437 EEG recordings, 168 (38.4\%) had no tag, reflecting normal findings; 24 (5.5\%) included generalized while 29 $(6.6 \%)$ focal epileptiform discharges. Convulsive status epilepticus was recorded in $7(1.6 \%)$ and NCSE in $8(1.8 \%)$. The most frequent confirmed aetiology was lesional $(n=73,16.7 \%)$ followed by dementia $(n=21$, $4.8 \%$ ). The report parsing is very fast also on older computers, requiring about $4 \mathrm{~s}$ to process the described reports.

Discussion and conclusions: Retrospective analysis of EEG features can be difficult but very useful for clinical research. Identifying all EEGs showing ictal discharges or periodic patterns in a certain data range can be a difficult task if data are not sorted while they are recorded. Tagging EEG reports and tag parsing and extraction allows easy and quick retrieval of structured information.

References:

- https://en.wikipedia.org/wiki/MHTML

- https://gitlab.com/luciomarinelli/eeg-report-parser

\section{DIFFERENT MOTOR LEARNING TASKS INDUCE DIFFERENTIAL EFFECTS ON THE PRIMARY MOTOR AND SENSORY CORTICES IN HEALTHY HUMANS}

\section{G. Paparella ${ }^{1}$, L. Rocchi ${ }^{2}$, M. Bologna ${ }^{3}$, A. Berardelli ${ }^{3}$, J. Rothwell $^{2}$}

${ }^{1}$ IRCSS Neuromed (Pozzilli - IS); ${ }^{2}$ Department of Clinical and Movement Neurosciences, UCL Queen Square Institute of Neurology (London-UK); ${ }^{3}$ Department of Human Neurosciences, Sapienza University of Rome, IRCCS Neuromed (Roma, Pozzilli-IS)

Objectives: A large variability in learning-related neurophysiological changes in the primary motor and sensory cortices has been observed. It is unclear whether these differential effects are due to the different tasks investigated or to interindividual variance. Only a few studies have assessed different motor learning tasks and their effects on neurophysiological features within the same group of participants, and several issues are unclear. Here, we compared the effects of different tasks within each individual. We also tested possible correlation between neurophysiological changes and motor performance.

Materials: As representative of a model-free learning task, we used a ballistic motor task; as samples of model-based learning tasks, we tested two visuomotor learning tasks. Transcranial magnetic stimulation techniques were used to record motor and sensory evoked potentials, to test intracortical excitability as assessed by shortinterval intracortical inhibition, and sensorimotor interaction, i.e. short-latency afferent inhibition.

Methods: Neurophysiological measure of primary motor and sensory cortices were assessed from 15 healthy subjects before and after the motor learning tasks in three randomized experimental sessions.

Results: The ballistic motor task induced an increase in corticospinal excitability but did not change motor cortex intracortical inhibition or sensorimotor integration. In addition, it decreased the amplitude of cortical components of the somatosensory evoked potentials. The visuomotor learning tasks induced a reduction in motor cortex intracortical inhibition but did not modulate corticospinal and sensory cortex excitability or sensorimotor integration. No correlations between neurophysiological changes and motor performance merged from the analysis.

Discussion: Our results demonstrate differential motor skill acquisition-related effects on cortical parameters, possibly reflecting the engagement of specific neurophysiological substrates. This differential modulation is likely secondary to the motor skill acquisition, since no correlation was observed between neurophysiological changes and motor performance.

Conclusion: Our study contributes in-depth knowledge of the mechanisms involved in different types of motor skill acquisition in humans. 


\section{References:}

- Hamada M, Galea JM, Di Lazzaro V, Mazzone P, Ziemann U \& Rothwell JC. Two distinct interneuron circuits in human motor cortex are linked to different subsets of physiological and behavioral plasticity. J Neurosci (2014);34:12837-849

- Bologna M, Guerra A, Paparella G, Colella D, Borrelli A, Suppa A, Di Lazzaro V, Brown P \& Berardelli A. Transcranial Alternating Current Stimulation Has Frequency-Dependent Effects on Motor Learning in Healthy Humans. Neuroscience (2019);411:130-39

- Berghuis KMM, Semmler JG, Opie GM, Post AK \& Hortobágyi T. Age-related changes in corticospinal excitability and intracortical inhibition after upper extremity motor learning: a systematic review and meta-analysis. Neurobiol Aging (2017);55:61-71

\section{PAINFUL STIMULATION INCREASES SPONTANEOUS BLINK RATE IN HEALTHY SUBJECTS}

G. Paparella ${ }^{1}$, G. Di Stefano ${ }^{2}$, A. Fasolino ${ }^{2}$, G. Di Pietro ${ }^{2}$, D. Colella ${ }^{2}$, A. Truini $^{2}$, G. Cruccu $^{2}$, A. Berardelli ${ }^{3}$, M. Bologna ${ }^{3}$

${ }^{1}$ IRCSS Neuromed (Pozzilli - IS); ${ }^{2}$ Department of Human Neurosciences, Sapienza University of Rome (Roma); ${ }^{3}$ Department of Human Neurosciences, Sapienza University of Rome, IRCCS Neuromed (Pozzilli-IS)

Objectives: Spontaneous blink rate is considered a biomarker of central dopaminergic activity. Recent evidence suggests that the central dopaminergic system plays a role in nociception. In the present study, we investigate whether pain modulates spontaneous blink rate in healthy subjects. We also tested whether laser stimulation effects depended on stimulation intensity and if laser stimulation induced any changes in the blink reflex recovery cycle. Finally, we instigated possible relationship between spontaneous blink rate modification and pain modulation effect during the cold pressor test.

Materials: We enrolled 15 participants. Spontaneous blink rate and the blink reflex recovery cycle were quantified by an optoelectronic system for movement analysis. To induce pain, we stimulated the skin on the dorsum of the right forearm with a Neodymium YAP stimulator. The acoustic startling stimulation was applied binaurally (Sony auricular MDR-201) with tone bursts of $120 \mathrm{~dB}$ SPL (ISO, frequency $1000 \mathrm{~Hz}$, duration $120 \mathrm{~ms}$ ). To evoke blinking movements, we transcutaneously applied electrical stimuli to the right supraorbital nerve. The water bath for the cold pressor test was a plastic box filled with ice and water.

Methods: We kinematically recorded the spontaneous blink rate before and after 1) a painful laser stimulation, and 2) an acoustic startling stimulation. In a first control experiment, we investigated the laser stimulation effects on the spontaneous blink rate at different intensities of stimulation. In second control experiment, we tested possible changes in the blink reflex recovery cycle induced by the laser stimulation. Finally, in a third control experiment, we investigated any relationship between spontaneous blink rate modification and pain modulation effect during the cold pressor test.

Results: Laser, but not acoustic, stimulation increased spontaneous blink rate. This effect was independent of stimulation intensity and negatively correlated with pain perception. No changes in trigeminal-facial reflex circuit excitability were elicited by laser stimulation. The cold pressor test also induced an increased spontaneous blink rate.
Discussion: Our study provides evidence on the role of dopamine in nociception and suggests that dopaminergic activity may be involved in pain modulation.

Conclusions: These findings lay the groundwork for further investigations in patients with pathological conditions characterized by dopaminergic deficit and pain.

References:

1. Schmidtke K., Büttner-Ennever J. A. Nervous control of eyelid function. A review of clinical, experimental and pathological data. Brain (1992);115:227-47

2. Cruccu G. et al. Brainstem reflex circuits revisited. Brain (2005);128:386-94

3. Bologna M. et al. Facial bradykinesia. J. Neurol. Neurosurg. Psychiatry (2013);84:681-85

\section{EARLY NEUROPHYSIOLOGICAL ABNORMALITIES IN GUILLAIN-BARRÉ SYNDROME: 4-YEAR EXPERIENCE OF VERONA CENTER}

\author{
A. Rasera ${ }^{1}$, S. Romito ${ }^{2}$, A. Segatti ${ }^{2}$, E. Concon $^{2}$, L. Alessandrini ${ }^{2}$, F. \\ Basaldella $^{2}$, A. Badari ${ }^{2}$, B. Bonetti ${ }^{2}$, G. Squintani ${ }^{2}$ \\ ${ }^{1}$ Neurology Unit, University of Verona (Verona); ${ }^{2}$ Neurology Unit, \\ AOUI Verona (Verona)
}

Objective: Guillain Barré syndrome (GBS) is an acute-onset disorder of the peripheral nervous system that may be difficult to identify in the initial stages because diagnostic criteria are not always fulfilled. Our aim is to identify the most common electrophysiological abnormalities in 'very early' and 'early' neurophysiological evaluations of patients affected by GBS.

Material: We reviewed clinical documents of all patients with confirmed diagnosis of GBS, admitted to our Neurology unit in the last 4 years. We included patients who underwent nerve conduction studies (NCS) within 15 days from symptoms onset.

Method: Our cohort was divided in two subgroups according to the interval between disease onset and NCS: "very early" (within 7 days) and "early" (7-15 days). We evaluated all these factors: $\mathrm{H}$ reflex from tibial nerves, motor conduction parameters (amplitude, conduction velocity, distal motor latency and cMAP [compound motor action potential] duration) from ulnar, median, tibial and fibular nerves and $\mathrm{F}$ wave from the same nerves. All parameters were considered pathological if they were outside normal values in at least 2 nerves. We also evaluated sensory conduction and the presence of "sural sparing" pattern.

Results: 32 patients fulfilled the diagnostic criteria of GBS: 17 patients belonged to the "very early" subgroup, the other 15 patients were considered as "early" subset. The main abnormal parameter was the absence of $\mathrm{H}$ reflex from tibial nerve: it was found in $76 \%$ of "very early" and in $93 \%$ of "early" patients, with an overall frequency of $84 \%$. F wave abnormalities were present with a frequency of $65-67 \%$ in all subgroups. Demyelinating features in motor nerve conduction were found in $47 \%$ of "very early" and in $67 \%$ of the "early" patients. cMAP amplitude decrease was seen in $41 \%$ of patients, although it was present in all patients fullfilling the acute motor axonal neuropathy (AMAN) diagnostic criteria at further electrodiagnostic evaluation. In all subgroups, sensory conductions involvement was present in $38 \%$ patients and "sural sparing" pattern was observed in half of them. 
Discussion: The most sensitive electrophysiological parameter in GBS patients is the bilateral absence of tibial nerve $\mathrm{H}$ reflex, followed by $\mathrm{F}$ wave abnormalities.

Conclusions: Our findings confirm that proximal segments are the most frequently affected in the early phases of the disease and strengthen the importance of an exhaustive NCS, including $\mathrm{H}$ reflex and $\mathrm{F}$ waves evaluations, in the diagnosis of GBS.

\section{PREDICTING ISCHEMIC STROKE 90-DAYS PROGNOSIS CHALLENGE. USEFULNESS OF SENSORS PARAMETERS}

\section{G. Reale, A. Zauli, C. Iacovelli, P. Caliandro}

Neurosciences Department, Cattolica del Sacro Cuore University (Roma)

Aims: Formulating a reliable prognosis for ischemic stroke patients is still difficult. Although an increasing interest towards sensors, no sensorbased prognostic parameter is available yet. We aimed to assess whether an actigraphic-based parameter (Asymmetry Index, AsI) obtained in the acute phase can improve 90-days prognosis accuracy.

Materials and Methods: We used two actigraphic wristwatchlike sensors to register upper limbs movement asymmetry (and the relative AsI) among 20 patients with acute ischemic stroke. We therefore collected their main stroke outcome predictors and their 90-days modified Rankin Score (mRS). Then, we showed the aforementioned data to six experienced stroke physicians, asking them to formulate a 90-days mRS prognosis.

Results: We found a positive correlation between AsI and the real 90-days mRS $(r=0.69, p<0.001)$. We found that an AsI $>32 \%$ predicts a 90-days $\mathrm{mRS}>2$ with sensitivity $=100 \%$ and specificity $=89 \%$. The agreement between the 90 -days mRS clinically predicted by stroke physicians in the acute phase and the real 90-days mRS was low (Cohen's k=0.23).

Discussion and conclusions: Making a reliable stroke 90-days prognosis is still challenging, even for experienced stroke physicians. In this view, a possible help might come from sensor-based parameters.

References:

- Iacovelli C, Caliandro P, Rabuffetti M, et al. Actigraphic measurement of the upper limbs movements in acute stroke patients. J Neuroeng Rehabil (2019);16:153

- Geurts M, de Kort FAS, de Kort PLM, et al. Predictive accuracy of physicians' estimates of outcome after severe stroke. PLoS ONE (2017);12:e0184894

- Le Heron C, Fang K, Gubbi J, et al. Wireless accelerometry is feasible in acute monitoring of upper limb motor recovery after ischemic stroke. Cerebrovasc Dis (2014);37:336-41

\section{SOMATOSENSORY EVOKED POTENTIALS RECORDED WITHIN THE INSULA TO MEDIAN NERVE STIMULATION}

\author{
M. Valeriani, L. De Palma, D. De Stefano, N. Specchio, C. Marras, F. \\ Vigevano, S. Pro
}

${ }^{1}$ Neurology Unit, Bambino Gesù Hospital (Roma); ${ }^{2}$ Neurophysiology, Bambino Gesù Hospital (Roma); ${ }^{3}$ Neurosurgery, Bambino Gesù Hospital (Roma)
Objective: Somatosensory evoked potentials (SEPs) originate from different regions of the brain, beyond the classical somatosensory cortices. However, non-nociceptive SEPs have never been recorded from the insular cortex. In the present study, we investigated whether SEPs could be recorded from the insula after non painful electrical stimulation of the median nerve.

Materials and Methods: Eight patients with drug resistant epilepsy were recruited. Intracerebral electrodes were implanted for a presurgical assessment of the epilepsy. Three patients were implanted in both the anterior and posterior insula, two patients only in the posterior insula, and three only in the anterior insula. SEPs were recorded from the intracerebral electrode contacts after stimulation of the median nerve contralateral to the implanted electrode at the wrist. Stimulation intensity was set at the motor threshold, thus it was not painful. Stimulation rate was $1 \mathrm{~Hz}$ and filter bandpass was 1-1500 Hz.

Results: Five components were recorded from the posterior insula: $\mathrm{N} 50$ (mean latency $47.5 \pm 3.4 \mathrm{~ms}$ ), P70 (mean latency $72.9 \pm 2.8 \mathrm{~ms}$ ), N100 (mean latency 101.6 $\pm 4.9 \mathrm{~ms}$ ), P160 (mean latency $158.2 \pm 11.9$ $\mathrm{ms}$ ), and N250 (mean latency $249.1 \pm 27.4 \mathrm{~ms}$ ). On the contrary, no SEPs could be evoked from the anterior insula.

Discussion and Conclusion: This is the first study in which nonnociceptive SEPs were recorded from the posterior insula. Our results agree with the findings of neurophysiological studies in animals and of neuroimaging investigations and support the key role of the posterior insula in the somatosensory input processing.

\section{CLINICAL NEUROPSYCHOLOGY}

\section{USABILITY OF THE NEGATIVE BINOMIAL MODEL FOR ANALYZING CEILING AND HIGHLY-INTER-INDIVIDUALLY VARIABLE COGNITIVE DATA}

\author{
E. N. Aiello ${ }^{1}$, E. Depaoli ${ }^{2}$, M. Gallucci ${ }^{3}$
}

${ }^{1}$ School of Medicine and Surgery, University of Milano-Bicocca (Milano); ${ }^{2}$ Department of Statistical Sciences, University of Padua (Padova); ${ }^{3}$ Department of Psychology, University of Milano-Bicocca (Milano)

Aims: Cognitive data from both mildly-impaired patients and healthy controls (HC) often do not meet assumptions for linear model (LM) analyses since they show left-skeweness and overdispersion - due to ceiling effect and high inter-individual variability, respectively. Normalizing/variance-stabilizing transformations and non-parametric tests alter the original metric of the outcome and may thus lead to loss of information. Moreover, non-parametric tests offer a restricted range of analytical options and might lack statistical power. This study aimed at providing a proof of concept that using generalized linear models (GLM) which assume overdispersed count-like distributions (Negative Binomial; NB) can obviate aforesaid issues.

Materials: Data regarding a noun naming task (range $=0-50$ ) from 30 motor neuron disease (MND) patients and $29 \mathrm{HCs}$ were taken into consideration [1] - since MND patients can show mild lexical-retrieval deficits [2].

Methods: The number of errors on the task was computed for each subject and regarded as the outcome variable. Likelihood ratio tests between non-nested models with parametric-bootstrap-inferred distributions [3] were implemented in order to assess the goodness of fit of the NB GLM compared to a classical LM and the Poisson GLM (a non- 
overdispersed GLM for count data) with regard to a case-control comparison with and without a covariate (age).

Results: The null hypothesis (H0) that the underlying distribution was a NB vs. a Gaussian could not be rejected $(\mathrm{p}=.378)$. The H0 that the underlying probability distribution was a NB vs. a Poisson could not be rejected ( $\mathrm{p}=.509$ ), whereas the $\mathrm{H} 0$ that the underlying probability distribution was a Poisson vs. a NB could be rejected $(\mathrm{p}<.001)$. Similar results were obtained without the covariate.

Discussion: NB GLM yields a better fit to overdispersed ceiling cognitive data when compared to both a classical LM and the Poisson GLM if a case-control comparison with and without a covariate is performed. NB GLM could be thus adopted in high-inter-individual-variability scenarios when patients show mild deficits and/or find it easy to score high on a test. Since the present method regards the number of errors as the outcome, caution should be exercised when applying it to closed-range neuropsychological tests. The presented method is ostensibly indicated for the majority of language tests.

Conclusions: NB GLM allows case-control comparisons of cognitive data from closed-range tests when ceiling effect and high inter-individual variability are present by regarding the number of errors as the outcome. This model is suitable whether a covariate is entered or not.

References:

1. Aiello, E., Luzzatti, C., Pain, D., Gallucci, M., Mora, G. Noun-verb dissociation in motor neuron disease: theoretical and clinical entailments [Poster presentation]. 8th Winter Seminar on Dementia and Neurodegenerative Disorders, Bressanone, BZ, Italy. (2020, January 22-24). http://sindem4juniors.it/program.php.

2. Pinto-Grau, M., Hardiman, O., Pender, N. The study of language in the amyotrophic lateral sclerosis - frontotemporal spectrum disorder: a systematic review of findings and new perspectives. Neuropsychology Review (2018);28:251-68

3. Hinde J. Choosing Between Non-nested Models: a Simulation Approach. In L. Fahrmeir, B. Francis, R. Gilchrist, G. Tutz (Eds.), Advances in GLIM and Statistical Modelling. (1992); pp. 119-124 Springer.

\section{ALTERED RESTING STATE DYNAMIC FUNCTIONAL CONNECTIVITY OF THE PRECUNEUS CONTRIBUTES TO COGNITION AND DEPRESSION IN NEUROMYELITIS OPTICA}

\author{
L. Cacciaguerra ${ }^{1}$, D. Mistri ${ }^{1}$, P. Valsasina ${ }^{1}$, V. Martinelli ${ }^{2}$, M. Filippi ${ }^{3}$, \\ M. Rocca ${ }^{4}$
}

${ }^{1}$ Neuroimaging Research Unit, Institute of Experimental Neurology, Division of Neuroscience, IRCCS San Raffaele Scientific Institute (Milano); ${ }^{2}$ Neurology Unit, IRCCS San Raffaele Scientific Institute (Milano); ${ }^{3}$ Neuroimaging Research Unit, Institute of Experimental Neurology, Division of Neuroscience; Neurology Unit and Neurophysiology Unit, IRCCS San Raffaele Scientific Institute; VitaSalute San Raffaele University (Milano); ${ }^{4}$ Neuroimaging Research Unit, Institute of Experimental Neurology, Division of Neuroscience; and Neurology Unit, IRCCS San Raffaele Scientific Institute (Milano)

Objectives: To explore the role of dynamic functional connectivity (dFC) of the precuneus (PCUN) at resting state (RS) to explain cognitive alterations in neuromyelitis optica spectrum disorders (NMOSD) patients.

Materials: 3.0 T RS functional MRI (fMRI) were acquired from 27 aquaporin4 (AQP4)-positive NMOSD patients and 30 age- and sex- matched healthy controls (HC). Patients underwent an extensive neuropsychological evaluation including the assessment of global and domainspecific cognitive impairment index (CII) and Beck Depression Inventory II (BDI-II) scores

Methods: DFC of the left (L) and right (R) PCUN was assessed by means of sliding-window seed-voxel correlation analysis. Standard deviation of $\mathrm{dFC}$ across windows was used as a measure of dynamicity (the higher the better). Age- and sex-adjusted between-group dFC comparisons and correlations with cognitive scores were assessed using SPM12 and full-factorial models. A $p$ value $<0.001$ was considered statistically significant.

Results: Compared to HC, NMOSD patients had reduced LPCUN dFC with caudate nucleus, rectus, olfactory bulb and occipital inferior gyrus and increased $\mathrm{dFC}$ between the L-PCUN and the middle temporal gyrus and between the R-PCUN the middle occipital gyrus. Global CII positively correlated with higher Lintra-PCUN dFC, as well as with higher $\mathrm{dFC}$ between the LPCUN and the middle temporal and middle frontal gyrus and between the R-PCUN and the middle cingulate gyrus. Impairment of information processing speed (IPS, 59.2\%) and depression $(63.0 \%)$ were the most common cognitive alterations. The IPS index positively correlated with a higher L-intra-PCUN$\mathrm{dFC}$, and a higher $\mathrm{dFC}$ between the R-PCUN and the middle cingulate gyrus. The BDI-II score positively correlated with a higher $\mathrm{dFC}$ between the R-PCUN and the middle frontal gyrus.

Discussion: In NMOSD, cognitive impairment is nowadays considered as a unique relapse-unrelated manifestation of the disease. As a proof-of-concept, AQP4-IgG seem to inhibit neuronal plasticity and long-term potentiation. Structural and fMRI studies have disclosed an association with damage of the PCUN and cognitive impairment CI in several neurological conditions.

Conclusions: The assessment of PCUN dFC supports the role of PCUN in NMOSD cognitive dysfunction. We observed a protective effect of higher dynamic connections with limbic regions for cognitive performance, while those with the frontal lobe were detrimental for depressive symptoms.

\section{DETECTING EARLY SIGNS OF COGNITIVE IMPAIRMENT IN ASYMPTOMATIC SUBJECTS: AN EXPLORATORY STUDY IN AN ITALIAN COHORT OF COGNITIVELY HEALTHY ELDERLY SUBJECTS}

\author{
E. Chipi ${ }^{1}$, C. Montanucci ${ }^{1}$, N. Salvadori ${ }^{1}$, L. Biscetti ${ }^{1}$, G. Bellomo ${ }^{2}$, L. \\ Parnetti $^{2}$
}

${ }^{1}$ Center for Memory Disturbances, University of Perugia (Perugia); ${ }^{2}$ Center for Memory Disturbances, Lab of Clinical Neurochemistry, University of Perugia (Perugia)

Objectives: Early identification of the first clinical symptoms in older healthy individuals represents a major issue in Alzheimer's Disease (AD) field. This could be achieved by means of sensitive neuropsychological tools able to detect subtle cognitive changes. Cambridge Neuropsychological Test Automated Battery (CANTAB) represents a computerized advanced tool for in-depth neuropsychological assessment. The aim of this study is to explore the percentage of healthy elderly subjects who had worse performance at CANTAB.

Materials: One hundred and eighty healthy and functionally independent subjects (114 F, $66 \mathrm{M}$; mean age: 70.54, SD: 5.55, median age 70.57, min age: 50 , max age: 82 ; median education: $13 \mathrm{yr}$ ) were recruited among 
relatives of patients attending our Memory Clinic or as volunteers after advertisement.

Method: All subjects underwent a standard battery of neuropsychological tests (Mini Mental State Examination, MMSE; Italian version of Repeatable Battery for the Assessment of Neuropsychological Status, RBANS) and CANTAB tests assessing visual-spatial memory (Pattern Recognition Memory, PRM; Paired Associative Learning, PAL) and executive functions (Spatial Working Memory, SWM). Pathological performance at CANTAB was considered as scores $\geq 1.5 \mathrm{SD}$ in at least one test.

Results: The highest percentage of impaired performance was found in PAL-total errors $(14.4 \% ; \mathrm{p}=0.02)$, while the lowest was found in PRM $(4.8 \%)$. CANTAB PAL total-error correlated with RBANS-delayed memory $(\mathrm{r}=0.39, \mathrm{p}<0.001,95 \% \mathrm{CI}=0.26-0.51)$ and with RBANS-total index $(\mathrm{r}=0.30, \mathrm{p}<0.001,95 \% \mathrm{CI}=0.16-0.43)$. PAL 6-shapes correlated with RBANS-delayed memory $(\mathrm{r}=0.40, \mathrm{p}<0.001,95 \% \mathrm{CI}=0.27-0.52)$. Participants with pathological performance at PAL-total errors also showed poorer scores at RBANS-total index, compared to those with normal performance at PAL $(89.7 \pm 9.8$ vs $95.0 \pm 12.3 ; p=0.039)$.

Discussion: Our findings suggest that a significant high percentage of impairment at CANTAB-PAL test can occur in cognitively asymptomatic elderly subjects and that its scores are significantly associated with global cognitive functioning. Since accumulating evidences demonstrates that impaired performance on CANTAB- PAL may be sensitive for early diagnosis of $\mathrm{AD}$, longitudinal observation of this cohort is crucial.

Conclusions: Current evidence suggests that there is a gap between the positivity of $\mathrm{AD}$ biomarkers and the emergence of clinical manifestation. In this perspective, tracking longitudinal change in cognitive functions in older individual is mandatory. We reported that a significant proportion of cognitively healthy elderly subjects shows subtle impairment at a computerized measure of associative learning. Longitudinal assessments are needed in order to understand whether poor performance in CANTAB tests may represent the first sign of cognitive impairment due to $\mathrm{AD}$.

References:

- Papp, K.V., Buckley, R., Mormino, E., Maruff, P., Villemagne, V.L., Masters, C.L., Johnson, K.A., Rentz, D.M., Sperling, R.A. and Amariglio, R.E. Clinical meaningfulness of subtle cognitive decline on longitudinal testing in preclinical AD. Alzheimers Dement. (2020);16(3):552-60

- Chipi E, Salvadori N, Farotti L, Parnetti L. Biomarker-Based Signature of Alzheimer's Disease in Pre-MCI Individuals. Brain Sci. (2019);9(9):213

- Soldan A, Pettigrew C, Moghekar A, Albert M; BIOCARD Research Team. Computerized Cognitive Tests Are Associated with Biomarkers of Alzheimer's Disease in Cognitively Normal Individuals 10 Years Prior. J Int Neuropsychol Soc. (2016) Nov;22(10):968-77

\section{OXFORD COGNITIVE SCREEN (OCS) FEASIBILITY IN THE STROKE UNIT SETTING}

\author{
N. Ciccarelli ${ }^{1}$, E. Pini $^{2}$, F. Pepe ${ }^{2}$, M. Silveri ${ }^{1}$, E. Magni ${ }^{2}$ \\ ${ }^{1}$ Department of Psychology, Catholic University (Milano); ${ }^{2}$ Department \\ of Neuroscience, Poliambulanza Foundation (Brescia)
}

Objectives: Cognitive screening is not widely used in acute stroke patients, and usually is based on tests developed for dementia. Our aim was to explore the feasibility of a new tool designed for stroke survivors, as the Oxford Cognitive Screen (OCS).
Materials: 3-10 days after stroke, patients underwent the OCS, providing a five domain-specific cognitive profile: attention and executive function, language, memory, number processing and praxis. Pre-morbid functional and cognitive status was evaluated by caregiver interview. The National Institute of Health and Stroke Scale (NIHSS) estimated the stroke severity.

Method: This cross-sectional study consecutively enrolled stroke patients (both ischemic and hemorrhagic). Exclusion criteria: Pre-morbid cognitive decay, difficulties with the Italian language, visual impairment or other preexisting conditions affecting cognitive status. OCS was considered non-applicable when patients failed the preliminary subtest. Ischemic brain lesions were classified according to the Bamford criteria: Total Anterior Circulation Stroke (TACS), Partial Anterior Circulation Stroke (PACS), Posterior Circulation Syndrome (POCS), Lacunar Syndrome (LACS).

Results: Between October and December 2019, 45 out of 62 patients admitted to the Stroke Unit of Poliambulanza Foundation of Brescia were enrolled. Eight patients were non-eligible, 2 died, and 7 did not fit the time window inclusion criteria. Characteristics of the sample were: 43 (95.6\%) Italian born, $33(73.3 \%)$ male, 37 (82.2\%) with ischemic stroke, mean age (years) 68.00 (DS 15.45), mean initial NIHSS score 7.02 (DS 7.67). Overall, 25 (55.6\%) patients had a left sided brain lesion, 13/37 (35\%) were affected by TACS, 19 (42.2\%) were discharged at home without any cognitive follow-up indications, and 9 (20\%) were transferred to neurologic rehabilitation. OCS was applicable in $37 / 45$ $(82.2 \%)$ patients. Twenty-eight $(75.7 \%)$ patients showed at least one impaired cognitive domain. At multivariate logistic regression analyses, higher age (OR 1.33; 95\% CI 1.02-1.74, $\mathrm{p}=0.038)$ and higher NIHSS score (for every 1-point increase, OR 1.56; 95\% CI 1.05-2.33, $\mathrm{p}=0.029$ ) were significantly associated to OCS non-applicability, after adjusting for type of stroke (ischemic vs hemorrhagic). In the ischemic subgroup, having TACS (compared to PACS, POCS, or LACS) showed a significant association to OCS non-applicability (OR 13.50, 95\% CI 1.19-153.33, $\mathrm{p}=$ 0.036). Brain lesion side wasn't a significant factor.

Discussion: OCS confirmed a very good applicability in acute stroke setting, uncontaminated by aphasia and neglect. Age, stroke severity and lesion extension (but not side) emerged as major determinants of its applicability.

Conclusions: OCS seems a promising tool in acute stroke setting.

\section{ANATOMICAL CORRELATES OF STRATEGIC MEMORY: A NEUROPSYCHOLOGICAL STUDY}

A. Cocuzza ${ }^{1}$, L. Sbrissa ${ }^{1}$, T. Difonzo ${ }^{2}$, M. Sirtori ${ }^{3}$, A. Di Fonzo ${ }^{2}$, G. Franco $^{2}$, G. Bertani ${ }^{4}$, B. Zarino ${ }^{4}$, N. Bresolin, M. Saetti ${ }^{1}$

${ }^{1}$ Department of Medical-Surgical Pathophysiology and Transplantation, IRCCS Foundation Ca' Granda Ospedale Maggiore Policlinico, University of Milan (Milano); ${ }^{2}$ Neurology Unit, IRCCS Foundation Ca' Granda Ospedale Maggiore Policlinico (Milano); ${ }^{3}$ Department of Psychology, University of Milano-Bicocca (Milano); ${ }^{4}$ Neurosurgery Unit, IRCCS Foundation Ca' Granda Ospedale Maggiore Policlinico (Milano)

Objective: During word lists learning tasks, patients affected by Parkinson's Disease (PD) are not significantly impaired in encoding items compared to Normal Controls (NC), but they don't benefit from semantic clues [1]. In similar tasks, Frontal Patients (FP) show an overall deficit in list learning, but patients with lesions involving Area 9 (A9) result significantly more impaired when compared to other groups with lesions in 
mDLPFC [2], due to poor semantic strategy use. Based on the evidence of an associative fronto-striatal circuit involving A9 [3], we aimed at evaluating the relation linking the impairment of the aforementioned neural circuit and the neuropsychological features between these two patients' groups (PD and FP).

Materials: 30 PD patients, $10 \mathrm{FP}$ and $\mathrm{NC}$ matched for age, sex and education were recruited and tested with a two-part verbal learning test: multi-trial words list learning in free recall and multi-trial words list learning preceded by an explicit semantical strategy cue. FP were divided into two groups: patients with frontal lesions involving (F9) or sparing (Fn) A9.

Methods: Bivariate between-group analyses were performed with age, sex and education as covariate in order to evaluate the performance of the four groups (NC, PD, F9, Fn) in terms of number of items recalled and effective use of learning strategies (cued or not).

Results: PD patients learned fewer words and found difficulties in using semantic strategies compared to NC. When the strategy was suggested by the examiner, PD patients improved their performance, but not as much as NC. F9 showed similar results to PD patients. Fn showed similar results to the NC.

Discussion: These results are in line with previous studies and show that the similarity between PD and F9 may be due to the degeneration of specific cortico-subcortical connections between Caudate Nuclei and A9. More specifically, impaired results in the learning task in PD could be due to subcortical degeneration that arises from the basal ganglia.

Conclusions: These findings support the hypothesis that A9 and its connections with Caudate Nuclei could have a prominent role in strategy implementation. Moreover, they show that A9, and not surrounding areas, could be responsible for the effective use of semantic strategies in learning tasks. The similarities between PD and F9 suggest that the connection between Caudate Nuclei and A9 could be an integral part of the dorsolateral cognitive circuit of the extrapyramidal system.

References:

1. Faglioni P, Saetti MC, Botti C, Modena U. Verbal Learning Strategies in Parkinson's Disease. Neuropsychology (2000);14(3):456-70

2. Alexander MP, Stuss D, Gillingham S. Impaired List Learning Is Not a General Property of Frontal Lesions. J Cogn Neurosci. (2009):1422-34

3. Duffau H. The anatomo-functional connectivity of language revisited New insights provided by electrostimulation and tractography. Neuropsychologia (2008);46:927-934

\section{UNRAVELING THE SUBSTRATES OF COGNITIVE IMPAIRMENT IN MULTIPLE SCLEROSIS: THE CONTRIBUTION OF A MULTIPARAMETRIC STRUCTURAL AND FUNCTIONAL MRI APPROACH}

\author{
L. Conti ${ }^{1}$, P. Preziosa ${ }^{2}$, A. Meani ${ }^{1}$, C. Vizzino ${ }^{1}$, G. Riccitelli ${ }^{1}$, E. Pagani ${ }^{1}$,

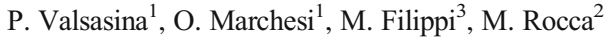

${ }^{1}$ Neuroimaging Research Unit, Institute of Experimental Neurology, Division of Neuroscience, IRCCS San Raffaele Scientific Institute (Milano); ${ }^{2}$ Neuroimaging Research Unit, Institute of Experimental Neurology, Division of Neuroscience; and Neurology Unit, IRCCS San Raffaele Scientific Institute (Milano); ${ }^{3}$ Neuroimaging Research Unit, Institute of Experimental Neurology, Division of Neuroscience; Neurology Unit and Neurophysiology Unit, IRCCS San Raffaele Scientific Institute; Vita-Salute San Raffaele University (Milano)
Objectives: To evaluate the combined contribution of white matter (WM) lesions, gray matter (GM) atrophy and resting state (RS) functional (f) MRI abnormalities in explaining cognitive impairment (CI) in a large cohort of multiple sclerosis (MS) patients.

Material: Brain 3T dual-echo, 3D T1-weighted and RS fMRI scans were acquired from 100 healthy controls (HC) and 276 MS patients. All MS patients underwent a neuropsychological evaluation through the administration of the Rao's Brief Repeatable Battery of Neuropsychological tests (BRB).

Methods: CI was defined with a z-score $<-1.5$ at least two tests of BRB. Distribution of brain WM lesions, GM atrophy and RS functional connectivity (FC) abnormalities within the default mode (DMN) and salience (SN) networks were compared between $\mathrm{HC}$ and MS patients at a voxel level. Using sex-, age- and phenotype-adjusted stepwise logistic regression models, the role of WM lesions (model 1), GM atrophy (model 2), RS FC (model 3) and their combination (model 4) in explaining CI was investigated. Model performances were assessed by the area under the curve (AUC).

Results: Eighty-three (30.1\%) MS patients were classified with CI. In model 1, lesions in left (L) superior longitudinal fasciculus (SLF) (odds ratio $[\mathrm{OR}]=1.84)$, $\mathrm{L}$ medial lemniscus $(\mathrm{OR}=1.79)$ and $\mathrm{L}$ inferior longitudinal fasciculus $(\mathrm{OR}=1.57)$ predicted $\mathrm{CI}(\mathrm{p} \leq 0.009)$. In model $2, \mathrm{~L}$ precuneus $(\mathrm{OR}=0.52)$ and $\mathrm{L}$ caudate $(\mathrm{OR}=0.56)$ volumes predicted $\mathrm{CI}$ $(\mathrm{p} \leq 0.007)$. In model 3 , increased RS FC in L caudate $(\mathrm{DMN})(\mathrm{OR}=1.77)$ and decreased RS FC in right $(\mathrm{R})$ thalamus $(\mathrm{DMN})(\mathrm{OR}=0.66)$ and $\mathrm{L}$ inferior frontal gyrus (IFG) $(\mathrm{SN})(\mathrm{OR}=0.68)$ predicted $\mathrm{CI}(\mathrm{p} \leq 0.02)$. In model 4, $\mathrm{R}$ middle cerebellar peduncle $(\mathrm{OR}=2.05)$ and $\mathrm{L}$ SLF $(\mathrm{OR}=1.84)$ lesions, $\mathrm{L}$ precuneus atrophy $(\mathrm{OR}=0.46)$, increased $\mathrm{RS} F C$ in $\mathrm{L}$ caudate $(\mathrm{DMN})(\mathrm{OR}=1.64)$, and decreased RS FC in L IFG (SN) $(\mathrm{OR}=0.64)$ predicted $\mathrm{CI}(\mathrm{p} \leq 0.02)$. Compared to demographic and clinical variables only ( $\mathrm{AUC}=0.73$ ), the separate models performed significantly better (AUC $=0.82,0.81$ and 0.80 , respectively, $\mathrm{p} \leq 0.003$ ), with model 4 having the best performance ( $\mathrm{AUC}=0.86, \mathrm{p}<0.001$ ).

Discussion: The combination of multiparametric MRI techniques suggests that the accumulation of focal WM lesions and GM atrophy in strategic brain regions together with maladaptive functional mechanisms explains $\mathrm{CI}$ in MS.

Conclusions: The combined assessment of structural and functional MRI abnormalities represents a rewarding strategy to reveal the pathophysiological substrates underpinning CI in MS patients.

\section{COGNITIVE RESERVE MODULATION OF FUNCTIONAL BRAIN CONNECTIVITY IN PARKINSON'S DISEASE}

\section{S. Di Tella ${ }^{1}$, M. De Marco $^{2}$, F. Baglio ${ }^{1}$, M. Silveri ${ }^{3}$, A. Venneri $^{2}$}

${ }^{1}$ IRCCS Fondazione Don Carlo Gnocchi (Milano); ${ }^{2}$ Department of Neuroscience, University of Sheffield, Medical School (Sheffield-UK); ${ }^{3}$ Department of Psychology, Catholic University of the Sacred Heart (Milano)

Objectives: The functional disconnection of the striato-thalamo-frontal pathways represents the neural correlate of the dysexecutive syndrome, that are the most common cognitive deficits observed in patients with Parkinson's Disease (PD) [1]. Multiple reserve-related processes may contribute to the resilience against neurodegeneration, such as cognitive reserve, a flexible construct that refers to the adaptability (intended as efficiency, capacity and flexibility) of cognitive processes, which are supported by more adaptable neurofunctional brain processes when performing a task [2]. In this study we explore if the functional connectivity of brain regions associated with the executive dysfunction in PD is modulated by the cognitive reserve. 
Materials and Methods: Twenty-six PD patients and 24 healthy controls (HC) underwent a resting-state functional magnetic resonance imaging (rs-fMRI) examination. Two approaches were implemented to explore the functional connectivity: an independent component analysis (ICA) and a seed-based approach. In the ICA approach targeted networks were selected: the default mode, the salience, the left and right frontoparietal, the sensorimotor, and the occipital visual networks. In the seedbased approach the substantia nigra, the dorsolateral and ventromedial frontal regions part of the frontostriatal loop were selected as regions of interest. Cognitive reserve was attained using education and level of occupation as proxy measures.

Results: With respect to HC, PD patients showed a significantly lower functional connectivity in sensorimotor regions and functional alteration of executive-attentional networks exhibiting lower coupling between prefrontal and inferior parietal regions than $\mathrm{HC}$. Modulation of functional brain connectivity by cognitive reserve was detected: PD patients with low cognitive reserve manifested reduced functional connectivity in different regions of the anterior cingulate and the basal ganglia (putamen and caudate) and a bilateral reduction of connectivity between fronto-parietal regions. However, hyperconnectivity was shown within medial prefrontal regions when comparing PD with low cognitive reserve to $\mathrm{HC}$.

Discussion: These findings suggest that cognitive reserve may exert a modulatory effect on functional connectivity involving basal ganglia and executive-attentional fronto-parietal networks. Attentional control networks seem to be downregulated in PD patients with low cognitive reserve; whereas the higher recruitment of medial frontal regions in $\mathrm{PD}$ patients with lower cognitive reserve than $\mathrm{HC}$ suggests a compensation by an upregulation mechanism. This upregulation might contribute to maintaining an efficient cognitive functioning when posterior cortical function begins to be reduced [3]

Conclusions: PD patients presented a disruption of networks of central importance for executive functioning with a relevant modulation exerted by cognitive reserve.

References:

1. Williams-Gray C. H., Foltynie T., Brayne C. E. G., Robbins T. W., Barker R. A., Evolution of cognitive dysfunction in an incident Parkinson's disease cohort. Brain (2007); 130(7): 1787-98

2. Stern Y., Arenaza-Urquijo E. M., Bartrés-Faz D., Belleville S., Cantilon M., Chetelat G., ... \& Okonkwo, O., Whitepaper: Defining and investigating cognitive reserve, brain reserve, and brain maintenance. Alzheimer's \& Dementia (2018);S15525260(18)33491-5

3. Cabeza R., Albert M., Belleville S., Craik F. I., Duarte A., Grady C. L., ... \& Rugg M. D., Maintenance, reserve and compensation: the cognitive neuroscience of healthy ageing. Nature Reviews Neuroscience (2018);19(11):701-10

\section{PRODUCTION OF NEGATIVE VALENCE EMOTIONS CONVEYED BY VOICE IN PARKINSON'S DISEASE: ASSOCIATION BETWEEN ACOUSTIC PARAMETERS AND WHITE MATTER MICROSTRUCTURAL INTEGRITY}

\author{
S. Di Tella ${ }^{1}$, I. Anzuino ${ }^{2}$, L. Pelizzari ${ }^{1}$, M. Cabinio ${ }^{1}$, F. Biassoni ${ }^{2}$, M.

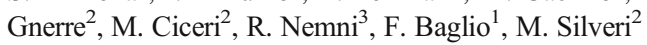 \\ ${ }^{1}$ IRCCS Fondazione Don Carlo Gnocchi (Milano); ${ }^{2}$ Department of \\ Psychology, Catholic University of the Sacred Heart (Milano); ${ }^{3}$ IRCCS, \\ Fondazione Don Carlo Gnocchi, Department of Pathophysiology and \\ Transplantation, University of Milan (Milano)
}

Objectives: Parkinson's Disease (PD) patients commonly manifested impaired expression of negative valence emotions through pitch, loudness, and speech rhythm alterations. Altered affective prosody may be due to the damage of speech articulatory systems and/or to the specific dysfunction of emotional processing conveyed by voice [1]. We explored: 1) PD vs healthy controls (HC) differences in affective negative prosody; 2) their association with white matter (WM) integrity of specific regions of interest (ROIs).

Materials and Methods: Affective prosody was rated in 15 PD and 15 $\mathrm{HC}$ participants through the vocal expression of negative emotions elicited by the reading of a short story. Mean (M) and standard deviation (SD) values of the fundamental frequency (F0) were extracted from the speech samples. A subset of participants $(n=25)$ were scanned with diffusionweighted imaging (DWI) and 3D-T1 images at 1.5T. After standard processing, Tract-Based Spatial Statistics was used to test voxel-wise PD-vs$\mathrm{HC}$ differences of fractional anisotropy (FA). Also, within each group, voxel-wise correlations between FA and F0 parameters were assessed. All voxel-wise analyses were restricted to ROIs related to prosody expression $[2,3]$ including: bilateral cortico-spinal tracts (CST), superior longitudinal (SLF), superior fronto-occipital (SFOF), inferior frontooccipital (IFOF), uncinate (UF) fascicules.

Results: PD patients showed lower SD-F0 values $(p=0.012)$ than HC. Significantly lower FA was observed in the CST of PD patients, bilaterally (pFWE, peak=0.020). Correlation analyses between F0 and white matter microstructural parameters showed in PD significant negative correlation between M-F0 and FA in bilateral CST and right SLF (pFWE, peak=0.007) and between SD-F0 and FA in bilateral CST and SLF (pFWE, peak<0.001).

Discussion: PD patients manifested altered affective prosody showing a reduction in the variability of F0 of speech. Acoustic parameters of negative emotions in PD group were predominantly associated with WM integrity in CST. Microstructural changes along the CST are likely to alter the communication between cortical and subcortical brain regions that are essential for voluntary voice control of speech production. Furthermore, the association between acoustic parameters and the FA of the SLF might suggest an involvement of a bundle of WM that connects cortical regions supposed to be implicated in emotional prosody.

Conclusions: The difficulty to convey negative emotions by voice in PD may be principally related to the articulatory deficits although the involvement of the SLF also suggests the damage of neural substrates implicated in the emotional processing.

References:

1. Möbes J., Joppich G., Stiebritz F., Dengler R., Schröder C., Emotional speech in Parkinson's disease. Movement Disorders (2008);23(6):824-29

2. Patel S., Oishi K., Wright A., Sutherland-Foggio H., Saxena S., Sheppard S. M., Hillis A. E., Right hemisphere regions critical for expression of emotion through prosody, Frontiers in Neurology (2018);9:224

3. Kostic V. S., Agosta F., Sarro L., Tomić A., Kresojević N., Galantucci S., ... Filippi M., Brain structural changes in spasmodic dysphonia: a multimodal magnetic resonance imaging study. Parkinsonism \& related disorders (2016);25:78-84

\section{STRIATAL PATHOLOGY AND RECOGNITION OF EMOTIONS CONVEYED BY VOICE IN PARKINSON'S DISEASE: AN MRI STRUCTURAL STUDY}

S. Di Tella ${ }^{1}$, I. Anzuino ${ }^{2}$, M. Cabinio ${ }^{1}$, F. Biassoni ${ }^{2}$, M. Gnerre ${ }^{2}$, M.

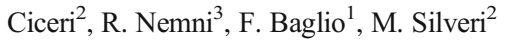


${ }^{1}$ IRCCS Fondazione Don Carlo Gnocchi (Milano); ${ }^{2}$ Department of Psychology, Catholic University of the Sacred Heart (Milano); ${ }^{3}$ IRCCS, Fondazione Don Carlo Gnocchi, Department of Pathophysiology and Transplantation, University of Milan (Milano)

Objectives: Emotion recognition supports communication and social interactions. Human beings express their emotions principally by the visual and auditory channels. Along with recognition of emotional facial expressions, identification of emotions from voices is fundamental. Some studies suggest that the emotional processing is compromised in Parkinson's Disease (PD), due to striatal pathology [1]. Although movement disorder is the principal symptom in this disease, non-motor impairment such as emotion recognition deficit in both facial and vocal expressions has been reported [2,3]. Aim of this study was to investigate whether emotion recognition conveyed by voice is impaired in $\mathrm{PD}$, and whether the impairment is related to striatal pathology.

Materials and Methods: Fifteen PD patients and 15 healthy controls (HC) were requested to listen to six audio tracks and to recognize the emotions expressed by a professional actor while reading a meaning-neutral sentence. Accuracy of different emotions was analyzed by a $2 \times 6$ ANOVA (Group x Emotion). All subjects also received a structural MRI examination. Volumetric measurements were extracted for the striatum, a key region involved in emotional processing and typically impaired in PD. Point biserial correlation coefficients (rbp) were calculated in $\mathrm{HC}$ and $\mathrm{PD}$ groups between the volume of the bilateral striatum normalized for the total grey volume and the accuracy of the task in which were reported differences between the two groups.

Results: The ANOVA showed a significant effect of interaction Group $x$ Emotion $(\mathrm{F}(5,140)=2.67, \mathrm{p}=0.024$; partial $\eta 2=0.09, \omega=$ $0.80)$. Post hoc comparisons revealed that the accuracy for the sadness was lower in PD than in HC group $(p=0.011)$. Significant correlation was found in the PD group ( $\mathrm{rbp}=0.549, \mathrm{p}=0.034)$; whereas no significant correlation emerged for the HC group ( $r b p=-0.034 ; p=0.909$ ).

Discussion: PD were specifically impaired in emotion recognition conveyed by voice and specifically in identifying the emotion of sadness compared to HC. The ability to correctly recognize sadness resulted positively associated with the volume of striatum bilaterally.

Conclusions: Our findings reinforce the view that the striatum might play an important role not only in motor symptomatology, but also in the network involved in emotional processing.

References:

1. Péron J., Dondaine T., Le Jeune F., Grandjean D., Vérin, M. Emotional processing in Parkinson's disease: a systematic review. Movement Disorders (2012);27(2):186-99

2. Dara C., Monetta L., Pell M. D., Vocal emotion processing in Parkinson's disease: reduced sensitivity to negative emotions. Brain Research (2008);1188:100-11

3. Pell M. D., Leonard C. L., Processing emotional tone from speech in Parkinson's disease: a role for the basal ganglia. Cognitive, Affective \& Behavioral Neuroscience (2003);3(4):275-88

\section{PSYCHOGENIC NON-EPILEPTIC SEIZURES AND MILD- MAJOR DEPRESSION DISORDER: A CORTICAL THICKNESS STUDY}

I. Martino ${ }^{1}$, F. Fortunato ${ }^{1}$, M. Caligiuri ${ }^{2}$, A. Bruni ${ }^{3}$, P. De Fazio ${ }^{3}$, A. Gambardella ${ }^{1}$, A. Labate ${ }^{1}$
${ }^{1}$ Institute of Neurology, Magna Graecia University (Catanzaro); ${ }^{2}$ Neuroscience Research Center, Magna Graecia University (Catanzaro); ${ }^{3}$ Institute of Psychiatry, Magna Graecia University (Catanzaro)

Objectives: Psychogenic non-epileptic seizures (PNES) consist of paroxysmal behavior that resembles epileptic seizures, without ictal epileptiform activity. [1] Although the high comorbidity between depression and PNES is known, to the best of our knowledge, there are no neuroimaging studies comparing patients with these two disorders [2] The aim of our work was to investigate morphometric brain MRI differences between psychogenic non epileptic seizures (PNES) patients and mild-Major Depressive Disorder (MDD) patients.

Materials and Methods: 42 PNES patients (mean age 42, $46 \pm 13,86$ years; 9/42 males) and 25 mild-MDD (mean age 42,53 $\pm 11,99$ years; 6/25 males), matched depression degree measured by BDI-II (Beck Depression Inventory-II), were consecutively recruited. Patients performed a wide neuropsychiatric assessment, including the following tests: Hamilton Anxiety Rating Scale (HAM-A), Toronto Alexithymia Scale (TAS-20) and Somatoform Dissociation Questionnaire (SDQ-20) [3] All patients underwent a brain 3T MRI and a cortical thickness analysis was carried out.

Results: PNES patients showed significantly higher scores in the following tests: HAM-A ( $\mathrm{p}=0.015)$, TAS-20 ( $\mathrm{p}=0.026)$ and SDQ-20 ( $<0.001$ ) with respect to the mild-MDD group. Cortical thickness analysis showed a significant bilateral cortical thinning in the medial orbitofrontal cortex (OFC) in patients with MDD compared to patients with PNES ( $\mathrm{p}<$ 0.05 FDR-corrected). No significant differences were found in other brain regions explored.

Discussion: Our results demonstrate differences in OFC thickness between the two groups. OFC is a key region involved in mood regulation and in the adaptive control of emotions. This could suggest that depressive symptoms experienced by PNES patients may lie on different neuroanatomic bases with respect of mild-MDD patients.

References:

1. LaFrance WC, Baker GA, Duncan R, Goldstein LH, Reuber M. Minimum requirements for the diagnosis of psychogenic nonepileptic seizures: a staged approach. A report from the International League against Epilepsy Nonepileptic Seizures Task Force. Epilepsia (2013);54: 2005-18

2. Labate A, Cerasa A, Mula M, et al., Neuroanatomic correlates of psychogenic nonepileptic seizures: a cortical thickness and VBM study. Epilepsia (2012);53:377-85

3. Martino A, Bruni A, Labate A, et al., Psychopatological constellation in patients with PNES: A new hypothesis. Epilepsy \& Nehavior (2018);78:297-301

\section{THE FUNCTIONAL NEUROANATOMY OF PURE WORD DEAFNESS}

G. Miceli ${ }^{1}$, P. Bartolomeo ${ }^{2}$, S. Beber ${ }^{1}$, L. Cohen ${ }^{2}$, B. Rapp ${ }^{3}$, M. Wolmetz $^{4}$

${ }^{1}$ Center for Mind/Brain Sciences, University of Trento (Rovereto-TN);

${ }^{2}$ Institut du Cerveau et de la Moelle Epinière, Hôpital de la Salpêtrière (Paris-F); ${ }^{3}$ Cognitive Science Department, Johns Hopkins University (Baltimore-USA); ${ }^{4}$ Research \& Exploratory Development Department, Johns Hopkins University (Baltimore-USA)

Introduction and Aims: Comprehension disorders in aphasia are occasionally more pronounced for speech than for reading. Rarely, 
auditory processing is selectively and globally disrupted (auditory agnosia). Exceptionally, damage only affects speech processing (pure word deafness, PWD). Even though PWD is typically caused by bilateral temporal lesions, approximately one-third of the patients have unilateral damage. In these cases, a strategically located left subcortical lesion disconnects Wernicke's area from both auditory cortices, and speech processing relies on the right (instead of the left, language-dominant) hemisphere. Consequently, a reversed right-ear advantage is observed in dichotic listening tasks: when syllables are presented simultaneously to both ears, patients with unilateral PWD report more stimuli delivered to the left than to the right ear - the opposite behavior to healthy subjects [1]. For a long time, primary auditory cortices (PACs) were deemed functionally symmetrical. More recently, PWD following unilateral temporal damage was taken to support functional asymmetry of PACs, left PAC being better suited to process speech properties (temporal analysis over short time windows), and right PAC more attuned to nonspeech sound processing (spectral analysis over longer time intervals) $[2,3]$. Since anatomical evidence relies on single-case reports, the functional neuroanatomy of PWD remains unclear. Here we report on MRI data in 4 PWD subjects with left temporal damage.

Case Reports: All participants had: unilateral left temporal damage; normal peripheral hearing; intact subcortical auditory pathways; normal reading comprehension; no signs of aphasia; normal recognition of nonverbal auditory stimuli (music and environmental sounds); poor auditory speech processing; left-ear advantage in dichotic listening.

Methods: Lesions were manually reconstructed in FSL (https://fsl. fmrib.ox.ac.uk/fsl/fslwiki/), drawing a region of interest (ROI) in the damaged area (T1-weighted images) and registered into MNI152 standard space $(1 \mathrm{x} 1 \mathrm{x} 1 \mathrm{~mm})$.

Results: Lesions overlap in two left temporal lobe clusters, in $4 / 4$ cases. A cluster $(-40 ;-19 ; 1)$ includes PAC, posterior insula and planum polare. A second, larger cluster $(-55 ;-38 ; 16)$ includes planum temporale, superior temporal and supramarginal gyrus. PAC damage is partial in all subjects.

Discussion/Conclusions: In 4 PWD patients with left temporal lesions, PAC was only partially damaged, and lesion reconstruction showed an additional involvement of language-specific areas. Two accounts can be entertained. On the first, PACs are functionally asymmetrical and left PAC damage suffices to yield PWD. On the second, PACs are functionally symmetrical and additional, posterior temporal damage to language-specific areas is necessary to cause PWD.

References:

1. Maffei C., Capasso R., Cazzolli G., Colosimo C., Dell'Acqua F., Piludu F., Catani M., Miceli G. Pure word deafness following left temporal damage: Behavioral and neuroanatomical evidence from a new case. Cortex (2017);97:240-54 https://doi.org/10.1016/j. cortex.2017.10.006

2. Hickok G., Poeppel D. The cortical organization of speech processing. Nature Reviews Neuroscience (2007);8(5): 393-402

3. Zatorre R. J., Belin P., Penhune V. B. Structure and function of auditory cortex: Music and speech. Trends in Cognitive Sciences (2002);6(1):37-46

\section{IMPACT OF MULTIPLE SCLEROSIS ON COGNITIVE AGING: A MULTICENTER STUDY}

D. Mistri ${ }^{1}$, A. Meani ${ }^{1}$, G. Tedeschi ${ }^{2}$, P. Pantano ${ }^{3}$, P. Zaratin ${ }^{4}$, N. De

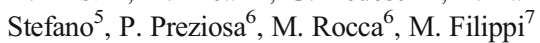

${ }^{1}$ Neuroimaging Research Unit, Institute of Experimental Neurology, Division of Neuroscience, IRCCS San Raffaele Scientific Institute (Milano); ${ }^{2}$ Department of Advanced Medical and Surgical Sciences, and 3T MRI Center, University of Campania "Luigi Vanvitelli" (Napoli); ${ }^{3}$ Department of Human Neuroscience, 'Sapienza' University of Rome (Roma); ${ }^{4}$ Fondazione Italiana Sclerosi Multipla (FISM) (Genova); ${ }^{5}$ Department of Medicine, Surgery and Neuroscience, University of Siena (Siena); ${ }^{6}$ Neuroimaging Research Unit, Institute of Experimental Neurology, Division of Neuroscience; and Neurology Unit, -IRCCS San Raffaele Scientific Institute (Milano); ${ }^{7}$ Neuroimaging Research Unit, Institute of Experimental Neurology, Division of Neuroscience; Neurology Unit and Neurophysiology Unit, -IRCCS San Raffaele Scientific Institute; Vita-Salute San Raffaele University (Milano)

Objectives: Cognitive deterioration affects a large proportion of multiple sclerosis (MS) patients, but it occurs also with healthy aging. The effects of aging on cognitive performance in MS have not been fully investigated yet. By evaluating a large multicentric cohort of healthy controls (HC) and MS patients, we compared the age-related decline of cognitive functions occurring in $\mathrm{HC}$ and MS patients.

Materials: Brief Repeatable Battery of Neuropsychological Tests (BRB-N) was evaluated in 301 healthy controls (HC) (150 females, age 18-76 years, mean education $=14.9$ years) and 664 MS patients (421 females; age 18-77 years; mean education $=13.3$ years; 536 relapsing-remitting and 128 progressive MS) recruited from 3 centers of the Italian Neuroimaging Network Initiative (INNI, www.inni-ms.org). BRB-N allowed to assess verbal memory (Selective Reminding Test [SRT]), visuospatial memory (10/36 Spatial Recall Test [SPART] and delayed-recall), information processing speed (Symbol Digit Modalities Test [SDMT], Paced Auditory Serial Addition Test [PASAT] 3" and 2") and verbal fluency (Word List Generation [WLG]).

Methods: Raw scores of each BRB-N test were converted to Z-scores, based on HC's cohort by running linear models to regress out the effects of sex, age, education and center. The residuals for both HC and MS patients were then divided by the HC's error term. Linear models were built for investigating the association of standardized scores with age in MS patients.

Results: In HC, scores of all the BRB-N tests except PASAT 3" and WLG declined significantly with aging ( $\mathrm{p}$ from $<0.0001$ to 0.003 ). Compared to HC, MS patients showed significant worse estimated mean performances already from the age of 20 years in all BRB-N tests ( $\mathrm{p}$ from $<0.0001$ to 0.003 ), except for SPART, SPART delayed-recall and PASAT 3", whose estimated mean scores significantly worsened later in age ( $\mathrm{p}$ from 0.03 to 0.04 ). MS patients showed also a steeper agerelated decline of SPART, SPART delayed-recall, SDMT, PASAT 3", PASAT 2" performances compared to HC ( $p$ from $<0.0001$ to 0.008 ). No differential effect of age compared to $\mathrm{HC}$ was detected in MS patients for WLG and SRT.

Discussion: Cognitive deficits already affect young adult MS patients and progress faster during patients' lifespan compared to healthy aging. A different susceptibility to age-effect exists in the cognitive tests currently used to assess cognition in MS patients.

Conclusions: The accumulation of MS-related damage combined with brain aging may have synergic detrimental effects on cognitive performances of MS patients.

This project has been supported by a research grant from the Fondazione Italiana Sclerosi Multipla (FISM2018/S/3), and financed or co-financed with the ' 5 per mille' public funding. 


\section{COVID-19 PANDEMIC AND MENTAL DISTRESS IN MULTIPLE SCLEROSIS: IMPLICATIONS FOR CLINICAL MANAGEMENT}

M. Petracca ${ }^{1}$, T. Costabile ${ }^{1}$, A. Carotenuto ${ }^{1}$, L. Lavorgna ${ }^{2}$, G. Borriello ${ }^{3}$, L. Moiola ${ }^{4}$, M. Inglese ${ }^{5}$, M. Petruzzo ${ }^{1}$, F. Trojsi ${ }^{2}$, A. Ianniello ${ }^{6}$, A. Nozzolillo $^{7}$, M. Cellerino ${ }^{5}$, G. Boffa ${ }^{5}$, L. Rosa ${ }^{1}$, G. Servillo ${ }^{1}$, M. Moccia $^{1}$, S. Bonavita ${ }^{2}$, M. Filippi ${ }^{7}$, R. Lanzillo ${ }^{1}$, V. Brescia Morra ${ }^{1}$

${ }^{1}$ Department of Neurosciences and Reproductive and Odontostomatological Sciences, University of Naples Federico II (Napoli); ${ }^{2}$ Second Division of Neurology, Multiple Sclerosis Center, University of Campania Luigi Vanvitelli (Napoli); ${ }^{3}$ MS Center, S. Andrea Hospital, Sapienza University (Roma); ${ }^{4}$ Neurology Unit, IRCCS San Raffaele Scientific Institute (Milano); ${ }^{5}$ Department of Neuroscience, Rehabilitation, Ophthalmology, Genetics, Maternal and Child Health, University of Genoa (Genova); ${ }^{6} \mathrm{MS}$ Center, S. Andrea Hospital, Sapienza University (Roma); ${ }^{7}$ Neurology Unit, IRCCS San Raffaele Scientific Institute (Milano)

Objective: To explore the relationship between mental distress, disability and coping strategies in the Italian MS population under lockdown during COVID-19 pandemic.

Material and Methods: Structural equation modeling (SEM) was applied to information collected via web-survey to identify modifiable factors that could account for mental distress.

Results: 845 subjects (497 MS and 348 controls) were included in the study. MS patients showed higher scores than controls for depression $(\mathrm{p}=0.005)$, but not for anxiety, emotional dyscontrol or sleep disturbances. The SEM explained $74 \%$ of the variance observed in depression score. Within the model, three latent factors were characterized from measured variables: motor disability and cognitive dysfunction contributed to disability (Beta $=0.509$ and Beta $=0.836, \mathrm{p}<0.001$ ); positive attitude and exercise contributed to active attitude (Beta $=0.386$ and Beta $=0.297, \mathrm{p}<0.001$ ); avoidance, social support and watching TV contributed to passive attitude (Beta $=0.301$, Beta $=0.243$ and Beta $=0.212$, $\mathrm{p}<0.001)$. As per the relationship between latent factors and their influence on depression, disability contributed to passive attitude (Beta $=0.855$, $\mathrm{p}<0.001)$ while both passive and active attitude significantly influenced depression (Beta $=0.729$ and Beta $=-0.456, \mathrm{p}<0.001$ ).

Discussion and Conclusion: As practical implication of our model, favoring exercise would enhance active attitude and its positive impact on mental well-being while, at the same time, reducing the negative impact of disability on depression, representing a valuable tool to face COVID-19 related mental distress.

\section{NEUROPSYCHOLOGICAL PREDICTORS OF MCI CONVERSION TO DEMENTIA AT DIFFERENT TIMEPOINTS}

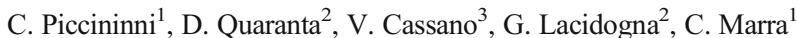

\footnotetext{
${ }^{1}$ Neurology, Catholic University of the Sacred Heart (Roma); ${ }^{2}$ Neurology, IRCCS Fondazione Policlinico Universitario "A. Gemelli" (Roma); ${ }^{3}$ Neurorehabilitation, IRCCS San Raffaele Pisana (Roma)
}

Objective: Episodic memory tests represent are considered as the most valuable tool to identify subjects with MCI who will convert to dementia, alongside with tests assessing executive functions and lexical-semantic system. It is conceivable that different neuropsychological markers may predict the conversion to dementia at different timepoints from the baseline assessment. The study was aimed at evaluating the predictive role of several neuropsychological markers at different follow-up times from the diagnosis of MCI.

Materials and methods: We recruited 192 individuals with amnesic $\mathrm{MCI}$ due to Alzheimer's Disease (AD) who underwent periodical clinical and neuropsychological assessment for 5 years. During the follow-up period, 121 individuals converted to dementia. They were subdivided into three groups: early converters (EC; conversion in the first two years of follow-up; $\mathrm{N}=63$ ); intermediate converters (IC; between 2 and 4 years; $\mathrm{N}=37$ ); late converters ( $\mathrm{LC}$; conversion over 4 years; $\mathrm{N}=21$ ). The analyses were performed using the baseline neuropsychological evaluation, that included: MMSE; Rey Auditory Verbal Learning Test; digit span; copy of drawing without and with landmarks; Raven's Matrices; Stroop's test; Multiple Features Targets Cancellation; phonological and semantic verbal fluency; nouns naming. The discrepancy between semantic and phonological verbal fluency (SPD) was computed. Multinomial logistic regression analyses were performed in which the groups of converters were set as dependent variables.

Results: On univariate analyses, EC was predicted by MMSE $(\mathrm{p}<0.001)$; RAVLT immediate $(\mathrm{p}=0.005)$ and delayed $(\mathrm{p}=0.007)$ recall; digit span backward $(\mathrm{p}=0.033)$. IC was predicted by the MMSE score $(\mathrm{p}<0.001)$; and semantic verbal fluency $(\mathrm{p}=0.012)$. LC was predicted by phonological verbal fluency $(\mathrm{p}=0.008)$ and SPD $(\mathrm{p}=0.05)$. On the multivariate model, EC was predicted by MMSE $(\mathrm{p}<0.001)$; RAVLT immediate recall $(\mathrm{p}=0.046)$; and SPD $(\mathrm{p}=0.026)$; IC was predicted by MMSE $(\mathrm{p}<0.001)$; LC was predicted by SPD $(\mathrm{p}=0.011)$.

Discussion: Different neuropsychological scores may predict conversion at different time points from the baseline. Episodic memory tests confirm to be reliable in detecting patients who will progress to dementia, but only in the first two years. The conversion over 4 years was predicted by the SPD, a marker of impairment of the lexical semantic system. This observation suggests that semantic memory may be affected in a very early phase of $\mathrm{AD}$, probably due to the involvement of medial temporal lobe structures like entorhinal and perirhinal cortices.

Conclusion: The predictive effect of neuropsychological markers varies at different timepoints from the diagnosis. The impairment of semantic memory could be a very early consequence of the neuropathological changes of Alzheimer's Disease.

\section{NEUROANATOMICAL CORRELATES OF SEMANTIC FEATURES OF NARRATIVE SPEECH IN SEMANTIC AND LOGOPENIC VARIANT OF PRIMARY PROGRESSIVE APHASIA}

\author{
D. Quaranta ${ }^{1}$, S. Di Tella ${ }^{2}$, F. L'Abbate ${ }^{1}$, C. Marra ${ }^{3}$, M. Silveri ${ }^{4}$
}

${ }^{1}$ Neurology, IRCCS Fondazione Policlinico Universitario "A. Gemelli" (Roma); ${ }^{2}$ Molecular Medicine and Imaging in Rehabilitation, IRCCS Fondazione Don Carlo Gnocchi (Milano); ${ }^{3}$ Neurology, Catholic University of the Sacred Heart (Roma); ${ }^{4}$ Psychology, Catholic University of the Sacred Heart (Milano)

Objective: Semantic variant of Primary Progressive Aphasia (sv-PPA) is characterized by a progressive disruption of semantic knowledge, leading to semantic errors, typically occurring in confrontation naming and single word comprehension. However, the progressive impairment of the semantic system could be explored also in narrative speech. The aims of the study were to compare the semantic features of words produced during narrative speech in sv-PPA and logopenic variant of PPA (lv-PPA), and to explore their neuroanatomical correlates. 
Materials and Methods: Six patients with sv-PPA and 16 lv-PPA patients underwent tasks of narrative speech (scene description). For all of the content words a Semantic Depth Index (SDI) was determined, based on the taxonomic structure of a large lexical database (Multiwordnet [1]). All of the study participants underwent a 1.5-T MRI examination including the acquisition of anatomical highresolution images (3D T1). Structural images were segmented and parcelled according to Desikan atlas [2] using Freesurfer [3]. Cortical thickness measures were extracted for all the regions included in the Desikan atlas [2] and correlations were computed between SDI and the thickness of all cortical regions in the whole sample.

Results: Median SDI was lower in sv-PPA than in lv-PPA (3.25 \pm 0.418 vs $4.03 \pm 0.694 ; p=0.019$ ). Correlation analyses showed a positive association between SDI and the thickness of the entorhinal cortex $(\mathrm{r}$ $=0.668, \mathrm{p}=0.001)$, fusiform gyrus $(\mathrm{r}=0.438, \mathrm{p}=0,047)$, inferior temporal cortex $(\mathrm{r}=0.504, \mathrm{p}=0.020)$, middle temporal cortex $(\mathrm{r}=0.558, \mathrm{p}=0.009)$, parahippocampal cortex $(\mathrm{r}=0.540, \mathrm{p}=0.011)$, superior temporal cortex $(r=0.559, p=0.008)$, and temporal pole $(r=0.662, p=0.001)$ in left hemisphere; and with entorhinal cortex $(\mathrm{r}=0.738, \mathrm{p}<0.001)$, parahippocampal cortex $(\mathrm{r}=0.555, \mathrm{p}=0.009)$ and temporal pole $(\mathrm{r}=0.551, \mathrm{p}=0.010)$ in right hemisphere. The correlation with left inferior frontal gyrus was not significant (pars opercularis: $r=0.199, p=0.387$; pars orbitalis: $r=0.162$, $\mathrm{p}=0.483$; pars triangularis: $\mathrm{r}=0.055, \mathrm{p}=0.812$ ).

Discussion: The disruption of semantic knowledge observed in svPPA leads to the production of generic terms and superordinate paraphasias in narrative speech, and the SDI my represent a useful tool to quantify the level of semantic impairment. The neuroanatomic correlates corroborates this hypothesis, since the measure was associated to the cortical thickness of brain regions associated to semantic memory, showing no association with regions involved in lexical selection.

Conclusion: Errors due to semantic disruption in sv-PPA can be detected and quantified in narrative using the SDI, that showed a robust association with brain regions devoted to semantic memory.

References:

1. Pianta E., Bentivogli L., Girardi C. MultiWordNet: developing an aligned multilingual database. in Proceedings of the $1 \mathrm{st}$ International WordNet Conference, January 21-25 2002, Mysore. URL: www.multiwordnet.fbk.eu

2. Desikan R. S., Ségonne F., Fischl B., Quinn B. T., Dickerson B. C., Blacker D. et al. An automated labeling system for subdividing the human cerebral cortex on MRI scans into gyral based regions of interest. Neuroimage (2006);31(3):968-80

3. Fischl B., Salat D. H., Busa E., Albert M., Dieterich M., Haselgrove C. et al. Whole brain segmentation: automated labeling of neuroanatomical structures in the human brain. Neuron (2002);33(3):341-55

\section{MEMORY DEFICITS IN AMNESTIC MILD COGNITIVE IMPAIRMENT REDUCE FACILITATION AND INHIBITION EFFECTS DURING RETRIEVAL OF SEMANTICALLY-RELATED ITEMS}

L. Serra ${ }^{1}$, C. Del Bono ${ }^{1}$, G. Bechi Gabrielli ${ }^{1}$, C. Di Domenico ${ }^{1}$, G. Bianchi $^{1}$, J. Pavia ${ }^{1}$, C. Caltagirone ${ }^{2}$, C. Marra ${ }^{3}$, M. Bozzali ${ }^{1,4}$

\footnotetext{
${ }^{1}$ Neuroimaging Laboratory, Fondazione Santa Lucia IRCCS (Roma); ${ }^{2}$ Laboratory of Clinical and Behavioural Neurology, Fondazione Santa Lucia IRCCS (Roma); ${ }^{3}$ Institute of Neurology, Catholic University (Roma); ${ }^{4}$ Department of Neuroscience "Rita Levi Montalcini" University of Torino (Torino)
}

Background: During the memory process, retrieval of significant information reduces the possibility to access semantically-related stimuli, thus inducing failure to remember [1]. In fact, the repetition (practice) of stimuli to remember produces an over-learning effect that interferes with retrieval of semantically-related items by frontal inhibition mechanisms that produce forgetting. These inhibition mechanisms have been observed during normal aging [2] but there is no clear evidence about what happens in pathological aging [3].

Objective: To investigate the retrieval-induced forgetting and its neural correlates in patients with amnestic Mild Cognitive Impairment (a$\mathrm{MCI})$.

Materials and Method: 31 a-MCI patients and 30 healthy subjects (HS) underwent Cognitive Examination of Addenbrooke (ACE-R) and Retrieval Practice Paradigm (RPP), that evaluates the retrieval-induced forgetting by producing three different indexes: the remember effect (Baseline-remembering), the retrievalinduced facilitatory (FAC) and the retrieval-induced forgetting (RIF) effects. A-MCI patients underwent 3T-MRI for voxel-based morphometry (VBM). One-way ANCOVAs were used to assess between-groups differences in ACE-R subtests and RPP indexes (formal education was used as covariate of no interest in all analyses). Correlations were performed separately in a-MCI and HS groups to assess associations between ACE-R subtests and RPP indexes. Finally, in patients only, one-sample t-test models were employed to investigate associations between regional grey matter (GM) volumes and RPP indexes.

Results: There were no between-group differences in age and gender distribution. Conversely, a-MCI patients were significantly more educated than HS. Patients showed significantly reduced performances, compared to HS in some ACE-R subtests, in the MMSE scores and in all RPP indexes (i.e., remembering and forgetting abilities). Significant correlations were found between ACE-R subtests and all RPP indexes in a-MCI patients, while HS showed significant association between ACE-R executive functions and RIF only. Finally, MCI patients showed significant positive correlations between baseline-remembering and GM volumes in the lateral occipital cortex bilaterally, and between RIF and GM volumes in the right fusiform cortex and hippocampus.

Discussion: The present study shows lower performances in all indexes of the RPP in a-MCI patients when compared to HS. These changes correlate with both cognitive functioning and GM volumes, mainly in the meso-temporal lobe structures.

Conclusions: The reduction of effects that are normally present in normal aging such as the facilitation in remembering due to overlearning and forgetting induced by inhibition mechanisms, are likely due to the widespread memory impairment that characterises a-MCI patients and does not allow them to process over-learned items in a different way.

References:

1. Anderson MC, Bjork RA, Bjork EL. Remembering can cause forgetting: retrieval dynamics in long-term memory. J Exp Psychol Learn Mem Cogn. (1994) Sep;20(5):1063-87

2. Moulin CJ, Perfect TJ, Conway MA, North AS, Jones RW, James N. Retrieval-induced forgetting in Alzheimer's disease. Neuropsychologia. (2002);40(7):862-7

3. Barber SJ, Mather M. Forgetting in context: the effects of age, emotion, and social factors on retrieval-induced forgetting. Mem Cognit. (2012);40(6):874-888. doi:10.3758/ s13421-012-0202-8 
PAIN

\section{CLINICAL AND GENETIC CHARACTERIZATION OF NEUROPATHIC PAIN IN PATIENTS WITH SUSPECTED SMALL FIBRE NEUROPATHY}

D. Cazzato, E. Salvi, M. Marchi, I. D'Amato, C. Pardo, R. Lombardi, M. Paolini, G. Lauria Pinter

Neuroalgology Unit, IRCCS Fundation Neurological Institute "C. Besta" (Milano)

This study is based on a deep clinical phenotyping of a cohort of patients with suspected small fibre neuropathy (SFN) in order to distinguish two clinical subsets defined as "painful SFN" and "neuropathic pain". Starting from this stratification, we aimed at comparing pain intensity in 1) normal versus abnormal intraepidermal nerve fibre density (IENFD) and 2) neuropathic versus non-neuropathic pain features. Furthermore, we explored the frequency of variants in sodium channels $(\mathrm{SCN})$ genes. All patients underwent a standardized neurological evaluation with assessment of sensory "positive" (allodynia and hyperalgesia) and "negative" (reduced sensation) signs, skin biopsy for IENFD quantification to confirm diagnosis of SFN, questionnaires for neuropathic pain (DN4) and average pain intensity at numeric rating scale (PI-NRS) reported for whole day and for different times of the day. Genetic variants in $\operatorname{SCN} \alpha$ and $\beta$ subunits, detected by next-generation-sequencing of 257 patients, were analyzed both as incidence of potentially pathogenic variants and comparing the allele frequencies of rare and common variants in our cohorts versus the reference population (GnomAD). We included 391 subjects. Differences in pain intensity were detected between patients with normal or abnormal IENFD, and with or without neuropathic pain. All patients experienced a worsening of pain intensity in the evening. "Negative" but not "positive" signs correlated with reduced IENFD at skin biopsy. Genetic analysis revealed 13 potentially pathogenic variants in SCN genes in 22 painful and 2 painless patients whereas the comparison analysis showed 7 rare and 6 common variants with higher frequency in our cohorts compared to general population. Our study revealed that pain intensity was higher in patients with neuropathic pain features and no significant correlation with IENFD. Reduced pin prick sensation better predicted IENFD reduction than positive signs suggesting a stronger role for negative sensory signs in prompting the suspect of SFN. Genetic analysis confirmed the presence of rare pathogenic variants and suggested a possible contribute of common polymorphisms as susceptibility factors. Pain is a key feature in SFN. It can be severe and associated with neuropathic features also in patients with normal skin innervation. Investigation of genetic variants in SCN genes might reveal molecular predictors for pain quality and intensity.

\section{LIFE WITH PAIN DURING THE COVID-19 PANDEMIC IN ITALY}

M. Consonni ${ }^{1}$, A. Telesca ${ }^{1}$, L. Grazzi ${ }^{1}$, D. Cazzato $^{1}$, G. Lauria $^{2}$

${ }^{1}$ Neuroalgology Department, IRCCS Foundation "Carlo Besta" Neurological Institute (Milano); ${ }^{2}$ Neuroalgology Unit, and Headache Center, IRCCS Foundation, University of Milan (Milano)

Background: The emergency due to the COVID-19 disease has strongly influenced physical and psychological health of the general population (1). Aim of this study was to evaluate COVID-19 related distress due to changes in habits and public health care reconfiguration in patients suffering from chronic pain, investigating any associations between pain outcomes, physical and psychological outcomes, loneliness, quality of life $(\mathrm{QoL})$ and attitude to cope with pain during the pandemic.

Methods: Between April 29 and June 11, 25 small fibre neuropathy (SFN) patients, 42 chronic migraine $(\mathrm{CM})$ patients, and 13 patients' healthy family members (HFM) completed the questionnaires assessing COVID-19 complains, changes in habits and clinical managements, behaviour, mood, loneliness, QoL, physical and mental health and coping strategies. Spearman rho correlations (Bonferroni correction, $\mathrm{p} \leq 0.003$ ) and Mann-Whitney $U$ tests $(\mathrm{p}<0.01)$ were performed for within and between-group analyses.

Results: Patients had lower QoL, lower physical health and higher catastrophizing attitude towards pain than HFM, with the SFN group having the worst profile. During the pandemic, SFN complained decline in clinical symptoms, worries about contagion and greater discomfort for disease management changes than CM patients. The SFN group showed higher levels of physical disability and greater suffering from changes in neurologist-patient relationship, whereas, $\mathrm{CM}$ patients complained of agitation/anxiety that was associated with feelings of loneliness, depressive mood and catastrophism. HFM participants experienced reduced QoL.

Discussion: Despite similar complains of change in habits and worries about COVID-19 pandemic, SFN and CM patients had distinct reactions. In SFN patients, pandemic distress impacted on physical health with worsening of clinical conditions, especially suffering from changes in their care. In CM patients, pandemic distress affected behaviour, mainly with psychological frailty, dysfunctional attitude toward negative selfstatement and excessively negative beliefs about the future, depressive mood and feeling of loneliness.

Conclusion: The COVID-19 pandemic distress raised the needs for a customization of the public health care for patients with distinct chronic pain conditions.

Reference:

- Brooks SK, Webster RK, Smith LE, Woodland L, Wessely S, Greenberg N, Rubin GJ. The psychological impact of quarantine and how to reduce it: rapid review of the evidence. Lancet (2020);395(10227):912-20

\section{VASCULAR COMPRESSION IN TRIGEMINAL NEURALGIA DISCLOSES TRIGEMINAL ROOT SOMATOTHOPIC ORGANIZATION}

G. De Stefano ${ }^{1}$, G. Di Stefano ${ }^{1}$, E. Ripiccini ${ }^{2}$, G. Di Pietro ${ }^{1}$, A. Di Lionardo $^{1}$, E. Sgro ${ }^{1}$, M. Fiorelli ${ }^{1}$, F. Caramia ${ }^{1}$, G. Cruccu ${ }^{1}$, A. Truini ${ }^{1}$

${ }^{1}$ Department of Human Neurosciences, Sapienza University (Roma); ${ }^{2}$ Advanced Quantum Architecture Laboratory, Swiss Federal Institute of Technology (Lausanne-CH)

Introduction and Objective: Trigeminal Neuralgia (TN) is a disorder characterized by recurrent paroxysmal episodes of unilateral facial pain in the distribution of one or more branches of the fifth cranial nerve. [1] A clinical hallmark of TN is the presence of discrete skin areas of tactile stimulation and typical manoeuvres that are able to trigger pain attacks. [2] In classical TN it is possible to recognize a vascular compression with morphological changes of trigeminal nerve root. In this neuroimaging study we investigate the distribution of the impacting vessel along the trigeminal root circumference and correlate it with the facial localization of pain and triggers. 
Materials and Methods: We enrolled 49 patients with clinically defined $\mathrm{TN}$, normal trigeminal reflexes testing, and evidence of neurovascular compression at 3-Tesla MRI with sequences dedicated to the anatomical study of cranial nerves. We reconstructed the images on a plane perpendicular to trigeminal root, and here we identified neurovascular interface. From the geometric center of the trigeminal root, we measured the polar coordinates of the impacting vessel on the trigeminal root circumference. All patients compiled a dedicated questionnaire aimed to identify pain distribution and the presence of specific trigger zones and manoeuvres.

Results: Neurovascular compression is mostly localized between $40^{\circ}$ and $90^{\circ}(\mathrm{p}<0.05)$. Pain in V1, V2 and V3 is associated, respectively, with vascular compression in the medial, superior and lateral aspect of the nerve $(\mathrm{p}<0.05)$. Cutaneous trigger zones are associated with discrete regions of the circumference $(\mathrm{p}<0.01)$.

Discussion: Our neuroimaging study showing that specific trigger zones are correlated with discrete zones of neurovascular compression highlights that neurovascular compression causing focal demyelination and ephaptic transmission in nerve fibres serving specific cutaneous trigger zones is the key pathogenetic mechanism in classical trigeminal neuralgia. [3]

References:

1. Merskey H, Bogduk N. Classification of chronic pain. Descriptions of chronic pain syndromes and definitions of pain terms. Seattle, WA: IASP Press (1994);59-71

2. Di Stefano G, Maarbjerg S, Nurmikko T et al. Triggering trigeminal neuralgia. Cephalalgia (2018);38(6):1049-56

3. Calvin WH, Devor M and Howe JF. Can neuralgias arise from minor demyelination? Spontaneous firing, mechanosensitivity, and afterdischarge from conducting axons. Exp Neurol (1982); 75(3): 755-63

\section{PAIN ASSESSMENT IN NON-COMMUNICATIVE PATIENTS}

\author{
R. Formisano, M. Contrada, M. Aloisi, S. Schiattone, G. Ferri, M.G. \\ Buzzi
}

IRCCS Santa Lucia Foundation (Roma)

Background: Recent evidences have shown that covert cognition may be present in around $15 \%$ of the patients with disorder of consciousness (DoC) [1]. The lack of overt behavioural responsiveness may be due to many clinical confounding factors, including severe spasticity [2] and diffuse pain [3].

Objective: Aim of the present study was to compare, in noncommunicative patients with DoC, NCS-R scores obtained with the standard pressure on fingernail bed (standard stimulus, SS) versus other personalized painful stimuli (PS), shared with the neurorehabilitation staff and to verify possible correlations between NCS-R and Coma Recovery Scale-Revised (CRS-R)

Methods: From a population of sixtysix patients ( $35 \mathrm{M}$ and $31 \mathrm{~F}$ ) enrolled in the study with a mean age of 42,74 years (range 14-69) diagnosed with DoC, according to the CRS-R, we selected a subgroup of 22 patients [10 M and $12 \mathrm{~F}$; mean age =40,72 years (range 14-69); etiology: 10 TBI; 3 anoxia; 7 vascular (hemorrhagic or ischemic); 1 encephalitis], who were assessed by means of NCS-R with personalized versus standard painful stimulation (NCS-R-SS).

Results: NCS-R-PS reached higher scores as compared to the standard stimulus, both for the total score and for motor and facial expression subscores $(\mathrm{p}<0.05)$. Significant correlations with CRS-R were found for both NCS-R-SS $(r=0.613, p=0.0031)$ and NCS-R-PS $(r=0.539$, $\mathrm{p}=0.0117)$.

Discussion: The preliminary results obtained in the present study suggest that NCS-R-PS may disclose pain perception in a larger number of non-communicative patients with DoC, compared to NCS-R-SS.

Conclusions: Standard painful stimulation may be affected by sensory deficits (hypoesthesia, anesthesia) of central and peripheral etiology and by the different pain sources. A personalized painful stimulation may be of some support to unveil covert cognition in non-communicative patients and to alleviate their possible sufferance by pain killers, thus improving their responsiveness and quality of life.

References:

1. Zasler N. D., Aloisi M., Contrada M., Formisano R. Disorders of consciousness terminology: history, evolution and future directions. Brain Injury (2019);1-6

2. Formisano R., Aloisi M., Contrada M., Spanedda F., Schiattone S., Niedbala S., Cobianchi M.R., Baldeschi G.C., Buzzi M.G. Late recovery of responsiveness after intra-thecal baclofen pump implantation and the role of diffuse pain and severe spasticity: a case report. Acta neurochirurgica, (2019),161(9):1965-67

3. Formisano R., Contrada M., Aloisi M., Ferri G., Schiattone S., Iosa M., Buzzi M. G. Nociception Coma Scale with personalized painful stimulation versus standard stimulus in non-communicative patients with disorders of consciousness. Neuropsychological Rehabilitation (2019);1-12.

\section{INTRAEPIDERMAL NERVE FIBRES (IENF) EVOLUTION IN A COHORT OF FIBROMYALGIC PATIENTS}

S. G. Quitadamo ${ }^{1}$, E. Vecchio ${ }^{1}$, G. Libro ${ }^{1}$, M. Delussi ${ }^{1}$, K. Ricci ${ }^{1}$, R. Lombardi $^{2}$, G. Lauria ${ }^{2}$, M. de Tommaso ${ }^{1}$

${ }^{1}$ Applied Neurophysiology and Neuropathic Pain Unit, Department of Basic Medical Sciences Neurosciences and Sensory Organs, University of Bari (Bari); ${ }^{2}$ Department of Clinical Neurosciences, 3rd Neurology Unit and Skin Biopsy, Peripheral Neuropathy and Neuropathic Pain Laboratory, IRCCS Foundation, 'Carlo Besta' Neurological Institute (Milano)

Background and objectives: The diagnostic criteria for Fibromyalgia [1] describe meticulously its clinical manifestations, however it remains a poorly defined disease entity in terms of physiopathological comprehension, although the research has already revealed a central and peripheral neurological involvement. In recent studies based on skin biopsy, a small fibers pathology has been demonstrated in most of fibromyalgic patients, more frequently with a non-length dependent pattern [3]. Our purpose is to observe the possible IENF modification two years after the first skin biopsy observation and to correlate with clinical hallmarks.

Methods: We have proposed a skin biopsy follow-up to a cohort of fibromyalgic patients monitored at Applied Neurophysiology and Neuropathic Pain of Bari Polyclinic from January 2018 to January 2020. 38 fibromyalgic patients, have signed the consent to performing the cutaneous sampling at the right ankle and ipsilateral thigh [2]. Exclusion criteria were active rheumatic and/or autoimmune disease and other neurologic condition with the exception of primary headache. The average age was 51 years, for a range of 26-71 years. At time T0 and T1 we have tested patients with skin punch biopsy with quantification of intraepidermal nerve fibre density (IENFD) at the thigh and distal leg 
(PTH and DL) and a battery of tests: The Fibromyalgia impact questionnaire (FIQ-I), the Multidimensional Assessment of Fatigue (MAF), the Neuropathic Pain 4 questionnaire (DN4)

Results: The fibre density is substantially unchanged at the control after 2 years, with a slight non-significant tendency to increase them, both for the evaluation of PTH and DL. Intraepidermal nerve fibre density was reduced in $87 \%$ of patients at the thigh and in $7,8 \%$ of patients at the distal leg, whereas it was normal in $7,8 \%$ of patients, data not significantly modified compared to our previous evaluation [3]. Two subjects with a reduced duration of illness and normal IENFD at first evaluation showed a reduction in PTH site at the follow up; two other subjects with reduced IENFD only at PTH showed reduced fiber density also at the distal site in the control. FIQ-I, MAF do not show a change in control after two years nor are they correlated with fiber density; DN4 was reduced at the follow up $(\mathrm{p}<0,01)$.

Conclusion: A variable and mild reduction of IENFD at the thigh, which might lead to the definition of associated proximal or non-lengthdependent small fibre pathology, is confirmed to be a phenotypic feature of fibromyalgic patients in our series.

References:

1. Wolfe F, Clauw DJ, Fitzcharles MA, Goldenberg DL, Katz RS, Mease P, Russell AS, Russell IJ, Winfield JB, Yunus MB. The American College of Rheumatology preliminary diagnostic criteria for fibromyalgia and measurement of symptom severity. Arthritis Care Res (Hoboken). (2010) May;62(5):600-10

2. Lauria G, Devigili G. Skin biopsy as a diagnostic tool in peripheral neuropathy. Nat Clin Pract Neurol. (2007);3(10):546-557. doi:10.1038/ncpneuro0630

3. Vecchio E, Lombardi R., Paolini M, Libro G, Delussi M, Ricci K, Quitadamo S., Gentile E, Girolamo F, Iannone F, Lauria G, de Tommaso M. Peripheral and central nervous system correlates in fibromyalgia. European Journal of Pain (2020);24(5);873-74. https://doi.org/10.1002/ejp.160

\section{CONDITIONED PAIN MODULATION AFFECTS THE N2/P2 COMPLEX BUT NOT THE N1 WAVE: A PILOT STUDY WITH LASER-EVOKED POTENTIALS}

\author{
A. Rasera ${ }^{1}$, G. Squintani ${ }^{2}$, A. Segatti ${ }^{2}$, E. Concon ${ }^{2}$, B. Bonetti ${ }^{2}$, M. \\ Valeriani ${ }^{3}$, M. Tinazzi ${ }^{1}$ \\ ${ }^{1}$ Neurology Unit, University of Verona (Verona); ${ }^{2}$ Neurology Unit, \\ AOUI Verona (Verona); ${ }^{3}$ Headache Center, Bambino Gesù Hospital \\ (Roma)
}

Objective: 'Pain-inhibits-pain' effect stems from neurophysiological mechanisms involving endogenous modulatory systems termed diffuse noxious inhibitory controls (DNIC) or conditioned pain modulation (CPM). Laser evoked potentials (LEPs) components, the N2/ $\mathrm{P} 2$ complex and N1 wave, reflect the medial and lateral pain pathway respectively: anatomically, the lateral thalamic nuclei (LT) project mainly to the somatosensory cortex (N1 generator) while the medial thalamic nuclei (MT) are bound to the limbic cortices (N2/ P2 generators).

Materials: We applied a CPM protocol in which the test stimulus was the laser stimulation and conditioning stimulus consisted in a cold pressor test.

Methods: LEPs recordings were obtained by 15 healthy subjects in three different phases: baseline, during heterotopic noxious conditioning stimulation (HNCS) and post-HNCS.
Results: We observed a significant reduction in N2/P2 amplitude during HNCS and a return to pre-test amplitude post-HNCS, while the N1 wave remained unchanged during and post-HNCS.

Discussion: Our results indicate that CPM affects only the medial pain system. The spinothalamic tract (STT) transmits to both LT and MT, while spinoreticulothalamic (SRT) projects only to the MT. The reduction in the amplitude of the N2/P2 complex, and the absence of change in the N1 wave, suggest that the DNIC inhibition on dorsal horn neurons affect only pain transmission via the SRT, while the neurons that give rise to the STT are not involved.

Conclusions: N1 wave can be a reliable neurophysiological parameter for assessment of STT function in clinical practice, as it is not influenced by CPM.

\section{THE USE OF MEDICAL GRADE CANNABIS IN ITALY: A NEUROLOGICAL PERSPECTIVE}

F. Saccà, A. Trinchillo, M. Caterino, S. De Pietro, G. De Michele, A. Carotenuto, V. Brescia Morra, C. Pane

\section{NSRO Department, Federico II University (Napoli)}

Introduction: In Italy, Medical Grade Cannabis (MGC) can be prescribed for different medical conditions, whenever standard and approved therapies have failed, or caused non-tolerable side effects. Here we describe our 5-year clinical experience in the management and prescription of MGC.

Methods: This is a retrospective observational study. MGC was prepared according to Italian laws and administered as either an olive oil extract (OOE), or as an oral non activated form. Cannabis was prescribed as either Bedrocan (22\% THC, <1\% CBD), Bediol (6.5\% THC, $8 \%$ CBD), or Bedrolite (0.4\% THC, 9\% CBD). Responders were classified as patients showing $\geq 20 \%$ reduction in the Numeric Rating Scale (NRS).

Results: We treated 111 patients (63\% female; mean age 47.4 years). Median FU for responders was 19 months. Prescription indications are reported in Figure 1. $70 \%$ of patients were treated with Bedrocan, $10.8 \%$ with Bediol, and $2.7 \%$ with Bedrolite. $57 \%$ of patients responded to MGC, whereas $43 \%$ dropped out for lack of response. Mean baseline NRS was $8.12 \pm 1.6$ and decreased at FU to $5.27 \pm 2.4(\mathrm{p}<0.001$; Figure 2). Patients receiving MGC for pain showed the best effect with -3.3 points NRS reduction. Patients with baseline NRS $>8$ had a higher therapy persistence $(H R=0.19$; $\mathrm{p}<0.001$; Figure 3$)$, and higher response to $(\mathrm{OR}=4.8 ; \mathrm{p}=0.001)$. MGC was overall safe and well tolerated.

Conclusions: Our experience shows that MGC can be successfully used in a neurological setting and that it is safe and well tolerated. Patients with higher NRS had a higher response probability and treatment persistence.

References:

Corli O, Davoli E, Medana C, Garattini S. Cannabis as a medicine. An update of the Italian reality. European Journal of Internal Medicine (2019);60:e9-e10

- Pane C, Sacca F. The use of medical grade cannabis in Italy for drugresistant epilepsy: a case series. Neurol Sci. (2020);41(3):695-8

- Sacca F, Pane C, Carotenuto A, Massarelli M, Lanzillo R, Florio EB, et al. The use of medical-grade cannabis in patients non-responders to Nabiximols. J Neurol Sci. (2016);368:349-51 
THE NEW, MICROPATTERNED INTERDIGITATED ELECTRODE 150IDE FOR THE ASSESSMENT OF EARLY CORTICAL ACTIVITY AFTER NOCICEPTIVE SYSTEM ACTIVATION

E. Sgro, G. Di Stefano, G. Di Pietro, A. Di Lionardo, G. De Stefano, E. Galosi, C. Leone, S. La Cesa, A. Fasolino, G. Cruccu, A. Truini

Department of Human Neurosciences, Sapienza University of Rome (Roma)

Background and aim: The new, micropatterned interdigitated electrode 150IDE has been recently developed to selectively activate the intraepidermal nociceptive fibers. This electrode, at low stimulation intensity, induces distinct pinprick sensations and evokes scalp responses comparable to laser-evoked potentials. Its nociceptive specificity depends on the peculiar interdigitated conformation, made of conductive rails arranged in a comb-like micropattern, situated only $150 \mu \mathrm{m}$ apart and alternately connected to the opposite poles of the stimulator. The short distance between anode and cathode generates an electric field which penetrates just a little depth in the skin, selectively activating intraepidermal free nerve endings. In this neurophysiological study, we aimed to record, for the first time, early components of brain evoked potentials after selective activation of nociceptive system, using the 150IDE.

Methods: We enrolled six healthy subjects (2F, 4M, age 22-31 years). In each participant, using the 150IDE electrode, we stimulated the hairy skin of the right-hand dorsum, delivering three separate blocks of 150 stimuli at $1 \mathrm{~Hz}$. Each stimulus consisted of bursts of ten electrical pulses at $1 \mathrm{kHz}$. We used a low-intensity electrical stimulation, slightly above the pinprick detection threshold and evoking a distinct pinprick sensation. Early components of evoked potentials were recorded using $32 \mathrm{Ag}-\mathrm{AgCl}$ electrodes placed in the scalp according to 10-20 international system.

Results: In all participants, low-intensity stimulation (2.3-9 mA) evoked two reproducible and lateralized components, corresponding to the parietal electrode $\mathrm{C} 3$ with $\mathrm{Fz}$ as a reference. The first component consisted of a positive deflection with a latency of $32.2 \pm 2.9 \mathrm{msec}$ and a peak amplitude of $1 \pm 1.2 \mu \mathrm{V}$; the second component consisted of a negative deflection with a latency of $42.7 \pm 5.7 \mathrm{msec}$ and a peak amplitude of $0.7 \pm .0 .3 \mu \mathrm{V}$.

Conclusions: The scalp topography and latency of these two components are consistent with the arrival and processing of nociceptive input into the somatosensory cortex. Therefore, our preliminary results indicate that IDE150 might be useful for assessing the early cortical activity after nociceptive system activation, thus representing a significative improvement in the assessment of nociceptive system in clinical practice.

References:

- G. Di Stefano, A. Di Lionardo, S. La Cesa, G. Di Pietro, A. Fasolino, E. Galosi, C. Leone, G. Cruccu, L. Marinelli, M. Leandri, A. Truini. The new micropatterned interdigitated electrode for selective assessment of the nociceptive system. European Journal of Pain. (2020);24(5): 956-66

- M. Leandri, L. Marinelli, A. Siri, L. Pellegrino. Micropatterned surface electrode for massive selective stimulation of intraepidermal nociceptive fibres. Journal of Neuroscience Methods (2018);293:17-26

- A. Mouraux, G.D. Iannetti, L. Plaghki. Low intensity intraepidermal electrical stimulation can activate $\mathrm{A} \delta$-nociceptors selectively. Pain (2010);150(1):199-207

\section{MULTIPLE SCLEROSIS}

\section{MULTIPLE SCLEROSIS AND GENETIC POLYMORPHISMS INVOLVED IN FIBRINOGEN-MEDIATED HEMOSTATIC PATHWAY: A CASE CONTROL STUDY}

G. Abbadessa ${ }^{1}$, L. Lavorgna ${ }^{2}$, G. Miele ${ }^{1}$, M. Sparaco ${ }^{1}$, A. Di Pietro ${ }^{1}$, R. Palladino $^{3}$, G. D’Elia ${ }^{4}$, I. Armetta ${ }^{4}$, E. Signoriello ${ }^{1}$, G. Lus ${ }^{1}$, S. Bonavita ${ }^{1}$

${ }^{1}$ II Division of Neurology, Department of Advanced Surgical and Medical Sciences, University of Campania Luigi Vanvitelli (Napoli); ${ }^{2}$ I Division of Neurology, Department of Advanced Medical and Surgical Sciences ,University of Campania Luigi Vanvitelli (Napoli); ${ }^{3}$ Department of Public Health, University Federico II, Department of Primary Care and Public Health, Imperial College London (Napoli, LondonUK); ${ }^{4}$ Clinical and Molecular Pathology, Department of Precision Medicine, University of Campania Luigi Vanvitelli (Napoli)

Introduction: Multiple Sclerosis (MS) is a chronic inflammatory disease of Central Nervous System (CNS). Increasing evidence suggested that blood coagulation constituents might exert immunomodulatory functions in the CNS with critical consequences in fostering and amplifying neuroinflammation and the demyelinating process. The strongest evidence is available for Fibrin(-ogen). It has revealed to leak out through compromised BBB at the earliest stage of MS lesion, preceding demyelination and axonal loss, and to correlate spatially with areas of demyelination and axonal damage [1]. Our purpose was to determine whether particular single nucleotide polymorphisms (SNPs), thought to be involved in fibrinogen-mediated hemostatic pathways, are over-represented in patients with MS compared with controls.

Methods: The case-control study consisted of 112 MS patients recruited consecutively at our clinic and 68 healthy controls. Risk associations between each risk genotype and MS susceptibility were estimated by odds ratio using three different genetic models (dominant, additive and recessive models) [2]. Afterwards, for each SNP, the genotypes were coded as 0,1 , or 2 indicating the number of hemostatic risk alleles in the genotype, in order to create a cumulative genetic risk score (CGRS) which included the 5 selected hemostatic risk alleles (BetaFibrinogen 455G/A, Glycoprotein IIIa P1A2, Factor V Leiden, Factor V H2R and Prothrombin 20210G/A). Multivariate ordinal logistic regression was applied to evaluate the effect of CGRS on MS susceptibility.

Results: The association analysis between individual SNPs and MS susceptibility revealed an increased likelihood of being in the MS status for subjects carrying the genotype A/A of the FGB polymorphism, either in the additive model (OR 8.994; IC95\% 1,108-72,976; $\mathrm{p}=0.040$ ) and in the recessive model (OR 10.101; IC95\% 1.265-80.646; $\mathrm{p}=0.029$ ), after adjustment for age and sex. Multivariate ordinal logistic regression found a significant association between CGRS and MS susceptibility ( $\mathrm{p}=0.010$ ).

Discussion: Our results disclosed a higher likelihood of being in the MS status for patients carrying the AA genotype of FGB polymorphism and for patients carrying at least 2 pro-hemostatic risk alleles strenghtnen the hypothesis that coagulation cascade could be involved in MS neuroinflammation [3].

Conclusion: This is the first study investigating the influence of SNPs involved in fibrinogen-mediated hemostatic pathway in MS susceptibility and severity. Potential future applications of our work will include verifying these findings in a larger population-based cohort in order to identify a further prognostic marker, possibly improving risk stratification of patients and the selection of those with a poor prognosis. 


\section{References:}

1. Lee NJ, Ha SK, Sati P, et al. Spatiotemporal distribution of fibrinogen in marmoset and human inflammatory demyelination. Brain (2018);141:1637-49

2. Zhao F, Song M, Wang Y, Wang W. Genetic model. J Cell Mol Med. (2016);20(4):765

3. Plantone D, Inglese M, Salvetti M, Koudriavtseva T. A Perspective of Coagulation Dysfunction in Multiple Sclerosis and in Experimental Allergic Encephalomyelitis [published correction appears in Front Neurol. 2019 Mar 12;10:210]. Front Neurol. (2019);9:1175. Published 2019 Jan 14. doi:10.3389/ fneur.2018.01175

\section{FATIGUE AND PSYCHOLOGICAL SYMPTOMS IN PEOPLE WITH MULTIPLE SCLEROSIS: DOES SEX MATTER?}

M. Altieri ${ }^{1}$, R. Capuano ${ }^{2}$, A. Bisecco ${ }^{2}$, A. d'Ambrosio ${ }^{2}$, R. Docimo ${ }^{2}, \mathrm{~S}$. Bonavita $^{2}$, L. Lavorgna ${ }^{2}$, M. della Corte ${ }^{2}$, R. Sacco ${ }^{2}$, G. Tedeschi ${ }^{2}$, G. Santangelo ${ }^{1}$, A. Gallo ${ }^{2}$

${ }^{1}$ Department of Psychology, University of Campania "Luigi Vanvitelli" (Caserta); ${ }^{2}$ Department of Advanced Medical and Surgical Sciences University of Campania "Luigi Vanvitelli" (Napoli)

Objective: Fatigue is experienced by $50-80 \%$ of people with Multiple Sclerosis (pwMS) and it is associated with depression and anxiety $[1,2]$. However, it is not clear whether sex has an impact on these associations. The aim of the study was to explore the relationships between fatigue, depression and anxiety in females and males with MS and healthy controls $(\mathrm{HC})$.

Materials and Methods: 271 pwMS (150 females, 121 males) and 69 HC (36 females, 33 males) matched for sex and age were included in the study. A neurological and neuropsychological assessment was conducted and the following tests/questionnaires were administered: the Symbol Digit Modalities Test (SDMT) to evaluate the cognitive status; the Modified Fatigue Impact Scale (MFIS) to evaluate physical, cognitive and psychosocial fatigue; the Beck Depression Inventory, second Edition (BDI-II) to evaluate depressive symptomatology and the StateTrait Anxiety Inventory (STAI-Y1 and STAI-Y2) to evaluate state and trait anxiety, respectively. Correlational analysis, t-test for independent sample (females pwMS vs males pwMS, females $\mathrm{HC}$ vs males $\mathrm{HC}$, females pwMS vs females HC, males pwMS vs males HC, pwMS whole group vs $\mathrm{HC}$ whole group), and comparison of correlations were performed. Benjamini-Hochberg procedure was applied to control for the false discovery rate.

Results: Females vs males pwMS comparison: no sex differences were found in pwMS on physical, cognitive and psychosocial fatigue scores; females scored higher than males only on STAI-Y1 and STAIY2. Females vs males HC comparison: females reported higher scores on the MFIS, BDI-II and STAI-Y scales. HC vs pwMS comparison: pwMS showed lower scores on SDMT and higher scores on MFIS, BDI-II and STAI-Y than HC in the whole and in the males' groups, whereas in the females group pwMS showed higher physical fatigue scores than HC. The correlational analysis between MFIS, BDI-II and STAI-Y scores showed strong associations in the pwMS group (both males and females), moderate associations in the $\mathrm{HC}$ females group and no association in the $\mathrm{HC}$ males group. In the pwMS group, the strength of association between MFIS and BDI-II $(z=1.73, p=.004)$ and STAI-Y2 $(z=-1.819, p=0.034)$ was higher in females than in males.
Conclusions: The results might indicate the need for a routine evaluation of fatigue in pwMS, especially in females, in order to identify patients with high levels of fatigue that may also have high levels of depression and anxiety, addressing them to psychoeducational and/or pharmacological interventions.

References:

1. Induruwa, I., Constantinescu, C. S., \& Gran, B. Fatigue in multiple sclerosis - A brief review. Journal of the Neurological Sciences (2012);323(1-2):9-15

2. Beiske, AG, et al. Depression and anxiety amongst multiple sclerosis patients. European Journal of Neurology (2008);15(3):239-45

\section{RELAPSE-FREE AND NEDA STATUS WITH CLADRIBINE IN A REAL LIFE POPULATION: A MULTICENTRE STUDY}

P. O. L. Annovazzi ${ }^{1}$, L. Prosperini ${ }^{2}$, R. Capuano ${ }^{3}$, A. Gallo ${ }^{3}$, J. Frau ${ }^{4}$, E. Cocco $^{4}$, A. Nozzolillo ${ }^{5}$, L. Moiola ${ }^{5}$, M. Margoni ${ }^{6}$, P. Perini ${ }^{6}$, M. Capobianco $^{7}$, M. Zaffaroni ${ }^{1}$

${ }^{1}$ Multiple Sclerosis Center, ASST Valle Olona (Gallarate-VA); ${ }^{2}$ Department of Neurosciences, S. Camillo-Forlanini Hospital (Roma); ${ }^{3}$ Multiple Sclerosis Center, I Division of Neurology, University of Campania "Luigi Vanvitelli" (Napoli); ${ }^{4}$ Multiple Sclerosis Center, Binaghi Hospital, ATS Sardinia-University of Cagliari (Cagliari); ${ }^{5}$ Department of Neurology, Multiple Sclerosis Center IRCCS Ospedale San Raffaele (Milano); ${ }^{6}$ Multiple Sclerosis Center of the Veneto Region (CeSMuV), University Hospital of Padua (Padova); ${ }^{7}$ Regional Referral Multiple Sclerosis Center, Dept. of Neurology, University-Hospital San Luigi (Orbassano-TO)

Background: Trials on Cladribine (CLD) for the treatment of Multiple Sclerosis (MS) were conducted over a decade ago: there is a need of proof of CLD efficacy and safety profile in the present MS landscape.

Objectives: To evaluate CLD efficacy and safety in the current MS population.

Materials and methods: Between CLD approval and its marketing in Italy, CLD was available through a Free Of Charge program. We asked all MS centres to contribute to the present study, collecting demographic, clinical and MRI data of the patients enrolled in the program.

Results: 56 MS centres recruited a total of 236 patients $(71 \% \mathrm{~F})$ (mean age: $39+/-11,5$ years; mean disease duration: $10+/-8,5$ years). Mean Annualized Relapse Rate (ARR) in the two years before CLD was $0,7+/-$ 0,6; median baseline EDSS was 3 (quartiles 1,5-3,5; range 0-6,5). 53 patients $(22,5 \%)$ were treatment naïve, $107(45,3 \%)$ switched to CLD from first-line DMDs (for inefficacy), 76 (32,2\%) switched to CLD after a second line therapy (33/76 for loss of tolerability or safety reason, 43/76 for inefficacy). Mean follow up was $12,2+/-5$ months. $84,7 \%$ of the patients were relapse-free at follow-up. Mean ARR at follow-up was 0,2 $+/-0,6$. Patients taking CLD as first therapy were less likely to experience a relapse (HR 0,6; 95\% CI: 0,2-0,8; $\mathrm{p}=0,04$ ) while a higher baseline ARR was a predictor of relapses (HR 2,7, 95\% CI: 1,4-5,6; $p=0,004$ ). Median EDSS at follow up was 2 (quartiles 1-3,5). EDSS was stable in $73.7 \%$, improved of at least 1 point in $21,6 \%$ and worsened of at least 1 point in $4,7 \%$ of the patients. 157/236 patients completed one year of follow-up. Of these, $92(59,7 \%)$ reached No Evidence of Disease Activity (NEDA-3); NEDA-3 was achieved more frequently by naive patients $(70 \%)$ than switchers from a first $(57 \%)$ or a second line $(50 \%)$ (HR 2,3; 95\% CI: $1,01-5,3 ; p=0,04) 33 / 236$ patients reported at least one adverse event (AE), most frequently infections (15 cases); other AEs included 
gastrointestinal side effects, cutaneous rash, aphthous stomatitis and headache. Two severe AEs were reported (one pneumonia, one melanoma).

Conclusions: Even with the limitations of a retrospective study, our data confirm CLD safety and efficacy profile. Consistently with studies on patients with a first demyelinating event, CLD efficacy is maximized when used early in the course of MS.

References:

- Giovannoni G, Comi G, Cook S et al. A placebo-controlled trial of oral cladribine for relapsing multiple sclerosis. N Engl J Med (2010) Feb 4;362(5):416-26

- Leist TP, Comi G, Cree BA et al. Effect of oral cladribine on time to conversion to clinically definite multiple sclerosis in patients with a first demyelinating event (ORACLE MS): a phase 3 randomised trial. Lancet Neurol. (2014) Mar;13(3):257-67

- Patti F, Visconti A, Capacchione A. et al. Long-term effectiveness in patients previously treated with cladribine tablets: a real-world analysis of the Italian multiple sclerosis registry (CLARINET-MS). Ther Adv Neurol Disord. (2020) Jun 10;13:1756286420922685

\section{SWITCHING TO RITUXIMAB FROM NATALIZUMAB AND FINGOLIMOD IN MULTIPLE SCLEROSIS PATIENTS: AN OBSERVATIONAL STUDY}

S. Arena, C. Chisari, S. Toscano, S. Lo Fermo, C. Finocchiaro, E. D'Amico, M. Zappia, F. Patti

GF Ingrassia, Section of Neurosciences, University of Catania (Catania)

Objectives: Natalizumab (NTZ) and fingolimod (FTY) are high effective treatments for relapsing-remitting multiple sclerosis (RRMS). However, in some cases, it may be necessary to suspend these drugs, potentially resulting in recrudescence of the disease activity. Our aim was to investigate the efficacy of RTX as rescue therapy in patients with MS who discontinued NTZ or FTY treatments because of efficacy or safety issues.

Materials: This observational retrospective study screened 54 off-label RTX-treated patients through a cohort of 3,302 RRMS patients, referring to the MS Centre of the University of Catania in the period between 2010 and 2020. Inclusion criteria were: diagnosis of MS according to the Mc Donald criteria 2010; NTZ or FTY treatment for at least six months followed by treatment with RTX for at least one year.

Methods: Data about expanded disability status scale (EDSS), number of relapses, number of new brain and spine T2 lesions, number of brain and spine Gd-enhanced lesions, during NTZ or FTY treatment, before starting RTX (T0) and after one year of RTX treatment (T1) were collected.

Results: Twenty-four MS patients (17 females, $70.8 \%$ ), with a mean age of $44.2 \pm 11.6$ years, age at onset $27.7 \pm 11.8$ years and age at diagnosis of $30.4 \pm 11.6$ years, were enrolled. Four $(16.7 \%)$ patients were secondary progressive (4 in FTY and 1 in NTZ). Twelve patients were previously treated with NTZ (mean treatment duration: $56.1 \pm 26.5$ months) and the other half with FTY (mean treatment duration: $35.6 \pm 17.6$ months). At T1, no differences in EDSS were found in NTZ group compared to T0 (4.0 \pm 2.0 vs $4.1 \pm 2.1, \mathrm{p}=0.9$ ), while a slight decrease was observed in FTY group, even if not statistically significant $(5.3 \pm 2.0$ vs $4.5 \pm 2.3, \mathrm{p}=0.8)$. Number of clinical relapses at $\mathrm{T} 1$ was similar to $\mathrm{T} 0$ in both groups. No differences in terms of new $\mathrm{T} 2$ brain lesions and $\mathrm{Gd}+$ enhanced lesions were found between T0 and T1 in both groups. No rebounds of MS activity were reported in our cohort.
Discussion: Our data showed that RTX is effective for the treatment MS patients demonstrating to be able to maintain the clinical stability achieved during the previous therapies.

Conclusion: Considering its favorable safety and cost-effectiveness profile compared to other DMTs, RTX could be a valid therapeutic option for MS treatment. Particularly, RTX may represent a rescue therapy for those patients requiring the suspension of highly active drugs and more vulnerable to relapse or disease progression.

References:

- P. Durozard, A. Maarouf, C. Boutiere, A. Ruet, B. Brochet, S. Vukusic, C. Carra-Dalliere, P. Labauge, G. Mathey, M. Debouverie, C. Papeix, E. Maillart, C. Lubetzki, C. Bensa, O. Gout, C. Giannesini, B. Stankoff, J. Ciron, D. Brassat, J. Pelletier, A. Rico Lamy and B. Audoin on behalf of the SFSEP and OFSEP groups Efficacy of rituximab in refractory RRMS. Multiple Sclerosis Journal (2019); 25(6):828-836

- Alping P, Frisell T, Novakova L, Islam-Jakobsson P, Salzer J, Björck A, Axelsson M, Malmeström C, Fink K, Lycke J, Svenningsson A, Piehl F. Rituximab versus fingolimod after natalizumab in multiple sclerosis patients. Ann Neurol. (2016) Jun;79(6):950-8

- Bassem I. Yamout, Nabil K. El-Ayoubi, Johny Nicolas, Yehya El Kouzi, Samia J. Khoury and Maya M. Zeineddine. Safety and Efficacy of Rituximab in Multiple Sclerosis: A Retrospective Observational Study Journal of Immunology Research Volume (2018);Nov 12;2018:9084759

\section{CORTICAL AND DEEP GREY MATTER VOLUME LOSS IS SPECIFICALLY LINKED TO CEREBROSPINAL FLUID INFLAMMATION IN EARLY MULTIPLE SCLEROSIS}

\author{
A. Bajrami ${ }^{1}$, R. Magliozzi ${ }^{2}$, A. Pisani ${ }^{2}$, F. Crescenzo ${ }^{2}$, D. Marastoni ${ }^{2}$, M. \\ Calabrese $^{2}$
}

${ }^{1}$ Regional Multiple Sclerosis Center, Neurology B, Department of Neurosciences, Biomedicine and Movement Sciences, University of Verona (Verona); ${ }^{2}$ Department of Neurosciences, Biomedicine and Movement Sciences, University of Verona (Verona)

Introduction: Reduction of cortical and deep grey matter has been demonstrated by neuropathology studies and replicated in-vivo with advanced MRI analysis, with the most severe abnormalities abutting the ventricular surface [1-3]. The involvement of cortical and deep grey matter volume loss in the early MS stages and the potential causes of this pathological alterations remain uncertain.

Aim: To verify whether soluble factors released from meningeal and cerebrospinal fluid (CSF) inflammation are considered the most probably triggers of superficial grey matter damage.

Methods: We evaluated the association, at diagnosis, between CSF protein levels of 32 inflammatory mediators, among 124 relapsing remitting naïve MS patients, who underwent blinded clinical and 3T-MRI evaluations at time of diagnosis of volume loss in hippocampus, insula, exterior cerebellum and thalamus.

Results: By using multivariate regression analysis, significant correlations were found between: hippocampus volume loss and the CSF protein levels of CXCL13 (beta $=-5.65 \times 10-3 ; \mathrm{p}<0.0001$ ), CXCL10 (beta $=-$ $1.46 \times 10-4 ; \mathrm{p}<0.05$ ) and $\mathrm{sCD} 163$ (beta $=-3.20 \times 10-6 ; \mathrm{p}<0.0001$ ); thalamic volume loss and the CSF protein levels of CXCL13 (beta $=-12.5$; $\mathrm{p}<0.0001)$, sTNF-R1 (beta $=-6.00 \times 10-2 ; \mathrm{p}<0.05), \mathrm{sCD} 163$ (beta $=-$ 
$1.33 \times 10-2 ; \mathrm{p}<0.0001)$ and fibrinogen (beta=-31.48; $\mathrm{p}<0.05$ ); cerebellum volume loss and the CSF protein levels of $\mathrm{sCD} 163$ (beta=-6.78x10-2; $\mathrm{p}<0.001$ ) and chitinase-3-like1 (beta $=-3.20 \times 10-2 ; \mathrm{p}<0.05$ ); finally, insula volume loss and the CSF protein levels of CXCL13 (beta=-5.92x10-3; $\mathrm{p}<0.001$ ), CCL20 (beta $=-1.14 \times 10-1 ; \mathrm{p}<0.001)$ and osteopontin (beta $=1.71 \times 10-6 ; \mathrm{p}<0.05$ )

Discussion: At time of diagnosis, elevated CSF levels of the mayor Bcell chemoattractant molecule CXCL13 is substantially linked to grey matter atrophy in all the deep and cortical grey matter examined areas. In addition, elevated levels of markers of glia activity, such as SCD163, osteopontin, chitinase-3-like1 and sTNFR1 have also a key role in grey matter volume loss.

Conclusion: These represent the first demonstration of a direct link between intrathecal CSF inflammatory milieu and superficial GM changes since early disease stages.

References:

1. Magliozzi R, Howell OW, Reeves C, et al. A gradient of neuronal loss and meningeal inflammation in multiple sclerosis. Ann Neurol (2010);68:477-93

2. Magliozzi R, Howell OW, Nicholas R, et al. Inflammatory intrathecal profiles and cortical damage in multiple sclerosis. Ann Neurol (2018);83:739-55

3. Magliozzi R., Scalfari A., Pisani A.I., et al. The CSF Profile Linked to Cortical Damage Predicts Multiple Sclerosis Activity. Ann Neurol. (2020);00:1-12

\section{ONSET OF B-CELL DEPLETION AND SUPPRESSION OF MRI ACTIVITY WITH OFATUMUMAB TREATMENT IN RELAPSING MULTIPLE SCLEROSIS: THE APLIOS STUDY}

\author{
A. Bar-Or ${ }^{1}$, E. Fox ${ }^{2}$, A. Goodyear ${ }^{3}$, I. Ludwig ${ }^{4}$, M. Bagger ${ }^{4}$, D. Häring ${ }^{4}$, \\ H. Kropshofer ${ }^{4}$, M. Merschhemke ${ }^{4}, \mathrm{H}$. Wiendl ${ }^{5}$
}

${ }^{1}$ Center for Neuroinflammation and Experimental Therapeutics and Department of Neurology, Perelman School of Medicine, University of Pennsylvania (Philadelphia -USA); ${ }^{2}$ Central Texas Neurology Consultants and Dell Medical School, The University of Texas at Austin (Round Rock-USA); ${ }^{3}$ Medical Department, Novartis Pharmaceuticals Corporation (East Hanover-USA); ${ }^{4}$ Medical Department, Novartis Pharma AG (Basel-CH); ${ }^{5}$ Department of Neurology, University of Muenster (Muenster-D)

Objectives: B cells play a major role in the pathogenesis of MS. Ofatumumab, the first fully human anti-CD20 monoclonal antibody with a monthly $20 \mathrm{mg}$ s.c. dosing regimen suppressed $94-98 \%$ of the gadolinium-enhancing $(\mathrm{Gd}+)$ lesions versus teriflunomide in the Phase 3 ASCLEPIOS I/II trials. In APLIOS, with monthly MRI scans, the onset of ofatumumab action on B-cell depletion and MRI activity suppression can be determined. The aim of this study is to evaluate the onset of ofatumumab $20 \mathrm{mg}$ subcutaneous (s.c) effect on B-cell depletion and suppression of magnetic resonance imaging (MRI) activity in relapsing multiple sclerosis (RMS) patients.

Materials: APLIOS was a 12-week, open-label, bioequivalence Phase 2 study in typical RMS patients ( $\mathrm{N}=284)$ who received an initial loading dose of ofatumumab $20 \mathrm{mg}(0.4 \mathrm{~mL})$ s.c. (initial loading dose on Days 1, 7 , and 14, and maintenance dose every 4 weeks from Week 4 onwards) via autoinjector or prefilled syringe.

Methods: Depletion of CD19+ B cells was measured before administration of every new injection. $\mathrm{Gd}+$ lesion counts at baseline and at Weeks $4,8,12$ were assessed.
Results: The loading doses of ofatumumab depleted B cells rapidly; the proportion of patients with B-cell concentrations of $<10$ cells $/ \mu \mathrm{L}$ was $>65 \%$ after the first injection by Day $7,94 \%$ by Week 4 , and sustained $>95 \%$ at all following injections. Ofatumumab treatment reduced the mean number of $\mathrm{Gd}+$ lesions from baseline (1.5) to $0.8,0.3$, and 0.1 by Weeks 4, 8, and 12, respectively; the proportion of patients free from $\mathrm{Gd}+$ lesions at the corresponding time points were $66.5 \%, 86.7 \%$, and $94.1 \%$, respectively.

Discussion: Ofatumumab $20 \mathrm{mg}$ s.c. monthly dosing regimen resulted in a rapid, close to complete and sustained B-cell depletion over 12 weeks, leading to a profound reduction of $\mathrm{Gd}+$ lesions in RMS patients, consistent with the effects observed in the pooled Phase 3 ASCLEPIOS I/II patient population.

This study has been first presented at the Annual American Academy of Neurology Meeting 2020.

Reference:

- S. L. Hauser, A. Bar-Or, J. A. Cohen, G. Comi, J. Correale, P. K. Coyle et al. for the ASCLEPIOS I and ASCLEPIOS II Trial Groups*; Ofatumumab versus Teriflunomide in Multiple Sclerosis. N Engl J Med (2020);383:546-557

\section{WHO IS THE BEST CANDIDATE TO CLADRIBINE TREATMENT IN MULTIPLE SCLEROSIS?}

E. Barbuti ${ }^{1}$, R. Nistri ${ }^{1}$, A. Ianniello ${ }^{1}$, G. Borriello
, Lozzilli ${ }^{1}$

${ }^{1}$ MS Centre, Sant'Andrea Hospital, Sapienza University (Roma); ${ }^{2}$ NCL, Institute of Neuroscience (Roma); ${ }^{3}$ Department of Neurosciences, San Camillo Forlanini Hospital (Roma)

Aim: The treatment of Multiple Sclerosis (MS) includes several molecules with different efficacy and safety profiles. The most recent drug approved for commercial use is Cladribine. We report our short- and long-term clinical experience with Cladribine in the treatment of MS, in the attempt to better define the best candidate to Cladribine treatment.

Materials and Methods: This study is a retrospective analysis on 63 patients treated with Cladribine in the MS Center of Sant'Andrea Hospital. We divided our cohort in 2 groups: the first one includes patients from the pivotal randomized clinical trials (RCTs) CLARITY, ONWARD and ORACLE-MS, who started with Cladribine from 2006 until 2010; the second one includes patients from the post-approval CLARIFY study and from real world.

Results: The first group $(n=20)$ had a mean age at baseline of $35.2 \pm 8.7$ years and a mean follow-up of $9.0 \pm 3.6$ years. Mean and median EDSS at baseline was 2,1 and 2,0. At follow-up was 3,0 and 2,0. Seven patients (35\%) had an increase $\geq 1$ point of EDSS. They included 1 patient out of 7 naïve patients, 2 patients out of 5 previously treated with one disease-modifying treatment (DMT) and 4 out of 8 having received $\geq 2$ DMTs before Cladribine. Seven $(35 \%)$ patients experienced one or more relapses after treatment $(1$ naïve, 3 previously treated with $1 \mathrm{DMT}$, and 3 previously treated with 2 or more DMTs). Magnetic resonance imaging (MRI) activity was present at the follow-up in half of the sample. Five pregnancies from four women occurred after Cladribine, without any fetal adverse event. The second group $(n=43)$ had a mean age at baseline of $36.0 \pm 8.8$ years. Most patients $(n=24)$ were treated before Cladribine with $\geq 2$ DMTs, 8 with only one DMT, and 9 were naïve. The mean and median baseline EDSS was 2,6 and 2,0. Over a shortterm period of $13.0 \pm 7.3$ months, $3(7.0 \%)$ patients relapsed and 4 out of 23 with available scan $(17,4 \%)$ had MRI activity. 
Discussion: Data from our patients included in the pivotal clinical trials suggest that naïve patients are the best long-term responders to the Cladribine treatment. On the other hand, the real-world data indicate that, up to now, the majority of patients started Cladribine after 2 or more DMTs.

Conclusion: We support the increasing evidence of the use of Cladribine as first treatment choice in naïve patients with "active" MS.

\section{EVALUATION OF FABRY DISEASE IN A COHORT OF PATIENTS WITH MULTIPLE SCLEROSIS}

S. Barca, E. Sunda, M. Fronza, D. Carmagnini, L. Lorefice, G. Coghe, G. Fenu, E. Cocco, J. Frau

Multiple Sclerosis Center, University of Cagliari (Cagliari)

Background: Various genetic disorders can mimic Multiple Sclerosis (MS). The implications of identifying genetic disorders are many, as choosing an appropriate therapy, changing disorder's natural history and offering a genetic counselling to the patient's family. Among genetic disorders, Fabry Disease (FD) can mimic MS. This pathology is an Xlinked lysosomal storage disorder caused by deficiency of $\alpha$ galactosidase $\mathrm{A}$, that leads to storage of neutral glycosphingolipids in many tissues and cell types, involving vascular endothelium in nervous system, kidneys, heart and skin [1]. Clinical presentation of FD is variable, neurological symptoms are common features and could be the initial manifestations of FD [2]. The age at onset, the episodes of severe neuropathic pain or paresthesia due to small fiber neuropathy, premature strokes, and cerebral multiples white matter lesions in magnetic resonance imaging studies are common manifestations and may suggest MS diagnosis in FD patients.

Objective: The aim of this study is verified in a group of MS patients the presence of FD mutations, which could signify either an overlap of the two pathologies, or an incorrect MS diagnosis.

Materials and Methods: We enrolled randomly subjects with diagnosis of MS according to McDonald criteria, referring to the Multiple Sclerosis Centre of Cagliari. The test of FD was made by CENTOGENE kits on dried blood samples, after the signature of the informed consent. Samples were processed at the Centogene Company, in Germany. In males the $\alpha$ galactosidase A concentration was tested using a fluorimetric assay (Sensitivity $100 \%$, Specifity $96 \%$ ); in females a genetic sequencing of the GLA gene using NGS-Illumina system was made.

Results: We enrolled 402 MS patients, 292 (72.63\%) females and 110 $(27.36 \%)$ males, mean age $45.36 \pm 12.3$. Among them, 349 (86.81\%) subjects had relapsing-remitting MS, $21(5.22 \%)$ clinically isolated syndrome, $9(2.23 \%)$ primary progressive MS, $23(5.72 \%)$ secondary progressive MS. Median EDSS score was 2 (range 0-7,5). In whole sample the FD test was negative.

Conclusions: Sardinia has the highest frequency of MS in Mediterranean Europe and one of the highest in the world [3]. Instead, FD is a rare pathology without any ethnic predisposition. This high prevalence of MS patients in the island can partially explain our result. Moreover, the subjects included didn't show clinical features or familiarity for FD, thus decreasing the possibility to find a positive test. We can conclude that, if clinical history and familiarity are negative, it is not necessary to test people with MS for FD.

References:

1. Zarate YA, Hopkin RJ. Fabry's disease. Lancet. (2008);372(9647):1427-35. doi:10.1016/S0140-6736(08)615895
2. Sims K, Politei J, Banikazemi M, Lee P. Stroke in Fabry disease frequently occurs before diagnosis and in the absence of other clinical events: natural history data from the Fabry Registry. Stroke (2009);40(3):788-94. doi:10.1161/ STROKEAHA.108.526293

3. Rosati G. The prevalence of multiple sclerosis in the world: an update. Neurol Sci. (2001);22(2):117-39. doi:10.1007/ s100720170011

\section{SARS-COV-2 INFECTION IN MULTIPLE SCLEROSIS PATIENTS: A SINGLE CENTER EXPERIENCE IN THE PROVINCE OF BERGAMO, ITALY}

\author{
V. Barcella, M. Radaelli, M. Conti, M. Grimoldi, M. Sessa
}

Neurology, ASST Papa Giovanni XXIII (Bergamo)

Objective: In Europe, the first case of severe acute respiratory syndrome coronavirus 2 (SARS-CoV-2) infection was reported in Lombardy region; the majority of cases were identified in Eastern Lombardy, particularly in Bergamo's province with 11,313 confirmed cases until April 30th 2020. The pandemic represents a challenge for neurologists treating Multiple Sclerosis (MS) because of the higher infectious risk of infections of MS and for the disease modifyng treatment (DMT) used. We report the experience of the MS center of Papa Giovanni XXIII hospital in Bergamo.

Material and Methods: We retrospectively and prospectively collected all MS patients with suggestive symptoms for SARS-CoV-2 infection since February 20201 st. We considered patients with a confirmed diagnosis on nasopharyngeal specimens and those with suggestive symptoms and signs of infection who did not performed swab and/or serological test. Since the beginning of pandemia, according to Italian reccomandation, we postponed the majority of treatment with ocrelizumab, rituximab, cladribine and alemtuzumab whereas all the other drugs were usually continued.

Results: Between February 1st and June 30th, we identified 153 patients with suspected SARS-CoV-2 infection, which represent the $18 \%$ of the whole population regularly followed at our center. The mean age was 45 years (range 20-71), the mean EDSS was 2,5 (range 1-8,5). $81 \%$ of patients were female, 92 patients $(60 \%)$ were on first line DMT, 51 patients $(33 \%)$ on second line treatment. Overall, only 11 patients performed a nasopharyngeal swab during the symptomatic phase; 7/11 were positive. In an additional patient the diagnosis was confirmed on bronchoalveolar lavage fluid obtained by bronchoscopy due to repeated negative nasopharyngeal swabs and a CT scan diagnostic for interstitial pneumoniae. Only 3 patients (2\%) presented a severe distress respiratory syndrome and were hospitalized in Intensive Care Unit. Two of these patients were female of 43 and 46 years old treated with Natalizumab and Ocrelizumab respectively both with mild disability (EDSS 2,5). The third patient was a 56 years-old with progressive MS (EDSS of 6.5). None of them had additional comorbidities and they all had complete recovery without sequaele.

Discussion and Conclusion: In our experience the course of infection was usually mild independently of the ongoing treatment. The good outcome observed in our cohort might support a possible protective role of immunomodulation during SARS-CoV-2 infection. However, the diagnosis was confirmed only in few patients, therefore we cannot draw definitive conclusions. We are collecting serological data to better characterize our cohort. 


\section{References:}

- G. Giovannoni, C. Hawkes, J. Lechner-Scott, M. Levy, E. Waubant, J. Gold. The COVID-19 pandemic and the use of MS disease-modifying therapies. Mult Scler Relat Disord. (2020) Apr; 39: 102073

- M. P. Sormani, Italian Study Group on COVID-19 infection in multiple sclerosis, An Italian programme for COVID-19 infection in multiple sclerosis. Lancet Neurol. (2020) Jun; 19(6): 481-482. Published online 2020 Apr 30. doi: 10.1016/S1474-4422(20)30147-2

- D. Baker, S. Amor, A. S. Kang, K. Schmierer, G. Giovannoni, The underpinning biology relating to multiple sclerosis disease modifying treatments during the COVID-19 pandemic. Mult Scler Relat Disord. (2020) Aug; 43: 102174. Published online 2020 May 12. doi: 10.1016/j.msard.2020.102174

\section{APPLICABILITY OF SNFL IN MULTIPLE SCLEROSIS AS ADDITIONAL MEASURE IN CLINICAL PRACTICE AND IMPLICATIONS IN NEDA-3 EVALUATION}

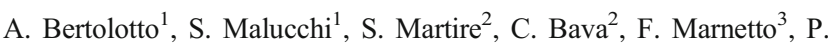 \\ Valentino $^{3}$ \\ ${ }^{1}$ Neurology and CRESM, University Hospital San Luigi Gonzaga \\ (Orbassano-TO); ${ }^{2}$ Neuroscience Institute Cavalieri Ottolenghi (NICO), \\ University Hospital San Luigi Gonzaga (Orbassano-TO); ${ }^{3}$ Department \\ of Neuroscience, NICO, University of Turin (Orbassano-TO)
}

Objectives: Despite the increased availability of efficient therapeutic options, early and effective intervention is still mandatory for successful clinical management in Multiple Sclerosis (MS). Speaking of which, a composite measure of disease status termed "no evidence of disease activity" (NEDA) has been proposed as target for treatment in MS. However, this definition needs to be implemented with biological measures for a more personalized medicine. Neurofilament light chain (NfL) is the most promising biomarker of disease activity and treatment efficacy in MS, reflecting axonal damage in the central nervous system. Recently, the ultrasensitive Simoa technology has allowed the quantification of serum NfL (sNfL). To date, sNFL correlation with clinical outcomes is well established in group analysis, yet, its implementation for individual patients in clinical practice is still to be addressed. The aim of the present real-life study is to investigate sNFL as additional measure of treatment efficacy in NEDA-3 patients.

Materials: We measured SNF-L in 79 healthy participants and in 582 cross-sectionally enrolled RRMS patients, including 61 naïve patients, and 521 patients treated with first- or second line therapies, demonstrating NEDA-3 status (no relapses, no disability progression, no MRI activity) during at least 12 months.

Method: sNFL were evaluated by Simoa assay (Quanterix).

Results: 1) We established clinically applicable cut-off values for each age decade testing healthy individuals, later used to interpret sNF-L levels in MS patients. 2) treatments notably lower SNF-L relative to untreated patients; 3) 53/521 (10\%) NEDA-3 patients still demonstrate high NFL levels, according to cut-off values. These patients were distinguished in different categories: a) patients showing borderline values; b) patients with concomitant pathologies; c) patients experiencing chronic ongoing fatigability (not classifiable as disability progression); d) patients with an very active disease history; e) patients with conceivable inflammation not detectable by RMN or clinics. 4) The percentage of NEDA-3 patients with high NFL lowers to $5 \%$ in patients with NEDA-3 status longer than 8 years.
Discussion: The present cross-sectional study identified a subgroup of NEDA-3 patients showing high sNFL levels. Indeed, high pathological sNFL levels, reflecting axonal damage, suggest the presence of chronic disease activity or subclinical transitory active inflammation not detectable by RMN or clinics in NEDA-3 patients.

Conclusion: The establishment of simple applicable cut-off values allows sNFL applicability in everyday clinical practice. In particular, sNFL could represent an additional clinical applicable measure to be included in NEDA assessment for monitoring therapeutic response in individual MS patients.

\section{BB-CRESM: AN INSTITUTIONAL BIOBANK TO SUPPORT QUALITY RESEARCH IN MULTIPLE SCLEROSIS}

\author{
A. Bertolotto ${ }^{1}$, P. Valentino ${ }^{2}$, M. Caldano ${ }^{3}$, A. Ficorilli ${ }^{1}$, A. Paudice ${ }^{4}$, F. \\ Marnetto $^{2}$
}

${ }^{1}$ Neurology and CRESM, University Hospital San Luigi Gonzaga, University of Turin (Orbassano-TO); ${ }^{2}$ Department of Neuroscience, NICO, University of Turin (Orbassano-TO); ${ }^{3}$ Pharmacy Division, University Hospital San Luigi Gonzaga (Orbassano-TO); ${ }^{4}$ Health Direction, Ordine Mauriziano Hospital (Torino)

Objectives: Collection and storage of biological samples following strict quality standards through biobanks has recently become a crucial tool in clinical research. In this context the Regional Referral Center for Multiple Sclerosis (CRESM) at the San Luigi Gonzaga Hospital (Orbassano, Italy), which provides comprehensive care for more than 2000 patients, has been able to transform the reserve of biological samples into a structured biobank. Aims of this study are to describe the steps for establishing a structured MS biobank starting from a collection of biological samples and the process for providing scientists with biological samples and associated data.

Methods: Collection of samples and data have been developed within appropriate legal, ethical, clinical, scientific, and technical guidelines, according to the "Biobanking and Biomolecular Resources and Research Infrastructure" (BBMRI) indications. All procedures were included into Standard Operating Procedures Manual.

Results: BB-CRESM is a structural part of the Piedmont Regional Health Service. The General Director of San Luigi Hospital approved its Regulation, selected the BB-CRESM director and the members of the Scientific Committee. BB-CRESM is a nonprofit organization supported by the Italian Multiple Sclerosis Foundation. Specific protocols regulate the timing and modalities of collection of biological samples. The protection of data was approved by the Data Protection Officer of San Luigi Hospital in compliance with the General Data Protection Regulation. A detailed description of the biobank is in-person explained to each patient or healthy subject with the help of a leaflet; following which the informed consent is obtained. The Ethical Committee approved both the leaflet and the informed consent, which were supervised by a bio-ethicist. Scientists can apply to the director of BB-CRESM specifying number, types and quantity of required samples and data. The samples can be distributed if a) they are used for a research project approved by an Ethic Committee; b) the research project is approved by the Scientific Committee; c) the scientist signs the Material Transfer Agreement; d) scientist agree to share crude data with BB-CRESM e) scientist contributes to BB-CRESM collection and shipment expenses. Since 2013, more than 1000 participants 
(healthy controls and MS patients) have enrolled in the BBCRESM; over 20000 tubes of biological material (cerebrospinal fluid, serum, plasma, PBMCs, RNA and DNA) have been collected and stored.

Discussion, Conclusions: BB-CRESM works to collect, store and distribute biological samples along with associated data ensuring quality of collection and bio-banking according to BBMRI.

MS researchers can contact B B-CRESM at biobanca.cresm@sanluigi.piemonte.it.

\section{NORMAL SERUM NFL LEVELS: A PROPOSAL OF CUT-OFF STRATEGY DEFINITION FOR THE CLINICAL PRACTICE}

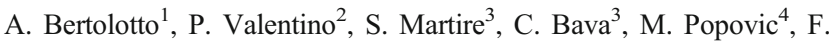 \\ Marnetto $^{2}$
}

${ }^{1}$ Neurology and CRESM, University Hospital San Luigi Gonzaga, University of Turin (Orbassano- TO); ${ }^{2}$ Department of Neuroscience, NICO, University of Turin (Orbassano-TO); ${ }^{3}$ Neuroscience Institute Cavalieri Ottolenghi (NICO), University Hospital San Luigi Gonzaga (Orbassano-TO); ${ }^{4}$ Department of Medical Sciences, University of Turin (Torino)

Objectives: Serum Neurofilament light (sNFL) protein is the most promising marker of disease activity and of treatment response in Multiple Sclerosis. To implement sNFL in clinical practice, the definition of normal widely accepted values represents a crucial step still to be addressed. Clinically applicable cut-off values need to take into account age dependency; in addition, recent evidences suggest that physical parameters as body mass index (BMI) and blood volume (BV) might influence sNFL levels. The present study aims to address these crucial needs.

Materials: We measured sNFL in 79 healthy individuals to define reference cut-off values. In addition, sNFL were evaluated after a shortterm follow-up time (median 67 days) to assess intra-individual variability: consecutive blood sample were tested in a subset of 27 participants ( $n=2-4$ samples for each individual). sNFL were tested in 23 naïve MS patients both at diagnostic time and immediately before treatment to evaluate the applicability of obtained cut-off values.

Method: sNFL were measured by Simoa assay (NF-light advantage Kit, Quanterix). Age, BMI and BV were correlated with sNFL levels. The coefficient of variation (CV) for sNFL levels from each participant during follow-up was evaluated to assess intra-individual variability of sNFL levels.

Results: 1) Our data confirm a strong correlation between SNFL levels and age. We found a negative correlation between sNFL levels and BV. 2) Short-term follow-up NFL assessments showed an overall intra-individual stability in sNFL levels in healthy population (median CV 15\%). 3) we defined specific age decade-related cut-off values. 4) In naïve MS patients, sNFL levels were higher than control values; in addition, a high variability between diagnostic time and the beginning of treatment (median CV 39\%) was shown. Cut-off values were applied in naïve MS patients to discriminate high from normal sNFL levels: at diagnostic time, $57 \%$ of MS patients showed high sNFL levels, while at treatment start, $70 \%$ of patients demonstrated normal NFL values.

Discussion and Conclusion: The present study suggests a strategy to define clinical applicable cut-offs to exploit sNFL as a personalised medicine tool in MS: specific cut-off values were calculated for each age decade. sNFL levels demonstrated an overall intra-individual stability in healthy participants in the short-term: this is relevant for a biomarker of disease activity and treatment response that, if successful, will be serially assessed during patient's follow-up.

\section{REAL-LIFE EXPERIENCE WITH SNFL IN MS PATIENTS, AS MONITORING AND TREATMENT DECISION BIOMARKER}

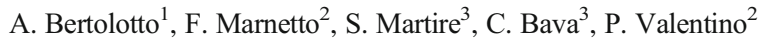

${ }^{1}$ Neurology and CRESM, University Hospital San Luigi Gonzaga, University of Turin (Orbassano- TO); ${ }^{2}$ Department of Neuroscience, NICO, University of Turin (Orbassano-TO); ${ }^{3}$ Neuroscience Institute Cavalieri Ottolenghi (NICO), University Hospital San Luigi Gonzaga (Orbassano-TO)

Objectives: Neurofilament light chain (NFL) are the most promising biomarkers to investigate clinical activity and treatment efficacy in multiple sclerosis (MS), for which, to date, clinical examination and magnetic resonances (MRI) are the only tools used for diagnosis and monitoring. NFL are released upon axonal damage in the cerebrospinal fluid and, in low concentration, in serum (sNFL). Whilst correlation between NFL and clinical outcomes is established, their implementation in clinical practice is still to be addressed. The aim of the present real-life cross-sectional study is to describe sNFL in a large cohort of MS patients as additional measure of disease activity and treatment efficacy, by establishing clinically applicable discriminating values

Materials: We measured sNF-L in 79 healthy participants and in 961 MS patients ( $\mathrm{n}=1130$ samples). sNFL were cross-sectionally evaluated in 830 relapsing remitting (RR), 53 primary progressive (PP) and $78 \mathrm{sec}-$ ondary progressive (SP) MS patients at different disease stages including diagnostic time, immediately before treatment, and during treatment with the main disease modifying treatments (DMTs), including Interferon-beta, Glatiramer acetate, Dimethyl-Fumarate, Teriflunomide, Natalizumab, Alemtuzumab, Fingolimod; anti-CD20.

Method: sNFL were measured by Simoa assay (NF-light advantage kit, Quanterix). Clinical assessment was performed to evaluate correlations between SNFL, MRI and relapses.

Results: 1) We established clinically applicable cut-off values for each age decade testing healthy individuals, later used to interpret sNF-L levels in individual MS patients. 2) Progressive MS patients showed higher sNF-L levels and a greater prevalence of high SNFL levels (32\% in PPMS and 26\% in SPMS) relative to RRMS patients (16\%). 3) Patients experiencing MRI and/or clinical activity close to NFL dosage (+/-60 days) showed higher levels than stable patients; according to cut-off values, high NFL levels were observed in a substantial percentage of MRI active patients $(72 \%)$ and clinically active patients (75\%). 4) All DMTs notably lower sNF-L in RRMS patients treated for more than 12 months relative to untreated patients; though, $12 \%$ of treated patients still demonstrated high NFL levels.

Discussion and Conclusion: This cross-sectional study provide a reallife picture of SNFL in a large cohort of MS patients. We defined clinically applicable cut-off values, able to introduce sNF-L dosing in the everyday practice on individual patients. Cut-offs were applied to discriminate patients' samples in different contexts, showing correlation with disease subtype, clinical activity and DMTs efficacy. Our study shows that implementation of SNFL in everyday clinical practice could be revolutionary in the monitoring of MS patients. 


\section{OPTIC CHIASM INVOLVEMENT IN MS, AQUAPORIN-4 ANTIBODY-POSITIVE NMOSD, AND MOG ANTIBODY- ASSOCIATED DISEASE} A. Bianchi ${ }^{1}$, R. Cortese
,, F. Prados

${ }^{1}$ Institute of Neurology, Department of Neuroinflammation, University College London (London-UK); ${ }^{2}$ Centre for Medical Imaging Computing, Medical Physics and Biomedical Engineering, University College London (London-UK); ${ }^{3}$ Department of Neurology, The Cleveland Clinic Abu Dhabi (Abu Dhabi-UAE); ${ }^{4}$ Division of Brain Sciences, Department of Medicine - Imperial College London (London-UK)

Aims: Inflammatory demyelination in the anterior optic pathway, including the optic chiasm (OC), occurs frequently in relapsingremitting multiple sclerosis (RRMS), aquaporin4 (AQP4) antibody (Ab) positive neuromyelitis optica spectrum disorder (NMOSD), and myelin oligodendrocyte glycoprotein- $\mathrm{Ab}$ associated-disease (MOGAD). The aim of the study was to evaluate the involvement of the OC in RRMS, AQP4-NMOSD and MOGAD using Magnetization Transfer Ratio (MTR) and explore its relationship with prior optic neuritis $(\mathrm{ON})$.

Methods and materials: We recruited 25 patients with RRMS (16 F, mean age: 44.6 yrs \pm 11.8 , median EDSS: 2.0 [range: $1.0-7.5$ ], mean number of previous episodes of $\mathrm{ON}$ : $0.44 \pm 0.58,9$ unilateral, 1 bilateral), 13 with AQP4-NMOSD (10 F, mean age: $45.3 \mathrm{yrs} \pm 11.2$, median EDSS: 3.0 [1.0-6.5], mean number of ON episodes: $1.54 \pm 1.13,4$ unilateral, 6 bilateral), 20 with MOGAD (13 F, mean age: 33.9 yrs \pm 16.4 , median EDSS: 2.0 [0.0-6.5], mean number of ON episodes: $2.85 \pm 2.80,6$ unilateral, 11 bilateral) and 29 healthy controls (HC) (23 F, mean age: 35.9 yrs \pm 12.8 ). We used T2-weighted, MTon and MToff images to obtain MTR maps of the OC. Age-, sex-, and disease duration-adjusted linear regression models were used to compare the measures between disease and healthy groups $(\mathrm{p}<0.05)$.

Results: Chiasmal MTR values in patients with previous ON were lower in AQP4-NMOSD $(p=0.040)$ and MOGAD $(p=0.001)$ than $\mathrm{HC}$ but not when compared to patients without previous ON. In patients with RRMS and previous ON, MTR values were lower than patients without prior $\mathrm{ON}(\mathrm{p}=0.003)$. No differences were found either between patients without $\mathrm{ON}$ and $\mathrm{HC}$ or between the disease groups. When considering all patients with demyelinating diseases, patients with previous ON had lower chiasmal MTR values when compared to $\mathrm{HC}$ (unilateral: $\mathrm{p}=0.037$; bilateral: $\mathrm{p}=0.002$ ) and to patients without $\mathrm{ON}$ (unilateral: $\mathrm{p}=0.019$; bilateral: $\mathrm{p}<0.001)$. This difference persisted when comparing both monophasic and relapsing ON patients to $\mathrm{HC}(\mathrm{p}=0.044 ; \mathrm{p}<0.001)$ and to patients without $\mathrm{ON}(\mathrm{p}=0.019 ; \mathrm{p}<0.001)$. No differences were found between patients without history of $\mathrm{ON}$ and HC. A correlation was found between MTR values and number of ON episodes $($ rho $=-0.55, \mathrm{p}<0.001)$.

Discussion: Microstructural damage in the OC correlated with the number of $\mathrm{ON}$ episodes across inflammatory demyelinating diseases. A higher number of episodes is associated with lower chiasmal MTR.

Conclusion: The findings support the role of OC as an accessible target for the assessment of the visual pathway in inflammatory diseases. References:

- Petzold A, Wong S, Plant GT. Autoimmunity in Visual Loss. Handbook of Clinical Neurology (2016);133:353-76
- Wang Y, van der Walt A, Paine M, Klistorner A, Butzkueven H, Egan GF, Kilpatrick TJ, Kolbe SC. Optic Nerve Magnetisation Transfer Ratio after Acute Optic Neuritis Predicts Axonal and Visual Outcomes. PLoS ONE (2012);7(12):e52291

- Klistorner A, Chaganti J, Garrick R, Moffat K, Yiannikas C. Magnetisation Transfer Ratio in Optic Neuritis Is Associated with Axonal Loss, but Not with Demyelination. NeuroImage (2011);56: $21-6$

\section{REGIONAL CHANGES IN THALAMIC SHAPE ARE RELATED TO UPPER EXTREMITY AND MULTIPLE SCLEROSIS FUNCTIONAL COMPOSITE PERFORMANCE IN MULTIPLE SCLEROSIS}

A. Bisecco ${ }^{1}$, R. Capuano ${ }^{1}$, A. d'Ambrosio ${ }^{1}$, M. Altieri ${ }^{1}$, R. Docimo ${ }^{1}, \mathrm{M}$.

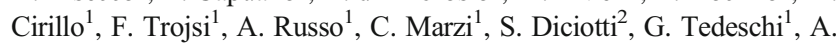
Gallo ${ }^{1}$

${ }^{1}$ Department of Advanced Medical and Surgical Sciences, University of Campania "Luigi Vanvitelli" (Napoli); ${ }^{2}$ Department of Electrical, Electronic, and Information Engineering "Guglielmo Marconi”, University of Bologna (Bologna)

Objectives: Previous studies reported inconsistent association between thalamic shape and disability levels as measured using the expanded disability status scale (EDSS). Multiple Sclerosis Functional Composite (MSFC) is an additional clinical measure of MS disability. It includes the assessment of leg function by moving a short walking distance ("Timed 25-Foot Walk", T25FT), the assessment of arm function using breadboard test ("9-Hole Peg Test", 9HPT) and an attention/concentration test to assess cognitive functions ("Paced Auditory Serial Addition test", PASAT). Aim of the present study is to assess the relationship between thalamic regional volumes/shape and disability as measured with EDSS and MSFC in MS patients.

Methods: One hundred and eighteen relapsing remitting MS (RRMS) patients and 52 sex, age and education-matched healthy controls (HC) underwent clinical evaluation (EDSS and MSFC), and a 3T-MRI protocol including high resolution 3D-T1 imaging. Thalamic volumes were calculated using FIRST tool of the FMRIB Software Library. A vertex analysis of thalamic shape was also performed, exploring regional differences between MS and HC. Correlation analyses were run between EDSS and MSFC and thalamic changes in shape/volume. Thalamic connectivitydefined subregions showing significant changes were labeled using an atlas previously derived that includes probability maps of thalamic parcellation in frontal, motor, parietal, occipital, and temporal connectivity defined regions.

Results: Compared to HC, MS patients showed a significant atrophy $(p<0.01)$ in the whole thalamus. MSFC performance correlated $(\mathrm{p}<0.01)$ with atrophy of bilateral frontal and temporalconnected and right motor and parietal-connected thalamic subregions. 9HPT performance correlated $(\mathrm{p}<0.01)$ with atrophy of bilateral frontal, motor, parietal and temporal-connected thalamic subregions. T25FT and EDSS score did not show correlation with thalamic shape/volume.

Conclusions: This study confirms that the thalamus is relevant for disability status of MS patients. In particular, upper extremity (as measured with 9HPT) and MSFC performance seems to be more sensitive to regional thalamic damage. 


\section{USING NIH TOOLBOX STANDING BALANCE TEST TO QUANTIFY SUBTLE BALANCE IMPAIRMENT IN PATIENTS WITH MULTIPLE SCLEROSIS}

G. Boffa, C. Lapucci, F. Tazza, E. Mancuso, E. Sbragia, N. Bruschi, M. Cellerino, M. Inglese

${ }^{1}$ Department of Neurosciences, Rehabilitation, Ophthalmology, Genetics, Maternal and Child Health, University of Genoa, Policlinico San Martino IRCCS (Genova)

Background: Balance impairment is frequent in Multiple Sclerosis (MS). Attempts to measure balance deficits in clinical practice have mostly relied on EDSS, but its scarce capability to detect subtle deficits is an important limitation.

Objectives: To compare NIH Toolbox Standing Balance Test (SBT) with EDSS in detecting balance impairment in MS and to assess the contribution of the different Functional Systems (FS) involved in balance on SBT metrics.

Methods: 128 consecutive MS patients and 36 age and sexmatched healthy controls (HCs) underwent NIH Toolbox SBT at the MS Center of the University of Genoa. Patients underwent clinical evaluation with EDSS FS recording and 3T brain MRI (Siemens Prisma). Theta scores were derived and corrected for age, sex, height and weight. T2 and T1 lesion volumes (LV) were obtained for the cerebellum and the total brain separately. A linear logistic regression model was performed to evaluate the relative contribution of cerebellar, sensory and brainstem impairment on balance performance.

Results: 92 (73.9\%) MS patients were females, mean (SD) age was 41.2 (11.6) years; 108 (84.4\%) patients had relapsing-remitting (RRMS) and $20(15.6 \%)$ progressive MS (PMS). Mean disease duration was 10.6 (9.3) years, median (IQR) baseline EDSS was 2.5 (1-4). According to their FS, $73(53.0 \%), 57$ (44.5\%) and 79 (61.7\%) patients had no evidence of cerebellar, sensory and brainstem dysfunction respectively. On brain MRI, $95(74.2 \%)$ patients exhibited cerebellar lesions [mean T2LV 0.31 (0.44) $\mathrm{mL}$; mean T1LV $0.20(0.31) \mathrm{mL}$. Patients had significantly lower theta scores compared with HCs $(-0.27 \mathrm{vs} 0.91 ; \mathrm{p}=0.003)$. RRMS had better performance than PMS patients $(-0.05 \mathrm{vs}-1.45 ; \mathrm{p}=0.006)$. Patients with a cerebellar and brainstem $\mathrm{FS}=0$ had higher theta scores than impaired patients $(0.34 \mathrm{vs}-1.07 ; \mathrm{p}<0.0001$ and $0.12 \mathrm{vs}-0.89 ; \mathrm{p}=0.008$ respectively). Patients without impairment in sensory and brainstem FS had worse performance than HCs $(0.08 \mathrm{vs} 0.91 ; \mathrm{p}=0.046$ and $0.12 \mathrm{vs} 0.91 ; \mathrm{p}=0.048$, respectively). Each 1 point increase in cerebellar FS independently determined a -0.50 decrease in theta score (95\% CI:-0.91-0.09; $\mathrm{p}=0.017)$. Theta scores correlated with cerebellar T2LV and T1LV (Spearman r$0.29, \mathrm{p}=0.001$ and $\mathrm{r}=-0.28, \mathrm{p}=0.001$ respectively) but not with global $\mathrm{T} 2 \mathrm{LV}$ and $\mathrm{T} 1 \mathrm{LV}$.

Discussion: NIH Toolbox SBT is able to detect subtle balance impairment in MS patients, not detected by clinical examination. Clinical and radiological cerebellar involvement seem to be specifically related to NIH Toolbox SBT metric.

\section{MOG-IGG ANTIBODIES: A POSSIBLE BIOMARKER IN MULTIPLE SCLEROSIS? NOT JUST NMOSD}

L. Bollo, P. Iaffaldano, M. Ruggieri, M. Mastrapasqua, M. Simone, A. Manni, D. Paolicelli, A. Frigeri, M. Trojano
Department of Basic Medical Sciences, Neurosciences and Sense Organs, University of Bari (Bari)

Background: Multiple sclerosis (MS) is the most common acquired demyelinating disorder of Central Nervous System. Neuromyelitis Optica Spectrum Disorders (NMOSD) was previously considered a form of MS involving predominantly the spinal cord and optic nerves. The discovery of specific autoantibodies, some unique clinical features, magnetic resonance imaging and other laboratory findings have led to consider them as distinct entities.

Objective: To compare clinical and laboratory characteristics between MOG-IgG negative MS patients (Group1), MOG-IgG positive MS patients (Group2) and MOG-IgG positive NMOSD patients (Group3).

Subjects and Methods: We included 305 patients, MOG-IgG and AQP4-IgG were evaluated by using an in-house Cell Based Assay in patients with first clinical episode suggestive of CNS demyelinating syndrome admitted to our Neurology department. The serum titer was determined using a fluorescent ratiometric method, ranging between 0 to 1 (cut-off values: 0.02 for MOG-IgG, 0.01 for AQP4IgG). AQP4-IgG positive patients were excluded. Clinical and CSF features were collected for each patient. Between groups comparisons were performed by using the Mann-Whitney test for continuous variables and the Chi-square for categorical variables. Cumulative probabilities of experiencing a relapse were evaluated by using the Kaplan-Meier method and the survival curves were compared using the log-rank (Mantel-Cox).

Results: According to 2017 McDonald criteria for MS and 2015 Wingerchuck criteria for NMOSD, 286 patients were diagnosed with MS, and 19 patients with NMOSD. MOG-IgG were found in 39/305 patients' sera. The median (range) follow-up time from onset was 4 (range 1-35 years). No significant differences were found in terms of age at onset ( $31.0 \pm 10.8$ vs $35.3 \pm 15.7$ years), EDSS at onset $(2.3 \pm 1.1$ vs $2.0 \pm 1.1)$ and at the last follow-up $(2.5 \pm 1.5$ vs $2.1 \pm 0.7)$ between Group 1 and Group2, however Group2 patients presented a lower frequency of CSF-restricted oligoclonal bands (47\% vs $78 \%$, $\mathrm{p}=0.0001)$. In Group 3 patients were older $(38.0 \pm 15.8$ vs $31.0 \pm 10.8$ years, $\mathrm{p}=0.03$ ) and more disabled at the last follow-up (EDSS: 4.0 \pm 2.4 vs $2.5 \pm 1.5, p=0.01$ ) than the Group 1 patients. Group2 patients presented more frequently than Group 1 optic neuritis (68\% vs $28 \%$, $\mathrm{p}<0.0001)$ or myelitis $(38 \%$ vs $20 \%, \mathrm{p}<0.0001)$ at onset. No one in the Group2 had supratentorial symptom at onset. Group2 had more frequently a monophasic disease course compared with Group 1 $(60 \%$ vs $10 \%, p<0.001)$ and they had a lower risk of experiencing a relapse $(\mathrm{p}=0.006)$.

Conclusion: MOG-IgG could represent a biomarker in MS to identify a subgroup of patients with opticospinal MS and might affect treatment decisions.

References:

- Wingerchuk DM, Banwell B, Bennett JL, et al. International consensus diagnostic criteria for neuromyelitis optica spectrum disorders. Neurology (2015);85(2):177-189. doi:10.1212/ WNL.0000000000001729

- Thompson AJ, Banwell BL, Barkhof F, et al. Diagnosis of multiple sclerosis: 2017 revisions of the McDonald criteria. Lancet Neurol. (2018);17(2):162-173. doi:10.1016/S1474-4422(17)30470-2

- Zamvil SS, Slavin AJ. Does MOG Ig-positive AQP4-seronegative opticospinal inflammatory disease justify a diagnosis of NMO spectrum disorder? Neurol Neuroimmunol Neuroinflammation (2015);2(1):e62. doi:10.1212/NXI.0000000000000062 


\section{PRIMARY PROGRESSIVE MULTIPLE SCLEROSIS CSF BIOMARKERS}

A. Bolzan, D. Marastoni, S. Rossi, A. Pisani, V. Mazziotti, M. Castellaro, R. Magliozzi, S. Monaco, M. Calabrese

Department of Neurosciences, Biomedicine and Movement Sciences, University of Verona (Verona)

Objective: Several evidences suggest that primary progressive multiple sclerosis (PPMS) is characterized by both a degenerative and inflammatory process, which, similarly to relapsing remitting multiple sclerosis (RRMS), become compartimentalized behind an intact blood brain barrier [1]. We evaluated the levels of 69 pro and anti-inflammatory cytokines and chemokines in the cerebrospinal fluid (CSF) of PPMS patients at the diagnosis in order to detect possible biomarkers of progressive course and their association with clinical and MRI variables.

Methods: Sixty-nine inflammatory mediators and NF-L CSF levels from 15 patients with PPMS (mean age 54.5y), 80 patients with RRMS (36.8y), and 12 with non-inflammatory neurological disorders (51y) were assessed at the diagnosis by mean of multiplex technique and ELISA [2]. All patients underwent clinical evaluation, including Expanded Disability Status Scale (EDSS) assessment, and 3-T Brain MRI with detection of white matter and cortical pathology.

Results: PPMS patients had higher EDSS compared to RRMS (mean 3.0 vs $2.0, \mathrm{p}<0.001)$ and older age $(54.5 \pm 9.6$ vs $36.8 \pm 11.9, \mathrm{p}<0.001)$. CSF of MS patients was characterized by overexpression of a pattern of inflammatory molecules compared to controls. A multivariate regression analysis showed that, at the diagnosis, levels of some B-cell related cytokines such as IL10 (OR=0.28, CI95\%[0.09-0.96]) and CXCL12 $(\mathrm{OR}=3.97, \mathrm{CI} 95 \%[1.34-11.7])$ and the monocyte-related osteopontin $(\mathrm{OR}=2.24, \mathrm{C} 195 \%[1.01-4.99])$ were predictive for a primary progressive course of the disease. We observed an association between CSF levels of lymphoid chemokine CXCL12 and both cortical lesion number and volume $(\mathrm{p}<0.005)$.

Discussion and Conclusions: These results confirm the presence of a significant inflammatory reaction in the CSF of PPMS patients. A detailed CSF profiling at the diagnosis could provide new insights into the pathogenesis of MS progression as well as suggest useful tools for clinical practice.

References:

1. Choi SR, Howell OW, Carassiti D, et al. Meningeal inflammation plays a role in the pathology of primary progressive multiple sclerosis. Brain (2012);135(Pt 10):2925-37

2. Magliozzi R, Howell OW, Nicholas R, et al. Inflammatory intrathecal profiles and cortical damage in multiple sclerosis. Ann Neurol. (2018);83(4):739-55

\section{AGE AT DISEASE ONSET INFLUENCES GREY MATTER AND WHITE MATTER INTEGRITY IN MULTIPLE SCLEROSIS}

\author{
R. Bonacchi ${ }^{1}$, A. Meani ${ }^{1}$, E. Pagani ${ }^{1}$, M. Filippi ${ }^{2}$, M. Rocca ${ }^{1}$
}

${ }^{1}$ Neuroimaging Research Unit, Institute of Experimental Neurology, Division of Neuroscience, IRCCS San Raffaele Scientific Institute (Milano); ${ }^{2}$ Neuroimaging Research Unit, Institute of Experimental Neurology, Division of Neuroscience; Neurology Unit and Neurophysiology Unit, IRCCS San Raffaele Scientific Institute; Vita-Salute San Raffaele University (Milano)
Aims: Natural history studies demonstrated clinical phenotype and course of multiple sclerosis (MS) are age-dependent. The comparison of pediatric- (POMS) vs adult-onset (AOMS) MS patients is a useful model for studying the effects of age on MS pathophysiology. We aimed to determine whether age of disease onset influences the extent, distribution and trajectories of development of brain grey matter volume (GMV) and white matter (WM) microstructural abnormalities in adult MS patients.

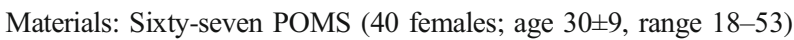
and 143 sex- and disease duration-matched AOMS ( 85 females; age 46 \pm 11 , range $20-70$ ) patients, together with 208 age- and sex-matched

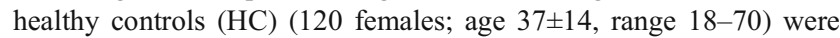
included.

Methods: All subjects underwent neurological examination with Expanded Disability Status Scale (EDSS) scoring, and same-day as 3T MRI acquisition, including dual echo, 3D T1weighted, and diffusion-weighted sequences. T2-lesion volumes, GMV and WM fractional anisotropy (FA) were derived and standardized based on distribution in $\mathrm{HC}$, to remove the effects of age and sex. Linear models were used to study associations with disease duration in POMS and AOMS patients. Time to reach clinical and radiological milestones was assessed with the productlimit approach.

Results: At disease duration=1year, GMV and WM FA were not abnormal in POMS, while they were already compromised in AOMS patients ( $\mathrm{p}$ ranging from 0.04 to $<0.001$ ) compared to $\mathrm{HC}$. Significant interaction of age at onset (POMS vs AOMS) into the association with disease duration was found for GMV $(\mathrm{p}=0.01)$ and WM FA $(\mathrm{p}=0.04)$. The crossing point of regression lines in POMS and AOMS was at 19 and 15 years of disease duration for GMV and WM FA, respectively. Median disease duration to reach EDSS $=3$ was 29 years for POMS and 19 years for AOMS patients $(\mathrm{p}<0.001)$, to reach brain GMV $\mathrm{z}$-score $=-1.645$ was 24 years for POMS and 19 years for AOMS $(\mathrm{p}=0.04)$, and to reach brain WM FA z-score=-1.645 was 19 years for POMS and 17 years for AOMS $(\mathrm{p}=0.31)$

Discussion: In POMS patients, disruption of WM integrity precedes GM damage and is initially less severe than in AOMS. The rate of WM damage accumulation is higher in POMS compared to AOMS, resulting in more severe WM damage with longer disease duration. Except for WM damage, POMS patients reach clinical and MRI milestones at younger age than AOMS, but take longer time.

Conclusions: Age at disease onset impacts on MS pathophysiology, affecting GMV, WM integrity, and clinical variables.

Funding. Partially supported by grants from Italian Ministry of Health (GR-2009-1529671) and Fondazione Italiana Sclerosi Multipla (FISM2016/R/23).

\section{CARDIOVASCULAR RISK FACTORS AFFECT BRAIN VOLUME IN YOUNG MS PATIENTS}

\author{
R. Bonacchi ${ }^{1}$, D. Mistri ${ }^{1}$, A. Meani ${ }^{1}$, A. Zanghi ${ }^{1}$, M. Filippi ${ }^{2}$, M. Rocca ${ }^{1}$ \\ ${ }^{1}$ Neuroimaging Research Unit, Institute of Experimental Neurology, \\ Division of Neuroscience, IRCCS San Raffaele Scientific Institute \\ (Milano); ${ }^{2}$ Neuroimaging Research Unit, Institute of Experimental \\ Neurology, Division of Neuroscience; Neurology Unit and \\ Neurophysiology Unit, IRCCS San Raffaele Scientific Institute; Vita- \\ Salute San Raffaele University (Milano)
}


Aims: Cardiovascular (CV) risk factors have been associated with changes in clinical and MRI outcomes in patients with multiple sclerosis (MS). However, previous studies have not set an age-limit, while older patients may be affected by cerebral small vessel disease-related damage in addition to MS. We aimed to investigate the impact of $\mathrm{CV}$ risk factors on brain atrophy in patients with MS under age 50 .

Materials: One-hundred and twenty-four (79 relapsing-remitting, 45 progressive) MS patients ( 74 females, age $36 \pm 8$, range $18-50$ ), and 95 age- and sex-matched healthy controls (HC) (47 females, age $35 \pm 8$, range $18-50$ ) were included in the study.

Methods: Subjects underwent brain 3T MRI with pulse sequences for assessing lesions and atrophy, and complete neurological examination. Traditional CV risk factors were assessed: having smoked $\geq 5$ pack-years (py), and presence of hypertension, dyslipidemia, diabetes/prediabetes. More stringent cut-offs were also assessed: having smoked $\geq 10 \mathrm{py}$, and hypertension, dyslipidemia or diabetes under treatment. Linear models adjusted for age, sex, disease duration, phenotype and treatment were used to determine the impact of CV risk factors on MRI variables.

Results: Nineteen $\mathrm{HC}$ and $48 \mathrm{MS}$ patients had one traditional CV risk factor, $4 \mathrm{HC}$ and $15 \mathrm{MS}$ patients had more than one. Ten $\mathrm{HC}$ and $30 \mathrm{MS}$ patients had one stringent $\mathrm{CV}$ risk factor, 3 and 8 had more than one. Most of our subjects had a smoking history as a CV risk factor (16 HC and $42 \mathrm{MS}$ patients among traditional, $8 \mathrm{HC}$ and $23 \mathrm{MS}$ patients among stringent). In MS patients, the presence of at least two traditional CV risk factors was associated with reduced normalized grey matter volume (NGMV) ( $\mathrm{p}=0.01)$, white matter volume (NWMV) $(\mathrm{p}=0.03)$ and brain volume $(\mathrm{NBV})(\mathrm{p}=0.003)$, and not with T2-lesion volume $(\mathrm{T} 2-\mathrm{LV})$ $(\mathrm{p}=0.27)$. Among traditional CV risk factors, only hypertension $(\mathrm{n}=8)$ was associated with MRI measures (NWMV and NBV). In MS patients, the presence of one stringent $\mathrm{CV}$ risk factor was associated with reduced NGMV $(p=0.006)$, NWMV $(p=0.003)$ and NBV $(p<0.001)$, and higher $\mathrm{T} 2-\mathrm{LV}(\mathrm{p}=0.03)$. In HC, no differences were observed according to either traditional or stringent risk factor presence.

Discussion: The presence of $\mathrm{CV}$ risk factors is associated with brain atrophy in MS patients, even under age 50. CV risk factors seem to have synergistic effects, determining brain atrophy even for levels of exposure that may often be overlooked by clinicians, when present in combination.

Conclusions: The systematic assessment of $\mathrm{CV}$ risk factor presence is important in MS patients, even under age 50, because of their effect on brain atrophy.

Funding: Fondazione Italiana Sclerosi Multipla (FISM/2018/R/16).

\section{EFFECTS OF DIMETHYLFUMARATE ON INTESTINAL AND BLOOD BRAIN BARRIER PERMEABILITY}

M. C. Buscarinu ${ }^{1}$, E. Morena ${ }^{1}$, A. Fornasiero ${ }^{1}$, A. Landi ${ }^{1}$, L. Lionetto ${ }^{2}$, M. Capi ${ }^{2}$, R. Mechelli ${ }^{3}$, F. Gargano ${ }^{4}$ L. Battistini ${ }^{4}$, M. Simmaco ${ }^{1}$, C. Fagnani $^{5}$, M. Salvetti ${ }^{1}$, G. Ristori ${ }^{1}$

${ }^{1}$ Department of Neuroscience, Mental Health and Sensory Organs, Sapienza University (Roma); ${ }^{2}$ Mass Spectrometry Laboratory Unit, Sant'Andrea University Hospital (Roma); ${ }^{3}$ Department of Human Science and Promotion of Quality of Life, San Raffaele Roma Open University (Roma); ${ }^{4}$ Neuroimmunology Unit, Santa Lucia Foundation (Roma); ${ }^{5}$ Center of Reference for Behavioral Sciences and Mental Health, Istituto Superiore di Sanità (Roma)

Multiple sclerosis is characterized by progressive demyelination of axons of the central nervous system and neuronal cell degeneration. The pathogenic process is immune-mediated and the etiology is probably multifactorial, in which heritable and non-heritable factors interact. Among this multiplicity of factors, microbiota and gut function are increasingly recognized as relevant in autoimmune disorders. Several studies have recently shown that the microbiota, as a part of the intestine-brain axis, could play a role in the etiopathogenesis of multiple sclerosis. Dimethylfumarate (DMF) that has neuroprotective and antiinflammatory effects, determines gastrointestinal tract irritation, as side effect.

Objective: The objectives of the study was to evaluate the effects of DMF on intestinal barrier permeability, defining variables and biomarkers of safety, efficacy and tolerability trough the analysis of mucosal immune responses (studying the frequency of CD8 + CD161high T cells).

Materials: We included 30 patients with relapsing-remitting MS before and during DMF therapy.

Methods: From each individual, we obtained urine for intestinal permeability test, performed through the measurement of lactulose and mannitol levels in urine samples, after an oral load. At the same time PBMCs were stained with an antibody panel to quantify CD8 + CD161 high T cells. All subjects underwent clinical evaluation and gadolinium (Gd)enhanced MRI (brain and spinal cord). All these parameters were evaluated and compared at different time points: baseline (T0), 1 month (T1) and 9 months (T2) after DMF.

Results: Under DMF therapy two significant changes emerged: the reduction in the percentage of CD161+CD8+ T cells in 16 cases out 22 (73\%) at $\mathrm{T} 1$ and $\mathrm{T} 2$ as well as the expected decrease of diseases activity at MRI. About $60 \%$ of patients had altered intestinal permeability (IP) at time $\mathrm{T} 1$ and $\mathrm{T} 2$, while no significant changes of IP occurred during DMF treatment.

Discussion and Conclusions: Preliminary data confirmed our previous work on the existence of IP changes in a substantial proportion of MS patients. The frequency of CD161+CD $8+T$ cells decreased significantly after 9 months of DMF treatment.

References:

- J. Correale, M. I Gaitán, M. C Ysrraelit, M. P Fiol. Progressive multiple sclerosis: from pathogenic mechanisms to treatment. Brain (2017);140(3):527-46. doi: 10.1093/brain/aww258

- Horai R, Zárate-Bladés CR, Dillenburg-Pilla P, et al. MicrobiotaDependent Activation of an Autoreactive T Cell Receptor Provokes Autoimmunity in an Immunologically Privileged Site. Immunity (2015); 43:343-53

- Camara-Lemarroy, C. R. et al. The intestinal barrier in multiple sclerosis: Implications for pathophysiology and therapeutics. Brain (2018);141(7):1900-16. doi: 10.1093/brain/awy131

\section{APPLICATION OF DEEP-LEARNING TO NMOSD AND TO UNCLASSIFIED SERONEGATIVE PATIENTS}

L. Cacciaguerra ${ }^{1}$, L. Storelli ${ }^{1}$, M. Radaelli ${ }^{2}$, S. Mesaros ${ }^{3}$, L. Moiola ${ }^{2}$, J. Drulovic $^{3}$, M. Rocca ${ }^{4}$, M. Filippi ${ }^{4}$

${ }^{1}$ Neuroimaging Research Unit, Institute of Experimental Neurology, Division of Neuroscience, IRCCS San Raffaele Scientific Institute (Milano); ${ }^{2}$ Neurology Unit, IRCCS San Raffaele Scientific Institute (Milano); ${ }^{3}$ Clinic of Neurology, Faculty of Medicine, University of Belgrade (Belgrade-SRB); ${ }^{4}$ Neuroimaging Research Unit, Institute of Experimental Neurology, Division of Neuroscience; and Neurology Unit, IRCCS San Raffaele Scientific Institute (Milano) 
Aims: To provide a deep-learning classification of neuromyelitis optica spectrum disorders (NMOSD) patients with different serological profiles and to compare these results with their clinical evolution.

Materials: 228 T2- and T1-weighted brain MRIs were acquired from patients with aquaporin-4 (AQP4)-seropositive NMOSD ( $\mathrm{n}=85$ ), early multiple sclerosis (MS, $n=95)$, AQP4-seronegative NMOSD ( $n=11,3$ with anti-myelin oligodendrocyte glycoprotein antibodies) and unclassified double-seronegative limited phenotypes ( $\mathrm{n}=17$ idiopathic recurrent optic neuritis [IRON], $n=20$ idiopathic recurrent myelitis [IRM]). The latter had a clinical re-evaluation after 4-year follow-up.

Methods: The neural network architecture was based on four 3D convolutional layers. It was trained and validated on MRI scans $(\mathrm{n}=180)$ from AQP4-seropositive NMOSD and MS patients. Then, it was applied to AQP4-seronegative NMOSD and double-seronegative patients with limited phenotypes to evaluate their classification as NMOSD or MS in comparison with their clinical follow-up.

Results: The final algorithm discriminated between AQP4seropositive NMOSD and MS with an accuracy of 0.95 . Forty-seven $/ 48$ $(97.9 \%)$ seronegative patients were classified as NMOSD (one patient with IRON was classified as MS) and all AQP4-seronegative NMOSD patients were correctly classified. Clinical follow-up was available in 27/ $37(73 \%)$ double-seronegative limited phenotypes: one patient evolved to MS, three developed NMOSD and the others did not change phenotype.

Discussion: Current diagnostic criteria of NMOSD allow the diagnosis of AQP4-seropositive patients with limited manifestations, whereas seronegative patients with limited phenotypes remain unclassified and are usually considered as prodromal phases of MS. According to our clinical and deep-learning results, these patients usually do not evolve to MS and their brain MRI is similar to that of NMOSD patients.

Conclusions: Deep-learning may help in the diagnostic work-up of NMOSD, especially in AQP4-seronegative patients. Our findings suggest the enlargement of the NMO spectrum to double-seronegative limited phenotypes.

\section{CLINICAL ACTIVITY AFTER FINGOLIMOD TREATMENT IN NAÏVE RRMS PATIENTS COMPARED TO PATIENTS PREVIOUSLY TREATED WITH OTHER DRUGS}

M. Cannizzaro, L. Ferrè, F. Sangalli, B. Colombo, L. Moiola, V. Martinelli, M. Filippi, F. Esposito

Neurology Unit, Department of Neurology, IRCCS San Raffaele Scientific Institute (Milano)

Objectives: Fingolimod (FTY) is a highly effective drug, with a significant proportion of subjects showing no evidence of disease activity (NEDA) at 2years follow-up. However, it is not known whether the previous treatment history may influence clinical outcomes under fingolimod. The aim of the present study is to assess NEDA status in an Italian cohort of relapsing remitting (RR) MS patients treated with FTY, comparing naïve patients with patients previously treated with first-line and second-line drugs.

Materials: 543 RRMS patients, who started fingolimod treatment at San Raffaele Hospital (Milan) between 2011 and 2018 were enrolled in the study. Patients were divided in three categories, according to the previous treatment: naïve patients (group $1, n=74$ ), patients previously treated with first-line drugs (group 2, n=315) and patients previously treated with second-line and immunosuppressive drugs (group 3, n=146).

Methods: NEDA status was assessed at two-year follow-up. Baseline characteristics were investigated in association with NEDA status, using logistic regression in univariable and multivariable models.
Results: Overall, $45.6 \%$ patients were NEDA $(n=227)$ at 2-year follow-up; specifically $62.1 \%$ patients belonging to group $1,47.4 \%$ to group 2 and $33.6 \%$ to group 3. In the multivariable analysis we observed that patients treated with a first-line and second-line therapy had an increased risk of being $\operatorname{EDA}(\mathrm{OR}=2.2, \mathrm{p}=0.036$ and $\mathrm{OR}=3.9, \mathrm{p}<0.001$ respectively $)$ compared to naïve patients. Moreover, a higher number of new/active MRI lesions (OR, 1.2, $\mathrm{p}=0.004)$, a higher annualized relapse rate in the 2 years before treatment start $(\mathrm{OR}=1.3, \mathrm{p}=0.03)$ and a younger age $(\mathrm{OR}=0.97, \mathrm{p}=0.01)$ at onset were independently associated with increased risk of being EDA.

Discussion: We confirmed FTY effectiveness in a large monocentric cohort of Italian RRMS patients. Moreover, patients treated with FTY as first drug had a higher probability of being NEDA after 2 years compared to patients previously treated with second-line but also first-line drugs.

Conclusions: Our data suggest an early use of FTY in naïve patients with high inflammatory activity, considering the better clinical outcomes observed at short-term follow-up.

References:

- Esposito, F., Ferrè, L., Clarelli, F. et al. Effectiveness and baseline factors associated to fingolimod response in a real-world study on multiple sclerosis patients. J Neurol (2018);265:896-905

- Comi, G., Pozzilli, C., Morra, V.B. et al. Effectiveness of fingolimod in real-world relapsing-remitting multiple sclerosis Italian patients: the GENIUS study. Neurol Sci (2020) Apr 21. doi: 10.1007/s10072020-04380-y

\section{PSYCHOLOGICAL CONSEQUENCES OF ITALIAN LOCKDOWN IN MS PATIENTS DURING COVID-19: BETTER THAN EXPECTED}

R. Capuano ${ }^{1}$, M. Altieri ${ }^{1}$, A. Bisecco ${ }^{1}$, A. d'Ambrosio ${ }^{1}$, R. Docimo ${ }^{1}$, D. Buonanno $^{1}$, F. Matrone ${ }^{1}$, F. Giuliano ${ }^{1}$, G. Tedeschi ${ }^{1}$, G. Santangelo ${ }^{2}$, A. Gallo ${ }^{1}$

${ }^{1}$ Department of Advanced Medical and Surgical Sciences, " DAMSS", University of Campania Luigi Vanvitelli (Napoli); ${ }^{2}$ Department of Psychology, University of Campania Luigi Vanvitelli (Napoli)

Objectives: Italy was strongly hit by COVID-19 pandemic, therefore the Italian Government decreed urgent measures promoting social distancing to limit the spread of the virus. In fact, since March 11th, all not indispensable work, social, sporting, retail and recreational activities were suspended or, where possible, converted to the so-called smart-working. Fear of getting sick from COVID-19, government s lockdown and the imposed social distancing might have an impact on anxiety, depression and quality of life (QoL) in people with Multiple Sclerosis (pwMS). Our study aimed to investigate anxiety, depression, and QoL changes in pwMS during SARSCoV-2 outbreak and lockdown in Italy.

Material and Methods: Sixty-seven pwMS with a previous (less than 6 months) neuropsychological evaluation before SARSCoV-2 outbreak (T0) were re-evaluated at the time of the outbreak and lockdown in Italy (T1). They underwent a clinical and neurological evaluation (at T0) and completed the State-Trait Anxiety Inventory (STAI-Y1), the Beck Depression Inventory second edition (BDI-II), and Multiple Sclerosis Quality of Life-54 (MsQoL-54) at T0 and T1. Bonferroni correction for multiple comparisons was applied.

Results: BDI-II and STAI-Y1 scores did not change between T0 and T1, whereas the satisfaction on the sexual function subscale of MsQoL-54 was significantly higher at $\mathrm{T} 1(\mathrm{p}<0.001)$. 
Discussion and Conclusions: Despite the tight Italian lockdown due to the COVID-19 pandemic and the fear of getting sick, we did not observe a relevant negative impact on anxiety, depression and QoL of our sample of pwMS. Contrariwise, we were even able to detect some positive effects on specific aspects of QoL, such as sexual satisfaction.

\section{COMPLICATIONS AFTER LUMBAR PUNCTURE: A PRELIMINARY COMPARATIVE ANALYSIS ON THE USE OF ATRAUMATIC VS STANDARD NEEDLE}

D. Carmagnini, L. Lorefice, G. Fenu, J. Frau, G. Coghe, M. Fronza, S. Barca, E. Cocco

Multiple Sclerosis Center, University of Cagliari (Cagliari)

Background: Atraumatic needles have been proposed to reduce complication rates after lumbar puncture (LP) [1]. The study aimed to evaluate the frequency of post procedural headache, low back pain and other complications in a cohort of multiple sclerosis (MS) patients who underwent LP by using atraumatic or standard needle.

Materials and methods: The study included patients who underwent the procedure of LP. Demographic (gender, age, BMI) and clinical features (disease duration) were collected for each patient. In addition, information on chronic headache and its treatment were also recorded. For each patient, it was indicated whether the LP was performed with the use of standard or atraumatic needle. Then, the occurrence of postprocedural complications (headache, low back pain and nausea) and the possible relationships with the type of needle used was investigated.

Results: The study included 100 patients (28\% male; mean age 42.3). $21 \%$ of these had a history of chronic headache with use of medications for $5 \%$. Regression analysis showed that lower body mass index (p 0.032) and younger age ( $p$ 0.002) were associated with the use of atraumatic needles, while no association was reported with gender. A lower frequency of postprocedural headache (31\% vs $50.7 \%$ ) and low back pain (34.5\% vs $40.8 \%$ ) were reported respectively by using atraumatic vs standard needles $(\mathrm{p}<0.05)$. Multivariate analyses showed that post-procedural headache, with a tendency toward statistical significance $(\mathrm{p}=0.058)$, but not low back pain and nausea were associated to the use of standard needles after controlling for other demographic variables. Finally, an association between lower back pain and female gender $(\mathrm{p}=0.018)$, and between nausea and lower BMI $(\mathrm{p}=0.032)$ were also reported.

Conclusions: Our data seem to suggest the usefulness of the atraumatic needle for PL to prevent post procedural headache. Further investigations into larger cohorts are needed.

Reference:

1. Nath S, Koziarz A, Badhiwala JH, et al. Atraumatic versus conventional lumbar puncture needles: a systematic review and meta-analysis. Lancet. (2018);391(10126):1197-1204. doi:10.1016/S01406736(17)32451-0

\section{SEXUAL DYSFUNCTION PREVALENCE IN MULTIPLE SCLEROSIS PATIENTS: VALIDATION OF THE ITALIAN VERSION OF THE MULTIPLE SCLEROSIS INTIMACY AND SEXUALITY QUESTIONNAIRE-19}

A. Carotenuto ${ }^{1}$, L. Del Giglio ${ }^{2}$, A. Chiodi ${ }^{1}$, M. Petracca ${ }^{1}$, L. Rosa ${ }^{1}$, M. Bianchi $^{1}$, I. Ferrante ${ }^{3}$, F. Lauro ${ }^{1}$, M. Moccia ${ }^{1}$, A. Ianniello ${ }^{3}$, C. Pozzilli ${ }^{3}$, V. Brescia Morra ${ }^{1}$, R. Lanzillo ${ }^{1}$
${ }^{1}$ Department of Neurosciences, Reproductive and Odontostomatological Sciences, 'Federico II' University (Napoli); ${ }^{2}$ Neurological Unit, 'S. Filippo' Hospital (Roma); ${ }^{3}$ Multiple Sclerosis Center, 'S. Andrea' Hospital (Roma)

Aim: People with Multiple sclerosis (MS) may experience sexual dysfunction throughout the disease course. Prevalence for sexual dysfunction in MS ranges from 70 to $90 \%$, higher than in healthy controls (about $50 \%$ ) [1]. Clinicians do not always ask for sexual dysfunction both for the limited timeframe for clinical assessment and the intimate nature of the subject. Hence, the use of self-reported questionnaires would be useful in clinical practice. However, validated scales to assess sexual dysfunction in MS for Italian patients are lacking. Hence, we aimed at validating Multiple Sclerosis Intimacy and Sexuality Questionnaire (MSISQ-19) for Italian MS patients.

Methods: We included both male and female MS patients. Each patient completed the Italian translation of the MSISQ-19, the Beck Depression Inventory (BDI-II), the Modified Fatigue Impact Scale (MFIS), the StateTrait Anxiety Inventory (STAI-Y) questionnaire, the International Consultation on Incontinence Questionnaire Lower Urinary Tract Symptoms Quality of Life Module (ICIQ-LUTSqol), the Female Sexual Function Index (FSFI, for female) and the International Index of Erectile Function (IIEF for male). Construct validity for the Italian version of the MSISQ-19 was explored by the exploratory factor analysis and the Cronbach's alpha coefficient. Test-retest stability and concurrent internal and external validity was examined by Pearson' correlation coefficients.

Results: We enrolled 369 MS patients (323 female). Mean MSISQ-19 total score was $37.2 \pm 15.2$ (range 18 - 89) with a sexual dysfunction prevalence of $59 \%$ in male MS patients and $41 \%$ in female MS patients. Cronbach's alpha was 0.92 for the MSISQ-19. MSISQ-19 test and retest total scores correlated between each other $(\mathrm{r}=0.48, \mathrm{p}=0.01)$. MSISQ-19 total score also correlated with primary, secondary and tertiary subscales $(\mathrm{p}<0.001)$, with the EDSS $(r=0.19, \mathrm{p}<0.001)$ and all other neuropsychological scales.

Discussion: We reported here for the first time that the Italian translation of the MSISQ-19 shows a three-subscales-structure as for the English original version, confirming its construct internal validity [2]. We also showed a correlation between MSISQ-19 and EDSS. As EDSS is a measure of physical disability this association is not surprising. Finally, we reported a strong association between MSISQ-19 and FSFI/IIES, confirming the external validity of the Italian MSISQ-19 questionnaire for MS patients.

Conclusion: The Italian Version of the MSISQ-19 showed satisfactory internal consistency and reliability with moderately adequate test-retest reproducibility, suggesting that the Italian MSISQ-19 questionnaire provides a valuable measure of sexual dysfunction in the Italian population. References:

1. Zorzon M, Zivadinov R, Bosco A, Bragadin LM, Moretti R, Bonfigli L, et al. Sexual dysfunction in multiple sclerosis: a casecontrol study. I. Frequency and comparison of groups. Mult Scler. (1999);5(6):418-27

2. Sanders AS, Foley FW, LaRocca NG, Zemon V. The Multiple Sclerosis Intimacy and Sexuality Questionnaire-19 (MSISQ-19). Sexuality and Disability (2000);18(1):3-26

\section{SEROPREVALENCE AND SEROCONVERSION OF JCV ANTIBODIES IN MS PATIENTS TREATED WITH NATALIZUMAB}

E. Casaglia, L. Lorefice, G. Fenu, D. Locci, M. Fronza, J. Frau, G. Coghe, E. Cocco 
Multiple Sclerosis Center, Binaghi Hospital, ATS Sardegna (Cagliari)

Background: Anti-John Cunningham virus (JCV) serology is a key component of the risk stratification algorithm of progressive multifocal leukoencephalopathy (PML) in Natalizumab (N) treated MS patients, together with previous immunosuppressants (IS) use and duration of $\mathrm{N}$ exposure.

Objective: To assess the JCV antibodies seroprevalence and the rates with timing of seroconversion in MS patients treated with N; to examine the possible relationship with demographics and MS clinical features as well as on the choice to continue N.

Methods: Retrospective analysis was conducted using records of MS patients with multiple JCV antibody determinations exposed to $\mathrm{N}$ for at least 4 years. Chi-square and independent t-tests were used to compare demographics and MS features on bases of JCV status. Rates of seroconversion over time, the association with previous IS exposure and the relationship of JCV seropositivity with $\mathrm{N}$ discontinuation were evaluated by multiple regression analyses.

Results: The study included 221 MS patients, of which $116(52.5 \%)$ were JCV seropositive. An association of JCV seropositivity with age (p value $=0.005)$, disease duration ( $\mathrm{p}$ value $=0.05)$ and $\mathrm{N}$ duration ( $\mathrm{p}$ value $=0.005$ ) were reported. 22 of the $105 \mathrm{JCV}$ seronegative patients converted over time, of these $20(90 \%)$ in the first 3 years of $\mathrm{N}$ exposure. Multiple regression analysis showed an association of JCV sieroconversion with previous IS exposure ( $\mathrm{p}$ value $=0.024$ ), independently from demographic and MS characteristics. Finally, a relationship of $\mathrm{N}$ discontinuation with baseline JCV seropositivity $(\mathrm{p}$ value $=0.001)$ and JCV seroconversion $(\mathrm{p}$ value $=0.001)$ over time was reported

Conclusions: Results suggest that previous IS exposure, important determinant for PML risk stratification in $\mathrm{N}$ treated patients, can also influence the JCV seroconversion over time. In addition, both baseline JCV status and JCV seroconversion seem to be crucial determinants influencing the choice of $\mathrm{N}$ treatment.

References:

- Schwab, N. et al. Anti-JCV serology during natalizumab treatment: Review and meta-analysis of 17 independent patient cohorts analyzing anti-John Cunningham polyoma virus sero- conversion rates under natalizumab treatment and differences between technical and biological se. Mult Scler (2018) Apr;24(5):563-73

- Major, E. O., Yousry, T. A. \& Clifford, D. B. Pathogenesis of progressive multifocal leukoencephalopathy and risks associated with treatments for multiple sclerosis: a decade of lessons learned. Lancet Neurol. (2018);17:467-80

\section{OCRELIZUMAB TREATMENT IN PATIENTS WITH PROGRESSIVE MULTIPLE SCLEROSIS: A SINGLE-CENTER REAL-WORLD EXPERIENCE}

M. Cellerino ${ }^{1}$, G. Boffa ${ }^{1}$, C. Lapucci ${ }^{2}$, E. Sbragia ${ }^{1}$, N. Bruschi ${ }^{1}$, E.

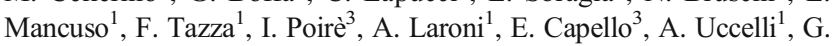
$\mathrm{Novi}^{3}$, M. Inglese ${ }^{1}$

${ }^{1}$ Department of Neuroscience, Rehabilitation, Ophthalmology, Genetics, and Mother-Child Health (DINOGMI), University of Genoa (Genova); ${ }^{2}$ Experimental Neurosciences Laboratory, Ospedale Policlinico San Martino IRCCS (Genoa); ${ }^{3}$ Department of Neurology, Ospedale Policlinico San Martino IRCCS (Genova)

Background: Ocrelizumab (OCR) treatment in pivotal trials of patients (pts) with progressive multiple sclerosis (PMS) has demonstrated to slow disability worsening, with a good safety profile. However, real-word data on efficacy and adverse events (AE) are still scarce.

Objectives: To provide first experience on PMS pts treated with OCR in a real-world setting.

Methods: We collected safety and efficacy data from all PMS pts treated with OCR at the MS Center of the University of Genoa. The probability of disability-, relapse- and MRI activity-free survival and NEDA-3 status was calculated with Kaplan-Meier estimator and Cox proportional hazards regression analysis. AE were recorded throughout the follow-up (FU).

Results: We recorded data from 59 PMS pts [42 (71\%) with primaryprogressive (PP) MS and 17 (29\%) with secondary progressive (SP) MS, 24 females (41), mean (SD) age 49.8 (8.2) years] with a mean disease duration (DD) of 12.1 (10.1) years, a median (IQR) baseline EDSS of 5.5 (3.5-6.0) and median number of previous DMTs 1 (0-2). SPMS patients had longer DD (20.8vs8.6; $\mathrm{p}=0.004)$ and had mean ARR of 0.24 (0.4). 21 $(36 \%)$ pts had not received any DMT prior to OCR. Mean FU was 2.0 (1.1) years. 14 (24\%) patients had an active MRI brain scan at baseline. At 1-year FU, MRI-inflammatory-activity-free survival was $87.3 \%$ (CI95\%: 76.9-97.7\%), relapse-free survival was $100 \%$ and progression-free survival was $82.7 \%$ (72.3-93.1\%). NEDA-3 status was achieved in $72.3 \%$ (59.0-85.5\%) of pts. No differences were noted between patients with PP and SPMS. At multivariate analyses, no baseline characteristic was found be predictive of a higher probability of progression-free survival, MRIactivity-free survival and NEDA-3 status. We recorded 69 AE in 36 pts (32 upper respiratory tract infections; 6 herpes simplex-1 reactivation; 7 lower urinary tract infections; 1 acute myeloid leukemia following myelodysplastic syndrome; 1 appendicitis treated with surgical procedure). No serious infusion-associated reactions were reported.

Discussion: We report short-medium term efficacy data in a realworld population of progressive patients treated with OCR, including a relatively high proportion of patients without MRI activity at baseline assessment. Our data suggest that OCR should be considered as treatment option in both patients with PPMS and SPMS.

Reference:

- Montalban X, Hauser SL, Kappos L, et al. Ocrelizumab versus Placebo in Primary Progressive Multiple Sclerosis. N Engl J Med. (2017);376(3):209-20. doi:10.1056/NEJMoa1606468

\section{CONCOMITANT ONSET OF MULTIPLE SCLEROSIS AND CELIAC DISEASE: ASSOCIATION OR NEW DISEASE ENTITY?}

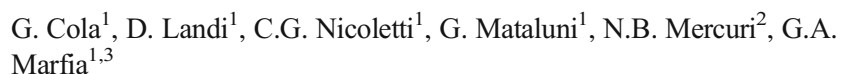

${ }^{1}$ Multiple Sclerosis Clinical and Research Unit, Department of Systems Medicine, Tor Vergata University (Roma); ${ }^{2}$ Neurology Unit, Department of Systems Medicine, Tor Vergata University (Roma); ${ }^{3}$ Unit of Neurology and Neurorehabilitation, IRCCS Neuromed (Pozzilli-IS)

Objective: Association between celiac disease (CD) and multiple sclerosis (MS) is uncommon, although CD and MS share some immunological mechanisms and are both considered T-cell mediated diseases. However, reported MS prevalence in CD population is low and CD prevalence in MS is even lower than in general population. Screening for $\mathrm{CD}$ is not part of the standard autoimmune screening in patients suspected for having MS as well as central nervous system MRI in celiac patients. We report clinical and MRI characteristics of two young female patients receving simultaneous diagnosis of MS and CD. 
Materials, methods and results: Both patients came to our attention for newly onset myelitis with EDSS 3.0 and 3.5 at onset. Both presented high annualised relapse rate evaluated retrospectively or prospectively. Diagnostic MRI unveiled multiple demyelinating enhancing lesions in the brain and extensive involvement of the spinal cord and cerebrospinal fluid was positive for oligoclonal bands, therefore RRMS was diagnosed. None of them referred gastrointestinal symptoms or malabsorbtion at that time or before. Family history was relevant for gluten sensitivity in one patient and unremarkable in the other. Autoimmune screening showed only a significant titre of CD antibodies (Anti tissue-Translutaminase $\operatorname{IgA}$, anti Deamidated Gliadin peptide $\operatorname{IgG}$ and $\mathrm{IgA}$, anti-endomysium $\operatorname{IgG})$; it was otherwise negative. Genetic screening demostrated HLADQ2 heterodimer. Duodenal biopsy confirmed CD diagnosis and gluten free diet was initiated. Screening for anti Acquaporin-4 and anti-MOG antibodies was negative.

Discussion: These cases add knowdledge about the concomitant occurrence of MS and CD, which is not strongly evinced in literature. An interesting question is whether such association is accidental in persons with genetic susceptibility or they are both part of the spectrum of the same illness. We cannot exclude that anti-gluten reaction triggers MS or that demyelinating lesions represent extra-intestinal manifestation of CD due to antigen cross-reactivity with CNS proteins or against celiac related CNS antigen (i.e. tranglutaminase 6). Additionally, if concomitant autoimmunity may amplify inflammatory cascade determining a more severe MS phenotype needs to be clarified.

Conclusions: Screening for celiac disease should be implemented in newly diagnosed MS patients, as part of routine autoimmune screening. This is relevant to better understanding pathogenesis and triggers of demyelinating disorder and to estimate patients prognosis.

References:

- Thomsen HL, Jessen EB, Passali M, Frederiksen JL. The role of gluten in multiple sclerosis: A systematic review. Mult Scler Relat Disord. (2019);27:156-63. doi:10.1016/j.msard.2018.10.019

- Piccini B, Ulivelli M, Amato MP, et al. Association of celiac disease in patients with multiple sclerosis in Tuscany. Rev Esp Enferm Dig. (2020);112(6):474-76. doi:10.17235/reed.2020.6123/2018

\section{MULTIPLE SCLEROSIS (MS) DURING COVID-19 PANDEMIC: EXPERIENCE OF BRESCIA MS CENTRE IN LOMBARDY, ITALY}

C. Cordioli ${ }^{1}$, L. Carmisciano ${ }^{2}$, C. Mancinelli ${ }^{1}$, S. Rasia $^{1}$, N. De Rossi ${ }^{1}$, R. Capra $^{1}$

${ }^{1}$ Multiple Sclerosis Center, ASST Spedali Civili of Brescia (Brescia); ${ }^{2}$ Dep of Health Sciences, University of Genoa (Genova)

Objectives: Lombardy is the Italian region most affected by SARS-CoV2 epidemic; cities of Milan, Bergamo, Brescia show the higher incidence. The MS Centre of Brescia, territory-based with high affiliation rate, takes care of $2300 \mathrm{MS}$ patients. We evaluate the rate of infection, clinical course and outcome in our MS patients.

Material/Methods: Since march 1st 2020 MS patient's report (by phone call, e-mail, hospital visit) of confirmed or suspected symptomatic Covid-19 occurring since February 2nd 2020 until june 13th 2020, were collected. Clinical features, disease duration and outcome were registered. We investigated the association between age, gender, and time to recovery with a Cox model and between age, gender, comorbidities, symptoms and severity using a composite time-dependent outcome (hospitalization, ICU admission, pneumonia or death).
Results: We collected 103 symptomatic patients ( $F / M=75 / 28$, mean age 45,8 years; 96 RRMS, 3 SPMS, 4 PPMS), 93 in immunomodulatory treatment ( $9 \%$ of all the 1036 treated patients of our Center), 10 untreated. Thirty-nine suspected cases underwent SARS-CoV-2 RNA-PCR oropharyngeal/nasopharyngeal swab (ONS), 16 of them (15.5\%) had at least one positive. Interferon, Glatiramer Acetate, Teriflunomide, and Fingolimod prevalence were slightly lower than expected $(12,6 \%, 9,7$ $\%, 9,7 \%, 6,8 \%$ respectively); the prevalence of Natalizumab, Dimethyl fumarate and Ocrelizumab appeared higher than the expected $(11,7 \%$, $25,2 \%, 14,6 \%$ respectively). After a median period of observation of 45 days per patient $(\mathrm{IQR}=22-66)$ we observed: $11(10.7 \%)$ hospitalizations, $21(20.4 \%)$ pneumonias, 2 (1.9\%) ICU admissions and 3 (2.9\%) deaths. At last follow-up 97 (91.3\%) patients were recovering or completely recovered. COVID-19 symptoms duration was at least 45 days $(95 \% \mathrm{CI}$ $=37,58)$ in half of the patients. A non-significative trend of worst COVID-19 progression (hospitalization, ICU admission, pneumonia or death) was observed in higher aged patients and in presence of comorbidities (doubling of the risk for each additional comorbidity; $\mathrm{HR}=2.32$; 95\% CI 1.21, 4.45; $\mathrm{p}=0.011$ ).

Discussion/conclusion: The majority of our symptomatic MS patients showed a mild infective disease and recovered. The presence and number of comorbidities is related with a worse outcome. The duration of the symptomatic phase is not influenced by age or gender. The course of infection did not show progression to the stage III (hyperinflammation phase) in none of the patients. The low rate of ONS performed in our patients is a point of uncertainty since it hampers the estimation of real prevalence of SARS-CoV-2.

\section{References:}

- M. P. Sormani on behalf of the Italian Study Group on COVID-19 infection in multiple sclerosis An Italian programme for COVID-19 infection in multiple sclerosis. (2020) Jun;19(6):481-82

- Hasan K. Siddiqi MD, MSCR , Mandeep R. Mehra MD COVID-19 Illness in Native and Immunosuppressed States: A ClinicalTherapeutic Staging Proposal., MSc. PII: S1053-2498(20)31473X. DOI: https://doi.org/10.1016/j.healun.2020.03.012

- Multiple Sclerosis International Federation. Global Covid-19 advice for people with MS. 09 jul 2020 https://www.msif.org/news/2020/ 02/10/the-coronavirus-and-ms-what-you-need-to-know/

\section{EVOLUTION OF NATALIZUMAB USE IN TWO ITALIAN MULTIPLE SCLEROSIS CENTERS}

P. A. Cortesi ${ }^{1}$, P. Cozzolino ${ }^{2}$, R. Capra $^{3}$, L. Santoni ${ }^{4}$, S. Masera ${ }^{4}$, F. Patti $^{5}$, L. Montovani ${ }^{2}$

${ }^{1}$ Research Centre on Public Health (CESP), University of Milan-Biccoca (Monza); ${ }^{2}$ IRCCS Multimedica (Sesto San Giovanni-MI); ${ }^{3}$ Multiple Sclerosis Centre, Spedali Civili di Brescia (Montichiari-BS); ${ }^{4}$ Biogen Italy (Milano); ${ }^{5}$ Department of Medical, Surgical Science and Advanced Technology “GF Ingrassia”, University of Catania (Catania)

Objective: Natalizumab is an effective treatment for relapsing remitting multiple sclerosis (RRMS). The aim of this study was to characterize evolution of natalizumab utilization between 2012 and 2016 exploring patients' characteristics and yearly infusions number.

Materials: A retrospective observational cohort study was conducted using two clinical registries from two large MS centers, one in North and one in South of Italy. 
Methods: All patients with RRMS and a first prescription of natalizumab between 2012-2016 were included in the study and followed-up until last visit at the MS center or December 2017. Natalizumab use was described with regards to clinical and demographic characteristics of patients in treatment and yearly infusions number.

Results: A total of 324 patients ( $72.5 \%$ female) starting natalizumab treatment between 2012-2016 were identified. The mean (SD) age at MS diagnosis and first natalizumab prescription was 33.5 (10.7) and 38.0 (10.9) respectively. Thirty-six patients reported a first prescription in 2012; from 2013 to 2016, we observed about 70 new patients each year. Within all new treated patients with Natalizumab, the percentage of naive patients increased from $17 \%$ in 2012 to $35 \%$ in 2016 (overall mean 29\%); moreover, $27.8 \%$ of patients reported an EDSS less or equal than 2, at last visit with EDSS available, before started natalizumab in 2012 and this number increased to $61.8 \%$ in 2016 . The mean (range) natalizumab yearly infusions number decreased from 13.7 (11.6-13.8) in 2012 to 9.8 (8.010.2) infusions in 2016 (overall mean 10.7 [9.7-11.3]). In the first year of treatment, natalizumab patients received mean 12.6 infusions. While, in patients with at least one year of treatment, the mean dosing decreased from 11.3 infusions in 2013 to 8.8 infusions in 2016.

Discussion: The study results support an evolution in natalizumab use in clinical practice, suggesting an earlier use of natalizumab in the disease course and a reduction of yearly infusions number since 2012 to 2016.

Conclusion: This data suggests a reduction of natalizumab consumption and treatment patients cost and must be taken into account for assessing the natalizumab value in the real world.

\section{THE DISABILITY PROGRESSION OF MULTIPLE SCLEROSIS PROGRESSIVE COURSE: A MARKOV MODEL APPROACH}

\author{
P. A. Cortesi ${ }^{1}$, C. Fornari ${ }^{1}$, R. Capra ${ }^{2}$, P. Cozzolino ${ }^{3}$, F. Patti ${ }^{4}$, L. \\ Mantovani ${ }^{3}$ \\ ${ }^{1}$ Research Centre on Public Health (CESP), University of Milan-Biccoca \\ (Monza); ${ }^{2}$ Multiple Sclerosis Centre, Spedali Civili di Brescia \\ (Montichiari-BS); ${ }^{3}$ IRCCS Multimedica (Sesto San Giovanni-MI); \\ ${ }^{4}$ Department of Medical, Surgical Science and Advanced Technology \\ "GF Ingrassia", University of Catania (Catania)
}

Objectives: Improving the understanding of multiple sclerosis (MS) mechanism and disability progression over time is essential to evaluate healthcare interventions. In detail, assessment of annual changes of patients Expanded Disability Status Scale (EDSS) level is necessary to project effectiveness and cost of clinical interventions. This data is widely available for relapsing remitting MS, while poor or no data are available for progressive courses. This study aims to fill this gap.

Materials: This is an observational study of a cohort of primary (PPMS) and secondary progressive (SPMS) MS patients. Data from the disease registries of two qualified Italian MS centers were used to select and follow patients over-time. The disease registries collect demographic, treatment and clinical data at each visit performed in the centers from 1983.

Methods: PPMS and SPMS patients with EDSS between 3.0 and 7.0 at first visit were enrolled. Patients were followed until last visit registered in the databases or February 2018. Patients' characteristics and disability progression were firstly analysed. Cox proportional hazard models were used to identify clinical characteristic associated to disability progression. Finally, annual transition probabilities among EDSS states 3.0 to 9.0 were estimated using continuous Markov models for PPMS and SPMS separately.
Results: The final study cohort included 758 patients of which 274 (36\%) with PPMS and 484 (64\%) with SPMS. Males accounted for the $45 \%$ of the cohort, mean age at first visit was 49 years for PPMS and 46 for SPMS patients. Median follow-up was 8.2 years. Relapse rate and EDSS level at first visit significantly affected disability progression in both disease courses. Disease-modifying treatment in the observational period was also significantly associated to disability progression in SPMS patients. Finally, annual transition probability matrix of SPMS patients reported a higher annual probability to move within EDSS levels than the PPMS matrix. Goodness of fit was acceptable when comparing predicted and observed EDSS profiles.

Discussion: This study provides a detailed description of disability progression and EDSS annual changes for PPMS and SPMS patients, using a rich database, with full access to original real-time disability assessment. These estimates are based on advanced statistical models, which allow us to include all valid disability assessments, regardless of their exact timing.

Conclusions: Transition EDSS probabilities estimates for progressive MS could be a useful tool for healthcare decision makers and clinicians to properly assess the impact of interventions on disability progression in order to select the most valuable ones.

\section{REWORD-MS: REAL WORLD STUDY TO ASSESS THE RESPONSE TO DIMETHYL-FUMARATE IN PATIENTS WITH MULTIPLE SCLEROSIS}

M. F. Creta ${ }^{1}$, I. Tramacere ${ }^{2}$, E. Tomas Roldan ${ }^{1}$, P. Confalonieri ${ }^{1}$, V. Torri Clerici $^{1}$, C. Antozzi ${ }^{1}$, R. Mantegazza ${ }^{1}$, A. Tozzo ${ }^{3}$, L. Brambilla ${ }^{1}$

${ }^{1}$ MS Center, Neuroimmunology and Neuromuscular Diseases Unit, IRCCS Foundation Neurological Institute Carlo Besta (Milano); ${ }^{2}$ Department of Research and Clinical Development, Scientific Directorate, IRCCS Foundation Neurological Institute Carlo Besta (Milano); ${ }^{3}$ Department of Pediatric Neuroscience, IRCCS Foundation Neurological Institute Carlo Besta (Milano)

Objectives: The response of relapsing-remitting Multiple Sclerosis patients (RRMSp) to disease-modifying drugs (DMDs) is heterogeneous. Tools able to predict response at the beginning of therapy are not available, but early treatment optimization is crucial to prevent disability accumulation. Scoring systems based on combination of relapse rate and new/enlarged T2 lesions (NT2) during the first year of treatment, as the MAGNIMS (Magnetic Resonance Imaging in MS) score, have been validated in RRMSp treated with interferon, to predict the individual response over time. Dimethyl-fumarate (DMF) is nowadays the leading treatment prescribed among first line DMDs. The aim of the study is to evaluate the validity of the MAGNIMS score in predicting therapeutic failure in RRMSp treated with DMF.

Materials: Data about clinical relapses and NT2 were collected from 195 RRMSp treated with DMF for at least 12 months and with $\geq 24$ months of follow-up. Methods: The MAGNIMS score was applied at month 12. Therapeutic failure was defined as EDSS (Expanded Disability Status Scale) worsening or change of therapy for inefficacy and loss of NEDA-3 status (No Evidence of Disease Activity: no relapse, no EDSS worsening, no NT2). The association between score and risk of treatment failure was evaluated by Cox proportional hazard model.

Results: At month 12, 175/195 (89.7\%) patients had score=0 (no clinical relapse and $<3$ NT2) and 20/195 (10.3\%) had score $\geq 1$ ( $\geq 1$ clinical relapse and/or $\geq 3$ NT2). Patients with score $\geq 1$ had a four times greater risk of EDSS worsening or change of therapy compared with patients 
with score $=0$ (HR 3.89, 95\% CI 1.80-8.43; $\mathrm{p}=0.001)$. Similarly, risk of losing NEDA-3 status was two times greater in patients scored $\geq 1$ (HR $2.19,95 \%$ CI 1.07-4.48; $\mathrm{p}=0.031$ ). Considering RRMSp treated with DMF as first therapy (naïve, 91), patients with score $\geq 1$ had a six times greater risk of EDSS worsening or change of therapy (HR 5.86, 95\% CI 2.03-16.92; $\mathrm{p}=0.001)$ and a two and a half times greater risk of losing NEDA-3 (HR 2.48, 95\% CI $1.01-6.11 ; \mathrm{p}=0.048$ ).

Discussion: Our data show that the MAGNIMS score is a reliable tool to predict response to DMF in a real-world setting, even in naïve patients. Early identification of non-responders is crucial to identify candidates for a therapeutic shift to prevent disease progression.

Conclusions: Due to the complexity of MS, future studies are needed to assess whether the addiction of other biomarkers (i.e. neurofilaments and brain atrophy) will improve the predictive power of clinicalradiological scores.

\section{LONGITUDINAL EVOLUTION OF CORTICAL LESIONS IN A COHORT OF MULTIPLE SCLEROSIS PATIENTS}

E. Curti $^{1}$, S. Graziuso ${ }^{2}$, V. Bazzurri ${ }^{1}$, A. Fiore ${ }^{1}$, C. Ganazzoli ${ }^{2}$, G.

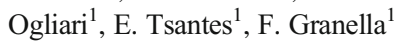

${ }^{1}$ Department of Medicine and Surgery, University of Parma (Parma); ${ }^{2}$ Department of Diagnostic, Neuroradiology Unit, Parma University Hospital (Parma)

Background and objectives: Cortical lesions (CLs) have recently acquired a great relevance in multiple sclerosis (MS), both at diagnosis and during the monitoring of the disease because of their impact on long-term prognosis. However, there is still limited knowledge about the evolution of CLs. The aim of the present observational study was to investigate, retrospectively, the evolution of CLs number in comparison to FLAIR-T2 hyperintense white matter lesions (WMLs) in a cohort of MS patients in a single MS centre.

Materials and methods: We included all consecutive patients with relapse-onset MS referred to MS centre of Parma who performed at least two MRI scans including double inversion recovery sequences from 2014 to 2019, collecting demographic, clinical and MRI data.

Results: We included 140 MS patients, $67.9 \%$ female. At first MRI they had a relapsing-remitting (RR) course in $84.3 \%$ and secondaryprogressive (SP) in $15.7 \%$ of cases, mean age $40.1 \pm 10.49$ years, mean disease duration $169.7 \pm 100.75$ months, mean EDSS $2.5 \pm 1.30$, mean number of WMLs and of CLs $24.8 \pm 16.5$ and $2.5 \pm 2.87$, respectively. After a mean follow-up of $51.8 \pm 8.32$ months we observed conversion to SP phase in 2.1\% and 3-mo-confirmed EDSS progression in $13.6 \%$ of patients and a mean number of relapses of $1.1 \pm 1.95$. During follow-up only $3.6 \%$ of patients did not take any therapy, while $47.1 \%$ and $49.3 \%$ were on first-line and second-line disease-modifying treatment (DMT), respectively. Occurrence of $\geq 1$ new WML or CL appeared in $37.9 \%$ and $12.9 \%$ of cases, respectively, with a mean number of new WMLs of 1.8 \pm 5.58 and new CLs of $0.2 \pm 0.6$. New CLs never appeared without concomitant WMLs, but $44.4 \%$ of cases with new CLs occurred in patients with 1-2 new WMLs and $26.7 \%$ of patients with 1-2 new WMLs had $\geq 1$ new CL. At multivariate analysis the risk of occurrence of new CLs was higher in patients with a higher number of new WMLs (OR 1.44, CI95\% 1.17-1.78, $\mathrm{p}=0.001)$ and lower in those who remained RR (OR 0.04, C195\% 0.002-0.76, $\mathrm{p}=0.03$ ).

Discussion: In our cohort we observed an overall low MRI activity, probably related to the high percentage of patients on DMT. New CLs were few and strictly related to new WMLs. Nevertheless, they added clinical relevance to several cases characterised by otherwise minimal MRI activity.

Conclusions: Even though in our cohort the occurrence of new CLs was low and strictly related to new WMLs, they added clinical relevance to MRI study.

References:

- Calabrese M, Rocca MA, Atzori M, et al. A 3-year MRI study of CLs in relapse onset MS. Ann Neurol (2010);67:376-83

- Rinaldi F, Perini P, Atzori M, et al. Disease-modifying drugs reduce cortical lesion accumulation and atrophy progression in relapsingremitting multiple sclerosis: results from a 48-month extension study. Mult Scler Int. (2015);2015:369348. doi:10.1155/2015/ 369348

- Faizy TD, Broocks G, Thaler C, et al. Development of Cortical Lesion Volumes on Double Inversion Recovery MRI in Patients With Relapse-Onset Multiple Sclerosis. Front. Neurol. (2019);10:133. doi: 10.3389/fneur.2019.00133

\section{EXPLORING THE POLYPHARMACY PHENOMENON IN NEWLY DIAGNOSED RELAPSING REMITTING MULTIPLE SCLEROSIS}

\section{E. D'amico, A. Zanghi', S. Lo Fermo, F. Patti, M. Zappia}

Department G.F. Ingrassia, University of Catania (Catania)

Objective: To examine the frequency of polypharmacy in a large cohort of patients at the time of RRMS diagnosis and to explore its effects on disease course after three years from the beginning of the first diseasemodifying treatment (DMT) assessed by the score no evidence of disease activity (NEDA-3).

Materials: We enroled RRMS patients starting their first DMT between January 1st, 2013 and December 31st, 2015.

Methods: According to the number of medicines prescribed (except DMTs) we divided patients in three groups: no-Poly-RRMS, minor-PolyRRMS (from one to three medications) and major Poly-RRMS ( $>3$ medications).

Results: 392 RRMS patients were enroled (mean age 41.1). MinorPoly-RRMS were 61 (15.6\%) and major-Poly-RRMS were 112 (28.6\%). Minor and major-poly-RRMS were older $(\mathrm{p}=0.00)$ and with higher median Body Mass Index $(\mathrm{BMI})(\mathrm{p}=0.00)$ than no-poly-RRMS patients. At multivariate regression analysis, higher age at onset was associated with minor and major-poly-RRMS (OR 1.050, CI 1.0-1-2, p=.015 and OR 1.063 , CI 1.0-1.1, respectively). BMI was associated with major polyRRMS (OR 1.186, CI 1.18-1.29, p=.000). Polypharmacy was not associated with disease activity after three years.

Discussion: Polypharmacy adds many variables in RRMS therapeutic algorithms. The choice of first DMT prescription should also take in account the presence of concomitant medical illnesses and the use of other medications which can expose patients to adverse drug reactions, and drug-drug or drug-disease interactions. In our cohort of newly diagnosed RRMS, polypharmacy was associated with an older age and higher BMI at the time of diagnosis.

Conclusions: Polypharmacy represents an emerging challenge in medical management in worldwide population, especially in the elderly. References:

- Sater N, White S, Venables R, Frisher M. Factors associated with polypharmacy in primary care: a cross-sectional analysis of data 
from The English Longitudinal Study of Ageing (ELSA). BMJ Open (2018);8(3):e020270-e

- Blonde L, Khunti K, Harris SB, Meizinger C, Skolnik NS. Interpretation and Impact of Real-World Clinical Data for the Practicing Clinician. Adv Ther. (2018);35(11):1763-74

- Frahm N, Hecker M, Zettl UK. Multi-drug use among patients with multiple sclerosis: A cross-sectional study of associations to clinicodemographic factors. Scientific Reports. (2019);9(1):3743

\section{INJECTABLE VERSUS ORAL FIRST-LINE DISEASE MODIFYING THERAPIES: RESULTS FROM ITALIAN MS REGISTER}

E. D'Amico ${ }^{1}$, A. Zanghi' ${ }^{1}$, M. Romeo ${ }^{2}$, E. $\mathrm{Cocco}^{3}$, G. Maniscalco ${ }^{4}, \mathrm{~V}$. Bresciamorra $^{4}$, D. Paolicelli ${ }^{5}$, G. De Luca ${ }^{6}$, S. Galgani ${ }^{7}$, M. Amato ${ }^{8}$, P. Confalonieri $^{9}$, G. Salemi ${ }^{10}$, M. Inglese ${ }^{11}$, G. Lus ${ }^{12}$, A. Gallo ${ }^{12}$, M. Vianello $^{13}$, M. Onofrj ${ }^{14}$, C. Pozzilli ${ }^{15}$, M. Filippi ${ }^{16}$, M. Trojano ${ }^{5}$, M. Zappia $^{1}$, F. Patti ${ }^{1}$

${ }^{1}$ Department G.F. Ingrassia, University of Catania (Catania); ${ }^{2}$ Neurology Dept, IRCCS San Raffaele (Milano); ${ }^{3}$ Neurology Dept, University of Cagliari (Cagliari); ${ }^{4}$ Neurology Dept, University of Naples (Napoli); ${ }^{5}$ Neurology Dept, University of Bari (Bari); ${ }^{6}$ Neurology Dept, University of Chieti (Chieti); ${ }^{7}$ Neurology Dept, Az. Osp. S. Camillo Forlanini (Roma); ${ }^{8}$ Neurology Dept, University of Florence (Firenze); ${ }^{9}$ IRCCS Carlo Besta, Neurology Dept (Milano); ${ }^{10}$ Neurology Dept, University of Palermo (Palermo); ${ }^{11}$ Neurology Dept, University of Genoa (Genova); ${ }^{12} \mathrm{MS}$ Center, University of Naples (Napoli); ${ }^{13}$ Neurology Unit, "Ca' Foncello" Hospital (Treviso); ${ }^{14}$ Neurology Dept, Policlinico SS Annunziata (Chieti); ${ }^{15}$ Neurology Dept, S. Andrea MS Center (Roma); ${ }^{16}$ Neuroimaging Research Unit, Institute of Experimental Neurology, Division of Neuroscience, IRCCS San Raffaele (Milano)

Objectives: To compare old injectable and oral first line disease modifying therapies (DMTs) for time to first relapse, time to confirmed disability progression (CDP), and time to discontinuation in a cohort of relapsing remitting multiple sclerosis (RRMS) patients extracted from the Italian MS Registry.

Materials: RRMS-naïve patients in the Italian MS Register starting injective or oral first line DMTs between 1 January 2010 and 31 December 2017 were enrolled.

Methods: Multicentre, observational, retrospectively acquired and propensity-adjusted cohort study to evaluate their impact on disability outcomes in patients. Enrolled patients were divided into two groups: injectable group (IG) and oral group (OG).

Results: From a cohort of 11,416 patients, 4,602 were enrolled (3,919 on IG and 683 on OG). IG had higher rate of women ( $67.3 \%$ vs $63.4 \%$, $\mathrm{p}<.05)$ and a lower mean age $(36.1 \pm 10.9$ vs $38.9 \pm 11.8, \mathrm{p}<.001)$. For the event time to first relapse, Cox models after PS adjustment revealed a lower risk for OG patients (HR 0.58 CI95\% 0.47-0.70, $\mathrm{p}<0.001$ ). About the risk of CDP, no differences were found in the two groups (HR 1.14 CI95\% 0.88-1.48, $\mathrm{p}=0.306$ ). About the risk of DMT discontinuation, OG patients showed lower risk (HR $0.70 \mathrm{CI} 95 \% 0.57-0.86 \mathrm{p}=0.001$ ) than IG patients.

Discussion: In this multicentre observational, retrospectively acquired cohort study, starting oral first-line DMTs (DMF and TRF) was associated to a lower risk of first relapse occurrence and treatment discontinuation rate, during the follow-up, in comparison to first-line injectable DMTs, but no significant difference was found in reaching CDP.
Conclusion: Real-world data from the Italian MS registry suggest that first line oral DMTs are associated to lower risks of experiencing a new relapse and of therapy discontinuation in comparison to injectable DMTs. References:

- Miller AE, Rhoades RW. Treatment of relapsing-remitting multiple sclerosis: current approaches and unmet needs. Current Opinion in Neurology (2012);25 Suppl:S4-10

- Burks J, Marshall TS, Ye X. Adherence to disease-modifying therapies and its impact on relapse, health resource utilization, and costs among patients with multiple sclerosis. Clinicoecon Outcomes Res. (2017);9:251-60

- Trojano M, Bergamaschi R, Amato MP, Comi G, Ghezzi A, Lepore $\mathrm{V}$, et al. The Italian multiple sclerosis register. Neurological sciences: official journal of the Italian Neurological Society and of the Italian Society of Clinical Neurophysiology (2019);40(1):155-65

\section{SERUM NEUROFILAMENTS PREDICT RECOVERY AFTER ACUTE OPTIC NEURITIS}

G. Dalla Costa, M. Pisa, L. Fabbella, M. Filippi, G. Comi, R. Furlan, L. Leocani

Institute of Experimental Neurology, Division of Neuroscience, San Raffaele Scientific Institute (Milano)

Background: Optic neuritis is an immune-mediated disease of the optic nerve, strongly associated with multiple sclerosis (MS). Although the visual prognosis of optic neuritis is generally favourable, the degree of remission varies considerably. The degree of clinical remission is associated with the degree of optic nerve axonal loss, that can be quantified accurately by Optic Coeherence Tomography (OCT). Neurofilament light chain (NfL) is part of the axonal cytoskeletal neurofilaments and is released upon immunemediated axonal damage during optic neuritis and MS. We aimed to investigate if NfL levels sampled close after symptom onset would predict the outcome after optic neuritis.

Methods: We included 31 patients with optic neuritis as a first demyelinating episode. Patients underwent visual tests, OCT, magnetic resonance imaging (MRI) and lumbar puncture. NfL levels were measured through use of a Simoa HD-1 instrument (Quanterix). Longitudinal changes in inter-ocular difference in visual acuity and OCT parameters were chosen as primary outcome measures of visual loss to account for their inter-individual variability. Multilevel mixed effect models have been used to assess the prognostic factor of baseline NfL levels on longitudinal changes in visual outcomes.

Results: Patients (mean age 37.3 years, SD $8.7,71 \%$ females) had a mean follow-up of 27.6 months (SD 12.3). The mean inter-ocular visual acuity difference decreased with the follow-up (baseline 2.8 SD 1.2, follow up 2.1 SD 1.5, p <0.05), while mean inter-ocular RNFL thickness difference significantly increased with time (3.2 SD 10.2 at baseline, 12.7 SD 15.2 at follow-up). Basel NfL levels above $75^{\circ}$ ile were significantly associated with an increase in interocular visual acuity difference (B 0.05 SE $0.02, \mathrm{p}<0.01)$ and interocular RNFL thickness difference (B 0.64 SE 0.20, p <0.01).

Conclusion: NfL is a promising biomarker of visual outcome after optic neuritis. This could aid neuroprotective/regenerative medical advancements. 


\section{ASSESSMENT OF BALANCE AND WALKING USING CLINICAL SCALE AND 3D-GAIT ANALYSIS IN PEOPLE WITH MULTIPLE SCLEROSIS AND SPASTICITY TREATED BY NABIXIMOLS}

P. De Blasiis, F. Siani, A. Fullin, A. Di Pietro, S. Sampaolo, E. Signoriello, G. Lus

Department of Advanced Medical and Surgical Sciences, 2nd Division of Neurology, University of Campania "Luigi Vanvitelli" (Napoli)

Background: Spasticity is one of the most disabling symptoms of Multiple Sclerosis (MS). Among the systemic antispastic drugs, Nabiximols (Sativex ${ }^{\circledR}$ ) showed a good tolerability and relevant efficacy. No study investigated short-term effects on people with MS (pwMS).

Objective: Aim of our study is to quantitatively evaluate the immediate effects of Sativex® and their persistence after 4 weeks in pwMS with spasticity.

Methods: pwMS were enrolled and randomized in 2 treatment groups: Sativex (SG) and control (CG) group. All patients were assessed at T0(before the first Sativex puff), T1(after 45 minutes) and T2 (after 4 weeks of Sativex treatment) using clinical scales (cs) and 3d-Gait Analysis (GA). Moreover, the SG patients were divided into 4 subgroups according to Numeric Rating Scale for spasticity (NRSs) and Berg Balance Score (BBS) response at T1

Results: 32 pwMS (22 SG;10 CG) were recruited. Significant improvements were found between $\mathrm{T} 0$ and $\mathrm{T} 1$ in SG vs CG in cs and GA parameters. Further significant differences were found for NRSs and BBS responders' groups versus CG. At T2 no significant differences were found for all parameters, suggesting the persistence of the changes emerged between T0-T1.

Conclusion: Our study shows the immediate effects of Nabiximols on balance and walking and their maintenance after 4 weeks in NRS-BBS responders pwMS. These results may suggest how to early select the real responders in order to improve the adherence and cost-effectiveness of the therapy.

References:

- Novotna A, Mares J, Ratcliffe S, et al. A randomized, double-blind, placebo-controlled, parallel-group, enriched-design study of nabiximols* (Sativex®), as add-on therapy, in subjects with refractory spasticity caused by multiple sclerosis. Eur J Neurol (2011) Sep;18(9):1122-31

- Gervasoni E. Minimal Clinically Important Difference of Berg Balance Scale in People with Multiple Sclerosis. Arch Phys Med Rehabil. (2017) Feb;98(2):337-40

- Coghe et al. Walking improvements with nabiximols in patients with multiple sclerosis. J Neurol. (2015) Nov;262(11):2472-7

\section{EARLY CLINICAL AND MRI PREDICTORS OF LONG-TERM DISABILITY IN PEDIATRIC MULTIPLE SCLEROSIS PATIENTS}

\author{
E. De $\mathrm{Meo}^{1}$, R. Bonacchi ${ }^{1}$, L. Moiola ${ }^{1}$, F. Sangalli ${ }^{1}$, B. Colombo ${ }^{1}$, G.

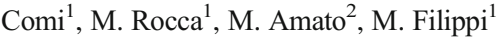

${ }^{1}$ San Raffaele Hospital, INSPE, Vita-Salute San Raffaele University (Milano); ${ }^{2}$ NEUROFARBA, University of Florence (Firenze)

Objectives: The main clinical and MRI features driving therapeutic choices are not as clear for pediatric multiple sclerosis (MS) patients as for adults. Inthie perspective, we aimed at assessing early predictors of long-term clinically-relevant outcomes in a large cohort of pediatric MS patients.

Materials: Clinical and MRI assessment was obtained at disease onset and after 1, 2 and 3 years, in a cohort of 123 pediatric MS patients. The longest clinical follow-up (mean $9.33+/-3.45$ years) was considered for long-term outcomes.

Methods: Cox proportional hazards models were used to assess predictors of time to first relapse, while multivariable logistic and linear regression models identified clinical and MRI predictors of long-term outcomes.

Results: Across baseline features, optic nerve involvement predicted a shorter time to first relapse (hazard ratio $=1.9, p=0.03$ ). Predictors of annualized relapse rate (ARR) were: at baseline, presence of cerebellar $(=-0.16, p=0.00)$ and number of cervical cord lesions $(=0.14, \mathrm{p}=0.01)$; considering short-term predictors, the same baseline variables together with time to first relapse (2-year: $=-0.12$, $\mathrm{p}=0.01 ; 3$-year: $=-0.08, \mathrm{p}=0.00)$ and the number of relapses $(1$-year: $=0.14, \mathrm{p}=0.00 ; 2$-year: $=0.06, \mathrm{p}=0.02$ ). Baseline predictors of 10 year disability worsening were: at baseline, presence of optic nerve [odds $\operatorname{ratio}(\mathrm{OR})=6.45, \mathrm{p}=0.01$ ] and brainstem lesions $(\mathrm{OR}=6.17$, $\mathrm{p}=0.04)$; considering short-term predictors, Expanded Disability Status Scale (EDSS) changes at $1(\mathrm{OR}=26.05, \mathrm{p}=0.00)$ and 2 $(\mathrm{OR}=16.38, \mathrm{p}=0.02)$ years and the detection of at least two new T2-lesions in 2 years (2-year: $\mathrm{OR}=4.91, \mathrm{p}=0.02$; 3-year: $\mathrm{OR}=5.49$, $\mathrm{p}=0.09$ ). Predictors of higher 10-year EDSS score were: at baseline, EDSS score $(=0.58, \mathrm{p}<0.001)$, presence of brainstem $(=0.31$, $\mathrm{p}=0.04)$ and number of cervical cord lesions $(=0.22, \mathrm{p}=0.05)$; considering short-term predictors, EDSS changes ( 1 -year: $=0.82, \mathrm{p}<0.001 ; 2$ year: $=0.79, p<0.001,3$-year: $=0.27, p=0.04)$, together with the detection of at least two new T2-lesions at $1(=0.28, \mathrm{p}=0.03)$ and $2(=0.35, \mathrm{p}=0.01)$ years.

Discussions: Baseline spinal cord, brainstem and optic nerve lesions have a major role in predicting long-term outcomes, both in term of disease activity and of disability worsening. In addition, an accurate clinical and MRI monitoring during the first 2 years of disease has proven to represent a powerful tool for counselling patients about long-term prognosis and personalizing treatment strategies.

Conclusions: A complete baseline MRI assessment and an accurate clinical and MRI monitoring are required to obtain prognostic information and to personalize treatment plans.

\section{IDENTIFYING DISTINCT COGNITIVE PHENOTYPES IN MULTIPLE SCLEROSIS}

E. De Meo ${ }^{1}$, E. Portaccio ${ }^{2}$, A. Giorgio ${ }^{3}$, L. Ruano ${ }^{4}$, B. Goretti ${ }^{2}$, C. Niccolai $^{2}$, F. Patti ${ }^{5}$, C. Chisari ${ }^{5}$, P. Gallo ${ }^{6}$, P. Grossi ${ }^{7}$, A. Ghezzi ${ }^{8}$, M. Roscio $^{8}$, F. Mattioli ${ }^{9}$, C. Stampatori ${ }^{9}$, M. Simone ${ }^{10}$, R. Viterbo ${ }^{10}$, M. Rocca $^{1}$, N. De Stefano ${ }^{3}$, M. Filippi ${ }^{1}$, M. Amato ${ }^{2}$

${ }^{1}$ San Raffaele Hospital, INSPE, Vita-Salute San Raffaele University (Milano); ${ }^{2}$ NEUROFARBA, University of Florence (Firenze); ${ }^{3}$ Department of Medicine, Surgery and Neuroscience, University of Siena (Siena); ${ }^{4}$ EPIUnit, Instituto de Saúde Pública, University of Porto (Porto-P); ${ }^{5}$ University of Catania (Catania); ${ }^{6}$ University of Padua (Padova); ${ }^{7}$ Neuroimmunology Center, Cardiocerebrovascular, ASST Crema (Crema-CR); ${ }^{8}$ Gallarate Hospital (Gallarate-VA); ${ }^{9}$ Neuropsychology Unit, ASST Spedali Civili (Brescia); ${ }^{10}$ Department of Basic Medical Sciences, Child and Adolescence Neuropsychiatry Unit, Neuroscience and Sense Organs, University of Bari (Bari) 
Objectives: Cognitive impairment is one of the most disabling symptoms of multiple sclerosis (MS), affecting about $50 \%$ of patients. We sought to define homogeneous cognitive phenotypes in a large cohort of MS patients by using a data-driven approach, and to assess their distinctive clinical and MRI features.

Materials: A cohort of 1212 MS patients and 196 healthy controls (HC) from 8 Italian MS centers underwent cognitive evaluation with Rao's Brief Repeatable Battery and Stroop Color Word Test. A subgroup (172 MS patients and $50 \mathrm{HC}$ ) also underwent a 3T MRI examination, including 3D T1-weighted and dual-echo sequences.

Methods: Latent-profile analysis was used on cognitive tests' z-scores for identifying cognitive phenotypes. Linear regression and mixed effects models were used to define the clinical and MRI features of each phenotype.

Results: Five cognitive phenotypes were identified, characterized by "preserved-cognition" (19\%), "mild verbal memory/semantic fluency" impairment (30\%), "mild-multi-domain" impairment (19\%), "severe-attention/executive" impairment with mild impairment of other domains (14\%), and "severe-multi-domain" impairment (18\%). "Preserved-cognition" patients had shorter disease duration and lower Expanded Disability Status Scale (EDSS) score than all other groups, and mildly impaired phenotypes included patients with shorter disease duration and less likely progressive disease compared to severely impaired groups. However, the "preserved-cognition" group also included patients with progressive disease and high EDSS scores, and severely impaired phenotypes were also represented in early MS stages. Comparing each phenotype to "preserved-cognition" group, distinctive MRI features emerged: "mild verbal memory/semantic fluency" patients had reduced hippocampal volume $(\mathrm{p}=0.02)$, "mild-multi-domain" reduced cortical gray matter volume $(p=0.04)$, "severe-attention/executive" higher lesion volume $(p=0.04)$ and severe-multi-domain" extensive brain damage $(\mathrm{p}<0.01$ for lesion, brain, gray matter and thalamic volumes).

Discussions: The cognitive characterization provided in the present study underscores the existence of different profiles in the MS cognitive spectrum. Importantly, we were able to identify separate underlying neuroanatomical substrates, supporting data-driven findings with a biological basis.

Conclusions: By defining homogenous and clinically meaningful groups, this characterization may be useful for future research on cognitive impairment in MS, and for defining personalized management approaches and rehabilitative strategies in clinical practice.

\section{FATIGUE AND DEPRESSION IN RELAPSING REMITTING MULTIPLE SCLEROSIS: THE CONTRIBUTION OF CORTICAL LESIONS}

R. Docimo ${ }^{1}$, R. Capuano ${ }^{1}$, A. Bisecco ${ }^{1}$, A. d'Ambrosio ${ }^{1}$, M. Altieri ${ }^{1}$, M.

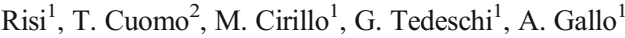

${ }^{1}$ Department of Advanced Medical and Surgical Sciences, " DAMSS", University of Campania Luigi Vanvitelli (Napoli); ${ }^{2}$ Umberto I Hospital, ASL Salerno (Nocera Inferiore-SA)

Objectives: Despite the high prevalence and debilitating nature of fatigue and depression in Relapsing Remitting Multiple Sclerosis (RRMS), the underlying pathophysiology is still far from being fully understood. While several findings highlighted the contribution of white matter lesion load (WMLL) and brain atrophy, the role of cortical lesions (CL) has been only marginally assessed. To investigate: i) the contribution of CL volume to fatigue and depression; ii) the relative contribution of total $\mathrm{CL}$ volume (tCLV), intracortical lesion volume (ICLV) and juxtacortical lesion volume (JCLV).

Materials and Methods: Sixty-five RRMS patients underwent: i) clinical evaluation including the Expanded Disability Status Scale (EDSS), ii) assessment of fatigue and depression trough the Modified Fatigue Impact Scale (MFIS) and the Beck Depression Inventory (BDI), iii) a 3T-MRI protocol including Double-Echo (DE) and 3D-Double Inversion Recovery (DIR) imaging to identify WMLL and CL. Correlation analyses were run between WMLL, CL and MFIS and BDI. A multiple linear regression model was applied to evaluate the contribution of CL to MFIS and BDI, controlling for clinico-demographic data and WMLL.

Results: The correlation analysis showed that tCLV and JCLV correlated with MFIS ( $\mathrm{rho}=0.31, \mathrm{p}=0.007$; $\mathrm{rho}=0.28, \mathrm{p}=0.01$ respectively) and BDI (rho $=0.24, \mathrm{p}=0.03$ and $\mathrm{rho}=0.23, \mathrm{p}=0.04$, respectively), while ICLV or WMLL did not correlate with neither MFIS nor BDI. Regression analysis did not reveal any CL volume as a significant predictor of fatigue or depression.

Discussion and Conclusions: This study shows a significant role of $\mathrm{CL}$ volume in determining these symptoms in RRMS.

\section{MAGNETIZATION TRANSFER IMAGING IN SECONDARY PROGRESSIVE MULTIPLE SCLEROSIS PATIENTS TREATED WITH SIPONIMOD: RESULTS FROM THE PHASE 3 EXPAND STUDY}

L.A. Douglas ${ }^{1}$, P. Vermersch ${ }^{2}$, B. Cree ${ }^{3}$, A. Bar-Or ${ }^{4}$, G. Giovannoni ${ }^{5}$, R. Gold $^{6}$, D. Meier ${ }^{7}$, S. Arnould ${ }^{7}$, S. Ritter ${ }^{8}$, G. Karlsson', L. Kappos ${ }^{9}$, R. Fox ${ }^{10}$

${ }^{1}$ NeuroRx Research (Montreal-CDN); ${ }^{2}$ INSERM U, CHU Lille, University Lille (Lille-F); ${ }^{3} \mathrm{UCSF}$ Weill Institute for Neurosciences, Department of Neurology, University of California San Francisco (San Francisco-USA); ${ }^{4}$ Center for Neuroinflammation and Experimental Therapeutics and Department of Neurology, Perelman School of Medicine, University of Pennsylvania (Philadelphia-USA); ${ }^{5}$ Blizard Institute, Barts and The London School of Medicine and Dentistry, Queen Mary University of London (London-UK); ${ }^{6}$ Department of Neurology, St Josef-Hospital/Ruhr-University Bochum (Bochum-D); ${ }^{7}$ Novartis Pharma AG (Basel-CH); ${ }^{8}$ Novartis Pharmaceuticals Corporation (East Hanover-USA); ${ }^{9}$ Neurologic Clinic and Policlinic, Departments of Medicine, Clinical Research, Biomedicine and Biomedical Engineering, University Hospital, University of Basel (Basel-CH); ${ }^{10}$ Mellen Center for Treatment and Research in Multiple Sclerosis, Neurological Institute (Cleveland-USA)

Objectives: Magnetization transfer ratio (MTR) is widely used for estimating myelin content in the brain. In the EXPAND study, siponimod significantly reduced disability progression, cognitive decline, and total brain volume loss versus placebo in secondary progressive multiple sclerosis patients. In preclinical studies, siponimod showed pro-myelinating effects. The aim of this study is to determine the effect of siponimod versus placebo on MTR changes as a marker of alterations in myelin density in different brain regions, and assess the degree of MTR recovery within MTR lesions.

Materials: This prospective exploratory MTR sub-study included 633 patients (siponimod [ $n=409]$; placebo [ $n=224]$ ). METHODS MTR changes were analyzed in normal appearing brain tissue (NABT), cortical grey matter (cGM) and normal appearing white matter (NAWM) at baseline, Month (M) 12, and M24. MTR variability across different scanners 
was reduced by MTR normalization. Absolute change from baseline in median normalized MTR (nMTR) expressed in percent units was derived from mixed models for repeated measures. MTR recovery metrics were assessed in new MTR lesions comparing decrease in nMTR from pre- to post-lesion time points in placebo and siponimod-treated patients.

Results: Absolute changes from baseline in median nMTR in brain tissues for siponimod versus placebo at M12 and M24 were: -0.016 versus $-0.024(-38 \%, \mathrm{P}=0.32)$ and -0.022 versus $-0.056(-61 \%$, $\mathrm{P}=0.019)$ for $\mathrm{NABT} ;-0.019$ versus $-0.026(-27 \% ; \mathrm{P}=0.42)$ and 0.025 versus $-0.056(-55 \%$; $\mathrm{P}=0.047)$ for $\mathrm{cGM}$; and 0.002 versus $0.019(-105 \% ; \mathrm{P}=0.021)$ and -0.001 versus $-0.045(-98 \% ; \mathrm{P}=0.0018)$ for NAWM. Lesion MTR recovery favored siponimod (-1.321) versus placebo (-1.506; difference 0.185 [0.056; 0.314]; $\mathrm{P}=0.005)$.

Conclusions: Siponimod demonstrated a consistent and significant effect versus placebo on the decrease of MTR over time in normalappearing tissues. Siponimod also improved MTR recovery in newly formed lesions, an effect that could be consistent with promotion of remyelination observed in preclinical studies.

This study has been first presented at the Annual American Academy of Neurology Meeting 2020.

References:

- M. Filippi, W. Brück, D. Chard, F. Fazekas, et al. Attendees of the Correlation between Pathological and MRI findings in MS workshop - Association Between Pathological and MRI Findings in Multiple Sclerosis; Lancet Neurol. (2019) Feb;18(2):198-210

- L. Kappos, A. Bar-Or, B.A.C. Cree, R. J. Fox, et al. EXPAND Clinical Investigators - Siponimod Versus Placebo in Secondary Progressive Multiple Sclerosis (EXPAND): A Double-Blind, Randomised, Phase 3 Study; Lancet (2018) Mar 31;391(10127):1263-73

\section{EATING HUBS IN MS: EXPLORING THE RELATIONSHIP BETWEEN MEDITERRANEAN DIET AND DISABILITY STATUS}

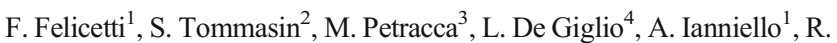
Nistri $^{1}$, S. Ruggieri ${ }^{2}$, C. Pozzilli ${ }^{2}$

${ }^{1}$ MS Center, Sant'Andrea Hospital (Roma); ${ }^{2}$ Department of Human Neurosciences, Sapienza University of Rome (Roma); ${ }^{3}$ Department of Neurosciences and Reproductive and Odontostomatological Sciences, University of Naples (Napoli); ${ }^{4}$ Neurology Unit, San Filippo Neri Hospital (Roma)

Objective: Multiple Sclerosis (MS) is a complex disease in which multiple factors contribute to disability accrual. Lifestyle factors affect the course of MS, while whether dietary habits may influence the course of the disease is still a matter of discussion and established MS therapeutical approach does not usually include any specific recommendation on diet. We aimed to evaluate whether the Mediterranean diet, i.e. the intake of specific foods, and cigarette smoking vary in MS patients compared to a group of healthy subjects and how it can affect disability.

Material and Method: Four hundred-two patients with MS [F:324;

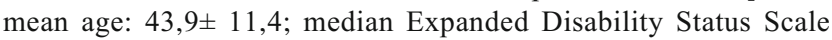
(EDSS): 2 (range:0-7,5)] were consecutively recruited. In addition, 165 age-, weight-, height-, gender-matched healthy subjects (HS) were recruited for the control group [F:112; mean age:40,9 $\pm 15,5]$. Data were obtained through the administration of questionnaires for the assessment of physical activity and the adequacy of Mediterranean diet. Food intake was further defined as healthy or not, according to scores derived from the diet questionnaire. We also evaluated the anthropometric indicators Body
Mass Index (BMI) and waist-hip ratio (WHR). Smoking habit was selfreported and entered in the analysis as smoking pack per year. EDSS was considered in the MS population. We inferred food networks in both MS cases and controls using mutual information matrices (MIM), a measure to detect nonlinear associations, and compared food consumption patterns and lifestyle features between groups. We then investigated main hubs separately according to group and into the MS population divided by EDSS upper of 1.5.

Results: MS patients showed higher total feeding score compare to HS, however there is no significant difference in MIM between groups. In the HS group, hubs are represented by WHR and meat, while in the MS cohort the only hub is embodied by cereals intake. When we explored hubs into the MS cohort, the group with no disability showed as only hub WHR, while in the group of patients with higher EDSS we found age, legume and cereal as main hubs.

Discussion and Conclusions: Patients with MS are more used to follow a healthy diet compared to HS. However, differences in diet networks in MS population suggest an eating habits toward carbohydrates especially in those patients with higher EDSS. Indeed, this attitude maybe associated with worse clinical outcomes and requires further investigation in randomized clinical trials.

Reference:

- Fitzgerald KC, Tyry T, Salter A, et al. Diet quality is associated with disability and symptom severity in multiple sclerosis. Neurology (2018);90(1):e1-e11. doi:10.1212/WNL.0000000000004768

\section{INTER-LABORATORY EVALUATION OF CEREBROSPINAL FLUID AND SERUM KAPPA FREE LIGHT CHAIN MEASUREMENTS}

D. Ferraro ${ }^{1}$, R. Bedin ${ }^{1}$, P. Natali ${ }^{2}$, D. Franciotta ${ }^{3}$, G. Bernardi ${ }^{4}$, E. Corsini $^{4}$, E. Cocco 5 , G. Costa ${ }^{5}$, I. Crespi ${ }^{6}$, M. Lamonaca ${ }^{6}$, A. Sala ${ }^{7}$, C. Nicolo $^{8}$, M. Di Filippo ${ }^{9}$, A. Villa ${ }^{10}$, V. Nociti ${ }^{11}$, T. De Michele ${ }^{12}$, P. Cavalla $^{13}$, P. Caropreso ${ }^{14}$, F. Vitetta ${ }^{15}$, M. Gastaldi ${ }^{3}$, T. Trenti ${ }^{2}$, S. Meletti ${ }^{1}$, P. Sola ${ }^{15}$

${ }^{1}$ Department of Biomedical, Metabolic and Neurosciences, -University of Modena and Reggio Emilia (Modena); ${ }^{2}$ Department of Laboratory Medicine, Azienda Ospedaliero-Universitaria and Azienda (Modena); ${ }^{3}$ Neuroimmunology Laboratory, IRCCS Mondino Foundation (Pavia); ${ }^{4}$ Laboratory Medicine Unit, Fondazione IRCCS Istituto Neurologico Carlo Besta (Milano); ${ }^{5}$ Multiple Sclerosis Center, ATS Sardegna/ University of Cagliari (Cagliari); ${ }^{6}$ Clinical Biochemistry Laboratory, Azienda Ospedaliero Universitaria Maggiore della Carità of Novara (Novara); ${ }^{7}$ Neurology Unit, CReSM, Azienda Ospedaliero Universitaria San Luigi Gonzaga (Orbassano-TO); ${ }^{8}$ Clinical Chemistry and Microbiology Laboratory, Azienda Ospedaliero Universitaria San Luigi Gonzaga (Orbassano-TO); ${ }^{9}$ Neurology Unit, Department of Medicine, University of Perugia (Perugia); ${ }^{10}$ Clinical Pathology and Haematology Laboratory, Azienda Ospedaliera of Perugia (Perugia); ${ }^{11}$ Multiple Sclerosis Center, Fondazione Policlinico Universitario "A. Gemelli" IRCCS, Catholic University (Roma); ${ }^{12}$ Clinical Chemistry, Biochemistry and Molecular Biology Laboratory, Fondazione Policlinico Universitario “A. Gemelli” IRCCS (Roma); ${ }^{13}$ Multiple Sclerosis Center, Department of Neurosciences and Mental Health, Azienda Ospedaliero-Universitaria Città della Salute e della Scienza of Torino (Torino); ${ }^{14}$ Clinical Biochemistry Laboratory, Department of Laboratory Medicine, Azienda OspedalieroUniversitaria Città della Salute e della Scienza of Torino (Torino);

${ }^{15}$ Neurology Unit, Azienda Ospedaliero-Universitaria of Modena (Modena) 
Objective: The kappa index, calculated by dividing the cerebrospinal (CSF)/serum kappa free light chain (KFLC) ratio by the CSF/serum albumin ratio, is gaining increasing interest as an indirect marker of intrathecal activation of the humoral immune response. The demonstration of intrathecal synthesis is of particular relevance in the diagnostic work-up of suspected Multiple Sclerosis. However, the lack of consistent data on inter-laboratory agreement in CSF and serum KFLC measurements is one of the factors that hamper the use of kappa index in routine practice. Our aim was to assess inter-laboratory agreement in CSF and serum KFLC measurements and kappa index values.

Methods: Fifteen paired CSF and serum samples were analyzed in all participating laboratories ( $\mathrm{nr}=8$ ). Four centers used Binding Site instruments and assays, 3 centers used Siemens instruments and assays, and one center used a Siemens instrument and a Binding Site assay. Absolute individual agreement between laboratories was calculated using a twoway mixed effects intraclass correlation coefficient (ICC). Cohen's kappa coefficient was used to measure inter-laboratory agreement on positive $(\geq 5.8)$ kappa index values.

Results: Within Binding Site laboratories, ICC for KFLC measurements was 0.96 (95\% CI: 0.9-0.98) for CSF, 0.93 (95\%CI: 0.63-0.98) for serum and 0.97 (95\% CI: 0.94-0.99) for kappa index values. Within Siemens laboratories, ICC for KFLC measurements was 0.99 (95\%CI: 0.97-100) for CSF, 0.93 (95\%CI: 0.48-0.98) for serum and 0.95 (95\%CI: $0.89-0.98)$ for kappa index values. ICC calculated for all laboratories was 0.93 (95\%CI: $0.87-0.97$ ) for CSF KFLC, 0.81 (95\%CI: 0.53-0.93) for serum KFLC and 0.65 (95\%CI: 0.43-0.84) for kappa index. Cohen's kappa coefficient for a positive kappa index was 0.89 across Binding Site laboratories, 0.70 across Siemens laboratories, and 0.77 across all laboratories.

Conclusion: There was an excellent agreement in CSF KFLC measurements and in kappa index values within laboratories using the same instrument and assay (Binding Site or Siemens), while serum KFLC measurements were less concordant. Agreement across all laboratories was decreased when including the laboratory using a Siemens instrument coupled with a Binding Site assay in the analyses. Concordance for a positive kappa index was substantial across all laboratories and within Siemens laboratories, and very good within Binding Site laboratories.

\section{RISKS ASSOCIATED WITH WASH-OUT DURATION WHEN SWITCHING FROM FINGOLIMOD TO CELL-DEPLETING AGENTS}

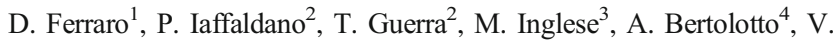
Brescia Morra ${ }^{5}$, M. Zaffaroni ${ }^{6}$, M. Mirabella ${ }^{7}$, G. Lus ${ }^{8}$, F. Patti ${ }^{9}$, P. Cavalla $^{10}$, M. Cellerino ${ }^{3}$, M. Capobianco ${ }^{4}$, F. Vitetta ${ }^{11}$, D. Paolicelli ${ }^{2}$, P. Sola ${ }^{11}$, M. Trojano ${ }^{2}$

${ }^{1}$ Department of Biomedical, Metabolic and Neurosciences, University of Modena and Reggio Emilia (Modena); ${ }^{2}$ Department of Basic Medical Sciences, Neuroscience and Sense Organs, University of Bari "Aldo Moro" (Bari); ${ }^{3}$ Department of Neuroscience, Rehabilitation, Ophthalmology, Genetics, and Mother-Child Health, University of Genoa (Genova); ${ }^{4}$ Regional Referral MS Center, University Hospital San Luigi (Orbassano-TO); ${ }^{5} \mathrm{MS}$ Center, OU Policlinico Federico II (Napoli); ${ }^{6}$ Multiple Sclerosis Center, Gallarate Hospital, ASST della Valle Olona (Gallarate-VA); ${ }^{7}$ Neurology Unit, Fondazione Policlinico Universitario A. Gemelli IRCCS (Roma); ${ }^{8}$ MS Center, II Division of Neurology, Univ. della Campania "L. Vanvitelli" (Napoli); ${ }^{9}$ Department of Medical and Surgical and Advanced Technologies, GF Ingrassia, University of Catania (Catania); ${ }^{10} \mathrm{MS}$ Centre, I Division of
Neurology, City of Health and Science Turin Univ. Hospital (Torino); ${ }^{11}$ Neurology Unit, Azienda Ospedaliero-Universitaria of Modena (Modena)

Objective: A wash-out duration lasting $>1-2$ months between the majority of sequential disease-modifying therapies (DMTs) is associated with an increased risk of disease reactivation in Multiple Sclerosis (MS) patients. Fingolimod (FTY) induces sequestration of lymphocytes in secondary lymphoid organs and the average lymphocyte recovery following discontinuation takes 1-2 months. It has been hypothesized that the therapeutic effects of subsequent cell-depleting agents may be compromised if lymphocyte recovery is still incomplete and that shorter wash-out periods do not affect the disease reactivation risk. Aim was to assess the risk of relapses following FTY discontinuation and the initiation of a B/T celldepleting agent initiation in relation to the duration of wash-out between the drugs using data from the Italian MS Registry.

Methods: Patients who initiated alemtuzumab, rituximab, ocrelizumab or cladribine within six months of FTY discontinuation, and with a follow-up of at least six months, or until a relapse occurred, were included in the study. The risk of relapses was assessed in relation to different wash-out durations $(<6,6-11,12-17$ and $>/=18$ weeks) using a Poisson regression analysis (and reported as incidence rate ratio - IRR) and a Cox proportional hazards model including age, disease duration, relapses during FTY treatment, EDSS and reason for FTY discontinuation as covariates.

Results: Inclusion criteria were met by 329 patients $(226 \mathrm{~F}, 103 \mathrm{M}$; mean age $41 \pm 10$ years). Following a median wash-out period of 11 weeks [IQR: 6-16], 175 patients started alemtuzumab, 69 rituximab, 68 ocrelizumab and 17 cladribine. Ninety patients relapsed during the wash-out period and 72 during the subsequent cell-depleting therapy. During the cell-depleting treatment, IRR for a relapse was significantly greater in patients with a washout-period of 12-17 (IRR (95\%CI): 2.4 $(1,1-5,5) ; \mathrm{p}=0.037)$ and $>/=18$ weeks $(6.0(2.8-12.7) ; \mathrm{p}<0.001)$ compared to the reference period ( $<6$ weeks). The multivariable Cox analysis showed that the time to a relapse was significantly influenced by the occurrence of relapses during FTY treatment (HR (95\% CI): $1.4(1.2-1.7) ; \mathrm{p}<0.001)$. Moreover, wash-out durations of $6-11,12-17$ and $>/=18$ weeks were associated with a higher risk of a relapse in comparison to wash-out durations shorter than 6 weeks (3.8 (1.1-13.2); $\mathrm{p}=0.037 ; 6.0$ (1.7-21.9); $\mathrm{p}=0.006 ; 16.3$ (4.8-56.3); $\mathrm{p}<0.001$, respectively).

Conclusion: The risk of relapses during a cell-depleting therapy following a sequestering agent, namely FTY, increases progressively with the duration of wash-out, underlining the need of a short wash-out period also in this type of treatment sequence.

\section{ASSESSMENT OF CLINICAL, GENETIC AND IMMUNE REPERTOIRE DATA TO PREDICT DISEASE ACTIVITY AND PROGRESSION IN RRMS}

L. Ferrè ${ }^{1}$, S. Santoro ${ }^{1}$, M. Tosi ${ }^{1}$, E. Mascia ${ }^{1}$, M. Sorosina ${ }^{1}$, F. Clarelli ${ }^{1}$, M. Lucia ${ }^{2}$, V. Martinelli ${ }^{2}$, G. Comi ${ }^{3}$, M. Filippi ${ }^{4}$, F. Esposito ${ }^{5}$

${ }^{1}$ Laboratory of Human Genetics of Neurological Disorders, INSPE, IRCCS San Raffaele Scientific Institute (Milano); ${ }^{2}$ Department of Neurology, IRCCS San Raffaele Scientific Institute (Milano); ${ }^{3}$ INSPE, IRCCS San Raffaele Scientific Institute (Milano); ${ }^{4}$ Department of Neurology, Neurophysiology Unit, Neuroimaging Research Unit, INSPE, IRCCS San Raffaele Scientific Institute and Vita Salute San Raffaele University (Milano); ${ }^{5}$ Department of Neurology and 
Neurorehabilitation, Laboratory of Human Genetics of Neurological Disorders, INSPE, IRCCS San Raffaele Scientific Institute (Milano)

Background: Multiple Sclerosis (MS) has a highly heterogeneous clinical course and, given the broad spectrum of approved therapies, there is a strong need to identify parameters that can guide treatment choice.

Objectives: The present study investigates clinical, genetic and immunological parameters associated with MS severity in order to combine them into a predictive model.

Patients and Methods: An "Extended" cohort of $\sim 1,000$ patients that started a first-line drug, with available clinical and genetic data, and a "Core" dataset of $\sim 200$ patients with clinical, genetic and immune repertoire information obtained before first-line treatment start were enrolled. The following outcomes were considered at the 4-year follow-up: NEDA-3 criterion, time to first relapse (TFR), EDSS and MS Severity Score (MSSS). A regression analysis was performed on both cohorts and results were meta-analyzed.

Results: A younger age at onset $(\mathrm{AAO})$ and a shorter disease duration strongly correlate with higher inflammatory activity; a higher baseline EDSS and AAO are the best prognostic markers of disability increase. The genetic study identified some interesting signals with suggestive association: rs6925307 is associated with NEDA status (OR 0.55, $\mathrm{p}: 1.53 \mathrm{e}-06$ ) and has an eQTL effect on CLVS2 gene, required for normal morphology of endosomes and lysosomes in neurons. Rs9264731, an intronic variant in the HLA-C gene, is associated with TFR (HR 1.49, $\mathrm{p}: 4.11 \mathrm{e}-06$ ). T-cell receptor (TCR) sequencing is ongoing and immune repertoire data are already available for 123 patients: overall more than 16.000 .000 sequences have been obtained, of which $81.5 \%$ are productive, corresponding on average to $\sim 77.000$ unique clonotypes per patient.

Discussion: We confirmed the association of clinical parameters with disease severity and we identified some interesting genetic markers whose association need to be replicated. TCR data are being generated and will be integrated in a predictive model of disease activity.

Conclusions: AAO and disease duration are associated with MS inflammatory activity while AAO and baseline EDSS correlate with disability progression during follow up. Some interesting genetic variants in regions coding for genes involved in neuronal and immunological functions also showed a suggestive association with disease severity, but replication in larger cohorts is warranted.

References:

- A Fromont, M Debouverie, G Le Teuff et al. Clinical parameters to predict response to interferon in relapsing multiple sclerosis. Neuroepidemiology (2008);31(3):150-6

- C. Confavreux, S. Vukusic, P. Adeleine. Early clinical predictors and progression of irreversible disability in multiple sclerosis: an amnesic process. Brain (2003) Apr;126(Pt 4):770-82

- T. Kalincik, A. Manouchehrinia, L. Sobisek et al. Towards personalized therapy for multiple sclerosis: prediction of individual treatment response. Brain (2017) Sep 1;140(9):2426-43

\section{EFFECT OF ORAL DISEASE-MODIFYING DRUGS ON GUT MICROBIOTA IN PATIENTS WITH RELAPSING REMITTING MULTIPLE SCLEROSIS}

\author{
C. Ferri ${ }^{1}$, R. Squarzoni ${ }^{1}$, E. Baldin ${ }^{1}$, M. Castellazzi ${ }^{2}$, L. Caniatti ${ }^{3}$, E. \\ Baldi $^{3}$, M. Pugliatti ${ }^{2}$
}

${ }^{1}$ Department of Biomedical and Specialist Surgical Sciences, University of Ferrara (Ferrara); ${ }^{2}$ Department of Biomedical and Specialist Surgical Sciences; Interdepartmental Research Center for the Study of Multiple
Sclerosis and Inflammatory and Degenerative Diseases of the Nervous System, University of Ferrara (Ferrara); ${ }^{3}$ Multiple Sclerosis Center, Department of Neuroscience and Rehabilitation, S. Anna Hospital (Ferrara)

Background: Considering the gut microbiota immunomodulatory properties and its influence in the development and course of experimental autoimmune encephalomyelitis, the interest in understanding its role in the pathogenesis of multiple sclerosis (MS) is increasing. The microbiota is potentially susceptible to the action of numerous factors including MS disease-modifying drugs (DMDs) which could be responsible for decreasing dysbiosis in MS patients. This mechanism may partially explain the therapeutic effect of DMDs. Nevertheless, only a few studies tried to directly investigate the effect of DMDs on the gut microbiota.

Objective: To evaluate the effects of the oral drugs dimethylfumarate (DMF) and teriflunomide (TFN) on the gut microbiota in patients with relapsing remitting MS (RRMS).

Methods: We conducted a prospective pilot study enrolling 24 consecutive patients with RRMS, with 19 being candidates to start DMF, and 5 to start TFN. Lifestyle, laboratory, clinical and MRI data were collected at the baseline and during the follow-up period. Stool specimens were collected before starting DMDs and after one, three and six months. The microbiota analysis was performed including the measurement of alpha diversity and taxonomic analysis at phylum, family and genus level. Furthermore, a bifidobacterial profiling was obtained.

Results: DMDs were not found to be associated to major changes in microbiota composition and alpha diversity. The patients assuming DMF showed transient fluctuations of the relative abundance of the phylum Proteobacteria, initially reduced, and in some genera belonging to the Clostridiales order of the phylum Firmicutes: the genera Anaerostipes and Ruminococcaceae UCG002 were transiently increased, the genus Dorea, after one month of treatment, showed a slight reduction while the genus Clostridium gradually decreased. TFN caused a transient reduction of the abundance of Firmicutes and the genus Roseburia, the genera Alistipes and Odoribacter after an initial increased were reduced, Sutterella was transiently increased and the subspecies Adolescentis of Bifibacterium was persistently reduced.

Conclusions: The studied DMDs did not significantly modified the overall gut microbiota composition. Nevertheless, minor alterations were observed, some transient and others persistent. At genus level, most of the alterations were involving the phyla Bacteroidetes in patients assuming TFN and Firmicutes in patients assuming DMF, especially Clostridiales order, which include subspecies known to have neurotoxic potential effect. Our findings suggest that these treatments partially influence the microbiota composition but whether and to what extent these are able to determine a therapeutic effect or a side effect remains to be elucidated.

References:

- Storm-Larsen C, Myhr K-M, Farbu E, Midgard R, Nyquist K, Broch L, et al. Gut microbiota composition during a 12-week intervention with delayed-release dimethylfumarate in multiple sclerosis - a pilot trial. Mult Scler J Exp Transl Clin. (2019) Nov 15;5(4):2055217319888767

- Katz Sand I, Zhu Y, Ntranos A, Clemente JC, Cekanaviciute E, Brandstadter R, et al. Disease-modifying therapies alter gut microbial composition in MS. Neurol Neuroimmunol Neuroinflamm. (2018) Oct 26;6(1):e517

- Rumah KR, Vartanian TK, Fischetti VA. Oral multiple sclerosis drugs inhibit the in vitro growth of epsilon toxin producing gut bacterium, Clostridium perfrigens. Front Cell Infect Microbiol. (2017) Jan 25;7:11 


\section{COMPARISON OF THE 2017 AND 2010 REVISIONS OF THE MCDONALD CRITERIA IN PATIENTS WITH CIS SUGGESTIVE OF MS: A MULTICENTRE MAGNIMS STUDY}

M. Filippi ${ }^{1}$, P. Preziosa ${ }^{1}$, A. Meani ${ }^{1}$, A. Rovira ${ }^{2}$, S. Mesaros ${ }^{3}$, O. Ciccarelli $^{4}$, J. Frederiksen ${ }^{5}$, C. Enzinger ${ }^{6}$, F. Barkhof ${ }^{7}$, C. Gasperini ${ }^{8}$, E. Fainardi $^{9}$, W. Brownlee ${ }^{4}$, J. Drulovic ${ }^{3}$, X. Montalban ${ }^{10}$, S. Cramer ${ }^{5}$, M. Khalil $^{6}$, M. Hagens ${ }^{11}$, S. Ruggieri ${ }^{8}$, G. Dalla Costa ${ }^{12}$, V. Martinelli ${ }^{12}, \mathrm{~K}$. Miszkiel $^{4}$, M. Tintorè ${ }^{10}$, G. Comi ${ }^{12}$, I. Dekker ${ }^{7}$, B. Uitdehaag ${ }^{11}$, J.

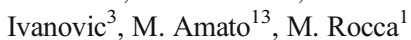

${ }^{1}$ Neuroimaging Research Unit, Institute of Experimental Neurology, Division of Neuroscience; and Neurology Unit, IRCCS San Raffaele Scientific Institute (Milano); ${ }^{2}$ Section of Neuroradiology, Department of Radiology, Hospital Universitari Vall d'Hebron (Barcelona-E); ${ }^{3}$ Clinic of Neurology, Faculty of Medicine, University of Belgrade (BelgradeSRB); ${ }^{4}$ NMR Research Unit, Queen Square MS Centre, Department of Neuroinflammation, UCL Institute of Neurology (London-UK); ${ }^{5}$ Clinic of Optic Neuritis and Clinic of Multiple Sclerosis, Department of Neurology, Rigshospitalet - Glostrup, University of Copenhagen (Copenhagen-D); ${ }^{6}$ Department of Neurology, Medical University of Graz (Graz-A); ${ }^{7}$ Department of Radiology and Nuclear Medicine, MS Center Amsterdam, Neuroscience Amsterdam UMC, (Amsterdam-NL); ${ }^{8}$ Department of Neurosciences, San Camillo Forlanini Hospital (Roma); ${ }^{9}$ Neuroradiology Unit, Department of Experimental and Clinical Biomedical Sciences 'Mario Serio', University of Florence (Firenze); ${ }^{10}$ Department of Neurology/Neuroimmunology, Multiple Sclerosis Center of Catalonia, Hospital Universitari Vall d'Hebron (Barcelona-E);

${ }^{11}$ Department of Neurology, MS Center Amsterdam, Neuroscience Amsterdam UMC (Amsterdam-NL); ${ }^{12}$ Neurology Unit, IRCCS San Raffaele Scientific Institute (Milano); ${ }^{13}$ Department of NEUROFARBA, University of Florence (Firenze)

Objectives: In 2017, a revision of the 2010 McDonald criteria for multiple sclerosis (MS) diagnosis in clinically isolated syndrome (CIS) patients has been proposed. However, its validation in a large multicenter cohort of CIS patients is still needed. In this study we aimed to compare the performance of 2017 and 2010 revisions of the McDonald criteria with respect to MS development in a large multicenter cohort of CIS suggestive of MS.

Materials: Brain and spinal cord magnetic resonance imaging (MRI) and cerebrospinal fluid (CSF) examination obtained $\leq 5$ months from CIS onset and a follow-up brain MRI acquired $\leq 15$ months from CIS onset were assessed in 626 CIS patients from 9 European MS centers.

Methods: The occurrence of a second clinical attack (clinically definite [CD] MS) was recorded. Performances of the 2017 and 2010 revisions of McDonald criteria for dissemination in space (DIS), time (DIT) and DIS plus DIT, also including OCB assessment, were evaluated with a time-dependent receiver operating characteristic curve analysis. Median time to MS diagnosis for the different sets of criteria was estimated through Kaplan-Meier curves.

Results: At the last evaluation (median=61.9 months [IQR=39.1-102.5]), 319 (51\%) of 626 patients had CDMS. At 36 months, for DIS, the 2017 MRI criteria had higher sensitivity $(0.84$ [95\% CI $=0.79-0.88]$ vs 0.77 [0.72-0.82]), lower specificity $(0.33[0.28-0.39]$ vs $0.40[0.35-0.46])$, and similar area under the curve values (AUC, 0.59 [0.55-0.62] for both). The 2017 DIS plus DIT MRI criteria had higher sensitivity (0.68 [0.63-0.74] vs 0.62 [0.56-0.68]), lower specificity $(0.55[0.49-0.61]$ vs $0.62[0.56-0.68])$, and similar AUC values (0.62 [0.58-0.66] for both). CSF-specific OCB assessment as part of the 2017 criteria revision, increased the sensitivity (0.81 [0.75-0.85]), decreased specificity $(0.40[0.34-0.46])$ and preserved AUC values $(0.60$
[0.56-0.64]). Median time to MS diagnosis was earlier with the 2017 revision compared to the 2010 or CDMS criteria, especially with OCB assessment (2017 revision with $\mathrm{OCBs}=3.6$ months [3.1-4.0], 2017 revision without $\mathrm{OCB}=11.6$ months [7.8-13.5], 2010 revision=13.9 months [12.4-15.3], $\mathrm{CDMS}=56.3$ months $[43.8-76.0])$.

Discussion: The 2017 revision of the McDonald criteria showed higher sensitivity, lower specificity, and overall similar accuracy to the 2010 McDonald criteria in predicting CDMS development. Moreover, it anticipates MS diagnosis, especially with the inclusion of OCB assessment.

Conclusions: The modifications suggested in the 2017 revision of the McDonald criteria are expected to simplify the clinical use of MRI criteria without reducing accuracy and allow an earlier diagnosis of MS.

\section{LONGER TREATMENT EXPOSURE MAY DELAY TIME TO WHEELCHAIR IN PRIMARY PROGRESSIVE MULTIPLE SCLEROSIS: A REAL-LIFE COHORT}

M. Fonderico ${ }^{1}$, E. Portaccio ${ }^{1}$, P. Iaffaldano ${ }^{3}$, L. Pastò $^{1}$, L. Razzolini ${ }^{1}$, A. Bellinvia $^{1}$, R. Fratangelo ${ }^{1}$, L. Tudisco ${ }^{1}$, G. De Luca ${ }^{4}$, P. Ragonese $^{5}$, F.

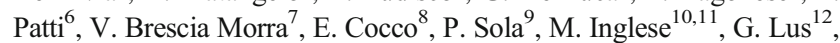
C. Pozzilli ${ }^{13}$, D. Maimone ${ }^{14}$, A. Lugaresi ${ }^{15,16}$, P. Gazzola $^{17}$, G. Comi ${ }^{18}$, I. Pesci $^{19}$, D. Spitalieri ${ }^{20}$, M. Rezzonico ${ }^{21}$, M. Vianello ${ }^{22}$, C. Avolio ${ }^{23}, \mathrm{~F}$. O. Logullo ${ }^{24}$, F. Granella ${ }^{25}$, M. Salvetti ${ }^{26,27}$, M. Zaffaroni ${ }^{28}$, C. Guaschino $^{29}$,A. Ghezzi ${ }^{30}$, G. Lucisano ${ }^{3,29}$, M. Filippi ${ }^{30,31,32}$, M. Trojano $^{3}$, M. P Amato ${ }^{1,2}$ on behalf of the Italian Multiple Sclerosis Register Centers Group

${ }^{1}$ Department NEUROFARBA, University of Florence (Firenze); ${ }^{2}$ IRCCS Fondazione Don Carlo Gnocchi (Firenze); ${ }^{3}$ Department of Basic Medical Sciences, Neurosciences and Sense Organs, University of Bari 'Aldo Moro' Policlinico (Bari); ${ }^{4} \mathrm{MS}$ Center, Neurologic Clinic, "SS. Annunziata" Hospital (Chieti); ${ }^{5}$ Department of Biomedicine, Neuroscience and Advanced Diagnostics, University of Palermo (Palermo); ${ }^{6}$ Department "G.F. Ingrassia", MS Center, University of Catania (Catania); ${ }^{7}$ Department of Neuroscience, Reproductive and Odontostomatological Sciences, Federico II University (Napoli); ${ }^{8}$ Multiple Sclerosis Center, Binaghi Hospital, Department of Medical Science and Public Health, University of Cagliari (Cagliari); ${ }^{9}$ Demyelinating Disease Center, Department of Neuroscience, Azienda Ospedaliero-Universitaria/OCSAE, Neurology Unit, University of Modena and Reggio Emilia (Modena); ${ }^{10}$ Department of Neurosciences, Rehabilitation, Ophthalmology, Genetics, Maternal and Child Health (DINOGMI), University of Genoa (Genova); ${ }^{11}$ Ospedale Policlinico San Martino, IRCCS (Genova); ${ }^{12}$ University of Campania Luigi Vanvitelli (Napoli); ${ }^{13}$ Multiple Sclerosis Center, S. Andrea Hospital, Dept. of Human Neuroscience, Sapienza University (Roma); ${ }^{14}$ Multiple Sclerosis Center, Neurology Unit, Garibaldi Hospital (Catania); ${ }^{15}$ IRCCS Istituto delle Scienze Neurologiche di Bologna (Bologna); ${ }^{16}$ Department of Biomedical and Neuromotor Sciences, University of Bologna (Bologna); ${ }^{17}$ Neurology Unit, P.A. Micone Hospital, ASL3 Genovese (Genova); ${ }^{18}$ San Raffaele Hospital - INSPE; Vita-Salute San Raffaele University (Milano); ${ }^{19}$ SM Center, Neurology, Hospital of Vaio, AUSL PR (Fidenza-PR); ${ }^{20}$ Multiple Sclerosis Center, Neurology, AORN San G. Moscati (Avellino); ${ }^{21}$ Multiple Sclerosis Center, Neurology, ASST Lariana S. Anna Hospital (Como); ${ }^{22}$ Multiple Sclerosis Center, Neurology Unit, Ospedale Regionale 'Ca' Foncello', (Treviso); ${ }^{23}$ Demyelinating Disease Center, AOU Ospedali Riuniti Di Foggia (Foggia); ${ }^{24}$ Multiple Sclerosis Center, Neurology, Hospital of Macerata (Macerata); ${ }^{25}$ Unit of Neurosciences, Department of Medicine and 
Surgery, University of Parma (Parma); ${ }^{26}$ Department of Neuroscience, Mental Health and Sensory Organs, Faculty of Medicine and Psychology, Centre for Experimental Neurological Therapies, S. Andrea Hospital/ Sapienza University (Roma); ${ }^{27}$ IRCCS Istituto Neurologico Mediterraneo (INM) Neuromed (Roma); ${ }^{28}$ ASST della Valle Olona, Multiple Sclerosis Center, S. Antonio Abate Hospital of Gallarate (Gallarate-VA); ${ }^{29}$ Center for Outcomes Research and Clinical Epidemiology (Pescara); ${ }^{30}$ Neuroimaging Research Unit, Division of Neuroscience, IRCCS San Raffaele Scientific Institute (Milano); ${ }^{31}$ VitaSalute San Raffaele University (Milano); ${ }^{32}$ Neurology and Neurophysiology Unit, IRCCS San Raffaele Scientific Institute (Milano)

Background: Except for ocrelizumab, treatment options in primary progressive multiple sclerosis (PPMS) are scarce, as randomized clinical trials failed to show efficacy in reducing disability progression in this patient population.

Objective: To investigate the effectiveness of disease-modifying treatment (DMT) on hard disability outcomes (EDSS 6 and 7) in a real-life population of PPMS patients.

Methods: Using the Italian MS Registry, we selected PPMS patients with at least three EDSS evaluations and three years of follow-up. Study baseline was defined as the first EDSS evaluation for untreated patients and the date of the first DMT initiation for treated patients. The impact of DMT on the risk of reaching EDSS 6 and 7 was assessed as a dichotomous variable (yes versus no) and as a time-dependent covariate through multivariable Cox regression models (adjusted for age at baseline, sex, first EDSS score, symptoms at onset, annualized visit rate, annualized relapse rate). We compared outcomes with an as-treated analysis and used propensity-score matching (PSM) to select cohorts with comparable baseline characteristics. DMT-exposure was also evaluated in terms of quartiles of exposure.

Results: Of the 1214 patients we included (671 females, mean \pm Standard Deviation [SD] baseline age $48.7 \pm 11.1$ years, mean EDSS score $4.1 \pm 1.8$ ), $790(65 \%)$ received a DMT during the follow-up (57\% platform and $43 \%$ highly active treatments). In the whole sample, after a mean follow-up of 11.6 \pm 6.3 years, 994 (82\%) patients reached EDSS 6 and 539 (44\%) EDSS 7. In the multivariable Cox regression models, the use of DMT analyzed as a dichotomous variable did not influence the risk of reaching EDSS 6 $(\mathrm{aHR}=1.1,95 \% \mathrm{CI} 0.95-1.28, \mathrm{p}=0.181)$ and EDSS 7 (aHR $=0.93,95 \%$ CI 0.77-1.12. $\mathrm{p}=0.454)$. However, longer DMT exposure significantly reduced the risk of reaching EDSS 7 (aHR $=0.73,95 \%$ CI $0.56-0.95, p=0.021)$. Of note, patients in the upper quartile of DMT exposure compared with those with shorter DMT exposure were younger at baseline (mean age 44.1 \pm 10.6 years; $\mathrm{p}<0.001$ ) and received the first DMT closer to the disease onset (mean time to first DMT 6.8 years $\pm 6.1 ; \mathrm{p}=0.002$ ). All these findings were confirmed in the PSM analysis.

Conclusion: Our results suggest that longer exposure to DMT may delay time to wheelchair in PPMS patients. Moreover, treating younger patients and reducing the delay to treatment initiation may improve the patients' long-term disability outcomes. To optimize treatment decision-making in PPMS further profiling of the best candidates to treatment is needed.

\section{SIPONIMOD REDUCES GREY MATTER ATROPHY IN PATIENTS WITH SECONDARY PROGRESSIVE MULTIPLE SCLEROSIS: SUBGROUP ANALYSES FROM THE EXPAND STUDY}

\author{
R. J. Fox ${ }^{1}$, D. L. Arnold ${ }^{2,3}$, G. Giovannoni ${ }^{4}$, B.A.C. Cree ${ }^{5}$, A. Bar-Or ${ }^{6}$, R.

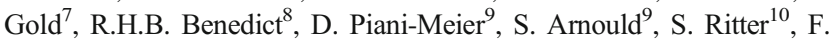 \\ Dahlke $^{9}$, G. Karlsson ${ }^{9}$, L. Kappos ${ }^{11}$, P. Vermersch ${ }^{12}$
}

${ }^{1}$ Mellen Center for Treatment and Research in Multiple Sclerosis, Neurological Institute (Cleveland-USA); ${ }^{2}$ Montreal Neurological Institute, McGill University (Montreal-CDN); ${ }^{3}$ Neuroimmunology Unit, Montreal Neurological Institute and Hospital, McGill University (Montreal - CDN); ${ }^{4}$ Blizard Institute, Barts and The London School of Medicine and Dentistry, Queen Mary University of London (LondonUK); ${ }^{5}$ UCSF Weill Institute for Neurosciences, Department of Neurology, University of California San Francisco (San FranciscoUSA); ${ }^{6}$ Center for Neuroinflammation and Experimental Therapeutics and Department of Neurology, Perelman School of Medicine, University of Pennsylvania (Philadelphia-USA); ${ }^{7}$ Department of Neurology, St Josef-Hospital/Ruhr-University Bochum (Bochum-D); ${ }^{8}$ Department of Neurology, University at Buffalo (Buffalo-USA); ${ }^{9}$ Novartis Pharma AG (Basel-CH); ${ }^{10}$ Novartis Pharmaceuticals Corporation (East Hanover-USA); ${ }^{11}$ Neurologic Clinic and Policlinic, Departments of Medicine, Clinical Research, Biomedicine and Biomedical Engineering, University Hospital, University of Basel (Basel-CH); ${ }^{12}$ Department of Neurology, University of Lille (Lille-F)

Objectives: Grey matter (GM) atrophy is more pronounced in progressive forms of multiple sclerosis and is associated with long-term irreversible disability accumulation. Siponimod significantly reduced GM atrophy in patients with SPMS, as reported previously. The aim of this study is to investigate the effect of siponimod versus placebo in reducing cortical grey matter (cGM) and thalamic atrophy in subgroups of secondary progressive multiple sclerosis (SPMS) patients in the EXPAND study.

Materials: This post-hoc analysis included the per-protocol EXPAND study data set $(\mathrm{N}=1560$, patients with major protocol deviations and data after treatment switch excluded).

Methods: Percent volume change in $\mathrm{cGM}$ and thalamus relative to baseline at Month (M)12 and M24 was assessed, and the effect of siponimod versus placebo determined using a mixedmodel for repeated measures in SPMS patient subgroups defined by baseline 'age' ( $\leq 45 />45$ years), 'disease duration' $(\leq 15 />15$ years), 'Expanded Disability Status Scale score' $(<6.0 / \geq 6.0)$, 'Symbol Digit Modalities Test score' (SDMT; $\leq 43 />43$ ), and 'pretreatment disease activity' (active/non-active).

Results: Over the course of treatment, in the placebo group, percent volume change from baseline to M24 of cGM was similar across all subgroups ( -1.17 to -0.94$)$; whereas for thalamus it differed ( -3.56 to $-1.31)$ and was more pronounced in subgroups 'with gadolinium-lesion activity' ( -3.56$)$, 'active disease' $(-2.15)$, 'age $\leq 45$ years' $(-2.12)$, 'disease duration $\leq 15$ years' $(-2.09)$, and 'SDMT score $\leq 43$ ' ( -1.99$)$. Across the subgroups studied, siponimod reduced cGM atrophy versus placebo by $48 \%$ to $116 \%$ ( $<<0.0001$ at both M12 and M24; except for "with gadolinium-lesions' $\mathrm{p}=0.0089$ at M24) and thalamic atrophy by $30 \%$ to $68 \%$ (p<0.05 at both M12 and M24; except for 'disease duration $>15$ years' $\mathrm{p}=0.1029$ at M12).

Discussion: Siponimod consistently reduced cGM and thalamic atrophy across SPMS subgroups, including those with less active and more advanced disease.

Conclusions: Combined with other analyses, these effects might potentially translate into a favorable impact on long-term clinical outcome including disability progression and cognitive decline. This study has been first presented at the Annual American Academy of Neurology Meeting 2020.

References:

- Eshaghi, F. Prados, W.J. Brownlee, D. R. Altmann, C. Tur, et al. Deep gray matter volume loss drives disability worsening in multiple sclerosis. Ann. Neurol. (2018) Feb;83(2):210-222. doi: 10.1002/ ana. 25145 
- D.L. Arnold, R. Fox, A. Bar-Or, B.A.C. Cree, G. Giovannoni, R. Gold, P. Vermersch, D. Piani Meier, S. Arnould, S. Ritter, F. Dahlke, D. Tomic, L. Kappos - Effect of siponimod on cortical grey matter and thalamic volume in patients with secondary progressive multiple sclerosis - results of the EXPAND study; ECTRIMS Online Library. Arnold D. 09/ 11/19; 278743; P382.

\section{CEREBELLAR GREY MATTER LESIONS ARE COMMON IN PEDIATRIC MULTIPLE SCLEROSIS AT CLINICAL ONSET}

\section{S. Franciotta ${ }^{1}$, M. Margoni ${ }^{2}$}

${ }^{1}$ Multiple Sclerosis Centre of the Veneto Region (CeSMuV), University Hospital of Padua, University of Padua (Padova); ${ }^{2}$ Multiple Sclerosis Centre of the Veneto Region (CeSMuV), University Hospital of Padua, Padova Neuroscience Centre (PNC), University of Padua (Padova)

Background: No data are available on the occurrence of grey matter lesions (GML) in the cerebellum of pediatric multiple sclerosis (pedMS).

Objectives: We analyzed frequency, number and topography of GML and their correlation with cerebellar-related disability in pedMS at clinical onset.

Methods: Fifteen adolescents with pedMS (12F/3M; mean age: 14.9 \pm 2.2 , range 11-17) were studied. Neurological and cognitive evaluations were done by means of EDSS, Trail Making Test - Part B (TMT-B) and Symbol Digit Modalities Test - oral version (SDMT). Cerebellar GML were investigated with double inversion recovery (DIR) and phase sensitive inversion recovery (PSIR) sequences obtained with a 3T-MRI scan.

Results: All patients had white matter lesions (WML) and/or GML in the cerebellum. A significantly higher GML number was observed on PSIR compared to DIR (mean: $2.3 \pm 2.3$ vs $1.1 \pm 1.6$; median: 2.0 (IQR, $1.0-2.0$ ) vs 1.0 (IQR, 0.0-0.0.1); $\mathrm{p}=0.004$ ). GML were observed in $14 / 15$ $(93.3 \%)$ patients and were more frequent in the posterior than in the anterior lobe (mean: $1.8 \pm 2.2$ vs $0.47 \pm 0.74$; median: 2.0 (IQR, 0.5-2.0) vs 0.0 (IQR, $0.0-1.0$ ); $\mathrm{p}=0.044$ ). No correlation was found between lesion number or topography and EDSS $(\mathrm{r}=0.12, \mathrm{p}=0.69)$, TMT-B and SDMT.

Conclusion: At clinical onset, cerebellar GML are common in pedMS, are very often asymptomatic, do not correlate with physical and cognitive disability and more frequently affect the posterior lobe.

\section{COGNITION AND SOCIO-PROFESSIONAL ATTAINMENT IN PAEDIATRIC ONSET MULTIPLE SCLEROSIS: A REAPPRAISAL AFTER 10 YEARS}

R. Fratangelo ${ }^{1}$, E. Portaccio ${ }^{1,2}$, A. Bellinivia ${ }^{1}$, L. Tudisco ${ }^{1}$, M. Fonderico $^{1}$, L. Razzolini ${ }^{1}$, L. Pastò ${ }^{2}$, B. Goretti ${ }^{1}$, C. Niccolai ${ }^{1}$, A. Ghezzi $^{3}$, M. Zaffaroni ${ }^{3}$, L. Pippolo ${ }^{3}$, L. Moiola ${ }^{4}$, M. Falautano ${ }^{4}$, R. Viterbo ${ }^{5}$, F. Patti ${ }^{6}$, C. Chisari ${ }^{6}$, P. Gallo ${ }^{7}$, A. Riccardi ${ }^{7}$, M. Borghi ${ }^{8}$, M. Simone $^{5}$, A. Bertolotto ${ }^{9}$, C. Pozzilli ${ }^{10}$, V. Bianchi ${ }^{10}$, M. Roscio ${ }^{11}$, V. Martinelli $^{4}$, G. Comi ${ }^{12}$, M. Filippi ${ }^{13}$, M. Trojano ${ }^{5}$, M. Amato ${ }^{1,2}$

${ }^{1}$ Department NEUROFARBA, Section of Neurosciences, University of Florence (Firenze); ${ }^{2}$ IRCCS Fondazione Don Carlo Gnocchi (Firenze); ${ }^{3}$ Multiple Sclerosis Center, ASST della Valle Olona, Gallarate Hospital (Gallarate-VA); ${ }^{4}$ Department of Neurology, Multiple Sclerosis Center, IRCCS Ospedale San Raffaele (Milano); ${ }^{5}$ Department of Basic Medical Sciences, Neurosciences and Sense Organs, University of Bari (Bari);
${ }^{6}$ Neuroscience, University of Catania (Catania); ${ }^{7}$ Department of Neurosciences, Multiple Sclerosis Centre, Veneto Region (CeSMuV), University Hospital of Padua (Padova); ${ }^{8}$ CRESM, Regional Reference Centre for Multiple Sclerosis, San Luigi Gonzaga Hospital (Torino); ${ }^{9}$ Neurobiology Unit, Neurology-CReSM (Regional Referring Center of Multiple Sclerosis), University of Turin, Neuroscience Institute Cavalieri Ottolenghi (NICO), AOU San Luigi Gonzaga (Orbassano-TO); ${ }^{10}$ Department of Neurology and Psychiatry, Sapienza University of Rome (Roma); ${ }^{11}$ MS Center, Hospital of Gallarate (Gallarate-VA); ${ }^{12}$ Department of Neurology, Scientific Institute University Vita-Salute San Raffaele, University Vita-Salute San Raffaele (Milano); ${ }^{13}$ Neuroimaging Research Unit, Division of Neuroscience, Neurology and Neurophysiology Unit, IRCCS San Raffaele Scientific Institute (Milano)

Background: Cognitive impairment (CI) affects nearly $30 \%$ of paediatric patients with Multiple Sclerosis (MS) and has a negative impact on school performance and participation in social activities. This study is a reappraisal of cognitive functioning and socio-professional attainment in adulthood in an Italian cohort of paediatric MS patients after 10 years from baseline neuropsychological assessment.

Objectives: To re-assess cognitive performance and its impact on socio-professional attainment in our cohort of paediatric MS patients after 10 years from baseline evaluation and to determine predictors of the individual outcomes.

Methods: Sixty-three paediatric patients were assessed at baseline and 48 followed-up after five years. To date, 31 out of these 48 patients (17 females, mean age $27.9 \pm 2.5$ years, mean EDSS $1.7 \pm 1.6$ ) were reassessed on an extensive neuropsychological battery and compared with a matched group of 31 healthy controls. CI was defined as the failure of $>2$ tests. Socio-professional attainment was evaluated on the Work and Social Assessment Scale (WSAS). Predictors of CI and WSAS score were assessed through multivariable logistic and linear models

Results: After a mean follow-up of $12.5 \pm 2.3$ years, 15 (54\%) subjects were classified as cognitively impaired. Patients with CI compared with those cognitively preserved at follow-up had higher Expanded Disability Status Scale (EDSS) score $(1.9 \pm 1.4$ vs $1.0 \pm 0.7 ; \mathrm{p}=0.046)$, lower baseline intelligence quotient (IQ) $(86.2 \pm 23.8$ vs $103.6 \pm 14.7 ; \mathrm{p}=0.025)$ and were less frequently treated with disease modifying therapy (DMT) at baseline [6 $(35.3 \%)$ vs $11(78.6 \%) ; p=0.016]$. In the regression model, CI after 10 years was related to lower IQ (OR 0.93, 95\% CI 0.87-0.99, p = 0. 027) and absence of DMT at baseline assessment (OR 17.78 95\%; 1.72-183.65, p = 0.017). As for the WSAS assessment, in the multivariable analysis the only correlate of poor functional outcome was the presence of $\mathrm{CI}$ after 10 years $(\mathrm{B}=5.2$, $\mathrm{p}=0.006$ ). Baseline predictors of worse socio-professional attainment in adulthood were $\mathrm{CI}(\mathrm{B}=6.3, \mathrm{p}=0.016)$, higher $\mathrm{EDSS}(\mathrm{B}=2.2, \mathrm{p}=0.023)$ and higher age at onset $(\mathrm{B}=0.6, \mathrm{p}=0.041)$.

Conclusions: Complete data collection is ongoing; available findings to date show that in paediatric onset subjects CI remains significant in adulthood, is related to lower cognitive reserve, higher levels of neurological impairment and delay in DMT initiation. Moreover, cognitive more than physical impairment plays a key role in predicting the subject social performance and professional outcome. Early treatment and promotion of strategies aimed at enhancing cognitive reserve are recommended in paediatric patients with MS.

Reference:

- M P Amato, B Goretti, A Ghezzi, S Lori, V Zipoli, E Portaccio, L Moiola, M Falautano, M F De Caro, M Lopez, F Patti, R Vecchio, C Pozzilli, V Bianchi, M Roscio, G Comi, M Trojano, Multiple Sclerosis Study Group of the Italian Neurological Society. Cognitive and psychosocial features of childhood and juvenile MS. Neurology (2008) May 13;70(20):1891-7 


\section{VARIATION OF RNFL THICKNESS IN MS PATIENTS AND DMTS: A LONGITUDINAL STUDY}

J. Frau, G. Coghe, B. Pintus, M. Fronza, L. Lorefice, G. Fenu, E. Cocco

Multiple Sclerosis Centre, ATS Sardegna (Cagliari)

Background: The measure of retinal nerve fiber layer (RNFL) thickness by OCT is a marker of neurodegeneration. RNFL is thinner in MS patients than in healthy controls and this process occurs over time. No study has explored the possible relation between RNFL thickness and the use of DMTs to date.

Objective: To evaluate the variation of RNFL over a follow-up of 2 years and its relation with DMTs in a group of relapsing MS patients.

Materials and methods: Patients with relapsing-remitting MS were included and underwent a spectral-domain OCT at baseline, after 6, 12 and 24 months. In patients taking DMTs, the baseline was the time of DMT initiation. Global $(\mathrm{G})$, temporal $(\mathrm{T})$, and papillo-macular bundle (PMB) sectors of RNFL have been measured. Age, gender, EDSS, age at onset, and DMTs taken during the study have been collected. DMTs were divided in first (interferon beta, glatiramer acetate, dimetilfumarate, teriflunomide) and second line (natalizumab, ocrelizumab, alemtuzumab, fingolimod). The variation of RNLF during the follow-up was studied by ANOVA. By linear regression we analysed the $G$ sector variation using as variables: DMT taken for longer time during the study by each patient, demographic and clinical features.

Results: One-hundred-one patients were included (78.2\%: females; mean age and mean age at onset: 41.3 years (SD:9.7) and 30.5 (SD:9.4), respectively). Seven patients did not take any DMT during the study, and one patient started interferon beta 17 month after the baseline. At baseline: 21 subjects started with a second line DMT; 72 started with a first line DMT, and 9 of them shifted to a second line after a mean time of 17.3 months (SD:7.1). An over-time reduction of all the RNFL sectors has been found both in right $(\mathrm{G}: \mathrm{p}<0.001$; $\mathrm{T}$ : $\mathrm{p}<0.001$; PMB: $\mathrm{p}=0.041)$ and in left eye $(\mathrm{G}: \mathrm{p}<0.001 ; \mathrm{T}: \mathrm{p}<0.001 ;$ PMB: $\mathrm{p}=0.002)$. No relation has been found between the $\mathrm{G}$ sector thinning and clinical and demographic features, but a trend versus less thinning in patients with second line DMT has been shown.

Discussion: We confirmed an over-time thinning of RNFL in patients with MS, also in a short follow-up of 2 years. No clinical and demographic variables seem to influence this phenomenon. Otherwise, even if our result is only a trend, the DMTs with more impact on the inflammation appear to slow down the thinning. A wider cohort with more patients taking second line DMTs is needed to better clarify this point.

\section{A COHORT ANALYSIS OF MS PATIENTS EXPOSED TO HIGH- DOSE CORTICOSTEROIDS}

M. Fronza, L. Lorefice, J. Frau, D. Carmagnini, G. Fenu, G. Coghe, M. Fois, E. Casaglia, S. Barca, E. Cocco

Multiple Sclerosis Center, Binaghi Hospital, University of Cagliari (Cagliari)

Background: Corticosteroids in high dose (HDC) is the recommended treatment for multiple sclerosis (MS) relapses. Most common first line treatment consists of three to five days course of $1 \mathrm{~g}$ intravenous methylprednisolone $[1,2]$. The choice of HDC duration might vary according to different clinical considerations.
Objectives: Our aim was to determine which demographic factors, comorbidities and MS clinical considerations led to the choice of a longer or shorter HDC course duration, also exploring the possible effect on clinical benefit reported by treated patients after one month.

Methods: MS subjects with a clinical relapse of MS or with MRI activity who underwent a treatment with HDC were enrolled. Demographics (sex and age), clinical features (type of relapse and EDSS) and medical history (occurrence of metabolic, immune and psychiatric comorbidities) were collected prior to HDC. After a month, subjective clinical benefit was also evaluated. Regression models were used to evaluate the relationships of HDC duration and clinical benefit after 1 month with demographic and medical variables.

Results: $101 \mathrm{MS}$ patients were enrolled (mean age 44 years, male $24.8 \%$, mean EDSS 3.1). Most of them (92.1\%) had a clinical relapse (31.1\% with multisystem involvement), $7.9 \%$ only had brain MRI activity without neurological symptoms. $66.3 \%$ were on DMDs. $36.6 \%$ had comorbidities (autoimmune comorbidities 16.8\%) and $45.8 \%$ had mood disorder. Linear regression showed that older age (p 0.039) and psychiatric comorbidities ( $p$ 0.019) correlate with the choice of shorter HDC treatment ( 3 days). Conversely, multisystem deficit relapses correlate with the choice of longer treatment (5 days) (p 0.001). Subjective clinical benefit after one month was only associated with EDSS score pre HCD treatment ( $p$ 0.004), while no association was reported with number of days of treatment.

Conclusions: Data suggests that some clinical factors, such as severity of relapse, age and comorbidities can affect the choice of HDC course duration. Shorter HDC treatments in selected patients appear to be an appropriate treatment option that does not affect the reported clinical benefit.

References:

1. Kupersmith MJ, Kaufman D, Paty DW, et al. Megadose corticosteroids in multiple sclerosis. Neurology (1994);44:1

2. Murray TJ. Diagnosis and treatment of multiple sclerosis. BMJ (2006); 332(7540):525-7

\section{COST-EFFECTIVENESS OF DIMETHYL-FUMARATE COMPARED TO TERIFLUNOMIDE FOR RELAPSING REMITTING MULTIPLE SCLEROSIS PATIENTS IN ITALY}

\author{
G. Furneri ${ }^{1}$, L. Mantovani ${ }^{2}$, P. Cortesi ${ }^{2}$, A. Cardillo ${ }^{3}$, L. Santoni ${ }^{4}$, L. \\ Prosperini $^{5}$
}

${ }^{1}$ EBMA Consulting SRL, Health Economics, Pricing \& Market Access Department (Melegnano-MI); ${ }^{2}$ Research Centre on Public Health (CESP), University of Milan - Bicocca (Monza); ${ }^{3}$ Medical Department, Biogen Italia (Milano); ${ }^{4}$ Health Economics \& Pricing, Biogen Italia (Milano); ${ }^{5}$ Department of Neurosciences, S. Camillo-Forlanini Hospital (Roma)

Objective: The objective of this economic analysis was to compare the cost-effectiveness of dimethyl-fumarate vs teriflunomide for the treatment of adult patients with relapsing-remitting multiple sclerosis (RRMS) in the Italian setting. Providing estimation of relapse burden and disability progression at different time points during patients' life.

Methods: A Markov model was used to conduct the cost-effectiveness analysis. The model measured health outcomes and costs of RRMS patients treated with either dimethyl-fumarate or teriflunomide. Data from a published mixed treatment comparison [1] were used for efficacy and safety input. Local economic data [2,3] were used to calculate costs. A supplementary analysis was carried out to assess ICER variability over time from the Italian National Healthcare Service (NHS) and societal 
perspectives. Further analyses were conducted to compare clinical effectiveness of the alternatives over time, in terms of incidence of relapse, percentage of patients with EDSS (Expanded Disability Status Scale) score $<=3$ and EDSS score $>=6$.

Results: In the base-case analysis (lifetime horizon; societal perspective) dimethyl-fumarate was dominant over teriflunomide (6.526 vs 5.953 QALYs; $€ 1.01 \mathrm{M}$ vs $€ 1.03 \mathrm{M}$, respectively). The most relevant costs savings (for patient) with dimethyl-fumarate regarded relapses (-€5,096), inpatient care $(-€ 5,767)$, informal care $(-€ 9,603)$, long-term absence/early retirement $(-€ 14,187)$. The additional analysis of ICER by time horizon shows that dimethyl-fumarate is cost-effective (i.e. ICER $<€ 50,000$ per QALY gained) at already 6 years and at 15 years in societal or NHS perspectives, respectively. Results favoured dimethyl-fumarate vs teriflunomide also for: cumulative burden of relapses $(-0.23$ and -1.37 relapses saved per patient at 1 year or 10 years, respectively), proportion of patients with $\mathrm{EDSS}<=3$ $(+4.0 \%$ at 10 years $)$ and with EDSS $>=6(-4,0 \%$ at 10 years $)$.

Conclusions: Dimethyl-fumarate is cost-effective or dominant (more effective and less costly) vs teriflunomide for the first-line treatment of relapsing-remitting multiple sclerosis in Italian setting.

References:

1. M. Hutchinson, R. J Fox, E. Havrdova, N. C Kurukulasuriya, S. P Sarda, S. Agarwal, M. Kashif Siddiqui, A. Taneja, B. Deniz. Efficacy and safety of BG-12 (dimethyl fumarate) and other disease-modifying therapies for the treatment of relapsing-remitting multiple sclerosis: a systematic review and mixed treatment comparison. Current Medical Research and Opinion (2014);30(4):613-27

2. M. Battaglia, G. Kobelt, M. Ponzio, J. Berg, D. Capsa, J. Dalén. New insights into the burden and costs of multiple sclerosis in Europe: Results for Italy. Multiple Sclerosis Journal (2017);23(2_suppl):104-16

3. K. Karampampa, A. Gustavsson, C. Miltenburger, C. Teruzzi, G. Fattore. Treatment experience, burden and unmet needs (TRIBUNE) in MS study: results from Italy. Multiple Sclerosis Journal (2012);18(2 Suppl):29-34

\section{LOWER LYMPHOCYTE COUNTS AND OLDER AGE ARE ASSOCIATED TO REDUCED MULTIPLE SCLEROSIS DISEASE ACTIVITY DURING DIMETHYL FUMARATE THERAPY}

\author{
R. Garbo ${ }^{1}$, S. Lorenzut ${ }^{2}$, I. Del Negro ${ }^{3}$, G. Merlino ${ }^{3}$, G. Gigli ${ }^{3}$, D. \\ Cargnelutti ${ }^{2}$, M. Valente ${ }^{3}$
}

${ }^{1}$ Clinic of Neurology and Neurorehabilitation, Department of Neuroscience, S. Maria della Misericordia University Hospital (Udine); ${ }^{2}$ Neurology Unit, Udine University Hospital (Udine); ${ }^{3}$ Clinical Neurology Unit, Udine University Hospital (Udine)

Aim of the study: To asses dimethyl fumarate (DMF) induced lymphopenia prevalence, risk factors and impact on disease activity in multiple sclerosis patients.

Materials: A retrospective observational study including 135 consecutive patients diagnosed with relapsing remitting multiple sclerosis, aged $>18$, treated with DMF for at least 4 months and with adequate therapy compliance was performed.

Methods: Patient's demographic, clinical, radiologic and laboratoristic data were retrospectively assessed. Absolute lymphocyte count (ALC) was acquired at baseline and was then measured periodically following routine clinical indications. Patients were considered lymphopenic if they presented at least 2 absolute lymphocyte counts under normal limit. Baseline characteristics association with lymphopenia development was assessed. Association between disease activity and basal clinical and demographic characteristic was explored. Patients were divided into two groups: non lymphopenic+lymphopenia grade 1 and lymphopenia grade $2+3$. The difference between these groups in time to first clinical relapse, first evidence of MRI disease activity and breakthrough disease activity was assessed.

Results: Mean DMF therapy duration was of $32,3 \pm 15,9$ months. 44 patients $(32,6 \%)$ developed lymphopenia, with $11(8,1 \%)$ grade 1,23 $(17,0 \%)$ grade 2 and $10(7,4 \%)$ grade 3 lymphopenia. Age at DMF therapy initiation and basal ALC were associated with lymphopenia development $(p<0,001 ; p=0,009)$. Disease activity free survival proved to be significantly longer in grade $2+3$ lymphopenia group $(p<0,001)$. On Cox regression model, age and lymphopenia grade $2+3$ were found to be predictors of disease activity (HR $=0,966 ; 95 \%$ C.I. $=0,942-0,992$; $\mathrm{p}=0,009$ for age; HR=7,308; 95\% C.I. $=2,276-23,469 ; \mathrm{p}=0,001$ for lymphopenia grade $2+3)$ and of MRI disease activity $(\mathrm{HR}=0,968 ; 95 \%$ C.I. $=0,941-0,997 ; p=0,030$ for age; HR=7,061; 95\% C.I. $=1,693-29,453$; $\mathrm{p}=0,007$ for lymphopenia grade $2+3$ ). Only lymphopenia grade $2+3$ proved to be a predictor of clinical relapses $(\mathrm{HR}=7,704 ; 95 \%$ C.I. $=1,171-64,389 ; p=0,034)$.

Discussion: DMF is a disease modifying therapy with anti-oxidant and anti-inflammatory properties. It is known to reduce ALC of about $30 \%$. Lymphopenia is a quite common adverse effect, and an important safety issue since some cases of progressive multifocal leukoencephalopathy were reported during fumaric esters treatment induced lymphopenia [1]. Whether ALC influences DMF treatment response is still a matter of debate, with some studies pointing toward a better disease control in patients showing lower ALCs [2] and others suggesting no relation between these two elements [3].

Conclusions: We found lymphopenia during DMF treatment to be protective against clinical and radiologic disease activity. Older age and lower basal lymphocyte count were found to be associated with lymphopenia development.

References:

1. Gieselbach RJ, Muller-Hansma AH, Wijburg MT, et al. Progressive multifocal leukoencephalopathy in patients treated with fumaric acid esters: a review of 19 cases. Journal of Neurology (2017); 264: 1155-64

2. Wright K, Winkler MD, Newton BD, et al. Patient outcomes influenced by reduced lymphocyte counts after dimethyl fumarate initiation. Neurol Neuroimmunol NeuroInflammation (2017); 4: 1-5

3. Longbrake EE, Naismith RT, Parks BJ, et al. Dimethyl fumarateassociated lymphopenia: Risk factors and clinical significance. Mult Scler J - Exp Transl Clin (2015);1: 205521731559699

\section{GENETIC AND ENVIRONMENTAL FACTORS IN PEDIATRIC MULTIPLE SCLEROSIS (PEDIGREE STUDY)}

A. Ghezzi ${ }^{1}$, M. Amato ${ }^{2}$, R. Bergamaschi ${ }^{3}$, E. Cocco ${ }^{4}$, S. D'Alfonso ${ }^{5}$, F. Martinelli Boneschi ${ }^{6}$, M. Pugliatti ${ }^{7}$, M. Trojano ${ }^{8}$ and the PEDIGREE Study Group

${ }^{1}$ Multiple Sclerosis Study Center, Gallarate Hospital (Gallarate-VA); ${ }^{2}$ Neurological Clinic, University of Firenze (Firenze); ${ }^{3}$ Neurological Clinic, Istituto Mondino (Pavia); ${ }^{4}$ Multiple Sclerosis Center, Binaghi Hospital (Cagliari); ${ }^{5}$ Scienze della Salute, Università Piemonte Orientale (Novara); ${ }^{6}$ Neurological Clinic, University of Milan (Milano); ${ }^{7}$ Neurological Clinic, University of Ferrara (Ferrara); ${ }^{8}$ Neurological Clinic, University of Bari (Bari) 
Background: Pediatric multiple sclerosis (PedMS) is a privileged condition to explore the mechanisms involved in MS development as close to exposure to etiological factors, with the possibility to study their role with respect to genetic background. The PEDIGREE study has been developed to explore the genetic architecture and the interaction between genes and environment predisposing to pediatric multiple sclerosis pedMS in a large Italian cohort.

Material and methods: The study is supported by FISM. The objectives are: a) to collect a cohort of PedMS patients (cohort-a) and healthy controls and to identify genetic risk factors associated with PedMS. A cohort of adult MS patients with onset before 18 years will also be included for the only genetic study (cohort-b). In particular we will i) measure the genetic burden of pediatric-onset; ii) identify genetic variants associated with the risk of PedMS by means of case-control association study at genome-wide and mitochondrial DNA levels; iii) evaluate the familial aggregation of the disease in PedMS. b) to identify the environmental factors associated with PedMS such as exposure to passive/active cigarette smoking, sunlight, vitamin D supplementation, early life nutrition, increased body mass index, poor physical activity, childhood infections, and vaccinations on the risk of MS. The exposure to environmental factors will be assessed by means of a modified version of an existing Questionnaire designed English. c) to explore gene-environment interactions associated with PedMS e) to explore the role of gut microbial in PedMS. $f$ ) to compare the gut microbial community profiles in incident cases of PedMS and control children matched for age and sex.

Results: The project has been initiated in February 2018, 28 Italian MS have obtained the approval of their own EC, and since February 2020 they are actively involved in the study. At present: 1. the questionnaire has been translated in Italian, 2. A pilot study including $>50$ healthy subjects $<18$ years and their relatives is ongoing to evaluate the feasibility of the questionnaire, 3 . participating centers have identified cases according to inclusion criteria for cohort-a and -b.

Conclusions: This study, coordinating a large number of MS centers involved in the management of pediatric MS, will provide information on the role of environmental and genetic factors in the development of MS.

Multicentric PEDIGREE Study, Supported by FISM-Fondazione Italiana Sclerosi Multipla-cod 2017/R/15

\section{EFFECT OF BDNF VAL66MET POLYMORPHISM ON MOTOR RECOVERY AFTER REHABILITATION IN PROGRESSIVE MS}

\author{
A. Giordano ${ }^{1}$, F. Clarelli ${ }^{1}$, L. Ferre' ${ }^{1}$, E. Mascia ${ }^{1}$, S. Santoro ${ }^{1}$, M. \\ Sorosina $^{1}$, M. Comola ${ }^{2}$, G. Comi ${ }^{3}$, M. Filippi ${ }^{4}$, L. Leocani ${ }^{2}$, F. Esposito ${ }^{1}$ \\ ${ }^{1}$ Laboratory of Human Genetics of Neurological Disorders, San Raffaele \\ Scientific Institute and University Hospital (Milano); \\ ${ }^{2}$ Neurorehabilitation Unit, San Raffaele Scientific Institute and \\ University Hospital (Milano); ${ }^{3}$ Institute of Experimental Neurology, \\ San Raffaele Scientific Institute and University Hospital (Milano); \\ ${ }^{4}$ Department of Neurology, San Raffaele Scientific Institute and \\ University Hospital (Milano)
}

Objectives: It is known that a single-nucleotide polymorphism mapping to the Brain-Derived Neurotrophic Factor (BDNF) gene and resulting in the valine to methionine change (Val66Met or V66M), impacts memory, cognition and motor learning [1]. Previous studies have shown that V66M polymorphism may exert a protective effect on grey matter atrophy in multiple sclerosis (MS) patients [2]; however, its influence on motor recovery after rehabilitation is not known. Our aim is to explore the possible influence of BDNF V66M polymorphism on motor recovery after rehabilitation in progressive MS subjects and to investigate the effect of two SNPs (rs2289656 and rs1212171) in NTRK2 gene, which encodes for BDNF receptor.

Materials: We retrospectively included in the study patients with primary progressive (PP) and secondary progressive (SP) MS, who were admitted to the Neurorehabilitation Unit and who had already available genetic data.

Methods: The results of tests for gait (Six-minutes Walking Test, 6MWT; 10-Meters Test, 10MT) and hand dexterity (Nine-Hole Peg Test, 9HPT) were collected at baseline and after a 4-week inpatient rehabilitation program. We used ANCOVA models to explore the effects of the selected SNPs on the change of such clinical outcomes after rehabilitation, expressed as ratio values.

Results: 100 patients (79 SP, 21 PP) with available clinical and genetic data were included in the study. Female:Male ratio was 1.27, mean age was $51 \pm 10$ years and median EDSS score was 6.0 (range: 5.5-6.5). Sixty-eight patients were carriers of the more common genotype (GG), while the remaining were heterozygote $(n=28)$ or homozygote $(n=4)$ carriers of the V66M polymorphism (Metcarriers). Among 89 subjects with available data on 6MWT, Metcarriers showed greater improvement after rehabilitation if compared to GG patients $(p=0.024$; mean variation $=0.16$ [CI: 0.02 $0.29]$ ). SNPs in NTRK2 did not show any association with 6MWT change after rehabilitation neither alone, nor in interaction with V66M. As regards data on 10MT $(n=42)$ and 9HPT $(n=45)$, no associations were found for V66M or SNPs in NTRK2.

Discussion: In the present pilot study, progressive MS patients carrying V66M polymorphism seem to have a greater improvement in walking performance at 6MWT after rehabilitation. Conclusions: These data need to be confirmed in larger and independent datasets, in order to better explore the effect of this polymorphism in MS patients undergoing intense rehabilitation program.

References:

1. Egan MF, Kojima M, Callicott JH, Goldberg TE, Kolachana BS, Bertolino A, Zaitsev E, Gold B, Goldman D, Dean M, Lu B, Weinberger DR. The BDNF val66met polymorphism affects activity-dependent secretion of BDNF and human memory and hippocampal function. Cell. (2003) Jan 24;112(2):257-69

2. Zivadinov R, Weinstock-Guttman B, Benedict R, Tamaño-Blanco M, Hussein S, Abdelrahman N, Durfee J, Ramanathan M. Preservation of gray matter volume in multiple sclerosis patients with the Met allele of the rs6265 (Val66Met) SNP of brain-derived neurotrophic factor. Hum Mol Genet. (2007) Nov 15;16(22):265968. Epub 2007 Jul 26

\section{CARDIOVASCULAR MONITORING DURING ALEMTUZUMAB INFUSION IN MULTIPLE SCLEROSIS PATIENTS}

R. Giossi ${ }^{1}$, E. Tomas Roldan ${ }^{2}$, M. Costanza ${ }^{2}$, C. Antozzi ${ }^{2}$, R. Frangiamore $^{2}$, V. Torri Clerici ${ }^{2}$, P. Confalonieri ${ }^{2}$, A. Tozzo ${ }^{3}$, R. Mantegazza $^{2}$, L. Brambilla²

${ }^{1}$ Department of Oncology and Onco-hematology, Postgraduate school of Clinical Pharmacology and Toxicology, University of Milan (Milano); ${ }^{2}$ Neuroimmunology and neuromuscular diseases Unit, IRCCS Foundation Neurological Institute Carlo Besta (Milano); ${ }^{3}$ Department of Pediatric Neuroscience, IRCCS Foundation Neurological Institute Carlo Besta (Milano) 
Objectives: Alemtuzumab (AL) is a monoclonal antibody approved for Relapsing Remitting Multiple Sclerosis (MS). Infusion adverse events (AEs) are common during AL administration. AL administration protocol was revised in 2019 by regulatory agencies due to serious cardiovascular (CV) AEs. The new recommendations included at least hourly vital signs (VS) measurement during infusion, daily electrocardiograms (EKGs) before infusion, and contraindication in patients with previous $\mathrm{CV}$ comorbidities. Our objective is to describe changes in VS and EKGs recordings in a cohort of MS patients during the first AL cycle treated at the same MS Center.

Materials: Since 2015, when AL was approved for MS in Italy, we consecutively monitored our patients for VS before and every 30 minutes during $\mathrm{AL}$ administration lasting at least 5 hours; EKGs were recorded daily before premedication. Methylprednisolone, chlorphenamine, acetaminophen, were given as premedication with ranitidine or omeprazole. All these data were recorded in clinical files at our center.

Methods: We collected heart rate (HR), systolic (SBP) and diastolic blood pressure (DBP) after premedication, before AL infusion and every 60 minutes thereafter up to hospital discharge. Bradycardia was defined as $\mathrm{HR}<60 \mathrm{bpm}$ or $\mathrm{HR}<20 \%$ if patient had bradycardia at day 1 admission. $\mathrm{HR}<45 \mathrm{bpm}$ was the cut-off for clinically relevant bradycardia. Data about clinical history, comorbidities, and concomitant medications were collected.

Results: From June 2015 to November 2019, 47 (31 female) MS patients received the first AL course. At baseline mean age was $20.1( \pm 8.1)$ years, disease duration $5.5( \pm 5.5)$ years and EDSS $2(0-4.5)$. 4 patients had CV comorbidities ( 2 hypertension, 2 premature ventricular contraction). A total of 153 EKGs were analyzed. We observed 30 nonspecific repolarization abnormalities and 18 inverted $\mathrm{T}$ waves, which were not present at baseline. Bradycardia was reported in 24 EKGs (20/ 24 at day 4 and 5). Bradycardia was recorded in 45/229 infusions. 24 patients showed at least 1 episode of bradycardia, 16/47 had clinically relevant bradycardia. SBP showed an increasing trend starting from the third day, but still within normal limits.

Discussion: AL administration was rarely associated with clinically relevant CV AEs. Nevertheless, bradycardia was frequently recorded even though usually asymptomatic, especially after the third infusion day.

Conclusion: Our data support the need for accurate monitoring of $\mathrm{AL}$ infusion. Careful monitoring should be continued for the whole protocol of $\mathrm{AL}$ infusion since $\mathrm{CV}$ events can be observed independently from the expected early infusion-related reactions likely associated to cytokine release.

\section{DEFINING THE DISEASE COURSE OF TNF BLOCKERS- ASSOCIATED MULTIPLE SCLEROSIS}

\author{
G. M. Giuffrè, M. Lucchini, V. Nociti, A. Bianco, C. De Fino, F. Losavio, \\ G. Presicce, P. Calabresi, M. Mirabella
}

UOC of Neurology, Catholic University of the Sacred Heart, Agostino Gemelli IRCCS University Hospital Foundation (Roma)

Background: Tumour Necrosis Factor alpha $(\mathrm{TNF} \alpha)$ blockers are common and effective therapeutic choices for several autoimmune diseases. Multiple studies described cases of neuroinflammatory events after exposure to this drug class, raising the hypothesis of a possible association.

Objective: To describe the disease course of patients who developed CNS demyelinating events while exposed to TNF $\alpha$ blockers.
Methods: We searched patients with previous history of autoimmune disease and exposure to TNF $\alpha$ blockers who subsequently developed an inflammatory CNS event through the electronic database of our Multiple Sclerosis Unit.

Results: We identified 10 patients ( 8 female) with a mean age of 36,8 years and a median time of exposure to TNF $\alpha$ blockers of 2 years. Psoriatic arthritis was the most common primary autoimmune disease, while adalimumab was the most used TNF $\alpha$ blocker. All patients stopped TNF $\alpha$ blockers after neurological onset. Based on clinical and radiological characteristics, the patients were divided in three groups: six clinically defined Multiple Sclerosis (CDMS), two isolated optic neuritis and two other monophasic demyelinating events. Focusing on the six CDMS patients, five were female, with a mean age at onset of 27,3 years, a median time to clinical conversion of 9 months, and a mean time of follow-up of 47.7 months. Median EDSS at onset was 2.5, while median EDSS at last follow-up was 1.0. All patients started a DMT after their second relapse, without developing any new clinical relapse nor disease progression afterwards.

Conclusions: Our study confirms the broad spectrum of CNS demyelinating disorders related to TNF $\alpha$ blockers. We describe the clinical course of TNF $\alpha$ blockers-related MS, characterized by an abrupt and highly inflammatory disease onset followed by a favourable mediumterm prognosis. These findings corroborate the hypothesis that these biological agents can trigger an inflammatory flare, especially in predisposed individuals.

\section{B-CELL DEPLETION AND EFFICACY OUTCOMES WITH OFATUMUMAB: SUBGROUP ANALYSIS FROM THE POOLED PHASE 3 ASCLEPIOS I AND II TRIALS}

S. Hauser ${ }^{1}$, A. Bar-Or ${ }^{2}$, J. Cohen ${ }^{3}$, G. Comi ${ }^{4}$, J. Correale ${ }^{5}$, P. Coyle ${ }^{6}$, A. Cross $^{7}$, J. de Seze ${ }^{8}$, D. Leppert ${ }^{9}$, X. Montalban ${ }^{10}$, K. Selmaj ${ }^{11}$, H. Wiendl $^{12}$, A. Kakarieka ${ }^{13}$, B. Li ${ }^{14}$, R. Willi ${ }^{13}$, D. Häring ${ }^{13}$, M. Merschhemke $^{13}$, L. Kappos ${ }^{9}$

${ }^{1}$ Department of Neurology, UCSF Weill Institute for Neurosciences, University of California (San Francisco-USA); ${ }^{2}$ Center for Neuroinflammation and Experimental Therapeutics and Department of Neurology, Perelman School of Medicine, University of Pennsylvania (Philadelphia-USA); ${ }^{3}$ Department of Neurology, Mellen MS Center, Neurological Institute, Cleveland Clinic (Cleveland-USA); ${ }^{4}$ University Vita-Salute San Raffaele (Milano); ${ }^{5}$ Institute for Neurological Research Dr. Raul Carrea (Buenos Aires-RA); ${ }^{6}$ Stony Brook University (Stony Brook-USA); ${ }^{7}$ Washington University School of Medicine (Saint Louis -USA); ${ }^{8}$ University Hospital of Strasbourg (Strasbourg-F); ${ }^{9}$ Neurologic Clinic and Policlinic, Departments of Medicine, Clinical Research, Biomedicine and Biomedical Engineering, University Hospital and University of Basel (Basel-CH); ${ }^{10}$ Centre d'Esclerosi Múltiple de Catalunya (Cemcat), Hospital Universitario Vall d'Hebron (BarcelonaE); ${ }^{11}$ Center for Neurology (Lodz-PL); ${ }^{12}$ University of Muenster (Muenster-D); ${ }^{13}$ Novartis Pharma AG (Basel-CH); ${ }^{15}$ Novartis Pharmaceuticals Corporation (East Hanover-USA)

Objectives: Ofatumumab, the first fully human anti-CD20 monoclonal antibody demonstrated superior efficacy versus teriflunomide in the Phase 3 ASCLEPIOS I/II relapsing multiple sclerosis trials. The aim is to evaluate the effect of subcutaneous (s.c.) ofatumumab on B-cell depletion and efficacy outcomes in subgroups of patients defined by baseline characteristics.

Materials: Patients in the ASCLEPIOS I/II trials were randomized to receive for up to 30 months either s.c. ofatumumab $20 \mathrm{mg}$ (initial loading 
regimen at Days 1, 7, and 14, maintenance doses every 4 weeks thereafter), or oncedaily oral teriflunomide $14 \mathrm{mg}$.

Methods: B-cell numbers were determined at baseline and over the course of 96 weeks in all patients and in subgroups by quartiles of baseline body weight $(\mathrm{kg})$ : Q1 $(<60.1), \mathrm{Q} 2(\geq 60.1-<70.8)$, Q3 $(\geq 70.8-<84.4)$, and Q4 ( $\geq 84.4)$. Annualized relapse rate (ARR), 3-month and 6-month confirmed disability worsening (3mCDW and $6 \mathrm{mCDW})$ were compared in different subgroups defined by demographics and baseline disease characteristics.

Results: In both the total population and across body weight subgroups, B-cell concentrations were below the lower limit of normal $(\leq 40$ cells $/ \mu \mathrm{L}$ ) for $>90 \%$ of ofatumumab-treated patients at Week 2 , for $>97 \%$ at Week 4 , and remaining between $96 \%$ and $100 \%$ over 96 weeks. Reductions in $\mathrm{ARR}, 3 \mathrm{mCDW}$, and $6 \mathrm{mCDW}$ favored ofatumumab versus teriflunomide across all subgroup analyzed. Similar efficacy was achieved in all subgroups.

Discussion: The ofatumumab dosing regimen chosen for this study achieved rapid B-cell depletion in all patients, regardless of body weight.

Conclusions: Ofatumumab demonstrated treatment benefits on clinical outcomes across different subgroups, similar to the effects observed in the overall pooled ASCLEPIOS I/II patient population.

This study has been first presented at the Annual American Academy of Neurology Meeting 2020.

ASCLEPIOS I: ClinicalTrials.gov Identifier: NCT02792218 https:// clinicaltrials.gov/ct2/show/NCT02792218

ASCLEPIOS II: ClinicalTrials.gov Identifier: NCT02792231 https:// clinicaltrials.gov/ct2/show/NCT02792231

Reference:

- S.L. Hauser, A. Bar-Or, J. Cohen, G. Comi, J. Correale, P.K. Coyle, A.H. Cross, J. de Seze, X. Montalban K. Selmaj, H. Wiendl, A. Goodyear, D.A. Häring, C. Kerloeguen, D. Tomic, R. Willi, K. Ramanathan, M. Merschhemke, L. Kappos. Efficacy and safety of ofatumumab versus teriflunomide in relapsing multiple sclerosis: results of the phase 3 ASCLEPIOS I and II trials. ECTRIMS Online Library. Hauser S. 09/13/19; 279581; 336

\section{COMPARING DISABILITY TRAJECTORIES IN RELAPSING MULTIPLE SCLEROSIS PATIENTS TREATED WITH EARLY INTENSIVE OR ESCALATION TREATMENT STRATEGIES}

P. Iaffaldano $^{1}$, G. Lucisano ${ }^{2}$, F. Caputo ${ }^{1}$, D. Paolicelli ${ }^{1}$, F. Patti ${ }^{3}$, M. Zaffaroni $^{4}$, V. Brescia Morra ${ }^{5}$, C. Pozzilli ${ }^{6}$, G. De Luca ${ }^{7}$, M. Inglese ${ }^{8}$,

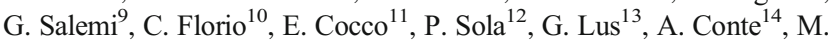
Amato $^{15}$, F. Granella ${ }^{16}$, S. Galgani ${ }^{17}$, D. Centonze ${ }^{18}$, R. Totaro ${ }^{19}, \mathrm{M}$. Rovaris $^{20}$, M. Salvetti ${ }^{21}$, R. Mantegazza ${ }^{22}$, M. Bergamaschi ${ }^{23}$, D. Maimone $^{24}$, E. Scarpini ${ }^{25}$, A. Bertolotto ${ }^{26}$, G. Comi ${ }^{27}$, M. Filippi ${ }^{27}$, M. Trojano $^{1}$ on behalf of the Italian MS Register

${ }^{1}$ Department of Basic Medical Sciences, Neurosciences and Sense Organs, University of Bari Aldo Moro (Bari); ${ }^{2}$ Center for Outcomes Research and Clinical Epidemiology, Coresearch (Pescara); ${ }^{3}$ Department of Neurosciences G.F. Ingrassia, University of Catania (Catania); ${ }^{4}$ Multiple Sclerosis Center, S. Antonio Abate Hospital (Gallarate-VA); ${ }^{5}$ Multiple Sclerosis Clinical Care and Research Center, Federico II University (Napoli); ${ }^{6}$ Multiple Sclerosis Center, S.Andrea Hospital, Dept. of Human Neuroscience, Sapienza University (Roma); ${ }^{7}$ Department of Neuroscience, Imaging, and Clinical Sciences, D'Annunzio University (Chieti); ${ }^{8}$ Department of Neurology, Rehabilitation, Ophthalmology, Genetics, Maternal and Child Health,
University of Genoa (Genova); ${ }^{9}$ Department of Biomedicine, Neuroscience and Advanced Diagnostics, University of Palermo (Palermo); ${ }^{10}$ Neurological Clinic and Multiple Sclerosis Center UA. Cardarelli Hospital (Napoli); ${ }^{11}$ Dpt Medical Science and Public Health, University of Cagliari (Cagliari); ${ }^{12}$ Department of Neurosciences, Neurology Unit, Nuovo Ospedale Civile S. Agostino/Estense, University of Modena and Reggio Emilia (Modena); ${ }^{13}$ Multiple Sclerosis Center, II Division of Neurology, Department of Clinical and Experimental Medicine, Second University of Naples (Napoli); ${ }^{14}$ Department of Human Neurosciences, Sapienza University (Roma); ${ }^{15}$ Department of NEUROFARBA, University of Florence (Firenze); ${ }^{16}$ Unit of Neurosciences, Department of Medicine and Surgery, University of Parma (Parma); ${ }^{17}$ Multiple Sclerosis Clinical Centre, San Camillo-Forlanini Hospital (Roma); ${ }^{18}$ Multiple Sclerosis Center, IRCCS Istituto Neurologico Mediterraneo (INM) Neuromed (Pozzilli-IS); ${ }^{19}$ Department of Neurology, Demyelinating Disease Center, San Salvatore Hospital (L'Aquila); ${ }^{20}$ Multiple Sclerosis Center, IRCCS Fondazione don Carlo Gnocchi ONLUS (Milano); ${ }^{21}$ CENTERS Centro Neurologico Terapie Sperimentali, Sapienza University, S.Andrea Hospital (Roma); ${ }^{22}$ Multiple Sclerosis Center, Fondazione IRCCS Istituto Neurologico 'Carlo Besta' (Milano); ${ }^{23}$ Multiple Sclerosis Center, IRCCS Mondino Foundation (Pavia); ${ }^{24}$ Multiple Sclerosis Center, Garibaldi Hospital (Nesima - CT); ${ }^{25}$ Multiple Sclerosis Center, Neurodegenerative Diseases Unit - Fondazione IRCCS Cà Granda Ospedale Maggiore Policlinico (Milano); ${ }^{26}$ Department of Neurology and Regional Referral Multiple Sclerosis Centre, University-Hospital San Luigi (Orbassano-TO); ${ }^{27}$ Department of Neurology, Vita-Salute San Raffaele University, San Raffaele Scientific Institute (Milano)

Background: Despite the therapeutic scenario for relapsing-remitting multiple sclerosis (RRMS) has widely expanded during the past 20 years, to date no consensus exists on how aggressively and timely treat RRMS patients.

Objectives: To evaluate the long-term disability outcome in a cohort of RRMS patients stratified according to two different disease modifying therapy (DMT) strategies: early intensive treatment (EIT) or moderateefficacy treatment followed by escalation to higher-efficacy DMT (ESC).

Methods: RRMS patients with $\geq 5$-year follow-up and $\geq 3$ visits after start DMT, and a first visit within 3 years from disease onset were selected from the Italian MS Registry. EIT group included patients who received, as first DMT, fingolimod, natalizumab, mitoxantrone, alemtuzumab, ocrelizumab, cladribine. ESC group included those who received the high efficacy DMT after $\geq 1$ year of glatiramer acetate, interferons, azathioprine, teriflunomide or dimethylfumarate treatment. Patients were 1:1 propensity score (PS)-matched for characteristics at the first DMT. The follow-up time from the first DMT start has been segmented into 12-month periods. The effect of early versus late start of high-efficacy DMT was assessed by the mean annual EDSS changes compared to baseline values (delta-EDSS) in EIT and ESC groups. The disability trajectories were evaluated by applying a longitudinal model for repeated measures with an autoregressive variance-covariance structure.

Results: The study cohort included 2,652 RRMS patients from 62 Italian MS centers. The PS matching procedure produced 365 pairs. The median (IQR) follow-up after the first DMT start was 8.5 (6.511.7) years. All of the ESC patients escalated to a higher-efficacy DMT after a median time of $5.1(3.1-8.4)$ years. The estimated baseline EDSS with relative confidence interval $(95 \% \mathrm{CI})$ value was $2.52(2.33-2.71)$ in the ESC group and 2.45 (2.26-2.64) in the EIT group. Mean delta-EDSS at each 12 -month period were all significantly $(\mathrm{p}<0.02)$ higher in the ESC group compared to the EIT group. In particular, the mean delta-EDSS 
differences between the two groups tend to increase from 0.1 (0.01-0.19, $\mathrm{p}=0.03)$ at 1 year to $0.30(0.07-0.53, \mathrm{p}=0.009)$ at 5 years and to 0.67 $(0.31-1.03, \mathrm{p}=0.0003)$ at 10 years.

Conclusions: Our results indicate that EIT strategy is more effective than ESC strategy in controlling disability progression and the effect tends to increase over time despite patients in the ESC group escalated to a higher-efficacy DMT.

\section{HOW TO DEFINE SECONDARY PROGRESSIVE MULTIPLE SCLEROSIS USING DIFFERENT DATA DRIVEN DEFINITIONS: A VALIDATION STUDY FROM THE ITALIAN MS REGISTER}

P. Iaffaldano ${ }^{1}$, G. Lucisano ${ }^{2}$, M. Filippi ${ }^{3}$, G. Comi ${ }^{3}$, M. Onofrj ${ }^{4}$, F. Patti ${ }^{5}$, V. Brescia Morra $^{6}$, M. Zaffaroni ${ }^{7}$, C. Pozzilli ${ }^{8}$, E. Cocco ${ }^{9}$, P. Sola ${ }^{10}$, G. Salemi ${ }^{11}$, M. Inglese ${ }^{12}$, R. Bergamaschi ${ }^{13}$, S. Galgani ${ }^{14}, \mathrm{M}$. Amato ${ }^{15}$, A. Conte ${ }^{16}, \mathrm{M}$.

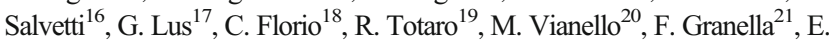
Ferraro $^{22}$, U. Aguglia ${ }^{23}$, M. Gatto ${ }^{24}$, B. Orlando ${ }^{1}$, F. Sangalli ${ }^{3}$, G. De Luca ${ }^{4}$, C. Chisari ${ }^{5}$, A. Carotenuto ${ }^{6}$, D. Baroncini ${ }^{7}$, D. Colombo ${ }^{25}$, M. Nica ${ }^{25}$, D. Paolicelli $^{1}$, M. Trojano ${ }^{1}$ on behalf of the Italian MS Register

${ }^{1}$ Department of Basic Medical Sciences, Neurosciences and Sense Organs, University of Bari Aldo Moro (Bari); ${ }^{2}$ Center for Outcomes Research and Clinical Epidemiology, Coresearch (Pescara); ${ }^{3}$ Department of Neurology, Vita-Salute San Raffaele University, San Raffaele Scientific Institute (Milano); ${ }^{4}$ Department of Neuroscience, Imaging, and Clinical Sciences, D'Annunzio University (Chieti); ${ }^{5}$ Department of Neurosciences G.F. Ingrassia, University of Catania (Catania); ${ }^{6}$ Multiple Sclerosis Clinical Care and Research Center, Federico II University (Napoli); ${ }^{7}$ Multiple Sclerosis Center, Hospital of Gallarate (Gallarate-VA); ${ }^{8}$ Multiple Sclerosis Center, S.Andrea Hospital, Dept. of Human Neuroscience, Sapienza University (Roma); ${ }^{9}$ Dpt Medical Science and Public Health, University of Cagliari (Cagliari); ${ }^{10}$ Department of Neurosciences, Neurology Unit, University of Modena and Reggio Emilia (Modena); ${ }^{11}$ Department of Biomedicine, Neuroscience and Advanced Diagnostics, University of Palermo (Palermo); ${ }^{12}$ Department of Neurology, Rehabilitation, Ophthalmology, Genetics, Maternal and Child Health, University of Genoa (Genova); ${ }^{13}$ Multiple Sclerosis Center, IRCCS Mondino Foundation (Pavia); ${ }^{14}$ Multiple Sclerosis Clinical Centre, San Camillo-Forlanini Hospital (Roma); ${ }^{15}$ Department of NEUROFARBA, University of Florence (Firenze); ${ }^{16}$ Department of Human Neurosciences, Sapienza University (Roma); ${ }^{17}$ Multiple Sclerosis Center, II Division of Neurology, Department of Clinical and Experimental Medicine, Second University of Naples Napoli); ${ }^{18}$ Department of Neurology and Stroke Unit, Multiple Sclerosis Centre (Napoli); ${ }^{19}$ Department of Neurology, Demyelinating Disease Center, San Salvatore Hospital (L'Aquila); ${ }^{20}$ O.U. Neurology, "Ca' Foncello" Hospital (Treviso); ${ }^{21}$ Unit of Neurosciences, Department of Medicine and Surgery, University of Parma (Parma); ${ }^{22}$ Centro SM, PO San Filippo Neri, ASL Roma 1 (Roma); ${ }^{23}$ Neurology Department, Magna Graecia University (Catanzaro); ${ }^{24}$ Neurology Unit, F. Miulli Hospital (Acquaviva delle Fonti-BA); ${ }^{25}$ Medical Department, Novartis Pharma Italia (Origgio-VA)

Background: A lack of prompt and accurate definition of secondary progressive multiple sclerosis (SPMS) makes difficult to recognize the transition from relapsing-remitting (RR) MS to SPMS.

Objectives: To apply two different data-driven SPMS definitions comparing their diagnostic performances.
Methods: Patient with RRMS with a follow-up $\geq 5$ years, with a current age $\geq 18$ years, and with $\geq 3$ EDSS scores recorded were selected from the Italian MS Registry. Annual incidence of SPMS conversion was reported as number of patients converting to SP every 100 patients/year. Three different SPMS definitions have been used. Data-driven definitions based on the Lorscheider's algorithm (LA) and on the EXPAND trial inclusion criteria were validated, using the neurologist's definition as gold standard, in terms of calibration, discrimination and goodness of fit by calculating: sensitivity, specificity, Positive Predictive Value (PPV), Negative Predictive Value (NPV), the Akaike information criterion (AIC), the Area Under the Curve (AUC). The overall calibration of the data-driven definitions was evaluated by the Calibration Slope test.

Results: A cohort of 10,240 RRMS patients was extracted from the Italian MS Registry. According to the neurologist judgment, 880 (8.59\%) patients were classified as SPMS in the dataset. By applying the LA and the EXPAND definition, 1,806 (17.64\%) and 1,134 (11.07\%) patients, respectively, were classified as SPMS. The annual rate of SP conversion during the follow-up was 0.74 every 100 patients/year based on the neurologist's definition, 1.57 every 100 patients/year using the LA and 0.94 every 100 patients/year applying the EXPAND definition. Both the data-driven definitions were well calibrated, with a p-value of the Calibration Slope test higher than 0.05 ( $\mathrm{LA}=0.55$; EXPAND definition=0.57). The AIC $(\mathrm{LA}=4301$; EXPAND definition=5510) and the R-Square (LA $=0.15$ vs EXPAND definition=0.05), were in favor of the LA. The LA showed a greater discrimination power (AUC: 0.83 vs 0.65 ) and a higher sensitivity (77.1\% vs $38.0 \%$ ) in comparison to the EXPAND definition. Both definitions showed similar specificity (88.0\% vs $91.5 \%)$. The PPV and the NPV were both higher using the LA than those obtained by the EXPAND definition $(37.5 \%$ vs $29.5 \%$; $97.6 \%$ vs $94.0 \%$, respectively).

Conclusions: An early identification of the SP transition is necessary to optimize management and treatment of SPMS patients. This study highlights how data-driven statistical models can improve diagnostic accuracy and efficiency to identifying the SP transition than neurologist's definition, particularly LA seems to be more accurate than a definition based on the EXPAND trial inclusion criteria.

References:

- Lublin FD, Reingold SC, Cohen JA, et al. Defining the clinical course of multiple sclerosis: the 2013 revisions. Neurology (2014) Jul 15;83(3):278-86. doi: 10.1212/WNL.0000000000000560

- Lorscheider J, Buzzard K, Jokubaitis V, et al. Defining secondary progressive multiple sclerosis. Brain (2016) Sep;139(Pt 9):2395405. doi: 10.1093/brain/aww173

- Kappos L, Bar-Or A, Cree BAC, et al. Siponimod versus placebo in secondary progressive multiple sclerosis (EXPAND): a doubleblind, randomised, phase 3 study [published correction appears in Lancet (2018) Nov 17;392(10160):2170]. Lancet. (2018);391(10127):1263-73

\section{LONG-TERM EFFICACY AND SAFETY OF SIPONIMOD IN PATIENTS WITH SPMS: EXPAND EXTENSION ANALYSIS UP TO 5 YEARS}

L. Kappos ${ }^{1}$, G. Giovannoni ${ }^{2}$, R. Gold ${ }^{3}$, R. Fox ${ }^{4}$, P. Vermersch ${ }^{5}$, R. Benedict $^{6}$, A. Bar-Or ${ }^{7}$, N. Rouyrre ${ }^{8}$, D. Piani-Meier ${ }^{8}$, S. Ritter ${ }^{9}$, A. $\mathrm{Kilaru}^{8}$, F. Dahlke ${ }^{8}$, G. Karlsson ${ }^{8}$, B. Cree ${ }^{10}$ 
${ }^{1}$ Neurologic Clinic and Policlinic, Departments of Medicine, Clinical Research, Biomedicine and Biomedical Engineering, University Hospital and University of Basel (Basel-CH); ${ }^{2}$ Blizard Institute, Barts and The London School of Medicine and Dentistry, Queen Mary University of London (London-UK); ${ }^{3}$ Department of Neurology, St Josef-Hospital/Ruhr-University Bochum (Bochum-D); ${ }^{4}$ Mellen Center for Treatment and Research in Multiple Sclerosis, Neurological Institute, Cleveland Clinic (Cleveland-USA); ${ }^{5}$ Department of Neurosurgery, 5Univ. Lille, INSERMU995, CHU Lille, FHU Imminent (Lille-F); ${ }^{6}$ Department of Neurology, University at Buffalo (BuffaloUSA); ${ }^{7}$ Center for Neuroinflammation and Experimental Therapeutics and Department of Neurology, Perelman School of Medicine, University of Pennsylvania (Philadelphia-USA); ${ }^{8}$ Medical Department, Novartis Pharma AG (Basel-CH); ${ }^{9}$ Medical Department, Novartis Pharmaceuticals Corporation (East Hanover-USA); ${ }^{10}$ Department of Neurology, UCSF Weill Institute for Neurosciences, University of California San Francisco (San Francisco-USA)

Objectives: In the EXPAND-Core, siponimod showed significant reductions in confirmed disability progression (3-/6-month $(\mathrm{m})$ CDP) and cognitive decline in typical SPMS patients. The aim of this study is to assess the long-term efficacy and safety of siponimod in patients with SPMS from the Core and Extension parts of the EXPAND study.

Materials: This analysis included patients who received $\geq 1$ dose of randomized treatment (Siponimod $2 \mathrm{mg} /$ placebo; $36 \mathrm{~m}$ Extension data cutoff [April-2019]; total study duration $\leq 5$ years).

Methods: Efficacy analyses included time-to-3m/6mCDP on Expanded Disability Status Scale, time-to-6m confirmed worsening ( $6 \mathrm{mCW}: \geq 4$ points in Symbol Digit Modalities Test) and annualized relapse rate (ARR) for continuous siponimod group (CSG: siponimod in Core + Extension) and switch group (PSG: placebo in Core/switched to siponimod in Extension). Safety was also evaluated.

Results: Of the 1224 (74\% of 1651 randomized) patients entering the Extension, 878 (72\%) were ongoing. Patients in CSG were less likely to experience $3 \mathrm{mCDP}(\mathrm{p}=0.0064)$ and $6 \mathrm{mCDP}(\mathrm{p}=0.0048)$ versus PSG. Time to $6 \mathrm{mCDP}$ was prolonged, corresponding to a delay of $54 \%$ for the 25 th percentile ( 21.0 vs. 13.6 months) in the CSG versus PSG. Median time to- $6 \mathrm{mCDP}$ was not reached for CSG (PSG: 51.7 months). Time-to-6mCW on SDMT was delayed in CSG versus PSG ( $p=0.0014)$. Risk of worsening on the SDMT was reduced by $23 \%(0.77$ [0.65-0.92]), corresponding to a delay of $62 \%$ for the 25 th percentile (CSG vs. PSG: 29.6 vs. 18.3 months). A reduction in ARR by $52 \%$ was observed in the CSG versus PSG ( 0.054 vs. $0.097 ; \mathrm{p}<0.0001)$. Incidence rates of $\mathrm{AEs} / 100$ patientyears in the long-term follow up were consistent with the controlled treatment period; no new safety findings were observed.

Discussion: Benefits of CSG over PSG gained during the controlled period are sustained for up to 5 years.

Conclusions: These results suggest a continuous effect of siponimod and underline the advantages of early treatment initiation with siponimod.

This study has been first presented at the Annual American Academy of Neurology Meeting 2020.

Reference:

- L. Kappos, A. Bar-Or, B.A.C. Cree, R.J. Fox, G. Giovannoni, R. Gold, P. Vermersch, D. L. Arnold, S. Arnould, T. Scherz, C. Wolf, E. Wallström, F. Dahlke, EXPAND Clinical Investigators Siponimod Versus Placebo in Secondary Progressive Multiple Sclerosis (EXPAND): A Double-Blind, Randomised, Phase 3 Study; Lancet (2018) Mar 31;391(10127):1263-73

\section{EVALUATION OF T AND B LYMPHOCYTOPENIA IN PATIENTS TREATED WITH OCRELIZUMAB SWITCHING FROM OTHER TREATMENTS COMPARED TO NAIVE}

D. Landi ${ }^{1}$, A. Grimaldi ${ }^{1}$, F. Bovis ${ }^{2}$, E. Signoriello ${ }^{3}$, C.G. Nicoletti ${ }^{1}$, G. Cola $^{1}$, D. Centonze ${ }^{4,5}$, G. Lus ${ }^{3}$, M.P. Sormani ${ }^{2}$, G.A. Marfia ${ }^{1,5}$

${ }^{1}$ Multiple Sclerosis Clinical and Research Unit, Department of Systems Medicine, Tor Vergata University (Roma); ${ }^{2}$ Section of Biostatistics, University of Genoa (Genova); ${ }^{3}$ Multiple Sclerosis Center, II Division of Neurology, Department of Clinical and Experimental Medicine, Second University of Naples (Napoli); ${ }^{4}$ Synaptic Immunopathology Lab, Department of System Medicine, Tor Vergata University (Roma); ${ }^{5}$ Unit of Neurology and Neurorehabilitation, IRCCS Neuromed (Pozzilli-IS)

Ocrelizumab (Ocre) is an anti-CD20 monoclonal antibody with a known major depleting effect on B cells and marginal on T cells. It is approved for clinical use in highly-active naïve multiple sclerosis (MS) patients and those not responder to previous treatment. In this study we aim to investigate the $\mathrm{B}$ and $\mathrm{T}$ cells subsets longitudinal dynamic during treatment with Ocrelizumab (Ocre) in patients with MS switching from Fingolimod (FTY) and other treatments (Dimetylfumarate, Interferon Beta, Glatiramer Acetate, Natalizumab, Teriflunomide) compared to naïve patients. A multicenter observational 2-year study was conducted in patients starting treatment with Ocre grouped in three arms: naïve (naïve), switching from FTY (pre-FTY), switching from other treatments (other). A sample of 135 patients was analysed ( 37 pre-FTY, 64 other, 34 naïve). Data about lymphocyte subtype count (CD3+, CD4+, CD8+ and CD20+) were collected at baseline and every 6 months after starting Ocre. Slope of reduction and proportion of patients with lymphocytes count below the normal lower limit was calculated. At baseline pre-FTY compared to naïve showed significant decrease of CD3+ $(1204.54+675.37$ cells $/ \mathrm{mm} 3$ vs $1735.53+653.56$, $\mathrm{p}=0.0003), \mathrm{CD} 4+(551.91+254.42$ vs $997.03+352.79, \mathrm{p}<0.0001), \mathrm{CD} 8+$ $(430.38+379.73$ vs $537.75+254.34, \mathrm{p}=0.027)$ and $\mathrm{CD} 20+(88.25+90.94$ vs $191.32+149.62, \mathrm{p}=0.021$ ) cells. During Ocre the slope of reduction of $\mathrm{CD} 3+$ in naïve patients was 5.45 cells $/ \mathrm{mm} 3 /$ week $(\mathrm{p}=0.003)$. Compared to naïve, the rate of decrease in $\mathrm{CD} 3+$ was -1.2 cells $/ \mathrm{mm} 3 /$ week in pre-FTY $(p=0.087)$ and $+0.19(p=0.012)$ in other. The slope of reduction of CD4+ was $2.00 \mathrm{cells} / \mathrm{mm} 3 /$ week in naïve $(\mathrm{p}=0.072)$. Compared to naïve the rate of reduction in $\mathrm{CD} 4+$ was +0.91 cells $/ \mathrm{mm} 3 /$ week in pre-FTY $(\mathrm{p}=0.061)$ and +1.70 cells $/ \mathrm{mm} 3 /$ week $(p=0.012)$ in other. $C D 8+$ and CD20+ cells decrease was similar among groups ( $\mathrm{p}$ for interaction between time and treatment $=$ 0.184 and 0.108 , respectively). In pre-FTY group compared to baseline the proportion of patients with $\mathrm{CD} 3+$ and $\mathrm{CD} 4+$ cells lymphopenia was unchanged (16.22\% versus $17.14 \%$; $32.35 \%$ versus $34.29 \%$ ), while the proportion of CD8+ cells was increased (8.82\% versus $25.71 \%)$. Our study confirms that Ocre may induce depletion of $\mathrm{T}$ cell subsets beyond $\mathrm{B}$ cells. Nevertheless, in pre-FTY we also observed a prolonged Tlymphocytopenia, as carry-over effect of the previous therapy. FTYinduced immunosenescence or slow immunoreconstitution may explain this finding. These results pose the need to carefully investigate the safety profile of treatment sequencing.

\section{JCV SEROSTATUS AND VIRAL REPLICATION IN PATIENTS WITH MULTIPLE SCLEROSIS TREATED WITH OCRELIZUMAB} D. Landi ${ }^{1}$, A. Grimaldi ${ }^{1}$, C. Prezioso

Pietropaolo $^{2,3}$, M.A. Marfia 
${ }^{1}$ Multiple Sclerosis Clinical and Research Unit, Department of Systems Medicine, Tor Vergata University (Roma); ${ }^{2}$ Department of Public Health and Infectious Diseases, Sapienza University (Roma); ${ }^{3}$ Microbiology of Chronic Neuro-Degenerative Pathologies, IRCCS San Raffaele Pisana (Roma); ${ }^{4}$ Virology Unit, Tor Vergata University (Roma); ${ }^{5}$ Infectious Diseases Clinic, Department of System Medicine, Tor Vergata University (Roma); ${ }^{6}$ Unit of Neurology and Neurorehabilitation, IRCCS Neuromed (Pozzilli-IS)

Rituximab has been associated with progressive multifocal leukoencephalopathy (PML) by John Cunningham Polyomavirus (JCPyV), while the long-term effects of ocrelizumab (Ocre) use, recently approved for multiple sclerosis (MS), are essentially unknown. Here we reported our preliminary data of an ongoing project aimed to explore the anti-JCPyV serostatus and the JCPyV replication in MS patients treated with Ocre. $30 \mathrm{MS}$ patients (age $41+/-9,6$ primary progressive, 16 naïve to treatments) starting treatment with Ocre were recruited. Anti-JCPyV index, JCPyV-DNA in urine and plasma samples, IgG and IgM titres and lymphocyte subsets were longitudinally assessed. At baseline $26 / 30$ patients were anti-JCPyV seropositive $(>0,4), 3 / 30$ seronegative $(<0,2)$, and $1 / 30$ was indeterminate $(>0,20$ and $<0,4) .8 / 25$ seropositive and $0 / 3$ seronegative patients had detectable JCPyV-DNA (range: $4 * 104-5 * 107$ copies) in urine; all were negative for plasma JCPyV-DNA. At 3 months (T3) 27/30 were positive for anti-JCPyV antibodies, $3 / 30$ negative. Mean anti$\mathrm{JCPyV}$ index was marginally reduced at T3 (t-test $\mathrm{p}=0.058$ ). Patients were persistently positive for urinary JCPyV DNA at T3. CD4, CD8 and $\mathrm{NK}$ counts and IgG titres did not significantly change from baseline to T3; CD19 counts were significantly lowered ( $<<0,001)$, as well as IgM titre. Our preliminary data indicate that Ocre is not associated with increased JCPyV replication; we found a discordance between anti-JCPyV titre and urine JCPyV-DNA load at baseline, suggesting a possible overestimation of PML risk. Validity of anti-JCPyV index to monitor PML risk during ocrelizumab treatment needs to be carefully assessed considering its potential longterm impact on immunoglobulin titres.

\section{PATIENT SATISFACTION AND SUSTAINABILITY SURVEY ON THE USE OF TELEMEDICINE IN MULTIPLE SCLEROSIS}

\author{
D. Landi ${ }^{1}$, C.G. Nicoletti ${ }^{1}$, G. Cola ${ }^{1}$, G. Cecchi ${ }^{1}$, A. Grimaldi ${ }^{1}$, G. \\ Mataluni $^{1}$, N.B. Mercuri ${ }^{2}$, G. Pacileo ${ }^{3}$, G.A. Marfia ${ }^{1,4}$
}

${ }^{1}$ Multiple Sclerosis Clinical and Research Unit, Department of Systems Medicine, Tor Vergata University (Roma); ${ }^{2}$ Neurology Unit, Department of Systems Medicine, Tor Vergata University (Roma); ${ }^{3}$ Centre of Research on Health and Social Care Management, SDA Bocconi School of Management, Bocconi University (Milano); ${ }^{4}$ Unit of Neurology and Neurorehabilitation, IRCCS Neuromed (Pozzilli-IS)

Restriction in the access to care during COVID-19 pandemic has raised the need for remote monitoring for several medical chronic conditions, including Multiple Sclerosis (MS). Several telemedicine applications and solutions are under development to guarantee continuity of care in these circumstances. Telemedicine might turn to be useful also in routinely management of MS patients with limited mobility, living in rural settings or far from the MS centre, lacking of transport facilities or caregivers, etc. Nevertheless, there are several potential barriers limiting wide use and uptake of telemedicine solutions (limited internet access, poor computer skills, lack of digital technology devices, etc.). In order to evaluate sustainability and patients' satisfaction, we built a web-based survey exploring patients' attitudes, awareness and opinions on telemedicine. A 37-item questionnaire was created using an electronic survey. A combination of forced choice (yes/no) and multiple-choice questions was used. The questionnaire collected data regarding demographics, employment status, residency, MS characteristics, attitude toward digital health and availability of digital devices. We emailed 1098 patients followed at the MS centre of Tor Vergata Hospital, Rome. Survey is still open. Among 408 respondents, 69\% were women, 65\% >40 years-old, 73\% not requiring assistance, $52 \%$ with disease duration $>10$ years, $53 \%$ with middle-high income, 57\% taking first-line therapies. $93 \%$ of them has home internet connection and they routinely connect by means of smartphone $(90 \%)$, PC (70\%) and tablet (30\%). The majority of respondents $(91 \%)$ use e-mail and social platforms (98\% in total, $93 \%$ WhatsApp, 35\% Skype, 25\% Zoom). 53\% of subjects have never heard about telemedicine and $98 \%$ have never attended a tele-visit, but $63 \%$ would appreciate it. Time-saving is considered the main advantage by $60 \%$ of respondents while limitation in evaluating neurological functions the main disadvantage (70\%). $30 \%$ of patients wouldn't replace in-person with tele-visit and 50\% would do it only in selected circumstances $(78 \%$ follow-up visits and $45 \%$ in case of relapses). $85 \%$ have never used digital wearable devices for home monitoring of motor or other neurological performances. These preliminary results show wide availability of digital devices and internet among MS patients and positive attitude towards the use of telemedicine making this approach sustainable in this population. Limited confidence in the use and validation of wearable devices for remote monitoring of neurological functioning represents the main cultural barrier for optimal suitability of tele-visits. Telemedicine solutions should be implemented in MS targeting specific needs and taking into account patients' ecological conditions.

\section{OCRELIZUMAB TREATMENT IN PATIENTS WITH RELAPSING-REMITTING MULTIPLE SCLEROSIS: A SINGLE- CENTER REAL-WORLD EXPERIENCE}

C. Lapucci ${ }^{1}$, G. Boffa ${ }^{2}$, M. Cellerino ${ }^{2}$, G. Novi ${ }^{3}$, E. Sbragia ${ }^{2}$, N.

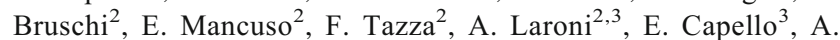
Uccelli $^{2,3}$, M. Inglese ${ }^{2,3}$

${ }^{1}$ Experimental Neurosciences Laboratory, Ospedale Policlinico San Martino IRCCS (Genova); ${ }^{2}$ DINOGMI, University of Genoa (Genova); ${ }^{3}$ Neurology, Ospedale Policlinico San Martino IRCCS (Genova)

Objectives: To provide first experience on pts with relapsing-remitting multiple sclerosis (RRMS) treated with ocrelizumab (OCR) in a realworld setting.

Methods: We collected safety and efficacy data from pts with RRMS treated with OCR at the MS Center of the University of Genoa. The probability of disability worsening-free survival, relapse-free survival, MRI-activity free-survival and NEDA-3 status was calculated with the Kaplan-Meier estimator and Cox proportional hazards regression analysis.

Results: 96 RRMS pts [60 females (62.5\%), mean (SD) age 37.3 (10.2) years] with a mean disease duration (DD) of 9.6 (9.3) years, a median (IQR) baseline EDSS of 2.5 (2-4) and a mean ARR of 0.79 (0.73). Median (IQR) number of previous DMTs was $1(0-2)$. The mean time from previous DMT discontinuation and OCR start of 209 (661) days. Reasons for previous DMTs discontinuation were (i) lack of efficacy for 45 (67\%), (ii) occurrence of adverse events for 7 (10\%) and (iii) high JCV titer during natalizumab treatment for $5(7.5 \%)$ pts. 28 pts $(29.5 \%)$ had not received any DMT prior to OCR. Naïve pts had 
significantly shorter disease duration ( 2.6 vs 12.5 years; $p<0.0001)$, had higher ARR (1.1 vs $0.7 ; \mathrm{p}=0.002$ ) and more frequently exhibited inflammatory activity on baseline MRI scan $(96.3 \%$ vs $74.6 \%$; $=0.019)$. Mean follow-up (FU) was 1.4 (1.2) years. At 1-year FU, MRI-inflammatory activity free survival was $75.9 \%$, relapse free survival was $95.9 \%$, progression free survival was $98.7 \%$. 2-years NEDA-3 status was achieved in $73.6 \%$ of pts. At multivariate analyses, adjusting for DD, ARR and baseline MRI activity, 2-years NEDA-3 status was significantly higher in naïve compared with treated pts $[90.7 \%$ versus $60.8 \%$ at the end of the observation period; HR (CI 95\%) 0.14 (0.03-0.65); $\mathrm{p}=0.012$ ]. We recorded 55 adverse events in 39 pts (4 lower respiratory tract infections; 18 upper respiratory tract infections; 7 herpes simplex-1 reactivation; 1 shingles; 8 upper urinary tract infections; 2 breast cancers). No serious infusion-associated reactions were reported.

Discussion: OCR treatment in pivotal trials of patients (pts) with RRMS was associated with high clinical efficacy and safety. However, real word data on efficacy and safety are still scarce. OCR treatment allows complete disease control in a high proportion of real-world RRMS pts, with a manageable safety profile.

Conclusions: Although ocrelizumab can control disease activity after failure of highly efficacy DMTs, its efficacy seems to be higher in naïve patients. References:

- E. Ellwardt, L. Rolfes, J. Klein, K. Pape, T. Ruck, et al. Ocrelizumab initiation in patients with MS: A multicenter observational study. Neurol Neuroimmunol Neuroinflamm (2020) Apr 9;7(4):e719. doi: 10.1212/NXI.0000000000000719

- Menge T, Dubey D, Warnke C, Hartung HP, Stüve O. Ocrelizumab for the treatment of relapsing-remitting multiple sclerosis. Expert Rev Neurother (2016);16:1131-39

- Hauser SL, Waubant E, Arnold DL, et al. B-cell depletion with rituximab in relapsing-remitting multiple sclerosis. N Engl J Med (2008);358:676-88

\section{SHORT-TERM EVALUATION OF ALEMTUZUMAB TO OCRELIZUMAB SWITCH IN MS PATIENTS WITH DISEASE ACTIVITY AFTER ALEMTUZUMAB: AN ITALIAN MULTICENTRIC STUDY}

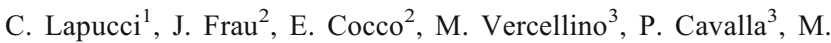
Petracca $^{4}$, R. Lanzillo ${ }^{4}$, P. Grossi ${ }^{5}$, M. Ferrò ${ }^{5}$, C. Guaschino ${ }^{6}$, M.

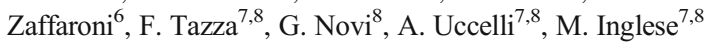

${ }^{1}$ Experimental Neurosciences Laboratory, Ospedale Policlinico San Martino IRCCS (Genova); ${ }^{2}$ MS Centre, Binaghi Hospital, ATS Sardegna (Cagliari); ${ }^{3}$ Neurology IU, Department of Neuroscience, AOU Città della Salute e della Scienza di Torino (Torino); ${ }^{4}$ Department of Neurosciences and Reproductive and Odontostomatological Sciences, University of Naples Federico II (Napoli); ${ }^{5}$ Neuroimmunology, Multiple Sclerosis Center, Neurological Department, Ospedale Maggiore (Crema$\mathrm{CR}) ;{ }^{6}$ Multiple Sclerosis Centre, ASST Valle Olona (Gallarate - VA); ${ }^{7}$ DINOGMI, University of Genoa (Genova); ${ }^{8}$ Neurology, Ospedale Policlinico San Martino IRCCS (Genova)

Objectives: To evaluate efficacy and safety in MS patients (pts) who switched to ocrelizumab (OCR) due to persistence of disease activity after 2 alemtuzumab (ALM) courses

Methods: MS pts who switched from ALM to OCR from March 2019 to March 2020 were retro- and prospectively recruited from different Italian MS Centers. Clinico-immunological and neuroradiological data about ALM treatment period, ALM-OCR interval and OCR treatment period were collected.

Results: We recruited $23 \mathrm{MS}$ pts [mean age: 35.7(SD \pm 6.8$)$; female, 40.1\%; Relapsing Remitting, (RR): $75.8 \%$, active Secondary progressive, (aSP): $24.2 \%$; mean time interval (days) from II alemtuzumab course: 87.4(SD \pm 108$)$; cumulative number of relapses: 21 ; mean number of new $\mathrm{T} 2$ and $\mathrm{Gd}+$ lesions: $4.1(\mathrm{SD} \pm 4.5)$ and 1.6( $\mathrm{SD} \pm 3.1)$; median EDSS:3(range 1-7)]. The mean follow-up (FU) from OCR start was 7.9 \pm 7.4 months. Efficacy: 4 (17.4\%) pts had a relapse after OCR start (1 pt relapsed during the interval between the first and the second OCR infusion and 3 pts after 3, 11 and 15 months from OCR start respectively), with complete recovery after steroid treatment. $4(17.4 \%)$ pts showed radiological activity with no clinical correlates at 3 months $(n=2), 4$ months $(n=1)$ and 9 months ( $=1)$. EDSS was stable except for 1 aSP patient who showed 1-year disability progression. Safety: I) Infusion Associated Reactions (IARs) occurrence was significantly lower with respect to alemtuzumab courses $(\mathrm{p}<0.05)$; (ii) infections: mild upper airways $(n=1)$, urinary infections $(n=1)$, appendicectomy $(n=1)$ and fever due to probable Sars-Cov2 infection $(n=1)$. For 12 pts, data about immunophenotype were available. Of them, no pts showed T CD4+ cell count decrease $<200 \mathrm{cell} / \mathrm{mm} 3$ at 3, 6-months and 1-year FU; complete B CD19+ cell depletion $(<5 \mathrm{cell} / \mathrm{mm} 3)$ was confirmed at 3,6 -months and 1year FU with the exception of $1 \mathrm{pt}$ that showed B CD19+ count of 12 cells/ $\mathrm{mm} 3$ at 6 month FU. 10 (43.4\%) pts developed hypogammaglobulinemia without developing associated infectious events. C) Autoimmunity: no alemtuzumab-related new complications occurred.

Discussion and Conclusions: Management of MS pts who show disease activity after 2 ALM courses represent an unsolved issue. No real-life data about the switch to OCR have been reported yet. Our study showed that, at short-term FU, the switch to OCR in MS patients who showed disease activity after 2 ALM courses is characterized by good safety and efficacy, although clinical and neuroradiological activity can be detected both in early and later phase of treatment. Longer follow-up is warranted and recruitment is still ongoing.

References:

Menge T, Dubey D, Warnke C, Hartung HP, StÃ 1/4ve O. Ocrelizumab for the treatment of relapsing-remitting multiple sclerosis. Expert Rev Neurother (2016);16:11311139

- Steingo B, Al Malik Y, Bass AD, Berkovich R, Carraro M, Fernández Ó, Ionete C, Massacesi L, Meuth SG, Mitsikostas DD, Pardo G, Simm RF, Traboulsee A, Choudhry Z, Daizadeh N, Compston DAS; CAMMS223, CAMMS03409, and TOPAZ Investigators, Long-term efficacy and safety of alemtuzumab in patients with RRMS: 12-year follow-up of CAMMS223 J Neurol (2020) Jun 24. doi: 10.1007/s00415-020-09983-1

- Rolla S, Maglione A, De Mercanti SF, Clerico M. Meaning of Immune Reconstitution after Alemtuzumab Therapy in Multiple Sclerosis. Cells. (2020) Jun 3;9(6):E1396. doi: 10.3390/ cells 9061396

\section{ALEXITHYMIA IN MULTIPLE SCLEROSIS PATIENTS: ASSOCIATION WITH DEPRESSION AND DYSFUNCTIONAL COPING}

M. Luca, C. Chisari, S. Toscano, S. Arena, E. D'Amico, S. Lo Fermo, M. Zappia, F. Patti

Department GF Ingrassia, Section of Neurosciences, University of Catania (Catania) 
Objective: Alexithymia, namely the difficulty in identifying and describing one's own feeling, may limit the individual's ability to cope with a chronic disorder. Alexithymic persons tend to misinterpret their bodily sensation and focus on the concrete aspects of life, thus showing a reduced ability to learn from past experiences. This study aims to investigate the prevalence of alexithymia in a sample of patients with multiple sclerosis (MS) and explore its possible association with depressive symptoms and dysfunctional coping.

Materials: Patients were consecutively recruited in the MS centre of the University Hospital "Policlinico-Vittorio Emanuele" of Catania and asked for their written informed consent.

Methods: All participants were administered the following scales: Toronto Alexithymia Scale (TAS-20): Consisting of three sub-scales (difficulty identifying feelings; difficulty describing feelings; externally-oriented thinking), it is the most used self-administered tool to assess alexithymic traits. Scoring is interpreted as follows: $<50$ : no alexithymia; 50-60: indeterminate alexithymia; $\geq 61$ : alexithymia; Coping orientation to problems experienced (COPE): A scale composed by 60 statements referring to coping strategies (e.g. praying, asking for help). The patient indicates the level of agreement with each statement. The "coping style" of each respondent is obtained; Hamilton rating scale for depression (HAM-D): The tool, rated by the clinician, investigates cognitive, affective and somatic symptoms of depression. Scores are interpreted as follows: $<7$ normal; 8-16: mild depression; 17-23: moderate depression; $>24$ : severe depression.

Results: Out of 83 MS patients screened, 70 patients with mean age $36.8 \pm 9.8$ years, 49 (70\%) females, disease duration $76.8 \pm 56.3$ months were finally enrolled. Relapsing-remitting type MS was present in $56(80 \%)$ patients while $14(20 \%)$ patients had secondary-progressive type. Alexithymia was found in $33(47.1 \%)$ patients. No differences in terms of demographical and clinical variables were found between alexithymic and non-alexithymic patients. HAM-D scale showed that depression symptoms were more frequent in alexithymic patients than in non-alexithymic. COPE scale evaluation showed that alexithymc patients had significantly lower score in "acceptance", "suppression", and "focalization" subitems, while they had higher scores in "negation".

Discussion: A prevalence of around $50 \%$ of alexithymia in our population of patients with MS was found. In terms of coping strategies, our results showed significantly higher rates of nonfunctional coping strategies in alexithimic patients compared to non-alexithymic.

Conclusion: Alexithymic traits are frequent among MS patients and is associated with depressive symptoms and dysfunctional coping. The assessment of alexithymic traits may represent an useful strategy to identify those patients that may need psychological support to cope with a burdening disorder such as MS.

References:

- Moussa A Chalah, Samar S Ayache. Alexithymia in multiple sclerosis: A systematic review of literature. Neuropsychologia (2017) Sep; 104:31-47

- Toshiyuki Tominaga, Hyungin Choi, Yasuhide Nagoshi, Yoshihisa Wada, Kenji Fukui. Relationship between alexithymia and coping strategies in patients with somatoform disorder. Neuropsychiatr Dis Treat . (2014);10:55-62. doi: 10.2147/NDT.S55956. Epub 2013 Dec 27

- F. Starita and G. di Pellegrino. Alexithymia and the Reduced Ability to Represent the Value of Aversively Motivated Actions. Front. Psychol. 18 December (2018) | https://doi.org/10.3389/fpsyg.2018.02587

\section{SAFETY OF NATALIZUMAB AND FINGOLIMOD TREATMENT IN MULTIPLE SCLEROSIS PATIENTS DURING COVID-19 PANDEMIC}

\author{
G. Mallucci, A. Zito, B. Dal Fabbro, M. Gastaldi, D. Franciotta, R. \\ Bergamaschi
}

IRCCS Mondino Foundation (Pavia)

Objective: The aim of the study is to verify the impact of SARS-CoV-2 on persons with multiple sclerosis (pwMS) treated with two "second line disease" modifying therapies (DMTs), that are natalizumab (NTZ) and fingolimod (FTY).

Materials and Methods: We conducted an observational cohort study on all pwMS treated with NTZ (NTZ group) or with FTY (FTY group) at the MS Center of IRCCS Mondino Foundation of Pavia, Italy. During the last week of Italian lookdown (27th April 2020-3rd May 2020), we collected data related to MS and to the possible COVID-19 infection from medical records of pwMS.

Results: We analyzed 142 pwMS: 61 in NTZ (F/M 1.90; mean age 36.7(+/-10.1); mean disease duration $10.7(+/-6.9)$; median EDSS 1.5) and 81 in FTY (F/M 1.90; mean age 45.1 (+/-9.2); mean disease duration $15.3(+/-.7)$; median EDSS 2.0). Active smokers were $16.4 \%$ and $25.9 \%$ in NTZ and FTY, respectively. Hypertension was found in $8.6 \%$ in FTY, but in none in NTZ. A clinical/laboratory pattern suggestive for COVID19 was observed in 23/61 (37.7\%) in NTZ and in 17/81 (21.0\%) in FTY. Notably, all patients with possible COVID-19 had mild symptoms, thus none of them was hospitalized.

Discussion: Currently, we do not know whether pwMS carry a different risk of SARS-CoV-2 contagion and developing serious complications when infected, especially if exposed to immunomodulatory therapies. Anyway, for some pwMS having their MS treated and well controlled may be more important than the potential danger of being exposed to SARS-CoV-2 infection. Therefore, DMT should not be a priori stopped during pandemic. Even if a possible SARS-CoV-2 infection occurred in $28 \%$ of our pwMS, none of them developed a severe COVID-19 despite they were on a "second-line" MS therapy.

Conclusions: So far, the use of NTZ and FTY does not seem to expose pwMS to a particular risk of unfavorable COVID-19 evolution.

\section{ALEMTUZUMAB FOLLOWING NATALIZUMAB: A MULTICENTRIC ITALIAN REAL-WORLD EXPERIENCE}

S. Malucchi ${ }^{1}$, M. Capobianco ${ }^{1}$, M. Malentacchi ${ }^{1}$, M. Lo Re ${ }^{1}$, R. Bottero ${ }^{1}$, F. Sperli ${ }^{1}$, A. Oggero ${ }^{1}$, F. Rinaldi ${ }^{2}$, P. Perini ${ }^{2}$, M. Radaelli ${ }^{3}$, A. Laroni ${ }^{4}$, A. Gallo ${ }^{5}$, A. Bertolotto ${ }^{1}$

${ }^{1}$ Multiple Sclerosis Centre, University Hospital San Luigi Gonzaga (Orbassano-TO); ${ }^{2}$ Multiple Sclerosis Centre, University Hospital (Padova); ${ }^{3}$ Multiple Sclerosis Centre, Papa Giovanni XIII Hospital (Bergamo); ${ }^{4}$ DINOGMI, University of Genoa (Genova); ${ }^{5}$ Department od Advanced Medical and Surgical Sciences, University of Campania (Napoli)

Background: Alemtuzumab was approved by EMA in 2013 for active relapsing-remitting multiple sclerosis patients (RRMS). Its mechanism of action, causing lymphocyte depletion followed by cellular repopulation makes it to be considered an induction treatment. For this reason the ideal candidate is an active patient in 
early phase of disease. In real world alemtuzumab is also used when many treatments before have failed. Patients with long-term natalizumab exposure and anti JCV seropositivity who stop natalizumab for the risk of PML are a category of patients for whom no specific therapeutic strategy has been established. In patients who stop natalizumab, therapeutic management is largely personalized on the individual patient and alemtuzumab can represent a choice.

Objectives: To evaluate the efficacy and safety of alemtuzumab when used in patients previously treated with natalizumab.

Methods: This is a multicentric retrospective observational study. Study population is composed by 50 RRMS patients (18 male and 32 female) with median EDSS of 2 (range 1-7) from five Italian Multiple Sclerosis Centres who stopped natalizumab treatment after a median number of 22 infusions (range 3-114). Five out of 50 patients were JCV seronegative and in these patients decision to stop natalizumab was due to radiological activity during natalizumab (2 patients), hypertransaminasemia (2 patients), patient request (1 patient); 45 patients were JCV seropositive and for these patients reason for stopping was the risk of PML. Switch to alemtuzumab was made after a median wash out period of 2 months (range 0,7-5 months). Patients underwent brain MRI at the end of natalizumab treatment, at 6 and 12 months after alemtuzumab infusion.

Results: Brain MRI at six months after alemtuzumab was available for 48 out of 50 patients and in 43 of them neither signs of disease activity nor new lesions were present; 3 patients showed new lesions and 1 patient had radiological activity. Clinical activity was absent in all the patients. Brin MRI at 12 months after alemtuzumab was available in 46 out of 50 patients and in 42 out of 48 there was no sign of disease activity. In 4 patients brain MRI showed disease activity (1 patient) or new lesions (2 pats) or both (1 pat). Clinical relapse after alemtuzumab therapy occurred in 1 out of 50 patients; this patient underwent the third infusion of drug. No patient developed PML.

Conclusions: Alemtuzumab started shortly after natalizumab interruption was highly efficacious in controlling disease course, as $87 \%$ of patients showed no evidence of clinical and radiological activity one year after treatment starting.

References:

- Plavina T, Subramanyam M, Bloomgren G, et al. Anti-JC virus antibody levels in serum or plasma further define risk of natalizumabassociated progressive multifocal leukoencephalopathy. Ann Neurol. (2014);76:802-12

- Giovannoni G, Marta M, Davis A, Turner B, et al. Switching patients at high risk of PML from natalizumab to another disease-modifying therapy. Pract Neurol. (2016);16:389-393. doi: 10.1136/ practneurol-2015-001355

- S. Malucchi, M. Capobianco, M. Lo Re et al. High-Risk PML Patients Switching from Natalizumab to Alemtuzumab: an Observational Study. Neurol Ther. (2017) Jun; 6(1): 145-52

\section{ATROPHY OF THE POSTERIOR CEREBELLAR LOBULES IS RELATED TO EPISODIC MEMORY DIFFICULTIES IN PATIENTS WITH MULTIPLE SCLEROSIS: A 3T MRI STUDY}

\author{
E. Mancuso, G. Boffa, C. Lapucci, M. Moro, E. Colombo, F. Tazza, M. \\ Cellerino, N. Bruschi, E. Sbragia, R. Meli, M. Inglese
}

Department of Neuroscience, Rehabilitation, Ophthalmology, Genetics, Maternal and Child Health (DINOGMI), University of Genoa, IRCCS Policlinic San Martino (Genova)
Background: Cerebellar pathology is associated with worse cognitive performances in patients with multiple sclerosis (MS), but the structural correlates (in terms of single cerebellar lobules) of different cognitive domains (processing speed, episodic memory and visuospatial memory) are still unknown.

Objectives: To investigate the association of specific cerebellar lobules with impairment in different cognitive domains in MS.

Methods: Patients underwent 3T brain MRI (Siemens,Prisma) and neuropsychological evaluation with assessment of the symbol digit modalities test (SDMT), the California verbal learning test (CVLT) and the Brief Visuospatial Memory Test (BVMT). 1x1x1mm T1-weighted images were used for cerebral and cerebellar segmentation. MS lesions were manually segmented on $\mathrm{T} 1$ and $\mathrm{T} 2$ weighted images.

Results: We included 70 pts [58(82.9\%) with relapsing-remitting MS]; 52 females $(74.3 \%)$, mean age $42.9( \pm 11.1)$ years] with a mean disease duration of 12.4( \pm 10.1 ) years and a median (IQR) baseline EDSS of 2.5(1-4). Mean SDMT score was 54.3( \pm 13.7$)$, mean CVLT score was $57.9( \pm 11.5)$ and mean BVMT score was $28.4( \pm 6.5)$. Mean brain T2 lesion volume (LV) was 12,2( \pm 12.3$) \mathrm{mL}$, mean brain T1LV was $7.9( \pm 9.0) \mathrm{mL}$, mean cerebellar T1LV and cerebellarT2LV were $0.2( \pm 0.3)$ and $0.3( \pm 0.5)$, respectively. 53(75.7\%) pts had at least one cerebellar MS lesion. Mean normalized brain volume (NBV) was $1389( \pm 131) \mathrm{mL}$ and mean normalized grey matter volume (NGMV) was $602( \pm 70) \mathrm{mL}$. Correlations were found between volumes of the posterior lobe of the cerebellum, lobule VIIIa, VIIIb, IX and X and CVLT scores $(0.24<\mathrm{r}<0.29,0.015<\mathrm{p}<0.046)$, with additional correlations of lobule $\mathrm{X}$ with BVMT values $(\mathrm{r}=0.29$, $\mathrm{p}=0.016$ ). Correlations were found between CVLT and BVMT scores and cerebellar T2 and T1LV $(-0.29<\mathrm{r}<-0.27,0.017<\mathrm{p}<0.024)$, but not with brain T2 and T1LV. NGM was associated with SDMT score $(r=0.26$, $\mathrm{p}=0.032$ ), but not with CVLT and BVMT scores. At multivariate analyses, accounting for the effects of age, sex, NGMV and cerebellarT2LV, atrophy of the posterior portion of the cerebellum was independently associated with worse performance at CVLT $(\mathrm{p}=0.038, \mathrm{r}=0.19)$.

Discussion: Atrophy of the cerebellar lobules VIIIa, VIIIb, IX and X is independently associated with episodic memory difficulties in MS patients, while processing speed seems to relate mostly to brain pathology. References:

- Franziska Hoche et al. The cerebellar cognitive affective/ Schmahmann syndrome scale. Brain (2018) Jan;141(1):248-70

- Sean M Tobyne et al. Cognitive impairment and the regional distribution of cerebellar lesions in multiple sclerosis. Mult Scler (2018) Nov;24(13):1687-95

\section{DIGITAL APPROACHES FOR A PATIENT-CENTRED REALITY DURING COVID19 OUTBREAK: QUESTIONS FROM A MULTIPLE SCLEROSIS CENTRE IN SOUTHERN ITALY}

A. Manni, F. Pastore, F. Caputo, B. Orlando, M. La Cava, P. Iaffaldano, M. Trojano, D. Paolicelli

Department of Basic Medical Sciences, Neurosciences and Sense Organs, University of Bari (Bari)

Objectives: The COVID-19 pandemic made patients with chronic diseases facing the difficulty of keeping in touch with their healthcare provider. Among these, people with multiple sclerosis (pwMS), who immediately were classified as at-risk population, because of their comorbidity, the use of disease modifying treatments (DMTs) affecting the immune system and their well known risk of death from respiratory and infectious diseases. This led to the improvement of telemedicine and video 
consultations. We aimed to quantify the number of patients who used Mobile Health (M-health) services during the lockdown period, evaluate their clinical and demographic characteristics and analysing the events managed by the Multiple Sclerosis (MS) staff remotely.

Materials and Methods: SMcare2.0 is an APP developed by the digital health staff of the MS Center of Bari, for an ECTRIMS MS nurse fellowship. The main objective of this tool is to provide remote M-health services: to perform video-consultations, to post videos illustrating rehabilitation and explaining major symptoms, to answer pwMS's questions in real time, sending them notifications directly via app. We collected data on the use of SMcare2.0 APP and compared them with the same period in the previous year. Using keywords, the main events managed by healthcare personnel during the pandemic phase were collected.

Results: From March to June 2020, 163 people (75\%female, 97\% RRMS, mean age 38.9 years) downloaded the app, using its services 107351 times. The most frequently reported questions were: Disease Modifying Drugs (DMDs) management (72 times), follow-up tests (MRI / blood exams, 66 times), Covid emergency (45 times), symptoms management (45 times), DMTs-related adverse events and therapy switch (18times each), medical certification (12times), pregnancy (6 times). The Smcare2.0 APP was also used to perform 21 tele-consultations. During the same period of the previous year a total of 19 pwMS (70\%female, $94 \%$ RRMS, mean age 37.8 years) installed the APP, using it 221 times.

Discussions and Conclusions: During Covid19 outbreak the use of Mhealth allowed physicians to guarantee social distancing, while providing the required assistance. However, we wonder why our tool did not address the expectations of individuals with progressive, disabling forms of MS, given the low number of downloads in this disease form. In progressive MS mobility limitations represent a major hurdle to access comprehensive and coordinated care, and these patients are exceedingly likely to develop infectious secondary complications. Therefore, home-based, patient-centred, comprehensive house call programs should be implemented to be easily accessible in progressive MS people.

References:

- Buonomo A, Brescia Morra V, et al. COVID-19 prevention and multiple sclerosis management: The SAFE pathway for the postpeak. Mult Scler Relat Disord. (2020) Jun 10;44:102282

- Brownlee W., Bourdette D., Broadley S., Killestein J., Ciccarelli O. Treating multiple sclerosis and neuromyelitis optica spectrum disorder during the COVID-19 pandemic. Neurology. (2020); 94(22):949-52

- Healey K, Zabad R, et al. Multiple Sclerosis at Home Access (MAHA): An Initiative to Improve Care in the Community. Int $\mathrm{J}$ MS Care (2019) May-Jun;21(3):101-12

\section{VERBAL FLUENCY DEFICITS IN MULTIPLE SCLEROSIS: SWITCHING AND CLUSTERING STRATEGIES}

\author{
M. C. Mantovan, M. Ravagnan ${ }^{2}$, A. Perri ${ }^{3}$, E. Cagliari ${ }^{3}$, A. Boccignone ${ }^{4}$, \\ R. De Gennaro ${ }^{2}$, G. Roversi ${ }^{2}$, S. De Biase ${ }^{2}$, R. Quatrale $^{2}$
}

Department of Neurological Sciences, Ospedale dell'Angelo, AULSS3 Serenissima (Venezia); ${ }^{2}$ Department of Neurology, AULSS3 Serenissima (Mestre-VE); ${ }^{3}$ Department of Neuroradiology, AULSS3 Serenissima (Mestre-VE); ${ }^{4}$ Department of Physical Medicine and Rehabilitation, AULSS3 Serenissima (Mestre-VE)

Objective: The aim of this study is to investigate the capacity of patients with Multiple Sclerosis (MS) to operate hypothetical switches between distant cortical areas through subcortical pathways while performing a verbal fluency task.
Materials and Methods: 20 patients with R-R MS and 20 healthy subjects (NC) matched for sex, age and educational level were recruited. The patients and the NC performed a multitasking verbal fluency task which consists of a phonemic fluency task and semantic switching. Clinical and brain magnetic resonance imaging (MRI) assessment were obtained from the patients. The phonological fluency word production and the semantic switches were recorded. A Wilcoxon non parametric test was used to compare the performance of controls and patients. A Spearman non parametric correlation test was used to investigate the correlations between fluency performance, age, school, disease duration, EDSS score. A subject by subject analysis was performed to compare verbal performance of the MS and the brain MRI lesions.

Results: The verbal fluency of MS patients is significantly reduced compared to $\mathrm{NC}$ ( $\mathrm{p}$ value 0.001 ), while the number of semantic categories is not significantly different in the two groups. The percentage of switches with respect to the words produced is not significantly different in the two groups. Otherwise, the percentage of switches compared to the number of semantic categories produced is significantly lower in MS patients than in NC (p value 0.004). An inverse correlation was found between the verbal performances of the MS and their age, disease duration and degree of disability. The load of frontal and periventricular lesions influences the verbal performance of patients with MS.

Discussion: In our MS group semantic memory is preserved, but lexical-semantic recovery during the fluency task occurs with a lower number of switches compared to NC. This result may suggest a different lexical recovery mechanism in MS patients through "nodes" of less extensive connections than in NC. The NC perform a greater number of switches, probably because they use more extensive subcortical connections. Therefore, we assume a different lexical-semantic access mechanism of "intra-territorial" connections in MS and a lexical-semantic access mechanism of connections "Inter-territorial" in healthy subjects.

Conclusions: Our results therefore confirm the hypothesis of damage to the long-way connectivity systems in MS. However, we cannot exclude a role of the frontal lobe pathology as a guide for the activation of network of associative areas where semantic knowledge is deposited.

References:

- R. Manca, B. Sharrack, D. Paling, Ian D Wilkinson, A Venneri. Brain connectivity and cognitive processing speed in multiple scelrosis: a systematic review. J of Neurological Sciences (2018);388:115-27

- R.G. Viterbo, P. Iaffaldano, M. Trojano. Verbal fluency deficits in clinically isolated syndrome suggestive of multiple sclerosis. J. of Neurological Sciences (2013);330:56-60

- E. Abaf, J. Sepulcre, E.H. Martinez-Lapiscina, I. Zubizareta, J. Garcia-Ojalvo, P. Villoslada. The analisys of semantic network in multiple sclerosis identifies preferential damage of longe-range connectivity. Multiple Sclerosis and Related Disorders (2015);4: $387-$ 94

\section{A MULTICENTRE, REAL-LIFE STUDY ON THE RISK OF LYMPHOPENIA AND INFECTIONS DISCLOSES A FAVOURABLE AND MANAGEABLE SAFETY PROFILE OF CLADRIBINE IN MS PATIENTS}

M. Margoni ${ }^{1}$, P. Annovazzi ${ }^{2}$, L. Prosperini ${ }^{3}$, R. Capuano ${ }^{4}$, A. Gallo ${ }^{4}$, J.

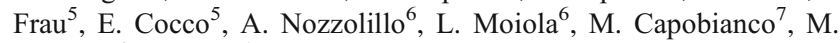
Zaffaroni $^{2}$, P. Perini ${ }^{1}$ 
${ }^{1}$ Department of Neuroscience, University of Padua (Padova); ${ }^{2}$ Multiple Sclerosis Centre, ASST Valle Olona (Gallarate-VA); ${ }^{3}$ Department of Neurosciences, S. Camillo-Forlanini Hospital (Roma); ${ }^{4}$ Multiple Sclerosis Centre, I Division of Neurology, University of Campania "Luigi Vanvitelli" (Napoli); ${ }^{5}$ Multiple Sclerosis Centre, Ospedale Binaghi, ATS Sardegna-Università di Cagliari (Cagliari); ${ }^{6}$ Neurology Unit, IRCCS San Raffaele Scientific Institute (Milano); ${ }^{7}$ Regional Referral Multiple Sclerosis Centre, Department of Neurology, University-Hospital San Luigi (Orbassano-TO)

Background: Lymphopenia monitoring during treatment with disease modifying drugs for MS is relevant because of the potential increased risk of infections. Lymphopenia is an anticipated effect of cladribine (CLD) treatment, given its mechanism of action.

Objectives: We aimed to i) characterize the absolute lymphocyte count (ALC) changes, and ii) evaluate the risk of infections in CLDtreated RRMS patients. ALCs were graded according to the Common Terminology Criteria for Adverse Events (CTCAE v5.0).

Materials and methods: In this observational multicentre study, demographic, clinical and MRI data of the patients included in the Free Of Charge CLD program were collected. ALC was also collected at baseline (before therapy initiation) and at month 3, 7, $12,15,19$ and 24 .

Results: 236 patients were enrolled in 56 Italian MS Centres (71\% F; mean age: $39+11.5$ years; mean disease duration: $10+8.5$ years). The median baseline EDSS was 3.0 (quartiles 1.5-3.5; range 0-6.5). 53 patients $(22.5 \%)$ were treatment naïve, $107(45.3 \%)$ switched to CLD from first line DMDs (for inefficacy), 76 (32.2\%) switched to CLD after a second line therapy (33/76 for safety reason, 43/76 for inefficacy). Mean follow up was $12.2+5$ months. At baseline, median ALC was $1615.0 \mathrm{cell} / \mathrm{mm} 3$ (quartiles, $1300.0-2200.0$ ). At month 3 , ALC was available in 190/236 and 101/190 had lymphopenia: 12 (6.3\%) grade 3, $47(24.7 \%)$ grade 2 and $42(22.1 \%)$ grade 1 . Among patients presenting grade 3 at month 3 , only one had persistent ALC $<500$ cell/mm3 at month 7 . At month 7, 1/180 $(0.6 \%)$ had grade 4 lymphopenia, $1 / 180(0.6 \%)$ grade $3,43 / 180(23.9 \%)$ grade 2 and $32 / 180$ $(17.8 \%)$ grade 1 . Up to date, $159 / 236$ patients were re-treated. No retreatment was delayed because of grade 4 lymphopenia. No patient presented grade 4 lymphopenia at month 15, 6/89 (6.6\%) experienced grade $3,37 / 89(40.7 \%)$ grade $2,17 / 89(18.7 \%)$ grade 1 . At month 19 , $1 / 38(2.6 \%)$ presented grade 3 lymphopenia, $11 / 38(29.0 \%)$ grade 2 and $9 / 38(23.7 \%)$ grade 1 . At month $24,1 / 9(11.1 \%)$ patient presented grade 3 and 1/9 (11.1\%) presented grade 4 lymphopenia. During treatment course, 15 patients experienced infections ( $1 \mathrm{VZV}, 3 \mathrm{HSV}$ ), none occurring in grade 3 or 4 lymphopenia.

Conclusions: In our study, the risk of grade 3 and 4 lymphopenia was lower compared to that observed in RCT. Moreover, grade 3 lymphopenia was transient in the majority of the patients. Compared to RTC, a more favourable CLD safety profile emerged in our study.

\section{ARE WE READY FOR PRECISION MEDICINE IN MULTIPLE SCLEROSIS? A WEB-BASED SURVEY ACROSS ITALY AND EUROPE}

\author{
F. Martinelli Boneschi ${ }^{1}$, S. D'Alfonso ${ }^{2}$, M. Pozzato ${ }^{3}$, A. Caserini ${ }^{4}$, B. \\ Hemmer $^{5}$, G. Celius ${ }^{6}$, P. Villoslada ${ }^{7}$, A. Goris ${ }^{8}$, M. Leone ${ }^{9}$, S. Kalra ${ }^{10}$, \\ P. Tienari ${ }^{11}$, F. Piehl ${ }^{12}$, I. Kochum ${ }^{12}$
}

${ }^{1}$ Department of Neurology, IRCCS Fondazione Ca' Granda Policlinico Maggiore, University of Milan (Milano); ${ }^{2}$ Department of Health
Sciences, University of Eastern Piedmont (Novara); ${ }^{3}$ Neurology Unit and MS Center, Dino Ferrari Centre, Neuroscience Section, Department of Pathophysiology and Transplantation, Fondazione IRCCS Ca' Granda Ospedale Maggiore Policlinico, University of Milan (Milano); ${ }^{4}$ University of Milano-Bicocca (Milano); ${ }^{5}$ Department of Neurology, Klinikum rechts der Isar, TUM School of Medicine, Technical University of Munich (Munich-D); ${ }^{6}$ Department of Neurology, Oslo University Hospital and University of Oslo (Oslo-N); ${ }^{7}$ Institut d'Investigacions Biomediques August Pi Sunyer, University of Barcelona (Barcelona- E); ${ }^{8}$ Department of Neurosciences, KU Leuven (Leuven-B); ${ }^{9}$ Department of Neurology, IRCCS Casa Sollievo della Sofferenza (San Giovanni Rotondo- FG); ${ }^{10}$ Royal Stoke MS Centre of Excellence, Neurology Department, University Hospital North Midlands NHS Trust; Institute for Science and Technology in Medicine, Keele University Medical School (Newcastle-UK); ${ }^{11}$ Department of Neurosciences, Helsinki University Hospital (Helsinki- FIN); ${ }^{12}$ The Karolinska Neuroimmunology \& Multiple Sclerosis Centre, Center for Molecular Medicine, Department of Clinical Neurosciences, Karolinska Insitutet (Stockholm-S)

Objectives: 1) To assess how neurologists across European countries view the role of body-fluid biomarkers in clinical practice; 2) To survey clinical practices of diagnostic work up, therapy selection and monitoring, and frequency of data collection and clinical and paraclinical measurements.

Material and Methods: We designed a web-based survey to assess the willingness and interest of European neurologists working with MS to implement precision medicine in their routine clinical practice. This study is a part of the EU-funded MULTIPLEMS grant. The survey had three parts: a) demographics of respondents; b) opinion of the role of predictive, diagnostic, disease-activity and treatment-response body-fluid biomarkers in clinical practice; c) survey of clinical practice and management of MS cases (including therapy choice and use of biomarkers) by evaluating 5 clinical cases with different characteristics (therapeutic management in drug naive patients and in patients displaying different forms of remaining disease activity, as well as stopping therapy in stable diasease since long).

Results: 194 neurologists across 11 European countries responded to the survey, with a mean response rate of $45 \% .57 .7 \%$ were male and the mean age was 49.8 years. The importance of biomarkers in clinical practice was rated from 1 (low) to 7 (high), and it was generally high: 4.1 for predictive and disease-activity biomarkers, 5.2 for treatment-response and 5.7 for diagnostic biomarkers, with neurologists in Belgium, Denmark, Spain, Sweden and UK being the most positive. Determination of cerebrospinal fluid (CSF) oligoclonal bands was considered the most established biomarker for diagnosis (98.5\% of neurologists), prediction $(56.7 \%)$ and disease activity $(36.5 \%)$, trailed by antiaquaporin $4(90.7 \%)$ and anti-myelin oligodendrocyte antibodies $(85.1 \%)$ for diagnosis. Anti-JC (93.8\%) and varicella virus (61.9\%) and anti-drug (natalizumab (74.7\%) and interferon-beta $(68.6 \%)$ ) were considered useful in context of therapy selection and monitoring by most neurologists, while neurofilament levels in CSF and serum and vitamin D levels were less established. Therapeutic management in the five case examples varied widely, likely as a result of differences in local and national guidelines.

Conclusions: European MS neurologists express a positive opinion on the role of body-fluid biomarkers to manage MS in clinical practice, however, these seem still to have had a limited impact on therapeutic management and selection, which also varied markedly across countries. This underscores the need for further research in this area. 


\section{HARMONIZATION OF REAL-WORLD STUDIES IN MULTIPLE SCLEROSIS: RETROSPECTIVE ANALYSIS FROM THE RIREMS GROUP}

M. Moccia ${ }^{1}$, P. Annovazzi ${ }^{2}$, M. Buscarinu ${ }^{3}$, M. Calabrese ${ }^{4}$, P. Cavalla ${ }^{5}$, C. Cordioli ${ }^{6}$, M. Di Filippo ${ }^{7}$, D. Ferraro ${ }^{8}$, A. Gajofatto ${ }^{4}$, A. Gallo ${ }^{9}$, R. Lanzillo $^{1}$, A. Laroni ${ }^{10}$, L. Lorefice ${ }^{11}$, S. Mallucchi ${ }^{12}$, V. Nociti ${ }^{13}$, D. Paolicelli $^{14}$, F. Pinardi ${ }^{15}$, L. Prosperini ${ }^{16}$, M. Radaelli ${ }^{17}$, P. Ragonese ${ }^{18}$,

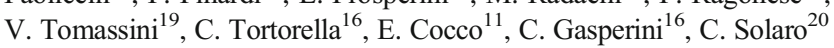

${ }^{1}$ Department of Neuroscience, Federico II University of Naples (Napoli); ${ }^{2}$ MS Centre, Gallarate Hospital (Varese); ${ }^{3}$ Department of Neurosciences, Sapienza University (Roma); ${ }^{4}$ Department of Neuroscience, University of Verona (Verona); ${ }^{5}$ Department of Neurosciences, City of Health \& Science University Hospital (Torino); ${ }^{6}$ Multiple Sclerosis Center, ASST Spedali Civili di Brescia (Brescia); ${ }^{7}$ Medicine Department, University of Perugia (Perugia); ${ }^{8}$ Department of Biomedical, Metabolic and Neurosciences, University of Modena and Reggio Emilia (Modena); ${ }^{9}$ Department of Advanced Medical and Surgical Sciences - University of Campania "L. Vanvitelli" (Napoli); ${ }^{10}$ Department of Neuroscience, University of Genoa (Genova); ${ }^{11}$ Department of Medical Sciences and Public Health, University of Cagliari (Cagliari); ${ }^{12}$ Neurology Unit, San Luigi Gonzaga Hospital (Orbassano-TO); ${ }^{13}$ Neurology Unit, 'A. Gemelli' Hospital (Roma); ${ }^{14}$ Department of Basic Medical Sciences, Neuroscience and Sense Organs, University of Bari (Bari); ${ }^{15}$ Multiple Sclerosis Centre Unit, Rehabilitation Hospital (Bologna); ${ }^{16}$ Department of Neurosciences, San Camillo Forlanini Hospital (Roma); ${ }^{17}$ Department of Neurology, San Raffaele Hospital (Milano); ${ }^{18}$ Department of Biomedicine Neurosciences and Advanced Diagnostic, University of Palermo (Palermo); ${ }^{19}$ Department of Neurosciences, University of Chieti-Pescara (Chieti);

${ }^{20}$ Rehabilitation Department, Moncrivello Hospital (Vercelli)

Background: Worldwide multiple sclerosis (MS) centers have coordinated their efforts to use data acquired in clinical practice for real-world observational studies. In this retrospective study, we aim to harmonize outcome measures, and to evaluate their heterogeneity within the Rising Italian Researchers in MS (RIReMS) study group.

Methods: RIReMS members filled in a structured questionnaire evaluating the use of different outcome measures in clinical practice. Thereafter, thirty-four already-published papers from RIReMS centers were used for heterogeneity analyses, using the DerSimonian and Laird random-effects method to compute the between-study variance $(\tau 2)$.

Results: Based on questionnaire results, we defined basic modules for diagnosis and follow-up, consisting of outcome measures recorded by all participating centers at the time of diagnosis, and, then, at least annually; we also defined more detailed/optional modules, with outcome measures recorded less frequently and/or in the presence of specific clinical indications. Looking at heterogeneity, we found 5 -year variance in age at onset $(\mathrm{ES}=27.34 ; 95 \% \mathrm{CI}=26.18,28.49 ; \mathrm{p}<0.01 ; \tau 2=4.76)$, and $7 \%$ in female percent $(\mathrm{ES}=66.42 ; 95 \% \mathrm{CI}=63.08,69.76 ; \mathrm{p}<0.01 ; \tau 2=7.15)$. EDSS variance was 0.2 in studies including patients with average age $<36.1$ years $(\mathrm{ES}=1.96 ; 95 \% \mathrm{CI}=1.69,2.24 ; \mathrm{p}<0.01 ; \tau 2=0.19)$, or from 36.8 to 41.1 years $(\mathrm{ES}=2.70 ; 95 \% \mathrm{CI}=2.39,3.01 ; \mathrm{p}<0.01 ; \tau 2=0.18)$, but increased to 3 in studies including patients aged $>41.4$ years $(\mathrm{ES}=4.37 ; 95 \% \mathrm{CI}=3.40$, $5.35 ; \mathrm{p}<0.01 ; \tau 2=2.96$ ). The lowest variance of relapse rate was found in studies with follow-up duration $\leq 2$ years $(\mathrm{ES}=9.07 ; 95 \% \mathrm{CI}=5.21,12.93$; $\mathrm{p}=0.02 ; \tau 2=5.53$ ), whilst the lowest variance in EDSS progression was found in studies with follow-up duration $>2$ years $(E S=5.41$; $95 \% \mathrm{CI}=3.22,7.60 ; \mathrm{p}=0.02 ; \tau 2=1.00$ ).
Discussion: We suggest common sets of biomarkers to be acquired in clinical practice, that can be used for research purposes. Also, we provide researchers with specific indications for improving inclusion criteria and data analysis, ultimately allowing data harmonization and high-quality collaborative studies.

\section{TELEMEDICINE IN/OUTSIDE THE PANDEMIC: A SURVEY ABOUT SATISFACTION OF THIS TOOL IN A COHORT OF MULTIPLE SCLEROSIS PATIENTS AND THEIR NEUROLOGISTS}

\author{
L. Moiola ${ }^{1}$, M. Di Cristinzi ${ }^{2}$, S. Guerrieri ${ }^{1}$, A. Nozzolillo ${ }^{1}$, M. Orrico ${ }^{1}, \mathrm{~S}$. \\ Gelibter $^{1}$, L. Bosco ${ }^{1}$, C. Zanetta ${ }^{1}$, F. Sangalli ${ }^{1}$, M. Filippi ${ }^{3}$
}

${ }^{1}$ Multiple Sclerosis Centre, Neurology Unit, IRCCS San Raffaele Scientific Institute (Milano); ${ }^{2}$ Multiple Sclerosis Center, Neurology Department, University of Florence (Firenze); ${ }^{3}$ Multiple Sclerosis Centre, Neurology, Neurophysiology and Neuroimaging Units, IRCCS San Raffaele Scientific Institute (Milano)

Objectives: Telemedicine is a live communication between patients and physicians through different technological tools. It became fundamental during COVID-19 pandemic to keep on taking care of patients in more medical fields than before. Given the chance for telemedicine to become a pivotal part of neurological routine practice, we investigated patients and neurologists satisfaction about this tool.

Materials and methods: We administered a brief survey to a cohort of Multiple Sclerosis (MS) patients and their neurologists at MS center of San Raffaele Hospital, Milan. Demographic and clinical data (EDSS, treatment) were collected.

Results: 151 patients filled out the survey: $75 \%$ females; mean age 42.2yy (18-73); median EDSS 1.5 (0-7). Treatments were almost equally distributed across first and second-line drugs $(10 \%$ interferon, $10 \%$ glatiramer acetate, $14 \%$ teriflunomide, $22 \%$ dimethylfumarate, $23 \%$ fingolimod, $3 \%$ cladribine, $8 \%$ alemtuzumab, $10 \%$ ocrelizumab). $87 \%$ of patients appreciated telemedicine during pandemic; $10 \%$ had a positive opinion but with a need for traditional evaluations, while $3 \%$ were not satisfied. Considering the possible routine use of telemedicine outside the pandemic, $82 \%$ firmly stated the importance of traditional evaluations. In particular, $44 \%$ would alternate in person and remote visits, while $38 \%$ strongly preferred traditional ones. Among the latters, the main reasons were the need for human empathy with the neurologist and the feeling that traditional evaluations may lead to better clinical outcomes. The remaining $18 \%$ would always use telemedicine except in the case of acute events. Neurologists and residents $(n=18)$ were inquired about telemedicine: no one would use it as the only tool, $33 \%$ would alternate it with traditional practice and $67 \%$ would use it only in special contexts.

Conclusions: In our survey, both patients and neurologists recognized the importance of telemedicine during a pandemic. Patients more than physicians seemed ready to use it in everyday clinical practice. These data may be biased by a still ongoing patients fear and physicians lack of confidence in this multifaceted tool. The pandemic spurred the development of institutional telematic platforms capable of providing legal protection and traceability of visits and communication between patients and physicians. So far, we can conclude that telemedicine is a useful tool to overcome space-time limits, giving the best care to all patients in any condition. However, it cannot replace but only integrate traditional medicine. 


\section{MULTIPLE SCLEROSIS CLINICAL COURSE IMPACTS ON THE ASSOCIATION BETWEEN GENERAL COGNITION AND MENTALIZING DEFICITS}

\author{
M. Pardini ${ }^{1}$, E. Colombo ${ }^{1}$, R. Meli ${ }^{1}$, C. Lapucci $^{2}$, N. Bruschi ${ }^{1}$, G. Boffa ${ }^{1}$, \\ M. Cellerino ${ }^{1}$, E. Sbragia ${ }^{1}$, A. Uccelli ${ }^{3}$, M. Inglese ${ }^{3}$
}

${ }^{1}$ Department of Neurosciences, Rehabilitation, Ophthalmology, Genetics, Maternal and Child Health, University of Genoa (Genova); ${ }^{2}$ Scientific Direction, Policlinico S. Martino (Genova); ${ }^{3}$ Department of Neurosciences, Rehabilitation, Ophthalmology, Genetics, Maternal and Child Health, University of Genoa and Policlinico S. Martino (Genova)

Objective: Theory of Mind (ToM, i.e the ability to decode emotional states) is a cognitive function that plays a key role in social functioning. While ToM deficits have been found to be frequent in subjects with MS, ToM is not routinely assessed in formal neuropsychological assessments in this population. The lack of inclusion of ToM assessment in routine neuropsychological evaluation of MS patients, stems from different causes, including a partial characterisation of the association between general cognition and ToM in the different phases of the disease. Here we thus decided to evaluate the interaction between clinical course (relapsing remitting MS (RRMS) vs progressive MS (PMS)), ToM deficits and general cognition. More in detail we aim to assess if the role played by general cognitive functioning on ToM is different in PMS rather than in RRMS

Methods: 120 MS patients (age: $44.9 \pm 11.6$ years, median EDSS 2.5 range 1-6; 75 subjects with RRMS and 45 with PMS) were assessed with the Symbol Digit Modalities Test (SDMT) to evaluate general cognition and the Reading the Mind in the Eyes Test (RMET) to evaluate ToM. The RMET, developed by Baron-Cohen and colleagues in 2001 is a standardised test that consists of 36 black and white picture of the eye region; the subject has to recognize the emotional state represented in the picture and choose one among four given words that depict an emotion [1].

Results: Comparing RRMS and PMS patients, there was a significant difference in SDMT $(55.3 \pm 12.0$ vs $40.2 \pm 11.0, \mathrm{p}=0.001)$ and in total RMET $(27.0 \pm 4.0$ vs $22.9 \pm 3.0 \mathrm{p}=0.001)$ scores. There was a significant correlation between SDMT and ToM in the whole sample $(\mathrm{p}<0.001$, $r=0.45$ ) and in the RRMS group ( $p=0.001, r=0.48$, but not in PMS $(\mathrm{p}=0.83)$ group.

Discussion: The association between SDMT and RMET is modulated by clinical course in MS. This observation suggests that a bigger role is played by general cognition on RMET in RRMS rather than in PMS possibly due to the presence of more diffuse damage in PMS. Our data suggest that these two metrics change differently over the disease course and thus provide complementary information in the study of cognitive deficits in MS.

Reference:

1. Baron-Cohen S, Wheelwright S, Hill J, Raste Y, Plumb I. The "Reading the Mind in the Eyes" Test revised version: a study with normal adults, and adults with Asperger syndrome or highfunctioning autism. J Child Psychol Psychiatry (2001);42(2):24151

\section{MILD GRAY MATTER ATROPHY IN PATIENTS WITH LONGSTANDING MULTIPLE SCLEROSIS AND FAVORABLE CLINICAL COURSE}

F. Parodi ${ }^{1}$, R. Cortese ${ }^{1}$, M. Battaglini ${ }^{1}$, M. Stromillo ${ }^{1}$, E. Portaccio ${ }^{2}$, L. Razzolini $^{3}$, A. Giorgio ${ }^{1}$, M. Sormani ${ }^{4}$, M. Amato ${ }^{3}$, N. De Stefano ${ }^{1}$
${ }^{1}$ Department of Medicine, Surgery and Neuroscience, University of Siena (Siena); ${ }^{2}$ IRCCS Fondazione Don Carlo Gnocchi (Firenze); ${ }^{3}$ Department of Neurological and Psychiatric Sciences (NEUROFARBA), University of Florence (Firenze); ${ }^{4}$ Department of Health Sciences, University of Genoa (Genova)

Objective: Understanding whether multiple sclerosis (MS) can have a favorable course is still challenging. However, a small group of patients who are not disabled after many years of disease can be identified. The mechanisms responsible for this 'benign' clinical course remain unclear, likely due to the lack of long-term studies. We aim to assess brain damage in MS patients with no or minimal disability after a longstanding clinical course.

Materials and methods: We compared 13 patients with long-term benign clinical course (LT-BMS, age $>55$ years, disease duration $>30$ years, Expanded Disability Status Scale $[$ EDSS] $<3.0$ ) and 27 nonbenign MS (non-BMS) patients (age $>55$ years, EDSS $>3.0$ ). MRI scans were retrospectively assessed (mean follow-up: 11 years, mean scan per patient: 3). Comparisons of brain volumes (BV) and total T2-lesion volume (LV) changes between the two groups were performed using a mixed effect model. Lesion probability maps (LPMs) of both groups were compared using a nonparametric permutation test.

Results: Patients with LT-BMS showed less over-time decrease in global BV $(p=0.02)$ and grey matter $(G M)$ volume $(p<0.001)$ than nonBMS. Lower atrophy was seen in LT-BMS with no or mild cognitive impairment. By contrast, there was no over-time difference between patient groups in T2-LV accumulation and lesion frequency across brain.

Conclusion: Global brain and GM atrophy changes were mild in this unique patient group with long-standing and no or minimal physical and cognitive disability. These results support the relevant role of GM atrophy in characterizing MS patients who may have favorable long-term disease evolution.

References:

Razzolini L, Portaccio E, Stromillo ML et al. The dilemma of benign multiple sclerosis: Can we predict the risk of losing the "benign status"? A 12-year follow-up study. Mult. Scler. Relat. Disord. (2018) Nov;26:71-73

- Cortese R, Collorone S, Ciccarelli O and Toosy AT. Advances in brain imaging in multiple sclerosis. Ther. Adv. Neurol. Disord. (2019) Jun 27;12:1756286419859722

- De Stefano N, Battaglini M, Stromillo ML. et al. Brain damage as detected by magnetization transfer imaging is less pronounced in benign than in early relapsing multiple sclerosis. Brain (2006) Aug;129(Pt 8):2008-16

\section{INFECTIOUS RISK MANAGEMENT IN MULTIPLE SCLEROSIS}

V. Perri ${ }^{1}$, P. Pasculli ${ }^{1}$, M. Zingaropoli ${ }^{1}$, M. Iannetta ${ }^{2}$, M. Tartaglia ${ }^{3}, \mathrm{~S}$. Crisafulli $^{3}$, V. Baione ${ }^{3}$, C. Merluzzo ${ }^{3}$, M. Altieri ${ }^{3}$, M. Frontoni ${ }^{3}$, A. Conte $^{4}$, C. Mastroianni ${ }^{1}$, M. Ciardi ${ }^{1}$

${ }^{1}$ Department of Public Health and Infectious Diseases, Sapienza University (Roma); ${ }^{2}$ Department of System Medicine, Tor Vergata University (Roma); ${ }^{3}$ Department of Human Neurosciences, Sapienza University (Roma); ${ }^{4}$ IRCSS Neuromed (Pozzilli-IS)

Objectives: Aim of the study was to define the infectious risk in multiple sclerosis (MS) patients receiving disease-modifying therapies (DMTs) with approaches tailored to individual patients. 
Material: At the Neuroinfectious Unit of Policlinico Umberto I (Rome), before starting or switching to DMTs, MS patients were evaluated for infectious risk.

Methods: Hepatitis B virus (HBV) and Mycobacterium tuberculosis (MTB) were investigated by serological test as hepatitis B surface antigen (HBsAg), hepatitis B surface antibody (HBsAb), hepatitis B core antibody $(\mathrm{HBcAb})$, and by quantiFERON®-TB Gold In-Tube (QFT) assay, respectively. HBV-DNA detection was performed in chronic hepatitis $\mathrm{B}$ MS patients and monthly monitored during DMTs. In QFT positive patients, active tuberculosis (TB) was excluded by medical history and chest $\mathrm{X}$-ray and when necessary, sputum smear microscopy, sputum culture and MTB PCR were also performed. All latent tuberculosis infection patients were undergone to TB prophylaxis (isoniazid+rifampicin) for 3 months. DMTs were started after one-month prophylaxis.

Results: One hundred thirty-eight MS patients (79 females, 59 males) with a median age [interquartile range (IQR)] of 47.5 (37-56), median years of disease (IQR) of 8 (2-18) and median EDSS (IQR) of 3.5 (2-6), were enrolled. Before starting DMTs, $10.1 \%$ (14/138) of patients had chronic B hepatitis and $10.1 \%$ (14/138) showed QFT positivity. During DMTs follow-up, one patient revealed HBV reactivation. The patient remained asymptomatic with liver enzymes unchanged. HBV-DNA became undetectable after 2 weeks of specific antiviral treatment without discontinuing ocrelizumab. Among QFT positive patients, no TB reactivation was observed. Interestingly, one of the QFT negative patients became positive during fingolimod treatment.

Discussion: HBV and TB screening should be recommended in MS patients candidate for DMTs. HBV monitoring may avoid a fatal event and the choice of a preemptive strategy spares HBV prophylaxis when unnecessary. Beside preventing TB reactivation, TB prophylaxis based on two active molecules, allows an earlier starting of DMTs.

Conclusions: TB screening may contribute to reduce the transmission, morbidity, and mortality of active disease in global population

\section{ASYMPTOMATIC ANTERIOR OPTIC PATHWAY INVOLVEMENT IN EARLY MULTIPLE SCLEROSIS AND CLINICALLY ISOLATED SYNDROMES}

M. Pisa, T. Croese, G. Dalla Costa, S. Guerrieri, S. Huang, L. Fabbella, A. Finardi, B. Colombo, F. Sangalli, L. Moiola, V. Martinelli, G. Comi, R. Furlan, L. Leocani

\section{San Raffaele Hospital, Vita-Salute San Raffaele University (Milano)}

Background and Objective: Optical coherence tomography is gaining increasing relevance in the assessment of people with multiple sclerosis. Converging evidence point to the view that neuro-retinal changes, in eyes without acute optic neuritis, reflect inflammatory and neurodegenerative processes taking place throughout the CNS. The present study aims at exploring the usefulness of optical coherence tomography as a marker of inflammation and disease burden in the earliest phases of the disease.

Methods: A cohort of 150 consecutive patients underwent clinical, neurophysiological and brain MRI assessment as well as lumbar puncture as part of their diagnostic workup for a neurological episode suggestive of inflammatory CNS disorder. For the present study, patients also received a visual pathway assessment - including OCT, VEP, visual acuity testing -, measurement of CSF inflammatory markers - a set of 17 cytokineschemokines and, count of extracellular vesicles of myeloid origin -, and dosage of serum neurofilaments.
Results: $19.2 \%$ of clinically isolated syndromes had abnormal visual evoked potentials in eyes without optic neuritis. Similarly, optical coherence tomography identified neuro-retinal thinning in $17.8 \%$ of patients without prior visual symptoms. The presence of asymptomatic involvement of the anterior optic pathway tested with either techniques was associated with a greater disease burden. A thinner ganglion cell layer in eyes without prior optic neuritis or instrumental evidences of it was correlated with higher EDSS, lower low contrast visual acuity, longer disease duration, higher brain lesion load, presence of gadolinium enhancing lesions, more severe abnormalities along motor and somatosensory evoked potentials, and higher frequency of CSF-specific oligoclonal bands. We also found that inner nuclear layer thickens in a post-acute (1.1 - 3.7 months) phase after a relapse, particularly in those who did not receive steroid treatment. Likewise, a longitudinal analysis on 65 patients, showed that this swelling is transient and returns to normal values after one year of follow-up. Notwithstanding, the clinical, MRI, serological and CSF markers of disease activity considered in the study were strictly associated with one-another but none of them was associated with inner nuclear layer volume.

Conclusions: The present findings suggest that instrumental evidence of asymptomatic optic nerve involvement is associated with a greater disease burden in early MS and clinically isolated syndrome. Neuroretinal changes are present since the earliest phases of the disease and yield important information regarding the neurodegenerative and inflammatory processes occurring in the CNS.

References:

- Balk LJ, Coric D, Knier B, et al. IMSVISUAL consortium. Retinal inner nuclear layer volume reflects inflammatory disease activity in multiple sclerosis; a longitudinal OCT study. Mult Scler J Exp Transl Clin. (2019) Sep 5;5(3):2055217319871582

- Nolan-Kenney RC, Liu M, Akhand O et al. International Multiple Sclerosis Visual System Consortium. Optimal intereye difference thresholds by optical coherence tomography in multiple sclerosis: An international study. Ann Neurol. (2019) May;8

- Pisa M, Guerrieri S, Di Maggio G, et al. No evidence of disease activity is associated with reduced rate of axonal retinal atrophy in MS. Neurology (2017) Dec 12;89(24):2469-2475. doi: 10.1212/ WNL.0000000000004736. Epub 2017 Nov 15

\section{INTRACORTICAL MOTOR CONDUCTION IS ASSOCIATED WITH DEXTERITY IN PROGRESSIVE MULTIPLE SCLEROSIS} M. Pisa, R. Chieffo, M. Congiu, F. Esposito, M. Romeo, M. Comola, G.
Comi, L. Leocani

San Raffaele Hospital, Vita-Salute San Raffaele University (Milano)

Background and Objectives: Hand dexterity dysfunction is a key feature of disability in people with progressive multiple sclerosis (PMS). It underlies corticospinal tract (CST) and cerebellar integrity but also disruption of cortical networks, which are hardly assessed by standard techniques. Transcranial magnetic stimulation is a promising tool for evaluating the integrity of intracortical motor pathways. The present study aims at investigating neurophysiological correlates of motor hand impairment in PMS and assess intracortical motor conduction through the use of a innovative TMS protocol.

Methods: Antero-posterior (AP) stimulation of the primary motor cortex activates the CST indirectly through polysynaptic pathways, while a direct CST activation occurs with latero-medial (LM) directed current. 30 
PMS and 15 healthy controls underwent dominant hand motor evoked potentials (MEP) using AP and LM-directed stimulation, and a clinical assessment of dexterity (nine-hole peg test) and strength (MRC scale, grip and pinch).

Results: PMS with AP-LM latency difference 2.5 standard deviation above the mean of controls (33\%) showed worse dexterity but no difference in upper limb strength. Accordingly, AP-LM latency shortening predicted dexterity ( $R 20.538, \mathrm{p}<0.001)$, but not strength impairment. On the contrary, absolute MEP latencies only correlated with strength (grip: R2 0.381, p=0.014; MRC: R2 0.184, p=0.041).

Conclusions: AP-LM latency shortening may be used to assess the integrity polysynaptic intracortical networks implicated in dexterity impairment.

References:

- Di Lazzaro V, Rothwell J, Capogna M. Noninvasive Stimulation of the Human Brain: Activation of Multiple Cortical Circuits. Neuroscientist (2018);24(3):246-60

- Hamada M, Murase N, Hasan A, et al. The role of interneuron networks in driving human motor cortical plasticity. Cereb Cortex (2013);23(7):1593-605

- Pisa M, Chieffo R, Giordano A, et al. Upper limb motor evoked potentials as outcome measure in progressive multiple sclerosis. Clin Neurophysiol (2020) Feb;131(2):401-05

\section{A CASE-CONTROL STUDY OF ENVIRONMENTAL RISK FACTORS IN AN ITALIAN COHORT OF MULTIPLE SCLEROSIS PATIENTS}

\begin{abstract}
M. Pozzato ${ }^{1}$, A. Mollica ${ }^{2}$, C. Starvaggi Cucuzza ${ }^{3}$, A. Cagol $^{3}$, M. Rodegher ${ }^{4}$, N. Bresolin ${ }^{5}$, A. Protti ${ }^{2}$, F. Martinelli Boneschi ${ }^{5}$

${ }^{1}$ Fondazione IRCCS Ca' Granda Ospedale Maggiore Policlinico, University of Milan (Milano); ${ }^{2}$ Department of Neurology and Multiple Sclerosis Research Unit, IRCCS Ospedale Niguarda, University of Milan (Milano); ${ }^{3}$ Department of Neurology, IRCCS Policlinico San Donato, University of Milan (Milano); ${ }^{4}$ Department of Neurology, IRCCS Fondazione Don Gnocchi (Milano); ${ }^{5}$ Neurology Unit \& MS Centre, Dino Ferrari Centre, Neuroscience Section, Department of Pathophysiology and Transplantation, Fondazione IRCCS Ca' Granda Ospedale Maggiore Policlinico, University of Milan (Milano)
\end{abstract}

Background and objectives: Multiple sclerosis (MS) is a demyelinating inflammatory disease of the central nervous system due to the interaction between genetic and environmental risk factors. Cigarette smoking, low serum vitamin D levels, previous EBV infection and obesity at young age are among known environmental risk factors. The aim of this work is to analyse the role of environmental risk factors in an Italian cohort of consecutive Multiple Sclerosis (MS) patients, through the administration of an Italian translation of an environmental questionnaire known to be associated with MS.

Materials and methods: The questionnaire has been developed by the Karolinska Institute, and it explores the role of different environmental risk factors including smoking habits, sun exposure, physical activity, diet, alcohol intake, working habits and questions specific for women. It has been administered to 136 consecutive MS patients and 136 age- and sex-matched healthy matched controls. Statistical analyses and Odds Ratios (ORs) have been calculated using SPSS software (version 25).

Results: Despite the reduced sample size, we found that smokers had an ORs of disease of $1.87(\mathrm{p}=0.01)$ with a significant dose-effect measured with pack-year index $(\mathrm{p}=0.03)$. A greater sun exposure before 30 years, a mixed diet at 20 years and a lower BMI were protective factors, while a higher vitamin $\mathrm{D}$ intake in cases were due to reverse causality.

Discussion and conclusion: Despite limitations, this study confirms the role of known environmental risk factors for MS like cigarette smoking and reduced sun exposure at young age also in the Italian setting, further supporting the importance to discuss lifestyle habits with persons at risk of MS like for example relatives of individuals with Multiple Sclerosis. There is an ongoing effort of replication in other Italian casecontrol samples to further confirm this trend.

\section{BDNF VAL66MET POLYMORPHISM EFFECT ON HIPPOCAMPAL SUBFIELDS IN MULTIPLE SCLEROSIS PATIENTS}

E. Prestipino ${ }^{1}$, E. De $\mathrm{Meo}^{2}$, E. Portaccio ${ }^{3}$, B. Nacmias ${ }^{1}$, S. Bagnoli ${ }^{1}$, L. Razzolini $^{1}$, L. Pasto ${ }^{13}$, C. Niccolai ${ }^{4}$, B. Goretti ${ }^{1}$, R. Fratangelo ${ }^{1}$, L. Tudisco $^{1}$, A. Bellinvia ${ }^{1}$, M. Fonderico ${ }^{1}$, A. Giorgio ${ }^{5}$, M. L Stromillo ${ }^{5}$, M. Filippi ${ }^{2}$, S. Sorbi ${ }^{1}$, N. De Stefano ${ }^{5}$, M. P. Amato ${ }^{1,4}$

${ }^{1}$ NEUROFARBA Department, University of Florence (Firenze); ${ }^{2}$ Institute of Experimental Neurology, Neuroimaging Research Unit, Department of Neurology, San Raffaele Hospital, Vita-Salute San Raffaele University (Milano); ${ }^{3}$ Operative Unit of Neurological Rehabilitation, Careggi University Hospital (Firenze); ${ }^{4}$ Unit of Neurological Rehabilitation, IRCCS Fondazione Don Carlo Gnocchi (Firenze); ${ }^{5}$ Neurology and Neurometabolic Unit, Department of Neurological and Behavioral Sciences (N.D.S.), University of Siena (Siena)

Background: Brain-derived neurotrophic factor (BDNF) can promote neuronal growth and repair, playing a key role in synaptic plasticity, especially in the hippocampus. The BDNF Val66Met polymorphism was shown to strongly affect BDNF function, but its role in modulating gray matter damage in multiple sclerosis (MS) patients is still not clear.

Objectives: Considering BDNF relevance on hippocampal function, we aimed to explore the effect of BDNF Val66Met polymorphism on the atrophy of hippocampal subfields and its role in cognitive functioning in MS patients.

Methods: Using a 3T scanner, we obtained dual-echo and 3DT1weighted sequences from $50 \mathrm{MS}$ patients and 15 healthy controls (HC). MS patients also underwent genotype analysis of BDNF and an extensive neuropsychological evaluation. Hippocampal subfields were segmented by using Freesurfer 7.0.1 software. Multiple linear regression models adjusted for age, sex and disease duration were used for between-group comparisons and analysis of associations.

Results: The BDNF Val66Met polymorphism was found in $22 \mathrm{MS}$ patients (44\%). Compared to HC, MS patients had reduced volumes of bilateral hippocampus-amygdala transition area (HATA); left cornus ammonis (CA)1, CA3 and granule cell layer of dentate gyrus (GCLDG); and right fimbria and presubiculum. BDNF Val66Met polymorphism carriers compared to wild-type (Val66Val) MS patients had higher volume of left hippocampal CA1, CA3, CA4, GCL-DG, molecular layer of subiculum and HATA; and of right hippocampal tail, fissure and presubiculum. In MS patients, higher volume in left CA3 and in right presubiculum correlated with better performance in semantic fluency, while higher volume in left GCL-DG correlated with better visuospatial memory performance.

Conclusions: The BNDF Val66Met polymorphism has a protective role in MS patients against both hippocampal atrophy and cognitive 
deterioration. BDNF genotype may be a potential biomarker for predicting cognitive prognosis, and an interesting target to study for neuroprotective strategies.

\section{SAFETY AND EFFICACY OF CLADRIBINE IN CLINICAL PRACTICE: A SINGLE CENTRE EXPERIENCE}

\author{
M. Radaelli, V. Barcella, M. Conti, M. Grimoldi, M. Sessa
}

Department of Neurology, ASST Papa Giovanni XXIII Hospital (Bergamo)

Objectives: Cladribine is available in Italy since 2018 as second-line drug for relapsing-remitting multiple sclerosis (RRMS). The safety and efficacy of Cladribine were confirmed by a phase III randomized clinical trial, the CLARITY study. The aim of the study is to confirm cladribine safety and efficacy in clinical practice, in a monocentric cohort of RRMS.

Material and Methods: We retrospectively and prospectively collected data of patients treated with cladribine at the MS center of HPG23 Hospital (Bergamo). Neurological evaluations were performed every 3 months, haematological blood tests were performed 2 months after the administration of the drug and then every 3 months. Brain MRIs were obtained every 6-9 months according to clinical practice.

Results: Starting from May 2018, 17 patients (9 females) were treated with cladribine. The mean age was 39 years (range 27-51) and the mean disease duration was 48 months (range 2 months- 24 years). The mean EDSS was 1,9 (range 1-4,5) and the mean number of relapses in the 2 years before cladribine was 1 (range 1-3). New T2-lesion and enhancing lesions were observed at basal brain MRI in 13 and 10 patients respectively. The drug was started after the failure of fingolimod treatment in 2 patients and after first-line DMT in additional 8 patients; seven subjects were treatment-naive. The mean follow-up is 13 months (range 1-26). Overall 9 patients have completed the retreatment in year 2. At last follow-up no relapses were observed and EDSS was stable in all patients. However, a neuroradiological reactivation was observed in 7 of the 13 patients $(54 \%)$ who performed at least one MRI after the beginning of the drug. No serious adverse events were observed. No patients reported a grade 3 or 4 lymphopenia. One patient reported a herpes simplex reactivation after treatment and 3 patients complained a transient cutaneous rash. One patient developed a suspected SARS CoV2 infection one month after completing the treatment in year 1 ; the infection was mild an she recovered without sequelae.

Discussion and conclusions: Our experience confirmed the good safety profile of the drug, that showed also a relative good efficacy. However, a neuroradiological reactivation was observed in about half of the patients. Further studies in a larger number of patients and with longer follow-up are needed to confirm the benefit-risk ratio of the drug in clinical practice and to assess the best management of patients treated with cladribine in the long-term follow-up.

References:

1. Giovannoni G, Soelberg Sorensen P, Cook S, Rammohan K, Rieckmann P, Comi G, Dangond F, Adeniji AK, Vermersch P. Safety and efficacy of cladribine tablets in patients with relapsing remi-ting multiple sclerosis: Results from the randomized extension trial of the CLARITY study. Mult Scler. (2018);24(12):1594-1604

2. Comi G, Cook S, Giovannoni G, Rieckmann P, Sørensen PS, Vermersch P, Galazka A, Nolting A, Hicking C, Dangond F. Effect of cladribine tablets on lymphocyte reduction and repopulation dynamics in patients with relapsing multiple sclerosis. Mult Scler Relat Disord. (2019); 29:168-74
3. Cook S, Vermersch P, Comi G, Giovannoni G, Rammohan K, Rieckmann P, Sørensen PS, Hamlett A, Miret M, Weiner J, Viglietta V, Musch B, Greenberg SJ; CLARITY Study Group. Safety and tolerability of cladribine tablets in multiple sclerosis: the CLARITY (CLAdRIbine Tablets treating multiple sclerosis orallY) study. Mult Scler. (2011);17(5):578-93

\section{SARS-COV-2 INFECTION IN MULTIPLE SCLEROSIS PATIENTS TREATED WITH NATALIZUMAB: A SINGLE CENTRE EXPERIENCE}

\author{
M. Radaelli, M. Conti, V. Barcella, M. Grimoldi, M. Sessa
}

Department of Neurology, ASST Papa Giovanni XXIII (Bergamo)

Objective: Since the beginning of SARS-CoV-2 pandemic, neurologists treating Multiple Sclerosis (MS) patients had to face the possible increased risk of infection related to disease modifying treatments (DMT). By general consensus, Natalizumab (NTZ) was considered the safest one. We report the outcome of suspected SARS-CoV-2 infection in NTZ-treated patients in the referral MS centre of the province of Bergamo.

Material and Methods: Starting from February 2020, we collected patients treated with NTZ who presented symptoms suggestive of SARS-CoV-2 infection. According to Italian guidelines, the treatment was continued and the infusions were delayed every 6-7 weeks. A nasal swab was routinely performed before NTZ infusion starting from the beginning of May. Before this date, in case of symptoms suggestive of SARS-CoV-2 infection, the treatment was postponed to 7-10 days from complete recovery.

Results: Among the 55 NTZ-treated patients, 22 (18 females) presented symptoms suggestive of SARS-CoV-2 infection. Mean age was 35 (range 18-51) and mean NTZ treatment duration was 47 months (range 11-144). All patients lived in the pandemic area of Bergamo province; 11 patients had at least one cohabitant or a close contact with a confirmed/suspected SARS-CoV-2 case. A nasal swab was performed during the symptomatic phase only in one patient, being positive. Four patients were positive at the nasal swab performed before NTZ infusion from the beginning of May. The mean interval between the onset of SARS-CoV-2 symptoms and the positive swab was 103 days (range 60-110 days). One additional patient showed a positive swab without clinical symptoms. Overall, the disease was considered mild in 21 patients, with a mean symptom duration of 7 days. The only patient with a severe disease was a 45 years old female who received last NTZ infusion one week before the beginning of symptoms. She developed a bilateral pneumonia and was admitted in ICU. She was treated with i.v. antibiotics, hydroxychloroquine and tocilizumab with complete recovery. NTZ was restarted in May 2020. No relapses were observed among these patients.

Discussion and conclusions: For patients under DMT the decision to whether continue or interrupt the treatment during SARSCoV-2 pandemic must be taken at the individual level, depending by the type of ongoing DMT and MS severity. Our experience confirmed NTZ safety in patients with suspected SARS-CoV-2 infection. Despite the absence of a confirmed diagnosis in the majority of patients, our data confirmed the positive benefit-risk ratio of continuing the drug, with an extended dose, even during the pandemic phase 


\section{References:}

- Borriello G, Ianniello A. COVID-19 occurring during Natalizumab treatment: a case report in a patient with extended interval dosing approach. Mult Scler Relat Disord. (2020) Jun;41:102165

- Aguirre C, Meca-Lallana V, Barrios-Blandino A, Del Río B, Vivancos J. Covid-19 in a patient with multiple sclerosis treated with natalizumab: May the blockade of integrins have a protective role? Mult Scler Relat Disord. (2020) Jun 3;44:102250

- Baker D, Amor S, Kang AS, Schmierer K, Giovannoni G. The underpinning biology relating to multiple sclerosis disease modifying treatments during the COVID-19 pandemic. Mult Scler Relat Disord. (2020); 43:102174

\section{VACCINAL IMMUNITY LOSS IN MULTIPLE SCLEROSIS PATIENT TREATED WITH OCRELIZUMAB}

\author{
L. Rapisarda ${ }^{1}$, S. Barone ${ }^{1}$, C. Torti ${ }^{2}$, V. La Gamba ${ }^{2}$, F. Fortunato ${ }^{1}$, I. \\ Sammarra $^{1}$, A. Gambardella ${ }^{1}$, P. Valentino ${ }^{1}$ \\ ${ }^{1}$ Institute of Neurology, Department of Medical and Surgical Sciences, \\ Magna Graecia University (Catanzaro); ${ }^{2}$ Infectious and Tropical Disease \\ Unit, Department of Medical and Surgical Sciences, Magna Graecia \\ University (Catanzaro)
}

Introduction: Ocrelizumab (OCR) is a novel humanized anti-CD20 antibody used for treatment of relapsing remitting (RRMS) and primary progressive multiple sclerosis with evidence of inflammatory activity [1]. Data on the effect of OCR on vaccinal immunity are uncertain and a recent study evidenced that patients treated with OCR might not reach an effective immunization after vaccination [2].

Case description: We describe the case of a 48 years old-man with a medical history of diabetes mellitus type 2, arterial hypertension, dyslipidemia and ischemic cardiopathy, affected by RRMS since 2005 . The patient discontinued several treatments, lately Natalizumab after the 24th administration for presence of high titer of JCV serum antibodies. Before starting OCR treatment, he performed serological screening including WBC count, lymphocyte subset assessment, immunoglobulin (Ig) dosage and an extended infectious screening. The patient received anti varicella zoster virus (VZV) vaccine. After 7 weeks brain MRI displayed a new periventricular occipital lesion with contrast enhancement but neurological examination was unchanged, thus he was treated with low dose of methylprednisolone for 5 days. Two weeks later serological test showed a protective titer of anti-VZV IgG and negative antiVZV IgM. Four months after the vaccination the patient received the initial dose of $300 \mathrm{mg}$ of OCR followed by the second one two weeks later. Before the subsequent dose of OCR the patient repeated serum screening tests and infectious screening. We found out that the patient had non-protective titer of anti-VZV antibodies. According with infectious disease consultant we decided to delay the next injection of OCR and seven months later the last administration, the patient received for the second time anti-VZV vaccine. We retested anti-VZV antibodies 8 weeks later which showed absence of vaccinal immunization. Thus, three months later the last vaccination the patient had new OCR infusion. We recommended prevention strategy through vaccination of close contacts and avoidance of subjects with active VZV infection.

Discussion and conclusion: We described the case of a man who loss vaccinal immunity for VZV after the first injection of OCR. Guidelines suggest assessing vaccination status and eventually vaccinate patients with multiple sclerosis before new disease modifying therapy initiation [3]. It is advisable to reassess vaccinal immunity after OCR treatment and recommend suitable preventive measures in non-immune patients such as cocoon immunization strategy (immunization of the family, cohabitants, and frequent contacts) and avoidance of contacts with active infection.

References:

1. S.L. Hauser et al. Ocrelizumab versus Interferon Beta-1a in Relapsing Multiple Sclerosis. N Engl J Med (2017); 376:221-34

2. A. Bar-Or et al. Effect of ocrelizumab on vaccine responses in patients with multiple sclerosis: The VELOCE study. Neurology (2020); 10.1212/WNL.0000000000010380

3. M.F. Farez et al. Practice guideline update summary: Vaccinepreventable infections and immunization in multiple sclerosis. Neurology (2019);93:584-94

\section{THE FRAMINGHAM CARDIOVASCULAR RISK SCORE AND 5- YEAR PROGRESSION OF MULTIPLE SCLEROSIS}

\author{
A. Reia ${ }^{1}$, M. Petruzzo ${ }^{1}$, G. T Maniscalco ${ }^{2}$, F. Luiso $^{1}$, R. Lanzillo ${ }^{1}$, C. V \\ Russo $^{1}$, A. Carotenuto ${ }^{1}$, L. Allegorico ${ }^{2}$, R. Palladino ${ }^{3}$, V. Brescia Morra ${ }^{1}$, \\ M. Moccia ${ }^{1}$
}

${ }^{1}$ Multiple Sclerosis Clinical Care and Research Centre, Department of Neuroscience, Reproductive Science and Odontostomatology, Federico II University (Napoli); ${ }^{2}$ Department of Neurology, Cardarelli Hospital (Napoli); ${ }^{3}$ Department of Public Health, Federico II University (Napoli)

Objectives: Cardiovascular risk factors and comorbidities can affect the prognosis of multiple sclerosis (MS). The Framingham risk score is an algorithm that can estimate the 10-year risk of developing macrovascular disease. We aimed to evaluate a possible association between the Framingham risk score at baseline, and MS relapses, disability and disease-modifying therapy choices over 5-year follow-up.

Materials and methods: This is a retrospective cohort study including 251 MS subjects. At baseline, we calculated the Framingham risk score considering the following variables: age, sex, diabetes, smoking, systolic blood pressure, and body mass index. MS outcomes including relapses, disability and treatments were collected over 5 years. Cox proportional regression models were employed to estimate hazard ratios (HR).

Results: 1-point increase in the Framingham risk score was associated with $31 \%$ higher risk of relapse $(\mathrm{HR}=1.31 ; 95 \% \mathrm{CI}=1.03,1.68), 19 \%$ higher risk of reaching of EDSS $6.0(\mathrm{HR}=1.19 ; 95 \% \mathrm{CI}=1.05,3.01)$, and $62 \%$ higher risk of disease modifying treatment escalation $(\mathrm{HR}=1.62 ; 95 \% \mathrm{CI}=1.22,3.01)$.

Discussion: Higher cardiovascular risk was associated with higher risk of relapses, disability, and treatment escalation in MS. Multiple mechanisms may contribute to explain the interactions between multiple cardiovascular comorbidities and MS, such as the association of cardiovascular comorbidities with increased brain atrophy and vascular oxidative stress.

Conclusions: Correction and treatment of cardiovascular risk factors, along with lifestyle changes, should be carefully considered in the longterm management of MS.

References:

- Marrie RA, Reider N, Cohen J, et al. A systematic review of the incidence and prevalence of cardiac, cerebrovascular, and peripheral vascular disease in multiple sclerosis. Mult. Scler. (2015); 21(3):318-31

- Moccia M, Lanzillo R, Palladino R, et al. The Framingham cardiovascular risk score in multiple sclerosis. Eur J Neurol. (2015);22(8):1176-83 
- D’Agostino RB, Vasan RS, Pencina MJ, et al. General cardiovascular risk profile for use in primary care: The Framingham heart study. Circulation (2008);117(6):743-53

\section{CHARACTERIZING 1-YEAR DEVELOPMENT OF CERVICAL CORD ATROPHY ACROSS DIFFERENT MS PHENOTYPES: A VOXEL-WISE, MULTICENTER ANALYSIS}

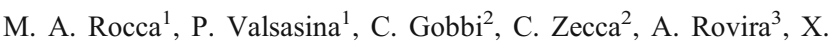 \\ Montalban ${ }^{4}$, H. Kearney ${ }^{5}$, O. Ciccarelli ${ }^{5}$, L. Matthews ${ }^{6}$, J. Palace ${ }^{6}$, A. \\ Gallo $^{7}$, A. Bisecco ${ }^{7}$, A. Gass ${ }^{8}$, P. Eisele ${ }^{8}$, M. Filippi ${ }^{9}$
}

${ }^{1}$ Neuroimaging Research Unit, Institute of Experimental Neurology, Division of Neuroscience, IRCCS San Raffaele Scientific Institute (Milano); ${ }^{2}$ Multiple Sclerosis Center, Department of Neurology, Neurocenter of Southern Switzerland, Civic Hospital (Lugano-CH); ${ }^{3}$ Section of Neuroradiology, Department of Radiology, Hospital Universitari Vall d'Hebron (Barcelona-E); ${ }^{4}$ Department of Neurology/ Neuroimmunology, Multiple Sclerosis Center of Catalonia, Hospital Universitari Vall d'Hebron (Barcelona-E); ${ }^{5} \mathrm{NMR}$ Research Unit, Queen Square MS Centre, Department of Neuroinflammation, UCL Institute of Neurology (London-UK); ${ }^{6}$ Nuffield Department of Clinical Neurosciences, University of Oxford (Oxford-UK); ${ }^{7}$ Department of Advanced Medical and Surgical Sciences, and 3T MRI Center, University of Campania "Luigi Vanvitelli" (Napoli); ${ }^{8}$ Department of Neurology, Universitätsmedizin Mannheim, University of Heidelberg (Mannheim-D); ${ }^{9}$ Neuroimaging Research Unit, Institute of Experimental Neurology, Division of Neuroscience; Neurology Unit and Neurophysiology Unit, IRCCS San Raffaele Scientific Institute; Vita-Salute San Raffaele University (Milano)

Objectives: Aim of this study was to use voxel-wise analysis to evaluate the distribution and changes over time of cervical cord atrophy in MS patients from a multicentre dataset acquired at 7 European sites.

Materials: Baseline and 1-year 3D T1-weighted cervical cord scans and clinical evaluation were obtained from $54 \mathrm{HC}$ and $110 \mathrm{MS}$ patients (13 clinically isolated syndromes [CIS], 75 relapsing-remitting [RR] and 22 progressive [P]MS).

Methods: A pipeline optimized for longitudinal analysis was used to coregister baseline and 1-year follow-up cervical cord scans to a cord template, obtained by averaging straightened HC images from all centers. Voxel-wise differences of cervical cord atrophy, their longitudinal changes and correlations with clinical variables were assessed using SPM12 and full factorial models (sex-, age-, center- and total cord volume-corrected).

Results: Compared to HC, MS patients exhibited significant $(\mathrm{p}<0.05$, family-wise error [FWE] corrected) baseline cervical cord atrophy, mainly located in anterior, posterior and lateral cord regions at $\mathrm{C} 1 / \mathrm{C} 2$, as well as in posterior regions between $\mathrm{C} 4$ and C6. While CIS patients showed a slight cord tissue expansion vs $\mathrm{HC}$ at posterior C4, RRMS presented significant clusters of cord atrophy vs CIS, mostly in lateral and posterior C2-C4 regions, and PMS showed widespread cord atrophy vs RRMS patients at C4-C5 and C7 levels. During the follow-up, a significant progression $(\mathrm{p}<0.05, \mathrm{FWE})$ of cord atrophy was detected in MS patients, predominantly in the posterior and lateral cord at $\mathrm{C} 2$, and between $\mathrm{C} 4$ and C6. Such pattern of cord atrophy progression was mainly driven by RRMS patients, while CIS patients did not show cord tissue loss at follow-up vs baseline, and PMS patients showed circumscribed tissue loss in posterior regions at $\mathrm{C} 2$ and C6. A strong relationship $(\mathrm{p}<0.05$, FWE) was found between baseline clinical disability and baseline cord atrophy in the posterior and lateral cord at $\mathrm{C} 2-\mathrm{C} 4$. Also, baseline atrophy in the lateral cord at $\mathrm{C} 3-\mathrm{C} 4$ was able to explain clinical disability at 1-year follow-up.

Discussion: A differential cord atrophy distribution was found across phenotypes, with circumscribed inflammation-related cord expansion in CIS, followed by tissue loss starting in the upper cord in RRMS, and subsequently spreading to the lowest cervical segments in PMS. Cord atrophy progressed over time in MS, and was more widespread in RRMS than in PMS patients.

Conclusions: Voxel-wise analysis of cervical atrophy characterized 1year evolution of tissue loss across phenotypes. Cord atrophy was clinically relevant and contributed to explain follow-up clinical disability.

\section{THE IMPACT OF BREASTFEEDING DURATION AND CHILD OBESITY IN THE ASSOCIATION BETWEEN ADULT MULTIPLE SCLEROSIS (MS) AND TYPE 1 DIABETES MELLITUS (T1DM): EVIDENCE FROM THE ENVIMS STUDY}

\author{
G. J. Rosa ${ }^{1}$, F. Colucci ${ }^{1}$, A. Montesano ${ }^{1}$, I. Casetta ${ }^{1}$, T. Riise ${ }^{2}$, K. Myhr ${ }^{3}$, \\ C. Wolfson ${ }^{4}$
}

${ }^{1}$ Department of Biomedical and Speciality-Surgical Sciences, University of Ferrara (Ferrara); ${ }^{2}$ Department of Global Public Health and Primary Care, University of Bergen (Bergen-N); ${ }^{3}$ Department of Clinical Medicine, University of Bergen (Bergen-N); ${ }^{4}$ School of Population and Global Health, McGill University (Montreal-CDN)

Objectives: MS is an inflammatory demyelinating disorder of the central nervous system likely resulting from a gene-environment interaction. We aimed to investigate the association between MS and T1DM and the impact of two early life exposures, i.e., breastfeeding duration and childhood obesity, on such association.

Materials: We benefitted from data collected within a multinational case-control study (the EnvIMS Study) exploring the role of environmental risk factors in the developing MS risk. We analyzed data from two different populations, Sardinians, insular Italy ( $\mathrm{N}=1536)$ and Norwegians $(\mathrm{N}=2478)$. Cases were selected from population-based MS data collection and controls were randomly selected through health statistics and sex and age-frequency matched to cases. A postal questionnaire, EnvIMS-Q, was administered.

Methods: We searched for T1DM comorbility among cases and controls, and analysed the association between breastfeeding duration ( $<4$ vs. $4+$ months=reference) and body size shape between 5-10 years old as a proxy for body mass index (BMI). Logistic regression analysis was performed to obtain odds ratio (OR) and $95 \%$ confidence intervals $(95 \% \mathrm{CI})$.

Results: MS was found associated with T1DM only in the Sardinian study population $(\mathrm{OR}=3.56 ; 95 \% \mathrm{CI} 1.48,8,54)$ and especially in the male population ( $\mathrm{OR}=9.49,95 \% \mathrm{CI} 1.10,81.93)$. The association in both sexes was even stronger when adjusting for $\mathrm{BMI}$ and sex $(\mathrm{OR}=5.11,95 \% \mathrm{CI}$ $1.30,20.04)$ and for breastfeeding duration and sex $(\mathrm{OR}=3.67,95 \% \mathrm{CI}$ $1.34,10.05)$. No association was detected in the Norvegian study population.

Discussion: We found a strong association between MS and T1DM among Sardinians but not among Norwegians. This evidence supports the protective role of breastfeeding likely and the detrimental role of childhood obesity towards both T1DM and MS. A different genetic make-up may explain the lack of MS and T1DM association in the Norwegian study population, as well as different early life exposures in this population (es. longer breastfeeding habit). 
Conclusion: The risk of associated MS and T1DM in the general population appears to be increased by childhood obesity and reduced breastfeeding in the first months of life.

References:

- Pugliatti M., Casetta I. Drulovic J., Granieri E. et al. A novel questionnaire for multinational case-control studies of environmental risk factors in multiple sclerosis (EnvIMS-Q). Acta Neurol Scand Suppl (2012);195:43-50

- Magalhaes S., Pugliatti M., Casetta I., Drulovic J. Et at. The EnvIMS Study: Design and Methodology of an International Case-Control study of Environmental Risk Factors in Multiple Sclerosis. Neuroepidemiology (2015); 44:173-81

\section{ASSOCIATION BETWEEN VITAMIN D CONCENTRATION, GENETIC POLYMORPHISM OF THE CYP24A1 AND CYP2R1 GENES, AND DISABILITY WORSENING IN MULTIPLE SCLEROSIS: A FOLLOW UP STUDY}

G. Salemi, C. Scazzone, M. Aprile, A. Bianchi, M. Cardilicchia, S. Fanara, E. Portera, M. Ciaccio, P. Ragonese

Dept of Biomedicine, Neuroscience and Advanced Diagnostic, University of Palermo (Palermo)

Background: Low blood levels of 25(OH)vitamin D (VD) are associated with an increased risk of Multiple Sclerosis (MS) [1]. The meaning of this association remains unclear. VD regulates the immune response. However, numerous studies and RCT failed to show a better MS course at the increase of blood levels of VD. We have confirmed that low levels of VD are associated with an increased risk of MS. Moreover, in two different studies we showed an association with the polymorphism rs2248137 of the gene CYP24A1 and the polymorphism rs10766197 of the gene CYP2R1 [2-3].

Objective: 1) to evaluate if the contemporary presence of the corresponding allele of these two genes play an additive role on VD concentration and on MS severity. 2) to determine if clinic-demographic features, VD concentration, and genetic status of the two polymorphisms influence MS course at a follow up of four years.

Methods: We included all the subjects with definite MS according to the $2010 \mathrm{McD}$ nald diagnostic criteria that possessed VD concentration at baseline and data on the polymorphism of the genes CYP24A1 and CYP2R1 $(\mathrm{N}=80, \mathrm{M} 17, \mathrm{~F}$ 63). Clinical informations were obtained four years after VD determination in 76 subjects. Both at baseline and at four years we determined EDSS and MSSS.

Results: At baseline we found an association between low concentration of $\mathrm{VD}$ and progressive forms $(\mathrm{p}=0.02)$. A higher concentration of VD was observed in those subjects carrying the allele $\mathrm{G}+$ of the polymorphism rs2248137 of the gene CYP24A1 and the allele A+ of the polymorphism rs 10766197 of the gene CYP2R1 $(p=0.005)$. No association was observed between MS severity and the genetic status of the two genes. At the follow up, we observed a major increase of EDSS and MSSS in those subjects with lower VD levels at baseline ( $\triangle$ EDSS 4-0 $\mathrm{yrs}<20 \mathrm{ng} / \mathrm{ml}$ vs $>20 \mathrm{ng} / \mathrm{ml}, \mathrm{p}=0.04,<20 / 20-30 />30 \mathrm{ng} / \mathrm{ml}$, chi square for linear trend $=0.005$ ). Again, MS worsening was not associated with CYP24A1 and CYP2R1 genes.

Discussion. Low concentration of VD is associated with an increased risk of MS, a more rapid worsening of disability, and with progressive forms. The role of genetic polymorphisms of VD metabolism seem to be marginal by a clinical point of view.
Conclusions: VD concentration could be a marker of disease activity. Studies targeted to this hypothesis are awaited.

References:

1. Simpson Jr S, van der Mei I, Taylor B: The Role of Vitamin D in Multiple Sclerosis: Biology and Biochemistry, Epidemiology and Potential Roles in Treatment. Medicinal Chemistry (2018);14:129143

2. Scazzone C, Agnello L, Ragonese P, Lo Sasso B, Bellia C, Bivona G, Schillaci R, Salemi G, Ciaccio M: Association of CYP2R1 rs10766197 with MS risk and disease progression. J Neurosci Res. (2018):96(2): 297-304

3. Agnello L, Scazzone C, Lo Sasso B, Ragonese P, Milano S, Salemi G, Ciaccio M: CYP27A1, CYP24A1, and RXR- $\alpha$ Polymorphisms, Vitamin D, and Multiple Sclerosis: a Pilot Study. J Mol Neurosci. (2018);66(1):77-84

\section{EMBRACING RESILIENCE IN MULTIPLE SCLEROSIS: A NEW PERSPECTIVE FROM COVID-19 PANDEMIC}

E. Sbragia ${ }^{1}$, E. Colombo ${ }^{1}$, C. Pollio ${ }^{1}$, M. Cellerino ${ }^{1}$, L. Caterina ${ }^{2}$, M. Inglese $^{3}$, G. Mancardi ${ }^{4}$, G. Boffa ${ }^{1}$

${ }^{1}$ Department of Neuroscience, Rehabilitation, Ophthalmology, Genetics, Maternal and Child Health (DiNOGMI), University of Genoa (Genova); ${ }^{2}$ Laboratorio di Neuroscienze Sperimentali, Ospedale Policlinico San Martino IRCCS (Genova); ${ }^{3}$ Department of Neuroscience, Rehabilitation, Ophthalmology, Genetics, Maternal and Child Health (DiNOGMI) and Department of Neurology, University of Genoa and Ospedale Policlinico San Martino IRCCS (Genova); ${ }^{4}$ Department of Neuroscience, Rehabilitation, Ophthalmology, Genetics, Maternal and Child Health (DiNOGMI), Scientific Clinical Institutes Maugeri IRCCS, University of Genoa and IRCCS Maugeri (Genova, Pavia)

Background: Coronavirus Disease 2019 (COVID-19), a severe acute respiratory syndrome due to Coronavirus 2 (SARS-CoV-2) infection, determined cross-sectional social and emotive consequences, representing an unprecedented social experiment. Past epidemiological experiences [1] and recent studies dealing with COVID-19 pandemic and healthy population [2,3] already showed the deep albeit heterogeneous psychological repercussions of pandemics. Nevertheless, little is known about the relationship between COVID-19 outbreak and patients with chronic diseases, Multiple Sclerosis (MS) in particular, and about the possible strategies for boosting resilience, a well-known protective and buffering instrument helping in facing the challenges of life.

Aims: To assess the changes in mental distress during COVID-19 outbreak in patients with MS (pw-MS) and to identify predictive factors that could help in developing resilience and facing COVID-19 pandemic.

Methods: We enrolled 106 pw-MS (69 relapsing-remitting, 20 secondaryprogressive and 17 primary-progressive) who had undergone neuropsychological assessment before outbreak (between January the 1st 2019 and March the 1st 2020): patients were previously tested with Brief International Cognitive Assessment for MS (BICAMS), Hospital Anxiety and Depression Scale (HADS) and patient-reported MS Neuropsychological Screening Questionnaire (MSNQ-P). All patients were asked to fulfill an online survey comprehensive of sociodemographic information (e.g. marital and employment status), HADS self-rating Scale, MSNQ-P Questionnaire and finally Connor-Davidson Resilience self-rating Scale (CD-RISC 25), in order to evaluate resilience. Statistical analyses (repeated-measures ANCOVA) were performed using SPSS 23. 
Results: Even if no significant changes in HADS and MSNQ-P scores were detected during COVID-19 pandemic in our population, preexisting lower HADS and MSNQ-P scores were found to be significantly $(\mathrm{p}<0.0001)$ and independently associated with a better resilience attitude; conversely, no demographic, disease- and treatment-related elements resulted predictive neither of anxiety, depression and perceived cognitive status nor of better resilient behaviour.

Discussion: The fundamental role of anxiety in adaptation to challenges and in growing resilience in MS prompt physicians to pay more attention to anxiety and mood disorders in patients with chronic neurological diseases. Moreover, considering that pandemic consequences did not determine any increase of mental distress in our population, as caregivers, we should inquire on "the other side of the coin" of disease management and perceive the daily commitment required to our patients.

Conclusions: Our study confirms the fundamental role of anxiety diagnosis and of neuropsychological evaluation in pw-MS, outlining its compelling role in predicting a resilient and positive response in case of pervasive commitment and the necessity of a comprehensive care for pw-MS. References:

1. Liao Q, et al. Anxiety, worry and cognitive risk estimate in relation to protective behaviors during the 2009 influenza A/H1N1 pandemic in Hong Kong: ten cross-sectional surveys. BMC Infect Dis. (2014) Mar 27;14:169. doi: 10.1186/1471-2334-14-169

2. Buzzi C, et al. The Psycho-Social Effects of COVID-19 on Italian Adolescents' Attitudes and Behaviors. Ital J Pediatr. (2020) May 24;46(1):69. doi: 10.1186/s13052-020-00833-4

3. Lu W, et al. Psychological Status of Medical Workforce During the COVID-19 Pandemic: A Cross-Sectional Study. Psychiatry Res. (2020) Jun; 288:112936. doi: 10.1016/j.psychres. 2020.112936

\section{RECOVERY OF MENSTRUAL CYCLE IN WOMEN WITH MULTIPLE SCLEROSIS TREATED WITH AUTOLOGOUS HAEMATOPOIETIC STEM CELL TRANSPLANTATION}

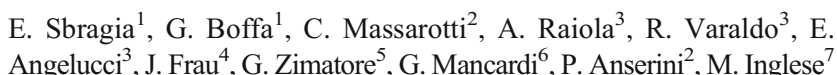

${ }^{1}$ Department of Neuroscience, Rehabilitation, Ophthalmology, Genetics, Maternal and Child Health (DiNOGMI), University of Genoa (Genova); ${ }^{2}$ Physiopathology of Human Reproduction Unit, IRCCS Policlinico San Martino (Genova); ${ }^{3}$ Hematology and Transplant Center, IRCCS Policlinico San Martino (Genova); ${ }^{4}$ Department of Medical Science and Public Health, Multiple Sclerosis Center, University of Cagliari (Cagliari); ${ }^{5}$ U.O.C. Neurology, Ospedale Generale Regionale (Bari); ${ }^{6}$ Department of Neuroscience, Rehabilitation, Ophthalmology, Genetics, Maternal and Child Health and Neuromotor Department, University of Genova and IRCCS ICS Maugeri, University of Genoa and IRCCS Maugeri (Genova, Pavia); ${ }^{7}$ Department of Neuroscience, Rehabilitation, Ophthalmology, Genetics, Maternal and Child Health (DiNOGMI) and Department of Neurology, University of Genoa and IRCCS Policlinico San Martino (Genova)

Background: Autologous haematopoietic stem cell transplantation (aHSCT) is increasingly taken into consideration as a treatment strategy for patients with aggressive multiple sclerosis (aMS) [1]. The chemotherapies used in the conditioning regimens for aHSCT are known to be responsible of amenorrhea in fertile women. Therefore, concerns on successful family planning in women with aggressive MS treated with aHSCT might rise.
Aims: To assess fertility outcomes in women treated with aHSCT for aMS considering different conditioning regimens.

Methods: We collected disease- and treatment-related characteristics from consecutive women with MS treated with aHSCT at the Italian MS centers of Genoa, Barletta and Cagliari. ANCOVA analyses and binary logistic regression were performed to assess the effects of baseline characteristics on fertility outcomes.

Results: We included 38 women [25(67\%) with relapsing-remitting MS] with a mean age at aHSCT of $31.7( \pm 6.7)$ years and a mean disease duration of $10.9( \pm 6.0)$ years. Mean age of menarche was $12.3( \pm 1.9)$ years. $31(82 \%)$ patients underwent transplant with a myeloablative conditioning regimen while $7(18 \%)$ patients were transplanted with a low-intensity lymphoablative regimen. $26(68.4 \%)$ patients recovered menstrual cycle after a mean time of 5.7(5.2) months. Among these, 10(38\%) patients had irregular periods ( $<21$ days; $>35$ days apart) and $11(42 \%)$ had changes in the menstrual flow. Patients who recovered menstrual cycle were younger at the time of transplant $(28.8 \mathrm{vs} 37.7$ years; $\mathrm{p}<0.0001)$ and had lower EDSS scores 1 year before aHSCT (4.7vs5.8; $\mathrm{p}=0.024)$. No significant differences were noted in terms of clinical phenotypes, age of menarche, body mass index, number of previous therapies, previous exposure to cyclophosphamide, mitoxantrone and the conditioning regimen used within aHSCT. A younger age was independently associated with a higher probability of recovery of menstrual cycle $(\mathrm{OR}=0.78 ; \mathrm{p}=0.006) .3 / 27$ patients $(11 \%)$ with $\leq 35$ years had persistent amenorrhea after aHSCT. We recorded 4 pregnancy after aHSCT ( 3 uncomplicated pregnancies and 1 spontaneous abortion). One patient repeatedly tried to conceive, both naturally and artificially, without success.

Discussion: Considering the increasing interest on fertility issues, aspiring to a comprehensive care of women with MS, and the worldwide growing role played by aHSCT in aMS, defining the fertility outcomes of different conditioning regimens represents a foundamental step in strenghten the safety of aHSCT.

Conclusions: Younger age is independently associated with the recovery of menstrual cycle in women with MS treated with aHSCT, which is obtained in $89 \%$ of women under 35 years old. The use of a myeloablative conditioning regimen doesn't seem to be associated with worse fertility outcomes.

Reference:

1. Mancardi G, et al. Intense immunosuppression followed by autologous haematopoietic stem cell transplantation as a therapeutic strategy in aggressive forms of multiple sclerosis. Mult Scler. (2018) Mar;24(3):245-55

\section{LOWER URINARY TRACT DISORDERS IN MULTIPLE SCLEROSIS PATIENTS: PREVALENCE, CLINICAL FEATURES AND RESPONSE TO TREATMENTS}

\author{
S. Seddone ${ }^{1}$, M. Marturano ${ }^{2}$, R. Bientinesi ${ }^{3}$, M. Lucchini ${ }^{1}$, P. Bassi ${ }^{3}$, M. \\ Mirabella ${ }^{1}$, V. Nociti ${ }^{1}$ \\ ${ }^{1}$ Institute of Neurology, “A. Gemelli” University Hospital IRCCS \\ (Roma); ${ }^{2}$ Urogynecology, Department of Woman and Child Health and \\ Public Health Sciences, "A. Gemelli" University Hospital IRCCS \\ (Roma); ${ }^{3}$ Department of Urology, "A. Gemelli" University Hospital \\ IRCCS (Roma)
}

Introduction: Bladder symptoms in patients with multiple sclerosis (MS) are common (from $32 \%$ up to $96 \%$ of patients) and have great impact on quality of life. First by frequency are overactive bladder (OAB) symptoms, such as frequency and/or urinary urgency associated or not with urge 
incontinence, followed by voiding disorders (hesitancy, interrupted voiding, feeling of incomplete bladder emptying). Urodynamic studies provide information about the physiopathology of these disorders. In MS patients, the most frequent urodynamic profile is detrusor overactivity. Symptomatic treatments are usually chosen empirically on patients' report.

Methods: In this cross-sectional retrospective study, conducted on consecutive MS patients who attended the MS Center of the Policlinico Universitario "A. Gemelli" IRCSS in Rome from 2nd of January 2018 to 31st of December 2018, we evaluated prevalence, clinical features and response to symptomatic treatments of lower urinary tract disorders. We also investigated the relationship between urological disorders and clinical and demographic features of our cohort. We evaluated the use of symptomatic treatments, adherence and response to therapy. Data of urodynamic studies performed in 2018 were collected.

Results: We enrolled 806 consecutive MS patients. The mean age was $45.84 \pm 12.86$ years with mean age at MS onset of $31.79 \pm 11.24$ years and mean disease duration of $12.54 \pm 9.60$ years. The overall prevalence of urological disorders was $52.9 \%(n=426)$ and urgency was the most frequent symptom in our cohort $(59.4 \%, \mathrm{n}=253)$. The most frequent clinical pattern was OAB syndrome $(54.2 \%, \mathrm{n}=231)$, followed by mixed symptomatology $(25.4 \%, \mathrm{n}=108)$ and voiding dysfunction $(20.4 \%, \mathrm{n}=87)$. The pattern of urological disorders differed in a statistically significant way in relation to the age at onset, earlier in patients with $\mathrm{OAB}$ syndrome and later in those with voiding dysfunction, and sex, as the presence of $\mathrm{OAB}$ symptoms was greater in women. Forty-six patients ( $11 \%$ of symptomatic ones) underwent an urodynamic study; the most frequent alterations were detrusor overactivity $(60.9 \%, \mathrm{n}=28)$ and increased proprioception $(43.5 \%, n=20)$. Only $41.8 \%$ ( $n=178)$ of symptomatic patients were under treatment for urological disorder. Of this group, $81.5 \%$ of patients reported an improvement of urinary symptoms during therapy.

Conclusions: These urinary disorders have a high prevalence with a significant impact on the patient's life, therefore. Lower urinary tract disorders in MS patients were more frequent in older patients, with longer disease duration, later onset of disease, progressive phenotype of disease and higher disability. It is essential to have an early and correct characterization of the type of symptoms and an early and targeted therapeutic strategy to improve the patient's quality of life and avoid future complications.

References:

- de Sèze M, Ruffion A, Denys P, et al. The neurogenic bladder in multiple sclerosis: review of the literature and proposal of management guidelines. Mult Scler Houndmills Basingstoke Engl (2007); 13: 915-28

- DasGupta R, Fowler CJ. Bladder, bowel and sexual dysfunction in multiple sclerosis: management strategies. Drugs (2003); 63: 153-66

- Mayo ME, Chetner MP. Lower urinary tract dysfunction in multiple sclerosis. Urology (1992);39:67-70

\section{DIMMI COME STAI. A WEB SURVEY ON PSYCHOLOGICAL REACTION OF PERSONS WITH MULTIPLE SCLEROSIS DURING THE COVID-19 EMERGENCY. THE ROLE OF MEDIA INFORMATION: STRENGTHS AND WEAKNESSES}

A. Sgoifo, M. Marazzi, E. Susani, L. Saraceno, M. Esposito, C. Campagna, E. Agostoni, A. Protti

\section{Dip. Neuroscience, ASST GOM Niguarda (Milano)}

Objective: We conducted a study to observe in persons with (pw) Multiple Sclerosis (MS) the psychological reaction during the Covid-19 emergency finding out possible patterns associated with psychological distress, concerning anxiety and depression (A/D), in this fragile population. The secondary objectives consider the effects of anamnestic and environmental factors on A/D. Resilience of pwMS, already studied for adaptation to MS, is also explored in this setting.

Materials: On April 10th 2020, 165 pwMS received by email an invitation to join the study by filling out an electronic case report form (e-crf). 88 e-crf were correctly returned. Moreover, 187 non MS affected subjects (no-MS-subj) joined voluntarily the study upon social network claim. The answers were referred to the last two weeks and contained: anamnestic data, lifestyle habits during the Covid-19 restrictions (job, family, media), "The 7-items Generalized Anxiety Disorder Scale" (GAD-7) and the "Patient Health Questionnaire-9" (PHQ-9) for depression.

Method: The study design is observational, retrospective, monocentric, focused on good clinical practice and conducted at the Niguarda Hospital. The principal outcome studied is the prevalence of PHQ-9 and GAD-7 scores in pwMS. The effects of environmental factors on $\mathrm{A} / \mathrm{D}$ were explored with nonparametric tests.

Results: Among pwMS (male $=27 \%$; mean age $=45$ years), the prevalence of depression is $27,5 \%$ and anxiety is $35 \%$. The mean A/D scores don't differ from no-MS-subj. The pwMS refer more fear for health ( $p=0.003$ ) considering themselves at higher risk. Respect to no-MS-subj, pwMS evaluate the media informations more useful $(\mathrm{p}=0.003)$ and reliable $(\mathrm{p}=0.017)$ motivating them to respect the restrictions $(\mathrm{p}=0.002)$. PwMS already suffering from depression have a more severe PHQ-9 score $(p<0.0001)$ and were supported with alternative strategies.

Discussion: The study had a good feedback by pwMS and the e-crf represented a very useful instrument, as other examples of telemedicine, in an outstanding period of restrictions. A/D scores were lower than expected maybe because not focused on MS and only screened whereas clinical evaluation is needed.

Conclusion: Despite the small sample, the results could suggest that pwMS showed a proper psychological resilience during the acute phase of the pandemic. They respected more easily the restrictions, maybe due to fear for health and sensibility to media informations. However, follow up is needed to check the psychological evolution.

References:

- Antonini A. Health care for chronic neurological patients after COVID-19. Lancet Neurol. (2020);19(7):562-563. doi:10.1016/ S1474-4422(20)30157-5

- Bloem BR, Dorsey ER, Okun MS. The Coronavirus Disease 2019 Crisis as Catalyst for Telemedicine for Chronic Neurological Disorders. JAMA Neurol. (2010) doi:10.1001/ jamaneurol.2020.1452

- Capuano R, Altieri M, Bisecco A, et al. Psychological consequences of COVID-19 pandemic in Italian MS patients: signs of resilience? J Neurol. (2020);1-8. doi:10.1007/s00415-020-10099-9

\section{CHANGES IN JCV INDEX VALUES IN MULTIPLE SCLEROSIS PATIENTS TREATED WITH DISEASE-MODIFYING DRUGS}

\author{
E. Sgralata, C. Chisari, S. Toscano, S. Arena, S. Lo Fermo, C. \\ Finocchiaro, E. D'Amico, M. Zappia, F. Patti
}

GF Ingrassia, Section of Neurosciences, University of Catania (Catania)

Objective: Natalizumab (NTZ) is one of the most efficacious drug for treating Multiple Sclerosis (MS). The decision of suspend NTZ is mainly 
driven by the risk of progressive multifocal leukoencephalopathy (PML), an opportunistic infection caused by John Cunningham virus (JCV). PML risk mainly depends on the anti-JCV antibody index (JCV index), which increases with age and number of NTZ infusions. However, no sufficient data about JCV status modification during other disease modifying drugs (DMDs) are to date available. Our aim was to evaluate JCV status modification during DMDs treatment.

Materials: This retrospective study screened all MS patients treated with DMDs and followed at the MS Centre of the University of Catania in the period between 2010 and 2020. Inclusion criteria were: diagnosis of MS according to the Mc Donald criteria 2010; treatment for at least six months with rituximab or ocrelizumab or alemtuzumab (RTX/OCR/ ALM), fingolimod (FTY), interferons or glatiramer (IFN/GA), teriflunomide (TFN), dimethyl fumarate (DMF); at least two determinations of JCV index during the treatment period.

Methods: JCV status was evaluated at baseline (T0), before starting treatment and during treatment (T1).

Results: Out of 200 patients screened, 152 (mean age $43.8 \pm 10.7$ years; 99 [65.1\%] females) were enrolled. Sixty-six (43.4\%) were treated with FTY, 31 (20.4\%) with RTX/OCR/ALM, 28 (18.4\%) with DMF, 16 $(10.5 \%)$ with TFN, and $11(7.2 \%)$ patients with IFN/GA. TFN-treated patients were older than IFN/GA, while FTY groups had a longer disease duration compared to IFN/GA and RTX/OCR/ALM. At T1, FTY group showed a significant increase in JCV index compared to $\mathrm{T} 0(2.14 \pm 1.28 \mathrm{vs}$ $2.62 \pm 1.39, \mathrm{p}=0.04)$. A reduction in JCV index, even if not statistically significant, was found in RTX/OCR/ALM $(2.64 \pm 1.30$ vs $2.28 \pm 1.55$, $\mathrm{p}=0.2$ ). In all the other treatment groups a slight and not statistically significant increase in JCV index between $\mathrm{T} 0$ and $\mathrm{T} 1$ was found.

Discussion: Treatment with DMDs may influence JCV status. In particular, FTY significantly increased JCV index, while B-cells depleting drugs induced a JCV index reduction, even if not statistically significant.

Conclusions: Considering that higher JCV index is associated to higher PML risk, our results may impact the decision making process, suggesting treatments different sequencing scenarios in which switching to different highly-effective DMDs should maximize disease control and minimize PML risk.

References:

- Aoyama S, Mori M, Uzawa A, Uchida T, Masuda H, Ohtani R, et al. Serum anti-JCV antibody indexes in Japanese patients with multiple sclerosis: elevations along with fingolimod treatment duration. $\mathrm{J}$ Neurol. (2018);265:1145-50

- Raffel J, Gafson AR, Malik O, Nicholas R. Anti-JC virus antibody titres increase over time with natalizumab treatment. Mult Scler Houndmills Basingstoke Engl. (2015);21:1833-8

- E. Sgarlata, C. G. Chisari, E. D’Amico, E. Millefiorini and F. Patti. Changes in Anti-JCV Antibody Status in a Large Population of Multiple Sclerosis Patients Treated with Natalizumab, CNS Drugs (2020) May;34(5):535-43

\section{ADIPONECTIN IS DETECTED IN CSF OF PATIENTS AFFECTED BY MULTIPLE SCLEROSIS}

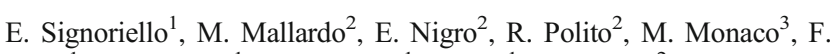
Rossi $^{1}$, A. Di Pietro ${ }^{1}$, S. Casertano ${ }^{1}$, G. Lus ${ }^{1}$, A. Daniele ${ }^{2}$

${ }^{1}$ Second Division of Neurology, University of Campania Luigi Vanvitelli (Napoli); ${ }^{2}$ Department of Pharmaceutical, Biological and Environmental Technologies Sciences, University of Campania Luigi Vanvitelli (Napoli); ${ }^{3}$ Advanced Biotechnology CEINGE (Napoli)
Background: Adiponectin, an adipose tissue secreted hormone, plays a key role as immunomodulatory protein in several inflammatory disorders. Recently, we demonstrated that basal serum adiponectin is associated to prognosis and progression of Multiple Sclerosis (MS) as in other autoimmune diseases. To our knowledge, no studies have analyzed so far the presence of adiponectin in cerebrospinal fluid (CSF) from MS patients as well as its oligomerization state. In serum, indeed, adiponectin is present as three different oligomers (low - LMW, medium - MMW, high HMW molecular weight).

Methods: 66 unrelated MS patients were recruited and compared to 32 age-and sex-matched controls. Sera and CSF obtained from patients were collected at the moment of diagnosis, before starting any treatment. Total adiponectin and its oligomers in sera and CSF were analyzed.

Results: Adiponectin and its oligomers were detected in CSF; importantly, adiponectin levels in CSF are higher in MS patients compared to matched controls $(9.91 \mathrm{ng} / \mathrm{ml}$ versus $6.02 \mathrm{ng} / \mathrm{ml})(\mathrm{p}<0.001)$. In addition, adiponectin oligomerization state is altered in MS, with an increase of HMW oligomers (those with the highest biological significance) compared to healthy controls. Interestingly, progressive MS patients have higher adiponectin levels in CSF compared to relapsing remitting $(15.51 \mathrm{ng} / \mathrm{ml}$ versus $8.9 \mathrm{ng} / \mathrm{ml})(\mathrm{p}=0.01)$ ones; CSF adiponectin positively correlates with serum adiponectin, higher EDSS at baseline and significantly higher MS severity score at 2.9 years follow-up. No difference in adiponectin expression was found between active and inactive patients.

Discussion and conclusions: For the first time our study detected adiponectin in CSF of MS patients. The correlation of CSF adiponectin with serum adiponectin and disease severity confirms and suggests that adiponectin plays an important role into MS pathogenesis suggesting that this adipokine could participate in neurodegenerative process of disease. References:

- E Signoriello, G Lus, R Polito, S Casertano, O Scudiero, M Coletta, M L Monaco, F Rossi, E Nigro, A Daniele. Adiponectin profile at baseline is correlated to progression and severity of multiple sclerosis. Eur J Neurol (2019) Feb;26(2):348-55

- Çoban A, Düzel B, Tüzün E, Tamam Y. Investigation of the prognostic value of adipokines in multiple sclerosis. Mult Scler Relat Disord (2017) Jul;15:11-14

- Guerrero-García JJ, Carrera-Quintanar L, López-Roa RI, MárquezAguirre AL, Rojas-Mayorquín AE, Ortuño-Sahagún D. Multiple Sclerosis and Obesity: Possible Roles of Adipokines. Mediators Inflamm (2016);2016:4036232

\section{DIMETHYL FUMARATE-INDUCED LYMPHOCYTE COUNT DROP IS ASSOCIATED WITH TREATMENT RESPONSE IN MULTIPLE SCLEROSIS: A REAL-LIFE MULTICENTRE STUDY}

E. Tsantes ${ }^{1}$, E. Curti ${ }^{1}$, D. Ferraro ${ }^{2}$, A. Lugaresi ${ }^{3}$, E. Baldi ${ }^{4}$, S. Montepietra $^{5}$, P. Immovilli ${ }^{6}$, A. Simone ${ }^{7}$, L. Mancinelli ${ }^{8}$, S. Strumia ${ }^{9}$, F. Vitetta ${ }^{10}$, M. Foschi ${ }^{11}$, C. Ferri ${ }^{12}$, C. Ferrarini ${ }^{1}$, P. Sola ${ }^{10}$, F. Granella ${ }^{1}$

${ }^{1}$ Neurosciences Unit, Department of Medicine and Surgery, University of Parma (Parma); ${ }^{2}$ Department of Biomedical, Metabolic and Neurosciences, University of Modena and Reggio Emilia (Modena); ${ }^{3}$ UOSI Rehabilitation Multiple Sclerosis, IRCCS Istituto delle Scienze Neurologiche of Bologna (Bologna); ${ }^{4}$ Neurology Unit, Department of Neuroscience/Rehabilitation Azienda Ospedaliera-Universitaria S. Anna (Ferrara); ${ }^{5}$ Neurology Unit, Arcispedale Santa Maria Nuova-IRCCS (Reggio Emilia); ${ }^{6}$ Neurology Unit, Guglielmo da Saliceto Hospital 
(Piacenza); ${ }^{7}$ Neurology Unit, Carpi Hospital, AUSL Modena (CarpiMO); ${ }^{8}$ Neurology Unit, Bufalini Hospital, AUSL Romagna (Cesena); ${ }^{9}$ Neurology Unit, G.B. Morgagni -L. Pierantoni Hospital, (Forli); ${ }^{10}$ Department of Neurosciences, Azienda Ospedaliero-Universitaria of Modena, Ospedale Civile of Baggiovara (Modena); ${ }^{11}$ Neurology Unit, S. Maria delle Croci Hospital, AUSL Romagna (Ravenna); ${ }^{12}$ Department of Biomedical and Specialty Surgical Sciences, University of Ferrara (Ferrara)

Background and Objective: Dimethyl fumarate (DMF) is an effective treatment for relapsing-remitting multiple sclerosis (RRMS) patients. Besides a partially known mechanism of action involving neuroprotective and antioxidant effects, it causes a mean lymphocyte drop of approximately $30 \%$, typically occurring within the first year of treatment. The relationship between this reduction and DMF effectiveness is still uncertain. Primary aim was to ascertain if lymphocyte decrease during DMF therapy is related to NEDA-3 ("no evidence of disease activity") status preservation. NEDA-3 is defined as absence of clinical relapses, MRI disease activity and confirmed disability progression. Secondary aims were to investigate ALC variations over time and the impact of baseline demographic and clinical factors on DMF-induced lymphopenia.

Materials and methods: Demographic, laboratory, clinical and MRI data were collected in this real-life, observational multicentre study, performed on RRMS patients treated with DMF for at least 6 months. Multivariate Cox models were performed to evaluate the impact of six month-ALC reduction on time to NEDA-3 status loss and Kaplan-Meier curves were generated to display the results. Multivariate logistic regression was carried out to investigate possible predictors of lymphopenia.

Results: 476 patients ( 312 females, age at DMF start $38.4 \pm 9.97$ years) were studied during a mean follow-up time of 29 months (range 6-61). A greater lymphocyte drop protected against the cumulative incidence of NEDA-3 status loss (HR 0.87, $\mathrm{p}=0.01$ ). A higher risk of disease activity $(\mathrm{p}=0.008)$ was observed in tertile with lower ALC reduction $(<11.5 \%)$, compared with other tertiles $(11.5-40.5 \%$ and $>40.5 \%$ ALC drop, respectively). A smaller six month-ALC decrease influenced a shorter relapse-free $(\mathrm{p}=0.03$, HR 0.85$)$ and MRI CUA-free $(\mathrm{p}<0.0001, \mathrm{HR} 0.80)$ survival. Starting DMF after the age of 40 was associated with longer NEDA-3 survival $(\mathrm{p}=0.02)$. Regarding secondary aims, the nadir of mean ALC drop $(-33.6 \%)$ and $35 \%$ of grade III lymphopenia cases were observed 24 months after treatment start. An older age at DMF start (OR 1.03, 95\% CI 1.00-1.06, $\mathrm{p}=0.009$ ) and lower ALC at baseline (OR 1.69, 95\% CI 1.34$2.14, \mathrm{p}<0.0001$ ) predicted a higher risk of lymphopenia.

Discussion: Our data showed an association between greater ALC reduction during treatment and longer NEDA-3 survival. One third of severely lymphopenic patients and the nadir of ALC drop occurred after 12 months of treatment.

Conclusions: A higher six month-lymphocyte count drop is related to better outcomes in DMF-treated patients. A careful ALC monitoring should be pursued up to 24 months of treatment.

References:

- Fox RJ, Kita M, Cohan SL, Henson LJ, Zambrano J, Scannevin RH, et al. BG-12 (dimethyl fumarate): a review of mechanism of action, efficacy, and safety. Curr Med Res Opin (2014); 30(2): 251-62

- Fox RJ, Chan A, Gold R, Phillips JT, Selmaj K, Chang I, et al. Characterizing absolute lymphocyte count profiles in dimethyl fumarate-treated patients with MS: patient management considerations. Neurol Clin Pract. (2016); 6:220-9

- Wright K, Winkler MD, Newton BD, Sormani MP, Okuda DT. Patient outcomes influenced by reduced lymphocyte counts after dimethyl fumarate initiation. Neurol Neuroimmunol Neuroinflamm. (2017);25:4(6):e397

\section{OCRELIZUMAB AS EXIT STRATEGY FROM NATALIZUMAB: RESULTS FROM A CLINICAL SERIES}

\author{
E. Tsantes, E. Curti, A. Fiore, V. Bazzurri, F. Granella
}

Neurosciences Unit, Department of Medicine and Surgery, University of Parma (Parma)

Background and Objective: Natalizumab (NTZ) is highly effective for relapsing-remitting (RR) multiple sclerosis (MS) treatment, but its prolonged use can increase the risk of progressive multifocal leukoencephalopathy (PML). Therefore, NTZ is mainly interrupted in the context of PML risk management strategy, but its discontinuation can precipitate disease activity. Currently, there is no consensus on either the duration of the washout period, or the best subsequent treatment approach. Few data are available on the use of anti-CD20 monoclonal antibodies (mostly rituximab) after NTZ withdrawal, whereas only a small case series of patients switching to ocrelizumab (OCR) has been published in literature. Aim of this study was to evaluate the effectiveness, tolerability and safety of OCR in a real-world cohort of MS patients switching from NTZ.

Materials and methods: Demographic, laboratory, clinical and MRI data were collected in this retrospective, observational study, performed on MS patients attending Parma MS centre.

Results: 23 patients (14 females, mean age $40.4 \pm 8.57$ years) were analysed during a mean follow-up of 15 months (range 5-34), since NTZ discontinuation. Patients had the following characteristics at OCR start: RR course in $74 \%$ and progressive active disease in 26\%, mean EDSS 3.1 (1.5-6.5), mean disease duration $11 \pm 5.67$ years and median number of NTZ infusions 28 (13-122). The reasons for switch included PML concern in $78 \%$ of cases and suboptimal response to NTZ in $22 \%$. The majority (83\%) of patients was $\mathrm{JCV}+$ and only 6 were treated with NTZ extended interval dosing for $\geq 18$ months. The median washout period was 50 days (41-251); all patients underwent a brain MRI immediately before OCR start. Only 4/23 (17\%) and 1/19 (5\%) patients experienced clinical relapses (all within the first 6 months of therapy) and MRI activity, respectively. ARR decreased from 0.41 (during NTZ) to 0.18 with OCR. Mild adverse events (mostly infusion reactions, hypogammaglobulinemia and leukopenia) occurred in $74 \%$ of cases. No patients discontinued OCR and no PML cases emerged.

Discussion: OCR is effective and well tolerated in highly active MS patients switching from NTZ. A median 6-week transition period appears reasonably safe to balance the risk of disease reactivation with that of opportunistic infections. Larger datasets are needed to confirm the risk of carryover PML in relationship with the length of washout period.

Conclusions: OCR start, after a median 6-week washout period, appears an effective, safe and well tolerated strategy in MS patients switching from NTZ.

References:

- Ho PR, Koendgen H, Campbell N, et al. Risk of natalizumabassociated progressive multifocal leukoencephalopathy in patients with multiple sclerosis: A retrospective analysis of data from four clinical studies. Lancet Neurol (2017); 16: 925-33

- Alping P, Frisell T, Novakova L, et al. Rituximab versus fingolimod after natalizumab in multiple sclerosis patients. Ann Neurol (2016); 79:950-58

- Levin SN, Ezuma C, Levine L, et al. Switching from Natalizumab to Ocrelizumab in Patients With Multiple Sclerosis Mult Scler (2020);1352458520927631. doi: 10.1177/1352458520927631 


\section{KAPPA FREE LIGHT CHAINS: MARKERS OF INTRATHECAL SYNTHESIS IN MULTIPLE SCLEROSIS}

\author{
D. Vecchio ${ }^{1}$, I. Crespi ${ }^{2}$, G. Bellomo ${ }^{2}$, R. Serino ${ }^{2}$, E. Virgilio ${ }^{1}$, U. \\ Dianzani $^{2}$, R. Cantello ${ }^{1}$, C. Comi ${ }^{1}$
}

${ }^{1}$ Neurology Unit, Department of Health Sciences, University of Piemonte Orientale (Novara); ${ }^{2}$ Clinical Biochemistry; Department of Health Sciences, University of Piemonte Orientale (Novara)

Background: Cerebrospinal fluid (CSF) kappa free light chain (KFLC) index has been described as a reliable marker of intrathecal synthesis in multiple sclerosis (MS).

Aims: 1) to compare the efficiency of KFLC through different interpretation approaches in diagnosing MS. 2) to evaluate the prognostic value of KFLC in radiological and clinical isolated syndromes (RIS-CIS).

Methods: We included 133 MS patients and 240 with other neurological diseases (93 inflammatory including 18 RIS-CIS, 147 non-inflammatory). Albumin, lambda (L) and KFLC were measured in the CSF and serum by nephelometry. We included two groups of markers: a) corrected for blood-brain barrier permeability: IgG, KFLC and LFLC indexes. b) "isolated CSF" ratios: KFLC/LFLC, KFLC/IgG, LFLC/IgG.

Results: KFLC were significantly higher in MS patients compared to those with other diseases (both inflammatory or not). KFLC index and "isolated CSF" KFLC/IgG ratio showed high sensitivity (93\% and $86.5 \%$ ) and moderate specificity (85\% and $88 \%$ ) in diagnosing MS. RIS-CIS patients who converted to MS showed greater KFLC index and "isolated CSF" KFLC/IgG.

Conclusions: Despite OB are confirmed to be the gold-standard to detect intrathecal synthesis, the KFLC confirmed their accuracy in MS diagnosis. A "K-oriented" response characterises MS and has a prognostic impact in the RIS-CIS population.

References:

- Kaplan B, Ganelin-Cohen E, Golderman S, Livneh A. Diagnostic utility of kappa free light chains in multiple sclerosis. Expert Review of Molecular Diagnostics (2019);19:4:277-79

- Crespi I, Sulas MG, Mora R, et al. Combined use of Kappa Free Light Chain Index and Isoelectrofocusing of Cerebro-Spinal Fluid in Diagnosing Multiple Sclerosis: Performances and Costs. Clinical Laboratories (2017);63:551-59

- Presslauer S, Milosavljevic D, Huebl W, et al. Validation of kappa free light chains as a diagnostic biomarker in multiple sclerosis and clinically isolated syndrome: a multicenter study. Multiple Sclerosis (2016);22:502-10

\section{CARDIOVASCULAR DYSFUNCTIONS IN A CUPRIZONE- INDUCED MOUSE MODEL OF MULTIPLE SCLEROSIS: A FOCUS ON ABERRANT EXPRESSION OF CARDIAC INWARDLY RECTIFYING POTASSIUM CHANNELS}

\section{Villa ${ }^{1}$, E. Akuyz ${ }^{2}$}

${ }^{1}$ Department of Medicine and Surgery, University of Milano-Bicocca (Monza); ${ }^{2}$ Department of Biophysics, Yozgat Bozok University (Yozgat-TR)

Aims: Multiple sclerosis (MS) is a chronic inflammatory autoimmune disease which affects the central nervous system (CNS) and is characterized by demyelination, astrogliosis and damage to oligodendrocytes.
Interestingly, MS patients showed an increased cardiovascular (CV) risk probably due to an impairment in the autonomic control of CV functions. However, the underlying molecular mechanisms are not completely understood. In this regard, inwardly-rectifying potassium (Kir) channels play a key role in cardiac excitability by contributing to the repolarization phase of action potential and were recently identified as target of the autoantibody response in MS patients. Thus, we aimed to investigate the role of cardiac Kir channels in the CV dysfunctions occurring in MS.

Methods: Electrocardiographic recordings (ECG) evaluated cardiac parameters and electrical activity in a cuprizone-fed C57BL/6 mice, a classic demyelination animal model. Relative transcript levels of cardiac Kir2.2, Kir4.1 and Kir6.2 channels in mice were analyzed using real-time PCR.

Results: The cuprizone-induced mouse model was confirmed by immunohistochemistry analysis showing demyelination in the corpus callosum. ECG recordings from mice showed a significant decreased duration of the $P$ wave and RR interval as well as an increase of the heart rate in cuprizonetreated mice as compared with the controls. Significant high expression levels of Kcnj11 and Kcnj12, encoding for Kir6.2 and Kir2.2 channels respectively, were observed in mouse heart tissue as compared with controls, whereas no differences in Kir4.1 channels were observed.

Discussion: To the best of our knowledge, these findings suggested a potential role of cardiac Kir channels explaining autonomic dysfunctions occurring in MS pathology.

Conclusions: Our data open more avenues to the development of novel therapeutic strategies based on targeting cardiac Kir channels.

\section{SERUM VITAMIN D LEVEL AT DIAGNOSIS AS A MARKER OF IMPAIRED INFORMATION PROCESSING SPEED AND EARLY DISABILITY IN MULTIPLE SCLEROSIS PATIENTS}

\author{
E. Virgilio ${ }^{1}$, D. Vecchio ${ }^{2}$, I. Crespi ${ }^{3}$, B. Caloni ${ }^{2}$, P. Naldi ${ }^{2}$, U. Dianzani ${ }^{3}$, \\ R. Cantello ${ }^{2}$, C. Comi ${ }^{4}$
}

${ }^{1}$ Multiple Sclerosis Center, Neurology Unit, Maggiore della Carità Hospital, Department of Translational Medicine, University of Eastern Piedmont (Novara); ${ }^{2}$ Neurology Unit, Maggiore della Carità Hospital, Department of Translational Medicine, University of Eastern Piedmont (Novara); ${ }^{3}$ Clinical Biochemistry, Department of Health Sciences, University of Eastern Piedmont (Novara); ${ }^{4}$ Neurology Unit, Sant'Andrea Hospital, Department of Translational Medicine, University of Eastern Piedmont (Vercelli)

Introduction: Cognitive impairment (CI) affects a large proportion of multiple sclerosis (MS) patients from disease onset to all stages (1). Slowed information processing speed (IPS) is the hallmark of CI in MS and the first cognitive domain to be altered at the diagnosis. Symbol Digit Modalities Test (SDMT) is the recommended test for IPS evaluation in MS at diagnosis $[1,2]$. Insufficient serum vitamin D was previously associated with risk of MS development, disease activity and progression but few data are reported regarding its effect on cognition. On the other hand, the association between serum vitamin D levels and CI in other neurodegenerative diseases is already established [3].

Aim: The aim of this study is to explore the possible correlation between vitamin D and cognition, in particular IPS, at MS diagnosis and to confirm the relationship between vitamin $\mathrm{D}$ and early disability at last follow-up.

Methods: We retrospectively collected vitamin D levels in serum at diagnosis. IPS was evaluated with SDMT at diagnosis. Raw scores were adjusted for age, sex and educational level using Italian brief international cognitive assessment for multiple sclerosis (BICAMS) Test Battery 
normative values, and z-scores were obtained [1]. Early disability at last clinical follow-up was evaluated with MS severity score (MSSS) and agerelated MSSS (ARMSS).

Results: We enrolled 60 newly diagnosed MS patients. 40 patients were female; the mean age at diagnosis was 39,5 years old $( \pm 12) .51$ patients $(85 \%)$ showed hypovitaminosis $\mathrm{D}$, with mean levels of $21,17 \mathrm{ng} / \mathrm{mL}( \pm 10,02) .16$ patients $(27 \%)$ had $\mathrm{CI}$, tracked with impaired IPS (defined as $\mathrm{z}$-score $\leq 1,5$ ). Mean SDMT raw score was $46,50( \pm 14,73)$ and $z$-score was $-0,62$ $( \pm 1,29)$. Patients with CI showed more severe hypovitaminosis $\mathrm{D}(\mathrm{p}=0,02)$, and no patients with sufficient vitamin D levels had CI. We found a positive correlation between vitamin D levels and both SDMT raw values $(\mathrm{R}=0,41 \mathrm{p}=0,001)$ and $\mathrm{z}$-score $(\mathrm{R}=0,34$ $\mathrm{p}=0,008)$. Mean follow-up was 2 years $( \pm 1)$ and we found a week correlation between vitamin $\mathrm{D}$ at diagnosis and MSSS $(\mathrm{R}=-0,29$, $\mathrm{p}=0,02)$ and ARMSS $(\mathrm{R}=-0,23 \mathrm{p}=0,08)$ at last clinical follow-up.

Conclusions: Low vitamin D levels may affect both cognition (in particular IPS) and early disability in newly diagnosed MS patients. This association might represent a marker of future disability, supporting the need for prompt supplementation. We plan, in the future, to replicate our findings in a bigger population and to reassess IPS after vitamin D supplementation.

References:

1. Goretti B, Niccolai C, Hakiki B, Sturchio A, et al. The Brief International Cognitive Assessment for Multiple Sclerosis (BICAMS): normative values with gender, age and education corrections in the Italian population. BMC Neurol. (2014) Sep $10 ; 14: 171$

2. Kalb R, Beier M, Benedict RH, Charvet L, et al. Recommendations for cognitive screening and management in multiple sclerosis care. Mult Scler. (2018) Nov;24(13):1665-1680. doi: 10.1177/ 1352458518803785. Epub 2018 Oct 10. PMID: 30303036; PMCID: PMC6238181

3. Chai B, Gao F, Wu R, Dong T, Gu C, Lin Q, Zhang Y. Vitamin D deficiency as a risk factor for dementia and Alzheimer's disease: an updated meta-analysis. BMC Neurol. (2019) Nov 13;19(1):284. doi: 10.1186/s12883-019-1500-6. PMID: 31722673; PMCID: PMC6854782

\section{DAMAGE OF THE SUBVENTRICULAR ZONE: RELATION WITH STRIATAL ATROPHY AND COGNITIVE PERFORMANCE IN MS}

\section{A. Zanghì ${ }^{1}$, L. Cacciaguerra ${ }^{2}$, A. Meani ${ }^{3}$, O. Marchesi ${ }^{3}$, M. Filippi ${ }^{4}$, M. Rocca $^{2}$}

${ }^{1}$ Neurology, University of Catania (Catania); ${ }^{2}$ Neuroimaging Research Unit, Institute of Experimental Neurology, Division of Neuroscience; and Neurology Unit, IRCCS San Raffaele Scientific Institute; Vita-Salute San Raffaele University (Milano); ${ }^{3}$ Neuroimaging Research Unit, Institute of Experimental Neurology, Division of Neuroscience, IRCCS San Raffaele Scientific Institute (Milano); ${ }^{4}$ Neuroimaging Research Unit, Institute of Experimental Neurology, Division of Neuroscience; Neurology Unit and Neurophysiology Unit, IRCCS San Raffaele Scientific Institute; Vita-Salute San Raffaele University (Milano)

Objectives: In this study, we characterized subventricular zone (SVZ) damage in terms of focal lesions and microstructural alterations in Multiple Sclerosis (MS) and assessed its association with striatal atrophy and cognitive dysfunction, evaluated with the Symbol Digit Modalities Test (SDMT).
Materials: 3.0 T brain magnetic resonance imaging (MRI) scans were acquired from 97 MS patients and 43 age- and sex-matched healthy controls (HC)

Methods: After lesion refilling, normalized (N-) brain volumes and cortical thickness (CT) were obtained. According to anatomical references, SVZ mask was segmented on T1-weighted images in the Montreal Neurological Institute space and then registered on fractional anisotropy (FA) and mean diffusivity (MD) maps. Age- and sex-adjusted linear models, partial correlations, and stepwise multiple linear regressions were used to assess SVZ damage and to identify predictors of $\mathrm{N}$-striatal volume and SDMT scores.

Results: In MS, mean SVZ percentage lesion volume (LV) was $4.2 \%$. Compared to HC, SVZ normal appearing (NA) tissue was characterized by increased MD ( 0.89 vs $0.86, p=0.04)$ and preserved FA values. Nstriatal volume correlated with all measures of brain damage ( $\mathrm{p}$ range: $<0.0001-0.02, \mathrm{r}$ absolute values range: $0.24-0.70$ ), while SDMT correlated with SVZ damage (percentage LV, lesional FA, NA MD, p range: 0.028-0.0028, $\mathrm{r}$ absolute values range: $0.33-0.36$ ) and brain T2-weighted $\mathrm{LV}(\mathrm{p}=0.0051, \mathrm{r}=-0.37)$. N-brain volume $(\mathrm{p}<0.0001)$, white matter MD $(p=0.0236)$, SVZ percentage LV $(p=0.0052)$, and mean CT $(p=0.0354)$ were independent predictors of $\mathrm{N}$-striatal volume $(\mathrm{R} 2=0.67)$. $\mathrm{SVZ}$ percentage LV was selected as the only predictor of SDMT performance $(\mathrm{p}=0.0018, \mathrm{R} 2=0.26)$.

Discussion: SVZ damage is associated with striatal atrophy and cognitive dysfunction in MS. MS can be considered as a disease-related model of SVZ injury since periventricular lesions involve this region. SVZ is likely to exert a neuroprotective role on striatal neurons and its damage has been associated with cognitive decline after brain radiation. In MS, cognitive dysfunction is common and information processing speed is affected from the earliest phases of the disease despite relatively low LV and atrophy.

Conclusions: These results might provide a novel key lecture on cognitive impairment in this disease, suggesting a possible role of periventricular injury in MS cognition.

References:

- Butti E, Bacigaluppi M, Rossi S, Cambiaghi M, Bari M, Cebrian Silla A, et al. Subventricular zone neural progenitors protect striatal neurons from glutamatergic excitotoxicity. Brain: a journal of neurology. (2012);135(Pt 11):3320-35

- Peiffer AM, Leyrer CM, Greene-Schloesser DM, Shing E, Kearns WT, Hinson WH, et al. Neuroanatomical target theory as a predictive model for radiation-induced cognitive decline. Neurology (2013);80(8):747-53

- Rocca MA, Amato MP, De Stefano N, et al. Clinical and imaging assessment of cognitive dysfunction in multiple sclerosis. Lancet Neurol. (2015);14(3):302-17

\section{FRAMINGHAM SCORE, SEDENTARY BEHAVIORS AND ASSOCIATION WITH DISEASE ACTIVITY IN RELAPSING REMITTING MULTIPLE SCLEROSIS}

\section{A. Zanghì, E. D'amico, M. Ciaorella, F. Patti, M. Zappia}

Department G.F. Ingrassia, University of Catania (Catania)

Objective: The aim of the study is the association between cardiovascular risk (measured with Framingham risk score (FRS)), sedentary behavior (measured with Godin Leisure-Time Excercise Questionnaire (GLTEQ)) and disease activity in MS patients. 
Materials: Patients with Relapsing Remitting MS (RRMS) consecutively admitted to the MS center of Catania from October 2019 to February 2020 have been enrolled.

Methods: According to the reason of access to the MS center, patients were divided in: active patients (patients with clinical relapses and/or radiological activity) and non-active patients (scheduled visit). The assessment included collection of demographical and clinical outcomes. To analyse any association between disease activity and FRS and GLTEQ a binary logistic regression model was built.

Results: 432 patients were enrolled. Out of them, 334 (77.8\%) were non active and $98(22.2 \%)$ were active. Active patients were younger, with higher number of relapses in the year before enrolment and higher level of disability ( $\mathrm{p}<.05$ for all). About FRS, no differences were found between the two groups in low $(54.3 \%$ vs 54.1$)$, intermediate ( $34 \%$ vs $34.2 \%)$ and high risk (11\% vs $11.7 \%)$ rates. About GLTEQ, no differences were found between the two groups in rates of full active (16\% vs $19.5 \%)$, sufficiently active (53.2\% vs $57.7 \%$ ) and inactive $(30.9 \%$ vs $22.8 \%$ ) patients. At logistic regression model, there was a correlation between a high GLTEQ value and disease activity (ExpB 2.462, IC 1.000-6.094, $\mathrm{p}=.049)$. No correlations were found with other clinical variables or with FRS.

Discussion: Cardiovascular comorbidities are one of the most leading causes of disability in the general population. Furthermore, sedentary behavior has been associated with putative outcomes of disability status in patients with MS and its correlation with disease activity needs to be further investigated.

Conclusions: Cardiovascular comorbidities and sedentary behavior deserve attention in all MS cohort. Further studies are needed.

References:

- Charron S, McKay KA, Tremlett H. Physical activity and disability outcomes in multiple sclerosis: A systematic review (2011-2016). Mult Scler Relat Disord. (2018);20:169-177. doi:10.1016/ j.msard.2018.01.021

- Roshanisefat H, Bahmanyar S, Hillert J, Olsson T, Montgomery S. Multiple sclerosis clinical course and cardiovascular disease risk Swedish cohort study. Eur J Neurol (2014); 21: 1353-1360. Doi: 10.1111/ene. 12518

- Jet JCS Veldhuijzen van Zanten, Lara A Pilutti and Joan L Duda. Sedentary behaviour in people with multiple sclerosis: Is it time to stand up against MS? Topical Reviews. (2016);1250-56

\section{IMPACT OF COVID-19 ON DMTS TREATED MULTIPLE SCLEROSIS PATIENTS}

M. A. Zingaropoli ${ }^{1}$, M. Iannetta ${ }^{2}$, V. Perri ${ }^{1}$, P. Pasculli ${ }^{1}$, L. Mazzocchi ${ }^{1}$, M. Tartaglia ${ }^{3}$, C. Merluzzo ${ }^{3}$, A. Conte ${ }^{4}$, F. Pauri ${ }^{3}$, D. Landi ${ }^{2}$, G. Marfia ${ }^{2}$, L. Sarmati ${ }^{2}$, M. Andreoni ${ }^{2}$, C. Mastroianni ${ }^{1}$, M. Ciardi ${ }^{1}$

${ }^{1}$ Department of Public Health and Infectious Diseases, Sapienza University (Roma); ${ }^{2}$ System Medicine, Tor Vergata University (Roma); ${ }^{3}$ Department of Human Neurosciences, Sapienza University (Roma); ${ }^{4}$ IRCCS Neuromed (Pozzilli-IS)

Objectives: To evaluate the potential exposure to SARS-CoV-2 of MS patients under disease-modifying therapies (DMTs) with confirmed or suspected COVID-19.

Materials and Methods: MS patients from Neuroinfectious Disease Unit of Policlinico Umberto I (Rome, Italy), have been interviewed by phone, asking about COVID-19 suggestive symptoms (fever, cough, rhinitis, sore throat, headache, anosmia, ageusia, arthromyalgia, dyspnea) occurred between January 2020 and May 2020. Moreover information on travels in high-risk zones of Northern Italy, epidemiological link with SARS-CoV-2 positive person and flu vaccination status were collected.

Results: Forty-seven MS patients (27 females/20 males) with a median age [interquartile range (IQR)] of 46 (36.5-52) years, median years of disease (IQR) of 8 (1-16) and median EDSS (IQR) of 4 (1.5-6) were interviewed. At the time of enrollment, $85.1 \%$ $(40 / 47)$ of patients were under DMTs: $57.5 \%$ (23/40) with ocrelizumab, $10 \%$ (4/40) with natalizumab, $7.5 \%$ (3/40) with dimethyl fumarate, $7.5 \%(3 / 40)$ with teriflunomide, $5 \%(2 / 40)$ with cladribine, $5 \%(2 / 40)$ with siponimod, $2.5 \%(1 / 40)$ with fingolimod, $2.5 \%(1 / 40)$ with glatiramer acetate, and $2.5 \%(1 / 40)$ with alemtuzumab. Among all patients, 10.6\% (5/47) reported symptoms suggestive for SARS-CoV-2 infection. One teriflunomide-treated multiple sclerosis patient with confirmed COVID-19 pneumonia was admitted at Policlinico Umberto I COVID-19 Unit because of fever, arthromyalgia and dyspnea. The patient was discharged after 10 days in a good health condition [1]. Two patients, hospitalized at Policlinico Torvergata because of dry cough and fever, developed mild COVID-19 pneumonia. The last two patients reported only fever. One of them had a close contact with COVID-19 positive subject and three nasopharyngeal swabs performed resulted negative for SARSCoV-2 on real-time reverse transcription-polymerase chain reaction (RT-PCR). None of the patients underwent flu vaccination. Two out of three patients with confirmed SARS-CoV-2 infections showed negative serology.

Discussion and conclusion: In line with to European data, [2] a low occurrence of severe symptoms suggestive of COVID-19 in MS patients was observed. The mild COVID-19 pneumonia observed in teriflunomide- and ocrelizumab-treated MS patients underline the low risk of developing a severe SARS-CoV-2 infection in MS patients treated with DMTs. Although DMTs may prevent a dysregulated immune response leading to severe and lifethreatening forms of COVID-19 [3], some DMTs may inhibit antibody production.

References:

1. Ciardi M. R. et al. The peripheral blood immune cell profile in a teriflunomide-treated multiple sclerosis patient with COVID-19 pneumonia. J. Neuroimmunol. (2020);346: 577323

2. Dalla Costa G. et al. Real-time assessment of COVID-19 prevalence among multiple sclerosis patients: a multicenter European study. Neurol. Sci. (2020);41(7):1647-50 doi:10.1007/s10072-020-04519-x

3. Berger J. R., Brandstadter R. \& Bar-Or A. COVID-19 and MS disease-modifying therapies. Neurol. Neuroimmunol. Neuroinflammation (2020);7(4):e761

\section{RED NUCLEUS ATROPHY IN EARLY RELAPSE-ONSET MULTIPLE SCLEROSIS}

S. Zywicki ${ }^{1}$, M. Margoni ${ }^{1}$, D. Poggiali ${ }^{2}$, M. Rubin ${ }^{1}$, A. Riccardi ${ }^{1}$, M.

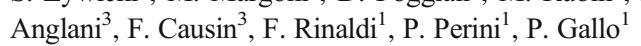

${ }^{1}$ Multiple Sclerosis Centre of the Veneto Region (CeSMuV), University Hospital of Padua, University of Padua (Padova); ${ }^{2}$ Department of Mathematics, University of Padua (Padova); ${ }^{3}$ Neuroradiology Unit, University Hospital of Padua (Padua) 
Background: No study has investigated red nucleus (RN) atrophy in MS despite cerebellum and its connections are elective sites of MS-related pathology.

Objective: To explore RN atrophy in early MS phases and whether it associated with cerebellar pathology, physical and cognitive disability.

Methods: Thirty-seven relapse-onset MS (RMS) patients (mean age: $35.6 \pm 8.5$ years; range: $18-56$ ) and 36 matched healthy controls (HC) (mean age: $33.8 \pm 9.9$ years; range: $23-63$ ) were studied. Cerebellar and $\mathrm{RN}$ volumes were analyzed on 3T-MRI images.

Results: RMS did not differ from $\mathrm{HC}$ in anterior and posterior cerebellar lobe volumes but had a significant reduction of both right (107.84 $\pm 13.95 \mathrm{~mm} 3$ vs $99.37 \pm 11.53 \mathrm{~mm} 3, \mathrm{p}=0.019$; $)$ and left $\mathrm{RN}(109.71 \pm 14.94$ $\mathrm{mm} 3$ vs $100.47 \pm 15.78 \mathrm{~mm} 3, \mathrm{p}=0.020$ ) volume. Moreover, correlations were observed between $R N$ volume and vermis VIIb ( $\mathrm{p}=0.011, \mathrm{r}=0.297)$, right interposed nucleus $(\mathrm{p}=0.039, \mathrm{r}=0.297)$ and vermis crus $\mathrm{I}(\mathrm{p}=0.032$, $\mathrm{r}=0.251$ ) volume.

Conclusion: Our findings indicate that $\mathrm{RN}$ atrophy occurs in early RMS and may be the result of both retrograde and anterograde axonal degeneration starting in cerebellar WM lesions. The prognostic value of $\mathrm{RN}$ atrophy is worth of further investigation since this nucleus is easily detectable and measurable on MRI.

\section{SLEEP}

\section{EFFECTS OF THE NUTRACEUTICAL SAFFRIL IGRIFFONIA, HUMULUS LUPULUS, CROCUS SATIVUS, PANTOTHENIC ACID, VIT. B6, RIBOFLAVIN, THIAMINE, FOLIC ACID) IN THE TREATMENT OF MILD/MODERATE ANXIETY AND/OR DEPRESSION PRELIMINARY REGIONAL OBSERVATIONAL MULTICENTRE STUDY}

\author{
L. Balzano ${ }^{1}$, B. Ciccone ${ }^{2}$, G. Sanges ${ }^{1}$ \\ ${ }^{1}$ Neurology Clinic, ASL NA3SUD (Torre del Greco-NA); \\ ${ }^{2}$ Neurophysiology Clinic (Saviano-NA)
}

Objective of the study: To evaluate the effect of the nutraceutical SAFFRIL $®$ in a population of patients diagnosed with mild-moderate anxiety and/or depression for the first time.

Endpoint: Evaluation at T0, T1 (after 60 days) and T2 (120 days) of the reduction in partial and/or total scores on the psychometric self-rating scales used (ZUNG A and ZUNG D, 1971).

Materials and methods: 73 consecutive patients diagnosed with generalised anxiety disorder and/or depression for the first time, were enrolled in the study. Each patient record features: an ID number, gender, age, totalised score on the ZUNG A and/or ZUNG D scales at time zero, time 1 and time 2 (T0, T1 and T2). Patients with anxiety disorder enrolled in the study (T0) presented a score between 1 and 60 on the Zung A; while patients enrolled in the study with depression had a score between 20 and 55 on the Zung D. The enrolled subjects were treated with 1 evening table of SAFFRIL at T0 and reassessed with the ZUNG A and/ or ZUNG D scales after 60 days (T1) and again after 120 days of treatment (T2). The analysis was carried out on the remaining 69, of whom 29 were male and 40 were female, with an average age of 49.

Results: Patients demonstrated an overall improvement in the Zung A scale at $\mathrm{T} 1$, which remained stable at T2 (p.value < 0.001), while the total numerical score for Zung D also showed a small reduction on average, although this reduction was not statistically significant $(\mathrm{p}$. value $=0.07)$. Quality of sleep improved significantly both for Q19 on the Zung A scale (p.value = 0.009) and for Q4 on the Zung D scale (p. value $=0.0007$ ). The results are achieved at different times: for Q19 on the Zung A scale it is necessary to wait until T2 before the improvement becomes statistically significant. Meanwhile, for Q4 on Zung D, the improvement is already significant at $\mathrm{T} 1$ and stable at $\mathrm{T} 2$.

Conclusions: Our preliminary observational study seems to confirm the data from scientific literature on the efficacy of the various nutraceutical components in Saffril ${ }^{\circledR}$ in regulating altered mood in subjects with mild and moderate depression and/or anxiety 1-2-3. A statistical analysis particularly highlights the synergic action of the abovementioned components of Saffril ${ }^{\circledR}$ in improving symptoms of anxiety and sleep quality, with a positive effect on the patients' quality of life.

References:

- Moshiri E. et al. Crocus Sativus L. (petal) in the treatment of mild to moderate depression: a double-blind randomized and placebocontrolled trial. Phytomedicine (2006);13(9-10):607-11

- Akhondzadeh Basti A. et al. Comparison of petal of Crocus Sativus $\mathrm{L}$ and fluoxetine in the treatment of depressed outpatients: a pilot double-blind randomized trial. Prog Neuropsychopharmacol Biol Psychiatry (2007);31(2):439-42

- Noorbala AA. et al. Hydro-alcoholic extract of crocus sativus L. versus fluoxetine in the treatment of mild to moderate depression: a pilot double-blind, randomized pilot trial. J Ethnopharmacol (2005);97(2):281-84

\section{NOCTURNAL HEART RATE VARIABILITY AFTER CPAP TREATMENT: POSSIBLE IMPLICATIONS FOR THE PREVENTION OF VASCULAR DISEASES}

L. Buratti ${ }^{1}$, D. Gambini ${ }^{1}$, S. Broggi ${ }^{1}$, C. Rocchi ${ }^{1}$, V. Totaro ${ }^{1}$, S. Lattanzi ${ }^{1}$, G. Viticchi ${ }^{1}$, L. Falsetti ${ }^{2}$, M. Silvestrini ${ }^{1}$

${ }^{1}$ Neurological Clinic, Marche Polytechnic University (Ancona); ${ }^{2}$ Internal and Subintensive Medicine, Ospedali Riuniti (Ancona)

Objective: The efficacy of CPAP for the prevention of vascular disease is unclear. Aim of this study was to evaluate the autonomic changes associated to CPAP treatment in order to better characterize cardiovascular risk in patients with obstructive sleep apnea syndrome (OSAS).

Materials and Methods: Twenty-one consecutive patients aged $65.43 \pm 3.75$ with a diagnosis of severe OSAS were selected and evaluated at baseline and after a three-month CPAP treatment period. Controls were subjects with a similar vascular risk profile, matched for age and sex.

Results: Patients differed from controls for the prevalence of restless legs syndrome, sleep scale scores and polygraphic parameters. After CPAP treatment, significant changes in all polygraphic and autonomic parameters were observed. When comparing controls and patients after CPAP, while polygraphic parameters were comparable, periodic limb movement index and autonomic activity remained significantly different. Further, considering the post-treatment evaluation, scores at the Pittsburgh Sleep Quality Index and at the Epworth sleepiness scale were significantly higher in patients with a hearth rate variability $(\mathrm{HRV})>2$ than in those with HRV $\leq 2(6.36 \pm 1.499$ vs. $3.00 \pm 1.83 ; \mathrm{p}=0.0001$ and $8.71 \pm 3.09$ vs. $4.86 \pm 1.68$; $\mathrm{p}=0.002$ ).

Discussion: Our findings suggest that the effects of CPAP can be modulated by the response of autonomic activity. Patients who maintain 
pathologic HRV values seem to have reduced benefit in terms of sleep quality and diurnal somnolence.

Conclusion: Based on these results, in the evaluation of the effect of OSAS treatment, the consideration of autonomic parameters in addition to the polygraphic respiratory data should be carefully considered.

References:

- Yoshihisa A, Takeishi Y Sleep Disordered Breathing and Cardiovascular Diseases. J AtherosclerThromb (2019); 26:315-27

- Buratti L, Cruciani C, Pulcini A, et al Lacunar stroke and heart rate variability during sleep. Sleep Medicine (2020) https://doi.org/10. 1016/j.sleep (in press)

- Chouchou F, Desseilles M Heart rate variability: a tool to explore the sleeping brain? Front Neurosci (2014);8:402

\section{SEX-RELATED DIFFERENCES IN POLYGRAPHIC PARAMETERS IN A POPULATION OF PATIENTS WITH OBSTRUCTIVE SLEEP APNEA SYNDROME}

\author{
L. Buratti ${ }^{1}$, C. Rocchi ${ }^{1}$, V. Totaro ${ }^{1}$, S. Broggi ${ }^{1}$, S. Lattanzi ${ }^{1}$, G. Viticchi ${ }^{1}$, \\ L. Falsetti ${ }^{2}$, M. Silvestrini ${ }^{1}$
}

${ }^{1}$ Neurological Clinic, Marche Polytechnic University (Ancona); ${ }^{2}$ Internal and Subintensive Medicine, Ospedali Riuniti (Ancona)

Objective: Sex-related differences in the prevalence and clinical presentation of obstructive sleep apnea syndrome (OSAS) have been widely documented. Aim of this study was to investigate the influence of patients' sex on polygraphic parameters with a particular attention to sleep autonomic changes in a population of OSAS patients.

Materials and Methods: Sixty OSAS patients aged 55-65 years (30 men, 30 women) were enrolled. Sleep quality was assessed with the Pittsburgh Sleep Quality Index (PSQI) and daytime sleepiness with the Epworth Sleepiness scale (ESS). The presence of respiratory events and autonomic changes during the night was investigated by a polygraphy.

Results: Men and women were similar for main cardiovascular risk factors prevalence. We observed a significant difference in PSQI (higher in women, $\mathrm{p}<0.0001$ ) and ESS (higher in men, $\mathrm{p}<0.004)$ scores. Snoring $(\mathrm{p}<0.033)$, supine $\operatorname{AHI}(\mathrm{p}<0.004)$, T90 $(\mathrm{p}<0.021)$, LO2 $(\mathrm{p}<0.0001)$, HRV and LF $(\mathrm{p}<0.0001)$ resulted significantly higher in men. The differences in PSQI mean score and in cardiac variability were preserved in all the subgroups of OSA severity.

Discussion: The influence of sex in modulating cardiovascular risk is a widely discussed topic. In our study, men had more severe polygraphic parameters and an increase in HRV compared to women.

Conclusions: The results of our investigation, suggest the relevance of delivering information about the different expression of OSAS in men and women in order to improve diagnostic skills and in-depth prevention approaches.

References:

- Marha RS, Katsuhisa B, Meir H K Differences Between Men and Women in the Clinical Presentation of Patients Diagnosed with Obstructive Sleep Apnea Syndrome. Sleep (2005);28:309-14

- Ozen K B, Mehmet ST. Gender Differences in Clinical and Polysomnographic Features of Obstructive Sleep Apnea: A
Clinical Study of 2827 Patients. Sleep Breath (2018);22:241249

- Valipour A. Gender-related Differences in the Obstructive Sleep Apnea Syndrome. Pneumologie (2012);66:584-8

\section{PITOLISANT FOR RESIDUAL EDS IN PATIENTS WITH OBSTRUCTIVE SLEEP APNEA SYNDROME (OSAS) TREATED WITH CONTINUOUS POSITIVE AIRWAY PRESSURE (CPAP): THE HAROSA I STUDY}

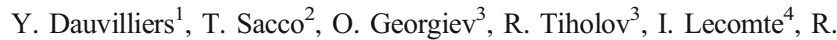
Tamisier $^{5}$, P. Levy ${ }^{6}$, C. Scart-Gres ${ }^{4}$, J.M. Lecomte ${ }^{4}$, J.C. Schwartz ${ }^{4}$, J.L. Pépin ${ }^{5}$

${ }^{1}$ National Reference Center for Narcolepsy, Sleep and Wake Unit, Department of Neurology, Gui-de-Chauliac Hospital, Montpellier University Hospital (Montpellier-F); ${ }^{2}$ Medical Department, Bioprojet Italia (Milano); ${ }^{3}$ Department of Internal Disease, Medical University of Sofia (Sofia-BG); ${ }^{4}$ Research Department, Bioprojet (Paris-F); ${ }^{5}$ HypoxiaPhysiopathology Laboratory, INSERM U1042, University Grenoble Alpes (Grenoble-F); ${ }^{6}$ Pulmonary Function Test Unit Sleep, University Grenoble Alpes (Grenoble-F)

Objectives: Pitolisant (PIT), a selective H3 receptor antagonists/ inverse agonist showed to improve wakefulness in other conditions and could contribute in reducing OSAS-related residual Excessive Daytime Sleepiness EDS (rEDS). The objective of this study was to evaluate efficacy and safety of PIT in the management of rEDS in patients with moderate to severe OSAS, adherent to CPAP treatment.

Materials and methods: The study was prospective, multicenter, randomized and double blind, comparing PIT and placebo (PLA) in patients (pts) with OSAS that had rEDS and underwent $\geq 3$ months of nasal CPAP (nCPAP). Selected patients were randomized 3:1 in the PIT and PLA groups. The treatment started with a 3 weeks dose titration, followed by 9 weeks of stabilized dose therapy. The main efficacy endpoint was the variation of Epworth Scale Score (ESS) from baseline to end of treatment. Secondary endpoints were: Osler test and fatigue Pichot's test outcomes and safety parameters.

Results: 244 pts have been randomized: average age was 51.3 years, $82.8 \%$ were males, with an Apnoea Hypopnea Index, under CPAP, of 4,18/hour. 183 pts received PIT (5, 10 or $20 \mathrm{mg} / \mathrm{d}$ ) and 61 PLA. A significant $(\mathrm{p}<0.001)$ reduction of ESS (from $14.9 \pm 2.7$ to $9.0 \pm 4.8$ ) was observed with PIT, compared to PLA (from $14.5 \pm 2.8$ to $12.1 \pm 6.4$ ), with a ESS reduction, in the PIT group, of -5.52 . Responders' (final ESS $\leq 10$ or ESS reduction $\geq 3$ ) rates were $71.0 \%$ with PIT vs $54.1 \%$ with PLA $(p=0.013)$. A significant improvement of the Osler test was found with PIT vs PLA in the Per Protocol population $p=0.0 .026$ (MMRM). The overall adverse effects (AEs) rates, were $26.8 \%$ with PIT vs $19.7 \%$ with PLA and the more frequent AEs were the same already observed in studies done with PIT in other indications, with rates not significantly different from PLA: headache (8.2\% PIT vs $8.2 \%$ PLA) and insomnia ( $4.9 \%$ PIT vs $1.6 \%$ PLA). No changes of hearth rate and blood pressure were detected with PIT or PLA.

Discussion: pts with OSAS-related rEDS, despite a good adherence to CPAP treatment, if treated with PLA still had a pathological level of ESS at the end of study.

Conclusion: PIT showed to be efficacious and safe in reducing rEDS in pts with moderate to severe OSAS treated with nCPAP. 


\section{PITOLISANT FOR DAYTIME SLEEPINESS IN PATIENTS WITH OBSTRUCTIVE SLEEP APNEA SYNDROME (OSAS) WHO REFUSE CONTINUOUS POSITIVE AIRWAY PRESSURE (CPAP) TREATMENT: THE HAROSA II STUDY}

Y. Dauvilliers, T. Sacco 2 , J. Verbraecken ${ }^{3}$, M. Partinen ${ }^{4}$, J. Hedner ${ }^{5}$, T.

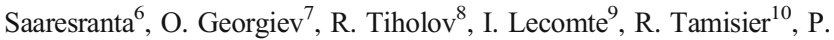
Lévy $^{10}$, C. Scart-Gres ${ }^{9}$, J.M. Lecomte ${ }^{9}$, J.C. Schwartz ${ }^{9}$, J.L. Pépin ${ }^{10}$

${ }^{1}$ Department of Neurology, Montpellier University Hospital (Montpellier-F); ${ }^{2}$ Medical Department, Bioprojet Italia (Milano); ${ }^{3}$ Multidisciplinary Sleep Disorders Center, Antwerp University Hospital (Antwerp- B); ${ }^{4}$ Department of Clinical Neurosciences, University of Helsinki (Helsinki-FIN); ${ }^{5}$ Department of Internal Medicine, University of Göteborg (Göteborg-S); ${ }^{6}$ Department of Pulmonary Diseases and Clinical Allergology, Turku University Hospital (Turku-FIN); ${ }^{7}$ Department of Internal Medicine, Alexandrovska Hospital Medical University (Sofia-BG); ${ }^{8}$ Department of Internal Diseases, Multiprofile Hospital for Active Treatment (Kozloduy-BG); ${ }^{9}$ Research Department, Bioprojet (Paris-F); ${ }^{10}$ Hypoxia-Physiopathology Laboratory, INSERM U1042, University Grenoble Alpes (Grenoble-F)

Objectives: Excessive Daytime Sleepiness (EDS) is a frequent OSAS's symptom which severely impacts on patients' life. Pitolisant (PIT), a selective $\mathrm{H} 3$ receptor antagonists/inverse agonist, showed to be effective on residual EDS in patients treated with CPAP and the objective of the present study was to evaluate its efficacy and safety in the patients that refuse or are not adherent to CPAP.

Materials and methods: The study was prospective, multicenter, randomized and double blind, comparing PIT, up to $20 \mathrm{mg} /$ day, to placebo (PLA) in patients with moderate to severe OSAS and EDS not treated with CPAP. Selected patients were randomized 3:1 in the PIT and PLA groups and underwent a 3 weeks dose titration phase, followed by 9 weeks of stabilized dose therapy. The main efficacy endpoint was the variation of Epworth Scale Score (ESS) from baseline to end of treatment. Secondary endpoints were: Osler test and fatigue Pichot's test outcomes and safety parameters.

Results: 268 OSAS patients were randomized: average age was 52.0 years, $75.4 \%$ were males, and average Apnoea Hypopnea Index was 49,3 /hour. In the intention to treat population, a significantly $(\mathrm{p}<0.001)$ higher reduction of ESS has been observed with PIT (from $15.7 \pm 3.1$ to $9.1 \pm 4.7$ ), compared to PLA (from $15.7 \pm 3.6$ to 12.2 \pm 6.1 ), with a marked ESS reduction, in the PIT group, of 6.3. Responders' (final ESS $\leq 10$ or ESS reduction $\geq 3$ ) rates were $80.6 \%$ with PIT vs $53.7 \%$ with PLA ( $\mathrm{p}=0.001$ ). No differences in OSleR test's outcomes were found, while Pichot's fatigue score significantly improved with PIT $(\mathrm{p}=0.005)$. The overall adverse effects (AEs) rates were $29.5 \%$ with PIT vs $25.4 \%$ with PLA and the more frequent AEs were the same already detected in studies done with PIT in other indications, with rates not significantly different from PLA: headache (8.5\% PIT vs $11.9 \%$ PLA) and insomnia (5.5 $\%$ PIT vs $3.0 \%$ PLA). No changes of hearth rate and blood pressure were detected with PIT or PLA.

Discussion: A unique aspect of our study was to targeted only OSAS pts that refused CPAP, in order to assess PIT's efficacy in these particular cases.

Conclusion: PIT showed to be efficacious in reducing EDS in OSAS patients that refused or were not adherent to CPAP, showing as well a good safety profile.

\section{FREQUENCY AND FEATURES OF SLEEP DISORDERS IN PATIENTS WITH AUTOIMMUNE ENCEPHALITIS}

\author{
M. P. Giannoccaro, R. Liguori, F. Pizza, P. Avoni, G. Plazzi
}

IRCCS Institute of Neurological Sciences of Bologna, University of Bologna (Bologna)

Objectives: Several sleep disorders have been reported in patients with autoimmune encephalitis (AE). However, despite sometimes they represent the most prominent clinical feature, they have been scarcely investigated until recently. Our aim is to study the frequency and characteristics of sleep disorders in patients with $\mathrm{AE}$ and their relationships with the antibody positivity status.

Methods: We retrospectively evaluated the clinical history, antibody testing and polysomnography (PSG) of patients diagnosed with $\mathrm{AE}$ at our tertiary care hospital.

Results: We included 30 patients $(11 \mathrm{~F}, 19 \mathrm{M}$, median age 67.5 years, range 31-88). Overall, 20 patients (66.7\%) were diagnosed with antibody positive AE (Ab-pos) and 10 (33.3\%) with seronegative AE (Ab-neg). Antibodies against intracellular antigens were identified in 5/20 patients, while $16 / 20$ cases had antibodies against neuronal surface antigens. Recent onset sleep-related symptoms were reported by 22 patients $(73.3 \%)$, and in 2 cases (9\%) these complaints preceded the other neurological manifestations. No difference was observed in the frequency of sleep disorders between patients with $\mathrm{Ab}$-pos and $\mathrm{Ab}$-neg $\mathrm{AE}(\mathrm{P}=0.68)$. Among patients with sleep symptoms $(\mathrm{n}=22)$, reported disorders included insomnia $(16,72.7 \%)$, hypersomnia $(2,9 \%)$ or daytime sleepiness $(4$, $18 \%)$ in one case associated with cataplexy, nocturnal agitation ( 7 , $31.8 \%)$, jerks $(6,27.2 \%)$, enacted dream $(2,9 \%)$, somniloquy $(2,9 \%)$, $\operatorname{RBD}(3,13.6 \%)$, RLS $(2,9 \%)$, snoring/apneas $(4,18)$. Up to 16 patients reported more than one sleep complaint. Video-PSG was performed in $13 / 22$ cases. In all cases but one, it was observed a marked reduction of sleep efficiency (median $25 \%$, range $0-50$ ), with a median sleep duration of 148 minutes. Agrypnia excitata was observed in 3 patients (two LGI/ CASPR2 positive); one patient presented an "undifferentiated" sleep. Obstructive sleep-related apneas were detected in 6 cases, mixed with central apneas in one patient and with laryngeal stridor in another case. Video-PSG documented PLM in 5 cases, REM without atonia in 3, and enacted dreams in 3 further cases. Seizures or epileptiform EEG abnormalities were observed in 4 patients. After immunotherapy, 7 patients reported an improvement of the sleep disorder.

Discussion: We observed a high frequency of sleep disorders in patients with $\mathrm{AE}$, with no difference in their distribution and features between seropositive and seronegative cases. Sleep disorders may also display a favourable outcome after/during immunotherapy and thus need to be carefully monitored. The recognition of specific sleep disorder could help in the diagnosis of AE, improving the overall patients' management and outcome.

\section{PUPILLOMETRY FINDINGS IN ISOLATED REM SLEEP BEHAVIOR DISORDER}

C. La Morgia ${ }^{1}$, E. Antelmi ${ }^{2}$, M. Romagnoli ${ }^{3}$, F. Pizza ${ }^{1}$, F. Biscarini ${ }^{4}$, M. Filardi $^{1}$, V. Donadio ${ }^{3}$, M. Carbonelli ${ }^{3}$, G. Amore ${ }^{1}$, V. Carelli ${ }^{1}$, R. Liguori $^{1}$, G. Plazzi ${ }^{1}$

${ }^{1}$ IRCCS Institute of Neurological Sciences of Bologna; Department of Biomedical and Neuromotor Sciences, University of Bologna (Bologna); ${ }^{2}$ Department of Neurosciences, Biomedicine and Movement, University 
of Verona (Verona); ${ }^{3}$ IRCCS Institute of Neurological Sciences of Bologna, Bellaria Hospital, AUSL Bologna (Bologna); ${ }^{4}$ Department of Biomedical and Neuromotor Sciences, University of Bologna (Bologna)

Objectives and background: Melanopsin retinal ganglion cells (mRGCs) are crucial for non-image forming functions of the eye, including photoentrainment of circadian rhythms and regulation of sleep and pupillary light reflex (PLR). Chromatic pupillometry, using light stimuli at different wavelengths, allows the isolation of the contribution of rods, cones and mRGCs to PLR. The presence of mRGC-mediated PLR impairment [1], as well as of mRGC loss in post mortem retinas have been demonstrated in $\mathrm{PD}$ and we recently described a reduction of the rod-mediated transient PLR in AD patients [2] suggesting a possible mRGC dendropathy. Idiopathic/ isolated REM sleep behavior disorder (iRBD) represents the strongest prodromal risk factor for alfa-synucleinopathies. We aim at investigating PLR in iRBD.

Materials: We evaluated with chromatic pupillometry [3] PLR in 16 iRBD patients compared to 21 age-matched controls. RBD and controls underwent an extensive ophthalmological evaluation including optical coherence tomography (OCT) and cognitive tests. The absence of sleep disorders was confirmed by sleep questionnaires and actigraphy in the control group. For the RBD cohort, PLR metrics were correlated to clinical variables including REM atonia index (RAI) and the presence of alfasynuclein deposition in skin biopsy.

Methods: Pupillometry data were analyzed by custom MATLAB scripts. Chi-square, independent-t and ANOVA tests were used to compare variables among groups. Analysis of covariance (ANCOVA) with age as covariate was used for comparisons between groups for pupil metrics. For OCT data, we followed one-eye approach evaluating the eye tested by pupillometry. Pearson correlation coefficients were used to measure the association between pupillometric parameters and RBD clinical data.

Results: We documented a higher baseline pupil diameter in the RBD group in all conditions and decreased rod-mediated transient PLR amplitude $(\mathrm{p}=0.007)$, whereas the post-illumination pupillary response did not differ between RBD and controls. Interestingly, we found a significant correlation between RAI and transient rod-mediated PLR amplitude. Moreover, the difference in pupillometry metrics compared to controls was evident only in the iRBD group with alfa-synuclein deposition. OCT findings did not differ between RBD and controls.

Discussion and conclusions: Our results show that rod-mediated contribution to PLR is impaired in idiopathic RBD and correlates with RAI suggesting a mRGC-mediated pupil dysfunction proportional to a REM sleep marker of the disease. Moreover, the higher baseline pupil diameter and reduced peak amplitude in RBD point to a parasympathetic dysfunction, as already reported for $\mathrm{PD}$. These pupil metrics can be envisaged as a possible biomarker for this sleep disorder.

References:

1. Joyce D.S., Feigl B., Kerr G., Roeder L., Zele A.J. Melanopsinmediated pupil function is impaired in Parkinson's disease. Sci Rep (2018);8:7796

2. M. Romagnoli, M. Stanzani Maserati, M. De Matteis, S. Capellari, M. Carbonelli, G. Amore, G. Cantalupo, C. Zenesini, R. Liguori, A. A. Sadun, V. Carelli, J. C. Park, C. La Morgia. Chromatic Pupillometry Findings in Alzheimer's Disease. Frontiers in Neuroscience (in press)

3. Park J.C., Moss H.E., McAnany J.J. The Pupillary Light Reflex in Idiopathic Intracranial Hypertension. Invest Ophthalmol Vis Sci (2016);57:23-9

\section{CLINICAL AND DOPAMINERGIC NIGRO-STRIATAL CORRELATES OF QUANTITATIVE SMARTPHONE MOTOR TESTING IN IDIOPATHIC REM SLEEP BEHAVIOUR DISORDER AND PARKINSON DISEASE}

P. Mattioli ${ }^{1}$, C. Lo $^{2}$, A. Lechiara ${ }^{3}$, R. Meli ${ }^{1}$, M. Pardini ${ }^{1}$, L. Filippi ${ }^{1}$, S. Grisanti $^{1}$, M. Grazzini ${ }^{1}$, F. Massa ${ }^{1}$, F. Famà ${ }^{1}$, L. Giorgetti ${ }^{1}$, S. Morbelli ${ }^{4}$, F. Nobili ${ }^{1}, \mathrm{M} . \mathrm{Hu}^{2}$, D. Arnaldi ${ }^{1}$

${ }^{1}$ Clinical Neurology, Dept. of Neuroscience (DINOGMI), University of Genoa, IRCCS San Martino Polyclinic Hospital (Genova); ${ }^{2}$ Oxford Parkinson's Disease Centre, Nuffield Department of Clinical Neurosciences, University of Oxford, John Radcliffe Hospital (OxfordUK); ${ }^{3}$ Clinical Neurology, Dept. of Neuroscience (DINOGMI), University of Genoa (Genova); ${ }^{4}$ Nuclear Medicine, Department of Health Sciences (DISSAL), University of Genoa, IRCCS San Martino Polyclinic Hospital (Genova)

Objective: To investigate clinical and dopaminergic nigro-striatal correlates of quantitative motor testing, performed by validated smartphone technology [1], in healthy controls, idiopathic REM sleep behaviour disorder (iRBD) and de novo, drug naïve Parkinson disease (PD) outpatients, with a single assessment. The original quantitative smartphonebased motor testing showed good discrimination ability in differentiating healthy controls, iRBD and PD patients [1].

Materials: We administered smartphone quantitative motor testing $[1,2]$ to twelve healthy controls $(69 \pm 4 \mathrm{y}, 10$ females), and $18 \mathrm{iRBD}(71$ $\pm 8 \mathrm{y}$, four females) and $13 \mathrm{PD}(73 \pm 10 \mathrm{y}$, six females $)$ consecutive patients. Seven tasks were performed: Balance, Gait, Rest and Postural Tremor, Finger-tapping, Reaction Time and Voice (the last not used in the present analysis). Tasks' values were derived from smartphone's accelerometer, gyroscope and touch-screen measurements. Nigro-striatal functioning was assessed in iRBD and PD patients with brain [123I]-FP-CIT Single Photon Emission Computed Tomography ([123I]-FP-CITSPECT). Clinical motor symptoms were measured with the Movement Disorder Society Unified Parkinson's Disease Rating Scale, part three (MDS-UPDRS-III).

Methods: To minimize multicollinearity and reduce the number of variables, a factorial analysis was applied to means and standard deviations of acceleration and angular velocity in $\mathrm{x}, \mathrm{y}$ and $\mathrm{z}$ axis of accelerometer and gyroscope data of each task, except for Finger-Tapping and Reaction-Time tasks in which a correct/incorrect ratio was used. We compared MDS-UPDRS-III, smartphone and [123I]-FP-CIT-SPECT data between groups by Kruskal-Wallis test. We correlated Smartphone data with MDS-UPDRS-III scores and [123I]-FP-CIT-SPECT data by Spearman analysis in the whole of $\mathrm{RBD}$ and PD patients.

Results: PD patients had higher MDS-UPDRS III scores $(\mathrm{p}<0.001)$ and lower putaminal $(\mathrm{p}<0.001)$ and caudate $(\mathrm{p}=0.035)$ [123I]-FP-CITSPECT specific to non-displaceable binding ratios (SBRs) values compared with iRBD. A significant effect of group belonging was found for Gait $(\mathrm{p}=0.008)$, left Rest Tremor $(\mathrm{p}=0.026)$, right Postural Tremor $(p=0.038)$ and Reaction Time $(p=0.024)$ scores. MDS-UPDRS-III showed significant inverse correlation with left $(\mathrm{p}<0.001$, rho $=-$ $0.47)$ and right $(\mathrm{p}=0.007$, rho $=-0.41)$ Finger Tapping. Putaminal SBR showed significant direct correlation with Gait $(\mathrm{p}=0.015$, rho=0.43) and inverse correlation with MDS-UPDRS-III $(\mathrm{p}<0.001$, rho=-0.69).

Conclusion: We showed for the first time the correlation of smartphone-based motor testing parameters with nigroputaminal dopaminergic deafferentation and standard clinical motor examination in $\mathrm{R} B \mathrm{BD}$ and PD patients. In the validation study [1], subjects performed several 
smartphone assessments over a maximum of 7 days, while in the present study the smartphone assessment was performed only once, and thus results need validation in larger series.

References:

1. Arora S, Baig F, Lo C, Barber TR, Lawton MA, Zhan A, et al. Smartphone motor testing to distinguish idiopathic REM sleep behavior disorder, controls, and PD. Neurology (2018);91:E1528-38

2. Arora S, Venkataraman V, Zhan A, Donohue S, Biglan KM, Dorsey ER, et al. Detecting and monitoring the symptoms of Parkinson's disease using smartphones: A pilot study. Park Relat Disord. (2015);21:650-3

\section{SLEEP INERTIA IN HYPERSOMNIAS OF CENTRAL ORIGIN: IMPORTANCE OF DEPRESSION AND SLEEP ARCHITECTURE}

\author{
A. Pagano, F. Placidi, C. Liguori, A. Castelli, L. Mari, N. Manfredi, V. \\ Bramato, N. B Mercuri, F. Izzi
}

Sleep Medicine Center, Department of Systems Medicine, Policlinico Tor Vergata, University of Rome "Tor Vergata" (Roma)

Objectives: Sleep inertia (SI) can be defined as difficulty becoming fully awake after sleep. To evaluate sleep inertia in mood disorders, the Sleep Inertia Questionnaire (SIQ) has been validated [1] and it has been very recently utilized also in hypersomnolence disorders [2]. Aim of the study is to assess SI, by means of SIQ, in patients affected by hypersomnias of central origin, including narcolepsy type 1 (NT1), narcolepsy type 2 (NT2) and idiopathic hypersomnia (IH) and to evaluate possible correlations among SI, depression and polysomnographic (PSG) parameters.

Methods: Patients with NT1, NT2 and IH, diagnosed in accordance to International Classification of Sleep Disorders-3 criteria were recruited at Sleep Medicine Center of Policlinico Tor Vergata. All patients underwent to nocturnal PSG recording, 5 naps Multi-sleep latency test (MSLT) and to evaluation of daytime somnolence using Epworth Sleepiness Scale (ESS). SIQ and Beck Depression Inventory (BDI-II) were administrated. SIQ total score (ranging from 21 to 105) and four SIQ subdomains (physiological, SIQ-P cognitive, SIQ-C, emotional, SIQ-E and responses to SI, SIQ-R) were assessed. Kruskal-Wallis test and Spearman correlation were used for statistical analysis.

Results: The analysis included 26 patients ( 15 females, 11 males, mean age $37.96 \pm 15.06$ years) with diagnosis of NT1 $(n=10)$, NT2 $(n=8), I H(n=8)$. No difference in terms of age, body mass index, ESS and BDI-II scores was found among NT1, NT2 and IH. Mean SIQ total score was higher in $\mathrm{IH}(63.3 \pm 14.8)$ with respect to NT1 $(50.8 \pm 20.8)$ and NT2 (53 \pm 25.5$)$ without reaching statistical significance. Strong positive correlation was observed between SIQ (total score and all SIQ subdomains) and BDI-II $(\mathrm{p}<0.01)$. Regarding PSG parameters, REM latency showed strong positive correlations with SIQ total score and SIQ-R $(\mathrm{p}<0.01)$ and a positive correlation with SIQ-E $(\mathrm{p}<0.05)$. SIQ total score was positively associated with percentage of stage N2 $(\mathrm{p}<0.05)$. SIQ-R negatively correlated with percentage of stage N3 $(\mathrm{p}<0.05)$, whereas was positively associated with sleep onset latency at $\operatorname{MSLT}(\mathrm{p}<0.05)$. Finally, SIQ-P negatively correlated with percentage of REM sleep $(\mathrm{p}<0.05)$.

Conclusions: SI assessed by SIQ is a common symptom reported by patients with NT1, NT2 and IH without significant differences among the three forms of hypersomnias. Depression can get worse physiological, cognitive, emotional factors of SIQ as well as responses to SI. Some PSG parameters, such as the increase of REM latency and stage N2, the reduction of stage N3 and REM sleep, seem to be associated with sleep inertia. References:

- Kanady JC, Harvey AG. Development and validation of the Sleep Inertia Questionnaire (SIQ) and assessment of Sleep Inertia in analogue and clinical depression. Cognit Ther Res. (2015) Oct;39(5):601-612. doi: 10.1007/s10608-015-9686-4

- Maness CB, Moron D, Bliwise D, Saini P, Rye D, Trotti LM. 0627 Initial Use of the Sleep Inertia Questionnaire in Hypersomnolence Disorders. Sleep (2019);42(Suppl.1):A249-A250, https://doi.org/ 10.1093/sleep/zsz067..625

\section{EASY TELEMEDICINE FOR NARCOLEPSY (EASY TENAR): A PHASE 2 OPEN TRIAL ASSESSING THE FEASIBILITY OF TELEVISIT FOR MULTIDISCIPLINARY CARE OF NARCOLEPSY DURING COVID-19 PANDEMIC}

\section{F. Pizza ${ }^{1}$, M. Moresco ${ }^{2}$, A. Mangiaruga ${ }^{3}$, C. Oriolo $^{3}$, L. Vignatelli $^{2}$, U.}

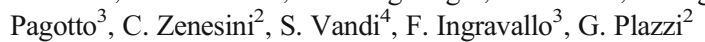

${ }^{1}$ Department of Biomedical and Neuromotor Sciences, University of Bologna (Bologna); ${ }^{2}$ IRCCS Istituto delle Scienze Neurologiche of Bologna (Bologna); ${ }^{3}$ Department of Medical and Surgical Sciences (DIMEC), University of Bologna (Bologna); ${ }^{4}$ Department of Biomedical and Neuromotor Sciences (DIBINEM), University of Bologna (Bologna)

Objective: Narcolepsy is a rare chronic hypersomnia of central origin, often associated with severe endocrine-metabolic and psychosocial issues, requiring a multidisciplinary approach. During the COVID-19 emergency, the Local Health Trust of Bologna suspended non-urgent office visits allowing telephone consultations (March-June 2020). However, some patients need a more structured intervention, including visual contact, to better interact with the physician. To assess the feasibility of multidisciplinary care by means of telemedicine (i.e. televisit, TV), a pragmatic open one-arm study was devised. The TENAR project is endorsed by the Italian Narcolepsy and Hypersomnias association (AIN onlus, www.narcolessia.org). We report preliminary data on the enrolment phase.

Materials: Consecutive patients with narcolepsy (age $\geq 14$ yrs) needing multidisciplinary care during the suspension of usual in-office visits at the Narcolepsy Center of Bologna were offered TV multidisciplinary assessment.

Methods: Phase 2 open trial. Neurologic evaluation by means of TV at baseline and at 2, 4, and 6 months, as well as metabolic, and psychosocial assessments. Feasibility is assessed calculating 1) the frequency of participants out of the total of invited people; 2 ) the frequency of participants completing the study at 6 months out of the total of enrolled patients. The technical quality of TVs and the perceived healthcare quality will be assessed.

Results: From May 9 to June 22, 2020, 44 patients were invited to a TV, $5(11 \%)$ refused for: not sufficient skillfulness in using electronic devices (3), preference for in-person visit (1), difficulty in scheduling the visit (1). Thirty-nine patients ( 38 NT1, 1 NT2 type) underwent TV (mean age $30.4 \mathrm{yrs}$, SD 13.7 ; F 17, 41.5\%; mean age at onset $16.4 \mathrm{yrs}$; mean age at diagnosis $22.7 \mathrm{yrs}$ ). Epworth sleepiness scale at baseline was 10.3 (SD 5.0, range 3-20). Eighteen pts (46\%) were on monotherapy, 17 (44\%) on bitherapy, and $4(10 \%)$ on tritherapy. The drugs used were the following: sodium oxybate (mean daily dosage $7.8 \mathrm{~g}$ ) in $32 \mathrm{pts}(82 \%)$, modafinil 
(169 mg) or pitolisant (36 mg) in $13 \mathrm{pts}(33 \%)$, antidepressant in $5 \mathrm{pts}$ $(13 \%)$.

Discussion: The high response rate indicate that TV was feasible in a vast majority of children and adults with narcolepsy, regardless the disease severity. Lack of sufficient skills in using electronic devices is the main barrier to telemedicine for narcolepsy management.

Conclusion: The study may be a model for the remote management of rare disorders, offering an alternative management approach aside routine in-person visit.

\section{HOW TO ORGANIZE AND IMPLEMENT TELEMEDICINE FOR NARCOLEPSY MANAGEMENT: FINDINGS FROM THE TELEMEDICINE FOR NARCOLEPSY (TENAR) RANDOMIZED CONTROLLED TRIAL COMPARING TELEVISIT VS STANDARD IN-PERSON OUTPATIENT VISIT}

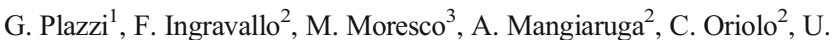
Pagotto $^{2}$, S. Vandi ${ }^{3}$, C. Zenesini ${ }^{4}$, F. Pizza ${ }^{3}$, L. Vignatelli ${ }^{4}$

${ }^{1}$ Department of Biomedical, Metabolic and Neural Sciences, University of Modena and Reggio Emilia (Modena); ${ }^{2}$ Department of Medical and Surgical Sciences (DIMEC), University of Bologna (Bologna); ${ }^{3}$ Department of Biomedical and Neuromotor Sciences (DIBINEM), University of Bologna (Bologna); ${ }^{4}$ IRCCS Institute of Neurological Sciences of Bologna, Ausl of Bologna (Bologna)

Objectives: Narcolepsy is a rare chronic disorder of hypersomnolence with excessive daytime sleepiness (EDS) as the main disabling symptom, often associated with severe endocrine-metabolic issues, requiring a multidisciplinary approach. The scarcity of reference sleep centres forces to travel for medical consultations, increasing disease burden. Telemedicine may facilitate patient care, however the performance of a televisit (TV) procedure and the procedural activities to apply telemedicine in clinical practice are poorly known. We aim to report the methodological and procedural milestones for the implementation of telemedicine research activities in a narcolepsy outpatient service.

Materials: Protocol of a randomized controlled trial comparing televisit vs standard in-person outpatient visit (Ingravallo 2020, clinicatrials.gov registration number NCT04316286) devised to enrol 202 narcolepsy patients (>14 y.o.) randomly allocated either to TV via videoconference or to standard in-person outpatient follow-up visits (control arm). The study is the part 2 of the TElemedicine for NARcolepsy (TENAR) project, endorsed by the Italian Narcolepsy and Hypersomnias association (AIN onlus, www.narcolessia.org), and publicly funded by the Italian Ministry of Health (RF-2016-02364742).

Methods: Qualitative analysis of the above reported protocol and the milestones for the implementation of telemedicine procedures (televisit, TV) in the narcolepsy center of of the Istituto delle Scienze Neurologiche di Bologna.

Results: The TENAR group designed the study as a non-inferiority randomized controlled trial whose primary outcome is EDS control at 12 months. Secondary outcomes are other symptoms control, compliance with treatment, metabolic control, quality of life, feasibility, patient and family satisfaction with care, safety, and disease-related costs. Patients undergo neurologic, metabolic, and psychosocial assessments at 3, 6, and 12 months through TV or in-person visit. The following were the organizational issue faced in order to implement telemedicine for the study purposes: 1) finding a data-safe video conferencing platform, compatible with the organizational and administrative aspects of the healthcare service; 2) setting up of a safe e-crf for the collection of patient-oriented outcomes (scales, questionnaires); 3 ) an efficient system for scheduling TVs and inviting patients to the TV in the context of the usual in-person outpatients; 4) education and training of health operators and patients.

Discussion: The TENAR project will assess the feasibility, accuracy, efficacy and safety of telemedicine procedures applied to the narcolepsy multidisciplinary care. Telemedicine implementation in clinical research includes technical, but also educational and organizational aspects.

Conclusion: The study may be a model for the remote management of other rare disorders, offering care access for patients living far from specialized centers.

Reference:

- Ingravallo F, Vignatelli L, Pagotto U, et al. Protocols of a diagnostic study and a randomized controlled non-inferiority trial comparing televisits vs standard in-person outpatient visits for narcolepsy diagnosis and care: TElemedicine for NARcolepsy (TENAR). BMC Neurol. (2020);20(1):176. Published 2020 May 11

\section{POST AUTHORIZATION SAFETY STUDY ON WAKIX (PASS- WAKIX): THE 2020 REPORT}

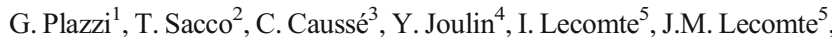 \\ J.C. Schwartz
}

${ }^{1}$ IRCCS Neurological Sciences, Bologna University (Bologna); ${ }^{2}$ Medical Department, Bioprojet Italia (Milano); ${ }^{3}$ Medical Department, Bioprojet (Paris-F); ${ }^{4}$ Pharmacovigilance Department, Bioprojet (Paris-F); ${ }^{5}$ Research Department, Bioprojet (Paris-F)

Objectives: In January 2020 it has been released the 3rd Interim Report of the Post Authorization Safety Study on Wakix ${ }^{\circledR}$ (PASS-Wakix), an innovative treatment for type 1 and type 2 narcolepsy, which improves wakefulness and reduces cataplexy enhancing central histamine pathways activation. Aim of this abstract is to summarize the main outcomes presented in the Report.

Materials: In Europe 33 Centers enrolled 370 type 1 or type 2 narcolepsy patients (pts), managed according to routine medical practice (RMP). The two Countries where most pts were included are Germany (143 pts) and Italy (192 pts).

Methods: PASS-Wakix is an international, multicenter, non-interventional, open-label, long-term study. Wakix ${ }^{\circledR}$ is prescribed in RMP, before and independently of the decision to enroll pts, alone or combined with other treatments. Primary objectives are: monitoring Wakix®'s safety and its use in RMP narcolepsy management and collecting pts' characteristics. Secondary objectives are evaluations of several variables concerning narcolepsy course and pts' life under treatment.

Results: On 2ndJanuary 2020, the numbers of pts that had follow-up at 3,6 12 and 24 months were, respectively, 121, 103, 122 and 30. The data below refer to the most numerous subgroups. At baseline, pts' main characteristics were: age $40.1 \pm 15.0$ years, $51 \%$ females and age at narcolepsy diagnosis of $33.6 \pm 14.4$ years. Wakix ${ }^{\circledR}$ was titrated up to $18 \mathrm{mg}$ in $55.8 \%$ of pts and up to $36 \mathrm{mg}$ in $35.5 \%$. In $33 \%$ of cases pts were switched to Wakix ${ }^{\circledR}$ and in $67 \%$ it was added to ongoing therapies. Percentages of pts with at least 1 adverse event (AE) were similar in Germany and in Italy (27.5\% vs $29.8 \%)$, while serious AEs and AEs leading to permanent Wakix ${ }^{\circledR}$ 
discontinuation were less frequent in Italy: $1.6 \%$ vs $2.8 \%$ and $5.8 \%$ vs $9.2 \%$, respectively. Epworth Sleepiness Scale average reduction at 3, 6 and 12 months were, respectively: $-2.8 \pm 4.4$, $2.7 \pm 5.2$ and $-3.4 \pm 4.6$. Cataplexy improved in $51.3 \%$ of pts at 3 months, in $68.5 \%$ at 6 and $60.5 \%$ at 12 months.

Discussion: The data of the 2020 Interim Report indicate that Wakix ${ }^{\circledR}$ is used, in RPM, mainly at $18 \mathrm{mg} / \mathrm{die}$, it is often added to other treatments and that severe AEs were rare in Italy. Symptoms trends were similar to the ones registered in clinical trials and impacted positively on pts' burden of disease and quality of life.

Conclusions: In PASS, Wakix® confirmed to be efficacious and safe in narcolepsy treatment.

\section{TREATMENT PATTERNS, CLINICAL OUTCOMES, AND HEALTHCARE RESOURCE UTILIZATION (HRU) AMONG PATIENTS WITH INSOMNIA TO WHOM TRAZODONE WAS PRESCRIBED IN THE UNITED STATES OF AMERICA (USA)}

\author{
I. Pochiero, M. Gorini, F. Torelli, A. Del Vecchio, A. Comandini, F. \\ Calisti, A. Cattaneo
}

Angelini RR\&D (Regulatory, Research, \& Development), Angelini Pharma (Roma)

Objectives: Insomnia is a common sleep disorder with serious impacts on quality of life and health outcomes. Current treatments include cognitive behavioral therapy and pharmacological treatments like zolpidem. Trazodone, an antidepressant SerotoninAntagonist/Reuptake Inhibitor, has shown to have positive effects on insomnia in depressed patients [1]. Nevertheless, its off-label use in insomnia is widely reported in literature. Moreover, some preliminary evidences on positive effects of trazodone in slowing cognitive decline in patients affected by dementia associated with Alzheimer Disease, may be mediated by its effects on sleep patterns [2]. Primary objective of this retrospective comparative cohort study is to describe patients suffering from insomnia and to whom trazodone was prescribed. Secondary objectives include description and comparison of demographic and clinical characteristics, including cognitive outcomes, in patients with insomnia treated with trazodone or zolpidem.

Materials: IBM Watson Health MarketScan ${ }^{\circledR}$ Commercial and Supplemental Medicare claims database is used for this study, retrieving the latest 10 years of data available (study period). This database captures person-specific clinical utilization, expenditures, and enrolment across inpatient, outpatient, prescription drug, and carve-out services in the related commercially insured USA population.

Methods: Patients of all ages are eligible if they have a prescription for either trazodone or zolpidem and a diagnosis of insomnia in the study period. Insomnia is identified using diagnosis codes from International Classification of Diseases, Clinical Modification, 9th or 10th Revision in the claims database.

Results: For primary objective, a descriptive analysis of demographics, clinical characteristics and exposure to drug will be conducted for trazodone cohort, counting 1.014.356 subjects (prior to enforce eligibility criteria). Both pediatric ( $<18$ years of age) and adult groups will be described. While for secondary objectives, comparative analysis on 1.481.869 subjects (prior to enforce eligibility criteria) will be conducted on demographics, clinical characteristics, medication adherence and persistence, HRU and healthcare costs for each cohort, including cognitive outcomes for a subgroup of patients aged 50 years and older.
Discussion: Defining characteristics of patients with insomnia treated with trazodone will be useful to drive future research on sleep disturbances. In addition, unveiling possible differences in terms of cognitive outcomes among patients with insomnia treated with trazodone or with zolpidem may provide insights on correlation between sleep disorders and cognitive impairment.

Conclusions: A deeper knowledge of insomnia treatments represents a further step towards improving sleep quality, daytime functioning and clinical outcomes. Sleep disturbances can impact long-term cognitive performance, but more remains to be clarified about their association. References:

1. Fagiolini A, Comandini A, Catena Dell'Osso M, Kasper S. Rediscovering trazodone for the treatment of major depressive disorder. CNS Drugs (2013) Aug;27(8):677]. CNS Drugs. 2012;26(12):1033-49

2. La AL, Walsh CM, Neylan TC, et al. Long-Term Trazodone Use and Cognition: A Potential Therapeutic Role for Slow-Wave Sleep Enhancers. J Alzheimers Dis. (2019);67(3):911-21

\section{SECONDARY NARCOLEPSY IN PARANEOPLASTIC LIMBIC ENCEPHALITIS IN A GENETICALLY PREDISPOSED PATIENT}

\author{
S. Rossi ${ }^{1}$, G. Asioli ${ }^{1}$, G. Sallemi ${ }^{1}$, C. Franceschini ${ }^{2}$, F. Pizza ${ }^{3}$, G. Plazzi ${ }^{3}$
}

${ }^{1}$ Department of Biomedical and Neuromotor Sciences, University of Bologna (Bologna); ${ }^{2}$ Deparment of Medicine and Surgery, University of Parma (Parma); ${ }^{3}$ IRCCS Istituto delle Scienze Neurologiche di Bologna, University of Bologna (Bologna)

Background and aims: Narcolepsy is a central hypersomnia caused by the selective loss of hypocretin-producing neurons in the lateral part of hypothalamus [1]. Secondary cases of narcolepsy are associated with focal lesions in hypothalamus, such as tumors and demyelinating disorders. Rarely, narcolepsy is reported in the context of paraneoplastic encephalitis, mainly in anti-Ma2 encephalitis [2]. Here we report a case of narcolepsy in a paraneoplastic limbic encephalitis associated with mediastinal thymic seminoma.

Materials and methods: A previously healthy 19-years-old Caucasian man presented with subacute-onset mood deflection, obsessivecompulsive disorder, and a hypothalamic syndrome with hyperphagia, sexual disabilities and a sleep disturbance characterized by excessive daytime sleepiness (EDS) and frequent sleep attacks. No hypnagogic hallucinations, sleep paralysis or cataplexy episodes were reported.

Results: Neurological examination was unremarkable. Magnetic resonance imaging showed T2-weighted hyperintense and contrast enhancing lesions involving limbic and hypothalamic regions. CSF studies revealed a mild pleocytosis ( 14 cells $/ \mathrm{mm} 3$ ). The search for onconeural and anti-neuronal-surface antibodies resulted negative. A total-body CT showed a mediastinal mass, which turned out to be a thymic seminoma at biopsy. The patient was treated with methylprendisolone ( $1 \mathrm{~g}$ /day for 5 days) without benefit. A few weeks later, he underwent to thoracotomy mass removal. Despite the progressive resolution of neuroradiological findings, the patient showed no neurological improvement. Given the persistence of severe EDS, the patient underwent a complete clinical and polysomnographic (24-h video-polysomnography and multiple sleep latency test) assessment, which disclosed hypersomnia with multiple sleep-onset REM periods. HLA typing was positive for DQB1*0602. CSF hypocretin-1 assay disclosed a level of $110 \mathrm{pg} / \mathrm{mL}$ (N.V. $>200$ 
$\mathrm{pg} / \mathrm{mL}$ ), leading to narcolepsy diagnosis. Sodium Oxybate was administered up to 9 grams per night with benefit on both nocturnal and daytime symptoms.

Discussion: Idiopathic narcolepsy is caused by a selective autoimmune attack to hypocretinergic neurons, mediated by T-cells, in a genetically susceptible population. The HLA-class-II allele DQB $1 * 0602$ is found in more than $90 \%$ of individuals with idiopathic narcolepsy [3]. In our patient, the presence of HLA DQ1B*0602 allele may have modulated the immune response, promoting hypocretinergic neurons destruction in the context of a limbic encephalitis.

Conclusion: Paraneoplastic limbic encephalitis triggered orexinergicneurons destruction in a genetically predisposed patient. Hypersomnolence in the context of an encephalitis could be due to a secondary narcolepsy and not merely to the brain inflammation. Prompt narcolepsy recognition in an encephalitic setting is thus essential, as it may benefit from specific symptomatic treatments, as it happened in our patient.

References:

1. Bassetti, C. L. A. et al. Narcolepsy - clinical spectrum, aetiopathophysiology, diagnosis and treatment. Nat. Rev. Neurol. (2019);15:519-39

2. Nishino, S. \& Kanbayashi, T. Symptomatic narcolepsy, cataplexy and hypersomnia, and their implications in the hypothalamic hypocretin/orexin system. Sleep Med. Rev. (2005);9: 269-310

3. Mahoney, C. E., Cogswell, A., Koralnik, I. J. \& Scammell, T. E. The neurobiological basis of narcolepsy. Nat. Rev. Neurosci. (2019);20:83-93

\section{COVID-19 AND SLEEP IN MEDICAL STAFF: OUR EXPERIENCE}

\section{Salsone ${ }^{1}$, M. Zucconi ${ }^{2}$, F. Casoni ${ }^{2}$, L. Ferini-Strambi ${ }^{2}$}

${ }^{1}$ Institute of Molecular Bioimaging and Physiology, National Research Council, Magna Graecia University (Catanzaro); ${ }^{2}$ Sleep Disorders Center, Division of Neuroscience, IRCCS San Raffaele Hospital, (Milano)

Objective: Coronavirus pandemic 2019 (COVID-19) is a condition which from December 2019 to today has upset our lives. Before the COVID-19 pandemic, the healthcare workers did not experience good sleep quality with relevant consequences on health, yet few data exist during this disastrous period. In this observational study, we aimed to assess the prevalence of sleep disorders among healthcare workers using a combined approach including actigraphic evaluation and the questionnaire survey.

Methods: Data were consecutively collected from 40 medical staff that treated patients with COVID-19 infection. Study participants were either doctors or nurses working in the Departments of Infectious Disease, Respiratory Medicine or the Intensive Care Unit of San Raffaele Hospital. All participants performed to seven nights of actigraphic recordings. Sleep quality was also evaluated using the Pittsburgh Sleep Quality Index (PSQI).

Results: We observed a gender-prevalence as about $70 \%$ of the participants were females, mainly nurses, with an age range from 26 to 50 years. Our analysis revealed that about $35 \%$ of healthcare workers were suffering from sleep disturbances having a sleep efficiency (SE) value less than $90 \%$ and PSQI scores ranging from 7 to 21 (PSQI global normal range: 0-21; PSQI abnormal score $>5$ ). We also found a significant negative correlation between SE and PSQI $(r=-0.54 ; \mathrm{p}=0.04)$ and $\mathrm{a}$ trend of a negative association between $\mathrm{SE}$ and age.

Conclusions: This study improves the knowledge on the impact of COVID-19 on the sleep among the medical staff, as our preliminary findings demonstrate that healthcare workers had a poor sleep quality during this disastrous period. A longitudinal investigation assessing whether and for how long sleep remains altered in medical staff, could be of interest to evaluate the temporal effect of the COVID-19 pandemic on the sleep and mental health.

\section{RESTLESS LEGS SYNDROME AND CEREBRAL SMALL VESSEL DISEASE LESIONS}

\author{
R. Sgobio ${ }^{1}$, M. Savarese ${ }^{1}$, D. Mezzapesa ${ }^{1}$, R. Ferri ${ }^{2}$, F. Federico ${ }^{1}$, M. \\ Trojano $^{1}$, I. Simone ${ }^{1}$
}

${ }^{1}$ Department of Basic Medical Sciences, Neurosciences and Sense Organs, University of Bari (Bari); ${ }^{2}$ Oasi Research Institute IRCCS (Troina-EN)

Introduction: In recent years, sleep disorders have emerged as vascular risk factors, particularly cerebrovascular. The objective of this study was to investigate the relationship between restless legs syndrome (RLS) and cerebrovascular disease, focusing on small vessel disease (SVD) lesions.

Methods: Forty-five patients with RLS (34 F) and 33 controls (24 F) aged 25-80 years were included in this retrospective cross-sectional study. White matter hyperintensities (WMH) were graded using Fazekas scale and measured in term of volume. The enlarged perivascular spaces (EPVS) were counted in both centrum semiovale and basal ganglia. The presence of RLS in combination with age and the effect of disease duration (longer or less than 10 years) on SVD lesions were analysed.

Results: RLS patients showed significantly higher WMH volumes $(p=0.044)$ and periventricular scores $(p=0.04)$ than controls. WMH volume correlated with age $(\mathrm{p}=0.005)$. Multivariate multiple regression revealed a combined effect of age and RLS on WMH volumes $(p=0.016)$. Patients with RLS longer than 10 years showed a higher number of EPVS into basal ganglia than both controls $(\mathrm{p}=0.018)$ and patients with shorter disease duration $(\mathrm{p}=0.018)$.

Conclusions: The present study showed an association between RLS and SVD lesions, especially in older patients and in patients with longer disease duration. Our conclusions suggest that early treatment of RLS might contribute to a reduction of SVD lesions. However, prospective and longitudinal studies on larger cohorts will be necessary to confirm these hypothesis.

References:

- Ferri R, Cosentino FI, Moussouttas M, Lanuzza B, Aricò D, Bagai K, Wang L, McLaughlin B, Walters AS. Silent cerebral small vessel disease in restless legs syndome. Sleep (2016); 39(7): 1371 - 77

- Wardlaw JM, Smith EE, Biessels GJ, Cordonnier C, et al. STandards for ReportIng Vascular changes on nEuroimaging (STRIVE v1). Neuroimaging standards for research into small vessel disease and its contribution to ageing and neurodegeneration. Lancet Neurology (2013) Aug;12(8):822-38

- Ferini - Strambi L, Walters AS, Sica D. The relationship among restless legs syndrome (Willis - Ekbom Disease), hypertension, cardiovascular disease, and carebrovascular disease. J Neurol. (2014);261(6):1051- 68 
INTER-PROCEDURE RELIABILITY OF TELEVISIT VS STANDARD IN-PERSON VISIT FOR DIAGNOSIS OF NARCOLEPSY. TELEMEDICINE FOR NARCOLEPSY (TENAR) TELE-TRIAGE STUDY

\author{
L. Vignatelli ${ }^{1}$, F. Pizza ${ }^{2}$, M. Moresco ${ }^{2}$, F. Ingravallo ${ }^{3}$, A. Mangiaruga ${ }^{3}$, \\ C. Oriolo ${ }^{3}$, C. Zenesini ${ }^{1}$, S. Vandi ${ }^{2}$, G. Plazzi ${ }^{1}$
}

${ }^{1}$ IRCCS Institute of Neurological Sciences of Bologna, AUSL of Bologna (Bologna); ${ }^{2}$ Department of Biomedical and Neuromotor Sciences (DIBINEM), University of Bologna (Bologna); ${ }^{3}$ Department of Medical and Surgical Sciences (DIMEC), University of Bologna (Bologna)

Objectives: Narcolepsy is a rare chronic disorder of hypersomnolence burdened by an extended diagnostic delay (mean 14 years) due to diagnostic complexity and scarcity of reference sleep centres. Patients and families are forced to travel for seeking medical consultations. Misdiagnoses and multiple referrals before receiving a proper diagnosis have negative consequences for untreated patients, their family and the healthcare system, increasing the economic and psychosocial disease burden. Telemedicine may facilitate patient access to sleep consultations accelerating the diagnostic process. However, the diagnostic performance of telemedicine procedures (e.g. televisit, TV) are unknown for patients with suspected narcolepsy. The TENAR-Tele-triage study aimed to evaluate reliability and diagnostic accuracy of a live interactive assessment through TV compared to an in-person standard visit and the full diagnostic process in patients with suspected narcolepsy. The TENAR project is endorsed by the Italian Narcolepsy and Hypersomnias association (AIN, www.narcolessia.org), and publicly funded by the Italian Ministry of Health (RF-2016-02364742). We present the preliminary data on the inter-procedure reliability for narcolepsy diagnosis.

Materials: Cross-sectional diagnostic study involving 250 adults and children with suspected narcolepsy.
Methods: Participants undergo a sleep assessment through in-person office visit and TV, by two independent physicians. Diagnostic judgment after examination is classified according to three levels: "probable", "possible" or "excluded" narcolepsy. Inter-procedure reliability is calculated with Kappa statistics.

Results: 54 patients included (mean age 34 yrs, range 11-73, 57\% females). Ten MDs were involved in the visits. According to TV 19 patients (35\%) had probable, $29(54 \%)$ possible, and $6(11 \%)$ excluded narcolepsy; according to in-person visit 17 (31\%) probable, $34(63 \%)$ possible, and $3(6 \%)$ excluded narcolepsy. Raw diagnostic agreement between the two procedures was $79.6 \%$, with a "substantial" agreement (Kappa 0.63).

Discussion: We found a substantial agreement between TV and inperson office visit when patients with suspected narcolepsy are assessed. On the basis of previous studies, this level of agreement is that expected when two observers classify the suspicion of narcolepsy in the same patient with the same procedure [1], suggesting that TV assessment is comparable to in-person assessment.

Conclusions: If data will be confirmed, the TV could be proposed as tele-triage procedure to prioritise patients with suspected narcolepsy to access qualified Sleep centres, reducing the diagnostic gap and its detrimental consequences. The study may be a model for the remote management of other rare disorders, offering care access for patients independently from their proximity to specialized centers.

Reference:

1. Vignatelli L, Plazzi G, Bassein L, et al. ICSD diagnostic criteria for narcolepsy: interobserver reliability. Intemational Classification of Sleep Disorders. Sleep (2002);25:193-96

Publisher's note Springer Nature remains neutral with regard to jurisdictional claims in published maps and institutional affiliations. 